Miscellaneous Data 


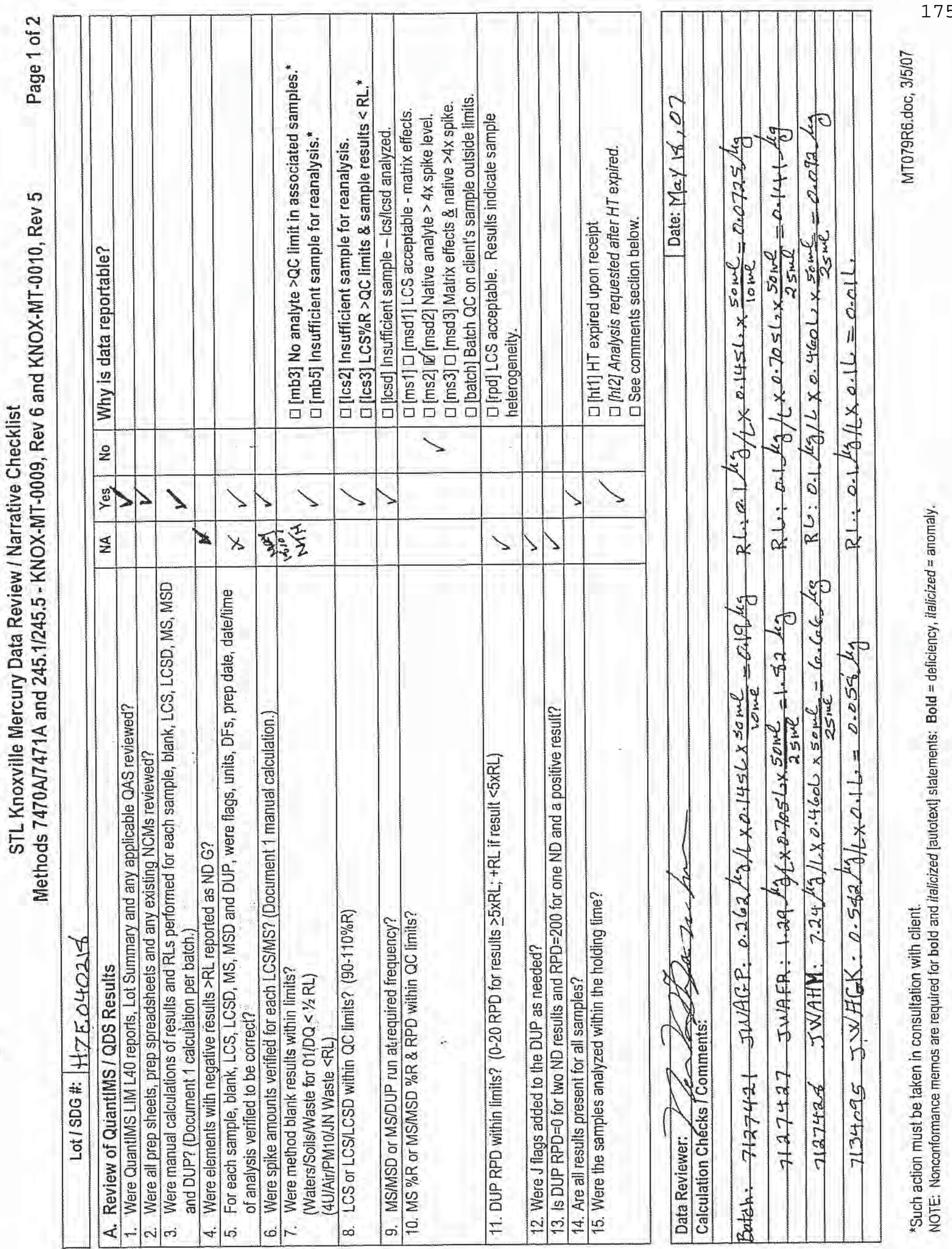




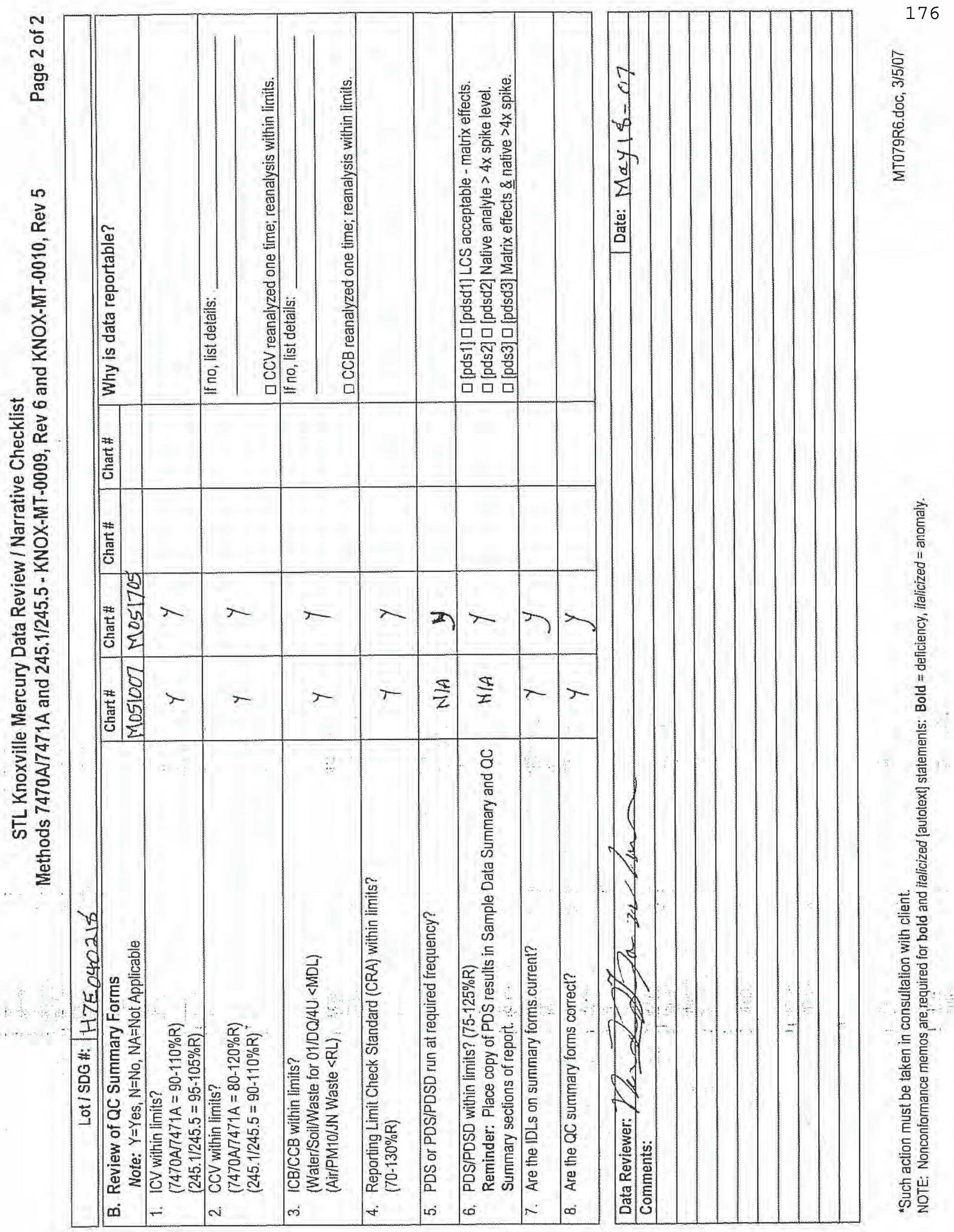




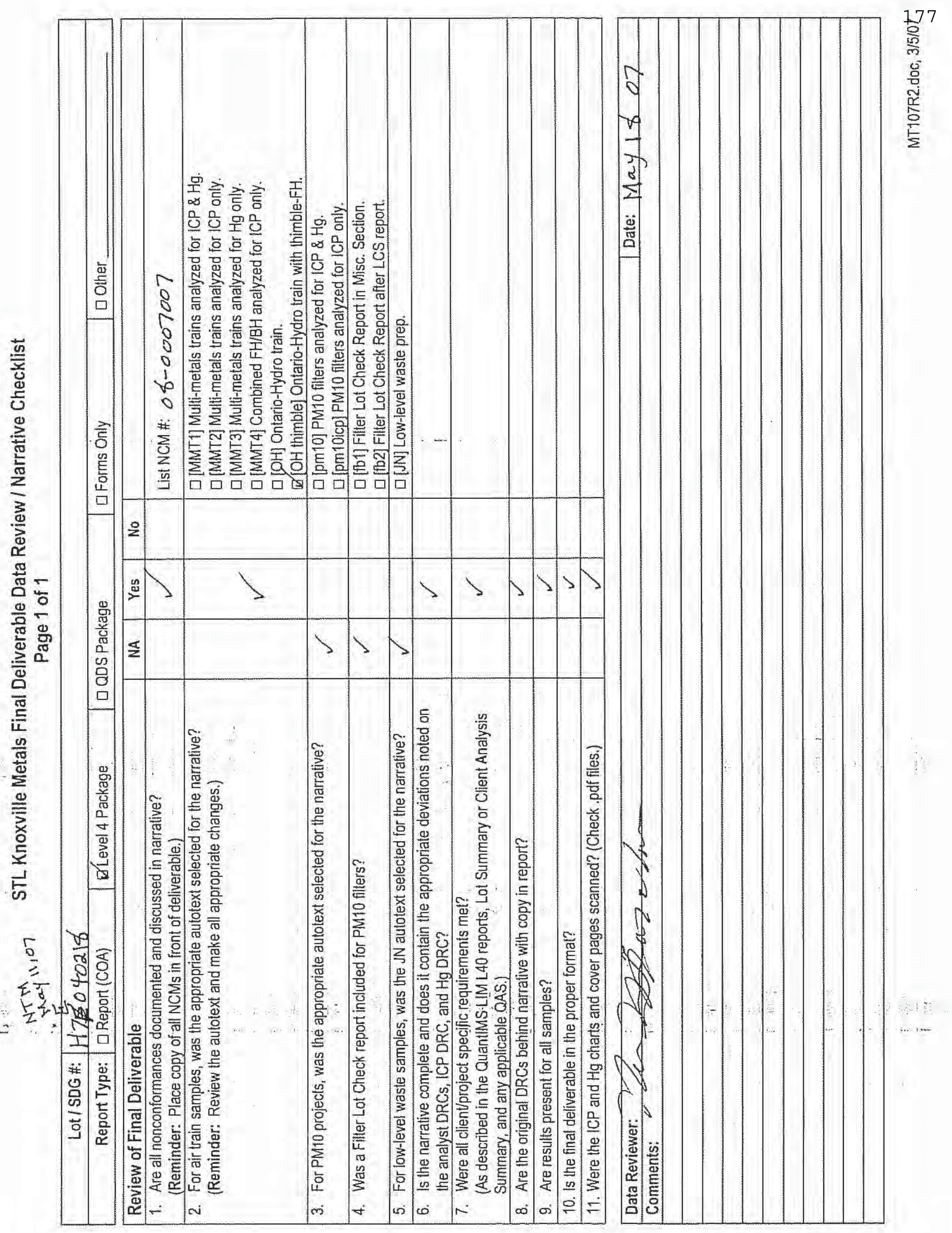




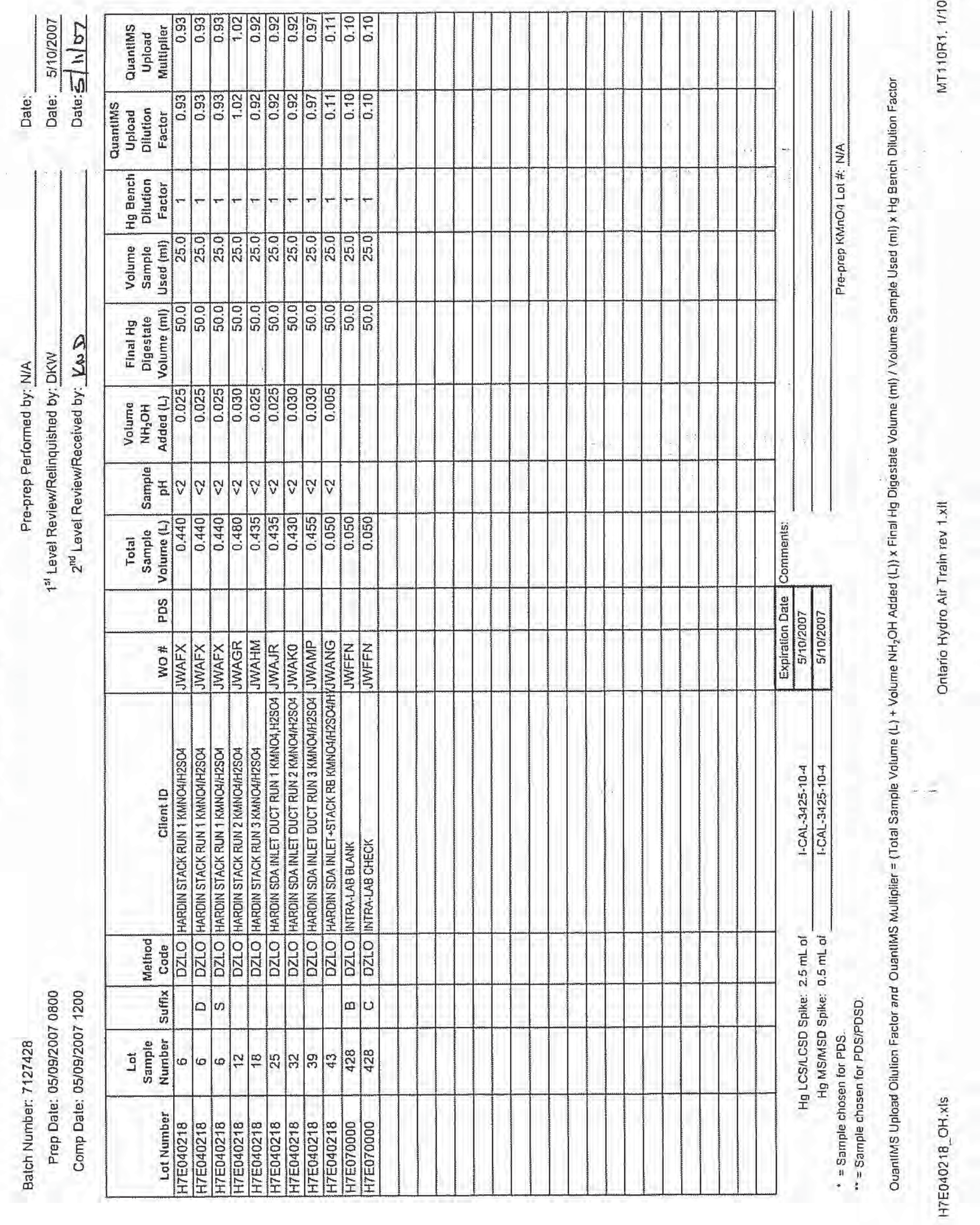


STL Knoxville

Analyst: DXe) Date: 050907 Batch:?127428

Bath A Temp:

Bath B Temp: $20 \mathrm{C}$

(Acceptance range $90^{\circ}-95^{\circ} \mathrm{C}$ )

口 Hg in PM10 filters: KNOX-IP-0003, Rev. 4

Ontario Hydro: KNOX-IP-0006, Rev. 3

口 MMT train: KNOX-MT-0006, Rev, 10

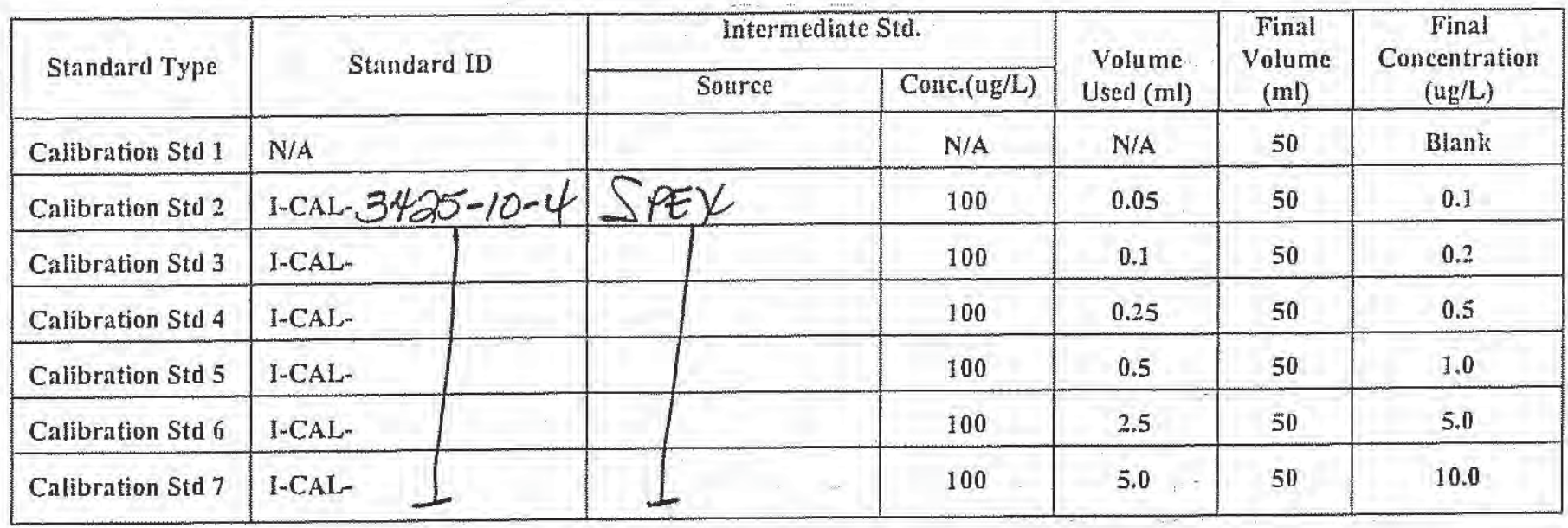

\begin{tabular}{|c|c|c|c|c|c|c|}
\hline \multirow{2}{*}{$\begin{array}{c}\text { Standard } \\
\text { Type }\end{array}$} & \multirow{2}{*}{ Standard ID } & \multicolumn{2}{|c|}{ Intermediate Std. } & \multirow{2}{*}{$\begin{array}{c}\text { Amount } \\
\text { Used (ml) }\end{array}$} & \multirow{2}{*}{$\begin{array}{c}\text { Final } \\
\text { Volume } \\
\text { (ml) }\end{array}$} & \multirow{2}{*}{$\begin{array}{c}\text { Final Conc } \\
(\mathrm{ug} / \mathrm{L})\end{array}$} \\
\hline & & Source & Conc.(ug/L) & & & \\
\hline ICV & IVVER- $3426-11-3$ & ULTRA & 100 & 1.25 & 50 & 2.5 \\
\hline $\mathrm{CCV}$ & $1-\mathrm{CAL}-3425-10-4$ & SPEX & 100 & 2.5 & 50 & 5.0 \\
\hline CRA & I-CAL- & & 100 & 0.1 & 50 & 0.2 \\
\hline
\end{tabular}

\begin{tabular}{|c|c|c|c|c|c|c|c|}
\hline \multirow{2}{*}{ Standard Type } & \multirow{2}{*}{ Standard ID } & \multicolumn{2}{|c|}{ Intermediate Std. } & \multirow{2}{*}{$\begin{array}{c}\text { Amount Used } \\
\text { @ ICP } \\
\text { prep/Iinal } \\
\text { yolume }\end{array}$} & \multirow{2}{*}{$\begin{array}{c}\text { ICP Sample } \\
\text { Volume } \\
\text { used @ Hg } \\
\text { prep (ml) }\end{array}$} & \multirow{2}{*}{$\begin{array}{c}\text { Final } \\
\text { Conc. @ } \\
\text { Hg prep } \\
\text { (ug/L) }\end{array}$} & \multirow{2}{*}{$\begin{array}{l}\text { Final } \\
\text { Amount } \\
\text { (ug) }\end{array}$} \\
\hline & & Source & Conc.(ug/L) & & & & \\
\hline Air (PM10) LCS & I-CAL- & & 10,000 & $0.250 \mathrm{ml} / 50 \mathrm{ml}$ & 5.0 & 5.0 & 11.25 \\
\hline Air (PM10) MS & I-CAL- & & 10,000 & $0.050 \mathrm{ml} / 50 \mathrm{ml}$ & 5.0 & 1.0 & 2.25 \\
\hline
\end{tabular}

\begin{tabular}{|c|c|c|c|}
\hline \multicolumn{4}{|c|}{ REAGENTS } \\
\hline Conc. $\mathrm{H}_{2} \mathrm{SO}_{4}$ Lot & 015045 & $5 \% \mathrm{KMnO}_{4}$ ID \#: & $B-3122-13-D$ \\
\hline Conc. $\mathrm{HNO}_{3}$ Lot \#: & 344032 & $5 \% \mathrm{~K}_{2} \mathrm{~S}_{2} \mathrm{O}_{8}$ ID H: & $12-2638-18-E$ \\
\hline Conc. HCL Lot H: & C42A22 & $\mathrm{SnCl}_{2}$ Solution ID \#: & $A-3382^{2} 26-D$ \\
\hline $12 \% \mathrm{NaCl} \cdot \mathrm{NH}_{2} \mathrm{OH} \cdot \mathrm{HCL} \mathrm{ID} \#:$ & $c-3267-4-c$ & & \\
\hline
\end{tabular}




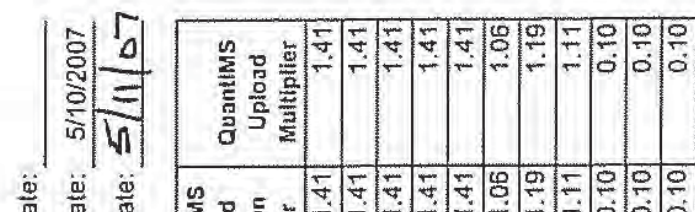

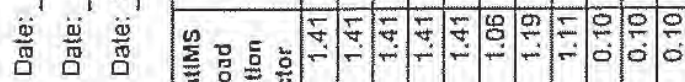

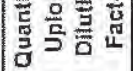

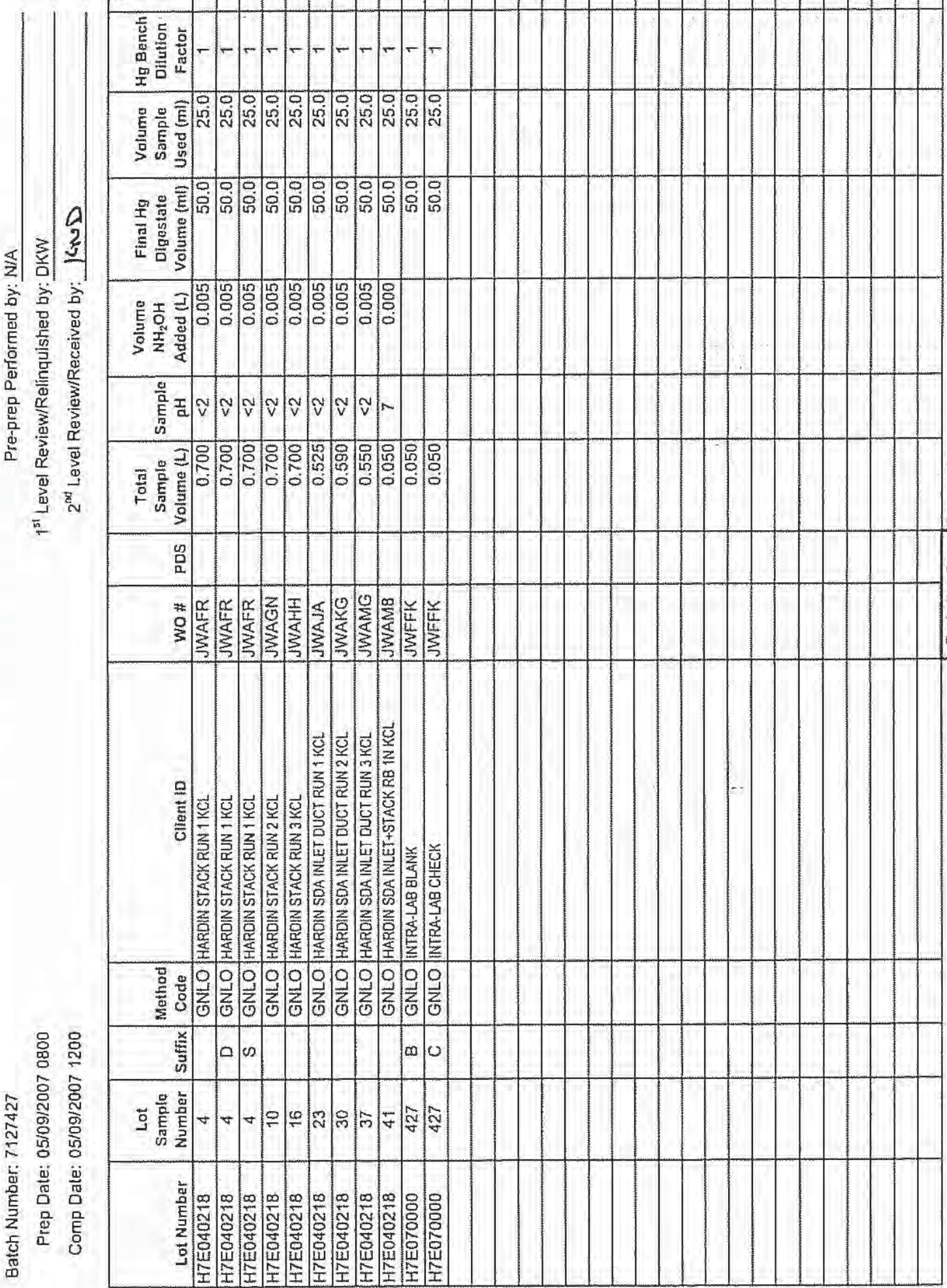


STL Knoxville

Analyst: TXe) Date: $050907 \quad$ Batch: 7127420

口 Hg in PM10 filters: KNOX-IP-0003, Rev. 4

$\downarrow$ Ontario Hydro: KNOX-IP-0006, Rev. 3

口 MMT train: KNOX-MT-0006, Rev. 10

Bath A Temp: $93^{\circ} \mathrm{C}$

Bath B Temp: 800

(Acceptance range $90^{\circ}-95^{\circ} \mathrm{C}$ )

\begin{tabular}{|c|c|c|c|c|c|c|}
\hline \multirow{2}{*}{ Standard Type } & \multirow{2}{*}{ Standard ID } & \multicolumn{2}{|c|}{ Intermediate Std. } & \multirow{2}{*}{$\begin{array}{c}\text { Volume } \\
\text { Used (ml) }\end{array}$} & \multirow{2}{*}{$\begin{array}{c}\text { Final } \\
\text { Volume } \\
\text { (ml) }\end{array}$} & \multirow{2}{*}{$\begin{array}{c}\text { Final } \\
\text { Concentration } \\
\text { (ug/L) }\end{array}$} \\
\hline & & Source & Conc.(ug/L) & & & \\
\hline Calibration Std 1 & N/A & & $\mathrm{N} / \mathrm{A}$ & N/A & 50 & Blank \\
\hline Calibration Std 2 & I-CAL-3425-10-4 & $S P E X$ & 100 & 0.05 & 50 & 0.1 \\
\hline Calibration Std 3 & I-CAL- & & 100 & 0.1 & 50 & 0.2 \\
\hline Calibration Std 4 & I-CAL- & & 100 & 0.25 & 50 & 0.5 \\
\hline Calibration Std 5 & I-CAL- & & 100 & 0.5 & s0 & 1.0 \\
\hline Calibration Std 6 & I-CAL- & & 100 & 2.5 & 50 & 5.0 \\
\hline Calibration Std 7 & 1-CAL- & $\perp$ & 100 & 5.0 & 50 & 10.0 \\
\hline
\end{tabular}

\begin{tabular}{|c|c|c|c|c|c|c|}
\hline \multirow{2}{*}{$\begin{array}{c}\text { Standard } \\
\text { Type }\end{array}$} & \multirow{2}{*}{ Standard ID } & \multicolumn{2}{|c|}{ Intermediate Std. } & \multirow{2}{*}{$\begin{array}{l}\text { Amount } \\
\text { Used (ml) }\end{array}$} & \multirow{2}{*}{$\begin{array}{c}\text { Final } \\
\text { Volume } \\
\text { (ml) }\end{array}$} & \multirow{2}{*}{$\begin{array}{c}\text { Final Conc. } \\
(\mathrm{ug} / \mathrm{L})\end{array}$} \\
\hline & & Source & Conc.(ug/L) & & & \\
\hline ICV & INVER-3426-11-3 & ULTRA & 100 & 1.25 & 50 & 2.5 \\
\hline $\mathrm{CCV}$ & I-CAL-3425-10-4 & SPEX & 100 & 2.5 & 50 & 5.0 \\
\hline CRA & I-CAL- & & 100 & 0.1 & 50 & 0.2 \\
\hline
\end{tabular}

\begin{tabular}{|c|c|c|c|c|c|c|c|}
\hline \multirow{2}{*}{ Standard Type } & \multirow{2}{*}{ Standard ID } & \multicolumn{2}{|c|}{ Intermediate Std. } & \multirow{2}{*}{$\begin{array}{l}\text { Amount Used } \\
\text { @ ICP } \\
\text { prep/inal } \\
\text { yolume } \\
\end{array}$} & \multirow{2}{*}{$\begin{array}{c}\text { ICP Sample } \\
\text { Volume } \\
\text { used @ Hg } \\
\text { prep (ml) }\end{array}$} & \multirow{2}{*}{$\begin{array}{c}\text { Final } \\
\text { Conc. (a) } \\
\text { Hg prep } \\
\text { (ug/L) } \\
\end{array}$} & \multirow{2}{*}{$\begin{array}{c}\text { Final } \\
\text { Amount } \\
\text { (ug) }\end{array}$} \\
\hline & & Source & Conc, $(\mathrm{ug} / \mathrm{L})$ & & & & \\
\hline Air (PM10) LCS & I-CAL- & & 10,000 & $0.250 \mathrm{ml} / 50 \mathrm{ml}$ & 5.0 & 5.0 & 11.25 \\
\hline Air (PM10) MS & I-CAL- & & 10,000 & $0.050 \mathrm{~m} / 50 \mathrm{ml}$ & 5.0 & 1.0 & 2.25 \\
\hline
\end{tabular}

\begin{tabular}{|c|c|c|c|}
\hline \multicolumn{4}{|c|}{ REAGENTS } \\
\hline Conc. $\mathrm{H}_{2} \mathrm{SO}_{4}$ Lot \#: & 015045 & $5 \% \mathrm{KMnO}_{4}$ ID H: & $B-3122-13-D$ \\
\hline Conc. $\mathrm{HNO}_{3}$ Lot \#: & $B 44032$ & $5 \% \mathrm{~K}_{2} \mathrm{~S}_{2} \mathrm{O}_{8} \mathrm{lD} \mathrm{H:}$ & $D-2638-18-E$ \\
\hline Conc. HCL Lot H: & $142 A 22$ & $\mathrm{SnCl}_{2}$ Solution ID \#: & $A-338226 D$ \\
\hline $12 \% \mathrm{NaCl} \cdot \mathrm{NH}_{2} \mathrm{OH} \cdot \mathrm{HCL}$ ID & $c-3267-4-c$ & & \\
\hline
\end{tabular}




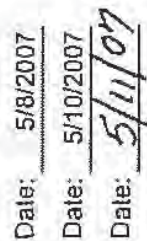

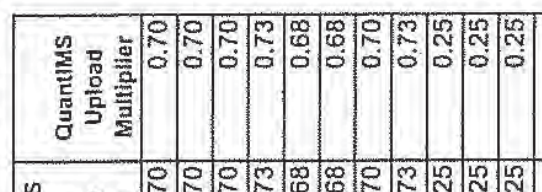

隹

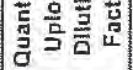

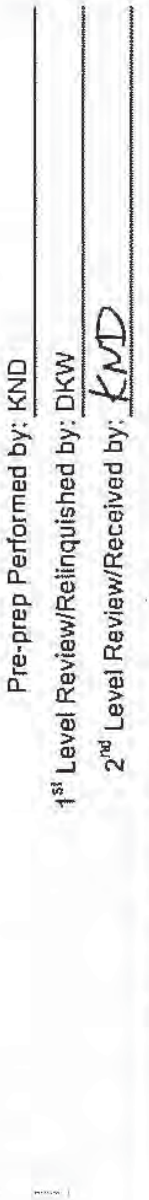

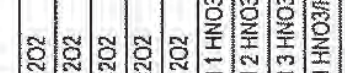

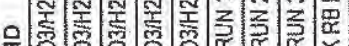

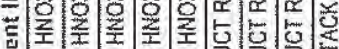

ثँ

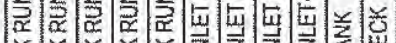

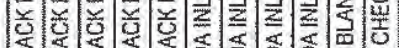

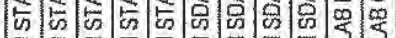

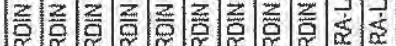

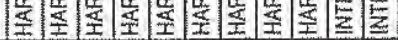

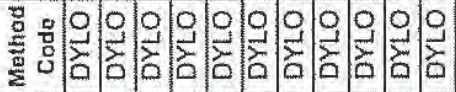

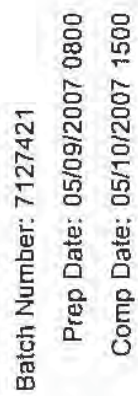

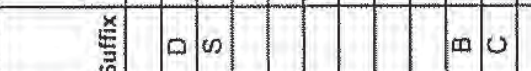

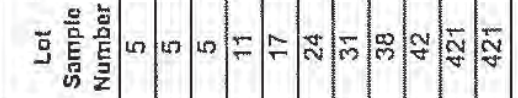

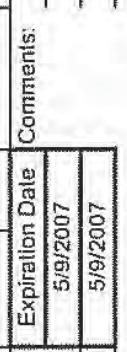

if

害言 点

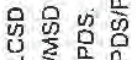

预 空

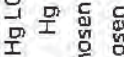


Analyst: $12 \mathrm{Ke}$

STL Knoxville

Mercury Air Prep Spiking Form

Date: 050907 Batch:
Bath A Temp:

Bath B Temp: $\$=C$

(Acceptance range $90^{\circ}-95^{\circ} \mathrm{C}$ )

$\square$ Hg in PM10 filters: KNOX-IP-0003, Rev. 4

Ontario Hydro: KNOX-IP-0006, Rev. 3

口 MMT train: KNOX-MT-0006, Rev. 10

\begin{tabular}{|c|c|c|c|c|c|c|}
\hline \multirow{2}{*}{ Standard Type } & \multirow{2}{*}{ Standard ID } & \multicolumn{2}{|c|}{ Intermediate Std. } & \multirow{2}{*}{$\begin{array}{l}\text { Volume } \\
\text { Used }(\mathrm{ml})\end{array}$} & \multirow{2}{*}{$\begin{array}{l}\text { Final } \\
\text { Volume } \\
(\mathrm{ml})\end{array}$} & \multirow{2}{*}{$\begin{array}{c}\text { Final } \\
\text { Concentration } \\
(\text { ug/L) }\end{array}$} \\
\hline & & Source & Conc.(ug/L) & & & \\
\hline Calibration Std 1 & N/A & & $\mathrm{N} / \mathrm{A}$ & N/A & 50 & Blank \\
\hline Calibration Std 2 & $1-\mathrm{CAL}-3425-10-4$ & SPEY & 100 & 0.05 & 50 & 0.1 \\
\hline Calibration Std 3 & 1-CAL- & & 100 & 0.1 & 50 & 0.2 \\
\hline Calibration Std 4 & I-CAL- & & 100 & 0.25 & 50 & 0.5 \\
\hline Calibration Std 5 & I-CAL- & & 100 & 0.5 & 50 & 1.0 \\
\hline Calibration Std 6 & I-CAL- & & 100 & 2.5 & 50 & 5.0 \\
\hline Calibration Std 7 & I-CAL- & $\perp$ & 100 & 5.0 & 50 & 10.0 \\
\hline
\end{tabular}

\begin{tabular}{|c|c|c|c|c|c|c|}
\hline \multirow{2}{*}{$\begin{array}{c}\text { Standard } \\
\text { Type } \\
\end{array}$} & \multirow{2}{*}{ Standard ID } & \multicolumn{2}{|c|}{ Intermediate Std. } & \multirow{2}{*}{$\begin{array}{l}\text { Amount } \\
\text { Used (ml) }\end{array}$} & \multirow{2}{*}{$\begin{array}{c}\text { Final } \\
\text { Volume } \\
\text { (ml) }\end{array}$} & \multirow{2}{*}{$\begin{array}{l}\text { Final Conc } \\
\quad(\mathrm{ug} / \mathrm{L})\end{array}$} \\
\hline & & Source & Conc.(ug/L) & & & \\
\hline $\mathrm{ICV}$ & I-VER- $3426-11-3$ & ULTRA & 100 & 1.25 & 50 & 2.5 \\
\hline $\mathrm{CCV}$ & I-CAL-3425-10-4 & SPEX & 100 & 2.5 & 50 & 5.0 \\
\hline CRA & I-CAL- $\quad L$ & & 100 & 0.1 & 50 & 0.2 \\
\hline
\end{tabular}

\begin{tabular}{|c|c|c|c|c|c|c|c|}
\hline \multirow{2}{*}{ Standard Type } & \multirow{2}{*}{ Standiard ID } & \multicolumn{2}{|c|}{ Intermediate Std. } & \multirow{2}{*}{$\begin{array}{c}\text { Amount Used } \\
\text { @ ICP } \\
\text { prep/rinal } \\
\text { yolume } \\
\end{array}$} & \multirow{2}{*}{$\begin{array}{l}\text { ICP Sample } \\
\text { Volume } \\
\text { used @ } \mathrm{Hg} \\
\text { prep (m) }\end{array}$} & \multirow{2}{*}{$\begin{array}{c}\text { Final } \\
\text { Conc. @ } \\
\text { Hg prep } \\
\text { (ug/L) } \\
\end{array}$} & \multirow{2}{*}{$\begin{array}{c}\text { Final } \\
\text { A mount } \\
\text { (ug) }\end{array}$} \\
\hline & & Source & Conc.(ug/L) & & & & \\
\hline Air (PM10) LCS & I-CAL- & & 10,000 & $0.250 \mathrm{ml} / 50 \mathrm{ml}$ & 5.0 & 5.0 & 11.25 \\
\hline Air (PM10) MS & 1-CAL- & & 10,000 & $0.050 \mathrm{ml} / 50 \mathrm{nl}$ & 5.0 & 1.0 & 2.25 \\
\hline
\end{tabular}

\begin{tabular}{|c|c|c|c|}
\hline \multicolumn{4}{|c|}{ REAGENTS } \\
\hline Conc. $\mathrm{H}_{2} \mathrm{SO}_{4}$ Lot H: & 015045 & $5 \% \mathrm{KMnO}_{4}$ ID & $B-3122-13-D$ \\
\hline Conc. $\mathrm{HNO}_{3}$ Lot \#: & 344032 & $5 \% \mathrm{~K}_{2} \mathrm{~S}_{2} \mathrm{O}_{8} \mathrm{ID}$ & $D-2038-18-E$ \\
\hline Conc, HCL Lot \#: & C42A23 & $\mathrm{SnCl}_{2}$ Solution ID H: & $A-3382-26-D$ \\
\hline $12 \% \mathrm{NaCl} \cdot \mathrm{NH}_{2} \mathrm{OH} \cdot \mathrm{HCL} \mathrm{ID} \#$ & $c-3267-4$ & & \\
\hline
\end{tabular}




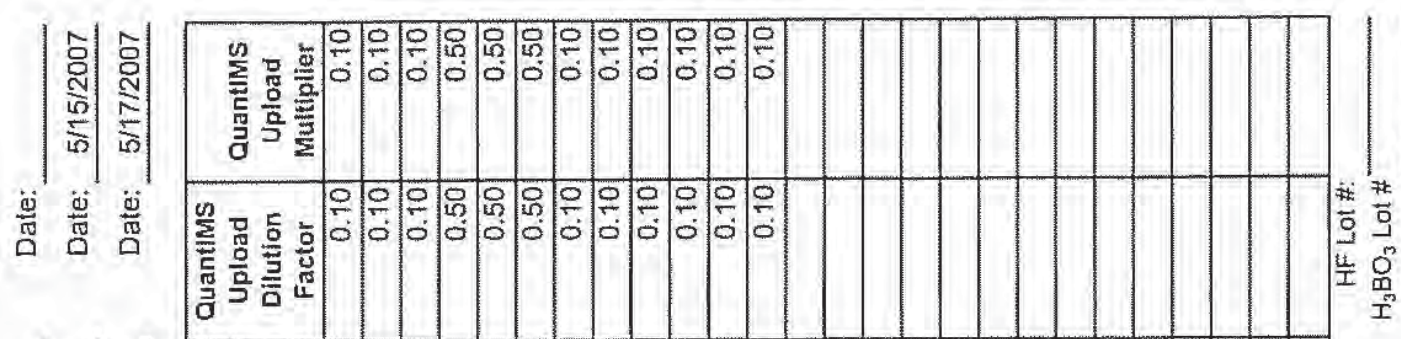

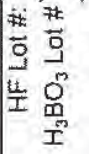

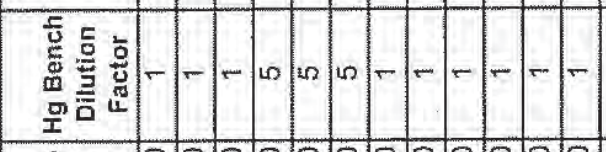

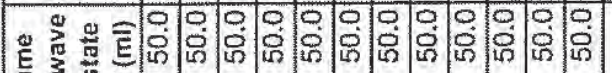

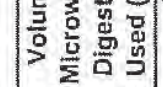

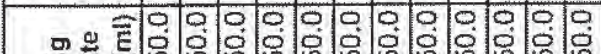

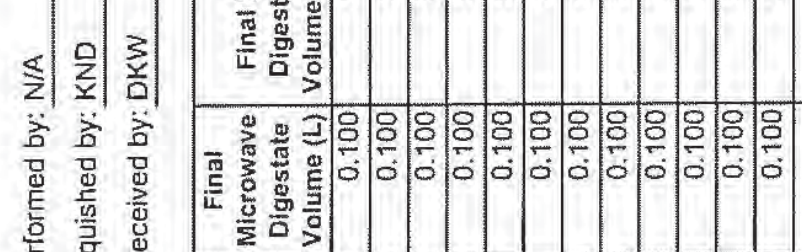

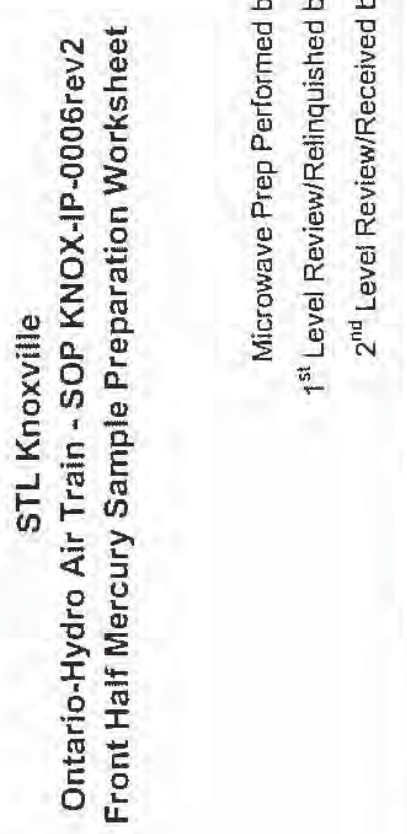

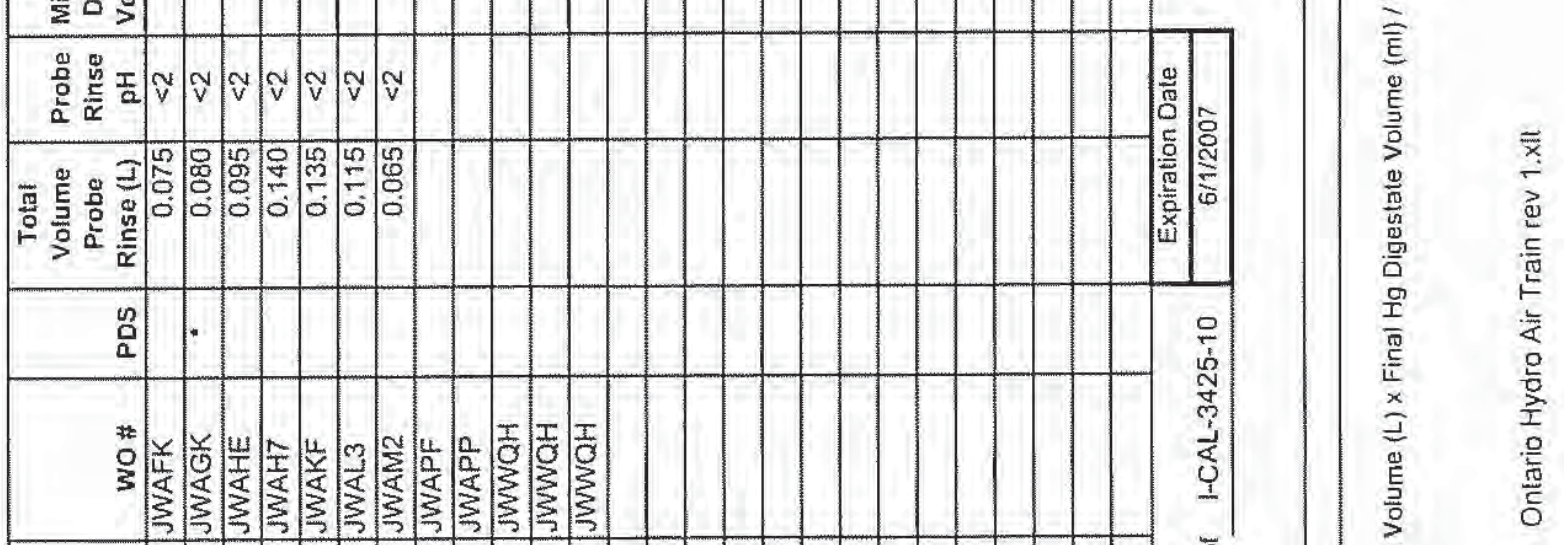

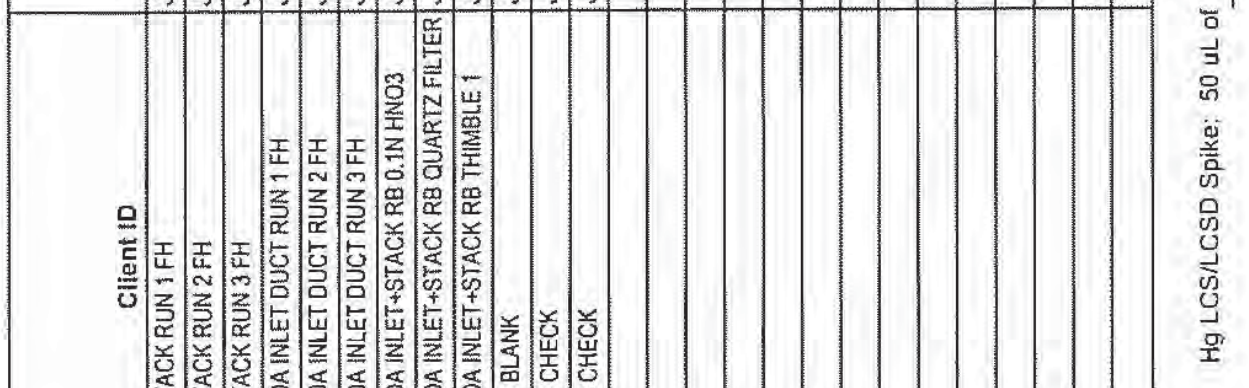

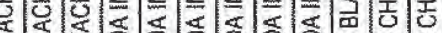

许

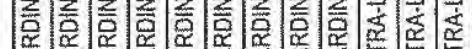

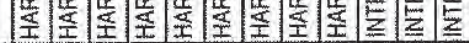

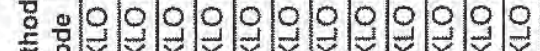

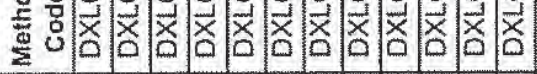

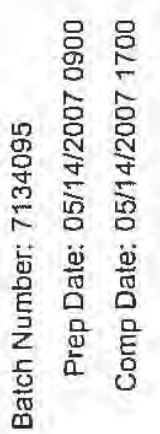

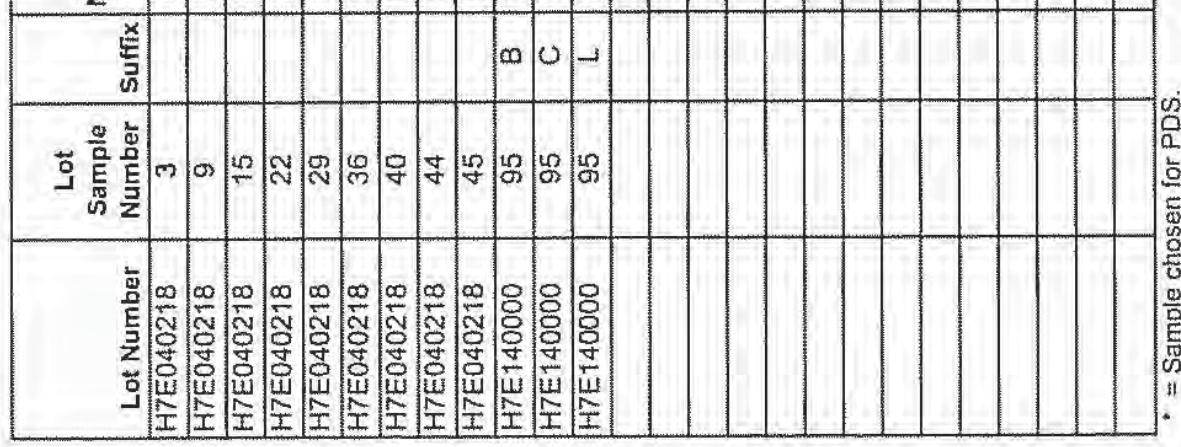

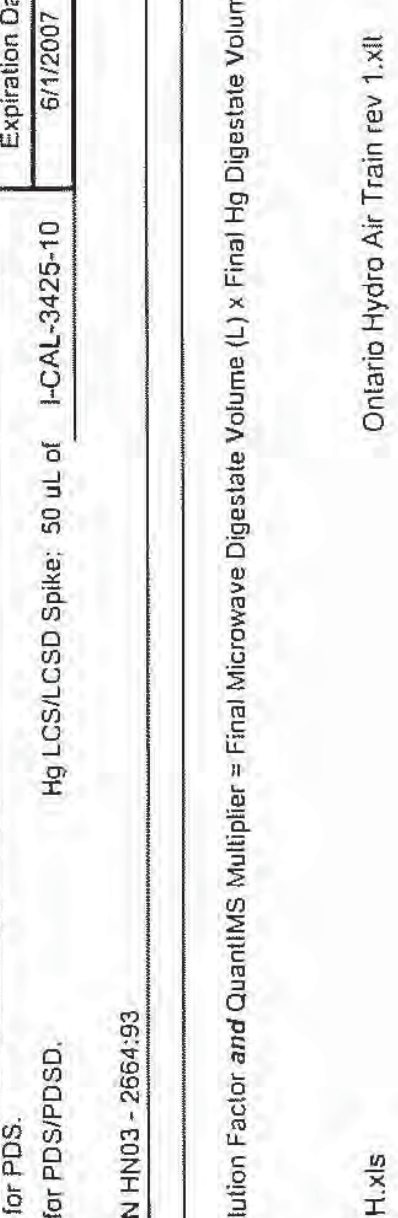

点 
STL Knoxville

Analyst: THW Mercury Air Prep Spiking Form

Date: 051607 Batch: 7/34095

Bath A Temp: $93^{\circ} \mathrm{C}$

Bath B Temp:

(Acceptance range $90^{\circ}-95^{\circ} \mathrm{C}$ )

$\square$ Hg in PM10 filters: KNOX-IP-0003, Rev. 4

Ontario Hydro: KNOX-IP-0006, Rev. 3

口 MMT train: KNOX-MT-0006, Rev, 10

\begin{tabular}{|c|c|c|c|c|c|c|}
\hline \multirow{2}{*}{ Standard Type } & \multirow{2}{*}{ Standard ID } & \multicolumn{2}{|c|}{ Intermediate Std. } & \multirow{2}{*}{$\begin{array}{l}\text { Volume } \\
\text { Used (mI) }\end{array}$} & \multirow{2}{*}{$\begin{array}{c}\text { Final } \\
\text { Volume } \\
\text { (ml) }\end{array}$} & \multirow{2}{*}{$\begin{array}{c}\text { Final } \\
\text { Concentration } \\
(u g / L)\end{array}$} \\
\hline & & Source & Conc.(ug/L) & & & \\
\hline Calibration Std 1 & N/A & & N/A & N/A & 50 & Blank \\
\hline Calibration Std 2 & I-CAL-3425-10-7 & PEA & 100 & 0.05 & 50 & 0.1 \\
\hline Calibration Std 3 & I-CAL- & 1 & 100 & 0.1 & 50 & 0.2 \\
\hline Calibration Std 5 & I-CAL- & & 100 & 0.5 & 50 & 1.0 \\
\hline Calibration Std 6 & I-CAL- & & 100 & 2.5 & 50 & 5.0 \\
\hline Calibration Std 7 & I-CAL- & $L$ & 100 & 5.0 & 50 & 10.0 \\
\hline
\end{tabular}

\begin{tabular}{|c|c|c|c|c|c|c|}
\hline \multirow{2}{*}{$\begin{array}{l}\text { Standard } \\
\text { Type }\end{array}$} & \multirow{2}{*}{ Standard ID } & \multicolumn{2}{|c|}{ Intermediate Std. } & \multirow{2}{*}{$\begin{array}{l}\text { Amount } \\
\text { Used (ml) }\end{array}$} & \multirow{2}{*}{$\begin{array}{c}\text { Final } \\
\text { Volume } \\
\text { (ml) }\end{array}$} & \multirow{2}{*}{$\begin{array}{l}\text { Final Conc } \\
\text { (ug/L) }\end{array}$} \\
\hline & & Source & Conc.(ug/L) & & & \\
\hline ICV & I-VER- $3426-11-6$ & KLTEA & 100 & 1.25 & 50 & 2.5 \\
\hline $\mathrm{CCV}$ & I-CAL- $3425-10-7$ & SPEK & 100 & 2.5 & 50 & 5.0 \\
\hline CRA & I-CAL- & $\perp$ & 100 & 0.1 & 50 & 0.2 \\
\hline
\end{tabular}

\begin{tabular}{|l|l|c|c|c|c|c|c|}
\hline \multirow{2}{*}{ Standard Type } & Standard ID & \multicolumn{2}{|c|}{ Intermediate Std. } & $\begin{array}{c}\text { Amount Used } \\
\text { @ ICP } \\
\text { prep/final } \\
\text { volume }\end{array}$ & $\begin{array}{c}\text { ICP Sample } \\
\text { Volume } \\
\text { used @ Hg } \\
\text { prep (ml) }\end{array}$ & $\begin{array}{c}\text { Final } \\
\text { Conc. @ } \\
\text { Hg prep } \\
\text { (ug/L) }\end{array}$ & $\begin{array}{c}\text { Final } \\
\text { Amount } \\
\text { (ug) }\end{array}$ \\
\cline { 2 - 8 } & Source & Conc.(ug/L) & & \\
\hline Air (PM10) LCS & I-CAL- & & 10,000 & $0.250 \mathrm{ml} / 50 \mathrm{ml}$ & 5.0 & 5.0 & 11.25 \\
\hline Air (PM110) MS & I-CAL- & & 10,000 & $0.050 \mathrm{ml} / 50 \mathrm{ml}$ & 5.0 & 1.0 & 2.25 \\
\hline
\end{tabular}

\begin{tabular}{|c|c|c|c|}
\hline \multicolumn{4}{|c|}{ REAGENTS } \\
\hline Conc. $\mathrm{H}_{2} \mathrm{SO}_{4}$ Lot $\#$ : & $C / 5045$ & $5 \% \mathrm{KMnO}_{4}$ ID & $B-3122-13-E$ \\
\hline Conc. $\mathrm{HNO}_{3}$ Lot \#: & B44032 & $5 \% \mathrm{~K}_{2} \mathrm{~S}_{2} \mathrm{O}_{8} \mathrm{ID} \#$ & $D-9638-P-A$ \\
\hline Conc. HCL Lot \#: & 142422 & $\mathrm{SnCl}_{2}$ Solution ID \#: & $A-3282-27-B$ \\
\hline 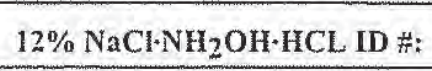 & $C-3267-4-D$ & & \\
\hline
\end{tabular}


Wet Chemistry 
Sample Summary 
Air Sampling Associates, Inc.

Client Sample ID: HARDIN STACK RUN 1 FILTKR A176

\section{General Chemistry}

Lot-Sample \#...: H7E040218-001

Date sampled...: $04 / 26 / 07$
Work order \#...: JWAEW

Date Received..: 05/01/07
Matrix........ A AIR

PREPARATION- PREP ANALYSIS DATE BATCH \#
PARAMETER

Particulates (total) 386
RESULT

$\frac{\mathrm{RL}}{0.50} \frac{\text { UNIT }}{\mathrm{mg}}$

METHOD

CFR60A 5 7130097 
Air Sampling Associates, Inc.

client Sample ID: HARDIN STACK RUN 1 ACBTONE

General Chemistry

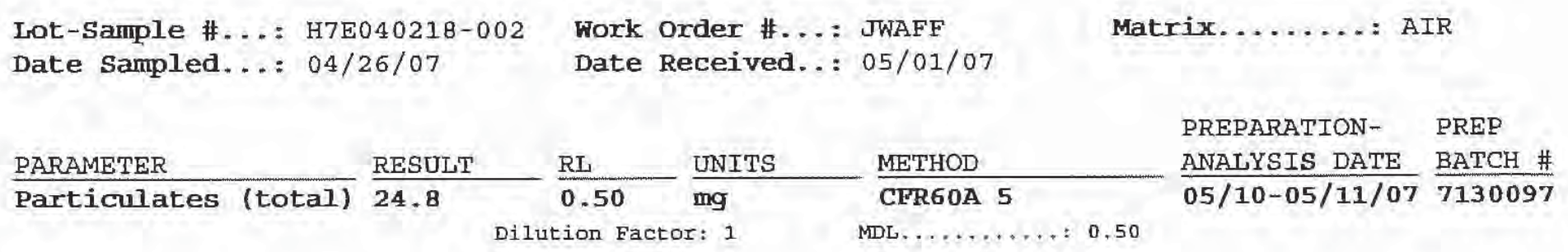


Air Sampling Associates, Inc.

client Sample ID: HARDIN STACK RUN 2 FILTRR Al77

\section{General Chemistry}

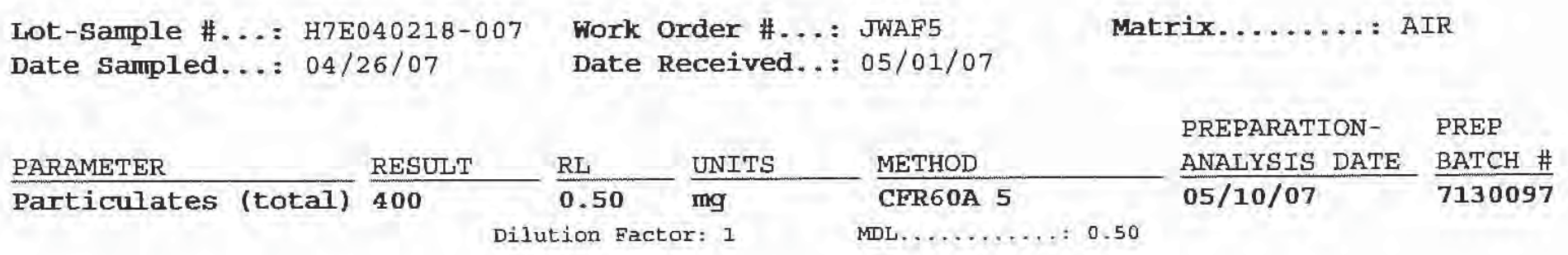


Air Sampling Associates, Inc.

client Sample ID: HARDIN STACK RUN 2 ACETONB

General Chemistry

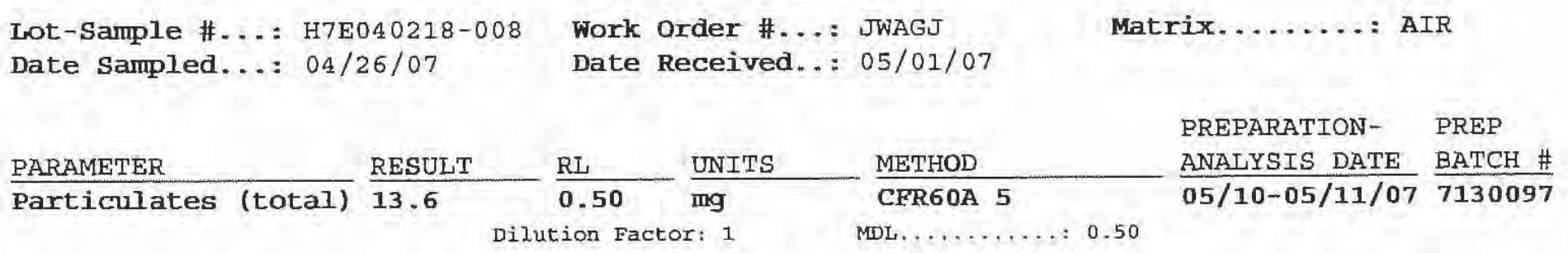


Air Sampling Associates, Inc.

Client Sample II: HARDIN STACK RUN 3 FILTER A778

General Chemistry

Lot-Sample \#. ..: H7E040218-013

Date Sampled... 04/26/07
Work Order \#...: JWAG8

Date Received..: 05/01/07
Matrix........ AIR

PREPARATION - PREP

ANALYSIS DATE BATCH \#
PARAMETER

RESULT

Particulates (total) 401
$\mathrm{RL}$

0.50

UNITS

mg

Dilution Factor: 1
METHOD

CFR60A 5

MDL ......... 0.50 
Air Sarmpling Associates, Inc.

Client Sample ID: HARDIN STACK RUN 3 ACIFIONB

General. Chemistry

Lot-Sample \#...: H7E040218-014 Work Order \#... : JWAHC

Date Sampled.... 04/26/07
Work Order \#...: JWAHC
Date Received... 05/01/07
Matrix........ AIR

PREPARATION- PREP ANALYSIS DATE BATCH \# 05/10-05/11/07 7130097
PARAMETER

Particulates (total)
RESULT

54.3
RL

0.50

ing
METHOD

CFR60A 5

MDL.......... 0.50 
Air Sampling Associates, Inc.

client Sample II: HARDIN SDA INLFT DUCT RUN 1 FIETKR (THMMRLE 50)

General Chemistry

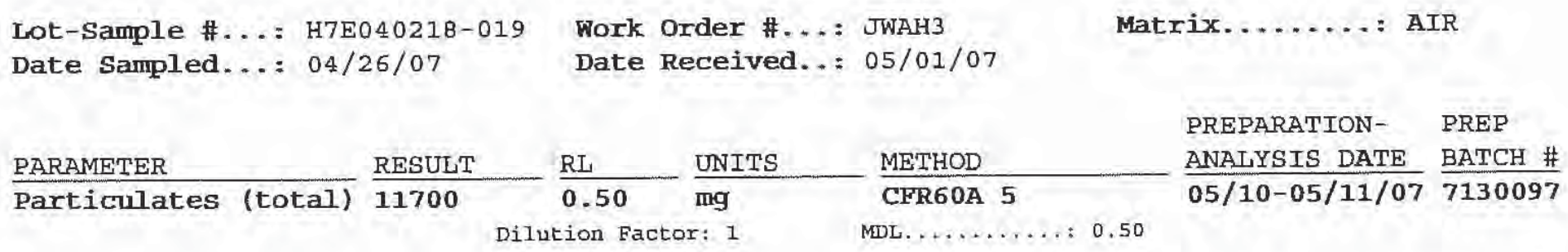


Air Sampling Associates, Inc.

Client sample ID: HARDIN SDA INLET DUCT RUN 1 FILTER (A173)

General Chemistry

Iot-Sample \#...: H7E040218-020

Date sampled...: 04/26/07
Hork Order \#.... JWAH5

Date Received..: 05/01/07
Matrix....... AIR

PREPARATION- PREP ANALYSIS DATE BATCH \# $\overline{05 / 10 / 07} \mathbf{7 1 3 0 0 9 7}$ 
Air Sampling Associates. Inc.

Client Sample ID: HARDIN SDA INLET DUCT RUN 1 ACETONB

General Chemistry

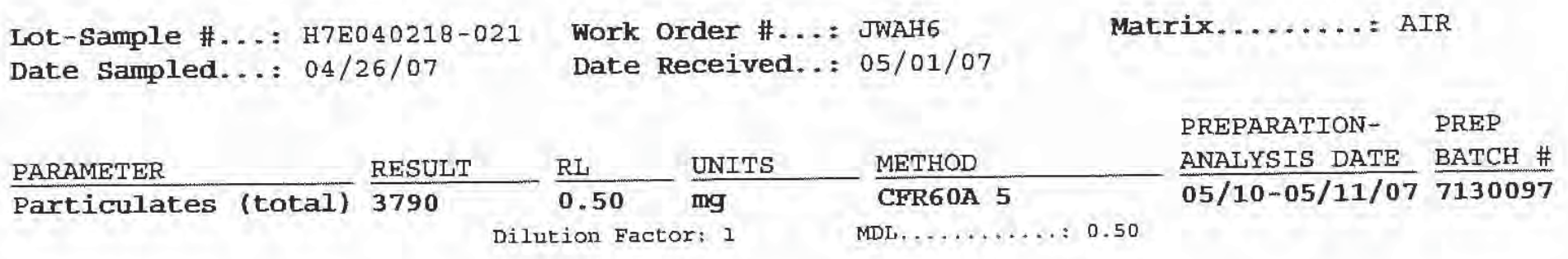


Air Sampling Associates, Inc.

Client Sample TD: HARDTN SDA INLET DUCT RUN 2 FILTKR (THTMBLR 52)

General Chemistry

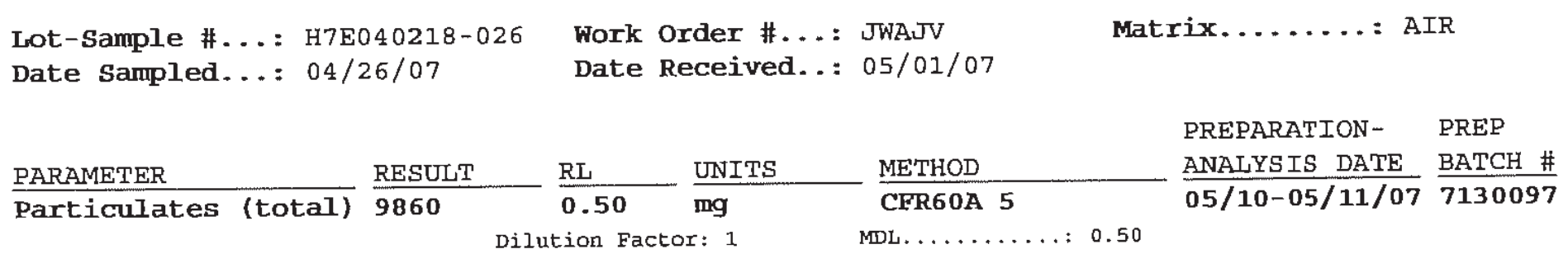


Air Sampling Associates, Inc.

Client Sample ID: HARDIN SDA INLFT DUCT RUN 2 FILTER (A174)

General Chemistry

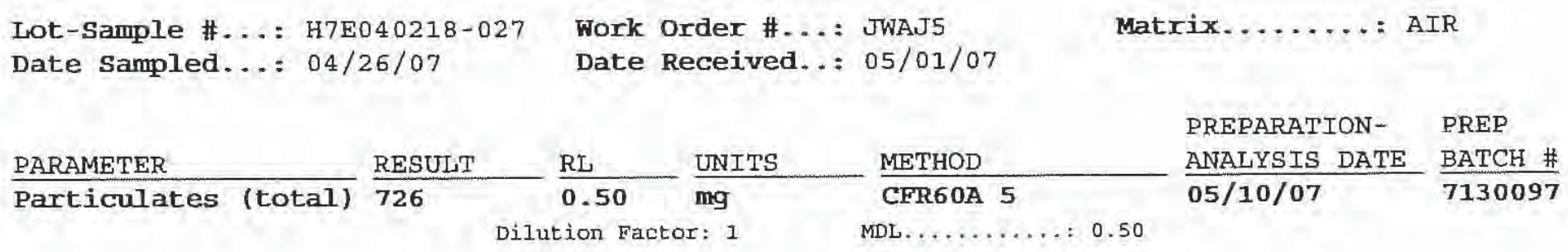


Air Sampling Associates, Inc.

Client Sample ID: HARDIN SDA INLFT DUCT RUN 2 ACBTONE

General Chemistry

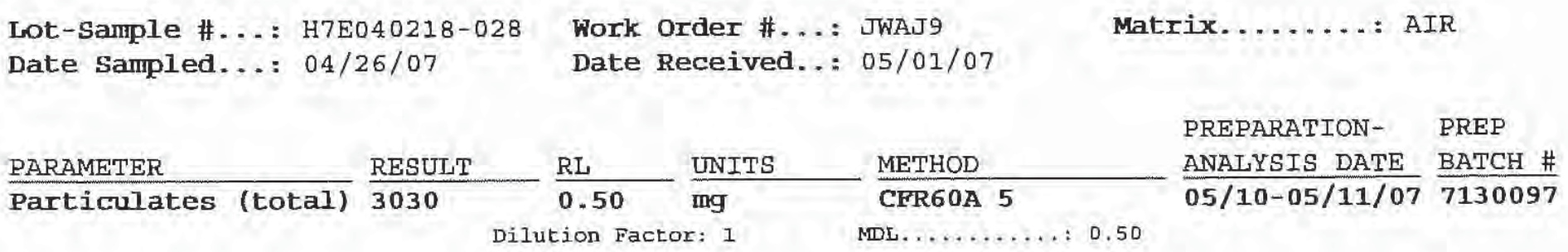


Air Sampling Associates, Inc.

client Sample ID: HARDIN SDA INLET DUCT RUN 3 FILTKR (THIMBLE 51)

General Chemistry

Irot-Sample \#...; H7E040218-033

Date Sampled.... 04/26/07
Work order \#...: JWAK9

Date Received..: 05/01/07
Matrix........ AIR

PREPARATION- PREP ANALYSIS DATE BATCH \#
PARAMETER

Particulates (total) $\mathbf{1 2 4 0 0}$
UNITS

mg

Dilution Factor: 1
METHOD

CFR60A 5 
Air Sampling Associates, Inc.

Client Sample ID: HARDIN SDA INLBT DUCT RUN 3 FILTTER (A175)

General Chemistry

Lot-Sample \#...: H7E040218-034 Work order \#...: JWALL

Date Sampled...: 04/26/07
Date Received..: 05/01/07
Matrix.......: AIR

PREPARATION- PREP

ANALYSIS DATE BATCH \#
PARAMETER

Particulates (total) 413
RESULT

RL

0.50

$\mathrm{mg}$

Dilution Factor: 1 05/10/07 7130097

CFR60A 5

MDL. . . . . . . . 0.50 
Air Sampling Associates, Inc.

\section{Client Sample WD: HARDIN SDA INLET DUCT RUN 3 ACETONE}

\section{General Chemistry}

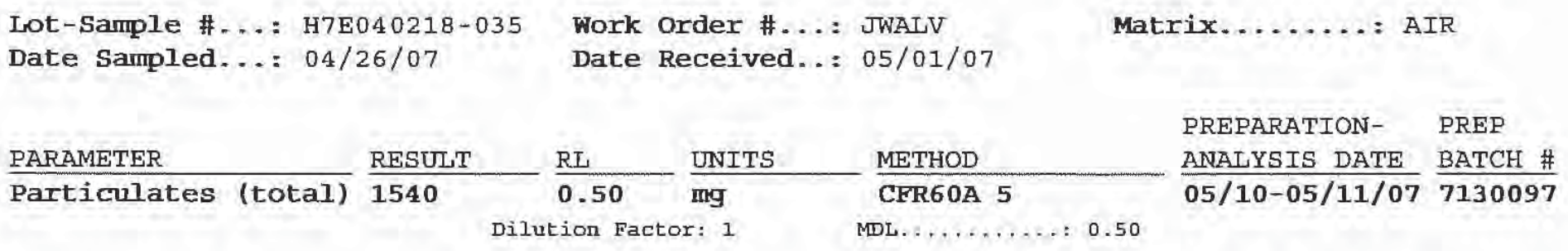


Raw Data 


\section{Total Particulates}




\section{STL Knoxville Particulate Matter Determination Data Review/Narrative Checklist Method: SW-846 Method 0050 by SOP KNOX-WC-0006, Rev. 2 Page 1 of 1}

\begin{tabular}{|r|l|}
\hline Batch Number: & 7130097 \\
\hline Lot Number(s): & 475040258 \\
\hline
\end{tabular}

\begin{tabular}{|c|c|c|c|c|c|}
\hline $\begin{array}{l}\text { Review Items } \\
\text { A. Calibration }\end{array}$ & N/A & $\mathrm{Y}$ & $\mathrm{N}$ & If No, why is data reportable? & 2nd \\
\hline $\begin{array}{l}\text { 1. Was the balance calibration checked with } 5,50 \& 100 \\
\text { gram weights prior to use? }\end{array}$ & & $\checkmark$ & & & \\
\hline $\begin{array}{l}\text { 2. Was the balance checked with } 100 \text { gram weight after } \\
\text { every } 10 \text { samples? }\end{array}$ & & $\checkmark$ & & & 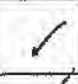 \\
\hline 3. Was the balance tared before each weighing? & & 7 & & & \\
\hline 4. Was the balance ID documented? & & $\checkmark$ & & & \\
\hline \multicolumn{6}{|l|}{ B. Client Sample and QC Sample Results } \\
\hline $\begin{array}{l}\text { 1. Were all special project requirements met? (Were PM } \\
\text { Checklists, Lot Summary, and any applicable QAS } \\
\text { reviewed?) }\end{array}$ & & $\mathcal{L}$ & & & \\
\hline 2. Were sample IDs verified? & & $\checkmark$ & & & $\checkmark$ \\
\hline 3. Were all transcriptions checked? & & $\mathcal{J}$ & & & \\
\hline 4. Calculations checked for error? & & $\checkmark$ & & & 7 \\
\hline $\begin{array}{l}\text { 5. Final report acceptable? (Results correct, units correct, } \\
\text { devintions noted in narrative, and analysis dates } \\
\text { correct.) }\end{array}$ & & $\checkmark$ & & & \\
\hline \multicolumn{6}{|l|}{ C. Preparation/Matrix QC } \\
\hline $\begin{array}{l}\text { 1. Were all sample weighings performed in duplicate (at } \\
\text { least)? }\end{array}$ & & $\mathcal{1}$ & & & 7 \\
\hline 2. Was the average of the last two weighings used? & & 7 & & & 7 \\
\hline \multicolumn{6}{|l|}{ D. Other } \\
\hline 1. Are all nonconformances documented appropriately? & $\mathcal{L}$ & & & NCM No.: & NSt \\
\hline
\end{tabular}

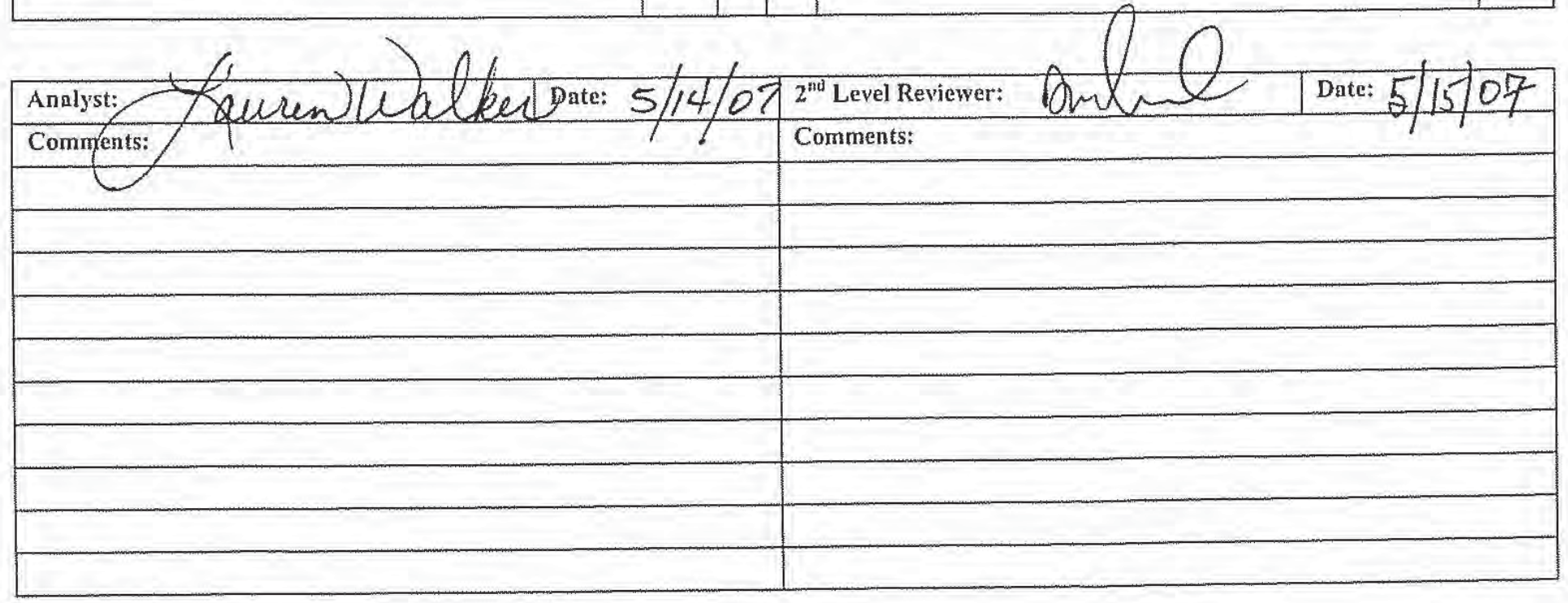




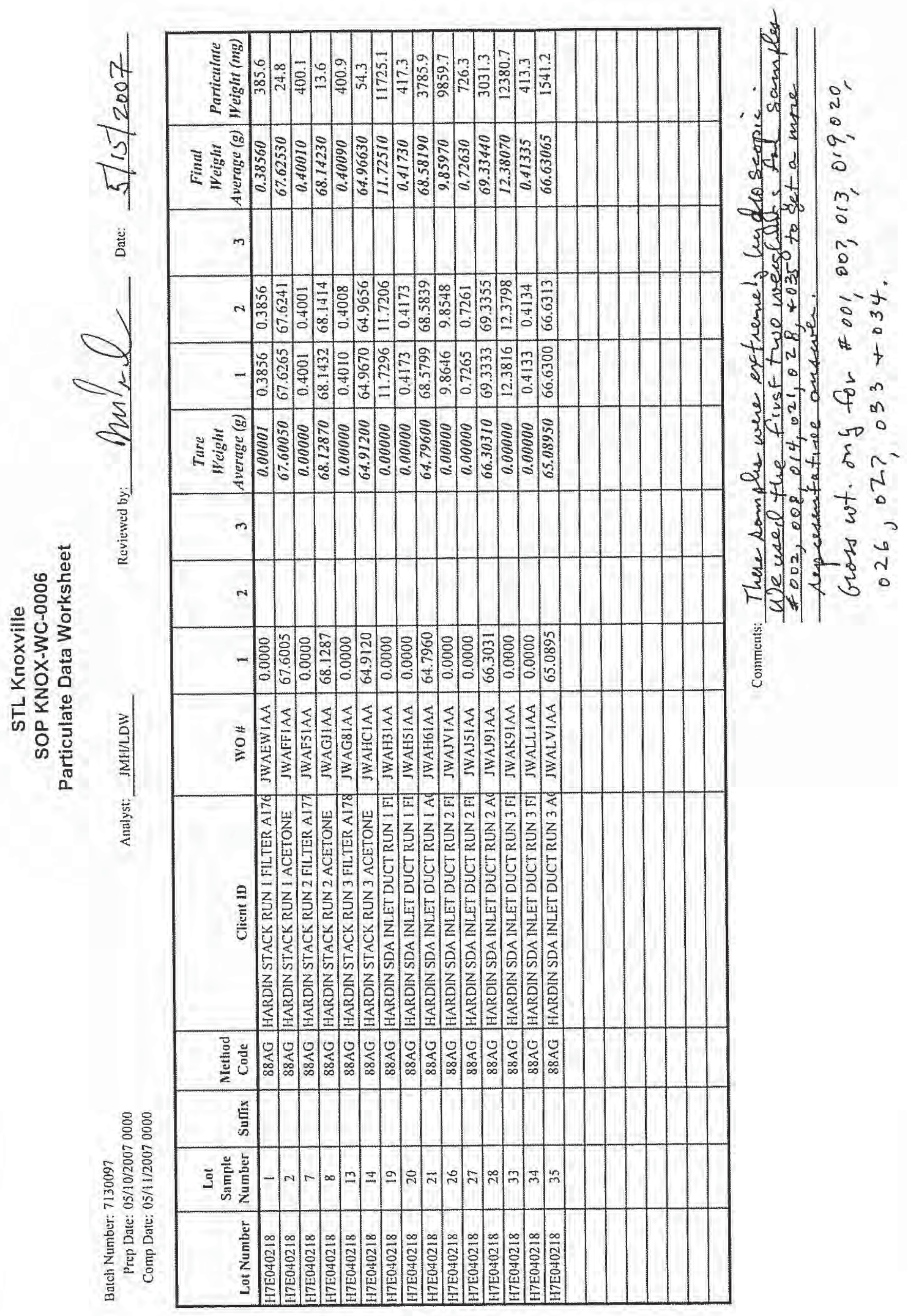




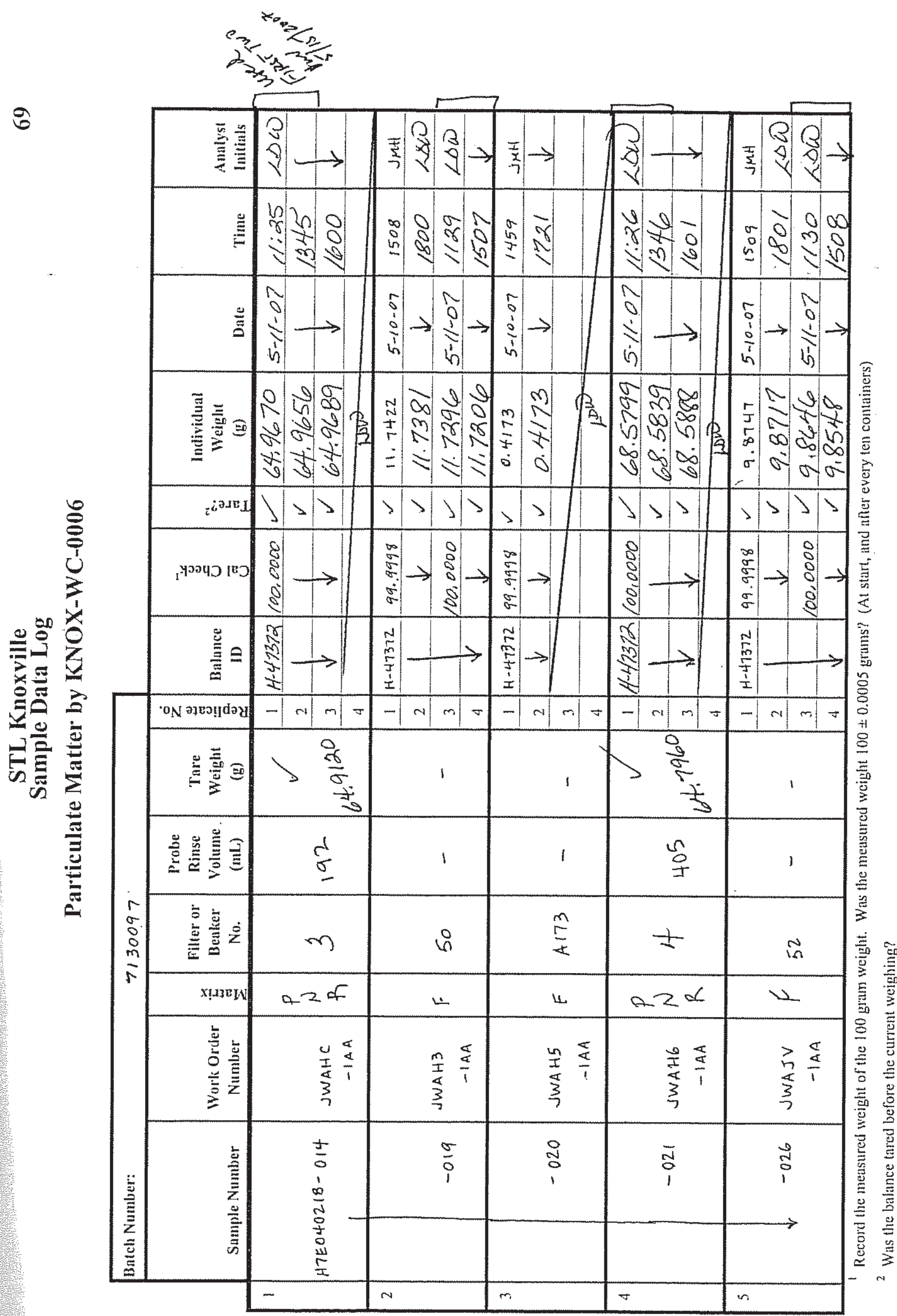




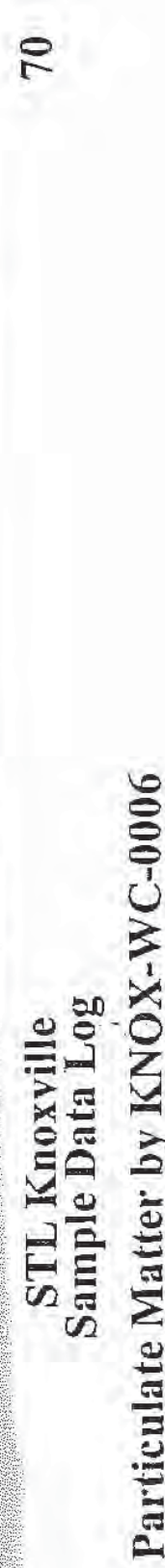

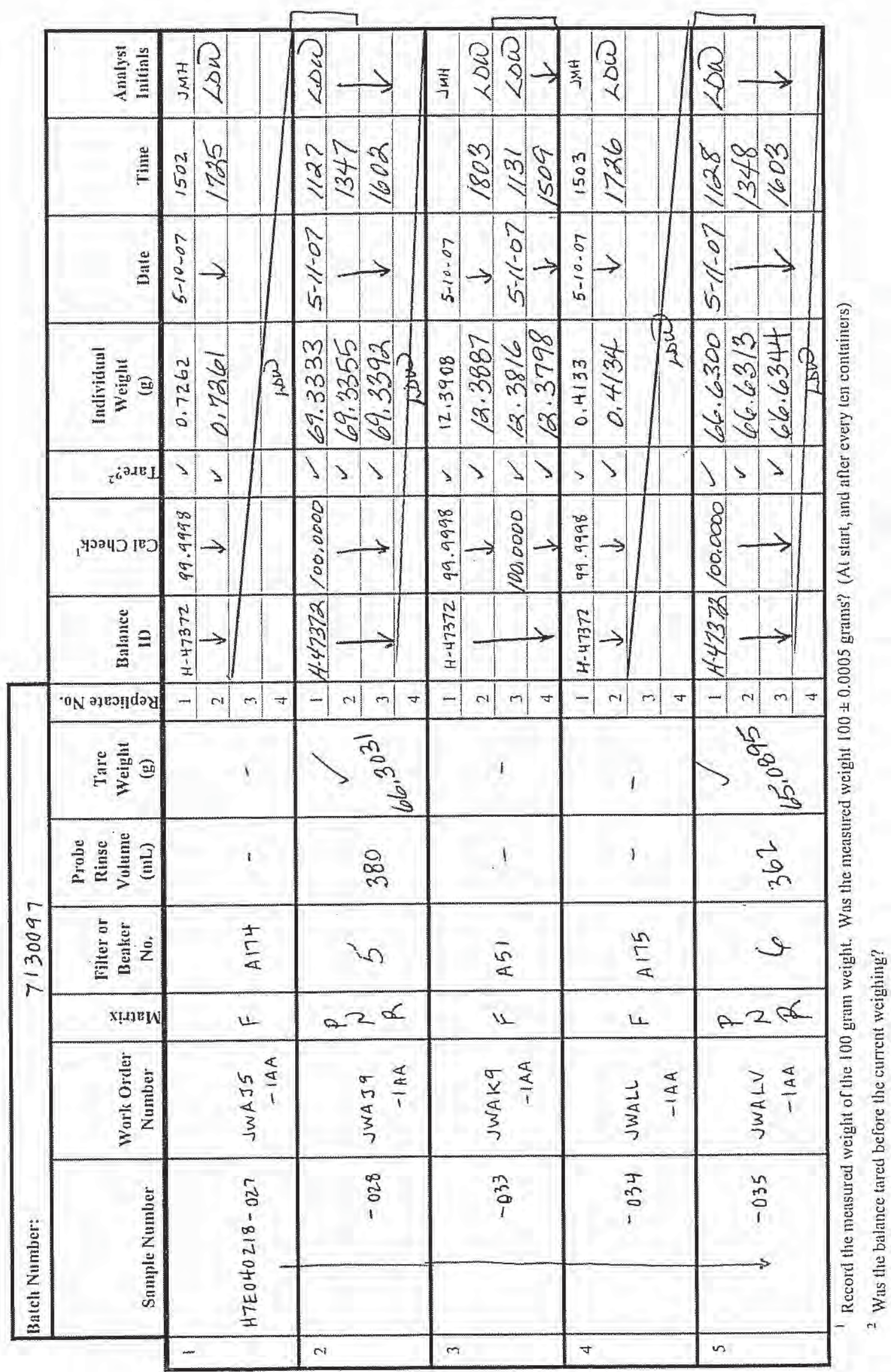


Tare Weight Log

Particulate Matter by KNOX-WC-0006

\begin{tabular}{|c|c|c|c|c|c|c|c|c|c|c|c|}
\hline & $\begin{array}{c}\text { Filter or } \\
\text { Beaker } \\
\text { No. }\end{array}$ & 总 & 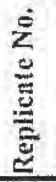 & Balance ID & 葛 & 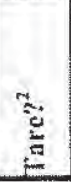 & $\begin{array}{l}\text { Individual } \\
\text { Weight (g) }\end{array}$ & Date & Time & $\begin{array}{l}\text { Analyst } \\
\text { Initials }\end{array}$ & $\begin{array}{c}\text { Tare } \\
\text { Weight } \\
\text { (g) } \\
\end{array}$ \\
\hline 1 & \multirow{4}{*}{1.1260} & \multirow{4}{*}{$f$} & 1 & 4.47372 & 99.9999 & $\checkmark$ & 79.8122 & 5.8 .07 & $\angle 2: 20$ & $\angle 0 \omega$ & \multirow{4}{*}{-79.8130} \\
\hline & & & 2 & 1 & 1 & $\checkmark$ & 79.8129 & 1 & $14: 45$ & 1 & \\
\hline & & & 3 & $y$ & $\checkmark$ & $\checkmark$ & 79.8131 & $\sqrt{ }$ & $17: 00$ & V & \\
\hline & & & 4 & & & & & & & & \\
\hline \multirow[t]{4}{*}{2} & \multirow{4}{*}{$1-1261$} & \multirow{4}{*}{$F$} & 1 & $A-47372$ & 99.9999 & 7 & 33.7370 & 5.8 .07 & $12: 21$ & $\angle \Delta \omega$ & \multirow{4}{*}{$33.7371^{5}$} \\
\hline & & & 2 & $k$ & $\downarrow$ & $\checkmark$ & 33.7373 & $k$ & $14: 46$ & $\downarrow$ & \\
\hline & & & 3 & & & 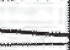 & $1 \Delta \omega$ & & & & \\
\hline & & & 4 & & & & 5.8 .07 & & & 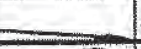 & \\
\hline \multirow[t]{4}{*}{3} & \multirow{4}{*}{1.1262} & \multirow{4}{*}{$F$} & 1 & 4.47372 & 99.9999 & $\checkmark$ & 34.1264 & 5.8 .07 & $12: 22$ & $\angle \Delta \omega$ & \multirow{4}{*}{$34.1264^{5}$} \\
\hline & & & 2 & $k$ & 1 & $\checkmark$ & 34.1265 & $k$ & $14: 47$ & $\downarrow$ & \\
\hline & & & 3 & & & 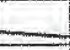 & 100 & & & & \\
\hline & & & 4 & & & & 5.8 .07 & & & & \\
\hline \multirow[t]{3}{*}{4} & \multirow{4}{*}{$1-1263$} & \multirow{4}{*}{$F$} & 1 & $A-47372$ & 49.4999 & $\checkmark$ & 31.0245 & 5.8 .07 & $12: 23$ & $\angle D W$ & \multirow{4}{*}{31.0247} \\
\hline & & & 2 & $\downarrow$ & $\downarrow$ & $\checkmark$ & 31.0249 & $k$ & $14: 48$ & $\downarrow$ & \\
\hline & & & 3 & & & 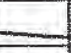 & $4 \Delta D$ & & & & \\
\hline & & & 4 & & & & 5.8 .07 & & & & \\
\hline \multirow{3}{*}{ r } & \multirow{4}{*}{$12-1264$} & \multirow{4}{*}{$F$} & 1 & $A-47372$ & 99.9999 & $\checkmark$ & 34.7858 & 5.8 .07 & $12: 24$ & $\angle \Delta \omega$ & \multirow{4}{*}{34.7860} \\
\hline & & & 2 & $\downarrow$ & $\downarrow$ & $\checkmark$ & 34.7862 & $k$ & $14: 49$ & $\downarrow$ & \\
\hline & & & 3 & & & $=$ & $\angle D W$ & & & & \\
\hline & & & 4 & & & & 5.8 .07 & & & & \\
\hline \multirow{2}{*}{ i } & & & 1 & $4-47372$ & 99.9999 & $\checkmark$ & 32.1083 & 5.8 .07 & $12: 25$ & $\angle D O U$ & \\
\hline & 1.1265 & $E$ & 2 & 1 & 1 & $\checkmark$ & 32.1088 & 1 & $14: 50$ & 1 & \\
\hline$\pi$ & & $F$ & 3 & $v$ & $\checkmark$ & r & 32.1089 & $v$ & $17: 01$ & $\sqrt{k}$ & \\
\hline & & & 4 & & & & & & & & 32.1088 \\
\hline 7 & & & 1 & $1-47372$ & 99.9999 & $\longleftarrow$ & 35.9456 & 5.8 .07 & $12: 26$ & $\angle \Delta \bar{\omega}$ & \\
\hline & 1,1066 & $F$ & 2 & $\downarrow$ & $\downarrow$ & $r$ & 35.9459 & $k$ & $14: 51$ & $k$ & \\
\hline 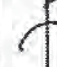 & $11 / 266$ & & 3 & & & 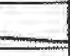 & 100 & & & & $25 a 4 m^{5}$ \\
\hline & & & 4 & & & & 5.8 .07 & & & 1 & $35.945 \%$ \\
\hline 8 & & & 1 & $1-47372$ & 100.0001 & $\checkmark$ & 67.6005 & $5-10-07$ & 0717 & $\mathrm{JMH}$ & \\
\hline & & $P_{u}$ & 2 & & & & & & & & \\
\hline & 1 & ${ }_{R}^{N}$ & 3 & & & & & JMII & & & 67.6005 \\
\hline & & & 4 & & & & & $5 \cdot 10-07$ & & & \\
\hline 9 & & $p$ & 1 & H- 47372 & 100.0001 & $r$ & 68.1287 & $5-10-07$ & 0718 & $\mathrm{JMH}$ & \\
\hline & 2 & $N_{R}$ & 2 & & & & & & & & 68. 1287 \\
\hline & 2 & & 3 & & & & & IMH & & & \\
\hline & & & 4 & & & & & $5-10-07$ & & $=$ & \\
\hline 10 & & & 1 & $H-47372$ & 100.0001 & $\checkmark$ & 64.9120 & $5-10-07$ & 0719 & JMH & \\
\hline & 3 & $N_{R}$ & 2 & $\longrightarrow$ & & & & & & & 64.9120 \\
\hline & & & 3 & & & & & JMH & & & \\
\hline & & & 4 & & & & & $5-10-07$ & & $=$ & \\
\hline
\end{tabular}

Record the measured weight of the 100 gram weight. Was the measured weight $100 \pm 0.0005$ grams?

(At star, and afier every ten containers)

- Was the balance cared belore the current weighing? 
Tare Weight Log

Particulate Matter by KNOX-WC-0006

\begin{tabular}{|c|c|c|c|c|c|c|c|c|c|c|c|}
\hline & $\begin{array}{c}\text { Filter or } \\
\text { Beaker } \\
\text { No. }\end{array}$ & $\stackrel{\frac{L}{E}}{E}$ & 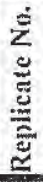 & Balance ID & 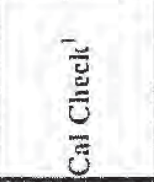 & Eั & $\begin{array}{l}\text { Individual } \\
\text { Weight (g) }\end{array}$ & Date & Time & $\begin{array}{l}\text { Analyst } \\
\text { Initials }\end{array}$ & $\begin{array}{c}\text { Tare } \\
\text { Weight } \\
\text { (g) }\end{array}$ \\
\hline \multirow[t]{4}{*}{1} & \multirow{4}{*}{4} & \multirow{4}{*}{$P_{N_{R}}$} & 1 & $\mathrm{H}-47372$ & 100.0001 & $r$ & 64.7960 & $5-10-07$ & 0720 & $\mathrm{JMH}$ & \multirow{4}{*}{64.7960} \\
\hline & & & 2 & 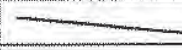 & & & & & & & \\
\hline & & & 3 & & & & & Jin & & & \\
\hline & & & 4 & & & & & $5-10-27$ & & {[} & \\
\hline \multirow[t]{4}{*}{2} & \multirow{4}{*}{5} & \multirow{4}{*}{$P_{N_{R}}$} & 1 & $1-47372$ & 100.0001 & $\checkmark$ & 66.3031 & $5-10-07$ & 0721 & $\mathrm{JMH}$ & \multirow{4}{*}{66.3031} \\
\hline & & & 2 & 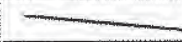 & & 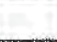 & & & & & \\
\hline & & & 3 & & & $=$ & & $\mathrm{juAl}$ & & & \\
\hline & & & 4 & & & & & $5+0-07$ & & $\square$ & \\
\hline \multirow[t]{4}{*}{3} & \multirow{4}{*}{6} & \multirow{4}{*}{$P_{N}$} & 1 & $H-47372$ & 100.0001 & $\checkmark$ & 65,0895 & $5-10-07$ & 0722 & $\mathrm{JMH}$ & \multirow{4}{*}{65.0895} \\
\hline & & & 2 & & & & & & & & \\
\hline & & & 3 & & & & & $J M H$ & & & \\
\hline & & & 4 & & & & & $5-10-07$ & & $=$ & \\
\hline \multirow[t]{4}{*}{4} & \multirow{4}{*}{7} & \multirow{4}{*}{$P_{N_{R}}$} & 1 & H. 47372 & 100.0001 & $\checkmark$ & b6. 1942 & $5-10-97$ & 0723 & JMH & \multirow{4}{*}{66.1942} \\
\hline & & & 2 & - & & & & & & & \\
\hline & & & 3 & & & 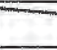 & & $\mathrm{J \mu H}$ & & & \\
\hline & & & 4 & & & & & $5-10-07$ & & $=$ & \\
\hline \multirow[t]{4}{*}{5} & \multirow{4}{*}{8} & \multirow{4}{*}{$P_{p}$} & 1 & $\mathrm{H}+47372$ & 100.0001 & $r$ & 84.3151 & $5-10-07$ & 0224 & $J$ MH & \multirow{4}{*}{84.3151} \\
\hline & & & 2 & 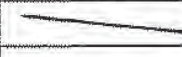 & & & & & & & \\
\hline & & & 3 & & & & & $\mathrm{MANH}$ & & & \\
\hline & & & 4 & & & & & $5-10-07$ & & 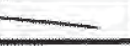 & \\
\hline \multirow[t]{4}{*}{6} & \multirow{4}{*}{9} & \multirow{4}{*}{$P_{N_{p}}$} & 1 & $\mathrm{H}-47372$ & 100.0001 & $\checkmark$ & 66.4565 & $5-10-07$ & 0725 & $J M H$ & \\
\hline & & & 2 & $\longrightarrow$ & $\mathrm{C}_{2}$ & 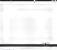 & & & & & \\
\hline & & & 3 & & & $=$ & & JMH & & & $6 t .4565$ \\
\hline & & & 4 & & & & & $5-10.07$ & & $=$ & \\
\hline 7 & & & 1 & $\mathrm{H}-42372$ & 100.0001 & $\checkmark$ & 67.4339 & $5-10-07$ & 0726 & JMH & \\
\hline & & & 2 & - & 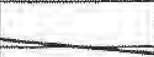 & & & & & & \\
\hline & 10 & $R$ & 3 & & & 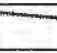 & 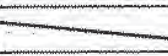 & JMH & & . & 67.4339 \\
\hline & & & 4 & & & & & $5-10-07$ & & 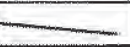 & \\
\hline 8 & & & 1 & $\mathrm{H}-47372$ & 100.0001 & $\checkmark$ & 65.1229 & $5-10-07$ & 0728 & $\mathrm{JMH}$ & \\
\hline & & $P_{N_{0}}$ & 2 & - & 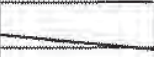 & & & & & & 15.729 \\
\hline & 11 & of & 3 & & & & & JMH & & & $65,12 \angle 7$ \\
\hline & & & 4 & & & & & $5-10-07$ & & $=$ & \\
\hline 9 & & & 1 & 62278 & 100.0001 & $\swarrow$ & 64.8821 & $5-10-07$ & $17: 00$ & $\angle O D$ & \\
\hline & $\Lambda$ & j & 2 & & $=$ & & & & & & \\
\hline & A & $R$ & 3 & & & & & $\angle 00$ & & & \\
\hline & & & 4 & & & & & & & $=$ & 64,8321 \\
\hline 10 & & & 1 & 62278 & 100.0001 & 5 & 67.1833 & $5-10-07$ & $17: 01$ & kow & \\
\hline & & $P$ & 2 & & 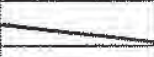 & & & & & & \\
\hline & $B$ & $N_{R}$ & 3 & & & & & $-1 D D$ & & & \\
\hline & & K & 4 & & & & & & & 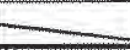 & 67.1833 \\
\hline
\end{tabular}

Record the measured weight of the 100 gram weight. Was the measured weight $100 \div 0.0005$ grams?

(At star, and afier every ten containers)

- Was the balance tared before the current weighing? 


\section{Sample Receipt Documentation}




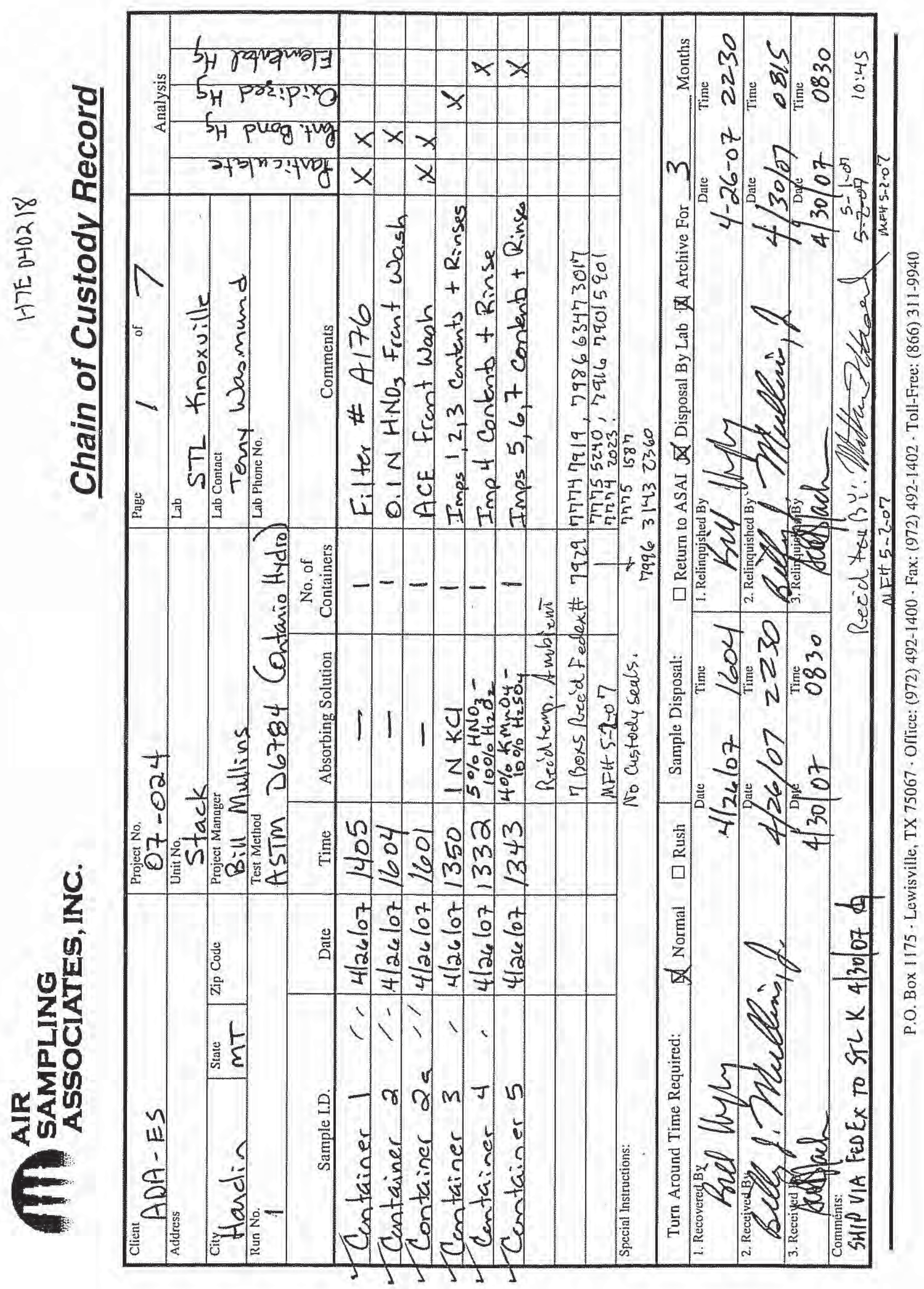




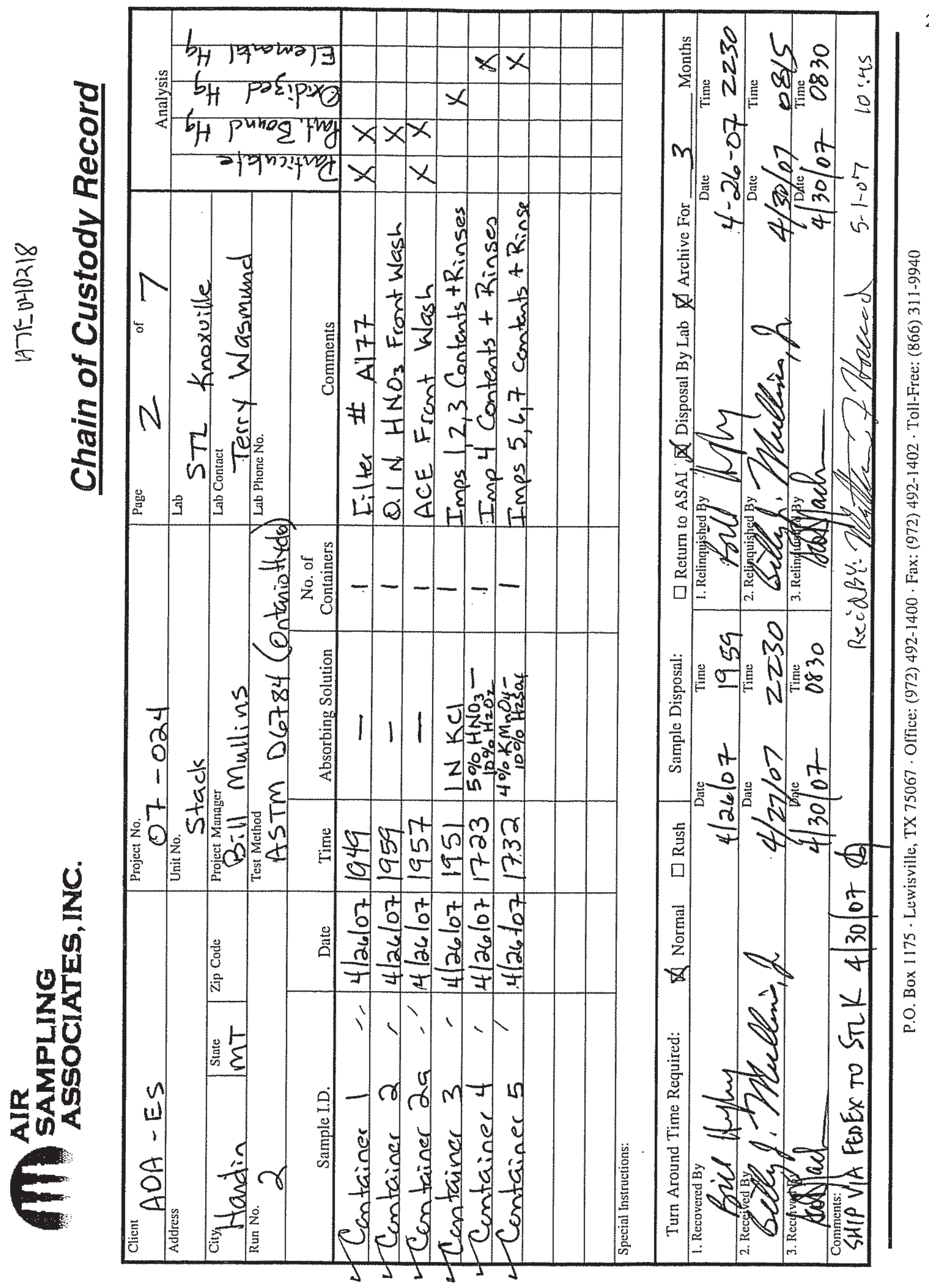




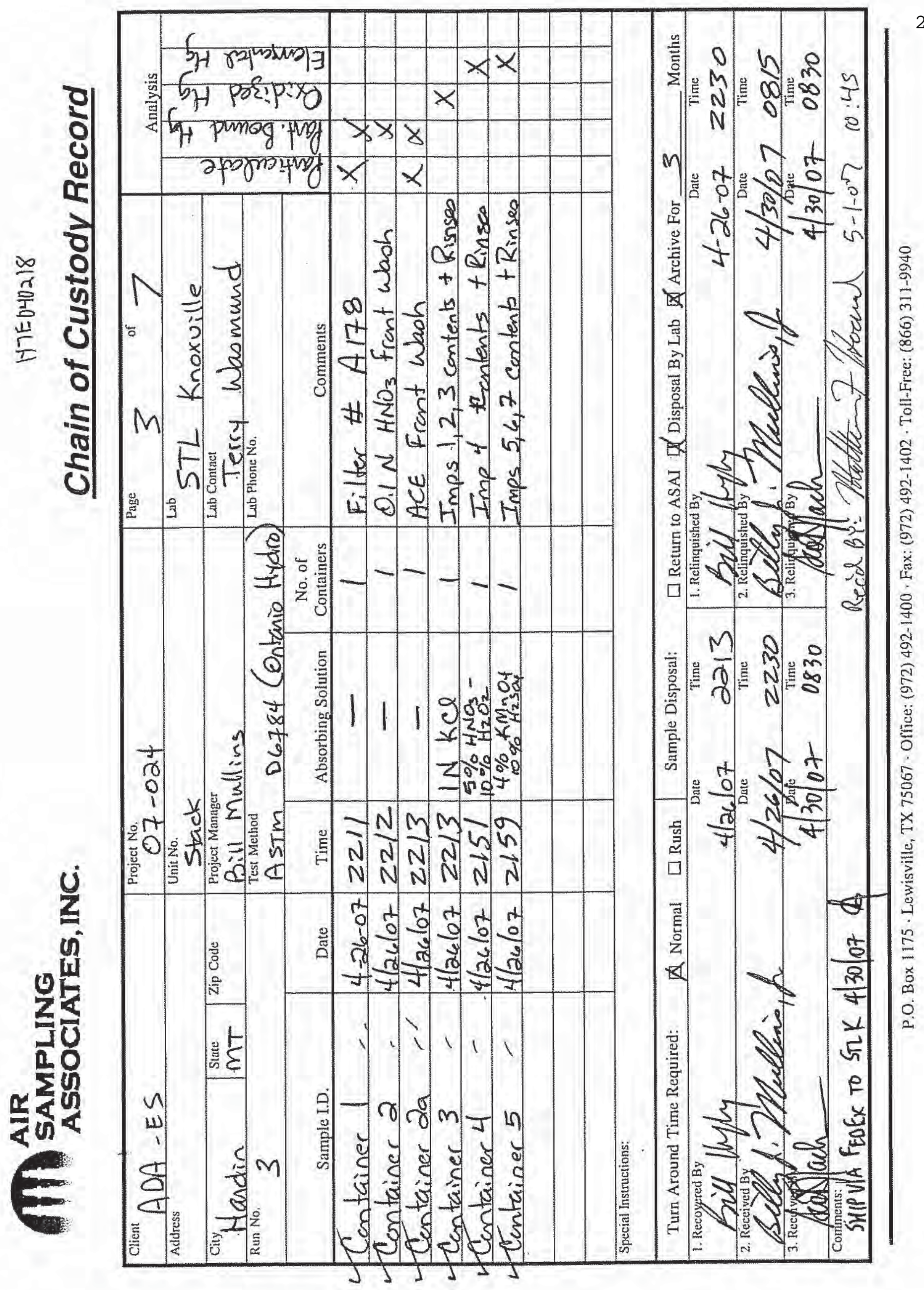




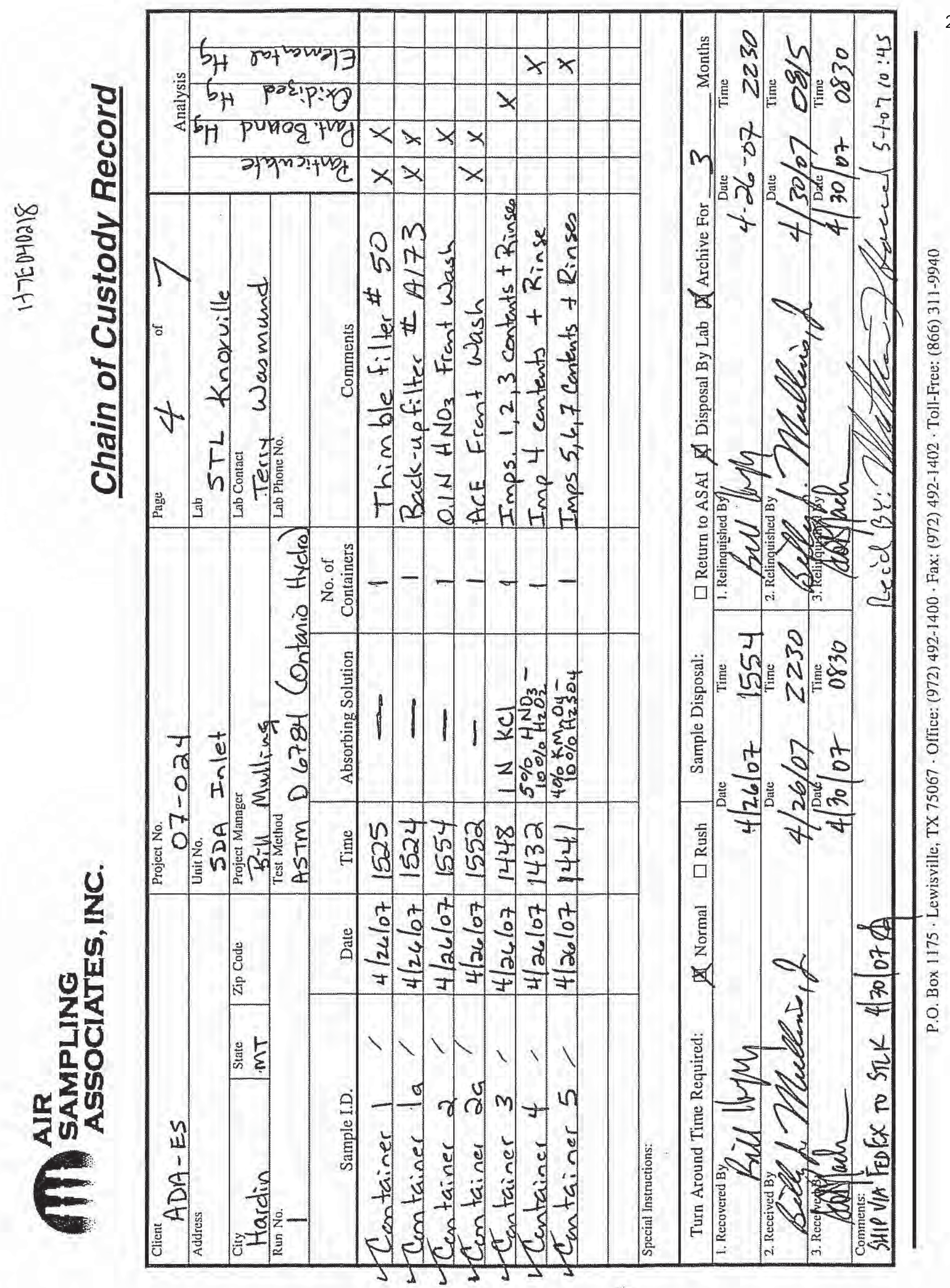




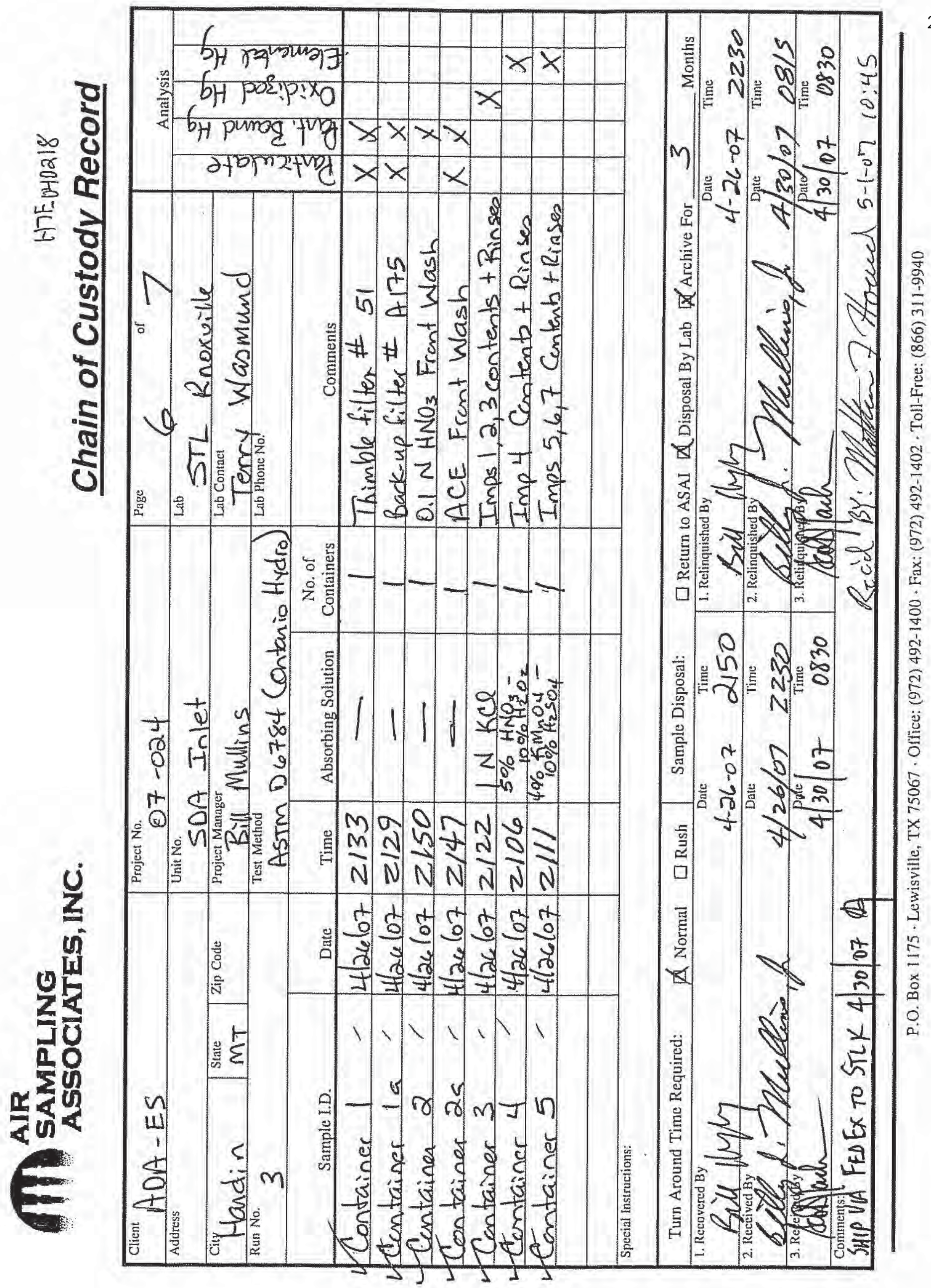




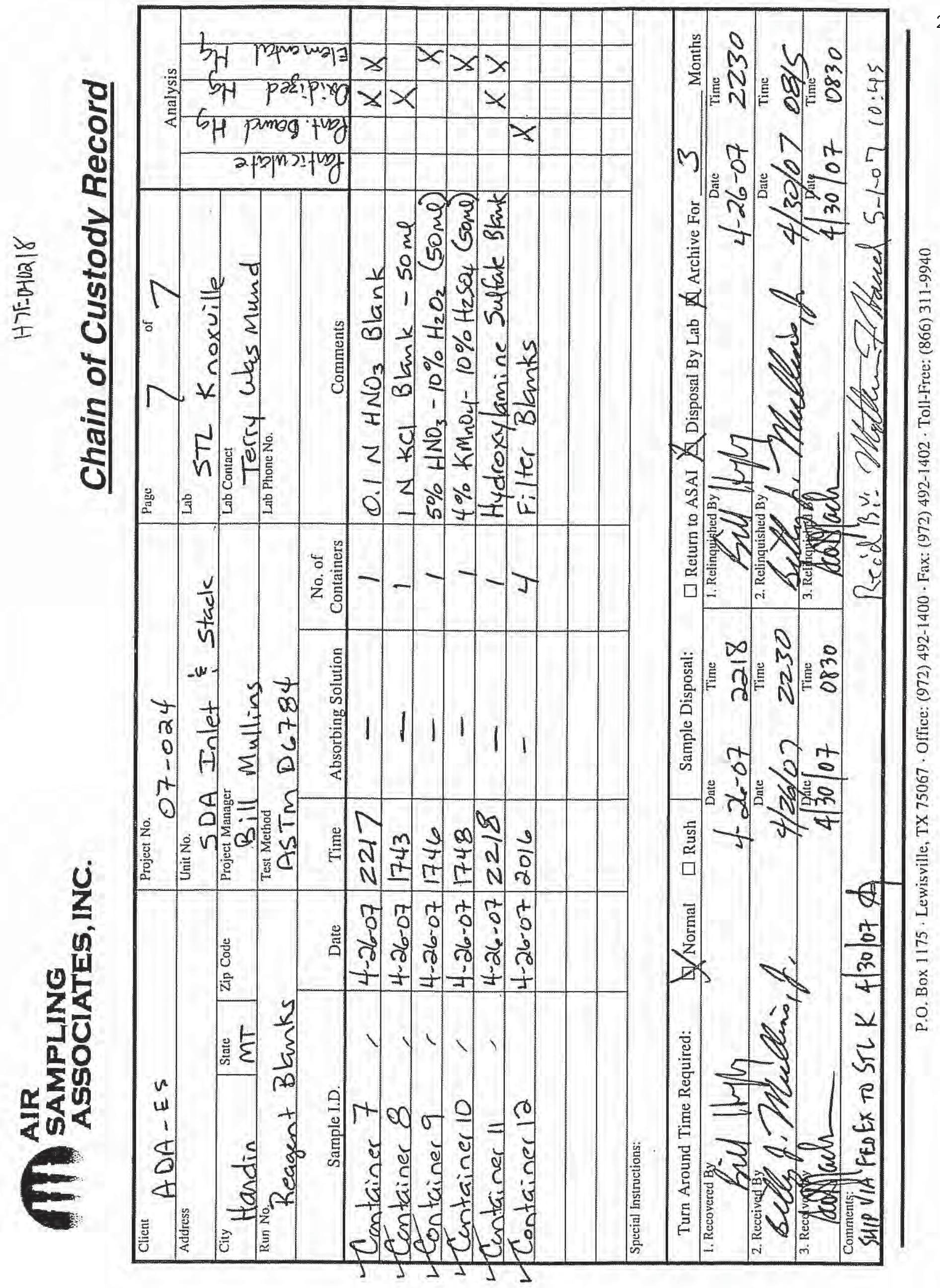




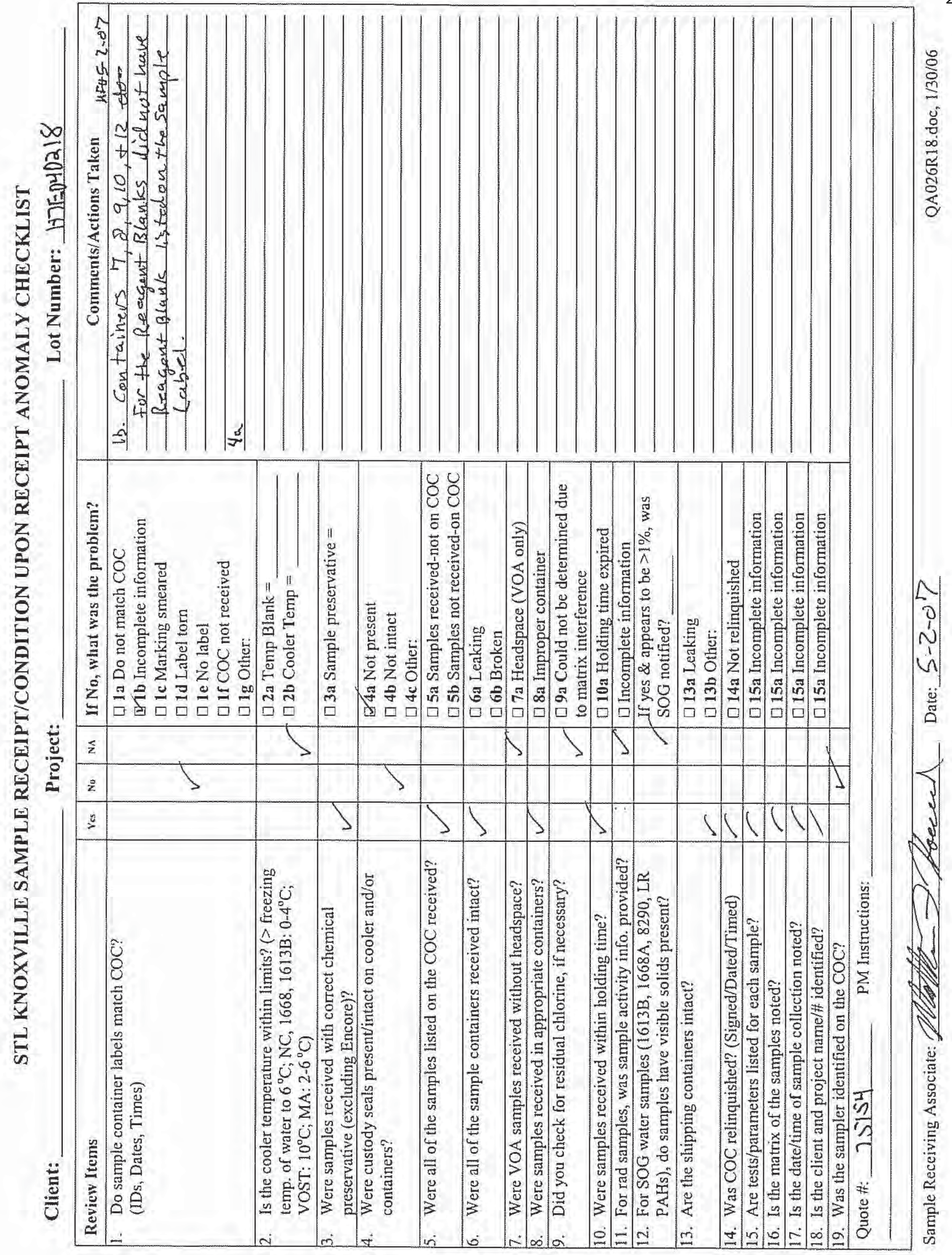


Appendix G:

Chain of Custodies 

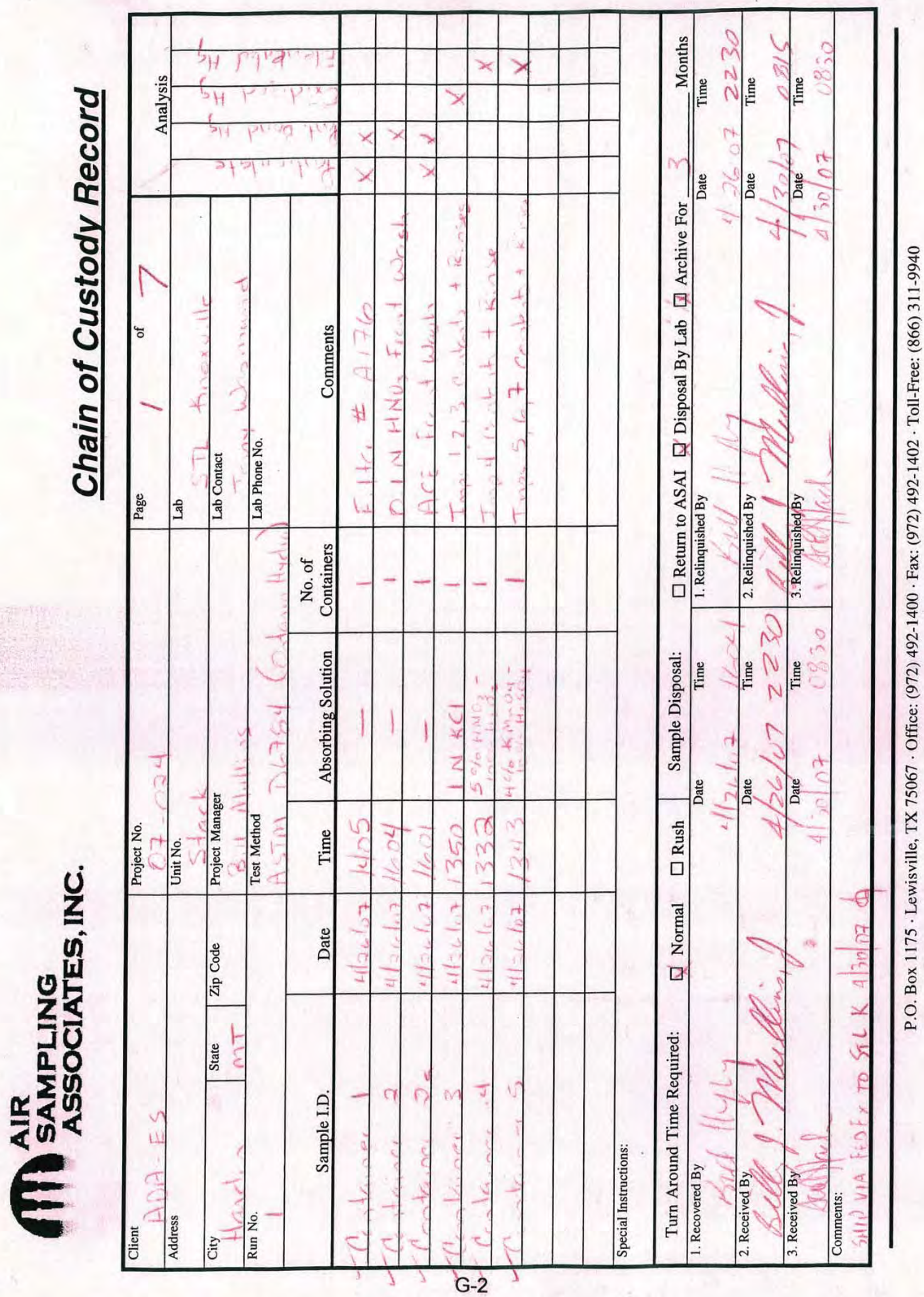


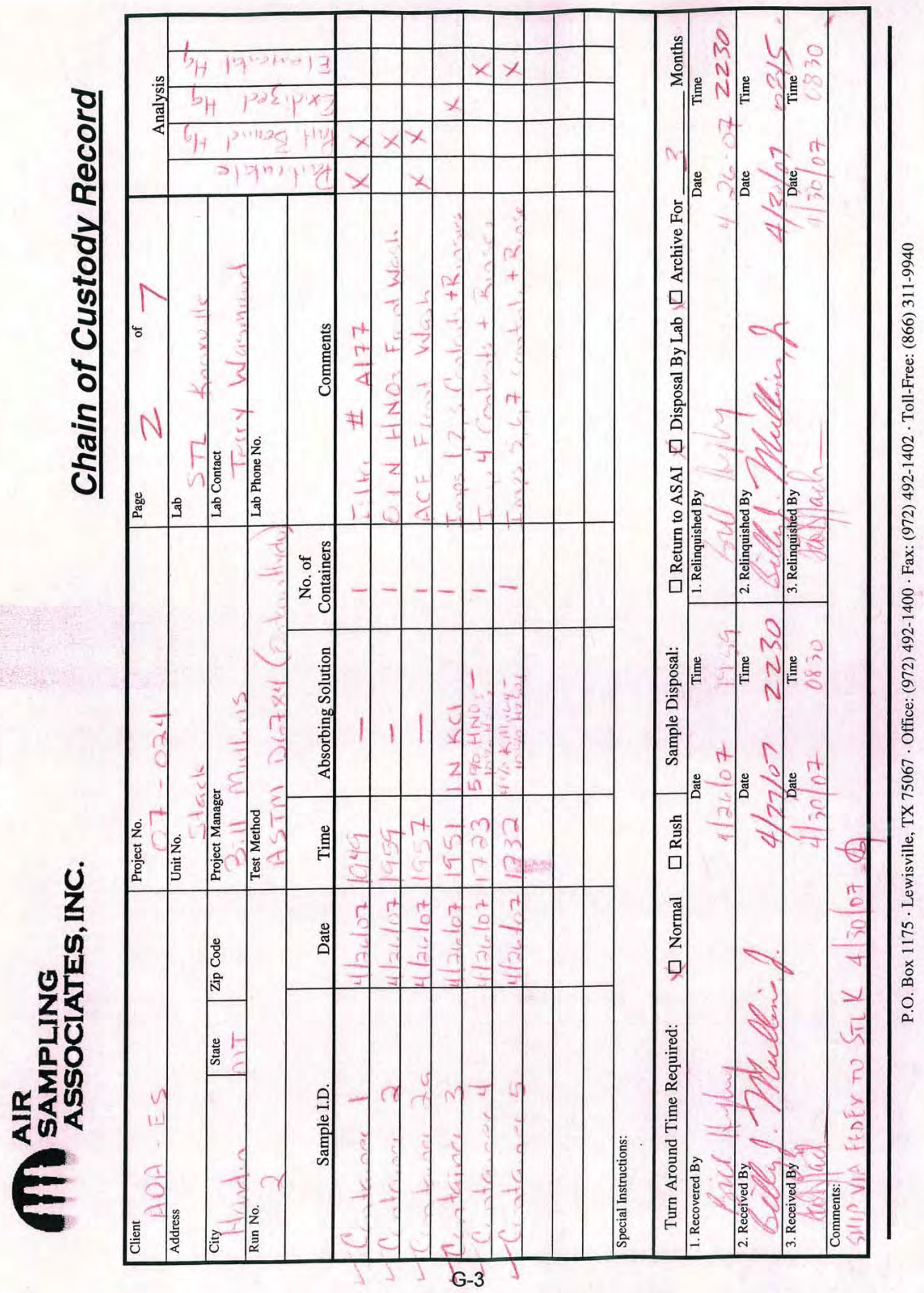




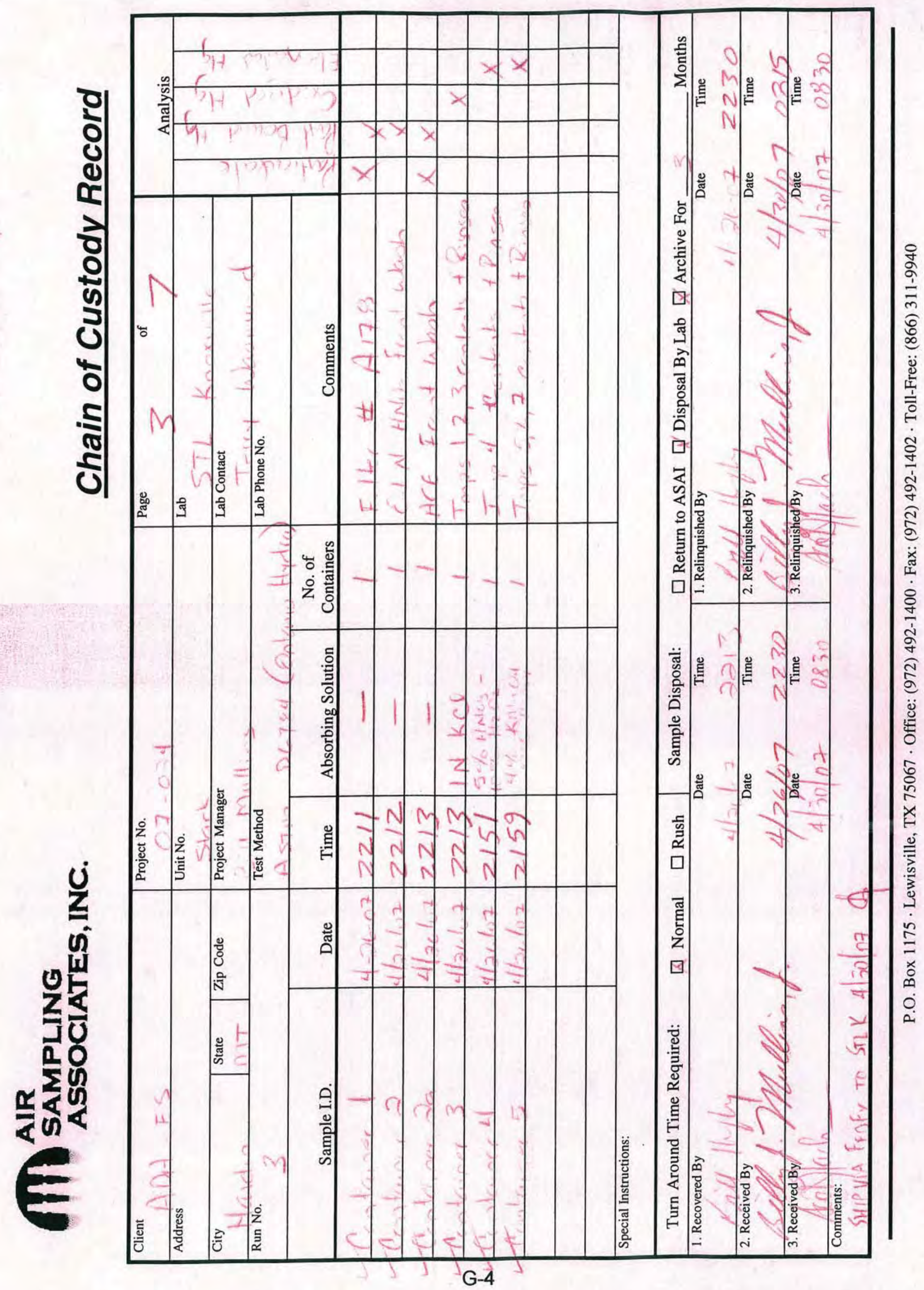




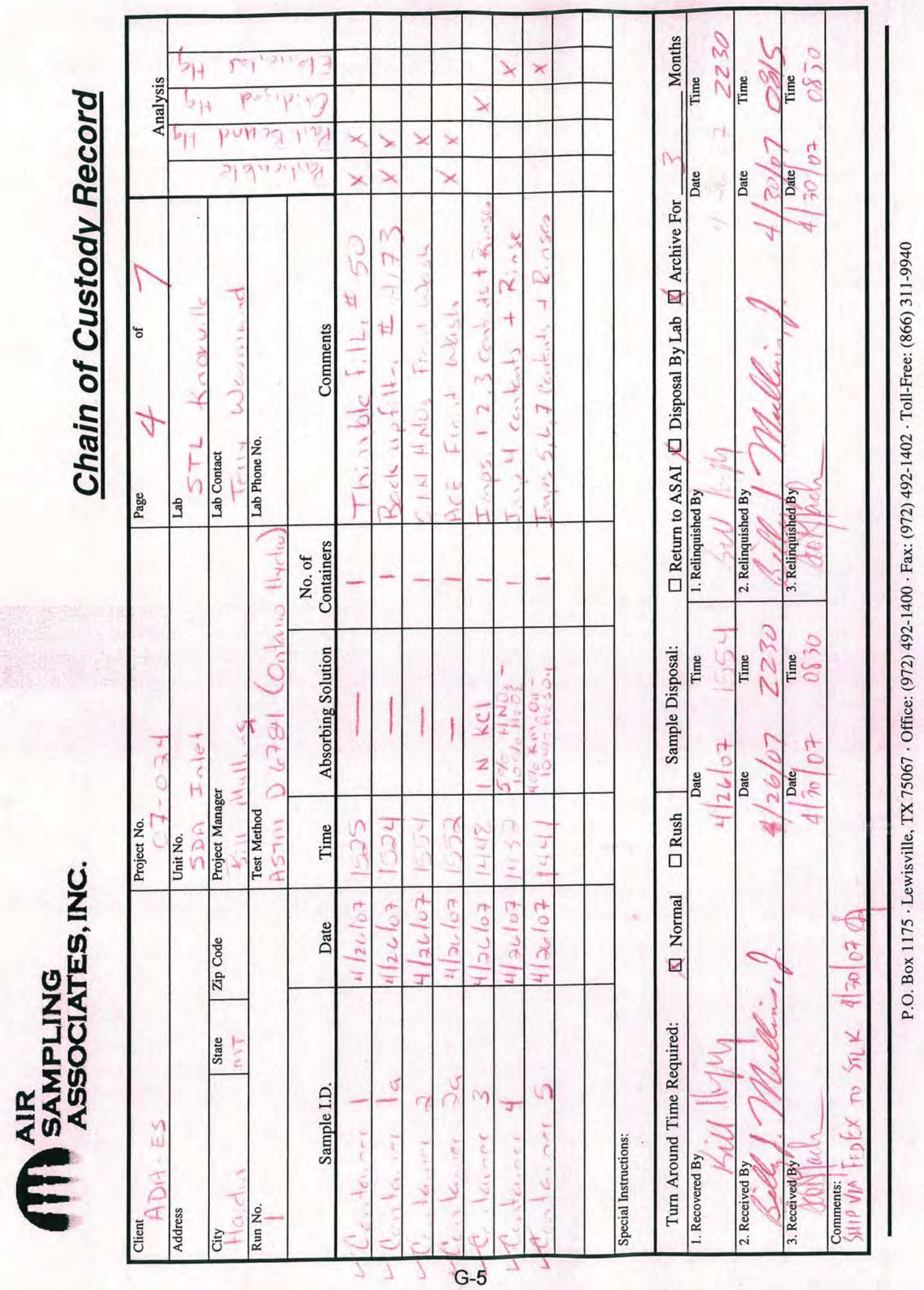



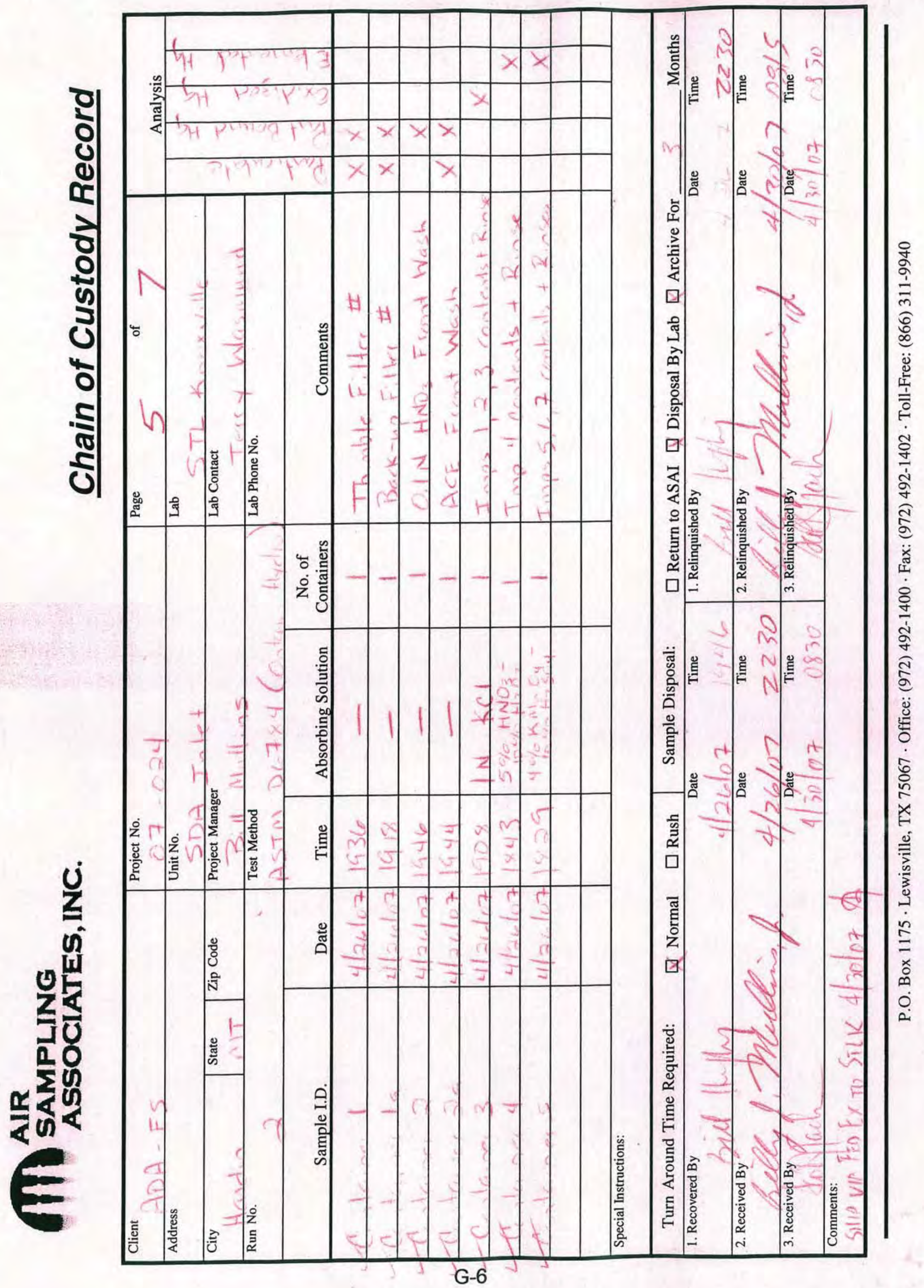

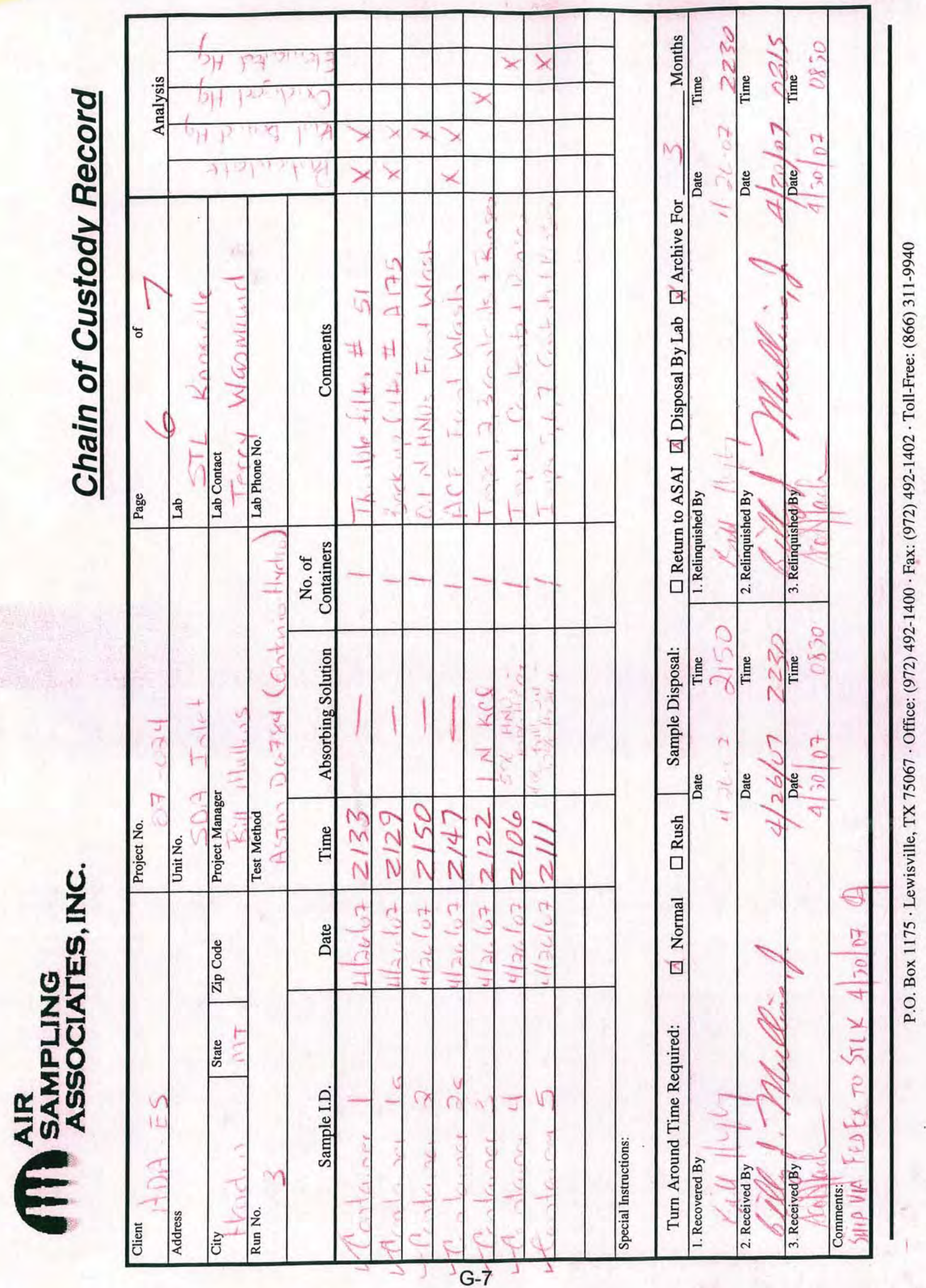


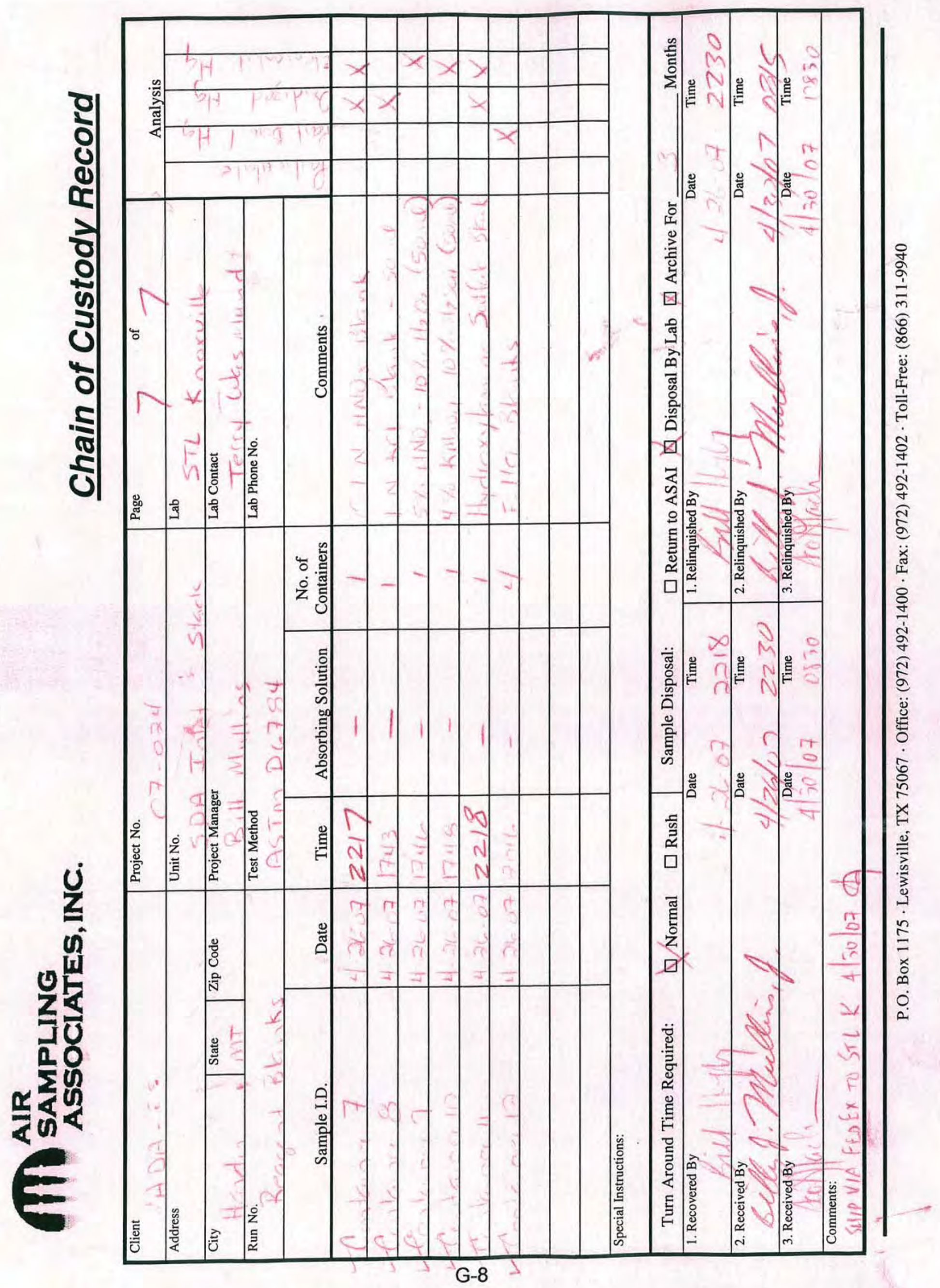



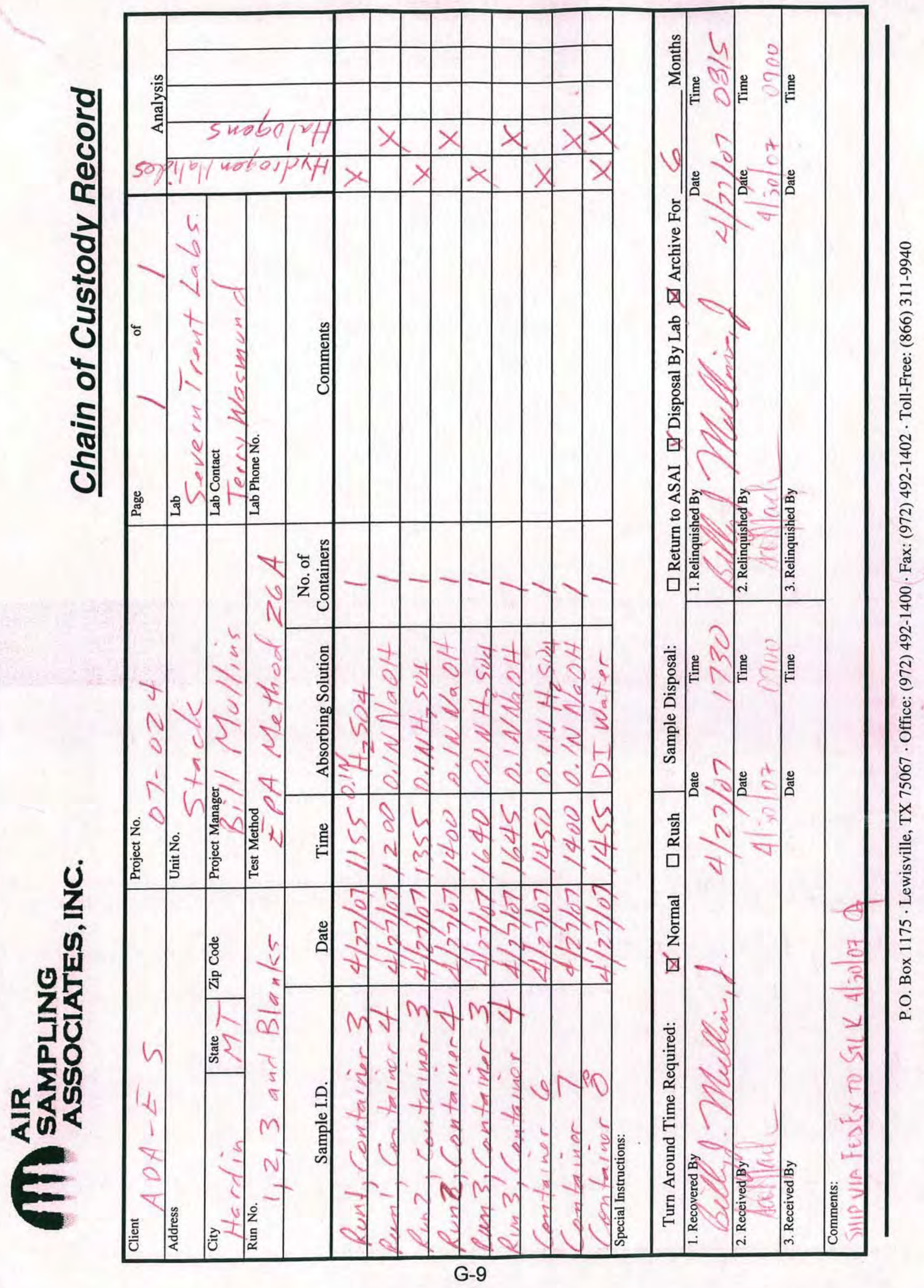


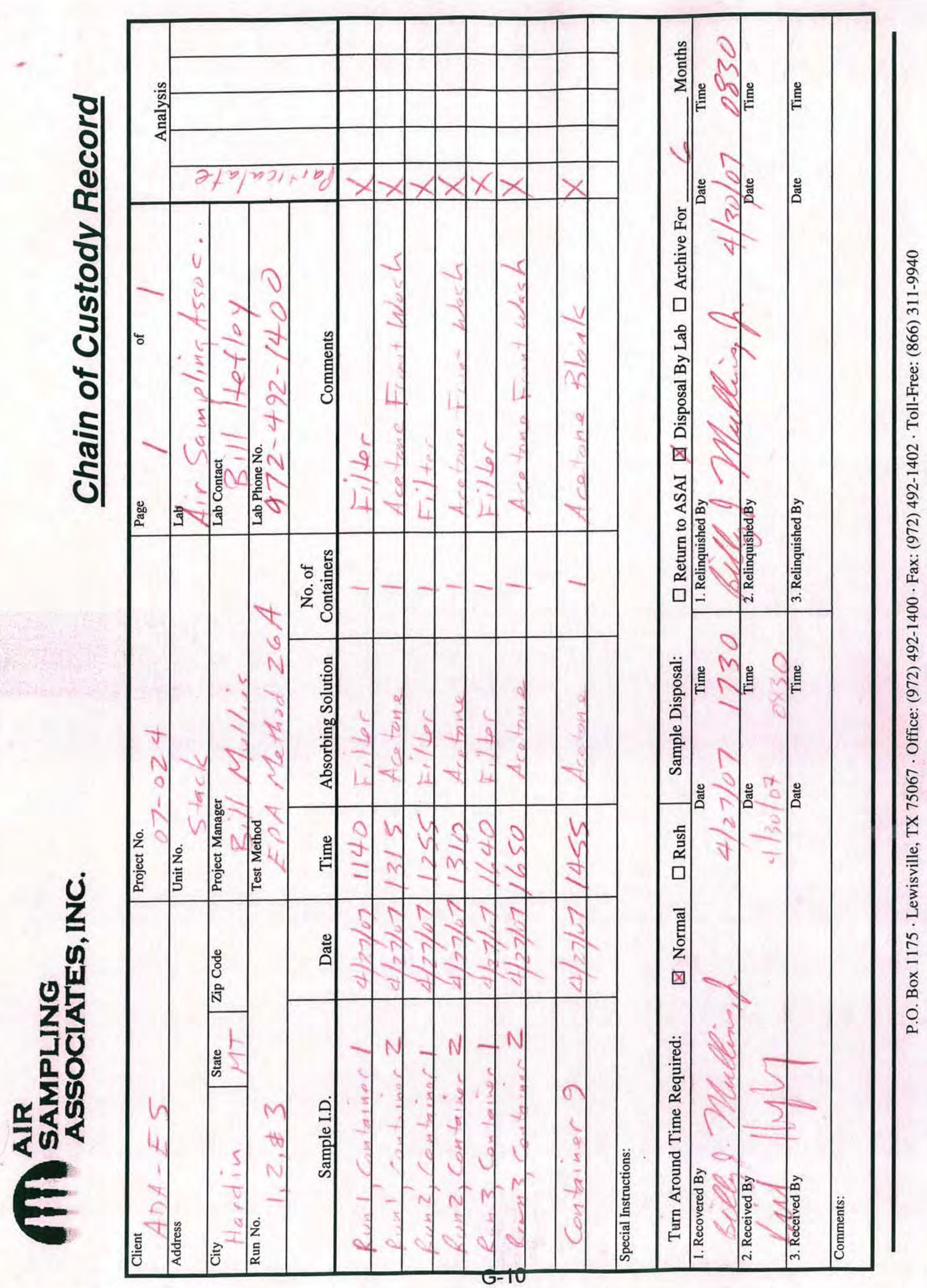




\section{Appendix H:}

\section{Resumes of Test Personnel}


BILLY J. MULLINS, JR.; President

Education

Post Graduate Study Environmental Engineering at Southern Methodist University; Dallas, Texas 1970.

M.S. 1969, New York University; New York, New York, in Civil Engineering (Air Resources).

B.S. 1968, Texas Tech University; Lubbock, Texas, in Civil Engineering (Water Resources). Studies in Engineering at the U.S. Naval Academy; Annapolis, Maryland, 1963-1964

Professional Attended Short Course on Air Pollution Engineering at the University

Training of Texas at Austin, February 1970.

$\underline{\text { Courses }}$

Attended four-week management course presented by the American Management Association, 1976.

Certification Registered Professional Engineer

Certified Visible Emissions Evaluator

Licensed Private Pilot (Multi-Engine-Land, Instrument)

Diplomat in the American Academy of Environmental Engineers

Inductee into the Stack Sampling Hall of Fame

Certified as Qualified Environmental Professional (QEP)

Professional Air \& Waste Management Association - Past Chairman, Past Vice Memberships Chairman, and Past Board of Directors of North Texas Chapter and Southwest Section; Past Chairman, Consultants Committee; Past Chairman, Source Measurement Committee

Source Evaluations Society - Past President, Past Board of Directors

\section{American Management Association}


MULLINS (p. 2)

Publications Authored "Real World Experience with USEPA's New Sampling and Analytical Methods for Conducting Risk Burn," May 1998.

Co-authored "Sulfur Compound Emissions of the Petroleum Production Industry," December 1974.

Co-authored "Field Procedure for Stabilizing Hydrogen Sulfide Samples to be Analyzed Using Modified Methylene Blue Technique," presented at the Conference on Ambient Air Quality Measurements, Austin, Texas, March 1975.

Co-authored "Atmospheric Emissions Survey of the Sour Gas Industry," October 1975.

Co-authored "Technique for Insuring the Validity of Samples for High Concentrations of Sulfur Dioxide Using the EPA Method 5 Sampling Train," presented at the Third National Conference on Energy and the Environment, College Corner, Ohio, September 1975.

Teaching Experience
Conducted training seminars on sampling methods periodically since 1974 to present.

Conducted a one-day seminar on Part 75 Testing over ten times in 1993 and 1994.

Served as a lecturer in the Environmental Protection Agency's (EPA) training course number 450, "Source Sampling for Particulate Pollutants," for two years from January 1974 to October 1975 and March, 1992.

Conducted a two-day training course entitled "technical Assistance in Source Sampling" at lowa State University, Ames, lowa, for the Environmental Protection Agency (EPA), October 1974.

Conducted Environmental Protection Agency's (EPA) training course number 450, "Source Sampling for Particulate Pollutants," at Research Triangle Park, North Carolina, September 1975. 
MULLINS (p. 3)

Teaching

Experience

(Cont'd)
Technical

Experience
Conducted a two-day short course entitled "Performing and Observing Source Sampling," Dallas, Texas, July 1976, May 1977, October 1977, November 1987 and November 1988; Lake Charles, Louisiana, May 1977; Casper Wyoming, May 1977; Point Comfort, Texas, November 1992.

Served as a lecturer in the Environmental Protection Agency's twoday seminar entitled "Asphalt Industry Environmental Solutions," presented in Dallas, Texas, March 21-22, 1979.

Conducted a two-day short course entitled "Performing and Observing Source Sampling," Phoenix, Arizona, August, 1990, for the State of Arizona, Department of Environmental Quality; Lincoln, Nebraska, March 1980, for the State of Nebraska, Air Quality Control Division.

Directed and performed stack sampling on over 2000 sources of which over 500 were sampled simultaneously using more than one sampling train at several points in the flue gas stream; 1972-present.

Directed and performed over 200 short-term ambient air studies using mobile sampling vans and various ambient air sampling equipment; 1972-present.

Designed, directed and operated over 20 permanent ambient air networks of various size and duration for a variety of parameters; 1972-present.

Designed surface and underground drainage systems for residential subdivisions, public works projects, and shopping centers; 19691972.

Designed several residential subdivisions including lot layout, street design, drainage design, and utility design; 1969-1972. 
MULLINS (p. 4)

Research

Projects

Related

$\underline{\text { Projects }}$
Supervised and conducted a study made by the Hawaiian Sugar Planters' Association to characterize the emissions for several bagasse-fired boilers, April-May 1976.

Supervised and conducted a study made by the Rio Grande Valley Sugar Growers, Inc. to determine the area affected by the burning of sugarcane fields prior to harvesting, November 1974-April 1975.

Supervised and conducted a study by a lightweight aggregate manufacturer to develop a material balance around the process through sampling and analysis of several parameters, November 1973.

Conducted a study in New York City to attempt to develop a correlation in the ambient air between carbon dioxide and sulfur dioxide to provide a tool for predicting air pollution predicting air pollution episodes, January-May 1969.

Served as Chairman of the Engineering Foundation Conference on Stack Sampling and Source Evaluation in Destin, Florida, 2002, and Santa Barbara, California, 1985.

Served as Co-Chairman of the Engineering Foundation Conference on Stack Sampling and Source Evaluation in Destin, Florida, 2001.

Served as Session Chairman at the Engineering Foundation Conference on Stack Sampling and Source Evaluation in Hershey, Pennsylvania, 1984; San Diego, California, 1993; and in Palm Coast, Florida, 1994. 
BILLY L. HEFLEY; Associate

Education

Professional

Training

$\underline{\text { Certification }}$

Professional

Memberships

Technical

Experience
B.S. 1992, East Central University; Ada, Oklahoma, in Environmental Science with a concentration in Environmental Management.

Attended 40-hour Occupational and Environmental Training Program on Hazardous Materials (CFR 1910.120) Dallas, Texas, April 1993.

Also attended an 8-hour refresher course January 1994, February 1995, January 1996, and May 2004.

Attended 8-hour Safe Hazardous Materials Transportation Training Program (HM-126F and HM-181) Dallas, Texas, October 1994.

Attended Bill Mullins' Performing and Observing Source Sampling Short Course; Dallas, Texas, January 1995.

Certified Visible Emissions Evaluator

\section{Air \& Waste Management Association Source Evaluations Society}

Participated in the sampling of over 750 sources, including several of which were sampled simultaneously using more than one sampling train. Thoroughly trained in all EPA testing procedures, 1992present.

Participated in an auditing program for a permanent eight-station sulfur dioxide ambient air network in East Texas, 1992-1993.

Participated in a semi-monthly ambient air monitoring survey for organic compounds at a petrochemical facility located in South Texas, 1992-1993.

Participated in EPA's Information Collection Request for Mercury conducting more than 46 simultaneous tests for Speciated Mercury using the "Standard Test Method for Elemental, Oxidized, ParticleBound, and Total Mercury in Flue Gas Generated from Coal-Fired Stationary Sources (Ontario Hydro Method)." September 1999-May 2000. 
$\operatorname{HEFLEY~(p.~2)~}$

Experienced in the analysis of commercial calibration gas cylinders for $\mathrm{NO}_{\mathrm{x}}, \mathrm{SO}_{2}, \mathrm{CO}_{2}$, and $\mathrm{O}_{2}$.

Experience with calibration techniques for all field testing equipment.

Thoroughly trained in the operation and routine maintenance of the following:

Anarad Model AR50-C Carbon Dioxide Analyzer

Anarad Model AR880 Oxides of Nitrogen Analyzer

Anarad Model AR23 Oxygen Analyzer

Anarad Model AR30C2 Sulfur Dioxide Analyzer

California Analytical Model 300-HFID Total Hydrocarbon Analyzer

Servomex Model 1440 Carbon Dioxide Analyzer

Servomex Model 1440 Oxygen Analyzer

Teledyne Model 326 Oxygen Analyzer

Thermo Environmental Model 10AR \& 10S Oxides of Nitrogen

Analyzer

Thermo Electron Model 46C Oxides of Nitrogen Analyzer

Thermo Electron Model 48C Oxides of Nitrogen Analyzer

Western Research Model 721A Sulfur Dioxide Analyzer 
PATRICK SELAKOVICH; Associate

Education

Professional

Training

Professional

Memberships

Technical

Experience
B.S.B.A. 1992, University of Arkansas; Fayetteville, Arkansas, in General Business.

Attended 24-hour Occupational and Environmental Training Program on Hazardous Materials (CFR 1910.120) Dallas, Texas, April 1997.

Attended 40-hour Occupational and Environmental Training Program on Hazardous Materials (CFR 1910.120) \& 'Train the Trainer', Gettysburg, Pennsylvania, July 1998.

Also attended an 8-hour refresher course January 2000, January 2001, and May 2004.

Attended OSHA General Industry Safety and Health Training, May 1999.

\section{Society For Human Resource Management}

Participated in the sampling of over 150 sources, including several of which were sampled simultaneously using more than one sampling train. Thoroughly trained in all EPA testing procedures, 1996present.

Participated in EPA's 3-D probe study. May - August 1997.

Experience with calibration techniques for all field testing equipment.

Thoroughly trained in the operation and routine maintenance of the following:

California Analytical Model 300-HFID Total Hydrocarbon Analyzer Servomex Model 1440 Carbon Dioxide Analyzer

Servomex Model 1440 Oxygen Analyzer

Thermo Electron Model 46C Oxides of Nitrogen Analyzer Thermo Electron Model 48C Oxides of Nitrogen Analyzer Western Research Model 721A Sulfur Dioxide Analyzer 
SCOT JACKSON; Associate

Education

Professional

$\underline{\text { Training }}$

Certification

Technical

Experience
B.S.B.A. May 1978, Mountain View Jr. College, in General Business.

Purchasing Supervisor for METCO Environmental, Inc. in charge of inventory and supplies. January 1995 - April 2005.

Attended 40-hour Occupational and Environmental Training Program on Hazardous Materials (CFR 1910.120), Dallas, Texas, May 2000.

Attended Fed-Ex Hazardous Goods Shipping Training, June 2004.

Certified Visible Emissions Evaluator

Participated in the sampling of over 100 sources, including several of which were sampled simultaneously using more than one sampling train. Thoroughly trained in all EPA testing procedures, 1995present.

Experience with calibration techniques for all field testing equipment.

Thoroughly trained in the operation and routine maintenance of the following:

California Analytical Model 300-HFID Total Hydrocarbon Analyzer Servomex Model 1440 Carbon Dioxide Analyzer

Servomex Model 1440 Oxygen Analyzer

Thermo Electron Model 42C Oxides of Nitrogen Analyzer Thermo Electron Model 48C Oxides of Nitrogen Analyzer Western Research Model 721A Sulfur Dioxide Analyzer 


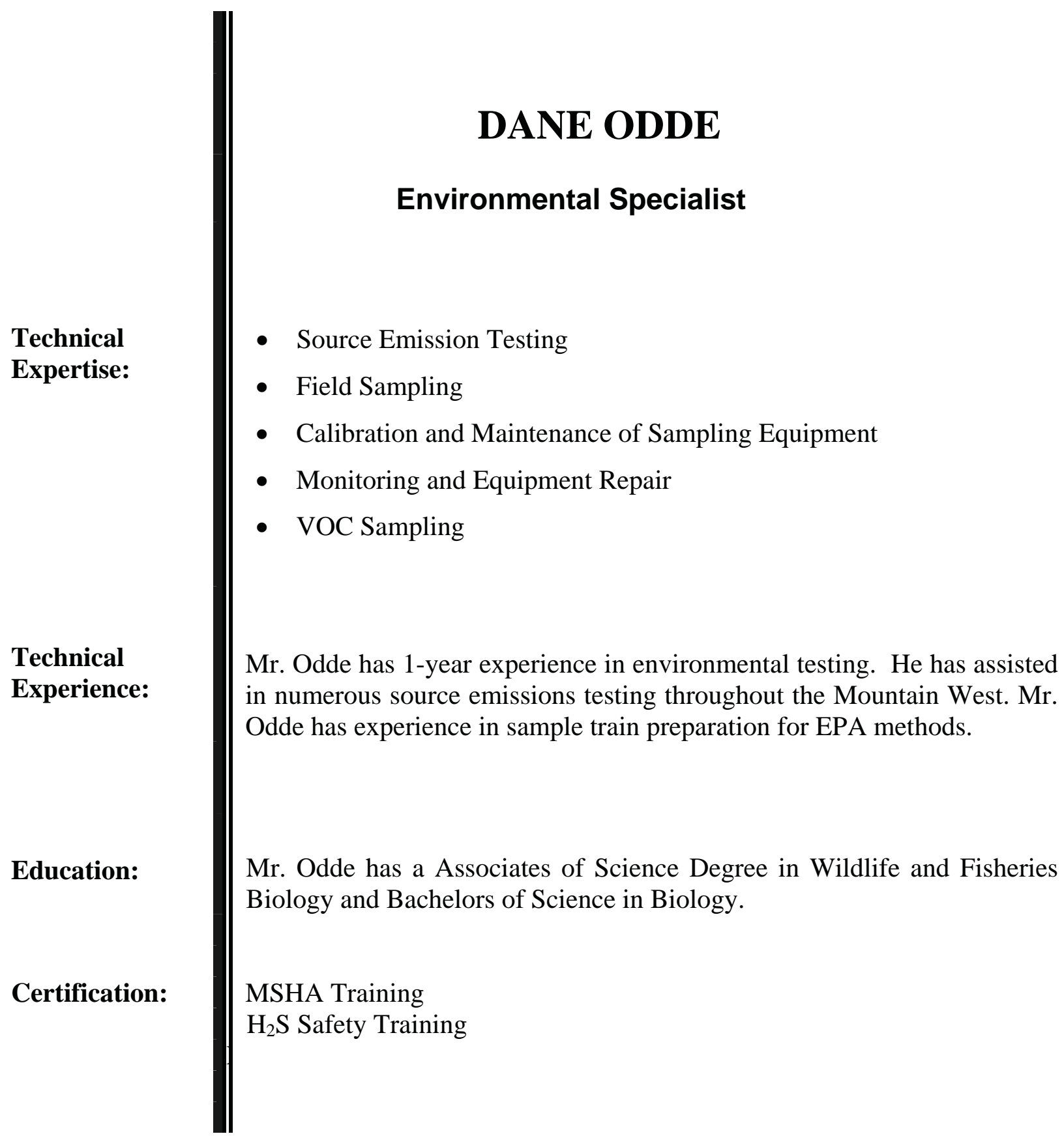




\section{STACK SAMPLING REPORT FOR}

PM10/2.5 and SULFUR TRIOXIDE TESTING ON THE

ROCKY MOUNTAIN POWER

HARDIN POWER PLANT

STACK

HARDIN, MONTANA

PROJECT NO. 07-057

SEPTEMBER 2007

PREPARED FOR:

ADA-ES, INC.

8100 SOUTHPARK WAY

LITTLETON, CO 80120

PREPARED BY:

AIR SAMPLING ASSOCIATES, INC.

P.O. BOX 1175

LEWISVILLE, TEXAS 75067

(Total Number of pages: 182) 


\section{TABLE OF CONTENTS}

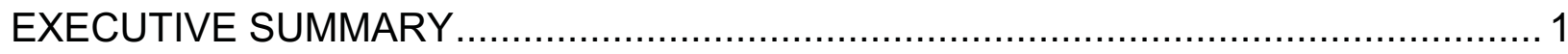

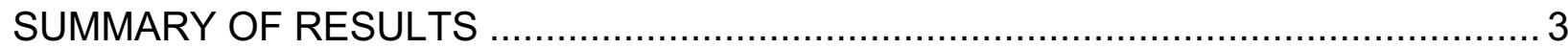

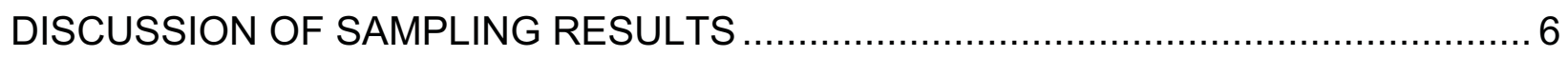

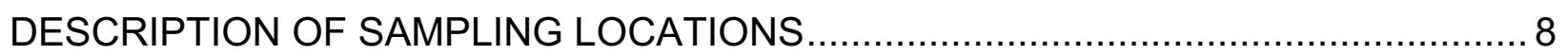

SAMPLING AND ANALYTICAL PROCEDURES …............................................ 10

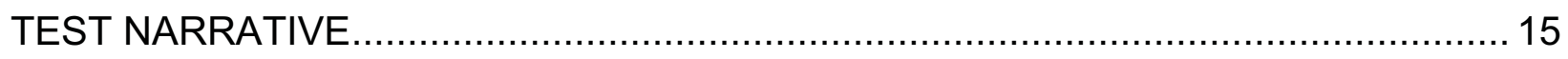

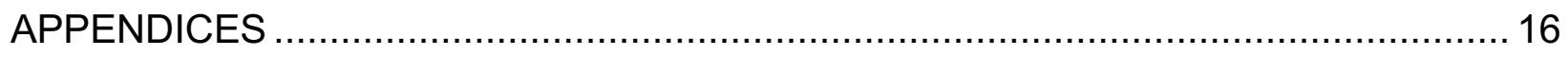

Appendix A: Location of Traverse Points ..........................................................

Appendix B: Nomenclature and Equations for Calculation of Source Emissions .......B-1

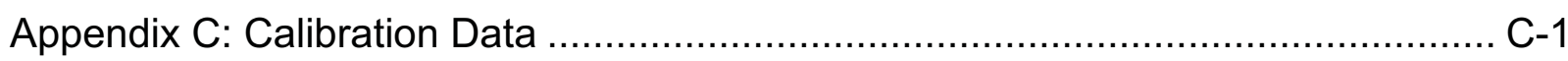

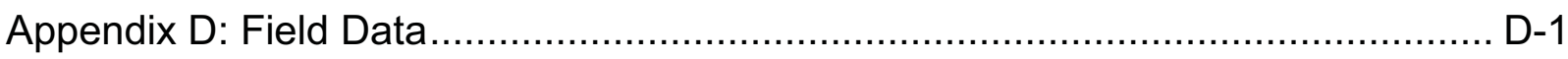

Appendix E: EPA Test Methods 201A and 202 (Particulate Matter) Analytical Data .E-1 Appendix F: Controlled Condensate Test Method (Sulfate) Analytical Data..............F-1

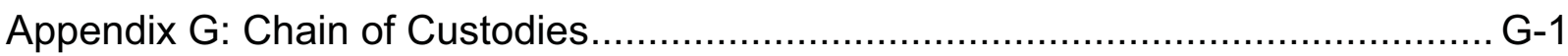

Appendix H: Resumes of Test Personnel .................................................... H-1 


\section{TABLES}

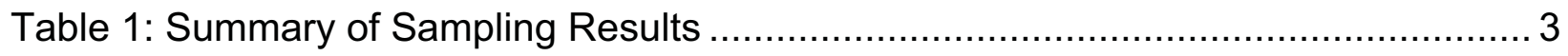

Table 2: Summary of Particulate Matter Sampling Results ........................................... 4

Table 3: Summary of Sulfur Trioxide Sampling Results ........................................... 5

Table 4: Reference Method 202 Sampling Train ................................................. 11

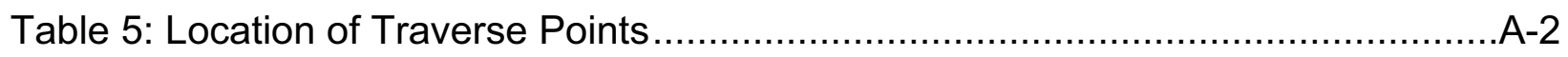

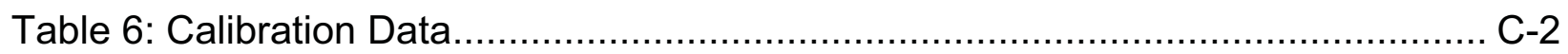

\section{FIGURES}

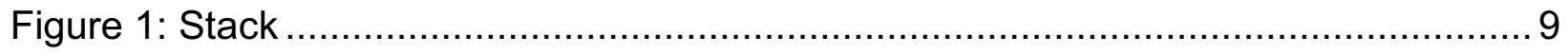

Figure 2: EPA Methods 201A and 202 Sampling Train ........................................ 12

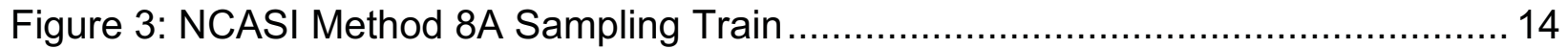

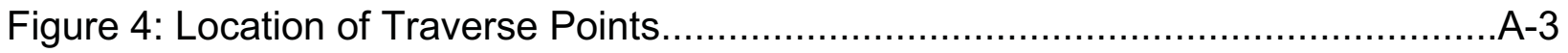




\section{EXECUTIVE SUMMARY}

Air Sampling Associates, Inc. of Lewisville, Texas conducted stack testing at the Rocky Mountain Power, Hardin Power Plant, located in Hardin, Montana. The purpose of the stack testing was to determine the particulate matter and sulfur trioxide being emitted to the atmosphere via the stack. The testing was conducted on September 5 and 6, 2007.

The sampling team consisted of Mr. Bill Mullins, Mr. Patrick Selakovich, Mr. Scot Jackson, and Mr. John Stanley. Mr. Mullins was the test team leader.

The procedures set forth in Title 40 of the Code of Federal Regulations, Part 60 (40CFR60), Appendix A, Test Methods 1, 2, 3, 4, 5, 201A and 202; and the National Council for Air and Stream Improvement, Inc. (NCASI) Method 8A were followed during the testing.

The average emission rate of particulate matter $>\mathrm{PM}_{10}$ was 0.0030 grains per dry standard cubic foot (gr/dscf). The average emission rate of particulate matter $>\mathrm{PM}_{10}$ was $7.90 \mathrm{lbs} / \mathrm{hr}$.

The average emission rate of particulate matter $<\mathrm{PM}_{10}>\mathrm{PM}_{2.5}$ was 0.0030 grains per dry standard cubic foot (gr/dscf). The average emission rate of particulate matter < $\mathrm{PM}_{10}>\mathrm{PM}_{2.5}$ was $7.90 \mathrm{lbs} / \mathrm{hr}$.

The average emission rate of particulate matter $\mathrm{PM}_{2.5}$ was 0.0023 grains per dry standard cubic foot (gr/dscf). The average emission rate of particulate matter $\mathrm{PM}_{2.5}$ was $6.31 \mathrm{lbs} / \mathrm{hr}$. 


\section{III}

The average emission rate of particulate matter total catch was 0.0494 grains per dry standard cubic foot $(\mathrm{gr} / \mathrm{dscf})$. The average emission rate of particulate matter total catch was $131.10 \mathrm{lbs} / \mathrm{hr}$.

The average $\mathrm{SO}_{3}$ concentrations at the Stack were $1.10 \mathrm{ppm}$. The average moisture content measured at the Stack using NCASI 8 A was $14.88 \%$.

Billy J. Mullins, Jr. P.E., Q.E.P., D.E.E. 


\section{SUMMARY OF RESULTS}

Table 1: Summary of Sampling Results

\begin{tabular}{|c|c|}
\hline $\begin{array}{l}\text { Parameter } \\
\text { - Results }\end{array}$ & Average \\
\hline $\begin{array}{l}\left.\text { Particulate Matter (> } \mathrm{PM}_{10}\right) \\
\text { - gr/dscf } \\
\text { - Ibs/hr }\end{array}$ & $\begin{array}{c}0.0030 \\
7.90\end{array}$ \\
\hline $\begin{array}{l}\text { Particulate Matter }\left(<\mathrm{PM}_{10}>\mathrm{PM}_{2.5}\right) \\
\text { - gr/dscf } \\
\text { - Ibs/hr }\end{array}$ & $\begin{array}{c}0.0030 \\
7.90\end{array}$ \\
\hline $\begin{array}{l}\text { Particulate Matter }\left(\mathrm{PM}_{2.5}\right) \\
\text { - gr/dscf } \\
\text { - Ibs/hr }\end{array}$ & $\begin{array}{c}0.0023 \\
6.31\end{array}$ \\
\hline $\begin{array}{l}\text { Particulate Matter (Total Catch) } \\
\text { - gr/dscf } \\
\text { - Ibs/hr }\end{array}$ & $\begin{array}{l}0.0494 \\
131.10\end{array}$ \\
\hline $\begin{array}{l}\text { Sulfur Trioxide } \\
\text { - ppm }\end{array}$ & 1.10 \\
\hline
\end{tabular}




\section{SAMPLING}

\section{SUMMARY OF RESULTS}

Table 2: Summary of Particulate Matter Sampling Results

\begin{tabular}{|c|c|c|c|c|}
\hline Run No. & 1 & 2 & 3 & Average \\
\hline Test Date & 09/06/07 & $09 / 06 / 07$ & 09/06/07 & $\begin{array}{l}---- \\
\end{array}$ \\
\hline Test Time & $1021-1124$ & $1310-1412$ & $1537-1641$ & ----- \\
\hline Flow Rate - DSCFM* & 307,112 & 307,778 & 318,655 & 311,182 \\
\hline Stack Temp. - ${ }^{\circ} \mathrm{F}$ & 227 & 217 & 217 & 220 \\
\hline $\mathrm{O}_{2}$ - \% Vol. (dry) & 6.7 & 6.7 & 6.8 & 6.7 \\
\hline $\mathrm{CO}_{2}-\%$ Vol. (dry) & 12.8 & 12.8 & 12.7 & 12.8 \\
\hline Percent Excess Air & 45.8 & 45.8 & 46.8 & 46.1 \\
\hline Moisture Content - \% & 15.12 & 15.38 & 15.05 & 15.18 \\
\hline Percent Isokinetic & 86.6 & 85.7 & 83.6 & 85.3 \\
\hline $\begin{array}{l}\text { Particulate Matter (> PM } 10) \\
\text { - gr/dscf } \\
\text { - lbs/hr }\end{array}$ & $\begin{array}{c}0.0046 \\
12.06\end{array}$ & $\begin{array}{c}0.0013 \\
3.38\end{array}$ & $\begin{array}{c}0.0030 \\
8.27\end{array}$ & $\begin{array}{c}0.0030 \\
7.90\end{array}$ \\
\hline $\begin{array}{l}\text { Particulate Matter }\left(<\mathrm{PM}_{10}>\mathrm{PM}_{2.5}\right) \\
\text { - gr/dscf } \\
\text { - lbs/hr }\end{array}$ & $\begin{array}{c}0.0037 \\
9.84\end{array}$ & $\begin{array}{c}0.0026 \\
6.75\end{array}$ & $\begin{array}{c}0.0026 \\
7.11\end{array}$ & $\begin{array}{c}0.0030 \\
7.90\end{array}$ \\
\hline $\begin{array}{l}\text { Particulate Matter }\left(\mathrm{PM}_{2.5}\right) \\
\text { - gr/dscf } \\
\text { - lbs/hr }\end{array}$ & $\begin{array}{c}0.0006 \\
1.67\end{array}$ & $\begin{array}{c}0.0006 \\
1.50\end{array}$ & $\begin{array}{c}0.0058 \\
15.77\end{array}$ & $\begin{array}{c}0.0023 \\
6.31\end{array}$ \\
\hline $\begin{array}{l}\text { Particulate Matter (Total Catch) } \\
\text { - gr/dscf } \\
\text { - lbs/hr }\end{array}$ & $\begin{array}{l}0.0465 \\
122.30\end{array}$ & $\begin{array}{l}0.0737 \\
194.47\end{array}$ & $\begin{array}{c}0.0280 \\
76.52\end{array}$ & $\begin{array}{l}0.0494 \\
131.10\end{array}$ \\
\hline
\end{tabular}

* 29.92" Hg, $68^{\circ} \mathrm{F}\left(760 \mathrm{~mm} \mathrm{Hg}, 20^{\circ} \mathrm{C}\right)$ 


\section{SUMMARY OF RESULTS}

Table 3: Summary of Sulfur Trioxide Sampling Results

\begin{tabular}{|c|c|c|c|c|}
\hline Run No. & 1 & 2 & 3 & Average \\
\hline Test Date & 09/06/07 & 09/06/07 & 09/06/07 & ----- \\
\hline Test Time & 1038-1138 & 1359-1459 & 1529-1629 & ----- \\
\hline $\mathrm{O}_{2}-\%$ Vol. (dry) & 6.7 & 6.7 & 6.8 & 6.7 \\
\hline $\mathrm{CO}_{2}-\%$ Vol. (dry) & 12.8 & 12.8 & 12.7 & 12.8 \\
\hline Percent Excess Air & 45.8 & 45.8 & 46.8 & 46.1 \\
\hline Moisture Content - \% & 14.82 & 14.94 & 14.87 & 14.88 \\
\hline Sample Volume - DSCM & 0.516 & 0.495 & 0.513 & 0.508 \\
\hline $\begin{array}{l}\text { Sulfur Trioxide } \\
\quad-\mu g \\
-p p m\end{array}$ & $\begin{array}{l}499 \\
0.49 \\
\end{array}$ & $\begin{array}{c}1,349 \\
1.32 \\
\end{array}$ & $\begin{array}{c}1,537 \\
1.49 \\
\end{array}$ & $\begin{array}{c}1,128 \\
1.10 \\
\end{array}$ \\
\hline
\end{tabular}

* 29.92" Hg, 68 ${ }^{\circ} \mathrm{F}\left(760 \mathrm{~mm} \mathrm{Hg}, 20^{\circ} \mathrm{C}\right)$ 


\section{DISCUSSION OF SAMPLING RESULTS}

\section{$\underline{\text { Particulate Matter }}$}

The three tests for particulate matter appeared to be an accurate representation of the actual emissions during the tests. All leak checks performed on the sampling train and the pitot tubes indicated no leaks before or after each test. The indicative parameters calculated from the field data were in reasonable agreement. The measured moisture contents for the three runs were within $1.3 \%$ of the mean value. The measured flow rates (DSCFM) for the tests were within $2.4 \%$ of the mean value. The rates of sampling for the three tests were within the specified limits ( 80 to 120 percent isokinetic). The greatest deviation from $100 \%$ isokinetic was $16.4 \%$. The particulate cut size was 9.17 microns, which was within the specified limits in EPA 201A of 9 to 11 microns.

The concentrations ( $\mathrm{gr} / \mathrm{dscf}$ ) of particulate matter $>\mathrm{PM}_{10}$ for the three tests showed a range of -56.18 percent to +55.06 percent variation from the mean value of 0.0030 gr/dscf.

The concentrations (gr/dscf) of particulate matter $<\mathrm{PM}_{10}>\mathrm{PM}_{2.5}$ for the three tests showed a range of -12.36 percent to +24.72 percent variation from the mean value of $0.0030 \mathrm{gr} / \mathrm{dscf}$.

The concentrations ( $\mathrm{gr} / \mathrm{dscf}$ ) of particulate matter $\mathrm{PM}_{2.5}$ for the three tests showed a range of -74.29 percent to +148.57 percent variation from the mean value of 0.0023 gr/dscf. 


\section{II}

The concentrations (gr/dscf) of particulate matter total catch for the three tests showed a range of -43.32 percent to +49.19 percent variation from the mean value of 0.0494 gr/dscf.

\section{Sulfur Trioxide}

The three tests for sulfur trioxide appeared to be a valid representation of the actual emissions during the tests. All leak checks performed on the reference method sampling train showed no leaks before or after testing. The measured moisture contents were within $0.4 \%$ of the mean value.

The concentrations (ppm) of sulfur trioxide for the three tests showed a range of -55.45 percent to +35.45 percent variation from the mean value of $1.10 \mathrm{ppm}$. 


\section{DESCRIPTION OF SAMPLING LOCATIONS}

The sampling ports on the Stack are approximately 123 feet $11 / 2$ inches above the ground. The sampling ports are located 33 feet $11 / 2$ inches (3.64 stack diameters) downstream from a constriction in the stack and $>18$ feet $23 / 8$ inches $(>2.00$ stack diameters) upstream from the outlet to the stack. 


\section{SAMPLING LOCATION}

Figure 1: Stack

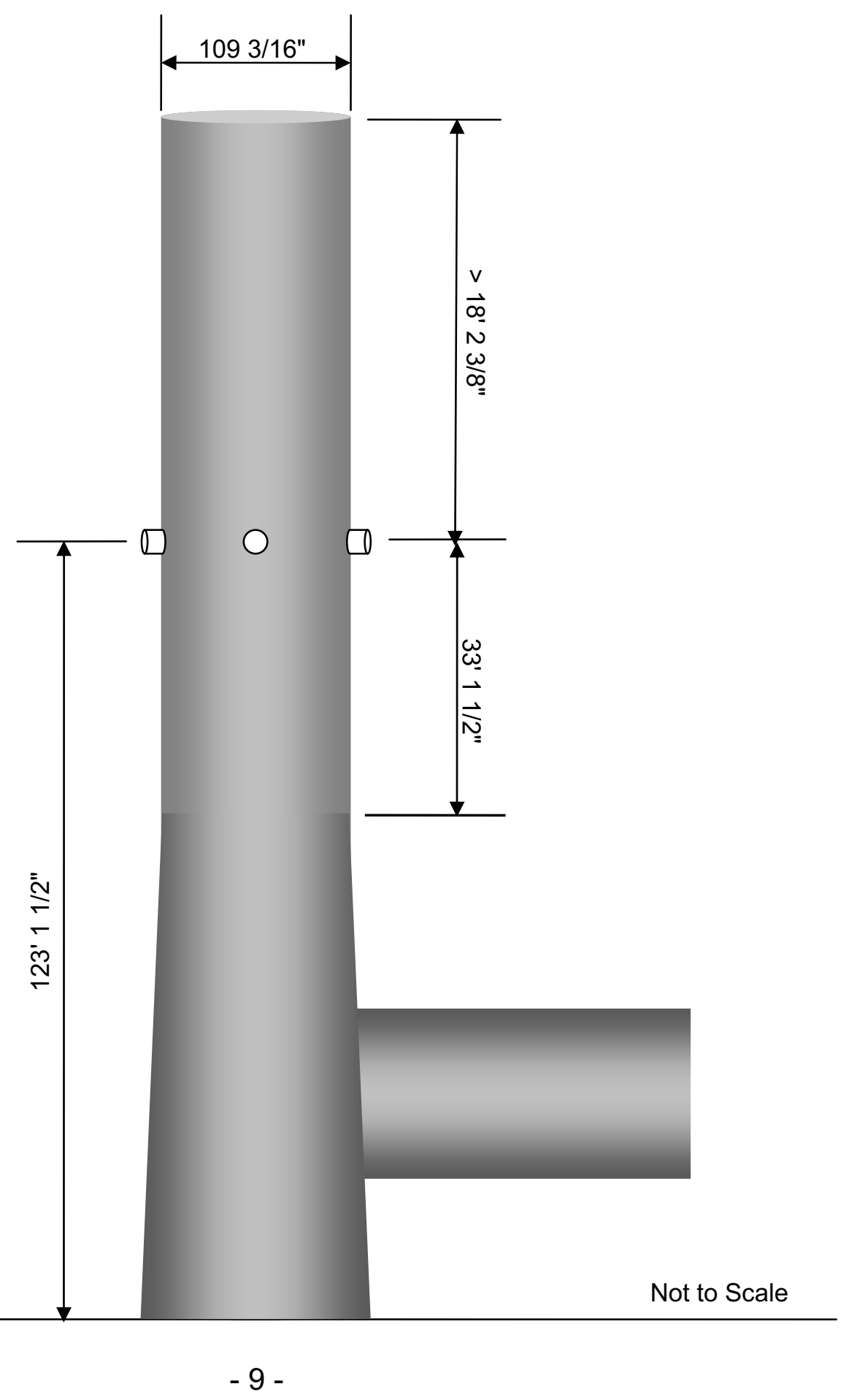




\section{SAMPLING AND ANALYTICAL PROCEDURES}

The procedures set forth in 40CFR60, Appendix A, Test Methods 1, 2, 3, 4, 5, 201A and 202; and NCASI Method 8A were followed during testing.

\section{Particulate Matter}

Six traverse points were sampled from each of the two ports on the stack for a total of twelve traverse points. All traverse points were previously checked for cyclonic flow and none was found to be present. For each run, samples of varying dwell time were taken at each of the twelve traverse points for a total sampling time of 60 minutes. The dwell time at each sampling point was proportional with the velocity at that point. Data was recorded at the beginning of each sample point.

The pitot tube lines were checked for leaks before and after each test under a vacuum and a pressure. The lines were also checked for clearance and the manometer was zeroed before each test.

The sampling train was leak checked at the end of the sampling probe at 15" of mercury vacuum before each test, and again at the conclusion of each test at the highest vacuum recorded during sampling. This was done to predetermine the possibility of a diluted sample.

The "front-half" of the sampling train contained the following components:

Stainless steel nozzle

$\mathrm{PM}_{10}$ Sampler

$\mathrm{PM}_{2.5}$ Sampler

Heated glass lined probe@ $250^{\circ} \mathrm{F} \pm 25^{\circ} \mathrm{F}$

Heated Teflon sample line @ $250^{\circ} \mathrm{F} \pm 25^{\circ} \mathrm{F}$ 


\section{III}

The "back-half" of the sampling train contained the following components:

Table 4: Reference Method 202 Sampling Train

\begin{tabular}{|ccccc|}
\hline Impinger No. & $\begin{array}{c}\text { Impinger } \\
\text { Type }\end{array}$ & $\begin{array}{c}\text { Impinger } \\
\text { Contents }\end{array}$ & Amount & $\begin{array}{c}\text { Parameter } \\
\text { Collected }\end{array}$ \\
\hline $\mathbf{1}$ & Modified & H.P.L.C. $\mathrm{H}_{2} \mathrm{O}$ & $100 \mathrm{ml}$ & $\mathrm{H}_{2} \mathrm{O}$ \\
\hline $\mathbf{2}$ & Greenburg-Smith & H.P.L.C. $\mathrm{H}_{2} \mathrm{O}$ & $100 \mathrm{ml}$ & $\mathrm{H}_{2} \mathrm{O}$ \\
\hline $\mathbf{3}$ & Modified & Empty & ----- & $\mathrm{H}_{2} \mathrm{O}$ \\
\hline $\mathbf{4}$ & Modified & Silica Gel & $250 \mathrm{~g}$ & $\mathrm{H}_{2} \mathrm{O}$ \\
\hline
\end{tabular}

At the completion of each run, the "back-half" of the sampling train was purged with nitrogen for 60 minutes at a rate of 20 liters per minute. 
Figure 2: EPA Methods 201A and 202 Sampling Train
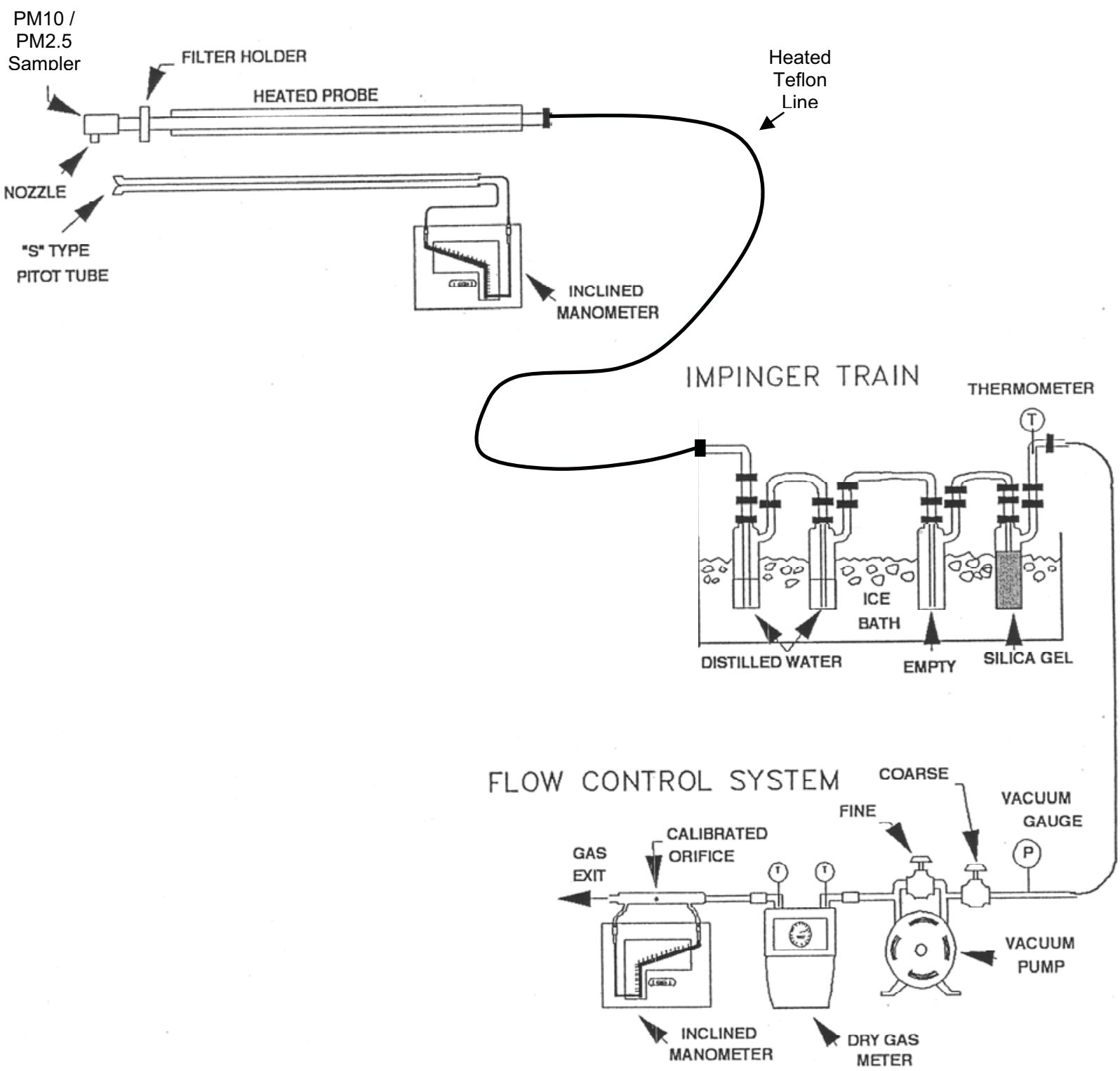


\section{III}

\section{Sulfur Trioxide}

All samples were taken at a single traverse point for a total sampling time of 60 minutes. Data was recorded at 5-minute intervals.

The sampling train was leak-checked at the end of the sampling probe at 15" of mercury vacuum before each test, and again at the conclusion of each test at the highest vacuum recorded during sampling. This was done to predetermine the possibility of a diluted sample.

The 'front-half' of the sampling train contained the following components:

Glass Sample Probe heated to $500^{\circ} \mathrm{F}$

Quartz Filter heated to $500^{\circ} \mathrm{F}$

Glass Condenser cooled to $140^{\circ} \mathrm{F}$

Teflon tubing connected to Impingers

The 'back-half' of the sampling train contained the following components:

\begin{tabular}{|c|c|c|}
\hline \multicolumn{3}{|c|}{ Condenser/Absorbing System } \\
\hline Impinger No. & Impinger Type & Absorbing Solution \\
\hline 1 & Modified & $100 \mathrm{ml} 3 \% \mathrm{H}_{2} \mathrm{O}_{2}$ \\
\hline 2 & Greenburg-Smith & $100 \mathrm{ml} 3 \% \mathrm{H}_{2} \mathrm{O}_{2}$ \\
\hline 3 & Modified & $100 \mathrm{ml} \mathrm{DI} \mathrm{H} \mathrm{H}_{2} \mathrm{O}$ \\
\hline 4 & Modified & $\sim 200 \mathrm{~g}$ silica gel \\
\hline
\end{tabular}




\section{AIR

Figure 3: NCASI Method 8A Sampling Train

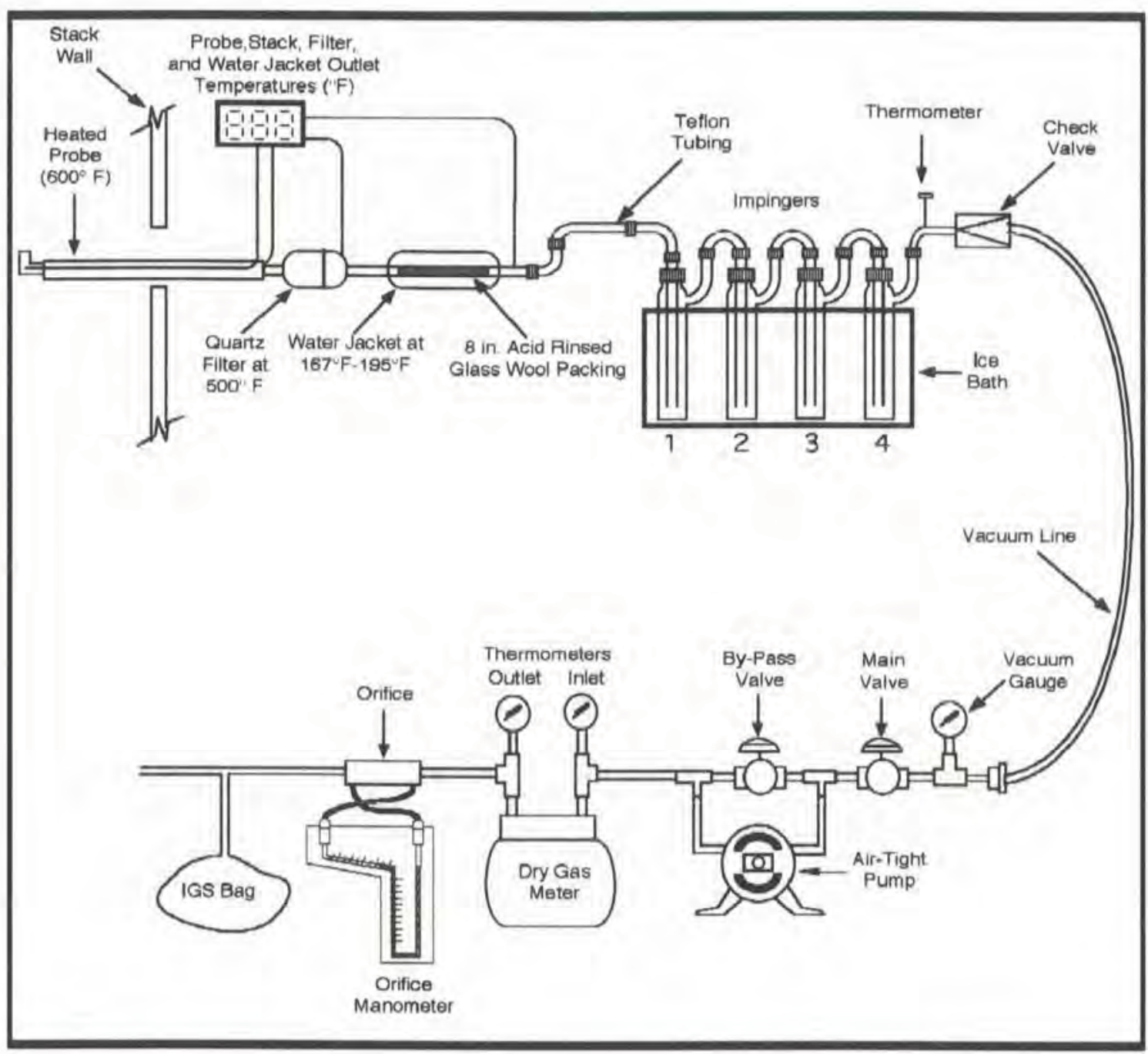




\section{TEST NARRATIVE}

Personnel from Air Sampling Associates, Inc arrived at the Rocky Mountain Power, Hardin Power Plant located in Hardin, Montana at 2:15 p.m. on Wednesday, September 5, 2007. Electrical power was established to the sampling trailer and the sampling equipment was moved onto the stack. Personnel departed the plant at 6:00 p.m.

On Thursday, September 6, 2007, personnel returned to the plant at 6:45 a.m. The sampling equipment was prepared for sampling and the first test for $\mathrm{PM}_{10}$ and $\mathrm{PM}_{2.5}$ began at 10:21 a.m. The first test utilizing the controlled condensate sampling procedure began at 10:38 a.m. Sampling continued until the completion of the third test for $\mathrm{PM}_{10}$ and $\mathrm{PM}_{2.5}$ at 4:41 p.m. and the completion of the third controlled condensate teat at 4:29 p.m.

The sampling equipment was moved off of the stack and loaded into the sampling trailer. The samples were recovered and taken to Air Sampling Associates, Inc.'s office in Lewisville, Texas for analysis and evaluation.

Operations at the Rocky Mountain Power, Harding Power Plant, located in Hardin, Montana, for ADA-ES, were completed at 6:30 p.m. on Thursday, September 6, 2007. 


\section{APPENDICES}

Appendix A: Location of Traverse Points

Appendix B: Nomenclature and Equations for Calculation of Source Emissions

Appendix C: Calibration Data

Appendix D: Field Data

Appendix E: EPA Test Methods 201A and 202 (Particulate Matter) Analytical Data

Appendix F: Controlled Condensate Test Method (Sulfate) Analytical Data

Appendix G: Chain of Custodies

Appendix H: Resumes of Test Personnel 


\section{Appendix A:}

Location of Traverse Points 


\section{Appendix A}

\section{Location of Traverse Points}

The sampling ports are located 33 feet 1 1/2 inches (3.64 stack diameters) downstream from a constriction in the stack and $>18$ feet $23 / 8$ inches ( $>2.00$ stack diameters) upstream from the outlet to the stack. The locations of the traverse points were calculated as follows:

Table 5: Location of Traverse Points

\begin{tabular}{|c|c|c|}
\hline \multicolumn{3}{|c|}{ Port \& Wall Thickness $=159 / 16$ inches } \\
\hline $\begin{array}{l}\text { Point } \\
\text { Number }\end{array}$ & $\begin{array}{c}\text { Percent of } \\
\text { Stack Diameter }\end{array}$ & $\begin{array}{l}\text { Distance } \\
\text { from Wall }\end{array}$ \\
\hline 1 & 4.4 & $413 / 16^{\prime \prime}$ \\
\hline 2 & 14.6 & 15 15/16" \\
\hline 3 & 29.6 & $325 / 16^{\prime \prime}$ \\
\hline 4 & 70.4 & 76 7/8" \\
\hline 5 & 85.4 & $931 / 4 "$ \\
\hline 6 & 95.6 & 104 3/8" \\
\hline
\end{tabular}




\section{III}

\section{Appendix A}

Figure 4: Location of Traverse Points

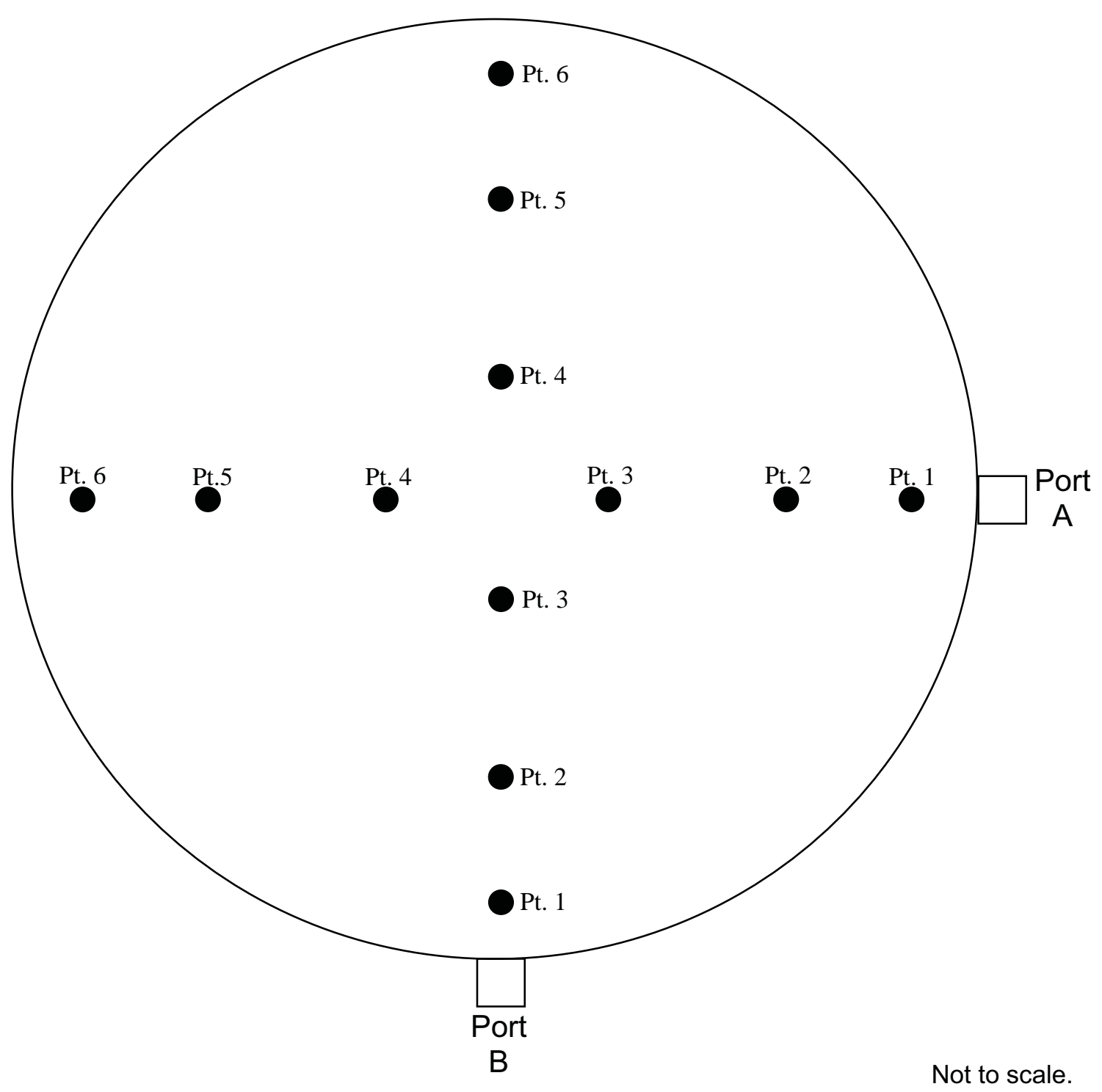




\section{Appendix B:}

Nomenclature and Equations for Calculation of Source Emissions 


\section{Nomenclature For Flow Rate and Moisture Calculations}

\begin{tabular}{|c|c|c|c|}
\hline Symbol & $\begin{array}{l}\text { English } \\
\underline{\text { Units }}\end{array}$ & $\begin{array}{l}\text { Metric } \\
\underline{\text { Units }}\end{array}$ & Description \\
\hline$A_{s}$ & in. $^{2}$ & $\mathrm{~m}^{2}$ & Stack Area \\
\hline $\mathrm{C}_{\mathrm{an}}$ & $\mathrm{gr} / \mathrm{dscf}{ }^{*}$ & $\mathrm{~g} / \mathrm{dscm}^{*}$ & $\begin{array}{l}\text { Particulate - probe, cyclone, } \\
\text { and filter }\end{array}$ \\
\hline $\mathrm{C}_{\mathrm{ao}}$ & $\mathrm{gr} / \mathrm{dscf}{ }^{*}$ & $\mathrm{~g} / \mathrm{dscm}^{*}$ & Particulate -total \\
\hline $\mathrm{C}_{\text {at }}$ & $\begin{array}{c}\mathrm{gr} / \mathrm{CF} @ \\
\text { stack conditions }\end{array}$ & $\mathrm{g} / \mathrm{m} 3$ & $\begin{array}{l}\text { Particulate - probe, cyclone, } \\
\text { and filter }\end{array}$ \\
\hline $\mathrm{C}_{\mathrm{au}}$ & $\begin{array}{c}\mathrm{gr} / \mathrm{CF} @ \\
\text { stack conditions }\end{array}$ & $\mathrm{g} / \mathrm{m} 3$ & Particulate - total \\
\hline $\mathrm{C}_{\mathrm{aw}}$ & lbs/hr & $\mathrm{kg} / \mathrm{hr}$ & $\begin{array}{l}\text { Particulate - probe, cyclone, } \\
\text { and filter }\end{array}$ \\
\hline $\mathrm{C}_{\mathrm{ax}}$ & $\mathrm{lbs} / \mathrm{hr}$ & $\mathrm{kg} / \mathrm{hr}$ & Particulate - total \\
\hline$C_{p}$ & & & Pitot Tube Calibration Factor \\
\hline$D_{n}$ & in. & $\mathrm{m}$ & Sampling Nozzle Diameter \\
\hline$\% \mathrm{EA}$ & & & $\begin{array}{l}\text { Percent Excess Air at } \\
\text { Sampling Point }\end{array}$ \\
\hline g & $32.2 \mathrm{ft} / \mathrm{sec}^{2}$ & & Acceleration of gravity \\
\hline$\% l$ & & & Percent Isokinetic \\
\hline$\% \mathrm{M}$ & & & $\begin{array}{l}\text { Percent Moisture in the Stack } \\
\text { Gas by Volume }\end{array}$ \\
\hline$M_{d}$ & & & Mole Fraction of Dry Gas \\
\hline$m_{f}$ & $\mathrm{mg}$ & $\mathrm{mg}$ & $\begin{array}{l}\text { Particulate - probe, cyclone, } \\
\text { and filter }\end{array}$ \\
\hline $\mathrm{mt}$ & $\mathrm{mg}$ & $\mathrm{mg}$ & Particulate -total \\
\hline$M_{\text {water }}$ & $18 \mathrm{lb} / \mathrm{lb}-\mathrm{mole}$ & & Molecular Weight of Water \\
\hline MW & Ib/lb-mole & g/g-mole & $\begin{array}{l}\text { Molecular Weight of Stack } \\
\text { Gas }\end{array}$ \\
\hline $\mathrm{MW}_{\text {air }}$ & $28.84 \mathrm{lb} / \mathrm{lb}-\mathrm{mole}$ & & Molecular Weight of Air \\
\hline $\mathrm{MW}_{\mathrm{d}}$ & lb/lb-mole & g/g-mole & $\begin{array}{l}\text { Molecular Weight of Dry Stack } \\
\text { Gas }\end{array}$ \\
\hline
\end{tabular}


Symbol

$P_{b}$

$P_{m}$

Ps

$\Delta \mathrm{P}$

$\mathrm{P}_{\text {std }}$

$\mathrm{Q}_{a}$

$Q_{s}$

R

$T_{m}$

$T_{t}$

$\mathrm{T}_{\mathrm{s}}$

$\mathrm{T}_{\text {std }}$

$V_{m}$

$V \mathrm{~m}_{\text {std }}$

$\mathrm{V}_{\mathrm{s}}$

$\mathrm{V}_{\mathrm{w}}$

$V w_{\text {gas }}$
English

Units

"Hg Absolute

$" \mathrm{H}_{2} \mathrm{O}$

"Hg Absolute

$" \mathrm{H}_{2} \mathrm{O}$

29.92" $\mathrm{Hg}$

ACFM

DSCFM $^{*}$

21.83" Hg-

$\mathrm{ft}^{3} / \mathrm{lb}$-mole ${ }^{\circ} \mathrm{R}$

${ }^{\circ} \mathrm{F}$

$\min$

${ }^{\circ} \mathrm{F}$

$528^{\circ} \mathrm{R}$

$\mathrm{ft}^{3}$

dscf* $^{*}$

fpm

$\mathrm{ml}$

$\operatorname{scf}^{*}$
Metric

$\underline{\text { Units }}$

$\mathrm{mm} \mathrm{Hg}$

$\mathrm{mm} \mathrm{H}_{2} \mathrm{O}$

$\mathrm{mm} \mathrm{Hg}$

$\mathrm{mm} \mathrm{H}_{2} \mathrm{O}$

$760 \mathrm{~mm} \mathrm{Hg}$

$\mathrm{m}^{3} / \mathrm{hr}$

$\mathrm{dscm} / \mathrm{hr} r^{*}$

${ }^{\circ} \mathrm{C}$

$\min$

${ }^{\circ} \mathrm{C}$

$293^{\circ} \mathrm{K}$

$\mathrm{m}^{3}$

$\mathrm{dscm}^{*}$

$\mathrm{m} / \mathrm{sec}$

$\mathrm{ml}$

$\mathrm{scm}^{*}$
Description

Barometric Pressure

Orifice Pressure drop

Stack Pressure

Velocity Head of Stack Gas

Standard Barometric Pressure

Stack Gas Volume at Actual Stack Conditions

Stack Gas Volume at 29.92"

$\mathrm{Hg}, 528^{\circ} \mathrm{R}$, dry

Universal Gas Constant

Average Gas Meter

Temperature

Net Time of Test

Stack Temperature

Standard Temperature

Volume of Dry Gas Sampled

@ Meter Conditions

Volume of Dry Gas Sampled @ Standard Conditions

Stack Velocity @ Stack Conditions

Total Water Collected in Impingers and Silica Gel

Volume of Water Vapor Collected @ Standard Conditions 
Symbol

$\rho_{\text {air }}$

$\rho_{\text {water }}$

$\rho_{\text {man }}$
English

Units

$0.0748 \mathrm{lbs} / \mathrm{ft}^{3}$

$1 \mathrm{~g} / \mathrm{ml}$

$62.32 \mathrm{lbs} / \mathrm{ft}^{3}$
Metric

$\underline{\text { Units }}$

Description

Density of Air

Density of Water

Density of Manometer Oil

(Inches of Water)

Standard Conditions: $29.92 " \mathrm{Hg}, 68^{\circ} \mathrm{F}\left(760 \mathrm{~mm} \mathrm{Hg}, 20^{\circ} \mathrm{C}\right)$ 


\section{EXAMPLE CALCULATIONS}

1. Volume of dry gas sampled at standard conditions. *

$$
\begin{aligned}
& V_{m s t d}=V_{m}\left(\frac{T_{s t d}}{T_{m}+460}\right)\left[\frac{P_{b}+\frac{P_{m}}{13.6}}{P_{s t d}}\right] \\
& V_{m s t d}=17.65 V_{m}\left[\frac{P_{b}+\frac{P_{m}}{13.6}}{T_{m}+460}\right]=d s c f \\
& V_{m s d}=d s c f \times 0.028317=d s c m
\end{aligned}
$$

2. Volume of water vapor collected at standard conditions. *

$$
\begin{aligned}
& V_{w_{\text {gas }}}=\frac{\left(V_{w}-g m s \mathrm{SO}_{2}-g m s \mathrm{H}_{2} S\right) \rho_{\text {water }} R T_{\text {std }}}{P_{\text {std }} M_{\text {water }} 453.6} \\
& V_{w_{\text {gas }}}=0.0472\left(V_{w}-g m s \mathrm{SO}_{2}-g m s \mathrm{H}_{2} S\right)=s c f \\
& V_{w_{\text {gas }}}=s c f \times 0.028317=s c m
\end{aligned}
$$

3. Percent moisture in stack gas.

$$
\% M=\frac{V_{\text {wgas }}}{V_{m_{s t d}}+V_{w_{g a s}}} \times 100=\%
$$


4. Mole fraction of dry gas.

$$
M_{d}=\frac{100-\% M}{100}
$$

5. Average molecular weight of dry stack gas.

$$
\begin{aligned}
M W_{d}=\left[\% \mathrm{CO}_{2} \times \frac{44}{100}\right]+\left[\% \mathrm{O}_{2} \times \frac{32}{100}\right]+\left[\% \mathrm{~N}_{2} \times \frac{28}{100}\right]+\left[\% \mathrm{CO} \times \frac{28}{100}\right] & =\mathrm{lb} / \mathrm{lb}-\text { mole } \\
& =\mathrm{g} / \mathrm{g}-\mathrm{mole}
\end{aligned}
$$

6. Molecular weight of stack gas.

$$
M W=M W_{d} \times M_{d}+18\left(1-M_{d}\right)=\frac{l b}{l b-m o l e}=g / g-\text { mole }
$$

7. Percent excess air at sampling point.

$$
\% E A=\frac{100\left[\% \mathrm{O}_{2}-(0.5 \% \mathrm{CO})\right]}{0.265\left(\% \mathrm{~N}_{2}\right)-\left[\% \mathrm{O}_{2}-(0.5 \% \mathrm{CO})\right]}
$$

8. Stack Pressure.

$$
P_{s}=P_{b}+\frac{\text { Stack Pressure " } \mathrm{H}_{2} \mathrm{O}}{13.6}=" \mathrm{Hg} \text { Absolute }
$$

$$
P_{s}=" H g \text { Abs. } \times 25.4=m m H g
$$

9. Stack velocity at stack conditions.

$$
\begin{aligned}
& V_{s}=C_{p} 60\left[\frac{2 g \times \rho_{\text {man }} \times P_{\text {std }} \times M W_{\text {air }} \times\left(T_{s}+460\right) \times \Delta P}{12 \times \rho_{\text {air }} \times P_{s} \times M W \times T_{\text {std }}}\right]^{1 / 2} \\
& V_{s}=5,123.8 C_{p}\left[\frac{\left(T_{s}+460\right)}{P_{s} \times M W}\right]^{1 / 2} \sqrt{\Delta P} \text { average }=\mathrm{fpm} \\
& V_{s}=f p m \times 0.00508=\mathrm{m} / \mathrm{sec}
\end{aligned}
$$


10. Dry stack gas volume at standard conditions. *

$$
\begin{aligned}
& Q_{s}=\frac{1}{144} V_{s} \times A_{s} \times M_{d} \times \frac{T_{s t d}}{T_{s}+460} \times \frac{P_{s}}{P_{s t d}} \\
& Q_{s}=\frac{0.123 V_{s} \times A_{s} \times M_{d} \times P_{s}}{T_{s}+460}=D S C F M \\
& Q_{s}=D S C F M \times 1.6990=d s c m / h r
\end{aligned}
$$

11. Actual stack gas volume at stack conditions.

$$
\begin{aligned}
& Q_{a}=\frac{V_{s} \times A_{s}}{144}=A C F M \\
& Q_{a}=A C F M \times 1.6990=m^{3} / h r
\end{aligned}
$$

12. Percent Isokinetic

$$
\begin{aligned}
& \% \mathrm{l}=\frac{V_{\text {mstd }} \times\left(T_{s}+460\right) \times P_{s t d} \times 100 \times 144 i n^{2} / f^{2}}{M_{d} \times T_{s t d} \times P_{s} \times T_{t} \times V_{s}\left(\frac{\Pi \times D_{n}{ }^{2}}{4}\right)} \\
& \% \mathrm{I}=\frac{1039 \times V_{\text {sstd }} \times\left(T_{s}+460\right)}{M_{d} \times P_{s} \times T_{t} \times V_{s} \times D_{n}{ }^{2}}
\end{aligned}
$$

* 29.92" Hg, $68^{\circ} \mathrm{F}\left(760 \mathrm{~mm} \mathrm{Hg}, 20^{\circ} \mathrm{C}\right)$ 
13. Particulate - Probe, cyclone, and filter.

$$
\begin{aligned}
& \mathrm{C}_{\mathrm{an}}=\frac{\mathrm{m}_{\mathrm{f}}}{\mathrm{V}_{\mathrm{mstd}}} \times \frac{1 \mathrm{gr}}{64.8 \mathrm{mg}} \\
& C_{a n}=0.0154 \times \frac{\mathrm{mf}}{\mathrm{V}_{\mathrm{mstd}}} \mathrm{gr} / \mathrm{dscf} \text { * } \\
& \mathrm{C}_{\text {an }}=\mathrm{gr} / \mathrm{dscf} \times 2.290=\mathrm{g} / \mathrm{dscm} \text { * }
\end{aligned}
$$

14. Particulate total.

$$
\begin{aligned}
& \mathrm{C}_{\mathrm{ao}}=0.0154 \times \frac{\mathrm{m}_{\mathrm{t}}}{\mathrm{V}_{\text {mstd }}}=g r / d s c f \text { * } \\
& \mathrm{C}_{\mathrm{ao}}=\mathrm{gr} / \mathrm{dscf} \times 2.290=\mathrm{g} / \mathrm{dscm} \text { * }
\end{aligned}
$$

15. Particulate - probe, cyclone, and filter at stack conditions.

$$
\begin{aligned}
& C_{a t}=C_{a n} \times \frac{P_{s}}{P_{\text {std }}} \times \frac{\left(T_{s t d}\right)}{\left(T_{s}+460\right)} \times M_{d} \\
& C_{a t}=\frac{17.65 \times C_{a n} \times P s \times M d}{T x+460}=g r / C F \\
& C_{a t}=g r / C F \times 2.290=g / m^{3}
\end{aligned}
$$

16. Particulate - total, at stack conditions.

$$
\begin{aligned}
& C_{a u}=\frac{17.65 \times C_{a o} \times P_{s} \times M_{d}}{T_{s}+460}=g r / C F \\
& C_{a u}=g r / C F \times 2.290=g / m^{3}
\end{aligned}
$$




\section{II}

17. Particulate - probe, cyclone, and filter.

$$
\begin{aligned}
& C_{a w}=C_{a n} \times Q_{s} \times \frac{60 \mathrm{~min} .}{1 \mathrm{hr}} \times \frac{1 \mathrm{lb}}{7,000 \mathrm{gr}} \\
& C_{a w}=0.00857 \times C_{a n} \times Q_{s}=\mathrm{lbs} / \mathrm{hr} \\
& C_{a w}=\mathrm{lbs} / \mathrm{hr} \times 0.4536=\mathrm{kg} / \mathrm{hr}
\end{aligned}
$$

18. Particulate - total.

$$
\begin{aligned}
& C_{a x}=0.00857 \times C_{a o} \times Q_{s}=l b s / h r \\
& C_{a x}=l b s / h r \times 0.4536=\mathrm{kg} / \mathrm{hr}
\end{aligned}
$$

*29.92" Hg, $68^{\circ} \mathrm{F}\left(760 \mathrm{~mm} \mathrm{Hg}, 20^{\circ} \mathrm{C}\right)$ 
$\underline{\mathrm{SO}}_{3}$ ppm Calculation

$$
\mathrm{ppm}=\frac{\mathrm{mgs}}{\mathrm{V}_{\mathrm{mstd}}(\mathrm{dscm})} \times \frac{24.04}{40}
$$

Where: $\quad \operatorname{mgs}=$ Concentration of ammonia collected in sample train, milligrams

$$
\begin{aligned}
24.04 & =\text { Conversion Constant } \\
40 & =\text { Molecular Weight of } \mathrm{SO}_{3} \text {, grams/gram-mole }
\end{aligned}
$$


SOURCE EMISSION SURVEY

JOB NUMBER: 07-057

JOB NAME: ADA-ES

LOCATION: Hardin, Montana

UNIT TESTED: Stack (PM 10/2.5)

SOURCE EMISSION CALCULATIONS

\begin{tabular}{|c|c|c|c|c|c|}
\hline \multirow{2}{*}{ SYMBOL } & \multirow[b]{2}{*}{ DESCRIPTION } & \multirow[b]{2}{*}{ UNITS } & \multicolumn{3}{|c|}{ RUN NUMBER } \\
\hline & & & 1 & 2 & 3 \\
\hline DATE & & & $09 / 06 / 07$ & 09/06/07 & 09/06/07 \\
\hline BEGIN TIME & & & 1021 & 1310 & 1537 \\
\hline END TIME & & & 1124 & 1412 & 1641 \\
\hline$P(b)$ & BAROMETRIC PRESSURE & $\begin{array}{l}\text { "Hg Abs. } \\
(\mathrm{mm} \mathrm{Hg})\end{array}$ & $\begin{array}{r}26.81 \\
(681.00) \\
\end{array}$ & $\begin{array}{r}26.87 \\
(682.00) \\
\end{array}$ & $\begin{array}{r}26.90 \\
(683.00) \\
\end{array}$ \\
\hline$P(m)$ & ORIFICE PRESSURE DROP & $\begin{array}{l}\text { " } \mathrm{H} 2 \mathrm{O} \\
(\mathrm{mm} \mathrm{H} 2 \mathrm{O})\end{array}$ & $\begin{array}{r}0.390 \\
(9.900) \\
\end{array}$ & $\begin{array}{r}0.390 \\
(9.900) \\
\end{array}$ & $\begin{array}{r}0.400 \\
(10.200) \\
\end{array}$ \\
\hline & DGM CALIBRATION FACTOR & & 1.004 & 1.004 & 1.004 \\
\hline$V(m)$ & $\begin{array}{l}\text { VOLUME DRY GAS SAMPLED } \\
\text { @ METER CONDITIONS } \\
\text { LEAK CHECK VOLUME }\end{array}$ & $\begin{array}{l}\text { ft. }{ }^{\wedge} \\
\left(\mathrm{m}^{\wedge} 3\right) \\
\mathrm{ft} .^{\wedge} 3\end{array}$ & $\begin{array}{c}24.806 \\
(0.702) \\
0.000 \\
\end{array}$ & $\begin{array}{c}24.865 \\
(0.704) \\
0.000 \\
\end{array}$ & $\begin{array}{c}25.173 \\
(0.713) \\
0.000 \\
\end{array}$ \\
\hline$T(m)$ & $\begin{array}{l}\text { AVERAGE GAS METER } \\
\text { TEMPERATURE }\end{array}$ & $\begin{array}{l}\text { DEG.F } \\
\text { (DEG.C) }\end{array}$ & $\begin{array}{r}78 \\
(26) \\
\end{array}$ & $\begin{array}{r}85 \\
(29) \\
\end{array}$ & $\begin{array}{r}87 \\
(31) \\
\end{array}$ \\
\hline$V(m[s t d])^{*}$ & $\begin{array}{l}\text { VOLUME DRY GAS SAMPLED } \\
\text { @ STANDARD CONDITIONS* }\end{array}$ & $\begin{array}{l}\text { DSCF } \\
\text { (DSCM) }\end{array}$ & $\begin{array}{l}21.841 \\
(0.618) \\
\end{array}$ & $\begin{array}{l}21.660 \\
(0.613) \\
\end{array}$ & $\begin{array}{l}21.874 \\
(0.619) \\
\end{array}$ \\
\hline$V(w)$ & $\begin{array}{l}\text { TOTAL WATER COLLECTED, } \\
\text { IMPINGERS \& SILICA GEL }\end{array}$ & $\mathrm{ml}$ & 82.4 & 83.4 & 82.1 \\
\hline$V(w[$ gas $])$ & $\begin{array}{l}\text { VOLUME WATER VAPOR } \\
\text { COLLECTED @ STANDARD } \\
\text { CONDITIONS* }\end{array}$ & $\begin{array}{l}\text { SCF } \\
\text { (SCM) }\end{array}$ & $\begin{array}{c}3.889 \\
(0.110)\end{array}$ & $\begin{array}{c}3.936 \\
(0.111)\end{array}$ & $\begin{array}{c}3.875 \\
(0.110)\end{array}$ \\
\hline$\% \mathrm{M}$ & $\begin{array}{l}\text { MOISTURE IN STACK GAS } \\
\text { BY VOLUME }\end{array}$ & $\%$ & 15.12 & 15.38 & 15.05 \\
\hline Md & MOL FRACTION OF DRY GAS & & 0.8488 & 0,8462 & 0.8495 \\
\hline $\mathrm{Tt}$ & NET TIME OF TEST & MINUTES & 60 & 60 & 60 \\
\hline
\end{tabular}

*68 Deg.F, 29.92 "Hg (20 Deg.C, 760 mm Hg) 


\section{SOURCE EMISSION CALCULATIONS}

JOB NUMBER: 07-057

JOB NAME: ADA-ES

LOCATION: Hardin, Montana

UNIT TESTED: Stack (PM 10/2.5)

\begin{tabular}{|c|c|c|c|c|c|}
\hline & & & & JNUMBER & \\
\hline SYMBOL & DESCRIPTION & UNITS & 1 & 2 & 3 \\
\hline $\mathrm{CO} 2$ & & $\%$ & 12.8 & 12.8 & 12.7 \\
\hline $\mathrm{O} 2$ & & $\%$ & 6.7 & 6.7 & 6.8 \\
\hline $\mathrm{CO}$ & & $\%$ & 0.0 & 0.0 & 0.0 \\
\hline N2 & & $\%$ & 80.5 & 80.5 & 80.5 \\
\hline$\%$ EA & $\begin{array}{l}\text { EXCESS AIR @ SAMPLING } \\
\text { POINT }\end{array}$ & $\%$ & 45.8 & 45.8 & 46.8 \\
\hline MWd & $\begin{array}{l}\text { MOLECULAR WEIGHT OF } \\
\text { DRY STACK GAS }\end{array}$ & $\begin{array}{l}\text { LB/LB-MOLE } \\
\text { (g/g-MOLE) }\end{array}$ & $\begin{array}{r}30.32 \\
(30.32) \\
\end{array}$ & $\begin{array}{r}30.32 \\
(30.32) \\
\end{array}$ & $\begin{array}{r}30.30 \\
(30.30) \\
\end{array}$ \\
\hline MW & $\begin{array}{l}\text { MOLECULAR WEIGHT OF } \\
\text { STACK GAS }\end{array}$ & $\begin{array}{l}\text { LB/LB-MOLE } \\
\text { (g/g-MOLE) }\end{array}$ & $\begin{array}{r}28.45 \\
(28.45) \\
\end{array}$ & $\begin{array}{c}28.42 \\
(28.42) \\
\end{array}$ & $\begin{array}{r}28.45 \\
(28.45) \\
\end{array}$ \\
\hline $\mathrm{Cp}$ & PITOT TUBE CALIBRATION & & 0.827 & 0.827 & 0.827 \\
\hline DELTAP & $\begin{array}{l}\text { VELOCITY HEAD OF STACK } \\
\text { GAS }\end{array}$ & $\begin{array}{l}\text { " } \mathrm{H} 2 \mathrm{O} \\
(\mathrm{mm} \mathrm{H} 20)\end{array}$ & $\begin{array}{r}4.033 \\
(102.400) \\
\end{array}$ & $\begin{array}{r}4.000 \\
(101.600) \\
\end{array}$ & $\begin{array}{r}4.250 \\
(108.000) \\
\end{array}$ \\
\hline DELTAP $\wedge(1 / 2)$ & & "H2O & 2.008 & 1.999 & 2.061 \\
\hline Ts & STACK TEMPERATURE & $\begin{array}{l}\text { DEG. F } \\
\text { (DEG. C) }\end{array}$ & $\begin{array}{r}227 \\
(108) \\
\end{array}$ & $\begin{array}{r}217 \\
(103) \\
\end{array}$ & $\begin{array}{r}217 \\
(103) \\
\end{array}$ \\
\hline Ps & STACK PRESSURE & $\begin{array}{l}\text { "Hg Abs. } \\
\text { (mm Hg) } \\
\text { "H2O }\end{array}$ & $\begin{array}{r}26.65 \\
(677.00) \\
-2.20 \\
\end{array}$ & $\begin{array}{r}26.75 \\
(679.00) \\
-1.70 \\
\end{array}$ & $\begin{array}{r}26.79 \\
(680.00) \\
-1.50 \\
\end{array}$ \\
\hline Vs & $\begin{array}{l}\text { STACK VELOCITY @ STACK } \\
\text { CONDITIONS }\end{array}$ & $\begin{array}{l}\text { FPM } \\
(\mathrm{m} / \mathrm{SEC} .) \\
\end{array}$ & $\begin{array}{r}8,099 \\
(41) \\
\end{array}$ & $\begin{array}{r}7,993 \\
(41) \\
\end{array}$ & $\begin{array}{r}8,231 \\
(42) \\
\end{array}$ \\
\hline As & STACK AREA & $\begin{array}{l}\text { (SQ.INCHES) } \\
\text { (SQ.METERS) }\end{array}$ & $\begin{array}{r}9,363 \\
(6) \\
\end{array}$ & $\begin{array}{r}9,363 \\
(6) \\
\end{array}$ & $\begin{array}{r}9,363 \\
(6) \\
\end{array}$ \\
\hline Qs & $\begin{array}{l}\text { DRY STACK GAS VOLUME @ } \\
\text { STANDARD CONDITIONS* }\end{array}$ & $\begin{array}{l}\text { DSCFM } \\
\text { (DSCM/HR) }\end{array}$ & $\begin{array}{c}307,112 \\
(521,783)\end{array}$ & $\begin{array}{r}307,778 \\
(522,915)\end{array}$ & $\begin{array}{c}318,655 \\
(541,395)\end{array}$ \\
\hline Qa & $\begin{array}{l}\text { ACTUAL STACK GAS VOLUME } \\
\text { @ STACK CONDITIONS }\end{array}$ & $\begin{array}{l}\text { ACFM } \\
\left(\mathrm{m}^{\wedge} 3 / \mathrm{HR}\right)\end{array}$ & $\begin{array}{r}526,626 \\
(894,738) \\
\end{array}$ & $\begin{array}{c}519,737 \\
(883,033) \\
\end{array}$ & $\begin{array}{r}535,174 \\
(909,261) \\
\end{array}$ \\
\hline Dn & SAMPLING NOZZLE DIAM. & $\begin{array}{l}\text { IN. } \\
(\mathrm{m})\end{array}$ & $\begin{array}{r}0.128 \\
(0.003) \\
\end{array}$ & $\begin{array}{r}0.128 \\
(0.003) \\
\end{array}$ & $\begin{array}{r}0.128 \\
(0.003) \\
\end{array}$ \\
\hline$\% 1$ & PERCENT ISOKINETIC & $\%$ & 86.6 & 85.7 & 83.6 \\
\hline
\end{tabular}

* 68 Deg.F, 29.92 "Hg (20 Deg.C, 760 mm Hg) 


\section{SOURCE EMISSION CALCULATIONS}

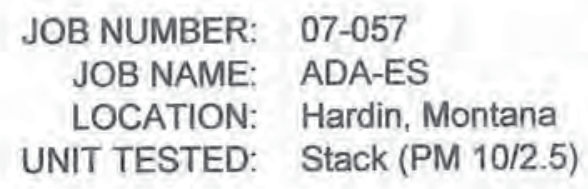

\begin{tabular}{|c|c|c|c|c|c|}
\hline \multirow{2}{*}{ SYMBOL } & \multirow[b]{2}{*}{ DESCRIPTION } & \multirow[b]{2}{*}{ UNITS } & \multicolumn{3}{|c|}{ RUN NUMBER } \\
\hline & & & 1 & 2 & 3 \\
\hline Mf & $\begin{array}{l}\text { PARTICULATE - PROBE, } \\
\text { CYCLONE AND FILTER }\end{array}$ & $\mathrm{mg}>\mathrm{PMIO}$ & 6.5 & 1.8 & 4.3 \\
\hline Mt & PARTICULATE - TOTAL & $\mathrm{mg}$ & 65.9 & 103.7 & 39.8 \\
\hline Can & $\begin{array}{l}\text { PARTICULATE - PROBE, } \\
\text { CYCLONE AND FILTER }\end{array}$ & $\begin{array}{l}\text { gr/DSCF* } \\
\text { (g/DSCM) }\end{array}$ & $\begin{array}{r}0.0046 \\
(0.0105) \\
\end{array}$ & $\begin{array}{r}0.0013 \\
(0.0029) \\
\end{array}$ & $\begin{array}{r}0.0030 \\
(0.0069) \\
\end{array}$ \\
\hline Cao & PARTICULATE - TOTAL & $\begin{array}{l}\text { gr/DSCF* } \\
\text { (g/DSCM) }\end{array}$ & $\begin{array}{r}0.0465 \\
(0.1064) \\
\end{array}$ & $\begin{array}{r}0.0737 \\
(0.1688) \\
\end{array}$ & $\begin{array}{r}0.0280 \\
(0.0642) \\
\end{array}$ \\
\hline Cat & $\begin{array}{l}\text { PARTIC.-PROBE, CYCLONE } \\
\text { AND FILTER @ STACK COND. }\end{array}$ & $\begin{array}{l}\mathrm{gr} / \mathrm{CF} \\
(\mathrm{g} / \mathrm{m} 3)\end{array}$ & $\begin{array}{c}0.0027 \\
(0.0062) \\
\end{array}$ & $\begin{array}{r}0.0008 \\
(0.0018) \\
\end{array}$ & $\begin{array}{r}0.0018 \\
(0.0041) \\
\end{array}$ \\
\hline Cau & $\begin{array}{l}\text { PARTICULATE - TOTAL @ } \\
\text { STACK CONDITIONS }\end{array}$ & $\begin{array}{l}\mathrm{gr} / \mathrm{CF} \\
(\mathrm{g} / \mathrm{m} 3)\end{array}$ & $\begin{array}{r}0.0270 \\
(0.0618) \\
\end{array}$ & $\begin{array}{r}0.0435 \\
(0.0996) \\
\end{array}$ & $\begin{array}{r}0.0166 \\
(0.0380) \\
\end{array}$ \\
\hline Caw & $\begin{array}{l}\text { PARTICULATE - PROBE, } \\
\text { CYCLONE AND FILTER }\end{array}$ & $\begin{array}{l}\text { LBS/HR } \\
(\mathrm{Kg} / \mathrm{HR})\end{array}$ & $\begin{array}{l}12.06 \\
(5.47) \\
\end{array}$ & $\begin{array}{r}3.38 \\
(1.53) \\
\end{array}$ & $\begin{array}{r}8.27 \\
(3.75) \\
\end{array}$ \\
\hline Cax & PARTICULATE - TOTAL & $\begin{array}{l}\text { LBS/HR } \\
(\mathrm{Kg} / \mathrm{HR})\end{array}$ & $\begin{array}{l}122.30 \\
(55.47) \\
\end{array}$ & $\begin{array}{l}194.47 \\
(88.21) \\
\end{array}$ & $\begin{array}{r}76.52 \\
(34.71) \\
\end{array}$ \\
\hline & & & & & \\
\hline
\end{tabular}

*68 Deg.F, 29.92 "Hg (20 Deg.C, 760 mm Hg) 


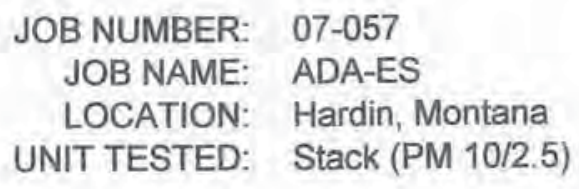

\begin{tabular}{|c|c|c|c|c|c|}
\hline \multirow{2}{*}{ SYMBOL } & \multirow[b]{2}{*}{ DESCRIPTION } & \multirow[b]{2}{*}{ UNITS } & \multicolumn{3}{|c|}{ RUN NUMBER } \\
\hline & & & 1 & \begin{tabular}{l|l}
2 \\
\end{tabular} & 3 \\
\hline Mf & $\begin{array}{l}\text { PARTICULATE - PROBE, } \\
\text { CYCLONE AND FILTER }\end{array}$ & $\begin{aligned} \mathrm{mg} & <P M 10 \\
& >P M 2.5\end{aligned}$ & 5.3 & 3.6 & 3.7 \\
\hline Mt & PARTICULATE - TOTAL & $\mathrm{mg} P M 2,5$ & 0.9 & 0.8 & 8.2 \\
\hline Can & $\begin{array}{l}\text { PARTICULATE - PROBE, } \\
\text { CYCLONE AND FILTER }\end{array}$ & $\begin{array}{l}\text { gr/DSCF* } \\
\text { (g/DSCM) }\end{array}$ & $\begin{array}{r}0.0037 \\
(0.0086) \\
\end{array}$ & $\begin{array}{r}0.0026 \\
(0.0059) \\
\end{array}$ & $\begin{array}{r}0.0026 \\
(0.0060) \\
\end{array}$ \\
\hline Cao & PARTICULATE - TOTAL & $\begin{array}{l}\text { gr/DSCF* } \\
\text { (g/DSCM) }\end{array}$ & $\begin{array}{r}0.0006 \\
(0.0015) \\
\end{array}$ & $\begin{array}{r}0.0006 \\
(0.0013) \\
\end{array}$ & $\begin{array}{r}0.0058 \\
(0.0132) \\
\end{array}$ \\
\hline Cat & $\begin{array}{l}\text { PARTIC.-PROBE, CYCLONE } \\
\text { AND FILTER @ STACK COND. }\end{array}$ & $\begin{array}{l}\mathrm{gr} / \mathrm{CF} \\
(\mathrm{g} / \mathrm{m} 3)\end{array}$ & $\begin{array}{c}0.0022 \\
(0.0050) \\
\end{array}$ & $\begin{array}{r}0.0015 \\
(0.0034) \\
\end{array}$ & $\begin{array}{c}0.0015 \\
(0.0034) \\
\end{array}$ \\
\hline Cau & $\begin{array}{l}\text { PARTICULATE - TOTAL @ } \\
\text { STACK CONDITIONS } \\
\end{array}$ & $\begin{array}{l}\mathrm{gr} / \mathrm{CF} \\
(\mathrm{g} / \mathrm{m} 3)\end{array}$ & $\begin{array}{r}0.0004 \\
(0.0009) \\
\end{array}$ & $\begin{array}{r}0.0003 \\
(0.0007) \\
\end{array}$ & $\begin{array}{c}0.0034 \\
(0.0078) \\
\end{array}$ \\
\hline Caw & $\begin{array}{l}\text { PARTICULATE - PROBE, } \\
\text { CYCLONE AND FILTER }\end{array}$ & $\begin{array}{l}\mathrm{LBS} / \mathrm{HR} \\
(\mathrm{Kg} / \mathrm{HR})\end{array}$ & $\begin{array}{r}9.84 \\
(4.46) \\
\end{array}$ & $\begin{array}{r}6.75 \\
(3.06) \\
\end{array}$ & $\begin{array}{c}7.11 \\
(3.23) \\
\end{array}$ \\
\hline Cax & PARTICULATE - TOTAL & $\begin{array}{l}\mathrm{LBS} / \mathrm{HR} \\
(\mathrm{Kg} / \mathrm{HR})\end{array}$ & $\begin{array}{r}1.67 \\
(0.76) \\
\end{array}$ & $\begin{array}{r}1.50 \\
(0.68) \\
\end{array}$ & $\begin{array}{l}15.77 \\
(7.15) \\
\end{array}$ \\
\hline & & & & & \\
\hline
\end{tabular}

*68 Deg.F, 29.92 "Hg (20 Deg.C, 760 mm Hg) 
SOURCE EMISSION SURVEY

JOB NUMBER: 07-057

JOB NAME: ADA-ES

LOCATION: Hardin, Montana

UNIT TESTED: Stack (Controlled Condensate)

SOURCE EMISSION CALCULATIONS

\begin{tabular}{|c|c|c|c|c|c|}
\hline \multirow{2}{*}{\multicolumn{3}{|c|}{ 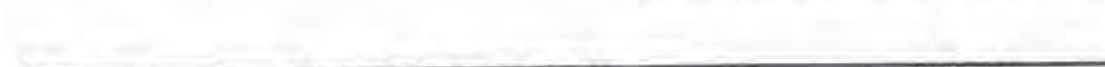 }} & \multirow{2}{*}{\multicolumn{3}{|c|}{ RUN NUMBER }} \\
\hline & & & & & \\
\hline SYMBOL & DESCRIPTION & UNITS & 1 & \begin{tabular}{l|l}
2 & \\
\end{tabular} & 3 \\
\hline DATE & & & 09/06/07 & $09 / 06 / 07$ & 09/06/07 \\
\hline BEGIN TIME & & & 1138 & 1359 & 1529 \\
\hline END TIME & & & 1238 & 1459 & 1629 \\
\hline \multirow[t]{2}{*}{$P(b)$} & \multirow[t]{2}{*}{ BAROMETRIC PRESSURE } & "Hg Abs. & 26.81 & 26.87 & \multirow{2}{*}{$\begin{array}{r}26.90 \\
(683.00) \\
\end{array}$} \\
\hline & & $(\mathrm{mm} \mathrm{Hg})$ & $(681.00)$ & $(682.00)$ & \\
\hline \multirow[t]{3}{*}{$P(m)$} & \multirow[t]{2}{*}{ ORIFICE PRESSURE DROP } & "H2O & 0.300 & 0.300 & \multirow{2}{*}{$\begin{array}{r}0.300 \\
(7.600) \\
\end{array}$} \\
\hline & & $(\mathrm{mm} \mathrm{H} 2 \mathrm{O})$ & $(7.600)$ & $(7.600)$ & \\
\hline & \multicolumn{2}{|l|}{ DGM CALIBRATION FACTOR } & 0.998 & 0.998 & 0.998 \\
\hline \multirow[t]{3}{*}{$V(m)$} & VOLUME DRY GAS SAMPLED & $\mathrm{ft} \wedge^{\wedge} 3$ & 20.610 & 20.025 & \multirow{3}{*}{$\begin{array}{r}20.789 \\
(0.589) \\
0.000 \\
\end{array}$} \\
\hline & @ METER CONDITIONS & $\left(m^{\wedge} 3\right)$ & $(0.584)$ & $(0,567)$ & \\
\hline & LEAK CHECK VOLUME & $\mathrm{ft},{ }^{\wedge} 3$ & 0.000 & 0.000 & \\
\hline \multirow[t]{2}{*}{$T(m)$} & AVERAGE GAS METER & DEG.F & 76 & 84 & \multirow{2}{*}{$\begin{array}{r}85 \\
(29) \\
\end{array}$} \\
\hline & TEMPERATURE & (DEG.C) & (24) & (29) & \\
\hline \multirow[t]{2}{*}{$V(m[s t d])^{*}$} & VOLUME DRY GAS SAMPLED & DSCF & 18.210 & 17.472 & \multirow{2}{*}{$\begin{array}{l}18.126 \\
(0.513) \\
\end{array}$} \\
\hline & @ STANDARD CONDITIONS* & (DSCM) & $(0.516)$ & $(0.495)$ & \\
\hline$V(w)$ & $\begin{array}{l}\text { TOTAL WATER COLLECTED, } \\
\text { IMPINGERS \& SILICA GEL }\end{array}$ & $\mathrm{ml}$ & 67.1 & 65.0 & 67.1 \\
\hline \multirow[t]{2}{*}{$\mathrm{V}($ w[gas $])$} & VOLUME WATER VAPOR & SCF & 3.167 & 3.068 & \multirow{2}{*}{$\begin{array}{l}3.167 \\
(0.090)\end{array}$} \\
\hline & $\begin{array}{l}\text { COLLECTED @ STANDARD } \\
\text { CONDITIONS* }\end{array}$ & (SCM) & $(0.090)$ & $(0.087)$ & \\
\hline$\% \mathrm{M}$ & $\begin{array}{l}\text { MOISTURE IN STACK GAS } \\
\text { BY VOLUME }\end{array}$ & $\%$ & 14.82 & 14.94 & 14.87 \\
\hline Md & MOL FRACTION OF DRY GAS & & 0.8518 & 0.8506 & 0.8513 \\
\hline $\mathrm{Tt}$ & NET TIME OF TEST & MINUTES & 60 & 60 & 60 \\
\hline
\end{tabular}

*68 Deg.F, 29.92 "Hg (20 Deg.C, 760 mm Hg) 


\section{SOURCE EMISSION CALCULATIONS}

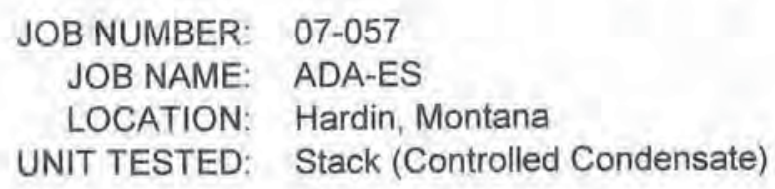

\begin{tabular}{|c|c|c|c|c|c|}
\hline \multicolumn{3}{|c|}{ 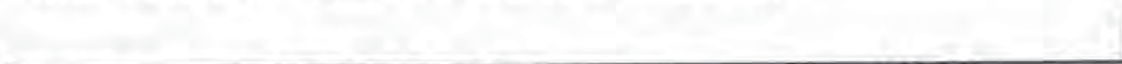 } & \multicolumn{3}{|c|}{ RUN NUMBER } \\
\hline SYMBOL & DESCRIPTION & UNITS & 1 & $2 \mid$ & 3 \\
\hline $\mathrm{CO} 2$ & & $\%$ & 12.8 & 12.8 & 12.7 \\
\hline $\mathrm{O} 2$ & & $\%$ & 6.7 & 6.7 & 6.8 \\
\hline $\mathrm{CO}$ & & $\%$ & 0.0 & 0.0 & 0.0 \\
\hline N2 & & $\%$ & 80.5 & 80.5 & 80.5 \\
\hline$\% E A$ & $\begin{array}{l}\text { EXCESS AIR @ SAMPLING } \\
\text { POINT }\end{array}$ & $\%$ & 45.8 & 45.8 & 46.8 \\
\hline MWd & $\begin{array}{l}\text { MOLECULAR WEIGHT OF } \\
\text { DRY STACK GAS }\end{array}$ & $\begin{array}{l}\text { LB/LB-MOLE } \\
\text { (g/g-MOLE) }\end{array}$ & $\begin{array}{r}30.32 \\
(30.32) \\
\end{array}$ & $\begin{array}{r}30.32 \\
(30.32) \\
\end{array}$ & $\begin{array}{c}30.30 \\
(30.30) \\
\end{array}$ \\
\hline MW & $\begin{array}{l}\text { MOLECULAR WEIGHT OF } \\
\text { STACK GAS }\end{array}$ & $\begin{array}{l}\text { LB/LB-MOLE } \\
\text { (g/g-MOLE) }\end{array}$ & $\begin{array}{r}28.49 \\
(28.49) \\
\end{array}$ & $\begin{array}{r}28.48 \\
(28.48) \\
\end{array}$ & $\begin{array}{r}28.47 \\
(28.47) \\
\end{array}$ \\
\hline $\mathrm{Cp}$ & PITOT TUBE CALIBRATION & & 0.000 & 0.000 & 0.000 \\
\hline DELTAP & $\begin{array}{l}\text { VELOCITY HEAD OF STACK } \\
\text { GAS }\end{array}$ & $\begin{array}{l}\text { "H2O } \\
(\mathrm{mm} \mathrm{H} 20)\end{array}$ & - & - & $\ldots$ \\
\hline DELTA $P^{\wedge}(1 / 2)$ & & "H2O & $\rightarrow$ & $\ldots$ & $\ldots$ \\
\hline Ts & STACK TEMPERATURE & $\begin{array}{l}\text { DEG.F } \\
\text { (DEG.C) }\end{array}$ & - & $\cdots$ & $\cdots$ \\
\hline Ps & STACK PRESSURE & $\begin{array}{l}\text { "Hg Abs. } \\
(\mathrm{mm} \mathrm{Hg}) \\
\text { "H2O }\end{array}$ & $\begin{array}{r}26.65 \\
(677.00) \\
-2.20 \\
\end{array}$ & $\begin{array}{r}26.75 \\
(679.00) \\
-1.70 \\
\end{array}$ & $\begin{array}{r}26.79 \\
(680.00) \\
-1.50 \\
\end{array}$ \\
\hline Vs & $\begin{array}{l}\text { STACK VELOCITY@ @TACK } \\
\text { CONDITIONS }\end{array}$ & $\begin{array}{l}\text { FPM } \\
(\mathrm{m} / \mathrm{SEC} .) \\
\end{array}$ & + & $\cdots$ & + \\
\hline As & STACK AREA & $\begin{array}{l}\text { (SQ.INCHES) } \\
\text { (SQ.METERS) }\end{array}$ & $\begin{array}{r}9,363 \\
(6) \\
\end{array}$ & $\begin{array}{r}9,363 \\
(6) \\
\end{array}$ & $\begin{array}{r}9,363 \\
(6) \\
\end{array}$ \\
\hline Qs & $\begin{array}{l}\text { DRY STACK GAS VOLUME @ } \\
\text { STANDARD CONDITIONS* } \\
\text { WET STACK GAS VOLUME @ } \\
\text { STANDARD CONDITIONS* }\end{array}$ & $\begin{array}{l}\text { DSCFM } \\
\text { (DSCM/HR) } \\
\text { WSCFH }\end{array}$ & - & - & - \\
\hline Qa & $\begin{array}{l}\text { ACTUAL STACK GAS VOLUME } \\
@ \text { STACK CONDITIONS }\end{array}$ & $\begin{array}{l}\text { ACFM } \\
\left(m^{\wedge} 3 / H R\right)\end{array}$ & - & - & - \\
\hline & & & & & \\
\hline
\end{tabular}

*68 Deg.F, 29.92 "Hg (20 Deg.C, 760 mm Hg) 


\section{$\underline{\mathrm{SO}_{3} \mathrm{ppm} \text { Calculations }}$}

$$
\mathrm{ppm}=\frac{\mathrm{mgs}}{\mathrm{V}_{\mathrm{mstd}}(\mathrm{dscm})} \times \frac{24.04}{40}
$$

Run No. $1=\frac{0.499 \mathrm{mg}}{0.618 \mathrm{dscm}} \times \frac{24.04}{40 \text { grams } / \text { gram-mole }}=0.49 \mathrm{ppm}$
Run No. $2=\frac{1.349 \mathrm{mg}}{0.613 \mathrm{dscm}} \times \frac{24.04}{40 \text { grams } / \text { gram-mole }}=1.32 \mathrm{ppm}$
Run No. $3=\frac{1.537 \mathrm{mg}}{0.619 \mathrm{dscm}} \times \frac{24.04}{40 \text { grams } / \text { gram-mole }}=1.49 \mathrm{ppm}$




\section{PM 10 CALCULATION SPREADSHEET}

This spreadsheet will calculate -1 ) nozzle size

2) delta $\mathrm{H}$ for $\mathrm{PM} 10$ sampling

3) dwell time calculation number (magic \#)

4) actual dwell time (if you dont have a calulator)

INSTRUCTIONS:

5) other cut sizes for different sample rates

1) Enter input data in -- INPUT DATA -

2) based on your nozzle size selection - see if the the $\min \& \max$ delta P's nozzle size. Do not use "MISC CALCS" section.

(That only uses the delta $P$ at a single point as if it was going to be your average delta $\mathrm{P}$ )

3) Start test - set delta $\mathrm{H}$ at the designated value. Calculate dwell time by by multiplying the "MAGIC NUMBER" by square root of the sample point delta $\mathrm{P}$ to calculate sample point dwell time (seconds).

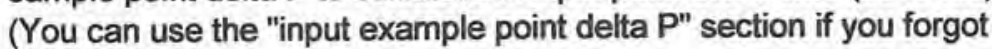
your calculator)

4) Copy column "C" over to the right if you want calc's for other data.

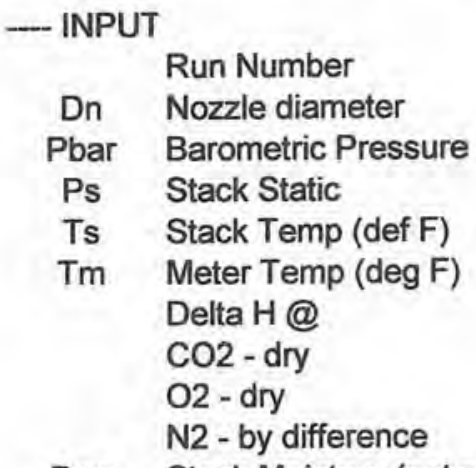

$\begin{array}{rrr}\text { RUN 1 } & \text { RUN 2 } & \text { RUN 3 } \\ 0.128 & 0.128 & 0.128 \\ 26.81 & 26.87 & 26.90 \\ -2.20 & -1.70 & -1.50 \\ 227.00 & 217.00 & 217.00 \\ 78.00 & 85.00 & 87.00 \\ 1.80 & 1.80 & 1.80 \\ 12.80 & 12.80 & 12.70 \\ 6.70 & 6.70 & 6.80 \\ 80.50 & 80.50 & 80.50 \\ 0.15 & 0.15 & 0.15 \\ 2.008 & 1.999 & 2.061 \\ & & \\ 12.00 & 12.00 & 12.00 \\ 60.00 & 60.00 & 60.00\end{array}$

Total No of Points
Total sampling time (min)

\section{- CALCULATED VALUES -}

MW-d Mol weight - dry

MW-w molecular weight - wet

Ps Stack Pressure

Meter pressure

Ts Stack Temp (deg R)

Tm Meter Temp $(\operatorname{deg} R)$
30.32

28.45

26.65

26.84

687.00

538.00

- CALCS AT 10um CUT POINT - CSR - mu Stack viscosity

Qs Sample flow (at the PSD head) delta $\mathrm{H}$

u stack gas velocity based on sample rate
200.88

0.55

0.39

103.25
30.32

28.42

26.75

26.90

677.00

545.00

198.18

0.54

0.40

101.35
30.30

28.45

26.79

26.93

677.00

547.00 
minimum stack gas velocity

maximum stack gas velocity

minimum delta $P$ (not square root)

maximum delta $P$ (not square root)
79.03

126.40

1.33

3.42

149.402

MAGIC NUMBER (multiply times sqrt delta
77.56

124.08

1.31

3.35

77.61

124.17

1.31

3.37

- DWELL TIME CALCULATOR -

\section{$\mathrm{P}$ at the point in question
to get dwell time in sec's) \\ $\mathrm{P}$ at the point in question
to get dwell time in sec's)}

- INPUT EXAMPLE DELTAP -

delta $\mathrm{P}$ sample point delta $\mathrm{P}$ (input this value)

150.08

145.56 sample point dwell time ( $\mathrm{min})$ sample point dwell time(sec)

$\begin{array}{rr}4.00 & 4.25 \\ 5.003 & 5.001 \\ 300.15 & 300.08\end{array}$

- MISC CALCs USING 100\% ISO SAMPE RATE FOR DELTA P IN ( vs PM10 BASED SAMPE RATE (Qs) (DO NOT USE THIS PART OF THE SPDSHT FOR NORMAL PM10 CALCs )

You can estimate cut size for different delta $\mathrm{H}$ (sample rate other than designated value ) based on $100 \%$ iso at "sample point delta P". You can input the average delta $\mathrm{P}$ in "sample point delta $\mathrm{P}$ " and your sample rate at the meter for $100 \%$ average iso's and the resulting cut size will be calculated. (meter cfm calc's do not include Pressure or Meter $\mathrm{Y}$ values - negligible)

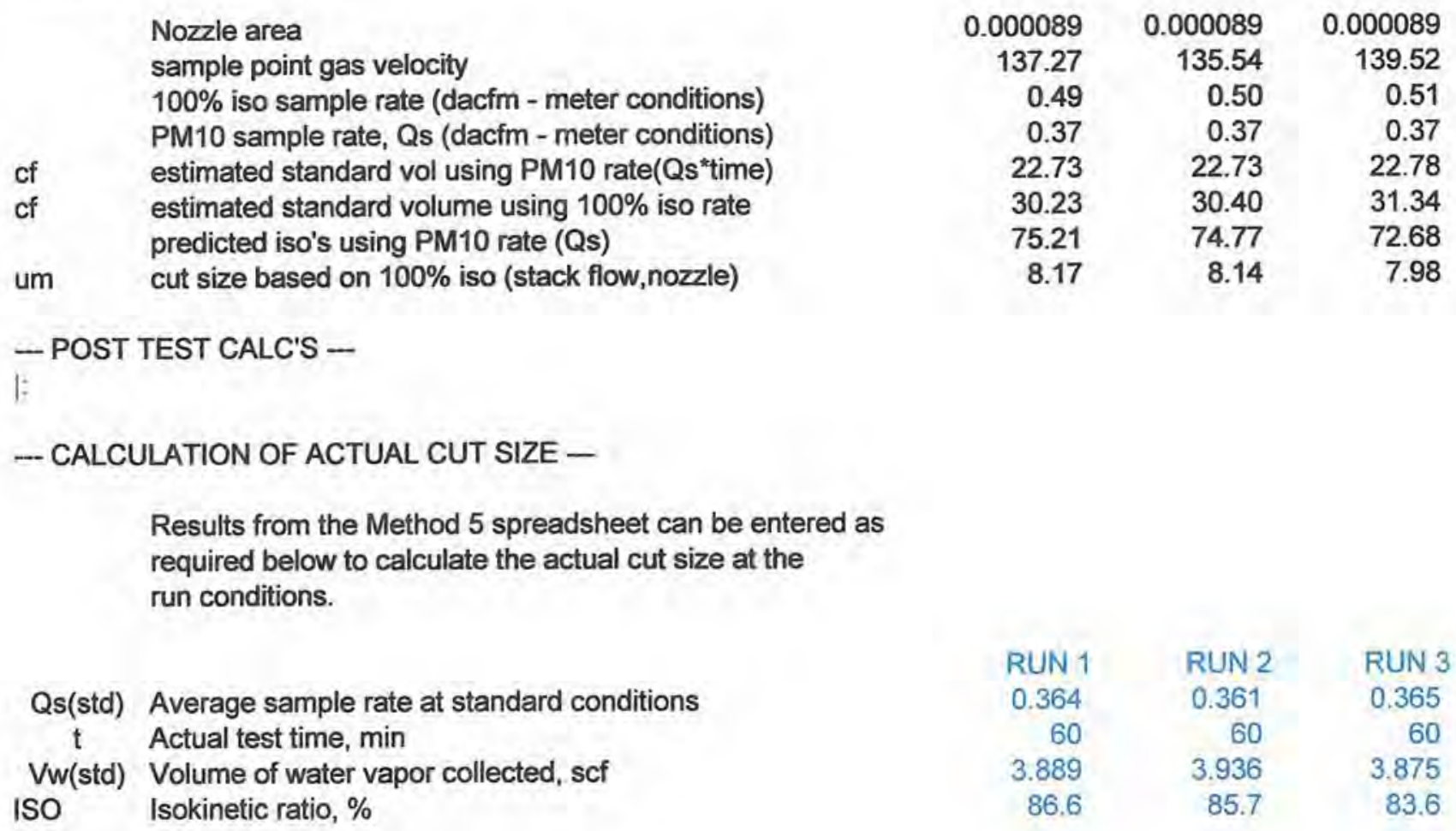


PM-10 RESULTS:

Run Number

Qs, Sample rate at the cyclone, cfm

D-50, Actual cut size, um

Isokinetic Rate, \%

RUN 1
0.6267
9.16
86.6

RUN 2

RUN 3

9.16
867

0.6122

9.19

0.6154

86.6

85.7

9.16

83.6 
Appendix C:

Calibration Data 


\section{Appendix C}

\section{Table 6: Calibration Data}

Pre-Test Calibrations:

Equipment

Dry Gas Meter 1-1

Digital Temperature Indicator 1-1

Dry Gas Meter 1-1 Orifice

Dry Gas Meter 1-2

Digital Temperature Indicator 1-2

Dry Gas Meter 1-2 Orifice

Pitot Tube PM-10

Nozzle PM-10-1
Calibration Factor

0.998

1.004

0.827

0.128

09/06/07

\section{Post-Test Calibrations:}

\section{Equipment}

Dry Gas Meter 1-1

Digital Temperature Indicator 1-1

Dry Gas Meter 1-1 Orifice

Dry Gas Meter 1-2

Digital Temperature Indicator 1-2

Dry Gas Meter 1-2 Orifice

Pitot Tube PM-10

Nozzle PM-10-1

\section{Calibration Factor $\quad \underline{\text { Calibration Date }}$}

0.998

09/11/07

$09 / 11 / 07$

09/11/07

0.997

09/11/07

$09 / 11 / 07$

09/13/07

0.825

09/10/07

0.128

09/06/07 
III AIR

SAMPLING

ASSOCIATES, INC.

Calibration Data

Dry Gas Meter Calibration

Meter Console No.

Date Calibration Performed:

$\frac{\text { ASA 1-1 }}{04 / 03 / 07}$

\begin{tabular}{lc}
$\begin{array}{c}\Delta H \text { Setting } \\
\left(" \mathrm{H}_{2} \mathrm{O}\right)\end{array}$ & $\mathrm{C}_{\mathrm{DG}}$ \\
\hline 0.50 & 1.002 \\
1.00 & 1.000 \\
1.50 & 1.002 \\
2.00 & 1.000 \\
3.00 & 0.992 \\
4.00 & 0.991 \\
Average & 0.998 \\
\hline
\end{tabular}

\begin{tabular}{ll} 
Variation +: & $0.40 \%$ \\
Variation -: & $-0.70 \%$ \\
\hline
\end{tabular}

Certified by:

Scot Jackson 04/03/07

Calibrator (Signature / Date)

Bill Hefley 04/03/07

QA Officer (Signature / Date)

C-3 
Calibration Data

Dry Gas Meter Calibration

\author{
Meter Console No. \\ ASAI 1-1 \\ Date Calibration Performed: \\ 04/03/07
}

$\begin{aligned} \text { Run } \frac{1}{\mathrm{~Pb}} & =29.61 \\ \text { Wet Test Meter } \mathrm{C}_{\mathrm{f}}=1.020 \quad \mathrm{Hg} & \text { Console Pump Vacuum }=-5.0 " \mathrm{Hg}\end{aligned}$

Wet Test Meter No. ASAl-0

Dry Gas Meter

Meter

Meter Temperature

\begin{tabular}{|c|c|c|c|c|c|c|c|c|c|c|c|c|c|c|}
\hline & Time & Reading & & Temp. & & $\underline{P_{m}}$ & & Reading & In & & Out & & $\underline{P_{m}}$ & \\
\hline End & $\overline{11: 11}$ & 5.367 & cf & 78 & ${ }^{\circ} \mathrm{F}$ & -0.2 & $" \mathrm{H}_{2} \mathrm{O}$ & 147.534 cf & 79 & ${ }^{\circ} \mathrm{F}$ & 76 & ${ }^{\circ} \mathrm{F}$ & 0.50 & $" \mathrm{H}_{2} \mathrm{O}$ \\
\hline art & $10: 58$ & $\underline{0.000}$ & cf & $\underline{78}$ & ${ }^{\circ} \mathrm{F}$ & -0.2 & $" \mathrm{H}_{2} \mathrm{O}$ & $142.100 \mathrm{cf}$ & $\underline{75}$ & ${ }^{\circ} \mathrm{F}$ & $\underline{74}$ & ${ }^{\circ} \mathrm{F}$ & $\underline{0.50}$ & $" \mathrm{H}_{2} \mathrm{O}$ \\
\hline & & 5.367 & cf & 78 & ${ }^{\circ} \mathrm{F}$ & -0.2 & $" \mathrm{H}_{2} \mathrm{O}$ & 5.434 & 77 & ${ }^{\circ} \mathrm{F}$ & 75 & ${ }^{\circ} \mathrm{F}$ & 0.50 & $" \mathrm{H}_{2} \mathrm{O}$ \\
\hline
\end{tabular}

Wet Test Meter $V_{m s d}=17.65 \times V_{m}\left[\frac{P_{b}+\frac{P_{m}}{13.6}}{\left(T_{m}+460\right)}\right] \times C_{f}=\underline{5.315} d c s f$
Dry Gas Meter $V_{m s s}=17.65 \times \mathrm{Vm}\left[\frac{P_{b}+\frac{P_{m}}{13.6}}{\left(T_{m}+460\right)}\right]=\underline{5.305} \mathrm{dcsf}$

Calibration Factor $(C D G)=\frac{\text { Wet Test Meter } V_{\text {mstd }}}{\text { Dry Gas Meter } V_{\text {mstd }}}=\underline{1.002}$ 


\author{
Calibration Data \\ Dry Gas Meter Calibration
}

Meter Console No.

ASAI 1-1

Date Calibration Performed:

04/03/07

$\begin{aligned} \text { Run } \frac{1}{\mathrm{~Pb}}= & =29.61 \\ \text { Wet Test Meter } \mathrm{C}_{\mathrm{f}}=1.020 \text { Console Pump Vacuum } & =-5.0 \mathrm{Hg}\end{aligned}$

Wet Test Meter No. ASAI-O Meter

\begin{tabular}{|c|c|c|c|c|c|c|c|}
\hline & Time & Reading & & Temp. & & $\underline{\mathrm{P}_{\mathrm{m}}}$ & \\
\hline & $11: 23$ & 5.099 & cf & 79 & ${ }^{\circ} \mathrm{F}$ & -0.3 & $" \mathrm{H}_{2} \mathrm{O}$ \\
\hline a & 11:14 & $\underline{0.000}$ & cf & 79 & ${ }^{\circ} \mathrm{F}$ & -0.3 & $" \mathrm{H}_{2} \mathrm{O}$ \\
\hline & & 5.099 & cf & 79 & ${ }^{\circ} \mathrm{F}$ & -0.3 & $" \mathrm{H}_{2} \mathrm{O}$ \\
\hline
\end{tabular}

Dry Gas Meter

Meter Temperature

Reading In $\underline{\text { Out }} \underline{\mathrm{P}_{\mathrm{m}}}$
153.187 cf $83{ }^{\circ} \mathrm{F} \quad 78 \quad{ }^{\circ} \mathrm{F} \quad 1.00 \quad{ }^{\prime} \mathrm{H}_{2} \mathrm{O}$ $\underline{148.001}$ cf $\quad \underline{79}{ }^{\circ} \mathrm{F} \quad \underline{77} \quad{ }^{\circ} \mathrm{F} \quad \underline{1.00} \quad \mathrm{H}_{2} \mathrm{O}$ $\begin{array}{llllllllll}5.186 & \text { of } & 81 & { }^{\circ} \mathrm{F} & 78 & { }^{\circ} \mathrm{F} & 1.00 & \quad \mathrm{H}_{2} \mathrm{O}\end{array}$

$$
\begin{gathered}
\text { Wet Test Meter Vmsdd }=17.65 \times V_{m}\left[\frac{P_{b}+\frac{P_{m}}{13.6}}{\left(T_{m}+460\right)}\right] \times C_{f}=\underline{5.039} \mathrm{dcsf} \\
\text { Dry Gas Meter } V_{m s d}=17.65 \times \mathrm{Vm}\left[\frac{P_{b}+\frac{P_{m}}{13.6}}{\left(T_{m}+460\right)}\right]=\underline{5.039} \mathrm{dcsf} \\
\text { Calibration Factor }\left(C_{D G}\right)=\frac{\text { Wet Test Meter } V_{\text {mstd }}}{\text { Dry Gas Meter } V_{m s t}}=\underline{1.000}
\end{gathered}
$$




\author{
Calibration Data \\ Dry Gas Meter Calibration
}

Meter Console No.

Date Calibration Performed:
ASAI 1-1

04/03/07

$\begin{aligned} \text { Run } \frac{1}{\mathrm{~Pb}}=29.61 & \text { at } 1.5^{\prime \prime} \Delta \mathrm{H} \\ \text { Wet Test Meter } \mathrm{C}_{\mathrm{f}}=1.020 \quad \text { Console Pump Vacuum } & =-5.0 " \mathrm{Hg}\end{aligned}$

Wet Test Meter No. ASAl-0 Meter

\begin{tabular}{|c|c|c|c|c|c|c|}
\hline & Time & Reading & Temp. & & $\underline{P_{m}}$ & \\
\hline End & $11: 41$ & $10.248 \mathrm{cf}$ & 79 & ${ }^{\circ} \mathrm{F}$ & -0.5 & $" \mathrm{H}_{2} \mathrm{O}$ \\
\hline tart & $11: 26$ & $\underline{0.000}$ & $\underline{79}$ & ${ }^{\circ} \mathrm{F}$ & $\underline{-0.5}$ & $" \mathrm{H}_{2} \mathrm{O}$ \\
\hline$A v$ & & 10.248 cf & 79 & ${ }^{\circ} \mathrm{F}$ & -0.5 & $" \mathrm{H}_{2} \mathrm{O}$ \\
\hline
\end{tabular}

Meter Temperature

Reading In Out $\quad \underline{P_{m}}$
164.136 of $87{ }^{\circ} \mathrm{F} \quad 80 \quad{ }^{\circ} \mathrm{F} \quad 1.50 \quad " \quad \mathrm{H}_{2} \mathrm{O}$ 153.704 cf $\quad \underline{81} \quad{ }^{\circ} \mathrm{F} \quad \underline{78}{ }^{\circ} \mathrm{F} \quad \underline{1.50} \quad " \mathrm{H}_{2} \mathrm{O}$ 10.432 cf $84{ }^{\circ} \mathrm{F} \quad 79{ }^{\circ} \mathrm{F} \quad 1.50 \quad " \mathrm{H}_{2} \mathrm{O}$

$$
\begin{gathered}
\text { Wet Test Meter } V_{m s d}=17.65 \times V_{m}\left[\frac{P_{b}+\frac{P_{m}}{13.6}}{\left(T_{m}+460\right)}\right] \times C_{f}=\underline{10.123} d c s f \\
\text { Dry Gas Meter Vmstd }=17.65 \times V_{m}\left[\frac{P_{b}+\frac{P_{m}}{13.6}}{\left(T_{m}+460\right)}\right]=\underline{10.106} \text { dcsf } \\
\text { Calibration Factor }(C D G)=\frac{\text { Wet Test Meter } V_{\text {mstd }}}{\text { Dry Gas Meter } V_{\text {mstd }}}=\underline{1.002}
\end{gathered}
$$




\author{
Calibration Data \\ Dry Gas Meter Calibration
}

Meter Console No.

Date Calibration Performed:

ASAI 1-1

04/03/07

$\begin{aligned} \text { Run } \frac{1}{\mathrm{~Pb}} & =29.61 \mathrm{Hg} \\ \text { Wet Test Meter } \mathrm{C}_{\mathrm{f}}=1.020 \quad \mathrm{Hg} & \text { Console Pump Vacuum }=\end{aligned}$

Wet Test Meter No. ASAI-0 Meter

Dry Gas Meter

\begin{tabular}{llllllllllllll}
\multicolumn{1}{c}{ Wet Test Meter No. ASAl-O } \\
Meter
\end{tabular}

Wet Test Meter Vmsd $=17.65 \times V_{m}\left[\frac{P_{b}+\frac{P_{m}}{13.6}}{\left(T_{m}+460\right)}\right] \times C_{f}=\underline{10.833} \mathrm{dcsf}$
Dry Gas Meter Vmsui $=17.65 \times \mathrm{Vm}\left[\frac{P_{b}+\frac{P_{m}}{13.6}}{\left(T_{m}+460\right)}\right]=\underline{10.833} \mathrm{dcsf}$ Calibration Factor $\left(C_{D G}\right)=\frac{\text { Wet Test Meter } V_{\text {mstd }}}{\text { Dry Gas Meter } V_{\text {msto }}}=1.000$ 
Calibration Data

Dry Gas Meter Calibration

$\begin{array}{rr}\text { Meter Console No. } & \text { ASAI 1-1 } \\ \text { Date Calibration Performed: } & 04 / 03 / 07\end{array}$

$\begin{aligned} \text { Run } \frac{1}{\mathrm{~Pb}} & =29.61 \\ \text { Wet Test Meter } \mathrm{C}_{\mathrm{f}}=1.020 \text { Console Pump Vacuum } & =-5.0 \mathrm{Hg}\end{aligned}$

Wet Test Meter No. ASAI-0 Meter

Time Reading Temp. $\quad \underline{\mathrm{P}_{\mathrm{m}}}$

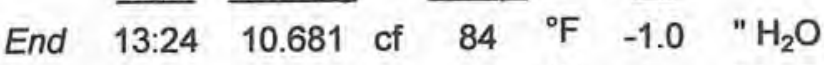

Start $\underline{13: 13} \quad \underline{0.000}$ of $\underline{84} \quad{ }^{\circ} \mathrm{F} \quad \underline{-1.0} \quad " \mathrm{H}_{2} \mathrm{O}$ $\begin{array}{lllllllll} & \text { Average } & 10.681 & \text { cf } & 84 & { }^{\circ} \mathrm{F} & -1.0 & \quad \mathrm{H}_{2} \mathrm{O}\end{array}$
Dry Gas Meter Meter Temperature

\begin{tabular}{|c|c|c|c|c|c|}
\hline$\underline{\text { Reading }}$ & In & & Out & & $\underline{P}_{m}$ \\
\hline $186.989 \mathrm{cf}$ & 87 & ${ }^{\circ} \mathrm{F}$ & 84 & ${ }^{\circ} \mathrm{F}$ & 3.00 \\
\hline $176.100 \mathrm{cf}$ & 84 & ${ }^{\circ} \mathrm{F}$ & 84 & ${ }^{\circ} \mathrm{F}$ & 3.00 \\
\hline 10.889 cf & 86 & ${ }^{\circ} \mathrm{F}$ & 84 & ${ }^{\circ} \mathrm{F}$ & 3.00 \\
\hline
\end{tabular}

$$
\begin{gathered}
\text { Wet Test Meter Vmstd }=17.65 \times V_{m}\left[\frac{P_{b}+\frac{P_{m}}{13.6}}{\left(T_{m}+460\right)}\right] x C_{f}=\underline{10.440} \mathrm{dcsf} \\
\text { Dry Gas Meter } V_{m s d}=17.65 \times V m\left[\frac{P_{b}+\frac{P_{m}}{13.6}}{\left(T_{m}+460\right)}\right]=\underline{10.524} \mathrm{dcsf} \\
\text { Calibration Factor }\left(C_{D G}\right)=\frac{\text { Wet Test Meter } V_{m s t d}}{\text { Dry Gas Meter } V_{m s t d}}=\underline{0.992}
\end{gathered}
$$




\author{
Calibration Data \\ Dry Gas Meter Calibration \\ Meter Console No. \\ ASAI 1-1 \\ Date Calibration Performed: \\ 04/03/07
}

Run 1 at $4.0^{\prime \prime} \Delta \mathrm{H}$

$\mathrm{Pb}=29.61 " \mathrm{Hg}$

Wet Test Meter $C_{f}=1.020$ Console Pump Vacuum $=-5.0 " \mathrm{Hg}$

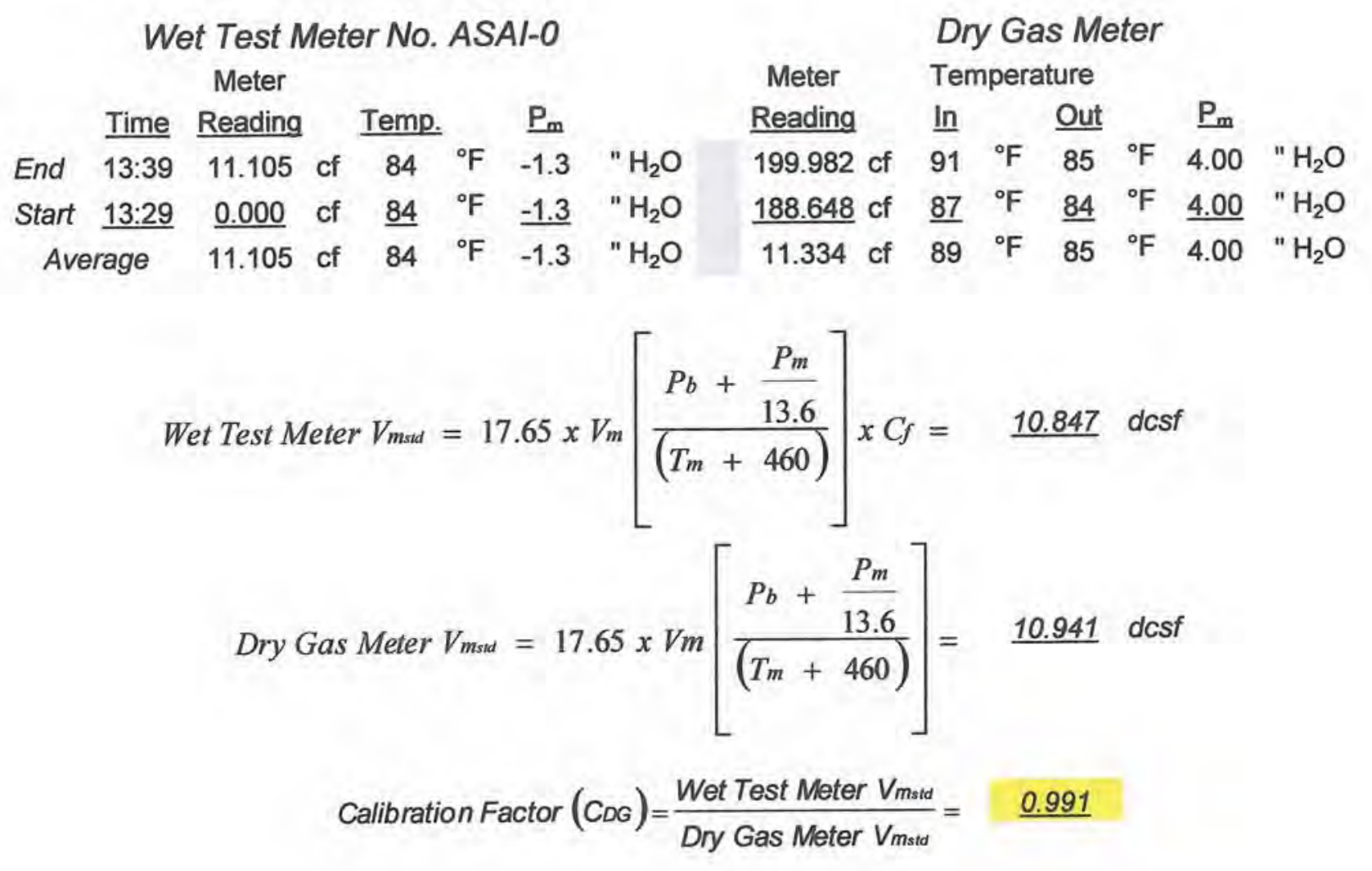




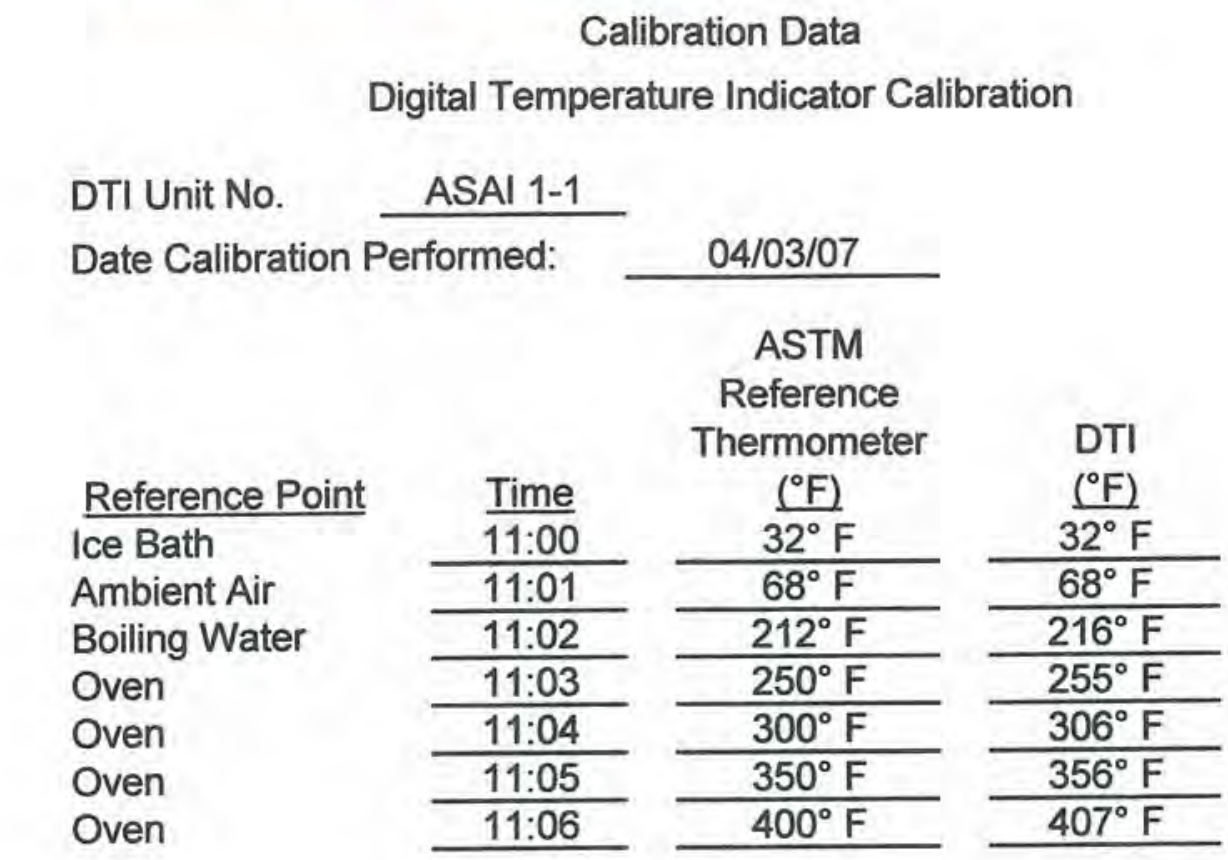

Meter Adjusted?

Yes

ASTM Reference Thermometer:

SN: 06000047

Range: $\quad-0.1^{\circ} \mathrm{C}$ to $1360.6^{\circ} \mathrm{C}$

Range:

Range:

Certified by:

Scot Jackson 04/03/07

Calibrator (Signature / Date)

Bill Hefley 04/03/07

QA Officer (Signature / Date) 


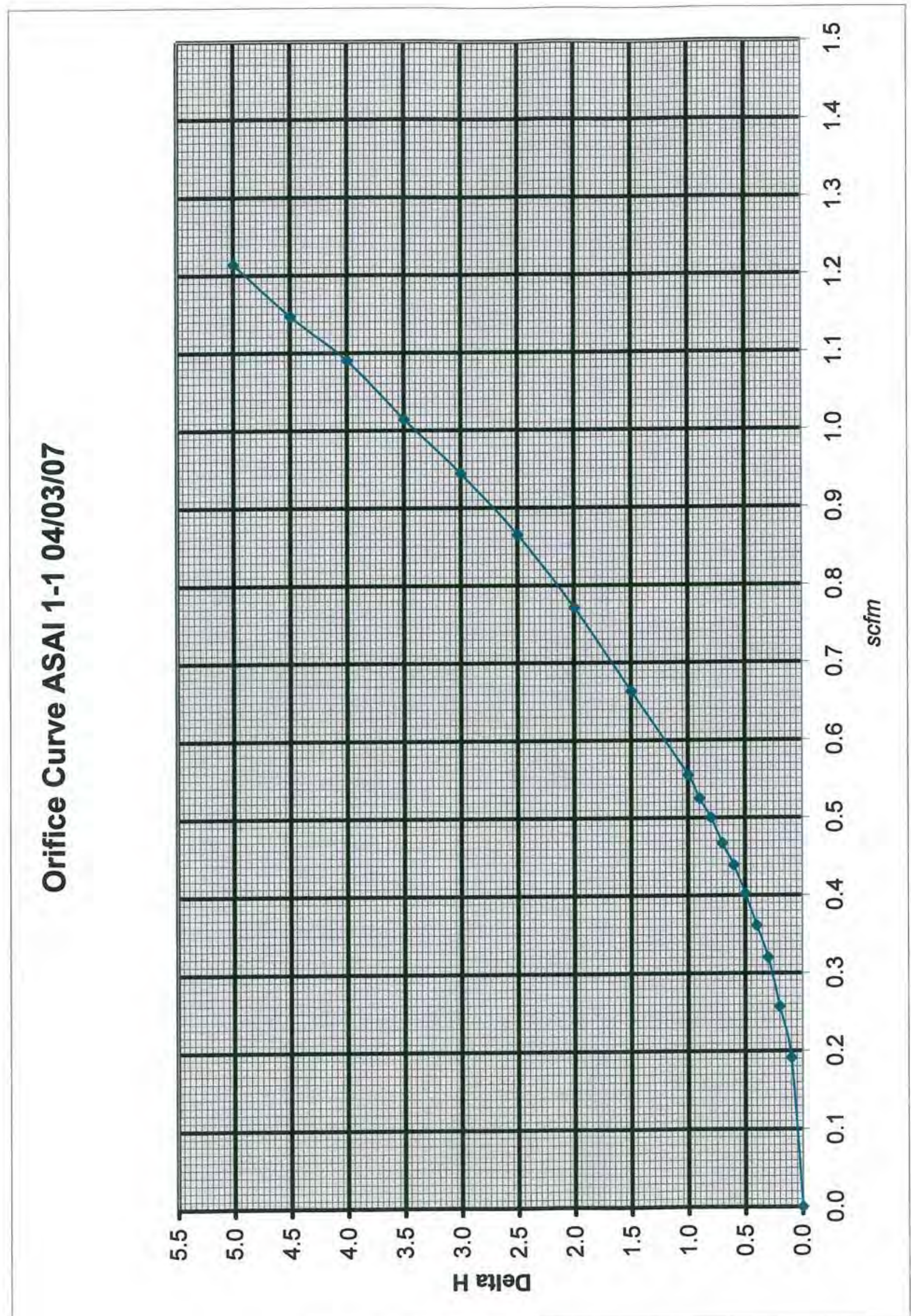

C-11 


\author{
Calibration Data \\ Dry Gas Meter Calibration \\ Meter Console No. \\ ASAI 1-2 \\ Date Calibration Performed: \\ 04/03/07 \\ $\Delta \mathrm{H}$ Setting

\begin{tabular}{c}
$\left(" \mathrm{H}_{2} \mathrm{O}\right)$ \\
\hline 0.50 \\
1.00 \\
1.50 \\
2.00 \\
3.00 \\
4.00 \\
Average
\end{tabular} \\ $\mathrm{C}_{\mathrm{DG}}$ \\ 1.007 \\ 1.008 \\ 1.002 \\ 1.001 \\ 1.001 \\ 1.002 \\ 1.004 \\ \begin{tabular}{ll} 
Variation +: & $0.40 \%$ \\
Variation -: & $-0.30 \%$ \\
\hline
\end{tabular} \\ Certified by: $\frac{\text { Scot Jackson 4/03/07 }}{\text { Calibrator (Signature I Date) }}$ \\ Bill Hefley 4/03/07 \\ QA Officer (Signature / Date)
}


Calibration Data

Dry Gas Meter Calibration

Meter Console No.

Date Calibration Performed:
ASAI 1-2

04/03/07

Run $\frac{1}{1}$ at $0.5^{\prime \prime} \Delta \mathrm{H}$

Wet Test Meter $C_{f}=1.020$ Console Pump Vacuum $=-5.0 " \mathrm{Hg}$

Wet Test Meter No. ASAI-0 Meter

\begin{tabular}{|c|c|c|c|c|c|c|c|}
\hline & Time & Reading & & Temp. & & $\underline{P_{m}}$ & \\
\hline End & $9: 02$ & 5.005 & cf & 74 & ${ }^{\circ} \mathrm{F}$ & -0.2 & $" \mathrm{H}_{2} \mathrm{O}$ \\
\hline Start & $\underline{8: 50}$ & $\underline{0.000}$ & cf & $\underline{74}$ & ${ }^{\circ} \mathrm{F}$ & $\underline{-0.2}$ & $" \mathrm{H}_{2} \mathrm{O}$ \\
\hline Ave & & 5.005 & cf & 74 & ${ }^{\circ} \mathrm{F}$ & -0.2 & $" \mathrm{H}_{2} \mathrm{O}$ \\
\hline
\end{tabular}

Dry Gas Meter

Meter Temperature

Reading In Out $\underline{P_{m}}$ 165.815 of $81 \quad{ }^{\circ} \mathrm{F} \quad \overline{78} \quad{ }^{\circ} \mathrm{F} \quad 0.50 \quad " \mathrm{H}_{2} \mathrm{O}$ $\underline{160.702}$ of $\underline{81}{ }^{\circ} \mathrm{F} \quad \underline{78}{ }^{\circ} \mathrm{F} \quad \underline{0.50} \quad \mathrm{H}_{2} \mathrm{O}$

5.113 of $81{ }^{\circ} \mathrm{F} \quad 78$ ' $\mathrm{F} \quad 0.50 \quad " \mathrm{H}_{2} \mathrm{O}$

Wet Test Meter $V_{m s t d}=17.65 \times V_{m}\left[\frac{P_{b}+\frac{P_{m}}{13.6}}{\left(T_{m}+460\right)}\right] \times C_{f}=\underline{4.985} d c s f$
Dry Gas Meter $V_{m s t d}=17.65 \times V m\left[\frac{P_{b}+\frac{P_{m}}{13.6}}{\left(T_{m}+460\right)}\right]=\underline{4.951} d c s f$ Calibration Factor $(C D G)=\frac{\text { Wet Test Meter } V_{m s t d}}{\text { Dry Gas Meter } V_{\text {mstd }}}=\underline{1.007}$ 


\author{
Calibration Data \\ Dry Gas Meter Calibration
}

Meter Console No.

ASAI 1-2

Date Calibration Performed:

$\begin{aligned} \text { Run } \frac{1}{\mathrm{~Pb}}=29.56 \mathrm{Hg} & \text { at } 1.0^{\prime \prime} \Delta \mathrm{H} \\ \text { Wet Test Meter } \mathrm{C}_{\mathrm{f}}=1.020 \text { Console Pump Vacuum } & =-5.0^{\mathrm{Hg}}\end{aligned}$

$$
\begin{aligned}
& \text { Wet Test Meter No. ASAI-0 } \\
& \text { Meter } \\
& \text { Meter Temperature } \\
& \text { Time Reading Temp. } \quad \underline{\mathrm{P}}_{\mathrm{m}} \\
& \text { Reading In Out } \underline{P_{m}}
\end{aligned}
$$

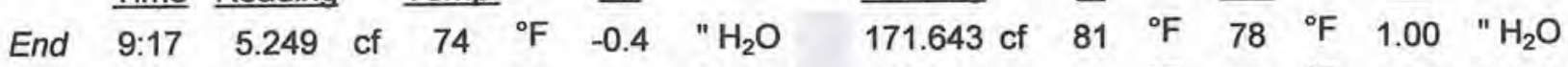

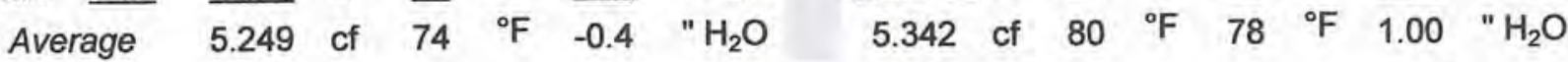

$$
\begin{aligned}
& \begin{array}{c}
\text { Wet Test Meter } V_{\text {msd }}=17.65 \times V_{m}\left[\frac{P_{b}+\frac{P_{m}}{13.6}}{\left(T_{m}+460\right)}\right] \times C_{f}=\underline{5.226} d c s f \\
\text { Dry Gas Meter Vmstd }=17.65 \times \mathrm{Vm}\left[\frac{P_{b}+\frac{P_{m}}{13.6}}{\left(T_{m}+460\right)}\right]=\underline{5.184} \mathrm{dcsf}
\end{array} \\
& \text { Calibration Factor }(C D G)=\frac{\text { Wet Test Meter } V_{m s t d}}{\text { Dry Gas Meter } V_{m s t d}}=1.008
\end{aligned}
$$


Calibration Data

Dry Gas Meter Calibration

Meter Console No.

Date Calibration Performed:
ASAI 1-2

04/03/07

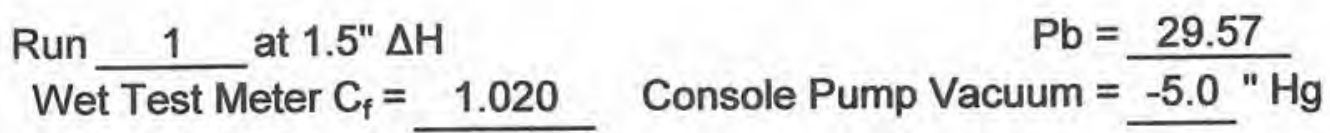

\begin{tabular}{|c|c|c|c|c|c|c|c|c|c|c|c|c|c|}
\hline \multirow{2}{*}{\multicolumn{7}{|c|}{$\begin{array}{l}\text { Wet Test Meter No. ASAI-O } \\
\text { Meter }\end{array}$}} & \multicolumn{7}{|c|}{ Dry Gas Meter } \\
\hline & & & & & & & Reading & In & & Out & & $\underline{P_{m}}$ & \\
\hline End & $9: 40$ & $10.523 \mathrm{cf}$ & 75 & ${ }^{\circ} \mathrm{F}$ & -0.6 & $" \mathrm{H}_{2} \mathrm{O}$ & $\overline{182.845}$ cf & 83 & ${ }^{\circ} \mathrm{F}$ & $\overline{78}$ & ${ }^{\circ} \mathrm{F}$ & 1.50 & " $\mathrm{H}_{2} \mathrm{O}$ \\
\hline Start & 9:25 & $\underline{0.000} \mathrm{cf}$ & $\underline{75}$ & ${ }^{\circ} \mathrm{F}$ & -0.6 & $" \mathrm{H}_{2} \mathrm{O}$ & $\underline{172.100 \mathrm{cf}}$ & $\underline{78}$ & ${ }^{\circ} \mathrm{F}$ & $\underline{78}$ & ${ }^{\circ} \mathrm{F}$ & 1.50 & $" \mathrm{H}_{2} \mathrm{O}$ \\
\hline Ave & rage & $10.523 \mathrm{cf}$ & 75 & ${ }^{\circ} \mathrm{F}$ & -0.6 & " $\mathrm{H}_{2} \mathrm{O}$ & $10.745 \mathrm{cf}$ & 81 & ${ }^{\circ} \mathrm{F}$ & 78 & ${ }^{\circ} \mathrm{F}$ & 1.50 & $" \mathrm{H}_{2} \mathrm{O}$ \\
\hline
\end{tabular}

$$
\begin{gathered}
\text { Wet Test Meter } V_{m s d}=17.65 \times V_{m}\left[\frac{P_{b}+\frac{P_{m}}{13.6}}{\left(T_{m}+460\right)}\right] \times C_{f}=\underline{10.455} \mathrm{dcsf} \\
\text { Dry Gas Meter } V_{m s d d}=17.65 \times \mathrm{Vm}\left[\frac{P_{b}+\frac{P_{m}}{13.6}}{\left(T_{m}+460\right)}\right]=\underline{10.438} \mathrm{dcsf}
\end{gathered}
$$$$
\text { Calibration Factor }\left(C_{D G}\right)=\frac{\text { Wet Test Meter } V_{\text {mstd }}}{\text { Dry Gas Meter } V_{\text {mstd }}}=\underline{1.002}
$$ 


\author{
Calibration Data \\ Dry Gas Meter Calibration \\ Meter Console No. \\ ASAI 1-2 \\ Date Calibration Performed: \\ 04/03/07
}

$\begin{aligned} \text { Run } \frac{1}{\mathrm{~Pb}} & =29.57 \mathrm{Hg} \\ \text { Wet Test Meter } \mathrm{C}_{\mathrm{f}}=1.020 \text { Console Pump Vacuum } & =-5.0 \mathrm{Hg}\end{aligned}$

Wet Test Meter No. ASAI-O Meter

$\underline{\text { Time }}$ Reading Temp. $\quad \underline{P_{m}}$

End 10:02 10.531 of $75{ }^{\circ} \mathrm{F} \quad-0.7 \quad " \mathrm{H}_{2} \mathrm{O}$

Start $\underline{9: 49} \quad \underline{0.000}$ of $\underline{\underline{75}} \quad{ }^{\circ} \mathrm{F} \quad \underline{\underline{-0.7}} \quad \mathrm{H}_{2} \mathrm{O}$

$\begin{array}{lllllllllllllll}\text { Average } & 10.531 & \text { of } & 75 & { }^{\circ} \mathrm{F} & -0.7 & \mathrm{H}_{2} \mathrm{O}\end{array}$
Dry Gas Meter

Meter Temperature

Reading In Out $\underline{\mathrm{P}_{m}}$ 193.955 cf $84{ }^{\circ} \mathrm{F} \quad 78 \quad{ }^{\circ} \mathrm{F} \quad 2.00 \quad \mathrm{H}_{2} \mathrm{O}$ $\underline{183.203} \mathrm{cf} \quad \underline{79}{ }^{\circ} \mathrm{F} \quad \underline{78}{ }^{\circ} \mathrm{F} \quad \underline{2.00} \quad \mathrm{H}_{2} \mathrm{O}$ 10.752 cf $82{ }^{\circ} \mathrm{F} \quad 78 \quad{ }^{\circ} \mathrm{F} \quad 2.00 \quad \mathrm{H}_{2} \mathrm{O}$

Wet Test Meter $V_{m s d}=17.65 \times V_{m}\left[\frac{P_{b}+\frac{P_{m}}{13.6}}{\left(T_{m}+460\right)}\right] x C_{f}=\underline{10.461} \mathrm{dcsf}$
Dry Gas Meter $V_{m s d}=17.65 \times \mathrm{Vm}\left[\frac{P_{b}+\frac{P_{m}}{13.6}}{\left(T_{m}+460\right)}\right]=\underline{10.448} \mathrm{dcsf}$ Calibration Factor $(C D G)=\frac{\text { Wet Test Meter } V_{\text {mstd }}}{\text { Dry Gas Meter } V_{m s t d}}=1.001$ 


\section{Calibration Data}

Dry Gas Meter Calibration

Meter Console No.

ASAI 1-2

Date Calibration Performed:

04/03/07

Run 1 at 3.0" $\Delta \mathrm{H}$

Wet Test Meter $C_{f}=1.020$

$\begin{aligned} \mathrm{Pb} & =29.57 \mathrm{Hg} \\ \text { Console Pump Vacuum } & =-5.0 \mathrm{Hg}\end{aligned}$

Wet Test Meter No. ASAl-0

Dry Gas Meter

Meter

Meter Temperature

$\underline{T i m e}$ Reading Temp. $\quad \underline{P_{m}}$ Reading In Out $\underline{P}_{m}$

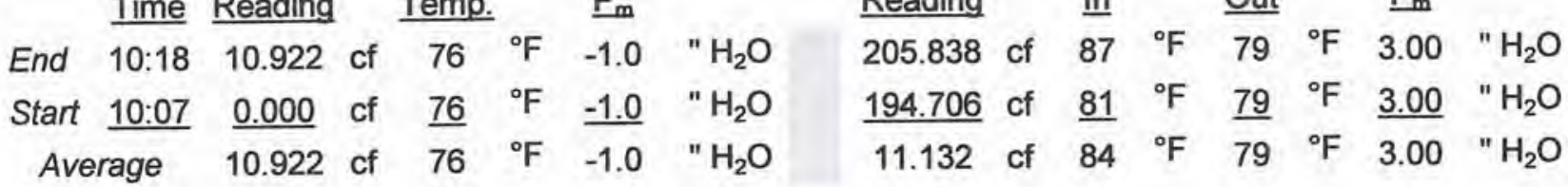

$$
\begin{gathered}
\text { Wet Test Meter } V_{m s s}=17.65 \times V_{m}\left[\frac{P_{b}+\frac{P_{m}}{13.6}}{\left(T_{m}+460\right)}\right] x C_{f}=\underline{10.821} \mathrm{dcsf} \\
\text { Dry Gas Meter } V_{m s d}=17.65 \times \mathrm{Vm}\left[\frac{P_{b}+\frac{P_{m}}{13.6}}{\left(T_{m}+460\right)}\right]=\underline{10.809} \mathrm{dcsf}
\end{gathered}
$$

$$
\text { Calibration Factor }(C D G)=\frac{\text { Wet Test Meter } V_{\text {mstd }}}{\text { Dry Gas Meter } V_{\text {mstd }}}=1.001
$$




\author{
Calibration Data \\ Dry Gas Meter Calibration \\ Meter Console No. \\ ASAI 1-2 \\ Date Calibration Performed: \\ 04/03/07
}

Run 1 at $4.0^{\prime \prime} \Delta \mathrm{H}$

Wet Test Meter $C_{f}=1.020$

$\begin{aligned} \mathrm{Pb} & =29.58 \mathrm{Hg} \\ \text { Console Pump Vacuum } & =-5.0 \mathrm{Hg}\end{aligned}$

Wet Test Meter No. ASAI-0 Meter

Meter Temperature

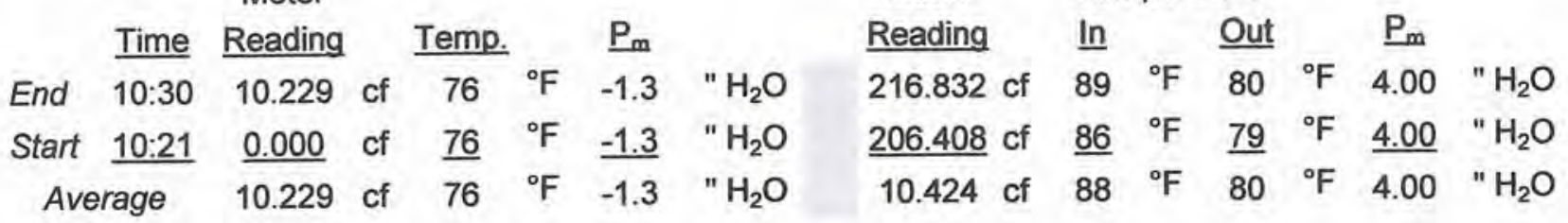

Wet Test Meter $V_{m s d}=17.65 \times V_{m}\left[\frac{P_{b}+\frac{P_{m}}{13.6}}{\left(T_{m}+460\right)}\right] x C_{f}=\underline{10.130} \mathrm{dcsf}$
Dry Gas Meter $V_{m s d}=17.65 \times \mathrm{Vm}\left[\frac{P_{b}+\frac{P_{m}}{13.6}}{\left(T_{m}+460\right)}\right]=\underline{10.113} \mathrm{dcsf}$

Calibration Factor $(C D G)=\frac{\text { Wet Test Meter } V_{\text {mstd }}}{\text { Dry Gas Meter } V_{m s t d}}=\underline{1.002}$ 


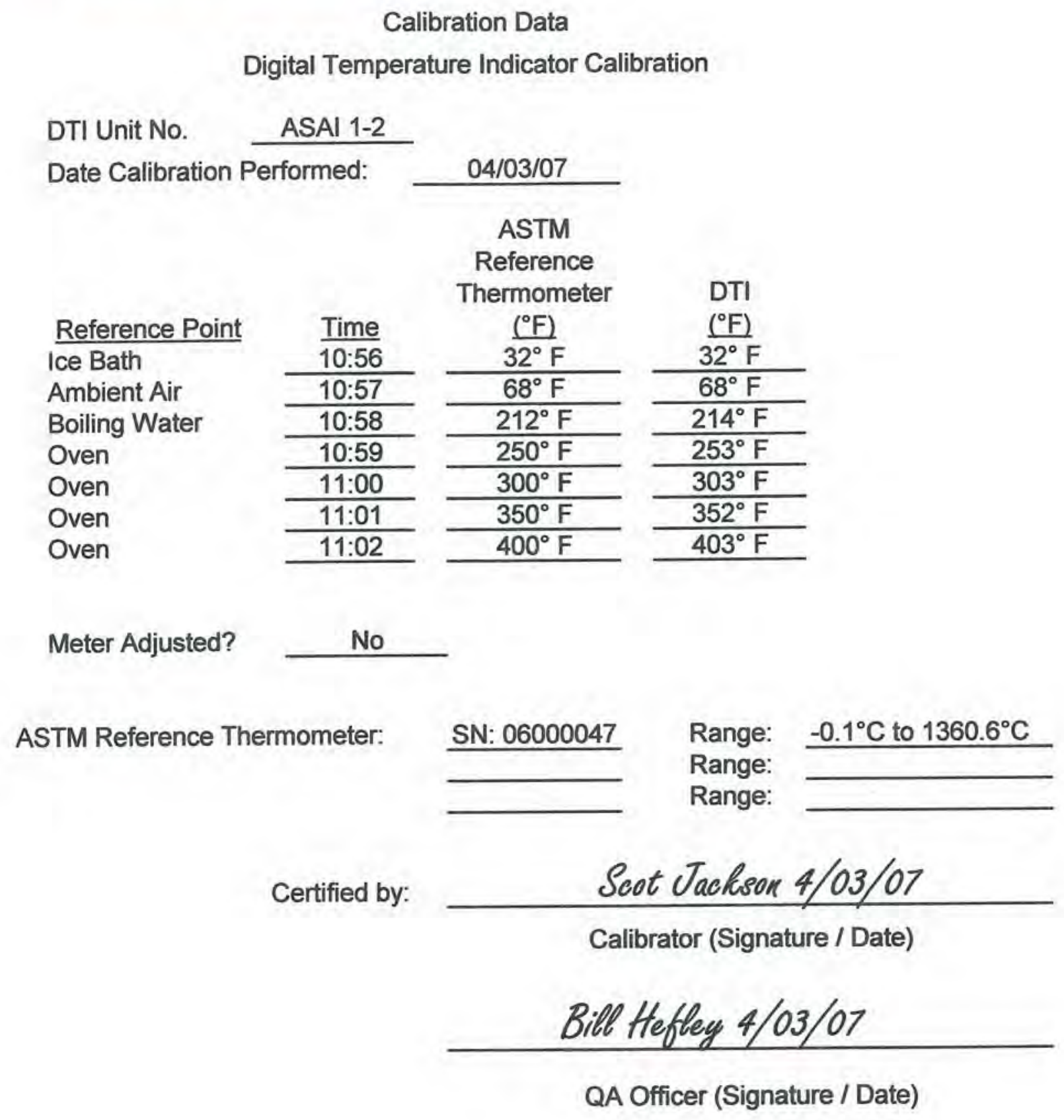




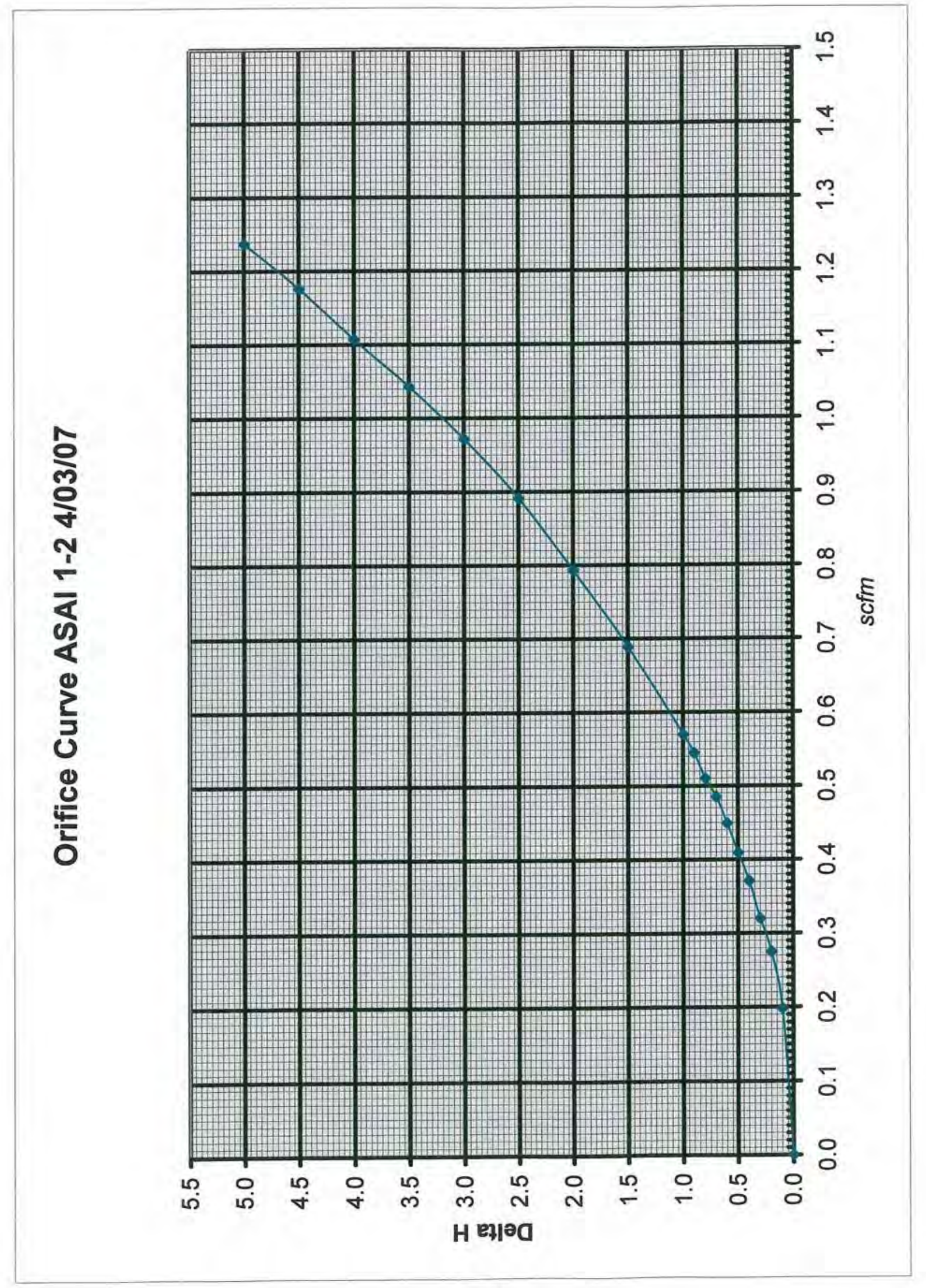




\section{II \\ ASSOCIATES, INC.}

CALIBRATION DATA

PITOT TUBE CALIBRATION DATA

\begin{tabular}{|c|c|}
\hline Date: & $08 / 31 / 07$ \\
\hline I.D. \# & PM-10 \\
\hline $\mathrm{C}_{\text {pstd: }}$ & 0.990 \\
\hline
\end{tabular}

Time: $13: 30$

$\mathrm{T}_{\mathrm{s}}: \frac{13.30}{9}{ }^{\circ} \mathrm{F}$

$\mathrm{Pb}: 29.72 \mathrm{Hg}$

\begin{tabular}{|c|c|c|c|c|c|c|c|c|c|c|}
\hline \multirow{2}{*}{$\begin{array}{c}\text { fps } \\
\text { Mark }\end{array}$} & \multirow{2}{*}{$\begin{array}{c}\text { Desired } \\
\text { Calibration } \\
\text { Standard }\end{array}$} & \multicolumn{2}{|c|}{$\begin{array}{c}\text { Calibration } \\
\text { Standard }\end{array}$} & \multirow{2}{*}{$\begin{array}{c}\text { v Standard } \\
\text { Average }\end{array}$} & \multirow[b]{2}{*}{ High } & \multirow[b]{2}{*}{ v High } & \multirow{2}{*}{$\begin{array}{l}\text { Cal. } \\
\text { Factor }\end{array}$} & \multirow[b]{2}{*}{ Low } & \multirow[b]{2}{*}{ v Low } & \multirow{2}{*}{$\begin{array}{l}\text { Cal. } \\
\text { Factor }\end{array}$} \\
\hline & & Start & End & & & & & & & \\
\hline 20 & 0.09 & 0.09 & 0.09 & 0.300 & 0.13 & 0.361 & 0.824 & 0.13 & 0.361 & 0.824 \\
\hline 30 & 0.19 & 0.19 & 0.19 & 0.436 & 0.27 & 0.520 & 0.830 & 0.27 & 0.520 & 0.830 \\
\hline 40 & 0.35 & 0.35 & 0.35 & 0.592 & 0.50 & 0.707 & 0.828 & 0.50 & 0.707 & 0.828 \\
\hline 50 & 0.54 & 0.54 & 0.54 & 0.735 & 0.78 & 0.883 & 0.824 & 0.78 & 0.883 & 0.824 \\
\hline 60 & 0.78 & 0.78 & 0.78 & 0.883 & 1.13 & 1.061 & 0.824 & 1.13 & 1.061 & 0.824 \\
\hline 70 & 1.06 & 1.05 & 1.05 & 1.025 & 1.50 & 1.225 & 0.828 & 1.50 & 1.225 & 0.828 \\
\hline 80 & 1.39 & 1.40 & 1.40 & 1.183 & 2.00 & 1.414 & 0.828 & 2.00 & 1.414 & 0.828 \\
\hline \multirow[t]{2}{*}{90} & 1.75 & 1.75 & $1, .75$ & 1.323 & 2.50 & 1.581 & 0.828 & 2.50 & 1.581 & 0.828 \\
\hline & & & & & & & & & & \\
\hline 50 & 0.54 & 0.54 & 0.54 & 0.735 & 0.78 & 0.883 & 0.824 & 0.78 & 0.883 & 0.824 \\
\hline 50 & 0.54 & 0.54 & 0.54 & 0.735 & 0.78 & 0.883 & 0.824 & 0.78 & 0.883 & 0.824 \\
\hline & & & & & & & & & & \\
\hline & & & & & & & & & & \\
\hline & Average & & & & & & 0.827 & & & 0.827 \\
\hline
\end{tabular}

Summary of Results:

Normal high side calibraiton factor: $\quad 0.827$

variation $+\quad 0.36 \%$

variation $-:-0.36 \%$

Normal low side calibration factor: $\quad 0.827$

variation $+0.36 \%$

variation $-: \frac{-0.36 \%}{-}$

Certification:

I certify that the Type S pitot tube, the standard type pitot tube, and the calibration setup meet or exceed all specifications, criteria and/or applicable design features and hereby assign a pitot tube calibration factor $\mathrm{C}_{\mathrm{p}}$ of: $\quad 0.827$

Certified by:

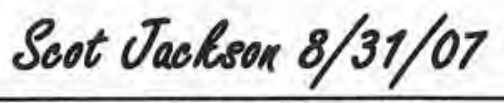

Calibrator (Signature/Date)

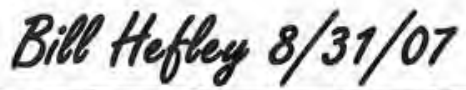

QA Officer (Signature/Date) 
Calibration Data

Nozzle Calibration

\begin{aligned} Nozzle Set No. & \multicolumn{2}{c}{$P M-10$} \\ Date Calibration Performed: & $\frac{9 / 6}{6 / 07}\end{aligned}$

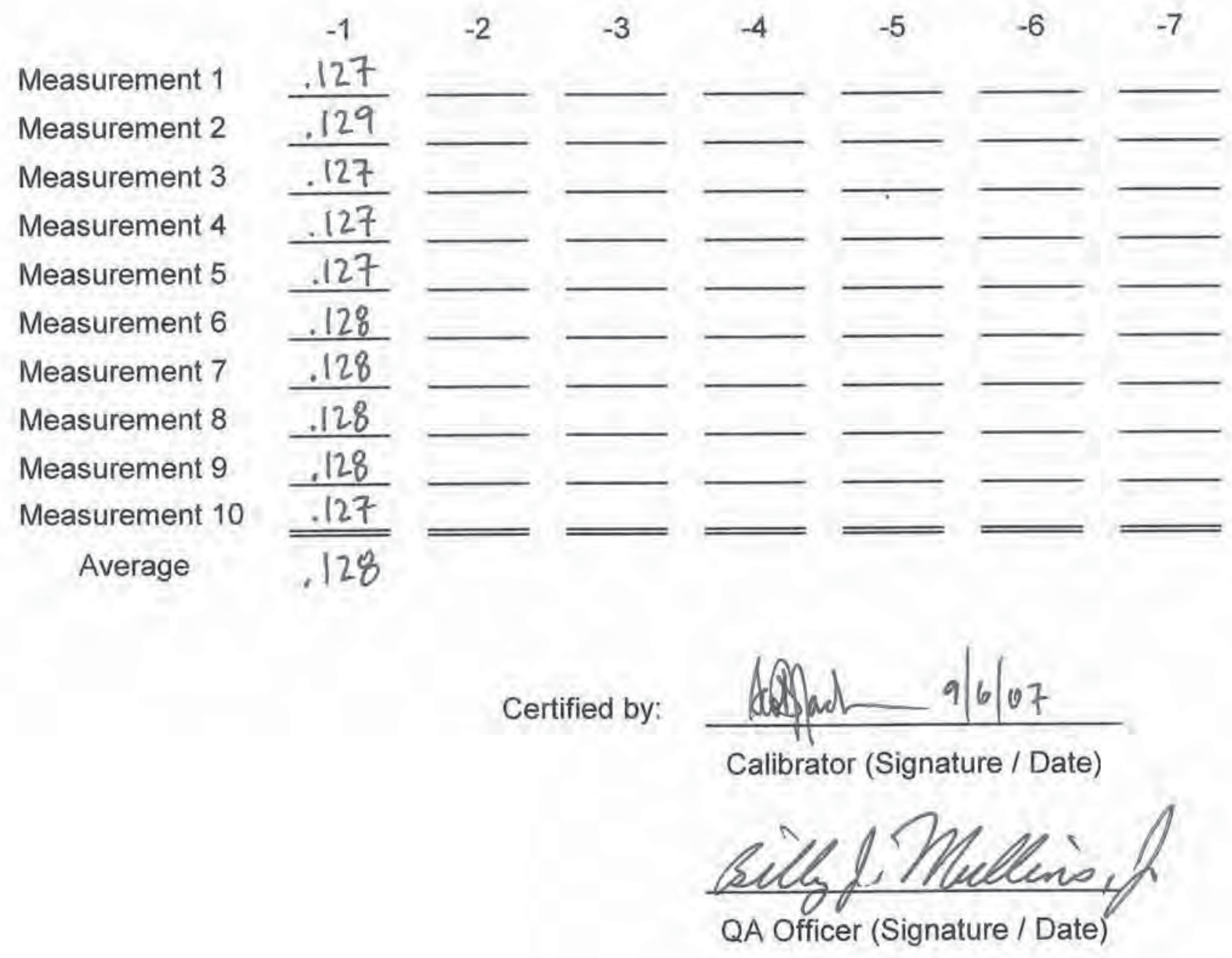




\section{Post-test Calibration Data}




\title{
II)
}

\author{
Calibration Data \\ Dry Gas Meter Calibration \\ Meter Console No. \\ Date Calibration Performed: \\ ASAI 1-1 \\ 09/11/07 \\ $\Delta \mathrm{H}$ Setting

\begin{tabular}{c}
$\left(" \mathrm{H}_{2} \mathrm{O}\right)$ \\
\hline 0.50 \\
1.00 \\
1.50 \\
2.00 \\
3.00 \\
4.00 \\
Average
\end{tabular} \\ Variation +: \\ Variation -:

\begin{tabular}{c}
$C_{D G}$ \\
\hline 0.995 \\
\hline 1.005 \\
\hline 1.002 \\
\hline 0.998 \\
\hline 0.994 \\
\hline 0.991 \\
\hline 0.998 \\
\hline
\end{tabular} \\ Certified by: \\ Scot Jackson 09/11/07 \\ Calibrator (Signature / Date) \\ $\frac{\text { Bill Hefley 09/11/07 }}{\text { QA Officer (Signature I Date) }}$
}




\author{
Calibration Data \\ Dry Gas Meter Calibration
}

Meter Console No.

Date Calibration Performed:
ASAI 1-1

09/11/07

Run $\quad 1$ at $0.5^{\prime \prime} \Delta \mathrm{H} \quad \mathrm{Pb}=29.77$ " $\mathrm{Hg}$

Wet Test Meter $\mathrm{C}_{\mathrm{f}}=1.020$ Console Pump Vacuum $=-5.0 \mathrm{Hg}$

Wet Test Meter No. ASAl-O

Meter

Time Reading Temp. $\quad \underline{P_{m}}$

End 11:10 5.281 cf $77 \quad{ }^{\circ} \mathrm{F} \quad-0.2 \quad " \mathrm{H}_{2} \mathrm{O}$

Start $\underline{10: 57} \quad \underline{0.000}$ cf $\quad \underline{77} \quad{ }^{\circ} \mathrm{F} \quad \frac{-0.2}{77} \quad{ }^{\circ} \mathrm{F} \quad \mathrm{H}_{2} \mathrm{O}$
Dry Gas Meter

Meter Temperature

Reading In $\underline{\text { Out }} \underline{\mathrm{P}_{\mathrm{m}}}$

694.214 cf $79{ }^{\circ} \mathrm{F} \quad 76 \quad{ }^{\circ} \mathrm{F} \quad 0.50 \quad " \mathrm{H}_{2} \mathrm{O}$

$\underline{688.800}$ of $\quad \underline{79}{ }^{\circ} \mathrm{F} \quad \underline{77} \quad{ }^{\circ} \mathrm{F} \quad \underline{0.50} \quad \mathrm{H}_{2} \mathrm{O}$

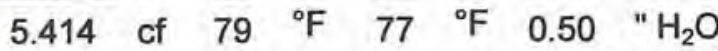

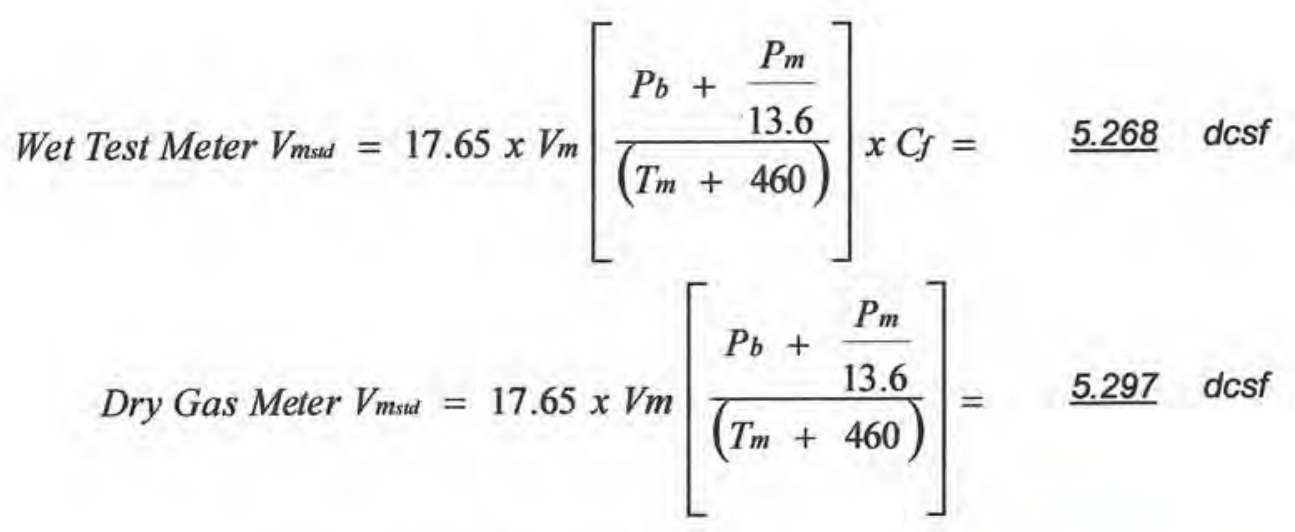

Calibration Factor $(C D G)=\frac{\text { Wet Test Meter } V_{\text {mstd }}}{\text { Dry Gas Meter } V_{\text {mstd }}}=\underline{0.995}$ 
Calibration Data
Dry Gas Meter Calibration

Meter Console No.

Date Calibration Performed:

ASAI 1-1

09/11/07

Run $\frac{1}{1}$ at $1.0^{\prime \prime} \Delta \mathrm{H} \quad \mathrm{Pb}=29.77^{\prime} \mathrm{Hg}$

Wet Test Meter $\mathrm{C}_{\mathrm{f}}=1.020$ Console Pump Vacuum $=-5.0$ " Hg

Wet Test Meter No. ASAl-0 Meter

\begin{tabular}{lllllllll} 
& $\underline{\text { Time }}$ & & Reading & \multicolumn{1}{c}{ Temp. } & \multicolumn{1}{c}{$\underline{P}_{m}$} & \\
End $9: 43$ & 5.086 & cf & 77 & ${ }^{\circ} \mathrm{F}$ & -0.3 & $" \mathrm{H}_{2} \mathrm{O}$ \\
Start & $\underline{9: 34}$ & $\underline{0.000}$ & cf & $\underline{77}$ & ${ }^{\circ} \mathrm{F}$ & $\underline{-0.3}$ & $" \mathrm{H}_{2} \mathrm{O}$ \\
Average & 5.086 & cf & $\frac{77}{77}$ & ${ }^{\circ} \mathrm{F}$ & $\underline{-0.3}$ & $" \mathrm{H}_{2} \mathrm{O}$
\end{tabular}

\section{Dry Gas Meter}

Meter Temperature

Reading $\underline{\text { In }} \quad \underline{\text { Out }} \quad \underline{P_{m}}$

642.773 cf $78{ }^{\circ} \mathrm{F} \quad 75{ }^{\circ} \mathrm{F} \quad 1.00 \quad " \mathrm{H}_{2} \mathrm{O}$

$\underline{637.638}$ of $\quad \underline{76}{ }^{\circ} \mathrm{F} \quad \underline{74}{ }^{\circ} \mathrm{F} \quad \underline{1.00} \quad " \mathrm{H}_{2} \mathrm{O}$

5.135 of $77{ }^{\circ} \mathrm{F} \quad 75 \quad{ }^{\circ} \mathrm{F} \quad 1.00 \quad " \mathrm{H}_{2} \mathrm{O}$

Wet Test Meter $V_{m s d}=17.65 \times V_{m}\left[\frac{P_{b}+\frac{P_{m}}{13.6}}{\left(T_{m}+460\right)}\right] x C_{f}=\underline{5.072} d c s f$
Dry Gas Meter $V_{m s d}=17.65 \times \mathrm{Vm}\left[\frac{P_{b}+\frac{P_{m}}{13.6}}{\left(T_{m}+460\right)}\right]=\underline{5.049} \mathrm{dcsf}$

Calibration Factor $(C D G)=\frac{\text { Wet Test Meter } V_{m s t d}}{\text { Dry Gas Meter } V_{m s t}}=1.005$ 


\author{
Calibration Data \\ Dry Gas Meter Calibration
}

Meter Console No.

ASAI 1-1

Date Calibration Performed:

09/11/07

$\begin{aligned} \text { Run } \frac{1}{\mathrm{~Pb}} & =29.77 \\ \text { Wet Test Meter } \mathrm{C}_{\mathrm{f}}=1.020 \quad \mathrm{Hg} & \text { Console Pump Vacuum }=\underline{-5.0^{\prime \prime} \mathrm{Hg}}\end{aligned}$

Wet Test Meter No. ASAI-0

Dry Gas Meter Meter

Meter Temperature

\begin{tabular}{|c|c|c|c|c|c|c|c|c|c|c|c|c|c|}
\hline & Time & Reading & & Temp. & & $\underline{\mathrm{P}_{\mathrm{m}}}$ & & Reading & In & & Out & & $\underline{P_{m}}$ \\
\hline End & 10:05 & 10.304 & cf & 77 & ${ }^{\circ} \mathrm{F}$ & -0.5 & " $\mathrm{H}_{2} \mathrm{O}$ & $653.935 \mathrm{cf}$ & 82 & ${ }^{\circ} \mathrm{F}$ & 76 & ${ }^{\circ} \mathrm{F}$ & 1.50 \\
\hline & 9:50 & $\underline{0.000}$ & cf & 77 & ${ }^{\circ} \mathrm{F}$ & -0.5 & $" \mathrm{H}_{2} \mathrm{O}$ & $\underline{643.500 \mathrm{cf}}$ & $\underline{75}$ & ${ }^{\circ} \mathrm{F}$ & $\underline{75}$ & ${ }^{\circ} \mathrm{F}$ & 1.50 \\
\hline & & 10.304 & cf & 77 & ${ }^{\circ} \mathrm{F}$ & -0.5 & $" \mathrm{H}_{2} \mathrm{O}$ & $10.435 \mathrm{cf}$ & 79 & ${ }^{\circ} \mathrm{F}$ & 76 & ${ }^{\circ} \mathrm{F}$ & 1.50 \\
\hline
\end{tabular}

Wet Test Meter Vmsd $=17.65 \times V_{m}\left[\frac{P_{b}+\frac{P_{m}}{13.6}}{\left(T_{m}+460\right)}\right] \times C_{f}=\underline{10.271} \mathrm{dcsf}$
Dry Gas Meter Vmssd $=17.65 \times \mathrm{Vm}\left[\frac{P_{b}+\frac{P_{m}}{13.6}}{\left(T_{m}+460\right)}\right]=\underline{10.248} \mathrm{dcsf}$

Calibration Factor $\left(C_{D G}\right)=\frac{\text { Wet Test Meter } V_{\text {mstd }}}{\text { Dry Gas Meter } V_{\text {mst }}}=\underline{1.002}$ 


\author{
Calibration Data \\ Dry Gas Meter Calibration
}

Meter Console No.

ASAI 1-1

Date Calibration Performed:

09/11/07

$\begin{aligned} \text { Run } \frac{1}{\mathrm{~Pb}} & =29.77 \mathrm{Hg} \\ \text { Wet Test Meter } \mathrm{C}_{\mathrm{f}}=1.020 \quad \mathrm{Hg} & \text { Console Pump Vacuum }=-5.0 " \mathrm{Hg}\end{aligned}$

Wet Test Meter No. ASAI-O Meter

\begin{tabular}{|c|c|c|c|c|c|c|c|}
\hline & Time & Reading & & Temp. & & $\underline{P_{m}}$ & \\
\hline ind & $10: 22$ & 10.258 & cf & 77 & ${ }^{\circ} \mathrm{F}$ & -0.7 & $" \mathrm{H}_{2} \mathrm{O}$ \\
\hline$a r$ & $10: 09$ & $\underline{0.000}$ & cf & $\underline{77}$ & ${ }^{\circ} \mathrm{F}$ & $\underline{-0.7}$ & $" \mathrm{H}_{2} \mathrm{O}$ \\
\hline & & 10.258 & cf & 77 & ${ }^{\circ} \mathrm{F}$ & -0.7 & $" \mathrm{H}_{2} \mathrm{O}$ \\
\hline
\end{tabular}

Dry Gas Meter

Meter Temperature

Reading In Out $\underline{P_{m}}$ 664.844 cf $83{ }^{\circ} \mathrm{F} \quad 76 \quad{ }^{\circ} \mathrm{F} \quad 2.00 \quad " \mathrm{H}_{2} \mathrm{O}$ $\underline{654.401} \mathrm{cf} \quad \underline{78}{ }^{\circ} \mathrm{F} \quad \underline{76}{ }^{\circ} \mathrm{F} \quad \underline{2.00} \quad " \mathrm{H}_{2} \mathrm{O}$ 10.443 cf $81{ }^{\circ} \mathrm{F} \quad 76 \quad{ }^{\circ} \mathrm{F} \quad 2.00 \quad \mathrm{H}_{2} \mathrm{O}$

$$
\begin{gathered}
\text { Wet Test Meter } V_{m s d}=17.65 \times V_{m}\left[\frac{P_{b}+\frac{P_{m}}{13.6}}{\left(T_{m}+460\right)}\right] x C_{f}=10.220 \mathrm{dcsf} \\
\text { Dry Gas Meter } V_{m s d}=17.65 \times \mathrm{Vm}\left[\frac{P_{b}+\frac{P_{m}}{13.6}}{\left(T_{m}+460\right)}\right]=\underline{10.245} \mathrm{dcsf}
\end{gathered}
$$

Calibration Factor $(C D G)=\frac{\text { Wet Test Meter } V_{\text {mstd }}}{\text { Dry Gas Meter } V_{\text {mstd }}}=\underline{0.998}$ 
Calibration Data

Dry Gas Meter Calibration

Meter Console No.

ASAl 1-1

Date Calibration Performed:

Run 1 at 3.0" $\Delta \mathrm{H}$

Wet Test Meter $\mathrm{C}_{\mathrm{f}}=1.020$

$\mathrm{Pb}=29.77^{\prime} \mathrm{Hg}$

Console Pump Vacuum $=-5.0 " \mathrm{Hg}$

Wet Test Meter No. ASAl-0 Meter

Dry Gas Meter

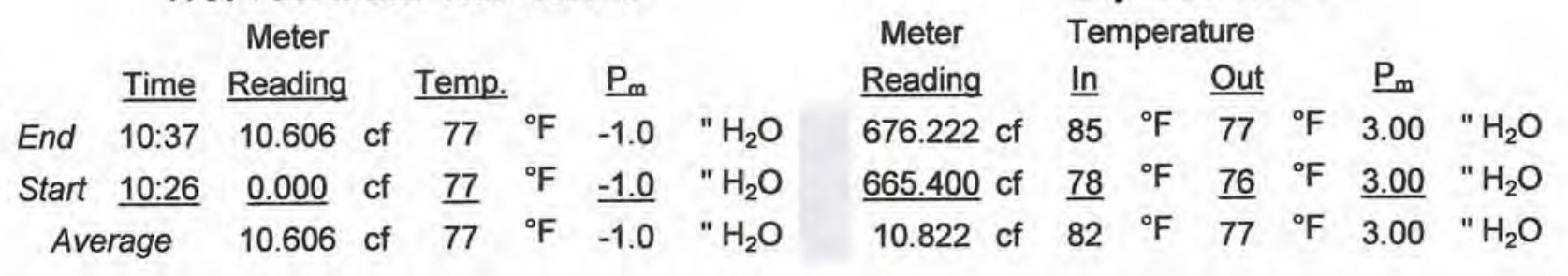

Wet Test Meter $V_{m s d}=17.65 \times V_{m}\left[\frac{P_{b}+\frac{P_{m}}{13.6}}{\left(T_{m}+460\right)}\right] \times C_{f}=\underline{10.559} \mathrm{dcsf}$
Dry Gas Meter $V_{m s d}=17.65 \times \mathrm{Vm}\left[\frac{P_{b}+\frac{P_{m}}{13.6}}{\left(T_{m}+460\right)}\right]=\underline{10.628} \mathrm{dcsf}$ Calibration Factor $(C D G)=\frac{\text { Wet Test Meter } V_{\text {mstd }}}{\text { Dy Gas Meter } V_{\text {msto }}}=\underline{0.994}$ 
Calibration Data

Dry Gas Meter Calibration

Meter Console No.

ASAI 1-1

Date Calibration Performed:

Run 1 at $4.0^{\prime \prime} \Delta \mathrm{H}$

Wet Test Meter $\mathrm{C}_{\mathrm{f}}=1.020$

$$
\begin{aligned}
\mathrm{Pb} & =29.77 \mathrm{Hg} \\
\text { Console Pump Vacuum } & =-5.0 \mathrm{Hg}
\end{aligned}
$$

Wet Test Meter No. ASAl-0 Meter

\section{Meter}

\begin{tabular}{|c|c|c|c|c|c|c|c|c|c|c|c|c|c|c|}
\hline & & & & & & & & & & & & & & \\
\hline & Time & Reading & & Temp. & & $\underline{\mathbf{P}_{m}}$ & & Reading & In & & Out & & $\underline{P_{m}}$ & \\
\hline & 10:51 & 11.019 & cf & 77 & ${ }^{\circ} \mathrm{F}$ & -1.3 & $" \mathrm{H}_{2} \mathrm{O}$ & 687.951 cf & 86 & ${ }^{\circ} \mathrm{F}$ & 77 & ${ }^{\circ} \mathrm{F}$ & 4.00 & \\
\hline art & 10:41 & $\underline{0.000}$ & cf & $\underline{77}$ & ${ }^{\circ} \mathrm{F}$ & -1.3 & $" \mathrm{H}_{2} \mathrm{O}$ & 676.700 of & $\underline{79}$ & ${ }^{\circ} \mathrm{F}$ & 77 & ${ }^{\circ} \mathrm{F}$ & 4.00 & $\theta$ \\
\hline & & 11.019 & 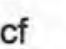 & 77 & ${ }^{\circ} \mathrm{F}$ & -1.3 & $" \mathrm{H}_{2} \mathrm{O}$ & 11.251 cf & 83 & ${ }^{\circ} \mathrm{F}$ & 77 & ${ }^{\circ} \mathrm{F}$ & 4.00 & \\
\hline
\end{tabular}

Dry Gas Meter

$$
\begin{gathered}
\text { Wet Test Meter } V_{m s d}=17.65 \times V_{m}\left[\frac{P_{b}+\frac{P_{m}}{13.6}}{\left(T_{m}+460\right)}\right] \times C_{f}=10.962 \mathrm{dcsf} \\
\text { Dry Gas Meter } V_{m s d}=17.65 \times \mathrm{Vm}\left[\frac{P_{b}+\frac{P_{m}}{13.6}}{\left(T_{m}+460\right)}\right]=\underline{11.061} \mathrm{dcsf}
\end{gathered}
$$

$$
\text { Calibration Factor }\left(C_{D G}\right)=\frac{\text { Wet Test Meter } V_{\text {mstd }}}{\text { Dry Gas Meter } V_{m s t d}}=\underline{0.991}
$$




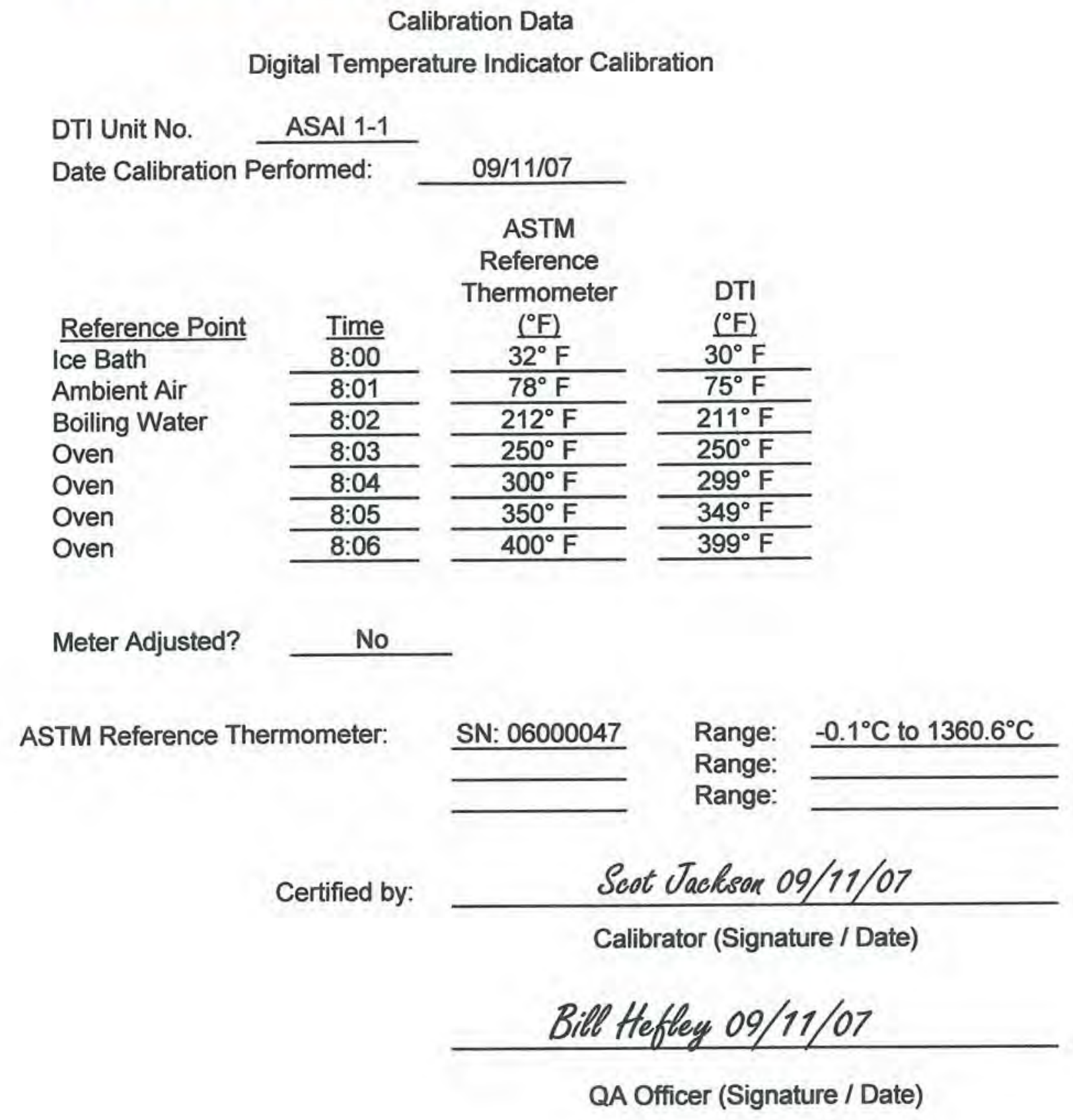




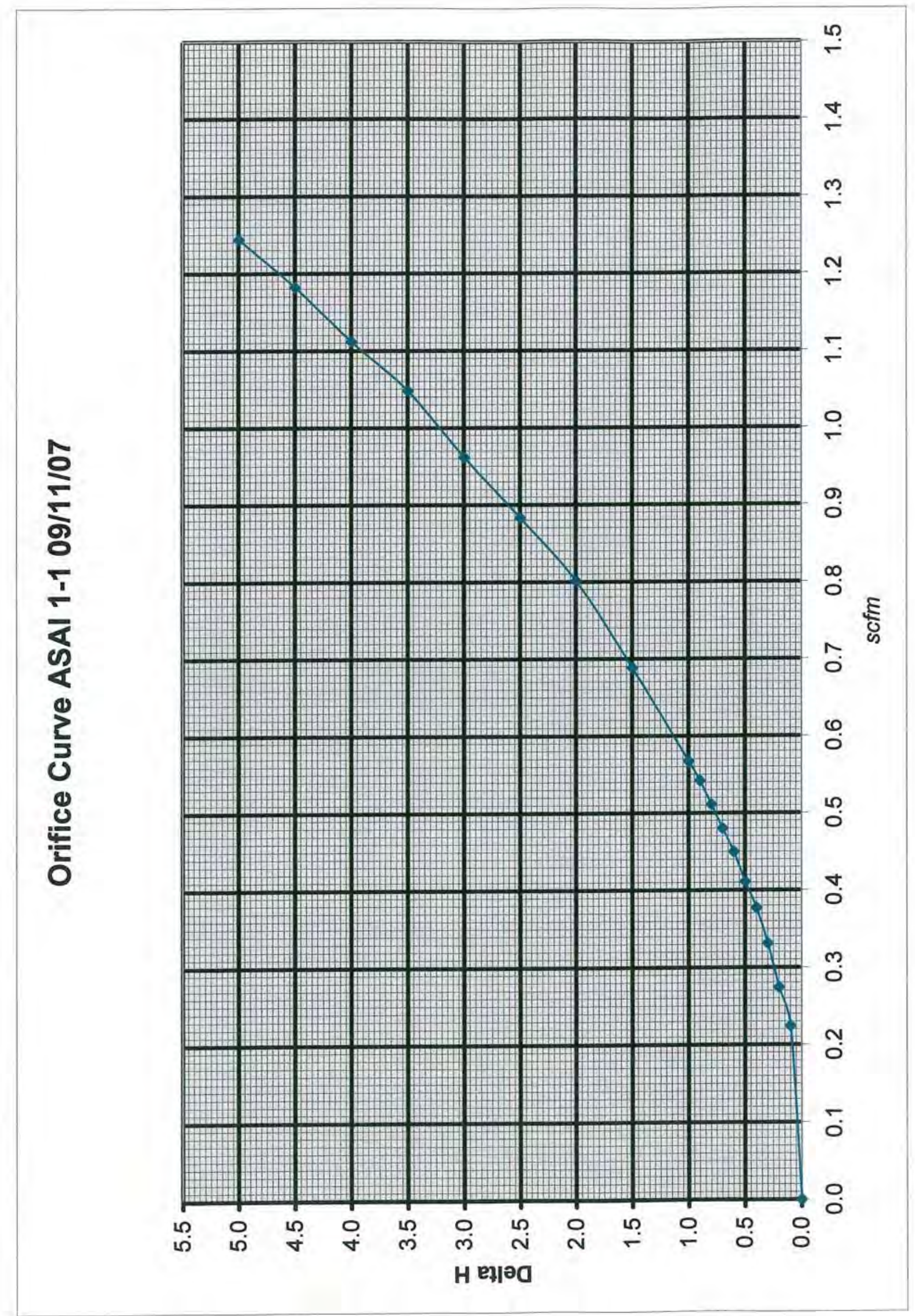




\author{
Calibration Data \\ Dry Gas Meter Calibration \\ Meter Console No. \\ ASAI 1-2 \\ Date Calibration Performed: \\ 09/11/07
}

\title{
$\triangle \mathrm{H}$ Setting
}

\begin{tabular}{c} 
(" $\left.\mathrm{H}_{2} \mathrm{O}\right)$ \\
\hline 0.50 \\
1.00 \\
1.50 \\
2.00 \\
3.00 \\
4.00 \\
Average
\end{tabular}

\begin{tabular}{c}
$\mathrm{C}_{\mathrm{DG}}$ \\
\hline 0.997 \\
\hline 1.001 \\
\hline 1.002 \\
\hline 0.997 \\
\hline 0.993 \\
\hline 0.991 \\
\hline 0.997 \\
\hline
\end{tabular}

\begin{tabular}{ll} 
Variation +: & $0.50 \%$ \\
Variation -: & $-0.60 \%$ \\
\hline
\end{tabular}

Certified by: $\quad$ Scot Jackson 9/11/07

$\frac{\text { Bill Hefley } 9 / 11 / 07}{\text { QA Officer (Signature / Date) }}$ 


\section{Calibration Data}

Dry Gas Meter Calibration

Meter Console No.

ASAI 1-2

Date Calibration Performed:

Run 1 at $0.5^{\prime \prime} \Delta \mathrm{H}$

Wet Test Meter $C_{f}=1.020$

$$
\begin{aligned}
\mathrm{Pb} & =29.80 \mathrm{Hg} \\
\text { Console Pump Vacuum } & =-5.0 \mathrm{Hg}
\end{aligned}
$$

$$
\begin{aligned}
& \text { Wet Test Meter No. ASAI-O } \\
& \text { Meter } \\
& \text { Meter Temperature }
\end{aligned}
$$

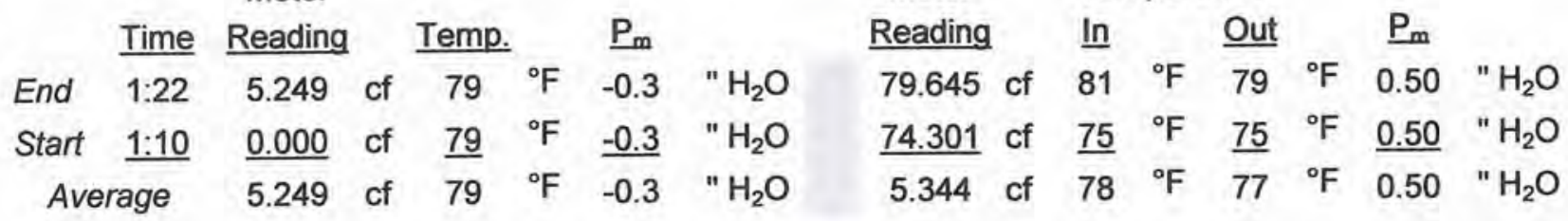

$$
\begin{aligned}
& \text { Wet Test Meter } V_{m s d}=17.65 \times V_{m}\left[\frac{P_{b}+\frac{P_{m}}{13.6}}{\left(T_{m}+460\right)}\right] x C_{f}=\underline{5.221} \text { dcsf } \\
& \text { Dry Gas Meter V } \text { msta }=17.65 \times \mathrm{Vm}\left[\frac{P_{b}+\frac{P_{m}}{13.6}}{\left(T_{m}+460\right)}\right]=\underline{5.236} \mathrm{dcsf} \\
& \text { Calibration Factor }\left(C_{D G}\right)=\frac{\text { Wet Test Meter } V_{m_{s t d}}}{\text { Dry Gas Meter } V_{m_{s t d}}}=\underline{0.997}
\end{aligned}
$$




\section{Calibration Data}

Dry Gas Meter Calibration

Meter Console No.

Date Calibration Performed:
ASAI 1-2

09/11/07

Run 1 at 1.0" $\Delta \mathrm{H}$

Wet Test Meter $\mathrm{C}_{\mathrm{f}}=1.020$ Console Pump Vacuum $=-5.0 \mathrm{Hg}$

Wet Test Meter No. ASAI-O

Meter

Time Reading Temp. $\quad \mathbf{P}_{\mathrm{m}}$

\begin{tabular}{|c|c|c|c|c|c|c|c|}
\hline & Time & Reading & & Temp. & & $\underline{P_{m}}$ & \\
\hline End & $1: 36$ & 5.502 & cf & 80 & ${ }^{\circ} \mathrm{F}$ & -0.4 & $" \mathrm{H}_{2} \mathrm{O}$ \\
\hline Start & $1: 27$ & $\underline{0.000}$ & cf & $\underline{79}$ & ${ }^{\circ} \mathrm{F}$ & -0.4 & $" \mathrm{H}_{2} \mathrm{O}$ \\
\hline Ave & & 5.502 & cf & 80 & ${ }^{\circ} \mathrm{F}$ & -0.4 & $" \mathrm{H}_{2} \mathrm{O}$ \\
\hline
\end{tabular}

Dry Gas Meter

Meter Temperature

Reading In Out $\underline{P_{m}}$ 85.708 cf $84{ }^{\circ} \mathrm{F} \quad 81 \quad{ }^{\circ} \mathrm{F} \quad 1.00 \quad " \mathrm{H}_{2} \mathrm{O}$ $\underline{80.100}$ cf $\underline{82}{ }^{\circ} \mathrm{F} \quad \underline{80}{ }^{\circ} \mathrm{F} \quad \underline{1.00} \quad \mathrm{H}_{2} \mathrm{O}$ 5.608 cf $83{ }^{\circ} \mathrm{F} \quad 81{ }^{\circ} \mathrm{F} \quad 1.00 \quad \mathrm{H}_{2} \mathrm{O}$

$$
\begin{gathered}
\text { Wet Test Meter } V_{m s d}=17.65 \times V_{m}\left[\frac{P_{b}+\frac{P_{m}}{13.6}}{\left(T_{m}+460\right)}\right] \times C_{f}=\underline{5.466} d c s f \\
\text { Dry Gas Meter } V_{m s d}=17.65 \times \mathrm{Vm}\left[\frac{P_{b}+\frac{P_{m}}{13.6}}{\left(T_{m}+460\right)}\right]=\underline{5.458} \mathrm{dcsf}
\end{gathered}
$$

$$
\text { Calibration Factor }(C D G)=\frac{\text { Wet Test Meter } V_{m s t d}}{\text { Dry Gas Meter } V_{\text {msto }}}=1.001
$$




\author{
Calibration Data \\ Dry Gas Meter Calibration
}

Meter Console No.

ASAI 1-2

Date Calibration Performed:

$$
\begin{aligned}
\text { Run } \frac{1}{\mathrm{~Pb}} & =29.80 \\
\text { Wet Test Meter } \mathrm{C}_{\mathrm{f}}=1.020 \quad \text { Console Pump Vacuum } & =-5 . \mathrm{N}^{\mathrm{N}} \mathrm{Hg}
\end{aligned}
$$

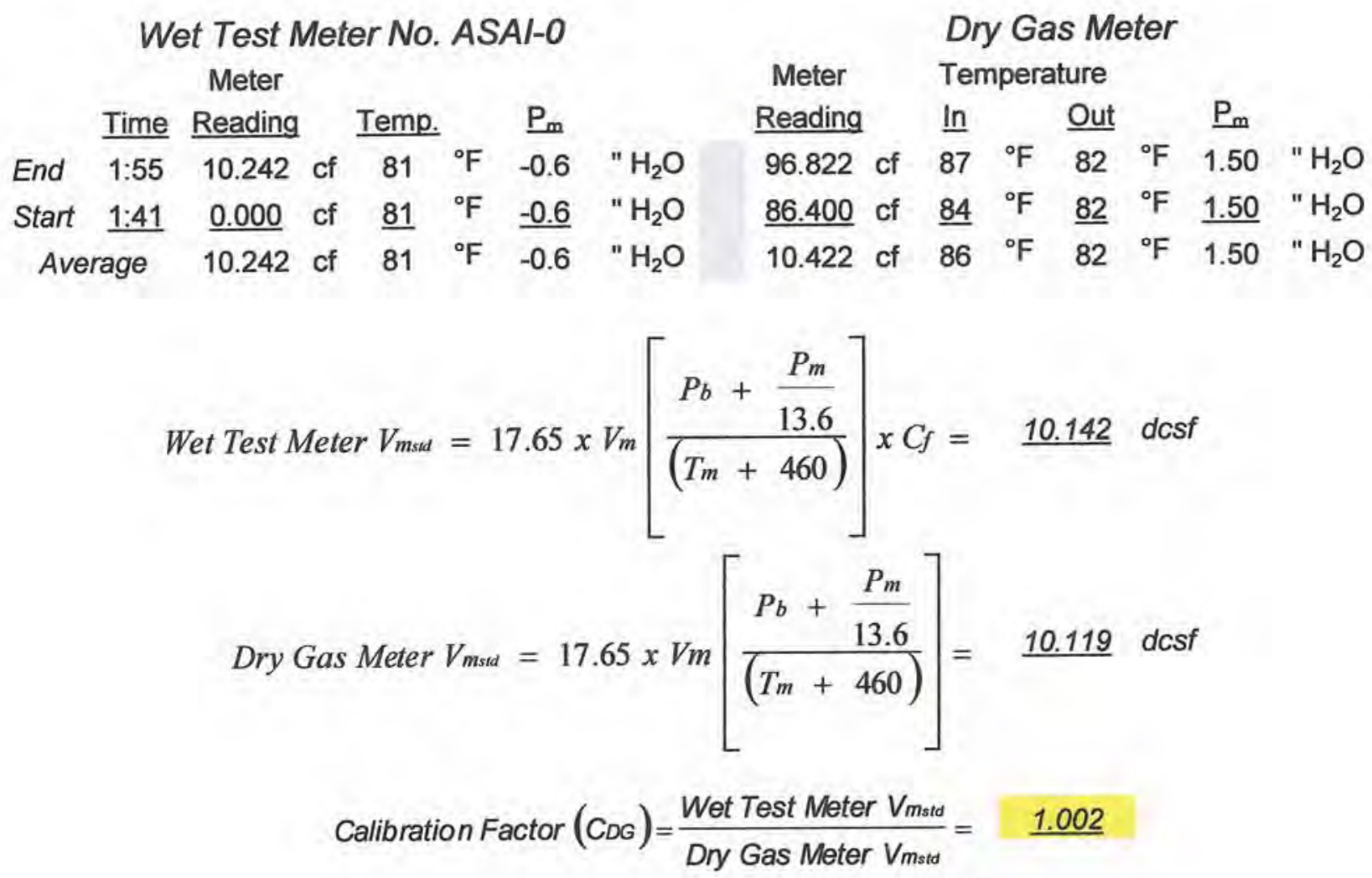


Calibration Data

Dry Gas Meter Calibration

Meter Console No.

Date Calibration Performed:
ASAI 1-2

09/11/07

$\begin{aligned} \text { Run } \frac{1}{\mathrm{~Pb}} & =29.80 \mathrm{Hg} \\ \text { Wet Test Meter } \mathrm{C}_{\mathrm{f}}=1.020 \text { Console Pump Vacuum } & =-5.0 \mathrm{Hg}\end{aligned}$

Wet Test Meter No. ASAl-0 Meter

Meter Temperature

\begin{tabular}{|c|c|c|c|c|c|c|c|c|c|c|c|c|c|}
\hline & Time & Reading & Temp. & & $\underline{\mathrm{P}_{\mathrm{m}}}$ & & Reading & In & & Out & & $\underline{P_{m}}$ & \\
\hline & $2: 12$ & $10.118 \mathrm{c}$ & 81 & ${ }^{\circ} \mathrm{F}$ & -0.7 & $" \mathrm{H}_{2} \mathrm{O}$ & 107.779 cf & 91 & ${ }^{\circ} \mathrm{F}$ & 83 & ${ }^{\circ} \mathrm{F}$ & 2.00 & " $\mathrm{H}_{2} \mathrm{O}$ \\
\hline art & 2:00 & $\underline{0.000}$ & $\underline{81}$ & ${ }^{\circ} \mathrm{F}$ & $\underline{-0.7}$ & $" \mathrm{H}_{2} \mathrm{O}$ & $\underline{97.403} \mathrm{cf}$ & $\underline{88}$ & ${ }^{\circ} \mathrm{F}$ & $\underline{82}$ & ${ }^{\circ} \mathrm{F}$ & $\underline{2.00}$ & $" \mathrm{H}_{2} \mathrm{O}$ \\
\hline & & 10.118 & 81 & ${ }^{\circ} \mathrm{F}$ & -0.7 & $" \mathrm{H}_{2} \mathrm{O}$ & 10.376 cf & 90 & ${ }^{\circ} \mathrm{F}$ & 83 & ${ }^{\circ} \mathrm{F}$ & 2.00 & $" \mathrm{H}_{2}$ \\
\hline
\end{tabular}

Wet Test Meter $V_{m s d}=17.65 \times V_{m}\left[\frac{P_{b}+\frac{P_{m}}{13.6}}{\left(T_{m}+460\right)}\right] \times C_{f}=\underline{10.016} \mathrm{dcsf}$
Dry Gas Meter Vmstd $=17.65 \times \mathrm{Vm}\left[\frac{P_{b}+\frac{P_{m}}{13.6}}{\left(T_{m}+460\right)}\right]=\underline{10.045} \mathrm{dcsf}$ Calibration Factor $(C D G)=\frac{\text { Wet Test Meter } V_{\text {mstd }}}{\text { Dry Gas Meter } V_{\text {mstd }}}=\underline{0.997}$ 


\author{
Calibration Data \\ Dry Gas Meter Calibration
}

Meter Console No.

ASAI 1-2

Date Calibration Performed:
Run 1 at 3.0" $\Delta \mathrm{H}$

Wet Test Meter $C_{f}=1.020$
$\mathrm{Pb}=29.80 " \mathrm{Hg}$

Console Pump Vacuum $=-5.0 " \mathrm{Hg}$

$$
\begin{aligned}
& \text { Wet Test Meter No. ASAl-0 } \\
& \text { Meter } \\
& \text { Meter Temperature }
\end{aligned}
$$

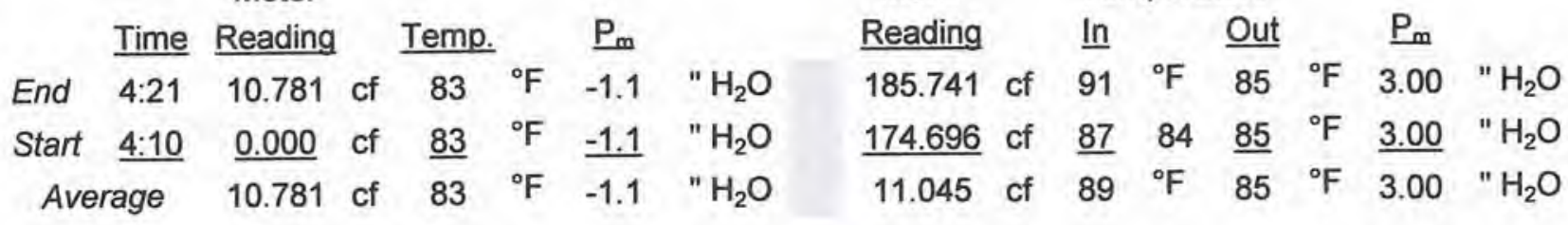

$$
\begin{aligned}
& \text { Wet Test Meter } V_{m s d}=17.65 \times V_{m}\left[\frac{P_{b}+\frac{P_{m}}{13.6}}{\left(T_{m}+460\right)}\right] x C_{f}=\underline{10.623} \mathrm{dcsf} \\
& \text { Dry Gas Meter Vmsd }=17.65 \times \mathrm{Vm}\left[\frac{P_{b}+\frac{P_{m}}{13.6}}{\left(T_{m}+460\right)}\right]=10.699 \mathrm{dcsf} \\
& \text { Calibration Factor }\left(C_{D G}\right)=\frac{\text { Wet Test Meter } V_{\text {mstd }}}{\text { Dry Gas Meter } V_{\text {mstd }}}=\underline{0.993}
\end{aligned}
$$




\title{
Calibration Data
}

Dry Gas Meter Calibration

\author{
Meter Console No. \\ ASAI 1-2 \\ Date Calibration Performed: \\ 09/11/07
}

$\begin{aligned} \text { Run } \frac{1}{\mathrm{~Pb}} & =29.76 \mathrm{Hg} \\ \text { Wet Test Meter } \mathrm{C}_{\mathrm{f}}=1.020 \quad \mathrm{Hg} & \text { Console Pump Vacuum }=-5.0 " \mathrm{Hg}\end{aligned}$

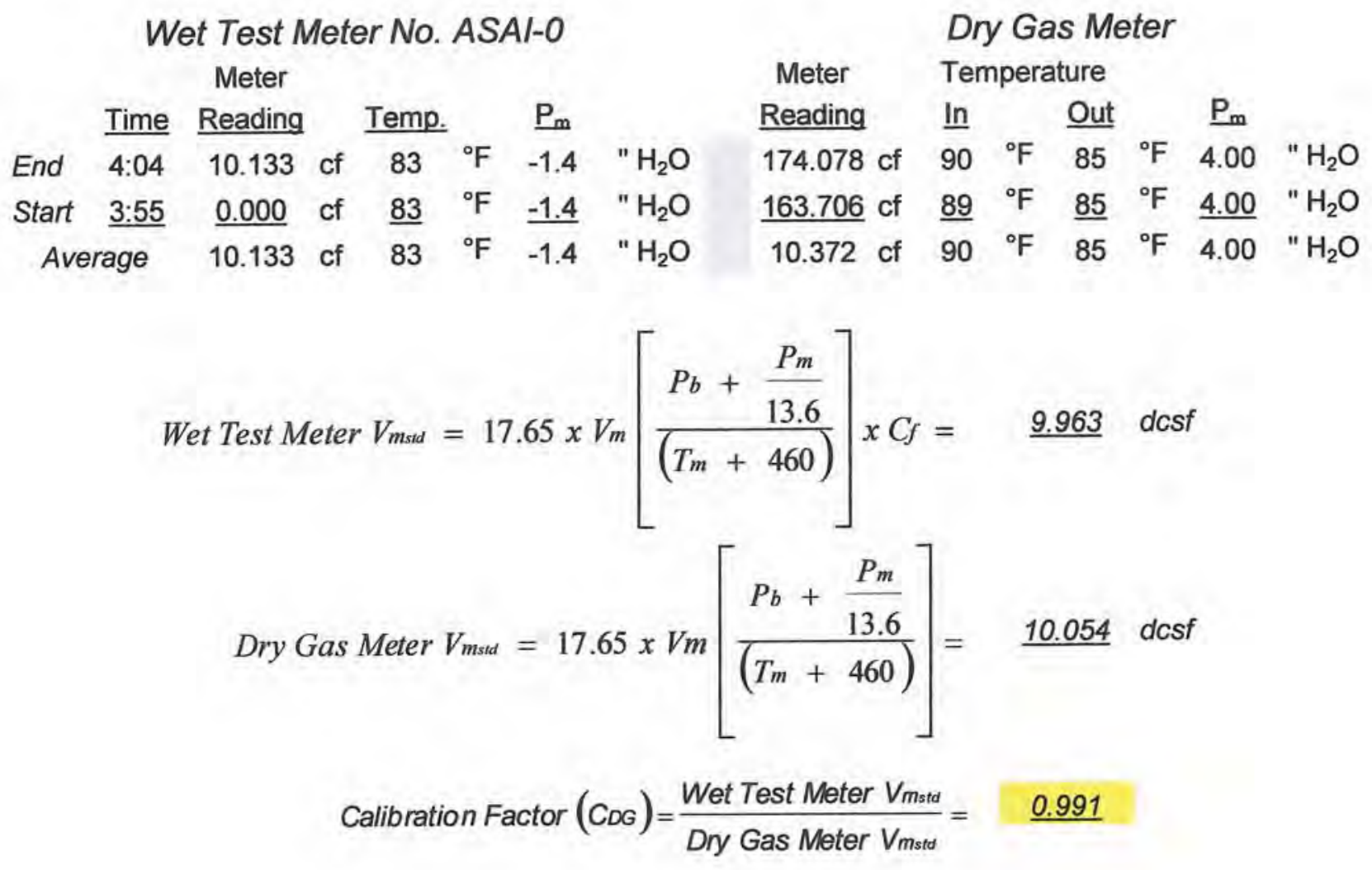


Calibration Data

Digital Temperature Indicator Calibration

DTI Unit No. ASAI 1-2

Date Calibration Performed:

\begin{tabular}{|c|c|}
\hline $09 / 11 / 07$ & \\
\hline $\begin{array}{c}\text { ASTM } \\
\text { Reference }\end{array}$ & \\
\hline Thermometer & DTI \\
\hline ( $\left.{ }^{\circ} \mathrm{F}\right)$ & $\left({ }^{\circ} \mathrm{F}\right)$ \\
\hline $32^{\circ} \mathrm{F}$ & $32^{\circ} \mathrm{F}$ \\
\hline $79^{\circ} \mathrm{F}$ & $79^{\circ} \mathrm{F}$ \\
\hline $212^{\circ} \mathrm{F}$ & $214^{\circ} \mathrm{F}$ \\
\hline $250^{\circ} \mathrm{F}$ & $253^{\circ} \mathrm{F}$ \\
\hline $300^{\circ} \mathrm{F}$ & $303^{\circ} \mathrm{F}$ \\
\hline $350^{\circ} \mathrm{F}$ & $352^{\circ} \mathrm{F}$ \\
\hline $400^{\circ} \mathrm{F}$ & $403^{\circ} \mathrm{F}$ \\
\hline
\end{tabular}

Meter Adjusted?

No

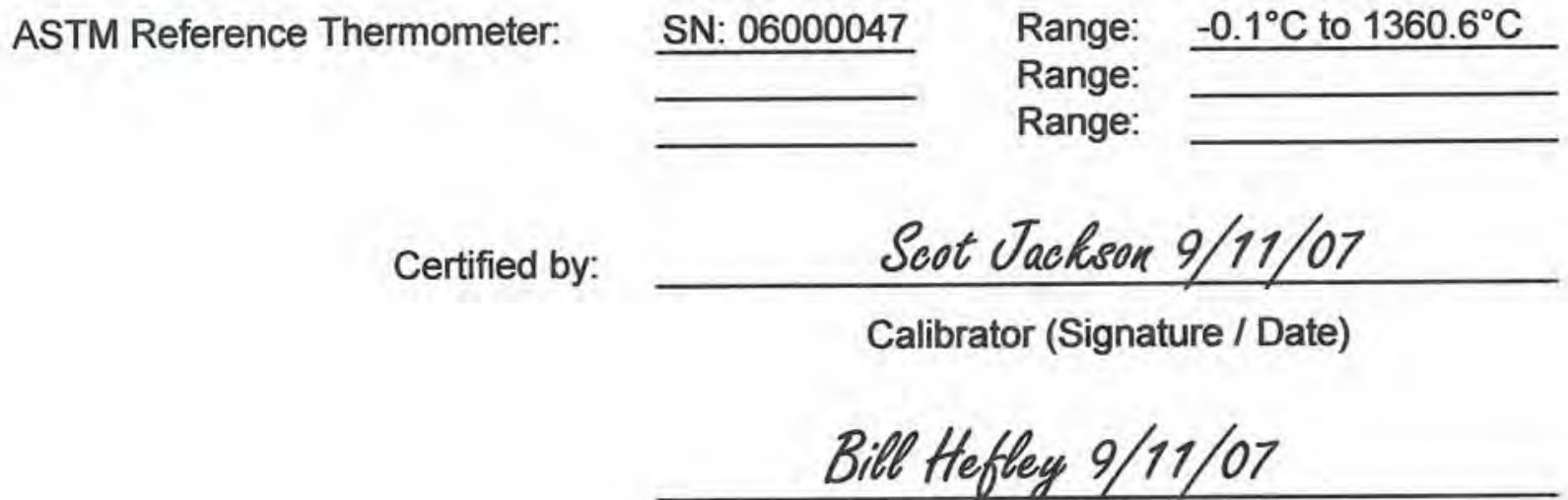

QA Officer (Signature / Date) 


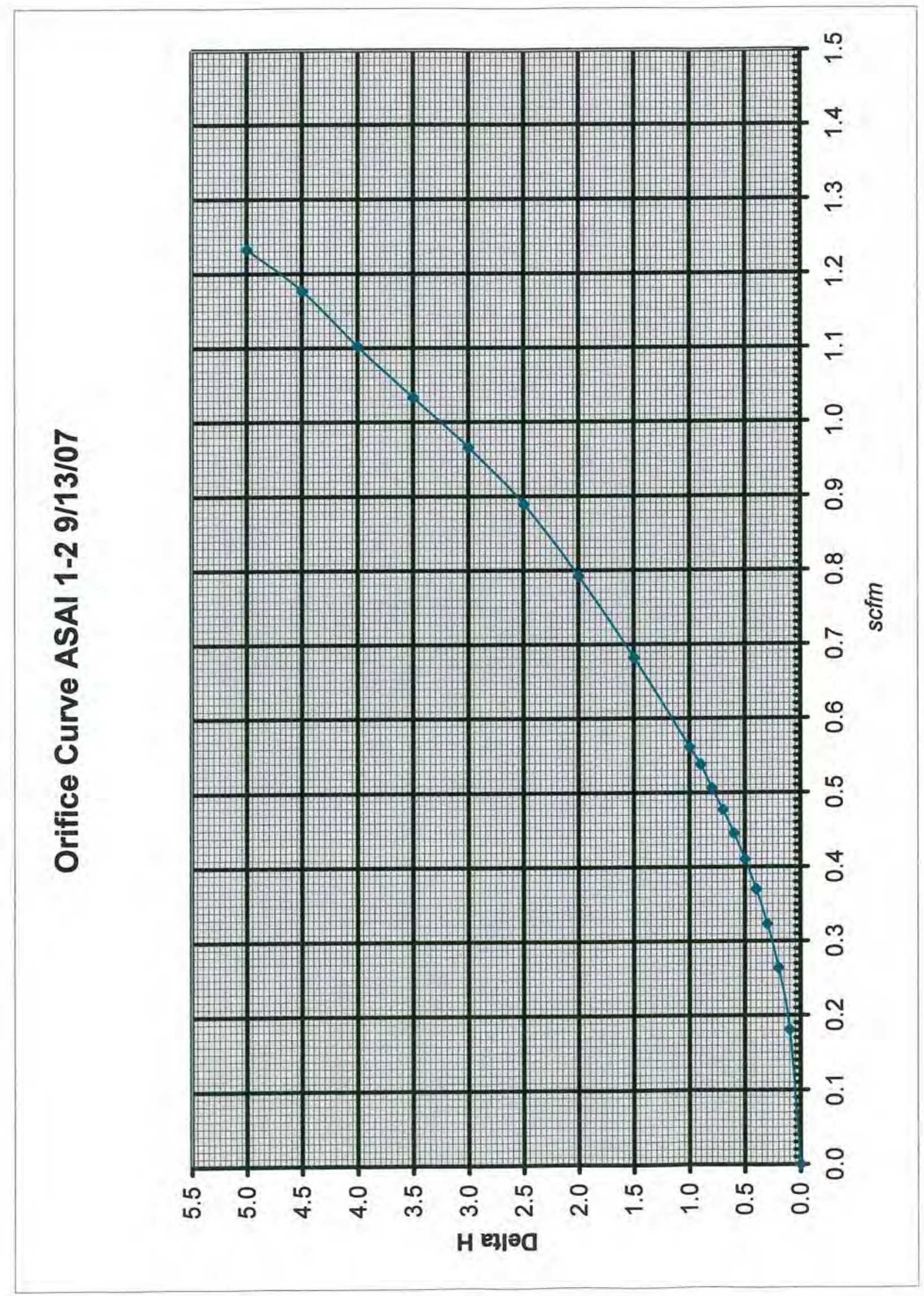


Calibration Data

Nozzle Calibration

Nozzle Set No.

Date Calibration Performed:

$\mathrm{PM}-10$

09/10/07

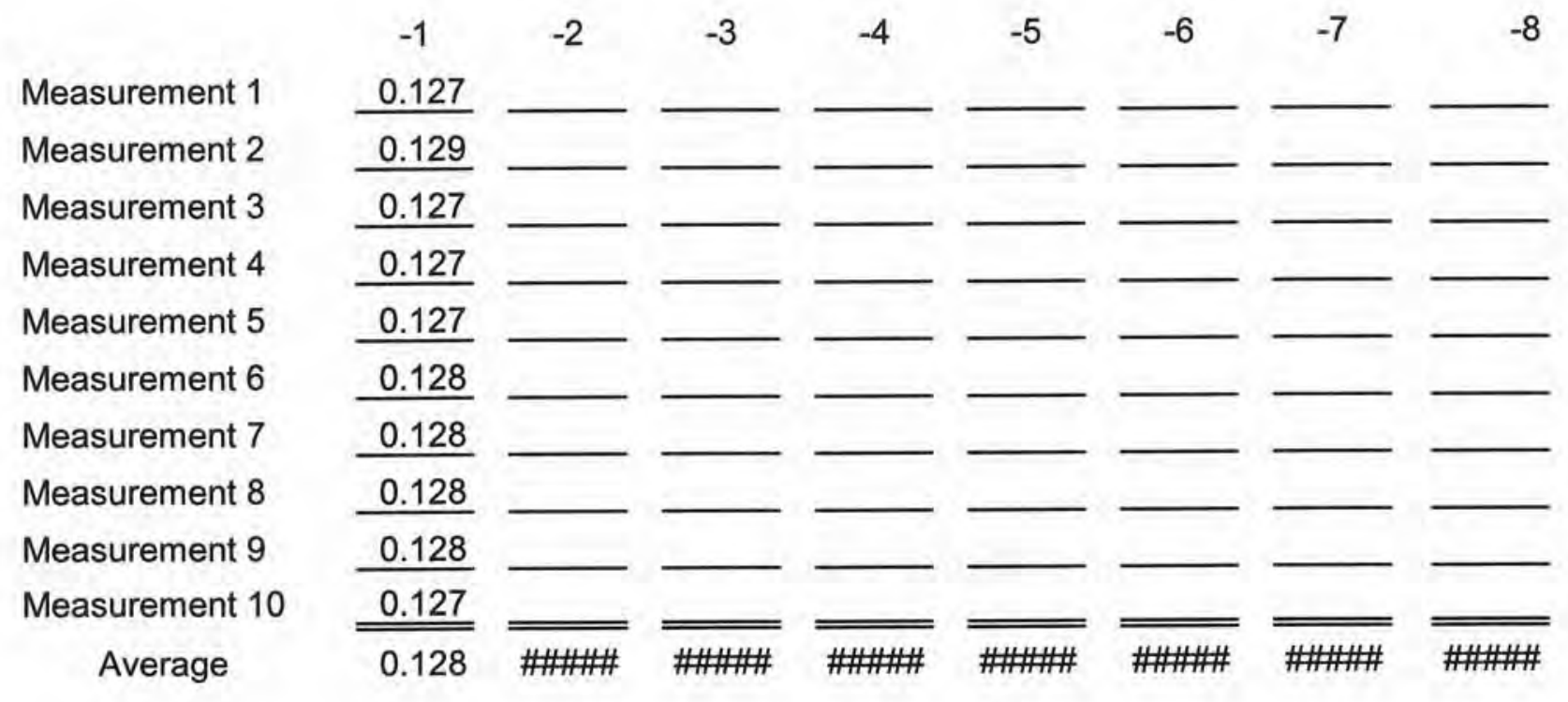

\section{Soot Jackson9/06/07}

Calibrator (Signature / Date)

\section{Bill Hefley 9/06/07}

QA Officer (Signature / Date) 


\section{SARMPLNG ASSOCIATES, INC.}

\section{CALIBRATION DATA \\ PITOT TUBE CALIBRATION DATA}

$\begin{array}{lc}\text { Date: } & 09 / 10 / 07 \\ \text { I.D. \# } \frac{P M-10}{P} & 0.990 \\ \text { C }_{\text {pstd: }} & \end{array}$
Time: $9: 00$

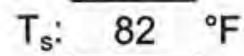

$\mathrm{Pb}: \underline{29.70} \mathrm{Hg}$

\begin{tabular}{|c|c|c|c|c|c|c|c|c|c|c|}
\hline fps & $\begin{array}{c}\text { Desired } \\
\text { Calibration }\end{array}$ & \multicolumn{2}{|c|}{$\begin{array}{l}\text { Calibration } \\
\text { Standard }\end{array}$} & \multirow{2}{*}{$\begin{array}{l}\text { v Standard } \\
\text { Average }\end{array}$} & \multirow[b]{2}{*}{ High } & \multirow[b]{2}{*}{ v High } & \multirow{2}{*}{$\begin{array}{l}\text { Cal. } \\
\text { Factor }\end{array}$} & \multirow[b]{2}{*}{ Low } & \multirow[b]{2}{*}{$\checkmark$ Low } & \multirow{2}{*}{$\begin{array}{l}\text { Cal. } \\
\text { Factor }\end{array}$} \\
\hline Mark & Standard & Start & End & & & & & & & \\
\hline 20 & 0.09 & 0.09 & 0.09 & 0.300 & 0.13 & 0.361 & 0.824 & 0.13 & 0.361 & 0.824 \\
\hline 30 & 0.20 & 0.20 & 0.20 & 0.447 & 0.29 & 0.539 & 0.822 & 0.29 & 0.539 & 0.822 \\
\hline 40 & 0.35 & 0.35 & 0.35 & 0.592 & 0.50 & 0.707 & 0.828 & 0.50 & 0.707 & 0.828 \\
\hline 50 & 0.55 & 0.55 & 0.55 & 0.742 & 0.79 & 0.889 & 0.826 & 0.79 & 0.889 & 0.826 \\
\hline 60 & 0.80 & 0.80 & 0.80 & 0.894 & 1.15 & 1.072 & 0.826 & 1.15 & 1.072 & 0.826 \\
\hline 70 & 1.08 & 1.10 & 1.10 & 1.049 & 1.58 & 1.257 & 0.826 & 1.58 & 1.257 & 0.826 \\
\hline 80 & 1.42 & 1.40 & 1.45 & 1.194 & 2.05 & 1.432 & 0.825 & 2.05 & 1.432 & 0.825 \\
\hline 90 & 1.79 & 1.80 & 1.80 & 1.342 & 2.60 & 1.612 & 0.824 & 2.60 & 1.612 & 0.824 \\
\hline & & & & & & & & & & \\
\hline & & & & & & 0880 & 0826 & 079 & 0889 & 0826 \\
\hline$\frac{50}{50}$ & 0.55 & 0.55 & 0.55 & $\frac{0.742}{0.712}$ & $\frac{0.79}{0.79}$ & 0.889 & $0.8<0$ & $\frac{0.19}{0.79}$ & 0.009 & $\frac{0.020}{0.826}$ \\
\hline 50 & 0.55 & 0.55 & 0.55 & 0.742 & 0.79 & 0.889 & 0.826 & & & 0.020 \\
\hline & & & & & & & & & & \\
\hline & verage & & & & & & 0.825 & & & 0.825 \\
\hline
\end{tabular}

Summary of Results:

$\begin{array}{rc}\text { Normal high side calibraiton factor: } & 0.825 \\ \text { variation } & 0.36 \% \\ \text { variation }-\frac{-0.36 \%}{\text { Normal low side calibration factor: }} & 0.825 \\ \text { variation } & 0.36 \% \\ \text { variation }-\frac{0.36 \%}{-0.36}\end{array}$

Certification:

I certify that the Type S pitot tube, the standard type pitot tube, and the calibration setup meet or exceed all specifications, criteria and/or applicable design features and hereby assign a pitot tube calibration factor $C_{p}$ of: $\quad \underline{0.825}$

Certified by:

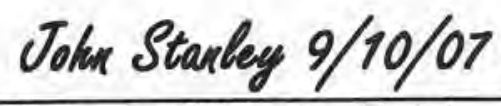

Calibrator (Signature/Date)

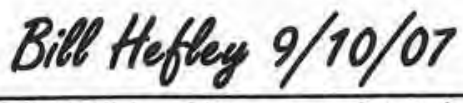

QA Officer (Signature/Date) 
Appendix D:

Field Data 

Impinger Box No.

$1-1$

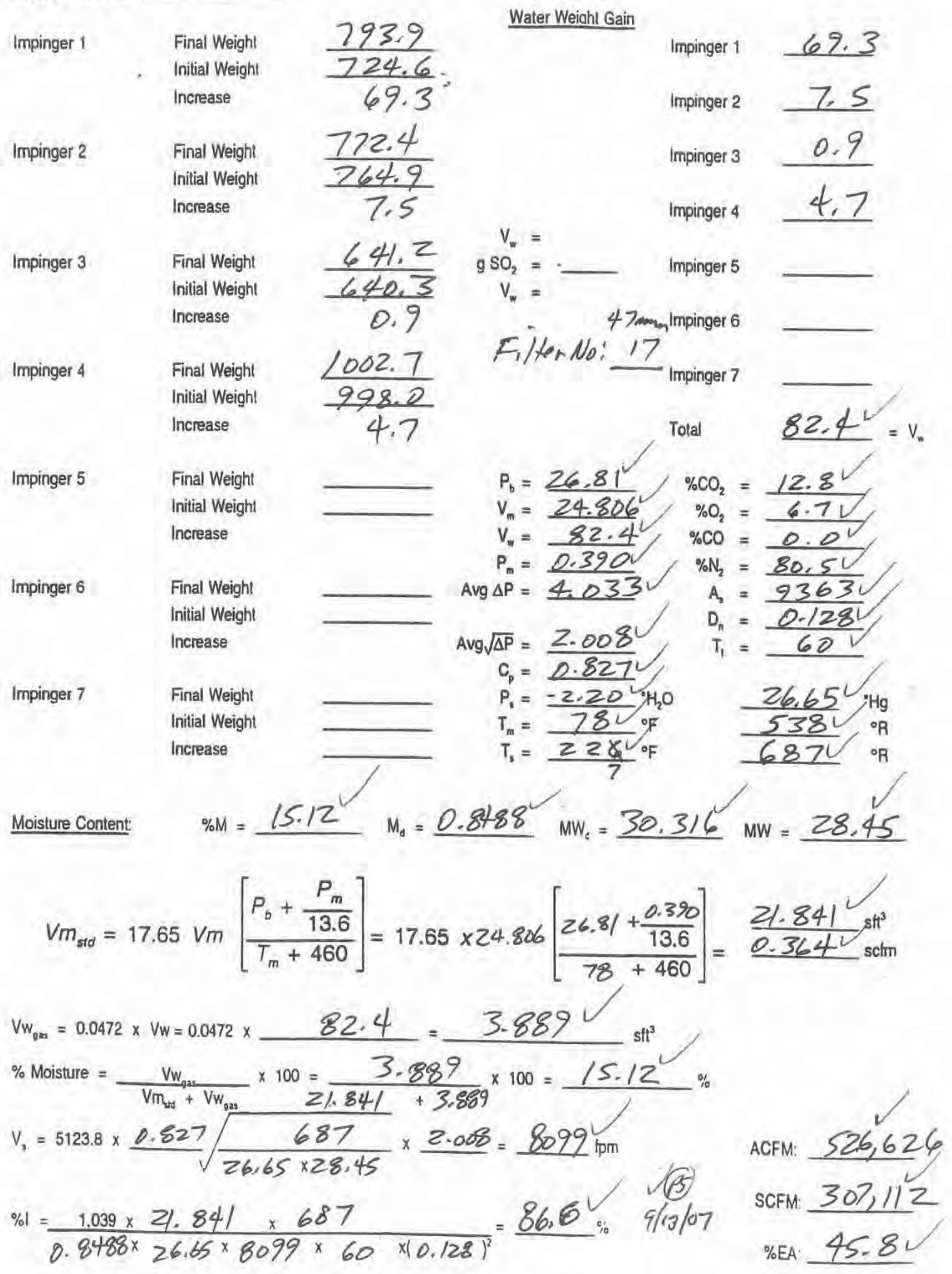


Impinger Box No. $1-2$

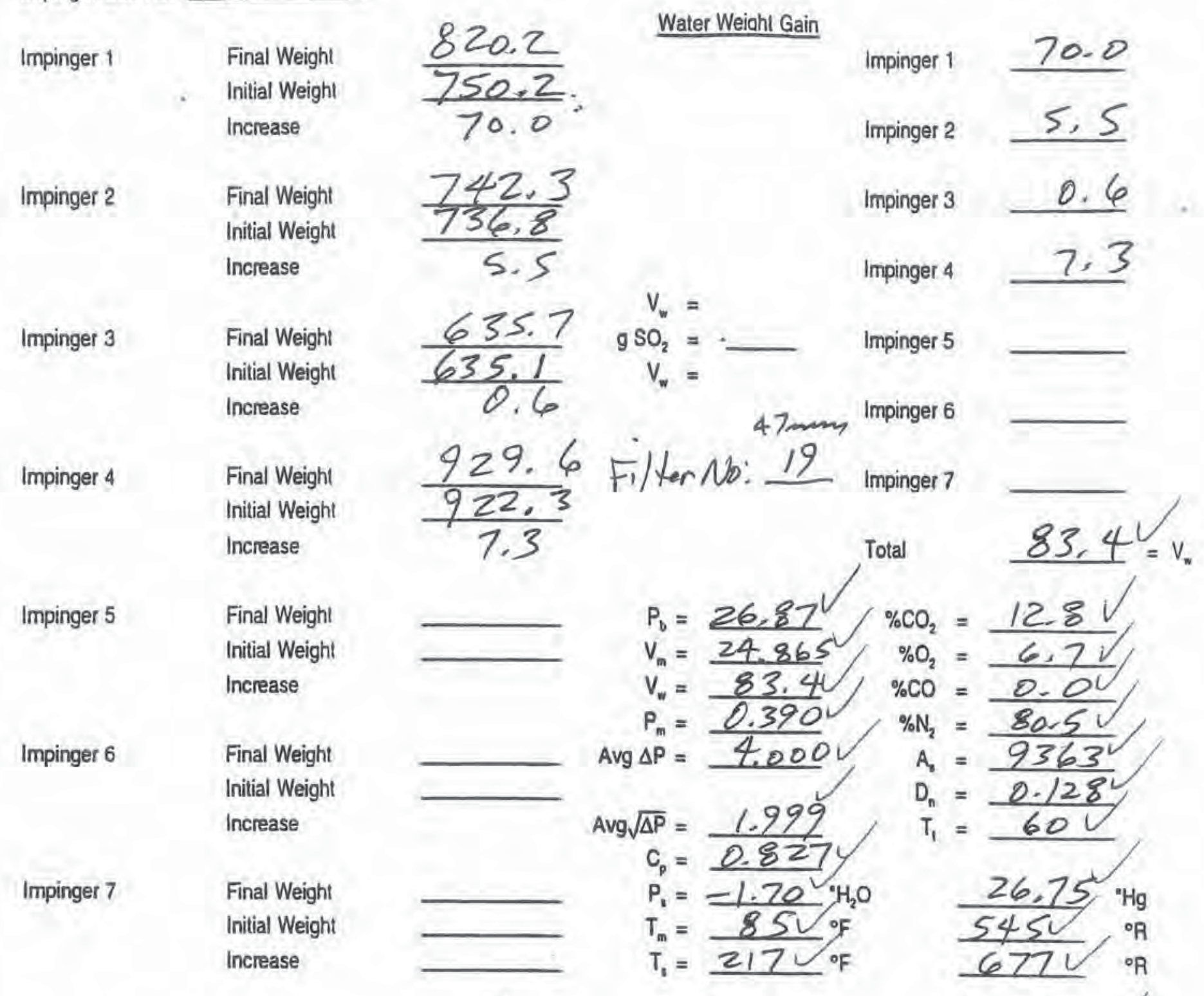

Moisture Content:

$\% M=15.38 \quad M_{d}=0.8462 \quad M_{c}=30.316^{\prime} \quad M W=28.42$ $V m_{s t d}=17.65 \mathrm{Vm}\left[\frac{P_{b}+\frac{P_{m}}{13.6}}{T_{m}+460}\right]=17.65 \times 24.865\left[\frac{26.87+\frac{0.390}{13.6}}{85+460}\right]=\frac{21.660^{\mathrm{sh}^{3}}}{0.361} \mathrm{scm}$

$V_{w_{0 s}}=0.0472 \times V_{w}=0.0472 \times-83.4=3.936$

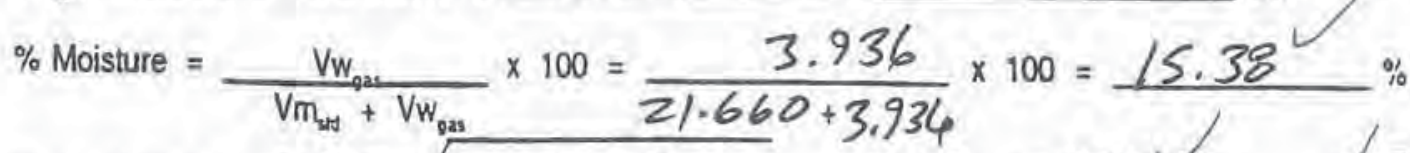

$v_{3}=5123.8 \times \frac{0.827}{\frac{677}{26.75 \times 28.42}} \times 1.999=7993 \mathrm{fm}$

$\%=\frac{1.039 \times 21.660 \times 677}{0.8462 \times 26.75 \times 7993 \times 60 \times(0.128)^{2}}=85.7 \%$

S/13/07 ACFM: $\frac{519,737}{307,728}$

$\%$ EA 45.8 


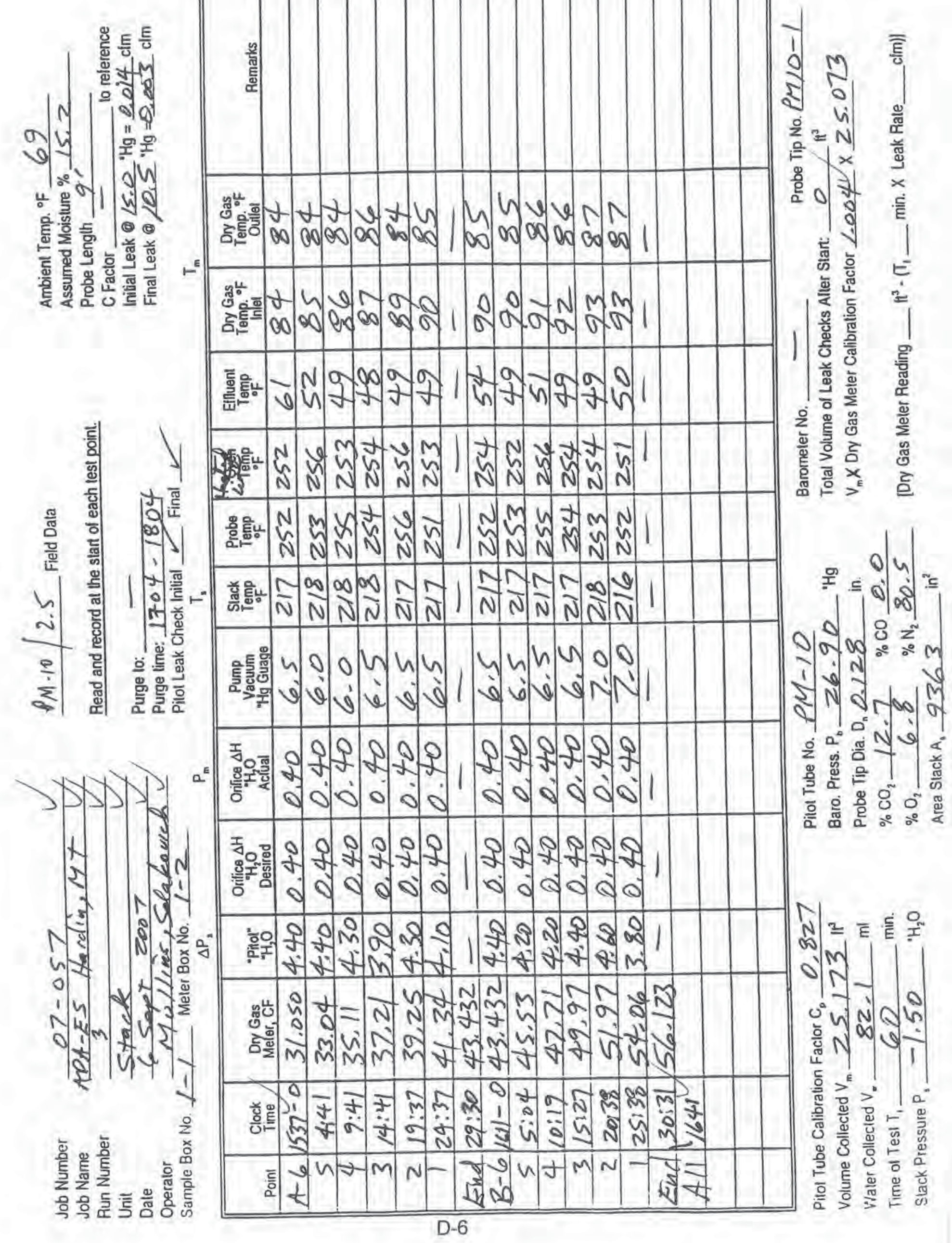


Impinger Box No.

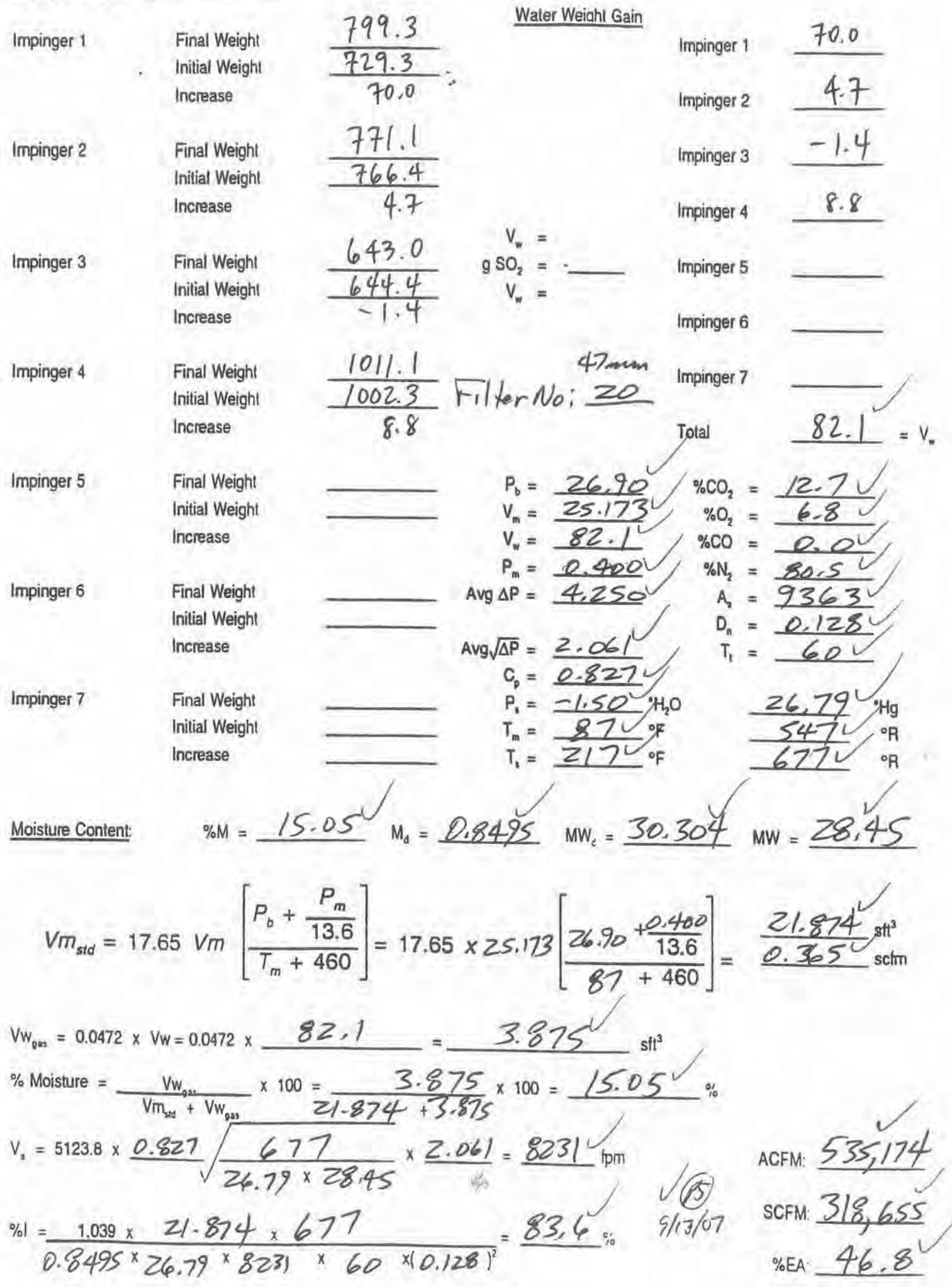



Impinger Box No. $3-1$

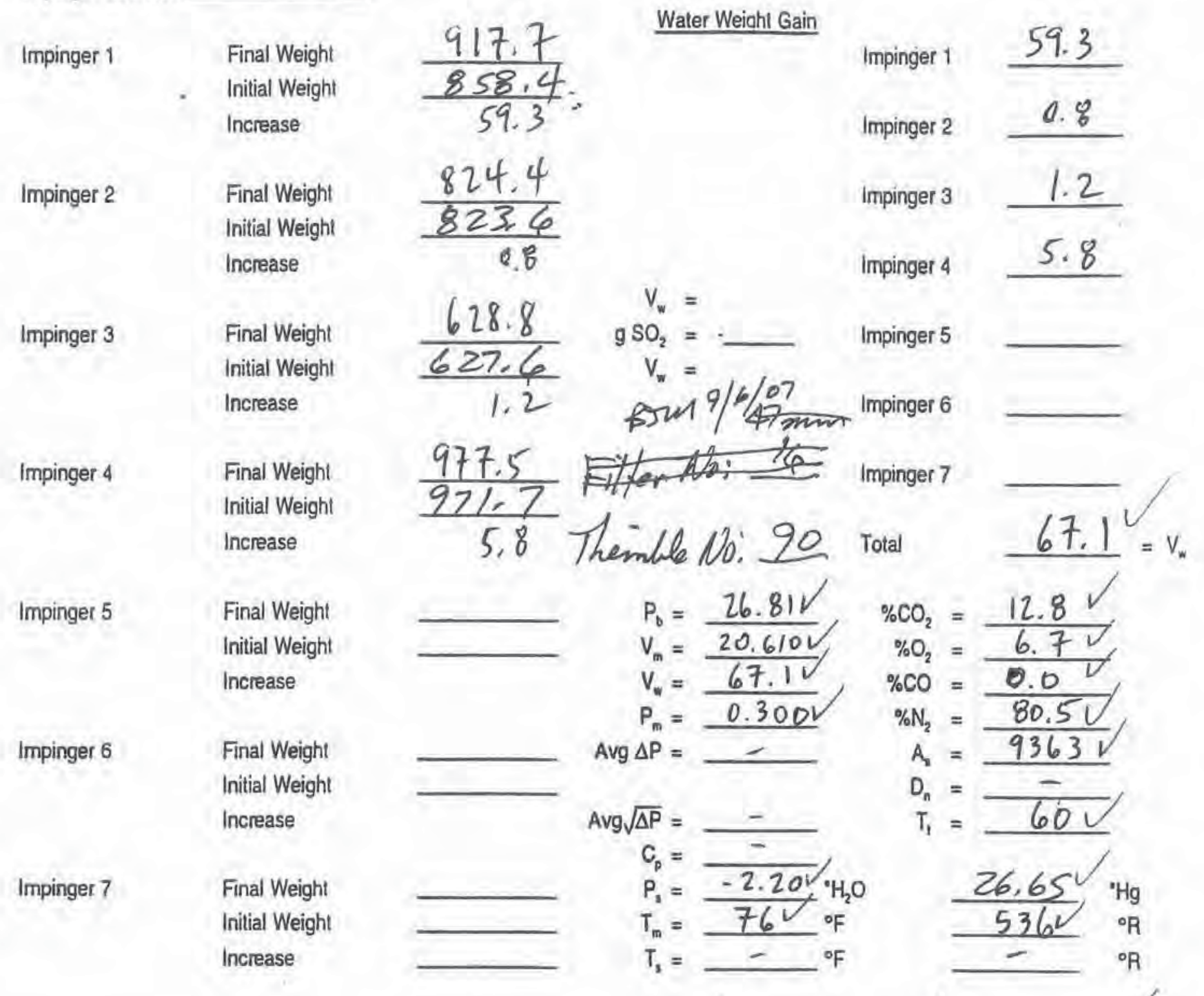

Moisture Content: $\quad \% \mathrm{M}=14.82^{\mathrm{V}} \quad \mathrm{M}_{\mathrm{d}}=0.8518 \quad \mathrm{MW}_{\mathrm{t}}=\underline{30.316} \mathrm{MW}=28.49$

$$
\begin{aligned}
& V m_{s i d}=17.65 \mathrm{Vm}\left[\frac{P_{b}+\frac{P_{m}}{13.6}}{T_{m}+460}\right]=17.65 \times 20.6 / 0\left[\frac{26.81+\frac{0.300}{13.6}}{76+460}\right]=\frac{18.210^{8 \mathrm{ft}^{3}}}{0.304^{2}} \mathrm{scm} \\
& V_{w_{0+3}}=0.0472 \times v_{w}=0.0472 \times-62,1=s^{3}
\end{aligned}
$$$$
\% \text { Moisture }=\frac{V_{w_{921}}}{V_{m_{x-1}}+V_{W_{0 a 3}}} \times 100=\frac{3.167}{18.210+3.167} \times 100=14.8 z^{\%} \%
$$$$
V_{1}=5123.8 \times
$$$$
\sqrt{\frac{1}{x}} \times 1 \mathrm{pm}
$$

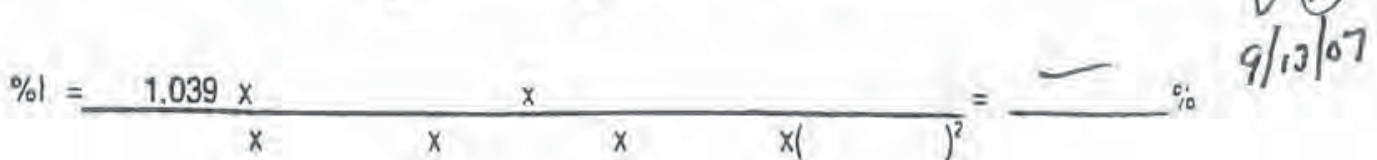
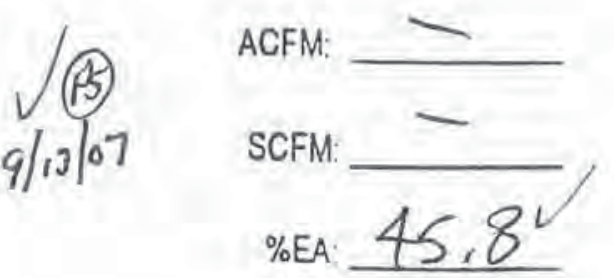


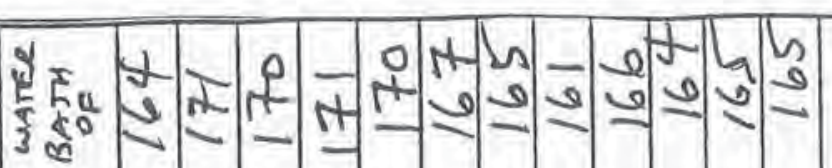

A 1 |

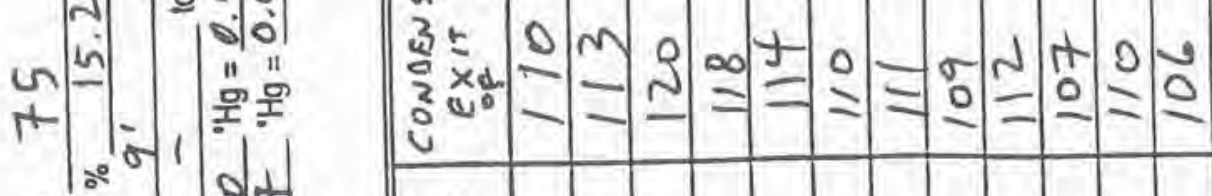

Q

는 늘

을햏항

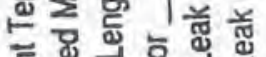

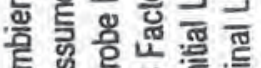

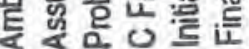

$\vdash$

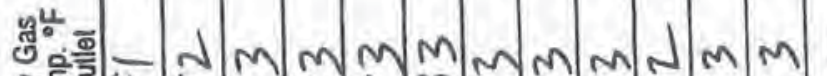

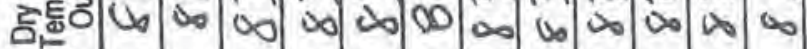

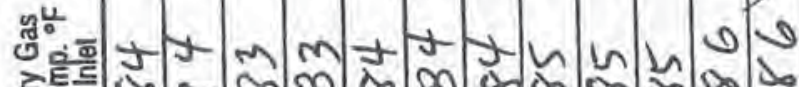

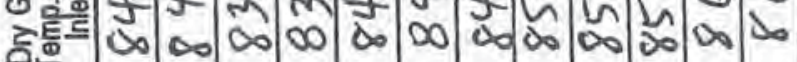

喜暿 +ु

1 क्षे

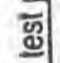

茄

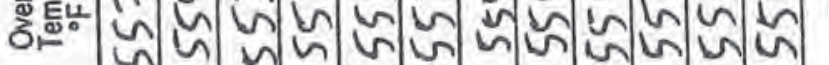

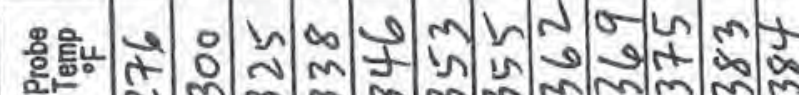

证娄

$x+x+10^{E}$

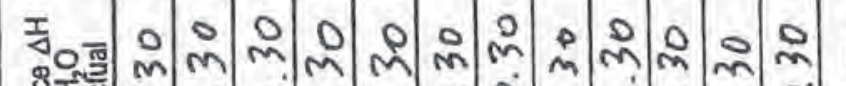

을

욤

맥옴음

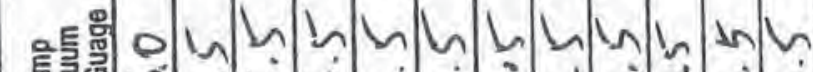

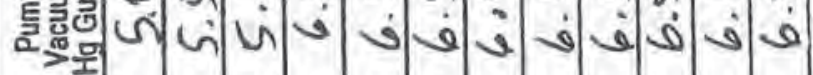

$a^{\mathrm{E}}$

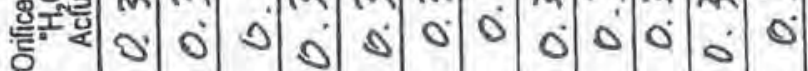

ह $>\frac{1}{11}$

द्य है

产

告

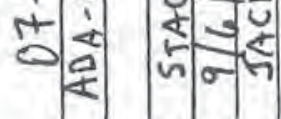

in

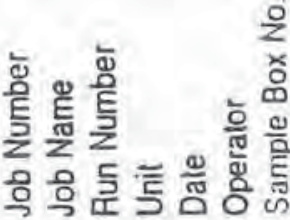

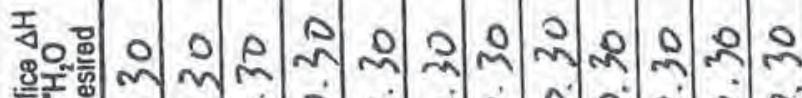

\begin{tabular}{l|l|l|lllll|lllllll} 
흔원 & 1 & 1 & 1 & 1 & 1 & 1 & 1 & 1 & 1 & 1 & 1 & 1 & 1
\end{tabular}

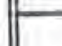

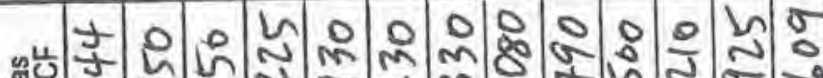

गु०

㖣 $m$ m

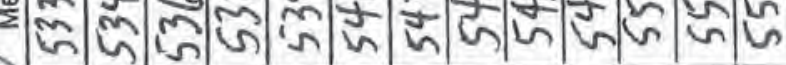

요의

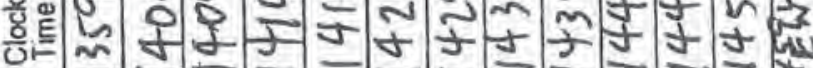

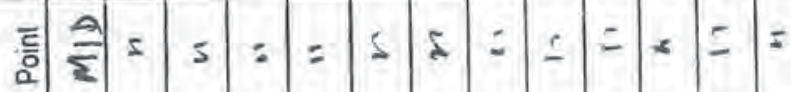

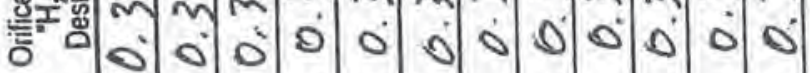

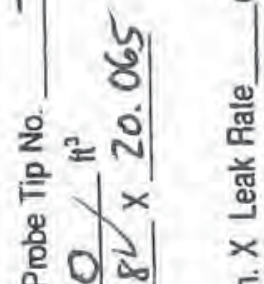

$\overline{\mathrm{E}}$

$\frac{0}{\infty} x$

o

ㄴํㅇ

कँ

西

둔

음

要

芯

히 立

之े है

क्ष

贾

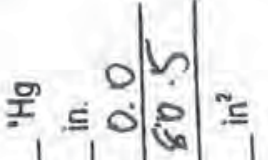

$1+10$

$\infty 11200$

1 ले

- 000

운 告

올 은

15

흘 옹

$$
3 \bar{E} \text { 高 }
$$

ด 0

0 No.

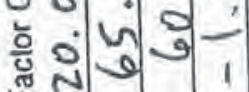

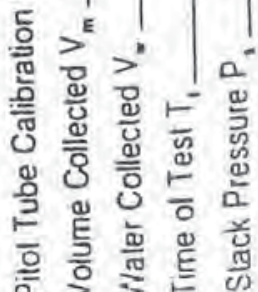


Impinger Box No.

$3-2$

Impinger 1

Final Weight

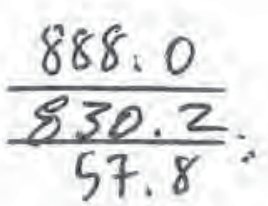

Water Weiahi Gain

Initial Weight

Increase

57.8 .

Impinger 2

Final Weight

Initial Weight

Increase

$\frac{\frac{842.2}{841.2}}{1.0}$

Impinger 1 57.8

Impinger $2 \quad 1.0$

Impinger 3

0.6

Impinger $4 \quad 5.6$

Impinger 3

Final Weight

$\frac{637.1}{636.5}$

$V_{w}=$

Initial Weight

Increase

0.6

Impinger 5

$\mathrm{gSO}_{2}=$
$\mathrm{V}_{\mathrm{w}}=$

Impinger 6

Impinger 4

Final Weight

962.0

Initial Weight

Increase

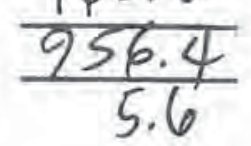

Impinger 7

Total

$65.0^{2}=v_{m}$

Impinger 5

Final Weight

Initial Weight

Increase

Impinger 6

Final Weight

Initial Weight

Increase

$P_{b}=26.87$

$\% \mathrm{CO}_{2}=$

$=12.8 \mathrm{\circ}$

$V_{m}=20.025$

$v_{w}=65.0 \mathrm{v}$

$\% \mathrm{O}_{2}=$

$\% \mathrm{CO}=$

$\frac{6.7}{0.0}$

$P_{m}=0.300 \mathrm{~V}$

$\% \mathrm{~N}_{2}=$

$=80.5$

Avg $\Delta P=-$

A $=$

$D_{n}=$

$$
\begin{aligned}
\operatorname{Avg} \sqrt{\Delta \mathrm{P}} & =-- \\
C_{p} & =-\frac{}{84.70}{ }^{\circ} \mathrm{H}_{2} \mathrm{O} \\
P_{1} & =-{ }^{\circ} \mathrm{F} \\
T_{m} & =-{ }^{\circ} \mathrm{F} \\
T_{1} & =-
\end{aligned}
$$$$
T_{\mathrm{t}}=
$$$$
93632
$$

Impinger 7

Final Weight

Initial Weight

Increase
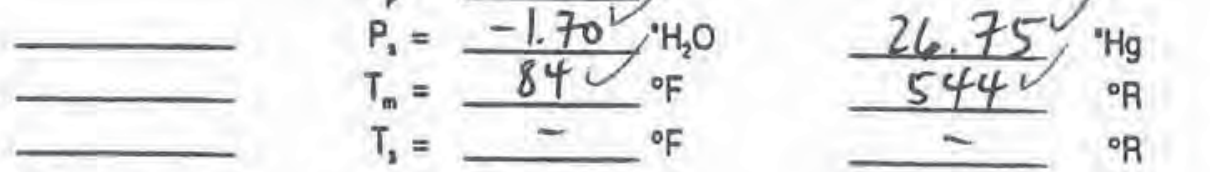

Moisture Content: $\quad \% M=14.94 \quad M_{d}=0.8506 \quad M_{c}=30.316^{\circ} \quad M W=28.48$

$$
V m_{s t d}=17.65 \mathrm{Vm}\left[\frac{P_{b}+\frac{P_{m}}{13.6}}{T_{m}+460}\right]=17.65 \times 20.025\left[\frac{26.87+\frac{0.300}{13.6}}{84+460}\right]=\frac{17.472}{0.291} \mathrm{stt}^{3}
$$

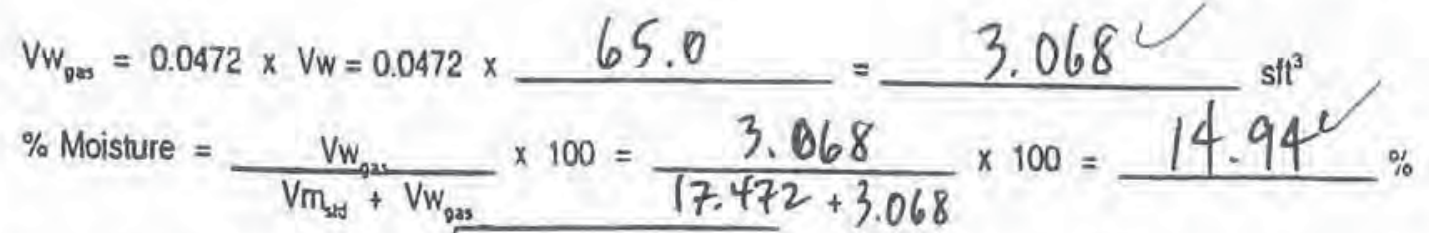

$V_{2}=5123.8 \times$

$x$

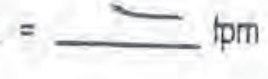

$\% 1=$

$x$

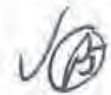

$5 / 13 / 07$
ACFM:

SCFM.

\%EA 45.8 
EE E

(4)

i. 10.01

$\ln _{+\rightarrow} \rightarrow$

운온

$\operatorname{lol}^{\circ}{ }^{2}$

는

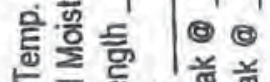

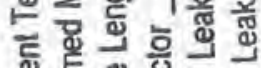

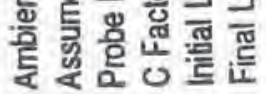

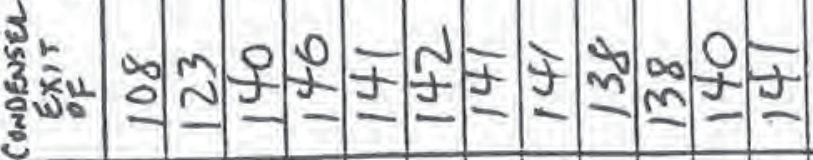

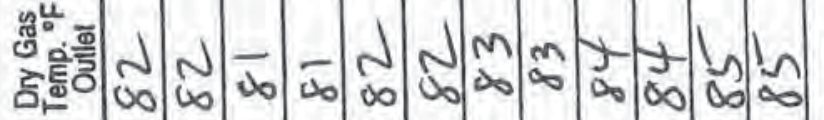

वृ.

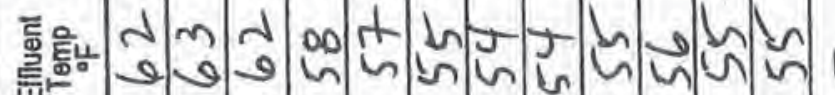

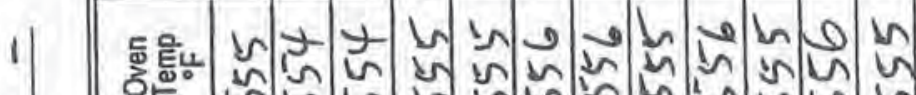

可

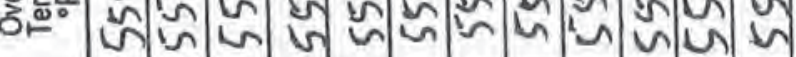

की

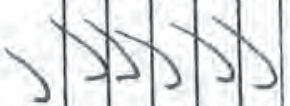

융.

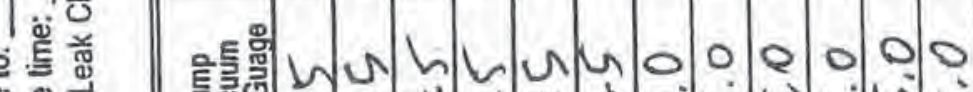

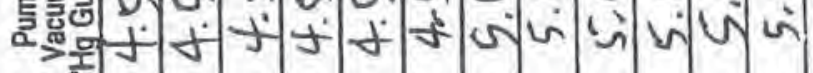

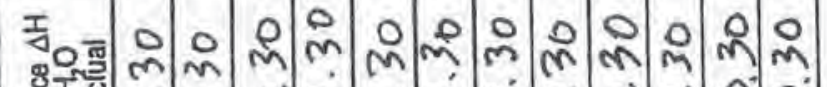

-

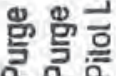

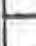

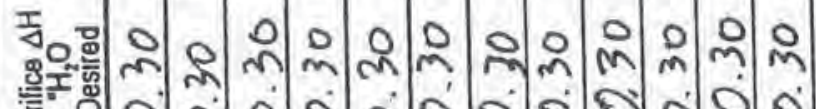

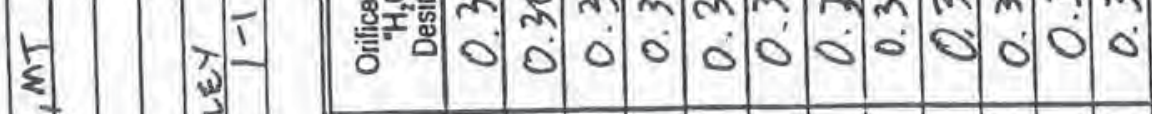

高

与

$+3 \sqrt{\frac{2}{2} \overline{\frac{9}{2}}}$

5.

iो

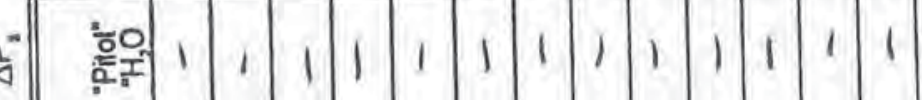

$=\bar{E} \cdot \stackrel{\text { C }}{\mathrm{E}}$

i :

\& 으 는

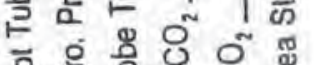

흘 윰

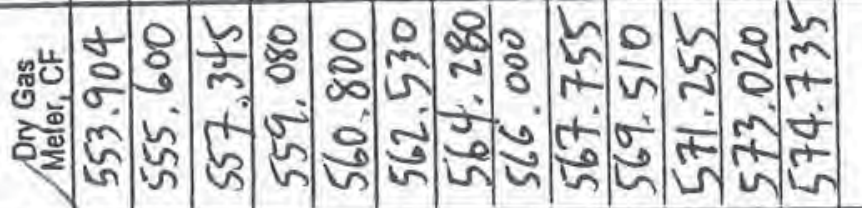

1

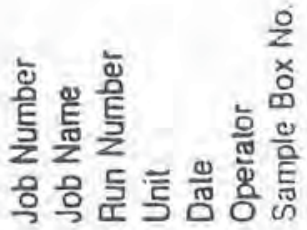

일

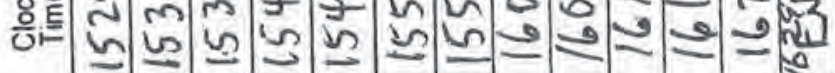

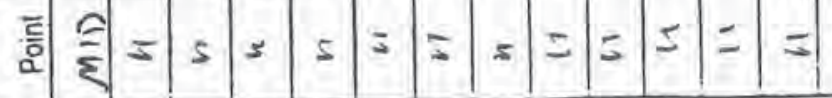

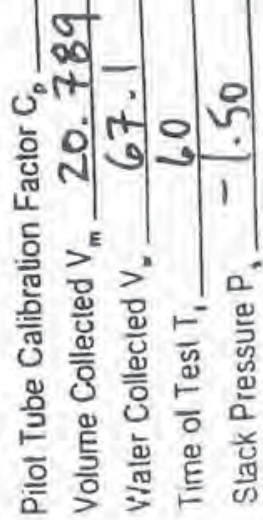


Impinger Box No.

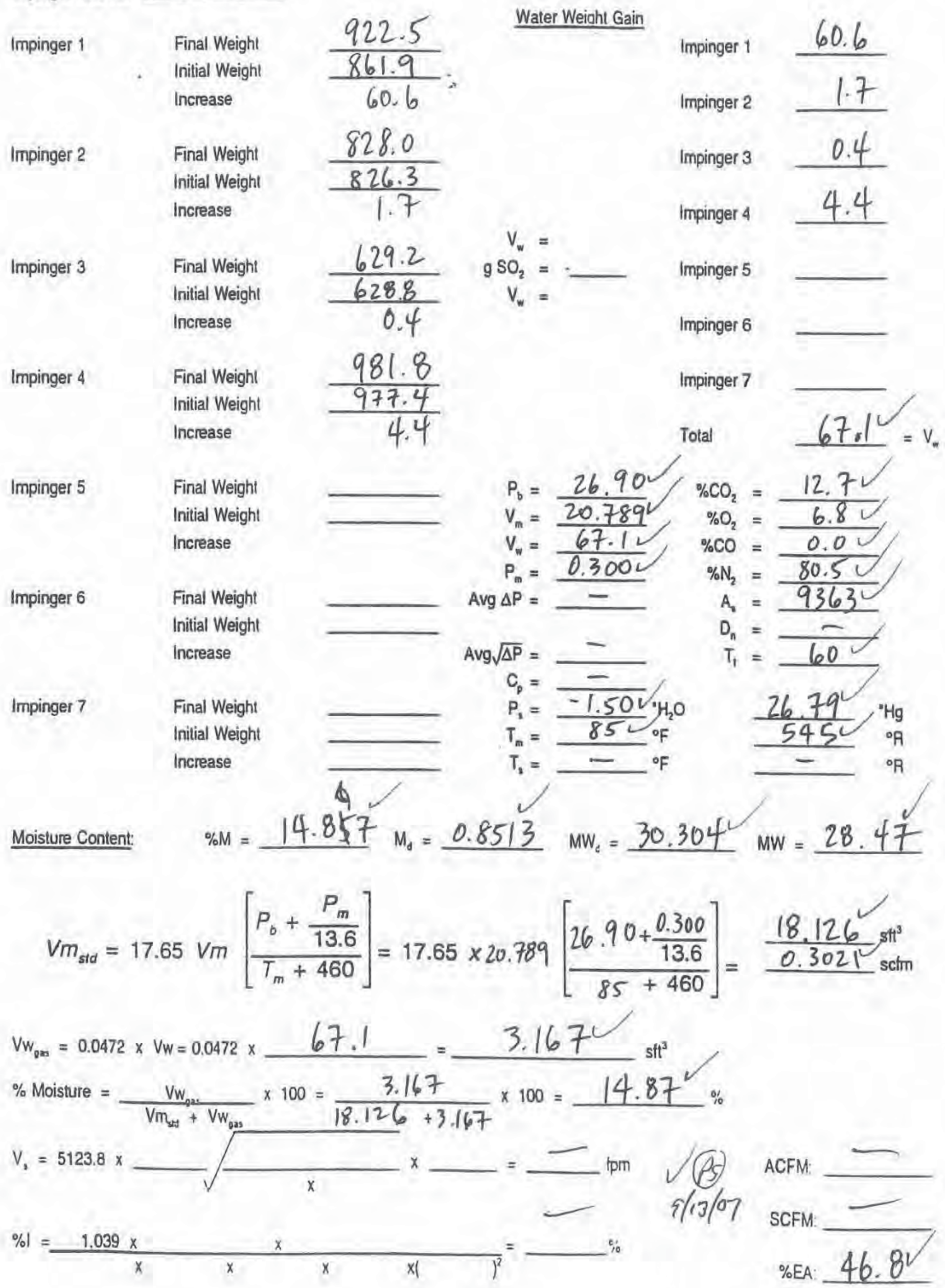


PRE MINARY VELOCITY TRAVERSE DATA

AND

SAMPLING LOCATION DATA

Job Number $07-057$

Job Name ADA-ES Hardin, MT

Sampling Location Stack

Date 6 Supt 2007 Time 0925-0940

Stack Height

fi.

Sampling Port Height Above Ground

it.

Port A

Port \& Inside Diameter (in.)

Port \& Wall Thickness (in.)

Inside Stack Diameter (in.)

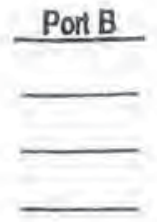

Port C

Port D

Average

Sampling Porls are 33 ค. $1 \frac{1 / 2}{}$ in.

Sampling Ports are 718 it. $23 / 8$ in.

(3,64 stack diameters) downstream from disturbance (inlel, constriction) bend, expansion)

172.00 slack diameters) upstream from disturbance (outhl, constriction, bend, expansion)

\begin{tabular}{|c|c|c|c|c|c|c|c|}
\hline $\begin{array}{c}\text { Point } \\
\text { Number }\end{array}$ & $\begin{array}{c}\text { Percent } \\
\text { Diameter }\end{array}$ & $\begin{array}{l}\text { Distance from } \\
\text { Ref. Point } \\
\text { (decimal in.) }\end{array}$ & $\begin{array}{l}\text { Distance lrom } \\
\text { Ref. Point } \\
\text { (fractional in.) }\end{array}$ & $\begin{array}{c}\text { Pont A } \\
\Delta \mathrm{P} / T_{d} / \alpha\end{array}$ & $\begin{array}{c}\text { Port B } \\
\Delta P / T_{d} \alpha\end{array}$ & $\begin{array}{l}\text { Port C } \\
\Delta \mathrm{P} / T_{\downarrow} \alpha\end{array}$ & $\begin{array}{l}\text { Port D } \\
\Delta \mathrm{P} / T_{\Omega} / \alpha\end{array}$ \\
\hline 1 & 4,4 & 4.804 & $413 / 16^{\prime \prime}$ & $3,80,229$ & $3.80 \mathrm{R2} 51$ & 11 & 11 \\
\hline 2 & 14.6 & 15.941 & $1515 / 16^{\prime \prime}$ & 3.551229 & $\$ 251024$ & 11 & 1 \\
\hline 9 & 29.6 & 32,320 & $325 / 16^{\prime \prime}$ & 3,6012251 & 4401229 & 11 & 1 \\
\hline 4 & 70,4 & 76.868 & $76^{918}$ & 4.001224 & 4901224 & 11 & 1 \\
\hline 5 & 85.4 & 93.246 & $931 / 4^{\prime \prime}$ & 4.10 R2S1 & 4.701229 & 11 & 11 \\
\hline 6 & 95.6 & $104: 383$ & $104^{3} / 8^{\prime \prime}$ & 3,801223 & 3901229 & 11 & 11 \\
\hline 7 & & & & 11 & 11 & 11 & 11 \\
\hline B & & & & 11 & 11 & 11 & 11 \\
\hline 9 & & & & 11 & 11 & 11 & 11 \\
\hline 10 & & & & 11 & 11 & 1 & 11 \\
\hline 11 & & & & 11 & 11 & 1 & 11 \\
\hline 12 & & & & 11 & 11 & 1 & 11 \\
\hline 13 & & & & 11 & 11 & 11 & 11 \\
\hline 14 & & & & 11 & 11 & 1 & 11 \\
\hline 15 & & & & 11 & 11 & 11 & 11 \\
\hline 16 & & & & 11 & 11 & 11 & 11 \\
\hline 17 & & & & 11 & 11 & 11 & 11 \\
\hline 18 & & & & 11 & 11 & 11 & 11 \\
\hline 19 & & & & 11 & 11 & 11 & 11 \\
\hline 20 & & & & 11 & 11 & 11 & 11 \\
\hline 21 & & & & 11 & 11 & 11 & 11 \\
\hline 22 & & & & 11 & 11 & 1. & 11 \\
\hline 28 & & & & 11 & 11 & 1 & 11 \\
\hline 24 & & & & 11 & 11 & 11 & 11 \\
\hline
\end{tabular}

Pitol Tube No,

$$
\begin{aligned}
& \mathrm{c}_{\mathrm{p}}=0.827 \\
& P_{b}=26.81 . \mathrm{Hg} \\
& P_{\mathrm{e}}=-2,2 \mathrm{O} \quad \mathrm{H}_{2} \mathrm{O} \text { Z6, } 65 \mathrm{mg} \\
& A_{1}=9363-\text { in. }^{2}
\end{aligned}
$$

Average $\triangle \mathrm{P} \quad 4,083$

Average $\Delta \mathrm{P}^{12} \geq .019$

Average $T_{x} \geq 25{ }^{\circ}$

Average $\alpha$ degrees 


\section{ORSAT ANALYSIS DATA FORM}

Job \#: $\quad 07-057$

Job Name: $A D A-E S$

Location: Herdin, $M T$

Date: $\quad 6$ Sept 2007

Operator: Mullins
Sample Location: Stack

Analytical Method:

Sample Type. Single Point/ Multi Point Grab Integrated Leak Check: Time:

Ambient Air Check:

\begin{tabular}{|l|c|}
\hline $\mathrm{CO}_{2}-\%$ Vol. & 0.0 \\
\hline $\mathrm{O}_{2}-\%$ Vol. & 20.9 \\
\hline $\mathrm{N}_{2}-\%$ Vol. & 79.1 \\
\hline
\end{tabular}

Run \#: 1

Run Time: $1221-1 / 24$

\begin{tabular}{|l|c|c|c|c|}
\hline $\mathrm{CO}_{2}-\%$ Vol. & 12.8 & 12.8 & 12.8 & $12-8$ \\
\hline $\mathrm{O}_{2}-\%$ Vol. & 6.7 & 6.7 & 6.7 & 6.7 \\
\hline $\mathrm{CO}-\%$ Vol. & 0.0 & 0.0 & 0.0 & 0.0 \\
\hline $\mathrm{N}_{2}-\% \mathrm{Vol}$. & 80.5 & 80.5 & 80.5 & 80.5 \\
\hline
\end{tabular}

Run \#: Z

Run Time: $13 / 0-14^{2}$ Analysis \#1 Analysis \#2 Analysis \#3 Average - $\%$ Volume

\begin{tabular}{|l|c|c|c|c|}
\hline $\mathrm{CO}_{2}-\%$ Vol. & 12.8 & 12.9 & 12.8 & 12.8 \\
\hline $\mathrm{O}_{2}-\% \mathrm{Vol}$. & 6.7 & 6.6 & 6.7 & 6.7 \\
\hline $\mathrm{CO}-\% \mathrm{Vol}$. & 0.0 & 0.0 & 0.0 & 0.0 \\
\hline $\mathrm{N}_{2}-\% \mathrm{Vol}$. & 80.5 & 80.5 & 80.5 & 80.5 \\
\hline
\end{tabular}

Run \#: $\frac{3}{\text { Run Time: } 1537-1641}$

\begin{tabular}{|l|c|c|c|c|}
\hline $\mathrm{CO}_{2}-\% \mathrm{Vol}$. & 12.7 & $12-7$ & 12.7 & 12,7 \\
\hline $\mathrm{O}_{2}-\% \mathrm{Vol}$. & 6.8 & 6.8 & 6.8 & 6.8 \\
\hline $\mathrm{CO}-\% \mathrm{Vol}$. & 0.0 & 0.0 & 0.0 & 0.0 \\
\hline $\mathrm{N}_{2}-\% \mathrm{Vol}$. & 80.5 & 80.5 & 80.5 & 80.5 \\
\hline
\end{tabular}




\section{Appendix E:}

EPA Test Methods 201A and 202 (Particulate Matter) Analytical Data 


\section{IIIR

Air Sampling Associates, Inc.

\section{Particulate Analysis Summary}

Project Number: $07-057$

Project Name: $4 D A-E S$

Project Location: Hardin, MT

Run Number:

Particulate Matter >PM10 (mgs):

Particulate Matter < PM10 > PM2.5 (mgs):

Particulate Matter PM2.5 Rinse (mgs):

Particulate Matter PM2.5 Filter (mgs):

Total Particulate Matter as PM2.5 (mgs):

Particulate Matter in “Back-Half” (mgs):

Total Particulate Matter in Sample - MT (mg):
Date Analysis Completed: $9 / 21 / 07$ Unit Tested:

\begin{tabular}{|c|c|c|}
\hline 1 & 2 & 3 \\
\hline .5 & 1.8 & 4.3 \\
\hline & 3.6 & 3.1 \\
\hline & 0,0 & 7.6 \\
\hline & 0.8 & .6 \\
\hline & 0.8 & \\
\hline
\end{tabular}

$53.2 \quad 97.5 \quad 23.6$

$\begin{array}{llll}65.9 & 103.7 & 39.8\end{array}$

Date:

Data Checked By 


\section{Particulate Analysis EPA Method PM 10/2,5}

\section{Front Wash}

Project No. $07-057$

Project Name

$A D A-E S$

Location Unit Tested

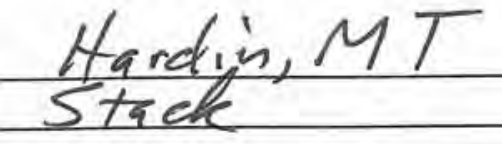

Desiccator Time In //16/0708509/17/07/310

Desiccator Time Out $0 / 12 \% 07 / 305$ 19/18/07 0720

\begin{tabular}{|c|c|c|c|}
\hline Run No. / & $>P M I O$ & Volume (ml) & Sample I.D. \\
\hline Final Weight $(\mathrm{g})$ & 4.1898 & 4.18954 & \\
\hline Initial Weight (g) & 4.1816 & 4.1816 & \\
\hline Particulate Weight (g) & & & \\
\hline
\end{tabular}

Particulate Average (mg) Less Acetone Blank (mg)

Total Particulate $(\mathrm{mg})$

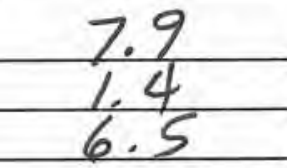

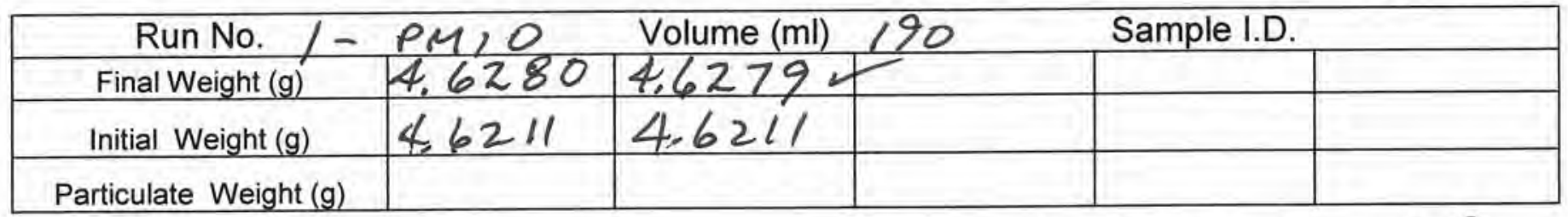

Particulate Average (mg) Less Acetone Blank (mg)

Total Particulate $(\mathrm{mg})$

6.8

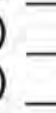

Sample I.D.

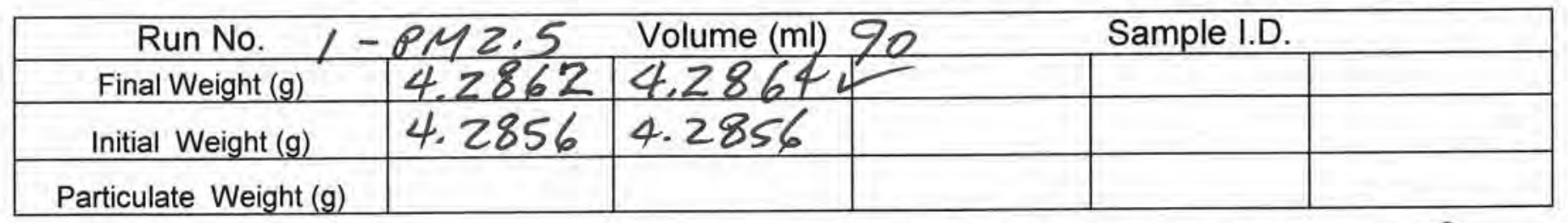

Particulate Average $(\mathrm{mg}) \quad 0.8$ Less Acetone Blank (mg) 0,7 Total Particulate $(\mathrm{mg})$

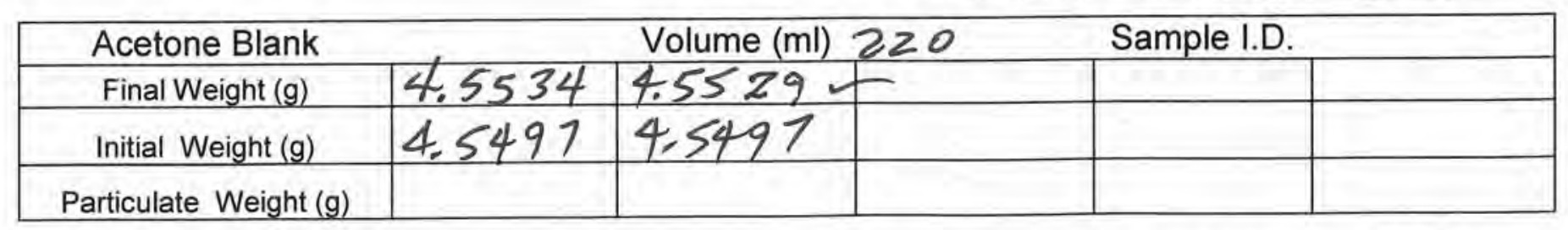

\footnotetext{
* Note: if greater than $7.9 \mathrm{mg} / \mathrm{l}$, use $7.9 \mathrm{mg} / \mathrm{l}$
}

$$
\begin{aligned}
& \text { Average (mg) } 3.2 \\
& { }^{*} \mathrm{mg} / \mathrm{l} 14.5
\end{aligned}
$$




\section{IIIIR

Particulate Analysis EPA Method PM $10 / 2.5$

Front Half Tare Weights

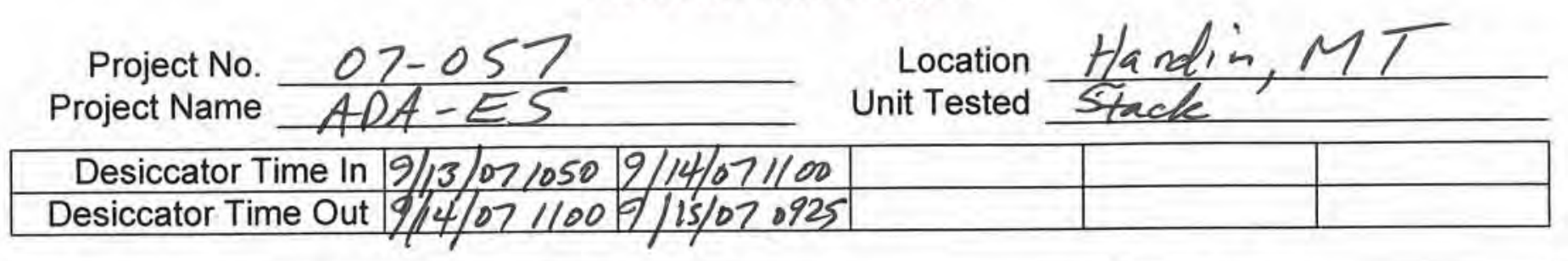

\begin{tabular}{|l|l|l|l|l|}
\hline Run No. $/ 7 P M / 0$ & \multicolumn{3}{|c|}{ Sample I.D. } \\
\hline Weight (g) & $4.18 / 6$ & $4.18 / 6$ & & \\
\hline
\end{tabular}

\begin{tabular}{|l|l|l|l|l|}
\hline Run No. $1-P M / O$ & \multicolumn{3}{|c|}{ Sample I.D. } \\
\hline Weight (g) & 4.6216 & $4,62 / 1$ & & \\
\hline
\end{tabular}

\begin{tabular}{|l|l|l|l|l|}
\hline Run No. $1-P M 2.5$ & Sample I.D. \\
\hline Weight (g) & 4.2859 & 4.28564 & & \\
\hline
\end{tabular}

Acetone Blank Weight (g)
Sample I.D.

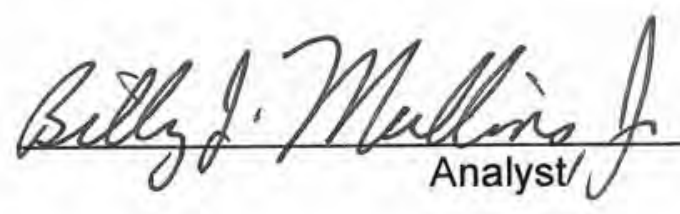




\section{IIIR

Particulate Analysis EPA Method PM $10 / 2.5$

Front Wash

Project No.
$07-057$

Project Name

$A D A-E S$

Location Unit Tested

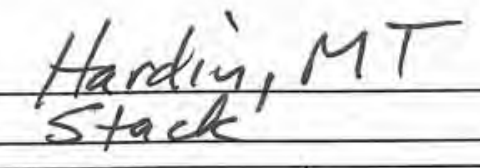

\begin{tabular}{|l|l|l|l|}
\hline Desiccator Time In $69 / 16 / 07085009 / 17 / 071320$ & & & \\
\hline Desiccator Time Out 69/17/07 $131509 / 18 / 070725$ & & & \\
\hline
\end{tabular}

\begin{tabular}{|c|c|c|c|c|c|}
\hline Run No. & $>P M / / 0$ & Volume $(\mathrm{ml})$ & 160 & Sample I.D. & \\
\hline Final Weight $(\mathrm{g})$ & 4.3979 & 4.3978 . & & & \\
\hline Initial Weight $(\mathrm{g})$ & 4.3947 & 4.3947 & & & \\
\hline Particulate Weight (g) & & & & & \\
\hline
\end{tabular}

Particulate Average (mg)

Less Acetone Blank (mg)

3. 1

Total Particulate $(\mathrm{mg})$

1.3

1.8

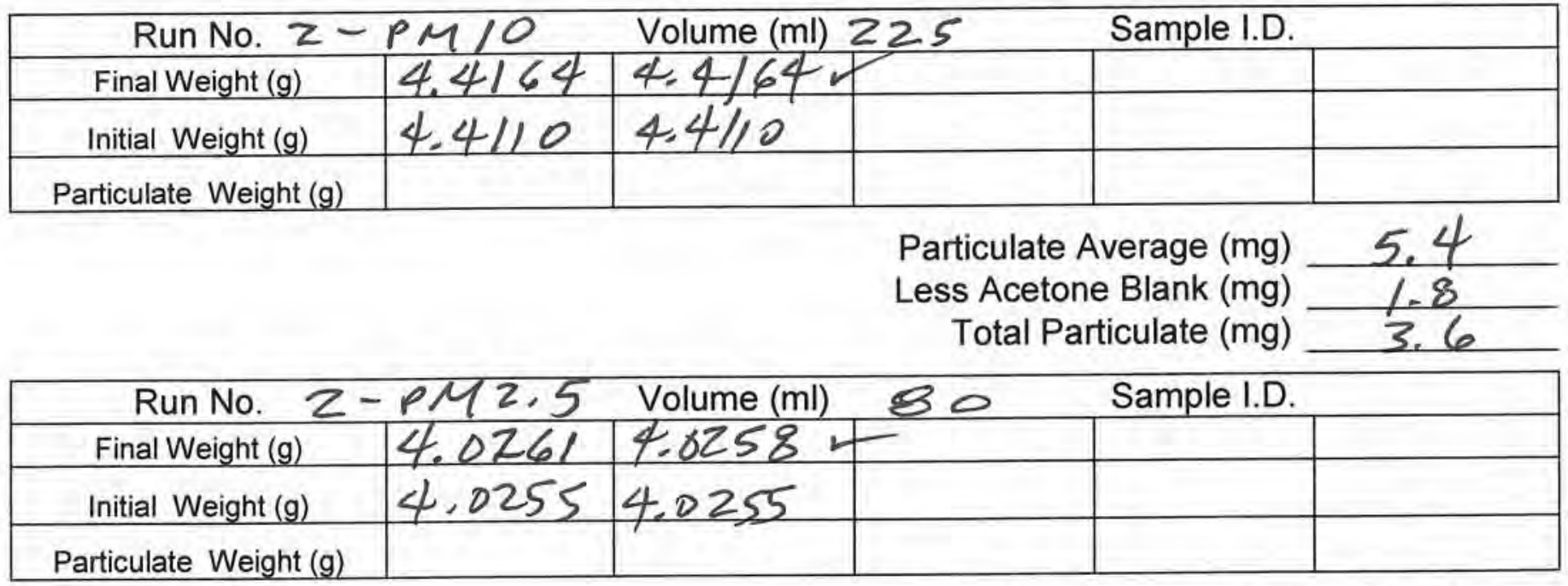

Particulate Average $(\mathrm{mg}) \quad 0,3$ Less Acetone Blank $(\mathrm{mg}) \quad 0,6$

Total Particulate $(\mathrm{mg}) \longrightarrow 0.0$

\begin{tabular}{|c|l|l|l|l|l|}
\hline Acetone Blank & & & \\
\hline Final Weight (g) & & & & & \\
\hline Initial Weight (g) & & & & & \\
\hline Particulate Weight (g) & & & & \\
\hline
\end{tabular}

Average (mg)

* Note: if greater than $7.9 \mathrm{mg} / \mathrm{l}$, use $7.9 \mathrm{mg} / \mathrm{l}$ 


\section{Im) \\ ASSOCIATES, INC.}

Particulate Analysis EPA Method PM $10 / 2.5$

Front Half Tare Weights

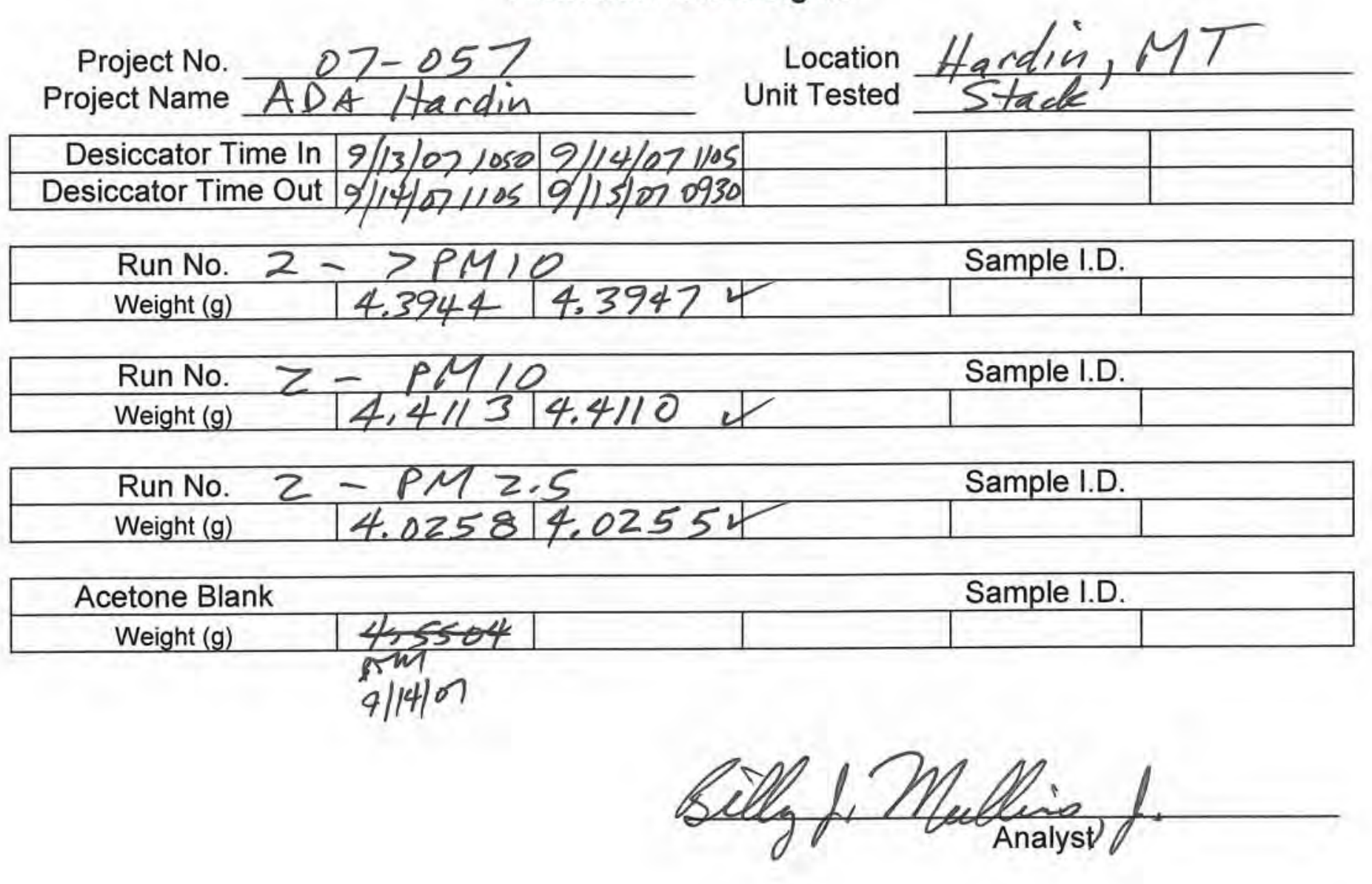


Particulate Analysis EPA Method PM-10/2.5

Page __ of

Front Wash

Project No. $07-057$

Project Name ADA-ES

Location Hardin, MT

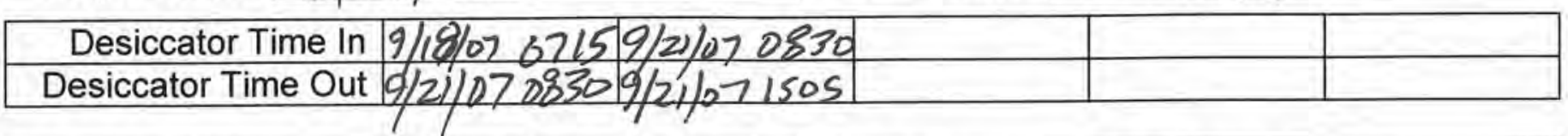

\begin{tabular}{|c|l|l|l|l|l|}
\hline \multicolumn{2}{|c|}{ Run No. 3 - JPM/10 Volume (ml) 150} & Sample I.D. \\
\hline Final Weight (g) & 4.3988 & 4.3993 & & & \\
\hline Initial Weight (g) & 4.3938 & 4.3938 & & & \\
\hline Particulate Weight (g) & & & & & \\
\hline
\end{tabular}

Particulate Average $(\mathrm{mg}) \quad 5.5$

Less Acetone Blank $(\mathrm{mg}) 1,2$

Total Particulate $(\mathrm{mg}) \quad 4.3$

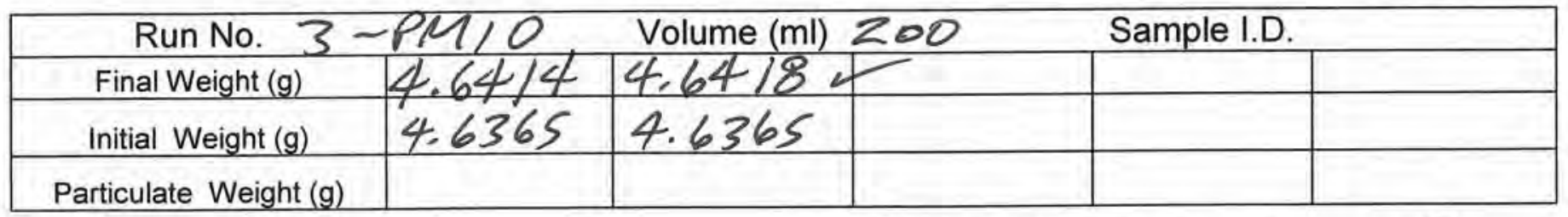

Particulate Average $(\mathrm{mg}) \quad 5.3$

Less Acetone Blank $(\mathrm{mg}) 1.6$

Total Particulate $(\mathrm{mg}) \quad 3,7$

\begin{tabular}{|c|c|c|c|c|c|}
\hline Run No. 3 & $8 M 2.5$ & Volume $(\mathrm{ml})$ & 70 & Sample I.D. & \\
\hline Final Weight $(\mathrm{g})$ & 4.4007 & 4.40084 & & & \\
\hline Initial Weight (g) & 4.3926 & 4.3926 & & & \\
\hline Particulate Weight (c & & & & & \\
\hline
\end{tabular}

Particulate Average $(\mathrm{mg}) \quad 8.2$

Less Acetone Blank (mg) 0.6

Total Particulate $(\mathrm{mg}) \quad 7,6$

\begin{tabular}{|c|l|l|l|l|l|}
\hline Acetone Blank & \multicolumn{3}{c|}{ Volume (ml) } & & \\
\hline Final Weight (g) & & & & & \\
\hline Initial Weight (g) & & & & & \\
\hline Particulate Weight (g) & & & & & \\
\hline
\end{tabular}

Average $(\mathrm{mg})$

* Note: if greater than $7.9 \mathrm{mg} / \mathrm{l}$, use $7.9 \mathrm{mg} / \mathrm{l}$

* $\mathrm{mg} / \mathrm{l}$

Version No.2 10/22/06

E-7

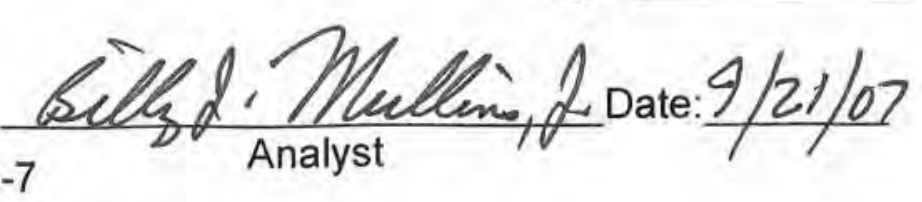




\section{IIIIR

Particulate Analysis EPA Method $P M 10 / 2.5$

Front Half Tare Weights

Project No.

Project Name

Location
Unit Tested Stack
Stan, MT

Desiccator Time In $9 / 13 / 07$ 1050 $9 / 14 / 07 / 1 / 5 / 9 / 15 / 070930$ 9/16/07 0845

Desiccator Time Out $/ / 14 / 0711 / 59 / 15 / 07093019 / 16 / 0708459 / 1710071300$

\begin{tabular}{|l|l|l|l|l|}
\hline Run No. $3->P M / 0$ & \multicolumn{3}{|c|}{ Sample I.D. } \\
\hline Weight (g) & 4.3940 & 4.39384 & & \\
\hline
\end{tabular}

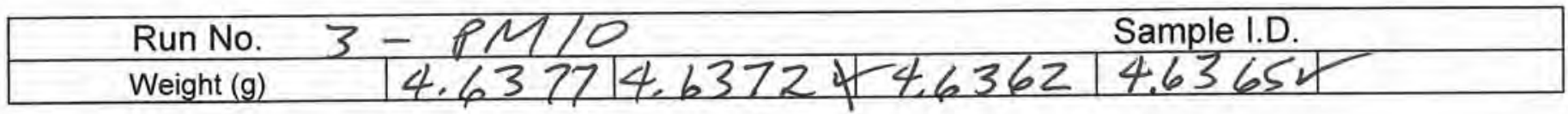

\begin{tabular}{|c|c|c|}
\hline Run No. & $3-P \mu Z .5$ & \multirow[t]{2}{*}{ Sample I.D. } \\
\hline Weight $(g)$ & $4,394214,3926$ & \\
\hline Acetone Blank & & $\begin{array}{l}6 \text { gom 9p/6/p7 } \\
\text { Sample I.D. }\end{array}$ \\
\hline Weight (g) & & \\
\hline
\end{tabular}

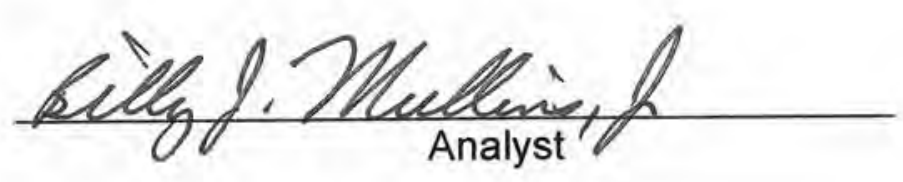


AIR

SAMPLING

ASSOCIATES, INC.

Page of

Particulate Analysis EPA Method PM 10/2.5

Stack Filters

Project No. $07-057$

Project Name ADA -ES
Location Hardin, MT Unit Tested Stack

Desiccator Time In 9/16/07 0850/9/18/070735

Desiccator Time Out 9/18/07 0730 9/18/07/520

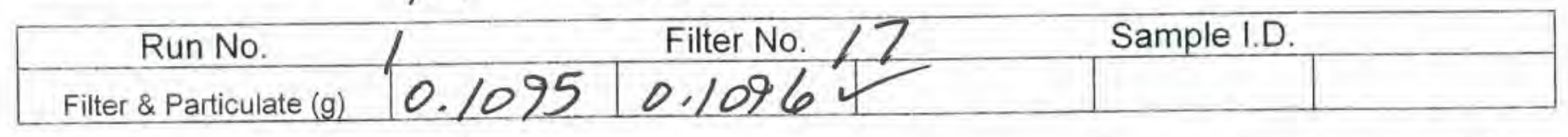

Filter \& Particulate Average (g) 0,1096 Initial Filter Weight $(\mathrm{g}) 0.1088$ Total Particulate $(\mathrm{mg})$ 0.8

Run No. 2

Filter \& Particulate $(\mathrm{g})$ 0.1083

Filter No. 0.1086
Sample I.D.

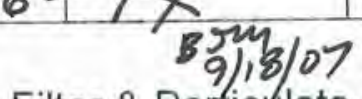

Filter \& Particulate Average $(\mathrm{g}) \quad 0.1086$ Initial Filter Weight $(\mathrm{g}) \quad 0.1078$ Total Particulate (mg) 0.8

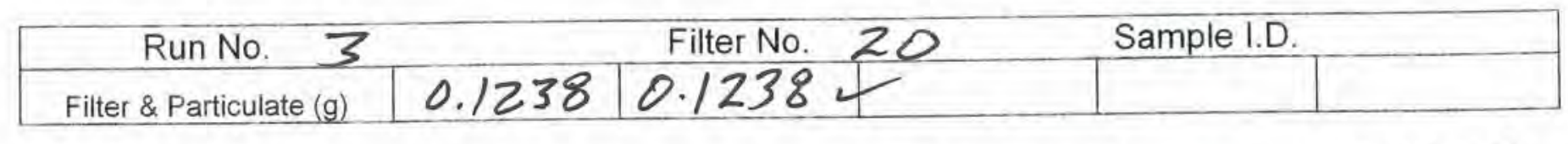

Filter \& Particulate Average (g) 0.1238 $\begin{array}{cr}\text { Initial Filter Weight }(\mathrm{g}) & 0.1232 \\ \text { Total Particulate }(\mathrm{mg}) & 0.6\end{array}$

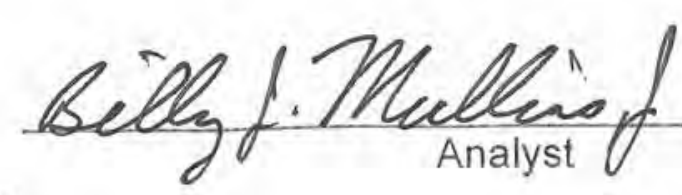

E-9

Version No. 2 2/13/07 


\section{AIR \\ SAMPLING}

ASSOCIATES, INC.

Filter Weight Log

$47 \mathrm{~mm}$

\begin{tabular}{|c|c|c|c|c|c|c|c|}
\hline \multirow[b]{3}{*}{ Filter №. } & Into Desicator & Weight & Weight & Weight & Weight & Weight & \multirow{3}{*}{$\begin{array}{l}\text { Weight } \\
\text { Used }\end{array}$} \\
\hline & Date & Date & Date & Date & Date & Date & \\
\hline & Time & Time & Time & Time & Time & Time & \\
\hline \multirow{3}{*}{16} & $8 / 1 / 07$ & 0.1214 & 0.1217 & $F$ & & & 0.1217 \\
\hline & 0800 & 8128107 & $8 / 28 / 07$ & & & & \\
\hline & & 0910 & 1520 & & & & \\
\hline \multirow{3}{*}{17} & 811107 & 0.1084 & 0,1088 & $\checkmark$ & & & 0.1088 \\
\hline & 0800 & $8 / 28 / 07$ & $8 / 28 / 07$ & & & & \\
\hline & & 0910 & 1520 & & & & \\
\hline \multirow{3}{*}{18} & 81107 & 0.1211 & 0.1213 & $\checkmark$ & & & 0.1213 \\
\hline & 0800 & $8 / 28 / 07$ & $8 / 28 / 07$ & & & & \\
\hline & & 6910 & 1520 & & & & \\
\hline \multirow{3}{*}{19} & $8 / 1 / 07$ & 0.1075 & 0,1078 & $r$ & & & p. 1078 \\
\hline & 6800 & $8 / 2 \xi / 07$ & $8 / 28 / 07$ & 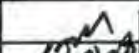 & 1 & & \\
\hline & & $\frac{0915}{1200}$ & 1525 & $\omega_{\xi} \mid+\infty$ & & & \\
\hline \multirow{2}{*}{20} & $\frac{8 / 1107}{0800}$ & $\frac{0.1229}{8.285107}$ & $\begin{array}{l}3728107 \\
0.1232\end{array}$ & $<$ & & & 0.1232 \\
\hline & & 0215 & 1525 & & & & \\
\hline \multirow{3}{*}{21} & $8 / 1107$ & $0.12 / 2$ & 0.1214 & $=$ & & & 0.1214 \\
\hline & 0800 & $8 \longdiv { 2 8 6 7 }$ & $8 / 28 \% 07$ & & & & \\
\hline & & 0915 & 1525 & & & & \\
\hline \multirow{3}{*}{22} & $8 / 1 / 07$ & $0.10^{87}$ & 0.1092 & Z & & & 0.1092 \\
\hline & 6800 & $8 / 28 / 07$ & $8 / 28 / 07$ & & & & \\
\hline & & 1.920 & $1 / 530$ & & & & \\
\hline \multirow{3}{*}{23} & $8 / 1 / 07$ & 0.1067 & 0.1067 & $\checkmark$ & & & 0.1067 \\
\hline & 0800 & $8 / 28 / 07$ & $8 / 28 / 07$ & & & & \\
\hline & & 6220 & 1530 & & & & \\
\hline \multirow{3}{*}{24} & $B / 1 / 07$ & $0.12 / 2$ & 0.1212 & 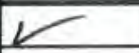 & & & p. $12 / 2$ \\
\hline & 0800 & $8 / 28) 7$ & $8 / 28 / 07$ & & & & \\
\hline & & 0920 & 1530 & & & & \\
\hline \multirow{3}{*}{25} & $8 / 1 / 07$ & 0.1188 & 0.1189. & & & & D. 1189 \\
\hline & 0800 & $8 / 28 / 07$ & $8 / 28 / 07$ & & & & \\
\hline & & 0925 & 1535 & & & & \\
\hline \multirow{2}{*}{26} & $8 / 1607$ & 0.1053 & 0.1053 & & & & 0.1053 \\
\hline & 6800 & $8 / 28 / 07$ & $8 / 28 / 27$ & & & & \\
\hline \multirow{3}{*}{27} & 811107 & 0.1211 & $\frac{1252}{0.1216}$ & r & & & 0.1216 \\
\hline & 0800 & $8 / 28 / 07$ & $8 / 28 / 107$ & & & & \\
\hline & & 0225 & 1535 & & & & \\
\hline \multirow{2}{*}{28} & & & & & & & \\
\hline & & & & & & & \\
\hline \multirow{2}{*}{29} & & & & & & & \\
\hline & & & & & & & \\
\hline \multirow{3}{*}{30} & & & & & & & \\
\hline & & & & & & & \\
\hline & & & & & & & \\
\hline
\end{tabular}




\author{
ADA-ES \\ Hardin, Montana \\ 202 - Back Half Particulate Analyses
}

\begin{tabular}{|c|c|c|c|c|c|c|c|}
\hline$\frac{\text { Run No. }}{1}$ & $\frac{\mathrm{MeCl}}{1.6}$ & $\begin{array}{c}\begin{array}{c}\mathrm{MeCl} \\
\text { Blank } \\
\text { Correction }\end{array} \\
(0.5)\end{array}$ & $\begin{array}{c}\text { DI } \\
\text { Water } \\
78.0\end{array}$ & $\begin{array}{c}\begin{array}{c}\text { DI Water } \\
\text { Blank } \\
\text { Correction }\end{array} \\
(4.3)\end{array}$ & $\begin{array}{c}\begin{array}{c}\text { Less } \\
\text { Chlorides }\end{array} \\
0.0\end{array}$ & $\begin{array}{c}\begin{array}{c}\text { Less } \\
\text { Sulfates }\end{array} \\
(21.6)\end{array}$ & $\frac{\text { Total }}{53.2}$ \\
\hline 2 & 1.3 & $(0.4)$ & 134.3 & (3.9) & 0.0 & (33.8) & 97.5 \\
\hline 3 & 0.7 & $(0.5)$ & 37.1 & (4.0) & 0.0 & (9.7) & 23.6 \\
\hline Blank & 1.8 & & 10.0 & & & & \\
\hline
\end{tabular}


Methylene Chloride Rinse

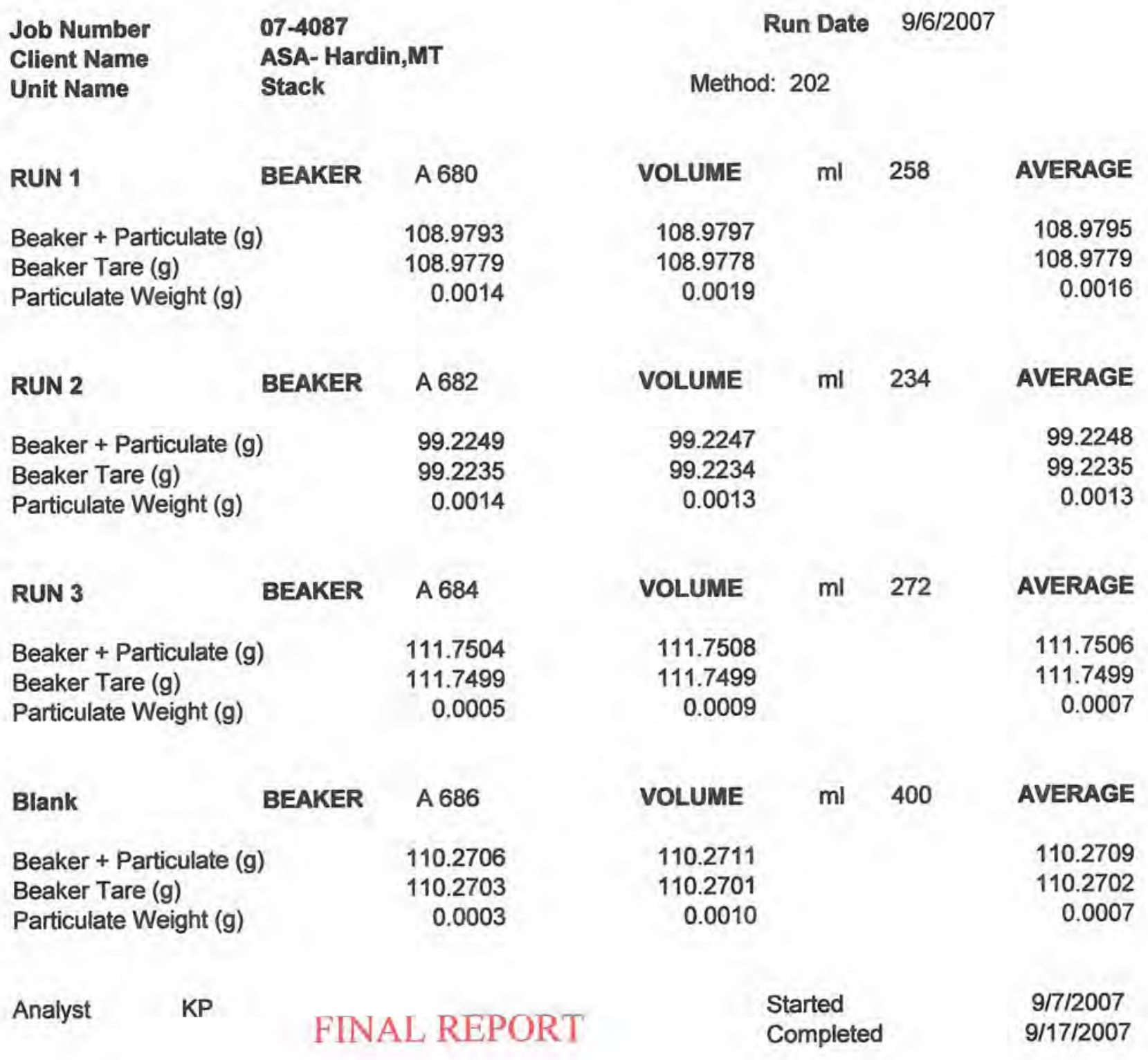




\section{Water Analysis (Back)}

\author{
Job Number \\ Client Name \\ Unit Name
}

07-4087

ASA- Hardin, MT

Stack
Run Date $\quad 9 / 6 / 2007$

Method: 202
RUN 1

BEAKER

A 679

107.4726

107.3944

0.0782

Particulate Weight (g)
VOLUME

107.4722

107.3944

0.0778

VOLUME $\mathrm{ml} 390$

109.2599

109.1256

0.1343

425

Particulate Weight (g)

09.2600

0.1343

RUN 3

BEAKER

Glass Fragments Removed

Beaker + Particulate (g)

Beaker Tare (g)

Particulate Weight (g)

Blank

BEAKER

Beaker + Particulate (g)

Beaker Tare (g)

Particulate Weight (g)

A 683

100.7996

100.7625

0.0371

A 685

100.9923

100.9898

0.0025
VOLUME

100.7996

100.7624

0.0372

VOLUME

100.9924

100.9897

0.0027
AVERAGE

107.4724

107.3944

0.0780

AVERAGE

109.2600

109.1257

0.1343

AVERAGE

100.7996

100.7625

0.0371

AVERAGE

100.9924

100.9898

0.0026

9/7/2007

9/17/2007 


$\begin{array}{ccccccc}\text { Sulfate } & \text { 07-4087 (07-057) } & \begin{array}{c}\text { Sample } \\ \text { Volume, } \\ \mathrm{ml}\end{array} & \begin{array}{c}\text { Lab } \\ \text { Volume, } \\ \mathrm{ml}\end{array} & \begin{array}{c}\text { Lab } \\ \text { Results, } \\ \text { ug/m! }\end{array} & \begin{array}{c}\text { Final } \\ \text { Results, } \\ \mathrm{mg}\end{array} \\ \text { Stack } & & \text { Sample No. } & & & & \\ & \text { Run 1 } & 4087-1 & 345 & 20 & 177 & 21.617 \\ & \text { Run 2 } & 4087-2 & 310 & 20 & 308 & 33.800 \\ & \text { Run 3 } & 4087-3 & 320 & 20 & 85.3 & 9.663\end{array}$




\section{LABORATORY ANALYTICAL. REPORT}

Client: Western Environmental

Site Name: $\quad 07-4087$

Report Date; 09/17/07

Lab ID: C07090271-001

Client Sample ID: 07-4087-1

Collection Date: Not Provided

Matrix: Aqueous

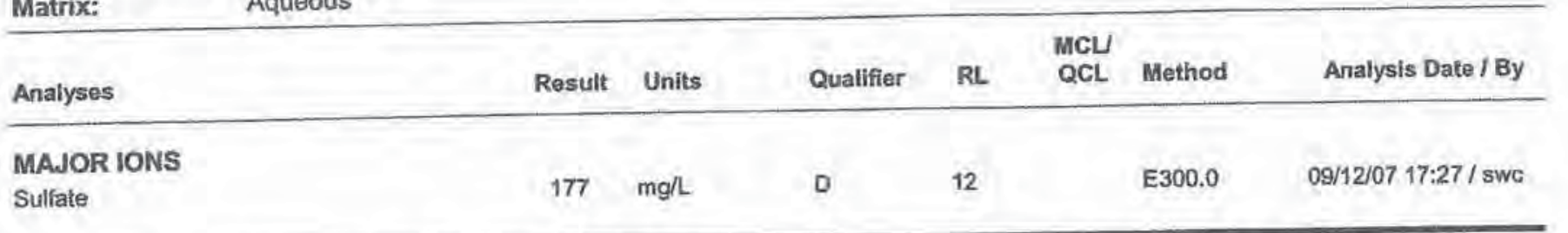

Lab ID:

C07090271-002

Client Sample ID: $07-4087-2$

Collection Date: Not Provided

Matrix:

Aqueous

DateReceived: 09/07/07

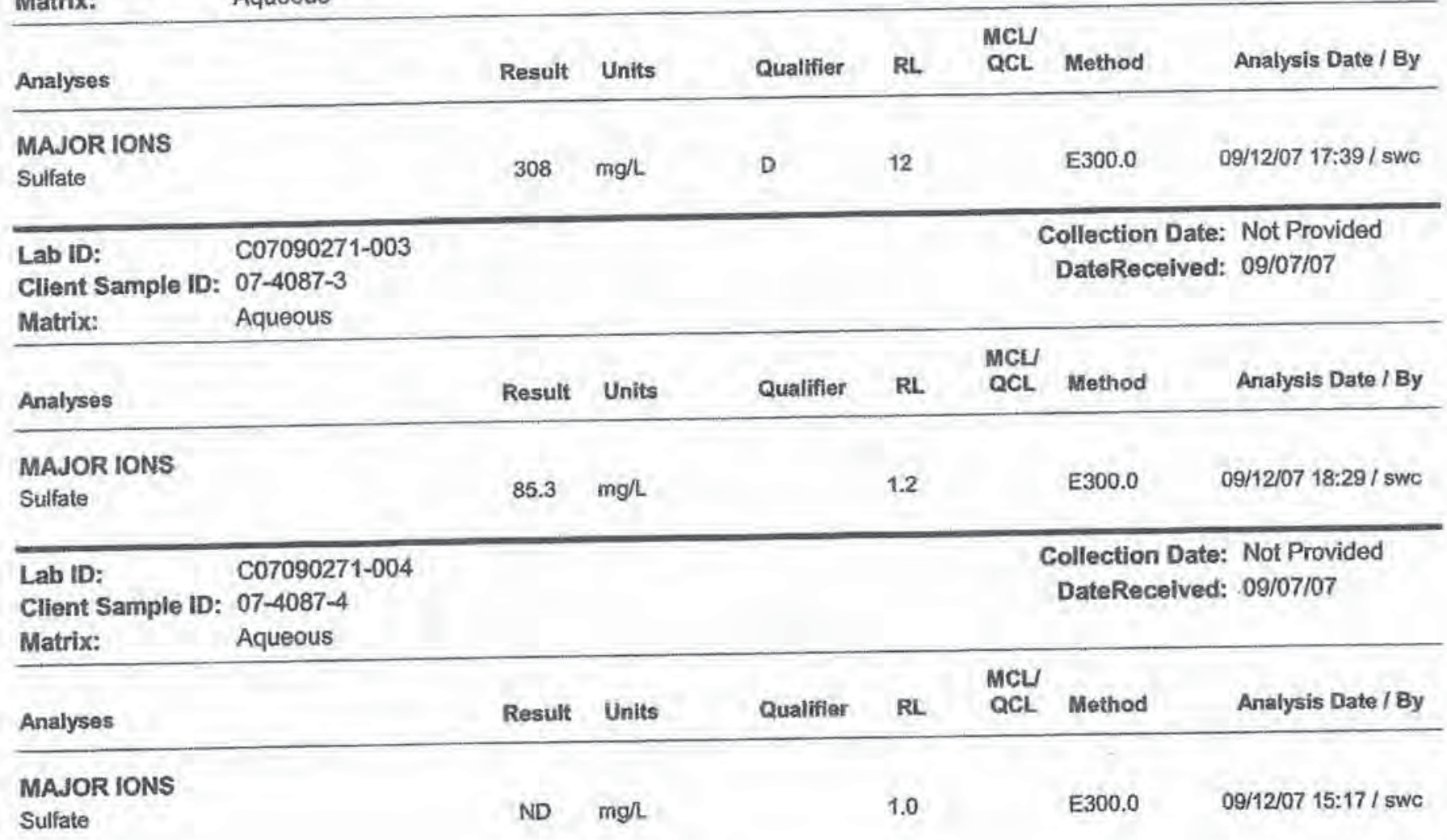

Report $\mathrm{RL}$-Analyte reporting limit.

Definitions: $Q C L$ - Quality control limit.

D-RL increased due to sample matrix interierence.
MCL - Maximum contaminant level.

ND - Not detected at the reporting limit. 


\section{Appendix F: \\ Controlled Condensate Test Method (Sulfate) Analytical Data}




\title{
Air Sampling Associates 407-2B Holford's Prairie Rd Lewisville, TX 75056-9582
}

\author{
ADA-ES \\ Hardin, MT \\ Client 07-057
}

\section{Analytical Report (0907-56)}

\section{HPLC Analysis \\ Sulfate}


I certify that to the best of my knowledge all analytical data presented in this report:

- Have been checked for completeness

- Are accurate, error-free, and legible

- Have been conducted in accordance with approved protocol, and that all deviations and analytical problems are summarized in the appropriate narrative(s)

- This analytical report was prepared in Portable Document Format (.PDF) and contains 53 pages.
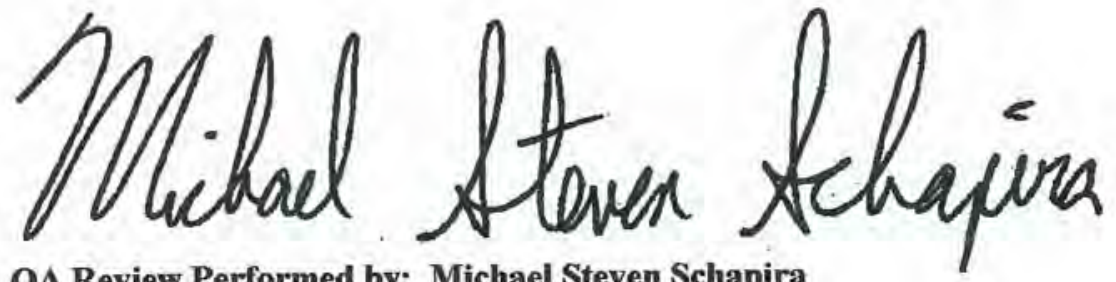

QA Review Performed by: Michael Steven Schapira 


\section{Summary of Results}

Page 3 of 53 


\begin{tabular}{|r|l|}
\hline Company & Air Sampling Associates \\
Analyst & KEH \\
Parameters & HPLC/IC Analysis \\
\# Samples & 3 Runs \& 1 blank \\
\hline
\end{tabular}

\begin{tabular}{|r|l|}
\hline Client \# & $07-057$ \\
Job \# & $0907-56$ \\
PO \# & Verbal \\
Report Date & $9 / 27 / 2007$ \\
\hline
\end{tabular}

Compound

Sample ID / Catch Weight (ug)

$\begin{array}{cccc} & \text { Run 1 - CC } & \text { Run 2 - CC } & \text { Run 3 - CC } \\ \text { Sulfate } & 499 & 1,349 & 1,537\end{array}$

HPLC Blank

Sulfate

2.92 ND 


\section{Results}

Page 5 of 53 

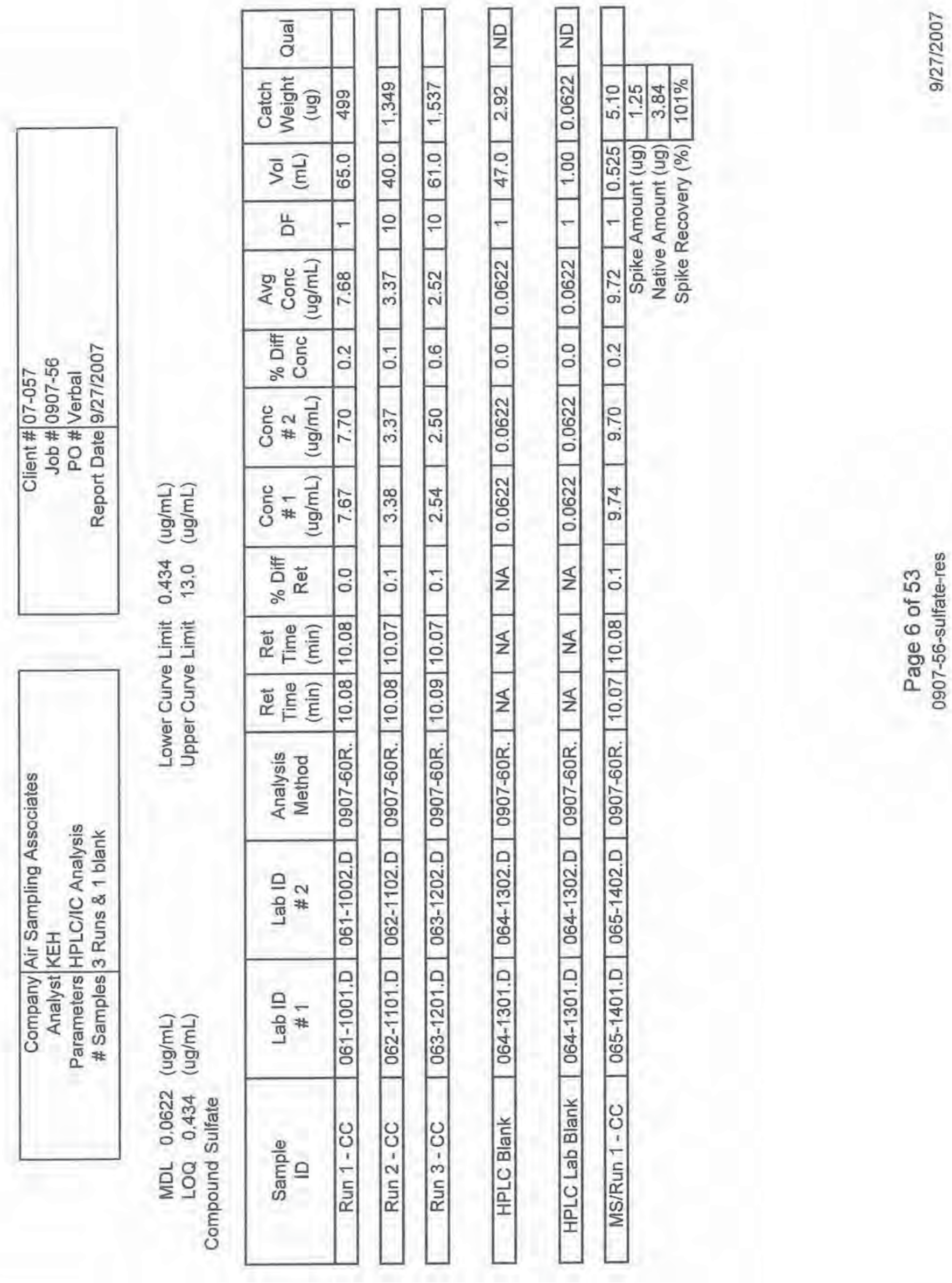


\section{Narrative Summary}

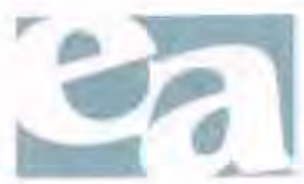

Page 7 of 53 


\section{Enthalpy Analytical Narrative Summary}

\begin{tabular}{|r|l|}
\hline Company & Air Sampling Associates \\
\hline Analyst & KEH \\
\hline Parameters & HPLC/IC Analysis \\
\hline \# Samples & 3 Runs \& 1 blank \\
\hline
\end{tabular}

\begin{tabular}{|r|l|}
\hline Client \# & $07-057$ \\
\hline Job \# & $0907-56$ \\
\hline PO \# & Verbal \\
\hline Report Date & September 27, 2007 \\
\hline
\end{tabular}

Custody Erin Ortiz of Enthalpy Analytical, Inc. received the samples on 9/13/2007 at $23.4^{\circ} \mathrm{C}$ after being relinquished by Air Sampling Associates. The blank was also received by Erin Ortiz, at a temperature of $17.4^{\circ} \mathrm{C}$ on $9 / 17 / 07$, after being relinquished by Air Sampling Associates. The samples were received in good condition. Prior to and during analysis, the samples were kept under lock with access only to authorized personnel by Enthalpy Analytical, Inc.

Analysis The samples were analyzed for sulfate using an Agilent Model 1100, High Performance Liquid Chromatograph ("Curly"). The analyzer was equipped as follows:

Front: DIONEX ED40 Electrochemical Detector

Front: dionex Ion Pac AS14A, 4 x 250 mm (S/N 005275)

Calibration The calibration curve(s) are located in the back of this report and referenced in the Analysis Method column on the Detailed Results page.

For each calibration curve used, the first page of the curve contains all method specific parameters (i.e., curve type, origin, weight, etc.) used to quantify the samples. The calibration curve section also includes a table with the Retention Time (RetTime), Level (Lvl), Amount (corresponding units), Area, Response Factor (Amt/Area) and the analyte Name. The calibration table is used to identify (by retention time) and quantify each target compound.

Chromatographic The acquisition method 0707-07.M is included in the Curve/QA Conditions Chromatograms section of this report.

Blanks

QC Notes

A blank sample (HPLC blank) was received and analyzed along with the samples. No blank corrections were made to any of the associated sample results.

A $0.500-\mathrm{mL}$ aliquot of $\boldsymbol{R}$ un $\mathbf{1}-\boldsymbol{C} \boldsymbol{C}$ was spiked with $1.25 \mu \mathrm{g}$ of sulfate $(50 \mu \mathrm{L}$ of a $50 \mu \mathrm{g} / \mathrm{mL}$ solution). The spiked sample was analyzed in the same manner as the samples and exhibited $101 \%$ recovery.

Reporting Notes None

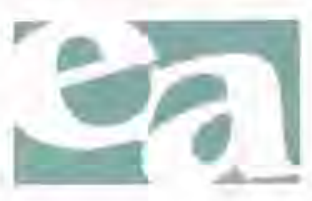




\section{General Reporting Notes}

The following are general reporting notes that are applicable to all Enthalpy Analytical, Inc. reports, unless specifically noted otherwise.

- The symbol $M D L$ represents the Minimum Detection Limit. Below this value the laboratory cannot confim the presence of the analyte of interest reliably.

- The symbol $L O Q$ represents the Limit of Quantification. Below this value the laboratory cannot quantitate the analyte of interest within the criteria of the method.

- The symbol ND following a value indicates a non-detect or analytical result below the MDL.

- The symbol $\boldsymbol{J}$ following a value indicates an analytical result between the MDL and the LOQ. A J flag indicates that the laboratory can positively identify the analyte of interest as present, but the value should be considered an estimate.

- The symbol $\boldsymbol{E}$ following a value indicates an analytical result exceeding $100 \%$ of the highest calibration point.

- The symbol $\boldsymbol{D F}$ represents a Dilution Factor. This number represents dilutions during the extraction and/or laboratory stages of sample treatment. The analytical result taken from a laboratory instrument is multiplied by the DF to get final results.

- The Sample ID $M S$ represents a Matrix Spike. An aliquot of an actual sample is spiked with a known amount of analyte so that a percent recovery value can be determined. This shows what effect the sample matrix may have on the target analyte, i.e. whether or not anything in the sample matrix prohibits analysis for the analyte(s).

- The Sample ID MSD represents a Matrix Spike Duplicate. Prepared in the same manner as an MS, the use of duplicate matrix spikes allows further confirmation of laboratory quality by showing the consistency of results gained by performing the same steps multiple times. Most methods performed by Enthalpy do not require analysis of an MSD.

- The Sample ID BS represents a Blind Spike. A member of the Quality Assurance department has created BS samples for many of the analytes Enthalpy tests for, and only QA and the Enthalpy Analytical ownership have access to the actual values of these samples. The laboratory analyzes them without knowledge of the actual value, and the spreadsheets get completed for these samples solely by the QA group.

- The Sample ID LCS represents a Laboratory Control Sample. Whenever spikes are prepared for our clients more spikes are prepared than needed. The extras (randomly chosen) are kept in-house at the appropriate temperature conditions. When the spike samples come back from the client for analysis, the LCSs (usually two are saved) are analyzed to confirm that the analyte could be recovered from the media, separate from the spike samples which were used on the project and which may have had issues caused during collection and/or transport.

- Significant Figures: Where the reported value is much greater than unity (1.00) in the units expressed (specifically values of 1,000 or greater), the number is rounded to a whole number of units, rather than to 3 significant figures. For example, a value of $10,456.45$ ug catch is rounded to 10,456 ug. There are five significant digits reported, but no confidence should be placed on more than three significant digits.

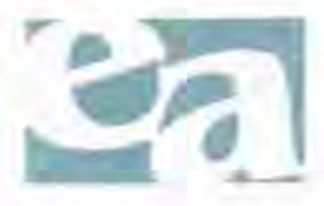


Sample Custody

Page 10 of 53 


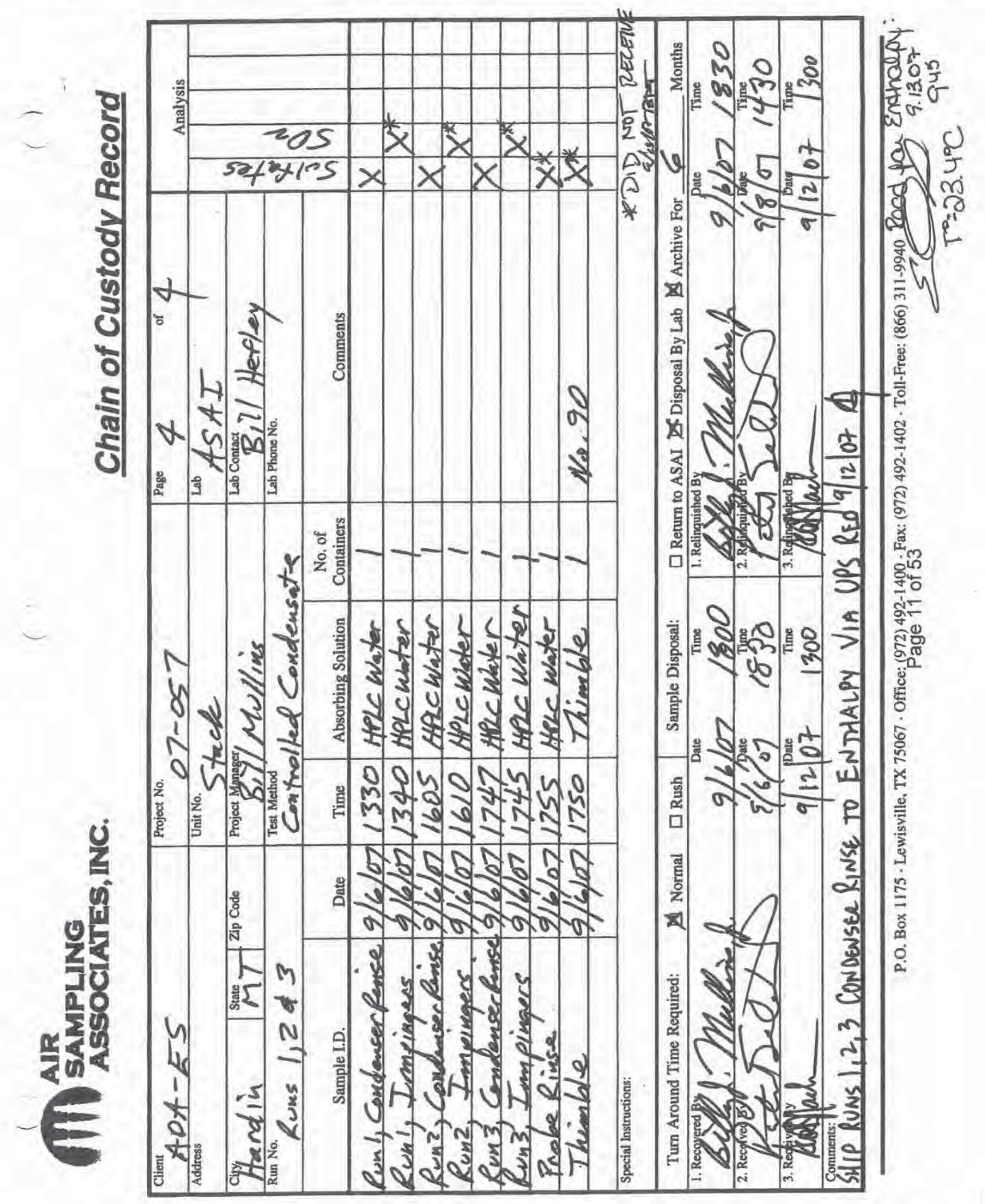




\section{CHAIN OF CUSTODY}

$\begin{array}{ll}\text { Project \#: } & \frac{07-057}{\text { Client: }} \\ \text { Location: } & \frac{\text { ADA-ES }}{\text { HAROIN,MT. }} \\ \text { Unit: } & \text { STACK }\end{array}$

Date: $9 / 13 / 07$

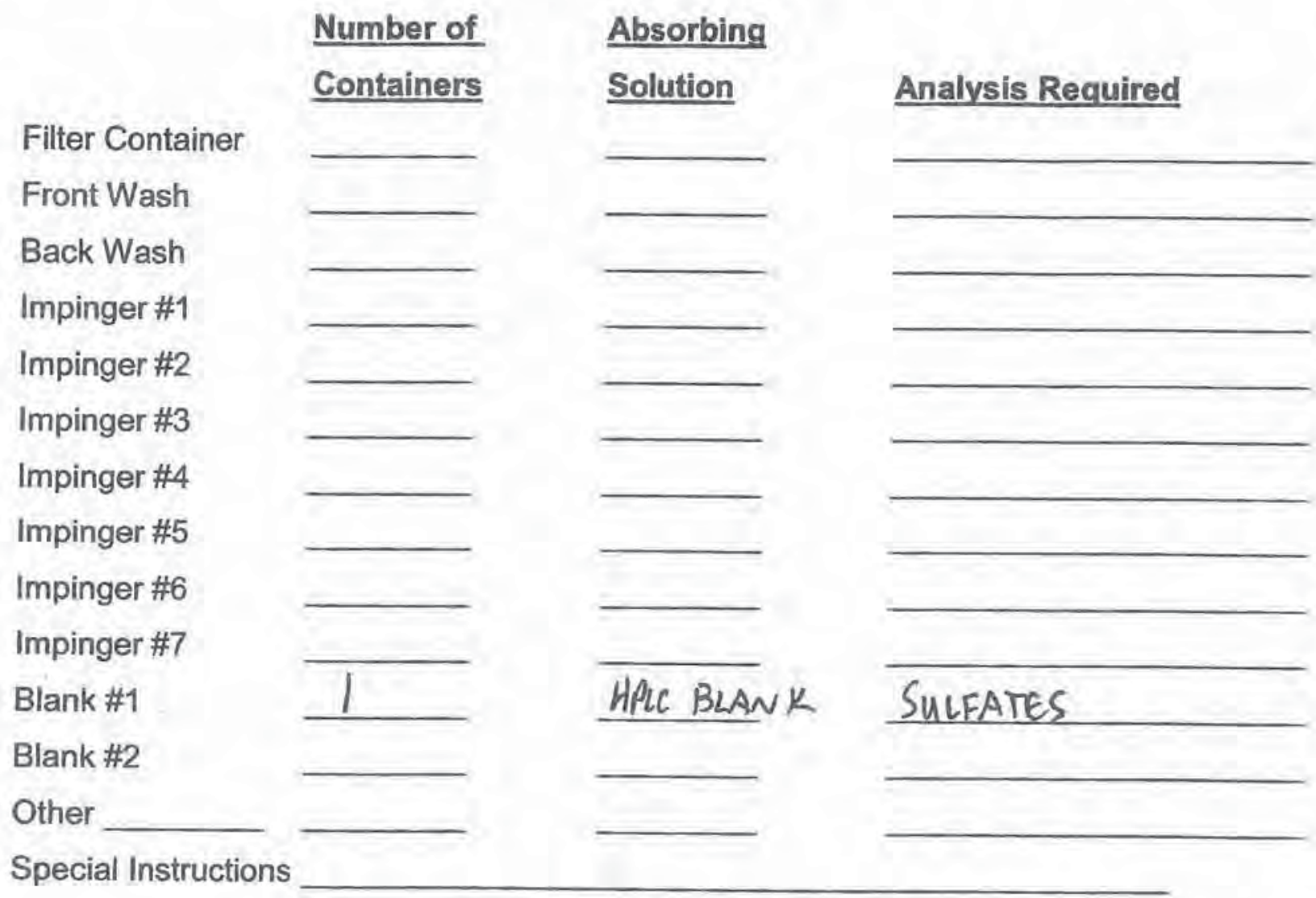

Sample \# I Recovered by Dod pach Date 9/13/07 Time 1115 Location ASA I Sample \#__Recovered by____ Date Time__L_Lation

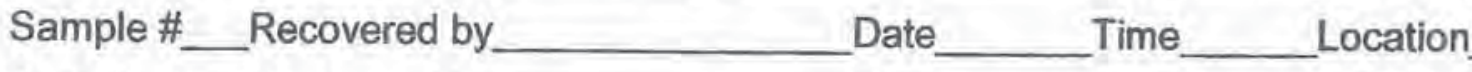

Sample \#__Recovered by____ Date___ Time___Location

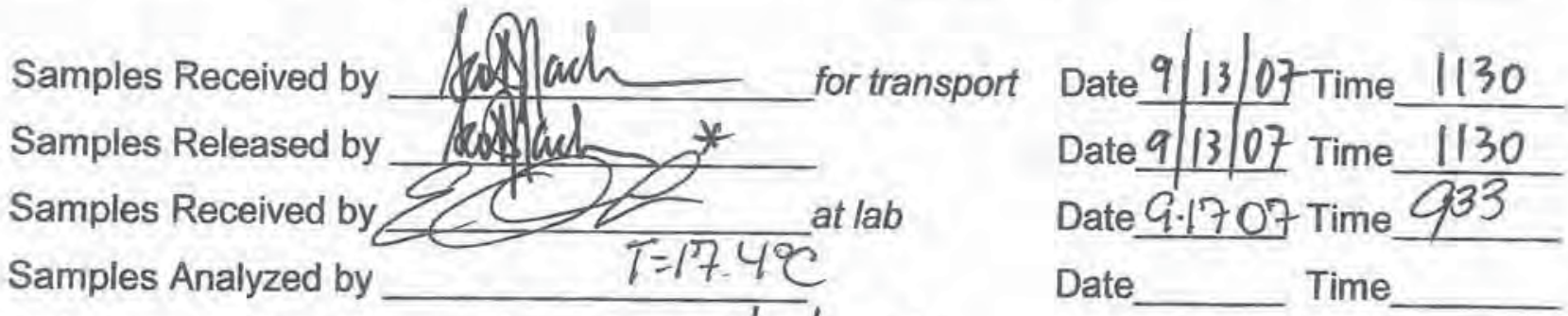

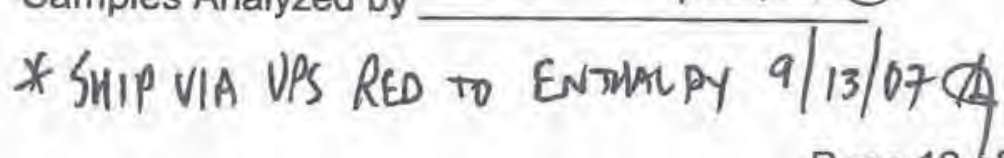




\section{Sample Chromatograms}

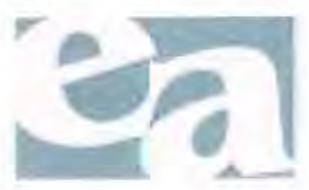

Page 13 of 53 
$0907-56$

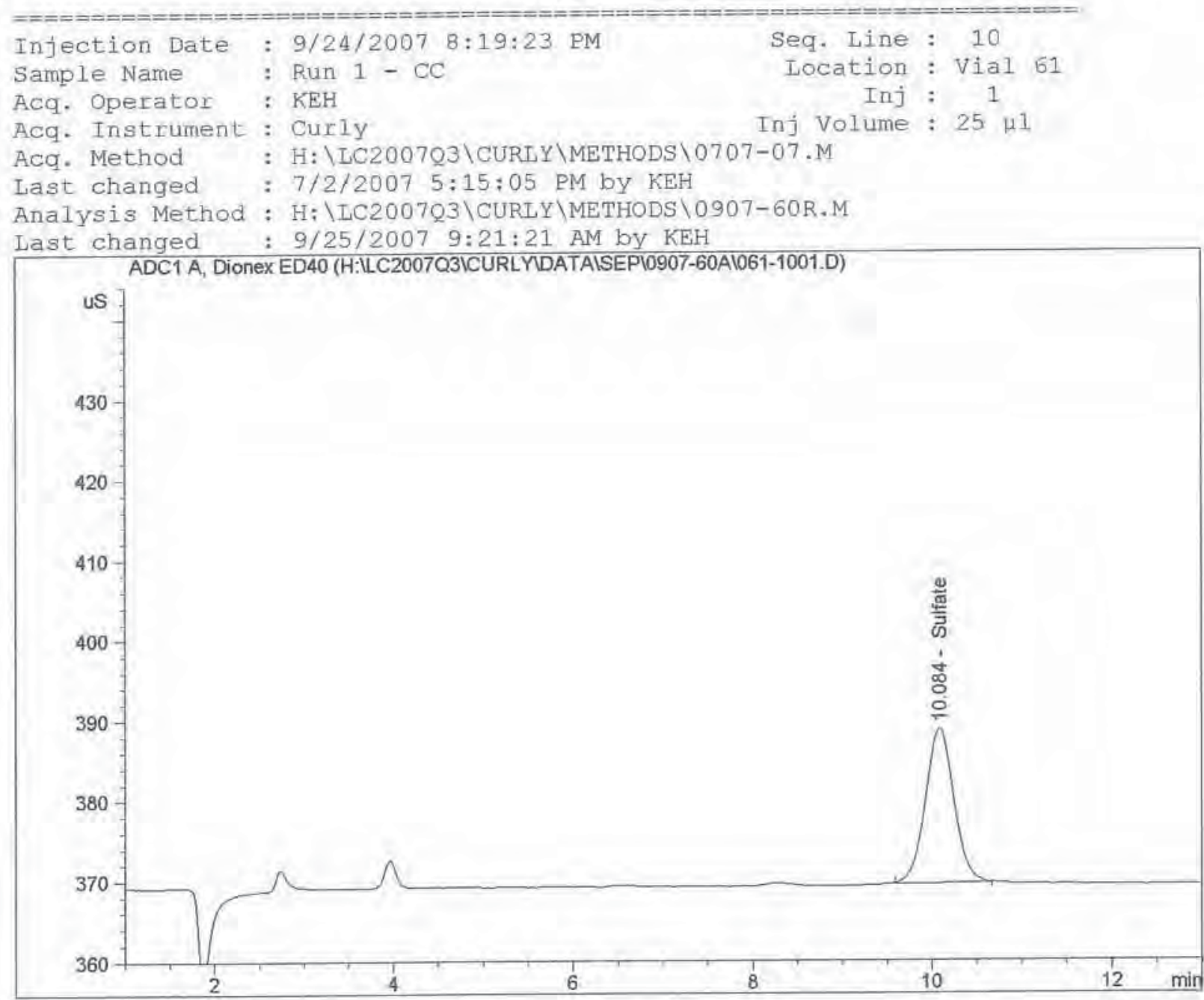

\section{External Standard Report}

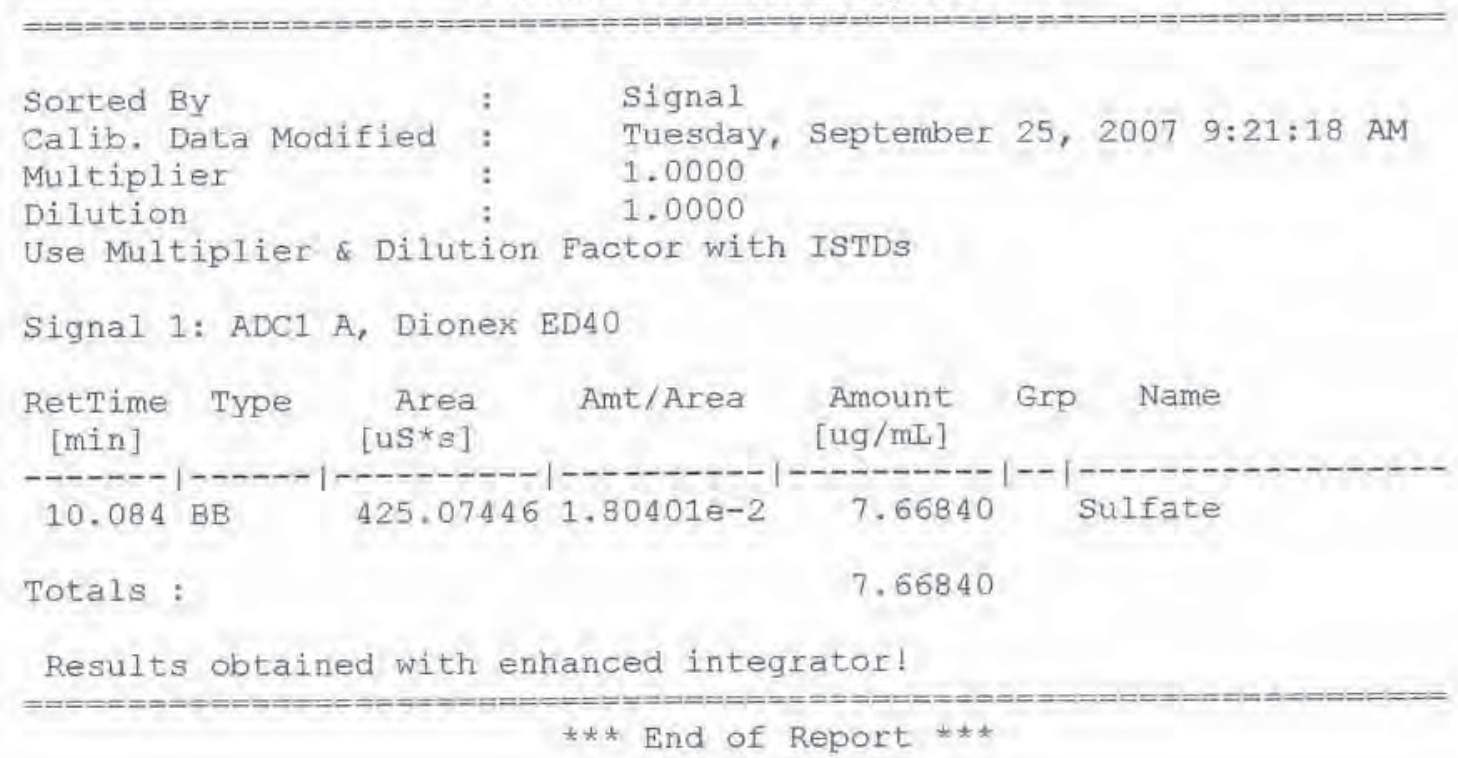

Page 14 of 53 
$0907-56$

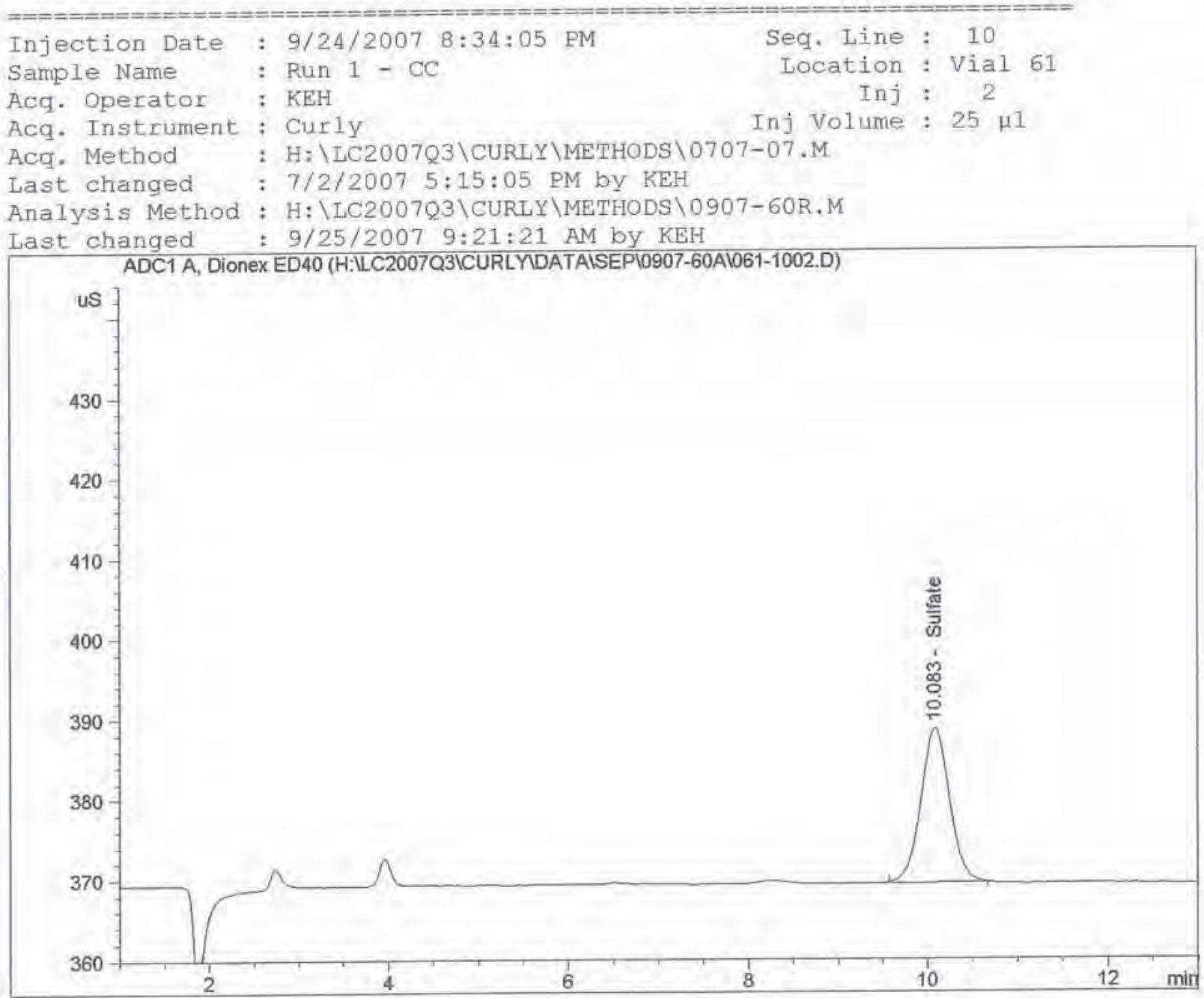

\section{External Standard Report}

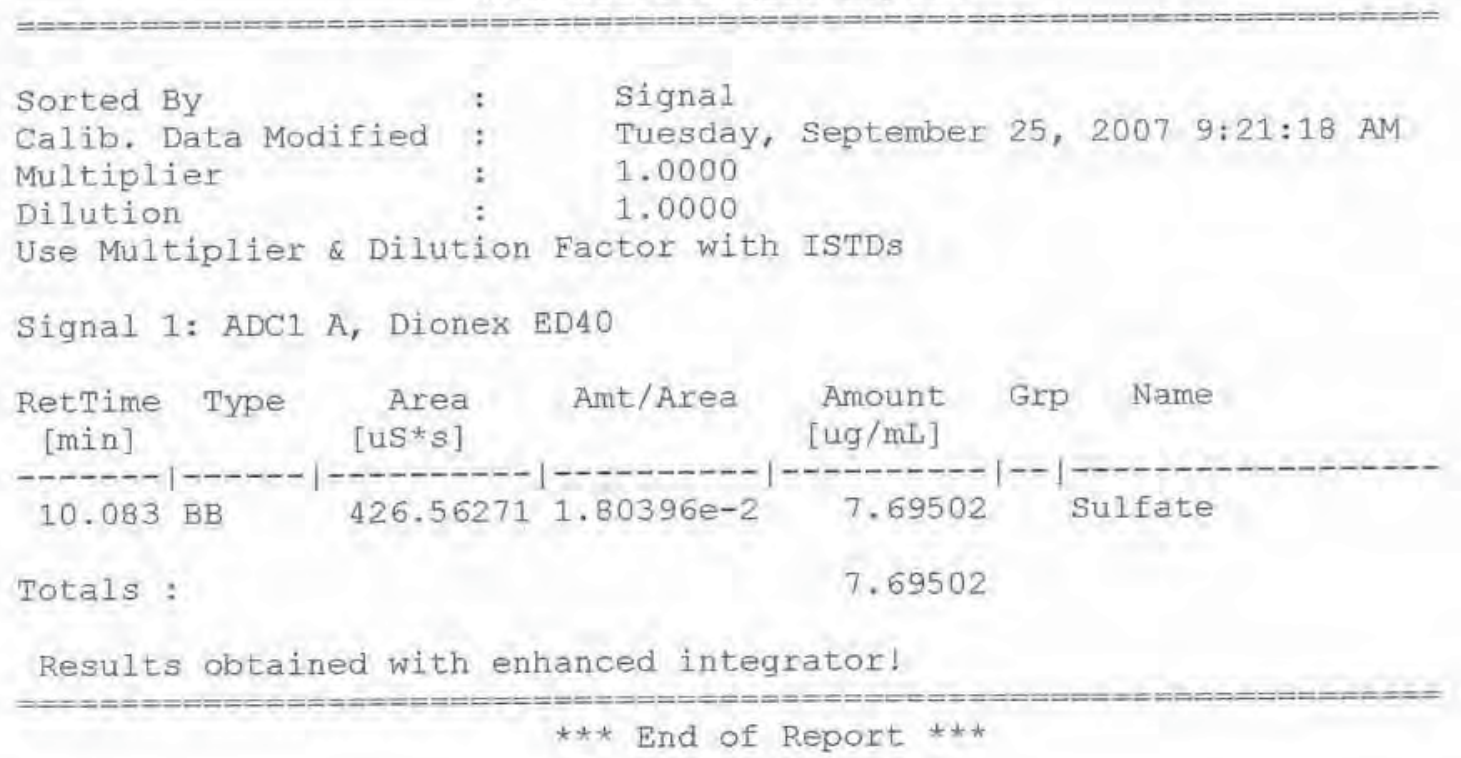




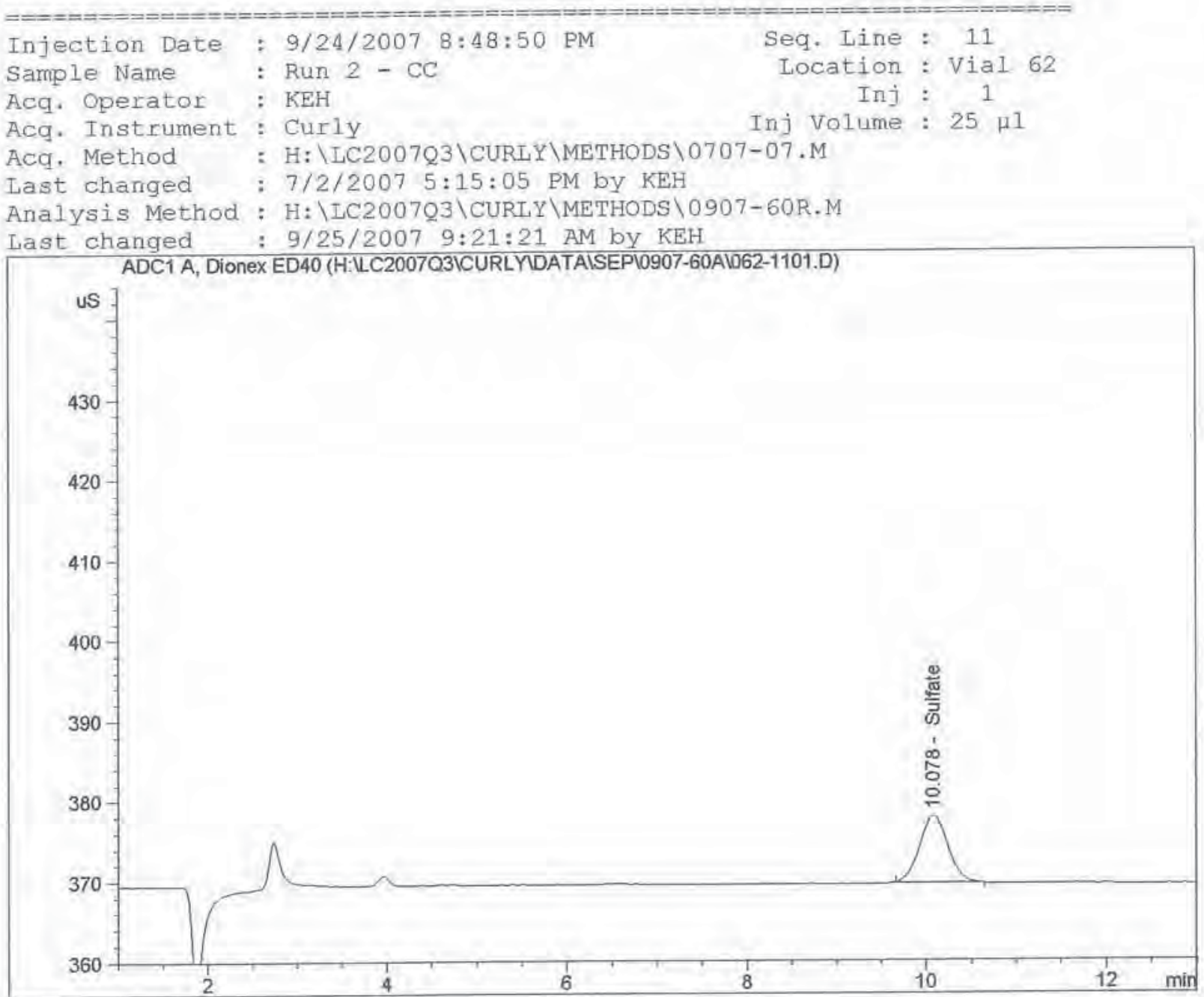

External Standard Report

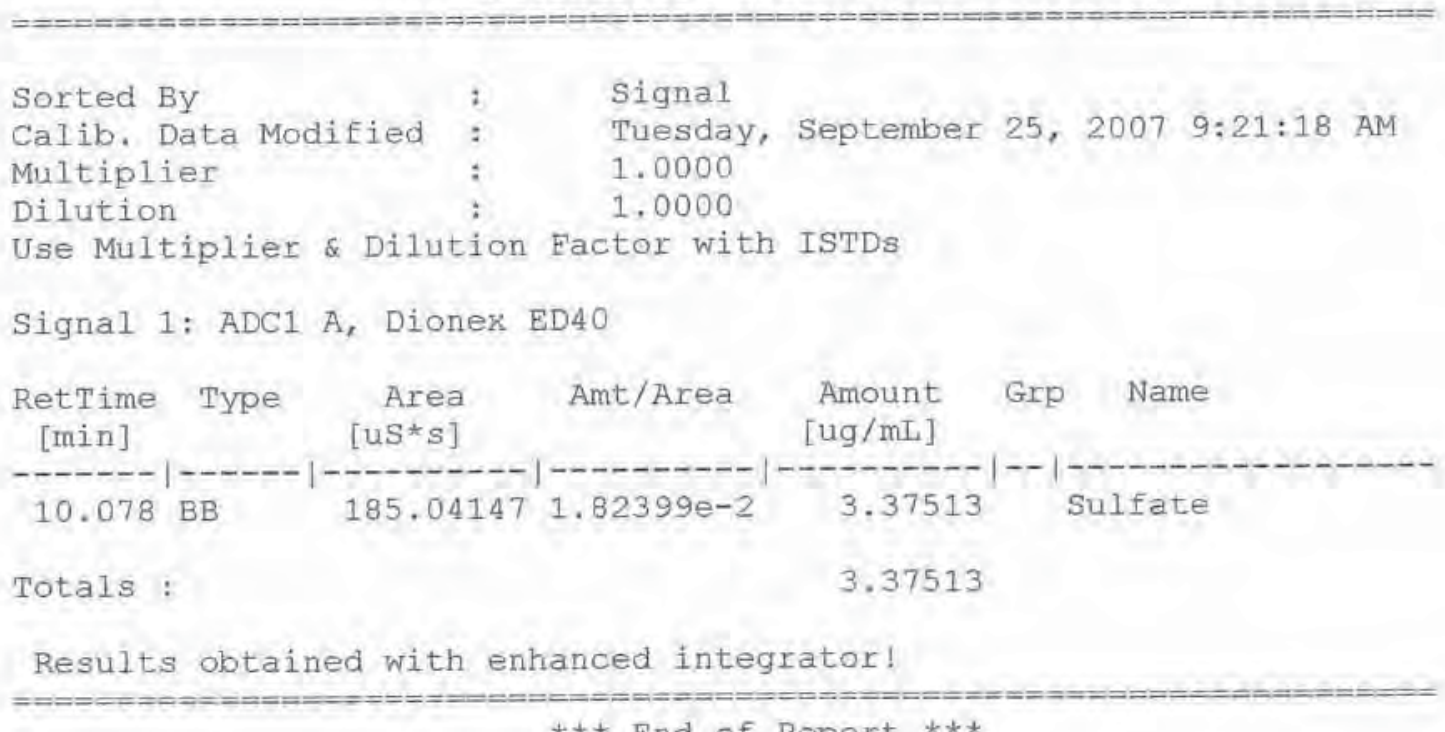




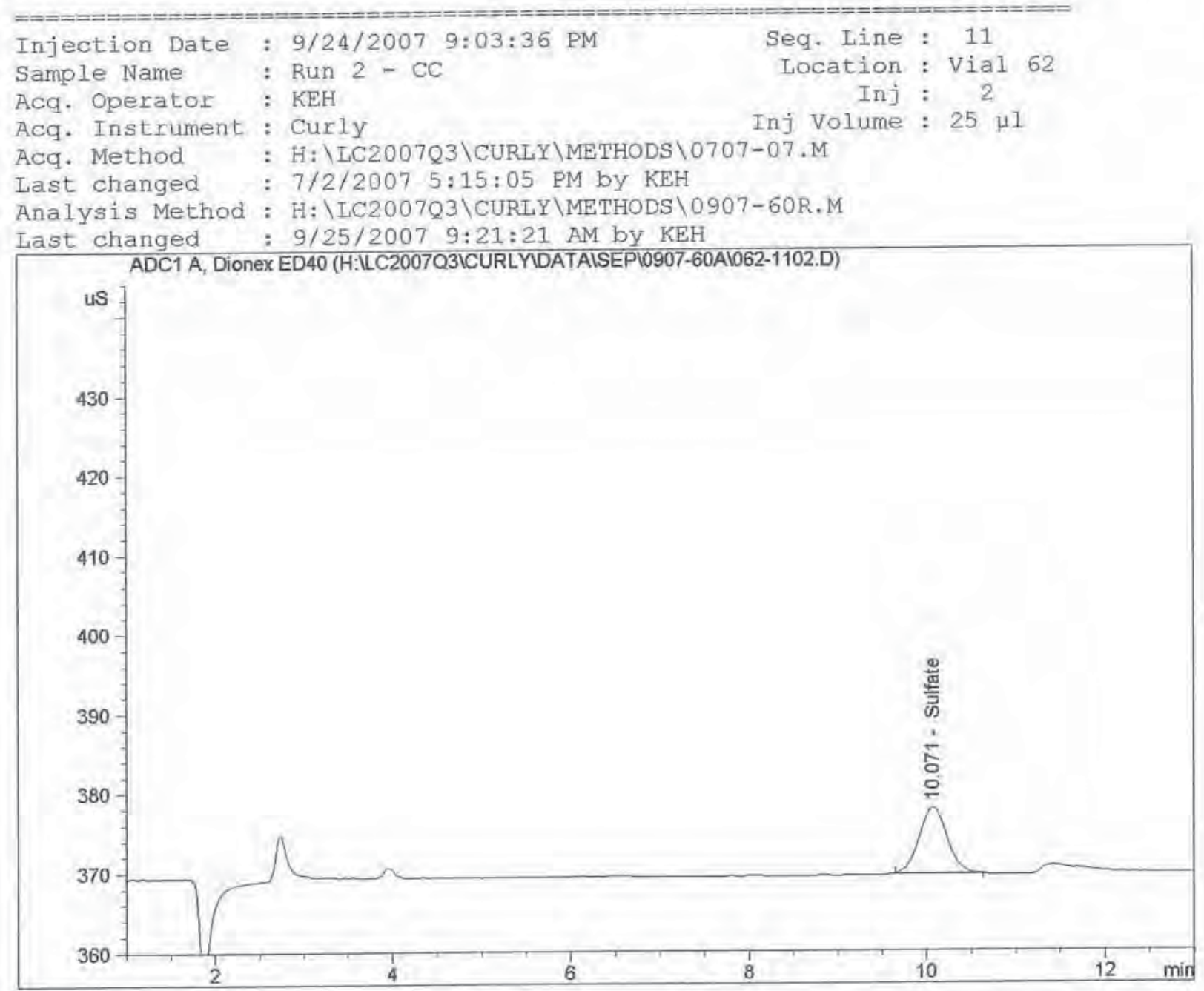

\section{External Standard Report}

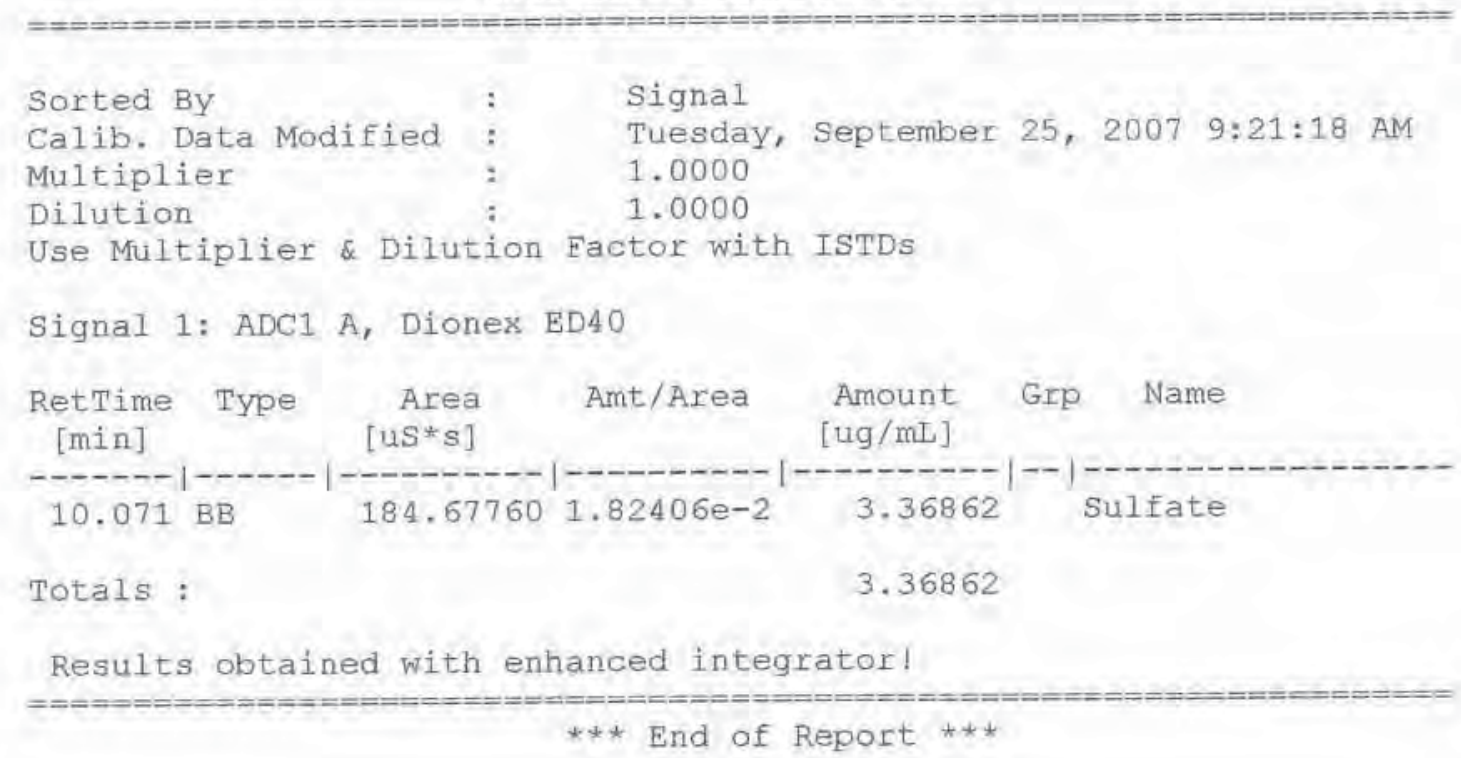

\section{Page 17 of 53}


$0907-56$

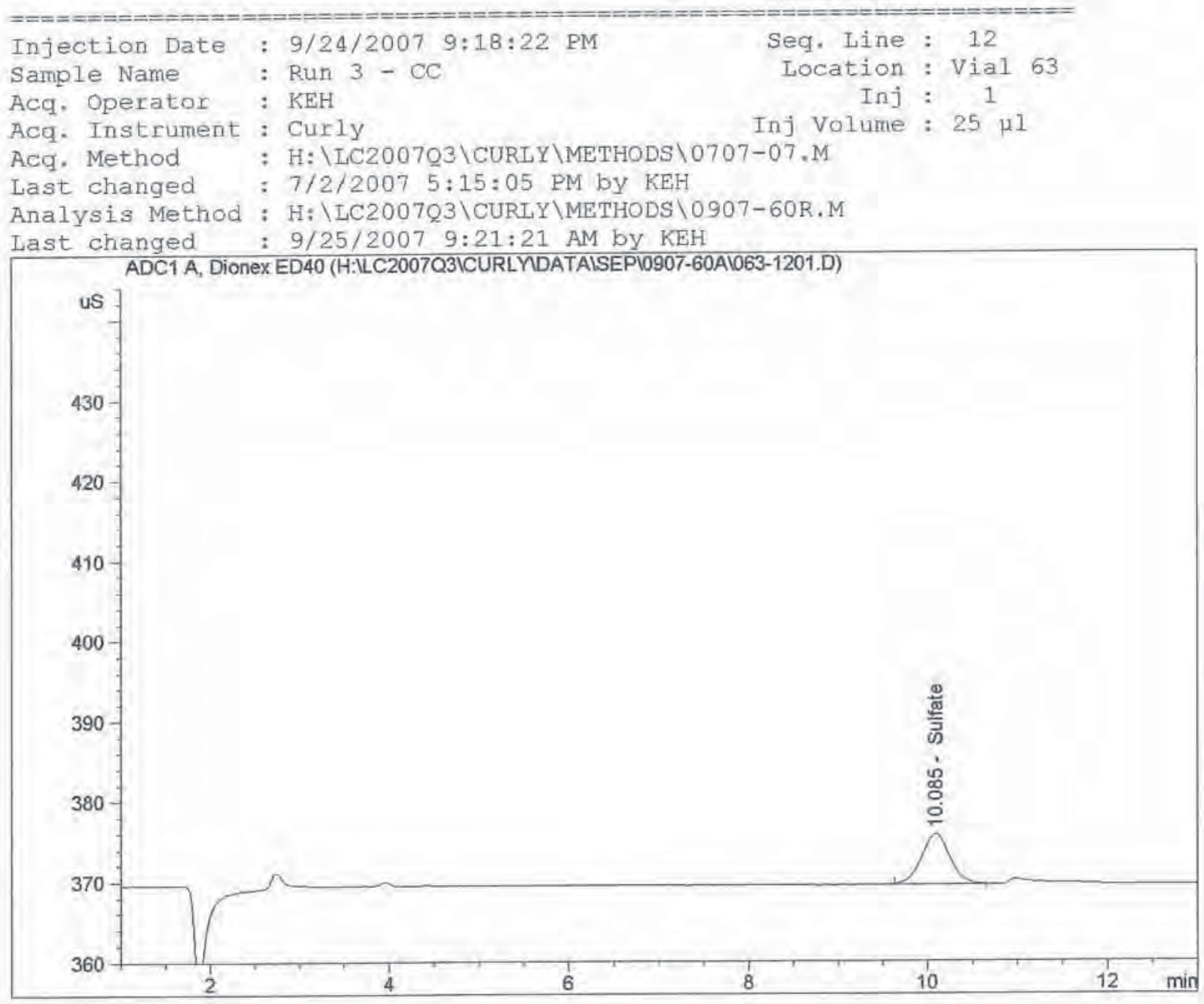

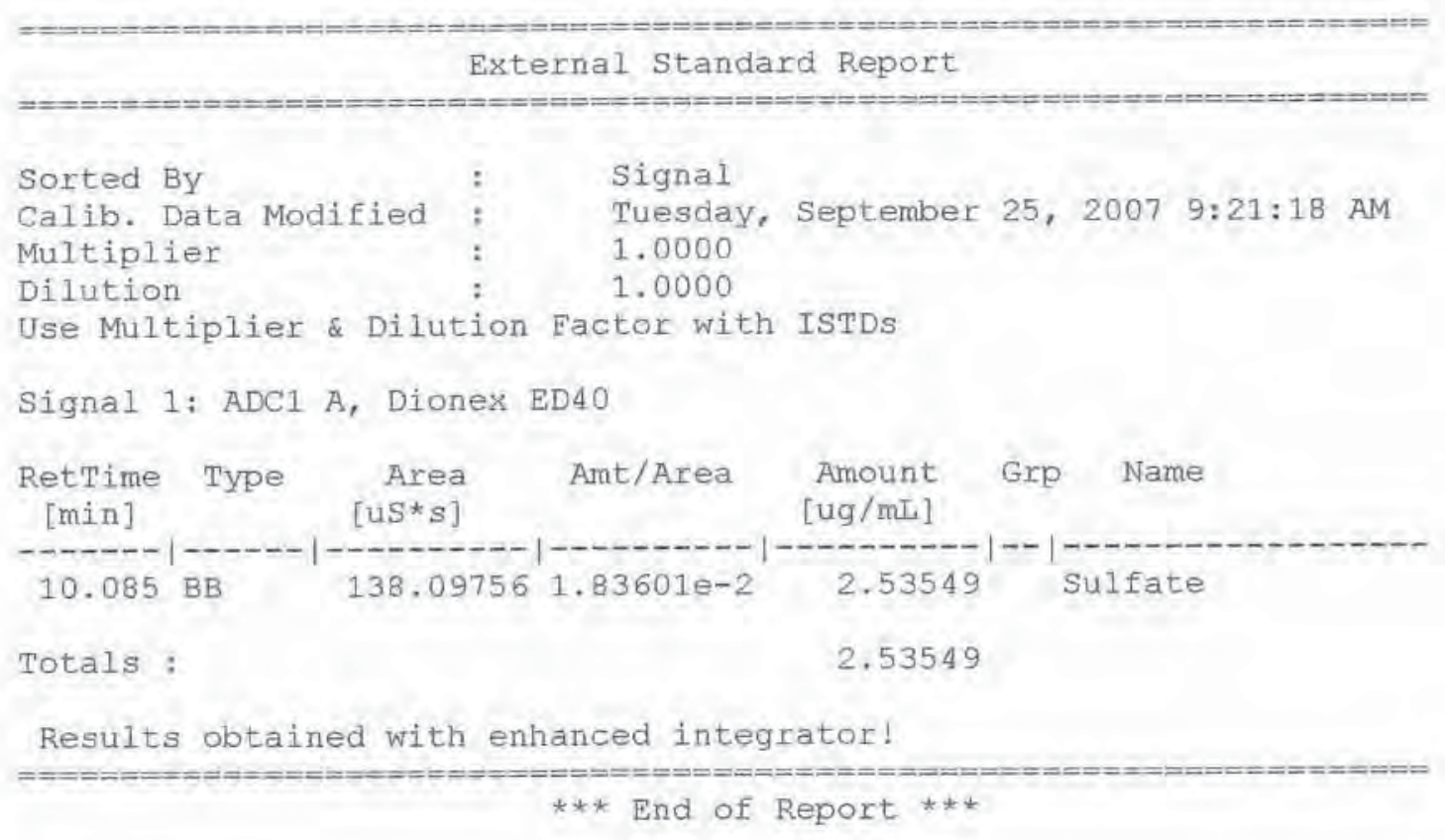

Page 18 of 53 
$0907-56$

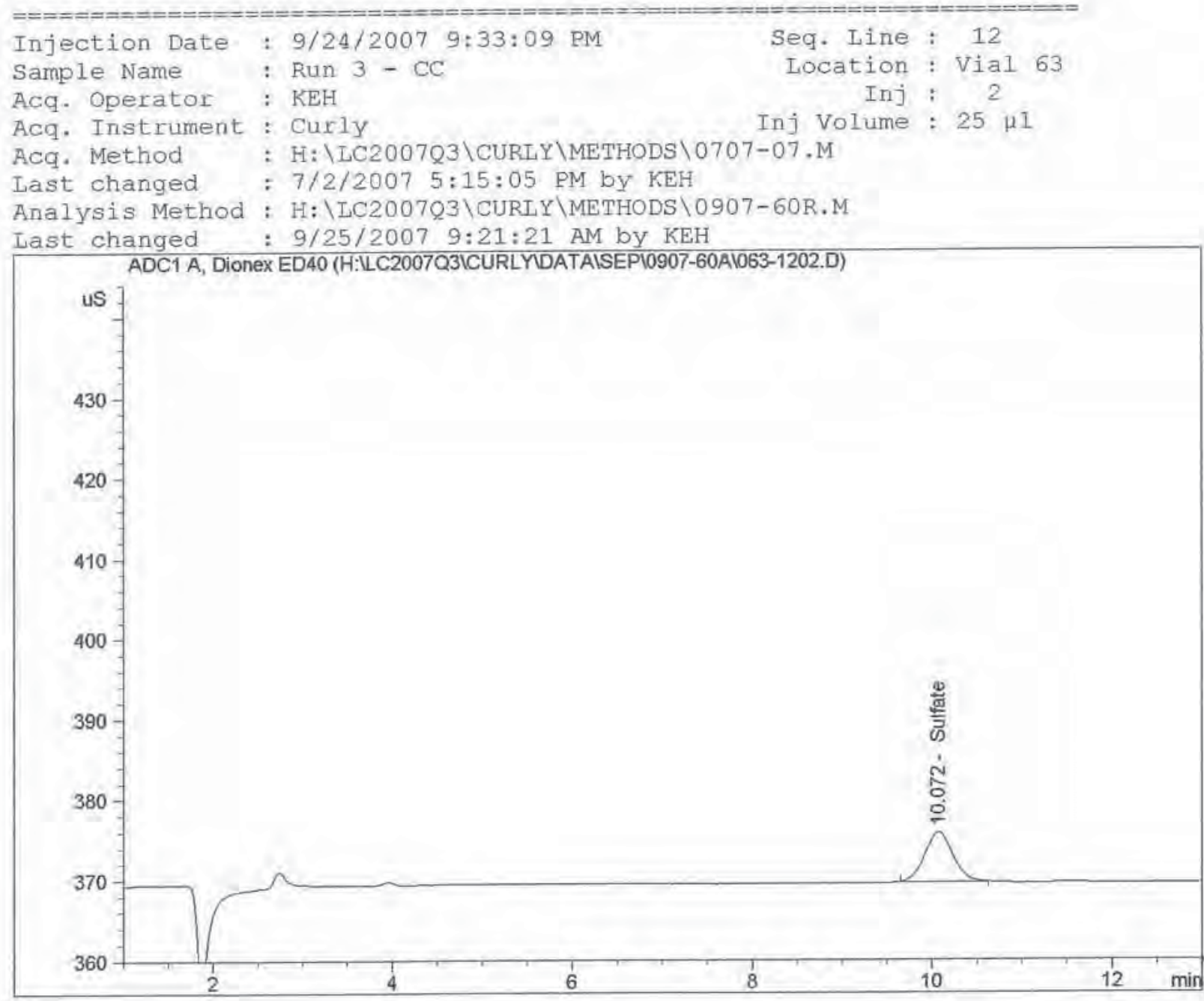

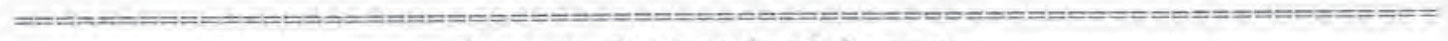

External Standard Report

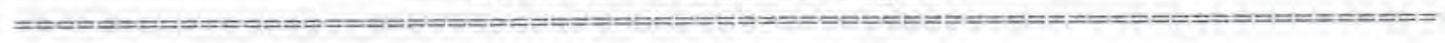

$\begin{array}{lll}\text { Sorted By } & : & \text { Signal } \\ \text { Calib. Data Modified } & : & \text { Tuesday, September 25, } 2007 \text { 9:21:18 AM } \\ \text { Multiplier } & : & 1.0000 \\ \text { Dilution } & \vdots & 1.0000\end{array}$

Use Multiplier \& Dilution Factor with ISTDS

Signal 1: ADC1 A, Dionex ED40

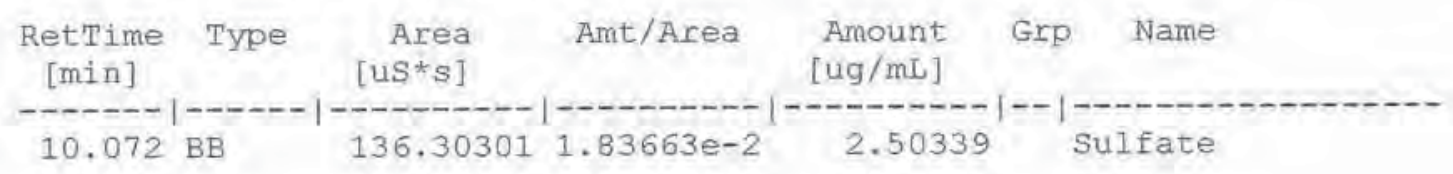

Totals: $\quad 2.50339$

Results obtained with enhanced integrator!

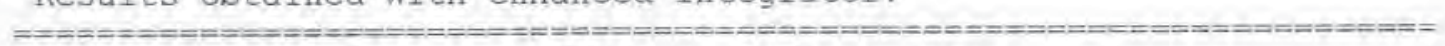

*** End of Report $\star * \star$
} 


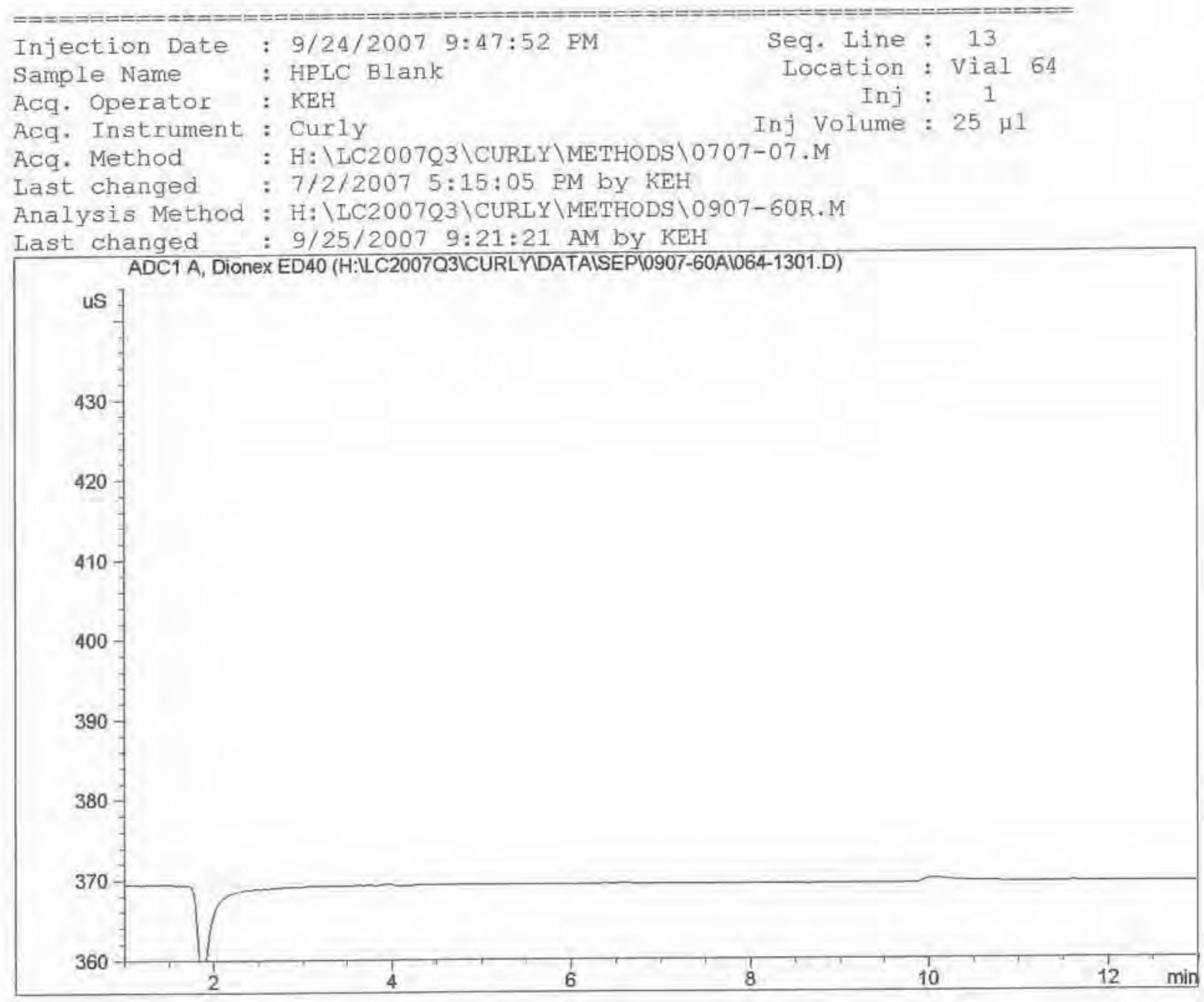

\section{External Standard Report}

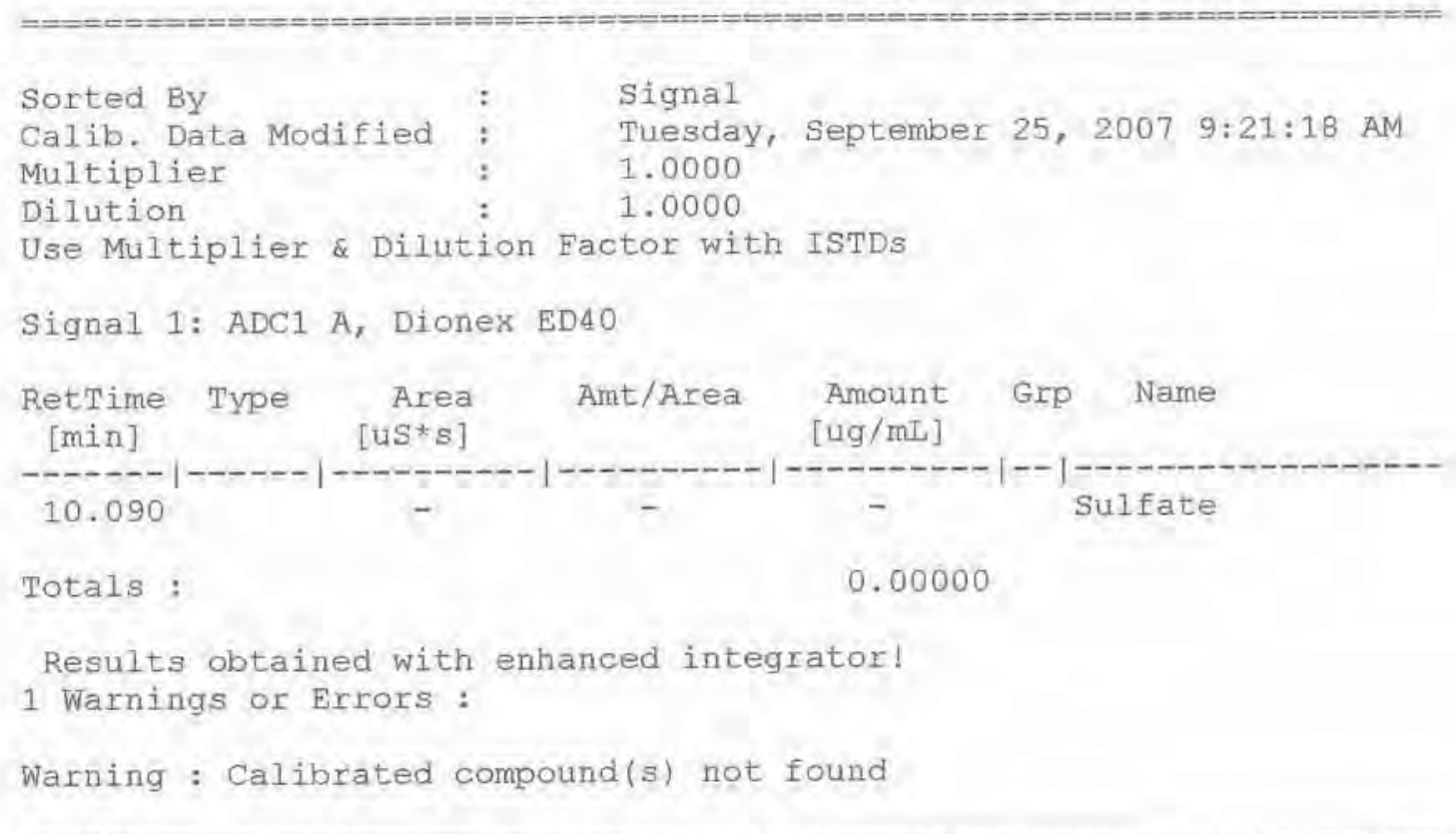




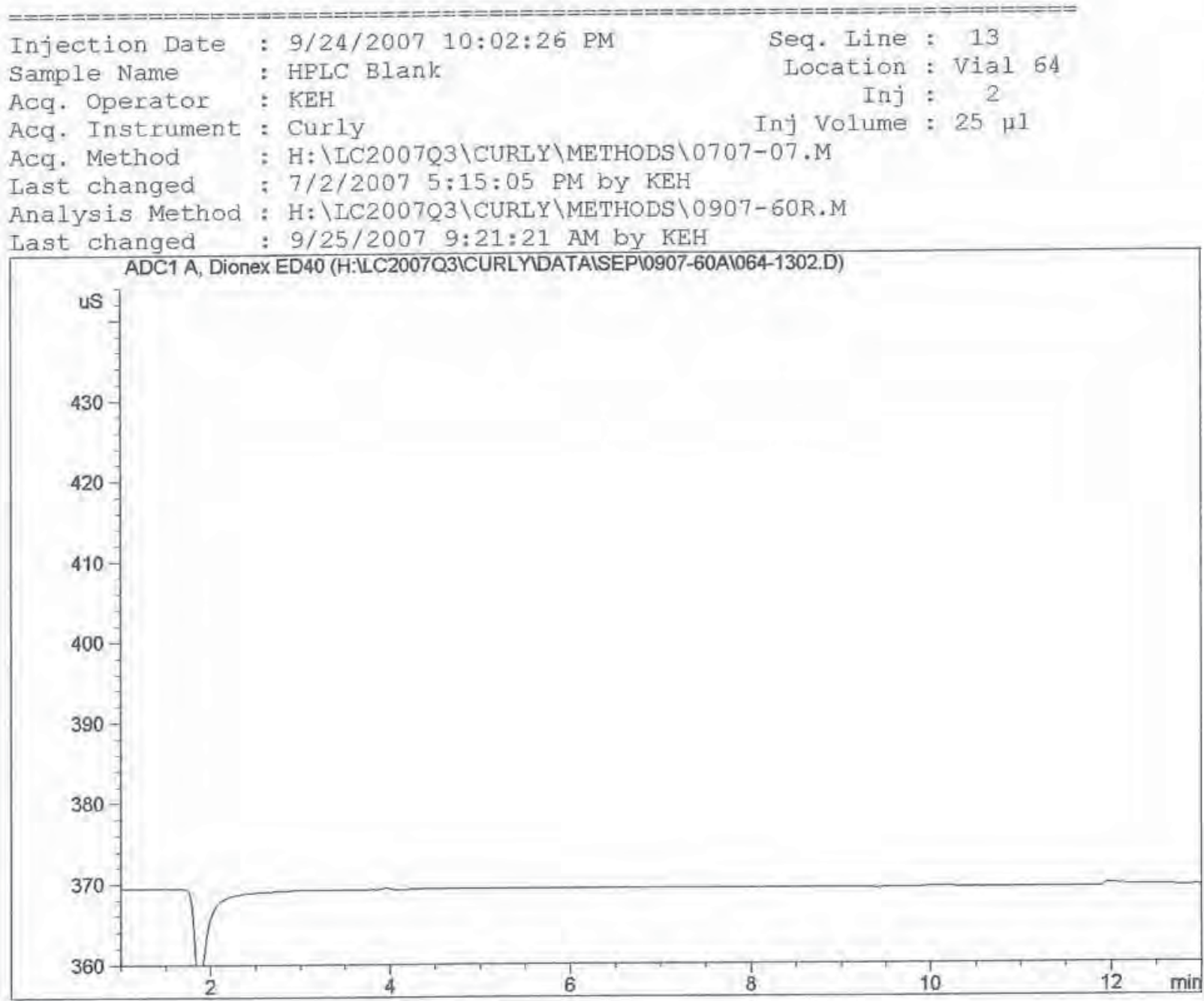

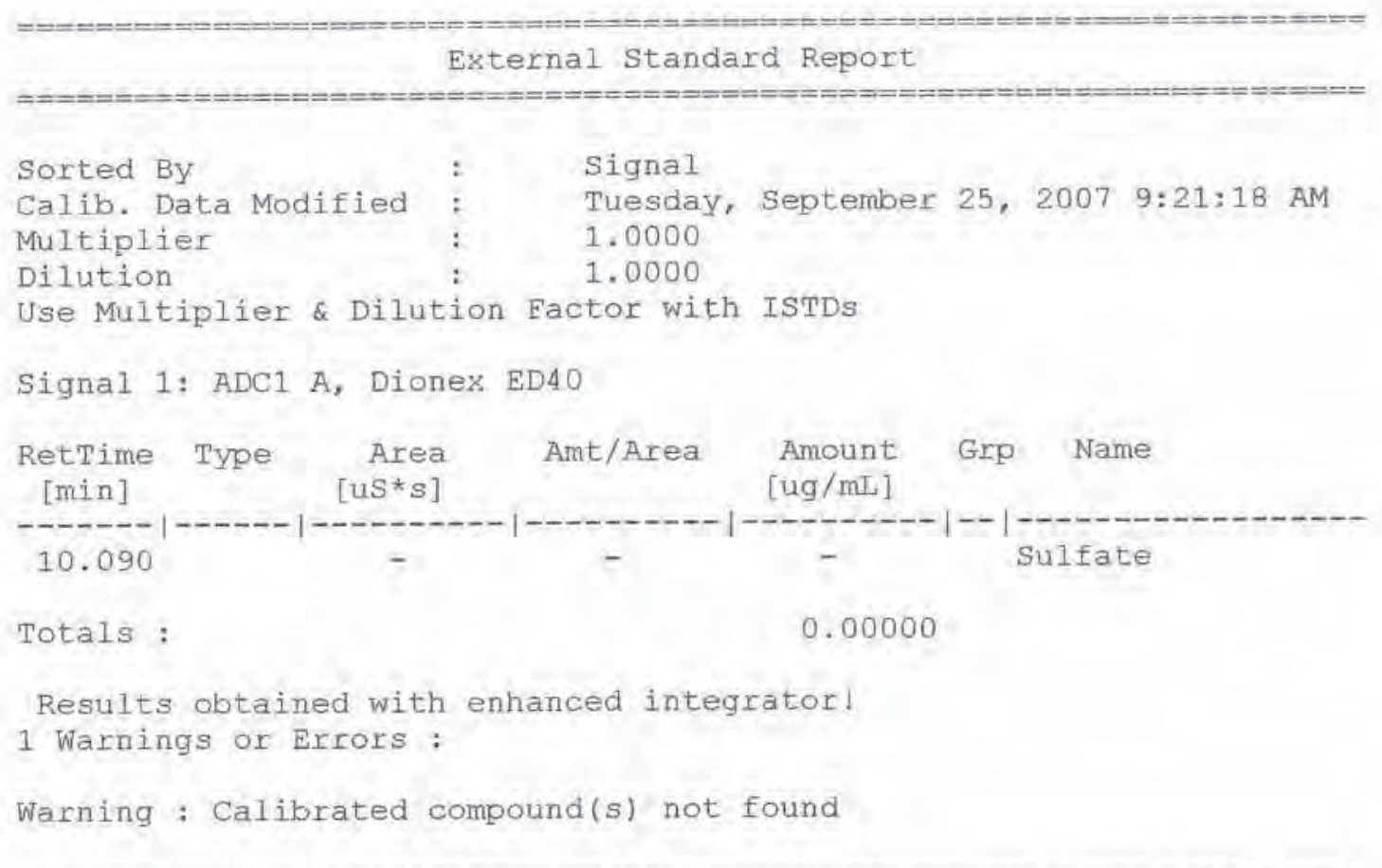




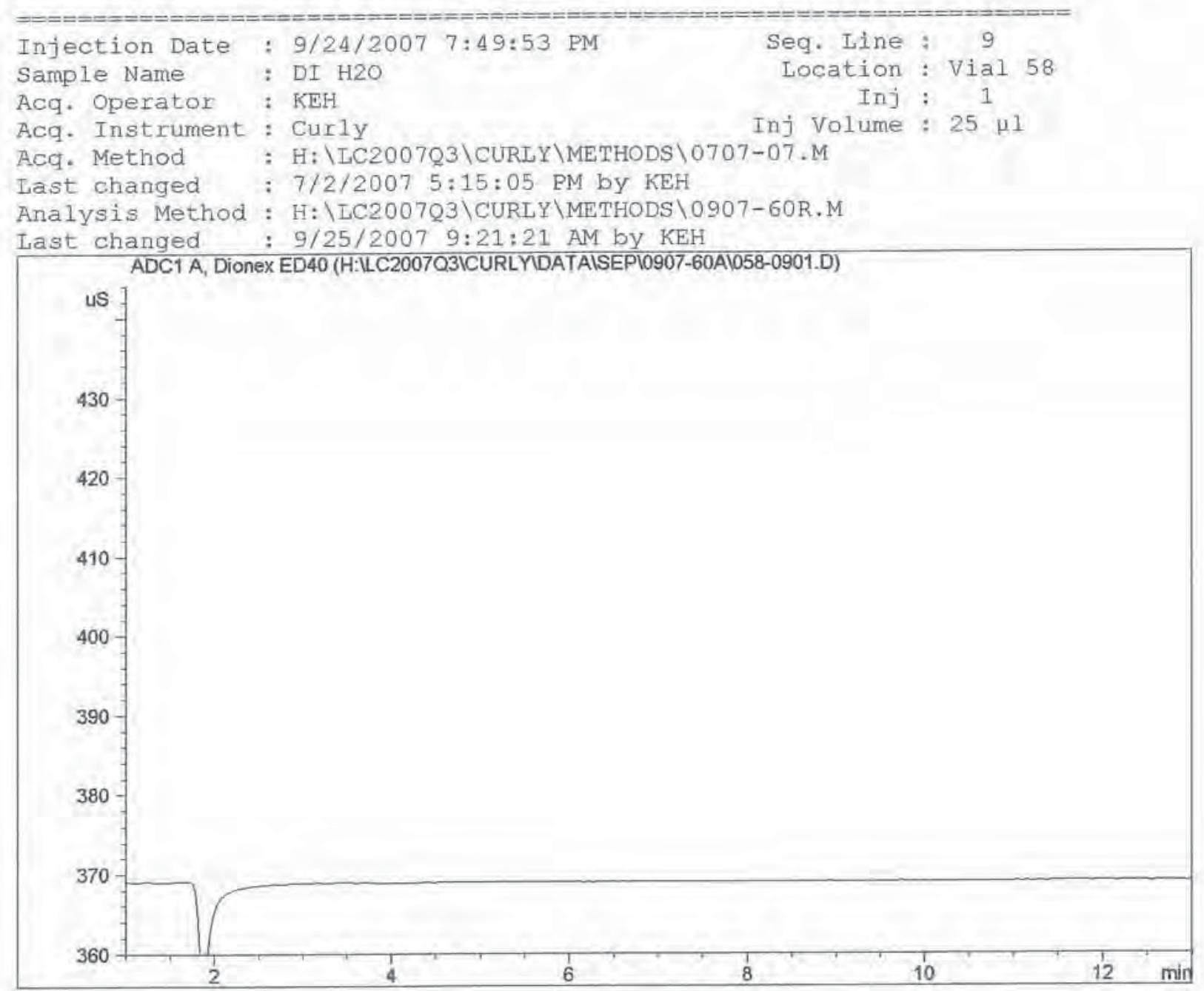

External Standard Report

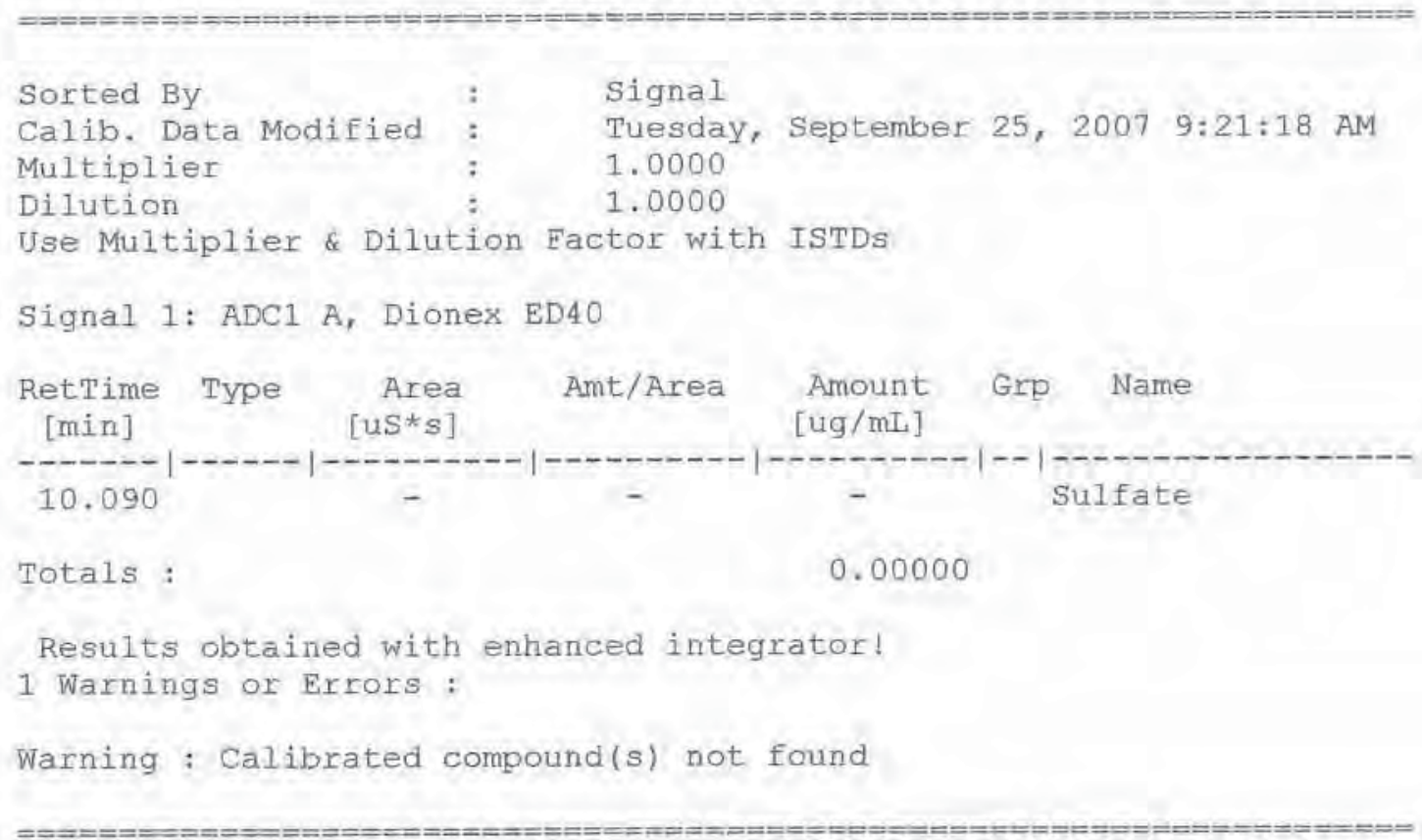




\section{Iab Blank DI H2O}

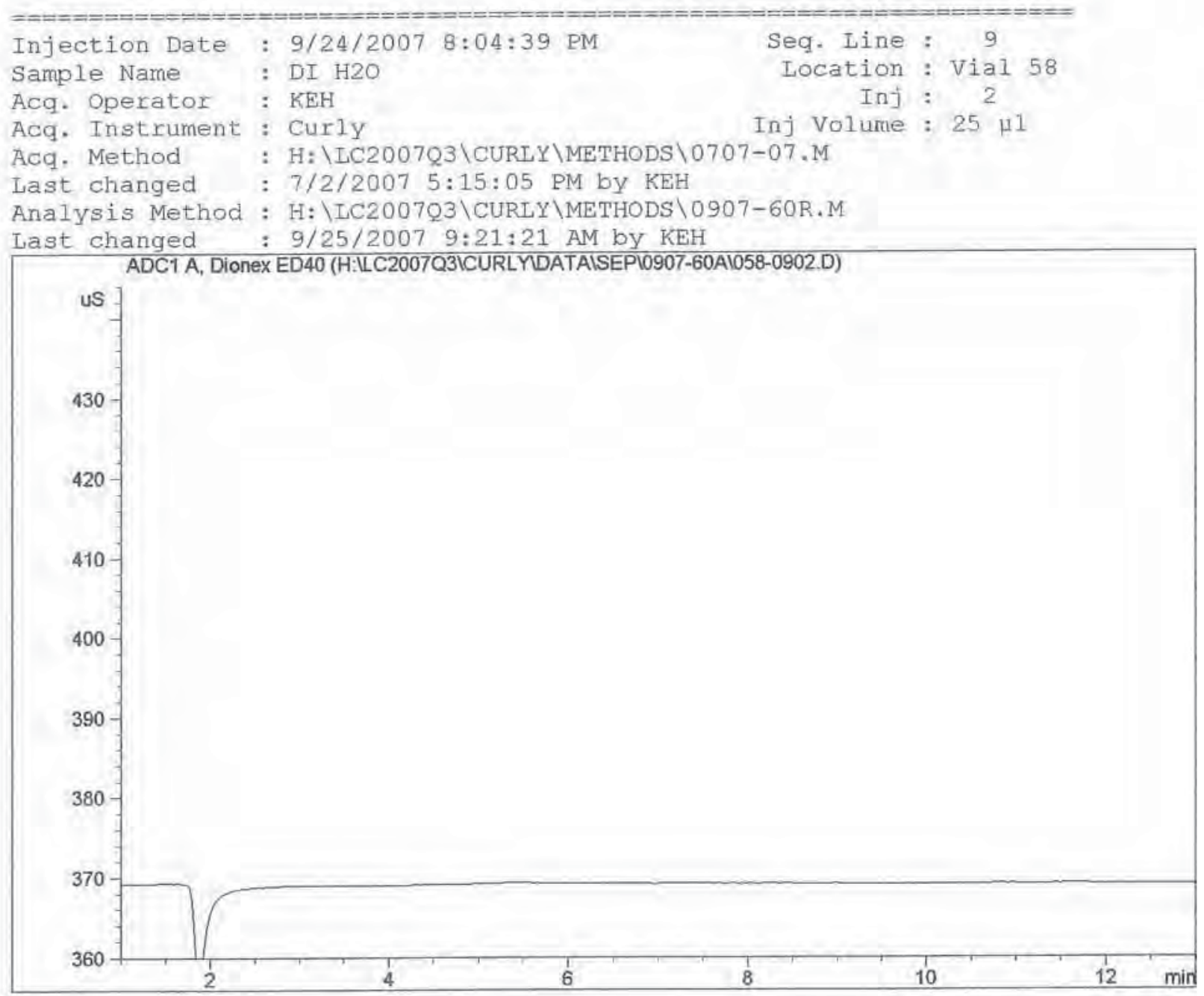

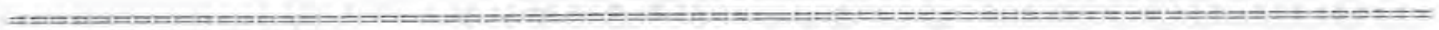

External standard Report

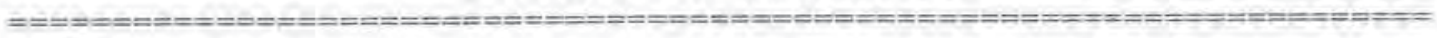

$\begin{array}{lcl}\text { Sorted By } & : & \text { Signal } \\ \text { Calib. Data Modified } & : & \text { Tuesday, September 25, 2007 9:21:18 AM } \\ \text { Multiplier } & \vdots & 1.0000 \\ \text { Dilution } & 1.0000 \\ \text { Use Multiplier \& Dilution Factor with ISTDs }\end{array}$

Use Multiplier \& Dilution Factor with ISTDs

Signal 1: ADC1 A, Dionex ED40

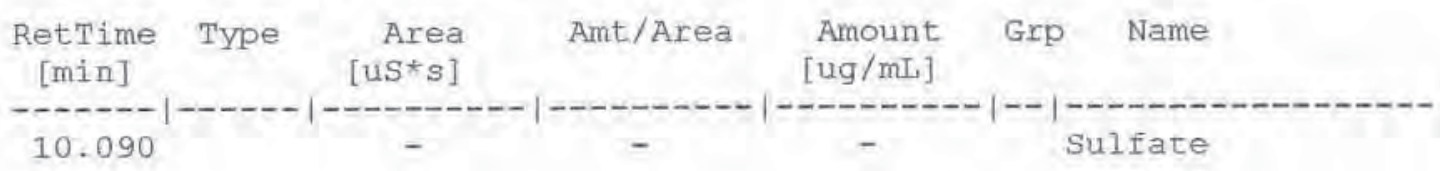

Totals : $\quad 0.00000$

Results obtained with enhanced integrator!

1 Warnings or Errors :

Warning : Calibrated compound(s) not found
} 



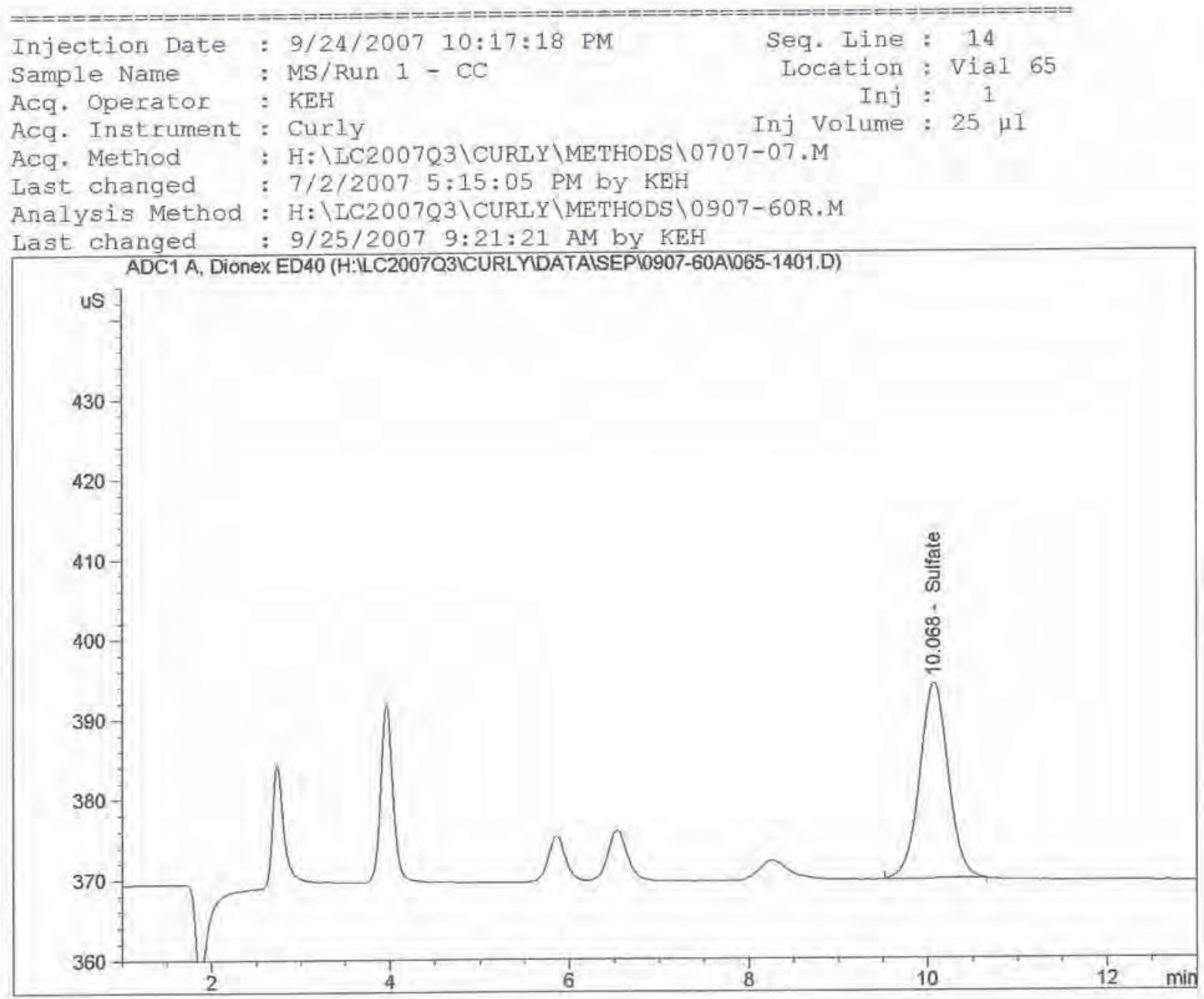

External Standard Report

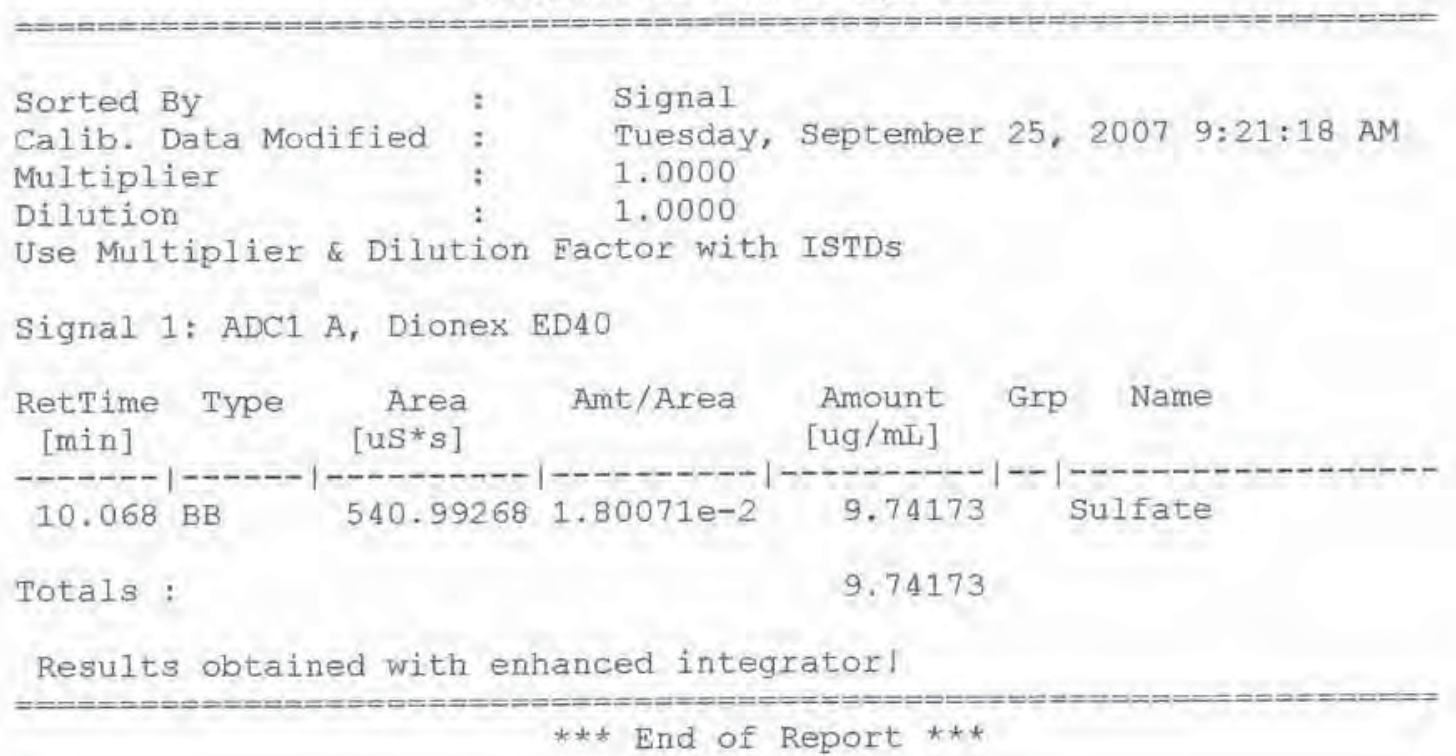




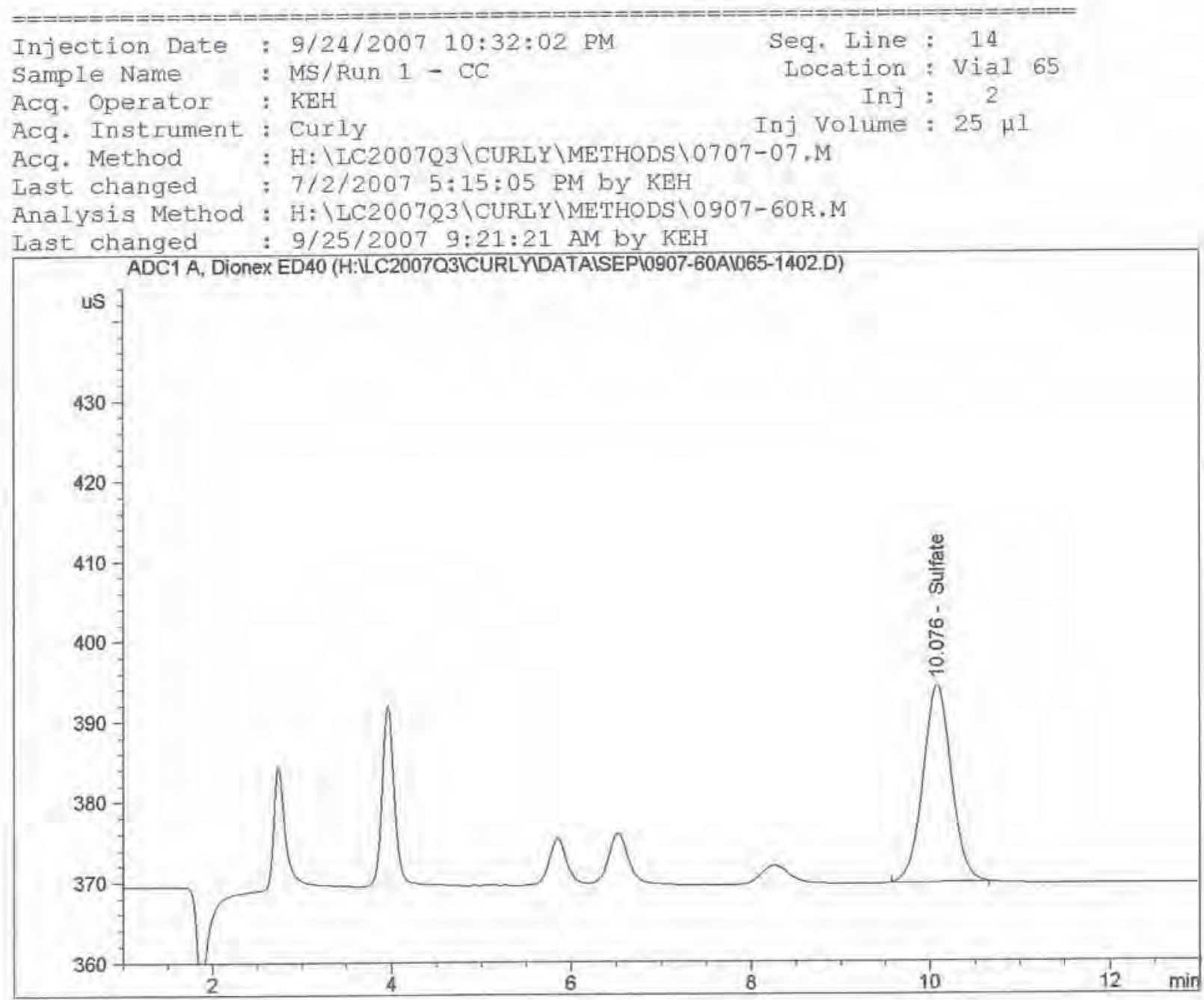

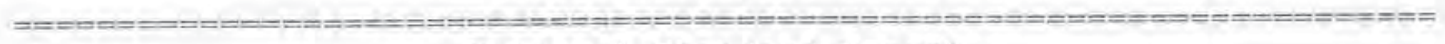

External Standard Report

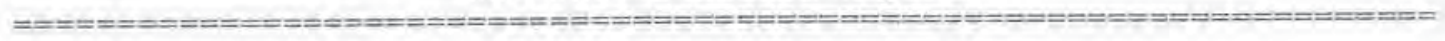

$\begin{array}{lll}\text { Sorted By } & : & \text { Signal } \\ \text { Calib. Data Modifled } & : & \text { Tuesday, September 25, } 2007 \text { 9:21:18 AM } \\ \text { Multiplier } & : & 1.0000 \\ \text { Dilution } & : & 1.0000\end{array}$

Use Multiplier \& Dilution Factor with ISTDs

Signal 1: ADC1 A, Dionex ED40

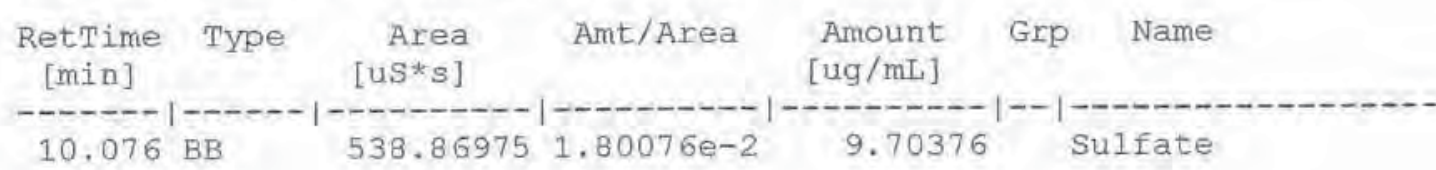

Totals :

9.70376

Results obtained with enhanced integrator!

$\star * \star$ End of Report $* \star *$
}

Page 25 of 53 


\section{Curve(s)/QA Point(s) Chromatograms}

Page 26 of 53 

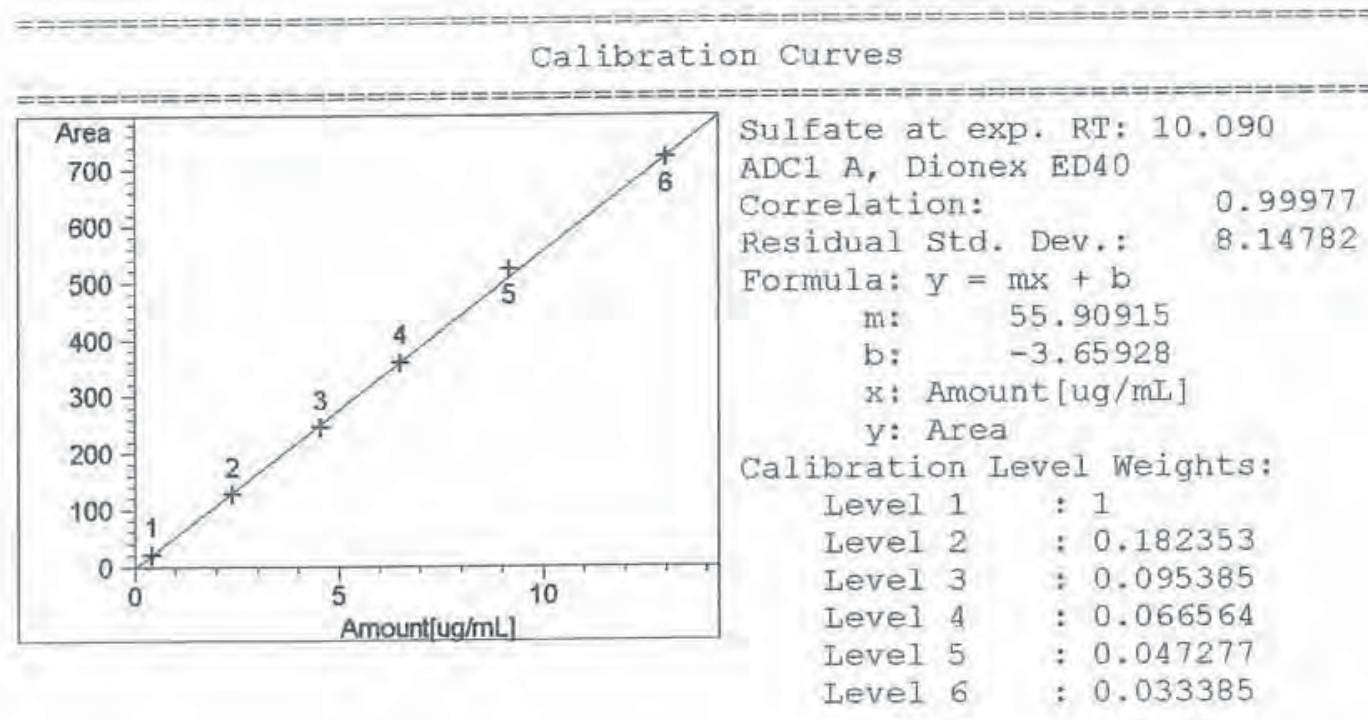


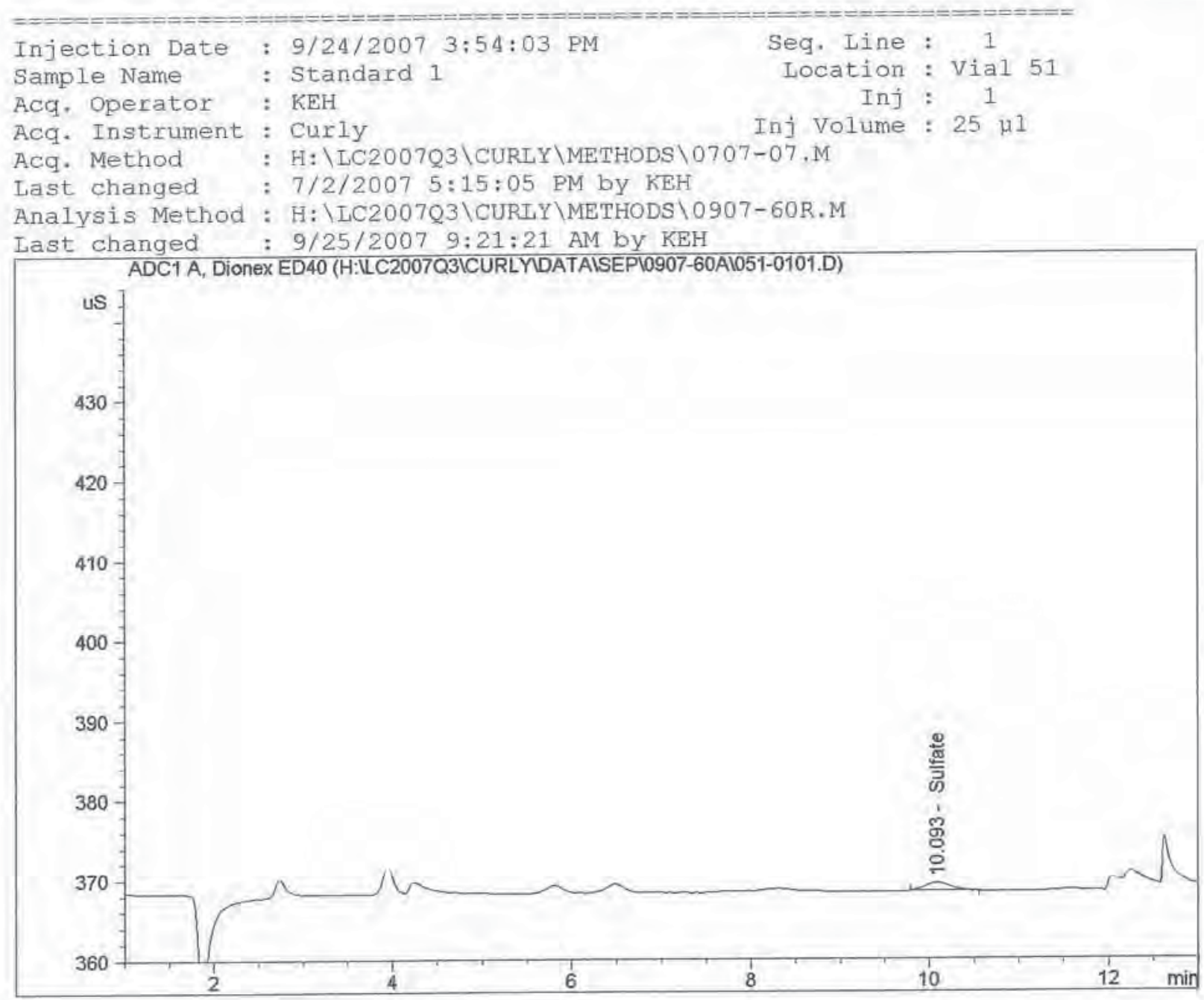

External Standard Report

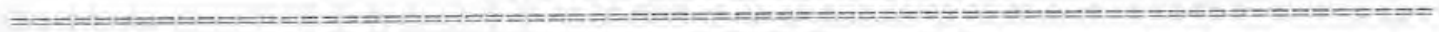

$\begin{array}{lll}\text { Sorted By } & : & \text { Signal } \\ \text { Calib. Data Modified } & : & \text { Tuesday, September 25, 2007 9:21:18 AM } \\ \text { Multiplier } & : & 1.0000 \\ \text { Dilution } & : & 1.0000\end{array}$

Use Multiplier \& Dilution Factor with ISTDS

Signal 1: ADC1 A, Dionex ED40

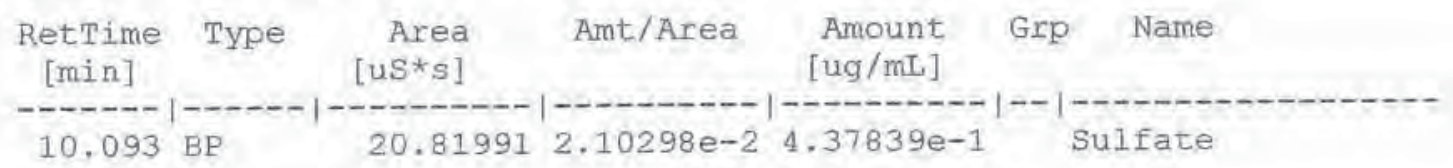

Totals: $\quad 4.37839 e-1$

Results obtained with enhanced integrator!

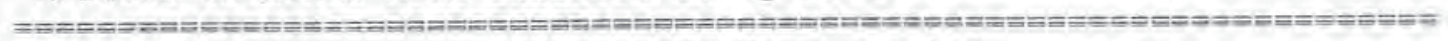

$\star \star *$ End of Report $* * *$

Page 29 of 53 


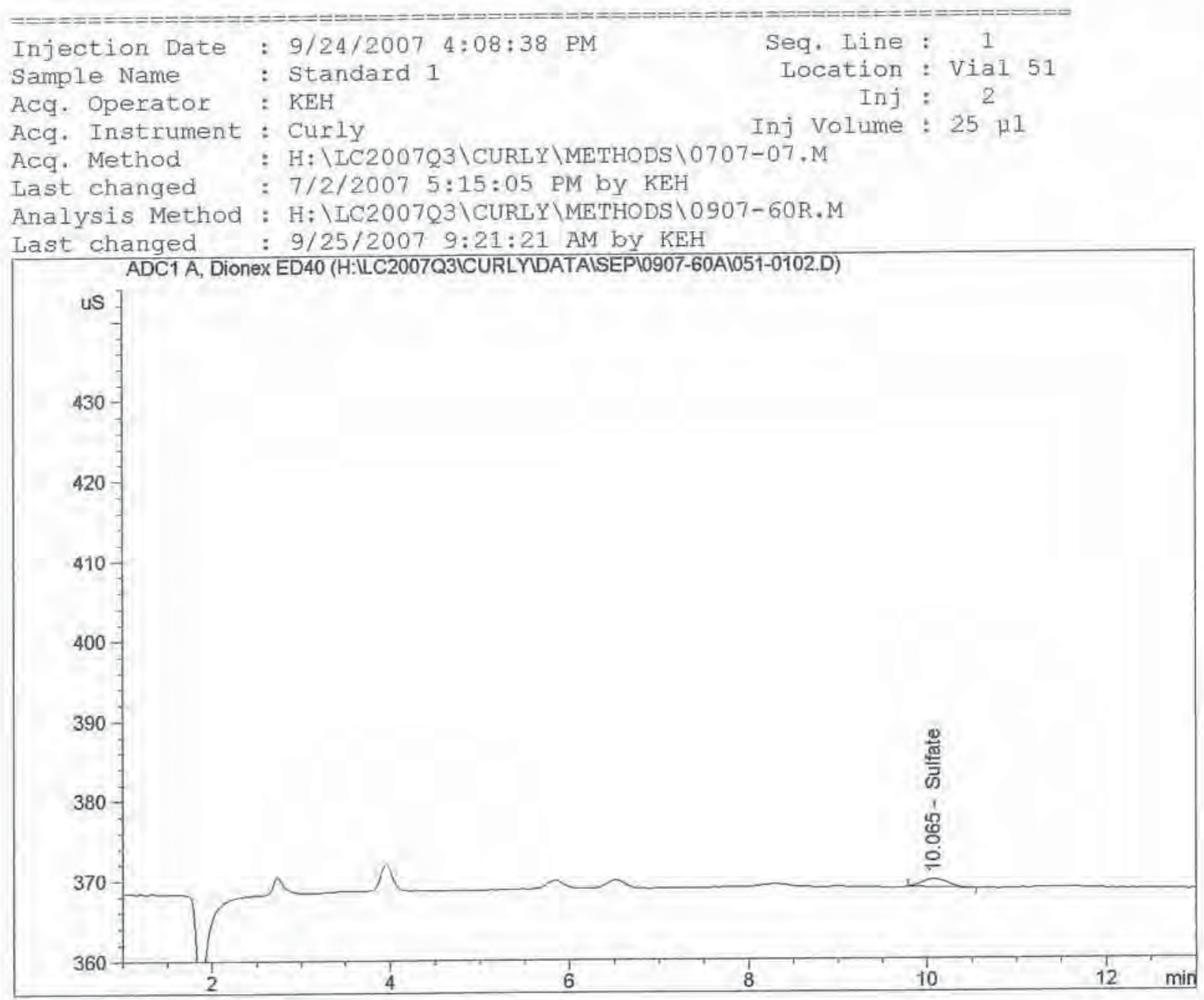

External Standard Report

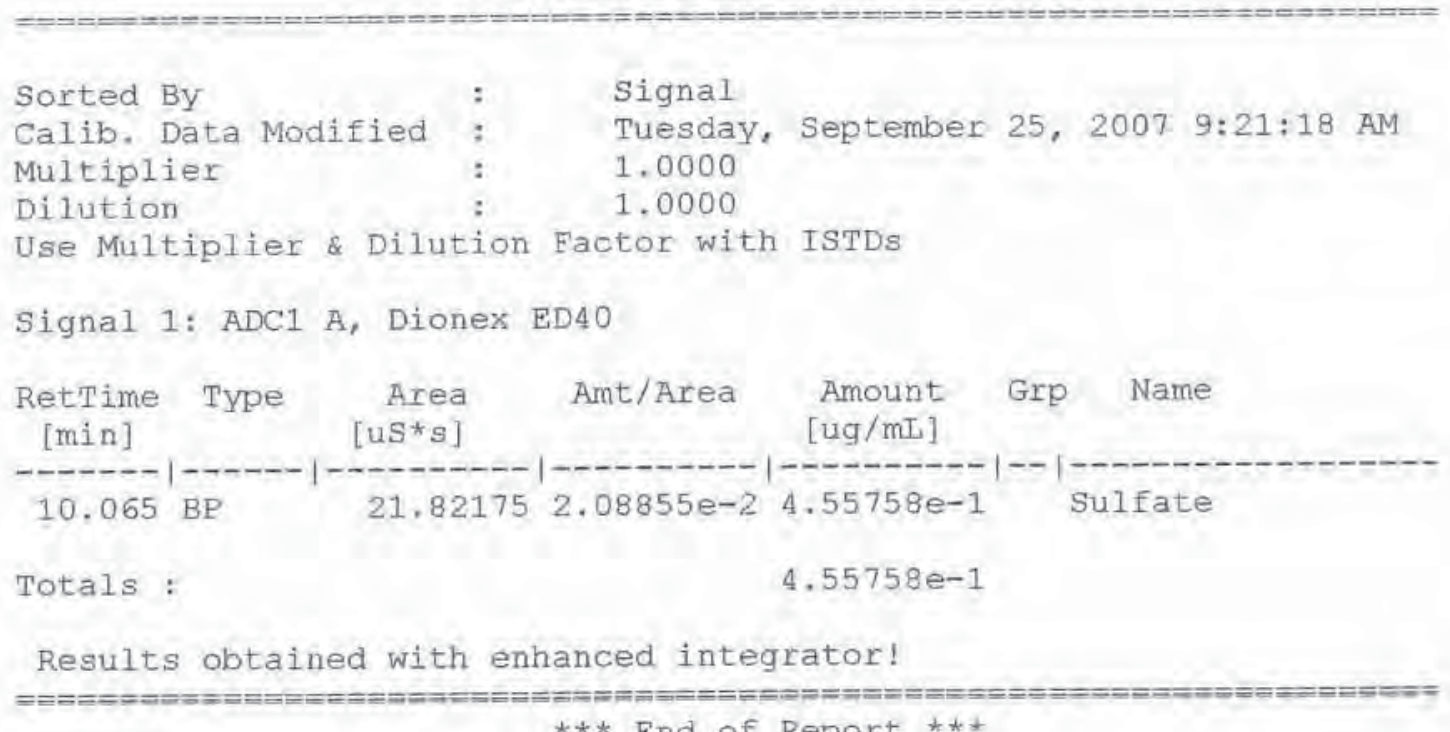

*** End of Report **t 


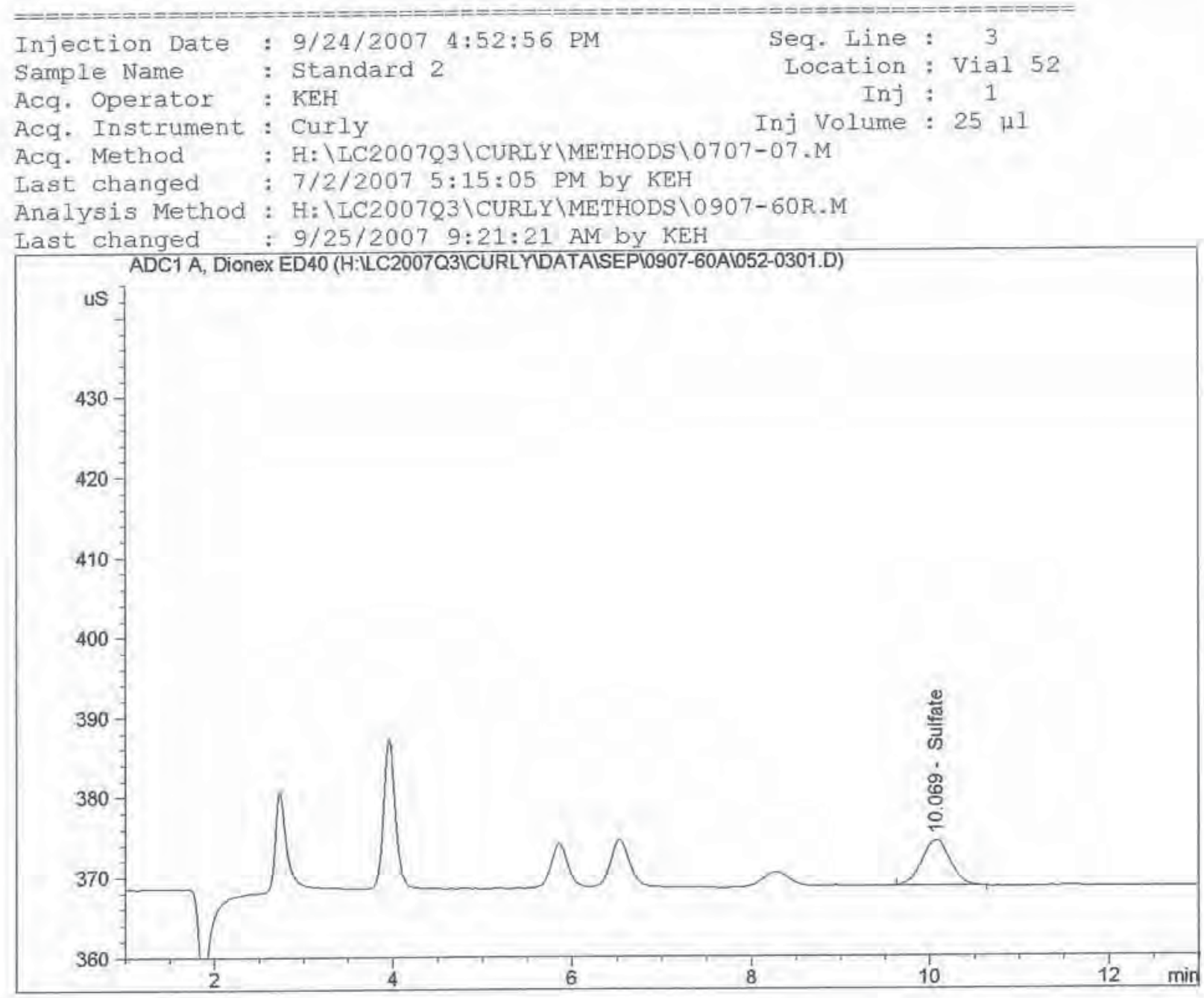

External standard Report

$\begin{array}{lll}\text { Sorted By } & : & \text { Signal } \\ \text { Calib. Data Modified } & : & \text { Tuesday, September 25, } 2007 \text { 9:21:18 AM } \\ \text { Multiplier } & \vdots & 1.0000 \\ \text { Dilution } & : & 1.0000 \\ \text { Use Multiplier \& Dilution Factor with ISTDS }\end{array}$

Signal 1: ADC1 A, Dionex ED4O

\begin{tabular}{|c|c|c|c|c|c|}
\hline $\begin{array}{l}\text { RetTime } \\
\text { [min] }\end{array}$ & Type & $\begin{array}{r}\text { Area } \\
\text { [us*s] }\end{array}$ & Amt/Area & $\begin{array}{l}\text { Amount } \\
{[\mathrm{ug} / \mathrm{mL}]}\end{array}$ & Grp Name \\
\hline 10.069 & $\mathrm{BB}$ & 126.60550 & $1.84031 e-2$ & 2.32994 & Sulfate \\
\hline Totals & & & & 2.32994 & \\
\hline
\end{tabular}

Results obtained with enhanced integrator!

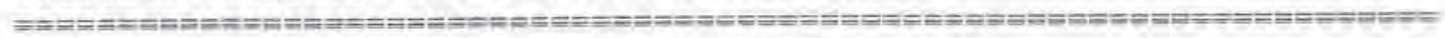

$* * *$ End of Report ***

Page 31 of 53 


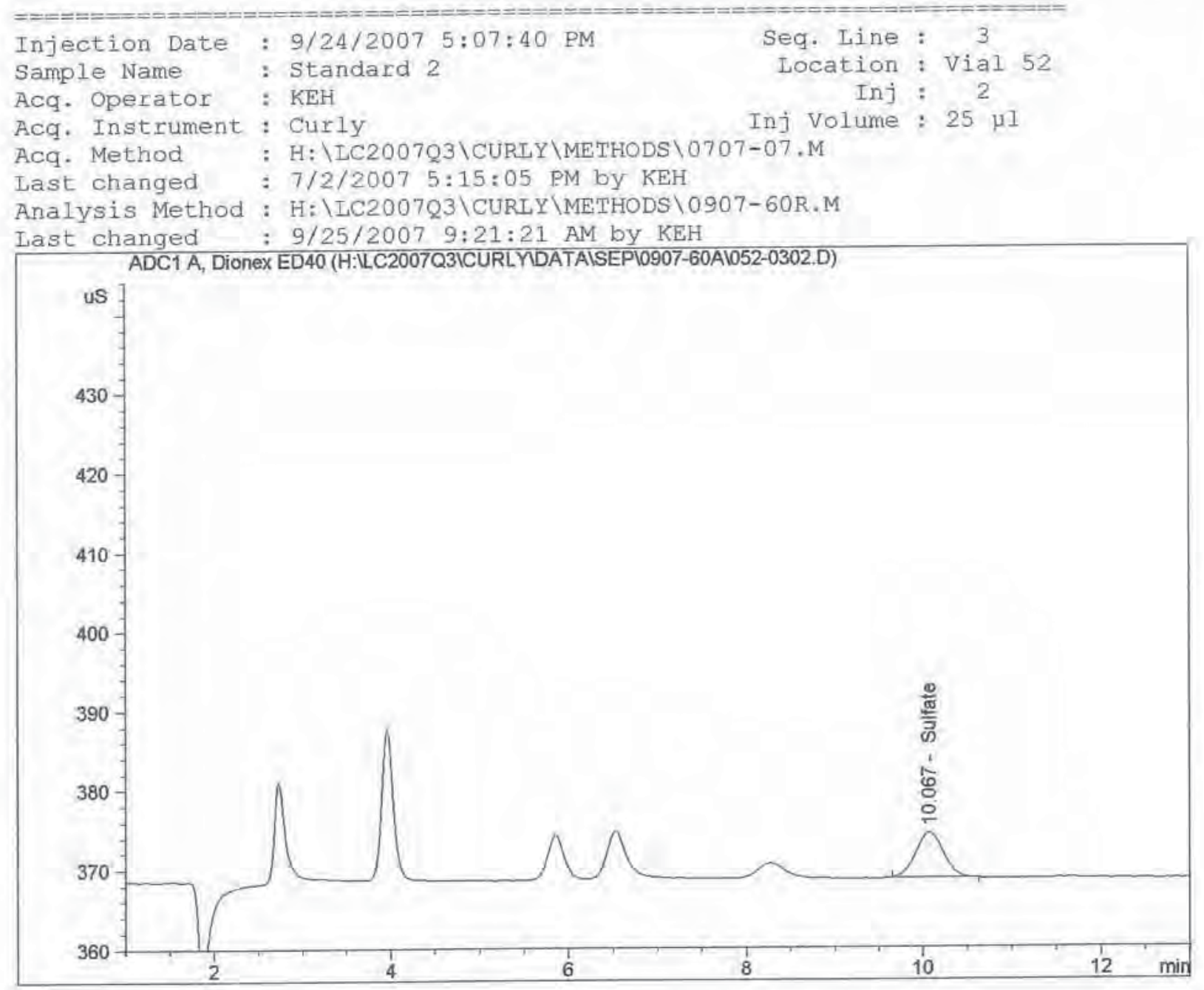

\section{External Standard Report}

$\begin{array}{lcl}\text { Sorted By } & : & \text { Signal } \\ \text { Calib. Data Modified } & : & \text { Tuesday, September 25, 2007 9:2.1:18 AM } \\ \text { Multiplier } & : & 1.0000 \\ \text { Dilution } & : \text { 1.0000 } \\ \text { Use Multiplier \& Dilution Factor with ISTDs }\end{array}$

Signal 1: ADC1 A, Dionex ED4O

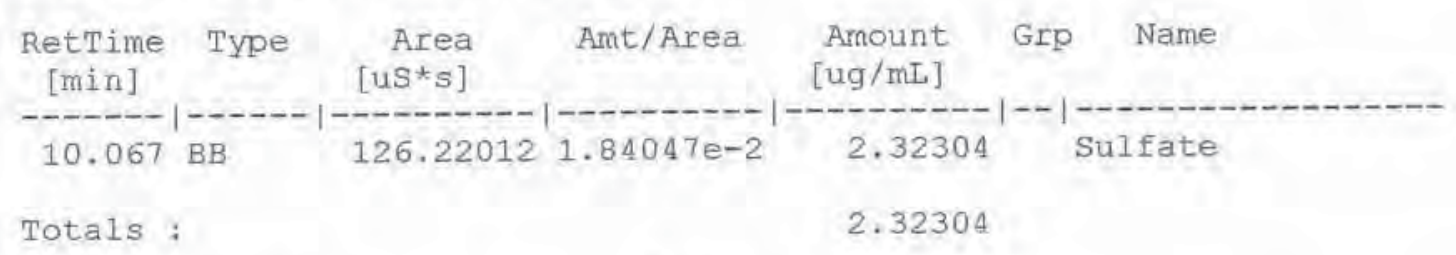

Results obtained with enhanced integrator!

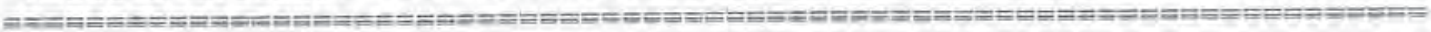
$\star * *$ End of Report ***

Page 32 of 53 


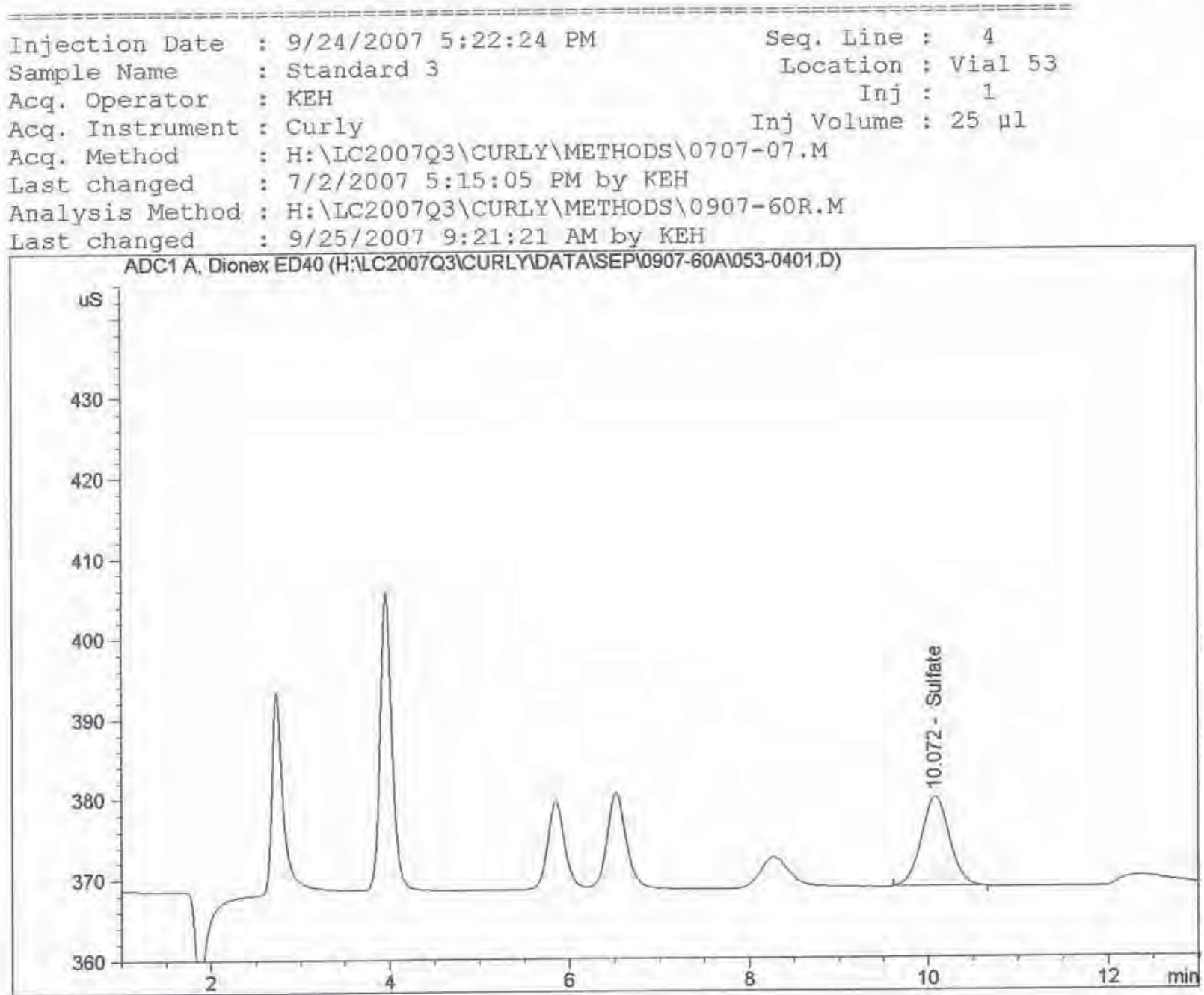

\section{External Standard Report}

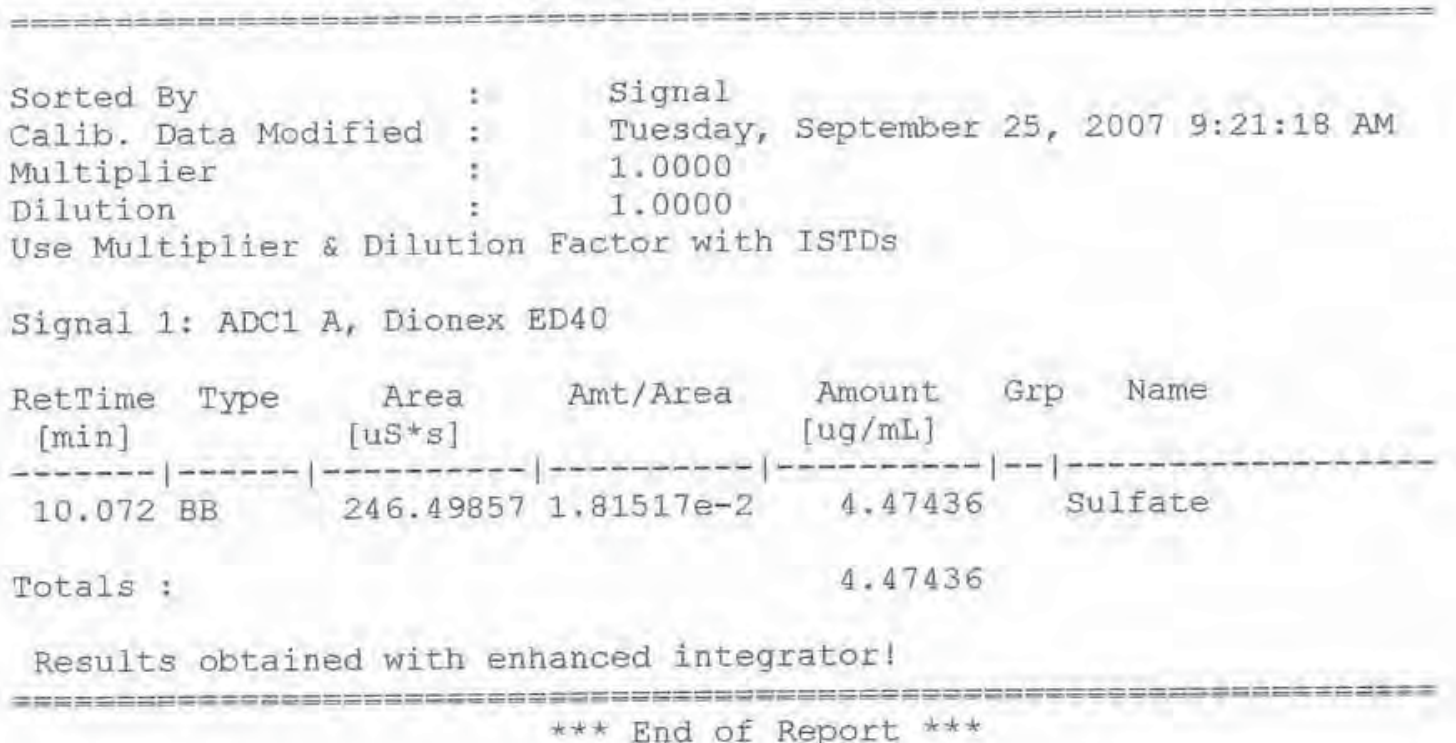




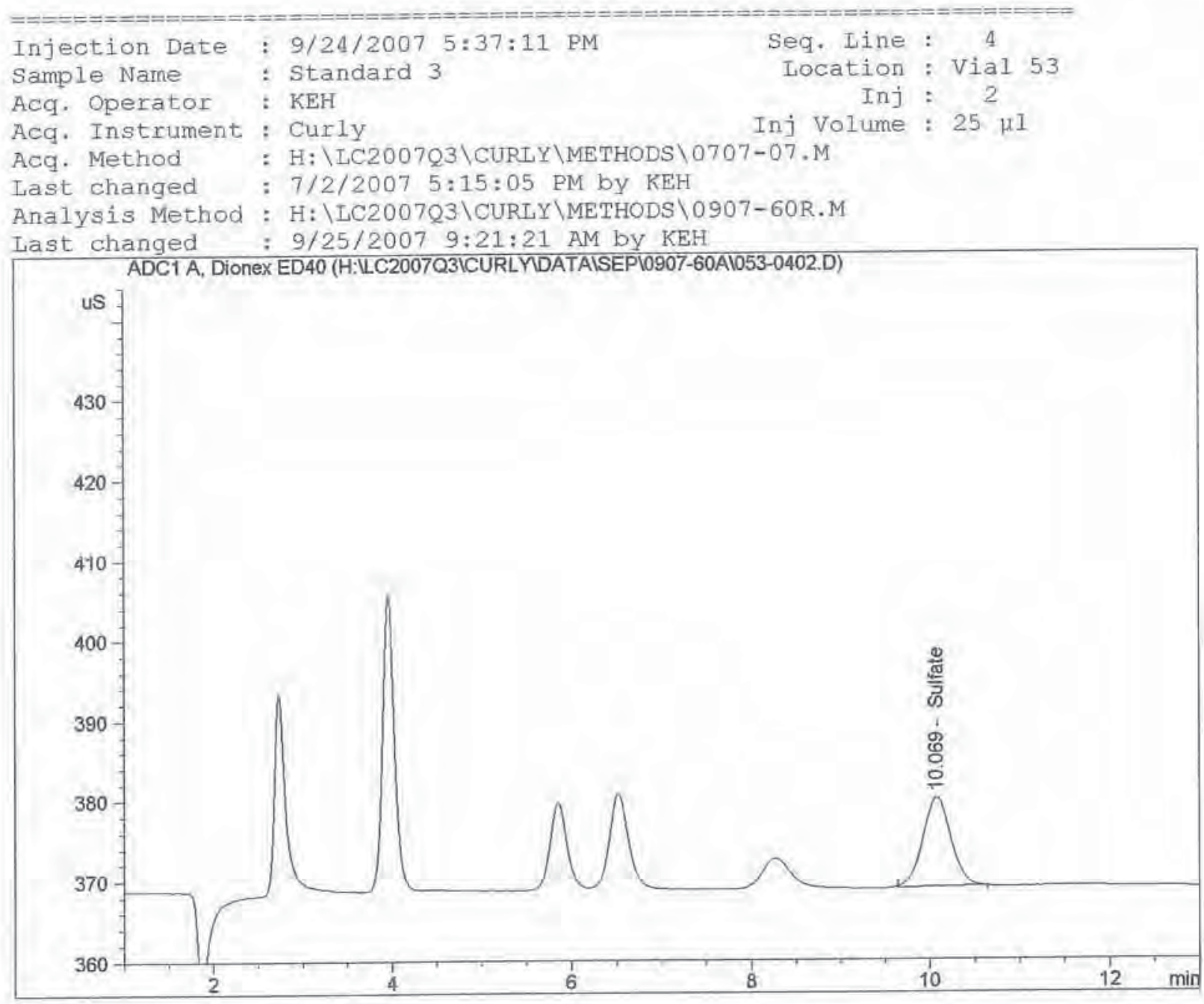

External Standard Report

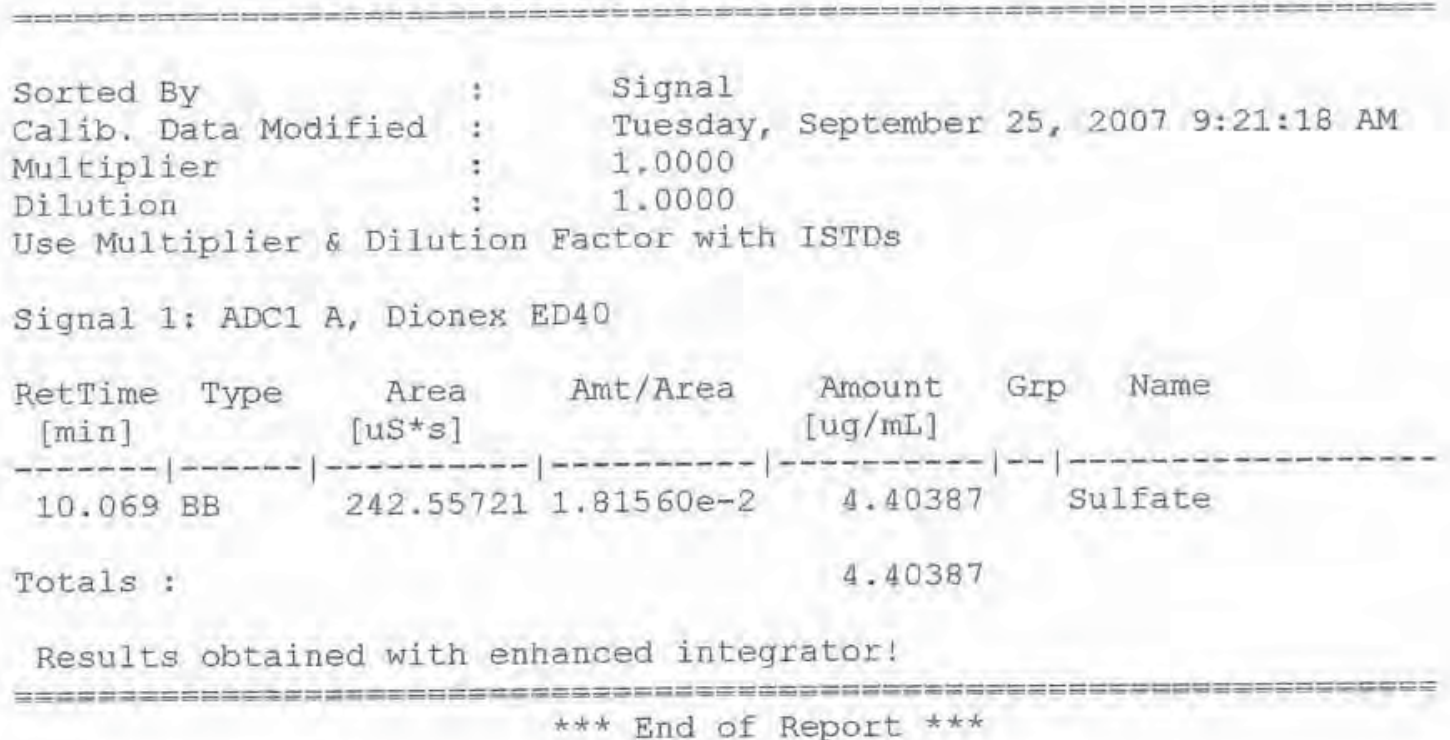




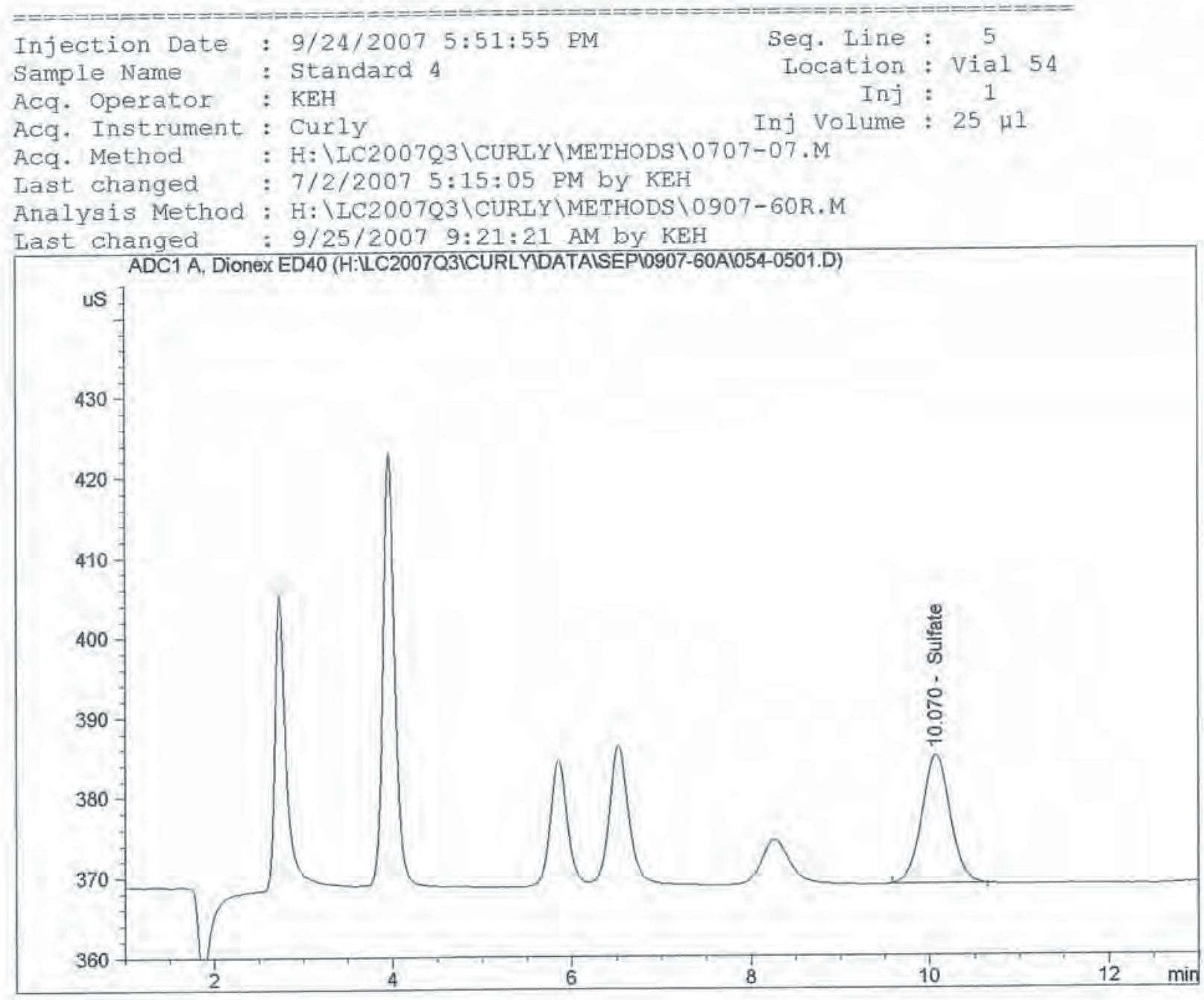

Esternal Standard Report

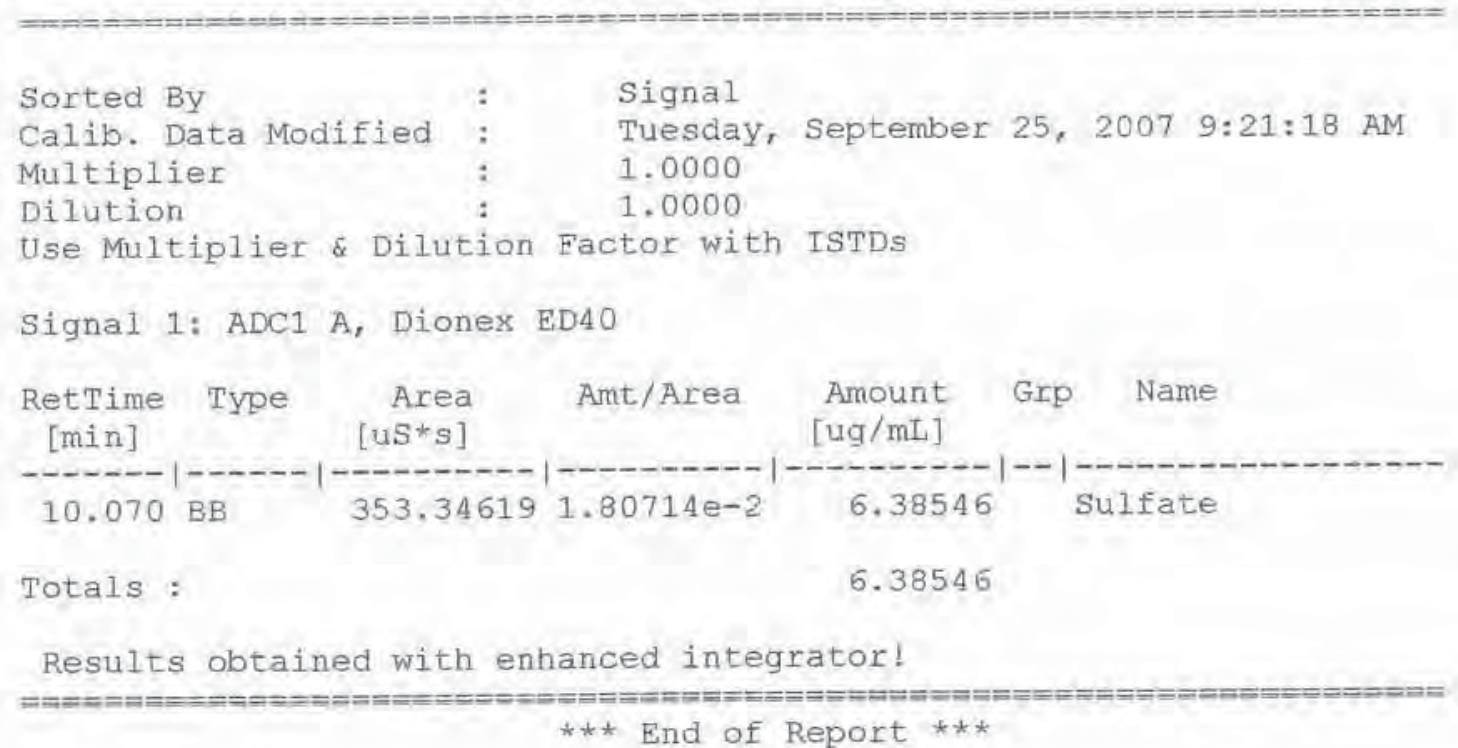




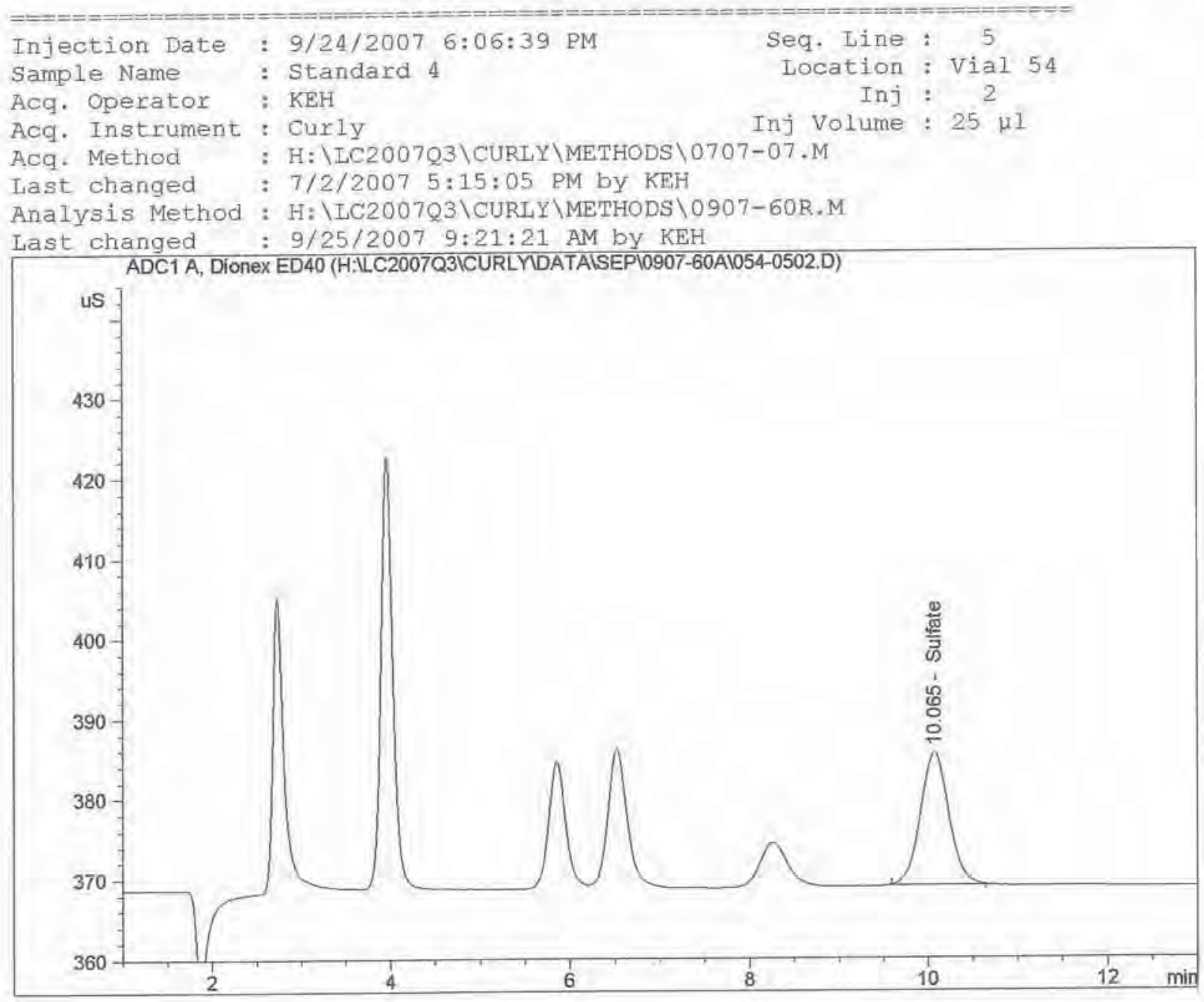

External Standard Report

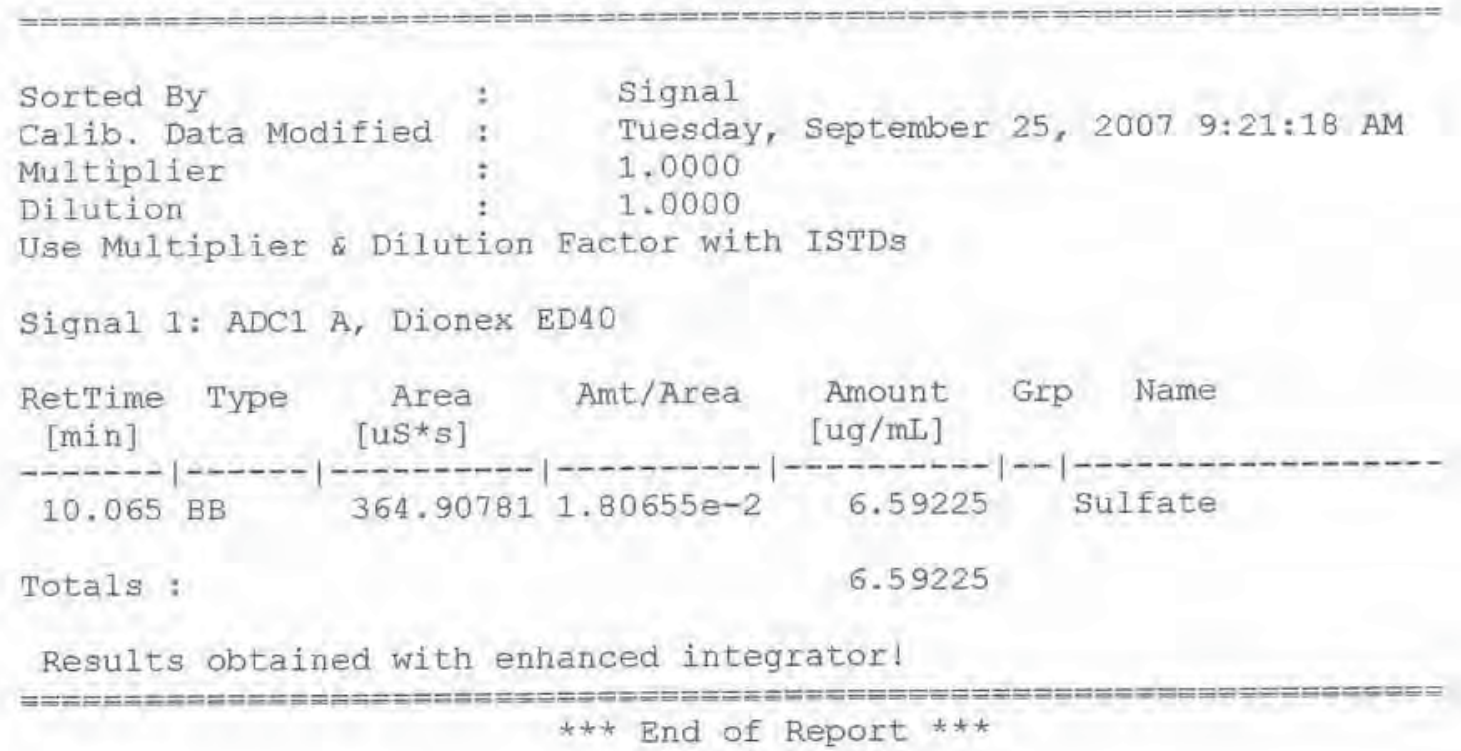




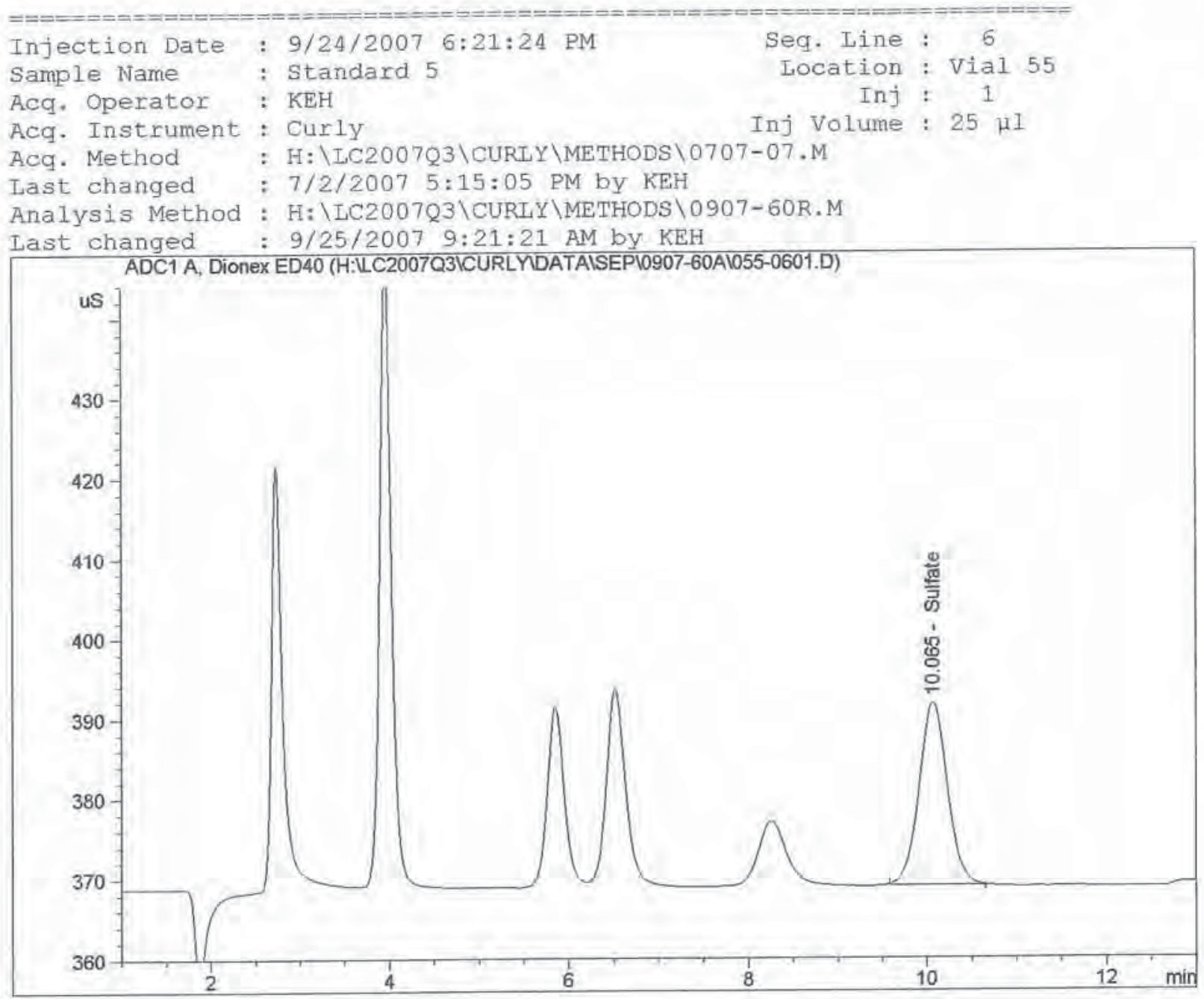

External standard Report

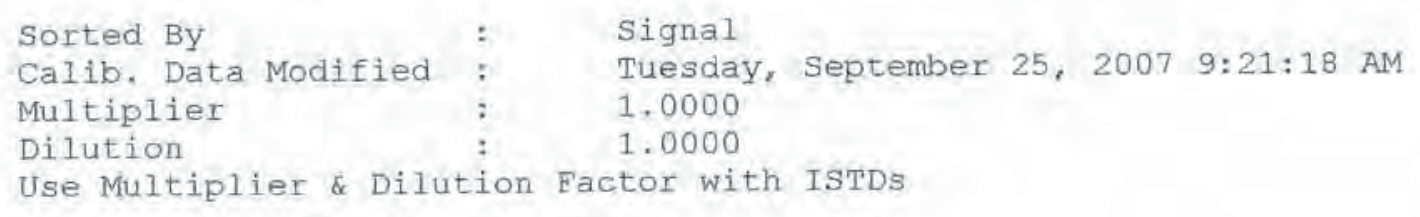

Signal 1: ADC1 A, Dionex ED40

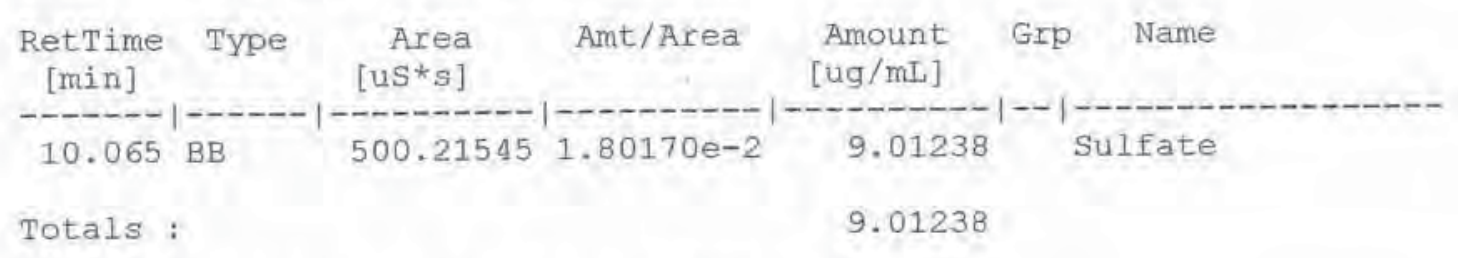

Results obtained with enhanced integrator!

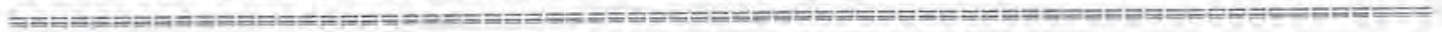

*** End of Report ***

Page 37 of 53 


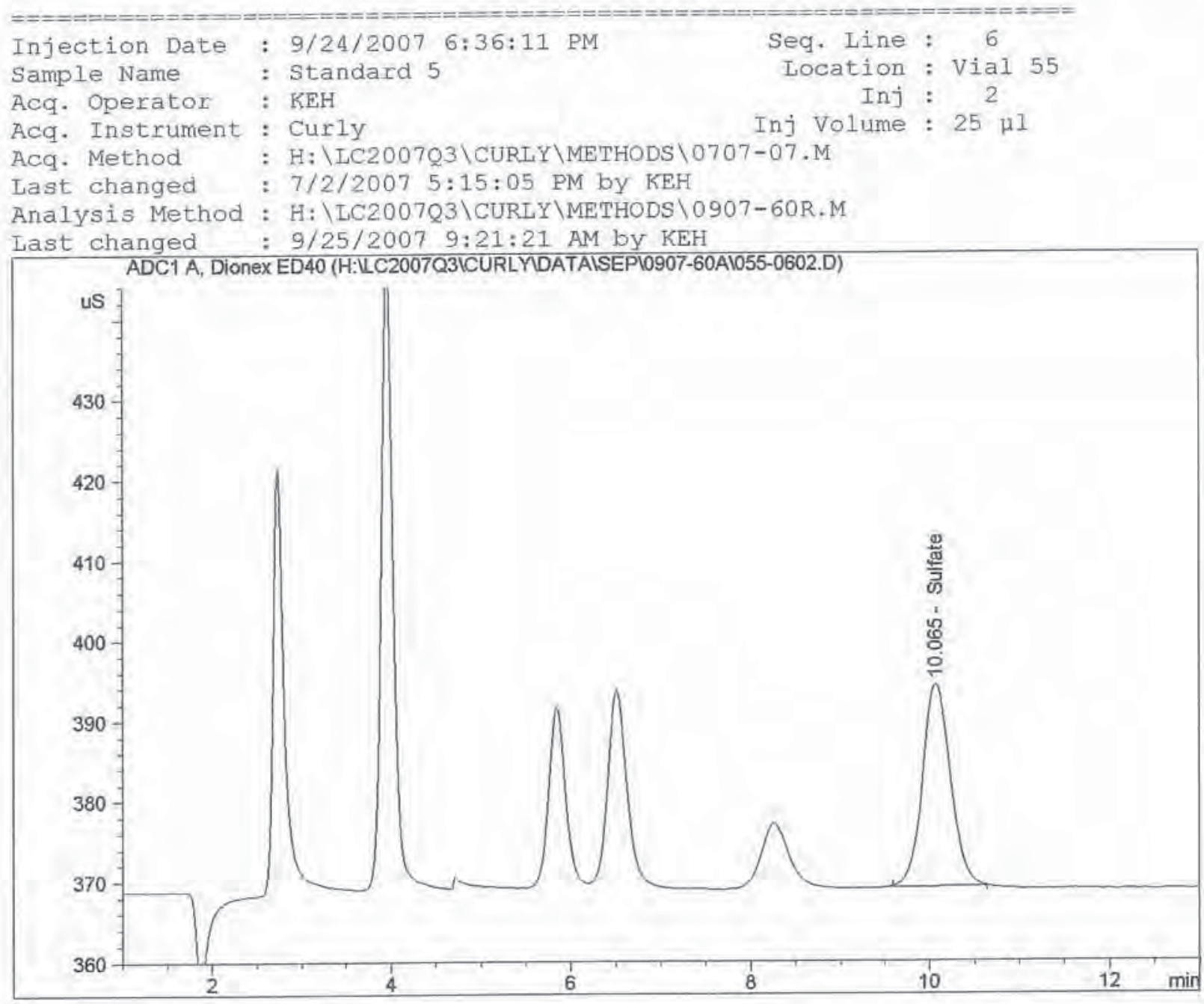

External standard Report

$\begin{array}{lll}\text { Sorted By } & : & \text { Signal } \\ \text { Calib. Data Modified }: & \text { Tuesday, September 25, } 2007 \text { 9:21:18 AM } \\ \text { Multiplier } & : & 1.0000 \\ \text { Dilution } & : & 1.0000 \\ \text { Use Multiplier \& Dilution Factor with ISTDS }\end{array}$

SignaI 1: ADC1 A, Dionex ED40

\begin{tabular}{|c|c|c|c|c|}
\hline $\begin{array}{l}\text { RetTime } \\
\text { [min] }\end{array}$ & Type & $\begin{array}{r}\text { Area } \\
{\left[u s^{*} s\right]}\end{array}$ & Amt/Area & $\begin{array}{l}\text { Amount } \\
\text { [ug/mL] }\end{array}$ \\
\hline 10.065 & $\mathrm{BB}$ & 547.19867 & $1,80058 e-2$ & 9.85273 \\
\hline
\end{tabular}

Results obtained with enhanced integrator!

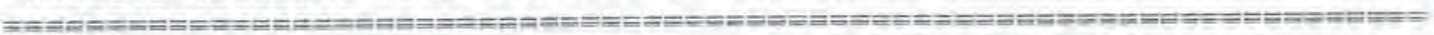

$\star * *$ End of Report $* \star *$ 


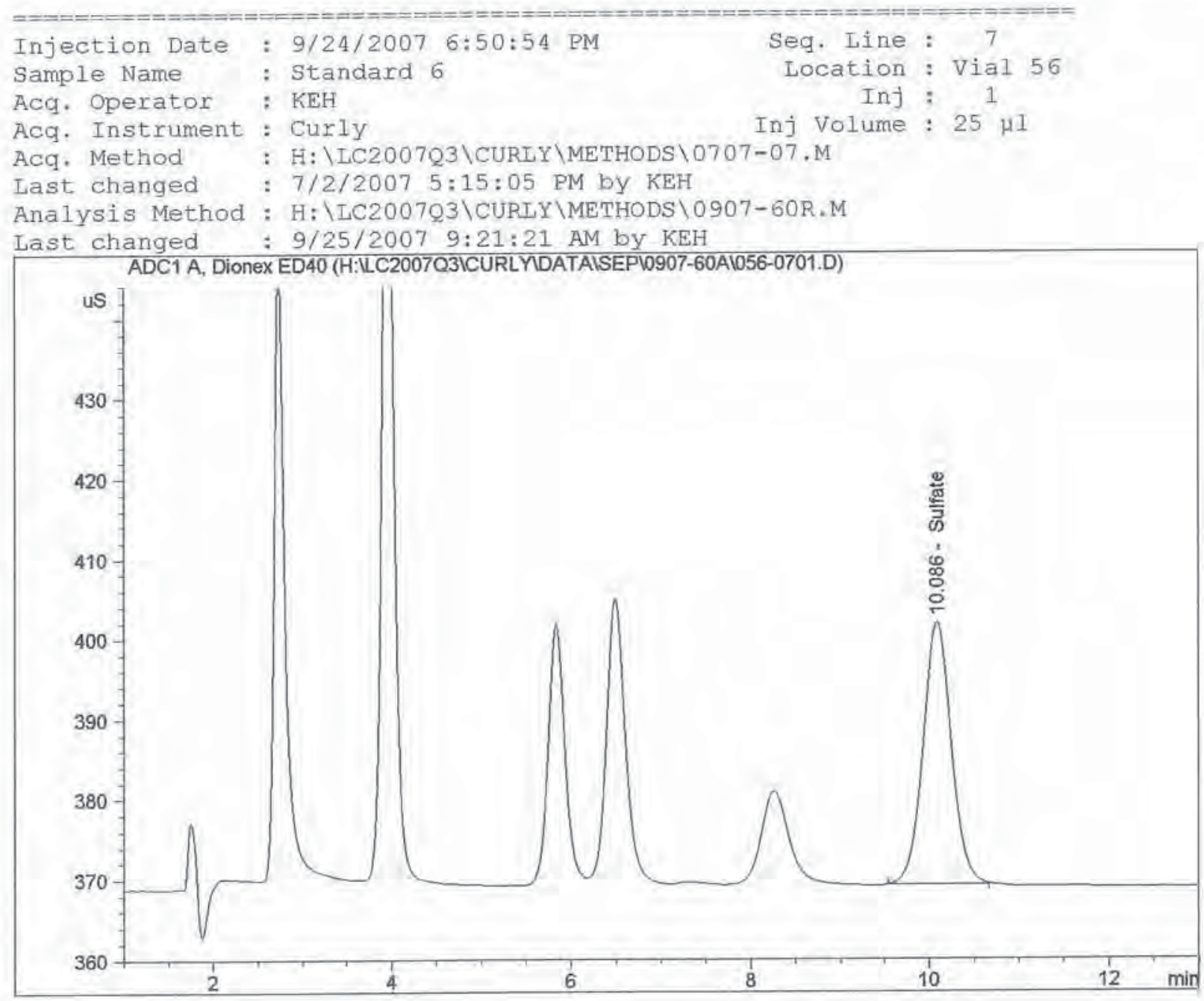

External Standard Report

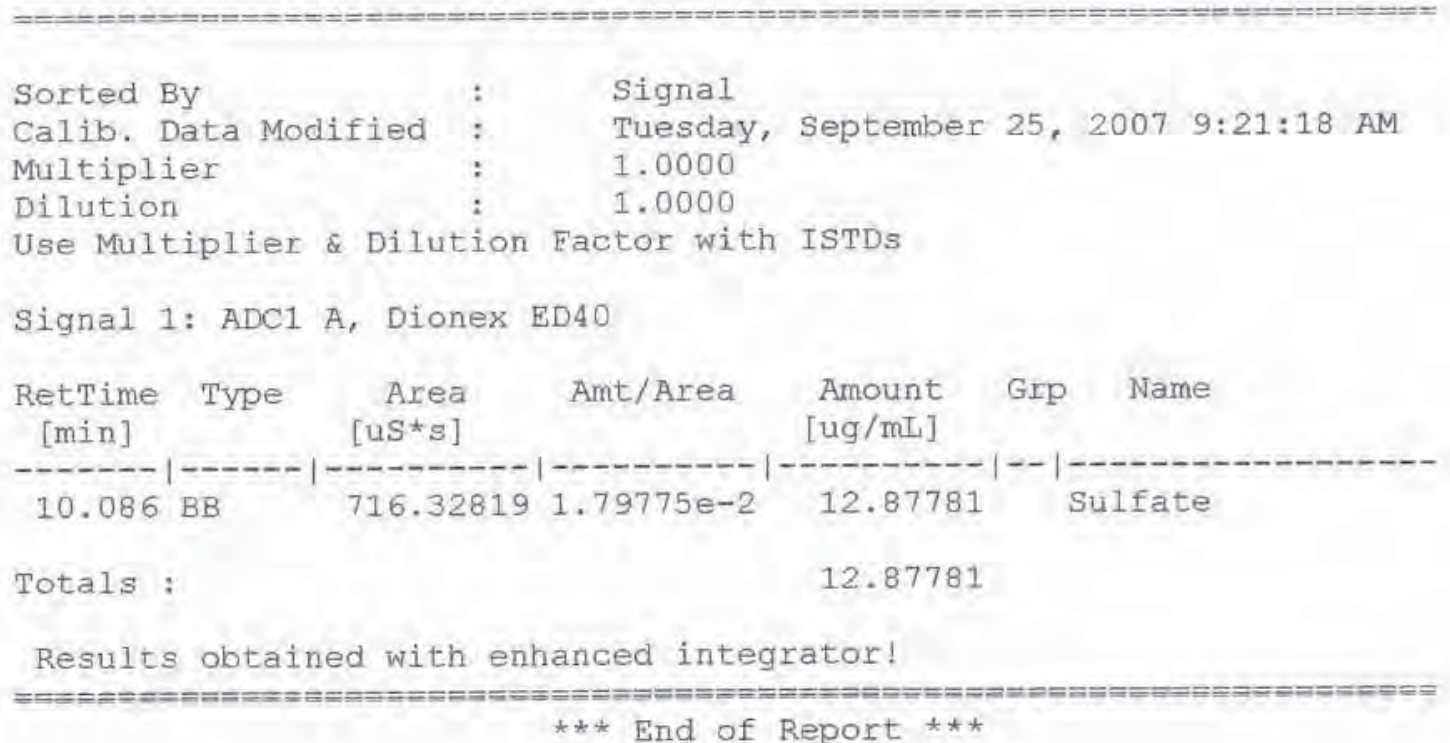



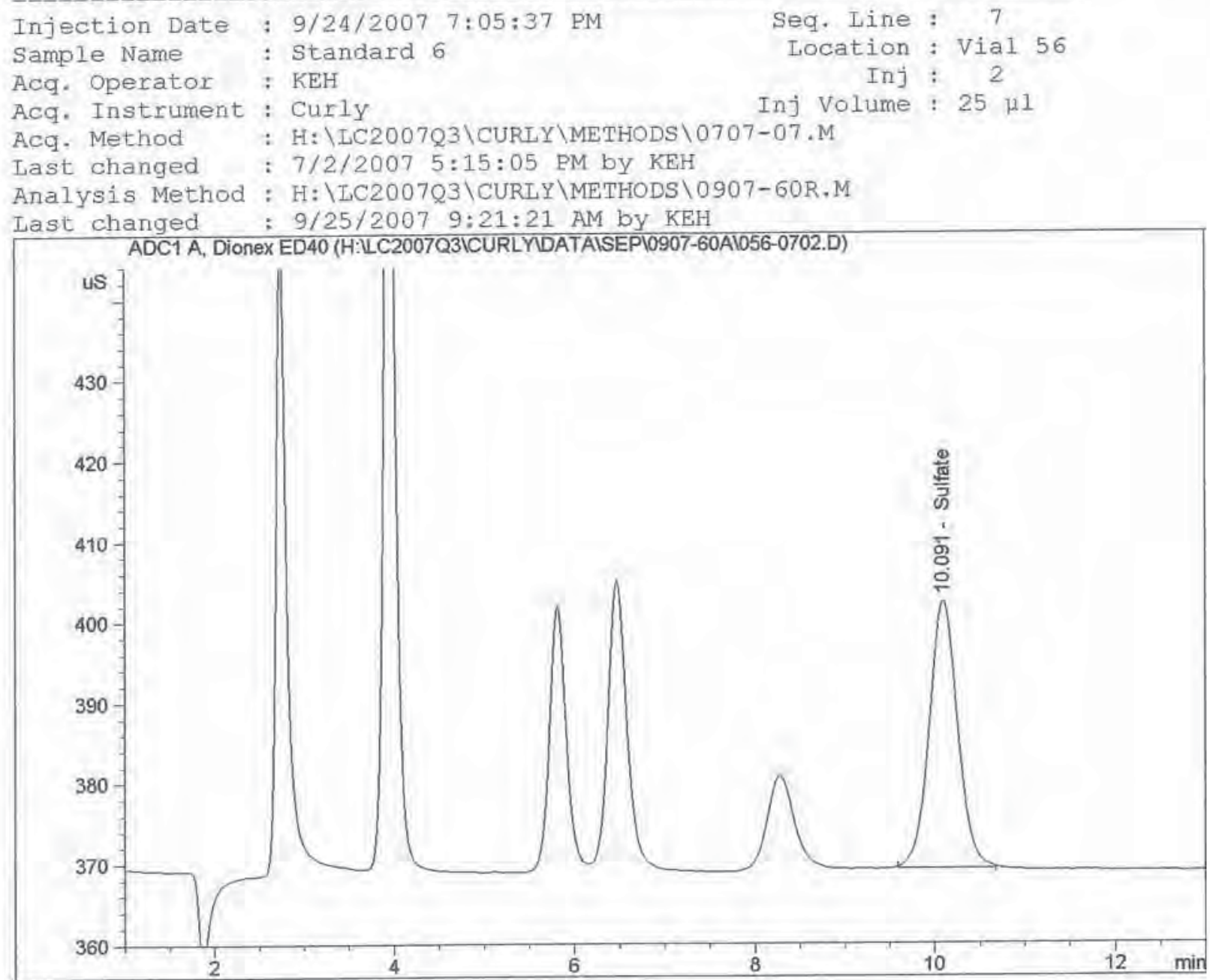

\section{External Standard Report}

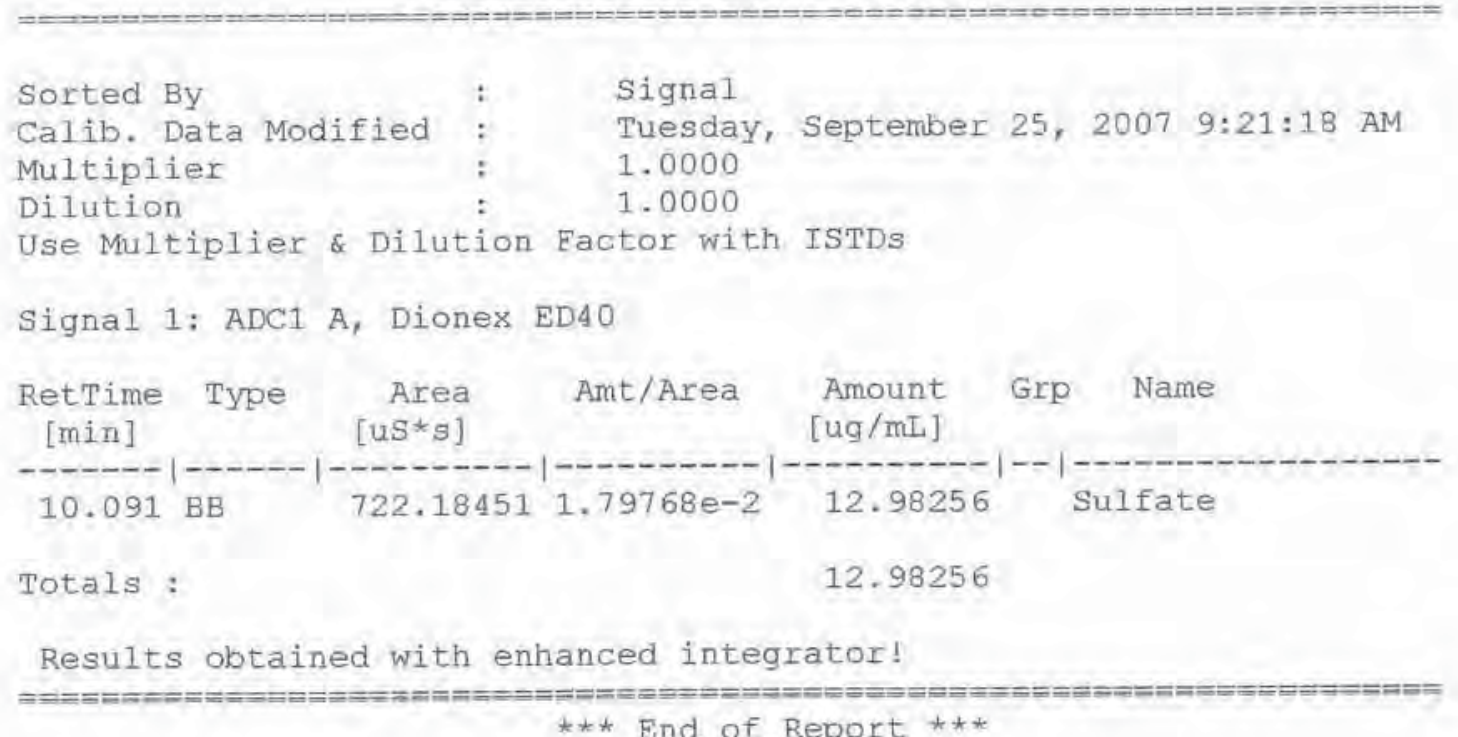




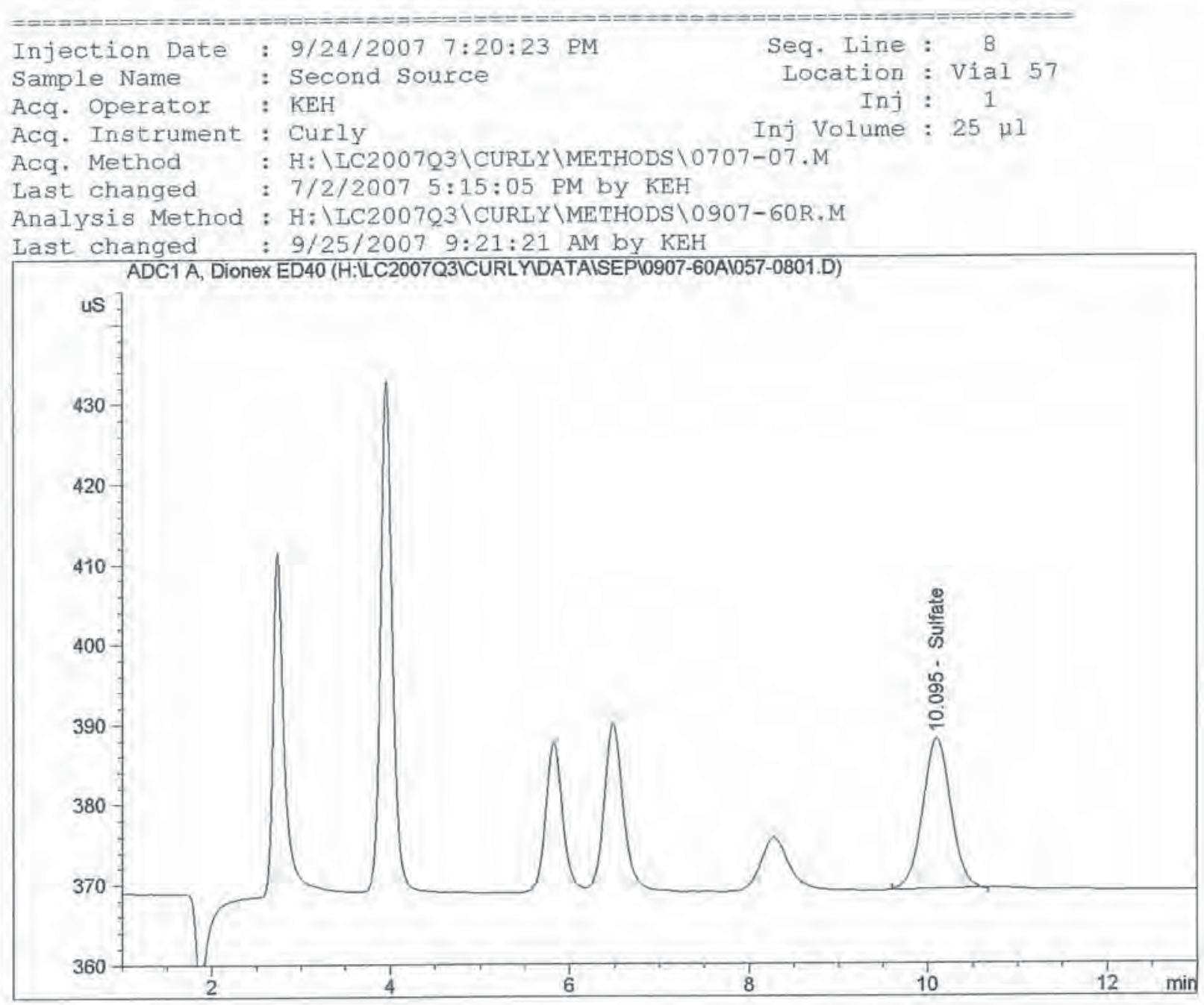

External Standard Report

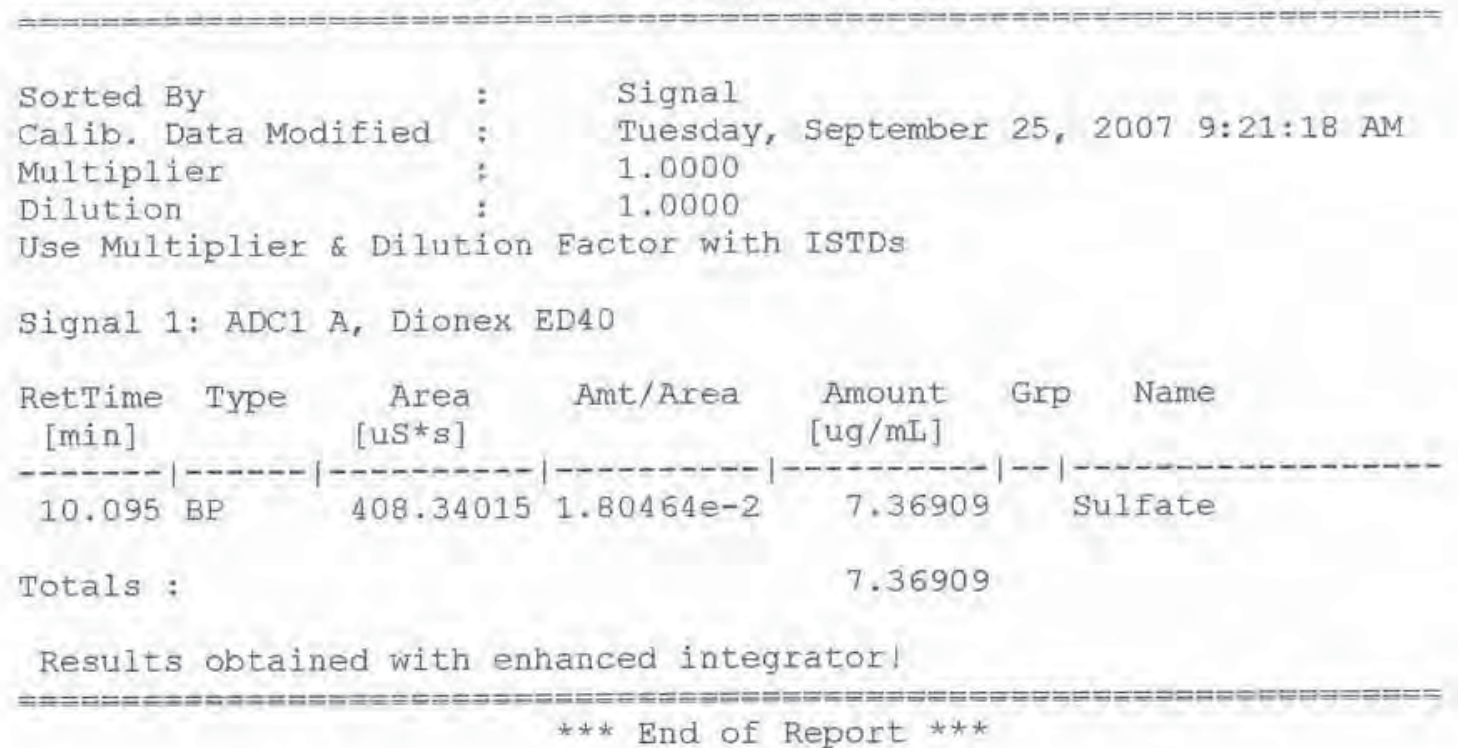




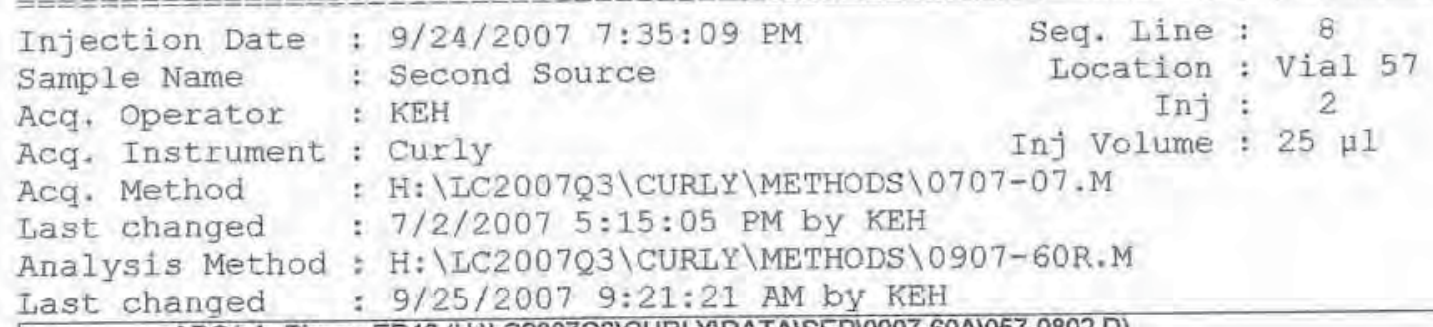




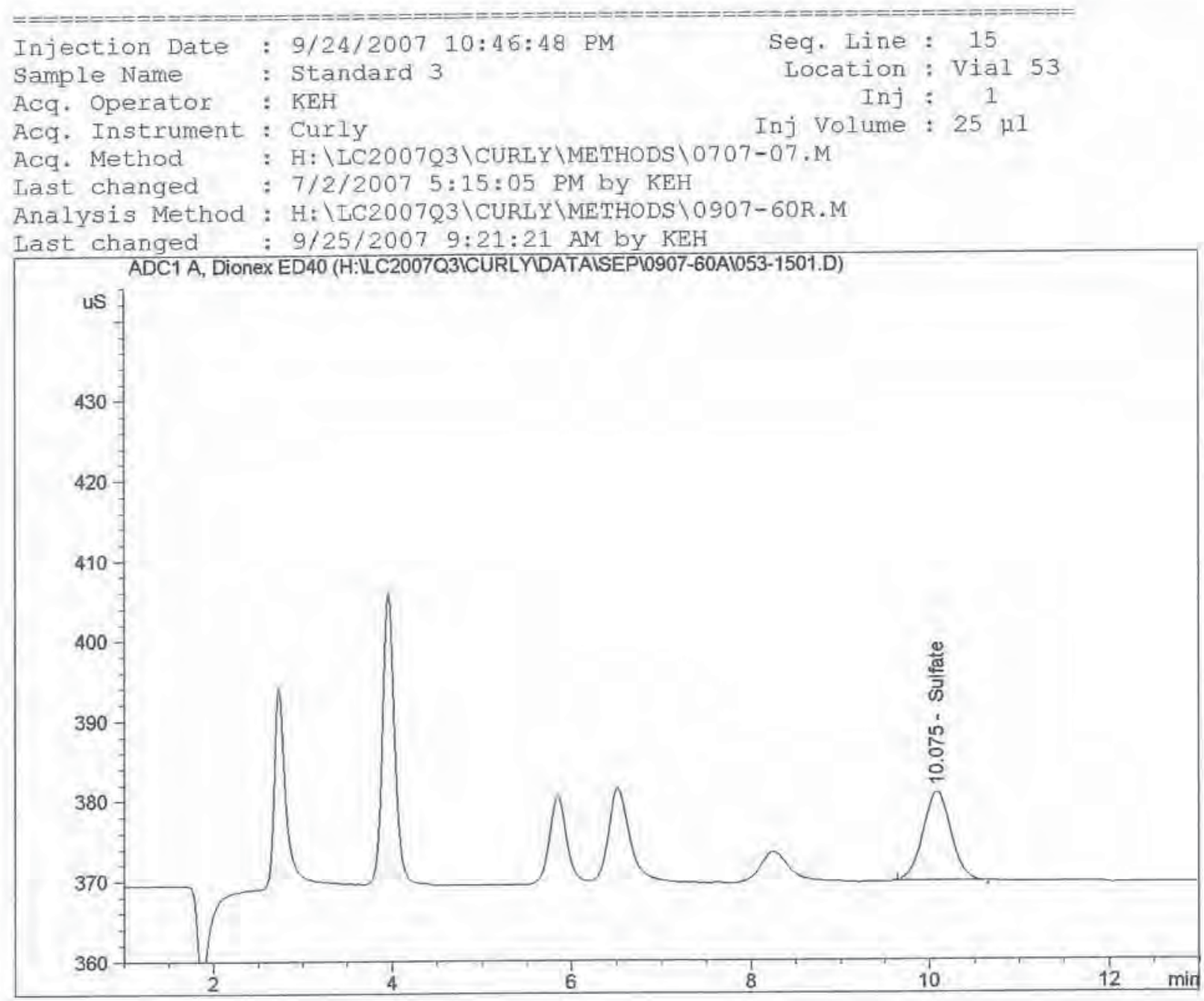

External Standard Report

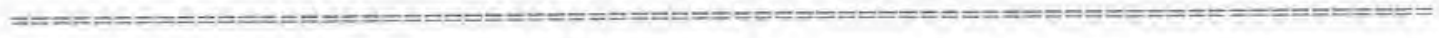

$\begin{array}{lll}\text { Sorted By } & : & \text { Signal } \\ \text { Calib. Data Modified }: & \text { Tuesday, September 25, 2007 9:21:18 AM } \\ \text { Multiplier } & : & 1.0000 \\ \text { Dilution } & : & 1.0000 \\ \text { Use Multiplier \& Dilution Eactor with ISTDS }\end{array}$

Signal 1: ADC1 A, Dionex ED40

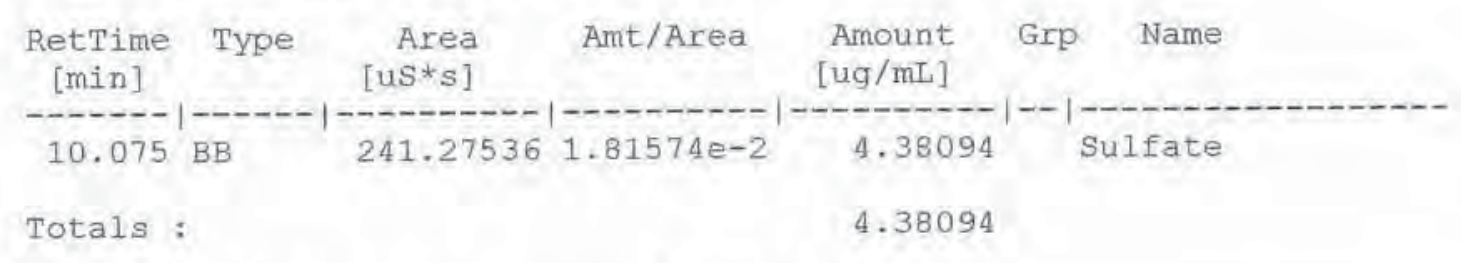

Results obtained with enhanced integrator!

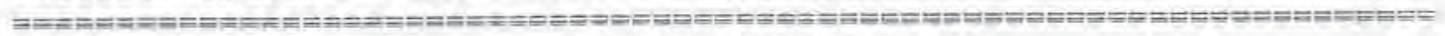
*** End of Report $* * \star$ 


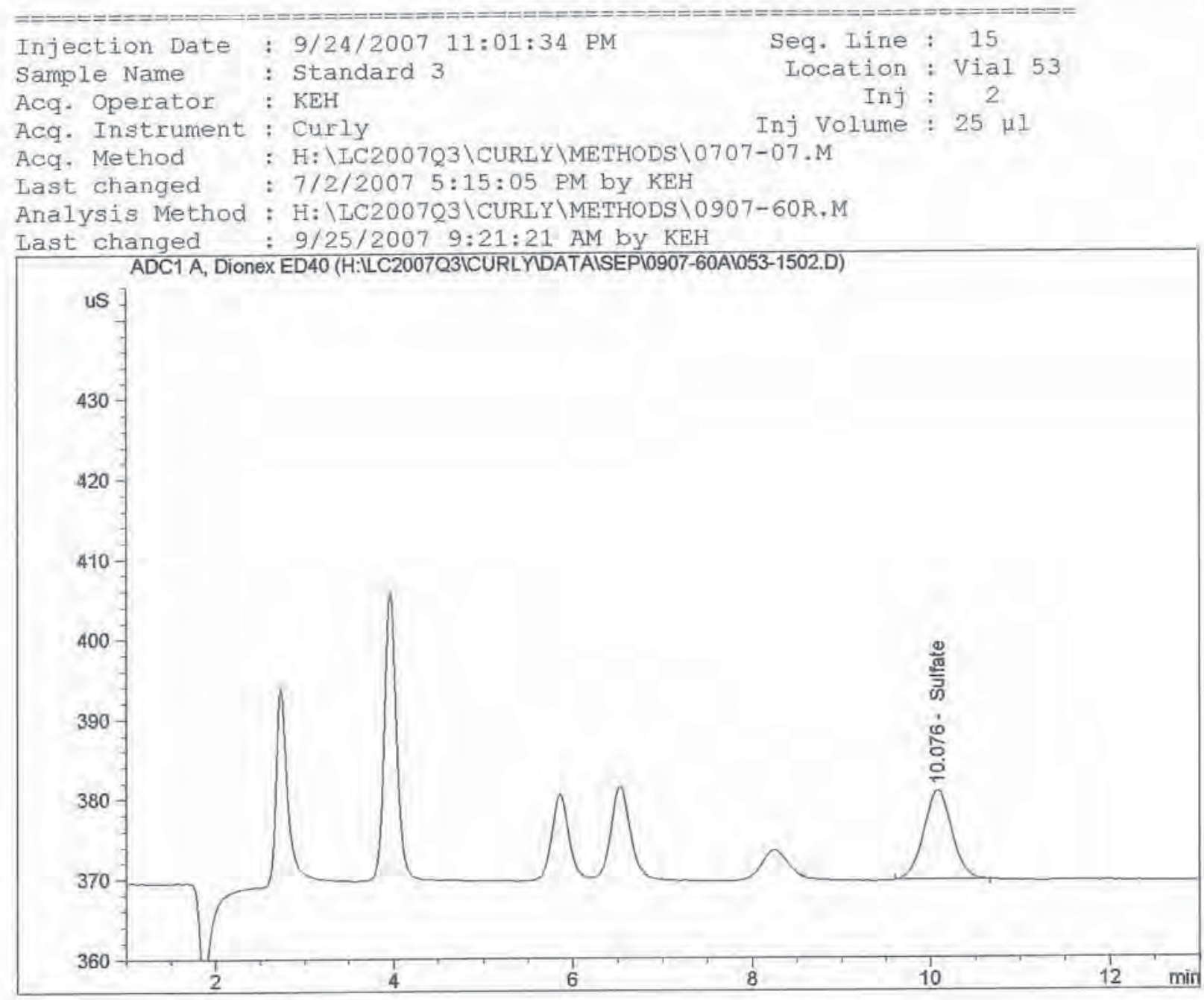

External Standard Report

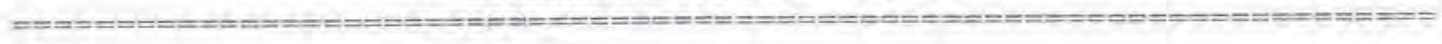

$\begin{array}{lll}\text { Sorted By } & : & \text { Signal } \\ \text { Calib. Data Modified } & \text { Tuesday, September 25, } 2007 \text { 9:21:18 AM } \\ \text { Multiplier } & \vdots & 1.0000 \\ \text { Dilution } & \vdots & 1.0000 \\ \text { Use Multiplier \& Dilution Factor with ISTDs }\end{array}$

Signal 1: ADC1 A, Dionex ED4O

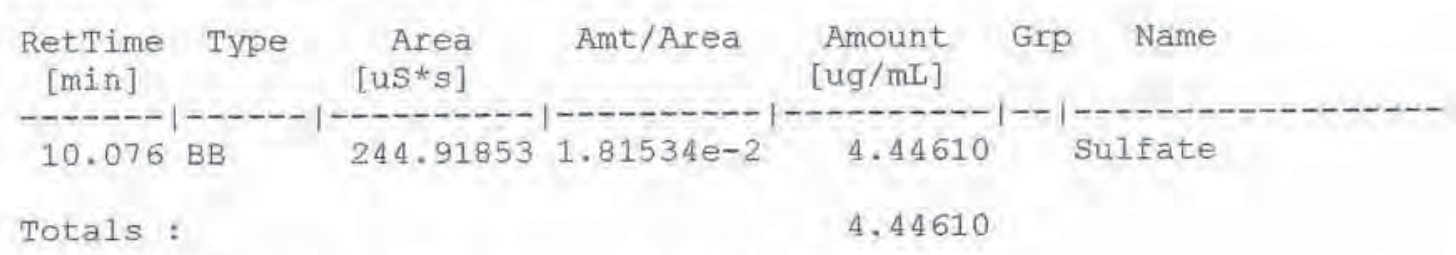

Results obtained with enhanced integrator!

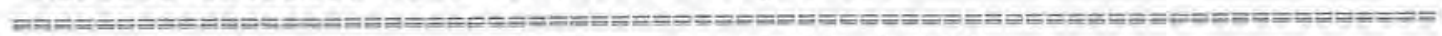
$\star \star \star$ End of Report $* * \star$ 


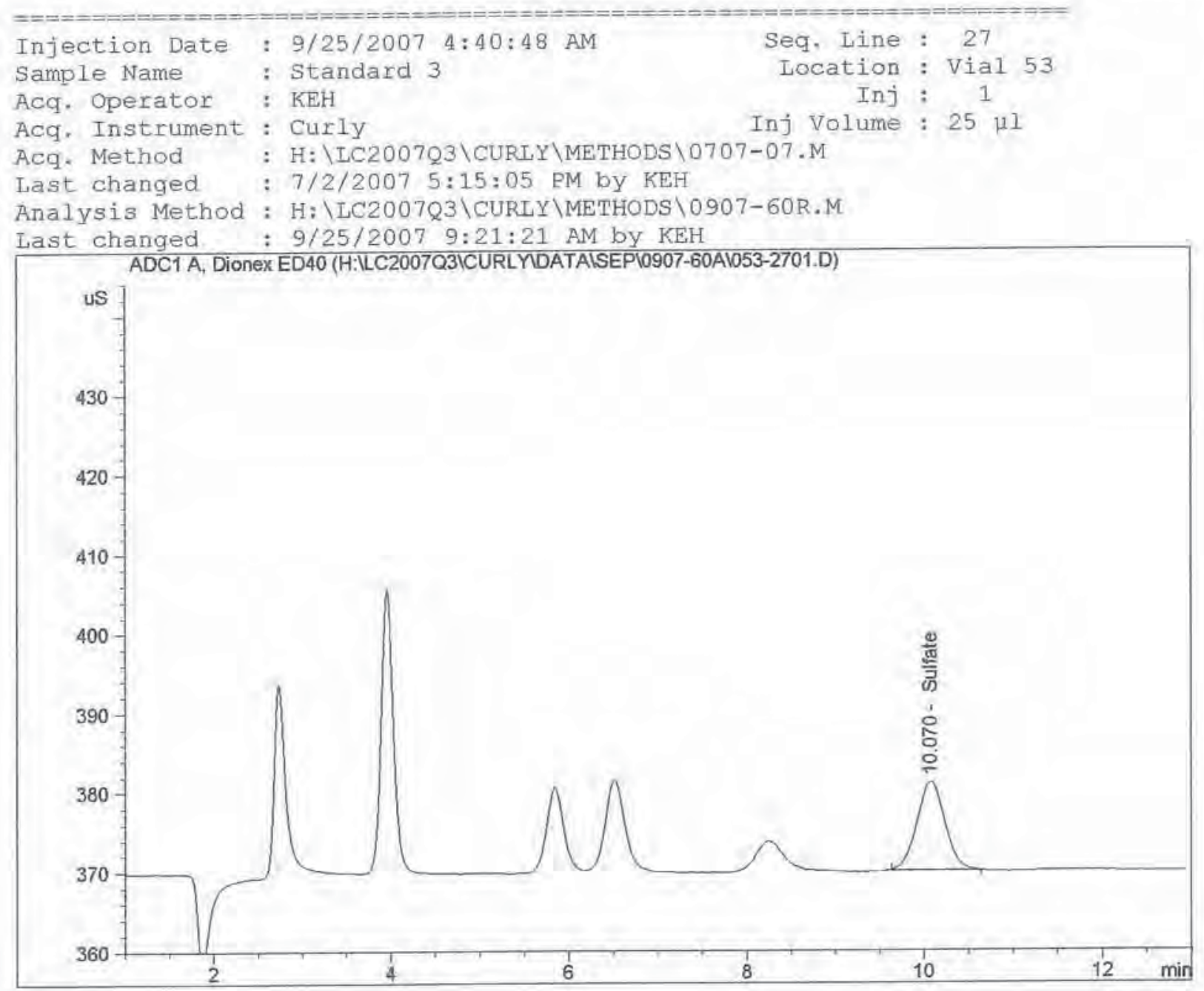

External Standard Report

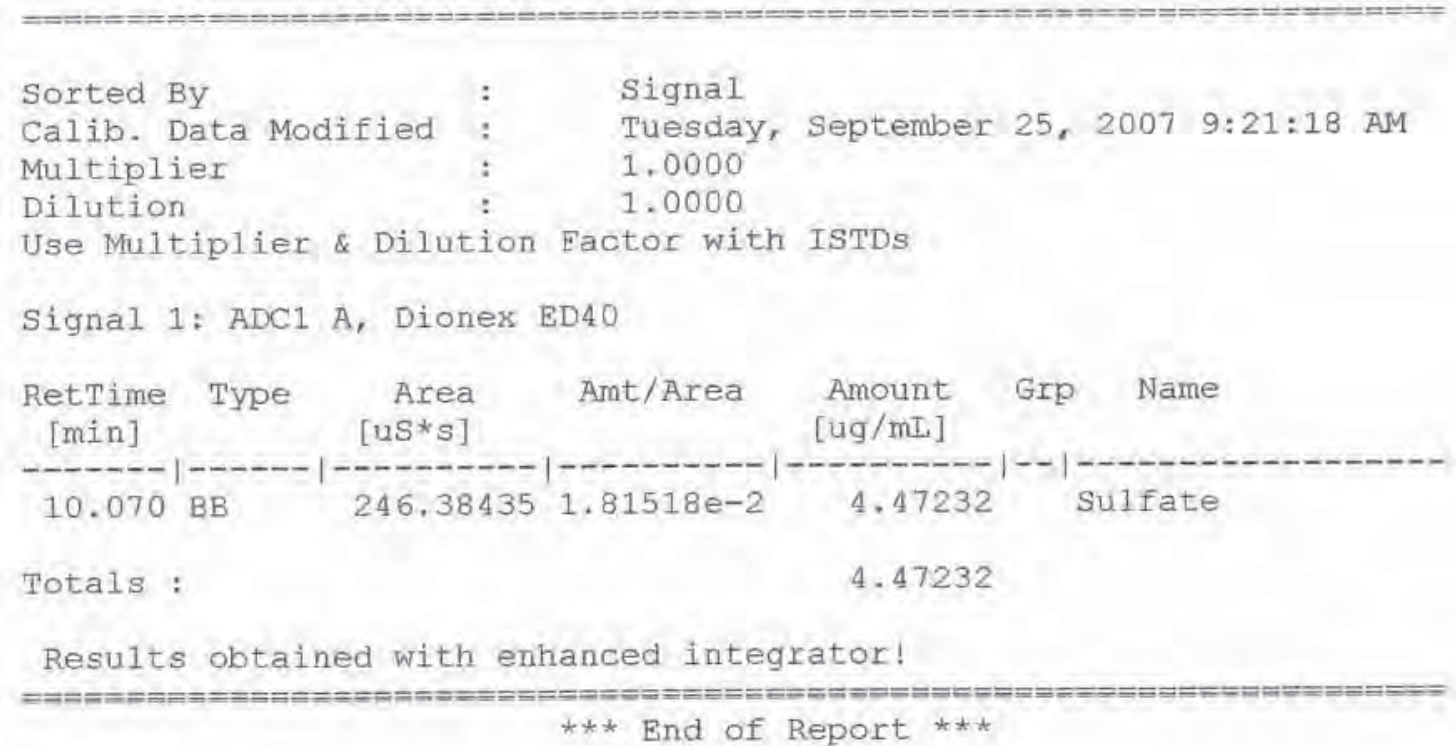




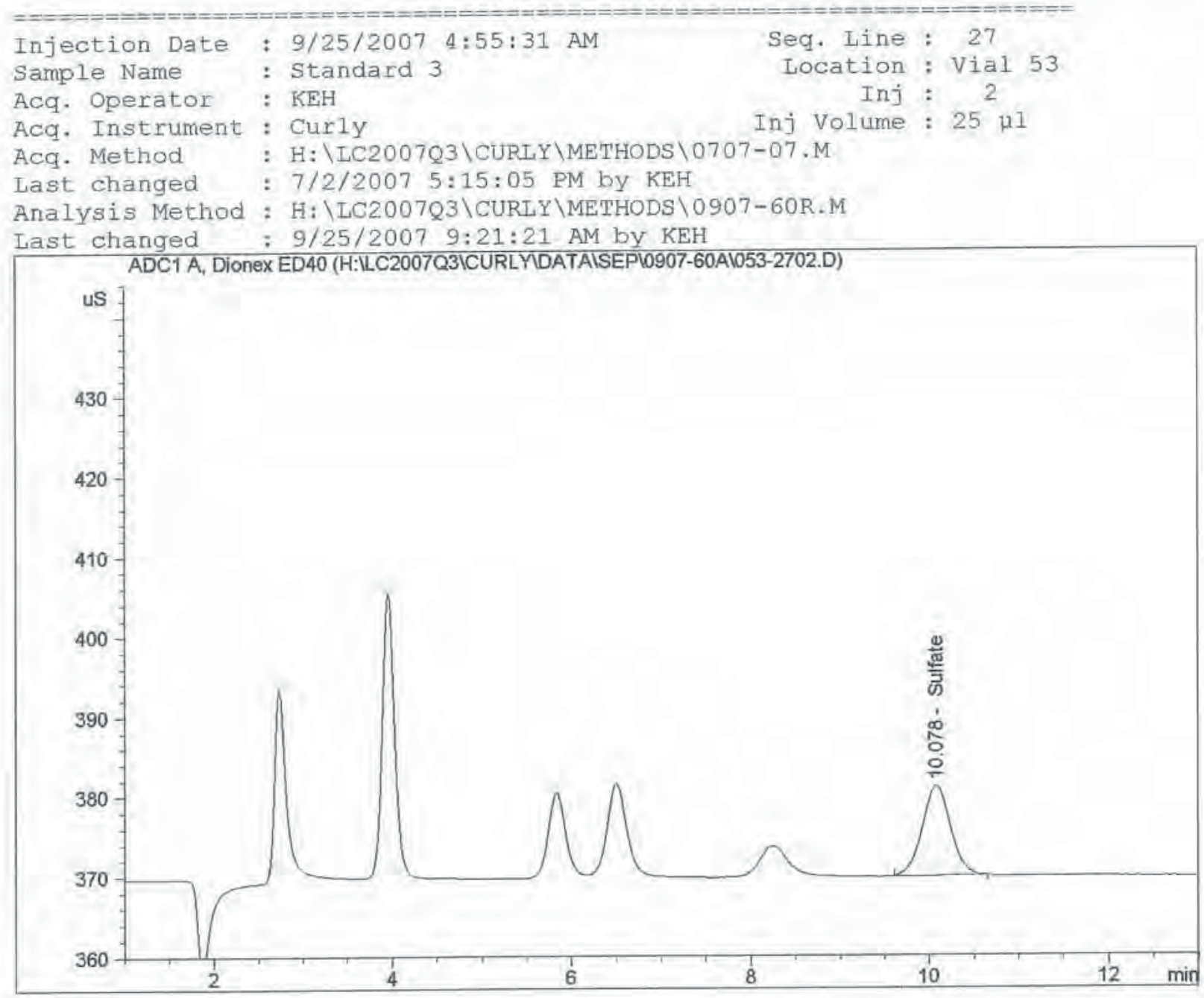

External Standard Report

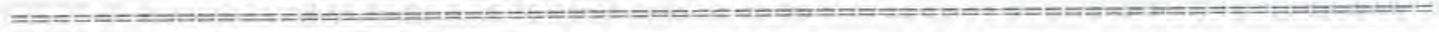

$\begin{array}{lcc}\text { Sorted By } & : & \text { Signal } \\ \text { Calib. Data Modified } & : & \text { Tuesday, September 25, } 2007 \text { 9:21:18 AM } \\ \text { Multiplier } & : & 1.0000 \\ \text { Dilution } & : & 1.0000 \\ \text { Use Multiplier \& Dilution Factor with ISTDs }\end{array}$

SignaI 1: ADC1 A, Dionex ED40

\begin{tabular}{|c|c|c|c|c|c|}
\hline $\begin{array}{l}\text { RetTime } \\
\text { [min] }\end{array}$ & Type & $\begin{array}{r}\text { Area } \\
{\left[u s^{*} s\right]}\end{array}$ & Amt/Area & $\begin{array}{l}\text { Anount } \\
\text { [ug/mL] }\end{array}$ & Name \\
\hline 10.078 & $\mathrm{BB}$ & 248.84175 & $1,81492 e-2$ & A. 51627 & Sulfate \\
\hline Totals & & & & 4.51627 & \\
\hline
\end{tabular}

Results obtained with enhanced integrator!

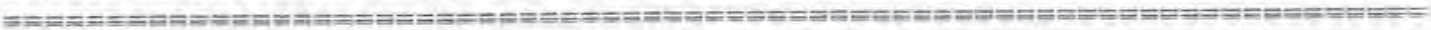

$* * *$ End of Report ***

Page 46 of 53 


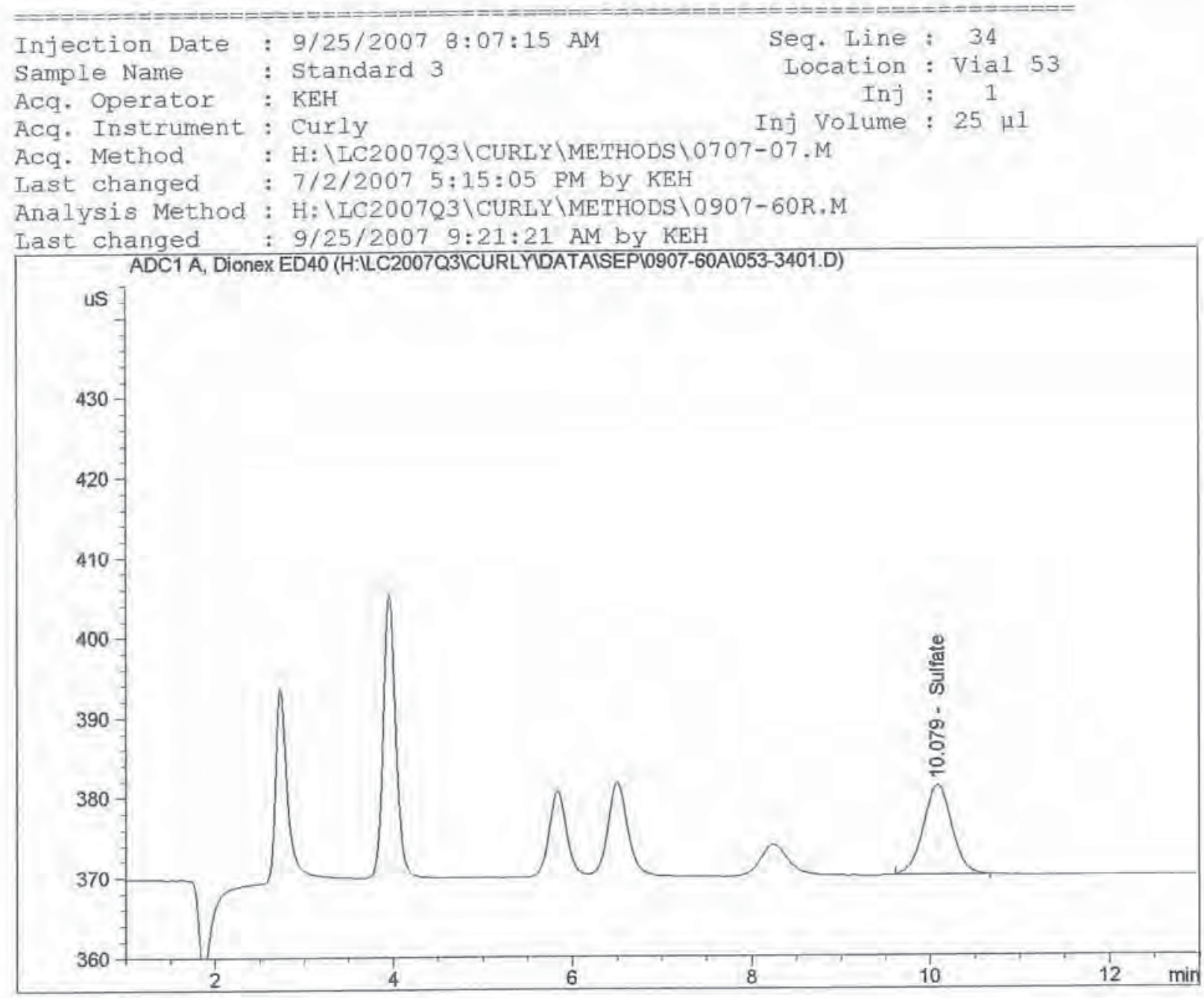

External Standard Report

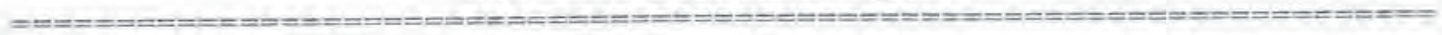

$\begin{array}{lll}\text { Sorted By } & : & \text { Signal } \\ \text { Calib. Data Modified } & : & \text { Tuesday, September 25, 2007 9:21:18 AM } \\ \text { Multiplier } & : & 1.0000 \\ \text { Dilution } & : & 1.0000\end{array}$

Use Multiplier \& Dilution Factor with ISTDs

Signal 1: ADC1 A, Dionex ED4O

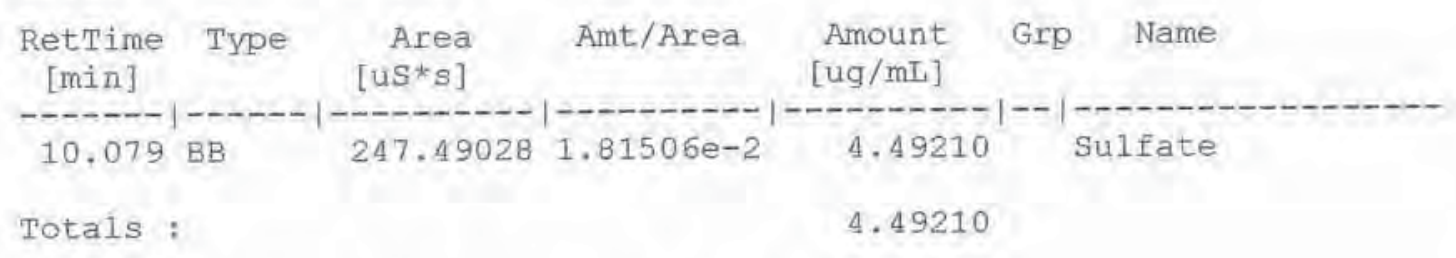

Results obtained with enhanced integrator!

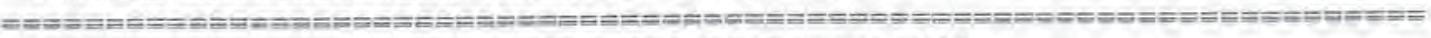

$* * *$ End of Report *** 

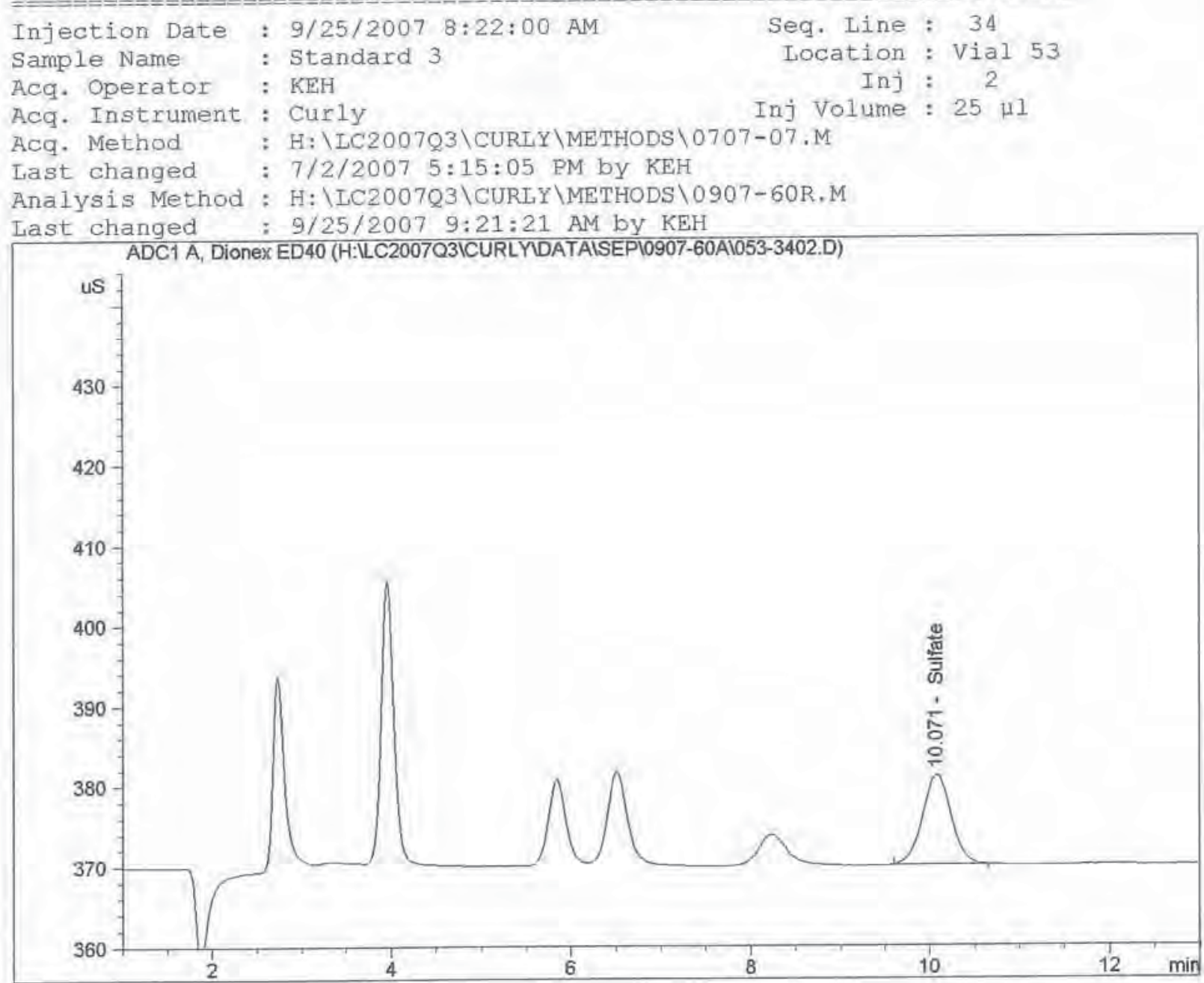

External Standard Report

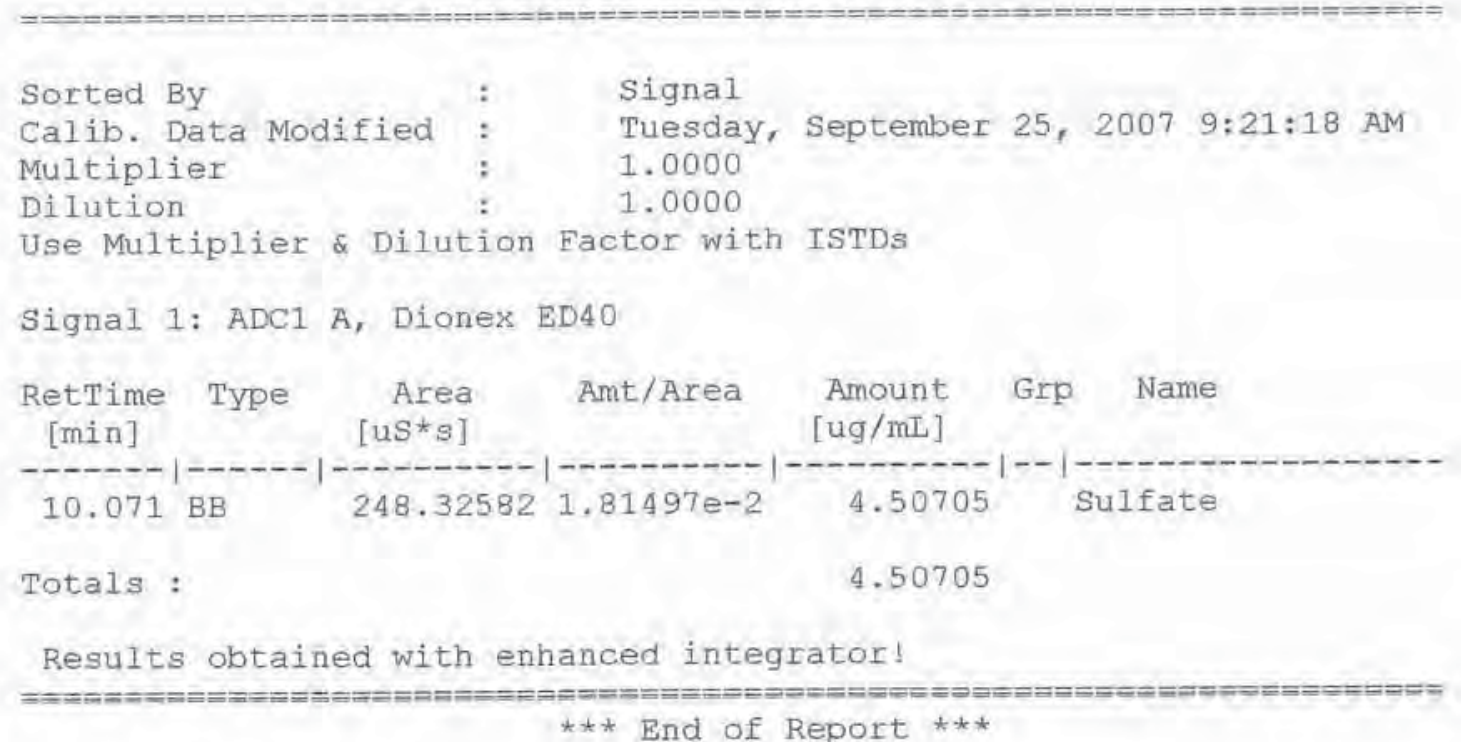


Method Information

Column: Dionex IonPac AS14A (250 mm x $4 \mathrm{~mm}$ )

Mobile Phase: 8mM Na2CO3/ImMNAHCO3

Detection: Suppressed Conductivity

Flow Rate: $1.2 \mathrm{mI} / \mathrm{min}$

Temp: $30 \mathrm{C}$

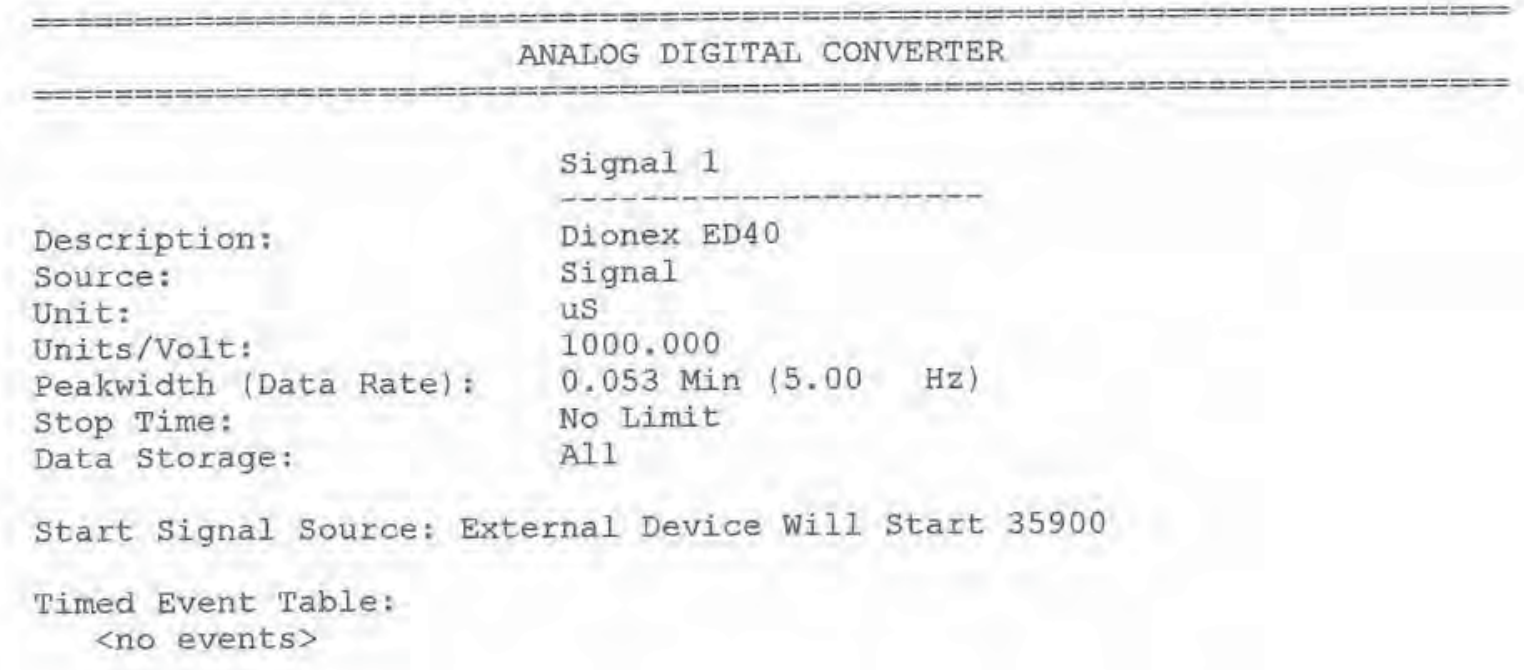




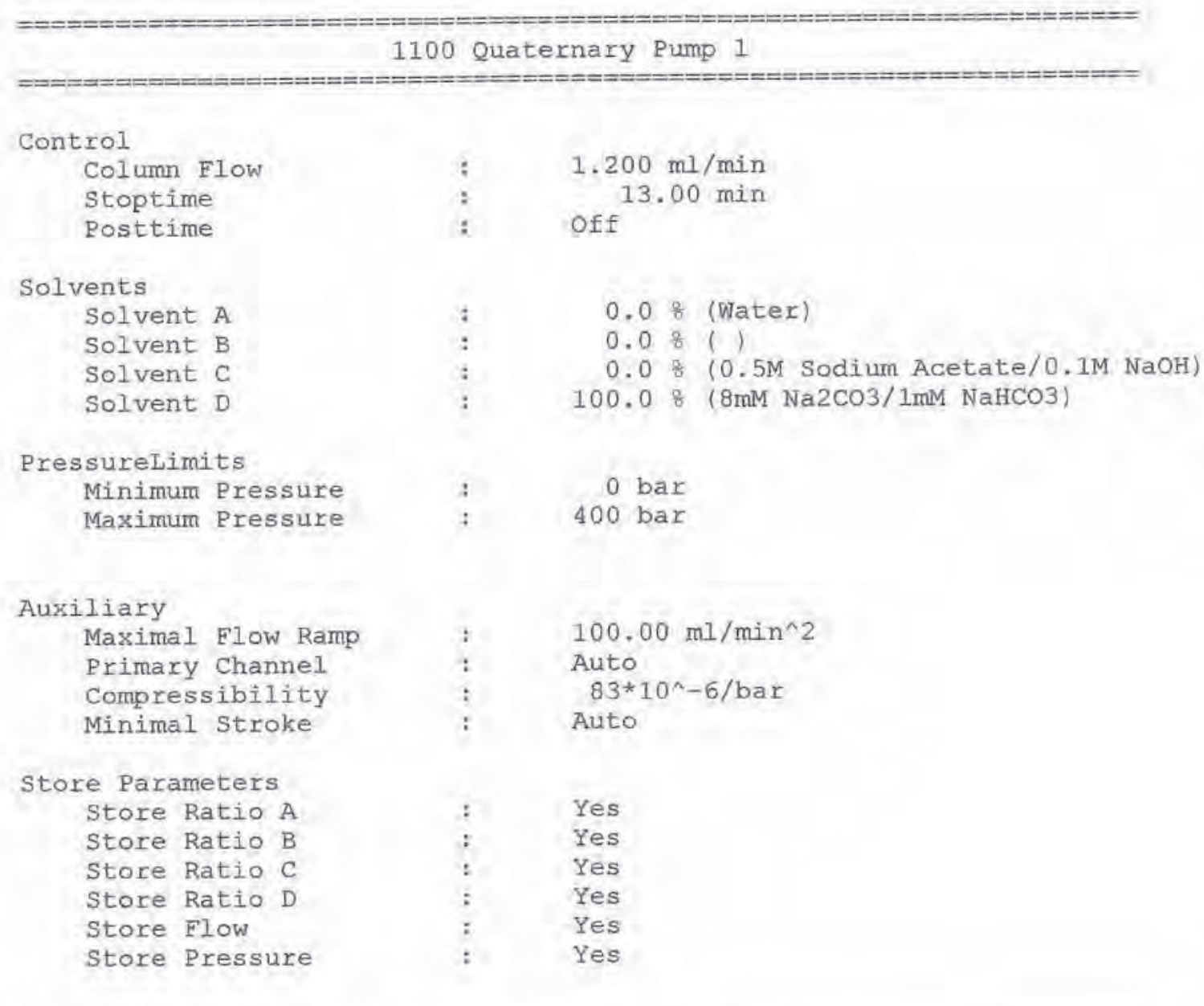

Agilent 1100 Autosampler 1

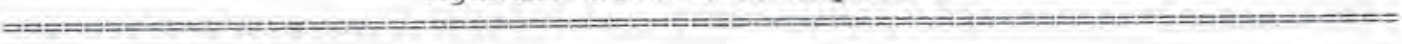

Injection

Injection Mode : Needle Wash

Injector volume : $\quad 25.00 \mu 1$

Wash Vial

Optimization

100

none

Auxiliary

Drawspeed

Ejectspeed

Draw position

$100 \mathrm{\mu l} / \mathrm{min}$

$1000 \mathrm{\mu l} / \mathrm{min}$

$2.0 \mathrm{rm}$

Time

Stoptime : : As Pump

posttime : off

Agilent 1100 Column Thermostat 1

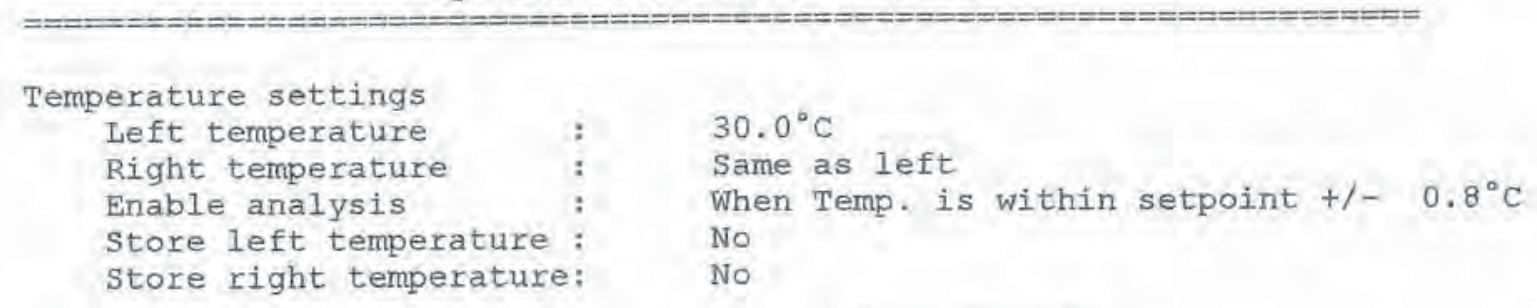

Time Stoptime As pump

\section{Page 50 of 53}


Method: H: \LC200703\CURLY\METHODS\0707-07.M of 7/2/2007 2:15:05. PM

Posttime : off

Column Switching Valve : Column I

Page 51 of 53 


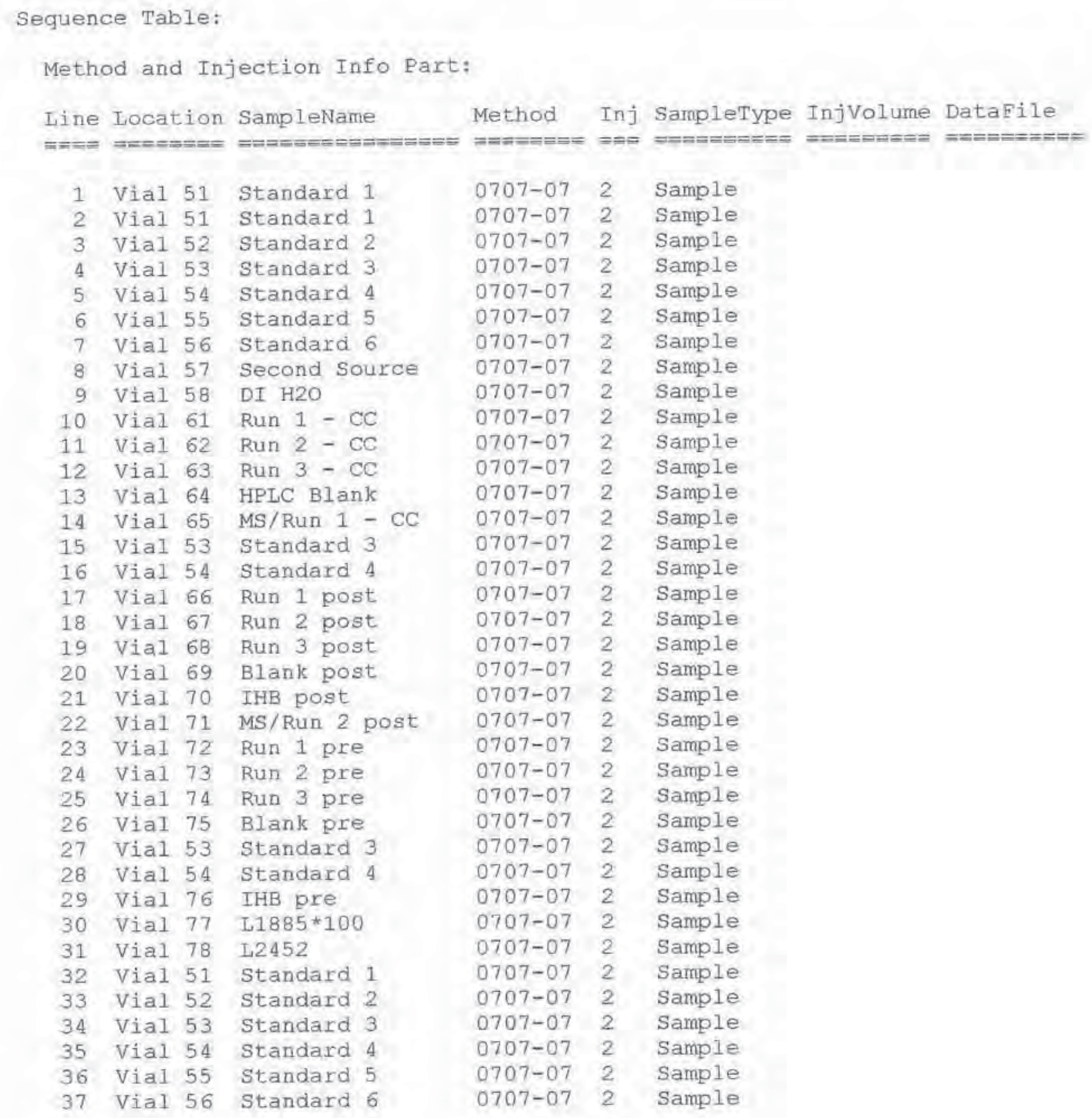




\section{This Is The Last Page Of This Report.}

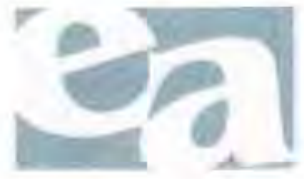


Appendix G:

Chain of Custodies 

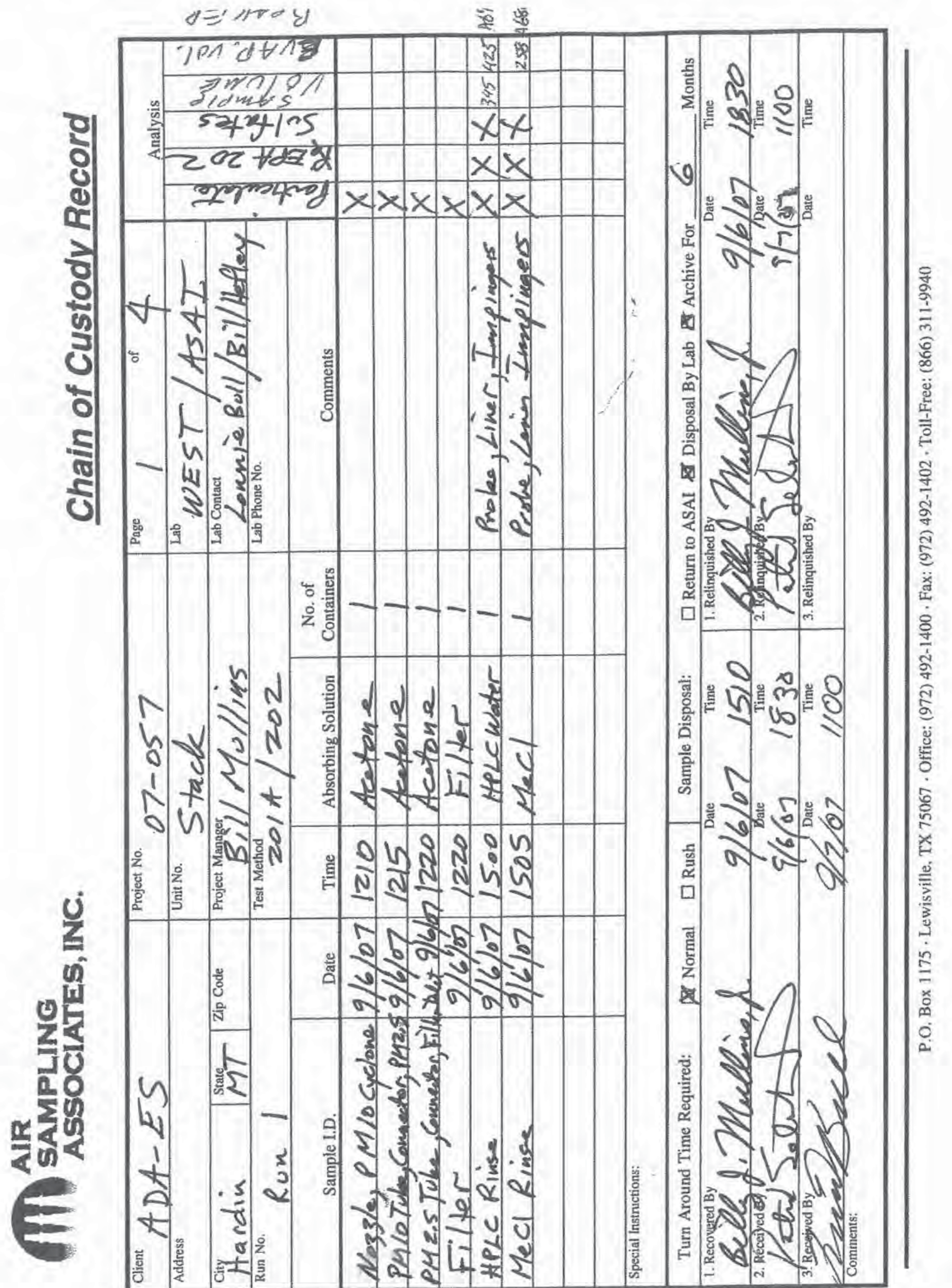


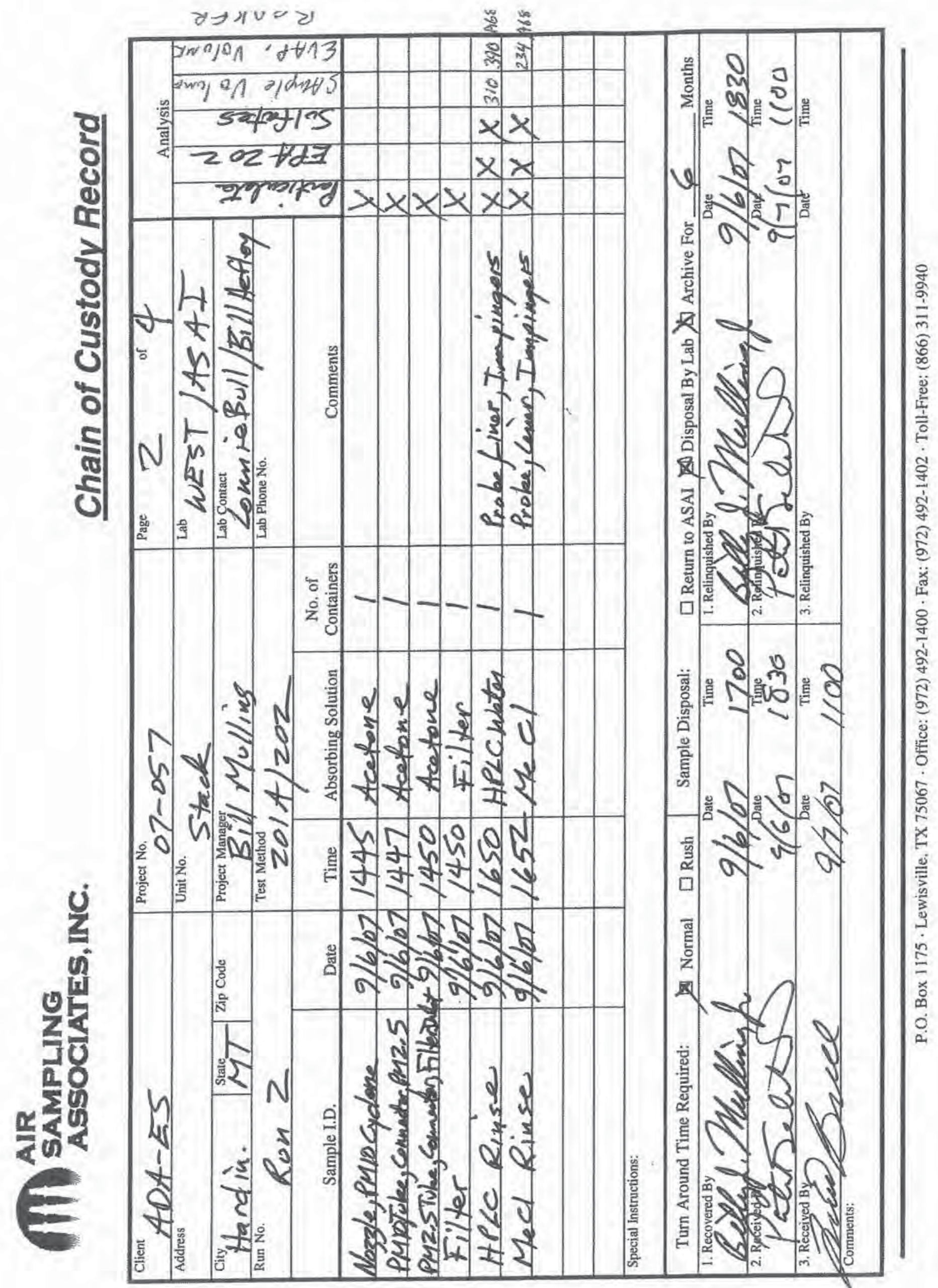



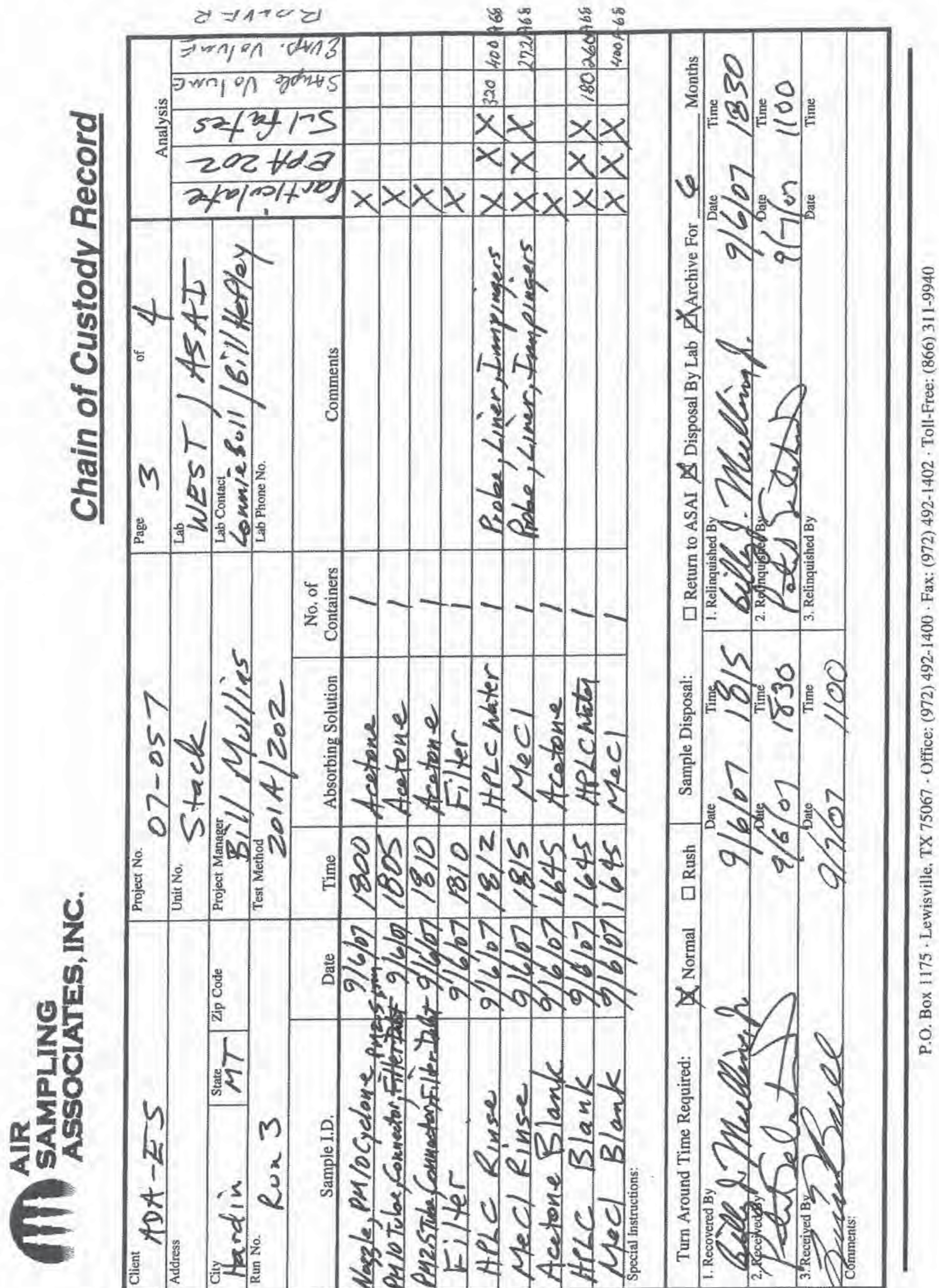


\section{Appendix H:}

\section{Resumes of Test Personnel}


BILLY J. MULLINS, JR.; President

Education

Post Graduate Study Environmental Engineering at Southern Methodist University; Dallas, Texas 1970.

M.S. 1969, New York University; New York, New York, in Civil Engineering (Air Resources).

B.S. 1968, Texas Tech University; Lubbock, Texas, in Civil Engineering (Water Resources). Studies in Engineering at the U.S. Naval Academy; Annapolis, Maryland, 1963-1964

Professional Attended Short Course on Air Pollution Engineering at the University

Training of Texas at Austin, February 1970.

$\underline{\text { Courses }}$

Attended four-week management course presented by the American Management Association, 1976.

Certification Registered Professional Engineer

Certified Visible Emissions Evaluator

Licensed Private Pilot (Multi-Engine-Land, Instrument)

Diplomat in the American Academy of Environmental Engineers

Inductee into the Stack Sampling Hall of Fame

Certified as Qualified Environmental Professional (QEP)

Professional Air \& Waste Management Association - Past Chairman, Past Vice Memberships Chairman, and Past Board of Directors of North Texas Chapter and Southwest Section; Past Chairman, Consultants Committee; Past Chairman, Source Measurement Committee

Source Evaluations Society - Past President, Past Board of Directors

\section{American Management Association}


MULLINS (p. 2)

Publications Authored "Real World Experience with USEPA's New Sampling and Analytical Methods for Conducting Risk Burn," May 1998.

Co-authored "Sulfur Compound Emissions of the Petroleum Production Industry," December 1974.

Co-authored "Field Procedure for Stabilizing Hydrogen Sulfide Samples to be Analyzed Using Modified Methylene Blue Technique," presented at the Conference on Ambient Air Quality Measurements, Austin, Texas, March 1975.

Co-authored "Atmospheric Emissions Survey of the Sour Gas Industry," October 1975.

Co-authored "Technique for Insuring the Validity of Samples for High Concentrations of Sulfur Dioxide Using the EPA Method 5 Sampling Train," presented at the Third National Conference on Energy and the Environment, College Corner, Ohio, September 1975.

Teaching Experience
Conducted training seminars on sampling methods periodically since 1974 to present.

Conducted a one-day seminar on Part 75 Testing over ten times in 1993 and 1994.

Served as a lecturer in the Environmental Protection Agency's (EPA) training course number 450, "Source Sampling for Particulate Pollutants," for two years from January 1974 to October 1975 and March, 1992.

Conducted a two-day training course entitled "technical Assistance in Source Sampling" at lowa State University, Ames, lowa, for the Environmental Protection Agency (EPA), October 1974.

Conducted Environmental Protection Agency's (EPA) training course number 450, "Source Sampling for Particulate Pollutants," at Research Triangle Park, North Carolina, September 1975. 
MULLINS (p. 3)

Teaching

Experience

(Cont'd)
Technical

Experience
Conducted a two-day short course entitled "Performing and Observing Source Sampling," Dallas, Texas, July 1976, May 1977, October 1977, November 1987 and November 1988; Lake Charles, Louisiana, May 1977; Casper Wyoming, May 1977; Point Comfort, Texas, November 1992.

Served as a lecturer in the Environmental Protection Agency's twoday seminar entitled "Asphalt Industry Environmental Solutions," presented in Dallas, Texas, March 21-22, 1979.

Conducted a two-day short course entitled "Performing and Observing Source Sampling," Phoenix, Arizona, August, 1990, for the State of Arizona, Department of Environmental Quality; Lincoln, Nebraska, March 1980, for the State of Nebraska, Air Quality Control Division.

Directed and performed stack sampling on over 2000 sources of which over 500 were sampled simultaneously using more than one sampling train at several points in the flue gas stream; 1972-present.

Directed and performed over 200 short-term ambient air studies using mobile sampling vans and various ambient air sampling equipment; 1972-present.

Designed, directed and operated over 20 permanent ambient air networks of various size and duration for a variety of parameters; 1972-present.

Designed surface and underground drainage systems for residential subdivisions, public works projects, and shopping centers; 19691972.

Designed several residential subdivisions including lot layout, street design, drainage design, and utility design; 1969-1972. 
MULLINS (p. 4)

Research

Projects

Related

$\underline{\text { Projects }}$
Supervised and conducted a study made by the Hawaiian Sugar Planters' Association to characterize the emissions for several bagasse-fired boilers, April-May 1976.

Supervised and conducted a study made by the Rio Grande Valley Sugar Growers, Inc. to determine the area affected by the burning of sugarcane fields prior to harvesting, November 1974-April 1975.

Supervised and conducted a study by a lightweight aggregate manufacturer to develop a material balance around the process through sampling and analysis of several parameters, November 1973.

Conducted a study in New York City to attempt to develop a correlation in the ambient air between carbon dioxide and sulfur dioxide to provide a tool for predicting air pollution predicting air pollution episodes, January-May 1969.

Served as Chairman of the Engineering Foundation Conference on Stack Sampling and Source Evaluation in Destin, Florida, 2002, and Santa Barbara, California, 1985.

Served as Co-Chairman of the Engineering Foundation Conference on Stack Sampling and Source Evaluation in Destin, Florida, 2001.

Served as Session Chairman at the Engineering Foundation Conference on Stack Sampling and Source Evaluation in Hershey, Pennsylvania, 1984; San Diego, California, 1993; and in Palm Coast, Florida, 1994. 


\section{PATRICK SELAKOVICH; Associate}

Education

Professional

Training

Professional

Memberships

Technical

Experience
B.S.B.A. 1992, University of Arkansas; Fayetteville, Arkansas, in General Business.

Attended 24-hour Occupational and Environmental Training Program on Hazardous Materials (CFR 1910.120) Dallas, Texas, April 1997.

Attended 40-hour Occupational and Environmental Training Program on Hazardous Materials (CFR 1910.120) \& 'Train the Trainer', Gettysburg, Pennsylvania, July 1998.

Also attended an 8-hour refresher course January 2000, January 2001, and May 2004.

Attended OSHA General Industry Safety and Health Training, May 1999.

\section{Society For Human Resource Management}

Participated in the sampling of over 150 sources, including several of which were sampled simultaneously using more than one sampling train. Thoroughly trained in all EPA testing procedures, 1996present.

Participated in EPA's 3-D probe study. May - August 1997.

Experience with calibration techniques for all field testing equipment.

Thoroughly trained in the operation and routine maintenance of the following:

California Analytical Model 300-HFID Total Hydrocarbon Analyzer Servomex Model 1440 Carbon Dioxide Analyzer

Servomex Model 1440 Oxygen Analyzer

Thermo Electron Model 46C Oxides of Nitrogen Analyzer Thermo Electron Model 48C Oxides of Nitrogen Analyzer Western Research Model 721A Sulfur Dioxide Analyzer 
SCOT JACKSON; Associate

Education

Professional

Training

Certification

Technical

Experience
B.S.B.A. May 1978, Mountain View Jr. College, in General Business.

Purchasing Supervisor for METCO Environmental, Inc. in charge of inventory and supplies. January 1995 - April 2005.

Attended 40-hour Occupational and Environmental Training Program on Hazardous Materials (CFR 1910.120), Dallas, Texas, May 2000.

Attended Fed-Ex Hazardous Goods Shipping Training, June 2004.

\section{Certified Visible Emissions Evaluator}

Participated in the sampling of over 100 sources, including several of which were sampled simultaneously using more than one sampling train. Thoroughly trained in all EPA testing procedures, 1995present.

Experience with calibration techniques for all field testing equipment.

Thoroughly trained in the operation and routine maintenance of the following:

California Analytical Model 300-HFID Total Hydrocarbon Analyzer Servomex Model 1440 Carbon Dioxide Analyzer Servomex Model 1440 Oxygen Analyzer

Thermo Electron Model 42C Oxides of Nitrogen Analyzer Thermo Electron Model 48C Oxides of Nitrogen Analyzer Western Research Model 721A Sulfur Dioxide Analyzer 
JOHN STANLEY; Associate

Education

B.A. June 1990, Hendrix College, in Business and Economics.

Technical

Participated in the sampling of over 25 sources, including several of

Experience which were sampled simultaneously using more than one sampling train.

Experience with calibration techniques for all field testing equipment.

Thoroughly trained in the operation and routine maintenance of the following:

California Analytical Model 300-HFID Total Hydrocarbon Analyzer Servomex Model 1440 Carbon Dioxide Analyzer

Servomex Model 1440 Oxygen Analyzer

Thermo Electron Model 42C Oxides of Nitrogen Analyzer Thermo Electron Model 48C Oxides of Nitrogen Analyzer Western Research Model 721A Sulfur Dioxide Analyzer 


\section{STACK SAMPLING REPORT FOR}

PARTICULATE MATTER, HYDROGEN HALIDE \& HALOGEN, AMMONIA, PM10/2.5, AND SULFUR TRIOXIDE TESTING ON THE COLORADO ENERGY MANAGEMENT, LLC HARDIN POWER PLANT SDA INLET DUCT AND STACK

HARDIN, MONTANA

PROJECT NO. 08-026

SEPTEMBER 2008

PREPARED FOR:

ADA-ES, INC. 8100 SOUTHPARK WAY

LITTLETON, CO 80120

PREPARED BY:

AIR SAMPLING ASSOCIATES, INC.

P.O. BOX 1175

LEWISVILLE, TEXAS 75067

(Total Number of pages: 522 ) 


\section{TABLE OF CONTENTS}

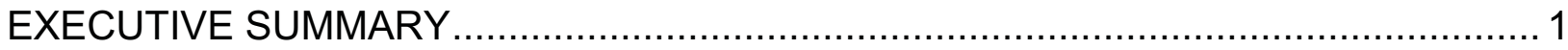

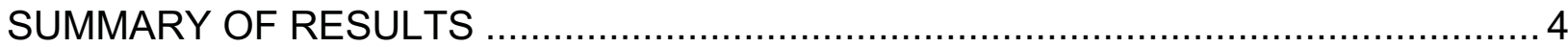

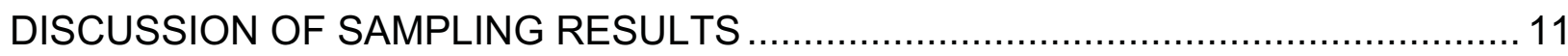

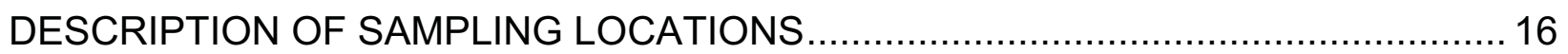

SAMPLING AND ANALYTICAL PROCEDURES …............................................. 19

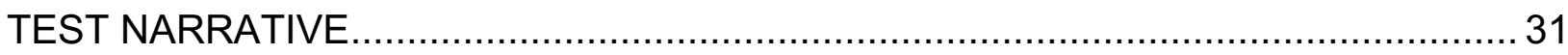

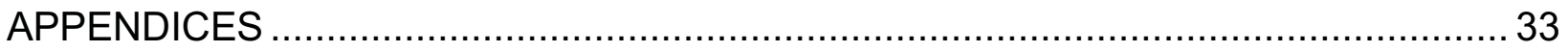

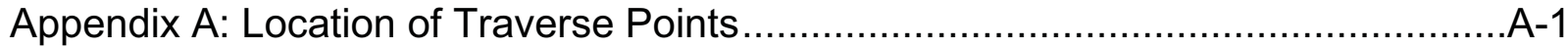

Appendix B: Nomenclature and Equations for Calculation of Source Emissions.......B-1

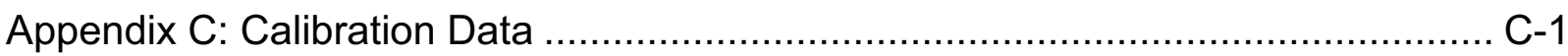

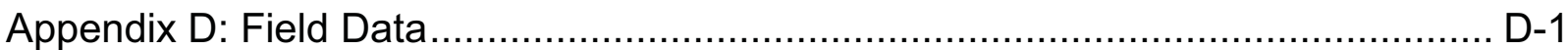

Appendix E: EPA Test Methods 5B and 202 (Particulate Matter) Analytical Data -

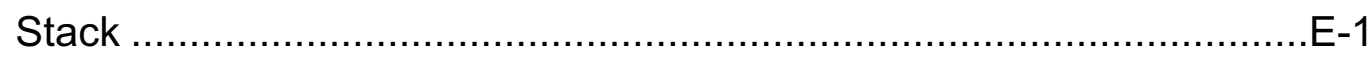

Appendix F: EPA Test Methods 8A, 26A, and CTM-027 (Sulfur Trioxide, Hydrogen Halides \& Halogens, and Ammonia) Analytical Data - SDA Inlet Duct and Stack $\mathrm{F}-1$

Appendix G: EPA Test Method 17 (Particulate Matter) Analytical Data - SDA Inlet

Duct G-1

Appendix H: EPA Test Methods 201A and 202 (PM10/2.5) Analytical Data- Stack . H-1 Appendix I: Chain of Custodies ...................................................................

Appendix J: Resumes of Test Personnel............................................................ 


\section{TABLES}

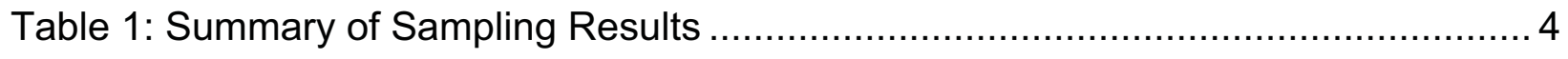

Table 2: Summary of PM10/2.5 Sampling Results - Stack ........................................... 4

Table 3: SDA Inlet Duct Particulate Matter, Hydrogen Halides \& Halogens, and Ammonia Summary of Sampling Results ................................................. 5

Table 4: SDA Inlet Duct Sulfur Trioxide Summary of Sampling Results ......................... 6

Table 5: Stack Particulate Matter Summary of Sampling Results ................................. 7

Table 6: Stack Hydrogen Halides \& Halogens Summary of Sampling Results .............. 8

Table 7: Stack Sulfur Trioxide Summary of Sampling Results ................................... 9

Table 8: Summary of Particulate Matter (PM10/2.5) Sampling Results........................ 10

Table 9: EPA Test Methods 5B / 202 Sampling Train Condenser/Absorbing System.. 20

Table 10: EPA Test Method 26A Condenser/Absorbing System ...............................2 23

Table 11: Reference Method 202 Sampling Train ................................................. 27

Table 12: NCASI Method 8A Condenser/Absorbing System...................................... 29

Table 13: Location of Velocity Traverse Points Boiler Stack .....................................-3

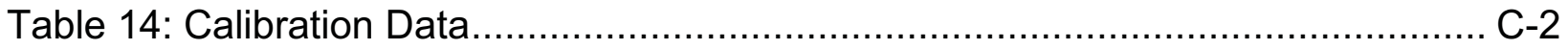




\section{FIGURES}

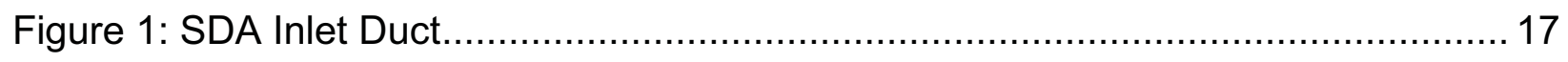

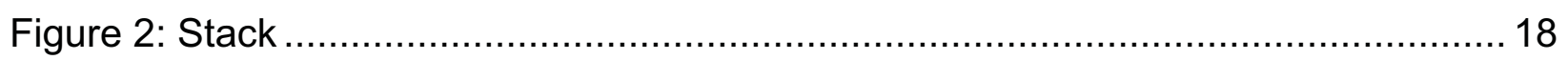

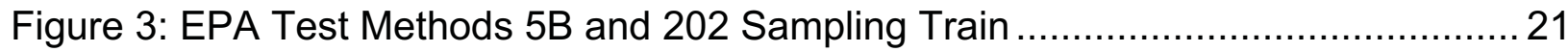

Figure 4: EPA Test Methods 17, 26A, and CTM-027 SDA Inlet Duct Sampling Train... 24

Figure 5: EPA Test Method 26A Stack Sampling Train - Stack................................... 25

Figure 6: EPA Methods 201A and 202 Sampling Train ......................................... 28

Figure 7: NCASI Method 8A SDA Inlet Duct and Stack Sampling Train........................ 30

Figure 8: Location of Traverse Points - SDA Inlet Duct ..........................................A-2

Figure 9: Location of Traverse Points - Stack ................................................... 


\section{EXECUTIVE SUMMARY}

Air Sampling Associates, Inc. of Lewisville, Texas conducted stack testing at the Colorado Energy Management, LLC, Hardin Power Plant, located in Hardin, Montana. The purpose of the stack testing was to determine the particulate matter emissions, hydrogen halide and halogen emissions, ammonia emissions, and sulfur trioxide emissions at the SDA Inlet Duct; and to determine the particulate matter emissions, hydrogen halide and halogen emissions, sulfur trioxide emissions, and PM10/2.5 emissions at the Stack. The testing was conducted on September 22, 23, and 24, 2008.

The sampling team consisted of Mr. Bill Mullins, Mr. Bill Hefley, Mr. Patrick Selakovich, Mr. John Stanley, and Mr. Dan Bert. Mr. Mullins was the test team leader.

Mr. Jerry Amrhein of ADA-ES was the project manager.

The procedures set forth in Title 40 of the Code of Federal Regulations, Part 60 (40CFR60), Appendix A, Test Methods 1, 2, 3B, 4, 5B, 17, 26A, 201A, 202, and Conditional Test Method 027 (CTM-027) and the National Council of the Paper Industry for Air and Stream Improvement, Inc. (NCASI) Method 8A were followed during testing.

Triplicate samples for particulate matter collected at the SDA Inlet Duct according to EPA Test Method 17, in conjunction with EPA Test Methods 26A and CTM-027, indicated an average of $11,786.20$ pounds per hour, based on the 'front half' analysis of the sampling train.

Triplicate samples for hydrogen halides \& halogens collected at the SDA Inlet Duct according to EPA Test Method 26A, in conjunction with EPA Test Method 17 and CTM027, indicated an average concentration of hydrogen bromide and an average concentration of bromine that were below the detectable limit of the sampling 08-026 


\section{II}

procedure. The sampling results indicated an average concentration of hydrogen chloride that was equal to $0.914 \mathrm{mg} / \mathrm{dscm}$ and an average concentration of chlorine that was equal to $0.836 \mathrm{mg} / \mathrm{dscm}$. The sampling results indicated an average concentration of hydrogen fluoride and an average concentration of fluoride that were below the detectable limit of the sampling procedure.

Triplicate samples for ammonia collected at the SDA Inlet Duct according to EPA CTM027, in conjunction with EPA Test Methods 17 and 26A, indicated an average concentration of ammonia equal to $57.34 \mathrm{ppm}$. The amount of ammonia that was injected into the process was reduced during each test.

Triplicate samples for sulfur trioxide collected at the SDA Inlet Duct according to NCASI Method $8 \mathrm{~A}$ indicated an average concentration of sulfur trioxide equal to $5.99 \mathrm{ppm}$.

Triplicate samples for particulate matter collected at the Stack according to EPA Test Methods 5B and 202 indicated an average of 20.07 pounds per hour, based on the analysis of the 'front half' of the sampling trains. The "back-half" of the sampling trains indicated a relatively large amount of particulate matter that had not been seen in previous testing. The analysis of the "back-half" particulate matter samples is suspect. Visual observations in the field indicated that the impinger solutions were clear with no cloudiness. The samples were purged for one-hour after the tests with pure nitrogen. Also, there were no visible emissions from the stack during the testing that would have indicated the level of emissions that were reported.

Triplicate samples for hydrogen halides \& halogens collected at the Stack indicated an average concentration of hydrogen bromide and an average concentration of bromine that were below the detectable limit of the sampling procedure. The sampling results indicated an average concentration of hydrogen chloride that was below the detectable limit of the sampling procedure for one test. However, the hydrogen chloride results for the other two tests indicated a concentration of hydrogen chloride that was equal to an 08-026 
average of $0.412 \mathrm{mg} / \mathrm{dscm}$. The sampling results indicated an average concentration of chlorine that was equal to $0.474 \mathrm{mg} / \mathrm{dscm}$. The sampling results indicated an average concentration of hydrogen fluoride and an average concentration of fluoride that were below the detectable limit of the sampling procedure.

Triplicate samples for particulate matter were collected at the Stack according to EPA Test Methods 201A and 202.

The average emission rate of particulate matter $>\mathrm{PM}_{10}$ was 0.0044 grains per dry standard cubic foot (gr/dscf). The average emission rate of particulate matter $>\mathrm{PM}_{10}$ was $10.76 \mathrm{lbs} / \mathrm{hr}$.

The average emission rate of particulate matter $<\mathrm{PM}_{10}>\mathrm{PM}_{2.5}$ was 0.0031 grains per dry standard cubic foot ( $\mathrm{gr} / \mathrm{dscf})$. The average emission rate of particulate matter < $\mathrm{PM}_{10}>\mathrm{PM}_{2.5}$ was $7.70 \mathrm{lbs} / \mathrm{hr}$.

The average emission rate of particulate matter $\mathrm{PM}_{2.5}$ was 0.0022 grains per dry standard cubic foot (gr/dscf). The average emission rate of particulate matter $\mathrm{PM}_{2.5}$ was $5.27 \mathrm{lbs} / \mathrm{hr}$.

The average emission rate of particulate matter total catch was 0.0656 grains per dry standard cubic foot (gr/dscf). The average emission rate of particulate matter total catch was $160.68 \mathrm{lbs} / \mathrm{hr}$.

Triplicate samples for sulfur trioxide collected at the Stack according to NCASI Method $8 \mathrm{~A}$ indicated an average concentration of sulfur trioxide equal to $1.22 \mathrm{ppm}$. 


\section{SAMpuing \\ ASSOCIATES, INC.}

\section{SUMMARY OF RESULTS}

Table 1: Summary of Sampling Results

\begin{tabular}{|l|c|c|}
\hline $\begin{array}{c}\text { Sampling Trains } \\
\text { - Parameters }\end{array}$ & $\begin{array}{c}\text { SDA Inlet Duct } \\
\text { Average }\end{array}$ & $\begin{array}{c}\text { Stack } \\
\text { Average }\end{array}$ \\
\hline EPA 5 / 17 / 26A / CTM027 & 11.12 & 15.50 \\
- Moisture (\%) & 293,196 & 285,170 \\
- Flow Rate (DSCFM) & $11,786.20$ & 20.07 \\
- Particulate Matter (Ibs/hr - front half) & 0.836 & 0.474 \\
- Chlorine (mg/dscm) & 0.914 & $<0.285$ \\
- Hydrogen Chloride (mg/dscm) & 54.15 & NA \\
- Ammonia (ppm) & & 15.66 \\
\hline NCASI 8A & 10.50 & 1.22 \\
- Moisture (\%) & 5.99 & \\
- Sulfur Trioxide (ppm) & & \\
\hline
\end{tabular}

Table 2: Summary of PM10/2.5 Sampling Results - Stack

\begin{tabular}{|l|c|}
\hline Parameter & \\
- Results & Average \\
\hline Particulate Matter (> PM 10$)$ & \\
- gr/dscf & 0.0044 \\
- Ibs/hr & 10.76 \\
\hline Particulate Matter (< PM 10 > PM 2.5$)$ & \\
- gr/dscf & 0.0031 \\
- Ibs/hr & 7.70 \\
\hline Particulate Matter $\left(\mathbf{P M}_{2.5}\right)$ & \\
- gr/dscf & 0.0022 \\
- Ibs/hr & 5.27 \\
\hline Particulate Matter (Total Catch) & \\
- gr/dscf & 0.0656 \\
- Ibs/hr & 160.68 \\
\hline
\end{tabular}




\section{SAMPLING $_{\text {ASSOCIATES,INC. }}^{\text {AlR }}$}

\section{SUMMARY OF RESULTS}

Table 3: SDA Inlet Duct Particulate Matter, Hydrogen Halides \& Halogens, and Ammonia Summary of Sampling Results

\begin{tabular}{|c|c|c|c|c|}
\hline Run No. & 1 & 2 & 3 & Average \\
\hline Test Date & $09 / 23 / 08$ & $09 / 23 / 08$ & $09 / 23 / 08$ & ---- \\
\hline Test Time & 0910-1013 & $1250-1354$ & $1744-1848$ & ---- \\
\hline Flow Rate-DSCFM* & 291,213 & 293,577 & 294,797 & 293,196 \\
\hline Stack Temp. - ${ }^{\circ} \mathrm{F}$ & 336 & 333 & 330 & 333 \\
\hline $\mathrm{O}_{2}-\%$ Vol. (dry) & 5.9 & 5.3 & 5.6 & 5.6 \\
\hline $\mathrm{CO}_{2}$ - \% Vol. (dry) & 13.4 & 14.2 & 14.1 & 13.9 \\
\hline Percent Excess Air & 38.1 & 33.1 & 35.7 & 35.6 \\
\hline Moisture Content - \% & 11.41 & 10.67 & 11.28 & 11.12 \\
\hline Percent Isokinetic & 108.3 & 97.4 & 97.6 & 101.1 \\
\hline Sample Volume - DSCM & 0.772 & 0.700 & 0.704 & 0.725 \\
\hline $\begin{array}{l}\text { Particulate Matter } \\
\text { - gr/dscf (Front Half) } \\
\text { - Ibs/hr (Front Half) }\end{array}$ & $\begin{array}{c}3.8488 \\
9,605.40\end{array}$ & $\begin{array}{c}4.8056 \\
12,090.55\end{array}$ & $\begin{array}{c}5.4079 \\
13,662.65\end{array}$ & $\begin{array}{c}4.6874 \\
11,786.20\end{array}$ \\
\hline $\begin{array}{l}\text { Hydrogen Bromide } \\
-\mathrm{mg} \\
-\mathrm{mg} / \mathrm{dscm}\end{array}$ & $\begin{array}{l}<0.022 \\
<0.029\end{array}$ & $\begin{array}{l}<0.024 \\
<0.035\end{array}$ & $\begin{array}{l}<0.027 \\
<0.038\end{array}$ & $\begin{array}{l}<0.024 \\
<0.034\end{array}$ \\
\hline $\begin{array}{l}\text { Bromine } \\
-m g \\
-m g / d s c m\end{array}$ & $\begin{array}{l}<0.077 \\
<0.099\end{array}$ & $\begin{array}{l}<0.081 \\
<0.115\end{array}$ & $\begin{array}{l}<0.081 \\
<0.115\end{array}$ & $\begin{array}{l}<0.080 \\
<0.1110\end{array}$ \\
\hline $\begin{array}{l}\text { Hydrogen Chloride } \\
-m g \\
-m g / d s c m\end{array}$ & $\begin{array}{l}1.118 \\
1.448\end{array}$ & $\begin{array}{l}0.317 \\
0.453\end{array}$ & $\begin{array}{l}0.592 \\
0.841\end{array}$ & $\begin{array}{l}0.676 \\
0.914\end{array}$ \\
\hline $\begin{array}{l}\text { Chlorine } \\
-m g \\
-m g / d s c m\end{array}$ & $\begin{array}{l}0.790 \\
1.023\end{array}$ & $\begin{array}{l}0.126 \\
0.180\end{array}$ & $\begin{array}{l}0.918 \\
1.304\end{array}$ & $\begin{array}{l}0.611 \\
0.836\end{array}$ \\
\hline $\begin{array}{l}\text { Hydrogen Fluoride } \\
\text { - mg } \\
-m g / d s c m\end{array}$ & $\begin{array}{l}<0.012 \\
<0.016\end{array}$ & $\begin{array}{l}<0.013 \\
<0.019\end{array}$ & $\begin{array}{l}<0.055 \\
<0.078\end{array}$ & $\begin{array}{l}<0.027 \\
<0.038\end{array}$ \\
\hline Ammonia - ppm & 96.04 & 47.98 & 28.00 & 57.34 \\
\hline
\end{tabular}

* 29.92" Hg, $68^{\circ} \mathrm{F}\left(760 \mathrm{~mm} \mathrm{Hg}, 20^{\circ} \mathrm{C}\right)$ 


\section{SUMMARY OF RESULTS}

Table 4: SDA Inlet Duct Sulfur Trioxide Summary of Sampling Results

\begin{tabular}{|l|c|c|c|c|}
\hline Run No. & 1 & 2 & 3 & Average \\
\hline Test Date & $09 / 23 / 08$ & $09 / 24 / 08$ & $09 / 24 / 08$ & ---- \\
Test Time & $1530-1625$ & $0745-0845$ & $0947-1047$ & ---- \\
Sample Volume - DSCM* & 0.480 & 0.532 & 0.529 & 0.514 \\
Moisture Content - \% & 10.42 & 10.82 & 10.26 & 10.50 \\
Sulfur Trioxide - ppm & 3.51 & 12.99 & 1.48 & 5.99 \\
\hline
\end{tabular}

* 29.92" Hg, $68^{\circ} \mathrm{F}\left(760 \mathrm{~mm} \mathrm{Hg}, 20^{\circ} \mathrm{C}\right)$ 


\section{ARR \\ ASSOCIATES, INC.}

\section{SUMMARY OF RESULTS}

Table 5: Stack Particulate Matter Summary of Sampling Results

\begin{tabular}{|c|c|c|c|c|}
\hline Run No. & 1 & 2 & 3 & Average \\
\hline Test Date & $09 / 23 / 08$ & $09 / 23 / 08$ & $09 / 24 / 08$ & ---- \\
\hline Test Time & 1058-1302 & $1551-1756$ & 0710-0914 & ---- \\
\hline Flow Rate-DSCFM ${ }^{*}$ & 287,645 & 282,484 & 285,381 & 285,170 \\
\hline Stack Temperature - ${ }^{\circ} \mathrm{F}$ & 206 & 204 & 210 & 207 \\
\hline $\mathrm{O}_{2}-\%$ Volume dry & 6.2 & 6.0 & 6.2 & 6.1 \\
\hline $\mathrm{CO}_{2}-\%$ Volume dry & 13.4 & 13.7 & 13.4 & 13.5 \\
\hline Percent Excess Air & 41.0 & 39.3 & 41.0 & 40.4 \\
\hline Moisture Content - \% & 15.36 & 15.22 & 15.91 & 15.50 \\
\hline Percent Isokinetic & 98.5 & 100.2 & 103.2 & 100.6 \\
\hline $\begin{array}{l}\text { Particulate Matter } \\
\text { - gr/dscf (Front Half) } \\
\text { - Ibs/mmBtu (Front Half)** } \\
\text { - Ibs/hr (Front Half) }\end{array}$ & $\begin{array}{c}0.0073 \\
0.0145 \\
17.94\end{array}$ & $\begin{array}{c}0.0079 \\
0.0155 \\
19.22\end{array}$ & $\begin{array}{c}0.0094 \\
0.0187 \\
23.05\end{array}$ & $\begin{array}{c}0.0082 \\
0.0162 \\
20.07\end{array}$ \\
\hline $\begin{array}{l}\text { Particulate Matter } \\
\text { - gr/dscf (Total) } \\
\text { - Ibs/mmBtu (Total) } \\
\text { - lbs } / \text { hr (Total) } \\
\end{array}$ & $\begin{array}{l}0.0775 \\
0.1539 \\
191.03 \\
\end{array}$ & $\begin{array}{l}0.0798 \\
0.1564 \\
193.14 \\
\end{array}$ & $\begin{array}{l}0.0746 \\
0.1482 \\
182.36 \\
\end{array}$ & $\begin{array}{l}0.0773 \\
0.1528 \\
188.84 \\
\end{array}$ \\
\hline
\end{tabular}

* 29.92" Hg, 68 ${ }^{\circ}$ F $\left(760 \mathrm{~mm} \mathrm{Hg}, 20^{\circ} \mathrm{C}\right)$

** Calculated using an $F_{d}$ Factor of 9,780

*** See Discussion of Results 


\section{SAMPLING}

\section{SUMMARY OF RESULTS}

Table 6: Stack Hydrogen Halides \& Halogens Summary of Sampling Results

\begin{tabular}{|c|c|c|c|c|}
\hline Run No. & 1 & 2 & 3 & Average \\
\hline Test Date & $09 / 23 / 08$ & $09 / 23 / 08$ & $09 / 23 / 08$ & ----- \\
\hline Test Time & 0910-1014 & $1354-1503$ & $1823-1926$ & ----- \\
\hline Flow Rate - DSCFM* & 305,692 & 302,476 & 302,117 & 303,428 \\
\hline Stack Temp. - ${ }^{\circ} \mathrm{F}$ & 207 & 208 & 207 & 207 \\
\hline $\mathrm{O}_{2}-\%$ Vol. (dry) & 6.1 & 6.1 & 6.0 & 6.1 \\
\hline $\mathrm{CO}_{2}-\%$ Vol. (dry) & 13.6 & 13.5 & 13.6 & 13.6 \\
\hline Percent Excess Air & 40.2 & 40.1 & 39.2 & 39.8 \\
\hline Moisture Content - \% & 16.03 & 15.73 & 14.81 & 15.52 \\
\hline Percent Isokinetic & 102.6 & 100.2 & 99.6 & 100.8 \\
\hline Sample Volume - DSCM & 1.070 & 1.034 & 1.027 & 1.044 \\
\hline $\begin{array}{l}\text { Hydrogen Bromide } \\
\text { - mg } \\
-m g / d s c m\end{array}$ & $\begin{array}{l}<0.030 \\
<0.028\end{array}$ & $\begin{array}{l}<0.033 \\
<0.032\end{array}$ & $\begin{array}{l}<0.032 \\
<0.031\end{array}$ & $\begin{array}{l}<0.032 \\
<0.030\end{array}$ \\
\hline $\begin{array}{l}\text { Bromine } \\
-m g \\
-m g / d s c m\end{array}$ & $\begin{array}{l}<0.087 \\
<0.081\end{array}$ & $\begin{array}{l}<0.084 \\
<0.081\end{array}$ & $\begin{array}{l}<0.076 \\
<0.074\end{array}$ & $\begin{array}{l}<0.082 \\
<0.079\end{array}$ \\
\hline $\begin{array}{l}\text { Hydrogen Chloride } \\
\text { - mg } \\
\text { - mg/dscm }\end{array}$ & $\begin{array}{l}0.761 \\
0.711\end{array}$ & $\begin{array}{l}0.116 \\
0.112\end{array}$ & $\begin{array}{l}<0.032 \\
<0.031\end{array}$ & $\begin{array}{l}<0.303 \\
<0.285\end{array}$ \\
\hline $\begin{array}{l}\text { Chlorine } \\
-m g \\
-m g / d s c m\end{array}$ & $\begin{array}{l}0.514 \\
0.480\end{array}$ & $\begin{array}{l}0.507 \\
0.490\end{array}$ & $\begin{array}{l}0.464 \\
0.452\end{array}$ & $\begin{array}{l}0.495 \\
0.474\end{array}$ \\
\hline $\begin{array}{l}\text { Hydrogen Fluoride } \\
\text { - mg } \\
\text { - mg/dscm }\end{array}$ & $\begin{array}{l}<0.017 \\
<0.016\end{array}$ & $\begin{array}{l}<0.018 \\
<0.018\end{array}$ & $\begin{array}{l}<0.017 \\
<0.017\end{array}$ & $\begin{array}{l}<0.017 \\
<0.017\end{array}$ \\
\hline
\end{tabular}

* $29.92 " \mathrm{Hg}, 68^{\circ} \mathrm{F}\left(760 \mathrm{~mm} \mathrm{Hg}, 20^{\circ} \mathrm{C}\right)$ 


\section{SUMMARY OF RESULTS}

Table 7: Stack Sulfur Trioxide Summary of Sampling Results

\begin{tabular}{|l|c|c|c|c|}
\hline Run No. & 1 & 2 & 3 & Average \\
\hline Test Date & $09 / 24 / 08$ & $09 / 24 / 08$ & $09 / 24 / 08$ & ---- \\
Test Time & $1407-1507$ & $1600-1700$ & $1745-1845$ & ---- \\
Sample Volume - DSCM* & 0.521 & 0.517 & 0.517 & 0.518 \\
Moisture Content - \% & 15.47 & 15.39 & 16.11 & 15.66 \\
Sulfur Trioxide - ppm & 1.29 & 0.87 & 1.49 & 1.22 \\
\hline
\end{tabular}

* 29.92" Hg, 68 ${ }^{\circ}$ F (760 mm Hg, $\left.20^{\circ} \mathrm{C}\right)$ 


\section{SAMPOCIATES,INC.}

\section{SUMMARY OF RESULTS}

Table 8: Summary of Particulate Matter (PM10/2.5) Sampling Results

\begin{tabular}{|c|c|c|c|c|}
\hline Run No. & 2 & 3 & 4 & Average \\
\hline Test Date & $09 / 24 / 08$ & $09 / 24 / 08$ & $09 / 24 / 08$ & ----- \\
\hline Test Time & $1308-1412$ & $1505-1610$ & $1700-1804$ & ----- \\
\hline Flow Rate - DSCFM* & 286,384 & 286,068 & 285,463 & 285,972 \\
\hline Stack Temp. - ${ }^{\circ} F$ & 211 & 214 & 211 & 212 \\
\hline $\mathrm{O}_{2}$ - \% Vol. (dry) & 6.2 & 6.2 & 6.2 & 6.2 \\
\hline $\mathrm{CO}_{2}$ - \%Vol. (dry) & 13.4 & 13.4 & 13.4 & 13.4 \\
\hline Percent Excess Air & 41.0 & 41.0 & 41.0 & 41.0 \\
\hline Moisture Content - \% & 15.26 & 15.01 & 15.46 & 15.24 \\
\hline Percent Isokinetic & 82.8 & 82.5 & 81.6 & 82.3 \\
\hline $\begin{array}{l}\text { Particulate Matter (> PM } 10) \\
\text { - gr/dscf } \\
\text { - Ibs/hr }\end{array}$ & $\begin{array}{c}0.0025 \\
6.19\end{array}$ & $\begin{array}{c}0.0051 \\
12.43\end{array}$ & $\begin{array}{c}0.0056 \\
13.67\end{array}$ & $\begin{array}{c}0.0044 \\
10.76\end{array}$ \\
\hline $\begin{array}{l}\text { Particulate Matter }\left(<\mathrm{PM}_{10}>\mathrm{PM}_{2.5}\right) \\
\text { - gr/dscf } \\
\text { - Ibs } / \mathrm{hr}\end{array}$ & $\begin{array}{c}0.0016 \\
4.01\end{array}$ & $\begin{array}{c}0.0045 \\
10.97\end{array}$ & $\begin{array}{c}0.0033 \\
8.13\end{array}$ & $\begin{array}{c}0.0031 \\
7.70\end{array}$ \\
\hline $\begin{array}{l}\text { Particulate Matter }\left(\mathrm{PM}_{2.5}\right) \\
\text { - gr/dscf } \\
\text { - Ibs/hr }\end{array}$ & $\begin{array}{c}0.0010 \\
2.37\end{array}$ & $\begin{array}{c}0.0017 \\
4.20\end{array}$ & $\begin{array}{c}0.0038 \\
9.24\end{array}$ & $\begin{array}{c}0.0022 \\
5.27\end{array}$ \\
\hline $\begin{array}{l}\text { Particulate Matter (Back-Half) } \\
\text { - gr/dscf } \\
\text { - lbs/hr }\end{array}$ & $\begin{array}{l}0.0556 \\
136.39\end{array}$ & $\begin{array}{l}0.0673 \\
165.05\end{array}$ & $\begin{array}{l}0.0447 \\
109.39\end{array}$ & $\begin{array}{l}0.0559 \\
136.94\end{array}$ \\
\hline $\begin{array}{l}\text { Particulate Matter (Total Catch) } \\
\text { - gr/dscf } \\
\text { - lbs/hr }\end{array}$ & $\begin{array}{l}0.0607 \\
148.95 \\
\end{array}$ & $\begin{array}{l}0.0786 \\
192.65 \\
\end{array}$ & $\begin{array}{l}0.0574 \\
140.43 \\
\end{array}$ & $\begin{array}{l}0.0656 \\
160.68 \\
\end{array}$ \\
\hline
\end{tabular}

* $29.92^{\prime \prime ~} \mathrm{Hg}, 68^{\circ} \mathrm{F}\left(760 \mathrm{~mm} \mathrm{Hg}, 20^{\circ} \mathrm{C}\right)$ 


\title{
DISCUSSION OF SAMPLING RESULTS
}

\author{
SDA Inlet Duct
}

\section{Particulate Matter, Hydrogen Halides \& Halogens, and Ammonia}

The three tests for particulate matter, hydrogen halides \& halogens, and ammonia taken at the SDA Inlet Duct appeared to be an accurate representation of the actual emissions during the tests. All leak checks performed on the reference method sampling train and pitot tubes showed no leaks before or after testing. The indicative parameters of the tests were in close agreement. The measured moisture contents $(\% \mathrm{M})$ were within $4.05 \%$ of the mean value. The measured flow rates (DSCFM) were within $0.68 \%$ of the mean value. The rates of sampling for the three tests were within the specified limits (90 to 110 percent isokinetic). The greatest deviation from $100 \%$ isokinetic was $8.3 \%$.

The concentrations ( $\mathrm{gr} / \mathrm{dscf}$ - front half) of particulate matter for the three tests showed a range of -17.89 percent to +15.37 percent variation from the mean value of 4.6874 gr/dscf - front half.

The concentrations $(\mathrm{mg} / \mathrm{dscm})$ of hydrogen bromide and bromine for the three tests were below the minimum reporting limit of the reference method.

The concentrations of hydrogen chloride $(\mathrm{mg} / \mathrm{dscm})$ for the three tests showed a range of $-50.44 \%$ to $+58.42 \%$ variation from the mean value of $0.914 \mathrm{mg} / \mathrm{dscm}$. The concentrations of chlorine $(\mathrm{mg} / \mathrm{dscm})$ of for the three tests showed a range of $-78.46 \%$ to $+56.04 \%$ variation from the mean value of $0.836 \mathrm{mg} / \mathrm{dscm}$. 
The concentrations $(\mathrm{mg} / \mathrm{dscm}$ ) of hydrogen fluoride and fluorine for the three tests were below the minimum reporting limit of the reference method.

The concentrations (ppm) of ammonia for the three tests varied as the rate of ammonia injection was reduced.

\section{Sulfur Trioxide}

The three tests for sulfur trioxide taken at the SDA Inlet Duct appeared to be an accurate representation of the actual emissions during the tests. All leak checks performed on the reference method sampling train showed no leaks before or after testing. The indicative parameters of the tests were in close agreement. The measured moisture contents $(\% \mathrm{M})$ were within $3.05 \%$ of the mean value.

The concentrations (ppm) of sulfur trioxide for the three tests showed a range of -75.31 percent to +116.74 percent variation from the mean value of $5.99 \mathrm{ppm}$.

\section{Stack}

\section{Particulate Matter}

The three tests for particulate matter at the Stack appeared to be an accurate representation of the actual emissions during the tests. All leak checks performed on the reference method sampling train and pitot tubes showed no leaks before or after testing. The indicative parameters of the tests were in close agreement. The measured moisture contents $(\% \mathrm{M})$ were within $2.67 \%$ of the mean value. The measured flow rates (DSCFM) were within $0.94 \%$ of the mean value. The rates of sampling for the 
tests were within the specified limits (90\% to $110 \%$ isokinetic). The greatest deviation from $100 \%$ isokinetic was $3.2 \%$.

The concentrations (lbs/hr - front half) of particulate matter for the three tests showed a range of -10.61 percent to +14.85 percent variation from the mean value of $20.07 \mathrm{lbs} / \mathrm{hr}$ - front half.

The analysis of the "back-half" particulate matter samples is suspect. Visual observations in the field indicated that the impinger solutions were clear with no cloudiness. The samples were purged for one-hour after the tests with pure nitrogen. Also, there were no visible emissions from the stack during the testing that would have indicated the level of emissions that were reported.

\section{Hydrogen Halides \& Halogens}

The three tests for particulate matter and hydrogen halides \& halogens taken at the Stack appeared to be an accurate representation of the actual emissions during the tests. All leak checks performed on the reference method sampling train and pitot tubes showed no leaks before or after testing. The indicative parameters of the tests were in close agreement. The measured moisture contents (\%M) were within $4.60 \%$ of the mean value. The measured flow rates (DSCFM) were within $0.75 \%$ of the mean value. The rates of sampling for the tests were within the specified limits $(90 \%$ to $110 \%$ isokinetic). The greatest deviation from $100 \%$ isokinetic was $2.6 \%$.

The concentrations $(\mathrm{mg} / \mathrm{dscm})$ of hydrogen bromide and bromine for the three tests were below the minimum reporting limit of the reference method.

The concentrations $(\mathrm{mg} / \mathrm{dscm})$ of hydrogen chloride for the third test was below the minimum reporting limit of the reference method. The concentrations of chlorine 
$(\mathrm{mg} / \mathrm{dscm})$ for the three tests showed a range of $-4.64 \%$ to $+3.38 \%$ variation from the mean value of $0.474 \mathrm{mg} / \mathrm{dscm}$.

The concentrations $(\mathrm{mg} / \mathrm{dscm})$ of hydrogen fluoride and fluorine for the three tests were below the minimum reporting limit of the reference method.

\section{Sulfur Trioxide}

The three tests for sulfur trioxide appeared to be a valid representation of the actual emissions during the tests. All leak checks performed on the reference method sampling train showed no leaks before or after testing. The measured moisture contents were within $2.90 \%$ of the mean value.

The concentrations (ppm) of sulfur trioxide for the three tests showed a range of -28.49 percent to +22.47 percent variation from the mean value of $1.22 \mathrm{ppm}$.

\section{$\underline{P M 10 / 2.5}$}

The first test for PM10/2.5 was not valid due to the sampling rate being below the required sampling rate of 80 percent. Therefore, an additional test was taken. The last three tests for PM10/2.5 appeared to be an accurate representation of the actual emissions during the tests. All leak checks performed on the sampling train and the pitot tubes indicated no leaks before or after each test. The indicative parameters calculated from the field data were in reasonable agreement. The measured moisture contents for the three runs were within $1.53 \%$ of the mean value. The measured flow rates (DSCFM) for the tests were within $0.18 \%$ of the mean value. The rates of sampling for the three tests were within the specified limits (80 to 120 percent isokinetic). The greatest deviation from $100 \%$ isokinetic was $18.4 \%$. The particulate cut 
size was 9.68 microns, which was within the specified limits in EPA 201A of 9 to 11 microns.

The concentrations ( $g r / d s c f)$ of particulate matter $>\mathrm{PM}_{10}$ for the three tests showed a range of -43.18 percent to +27.27 percent variation from the mean value of 0.0044 gr/dscf.

The concentrations (gr/dscf) of particulate matter $<\mathrm{PM}_{10}>\mathrm{PM}_{2.5}$ for the three tests showed a range of -48.94 percent to +43.62 percent variation from the mean value of $0.0031 \mathrm{gr} / \mathrm{dscf}$.

The concentrations (gr/dscf) of particulate matter $\mathrm{PM}_{2.5}$ for the three tests showed a range of -53.85 percent to +75.38 percent variation from the mean value of 0.0022 gr/dscf.

The concentrations ( $\mathrm{gr} / \mathrm{dscf}$ ) of particulate matter total catch for the three tests showed a range of -12.46 percent to +19.88 percent variation from the mean value of 0.0656 gr/dscf. 


\section{DESCRIPTION OF SAMPLING LOCATIONS}

The sampling ports on the SDA Inlet Duct are approximately 36 feet $91 / 2$ inches above the ground. The sampling ports are located 13 feet 4 inches (1.12 equivalent duct diameters) downstream from a bend in the duct and 56 feet 3 inches (4.75 equivalent duct diameters) upstream from a bend in the duct.

The sampling ports on the Hardin Unit No. 1 Stack (Boiler Stack) are approximately 123 feet 1 1/2 inches above the ground. The sampling ports are located 78 feet $(8.57$ stack diameters) downstream from the inlet to the stack and 127 feet (13.96 stack diameters) upstream from the outlet to the stack. 


\section{SAMPLING LOCATION}

Figure 1: SDA Inlet Duct

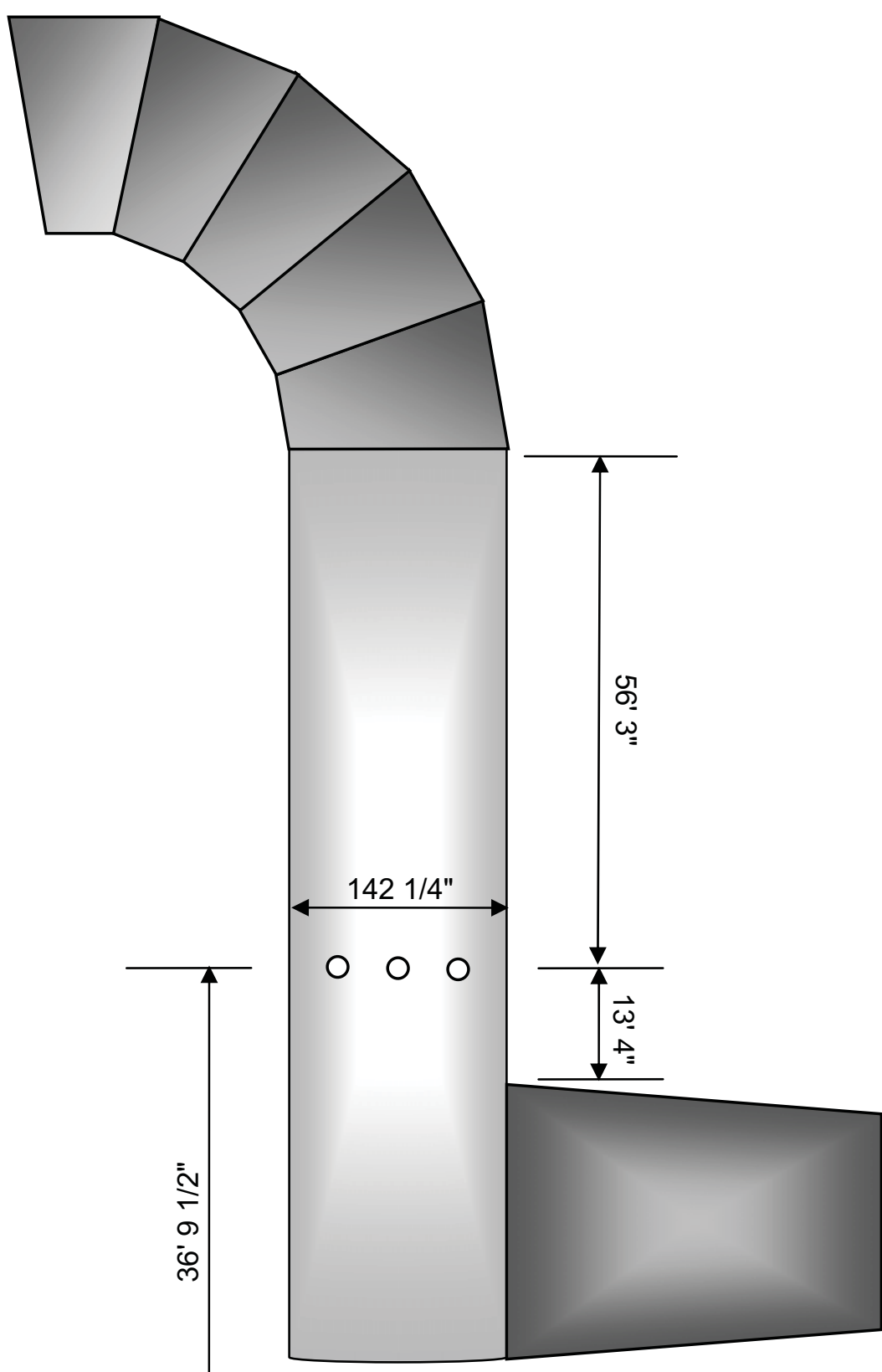




\section{SAMPLING LOCATION}

Figure 2: Stack

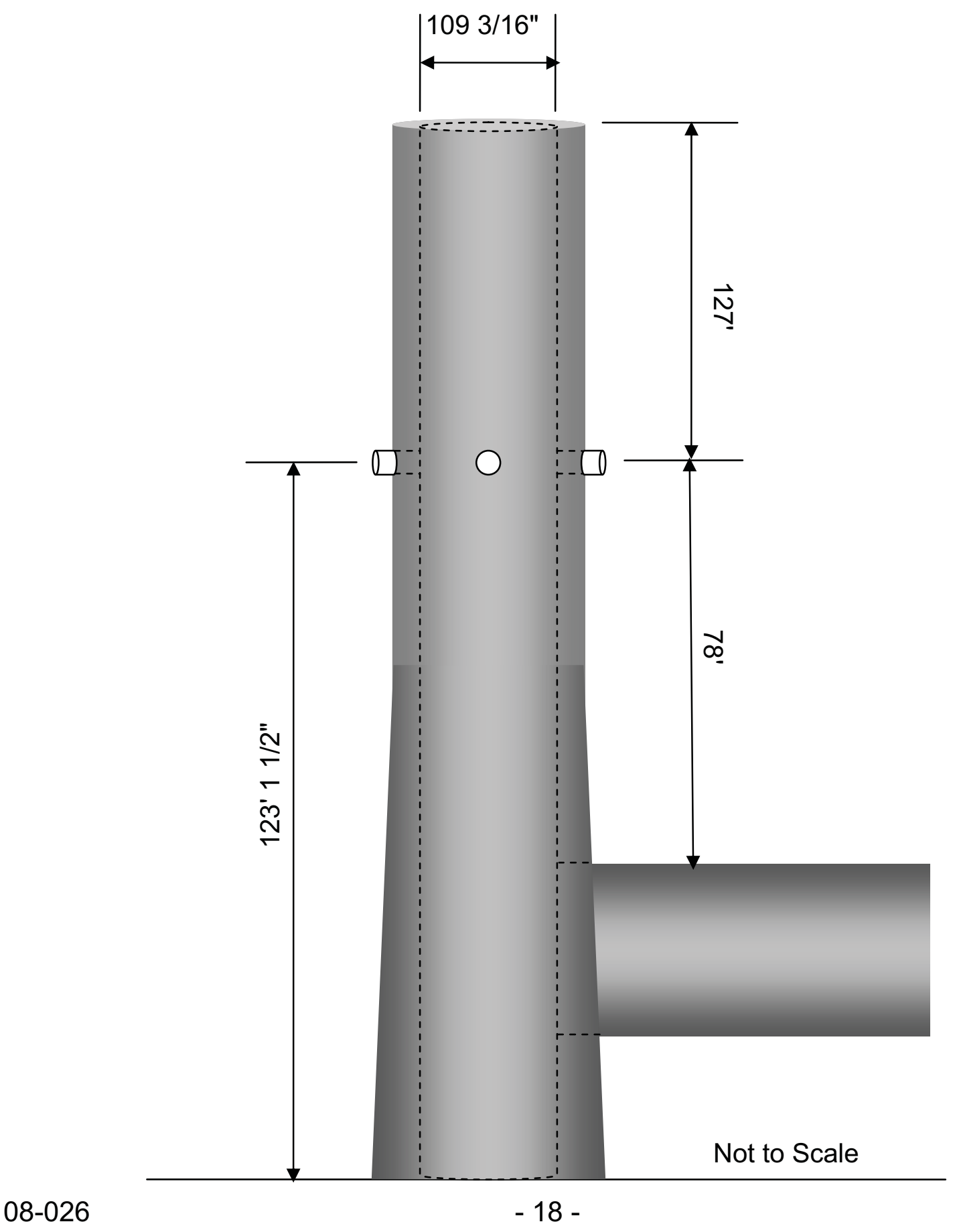




\section{SAMPLING AND ANALYTICAL PROCEDURES}

The procedures set forth in Title 40 of the Code of Federal Regulations, Part 60 (40CFR60), Appendix A, Test Methods 1, 2, 3B, 4, 5B, 17, 26A, 201A, 202, and Conditional Test Method CTM027; and the National Council of the Paper Industry for Air and Stream Improvement, Inc. (NCASI) Method 8A were followed during testing.

Four Traverse points were sampled from three ports on the SDA Inlet Duct for a total of twelve traverse points. All traverse points were checked for cyclonic flow and the average angle was equal to 2.4 degrees. The pitot tube lines were checked for leaks before and after each test under a vacuum and a pressure. The lines were also checked for clearance and the manometer was zeroed before each test.

Six traverse points were sampled from two ports on the Stack for a total of twelve traverse points. All traverse points were checked for cyclonic flow and the average angle was equal to 6.0 degrees. The pitot tube lines were checked for leaks before and after each test under a vacuum and a pressure. The lines were also checked for clearance and the manometer was zeroed before each test.

\section{Particulate Matter}

Particulate matter samples were taken at the Stack sampling location according to EPA Test Methods 5B and 202. For each run, samples of ten minute duration were taken at each of the twelve traverse points for a total sampling time of 120 minutes. Data was recorded at five minute intervals.

The sampling train was leak checked at the end of the sampling probe at 15" of mercury vacuum before each test, and again at the conclusion of each test at the highest 08-026 


\section{III}

vacuum recorded during sampling. This was done to predetermine the possibility of a diluted sample.

The "front half" of the sampling train at the Stack sampling location contained the following components:

Stainless steel nozzle

Heated glass lined probe@ $320^{\circ} \mathrm{F} \pm 25^{\circ} \mathrm{F}$

Heated glass fiber filter @ $320^{\circ} \mathrm{F} \pm 25^{\circ} \mathrm{F}$

The "back half" of the sampling train at the Stack sampling location contained the following components:

\section{Table 9: EPA Test Methods 5B / 202 Sampling Train Condenser/Absorbing System}

\begin{tabular}{|ccccc|}
\hline Impinger No. & $\begin{array}{c}\text { Impinger } \\
\text { Type }\end{array}$ & $\begin{array}{c}\text { Impinger } \\
\text { Contents }\end{array}$ & Amount & $\begin{array}{c}\text { Parameter } \\
\text { Collected }\end{array}$ \\
\hline $\mathbf{1}$ & Modified & H.P.L.C. $\mathrm{H}_{2} \mathrm{O}$ & $100 \mathrm{ml}$ & $\begin{array}{c}\text { Condensable } \\
\text { PM \& } \mathrm{H}_{2} \mathrm{O}\end{array}$ \\
\hline $\mathbf{2}$ & Greenburg-Smith & H.P.L.C. $\mathrm{H}_{2} \mathrm{O}$ & $100 \mathrm{ml}$ & $\begin{array}{c}\text { Condensable } \\
\text { PM \& } \mathrm{H}_{2} \mathrm{O}\end{array}$ \\
\hline $\mathbf{3}$ & Modified & Empty & ----- & $\mathrm{H}_{2} \mathrm{O}$ \\
\hline $\mathbf{4}$ & Modified & Silica Gel & $\sim 200 \mathrm{~g}$ & $\mathrm{H}_{2} \mathrm{O}$ \\
\hline
\end{tabular}

At the completion of each run, the "back half" of the sampling train was purged with nitrogen for 60 minutes at a rate of 20 liters per minute.

Integrated Orsat samples were collected during each test. The samples were analyzed according to EPA Method 3 to determine the stack gas molecular weight. 
Figure 3: EPA Test Methods 5B and 202 Sampling Train

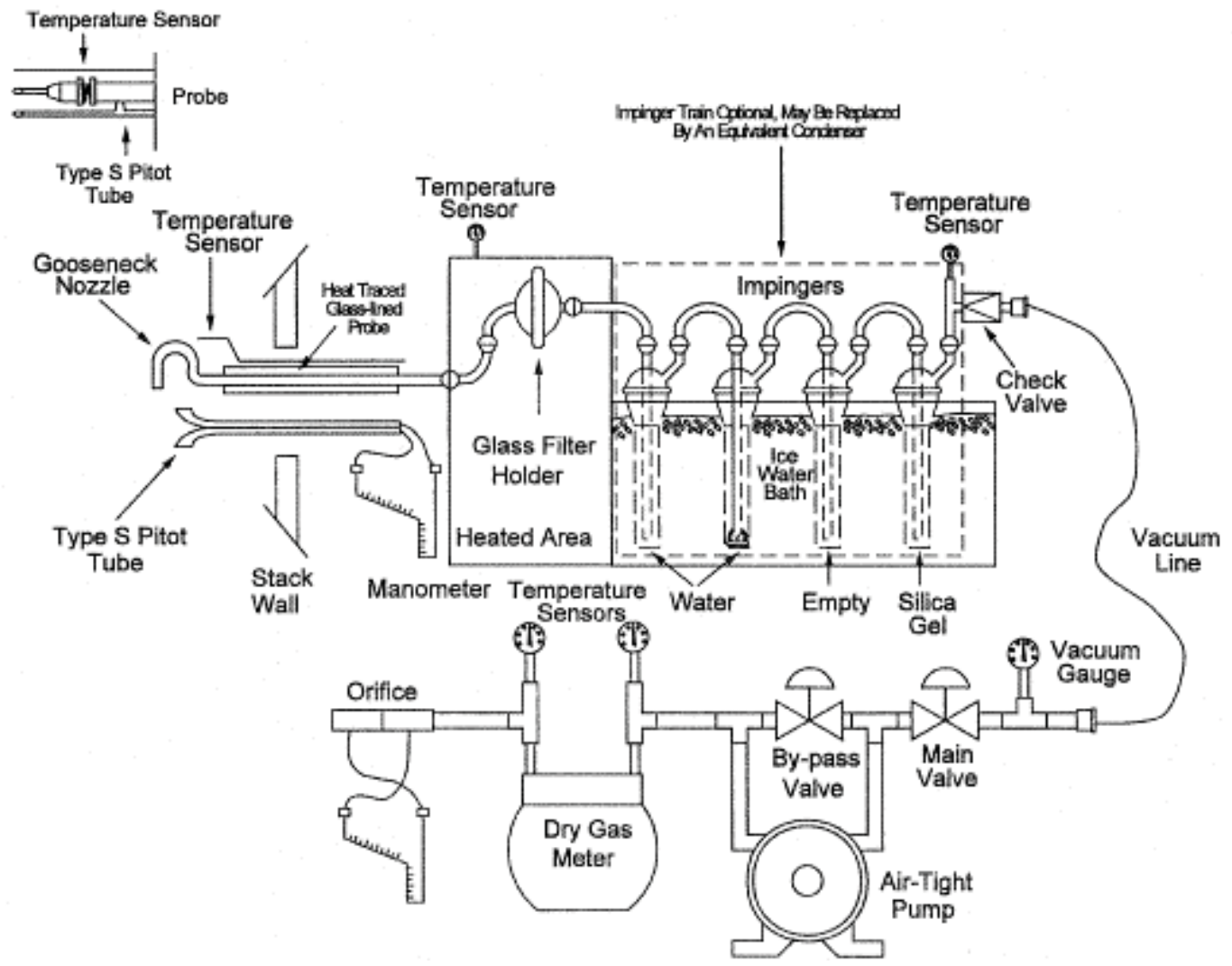




\section{Particulate Matter, Hydrogen Halides \& Halogens, and Ammonia}

Particulate matter, hydrogen halides \& halogens, and ammonia samples were taken at the SDA Inlet Duct and Stack sampling locations according to EPA Test Methods 1, 2, $3,4,5,17,26 \mathrm{~A}$, and CTM-027. For each run at the SDA Inlet Duct, samples of five minute duration were taken at each of twelve traverse points for a total test time of 60 minutes. Data was recorded at five minute intervals. For each run at the Stack, samples of five minute duration were taken at each of twelve traverse points for a total test time of 60 minutes. Data was recorded at five minute intervals.

The sampling trains were leak checked at the end of the sampling probe at 15" of mercury vacuum before each test, and again at the conclusion of each test at the highest vacuum recorded during sampling. This was done to predetermine the possibility of a diluted sample.

The 'front half' of the sampling train at the SDA Inlet Duct contained the following components:

Glass nozzle

Fiberglass Thimble

Heated glass lined probe @ $248^{\circ} \mathrm{F} \pm 25^{\circ} \mathrm{F}$

Heated glass filter bypass @ $248^{\circ} \mathrm{F} \pm 25^{\circ} \mathrm{F}$

The 'front half' of the sampling train at the Stack contained the following components:

Glass nozzle

Heated glass lined probe @ $248^{\circ} \mathrm{F} \pm 25^{\circ} \mathrm{F}$

Heated fiberglass filter @ $248^{\circ} \mathrm{F} \pm 25^{\circ} \mathrm{F}$ 
The 'back half' of the sampling trains at both sampling locations contained the following components:

Table 10: EPA Test Method 26A Condenser/Absorbing System

\begin{tabular}{|ccccc|}
\hline Impinger No. & $\begin{array}{c}\text { Impinger } \\
\text { Type }\end{array}$ & $\begin{array}{c}\text { Impinger } \\
\text { Contents }\end{array}$ & Amount & $\begin{array}{c}\text { Parameter } \\
\text { Collected }\end{array}$ \\
\hline $\mathbf{1}$ & Greenburg-Smith & $0.1 \mathrm{~N} \mathrm{H}_{2} \mathrm{SO}_{4}$ & $100 \mathrm{ml}$ & $\begin{array}{c}\text { Hydrogen } \\
\text { Halides }\end{array}$ \\
\hline $\mathbf{2}$ & Greenburg-Smith & $0.1 \mathrm{~N} \mathrm{H}_{2} \mathrm{SO}_{4}$ & $100 \mathrm{ml}$ & $\begin{array}{c}\text { Hydrogen } \\
\text { Halides }\end{array}$ \\
\hline $\mathbf{3}$ & Modified & $0.1 \mathrm{~N} \mathrm{NaOH}$ & $100 \mathrm{ml}$ & Halogens \\
\hline $\mathbf{4}$ & Modified & $0.1 \mathrm{~N} \mathrm{NaOH}$ & $100 \mathrm{ml}$ & Halogens \\
\hline $\mathbf{5}$ & Modified & Silica Gel & $\sim 200 \mathrm{~g}$ & $\mathrm{H}_{2} \mathrm{O}$ \\
\hline
\end{tabular}

A 100 milliliter aliquot of the Impinger No. 1 and No. 2 contents was taken from each run at the SDA Inlet Duct for lon Chromatograph analysis to determine the concentrations of ammonia.

Integrated Orsat samples were collected at each sampling location during each test. The samples were analyzed according to EPA Method 3 to determine the stack gas molecular weight. 


\section{AARMPLNG ASSOCIATES, INC.}

Figure 4: EPA Test Methods 17, 26A, and CTM027 SDA Inlet Duct Sampling Train

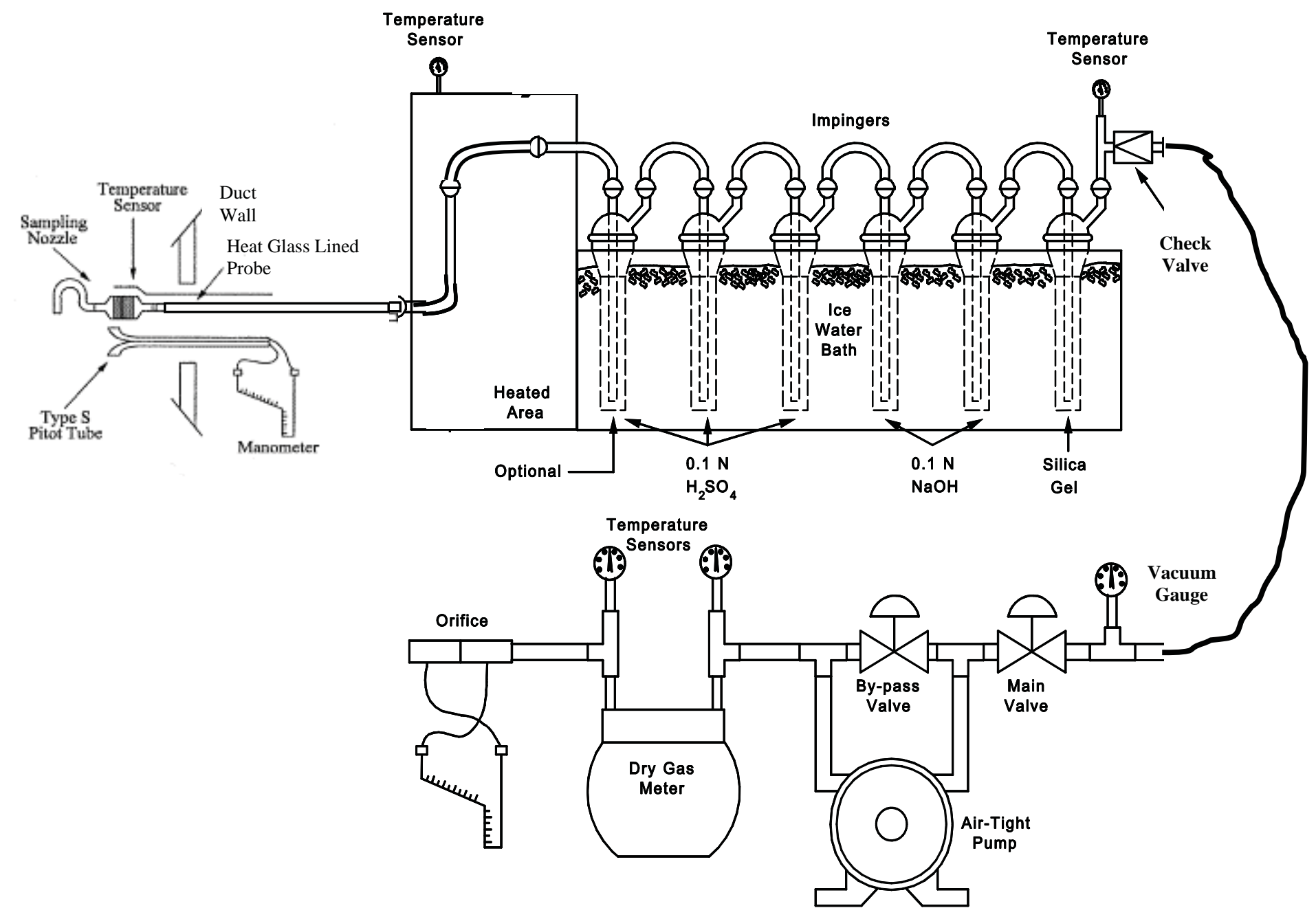




\section{III

Figure 5: EPA Test Method 26A Stack Sampling Train - Stack

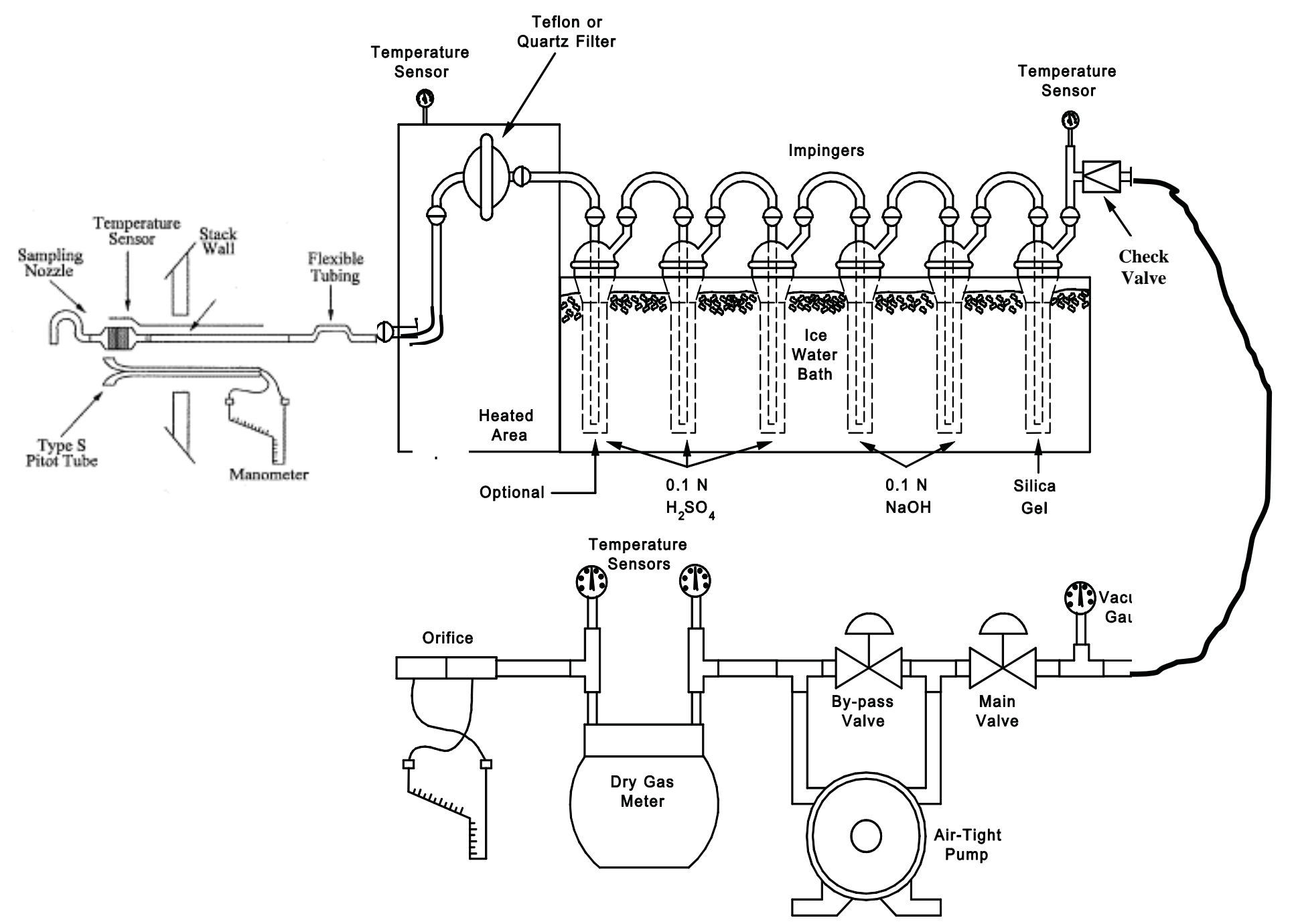




\section{$\underline{P M 10 / 2.5}$}

Six traverse points were sampled from each of the two ports on the stack for a total of twelve traverse points. All traverse points were previously checked for cyclonic flow and none was found to be present. For each run, samples of varying dwell time were taken at each of the twelve traverse points for a total sampling time of 61.2 minutes. The dwell time at each sampling point was proportional with the velocity at that point. Data was recorded at the beginning of each sample point.

The pitot tube lines were checked for leaks before and after each test under a vacuum and a pressure. The lines were also checked for clearance and the manometer was zeroed before each test.

The sampling train was leak checked at the end of the sampling probe at 15" of mercury vacuum before each test. This was done to predetermine the possibility of a diluted sample.

The "front-half" of the sampling train contained the following components:

Stainless steel nozzle

$\mathrm{PM}_{10}$ Sampler

$\mathrm{PM}_{2.5}$ Sampler

Heated glass lined probe@ $250^{\circ} \mathrm{F} \pm 25^{\circ} \mathrm{F}$ 
The "back-half" of the sampling train contained the following components:

Table 11: Reference Method 202 Sampling Train

\begin{tabular}{|ccccc|}
\hline Impinger No. & $\begin{array}{c}\text { Impinger } \\
\text { Type }\end{array}$ & $\begin{array}{c}\text { Impinger } \\
\text { Contents }\end{array}$ & Amount & $\begin{array}{c}\text { Parameter } \\
\text { Collected }\end{array}$ \\
\hline $\mathbf{1}$ & Modified & H.P.L.C. $\mathrm{H}_{2} \mathrm{O}$ & $100 \mathrm{ml}$ & $\begin{array}{c}\text { Condensable } \\
\text { PM \& } \mathrm{H}_{2} \mathrm{O}\end{array}$ \\
\hline $\mathbf{2}$ & Greenburg-Smith & H.P.L.C. $\mathrm{H}_{2} \mathrm{O}$ & $100 \mathrm{ml}$ & $\begin{array}{c}\text { Condensable } \\
\text { PM \& } \mathrm{H}_{2} \mathrm{O}\end{array}$ \\
\hline $\mathbf{3}$ & Modified & Empty & ----- & $\mathrm{H}_{2} \mathrm{O}$ \\
\hline $\mathbf{4}$ & Modified & Silica Gel & $\sim 200 \mathrm{~g}$ & $\mathrm{H}_{2} \mathrm{O}$ \\
\hline
\end{tabular}

At the completion of each run, the "back-half" of the sampling train was purged with nitrogen for 60 minutes at a rate of 20 liters per minute. 
Figure 6: EPA Methods 201A and 202 Sampling Train

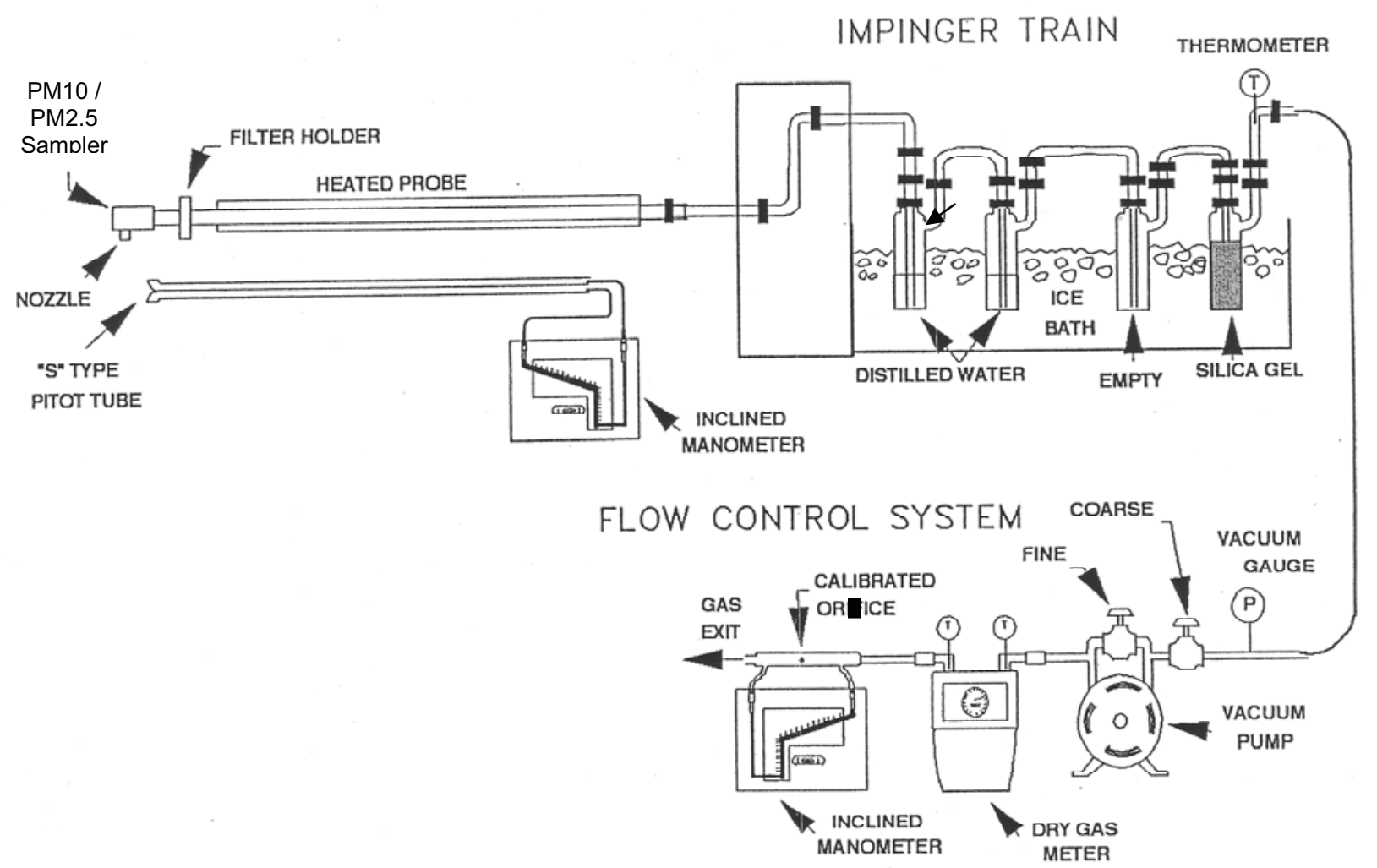




\section{III}

\section{$\underline{\text { Sulfur Trioxide }}$}

Triplicate samples for sulfur trioxide were taken at the SDA Inlet Duct and at the Stack sampling locations according to NCASI Method 8A. For each run, samples of 60 minute duration were taken at a single traverse point. Data was recorded at five minute intervals.

The sampling train was leak checked at the end of the sampling probe at 15" of mercury vacuum before each test, and again at the conclusion of each test at the highest vacuum recorded during sampling. This was done to predetermine the possibility of a diluted sample.

The 'front half' of the sampling train contained the following components:

Glass Sample Probe heated to $500^{\circ} \mathrm{F}$

Quartz Filter heated to $500^{\circ} \mathrm{F}$

Glass Condenser cooled to $140^{\circ} \mathrm{F}$

Teflon tubing connected to Impingers

The 'back half' of the sampling train contained the following components:

Table 12: NCASI Method 8A Condenser/Absorbing System

\begin{tabular}{|ccccc|}
\hline Impinger No. & $\begin{array}{c}\text { Impinger } \\
\text { Type }\end{array}$ & $\begin{array}{c}\text { Impinger } \\
\text { Contents }\end{array}$ & Amount & $\begin{array}{c}\text { Parameter } \\
\text { Collected }\end{array}$ \\
\hline $\mathbf{1}$ & Greenburg-Smith & $3 \% \mathrm{H}_{2} \mathrm{O}_{2}$ & $100 \mathrm{ml}$ & $\mathrm{H}_{2} \mathrm{O}$ \\
\hline $\mathbf{2}$ & Modified & $3 \% \mathrm{H}_{2} \mathrm{O}_{2}$ & $100 \mathrm{ml}$ & $\mathrm{H}_{2} \mathrm{O}$ \\
\hline $\mathbf{3}$ & Greenburg-Smith & $\mathrm{DI} \mathrm{H} \mathrm{H}_{2} \mathrm{O}$ & $100 \mathrm{ml}$ & $\mathrm{H}_{2} \mathrm{O}$ \\
\hline $\mathbf{4}$ & Modified & Silica Gel & $\sim 200 \mathrm{~g}$ & $\mathrm{H}_{2} \mathrm{O}$ \\
\hline
\end{tabular}


The sampling train was purged from the inlet of the condenser with clean ambient air for 15 minutes at the completion of each test run.

\section{Figure 7: NCASI Method 8A SDA Inlet Duct and Stack Sampling Train}

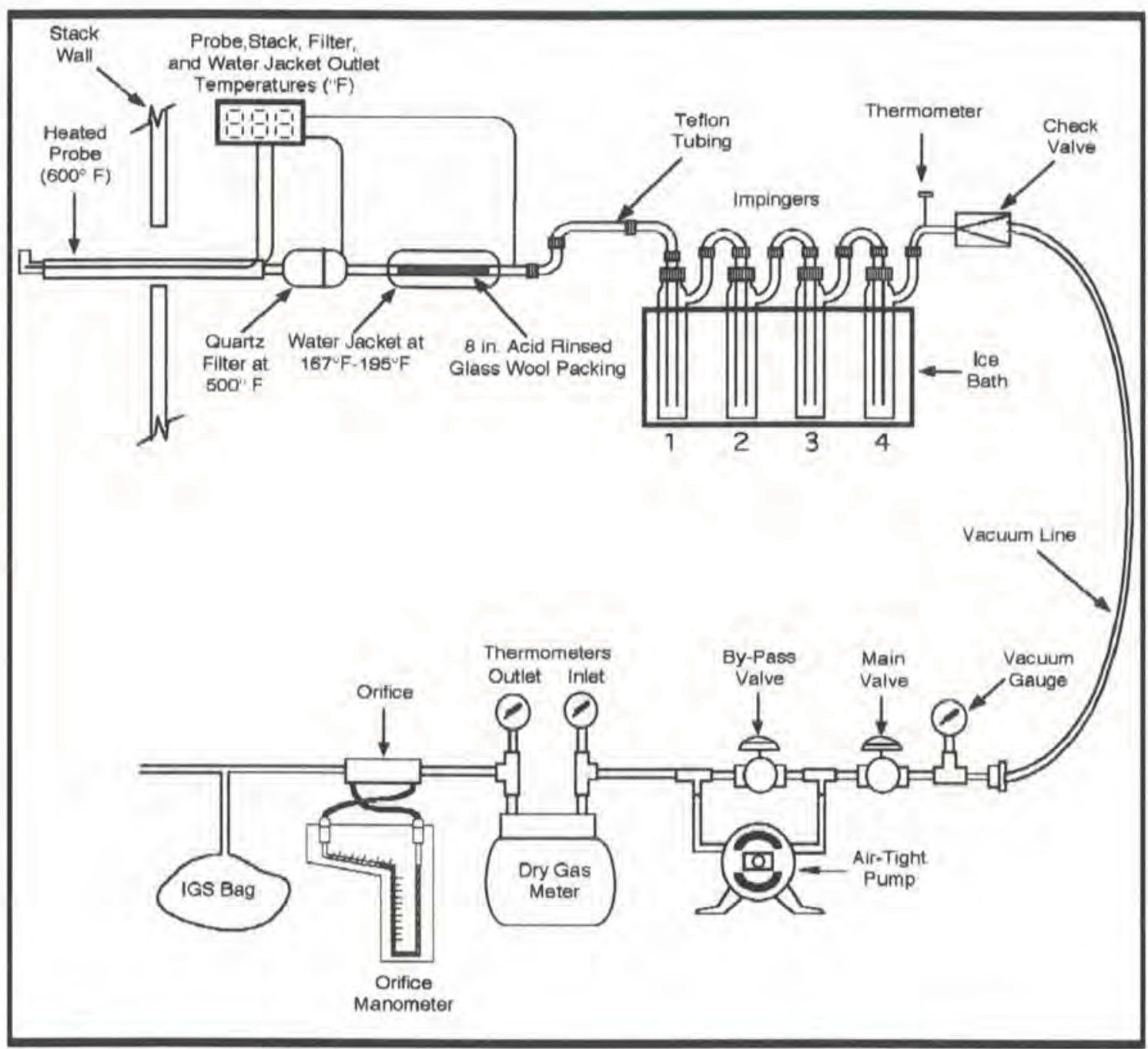




\section{TEST NARRATIVE}

Personnel from Air Sampling Associates, Inc. arrived at the Colorado Energy Management, LLC, Hardin Power Plant, located in Hardin, Montana at 1:30 p.m. on Monday, September 22, 2008. The sampling trailer was placed into position and electrical power was established. The sampling equipment was moved onto the SDA Inlet Duct and the Stack before securing the equipment for the night at 5:30 p.m.

On Tuesday, September 23, 2008, personnel returned to the plant at 6:30 a.m. The sampling equipment was prepared for testing and the first test for halogens on the stack and the first test for particulate matter, halogens, and ammonia on the SDA Inlet Duct began at 9:10 a.m. Testing continued until three tests for particulate matter, halogens, and ammonia and one test for sulfur trioxide had been taken on the SDA Inlet Duct as well as three tests for halogens and two tests for particulate matter on the Stack had been taken by 7:26 p.m. The samples were recovered and personnel departed the plant at 8:15 p.m.

On Wednesday, September 24, 2008, personnel returned to the plant at 6:15 a.m. The sampling equipment was prepared for testing and two tests for sulfur trioxide were taken on the SDA Inlet Duct before moving the sampling equipment to the Stack location. The third test for particulate matter was taken on the Stack as well as four tests for PM10/2.5 and three tests for sulfur trioxide. A fourth test for PM10/2.5 was taken due to the first test being sampled at less than the desired isokinetic sampling rate. The last test was completed at 6:45 p.m. 


\section{III}

The sampling equipment was moved off of the SDA Inlet Duct and the Stack and loaded into the sampling trailer. The samples were recovered and taken to Air Sampling Associates, Inc.'s office in Lewisville, Texas for analysis and shipment to the subcontracted laboratories.

Operations at the Colorado Energy Management, LLC, Hardin Power Plant, SDA Inlet Duct and Stack, located in Hardin, Montana, were completed at 8:15 p.m. on Wednesday, September 24, 2008. 


\section{APPENDICES}

Appendix A: Location of Traverse Points

Appendix B: Nomenclature and Equations for Calculation of Source Emissions

Appendix C: Calibration Data

Appendix D: Field Data

Appendix E: EPA Test Methods 5B and 202 (Particulate Matter) Analytical Data -Stack

Appendix F: EPA Test Methods 8A, 26A, and CTM027 (Sulfur Trioxide, Hydrogen Halides \& Halogens, and Ammonia) Analytical Data - SDA Inlet Duct and Stack

Appendix G: EPA Test Method 17 (Particulate Matter) Analytical Data - SDA Inlet Duct

Appendix H: EPA Test Methods 201A and 202 (PM10/2.5) Analytical Data- Stack

Appendix I: Chain of Custodies

Appendix J: Resumes of Test Personnel 


\section{Appendix A:}

Location of Traverse Points 


\section{Appendix A}

\section{Location of Traverse Points SDA Inlet Duct}

The sampling ports are located 13 feet 4 inches (1.12 equivalent duct diameters) downstream from a bend in the duct and 56 feet 3 inches (4.75 equivalent duct diameters) upstream from a bend in the duct. The locations of the traverse points were calculated as follows:

Figure 8: Location of Traverse Points - SDA Inlet Duct

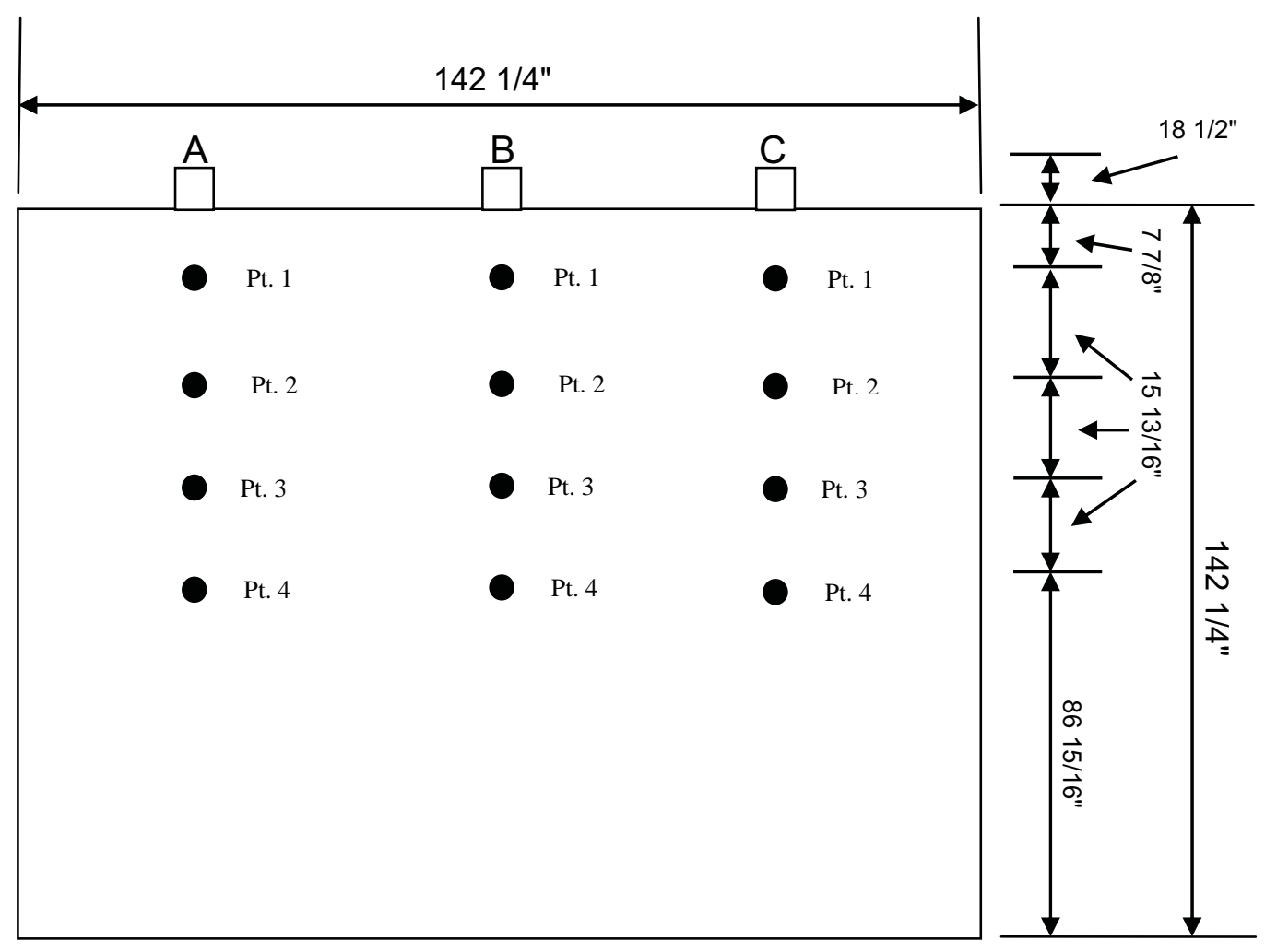

Note: Physical limitations prevented the complete traversing of the SDA Inlet Duct. 


\section{Appendix A}

\section{Location of Traverse Points Stack}

The sampling ports are located 78 feet (8.57 stack diameters) downstream from the inlet to the stack and 127 feet (13.96 stack diameters) upstream from the outlet to the stack. The locations of the traverse points were calculated as follows:

Table 13: Location of Velocity Traverse Points Boiler Stack

\begin{tabular}{|ccc|}
\hline \multicolumn{2}{|c|}{ Port \& Wall Thickness $=159 / 16$ inches } \\
\hline \multicolumn{2}{|c|}{ Inside Stack Diameter $=1093 / 16$ inches } \\
\hline $\begin{array}{c}\text { Point } \\
\text { Number }\end{array}$ & $\begin{array}{c}\text { Percent of } \\
\text { Stack Diameter }\end{array}$ & $\begin{array}{c}\text { Distance } \\
\text { from Wall }\end{array}$ \\
\hline 1 & 4.4 & $413 / 16^{\prime \prime}$ \\
\hline 2 & 14.6 & $1515 / 16^{\prime \prime}$ \\
\hline 3 & 29.6 & $325 / 16^{\prime \prime}$ \\
\hline 4 & 70.4 & $767 / 8^{\prime \prime}$ \\
\hline 5 & 85.4 & $931 / 4 "$ \\
\hline 6 & 95.6 & $1043 / 8^{\prime \prime}$ \\
\hline
\end{tabular}


AIR
SAMPling
ASSOCIATES, INC.

Appendix A

Figure 9: Location of Traverse Points - Stack

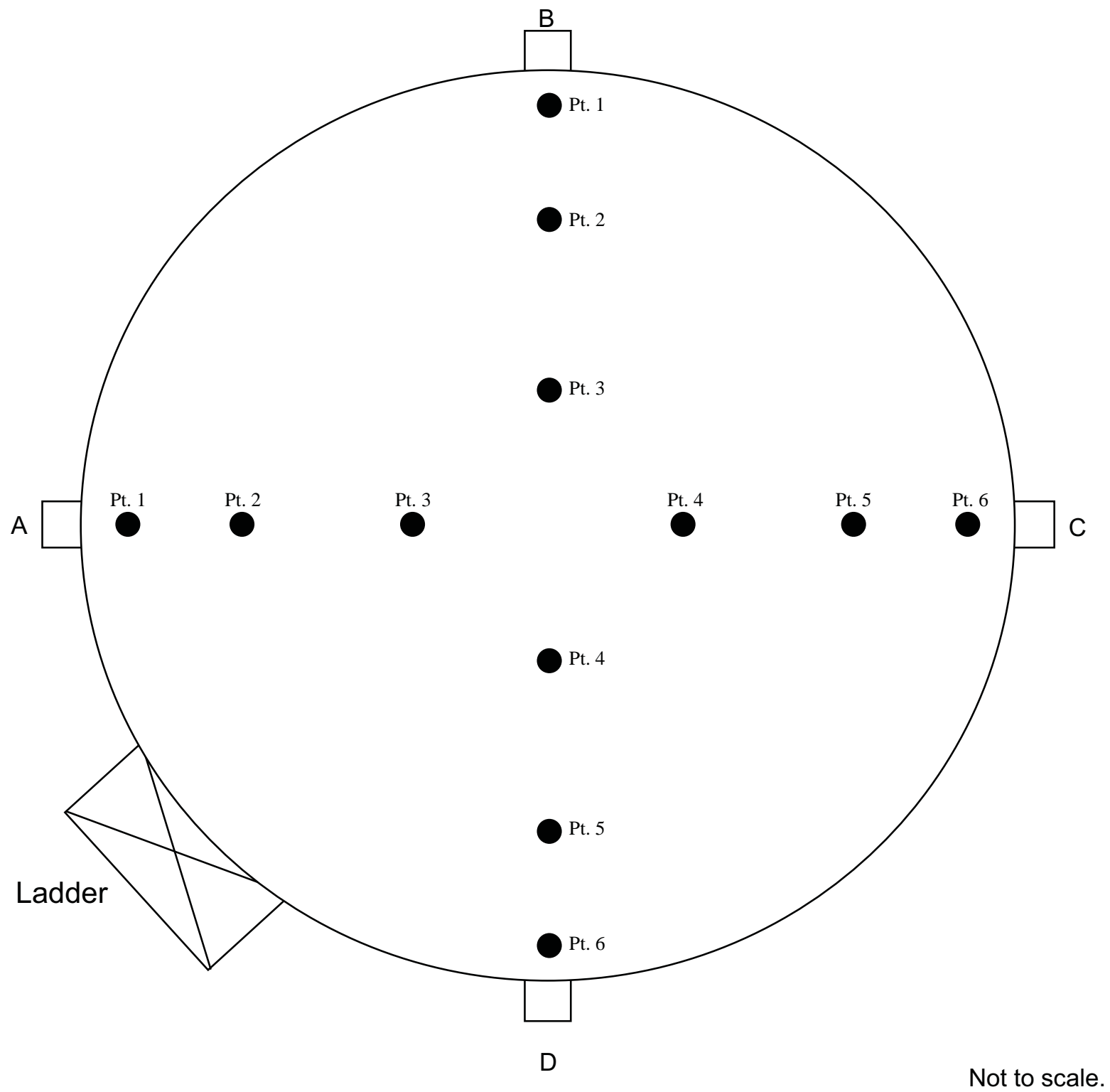

08-026

A-4 


\section{Appendix B:}

Nomenclature and Equations for Calculation of Source Emissions 


\section{Nomenclature For Flow Rate and Moisture Calculations}

\begin{tabular}{|c|c|c|c|}
\hline Symbol & $\begin{array}{l}\text { English } \\
\underline{\text { Units }}\end{array}$ & $\begin{array}{l}\text { Metric } \\
\underline{\text { Units }}\end{array}$ & Description \\
\hline$A_{s}$ & in. $^{2}$ & $\mathrm{~m}^{2}$ & Stack Area \\
\hline $\mathrm{C}_{\mathrm{an}}$ & $\mathrm{gr} / \mathrm{dscf}{ }^{*}$ & $\mathrm{~g} / \mathrm{dscm}^{*}$ & $\begin{array}{l}\text { Particulate - probe, cyclone, } \\
\text { and filter }\end{array}$ \\
\hline $\mathrm{C}_{\mathrm{ao}}$ & $\mathrm{gr} / \mathrm{dscf}{ }^{*}$ & $\mathrm{~g} / \mathrm{dscm}^{*}$ & Particulate -total \\
\hline $\mathrm{C}_{\text {at }}$ & $\begin{array}{c}\mathrm{gr} / \mathrm{CF} @ \\
\text { stack conditions }\end{array}$ & $\mathrm{g} / \mathrm{m} 3$ & $\begin{array}{l}\text { Particulate - probe, cyclone, } \\
\text { and filter }\end{array}$ \\
\hline $\mathrm{C}_{\mathrm{au}}$ & $\begin{array}{c}\mathrm{gr} / \mathrm{CF} @ \\
\text { stack conditions }\end{array}$ & $\mathrm{g} / \mathrm{m} 3$ & Particulate - total \\
\hline $\mathrm{C}_{\mathrm{aw}}$ & lbs/hr & $\mathrm{kg} / \mathrm{hr}$ & $\begin{array}{l}\text { Particulate - probe, cyclone, } \\
\text { and filter }\end{array}$ \\
\hline $\mathrm{C}_{\mathrm{ax}}$ & $\mathrm{lbs} / \mathrm{hr}$ & $\mathrm{kg} / \mathrm{hr}$ & Particulate - total \\
\hline$C_{p}$ & & & Pitot Tube Calibration Factor \\
\hline$D_{n}$ & in. & $\mathrm{m}$ & Sampling Nozzle Diameter \\
\hline$\% \mathrm{EA}$ & & & $\begin{array}{l}\text { Percent Excess Air at } \\
\text { Sampling Point }\end{array}$ \\
\hline g & $32.2 \mathrm{ft} / \mathrm{sec}^{2}$ & & Acceleration of gravity \\
\hline$\% l$ & & & Percent Isokinetic \\
\hline$\% \mathrm{M}$ & & & $\begin{array}{l}\text { Percent Moisture in the Stack } \\
\text { Gas by Volume }\end{array}$ \\
\hline$M_{d}$ & & & Mole Fraction of Dry Gas \\
\hline$m_{f}$ & $\mathrm{mg}$ & $\mathrm{mg}$ & $\begin{array}{l}\text { Particulate - probe, cyclone, } \\
\text { and filter }\end{array}$ \\
\hline $\mathrm{mt}$ & $\mathrm{mg}$ & $\mathrm{mg}$ & Particulate -total \\
\hline$M_{\text {water }}$ & $18 \mathrm{lb} / \mathrm{lb}-\mathrm{mole}$ & & Molecular Weight of Water \\
\hline MW & Ib/lb-mole & g/g-mole & $\begin{array}{l}\text { Molecular Weight of Stack } \\
\text { Gas }\end{array}$ \\
\hline $\mathrm{MW}_{\text {air }}$ & $28.84 \mathrm{lb} / \mathrm{lb}-\mathrm{mole}$ & & Molecular Weight of Air \\
\hline $\mathrm{MW}_{\mathrm{d}}$ & lb/lb-mole & g/g-mole & $\begin{array}{l}\text { Molecular Weight of Dry Stack } \\
\text { Gas }\end{array}$ \\
\hline
\end{tabular}




\begin{tabular}{|c|c|c|c|}
\hline Symbol & $\begin{array}{l}\text { English } \\
\text { Units }\end{array}$ & $\begin{array}{l}\text { Metric } \\
\text { Units }\end{array}$ & Description \\
\hline $\mathrm{Pb}_{\mathrm{b}}$ & "Hg Absolute & $\mathrm{mm} \mathrm{Hg}$ & Barometric Pressure \\
\hline$P_{m}$ & $" \mathrm{H}_{2} \mathrm{O}$ & $\mathrm{mm} \mathrm{H} \mathrm{H}_{2} \mathrm{O}$ & Orifice Pressure drop \\
\hline Ps & "Hg Absolute & $\mathrm{mm} \mathrm{Hg}$ & Stack Pressure \\
\hline$\Delta \mathrm{P}$ & $" \mathrm{H}_{2} \mathrm{O}$ & $\mathrm{mm} \mathrm{H} \mathrm{H}_{2} \mathrm{O}$ & Velocity Head of Stack Gas \\
\hline$P_{\text {std }}$ & 29.92" Hg & $760 \mathrm{~mm} \mathrm{Hg}$ & Standard Barometric Pressure \\
\hline $\mathrm{Q}_{\mathrm{a}}$ & ACFM & $\mathrm{m}^{3} / \mathrm{hr}$ & $\begin{array}{l}\text { Stack Gas Volume at Actual } \\
\text { Stack Conditions }\end{array}$ \\
\hline$Q_{s}$ & DSCFM* & $\mathrm{dscm} / \mathrm{hr} r^{*}$ & $\begin{array}{l}\text { Stack Gas Volume at } 29.92 " \\
\mathrm{Hg}, 528^{\circ} \mathrm{R} \text {, dry }\end{array}$ \\
\hline $\mathrm{R}$ & $\begin{array}{l}21.83 " \mathrm{Hg}- \\
\mathrm{ft}^{3} / \mathrm{lb}-\mathrm{mole}{ }^{\circ} \mathrm{R}\end{array}$ & & Universal Gas Constant \\
\hline$T_{m}$ & ${ }^{\circ} \mathrm{F}$ & ${ }^{\circ} \mathrm{C}$ & $\begin{array}{l}\text { Average Gas Meter } \\
\text { Temperature }\end{array}$ \\
\hline$T_{t}$ & $\min$ & $\min$ & Net Time of Test \\
\hline $\mathrm{T}_{\mathrm{s}}$ & ${ }^{\circ} \mathrm{F}$ & ${ }^{\circ} \mathrm{C}$ & Stack Temperature \\
\hline$T_{\text {std }}$ & $528^{\circ} \mathrm{R}$ & $293^{\circ} \mathrm{K}$ & Standard Temperature \\
\hline$V_{m}$ & $\mathrm{ft}^{3}$ & $\mathrm{~m}^{3}$ & $\begin{array}{l}\text { Volume of Dry Gas Sampled } \\
@ \text { Meter Conditions }\end{array}$ \\
\hline $\mathrm{Vm}_{\text {std }}$ & $d s c f^{*}$ & $\mathrm{dscm}^{*}$ & $\begin{array}{l}\text { Volume of Dry Gas Sampled } \\
\text { @ Standard Conditions }\end{array}$ \\
\hline $\mathrm{V}_{\mathrm{s}}$ & fpm & $\mathrm{m} / \mathrm{sec}$ & $\begin{array}{l}\text { Stack Velocity @ Stack } \\
\text { Conditions }\end{array}$ \\
\hline$V_{w}$ & $\mathrm{ml}$ & $\mathrm{ml}$ & $\begin{array}{l}\text { Total Water Collected in } \\
\text { Impingers and Silica Gel }\end{array}$ \\
\hline$V w_{\text {gas }}$ & $\operatorname{scf}^{*}$ & $\mathrm{scm}^{*}$ & $\begin{array}{l}\text { Volume of Water Vapor } \\
\text { Collected @ Standard } \\
\text { Conditions }\end{array}$ \\
\hline
\end{tabular}


Symbol

$\rho_{\text {air }}$

$\rho_{\text {water }}$

$\rho_{\text {man }}$
English

Units

$0.0748 \mathrm{lbs} / \mathrm{ft}^{3}$

$1 \mathrm{~g} / \mathrm{ml}$

$62.32 \mathrm{lbs} / \mathrm{ft}^{3}$
Metric

$\underline{\text { Units }}$

Description

Density of Air

Density of Water

Density of Manometer Oil

(Inches of Water)

Standard Conditions: $29.92 " \mathrm{Hg}, 68^{\circ} \mathrm{F}\left(760 \mathrm{~mm} \mathrm{Hg}, 20^{\circ} \mathrm{C}\right)$ 


\section{EXAMPLE CALCULATIONS}

1. Volume of dry gas sampled at standard conditions. *

$$
\begin{aligned}
& V_{m s t d}=V_{m}\left(\frac{T_{s t d}}{T_{m}+460}\right)\left[\frac{P_{b}+\frac{P_{m}}{13.6}}{P_{s t d}}\right] \\
& V_{m s t d}=17.65 V_{m}\left[\frac{P_{b}+\frac{P_{m}}{13.6}}{T_{m}+460}\right]=d s c f \\
& V_{m s d}=d s c f \times 0.028317=d s c m
\end{aligned}
$$

2. Volume of water vapor collected at standard conditions. *

$$
\begin{aligned}
& V_{w_{\text {gas }}}=\frac{\left(V_{w}-g m s \mathrm{SO}_{2}-g m s \mathrm{H}_{2} S\right) \rho_{\text {water }} R T_{\text {std }}}{P_{\text {std }} M_{\text {water }} 453.6} \\
& V_{w_{\text {gas }}}=0.0472\left(V_{w}-g m s \mathrm{SO}_{2}-g m s \mathrm{H}_{2} S\right)=s c f \\
& V_{w_{\text {gas }}}=s c f \times 0.028317=s c m
\end{aligned}
$$

3. Percent moisture in stack gas.

$$
\% M=\frac{V_{\text {wgas }}}{V_{m_{s t d}}+V_{w_{g a s}}} \times 100=\%
$$


4. Mole fraction of dry gas.

$$
M_{d}=\frac{100-\% M}{100}
$$

5. Average molecular weight of dry stack gas.

$$
\begin{aligned}
M W_{d}=\left[\% \mathrm{CO}_{2} \times \frac{44}{100}\right]+\left[\% \mathrm{O}_{2} \times \frac{32}{100}\right]+\left[\% \mathrm{~N}_{2} \times \frac{28}{100}\right]+\left[\% \mathrm{CO} \times \frac{28}{100}\right] & =\mathrm{lb} / \mathrm{lb}-\text { mole } \\
& =\mathrm{g} / \mathrm{g}-\mathrm{mole}
\end{aligned}
$$

6. Molecular weight of stack gas.

$$
M W=M W_{d} \times M_{d}+18\left(1-M_{d}\right)=\frac{l b}{l b-m o l e}=g / g-\text { mole }
$$

7. Percent excess air at sampling point.

$$
\% E A=\frac{100\left[\% \mathrm{O}_{2}-(0.5 \% \mathrm{CO})\right]}{0.265\left(\% \mathrm{~N}_{2}\right)-\left[\% \mathrm{O}_{2}-(0.5 \% \mathrm{CO})\right]}
$$

8. Stack Pressure.

$$
P_{s}=P_{b}+\frac{\text { Stack Pressure " } \mathrm{H}_{2} \mathrm{O}}{13.6}=" \mathrm{Hg} \text { Absolute }
$$

$$
P_{s}=" H g \text { Abs. } \times 25.4=m m H g
$$

9. Stack velocity at stack conditions.

$$
\begin{aligned}
& V_{s}=C_{p} 60\left[\frac{2 g \times \rho_{\text {man }} \times P_{\text {std }} \times M W_{\text {air }} \times\left(T_{s}+460\right) \times \Delta P}{12 \times \rho_{\text {air }} \times P_{s} \times M W \times T_{\text {std }}}\right]^{1 / 2} \\
& V_{s}=5,123.8 C_{p}\left[\frac{\left(T_{s}+460\right)}{P_{s} \times M W}\right]^{1 / 2} \sqrt{\Delta P} \text { average }=\mathrm{fpm} \\
& V_{s}=f p m \times 0.00508=\mathrm{m} / \mathrm{sec}
\end{aligned}
$$


10. Dry stack gas volume at standard conditions. *

$$
\begin{aligned}
& Q_{s}=\frac{1}{144} V_{s} \times A_{s} \times M_{d} \times \frac{T_{s t d}}{T_{s}+460} \times \frac{P_{s}}{P_{s t d}} \\
& Q_{s}=\frac{0.123 V_{s} \times A_{s} \times M_{d} \times P_{s}}{T_{s}+460}=D S C F M \\
& Q_{s}=D S C F M \times 1.6990=d s c m / h r
\end{aligned}
$$

11. Actual stack gas volume at stack conditions.

$$
\begin{aligned}
& Q_{a}=\frac{V_{s} \times A_{s}}{144}=A C F M \\
& Q_{a}=A C F M \times 1.6990=m^{3} / h r
\end{aligned}
$$

12. Percent Isokinetic

$$
\begin{aligned}
& \% \mathrm{l}=\frac{V_{\text {mstd }} \times\left(T_{s}+460\right) \times P_{s t d} \times 100 \times 144 i n^{2} / f^{2}}{M_{d} \times T_{s t d} \times P_{s} \times T_{t} \times V_{s}\left(\frac{\Pi \times D_{n}{ }^{2}}{4}\right)} \\
& \% \mathrm{I}=\frac{1039 \times V_{\text {sstd }} \times\left(T_{s}+460\right)}{M_{d} \times P_{s} \times T_{t} \times V_{s} \times D_{n}{ }^{2}}
\end{aligned}
$$

* 29.92" Hg, $68^{\circ} \mathrm{F}\left(760 \mathrm{~mm} \mathrm{Hg}, 20^{\circ} \mathrm{C}\right)$ 
13. Particulate - Probe, cyclone, and filter.

$$
\begin{aligned}
& \mathrm{C}_{\mathrm{an}}=\frac{\mathrm{m}_{\mathrm{f}}}{\mathrm{V}_{\mathrm{mstd}}} \times \frac{1 \mathrm{gr}}{64.8 \mathrm{mg}} \\
& C_{a n}=0.0154 \times \frac{\mathrm{mf}}{\mathrm{V}_{\mathrm{mstd}}} \mathrm{gr} / \mathrm{dscf} \text { * } \\
& \mathrm{C}_{\text {an }}=\mathrm{gr} / \mathrm{dscf} \times 2.290=\mathrm{g} / \mathrm{dscm} \text { * }
\end{aligned}
$$

14. Particulate total.

$$
\begin{aligned}
& \mathrm{C}_{\mathrm{ao}}=0.0154 \times \frac{\mathrm{m}_{\mathrm{t}}}{\mathrm{V}_{\text {mstd }}}=g r / d s c f \text { * } \\
& \mathrm{C}_{\mathrm{ao}}=\mathrm{gr} / \mathrm{dscf} \times 2.290=\mathrm{g} / \mathrm{dscm} \text { * }
\end{aligned}
$$

15. Particulate - probe, cyclone, and filter at stack conditions.

$$
\begin{aligned}
& C_{a t}=C_{a n} \times \frac{P_{s}}{P_{\text {std }}} \times \frac{\left(T_{s t d}\right)}{\left(T_{s}+460\right)} \times M_{d} \\
& C_{a t}=\frac{17.65 \times C_{a n} \times P s \times M d}{T x+460}=g r / C F \\
& C_{a t}=g r / C F \times 2.290=g / m^{3}
\end{aligned}
$$

16. Particulate - total, at stack conditions.

$$
\begin{aligned}
& C_{a u}=\frac{17.65 \times C_{a o} \times P_{s} \times M_{d}}{T_{s}+460}=g r / C F \\
& C_{a u}=g r / C F \times 2.290=g / m^{3}
\end{aligned}
$$

* 29.92" Hg, $68^{\circ} \mathrm{F}\left(760 \mathrm{~mm} \mathrm{Hg}, 20^{\circ} \mathrm{C}\right)$ 
17. Particulate - probe, cyclone, and filter.

$$
\begin{aligned}
& C_{a w}=C_{a n} \times Q_{s} \times \frac{60 \mathrm{~min} .}{1 \mathrm{hr}} \times \frac{1 \mathrm{lb}}{7,000 \mathrm{gr}} \\
& \mathrm{C}_{\mathrm{aw}}=0.00857 \times \mathrm{C}_{\mathrm{an}} \times \mathrm{Q}_{\mathrm{s}}=\mathrm{lbs} / \mathrm{hr} \\
& \mathrm{C}_{\mathrm{aw}}=\mathrm{lbs} / \mathrm{hr} \times 0.4536=\mathrm{kg} / \mathrm{hr}
\end{aligned}
$$

18. Particulate - total.

$$
\begin{aligned}
& C_{a x}=0.00857 \times C_{a o} \times Q_{s}=\mathrm{lbs} / \mathrm{hr} \\
& C_{a x}=\mathrm{lbs} / \mathrm{hr} \times 0.4536=\mathrm{kg} / \mathrm{hr}
\end{aligned}
$$

19. Ammonia-ppm.

$$
\mathrm{ppm}=\frac{\mathrm{mgs}}{\mathrm{Vm}_{\mathrm{std}}(\mathrm{dscm})} \times \frac{24.04}{17}
$$

*29.92" Hg, $68^{\circ} \mathrm{F}\left(760 \mathrm{~mm} \mathrm{Hg}, 20^{\circ} \mathrm{C}\right)$ 
SOURCE EMISSION SURVEY

JOB NUMBER: 08-026

JOB NAME: ADA-ES

LOCATION: Hardin, Montana

UNIT TESTED: SDA Inlet Duct (EPA 17, 26A, CTM-027)

SOURCE EMISSION CALCULATIONS

\begin{tabular}{|c|c|c|c|c|c|}
\hline & & & \multicolumn{3}{|c|}{ RUN NUMBER } \\
\hline SYMBOL & DESCRIPTION & UNITS & 1 & 2 & 3 \\
\hline DATE & & & 09/23/08 & 09/23/08 & $09 / 23 / 08$ \\
\hline BEGIN TIME & & & 0910 & 1250 & 1744 \\
\hline END TIME & & & 1013 & 1354 & 1848 \\
\hline$P(b)$ & BAROMETRIC PRESSURE & $\begin{array}{l}\text { "Hg Abs. } \\
(\mathrm{mm} \mathrm{Hg})\end{array}$ & $\begin{array}{r}27.07 \\
(688.00) \\
\end{array}$ & $\begin{array}{r}27.11 \\
(689.00) \\
\end{array}$ & $\begin{array}{r}27.10 \\
(688.00) \\
\end{array}$ \\
\hline$P(m)$ & ORIFICE PRESSURE DROP & $\begin{array}{l}\text { " } \mathrm{H} 2 \mathrm{O} \\
(\mathrm{mm} \mathrm{H} 2 \mathrm{O})\end{array}$ & $\begin{array}{r}0.730 \\
(18.500) \\
\end{array}$ & $\begin{array}{r}0.551 \\
(14.000) \\
\end{array}$ & $\begin{array}{r}0.560 \\
(14.200) \\
\end{array}$ \\
\hline & DGM CALIBRATION FACTOR & & 0.974 & 0.974 & 0.974 \\
\hline$V(m)$ & $\begin{array}{l}\text { VOLUME DRY GAS SAMPLED } \\
\text { @ METER CONDITIONS } \\
\text { LEAK CHECK VOLUME }\end{array}$ & $\begin{array}{l}\text { ft.^3 } \\
\left(m^{\wedge} 3\right) \\
\text { ft.^3 }\end{array}$ & $\begin{array}{c}28.989 \\
(0.821) \\
1.240 \\
\end{array}$ & $\begin{array}{c}26.502 \\
(0.750) \\
0.842 \\
\end{array}$ & $\begin{array}{c}26.734 \\
(0.757) \\
0.631 \\
\end{array}$ \\
\hline$T(m)$ & $\begin{array}{l}\text { AVERAGE GAS METER } \\
\text { TEMPERATURE }\end{array}$ & $\begin{array}{l}\text { DEG.F } \\
\text { (DEG.C) }\end{array}$ & $\begin{array}{r}49 \\
(9) \\
\end{array}$ & $\begin{array}{r}54 \\
(12) \\
\end{array}$ & $\begin{array}{r}55 \\
(13) \\
\end{array}$ \\
\hline$V(m[s t d])^{*}$ & $\begin{array}{l}\text { VOLUME DRY GAS SAMPLED } \\
\text { @ STANDARD CONDITIONS* }\end{array}$ & $\begin{array}{l}\text { DSCF } \\
\text { (DSCM) }\end{array}$ & $\begin{array}{l}27.265 \\
(0.772) \\
\end{array}$ & $\begin{array}{l}24.708 \\
(0.700) \\
\end{array}$ & $\begin{array}{l}24.867 \\
(0.704) \\
\end{array}$ \\
\hline$V(w)$ & $\begin{array}{l}\text { TOTAL WATER COLLECTED, } \\
\text { IMPINGERS \& SILICA GEL } \\
\end{array}$ & $\mathrm{ml}$ & 74.4 & 62.5 & 67.0 \\
\hline $\mathrm{V}($ w[gas] $)$ & $\begin{array}{l}\text { VOLUME WATER VAPOR } \\
\text { COLLECTED @ STANDARD } \\
\text { CONDITIONS* }\end{array}$ & $\begin{array}{l}\text { SCF } \\
(\mathrm{SCM})\end{array}$ & $\begin{array}{r}3.512 \\
(0.099)\end{array}$ & $\begin{array}{c}2.950 \\
(0.084)\end{array}$ & $\begin{array}{c}3.162 \\
(0.090)\end{array}$ \\
\hline$\% \mathrm{M}$ & $\begin{array}{l}\text { MOISTURE IN STACK GAS } \\
\text { BY VOLUME }\end{array}$ & $\%$ & 11.41 & 10.67 & 11.28 \\
\hline Md & MOL FRACTION OF DRY GAS & & 0.8859 & 0.8933 & 0.8872 \\
\hline Tt & NET TIME OF TEST & MINUTES & 60 & 60 & 60 \\
\hline
\end{tabular}

*68 Deg.F, 29.92 "Hg (20 Deg.C, 760 mm Hg) 
SOURCE EMISSION CALCULATIONS

JOB NUMBER: 08-026

JOB NAME: ADA-ES

LOCATION: Hardin, Montana

UNIT TESTED: SDA Inlet Duct (EPA 17, 26A, CTM-027)

\begin{tabular}{|c|c|c|c|c|c|}
\hline \multirow[b]{2}{*}{ SYMBOL } & \multirow[b]{2}{*}{ DESCRIPTION } & \multirow[b]{2}{*}{ UNITS } & \multicolumn{3}{|c|}{ RUN NUMBER } \\
\hline & & & 1 & \begin{tabular}{l|l}
2 & \\
\end{tabular} & 3 \\
\hline $\mathrm{CO} 2$ & & $\%$ & 13.4 & 14.2 & 14.1 \\
\hline $\mathrm{O} 2$ & & $\%$ & 5.9 & 5.3 & 5.6 \\
\hline $\mathrm{CO}$ & & $\%$ & 0.0 & 0.0 & 0.0 \\
\hline $\mathrm{N} 2$ & & $\%$ & 80.7 & 80.5 & 80.3 \\
\hline$\% \mathrm{EA}$ & $\begin{array}{l}\text { EXCESS AIR @ SAMPLING } \\
\text { POINT }\end{array}$ & $\%$ & 38.1 & 33.1 & 35.7 \\
\hline MWd & $\begin{array}{l}\text { MOLECULAR WEIGHT OF } \\
\text { DRY STACK GAS }\end{array}$ & $\begin{array}{l}\text { LB/LB-MOLE } \\
\text { (g/g-MOLE) }\end{array}$ & $\begin{array}{r}30.38 \\
(30.38) \\
\end{array}$ & $\begin{array}{c}30.48 \\
(30.48) \\
\end{array}$ & $\begin{array}{r}30.48 \\
(30.48) \\
\end{array}$ \\
\hline MW & $\begin{array}{l}\text { MOLECULAR WEIGHT OF } \\
\text { STACK GAS }\end{array}$ & $\begin{array}{l}\text { LB/LB-MOLE } \\
\text { (g/g-MOLE) }\end{array}$ & $\begin{array}{c}28.97 \\
(28.97) \\
\end{array}$ & $\begin{array}{r}29.15 \\
(29.15) \\
\end{array}$ & $\begin{array}{r}29.07 \\
(29.07) \\
\end{array}$ \\
\hline Cp & PITOT TUBE CALIBRATION & & 0.821 & 0.821 & 0.821 \\
\hline DELTAP & $\begin{array}{l}\text { VELOCITY HEAD OF STACK } \\
\text { GAS }\end{array}$ & $\begin{array}{l}\text { " } \mathrm{H} 2 \mathrm{O} \\
\left(\mathrm{mm} \mathrm{H}_{2} \mathrm{O}\right)\end{array}$ & $\begin{array}{r}0.896 \\
(22.800) \\
\end{array}$ & $\begin{array}{r}0.894 \\
(22.700) \\
\end{array}$ & $\begin{array}{r}0.908 \\
(23.100) \\
\end{array}$ \\
\hline DELTAP $\wedge(1 / 2)$ & & $\mathrm{H} 2 \mathrm{O}$ & 0.939 & 0.938 & 0.946 \\
\hline Ts & STACK TEMPERATURE & $\begin{array}{l}\text { DEG. F } \\
\text { (DEG. C) }\end{array}$ & $\begin{array}{r}336 \\
(169) \\
\end{array}$ & $\begin{array}{r}333 \\
(167) \\
\end{array}$ & $\begin{array}{r}330 \\
(166) \\
\end{array}$ \\
\hline Ps & STACK PRESSURE & $\begin{array}{l}\text { "Hg Abs. } \\
(\mathrm{mm} \mathrm{Hg}) \\
\text { "H2O }\end{array}$ & $\begin{array}{r}25.78 \\
(655.00) \\
-17.50 \\
\end{array}$ & $\begin{array}{r}25.88 \\
(657.00) \\
-16.70 \\
\end{array}$ & $\begin{array}{r}25.85 \\
(657.00) \\
-17.00 \\
\end{array}$ \\
\hline Vs & $\begin{array}{l}\text { STACK VELOCITY @ STACK } \\
\text { CONDITIONS }\end{array}$ & $\begin{array}{l}\text { FPM } \\
(\mathrm{m} / \mathrm{SEC} .) \\
\end{array}$ & $\begin{array}{r}4,078 \\
(21) \\
\end{array}$ & $\begin{array}{r}4,046 \\
(21) \\
\end{array}$ & $\begin{array}{r}4,080 \\
(21) \\
\end{array}$ \\
\hline As & STACK AREA & $\begin{array}{l}\text { (SQ.INCHES) } \\
\text { (SQ.METERS) }\end{array}$ & $\begin{array}{r}20,235 \\
(13) \\
\end{array}$ & $\begin{array}{r}20,235 \\
(13) \\
\end{array}$ & $\begin{array}{r}20,235 \\
(13) \\
\end{array}$ \\
\hline Qs & $\begin{array}{l}\text { DRY STACK GAS VOLUME @ } \\
\text { STANDARD CONDITIONS* } \\
\text { WET STACK GAS VOLUME @ } \\
\text { STANDARD CONDITIONS* } \\
\end{array}$ & $\begin{array}{l}\text { DSCFM } \\
\text { (DSCM/HR) } \\
\text { WSCFH }\end{array}$ & $\begin{array}{r}291,213 \\
(494,771) \\
19,723,197\end{array}$ & $\begin{array}{r}293,577 \\
(498,787) \\
19,718,594\end{array}$ & $\begin{array}{r}294,797 \\
(500,860) \\
19,936,677\end{array}$ \\
\hline Qa & $\begin{array}{l}\text { ACTUAL STACK GAS VOLUME } \\
@ \text { STACK CONDITIONS }\end{array}$ & $\begin{array}{l}\text { ACFM } \\
\left(m^{\wedge} 3 / H R\right)\end{array}$ & $\begin{array}{c}573,037 \\
(973,590) \\
\end{array}$ & $\begin{array}{r}568,479 \\
(965,846) \\
\end{array}$ & $\begin{array}{c}573,361 \\
(974,140) \\
\end{array}$ \\
\hline Dn & SAMPLING NOZZLE DIAM. & $\begin{array}{l}\text { IN. } \\
\text { (m) }\end{array}$ & $\begin{array}{c}0.193 \\
(0.005) \\
\end{array}$ & $\begin{array}{c}0.193 \\
(0.005) \\
\end{array}$ & $\begin{array}{c}0.193 \\
(0.005) \\
\end{array}$ \\
\hline$\% 1$ & PERCENT ISOKINETIC & $\%$ & 108.3 & 97.4 & 97.6 \\
\hline
\end{tabular}

* 68 Deg.F, 29.92 "Hg (20 Deg.C, 760 mm Hg) 
SOURCE EMISSION CALCULATIONS

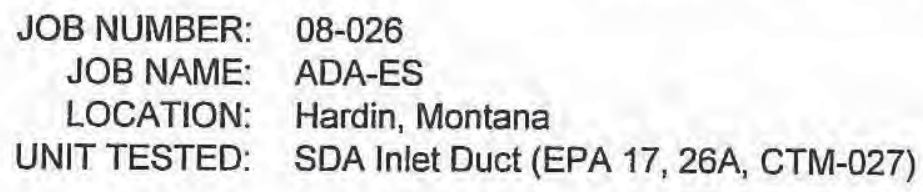

JOB NUMBER: 08-026

JOB NAME: ADA-ES

LOCATION: Hardin, Montana

UNIT TESTED: SDA Inlet Duct (EPA 17, 26A, CTM-027)

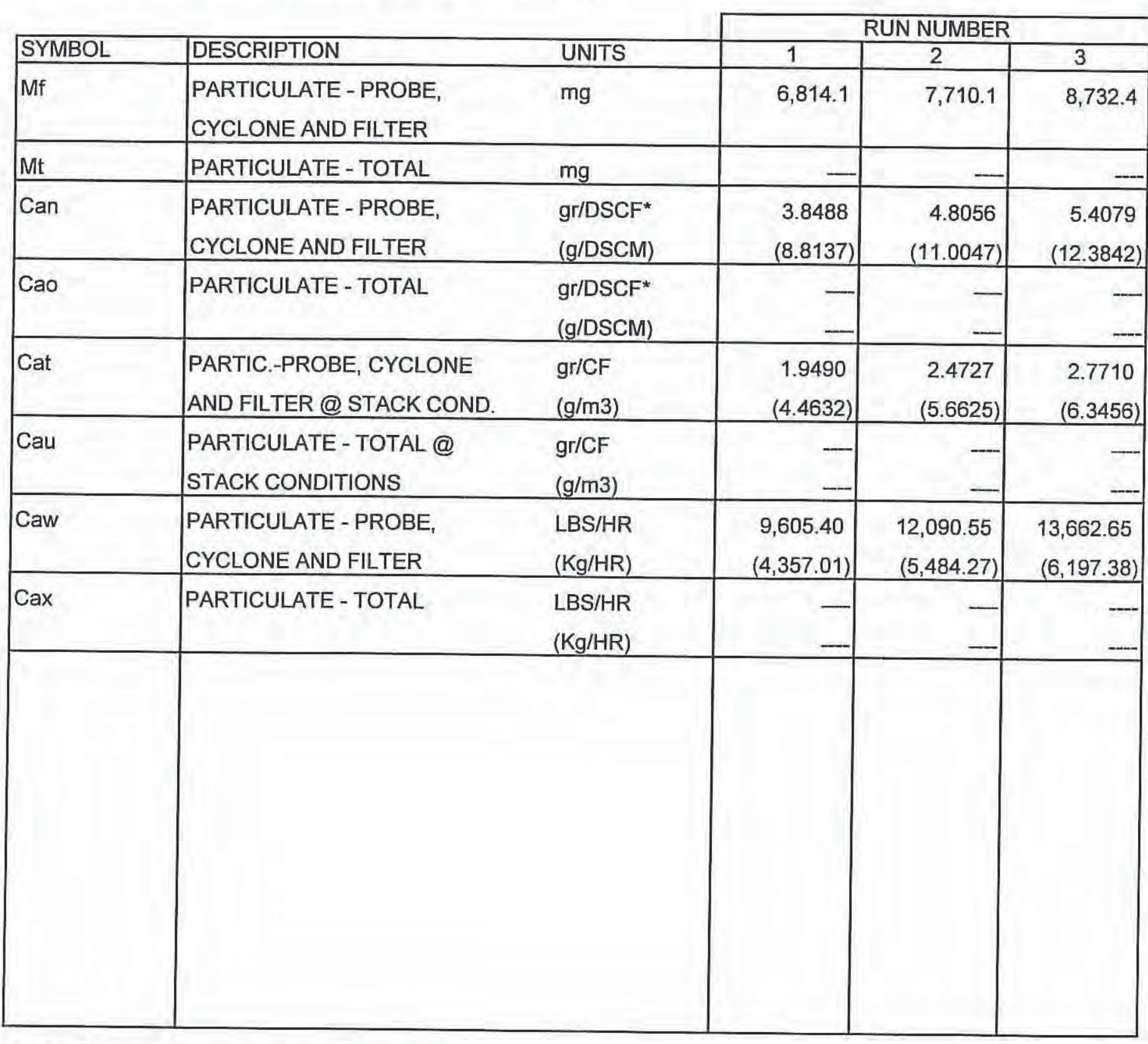

* 68 Deg.F, 29.92 "Hg (20 Deg.C, 760 mm Hg) 
SOURCE EMISSION SURVEY
JOB NUMBER: 08-026
JOB NAME: ADA-ES
LOCATION: Hardin, Montana
UNIT TESTED: SDA Inlet Duct (CCT)

SOURCE EMISSION CALCULATIONS

\begin{tabular}{|c|c|c|c|c|c|}
\hline \multirow[b]{2}{*}{ SYMBOL } & \multirow[b]{2}{*}{ DESCRIPTION } & \multirow[b]{2}{*}{ UNITS } & \multicolumn{3}{|c|}{ RUN NUMBER } \\
\hline & & & 1 & 2 & 3 \\
\hline DATE & & & $09 / 23 / 08$ & $09 / 24 / 08$ & $09 / 24 / 08$ \\
\hline BEGIN TIME & & & 1530 & 0745 & 0947 \\
\hline END TIME & & & 1625 & 0845 & 1047 \\
\hline$P(b)$ & BAROMETRIC PRESSURE & $\begin{array}{l}\text { "Hg Abs. } \\
(\mathrm{mm} \mathrm{Hg})\end{array}$ & $\begin{array}{r}27.11 \\
(689.00) \\
\end{array}$ & $\begin{array}{r}27.10 \\
(688.00) \\
\end{array}$ & $\begin{array}{r}27.10 \\
(688.00) \\
\end{array}$ \\
\hline$P(m)$ & ORIFICE PRESSURE DROP & $\begin{array}{l}\text { " } \mathrm{H} 2 \mathrm{O} \\
(\mathrm{mm} \mathrm{H} 2 \mathrm{O})\end{array}$ & $\begin{array}{r}0.300 \\
(7.600) \\
\end{array}$ & $\begin{array}{c}0.300 \\
(7.600) \\
\end{array}$ & $\begin{array}{r}0.300 \\
(7.600) \\
\end{array}$ \\
\hline & DGM CALIBRATION FACTOR & & 0.974 & 0.974 & 0.974 \\
\hline$V(m)$ & $\begin{array}{l}\text { VOLUME DRY GAS SAMPLED } \\
\text { @ METER CONDITIONS } \\
\text { LEAK CHECK VOLUME }\end{array}$ & $\begin{array}{l}\text { ft.^3 } \\
\left(\mathrm{m}^{\wedge} 3\right) \\
\text { ft. } 3\end{array}$ & $\begin{array}{c}18.177 \\
(0.515) \\
0.000 \\
\end{array}$ & $\begin{array}{c}19.476 \\
(0.552) \\
0.000 \\
\end{array}$ & $\begin{array}{c}19.758 \\
(0.559) \\
0.000 \\
\end{array}$ \\
\hline $\mathrm{T}(\mathrm{m})$ & $\begin{array}{l}\text { AVERAGE GAS METER } \\
\text { TEMPERATURE }\end{array}$ & $\begin{array}{l}\text { DEG.F } \\
\text { (DEG.C) }\end{array}$ & $\begin{array}{c}53 \\
(12) \\
\end{array}$ & $\begin{array}{c}36 \\
(2) \\
\end{array}$ & $\begin{array}{l}46 \\
(8) \\
\end{array}$ \\
\hline$V(m[s t d])^{*}$ & $\begin{array}{l}\text { VOLUME DRY GAS SAMPLED } \\
\text { @ STANDARD CONDITIONS* }\end{array}$ & $\begin{array}{l}\text { DSCF } \\
\text { (DSCM) }\end{array}$ & $\begin{array}{l}16.968 \\
(0.480) \\
\end{array}$ & $\begin{array}{l}18.797 \\
(0.532) \\
\end{array}$ & $\begin{array}{l}18.692 \\
(0.529) \\
\end{array}$ \\
\hline$V(w)$ & $\begin{array}{l}\text { TOTAL WATER COLLECTED, } \\
\text { IMPINGERS \& SILICA GEL }\end{array}$ & $\mathrm{ml}$ & 41.8 & 48.3 & 45.3 \\
\hline $\mathrm{V}(\mathrm{w}$ [gas] $)$ & $\begin{array}{l}\text { VOLUME WATER VAPOR } \\
\text { COLLECTED @ STANDARD } \\
\text { CONDITIONS* }\end{array}$ & $\begin{array}{l}\text { SCF } \\
\text { (SCM) }\end{array}$ & $\begin{array}{r}1.973 \\
(0.056)\end{array}$ & $\begin{array}{r}2.280 \\
(0.065)\end{array}$ & $\begin{array}{c}2.138 \\
(0.061)\end{array}$ \\
\hline$\% \mathrm{M}$ & $\begin{array}{l}\text { MOISTURE IN STACK GAS } \\
\text { BY VOLUME }\end{array}$ & $\%$ & 10.42 & 10.82 & 10.26 \\
\hline Md & MOL FRACTION OF DRY GAS & & 0.8958 & 0.8918 & 0.8974 \\
\hline $\mathrm{Tt}$ & NET TIME OF TEST & MINUTES & 55 & 60 & 60 \\
\hline
\end{tabular}

*68 Deg.F, 29.92 "Hg (20 Deg.C, 760 mm Hg) 
JOB NUMBER: 08-026

JOB NAME: ADA-ES

LOCATION: Hardin, Montana

UNIT TESTED: SDA Inlet Duct (CCT)

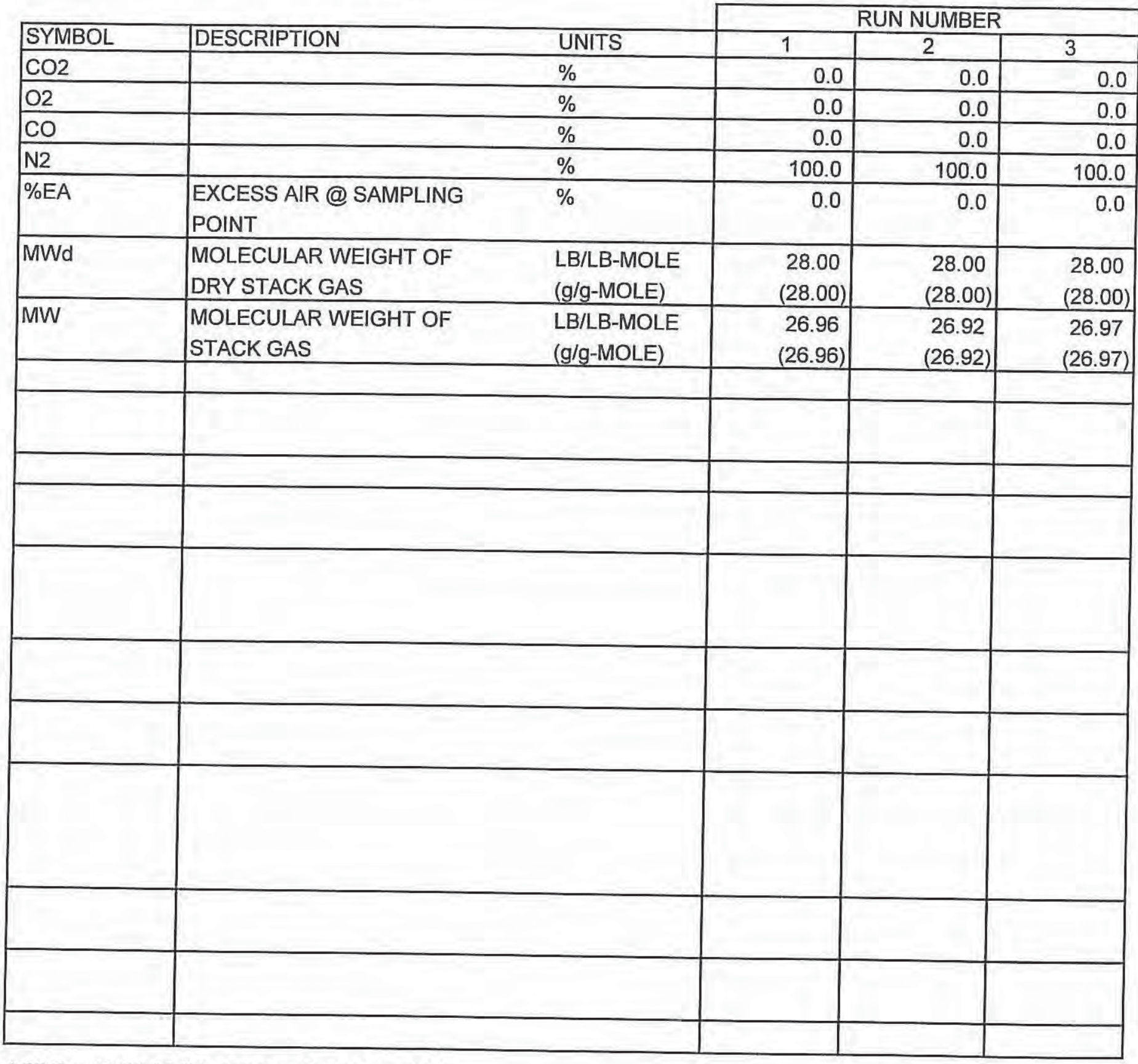

* 68 Deg.F, 29.92 "Hg (20 Deg.C, 760 mm Hg) 


\section{SULFUR TRIOXIDE EMISSION DATA}

\begin{tabular}{ll} 
JOB NUMBER: & $08-026$ \\
JOB NAME: & ADA-ES \\
LOCATION: & Hardin, Montana \\
UNIT TESTED: & SDA Inlet Duct (CCT) \\
RUN NUMBER & \\
\hline
\end{tabular}

\begin{tabular}{|c|c|c|c|}
\hline RUN NUMBER & \multicolumn{3}{|c|}{ RUN NUMBER } \\
\hline & 1 & \begin{tabular}{|l|}
2 \\
\end{tabular} & 3 \\
\hline DATE & $09 / 23 / 08$ & $09 / 24 / 08$ & $09 / 24 / 08$ \\
\hline START TIME & 1530 & 0745 & 0947 \\
\hline END TIME & 1625 & 0845 & 1047 \\
\hline $\mathrm{N}$ - NORMALITY OF Ba(ClO4)2 & 0.0000 & 0.0000 & 0.0000 \\
\hline ML(I) - mI IN IMPINGER & 0.00 & 0.00 & 0.00 \\
\hline ML(A) - mI IN ALIQUOT & 0.00 & 0.00 & 0.00 \\
\hline $\mathrm{ML}(\mathrm{B})$ - $\mathrm{ml}$ OF $\mathrm{Ba}(\mathrm{ClO} 4) 2$ TO TITRATE & 0.00 & 0.00 & 0.00 \\
\hline $\mathrm{ML}(\mathrm{BB})$ - $\mathrm{ml}$ OF $\mathrm{Ba}(\mathrm{ClO} 4) 2$ TO TITRATE BLANK & 0.00 & 0.00 & 0.00 \\
\hline Tm - AVERAGE GAS METER TEMPERATURE, DEG.F & 53 & 36 & 46 \\
\hline $\begin{array}{l}\text { Vm - VOLUME OF DRY GAS SAMPLED @ METER } \\
\text { CONDITIONS, FT^3 }\end{array}$ & 18.177 & 19.476 & 19.758 \\
\hline Pb - BAROMETRIC PRESSURE, "Hg Abs. & 27.11 & 27.10 & 27.10 \\
\hline $\begin{array}{l}\text { QS - STACK GAS VOLUME DRY @ STANDARD } \\
\text { CONDITIONS, *SCFM }\end{array}$ & - & - & - \\
\hline C(I) - SO3 IN IMPINGERS IN mgs & 5,606 & 22,952 & 2.606 \\
\hline ppm SO3 & 3,51 & 12.99 & 1.48 \\
\hline C(SO3) - EMISSION RATE OF SO3, Ibs/day & - & - & - \\
\hline Cs - EMISSION RATE OF SULFUR, Ibs/day & - & - & - \\
\hline
\end{tabular}

*68 Deg.F, 29.92 "Hg (20 Deg.C, 760 mm Hg)

$C(I)=M L(I) *[M L(B)-M L(B B)] * N * 40 / M L(A)$

$\mathrm{ppm} \mathrm{SO3}=0.6021{ }^{*} \mathrm{C}(\mathrm{I}) *[\mathrm{Tm}+460] / \mathrm{Vm}{ }^{*} \mathrm{~Pb}$

$\mathrm{C}(\mathrm{SO} 3)=\mathrm{ppm} \mathrm{SO} 3$ * Qs * 0.0002991

$\mathrm{Cs}=\mathrm{C}(\mathrm{SO} 3) / 2.5$ 
SOURCE EMISSION SURVEY

$\begin{aligned} \text { JOB NUMBER: } & 08-026 \\ \text { JOB NAME: } & \text { ADA-ES } \\ \text { LOCATION: } & \text { Hardin, Montana } \\ \text { UNIT TESTED: } & \text { Stack (Method } 5 / 202 \text { ) }\end{aligned}$

SOURCE EMISSION CALCULATIONS

\begin{tabular}{|c|c|c|c|c|c|}
\hline & & & \multicolumn{3}{|c|}{ RUN NUMBER } \\
\hline SYMBOL & DESCRIPTION & UNITS & 1 & 2 & 3 \\
\hline DATE & & & $09 / 23 / 08$ & $09 / 23 / 08$ & $09 / 24 / 08$ \\
\hline BEGIN TIME & & & 1058 & 1551 & 0710 \\
\hline END TIME & & & 1302 & 1756 & 0914 \\
\hline$P(b)$ & BAROMETRIC PRESSURE & $\begin{array}{l}\text { "Hg Abs. } \\
(\mathrm{mm} \mathrm{Hg})\end{array}$ & $\begin{array}{r}27.11 \\
(689.00) \\
\end{array}$ & $\begin{array}{r}27.14 \\
(689.00) \\
\end{array}$ & $\begin{array}{r}27.14 \\
(689.00) \\
\end{array}$ \\
\hline$P(m)$ & ORIFICE PRESSURE DROP & $\begin{array}{l}\text { "H2O } \\
(\mathrm{mm} \mathrm{H} 2 \mathrm{O})\end{array}$ & $\begin{array}{r}1.633 \\
(41.500) \\
\end{array}$ & $\begin{array}{r}1.617 \\
(41.100) \\
\end{array}$ & $\begin{array}{r}1.700 \\
(43.200) \\
\end{array}$ \\
\hline & DGM CALIBRATION FACTOR & & 0.997 & 0.997 & 0.997 \\
\hline$V(m)$ & $\begin{array}{l}\text { VOLUME DRY GAS SAMPLED } \\
\text { @ METER CONDITIONS } \\
\text { LEAK CHECK VOLUME }\end{array}$ & $\begin{array}{l}\text { ft.^3 } \\
\left(m^{\wedge} 3\right) \\
\text { ft. } 3\end{array}$ & $\begin{array}{c}91.120 \\
(2.580) \\
0.000 \\
\end{array}$ & $\begin{array}{c}91.433 \\
(2.589) \\
0.000 \\
\end{array}$ & $\begin{array}{c}91.612 \\
(2.594) \\
0.000 \\
\end{array}$ \\
\hline $\mathrm{T}(\mathrm{m})$ & $\begin{array}{l}\text { AVERAGE GAS METER } \\
\text { TEMPERATURE }\end{array}$ & $\begin{array}{l}\text { DEG.F } \\
\text { (DEG.C) }\end{array}$ & $\begin{array}{r}86 \\
(30) \\
\end{array}$ & $\begin{array}{r}89 \\
(32) \\
\end{array}$ & $\begin{array}{r}69 \\
(21) \\
\end{array}$ \\
\hline$V(m[s t d])^{*}$ & $\begin{array}{l}\text { VOLUME DRY GAS SAMPLED } \\
\text { @ STANDARD CONDITIONS* }\end{array}$ & $\begin{array}{l}\text { DSCF } \\
\text { (DSCM) }\end{array}$ & $\begin{array}{l}80.207 \\
(2.271) \\
\end{array}$ & $\begin{array}{l}80.128 \\
(2.269) \\
\end{array}$ & $\begin{array}{l}83.339 \\
(2.360) \\
\end{array}$ \\
\hline$V(w)$ & $\begin{array}{l}\text { TOTAL WATER COLLECTED, } \\
\text { IMPINGERS \& SILICA GEL }\end{array}$ & $\mathrm{ml}$ & 308.4 & 304.7 & 334.1 \\
\hline$V(w[g a s])$ & $\begin{array}{l}\text { VOLUME WATER VAPOR } \\
\text { COLLECTED @ STANDARD } \\
\text { CONDITIONS }{ }^{\star}\end{array}$ & $\begin{array}{l}\text { SCF } \\
\text { (SCM) }\end{array}$ & $\begin{array}{l}14.556 \\
(0.412)\end{array}$ & $\begin{array}{l}14.382 \\
(0.407)\end{array}$ & $\begin{array}{l}15.770 \\
(0.447)\end{array}$ \\
\hline$\% \mathrm{M}$ & $\begin{array}{l}\text { MOISTURE IN STACK GAS } \\
\text { BY VOLUME }\end{array}$ & $\%$ & 15.36 & 15.22 & 15.91 \\
\hline Md & MOL FRACTION OF DRY GAS & & 0.8464 & 0.8478 & 0.8409 \\
\hline Tt & NET TIME OF TEST & MINUTES & 120 & 120 & 120 \\
\hline
\end{tabular}

*68 Deg.F, 29.92 "Hg (20 Deg.C, 760 mm Hg) 


\section{SOURCE EMISSION CALCULATIONS}

JOB NUMBER: $\quad 08-026$

JOB NAME: ADA-ES

LOCATION: Hardin, Montana

UNIT TESTED: Stack (Method 5/202)

\begin{tabular}{|c|c|c|c|c|c|}
\hline & & & \multicolumn{3}{|c|}{ RUN NUMBER } \\
\hline SYMBOL & DESCRIPTION & UNITS & 1 & 2 & 3 \\
\hline $\mathrm{CO} 2$ & & $\%$ & 13.4 & 13.7 & 13.4 \\
\hline $\mathrm{O} 2$ & & $\%$ & 6.2 & 6.0 & 6.2 \\
\hline $\mathrm{CO}$ & & $\%$ & 0.0 & 0.0 & 0.0 \\
\hline N2 & & $\%$ & 80.4 & 80.3 & 80.4 \\
\hline$\% \mathrm{EA}$ & $\begin{array}{l}\text { EXCESS AIR @ SAMPLING } \\
\text { POINT }\end{array}$ & $\%$ & 41.0 & 39.3 & 41.0 \\
\hline MWd & $\begin{array}{l}\text { MOLECULAR WEIGHT OF } \\
\text { DRY STACK GAS }\end{array}$ & $\begin{array}{l}\text { LB/LB-MOLE } \\
\text { (g/g-MOLE) }\end{array}$ & $\begin{array}{r}30.39 \\
(30.39) \\
\end{array}$ & $\begin{array}{r}30.43 \\
(30.43) \\
\end{array}$ & $\begin{array}{r}30.39 \\
(30.39) \\
\end{array}$ \\
\hline MW & $\begin{array}{l}\text { MOLECULAR WEIGHT OF } \\
\text { STACK GAS }\end{array}$ & $\begin{array}{l}\text { LB/LB-MOLE } \\
\text { (g/g-MOLE) }\end{array}$ & $\begin{array}{r}28.49 \\
(28.49) \\
\end{array}$ & $\begin{array}{c}28.54 \\
(28.54) \\
\end{array}$ & $\begin{array}{r}28.42 \\
(28.42) \\
\end{array}$ \\
\hline Cp & PITOT TUBE CALIBRATION & & 0.823 & 0.823 & 0.823 \\
\hline DELTAP & $\begin{array}{l}\text { VELOCITY HEAD OF STACK } \\
\text { GAS }\end{array}$ & $\begin{array}{l}\text { "H2O } \\
(\mathrm{mm} \mathrm{H} 20) \\
\end{array}$ & $\begin{array}{r}3.444 \\
(87.500) \\
\end{array}$ & $\begin{array}{r}3.304 \\
(83.900) \\
\end{array}$ & $\begin{array}{r}3.442 \\
(87.400) \\
\end{array}$ \\
\hline DELTAP^(1/2) & & "H2O & 1.855 & 1.816 & 1.853 \\
\hline Ts & STACK TEMPERATURE & $\begin{array}{l}\text { DEG. F } \\
\text { (DEG.C) }\end{array}$ & $\begin{array}{c}206 \\
(97) \\
\end{array}$ & $\begin{array}{c}204 \\
(96) \\
\end{array}$ & $\begin{array}{l}210 \\
(99) \\
\end{array}$ \\
\hline Ps & STACK PRESSURE & $\begin{array}{l}\text { "Hg Abs. } \\
\text { (mm Hg) } \\
\text { "H2O }\end{array}$ & $\begin{array}{r}27.00 \\
(686.00) \\
-1.50 \\
\end{array}$ & $\begin{array}{r}27.05 \\
(687.00) \\
-1.20 \\
\end{array}$ & $\begin{array}{r}27.08 \\
(688.00) \\
-0.88 \\
\end{array}$ \\
\hline Vs & $\begin{array}{l}\text { STACK VELOCITY @ STACK } \\
\text { CONDITIONS }\end{array}$ & $\begin{array}{l}\text { FPM } \\
\text { (m/SEC.) }\end{array}$ & $\begin{array}{r}7,279 \\
(37) \\
\end{array}$ & $\begin{array}{r}7,102 \\
(36) \\
\end{array}$ & $\begin{array}{r}7,291 \\
(37) \\
\end{array}$ \\
\hline As & STACK AREA & $\begin{array}{l}\text { (SQ.INCHES) } \\
\text { (SQ.METERS) }\end{array}$ & $\begin{array}{r}9,363 \\
(6) \\
\end{array}$ & $\begin{array}{r}9,363 \\
(6) \\
\end{array}$ & $\begin{array}{r}9,363 \\
(6) \\
\end{array}$ \\
\hline Qs & $\begin{array}{l}\text { DRY STACK GAS VOLUME @ } \\
\text { STANDARD CONDITIONS* } \\
\text { WET STACK GAS VOLUME @ } \\
\text { STANDARD CONDITIONS* } \\
\end{array}$ & $\begin{array}{l}\text { DSCFM } \\
\text { (DSCM/HR) } \\
\text { WSCFH }\end{array}$ & $\begin{array}{r}287,645 \\
(488,709) \\
20,390,714\end{array}$ & $\begin{array}{r}282,484 \\
(479,940) \\
19,991,791\end{array}$ & $\begin{array}{r}285,381 \\
(484,862) \\
20,362,540\end{array}$ \\
\hline Qa & $\begin{array}{l}\text { ACTUAL STACK GAS VOLUME } \\
\text { @ STACK CONDITIONS }\end{array}$ & $\begin{array}{l}\text { ACFM } \\
\left(m^{\wedge} 3 / H R\right)\end{array}$ & $\begin{array}{c}473,257 \\
(804,064) \\
\end{array}$ & $\begin{array}{c}461,779 \\
(784,563) \\
\end{array}$ & $\begin{array}{c}474,047 \\
(805,406) \\
\end{array}$ \\
\hline $\mathrm{Dn}$ & SAMPLING NOZZLE DIAM. & $\begin{array}{l}\text { IN. } \\
(\mathrm{m})\end{array}$ & $\begin{array}{c}0.168 \\
(0.004) \\
\end{array}$ & $\begin{array}{c}0.168 \\
(0.004) \\
\end{array}$ & $\begin{array}{c}0.168 \\
(0.004) \\
\end{array}$ \\
\hline$\% 1$ & PERCENT ISOKINETIC & $\%$ & 98.5 & 100.2 & 103.2 \\
\hline
\end{tabular}

*68 Deg.F, 29.92 "Hg (20 Deg.C, 760 mm Hg) 
SOURCE EMISSION CALCULATIONS

JOB NUMBER: $\quad$ 08-026

JOB NAME: ADA-ES

LOCATION: Hardin, Montana

UNIT TESTED: Stack (Method 5/202)

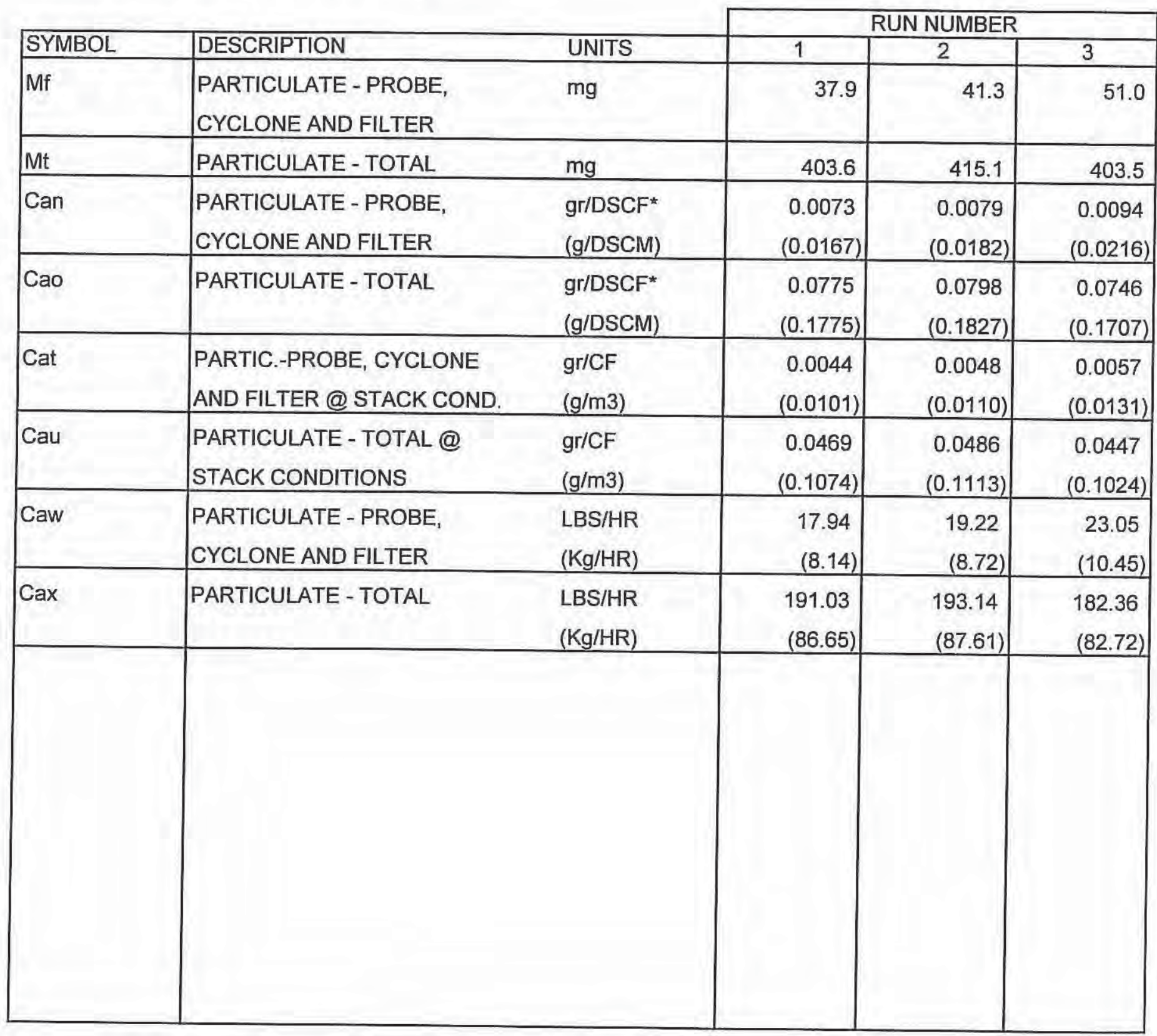

*68 Deg.F, 29.92 "Hg (20 Deg.C, 760 mm Hg) 


\section{SOURCE EMISSION SURVEY}

JOB NUMBER: $\quad 08-026$

JOB NAME: ADA-ES

LOCATION: Hardin, Montana

UNIT TESTED: Stack (26A)

SOURCE EMISSION CALCULATIONS

\begin{tabular}{|c|c|c|c|c|c|}
\hline & & & \multicolumn{3}{|c|}{ RUN NUMBER } \\
\hline SYMBOL & DESCRIPTION & UNITS & 1 & 2 & 3 \\
\hline DATE & & & 09/23/08 & $09 / 23 / 08$ & $09 / 23 / 08$ \\
\hline BEGIN TIME & & & 0910 & 1354 & 1823 \\
\hline END TIME & & & 1014 & 1503 & 1926 \\
\hline$P(b)$ & BAROMETRIC PRESSURE & $\begin{array}{l}\text { "Hg Abs. } \\
(\mathrm{mm} \mathrm{Hg})\end{array}$ & $\begin{array}{r}27.11 \\
(689.00) \\
\end{array}$ & $\begin{array}{r}27.11 \\
(689.00) \\
\end{array}$ & $\begin{array}{r}27.14 \\
(689.00) \\
\end{array}$ \\
\hline$P(m)$ & ORIFICE PRESSURE DROP & $\begin{array}{l}\text { " } \mathrm{H} 2 \mathrm{O} \\
(\mathrm{mm} \mathrm{H} 2 \mathrm{O})\end{array}$ & $\begin{array}{r}1.379 \\
(35.000) \\
\end{array}$ & $\begin{array}{r}1.317 \\
(33.500) \\
\end{array}$ & $\begin{array}{r}1.283 \\
(32.600) \\
\end{array}$ \\
\hline & DGM CALIBRATION FACTOR & & 0.997 & 0.997 & 0.997 \\
\hline$V(m)$ & $\begin{array}{l}\text { VOLUME DRY GAS SAMPLED } \\
\text { @ METER CONDITIONS } \\
\text { LEAK CHECK VOLUME }\end{array}$ & $\begin{array}{l}\mathrm{ft} \wedge^{\wedge} 3 \\
\left(\mathrm{~m}^{\wedge} 3\right) \\
\mathrm{ft} \wedge 3\end{array}$ & $\begin{array}{c}41.543 \\
(1.176) \\
0.000 \\
\end{array}$ & $\begin{array}{c}41.524 \\
(1.176) \\
0.000 \\
\end{array}$ & $\begin{array}{c}41.261 \\
(1.168) \\
0.000 \\
\end{array}$ \\
\hline $\mathrm{T}(\mathrm{m})$ & $\begin{array}{l}\text { AVERAGE GAS METER } \\
\text { TEMPERATURE } \\
\end{array}$ & $\begin{array}{l}\text { DEG.F } \\
\text { (DEG.C) }\end{array}$ & $\begin{array}{r}68 \\
(20) \\
\end{array}$ & $\begin{array}{r}86 \\
(30) \\
\end{array}$ & $\begin{array}{r}87 \\
(31) \\
\end{array}$ \\
\hline$V(m[s t d])^{*}$ & $\begin{array}{l}\text { VOLUME DRY GAS SAMPLED } \\
\text { @ STANDARD CONDITIONS* }\end{array}$ & $\begin{array}{l}\text { DSCF } \\
\text { (DSCM) }\end{array}$ & $\begin{array}{l}37.788 \\
(1.070)\end{array}$ & $\begin{array}{l}36.520 \\
(1.034) \\
\end{array}$ & $\begin{array}{r}36.259 \\
(1.027) \\
\end{array}$ \\
\hline$V(w)$ & $\begin{array}{l}\text { TOTAL WATER COLLECTED, } \\
\text { IMPINGERS \& SILICA GEL }\end{array}$ & $\mathrm{ml}$ & 152.8 & 144.4 & 133.5 \\
\hline $\mathrm{V}(\mathrm{w}[$ gas $])$ & $\begin{array}{l}\text { VOLUME WATER VAPOR } \\
\text { COLLECTED @ STANDARD } \\
\text { CONDITIONS* }\end{array}$ & $\begin{array}{l}\text { SCF } \\
(\mathrm{SCM})\end{array}$ & $\begin{array}{c}7.212 \\
(0.204)\end{array}$ & $\begin{array}{c}6.816 \\
(0.193)\end{array}$ & $\begin{array}{c}6.301 \\
(0.178)\end{array}$ \\
\hline$\% M$ & $\begin{array}{l}\text { MOISTURE IN STACK GAS } \\
\text { BY VOLUME }\end{array}$ & $\%$ & 16.03 & 15.73 & 14.81 \\
\hline Md & MOL FRACTION OF DRY GAS & & 0.8397 & 0.8427 & 0.8519 \\
\hline Tt & NET TIME OF TEST & MINUTES & 60 & 60 & 60 \\
\hline
\end{tabular}

* 68 Deg.F, 29.92 "Hg (20 Deg.C, 760 mm Hg) 
JOB NUMBER: $\quad$ 08-026

JOB NAME: ADA-ES

LOCATION: Hardin, Montana

UNIT TESTED: Stack (26A)

\begin{tabular}{|c|c|c|c|c|c|}
\hline & & & & RUN NUMBER & \\
\hline SYMBOL & DESCRIPTION & UNITS & 1 & \begin{tabular}{l|l}
2 \\
\end{tabular} & 3 \\
\hline $\mathrm{CO} 2$ & & $\%$ & 13.6 & 13.5 & 13.6 \\
\hline $\mathrm{O} 2$ & & $\%$ & 6.1 & 6.1 & 6.0 \\
\hline $\mathrm{CO}$ & & $\%$ & 0.0 & 0.0 & 0.0 \\
\hline N2 & & $\%$ & 80.3 & 80.4 & 80.4 \\
\hline \%EA & $\begin{array}{l}\text { EXCESS AIR @ SAMPLING } \\
\text { POINT }\end{array}$ & $\%$ & 40.2 & 40.1 & 39.2 \\
\hline MWd & $\begin{array}{l}\text { MOLECULAR WEIGHT OF } \\
\text { DRY STACK GAS }\end{array}$ & $\begin{array}{l}\text { LB/LB-MOLE } \\
\text { (g/g-MOLE) }\end{array}$ & $\begin{array}{r}30.42 \\
(30.42) \\
\end{array}$ & $\begin{array}{r}30.40 \\
(30.40) \\
\end{array}$ & $\begin{array}{r}30.42 \\
(30.42) \\
\end{array}$ \\
\hline MW & $\begin{array}{l}\text { MOLECULAR WEIGHT OF } \\
\text { STACK GAS }\end{array}$ & $\begin{array}{l}\text { LB/LB-MOLE } \\
\text { (g/g-MOLE) }\end{array}$ & $\begin{array}{r}28.43 \\
(28.43) \\
\end{array}$ & $\begin{array}{c}28.45 \\
(28.45) \\
\end{array}$ & $\begin{array}{r}28.58 \\
(28.58) \\
\end{array}$ \\
\hline $\mathrm{Cp}$ & PITOT TUBE CALIBRATION & & 0.823 & 0.823 & 0.823 \\
\hline DELTAP & $\begin{array}{l}\text { VELOCITY HEAD OF STACK } \\
\text { GAS }\end{array}$ & $\begin{array}{l}\text { " } \mathrm{H} 2 \mathrm{O} \\
(\mathrm{mm} \mathrm{H} 20)\end{array}$ & $\begin{array}{r}3.954 \\
(100.400) \\
\end{array}$ & $\begin{array}{r}3.850 \\
(97.800) \\
\end{array}$ & $\begin{array}{r}3.763 \\
(95.600) \\
\end{array}$ \\
\hline DELTA $P \wedge(1 / 2)$ & & "H2O & 1.987 & 1.961 & 1.939 \\
\hline Ts & STACK TEMPERATURE & $\begin{array}{l}\text { DEG. F } \\
\text { (DEG. C) }\end{array}$ & $\begin{array}{c}207 \\
(97) \\
\end{array}$ & $\begin{array}{l}208 \\
(98) \\
\end{array}$ & $\begin{array}{l}207 \\
(97) \\
\end{array}$ \\
\hline Ps & STACK PRESSURE & $\begin{array}{l}\text { "Hg Abs. } \\
(\mathrm{mm} \mathrm{Hg}) \\
\text { "H2O }\end{array}$ & $\begin{array}{r}26.99 \\
(686.00) \\
-1.60 \\
\end{array}$ & $\begin{array}{r}27.00 \\
(686.00) \\
-1.50 \\
\end{array}$ & $\begin{array}{c}27.04 \\
(687.00) \\
-1.30 \\
\end{array}$ \\
\hline Vs & $\begin{array}{l}\text { STACK VELOCITY @ STACK } \\
\text { CONDITIONS }\end{array}$ & $\begin{array}{l}\text { FPM } \\
(\mathrm{m} / \mathrm{SEC} .) \\
\end{array}$ & $\begin{array}{r}7,812 \\
(40) \\
\end{array}$ & $\begin{array}{r}7,711 \\
(39) \\
\end{array}$ & $\begin{array}{r}7,596 \\
(39) \\
\end{array}$ \\
\hline As & STACK AREA & $\begin{array}{l}\text { (SQ.INCHES) } \\
\text { (SQ.METERS) }\end{array}$ & $\begin{array}{r}9,363 \\
(6) \\
\end{array}$ & $\begin{array}{r}9,363 \\
(6) \\
\end{array}$ & $\begin{array}{r}9,363 \\
(6) \\
\end{array}$ \\
\hline Qs & $\begin{array}{l}\text { DRY STACK GAS VOLUME @ } \\
\text { STANDARD CONDITIONS* } \\
\text { WET STACK GAS VOLUME @ } \\
\text { STANDARD CONDITIONS* }\end{array}$ & $\begin{array}{l}\text { DSCFM } \\
\text { (DSCM/HR) } \\
\text { WSCFH }\end{array}$ & $\begin{array}{r}305,692 \\
(519,371) \\
21,842,944\end{array}$ & $\begin{array}{r}302,476 \\
(513,907) \\
21,536,205\end{array}$ & $\begin{array}{r}302,117 \\
(513,297) \\
21,278,343\end{array}$ \\
\hline Qa & $\begin{array}{l}\text { ACTUAL STACK GAS VOLUME } \\
@ \text { STACK CONDITIONS } \\
\end{array}$ & $\begin{array}{l}\text { ACFM } \\
\left(m^{\wedge} 3 / H R\right)\end{array}$ & $\begin{array}{r}507,944 \\
(862,997) \\
\end{array}$ & $\begin{array}{r}501,403 \\
(851,884) \\
\end{array}$ & $\begin{array}{c}493,913 \\
(839,158) \\
\end{array}$ \\
\hline Dn & SAMPLING NOZZLE DIAM. & $\begin{array}{l}\text { IN. } \\
(\mathrm{m})\end{array}$ & $\begin{array}{r}0.155 \\
(0.004) \\
\end{array}$ & $\begin{array}{r}0.155 \\
(0.004) \\
\end{array}$ & $\begin{array}{r}0.155 \\
(0.004) \\
\end{array}$ \\
\hline$\% 1$ & PERCENT ISOKINETIC & $\%$ & 102.6 & 100.2 & 99.6 \\
\hline
\end{tabular}

*68 Deg.F, 29.92 "Hg (20 Deg.C, 760 mm Hg) 
SOURCE EMISSION SURVEY

JOB NUMBER: $\quad$ 08-026

JOB NAME: ADA-ES

LOCATION: Hardin, Montana

UNIT TESTED: Stack (CCT)

SOURCE EMISSION CALCULATIONS

\begin{tabular}{|c|c|c|c|c|c|}
\hline & & & \multicolumn{3}{|c|}{ RUN NUMBER } \\
\hline SYMBOL & DESCRIPTION & UNITS & 1 & 2 & 3 \\
\hline DATE & & & $09 / 24 / 08$ & $09 / 24 / 08$ & $09 / 24 / 08$ \\
\hline BEGIN TIME & & & 1407 & 1600 & 1745 \\
\hline END TIME & & & 1507 & 1700 & 1845 \\
\hline$P(b)$ & BAROMETRIC PRESSURE & $\begin{array}{l}\text { "Hg Abs. } \\
(\mathrm{mm} \mathrm{Hg})\end{array}$ & $\begin{array}{r}27.08 \\
(688.00) \\
\end{array}$ & $\begin{array}{r}27.02 \\
(686.00) \\
\end{array}$ & $\begin{array}{r}26.99 \\
(686.00) \\
\end{array}$ \\
\hline$P(m)$ & ORIFICE PRESSURE DROP & $\begin{array}{l}\text { "H2O } \\
(\mathrm{mm} \mathrm{H} 2 \mathrm{O}) \\
\end{array}$ & $\begin{array}{r}0.300 \\
(7.600) \\
\end{array}$ & $\begin{array}{r}0.300 \\
(7.600) \\
\end{array}$ & $\begin{array}{r}0.300 \\
(7.600) \\
\end{array}$ \\
\hline & DGM CALIBRATION FACTOR & & 0.974 & 0.974 & 0.974 \\
\hline $\mathrm{V}(\mathrm{m})$ & $\begin{array}{l}\text { VOLUME DRY GAS SAMPLED } \\
\text { @ METER CONDITIONS } \\
\text { LEAK CHECK VOLUME }\end{array}$ & $\begin{array}{l}\text { ft. }{ }^{\wedge} \\
\left(m^{\wedge} 3\right) \\
\text { ft. }{ }^{\wedge} 3 \\
\end{array}$ & $\begin{array}{c}20.209 \\
(0.572) \\
0.000\end{array}$ & $\begin{array}{c}20.321 \\
(0.575) \\
0.000 \\
\end{array}$ & $\begin{array}{c}20.266 \\
(0.574) \\
0.000 \\
\end{array}$ \\
\hline$T(m)$ & $\begin{array}{l}\text { AVERAGE GAS METER } \\
\text { TEMPERATURE }\end{array}$ & $\begin{array}{l}\text { DEG.F } \\
\text { (DEG.C) }\end{array}$ & $\begin{array}{r}65 \\
(18) \\
\end{array}$ & $\begin{array}{c}71 \\
(22) \\
\end{array}$ & $\begin{array}{r}69 \\
(21) \\
\end{array}$ \\
\hline$V(m[s t d])^{*}$ & $\begin{array}{l}\text { VOLUME DRY GAS SAMPLED } \\
\text { @ STANDARD CONDITIONS* }\end{array}$ & $\begin{array}{l}\text { DSCF } \\
\text { (DSCM) }\end{array}$ & $\begin{array}{l}18.413 \\
(0.521) \\
\end{array}$ & $\begin{array}{l}18.266 \\
(0.517) \\
\end{array}$ & $\begin{array}{l}18.265 \\
(0.517) \\
\end{array}$ \\
\hline$V(w)$ & $\begin{array}{l}\text { TOTAL WATER COLLECTED, } \\
\text { IMPINGERS \& SILICA GEL }\end{array}$ & $\mathrm{ml}$ & 71.4 & 70.4 & 74.3 \\
\hline$V(w[$ gas $])$ & $\begin{array}{l}\text { VOLUME WATER VAPOR } \\
\text { COLLECTED @ STANDARD } \\
\text { CONDITIONS* }\end{array}$ & $\begin{array}{l}\text { SCF } \\
\text { (SCM) }\end{array}$ & $\begin{array}{c}3.370 \\
(0.095)\end{array}$ & $\begin{array}{c}3.323 \\
(0.094)\end{array}$ & $\begin{array}{c}3.507 \\
(0.099)\end{array}$ \\
\hline$\% \mathrm{M}$ & $\begin{array}{l}\text { MOISTURE IN STACK GAS } \\
\text { BY VOLUME }\end{array}$ & $\%$ & 15.47 & 15.39 & 16.11 \\
\hline Md & MOL FRACTION OF DRY GAS & & 0.8453 & 0.8461 & 0.8389 \\
\hline $\mathrm{Tt}$ & NET TIME OF TEST & MINUTES & 60 & 60 & 60 \\
\hline
\end{tabular}

* 68 Deg.F, 29.92 "Hg (20 Deg.C, 760 mm Hg) 


\section{SOURCE EMISSION CALCULATIONS}

JOB NUMBER: 08-026

JOB NAME: ADA-ES

LOCATION: Hardin, Montana

UNIT TESTED: Stack (CCT)

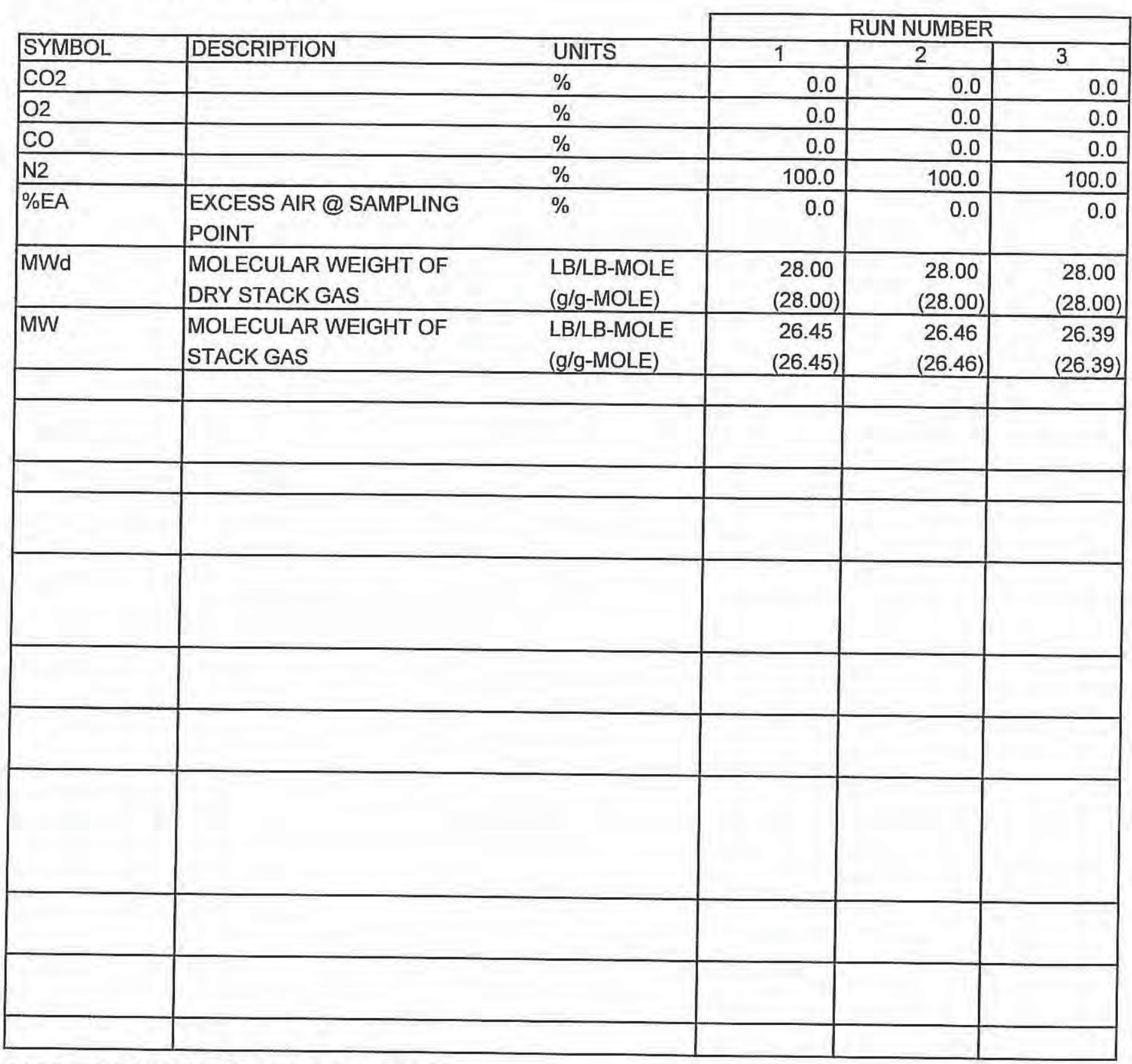

* 68 Deg.F, 29.92 "Hg (20 Deg.C, 760 mm Hg) 


\section{SULFUR TRIOXIDE EMISSION DATA}

JOB NUMBER: $\quad$ 08-026

JOB NAME: $\quad$ ADA-ES

LOCATION: Hardin, Montana

UNIT TESTED: $\quad$ Stack (CCT)

\begin{tabular}{|c|c|c|c|}
\hline RUN NUMBER & \multicolumn{3}{|c|}{ RUN NUMBER } \\
\hline & 1 & \begin{tabular}{l|l}
2 & \\
\end{tabular} & 3 \\
\hline DATE & $09 / 24 / 08$ & $09 / 24 / 08$ & $09 / 24 / 08$ \\
\hline START TIME & 1407 & 1600 & 1745 \\
\hline END TIME & 1507 & 1700 & 1845 \\
\hline $\mathrm{N}$ - NORMALITY OF $\mathrm{Ba}(\mathrm{ClO} 4) 2$ & 0.0000 & 0.0000 & 0.0000 \\
\hline ML(I) - mI IN IMPINGER & 0.00 & 0.00 & 0.00 \\
\hline ML(A) - mI IN ALIQUOT & 0.00 & 0.00 & 0.00 \\
\hline $\mathrm{ML}(\mathrm{B})$ - $\mathrm{ml}$ OF $\mathrm{Ba}(\mathrm{ClO} 4) 2$ TO TITRATE & 0.00 & 0.00 & 0.00 \\
\hline $\mathrm{ML}(\mathrm{BB})$ - $\mathrm{ml}$ OF $\mathrm{Ba}(\mathrm{ClO} 4) 2$ TO TITRATE BLANK & 0.00 & 0.00 & 0.00 \\
\hline Tm - AVERAGE GAS METER TEMPERATURE, DEG.F & 65 & 71 & 69 \\
\hline $\begin{array}{l}\text { Vm - VOLUME OF DRY GAS SAMPLED @ METER } \\
\text { CONDITIONS, FT^3 }\end{array}$ & 20.209 & 20.321 & 20.266 \\
\hline Pb - BAROMETRIC PRESSURE, "Hg Abs. & 27.08 & 27.02 & 26.99 \\
\hline $\begin{array}{l}\text { QS - STACK GAS VOLUME DRY @ STANDARD } \\
\text { CONDITIONS, *SCFM }\end{array}$ & - & - & \\
\hline C(I) - SO3 IN IMPINGERS IN mgS & 2.233 & 1.490 & 2.561 \\
\hline ppm SO3 & 1.29 & 0,87 & 1.49 \\
\hline C(SO3) - EMISSION RATE OF SO3, Ibs/day & & & \\
\hline Cs - EMISSION RATE OF SULFUR, Ibs/day & & & \\
\hline & & & \\
\hline
\end{tabular}

*68 Deg.F, 29.92 "Hg (20 Deg.C, $760 \mathrm{~mm} \mathrm{Hg}$ )

$C(I)=M L(I) *[M L(B)-M L(B B)] * N * 40 / M L(A)$

$\mathrm{ppm} \mathrm{SO} 3=0.6021 * \mathrm{C}(\mathrm{I}) *[\mathrm{Tm}+460] / \mathrm{Vm} * \mathrm{~Pb}$

$\mathrm{C}(\mathrm{SO} 3)=\mathrm{ppm} \mathrm{SO} 3{ }^{*} \mathrm{Qs} * 0.0002991$

$\mathrm{Cs}=\mathrm{C}(\mathrm{SO} 3) / 2.5$ 
SOURCE EMISSION SURVEY

JOB NUMBER: 08-026

JOB NAME: ADA-ES

LOCATION: Hardin, Montana

UNIT TESTED: Stack (201A/202)

SOURCE EMISSION CALCULATIONS

\begin{tabular}{|c|c|c|c|c|c|}
\hline & & & \multicolumn{3}{|c|}{ RUN NUMBER } \\
\hline SYMBOL & DESCRIPTION & UNITS & 1 & 2 & 3 \\
\hline DATE & & & $09 / 24 / 08$ & $09 / 24 / 08$ & $09 / 24 / 08$ \\
\hline BEGIN TIME & & & 1030 & 1308 & 1505 \\
\hline END TIME & & & 1135 & 1412 & 1610 \\
\hline$P(b)$ & BAROMETRIC PRESSURE & $\begin{array}{l}\text { "Hg Abs. } \\
(\mathrm{mm} \mathrm{Hg})\end{array}$ & $\begin{array}{r}27.14 \\
(689.00) \\
\end{array}$ & $\begin{array}{r}27.08 \\
(688.00) \\
\end{array}$ & $\begin{array}{r}27.02 \\
(686.00) \\
\end{array}$ \\
\hline$P(m)$ & ORIFICE PRESSURE DROP & $\begin{array}{l}\text { "H2O } \\
(\mathrm{mm} \mathrm{H} 2 \mathrm{O})\end{array}$ & $\begin{array}{r}0.410 \\
(10.400) \\
\end{array}$ & $\begin{array}{r}0.370 \\
(9.400) \\
\end{array}$ & $\begin{array}{r}0.370 \\
(9.400) \\
\end{array}$ \\
\hline & DGM CALIBRATION FACTOR & & 0.997 & 0.997 & 0.997 \\
\hline$V(m)$ & $\begin{array}{l}\text { VOLUME DRY GAS SAMPLED } \\
\text { @ METER CONDITIONS } \\
\text { LEAK CHECK VOLUME }\end{array}$ & $\begin{array}{l}\mathrm{ft} .^{\wedge} 3 \\
\left(\mathrm{~m}^{\wedge} 3\right) \\
\mathrm{ft} .^{\wedge} 3\end{array}$ & $\begin{array}{c}24.297 \\
(0.688) \\
0.000 \\
\end{array}$ & $\begin{array}{c}23.211 \\
(0.657) \\
0.000 \\
\end{array}$ & $\begin{array}{c}23.365 \\
(0.662) \\
0.000 \\
\end{array}$ \\
\hline$T(m)$ & $\begin{array}{l}\text { AVERAGE GAS METER } \\
\text { TEMPERATURE }\end{array}$ & $\begin{array}{l}\text { DEG.F } \\
\text { (DEG.C) }\end{array}$ & $\begin{array}{r}70 \\
(21) \\
\end{array}$ & $\begin{array}{r}75 \\
(24) \\
\end{array}$ & $\begin{array}{r}80 \\
(27) \\
\end{array}$ \\
\hline $\mathrm{V}(\mathrm{m}[\mathrm{std}])^{*}$ & $\begin{array}{l}\text { VOLUME DRY GAS SAMPLED } \\
@ \text { STANDARD CONDITIONS* }\end{array}$ & $\begin{array}{l}\text { DSCF } \\
\text { (DSCM) }\end{array}$ & $\begin{array}{l}21.984 \\
(0.623) \\
\end{array}$ & $\begin{array}{r}20.757 \\
(0.588) \\
\end{array}$ & $\begin{array}{l}20.656 \\
(0.585) \\
\end{array}$ \\
\hline$V(w)$ & $\begin{array}{l}\text { TOTAL WATER COLLECTED, } \\
\text { IMPINGERS \& SILICA GEL }\end{array}$ & $\mathrm{ml}$ & 89.6 & 79.2 & 77.3 \\
\hline $\mathrm{V}(w[$ gas $])$ & $\begin{array}{l}\text { VOLUME WATER VAPOR } \\
\text { COLLECTED @ STANDARD } \\
\text { CONDITIONS* }\end{array}$ & $\begin{array}{l}\text { SCF } \\
\text { (SCM) }\end{array}$ & $\begin{array}{r}4.229 \\
(0.120)\end{array}$ & $\begin{array}{c}3.738 \\
(0.106)\end{array}$ & $\begin{array}{r}3.649 \\
(0.103)\end{array}$ \\
\hline$\% \mathrm{M}$ & $\begin{array}{l}\text { MOISTURE IN STACK GAS } \\
\text { BY VOLUME }\end{array}$ & $\%$ & 16.13 & 15.26 & 15.01 \\
\hline Md & MOL FRACTION OF DRY GAS & & 0.8387 & 0.8474 & 0.8499 \\
\hline $\mathrm{Tt}$ & NET TIME OF TEST & MINUTES & 61 & 61 & 61 \\
\hline
\end{tabular}

*68 Deg.F, 29.92 "Hg (20 Deg.C, 760 mm Hg) 
SOURCE EMISSION CALCULATIONS

JOB NUMBER: 08-026

JOB NAME: ADA-ES

LOCATION: Hardin, Montana

UNIT TESTED: Stack (201A/202)

\begin{tabular}{|c|c|c|c|c|c|}
\hline & & & \multicolumn{3}{|c|}{ RUN NUMBER } \\
\hline SYMBOL & DESCRIPTION & UNITS & 1 & \begin{tabular}{|l|}
2 \\
\end{tabular} & 3 \\
\hline $\mathrm{CO} 2$ & & $\%$ & 13.4 & 13.4 & 13.4 \\
\hline $\mathrm{O} 2$ & & $\%$ & 6.2 & 6.2 & 6.2 \\
\hline $\mathrm{CO}$ & & $\%$ & 0.0 & 0.0 & 0.0 \\
\hline N2 & & $\%$ & 80.4 & 80.4 & 80.4 \\
\hline$\% \mathrm{EA}$ & $\begin{array}{l}\text { EXCESS AIR @ SAMPLING } \\
\text { POINT }\end{array}$ & $\%$ & 41.0 & 41.0 & 41.0 \\
\hline MWd & $\begin{array}{l}\text { MOLECULAR WEIGHT OF } \\
\text { DRY STACK GAS }\end{array}$ & $\begin{array}{l}\text { LB/LB-MOLE } \\
\text { (g/g-MOLE) }\end{array}$ & $\begin{array}{r}30.39 \\
(30.39) \\
\end{array}$ & $\begin{array}{r}30.39 \\
(30.39) \\
\end{array}$ & $\begin{array}{r}30.39 \\
(30.39) \\
\end{array}$ \\
\hline MW & $\begin{array}{l}\text { MOLECULAR WEIGHT OF } \\
\text { STACK GAS }\end{array}$ & $\begin{array}{l}\text { LB/LB-MOLE } \\
\text { (g/g-MOLE) }\end{array}$ & $\begin{array}{r}28.39 \\
(28.39) \\
\end{array}$ & $\begin{array}{r}28.50 \\
(28.50) \\
\end{array}$ & $\begin{array}{r}28.53 \\
(28.53) \\
\end{array}$ \\
\hline $\mathrm{Cp}$ & PITOT TUBE CALIBRATION & & 0.823 & 0.823 & 0.823 \\
\hline DELTAP & $\begin{array}{l}\text { VELOCITY HEAD OF STACK } \\
\text { GAS }\end{array}$ & $\begin{array}{l}\text { "H2O } \\
(\mathrm{mm} \mathrm{H} 20)\end{array}$ & $\begin{array}{r}3.442 \\
(87.400) \\
\end{array}$ & $\begin{array}{r}3.442 \\
(87.400) \\
\end{array}$ & $\begin{array}{r}3.442 \\
(87.400) \\
\end{array}$ \\
\hline DELTAP $\wedge(1 / 2)$ & & "H2O & 1.853 & 1.853 & 1.853 \\
\hline Ts & STACK TEMPERATURE & $\begin{array}{l}\text { DEG. F } \\
\text { (DEG. C) }\end{array}$ & $\begin{array}{l}210 \\
(99) \\
\end{array}$ & $\begin{array}{c}211 \\
(99) \\
\end{array}$ & $\begin{array}{c}214 \\
(101) \\
\end{array}$ \\
\hline Ps & STACK PRESSURE & $\begin{array}{l}\text { "Hg Abs. } \\
(\mathrm{mm} \mathrm{Hg}) \\
\text { "H2O }\end{array}$ & $\begin{array}{c}27.05 \\
(687.00) \\
-1.20 \\
\end{array}$ & $\begin{array}{c}26.97 \\
(685.00) \\
-1.50 \\
\end{array}$ & $\begin{array}{r}26.90 \\
(683.00) \\
-1.60 \\
\end{array}$ \\
\hline Vs & $\begin{array}{l}\text { STACK VELOCITY @ STACK } \\
\text { CONDITIONS }\end{array}$ & $\begin{array}{l}\text { FPM } \\
(\mathrm{m} / \mathrm{SEC} .) \\
\end{array}$ & $\begin{array}{r}7,299 \\
(37) \\
\end{array}$ & $\begin{array}{r}7,301 \\
(37) \\
\end{array}$ & $\begin{array}{r}7,323 \\
(37) \\
\end{array}$ \\
\hline As & STACK AREA & $\begin{array}{l}\text { (SQ.INCHES) } \\
\text { (SQ.METERS) }\end{array}$ & $\begin{array}{r}9,363 \\
(6) \\
\end{array}$ & $\begin{array}{r}9,363 \\
(6) \\
\end{array}$ & $\begin{array}{r}9,363 \\
(6) \\
\end{array}$ \\
\hline Qs & $\begin{array}{l}\text { DRY STACK GAS VOLUME @ } \\
\text { STANDARD CONDITIONS* } \\
\text { WET STACK GAS VOLUME @ } \\
\text { STANDARD CONDITIONS* }\end{array}$ & $\begin{array}{l}\text { DSCFM } \\
\text { (DSCM/HR) } \\
\text { WSCFH }\end{array}$ & $\begin{array}{r}284,631 \\
(483,588) \\
20,362,299\end{array}$ & \begin{tabular}{|}
286,384 \\
$(486,566)$ \\
$20,277,366$
\end{tabular} & $\begin{array}{r}286,068 \\
(486,030) \\
20,195,411\end{array}$ \\
\hline Qa & $\begin{array}{l}\text { ACTUAL STACK GAS VOLUME } \\
\text { @ STACK CONDITIONS } \\
\end{array}$ & $\begin{array}{l}\text { ACFM } \\
\left(m^{\wedge} 3 / H R\right)\end{array}$ & $\begin{array}{c}474,560 \\
(806,277) \\
\end{array}$ & $\begin{array}{c}474,699 \\
(806,514) \\
\end{array}$ & $\begin{array}{c}476,127 \\
(808,940) \\
\end{array}$ \\
\hline $\mathrm{Dn}$ & SAMPLING NOZZLE DIAM. & $\begin{array}{l}\text { IN. } \\
\text { (m) }\end{array}$ & $\begin{array}{c}0.160 \\
(0.004) \\
\end{array}$ & $\begin{array}{c}0.131 \\
(0.003) \\
\end{array}$ & $\begin{array}{c}0.131 \\
(0.003) \\
\end{array}$ \\
\hline$\% !$ & PERCENT ISOKINETIC & $\%$ & 59.2 & 82.8 & 82.5 \\
\hline
\end{tabular}

* 68 Deg.F, 29.92 "Hg (20 Deg.C, 760 mm Hg) 
SOURCE EMISSION SURVEY

JOB NUMBER: $\quad$ 08-026

JOB NAME: ADA-ES

LOCATION: Hardin, Montana

UNIT TESTED: Stack (201A/202)

SOURCE EMISSION CALCULATIONS

\begin{tabular}{|c|c|c|c|c|c|}
\hline & & & \multicolumn{3}{|c|}{ RUN NUMBER } \\
\hline SYMBOL & DESCRIPTION & UNITS & 4 & & \\
\hline DATE & & & $09 / 24 / 08$ & & \\
\hline BEGIN TIME & & & 1700 & & \\
\hline END TIME & & & 1804 & & \\
\hline$P(b)$ & BAROMETRIC PRESSURE & $\begin{array}{l}\text { "Hg Abs. } \\
(\mathrm{mm} \mathrm{Hg})\end{array}$ & $\begin{array}{r}26.99 \\
(686.00) \\
\end{array}$ & & \\
\hline$P(m)$ & ORIFICE PRESSURE DROP & $\begin{array}{l}\text { " } \mathrm{H} 2 \mathrm{O} \\
(\mathrm{mm} \mathrm{H} 2 \mathrm{O})\end{array}$ & $\begin{array}{c}0.370 \\
(9.400) \\
\end{array}$ & & \\
\hline & DGM CALIBRATION FACTOR & & 0.997 & & \\
\hline$V(m)$ & $\begin{array}{l}\text { VOLUME DRY GAS SAMPLED } \\
\text { @ METER CONDITIONS } \\
\text { LEAK CHECK VOLUME }\end{array}$ & $\begin{array}{l}\mathrm{ft} .^{\wedge} 3 \\
\left(\mathrm{~m}^{\wedge} 3\right) \\
\mathrm{ft} .^{\wedge} 3\end{array}$ & $\begin{array}{c}23.004 \\
(0.651) \\
0.000 \\
\end{array}$ & & \\
\hline $\mathrm{T}(\mathrm{m})$ & $\begin{array}{l}\text { AVERAGE GAS METER } \\
\text { TEMPERATURE }\end{array}$ & $\begin{array}{l}\text { DEG.F } \\
\text { (DEG.C) }\end{array}$ & $\begin{array}{c}78 \\
(26) \\
\end{array}$ & & \\
\hline$V(m[s t d])^{*}$ & $\begin{array}{l}\text { VOLUME DRY GAS SAMPLED } \\
\text { @ STANDARD CONDITIONS* }\end{array}$ & $\begin{array}{l}\text { DSCF } \\
\text { (DSCM) }\end{array}$ & $\begin{array}{l}20.389 \\
(0.577) \\
\end{array}$ & & \\
\hline$V(w)$ & $\begin{array}{l}\text { TOTAL WATER COLLECTED, } \\
\text { IMPINGERS \& SILICA GEL }\end{array}$ & $\mathrm{ml}$ & 79.0 & & \\
\hline$V(w[$ gas $])$ & $\begin{array}{l}\text { VOLUME WATER VAPOR } \\
\text { COLLECTED @ STANDARD } \\
\text { CONDITIONS* }\end{array}$ & $\begin{array}{l}\text { SCF } \\
\text { (SCM) }\end{array}$ & $\begin{array}{c}3.729 \\
(0.106)\end{array}$ & & \\
\hline$\% M$ & $\begin{array}{l}\text { MOISTURE IN STACK GAS } \\
\text { BY VOLUME }\end{array}$ & $\%$ & 15.46 & & \\
\hline Md & MOL FRACTION OF DRY GAS & & 0.8454 & & \\
\hline $\mathrm{Tt}$ & NET TIME OF TEST & MINUTES & 61 & & \\
\hline
\end{tabular}

*68 Deg.F, 29.92 "Hg (20 Deg.C, 760 mm Hg) 
SOURCE EMISSION CALCULATIONS

JOB NUMBER: $\quad$ 08-026

JOB NAME: ADA-ES

LOCATION: Hardin, Montana

UNIT TESTED: Stack (201A/202)

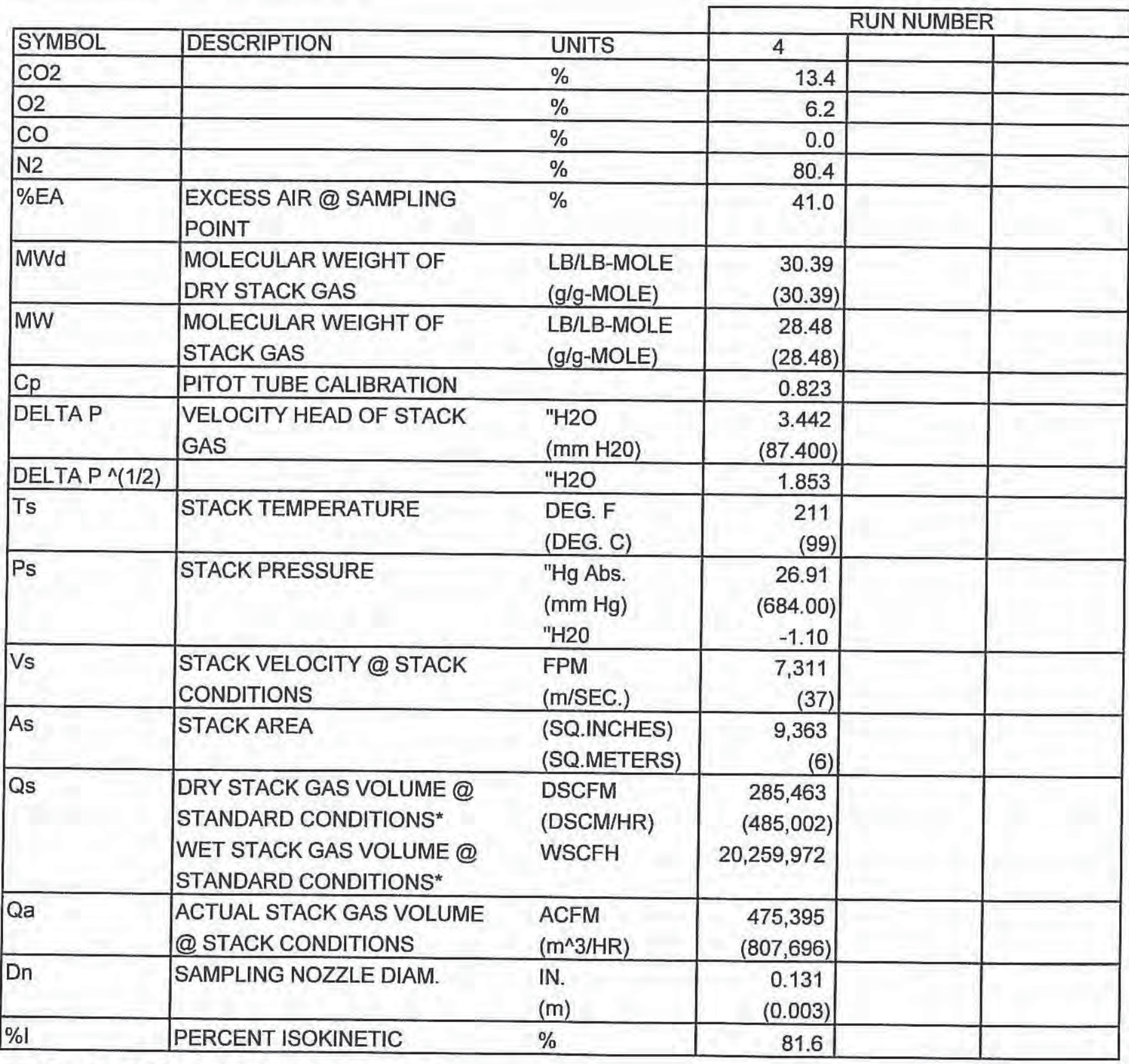

* 68 Deg.F, 29.92 "Hg (20 Deg.C, 760 mm Hg) 


\section{Appendix C:}

Calibration Data 


\section{Appendix C}

\section{Table 14: Calibration Data}

Pre-Test Calibrations:

\section{Equipment}

Dry Gas Meter 2-1

Digital Temperature Indicator 2-1

Dry Gas Meter 2-1 Orifice

Dry Gas Meter 2-2

Digital Temperature Indicator 2-2

Dry Gas Meter 2-2 Orifice

Pitot Tube 3-2

Pitot Tube 3-3

Pitot Tube 3-4

Nozzle 3-2

Nozzle 7-2

Nozzle 08-026-1

Nozzle 08-026-2

Nozzle 08-026-3
Calibration Factor

0.997

0.974

0.823

0.823

0.821

0.168

0.193

0.155

0.160

0.131
Calibration Date

08/19/08

$08 / 19 / 08$

08/19/08

08/18/08

$08 / 18 / 08$

08/18/08

07/03/08

07/03/08

05/01/08

03/03/08

01/18/08

09/24/08

09/24/08

09/24/08 


\section{Post-Test Calibrations:}

\section{Equipment}

Dry Gas Meter 2-1

Digital Temperature Indicator 2-1

Dry Gas Meter 2-2

Digital Temperature Indicator 2-2

Pitot Tube 3-2

Pitot Tube 3-3

Pitot Tube 3-4

Nozzle 3-2

Nozzle 7-2

Nozzle 08-026-1

Nozzle 08-026-2

Nozzle 08-026-3
Calibration Factor

0.982

0.981

0.823

0.824

0.821

0.169

0.192

0.155

0.160

0.131

\section{Calibration Date}

$10 / 03 / 08$

$10 / 03 / 08$

$10 / 03 / 08$

$10 / 03 / 08$

10/03/08

10/06/08

10/06/08

10/10/08

10/10/08

09/30/08

09/30/08

09/30/08 


\author{
Calibration Data \\ Dry Gas Meter Calibration
}

$\begin{aligned} \text { Meter Console No. } & \text { ASAI 2-1 } \\ \text { Date Calibration Performed: } & \underline{08 / 19 / 08}\end{aligned}$

$\triangle \mathrm{H}$ Setting

\begin{tabular}{c}
$\left(" \mathrm{H}_{2} \mathrm{O}\right)$ \\
\hline 0.50 \\
1.00 \\
1.50 \\
2.00 \\
3.00 \\
4.00 \\
Average
\end{tabular}

\begin{tabular}{c}
$\mathrm{C}_{\mathrm{DG}}$ \\
\hline 1.009 \\
\hline 1.012 \\
\hline 0.994 \\
\hline 0.990 \\
\hline 0.989 \\
\hline 0.987 \\
\hline 0.997 \\
\hline
\end{tabular}

Variation +: $1.50 \%$

Variation -: $-1.00 \%$

Certified by:

Patrich Selahovich 8/19/08

Calibrator (Signature / Date)

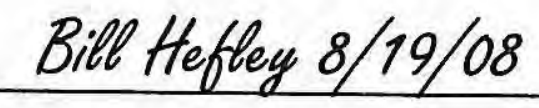

QA Officer (Signature / Date) 


\author{
Calibration Data \\ Dry Gas Meter Calibration
}

Meter Console No.

Date Calibration Performed:

ASAI 2-1

08/19/08

$\begin{aligned} \text { Run } \frac{1}{\mathrm{~Pb}} & =29.55 \mathrm{Hg} \\ \text { Wet Test Meter } \mathrm{C}_{\mathrm{f}}=1.009 \text { Console Pump Vacuum } & =-5.0^{\prime \prime} \mathrm{Hg}\end{aligned}$

Wet Test Meter No. ASAl-0 Meter

\begin{tabular}{|c|c|c|c|c|c|c|c|}
\hline & Time & Reading & & Temp. & & $\underline{P}_{m}$ & \\
\hline End & $10: 34$ & 5.005 & cf & 80 & ${ }^{\circ} \mathrm{F}$ & -0.2 & $" \mathrm{H}_{2} \mathrm{O}$ \\
\hline Start & $10: 22$ & $\underline{0.000}$ & cf & $\underline{80}$ & ${ }^{\circ} \mathrm{F}$ & -0.2 & " $\mathrm{H}_{2} \mathrm{O}$ \\
\hline Ave & rage & 5.005 & cf & 80 & ${ }^{\circ} \mathrm{F}$ & -0.2 & " $\mathrm{H}_{2} \mathrm{O}$ \\
\hline
\end{tabular}

Dry Gas Meter Meter Temperature Reading In Out $\underline{P}_{m}$ 293.205 of $83 \quad{ }^{\circ} \mathrm{F} \quad 79 \quad{ }^{\circ} \mathrm{F} \quad 0.50 \quad " \mathrm{H}_{2} \mathrm{O}$ $\underline{288.202}$ of $\quad \underline{81}{ }^{\circ} \mathrm{F} \quad \underline{79}{ }^{\circ} \mathrm{F} \quad \underline{0.50} \quad " \mathrm{H}_{2} \mathrm{O}$ 5.003 cf $82{ }^{\circ} \mathrm{F} \quad 79 \quad{ }^{\circ} \mathrm{F} \quad 0.50 \quad " \mathrm{H}_{2} \mathrm{O}$

Wet Test Meter $V_{m s s i}=17.65 \times V_{m}\left[\frac{P_{b}+\frac{P_{m}}{13.6}}{\left(T_{m}+460\right)}\right] \times C_{f}=\underline{4.875} d c s f$
Dry Gas Meter $V_{m s t i}=17.65 \times V m\left[\frac{P_{b}+\frac{P_{m}}{13.6}}{\left(T_{m}+460\right)}\right]=\underline{4.834} d c s f$ Calibration Factor $\left(C_{D G}\right)=\frac{\text { Wet Test Meter } V_{m s t d}}{\text { Dry Gas Meter } V_{\text {msta }}}=1.009$ 


\author{
Calibration Data \\ Dry Gas Meter Calibration
}

Meter Console No.

Date Calibration Performed:

ASAI 2-1

08/19/08

Run 1 at $1.0^{\prime \prime} \Delta H$

Wet Test Meter $C_{f}=1.009$ Console Pump Vacuum $=-5.0 " \mathrm{Hg}$

Wet Test Meter No. ASAI-O Meter

\begin{tabular}{|c|c|c|c|c|c|c|c|}
\hline & Time & Reading & & Temp & & $\underline{P}_{m}$ & \\
\hline & & 5.202 & cf & 80 & ${ }^{\circ} \mathrm{F}$ & -0.4 & $" \mathrm{H}_{2} \mathrm{C}$ \\
\hline & & $\underline{0.000}$ & cf & $\underline{80}$ & ${ }^{\circ} \mathrm{F}$ & -0.4 & $\mathrm{H}_{2}$ \\
\hline & & 5.202 & cf & 80 & ${ }^{\circ} \mathrm{F}$ & -0.4 & $" \mathrm{H}_{2}$ \\
\hline
\end{tabular}

Meter Temperature

Reading In $\underline{\text { Out }} \quad \underline{P_{m}}$

298.897 of $86 \quad{ }^{\circ} \mathrm{F} \quad 81 \quad{ }^{\circ} \mathrm{F} \quad 1.00 \quad \mathrm{H}_{2} \mathrm{O}$

$\underline{293.704}$ of $\quad \underline{83}{ }^{\circ} \mathrm{F} \quad \underline{80}{ }^{\circ} \mathrm{F} \quad \underline{1.00} \quad " \mathrm{H}_{2} \mathrm{O}$

5.193 cf $85{ }^{\circ} \mathrm{F} \quad 81 \quad{ }^{\circ} \mathrm{F} \quad 1.00 \quad " \mathrm{H}_{2} \mathrm{O}$

Wet Test Meter $V_{m s t}=17.65 \times V_{m}\left[\frac{P_{b}+\frac{P_{m}}{13.6}}{\left(T_{m}+460\right)}\right] \times C_{f}=\underline{5.065} d c s f$
Dry Gas Meter $V_{m s u l}=17.65 \times V m\left[\frac{P_{b}+\frac{P_{m}}{13.6}}{\left(T_{m}+460\right)}\right]=\underline{5.005} \mathrm{dcsf}$

Calibration Factor $(C D G)=\frac{\text { Wet Test Meter } V_{m s t d}}{D r y \text { Gas Meter } V_{m s t d}}=\underline{1.012}$ 


\author{
Calibration Data \\ Dry Gas Meter Calibration
}

Meter Console No.

Date Calibration Performed:

ASAI 2-1

08/19/08

Run 1 at $1.5^{\prime \prime} \Delta \mathrm{H}$

Wet Test Meter $C_{f}=1.009$

$\mathrm{Pb}=\underline{29.55} " \mathrm{Hg}$

Console Pump Vacuum $=-5.0 \mathrm{Hg}$

Wet Test Meter No. ASAI-O Meter

\begin{tabular}{|c|c|c|c|c|c|c|c|}
\hline & Time & Reading & & Temp. & & $\underline{P}_{m}$ & \\
\hline End & $11: 03$ & 10.450 & cf & 80 & ${ }^{\circ} \mathrm{F}$ & -0.6 & $" \mathrm{H}_{2} \mathrm{O}$ \\
\hline Start & $10: 48$ & $\underline{0.000}$ & cf & 80 & ${ }^{\circ} \mathrm{F}$ & $\underline{-0.6}$ & $" \mathrm{H}_{2} \mathrm{O}$ \\
\hline & & 10.450 & cf & 80 & ${ }^{\circ} \mathrm{F}$ & -0.6 & $" \mathrm{H}_{2} \mathrm{O}$ \\
\hline
\end{tabular}

Dry Gas Meter

Meter Temperature

Reading In $\underline{\text { Out }} \quad \mathrm{P}_{\mathrm{m}}$ 309.850 cf $91 \quad{ }^{\circ} \mathrm{F} \quad 82 \quad{ }^{\circ} \mathrm{F} \quad 1.50 \quad " \mathrm{H}_{2} \mathrm{O}$ $\underline{299.205}$ cf $\underline{85}{ }^{\circ} \mathrm{F} \quad \underline{81}{ }^{\circ} \mathrm{F} \quad \underline{1.50} \quad \mathrm{H}_{2} \mathrm{O}$ 10.645 of $88{ }^{\circ} \mathrm{F} \quad 82{ }^{\circ} \mathrm{F} \quad 1.50 \quad " \mathrm{H}_{2} \mathrm{O}$

$$
\begin{gathered}
\text { Wet Test Meter } V_{m s t}=17.65 \times V_{m}\left[\frac{P_{b}+\frac{P_{m}}{13.6}}{\left(T_{m}+460\right)}\right] x C_{f}=\underline{10.169} d c s f \\
\text { Dry Gas Meter } V_{m_{s s d}}=17.65 \times \mathrm{Vm}\left[\frac{P_{b}+\frac{P_{m}}{13.6}}{\left(T_{m}+460\right)}\right]=\underline{10.230} d c s f
\end{gathered}
$$$$
\text { Calibration Factor }\left(C_{D G}\right)=\frac{\text { Wet Test Meter } V_{m_{s t d}}}{\text { Dry Gas Meter } V_{m_{s t d}}}=\underline{0.994}
$$ 


\author{
Calibration Data \\ Dry Gas Meter Calibration
}

Meter Console No.

Date Calibration Performed:
ASAI 2-1

08/19/08

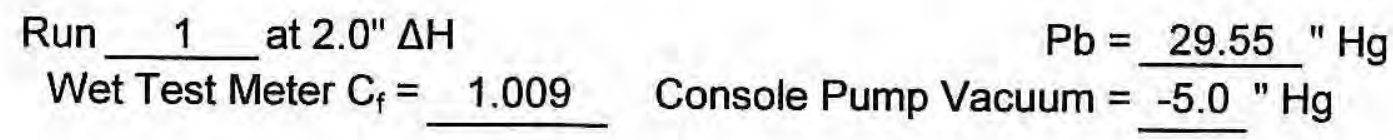

Wet Test Meter No. ASAI-O Meter

\begin{tabular}{|c|c|c|c|c|c|c|}
\hline & Time & Reading & Temp. & & $\underline{P}_{m}$ & \\
\hline End & $11: 20$ & 10.380 & 80 & ${ }^{\circ} \mathrm{F}$ & -0.8 & $" \mathrm{H}_{2} \mathrm{O}$ \\
\hline Start & 11:07 & $\underline{0.000}$ & 80 & ${ }^{\circ} \mathrm{F}$ & -0.8 & " $\mathrm{H}_{2} \mathrm{O}$ \\
\hline Ave & rage & 10.380 & 80 & ${ }^{\circ} \mathrm{F}$ & -0.8 & $" \mathrm{H}_{2} \mathrm{O}$ \\
\hline
\end{tabular}

\section{Dry Gas Meter} Meter Temperature

Reading In $\underline{\text { Out }} \quad \underline{P_{m}}$ 321.049 of $\quad 93 \quad{ }^{\circ} \mathrm{F} \quad 84 \quad{ }^{\circ} \mathrm{F} \quad 2.00 \quad " \mathrm{H}_{2} \mathrm{O}$ $\underline{310.408}$ of $\quad \underline{88}{ }^{\circ} \mathrm{F} \quad \underline{82}{ }^{\circ} \mathrm{F} \quad \underline{2.00} " \mathrm{H}_{2} \mathrm{O}$ 10.641 of $91{ }^{\circ} \mathrm{F} \quad 83 \quad{ }^{\circ} \mathrm{F} \quad 2.00 \quad " \mathrm{H}_{2} \mathrm{O}$

$$
\begin{gathered}
\text { Wet Test Meter Vmsda }=17.65 \times V_{m}\left[\frac{P_{b}+\frac{P_{m}}{13.6}}{\left(T_{m}+460\right)}\right] \times C_{f}=\underline{10.096} \mathrm{dcsf} \\
\text { Dry Gas Meter } V_{m s s u}=17.65 \times V_{m}\left[\frac{P_{b}+\frac{P_{m}}{13.6}}{\left(T_{m}+460\right)}\right]=\underline{10.201} \mathrm{dcsf}
\end{gathered}
$$

$$
\text { Calibration Factor }\left(C_{D G}\right)=\frac{\text { Wet Test Meter } V_{m s t d}}{\text { Dry Gas Meter } V_{\text {msto }}}=\underline{0.990}
$$




\author{
Calibration Data \\ Dry Gas Meter Calibration
}

Meter Console No.

Date Calibration Performed:

ASAl 2-1

08/19/08

$\begin{aligned} \text { Run } \frac{1}{\mathrm{~Pb}} & =29.55 \mathrm{at} 3 . \mathrm{O}^{\prime \prime} \mathrm{\Delta H} \\ \text { Wet Test Meter } \mathrm{C}_{\mathrm{f}}=1.009 \text { Console Pump Vacuum } & =-5.0 \mathrm{Hg}\end{aligned}$

Wet Test Meter No. ASAI-O

Dry Gas Meter

Meter

Meter Temperature

Time Reading Temp. $\quad \underline{P}_{m}$

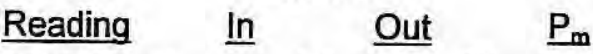

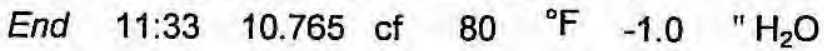

Start $\underline{11: 22} \underline{0.000}$ of $\underline{80} \quad{ }^{\circ} \mathrm{F} \quad \underline{-1.0} \quad " \mathrm{H}_{2} \mathrm{O}$

332.586 of $96 \quad{ }^{\circ} \mathrm{F} \quad 85 \quad{ }^{\circ} \mathrm{F} \quad 3.00 \quad " \mathrm{H}_{2} \mathrm{O}$

Average 10.765 cf $80 \quad{ }^{\circ} \mathrm{F} \quad-1.0 \quad$ " $\mathrm{H}_{2} \mathrm{O}$

$\underline{321.529} \mathrm{cf} \quad \underline{91}{ }^{\circ} \mathrm{F} \quad \underline{84} \quad{ }^{\circ} \mathrm{F} \quad \underline{3.00} \quad " \mathrm{H}_{2} \mathrm{O}$

11.057 of $94{ }^{\circ} \mathrm{F} \quad 85{ }^{\circ} \mathrm{F} \quad 3.00 \quad " \mathrm{H}_{2} \mathrm{O}$

Wet Test Meter $V_{m s d}=17.65 \times V_{m}\left[\frac{P_{b}+\frac{P_{m}}{13.6}}{\left(T_{m}+460\right)}\right] x C_{f}=\underline{10.465} \mathrm{dcsf}$
Dry Gas Meter $V_{m s t a}=17.65 \times \mathrm{Vm}\left[\frac{P_{b}+\frac{P_{m}}{13.6}}{\left(T_{m}+460\right)}\right]=\underline{10.583} \mathrm{dcsf}$

Calibration Factor $\left(C_{D G}\right)=\frac{\text { Wet Test Meter } V_{m s t d}}{\text { Dry Gas Meter } V_{m s t d}}=\underline{0.989}$ 


\author{
Calibration Data \\ Dry Gas Meter Calibration
}

Meter Console No.

Date Calibration Performed:

ASAI 2-1

08/19/08

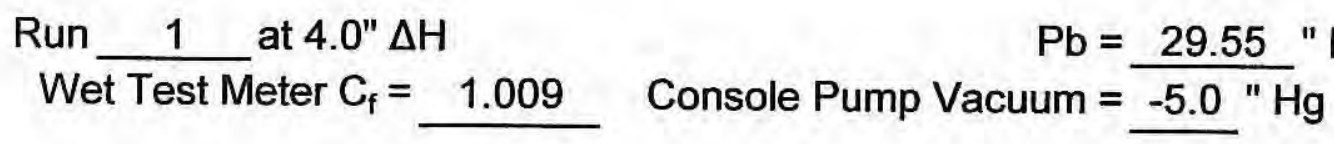

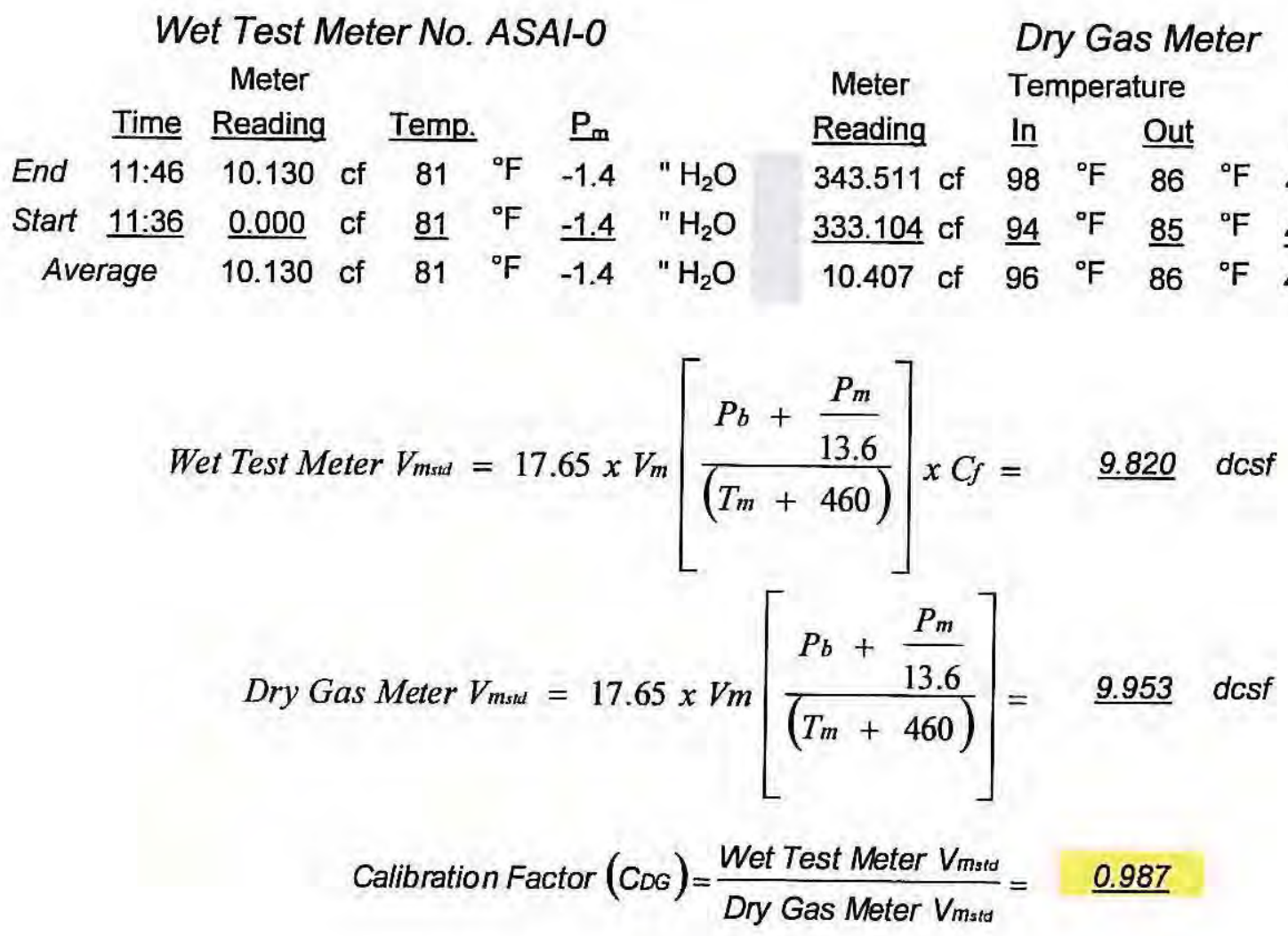


Calibration Data

Digital Temperature Indicator Calibration

DTI Unit No. ASAl 2-1

Date Calibration Performed:

\begin{tabular}{|c|c|}
\hline 08/19/08 & \\
\hline $\begin{array}{c}\text { ASTM } \\
\text { Reference }\end{array}$ & \\
\hline Thermometer & DTI \\
\hline$\frac{\left({ }^{\circ} \mathrm{F}\right)}{200-}$ & $\left({ }^{\circ} \mathrm{F}\right)$ \\
\hline $32^{\circ} \mathrm{F}$ & $32^{\circ} \mathrm{F}$ \\
\hline $80^{\circ} \mathrm{F}$ & $80^{\circ} \mathrm{F}$ \\
\hline $212^{\circ} \mathrm{F}$ & $214^{\circ} \mathrm{F}$ \\
\hline $250^{\circ} \mathrm{F}$ & $252^{\circ} \mathrm{F}$ \\
\hline $300^{\circ} \mathrm{F}$ & $303^{\circ} \mathrm{F}$ \\
\hline $350^{\circ} \mathrm{F}$ & $352^{\circ} \mathrm{F}$ \\
\hline $400^{\circ} \mathrm{F}$ & $402^{\circ} \mathrm{F}$ \\
\hline
\end{tabular}

Meter Adjusted?

No

ASTM Reference Thermometer:

SN: 06000047 SN:

SN:

Range: $\quad-0.1^{\circ} \mathrm{C}$ to $1360.6^{\circ} \mathrm{C}$

Range:

Range:

Certified by:

Patrick Selalovich 8/19/08

Calibrator (Signature / Date)

Bill Heffley 8/19/08

QA Officer (Signature / Date) 


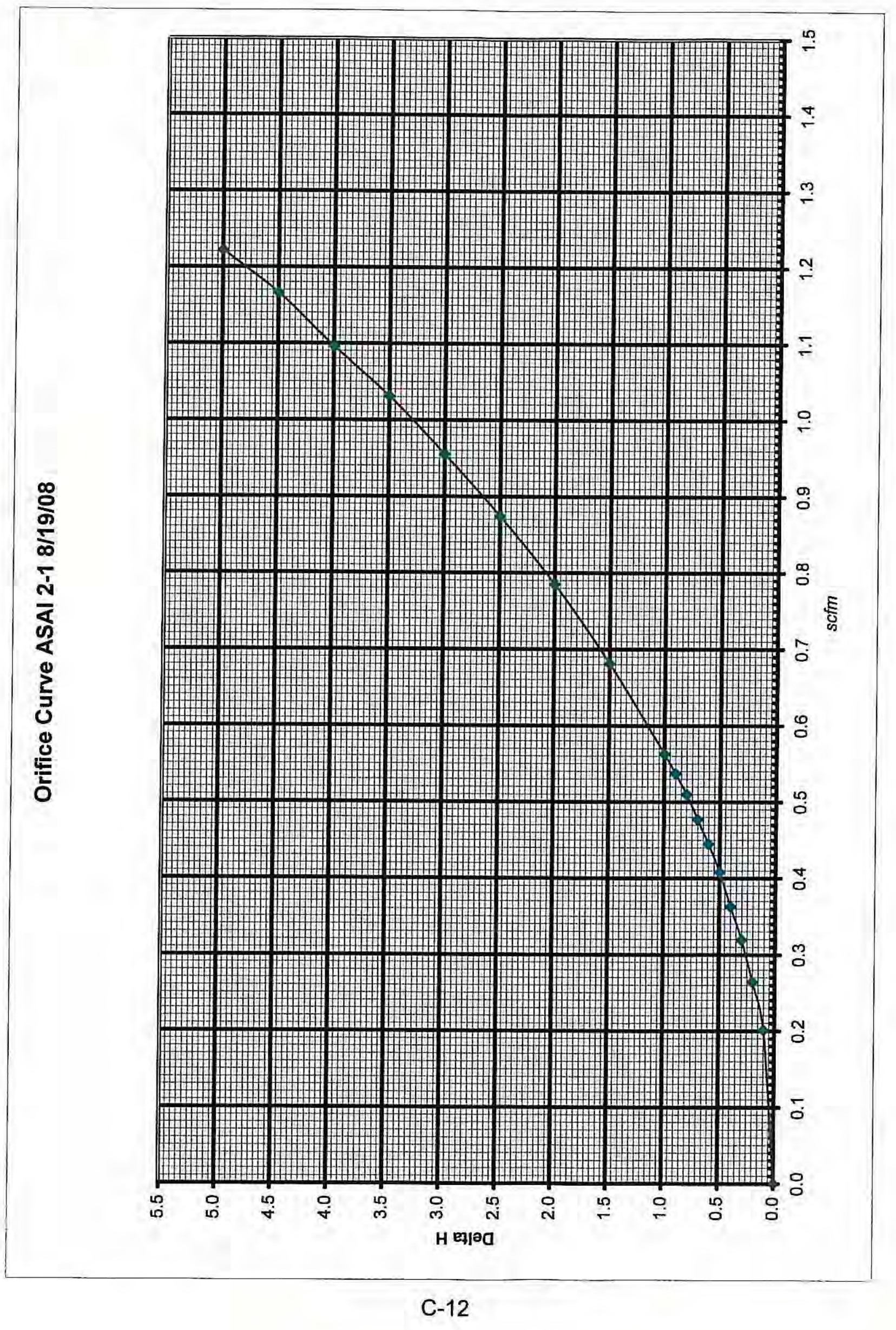




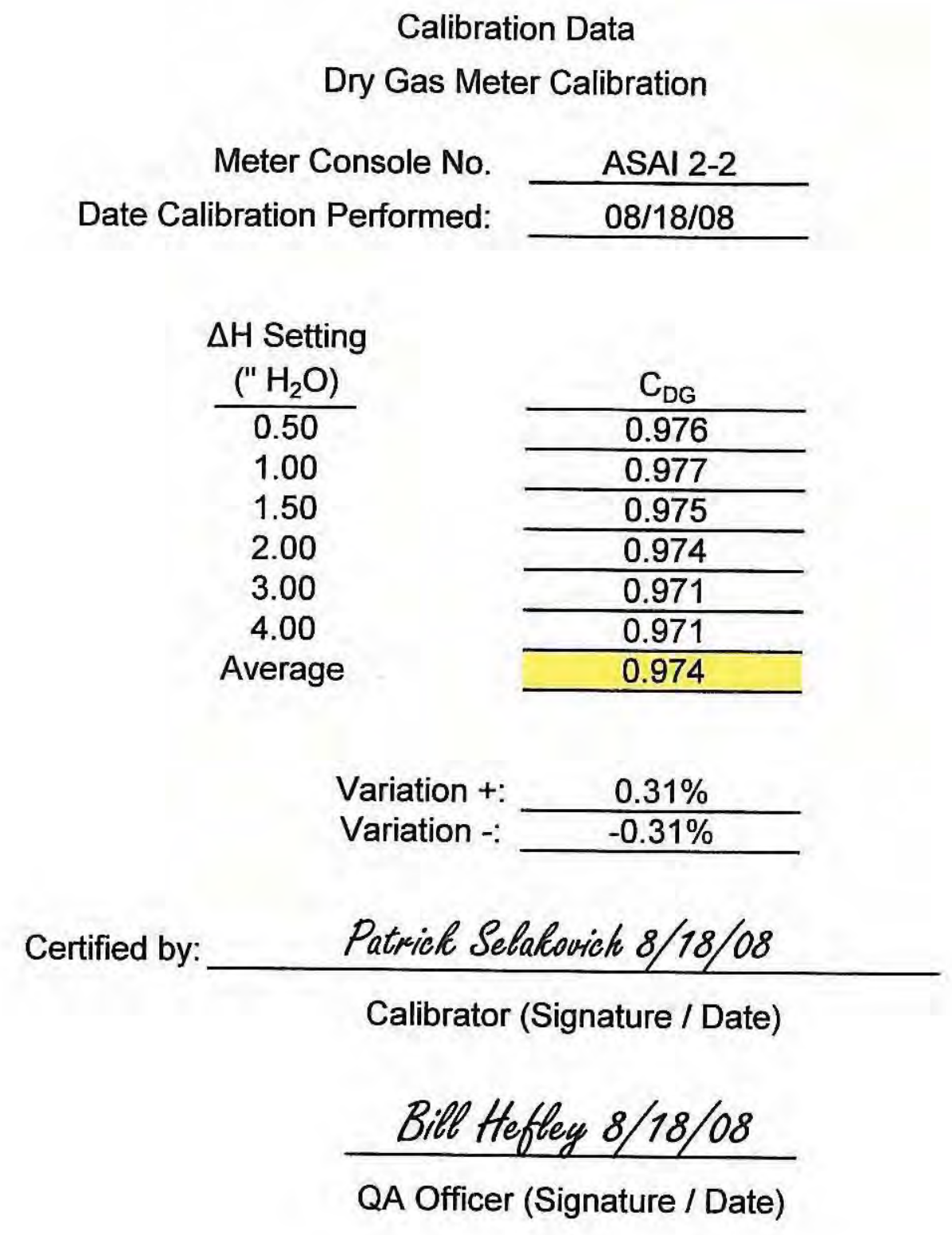


Calibration Data
Dry Gas Meter Calibration

Meter Console No.

Date Calibration Performed:
ASAI 2-2

08/18/08

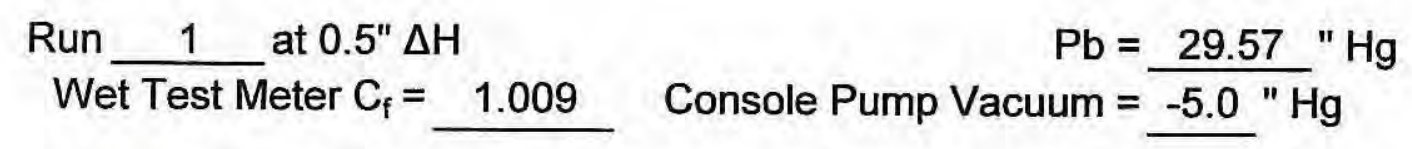

Wet Test Meter No. ASAI-O Meter

Time Reading Temp. $\quad \underline{P}_{m}$

\begin{tabular}{|c|c|c|c|c|c|c|c|c|c|c|c|c|c|c|}
\hline & Time & Reading & & Temp. & & $\underline{P_{m}}$ & & $\underline{\text { Reading }}$ & $\underline{\text { In }}$ & & Out & & $\underline{P_{m}}$ & \\
\hline End & $9: 18$ & 5.040 & cf & 82 & ${ }^{\circ} \mathrm{F}$ & -0.4 & $" \mathrm{H}_{2} \mathrm{O}$ & $400.350 \mathrm{cf}$ & 77 & ${ }^{\circ} \mathrm{F}$ & 76 & ${ }^{\circ} \mathrm{F}$ & 0.50 & $" \mathrm{H}_{2} \mathrm{O}$ \\
\hline Start & $\underline{9: 05}$ & $\underline{0.000}$ & cf & $\underline{82}$ & ${ }^{\circ} \mathrm{F}$ & $\underline{-0.4}$ & $" \mathrm{H}_{2} \mathrm{O}$ & $\underline{395.205} \mathrm{cf}$ & $\underline{77}$ & ${ }^{\circ} \mathrm{F}$ & $\underline{76}$ & ${ }^{\circ} \mathrm{F}$ & $\underline{0.50}$ & " $\mathrm{H}_{2} \mathrm{O}$ \\
\hline$A v \in$ & & 5.040 & cf & 82 & ${ }^{\circ} \mathrm{F}$ & -0.4 & " $\mathrm{H}_{2} \mathrm{O}$ & $5.145 \mathrm{cf}$ & 77 & ${ }^{\circ} \mathrm{F}$ & 76 & ${ }^{\circ} \mathrm{F}$ & 0.50 & $" \mathrm{H}_{2} \mathrm{O}$ \\
\hline
\end{tabular}

Wet Test Meter $V_{m s t}=17.65 \times V_{m}\left[\frac{P_{b}+\frac{P_{m}}{13.6}}{\left(T_{m}+460\right)}\right] x C_{f}=\underline{4.892} d c s f$
Dry Gas Meter $V_{m s t d}=17.65 \times V m\left[\frac{P_{b}+\frac{P_{m}}{13.6}}{\left(T_{m}+460\right)}\right]=\underline{5.011} d c s f$ Calibration Factor $(C D G)=\frac{\text { Wet Test Meter } V_{m_{s t d}}}{\text { Dry Gas Meter } V_{\text {mstd }}}=\underline{0.976}$ 


\author{
Calibration Data \\ Dry Gas Meter Calibration
}

Meter Console No.

Date Calibration Performed:
ASAI 2-2

$08 / 18 / 08$

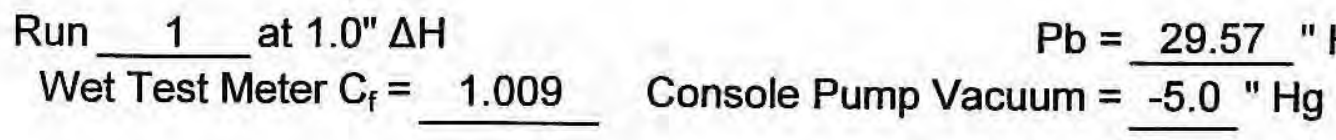

$$
\begin{aligned}
& \text { Wet Test Meter No. ASAI-O } \\
& \text { Meter } \\
& \text { End } \frac{\text { Time }}{9: 29} \frac{\text { Reading }}{5.235} \text { cf } \frac{\text { Temp. }}{82}{ }^{\circ} \mathrm{F} \quad \frac{\mathrm{P}_{\mathrm{m}}}{-0.5} \quad \mathrm{H}_{2} \mathrm{O} \\
& \text { Start } \underline{9: 20} \quad \underline{0.000} \text { of } \underline{82} \quad{ }^{\circ} \mathrm{F} \quad \underline{\underline{-0.5}} \quad " \mathrm{H}_{2} \mathrm{O} \\
& \text { Average } \quad 5.235 \text { of } 82 \quad{ }^{\circ} \mathrm{F} \quad-0.5 \quad \mathrm{H}_{2} \mathrm{O} \\
& \text { Wet Test Meter } V_{m s d}=17.65 \times V_{m}\left[\frac{P_{b}+\frac{P_{m}}{13.6}}{\left(T_{m}+460\right)}\right] x C_{f}=\underline{5.080} \text { dcsf } \\
& \text { Dry Gas Meter Vmsti }=17.65 \times \mathrm{Vm}\left[\frac{P_{b}+\frac{P_{m}}{13.6}}{\left(T_{m}+460\right)}\right]=\underline{5.200} \mathrm{dcsf} \\
& \text { Calibration Factor }\left(C_{D G}\right)=\frac{\text { Wet Test Meter } V_{\text {mstd }}}{D r y \text { Gas Meter } V_{\text {mstd }}}=\underline{0.977}
\end{aligned}
$$

\section{Dry Gas Meter}




\author{
Calibration Data \\ Dry Gas Meter Calibration
}

Meter Console No.

Date Calibration Performed:

ASAI 2-2

08/18/08

$\begin{aligned} \text { Run } \frac{1}{\mathrm{~Pb}}=29.57 & \text { at 1.5" } \mathrm{AH} \\ \text { Wet Test Meter } \mathrm{C}_{\mathrm{f}}=1.009 \quad \text { Console Pump Vacuum } & =-5.0^{\mathrm{Ng}} \mathrm{Hg}\end{aligned}$

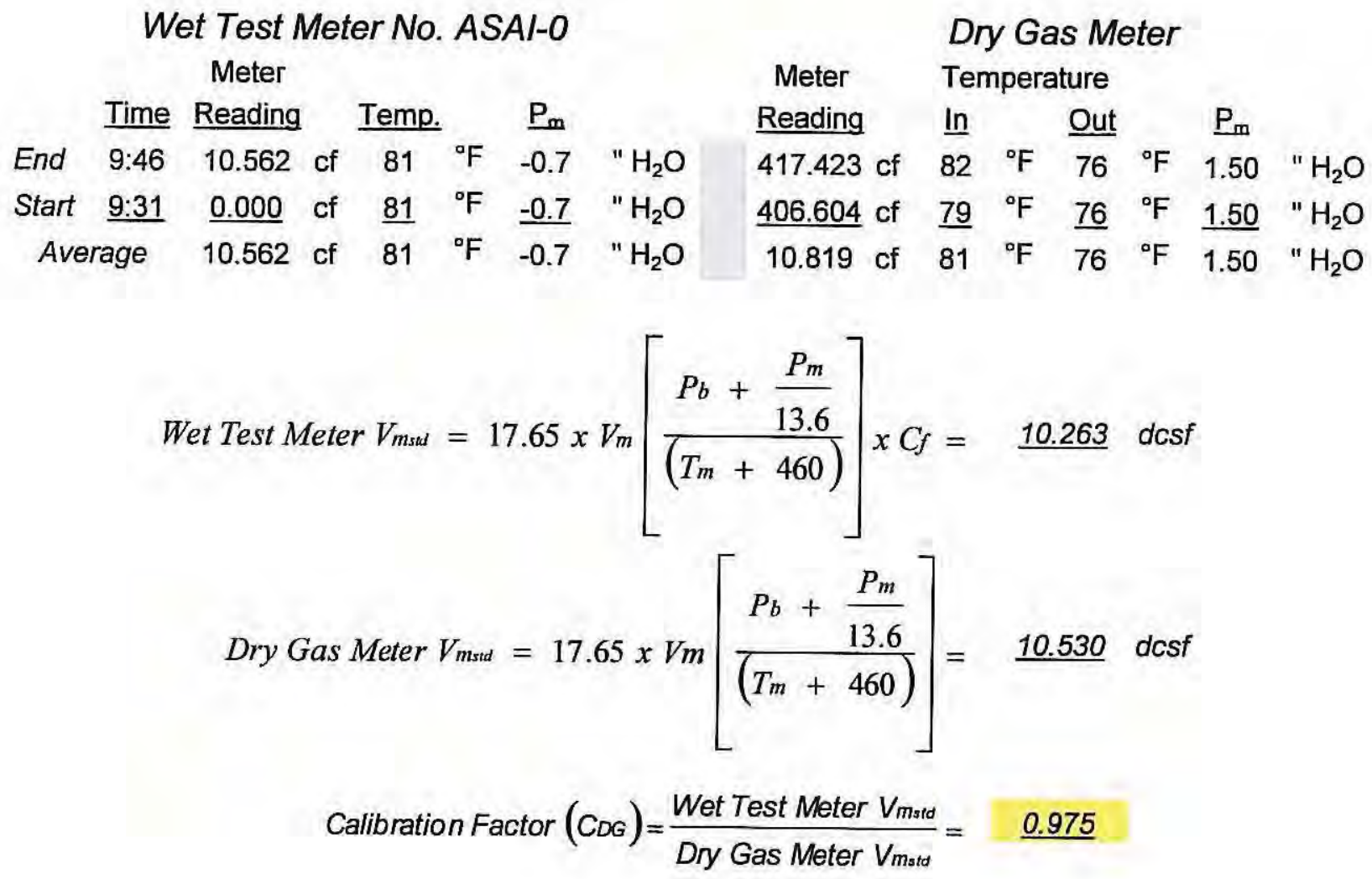




\author{
Calibration Data \\ Dry Gas Meter Calibration \\ Meter Console No. \\ ASAI 2-2 \\ Date Calibration Performed: \\ $08 / 18 / 08$
}

Run 1 at 2.0 " $\Delta \mathrm{H}$

Wet Test Meter $\mathrm{C}_{\mathrm{f}}=1.009$ Console Pump Vacuum $=-5.0 \mathrm{Hg}$

Wet Test Meter No. ASAI-O

Meter

Time Reading Temp. $\quad P_{m}$

End 10:02 10.531 of $81 \quad{ }^{\circ} \mathrm{F} \quad-0.8 \quad " \mathrm{H}_{2} \mathrm{O}$

Start $\underline{9: 49} \underline{\underline{0.000}}$ of $\underline{81} \quad{ }^{\circ} \mathrm{F} \quad \underline{-0.8} \quad " \mathrm{H}_{2} \mathrm{O}$

Average

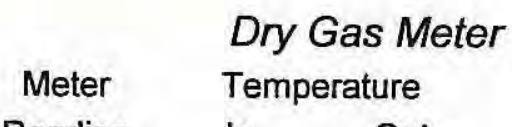

Reading In $\underline{\text { Out }} \quad \underline{P_{m}}$ 428.809 cf $83 \quad 3^{\circ} \mathrm{F} \quad 77^{\circ} \mathrm{F} \quad 2.00 \quad " \mathrm{H}_{2} \mathrm{O}$ $\underline{418.015}$ of $\underline{81}{ }^{\circ} \mathrm{F} \quad \underline{76} \quad{ }^{\circ} \mathrm{F} \quad \underline{2.00} \quad \mathrm{H}_{2} \mathrm{O}$ 10.794 cf $82{ }^{\circ} \mathrm{F} \quad 77^{\circ} \mathrm{F} \quad 2.00 \quad " \mathrm{H}_{2} \mathrm{O}$

$$
\begin{gathered}
\text { Wet Test Meter } V_{m s t}=17.65 \times V_{m}\left[\frac{P_{b}+\frac{P_{m}}{13.6}}{\left(T_{m}+460\right)}\right] \times C_{f}=\underline{10.230} \mathrm{dcsf} \\
\qquad \text { Dry Gas Meter Vmsd }=17.65 \times \mathrm{Vm}\left[\frac{P_{b}+\frac{P_{m}}{13.6}}{\left(T_{m}+460\right)}\right]=\underline{10.499} \mathrm{dcsf}
\end{gathered}
$$

$$
\text { Calibration Factor }\left(C_{D G}\right)=\frac{\text { Wet Test Meter } V_{m s t d}}{\text { Dry Gas Meter } V_{m s t d}}=\underline{0.974}
$$




\section{Calibration Data}

Dry Gas Meter Calibration

Meter Console No.

Date Calibration Performed:
ASAI 2-2

$08 / 18 / 08$

Run 1 at 3.0" $\Delta \mathrm{H}$

Wet Test Meter $\mathrm{C}_{\mathrm{f}}=1.009$

$\mathrm{Pb}=29.57 " \mathrm{Hg}$

Console Pump Vacuum $=-5.0 \mathrm{Hg}$

Wet Test Meter No. ASAI-O

Meter

Meter Temperature

Time Reading Temp. $\quad \mathbf{P}_{\mathrm{m}}$

End 10:15 10.857 of $81{ }^{\circ} \mathrm{F} \quad-1.1 \quad " \mathrm{H}_{2} \mathrm{O}$

Reading In Out $\underline{P_{m}}$

Start $\underline{10: 04} \underline{0.000}$ of $\underline{81} \quad{ }^{\circ} \mathrm{F} \quad \underline{-1.1} \quad " \mathrm{H}_{2} \mathrm{O}$ $440.651 \mathrm{cf} \quad 84{ }^{\circ} \mathrm{F} \quad 77 \quad{ }^{\circ} \mathrm{F} \quad 3.00 \quad " \mathrm{H}_{2} \mathrm{O}$

Average

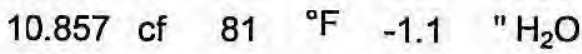

$\underline{429.505}$ of $\quad \underline{82}{ }^{\circ} \mathrm{F} \quad \underline{77} \quad{ }^{\circ} \mathrm{F} \quad \underline{3.00} \quad " \mathrm{H}_{2} \mathrm{O}$

11.146 of $83{ }^{\circ} \mathrm{F} \quad 77{ }^{\circ} \mathrm{F} \quad 3.00 \quad " \mathrm{H}_{2} \mathrm{O}$

Wet Test Meter $V_{m s t}=17.65 \times V_{m}\left[\frac{P_{b}+\frac{P_{m}}{13.6}}{\left(T_{m}+460\right)}\right] \times C_{f}=\underline{10.539} \mathrm{dcsf}$
Dry Gas Meter $V_{m s d}=17.65 \times V_{m}\left[\frac{P_{b}+\frac{P_{m}}{13.6}}{\left(T_{m}+460\right)}\right]=\underline{10.853} \mathrm{dcsf}$

Calibration Factor $($ CDG $)=\frac{\text { Wet Test Meter } V_{m s t d}}{D r y \text { Gas Meter } V_{m s t a}}=\underline{0.971}$ 


\author{
Calibration Data \\ Dry Gas Meter Calibration \\ Meter Console No. \\ ASAI 2-2 \\ Date Calibration Performed: \\ $08 / 18 / 08$
}

Run $\frac{1}{1}$ at $4.0 " \Delta H$

Wet Test Meter $C_{f}=1.009$

$\begin{aligned} \mathrm{Pb} & =29.57 \mathrm{Hg} \\ \text { Console Pump Vacuum } & =-5.0 \mathrm{Hg}\end{aligned}$

Wet Test Meter No. ASAI-O Meter

Dry Gas Meter

Meter Temperature

Time Reading Temp. $\quad \underline{P}_{m}$

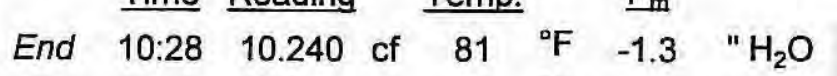

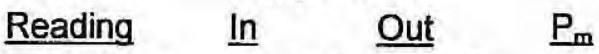

Start $\underline{10: 18} \underline{0.000}$ of $\underline{81}{ }^{\circ} \mathrm{F} \quad \underline{-1.3} \quad " \mathrm{H}_{2} \mathrm{O}$ 452.010 of $86 \quad{ }^{\circ} \mathrm{F} \quad 77 \quad{ }^{\circ} \mathrm{F} \quad 4.00 \quad \mathrm{H}_{2} \mathrm{O}$

Average $\begin{array}{llllll}10.240 & \text { cf } & 81 & { }^{\circ} \mathrm{F} & -1.3 & \quad \mathrm{H}_{2} \mathrm{O}\end{array}$

$\underline{441.509}$ cf $\underline{8}$

10.501 ef $85{ }^{\circ} \mathrm{F} \quad 77 \quad{ }^{\circ} \mathrm{F} \quad 4.00 \quad \mathrm{H}_{2} \mathrm{O}$

Wet Test Meter $V_{m s d}=17.65 \times V_{m}\left[\frac{P_{b}+\frac{P_{m}}{13.6}}{\left(T_{m}+460\right)}\right] x C_{f}=\underline{9.935} \mathrm{dcsf}$
Dry Gas Meter $V_{m s t d}=17.65 \times \mathrm{Vm}\left[\frac{P_{b}+\frac{P_{m}}{13.6}}{\left(T_{m}+460\right)}\right]=\underline{10.231} \mathrm{dcsf}$

Calibration Factor $\left(C_{D G}\right)=\frac{\text { Wet Test Meter } V_{\text {mstd }}}{\text { Dry Gas Meter } V_{m s t}}=\underline{0.971}$ 
Calibration Data

Digital Temperature Indicator Calibration

DTI Unit No. ASAI 2-2

Date Calibration Performed:

\begin{tabular}{|c|c|}
\hline 08/18/08 & \\
\hline $\begin{array}{c}\text { ASTM } \\
\text { Reference }\end{array}$ & \\
\hline $\begin{array}{l}\text { Thermometer } \\
\qquad \frac{\left({ }^{\circ} \mathrm{F}\right)}{32^{\circ} \mathrm{F}}\end{array}$ & $\begin{array}{l}\begin{array}{l}\mathrm{DTI} \\
\left({ }^{\circ} \mathrm{F}\right)\end{array} \\
32^{\circ} \mathrm{F}\end{array}$ \\
\hline $81^{\circ} \mathrm{F}$ & $81^{\circ} \mathrm{F}$ \\
\hline $212^{\circ} \mathrm{F}$ & $212^{\circ} \mathrm{F}$ \\
\hline $250^{\circ} \mathrm{F}$ & $251^{\circ} \mathrm{F}$ \\
\hline $300^{\circ} \mathrm{F}$ & $301^{\circ} \mathrm{F}$ \\
\hline $350^{\circ} \mathrm{F}$ & $350^{\circ} \mathrm{F}$ \\
\hline $400^{\circ} \mathrm{F}$ & $400^{\circ} \mathrm{F}$ \\
\hline
\end{tabular}

Meter Adjusted?

Yes

Reference Point

Ice Bath

Ambient Air

Boiling Water

Oven

Oven

Oven

Oven

\begin{tabular}{c} 
\\
\hline Time \\
\hline $7: 41$ \\
\hline $7: 42$ \\
\hline $7: 43$ \\
\hline $7: 44$ \\
\hline $7: 45$ \\
\hline $7: 46$ \\
\hline $7: 47$ \\
\hline
\end{tabular}

Yes

ASTM Reference Thermometer:

SN: 06000047

Range: $\quad-0.1^{\circ} \mathrm{C}$ to $1360.6^{\circ} \mathrm{C}$

Range:

Range:

Certified by:

Patrick Selakovich 8/18/08

Calibrator (Signature / Date)

Bill Hefley 8/18/08

QA Officer (Signature / Date) 


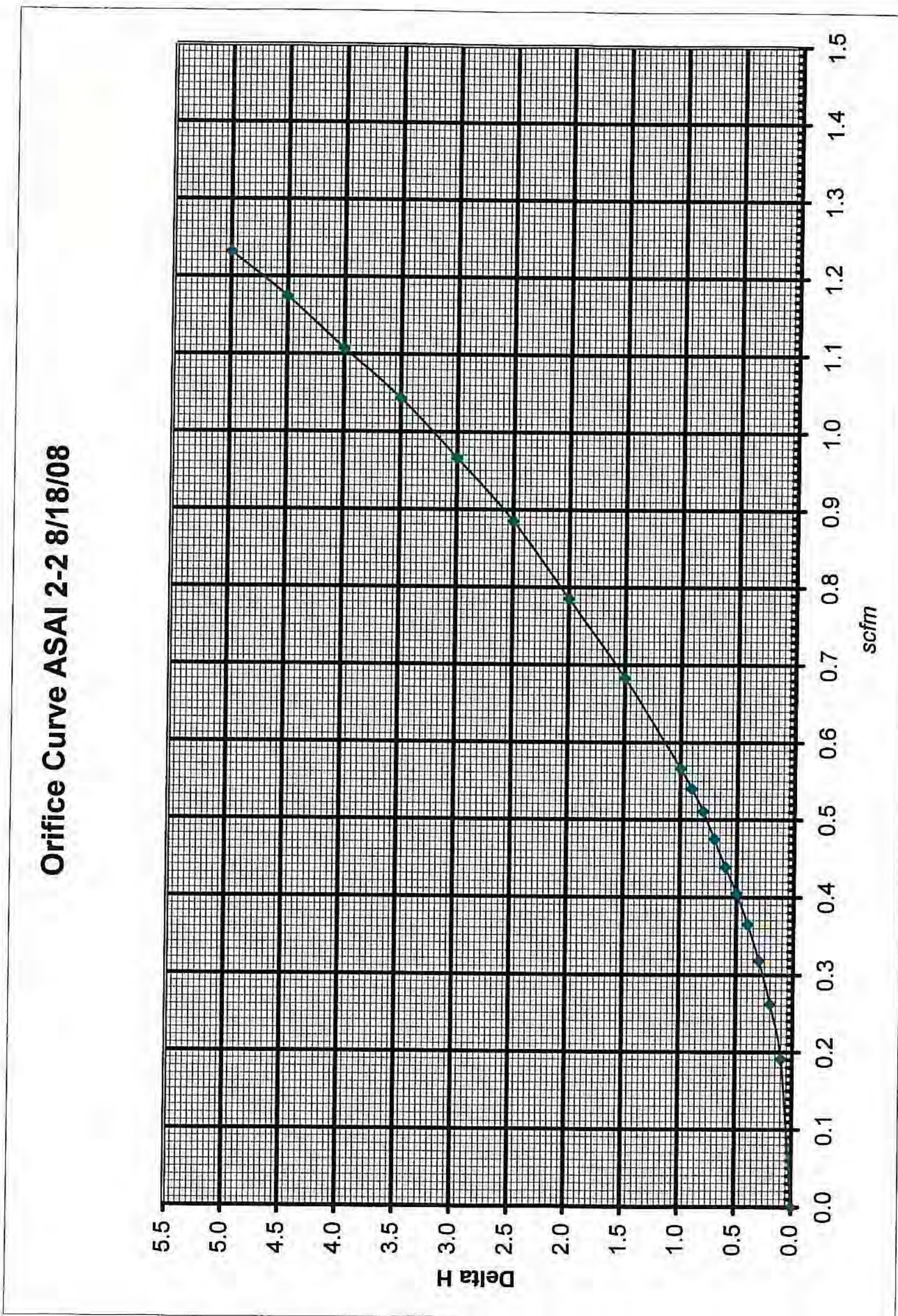




\section{AIR
CALIBRATION DATA
PITOT TUBE CALIBRATION DATA

$\begin{array}{cc}\text { Date: } & 07 / 03 / 08 \\ \text { I.D. \# } \frac{\text { ASAI 3-2 }}{0.990} \\ \text { C }_{\text {pstd: }}\end{array}$
Time: $6: 00$

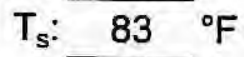
$\mathrm{Pb}: \overline{29.65} \mathrm{Hg}$

\begin{tabular}{|c|c|c|c|c|c|c|c|c|c|c|}
\hline $\begin{array}{c}\text { fps } \\
\text { Mark }\end{array}$ & $\begin{array}{c}\text { Desired } \\
\text { Calibration } \\
\text { Standard }\end{array}$ & \multicolumn{2}{|c|}{$\begin{array}{c}\text { Calibration } \\
\text { Standard } \\
\text { Start }\end{array}$} & $\begin{array}{c}\text { V Standard } \\
\text { Average }\end{array}$ & High & V High & $\begin{array}{c}\text { Cal. } \\
\text { Factor }\end{array}$ & Low & V Low & $\begin{array}{c}\text { Cal. } \\
\text { Factor }\end{array}$ \\
\hline 20 & 0.09 & 0.09 & 0.09 & 0.300 & 0.13 & 0.361 & 0.824 & 0.13 & 0.361 & 0.824 \\
\hline 30 & 0.20 & 0.20 & 0.20 & 0.447 & 0.29 & 0.539 & 0.822 & 0.29 & 0.539 & 0.822 \\
\hline 40 & 0.35 & 0.35 & 0.35 & 0.592 & 0.50 & 0.707 & 0.828 & 0.50 & 0.707 & 0.828 \\
\hline 50 & 0.55 & 0.55 & 0.55 & 0.742 & 0.79 & 0.889 & 0.826 & 0.79 & 0.889 & 0.826 \\
\hline 60 & 0.79 & 0.79 & 0.79 & 0.889 & 1.15 & 1.072 & 0.821 & 1.15 & 1.072 & 0.821 \\
\hline 70 & 1.08 & 1.10 & 1.10 & 1.049 & 1.60 & 1.265 & 0.821 & 1.60 & 1.265 & 0.821 \\
\hline 80 & 1.41 & 1.40 & 1.40 & 1.183 & 2.05 & 1.432 & 0.818 & 2.05 & 1.432 & 0.818 \\
\hline 90 & 1.78 & 1.80 & 1.80 & 1.342 & 2.60 & 1.612 & 0.824 & 2.60 & 1.612 & 0.824 \\
\hline & & & & & & & & & & \\
\hline & & & & & & & & & & \\
\hline 50 & 0.55 & 0.55 & 0.55 & 0.742 & 0.80 & 0.894 & 0.821 & 0.80 & 0.894 & 0.821 \\
\hline 50 & 0.55 & 0.55 & 0.55 & 0.742 & 0.80 & 0.894 & 0.821 & 0.80 & 0.894 & 0.821 \\
\hline & & & & & & & & & & \\
\hline & & & & & & & & & & \\
\hline \multicolumn{2}{|l|}{ Average } & & & & & & 0.823 & & & 0.823 \\
\hline
\end{tabular}

Summary of Results:

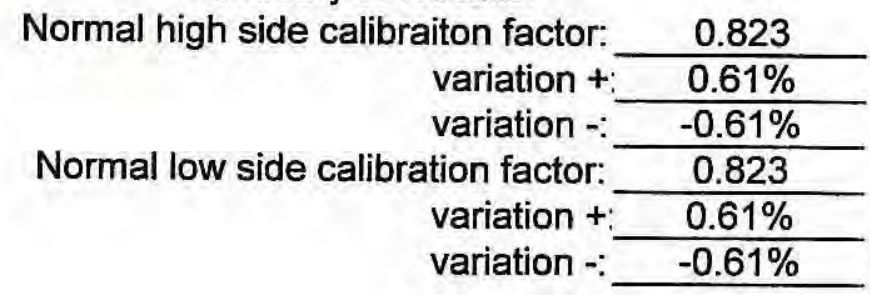

Certification:

I certify that the Type S pitot tube, the standard type pitot tube, and the calibration setup meet or exceed all specifications, criteria and/or applicable design features and hereby assign a pitot tube calibration factor $\mathrm{C}_{\mathrm{p}}$ of: $\quad 0.823$

Certified by:

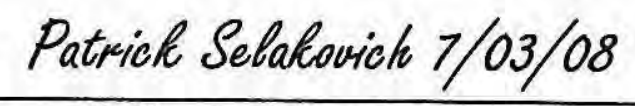

Calibrator (Signature/Date)
Bill Hefley $7 / 03 / 08$

QA Officer (Signature/Date) 


\section{ming
CALIBRATION DATA
PITOT TUBE CALIBRATION DATA

Time: $6: 30$

$\mathrm{T}_{\mathrm{s}}: \mathrm{84}^{\circ} \mathrm{F}$

$\mathrm{Pb}: \overline{29.65} " \mathrm{Hg}$

\begin{tabular}{|c|c|c|c|c|c|c|c|c|c|c|}
\hline \multirow{2}{*}{\begin{tabular}{|c|} 
fps \\
Mark \\
\end{tabular}} & \multirow{2}{*}{$\begin{array}{l}\text { Desired } \\
\text { Calibration } \\
\text { Standard }\end{array}$} & \multicolumn{2}{|c|}{$\begin{array}{l}\text { Calibration } \\
\text { Standard }\end{array}$} & \multirow{2}{*}{$\begin{array}{c}\sqrt{ } \text { Standard } \\
\text { Average }\end{array}$} & \multirow[b]{2}{*}{ High } & \multirow[b]{2}{*}{$\sqrt{ }$ High } & \multirow{2}{*}{$\begin{array}{l}\text { Cal. } \\
\text { Factor }\end{array}$} & \multirow[b]{2}{*}{ Low } & \multirow[b]{2}{*}{$\sqrt{ }$ Low } & \multirow{2}{*}{$\begin{array}{c}\text { Cal. } \\
\text { Factor }\end{array}$} \\
\hline & & Start & End & & & & & & & \\
\hline 20 & 0.09 & 0.09 & 0.09 & 0.300 & 0.13 & 0.361 & 0.824 & 0.13 & 0.361 & 0.824 \\
\hline 30 & 0.20 & 0.20 & 0.20 & 0.447 & 0.29 & 0.539 & 0.822 & 0.29 & 0.539 & 0.822 \\
\hline 40 & 0.35 & 0.35 & 0.35 & 0.592 & 0.51 & 0.714 & 0.820 & 0.51 & 0.714 & 0.820 \\
\hline 50 & 0.55 & 0.55 & 0.55 & 0.742 & 0.79 & 0.889 & 0.826 & 0.79 & 0.889 & 0.826 \\
\hline 60 & 0.79 & 0.79 & 0.79 & 0.889 & 1.15 & 1.072 & 0.821 & 1.15 & 1.072 & 0.821 \\
\hline 70 & 1.08 & 1.10 & 1.10 & 1.049 & 1.60 & 1.265 & 0.821 & 1.60 & 1.265 & 0.821 \\
\hline 80 & 1.41 & 1.40 & 1.40 & 1.183 & 2.00 & 1.414 & 0.828 & 2.00 & 1.414 & 0.828 \\
\hline \multirow[t]{2}{*}{90} & 1.78 & 1.80 & 1.80 & 1.342 & 2.60 & 1.612 & 0.824 & 2.60 & 1.612 & 0.824 \\
\hline & & & & & & & & & & \\
\hline 50 & 0.55 & 0.55 & 0.55 & 0742 & 079 & 0.889 & 0826 & 0.79 & 0880 & 0826 \\
\hline 50 & 0.55 & 0.55 & 0.55 & 0.742 & 0.79 & 0.889 & 0.826 & 0.79 & 0.889 & $\frac{0.020}{0.826}$ \\
\hline & & & & & & & & & & \\
\hline & & & & & & & & & & \\
\hline & verage & & & & & & 0.823 & & & 0.823 \\
\hline
\end{tabular}

Summary of Results:

Normal high side calibraiton factor: $\quad 0.823$

variation $+\quad 0.61 \%$

variation -:

Normal low side calibration factor:

$-0.36 \%$

variation $+\quad 0.61 \%$

variation $-\frac{-0.36 \%}{-}$

Certification:

I certify that the Type S pitot tube, the standard type pitot tube, and the calibration setup meet or exceed all specifications, criteria and/or applicable design features and hereby assign a pitot tube calibration factor $C_{p}$ of: $\quad 0.823$

Certified by: $\frac{\text { Patrick Selakovich } 7 / 03 / 08}{\text { Calibrator (Signature/Date) }}$

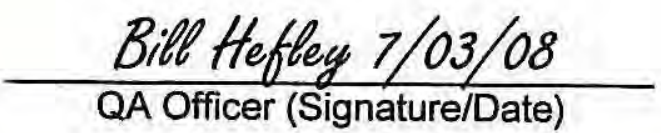




$\begin{array}{ll}\text { I.D. \# } & \text { ASAI 3-4 } \\ \text { C }_{\text {pstd: }} & 0.990\end{array}$

\section{PITOT TUBE CALIBRATION DATA}

Time: $12: 45$

$$
\begin{aligned}
& \mathrm{T}_{\mathrm{s}} \text { : } 82{ }^{\circ} \mathrm{F} \\
& \text { Pb: } 29.29 " \mathrm{Hg}
\end{aligned}
$$

\begin{tabular}{|c|c|c|c|c|c|c|c|c|c|c|}
\hline $\begin{array}{c}\text { fps } \\
\text { Mark }\end{array}$ & $\begin{array}{c}\text { Desired } \\
\text { Calibration } \\
\text { Standard }\end{array}$ & \multicolumn{2}{c|}{$\begin{array}{c}\text { Calibration } \\
\text { Standard } \\
\text { Start }\end{array}$} & $\begin{array}{c}\text { V Standard } \\
\text { Average }\end{array}$ & High & V High & $\begin{array}{c}\text { Cal. } \\
\text { Factor }\end{array}$ & Low & V Low & $\begin{array}{c}\text { Cal. } \\
\text { Factor }\end{array}$ \\
\hline 20 & 0.09 & 0.09 & 0.09 & 0.300 & 0.13 & 0.361 & 0.824 & 0.13 & 0.361 & 0.824 \\
\hline 30 & 0.20 & 0.20 & 0.20 & 0.447 & 0.29 & 0.539 & 0.822 & 0.29 & 0.539 & 0.822 \\
\hline 40 & 0.35 & 0.35 & 0.35 & 0.592 & 0.50 & 0.707 & 0.828 & 0.50 & 0.707 & 0.828 \\
\hline 50 & 0.55 & 0.55 & 0.55 & 0.742 & 0.80 & 0.894 & 0.821 & 0.80 & 0.894 & 0.821 \\
\hline 60 & 0.78 & 0.78 & 0.78 & 0.883 & 1.15 & 1.072 & 0.815 & 1.15 & 1.072 & 0.815 \\
\hline 70 & 1.07 & 1.10 & 1.10 & 1.049 & 1.60 & 1.265 & 0.821 & 1.60 & 1.265 & 0.821 \\
\hline 80 & 1.40 & 1.40 & 1.40 & 1.183 & 2.05 & 1.432 & 0.818 & 2.05 & 1.432 & 0.818 \\
\hline 90 & 1.77 & 1.80 & 1.80 & 1.342 & 2.65 & 1.628 & 0.816 & 2.65 & 1.628 & 0.816 \\
\hline & & & & & & & & & & \\
\hline & & & & & & & & & & \\
\hline 50 & 0.55 & 0.55 & 0.55 & 0.742 & 0.80 & 0.894 & 0.821 & 0.80 & 0.894 & 0.821 \\
\hline 50 & 0.55 & 0.55 & 0.55 & 0.742 & 0.80 & 0.894 & 0.821 & 0.80 & 0.894 & 0.821 \\
\hline & & & & & & & & & & \\
\hline & & & & & & & & & & \\
\hline \multicolumn{2}{|l|}{ Average } & & & & & & 0.821 & & & 0.821 \\
\hline
\end{tabular}

Summary of Results:

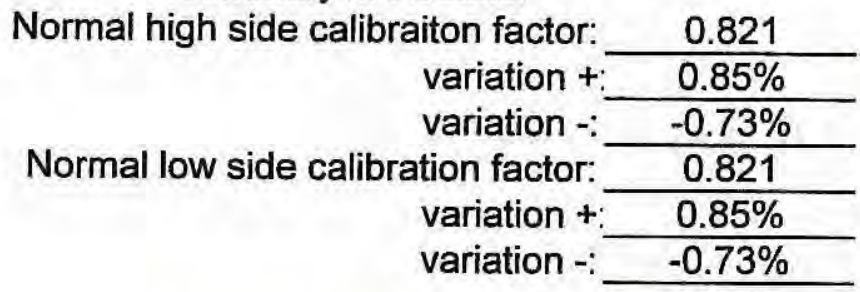

Certification:

I certify that the Type S pitot tube, the standard type pitot tube, and the calibration setup meet or exceed all specifications, criteria and/or applicable design features and hereby assign a pitot tube calibration factor $C_{p}$ of: $\quad 0.821$

Certified by: $\quad \frac{\text { Patrick Selakovick 5/01/08 }}{\text { Calibrator (Signature/Date) }}$

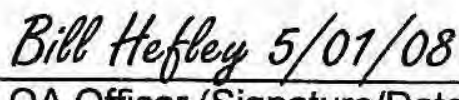

QA Officer (Signature/Date) 


\section{Calibration Data \\ Nozzle Calibration}

Nozzle Set No.

Date Calibration Performed:

ASAI - 3
$03 / 03 / 08$

$\begin{array}{llllllll}-1 & -2 & -3 & -4 & -5 & -6 & -7 & -8\end{array}$

Measurement 1

$\begin{array}{llllllll}0.121 & 0.168 & 0.233 & 0.316 & 0.364 & 0.397 & 0.497 & 0.231\end{array}$

Measurement 2

$\begin{array}{llllllllllllllllllllll}0.122 & 0.169 & 0.230 & 0.317 & 0.364 & 0.396 & 0.496 & 0.234\end{array}$

Measurement 3

$\begin{array}{llllllllllll}0.122 & 0.168 & 0.232 & 0.318 & 0.364 & 0.397 & 0.497 & 0.232\end{array}$

Measurement 4

$\begin{array}{llllllllllllllllll}0.122 & 0.169 & 0.231 & 0.319 & 0.365 & 0.397 & 0.495 & 0.233\end{array}$

Measurement 5

$\begin{array}{llllllllll}0.121 & 0.168 & 0.234 & 0.319 & 0.367 & 0.396 & 0.498 & 0.231\end{array}$

Measurement 6

Measurement 7

Measurement 8

Measurement 9

Measurement 10

Average

$\begin{array}{llllllllllllllllllllll}0.122 & 0.167 & 0.231 & 0.317 & 0.366 & 0.398 & 0.496 & 0.231\end{array}$

$\begin{array}{llllllllllllllllllllllll}0.120 & 0.168 & 0.234 & 0.318 & 0.367 & 0.396 & 0.496 & 0.232\end{array}$

$\begin{array}{llllllllll}0.122 & 0.168 & 0.231 & 0.319 & 0.364 & 0.396 & 0.496 & 0.231\end{array}$

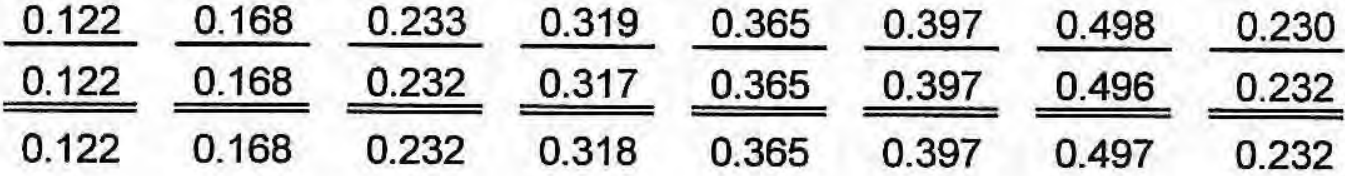

Patrick Selakovich 3/03/08

Calibrator (Signature / Date)

Bill Hefley 3/03/08

QA Officer (Signature / Date) 


\section{Calibration Data \\ Nozzle Calibration}

Nozzle Set No. ASAI 08-026

Date Calibration Performed: $09 / 24 / 08$

$\begin{array}{lllllll}-1 & -2 & -3 & -4 & -5 & -6 & -7\end{array}$

\begin{tabular}{|c|c|c|c|c|c|}
\hline Measurement 1 & 0.156 & 0.160 & 0.131 & & \\
\hline Measurement 2 & 0.155 & 0.161 & 0.131 & & \\
\hline Measurement 3 & 0.156 & 0.161 & 0.131 & & \\
\hline Measurement 4 & 0.154 & 0.160 & 0.131 & & \\
\hline Measurement 5 & 0.156 & 0.160 & 0.131 & & \\
\hline Measurement 6 & 0.155 & 0.161 & 0.131 & & \\
\hline Measurement 7 & 0.156 & 0.161 & 0.131 & & \\
\hline Measurement 8 & 0.155 & 0.160 & 0.131 & & \\
\hline Measurement 9 & 0.154 & 0.160 & 0.131 & & \\
\hline Measurement 10 & 0.156 & 0.160 & 0.131 & & \\
\hline Average & 0.155 & 0.160 & 0.131 & \#\#\#\# & \#\#\# \\
\hline
\end{tabular}

Certified by:

Jolon Stanley 9-24-08

Calibrator (Signature / Date)

Bill Hefley 9-24-08

QA Officer (Signature / Date) 


\section{Post-test Calibration Data}




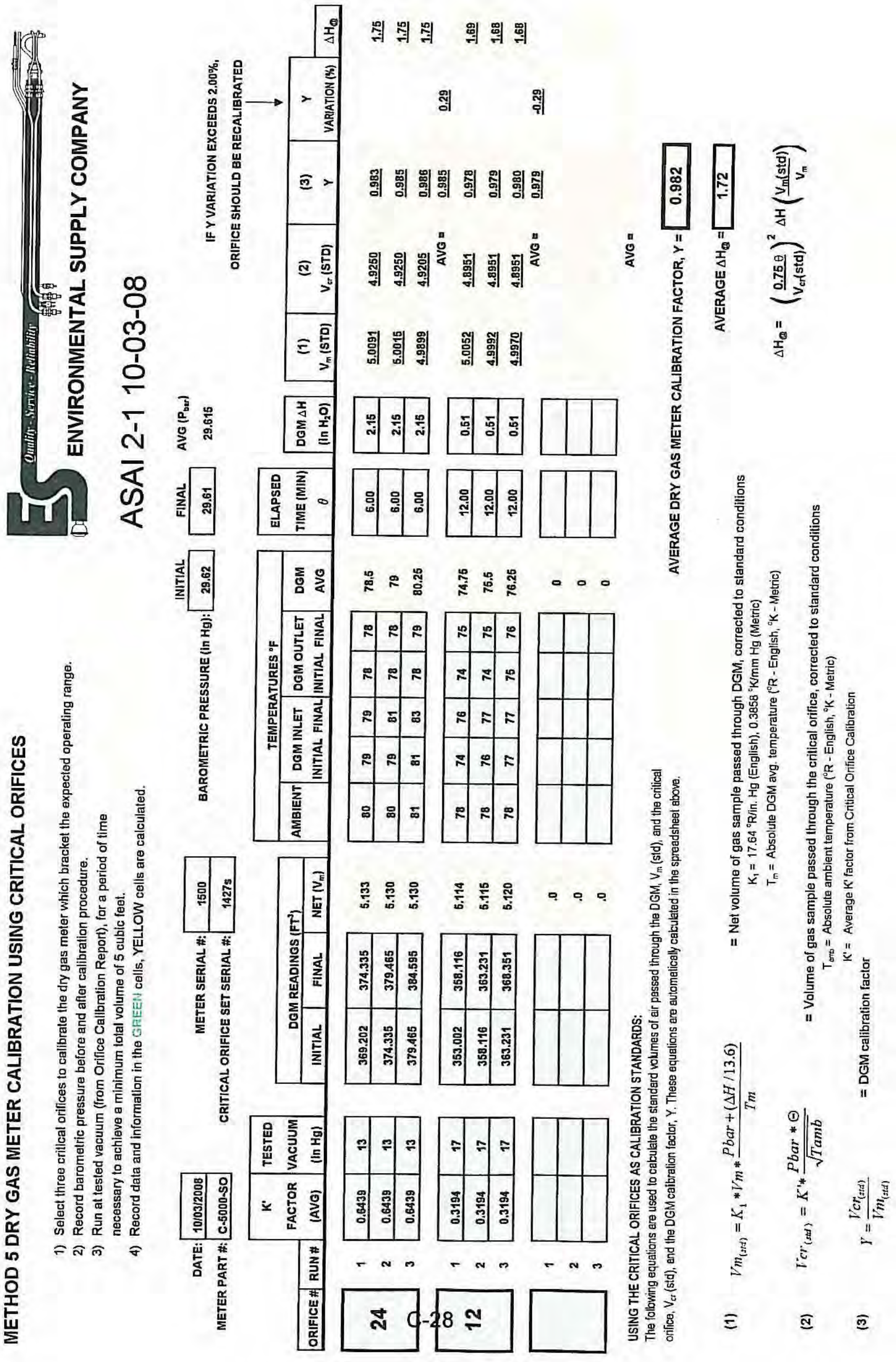


Calibration Data

Digital Temperature Indicator Calibration

DTI Unit No. ASAI 2-1

Date Calibration Performed:

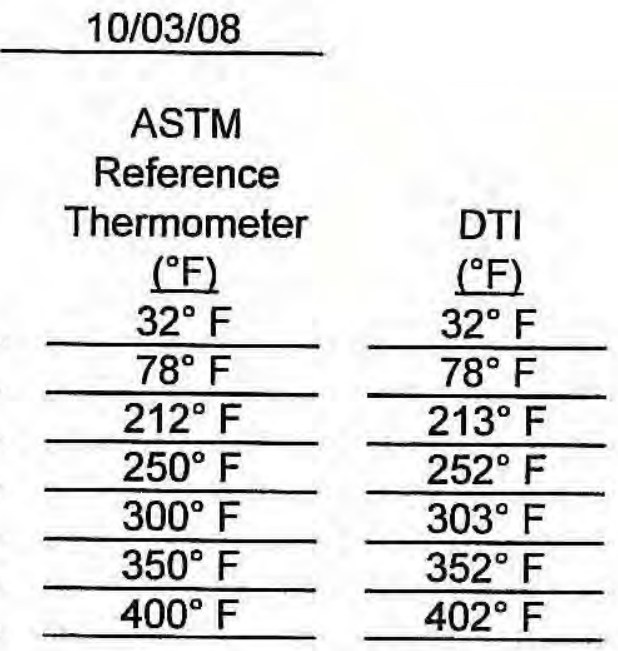

Meter Adjusted?

No

Reference Point

Ice Bath

Ambient Air

Boiling Water

Oven

Oven

Oven

Oven

\begin{tabular}{c}
$\frac{\text { Time }}{11: 45}$ \\
\hline $11: 46$ \\
\hline $11: 47$ \\
\hline $11: 48$ \\
\hline $11: 49$ \\
\hline $11: 50$ \\
\hline $11: 51$ \\
\hline
\end{tabular}

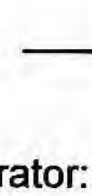

NIST Reference Calibrator:

SN: 06000047

Range:

Range:

Range:

$-0.2^{\circ} \mathrm{C}$ to $1,360.6^{\circ} \mathrm{C}$

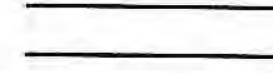

Certified by:

Patrick Selakovich 10/03/08

Calibrator (Signature / Date)

Bill Hefley 10/03/08

QA Officer (Signature / Date) 


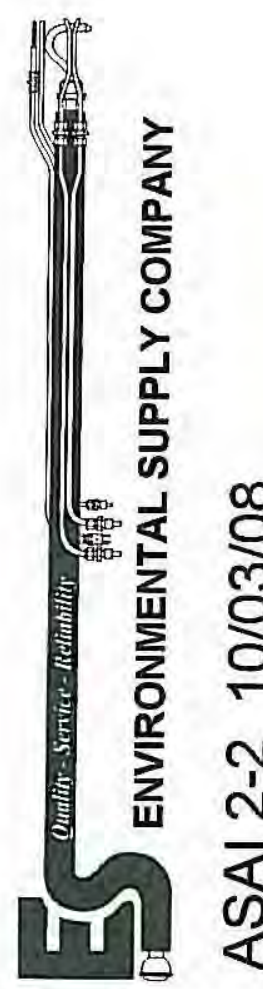

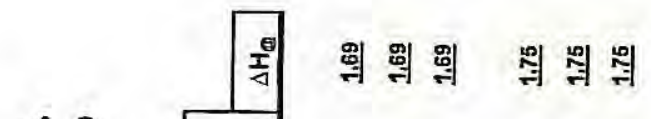

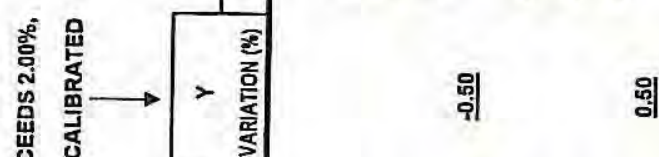

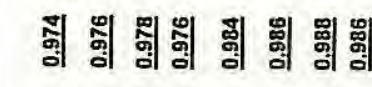

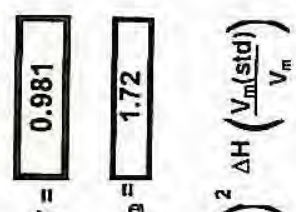

离

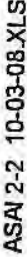

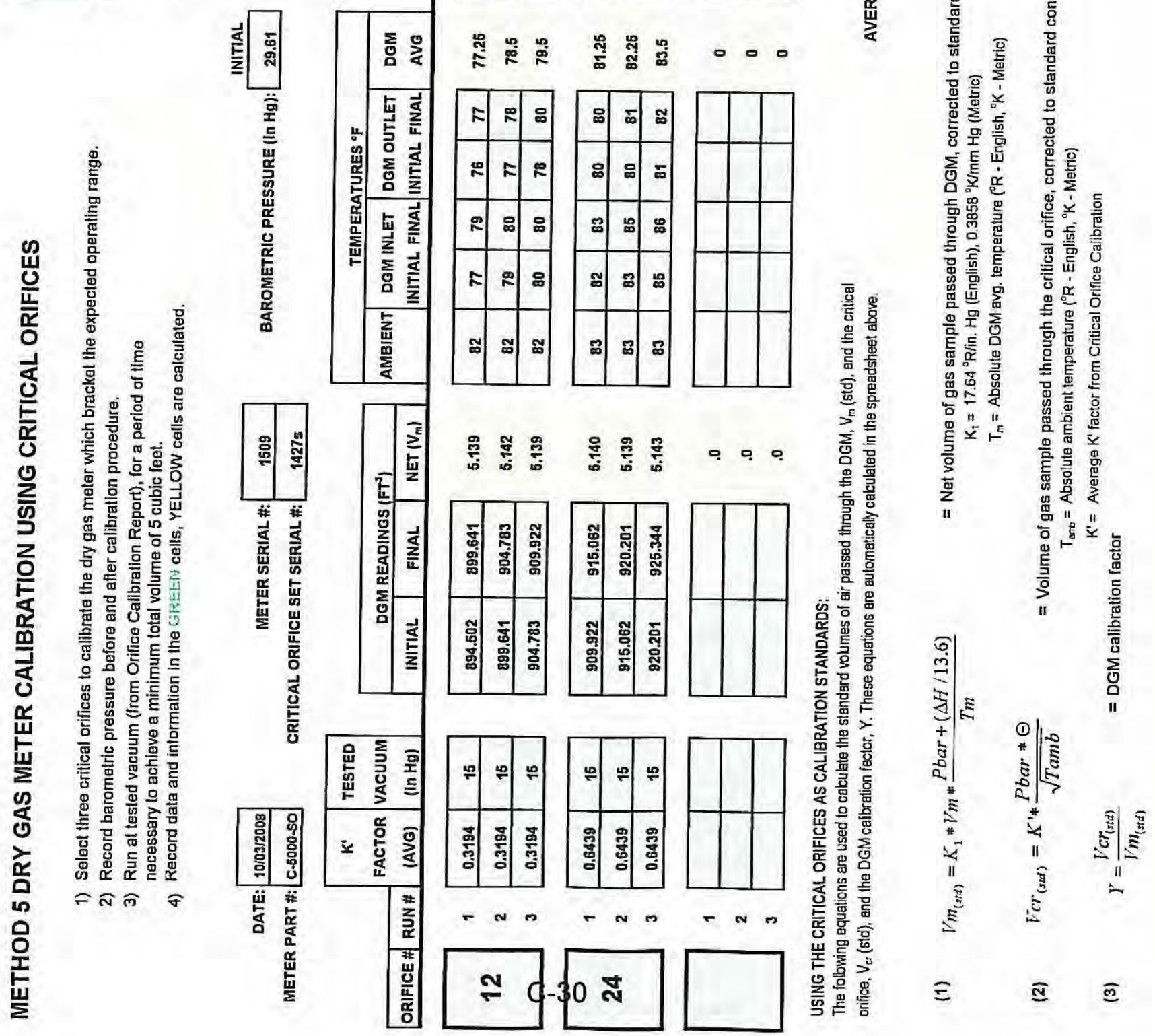


Calibration Data

Digital Temperature Indicator Calibration

DTI Unit No. ASAI 2-2

Date Calibration Performed: $10 / 03 / 08$

ASTM

Reference

Thermometer

Reference Point

$\left({ }^{\circ} \mathrm{F}\right)$

Ice Bath

Ambient Air

Boiling Water

Oven

Oven

Oven

Oven
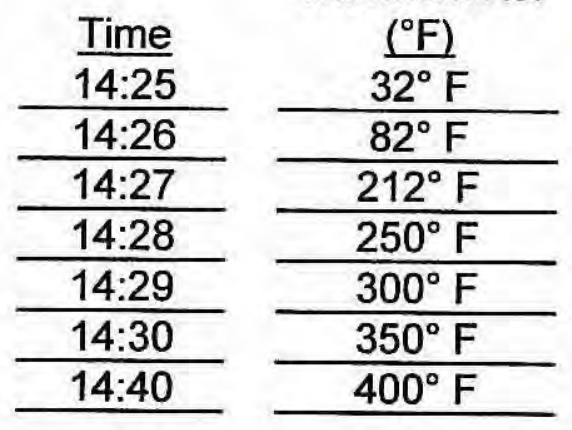

\begin{tabular}{c}
$\begin{array}{c}\text { DTI } \\
\left({ }^{\circ} \mathrm{F}\right)\end{array}$ \\
$31^{\circ} \mathrm{F}$ \\
\hline $81^{\circ} \mathrm{F}$ \\
\hline $212^{\circ} \mathrm{F}$ \\
\hline $250^{\circ} \mathrm{F}$ \\
\hline $300^{\circ} \mathrm{F}$ \\
\hline $350^{\circ} \mathrm{F}$ \\
\hline $399^{\circ} \mathrm{F}$ \\
\hline
\end{tabular}

Meter Adjusted?

No

ASTM Reference Thermometer:

SN: 06000047

Range: $\quad-0.1^{\circ} \mathrm{C}$ to $1,360.6^{\circ}$

Range:

Range:

Certified by:

Patrick Selakovich 10/03/08

Calibrator (Signature / Date)

Bill Heffey 10/03/08

QA Officer (Signature / Date) 


\section{II) SARMPLING \\ ASSOCIATES, INC.}
CALIBRATION DATA
$\begin{array}{lc}\text { Date: } \frac{10 / 03 / 08}{\text { I.D. \# }} & \frac{\text { ASAI 3-2 }}{0.990} \\ \text { C }_{\text {pstd: }} & 0.990\end{array}$
Time: $16: 00$
$\mathrm{T}_{\mathrm{s}}:{ }_{87}^{\circ} \mathrm{F}$
Pb: 29.57 " $\mathrm{Hg}$

\begin{tabular}{|c|c|c|c|c|c|c|c|c|c|c|}
\hline $\begin{array}{c}\text { fps } \\
\text { Mark }\end{array}$ & $\begin{array}{c}\text { Desired } \\
\text { Calibration } \\
\text { Standard }\end{array}$ & \multicolumn{2}{|c|}{$\begin{array}{c}\text { Calibration } \\
\text { Standard } \\
\text { Start }\end{array}$} & $\begin{array}{c}\text { End } \\
\text { V Standard } \\
\text { Average }\end{array}$ & High & V High & $\begin{array}{c}\text { Cal. } \\
\text { Factor }\end{array}$ & Low & V Low & $\begin{array}{c}\text { Cal. } \\
\text { Factor }\end{array}$ \\
\hline 20 & 0.09 & 0.09 & 0.09 & 0.300 & 0.13 & 0.361 & 0.824 & 0.13 & 0.361 & 0.824 \\
\hline 30 & 0.20 & 0.20 & 0.20 & 0.447 & 0.29 & 0.539 & 0.822 & 0.29 & 0.539 & 0.822 \\
\hline 40 & 0.35 & 0.35 & 0.35 & 0.592 & 0.50 & 0.707 & 0.828 & 0.50 & 0.707 & 0.828 \\
\hline 50 & 0.55 & 0.55 & 0.55 & 0.742 & 0.79 & 0.889 & 0.826 & 0.79 & 0.889 & 0.826 \\
\hline 60 & 0.79 & 0.79 & 0.79 & 0.889 & 1.15 & 1.072 & 0.821 & 1.15 & 1.072 & 0.821 \\
\hline 70 & 1.07 & 1.10 & 1.10 & 1.049 & 1.60 & 1.265 & 0.821 & 1.60 & 1.265 & 0.821 \\
\hline 80 & 1.40 & 1.40 & 1.40 & 1.183 & 2.05 & 1.432 & 0.818 & 2.05 & 1.432 & 0.818 \\
\hline 90 & 1.77 & 1.80 & 1.80 & 1.342 & 2.60 & 1.612 & 0.824 & 2.60 & 1.612 & 0.824 \\
\hline & & & & & & & & & & \\
\hline & & & & & & & & & & \\
\hline 50 & 0.55 & 0.55 & 0.55 & 0.742 & 0.79 & 0.889 & 0.826 & 0.79 & 0.889 & 0.826 \\
\hline 50 & 0.55 & 0.55 & 0.55 & 0.742 & 0.79 & 0.889 & 0.826 & 0.79 & 0.889 & 0.826 \\
\hline & & & & & & & & & & \\
\hline & & & & & & & & & & \\
\hline \multicolumn{2}{|l|}{ Average } & & & & & & 0.823 & & & 0.823 \\
\hline
\end{tabular}

Summary of Results:

\begin{tabular}{rc} 
Normal high side calibraiton factor: & 0.823 \\
variation + & $0.61 \%$ \\
variation -: & $-0.61 \%$ \\
Normal low side calibration factor: & 0.823 \\
variation $+:$ & $0.61 \%$ \\
variation $-:$ & $-0.61 \%$ \\
\hline
\end{tabular}

\section{Certification:}

I certify that the Type S pitot tube, the standard type pitot tube, and the calibration setup meet or exceed all specifications, criteria and/or applicable design features and hereby assign a pitot tube calibration factor $\mathrm{C}_{\mathrm{p}}$ of: $\quad 0.823$

Certified by:

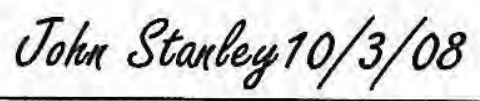

Calibrator (Signature/Date)
Bill Hefley 10/3/08

QA Officer (Signature/Date) 


\section{AIR

CALIBRATION DATA

PITOT TUBE CALIBRATION DATA

Date: $\quad 10 / 06 / 08$

I.D. \# $\frac{\text { ASAI 3-3 }}{0.990}$

Time: $10: 00$

$\mathrm{T}_{\mathrm{s}}: \frac{10: 00}{75}{ }^{\circ} \mathrm{F}$

$\mathrm{Pb}: 29.57 \mathrm{Hg}$

\begin{tabular}{|c|c|c|c|c|c|c|c|c|c|c|}
\hline $\begin{array}{c}\text { fps } \\
\text { Mark }\end{array}$ & $\begin{array}{c}\text { Desired } \\
\text { Calibration } \\
\text { Standard }\end{array}$ & \multicolumn{2}{c|}{$\begin{array}{c}\text { Calibration } \\
\text { Standard } \\
\text { Start }\end{array}$} & $\begin{array}{c}\text { V Standard } \\
\text { Everage }\end{array}$ & High & V High & $\begin{array}{c}\text { Cal. } \\
\text { Factor }\end{array}$ & Low & V Low & $\begin{array}{c}\text { Cal. } \\
\text { Factor }\end{array}$ \\
\hline 20 & 0.09 & 0.09 & 0.09 & 0.300 & 0.13 & 0.361 & 0.824 & 0.13 & 0.361 & 0.824 \\
\hline 30 & 0.20 & 0.20 & 0.20 & 0.447 & 0.29 & 0.539 & 0.822 & 0.29 & 0.539 & 0.822 \\
\hline 40 & 0.36 & 0.36 & 0.36 & 0.600 & 0.52 & 0.721 & 0.824 & 0.52 & 0.721 & 0.824 \\
\hline 50 & 0.56 & 0.56 & 0.56 & 0.748 & 0.81 & 0.900 & 0.823 & 0.81 & 0.900 & 0.823 \\
\hline 60 & 0.80 & 0.80 & 0.80 & 0.894 & 1.15 & 1.072 & 0.826 & 1.15 & 1.072 & 0.826 \\
\hline 70 & 1.09 & 1.10 & 1.10 & 1.049 & 1.60 & 1.265 & 0.821 & 1.60 & 1.265 & 0.821 \\
\hline 80 & 1.43 & 1.40 & 1.40 & 1.183 & 2.00 & 1.414 & 0.828 & 2.00 & 1.414 & 0.828 \\
\hline 90 & 1.81 & 1.80 & 1.80 & 1.342 & 2.60 & 1.612 & 0.824 & 2.60 & 1.612 & 0.824 \\
\hline & & & & & & & & & & \\
\hline & & & & & & & & & & \\
\hline 50 & 0.56 & 0.56 & 0.56 & 0.748 & 0.81 & 0.900 & 0.823 & 0.81 & 0.900 & 0.823 \\
\hline 50 & 0.56 & 0.56 & 0.56 & 0.748 & 0.81 & 0.900 & 0.823 & 0.81 & 0.900 & 0.823 \\
\hline & & & & & & & & & & \\
\hline & & & & & & & & & & \\
\hline \multicolumn{2}{l}{ Average } & & & & & & 0.824 & & & 0.824 \\
\hline
\end{tabular}

Summary of Results:

Normal high side calibraiton factor:

0.824

variation +

variation -:

Normal low side calibration factor:

$0.49 \%$

$-0.36 \%$

variation +

0.824

variation -

$0.49 \%$

$-0.36 \%$

\section{Certification:}

I certify that the Type S pitot tube, the standard type pitot tube, and the calibration setup meet or exceed all specifications, criteria and/or applicable design features and hereby assign a pitot tube calibration factor $\mathrm{C}_{\mathrm{p}}$ of: $\quad 0.824$

Certified by: $\quad$ Tolun Stanley 10/6/08 Calibrator (Signature/Date)
Bill Hefley 10/6/08

QA Officer (Signature/Date) 


\section{AIR

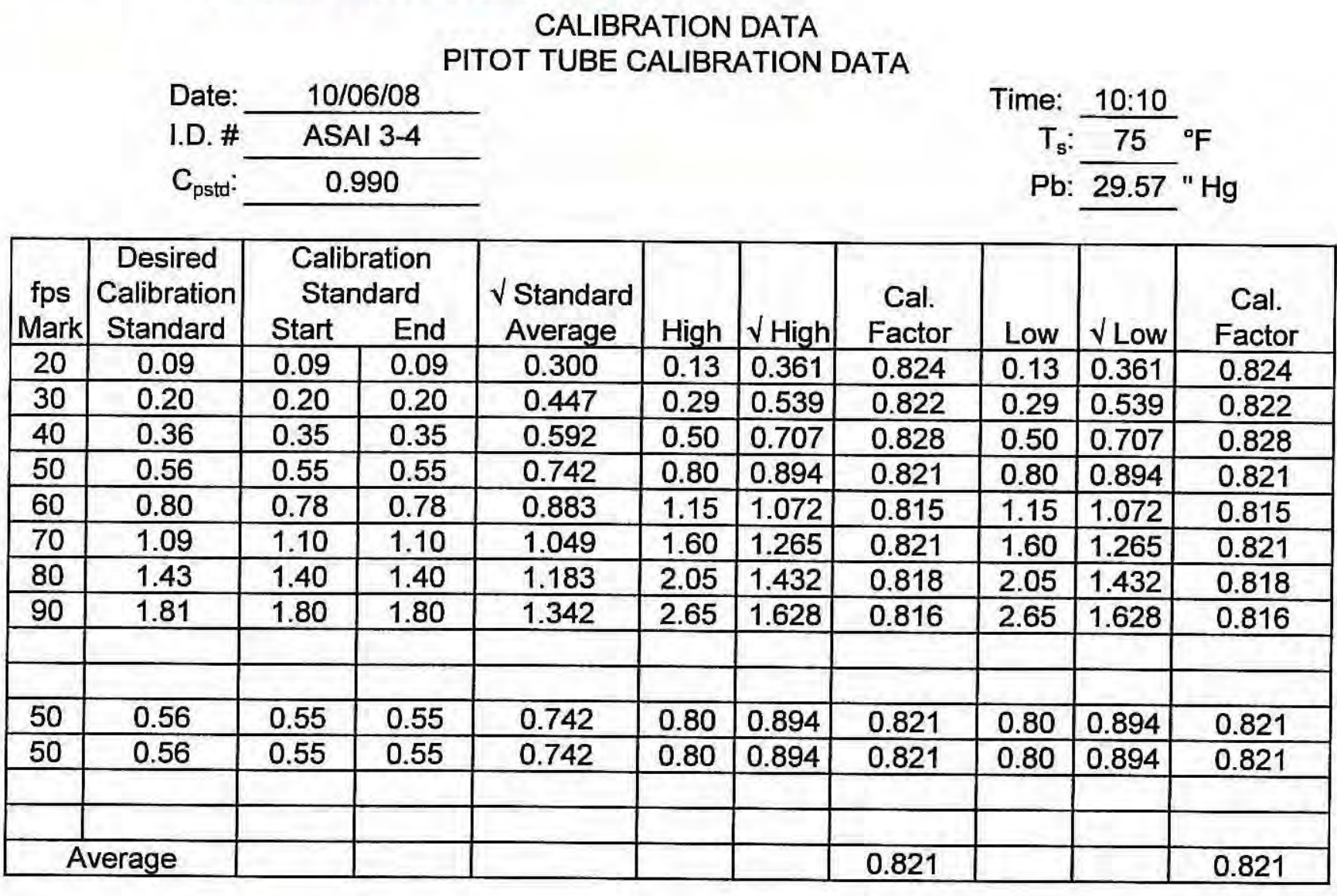

Summary of Results:

$\begin{array}{rc}\text { Normal high side calibraiton factor: } & 0.821 \\ \text { variation } & 0.85 \% \\ \text { variation }-\frac{-0.73 \%}{\text { Normal low side calibration factor: }} & \frac{0.821}{0.85 \%} \\ \text { variation }+\frac{0.73 \%}{\text { variation }-:} & -0.73\end{array}$

Certification:

I certify that the Type S pitot tube, the standard type pitot tube, and the calibration setup meet or exceed all specifications, criteria and/or applicable design features and hereby assign a pitot tube calibration factor $C_{p}$ of: $\quad 0.821$

Certified by: $\quad \frac{\text { Tohn Stanley 10/06/08 }}{\text { Calibrator (Signature/Date) }}$

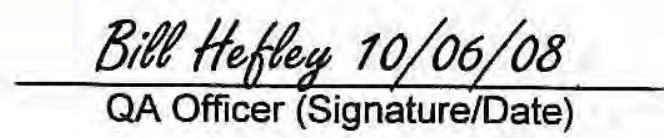


Nozzle Set No.

Date Calibration Performed:

ASAl - 3

$10 / 10 / 08$

$\begin{array}{llllllll}-1 & -2 & -3 & -4 & -5 & -6 & -7 & -8\end{array}$

Measurement 1

$\frac{0.121}{0.122} \frac{0.170}{0.169} \frac{0.233}{0.230} \frac{0.316}{0.317} \frac{0.364}{0.364} \frac{0.397}{0.396} \frac{0.497}{0.496} \frac{0.231}{0.234}$

Measurement 2

Measurement 3

Measurement 4

Measurement 5

Measurement 6

Measurement 7

Measurement 8

Measurement 9

Measurement 10

Average

\begin{tabular}{|c|c|c|c|c|c|c|c|}
\hline 0.122 & 0.169 & & 0.317 & 0.364 & 0.396 & 0.496 & 0.234 \\
\hline & & & & & & & \\
\hline & & & & & & & \\
\hline & & & & & & & .231 \\
\hline & & & & & & & 231 \\
\hline 0.12 & & & & & & & \\
\hline 120 & & & & & & & 0.231 \\
\hline & 0.169 & & & & & & 0.230 \\
\hline 0.122 & 0.170 & 0.232 & 0.317 & & & & \\
\hline 0.122 & 0.169 & 0.232 & 0.318 & 0.365 & 0.397 & 0.497 & 0.232 \\
\hline
\end{tabular}

Patrich Selakovich 10/10/08

Calibrator (Signature / Date)

Bill Hefley 10/10/08

QA Officer (Signature / Date) 


\section{Calibration Data}

Nozzle Calibration

Nozzle Set No.

Date Calibration Performed:

ASAl - 7

$10 / 10 / 08$

\begin{tabular}{|c|c|c|c|c|c|c|c|c|}
\hline & -1 & -2 & -3 & -4 & -5 & -6 & -7 & -8 \\
\hline Measurement 1 & 0.113 & 0.190 & 0.258 & 0.306 & 0.377 & 0.424 & 0.486 & 0.257 \\
\hline Measurement 2 & 0.114 & 0.193 & 0.258 & 0.308 & 0.376 & 0.425 & 0.488 & 0.260 \\
\hline Measurement 3 & 0.114 & 0.193 & 0.257 & 0.307 & 0.375 & 0.427 & 0.486 & 0.259 \\
\hline Measurement 4 & 0.115 & 0.193 & 0.257 & 0.308 & 0.377 & 0.427 & 0.488 & 0.259 \\
\hline Measurement 5 & 0.114 & 0.191 & 0.258 & 0.309 & 0.377 & 0.426 & 0.486 & 0.259 \\
\hline Measurement 6 & 0.114 & 0.191 & 0.255 & 0.308 & 0.377 & 0.425 & 0.487 & 0.259 \\
\hline Measurement 7 & 0.115 & 0.192 & 0.258 & 0.309 & 0.378 & 0.425 & 0.488 & 0.259 \\
\hline Measurement 8 & 0.114 & 0.191 & 0.257 & 0.308 & 0.377 & 0.426 & 0.487 & 0.258 \\
\hline Measurement 9 & 0.115 & 0.193 & 0.258 & 0.309 & 0.377 & 0.426 & 0.486 & 0.257 \\
\hline Measurement 10 & 0.115 & 0.190 & 0.258 & 0.307 & 0.377 & 0.424 & 0.487 & 0.259 \\
\hline Average & 0.114 & 0.192 & 0.257 & 0.308 & 0.377 & 0.426 & 0.487 & 0.259 \\
\hline
\end{tabular}

Patrick Selatovick 10/10/08

Calibrator (Signature / Date)

Bill Hefley 10/10/08

QA Officer (Signature / Date) 
Calibration Data

Nozzle Calibration

Nozzle Set No.

Date Calibration Performed:

ASAI 08-026

$09 / 30 / 08$

\begin{tabular}{|c|c|c|c|c|c|c|c|}
\hline & -1 & -2 & -3 & -4 & -5 & -6 & -7 \\
\hline Measurement 1 & 0.156 & 0.160 & 0.131 & & & & \\
\hline Measurement 2 & 0.156 & 0.161 & 0.132 & & & & \\
\hline Measurement 3 & 0.155 & 0.160 & 0.130 & & & & \\
\hline Measurement 4 & 0.155 & 0.162 & 0.131 & & & & \\
\hline Measurement 5 & 0.156 & 0.160 & 0.132 & & & & \\
\hline Measurement 6 & 0.155 & 0.162 & 0.131 & & & & \\
\hline Measurement 7 & 0.155 & 0.162 & 0.131 & & & & \\
\hline Measurement 8 & 0.155 & 0.160 & 0.132 & & & & \\
\hline Measurement 9 & 0.155 & 0.161 & 0.130 & & & & \\
\hline Measurement 10 & 0.156 & 0.162 & 0.131 & & & & \\
\hline Average & 0.155 & 0.161 & 0.131 & & \#\#\#\# & \#\#\#\# & \#\#\#\# \\
\hline
\end{tabular}

Certified by:

$\frac{\text { Jotur Stanley 9-30-08 }}{\text { Calibrator (Signature I Date) }}$
$\frac{\text { Bill Hefley 9-30-08 }}{\text { QA Officer (Signature I Date) }}$


Appendix D:

Field Data 


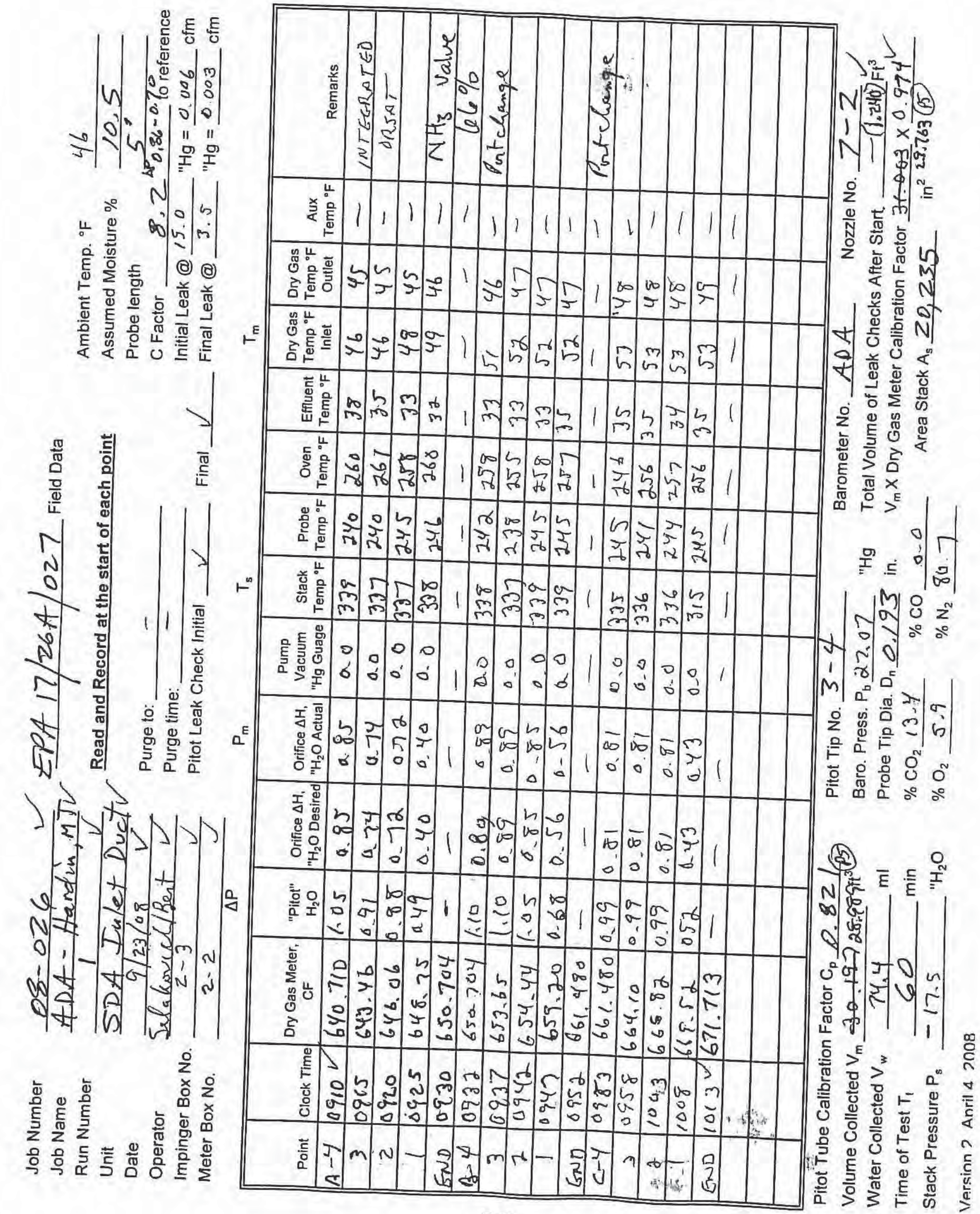


Impinger Bpx No. $2-3$

Impinger 1 Final Weight 786.6

Initial Weight
Increase $\frac{735.5}{56.2}$

Impinger 2 Final Weight 758.3 Initial Weight 749.9 Increase 8.4

Water Weight Gain

$\begin{array}{ll}\text { Impinger } 3 & \text { Final Weight } \\ & \begin{array}{l}\text { Initial Weight } \\ \text { Increase }\end{array}\end{array}$

Impinger 4 Final Weight 753.4 Initial Weight $\frac{752,7}{17}$
Increase

Impinger 5 Final Weight 635.3 Initial Weight 634.7 Increase ab

Impinger 6

Final Weight 978.6 Initial Weight 967,2 Increase 17,4

Impinger $7 \quad$ Final Weight Initial Weight Increase

Impinger $8 \quad$ Final Weight Initial Weight Increase

Moisture

Content:

$$
\begin{aligned}
& \% \mathrm{M}=11.4120 \\
& M_{0}=0 \begin{array}{l}
0.8859 \mathrm{~L} / \\
0.8900
\end{array} \\
& \mathrm{MW}_{\mathrm{d}}=30.380^{\circ}
\end{aligned}
$$

Impinger $1 S L /$ Impinger 28.4 Impinger 32,2

Impinger 40.7

Impinger 5 ‥

Impinger 611.4

Impinger 7

Impinger 8

Filter No. THIMACG 58 Total $\quad \underline{74.4^{\nu}}=v_{w}$<smiles>CC[14C][Te]=P</smiles>
$V_{m}=30.19728 .989$

$$
\begin{aligned}
\% \mathrm{CO}_{2} & =13.4 V \\
\% \mathrm{O}_{2} & =\frac{5.9 V}{0.0 V} \\
\% \mathrm{CO} & =0.7 V \\
\% \mathrm{~N}_{2} & =80.7 V \\
\mathrm{~A}_{\mathrm{s}} & =20.235 \\
\mathrm{D}_{\mathrm{n}} & =0.193 \mathrm{~V} \\
\mathrm{~T}_{1} & =60 \mathrm{~V}
\end{aligned}
$$$$
P_{m}=0,73 \Delta \nu
$$$$
C_{p}=0.82 / 2
$$$$
\mathrm{P}_{\mathrm{s}}=-17,5 \mathrm{H} \mathrm{H}_{2} \mathrm{O}
$$$$
\mathrm{T}_{\mathrm{m}}=49 \precsim{ }^{\circ} \mathrm{F}
$$$$
T_{s}=3 \sqrt{6 V}{ }^{\circ} \mathrm{F}
$$

$V m_{s t d}=17.65 \times V m\left[\frac{P_{b}+\frac{P_{m}}{13.6}}{T_{m}+460}\right]=17.65 \times 30.197$

$$
\left[\frac{27.07+\frac{0.730}{13.6}}{49+460}\right]=\frac{\frac{27.265}{28.407}}{\frac{8.497}{0.45465}} \mathrm{stt}^{\mathrm{scfm}}
$$

$V_{w_{\text {gas }}}=0.0472 \times V w=0.0472 \times$ $3.512^{2}$

$\%$ Moisture $=\frac{V_{w_{\text {gas }}}}{V m_{\text {std }}+V_{w_{\text {gas }}}} \times 100=$<smiles>CCCCCCCCCCC</smiles>

$V_{s}=5123.8 \times \frac{0.821}{\frac{796}{25.78 \times 79.02}} \times 0.939=4874 \mathrm{fpm}$ $\% 1=\frac{1039 \times 8.265 \times 79628.97}{0.8900 \times 2578 \times 4044 \times 60 \times(0193)^{2}}$

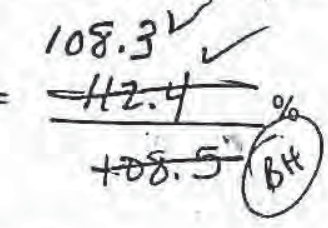

D-3
573,037

ACFM: 572,543 SCFM: $292273291213 \mathrm{~V}$

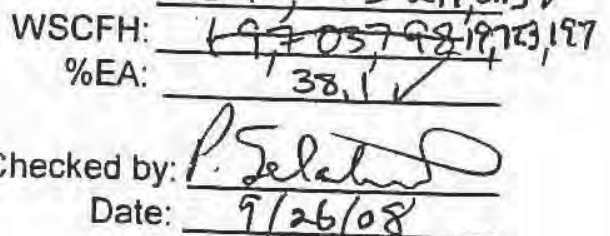




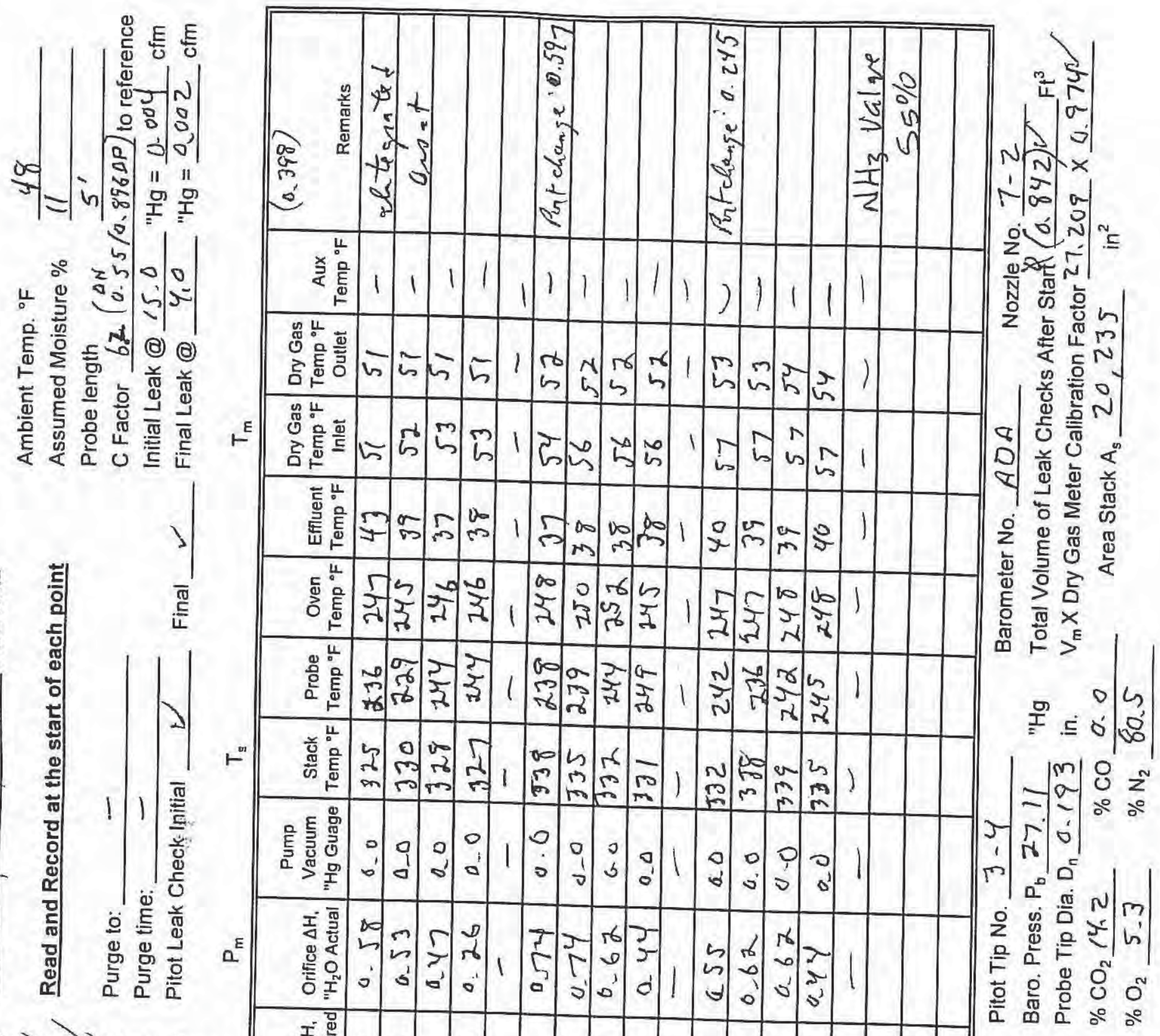

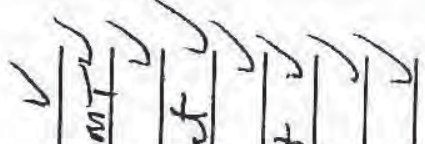

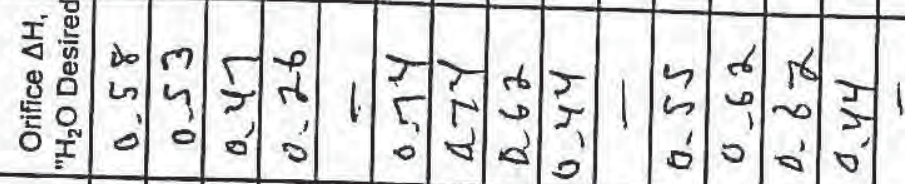

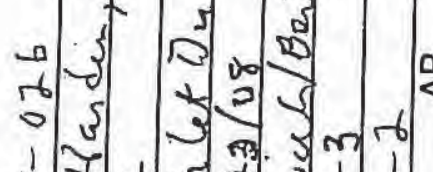

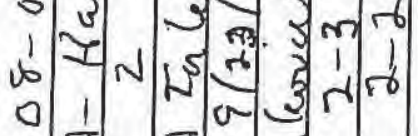

$\frac{0}{4}$

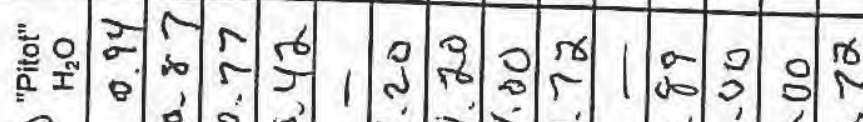

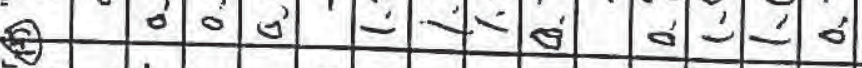

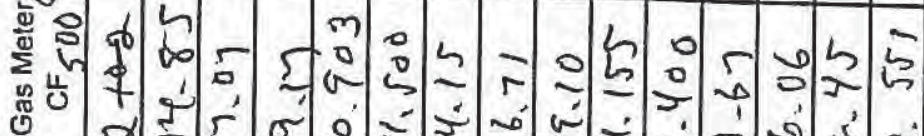

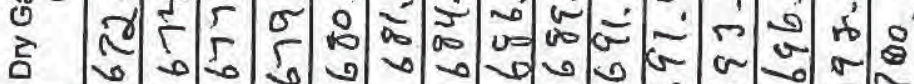

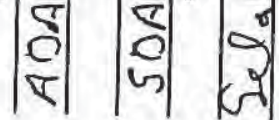

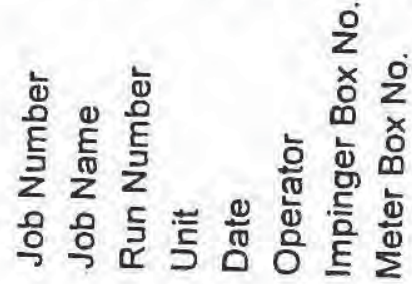

- 0 - $\rightarrow$ ?

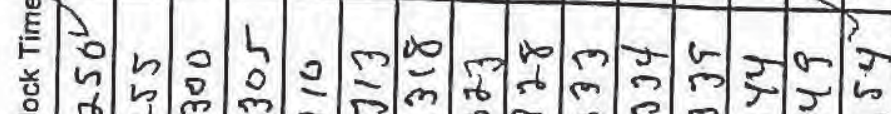

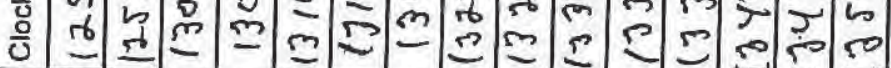

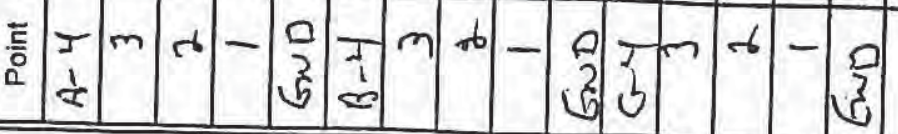

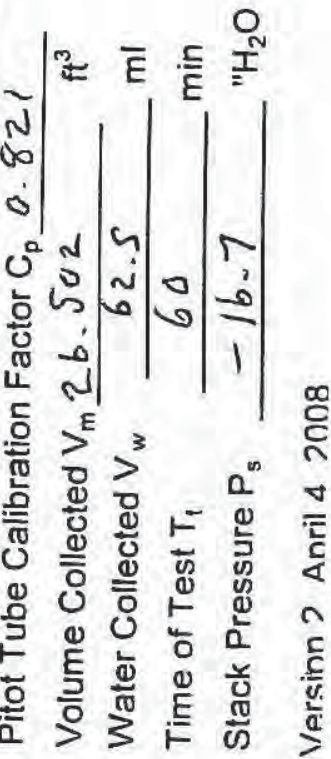


Impinger Bpx No. $2-3$

\begin{tabular}{|c|c|c|}
\hline Impinger 1 & Final Weight & 792.8 \\
\hline & Initial Weight & 745.3 \\
\hline & Increase & 47.5 \\
\hline Impinger 2 & Final Weight & 765.0 \\
\hline & Initial Weight & 758.4 \\
\hline & Increase & 6.6 \\
\hline
\end{tabular}

Impinger 3 Final Weight 745.5

Initial Weight 743.9

Increase

$\frac{743.9}{1.6}$

Impinger $4 \quad$ Final Weight $76 \% .4$

Initial Weight 763.2

Increase 1.2

Impinger 5

Final Weight
$\begin{aligned} & \text { Initial Weight } \\ & \text { Increase }\end{aligned} \frac{635.4}{635.3}$

Impinger 6

Final Weight

Initial Weight

Increase

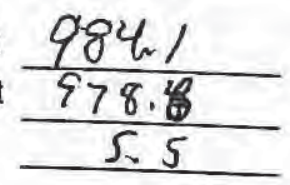

Impinger 7

Final Weight

Initial Weight

Increase

Impinger 8

Final Weight

Initial Weight

Increase
Filter No. Thunble

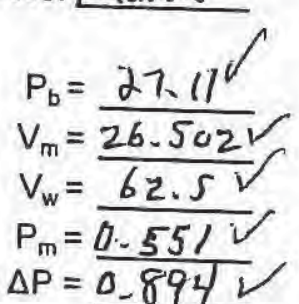

Avg $\triangle P=0.894 \mathrm{~V}$

Avg $\sqrt{\Delta \mathrm{P}}=0.938 \mathrm{~V}$

$C_{p}=\frac{0.821 \mathrm{~V}}{-1620}$

$P_{s}=-16,70^{2} " \mathrm{H}_{2} \mathrm{O}$

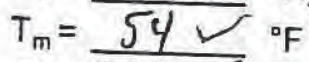

$T_{s}=333 \checkmark{ }^{\circ} \mathrm{F}$
Water Weight Gain

Impinger 147,5

Impinger $2 \quad 6.6$

Impinger 31.6

Impinger $4 l_{2} 2$

Impinger 5 0. I

Impinger 6 . 5

Impinger 7

Impinger 8

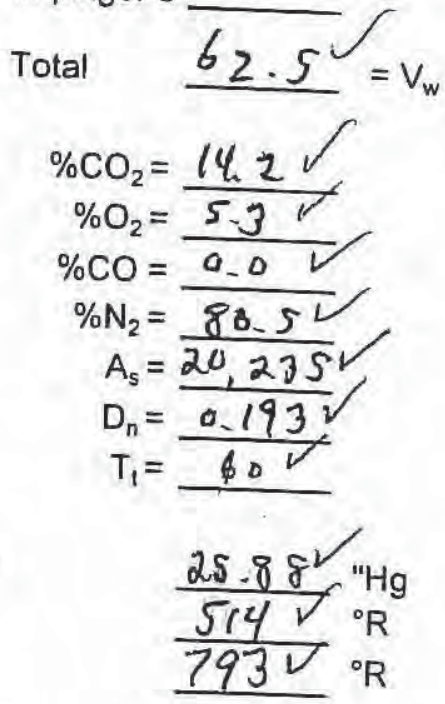

Moisture

Content:

$$
\begin{aligned}
& \% M=10.67 \\
& {\left[\frac{P_{b}+\frac{P_{m}}{13.6}}{T_{m}+460}\right]=17.65 \times 26.5 \mathrm{az}}
\end{aligned}
$$

$\mathrm{MW}_{\mathrm{d}}=30.484^{\curvearrowright}$

$\mathrm{MW}=29.25$

$V m_{\text {std }}=17.65 \times V m\left[\frac{P_{b}+\frac{P_{m}}{13.6}}{T_{m}+460}\right]=17.65 \times 26.5 \mathrm{aZ}$

$\left[\frac{27.11+\frac{0.551}{13.6}}{54+460}\right]=\frac{24.708^{\nu}}{0.412 V} \mathrm{sft}^{\mathrm{s}}$

$V w_{\text {gas }}=0.0472 \times V w=0.0472 \times 62.5$

$=$

$2.950^{\circ}$

$\mathrm{sft}^{3}$

$\%$ Moisture $=\frac{V w_{\text {gas }}}{V m_{\text {std }}+V w_{\text {gas }}} \times 100=\frac{2.950}{24.708+2.950} \times 100=10.67^{\sqrt{2}} \%$

$V_{s}=5123.8 \times 0.821 \sqrt{\frac{793}{25.88 \times 29.75}} \times 0.938=4046 \mathrm{fpm}$

$\% 1=\frac{1039 \times 24.708 \times 793}{98933 \times 25.88 \times 41046 \times 60 \times(0.193)^{2}}=97.4^{2} \%$

ACFM: 568,479

SCFM: $293,577 \mathrm{~V}$

WSCFH: $\frac{19,718,594}{33.1}$

Checked by: Pselats

Date: $9 / 26 / 08$ 


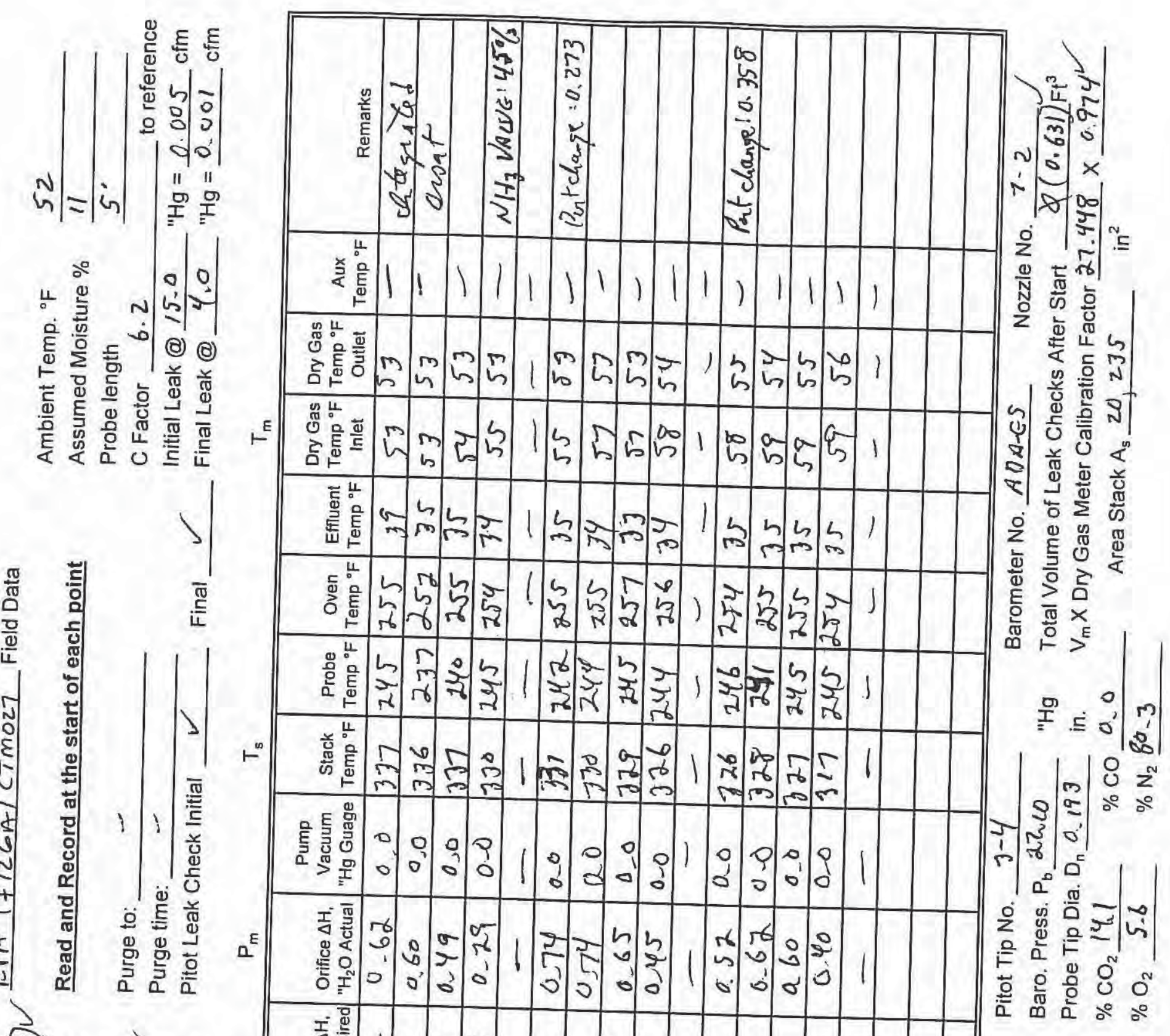

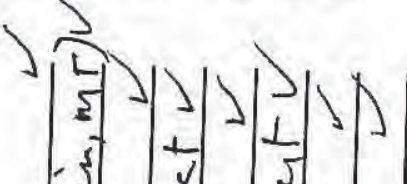

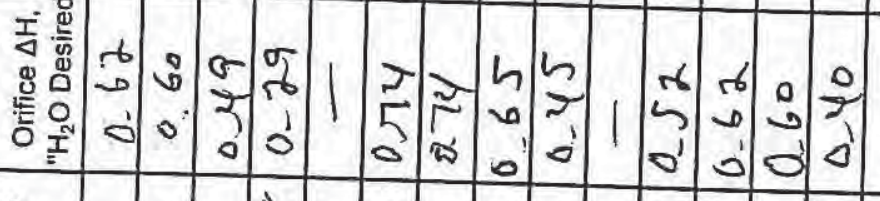

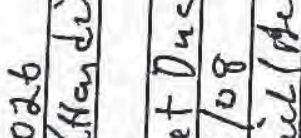

0 उका

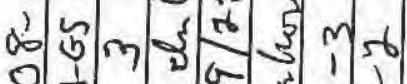

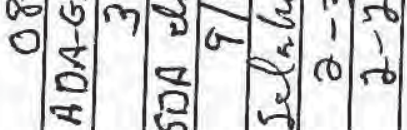

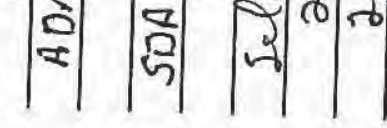

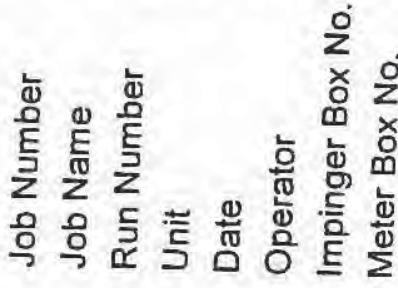

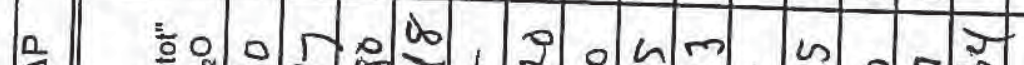

\&

(2)

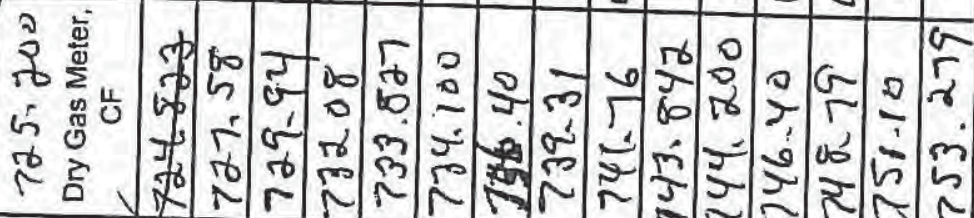

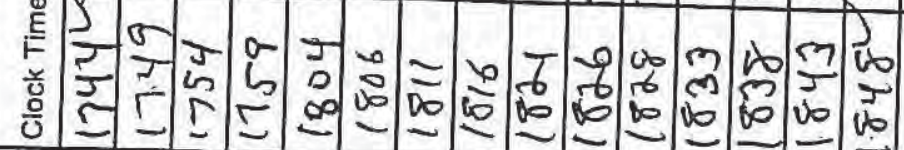

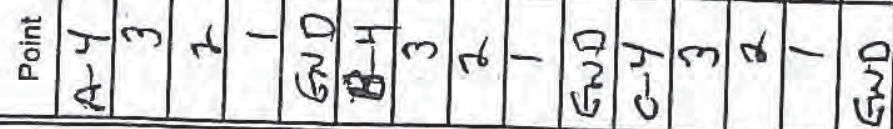

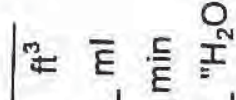

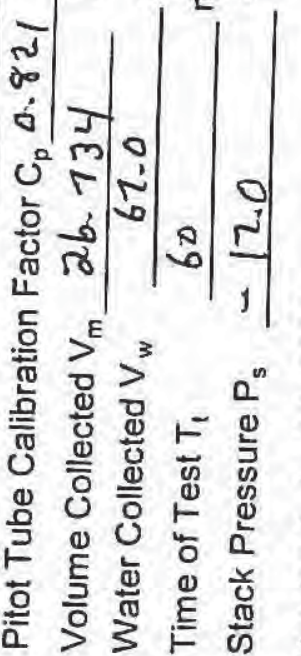

$\stackrel{\infty}{\circ}$

N

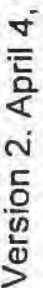


Impinger $\mathrm{Bpx}$ No

$2-3$

\begin{tabular}{|c|c|c|}
\hline \multirow[t]{2}{*}{ Impinger 1} & Final Weight & -798.5 \\
\hline & $\begin{array}{l}\text { Initial Weight } \\
\text { Increase }\end{array}$ & $\begin{array}{r}746.3 \\
52.2\end{array}$ \\
\hline \multirow[t]{2}{*}{ Impinger 2} & Final Weight & 768.6 \\
\hline & $\begin{array}{l}\text { Initial Weight } \\
\text { Increase }\end{array}$ & $\frac{762.7}{5.9}$ \\
\hline \multirow[t]{3}{*}{ Impinger 3} & Final Weight & .754 .2 \\
\hline & Initial Weight & 752.4 \\
\hline & Increase & 1.8 \\
\hline \multirow[t]{3}{*}{ Impinger 4} & Final Weight & 754.8 \\
\hline & Initial Weight & 753.7 \\
\hline & Increase & $1-1$ \\
\hline \multirow[t]{3}{*}{ Impinger 5} & Final Weight & 635.9 \\
\hline & Initial Weight & 635.4 \\
\hline & Increase & 0.5 \\
\hline
\end{tabular}

Impinger $6 \quad$ Final Weight Initial Weight Increase

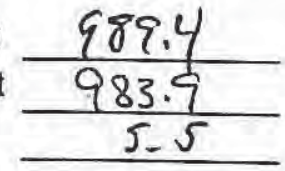

Impinger 7

Impinger 8
Final Weight Initial Weight Increase

Final Weight Initial Weight Increase
Thimble

Filter No

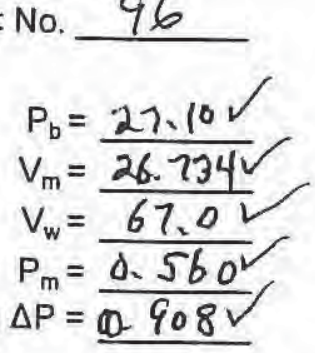

Avg $\triangle P=0.908 \mathrm{~V}$

Avg $\sqrt{\triangle \mathrm{P}}=0-946$

$C_{p}=0.8211$

$P_{s}=-17.02 / 1 \mathrm{H}_{2} \mathrm{O}$

$\mathrm{T}_{\mathrm{m}}=5 \mathrm{SV}{ }^{\circ} \mathrm{F}$

$\mathrm{T}_{\mathrm{s}}=334 \mathrm{~V}{ }^{\circ} \mathrm{F}$
Water Weight Gain

Impinger 152.2

Impinger $2 \quad 5.9$

Impinger $3 \quad L 28$

Impinger 4 hl

Impinger 5 a $S$

Impinger 6 5. 5

Impinger 7

Impinger 8

Total $\underline{67.0 \mathrm{~V}}=\mathrm{V}_{\mathrm{w}}$

$$
\begin{aligned}
\% \mathrm{CO}_{2} & =\frac{|4| V}{5.6 V} \\
\% \mathrm{O}_{2} & =\frac{14}{0.0}
\end{aligned}
$$$$
\% \mathrm{CO}=0.0
$$$$
\% \mathrm{~N}_{2}=80.3 \mathrm{~L}
$$$$
A_{s}=20,235 \mathrm{~V}
$$$$
D_{n}=0.193 \mathrm{~V}
$$$$
T_{1}=60
$$

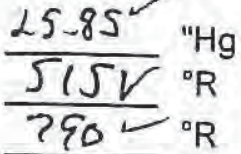

Moisture

Content:

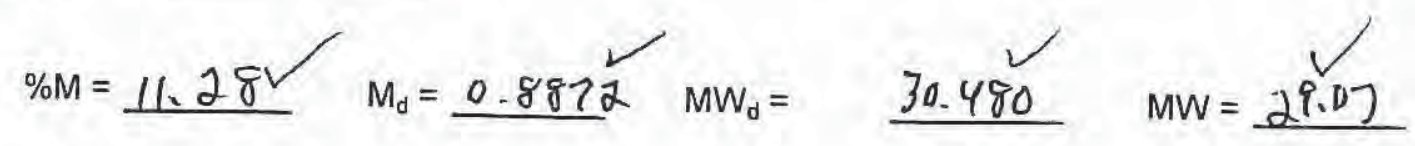

$V m_{\text {std }}=17.65 \times V m\left[\frac{P_{b}+\frac{P_{m}}{13.6}}{T_{m}+460}\right]=17.65 \times 26.734$

$\left[\frac{27.10+\frac{0.560}{13.6}}{s s+460}\right]=\frac{24.867^{\nu}}{0.414} \mathrm{sft}^{5}$

$V_{w_{\text {gas }}}=0.0472 \times V_{w}=0.0472 \times$

67.0 $=3.162$ $\mathrm{sft}^{3}$

$\%$ Moisture $=\frac{V w_{\text {gas }}}{V m_{\text {std }}+V w_{\text {gas }}} \times 100=\frac{3.162}{24.867+3.162} \times 100=11.28^{\prime} \%$

$V_{s}=5123.8 \times 0.821 \sqrt{\frac{790}{25.85 \times 29.07}} \times \underline{0.946}=4080^{\nu} \mathrm{fpm}$

$\% 1=\frac{1039 \times 24.867 \times 990}{4.8872 \times 25.85 \times 4080 \times 60 \times(0.193)^{2}}=97.6^{2} \%$

ACFM

$573,361 \mathrm{~V}$

SCFM: $294,797 /$

WSCFH: $19,936,677^{2}$

$\%$ EA:

35.7

Checked by: Eelats

Date: 


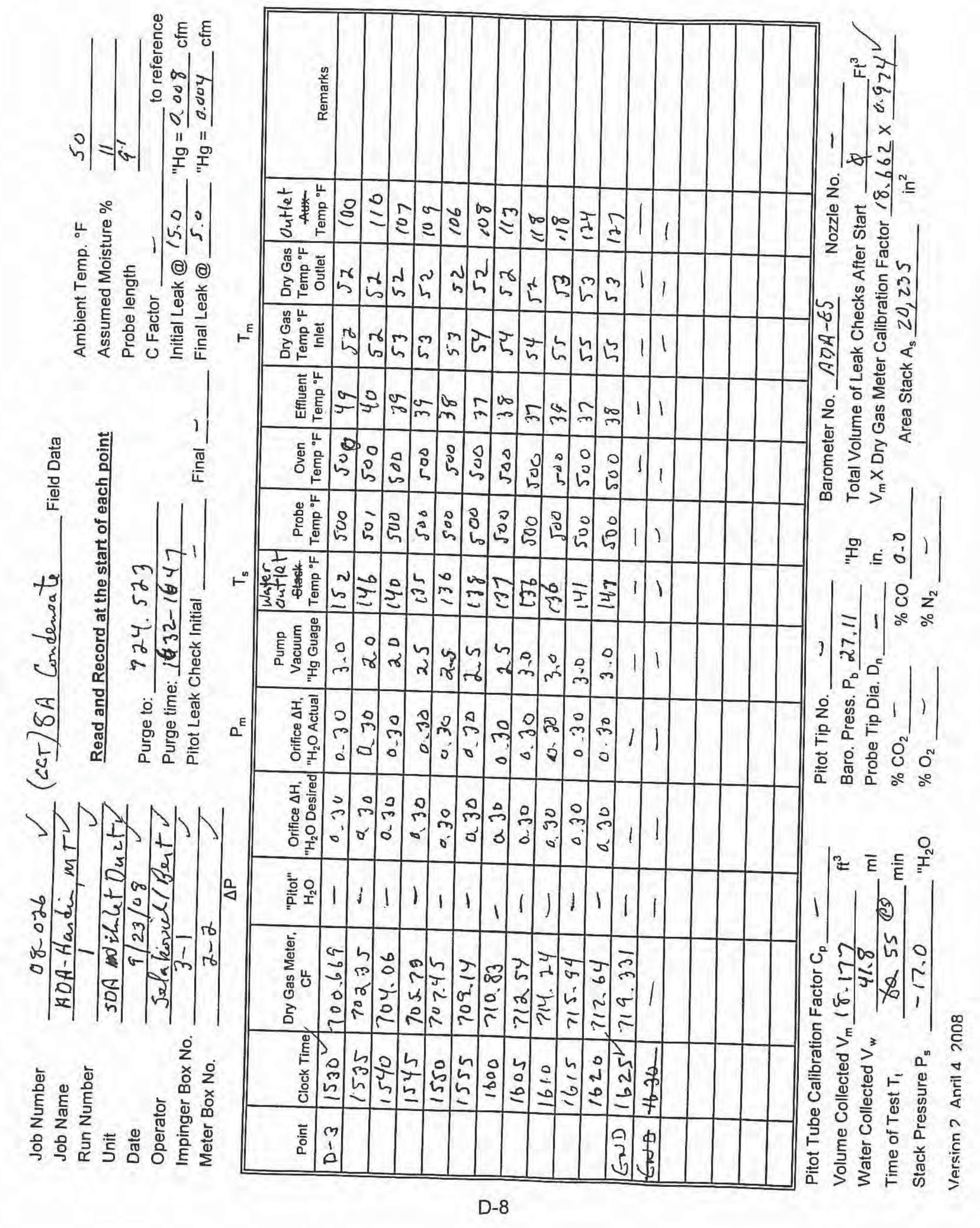


Impinger Bpx No. $3-1 \quad(C \subset T-1)$

Water Weight Gain

Impinger 1 Final Weight 754.9

Initial Weight $\frac{719.8}{35.1}$
Increase

Impinger 1

Impinger 2

$\begin{array}{ll}\text { Impinger } 2 & \text { Final Weight } \\ & \text { Initial Weight } \\ & \text { Increase }\end{array}$

Impinger 3

Impinger 4

Impinger 3 Final Weight 627.6

Initial Weight
Increase $\frac{626.8}{0.8}$

Impinger 5

Impinger 6

\begin{tabular}{ll} 
Impinger 4 & $\begin{array}{l}\text { Final Weight } \\
\text { Initial Weight } \\
\text { Increase }\end{array} \frac{942.3}{937.5}$ \\
\hline
\end{tabular}

Impinger $5 \quad$ Final Weight

Initial Weight Increase

Filter No.

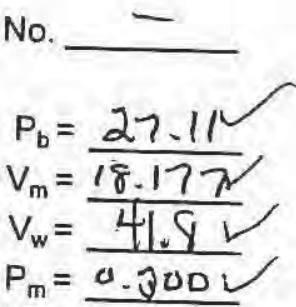

Avg $\triangle P=$

-

Impinger 7

Impinger 8

Total $41.8=v_{w}$

Impinger $6 \quad$ Final Weight

Initial Weight

Increase

Final Weight

Initial Weight

Increase

Avg $\sqrt{\Delta \mathrm{P}}$

$$
\begin{aligned}
& \sqrt{\Delta \mathrm{P}}=- \\
& \mathrm{C}_{\mathrm{p}}=- \\
& \mathrm{P}_{\mathrm{s}}=-17.0-{ }^{\mathrm{N}} \mathrm{H}_{2} \mathrm{O} \\
& \mathrm{T}_{\mathrm{m}}={ }^{\circ} \mathrm{F} \\
& \mathrm{T}_{\mathrm{s}}=--{ }^{\circ} \mathrm{F}
\end{aligned}
$$

$\begin{aligned} \% \mathrm{CO}_{2} & =-- \\ \% \mathrm{O}_{2} & =-- \\ \% \mathrm{CO} & =-- \\ \% \mathrm{~N}_{2} & =-\end{aligned}$

$A_{s}=20,235$

$D_{n}=$

$T_{1}=55$.

Impinger $8 \quad$ Final Weight

Initial Weight

Increase

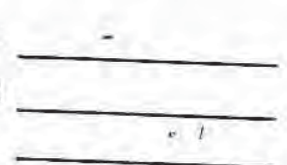

Moisture

Content:
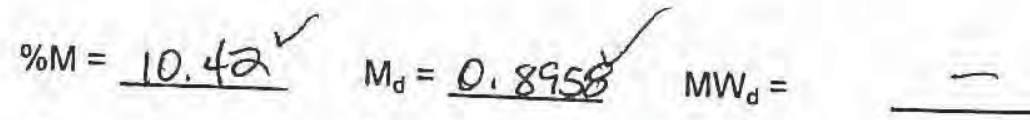

$\mathrm{MW}=$

$V m_{\text {std }}=17.65 \times V m\left[\frac{P_{b}+\frac{P_{m}}{13.6}}{T_{m}+460}\right]=17.65 \times 18.177$

$\left[\frac{27.11+\frac{0.300}{13.6}}{53+460}\right]=\frac{16.968}{\frac{0.309}{\mathrm{scfm}}} \mathrm{st}^{\mathrm{s}}$

$V w_{\text {gas }}=0.0472 \times V w=0.0472 \times 41.8$ $=1.973$ $\mathrm{sft}^{3}$

$\%$ Moisture $=\frac{V w_{\text {gas }}}{V m_{\text {std }}+V w_{\text {gas }}} \times 100=$

$\frac{1.973}{16.968+1.973} \times 100=10.42 \%$

$V_{5}=5123.8 \times$ $x-\sqrt{\frac{16}{x}} x$

$\% 1=1039 x$
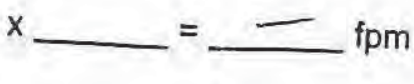

ACFM
SCFM
WSCFH

$x$

$\mathrm{x}$

$=-\%$

Checked by:

Date:

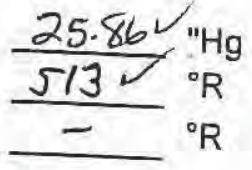

${ }^{\circ} \mathrm{R}$ 


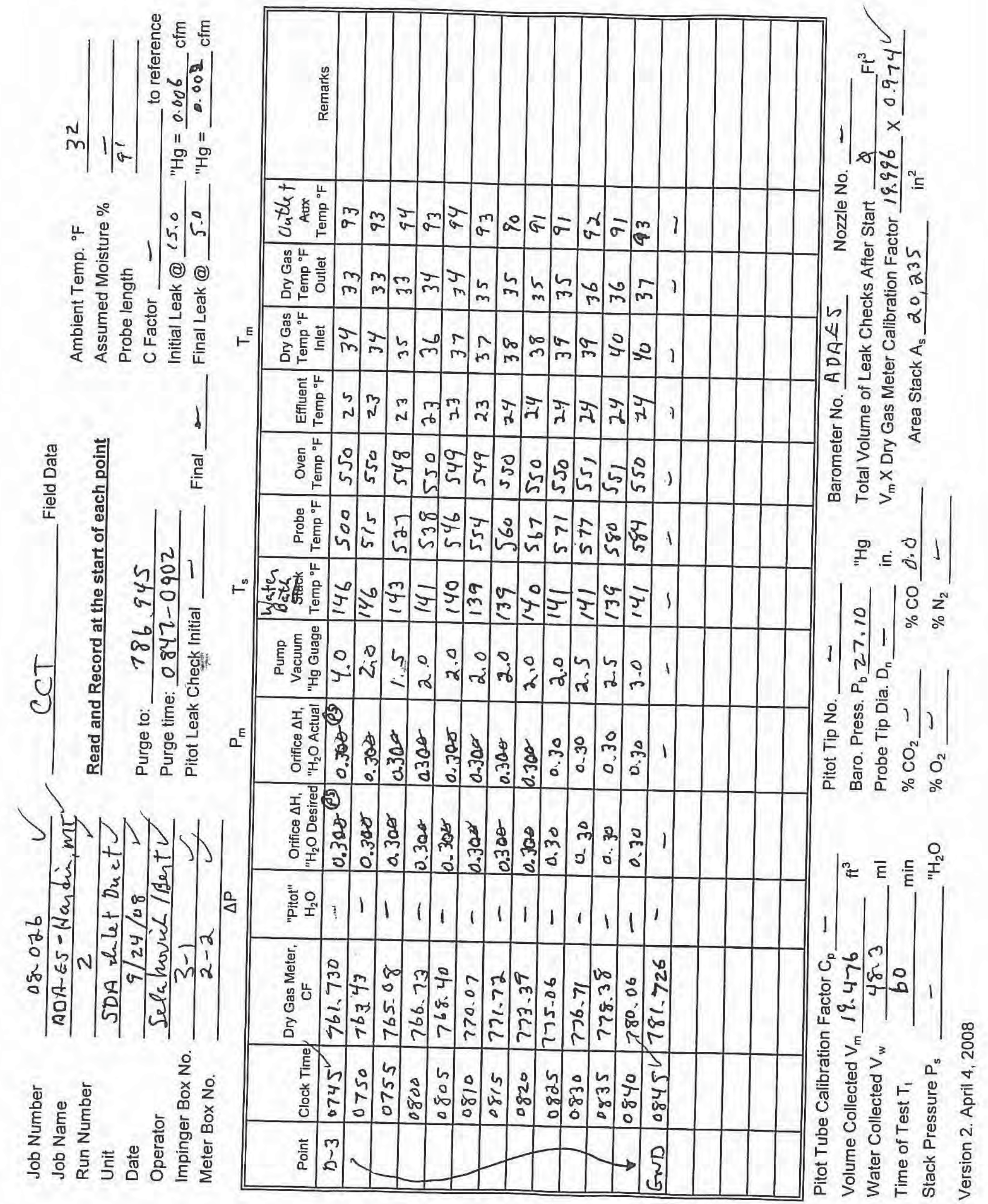




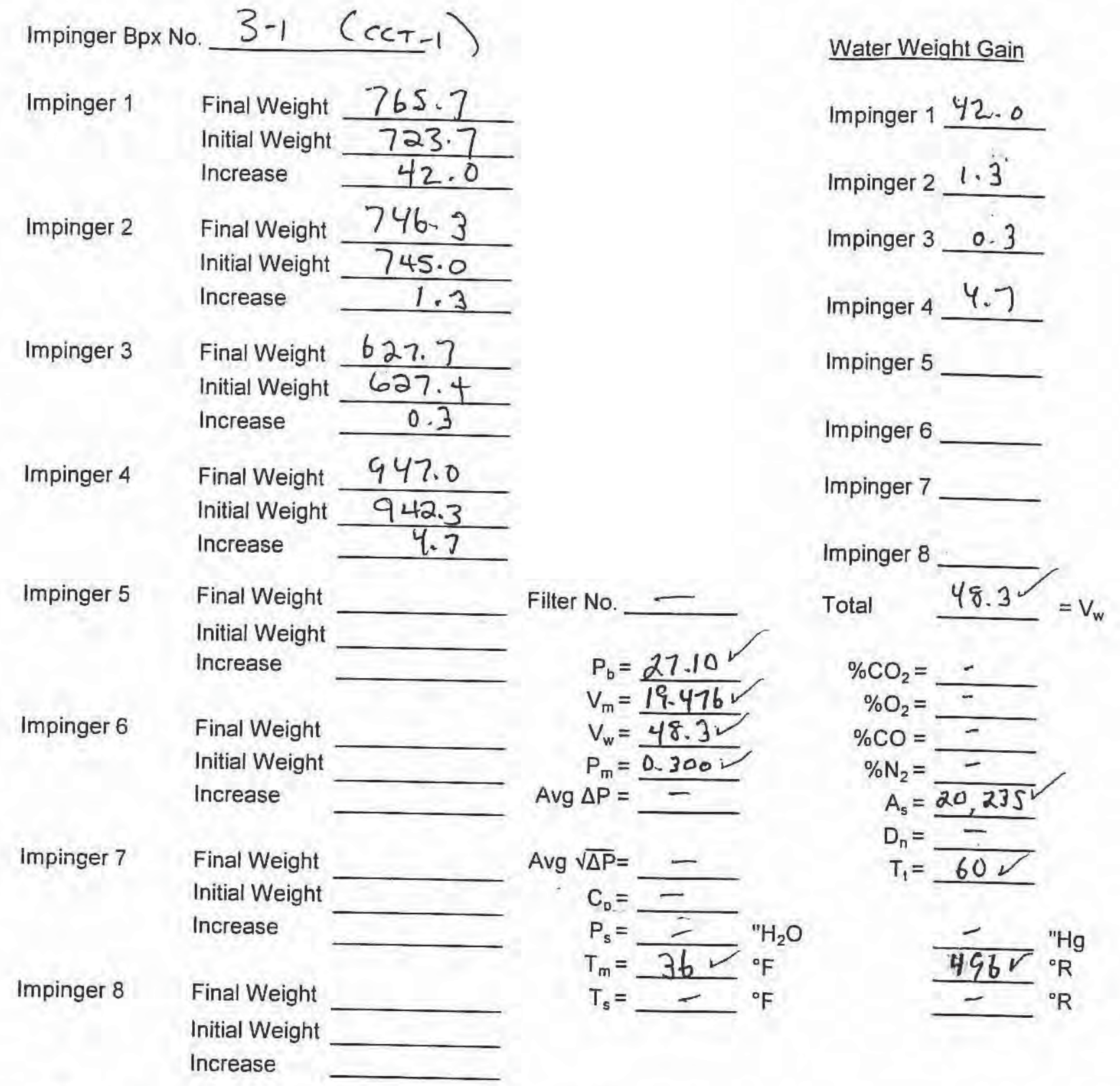

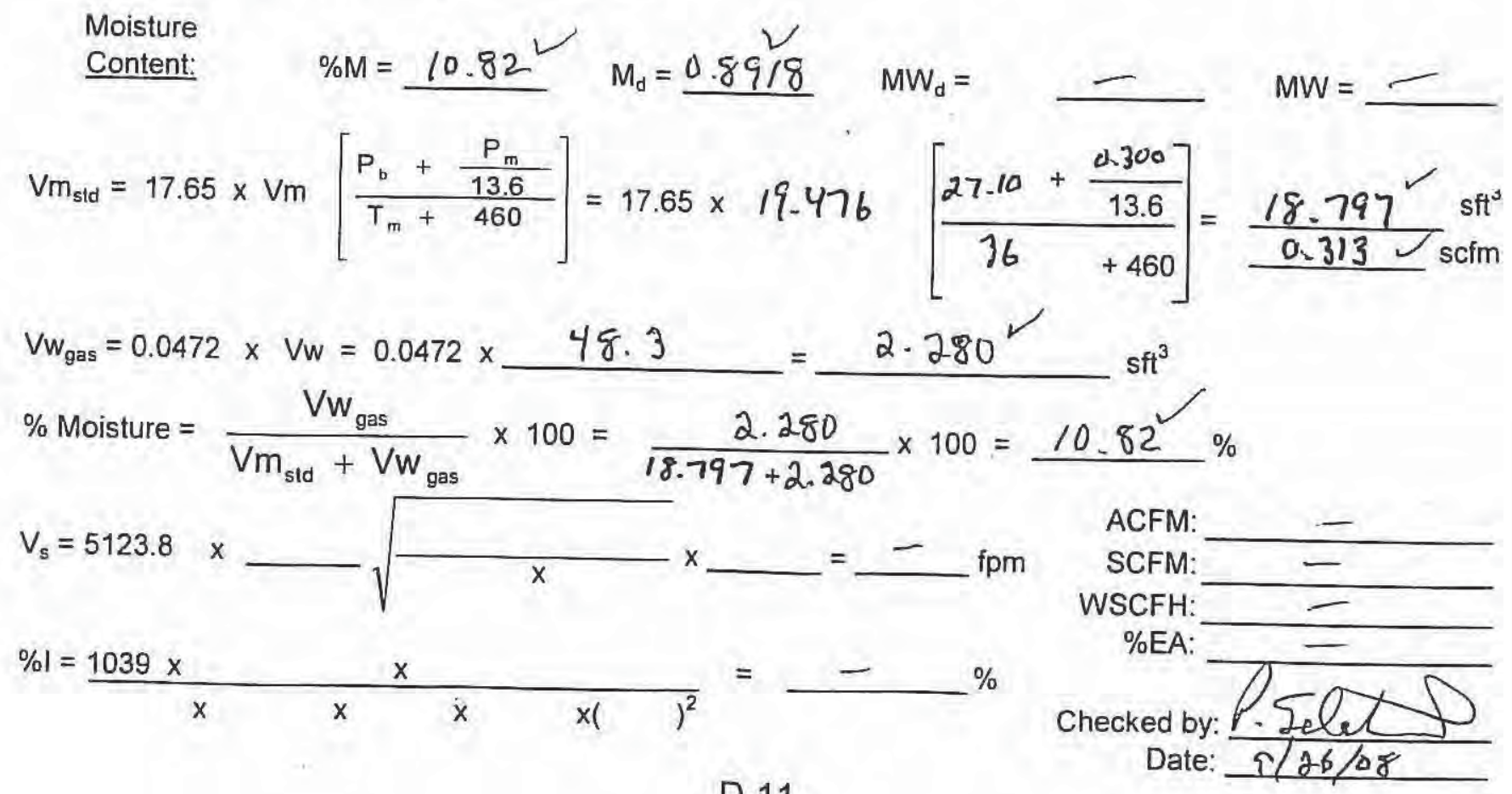



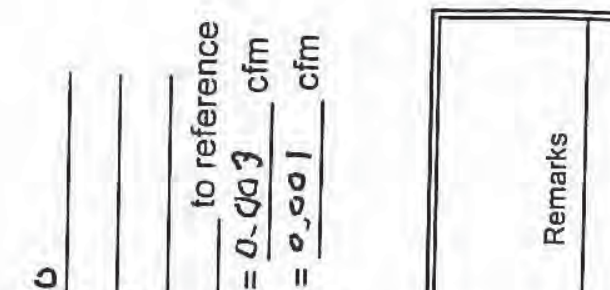

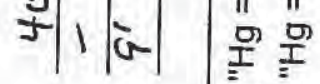

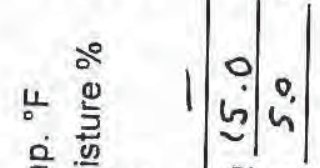

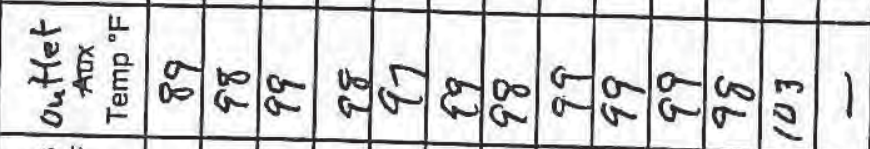

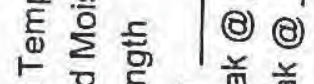

年

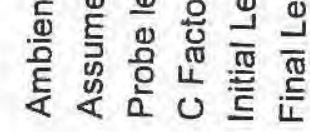

t

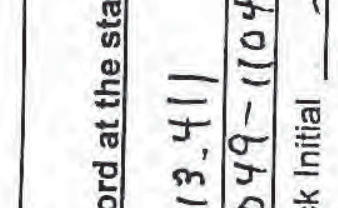

u

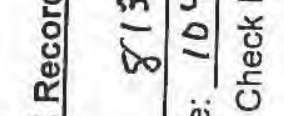

$\because \ddot{0}$

으 है

वृ.

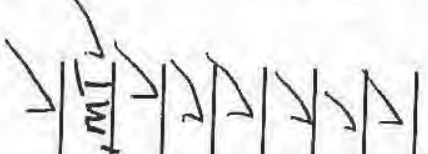

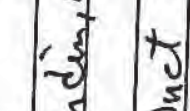

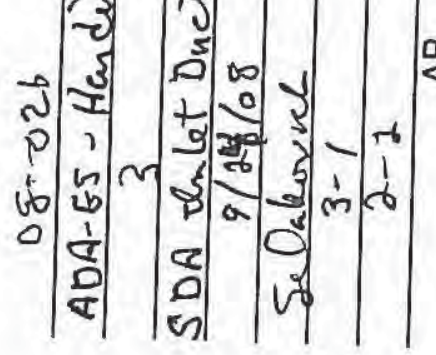

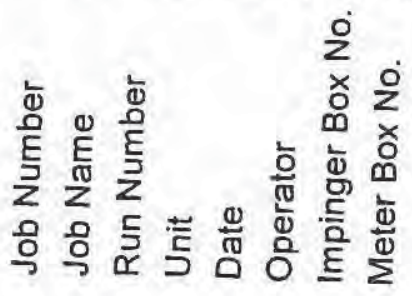

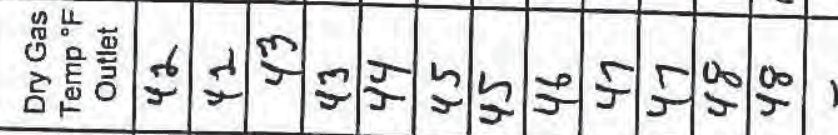

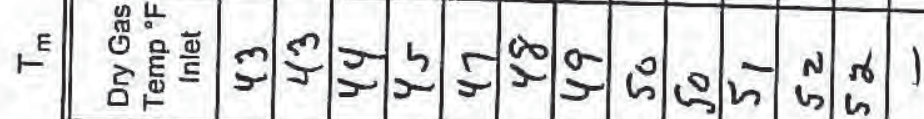

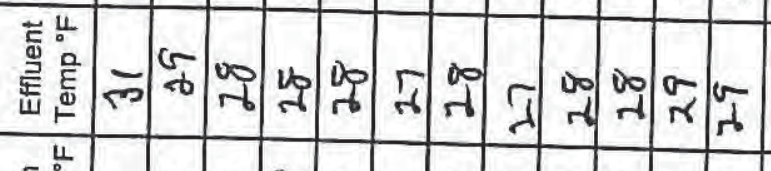

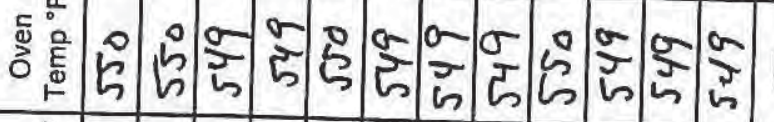

두을

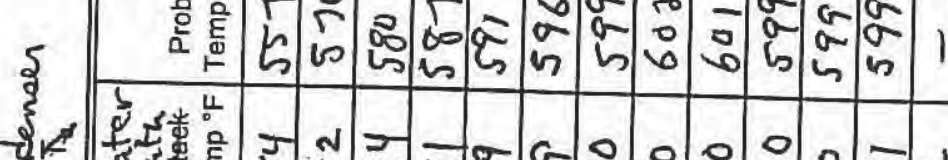

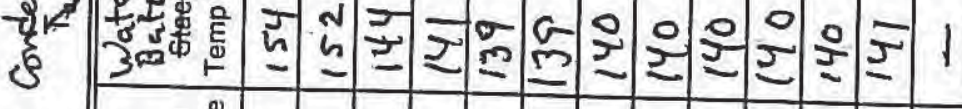

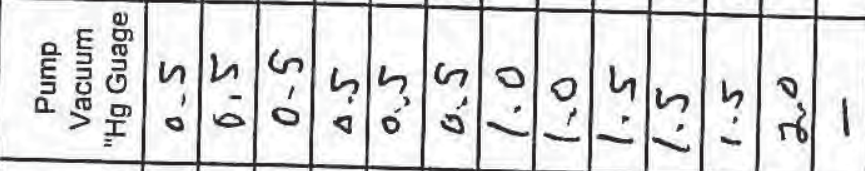

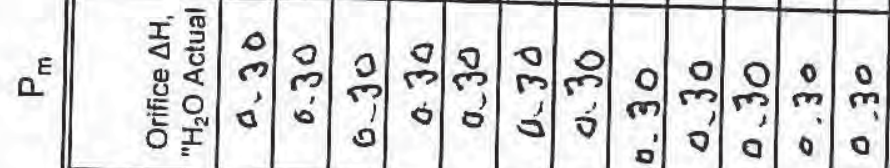

宅总

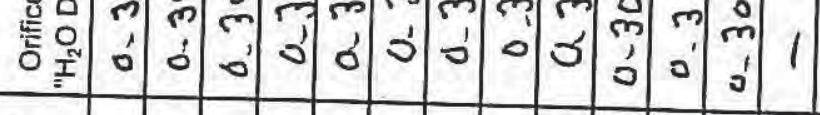

过

产苗

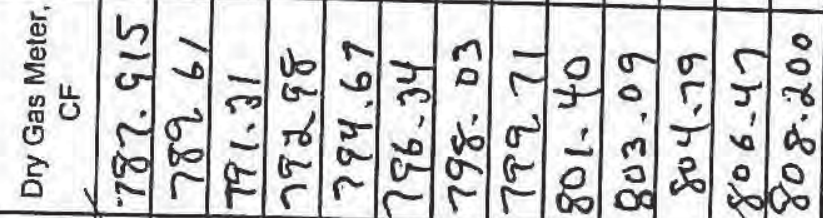

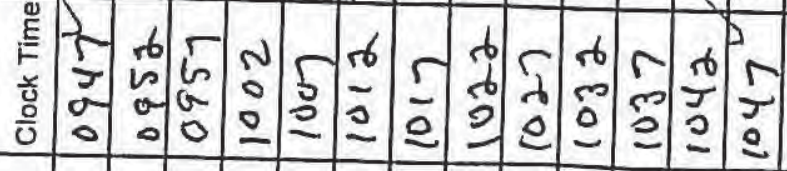

咅 $\begin{aligned} & 1 \\ & 1 \\ & 0\end{aligned}$

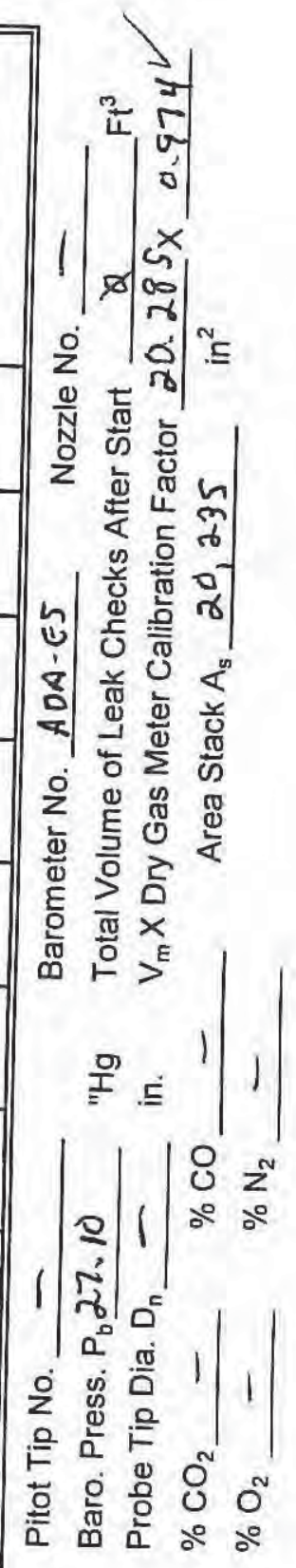


Impinger Bpx No

$3-1$

\begin{tabular}{|c|c|c|}
\hline \multirow[t]{3}{*}{ Impinger 1} & \multirow{3}{*}{$\begin{array}{l}\text { Final Weight } \\
\text { Initial Weight } \\
\text { Increase }\end{array}$} & 763.2 \\
\hline & & 724.6 \\
\hline & & 38.6 \\
\hline \multirow[t]{3}{*}{ Impinger 2} & \multirow{3}{*}{$\begin{array}{l}\text { Final Weight } \\
\text { Initial Weight } \\
\text { Increase }\end{array}$} & 743.7 \\
\hline & & 742.4 \\
\hline & & 1.3 \\
\hline \multirow[t]{3}{*}{ Impinger 3} & \multirow{3}{*}{$\begin{array}{l}\text { Final Weight } \\
\text { Initial Weight } \\
\text { Increase }\end{array}$} & 628.1 \\
\hline & & 622.7 \\
\hline & & 0.4 \\
\hline \multirow[t]{3}{*}{ Impinger 4} & \multirow{3}{*}{$\begin{array}{l}\text { Final Weight } \\
\text { Initial Weight } \\
\text { Increase }\end{array}$} & 952.0 \\
\hline & & $947=0$ \\
\hline & & 5.0 \\
\hline
\end{tabular}

Impinger 5 Final Weight

Initial Weight

Increase

Impinger $6 \quad$ Final Weight

Initial Weight

Increase

Impinger $7 \quad$ Final Weight

Initial Weight

Increase

Impinger $8 \quad$ Final Weight

Initial Weight

Increase
Filter No.

$$
\begin{aligned}
P_{b} & =27.10 \\
V_{m} & =19.7582 \\
V_{w} & =45.3 \mathrm{~V} \\
P_{m} & =0.300 \mathrm{~V}
\end{aligned}
$$$$
\text { Avg } \Delta \mathrm{P}=
$$

Avg $\sqrt{\Delta \mathrm{P}}=$

$$
\begin{aligned}
& C_{p}=- \\
& \mathrm{P}_{\mathrm{s}}=-\frac{-}{46 \mathrm{~V}}{ }^{\mathrm{N}} \mathrm{H}_{\mathrm{m}} \mathrm{O} \\
& T_{s}=\simeq{ }^{\circ} \mathrm{F}
\end{aligned}
$$

Water Weight Gain

Impinger 138.6

Impinger 2 t. 3

Impinger $3 \quad 0.4$

Impinger 4

Impinger 5

Impinger 6

Impinger 7

Impinger 8

Total $45.3^{2}=v_{w}$

$\begin{aligned} \% \mathrm{CO}_{2} & ==- \\ \% \mathrm{O}_{2} & == \\ \% \mathrm{CO} & == \\ \% \mathrm{~N}_{2} & =-\end{aligned}$

$A_{5}=20,235$

$D_{\mathrm{n}}=$

$T_{1}=60 V$

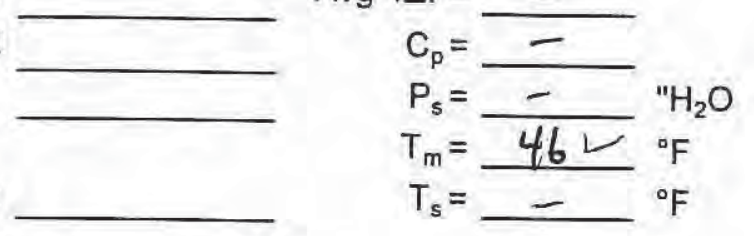

Moisture

Content:

$$
\% M=10.26 \quad M_{d}=0.8974 \quad M_{d}=
$$

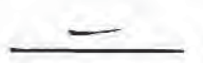

$\mathrm{MW}=$

$\mathrm{Vm}_{\mathrm{std}}=17.65 \times \mathrm{Vm}\left[\frac{\mathrm{P}_{\mathrm{b}}+\frac{\mathrm{P}_{\mathrm{m}}}{13.6}}{\mathrm{~T}_{\mathrm{m}}+460}\right]=17.65 \times 19.758\left[\frac{27.10+\frac{0.300}{13.6}}{46+460}\right]=\frac{18.692^{2}}{0.312} \mathrm{sft}^{\mathrm{scfm}}$

$V_{w_{\text {gas }}}=0.0472 \times V w=0.0472 \times$

45.3

$=2.138$

$\mathrm{sft}^{3}$

$\%$ Moisture $=\frac{V w_{\text {gas }}}{V m_{\text {std }}+V w_{\text {gas }}} \times 100=\frac{2.138}{18.692+2.138} \times 100=10.26 \int_{\%} \%$

$V_{s}=5123.8 \times \sqrt{\frac{x}{x}} \times-\frac{}{} \mathrm{fpm}$

$\% 1=1039 x$

$x$

$\begin{array}{llll}x & x & x\end{array}$

$=-\frac{}{2} \%$

ACFM:
SCFM:
WSCFH:
\%EA:

Date: 


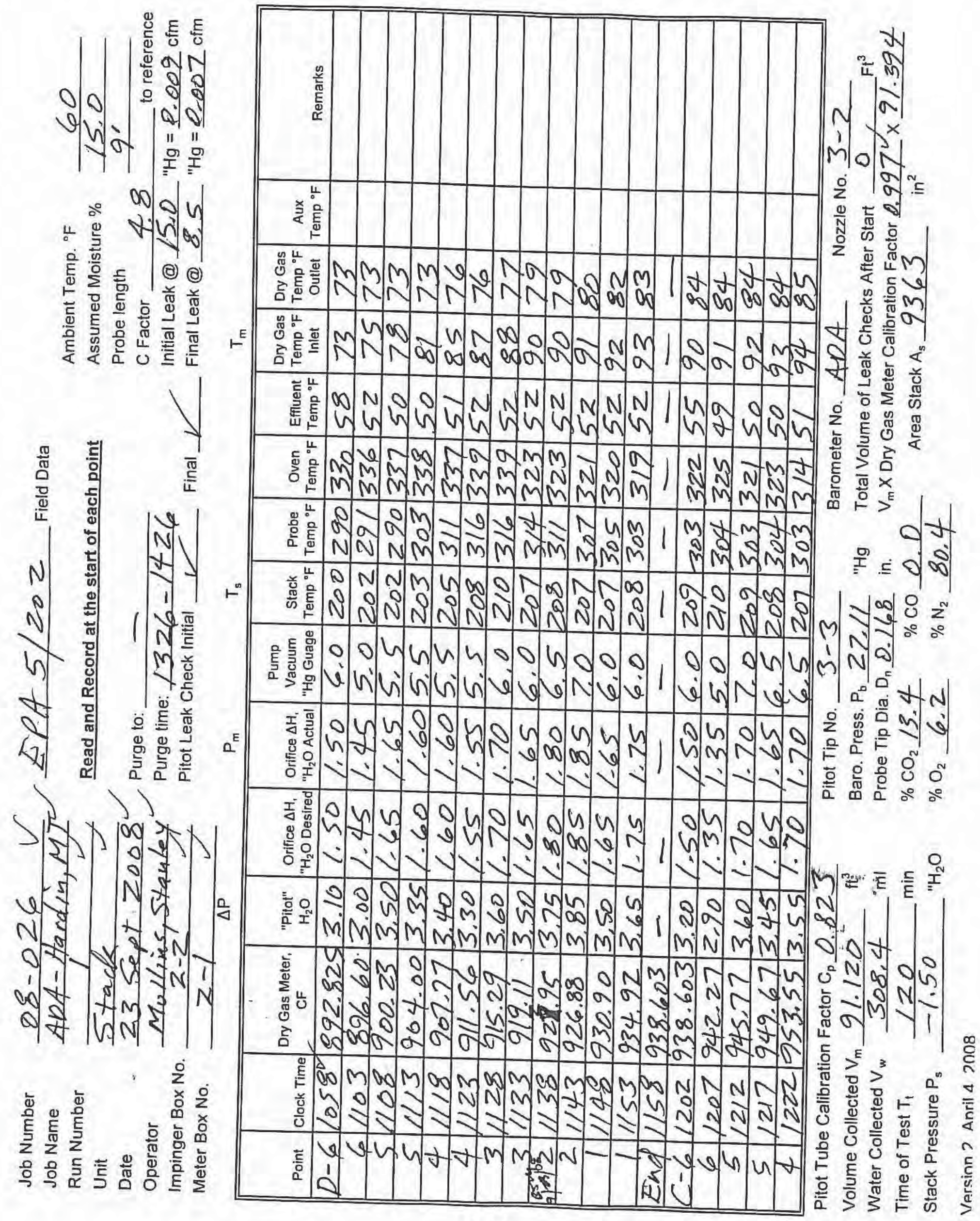


Impinger Bpx No. Z - Z

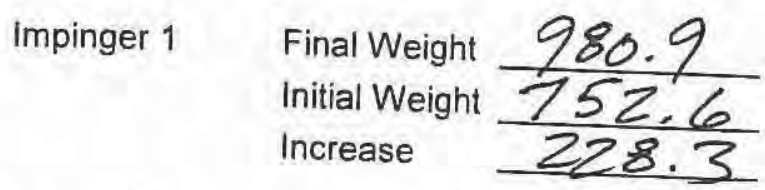

Impinger 2 Final Weight $8 / 4.5$

$$
\text { Initial Weight } \frac{757.4}{57.1}
$$

Impinger 3

Final Weight Increase

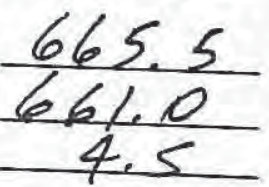

Impinger 4

Final Weight Initial Weight Increase

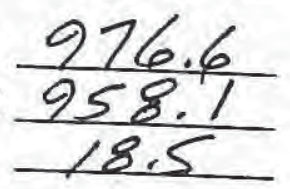

Impinger $5 \quad$ Final Weight Initial Weight Increase

Impinger 6

Final Weight Initial Weight Increase

Impinger 7

Final Weight Initial Weight Increase

Impinger 8

Final Weight Initial Weight Increase
Filter No. 4,239

$$
\begin{aligned}
& P_{b}=27.11 \\
& V_{m}=91.120 \\
& V_{w}=308.4 \\
& P_{m}=1.633
\end{aligned}
$$

Avg $\Delta \mathrm{P}=3.444$

Avg $\sqrt{\Delta \mathrm{P}}=1.855$

$C_{p}=0.823 L$

$P_{s}=-1.50^{2} \mathrm{H}_{2} \mathrm{O}$

$T_{m}=86 \mathrm{~V} \%$

$T_{s}=206{ }^{\circ} \mathrm{F}$
Water Weight Gain

Impinger $1 \mathrm{ZZ2} 3$

Impinger 2521

Impinger 3 4.5

Impinger 4

Impinger 5

Impinger 6

Impinger 7

Impinger 8

Total $308,4^{2}=v_{w}$

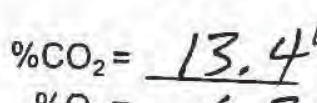$$
\% \mathrm{O}_{2}=6,2
$$$$
\% \mathrm{CO}=0.0
$$$$
\% \mathrm{~N}_{2}=80,4
$$

$A_{s}=9363$

$D_{n}=0.168$

$T_{1}=120$

$27.00^{\circ} \mathrm{Hg}$

$546 \%$ $666^{\circ} \mathrm{R}$

Moisture

Content:

$$
\% \mathrm{M}=15.36 \mathrm{~L} \quad \mathrm{~m}_{\mathrm{d}}=0.8464 \quad \mathrm{mw}_{\mathrm{d}}=\underline{0.392} \quad \mathrm{mw}=\underline{28.49}
$$

$\mathrm{Vm}_{\text {std }}=17.65 \times \mathrm{Vm}\left[\frac{\mathrm{P}_{\mathrm{b}}+\frac{\mathrm{P}_{\mathrm{m}}}{13.6}}{\mathrm{~T}_{\mathrm{m}}+460}\right]=17.65 \times 91.120\left[\frac{27.11+\frac{1.633}{13.6}}{86+460}\right]=\frac{80.207}{0.668} \mathrm{sft}^{\mathrm{s}}$

$v_{w_{\text {gas }}}=0.0472 \times v_{w}=0.0472 \times 308.4=14,556 \mathrm{~V} \mathrm{stl}^{3}$ $\%$ Moisture $=\frac{V w_{\text {gas }}}{V m_{\text {std }}+V w_{\text {gas }}} \times 100=\frac{14.556}{80,207+14.556} \times 100=15.36^{V} \%$

$v_{s}=5123.8 \times 0.823 \sqrt{\frac{666}{27.00^{\times} 28.49} \times 1.855}=7279 \mathrm{fpm}$

$\% 1=\frac{1039 \times 80.207 \times 666}{0.8464 \times 27.00^{\times} 7279 \times 120 \times(0.168)^{2}}=98,5^{1} \%$

ACFM: 473,257

SCFM: 287645

WSCFH: $\frac{20,370,21,4}{41.0}$

Checked by: Delst

Date: $9 / 26 / 08$ 
U. ง. II II

$\therefore$ 늠

彭

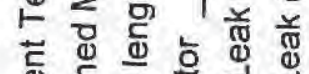

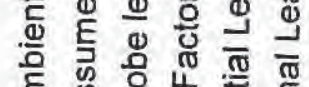

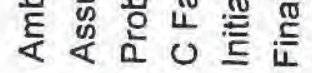

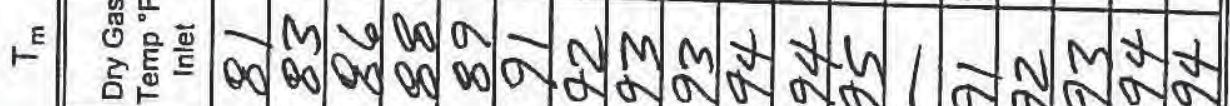

סु

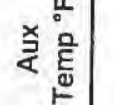

:

|

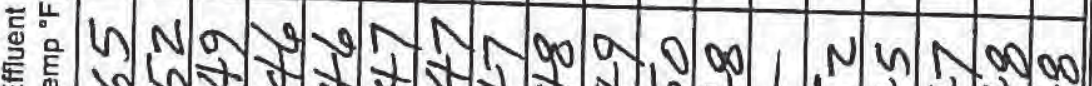

密导

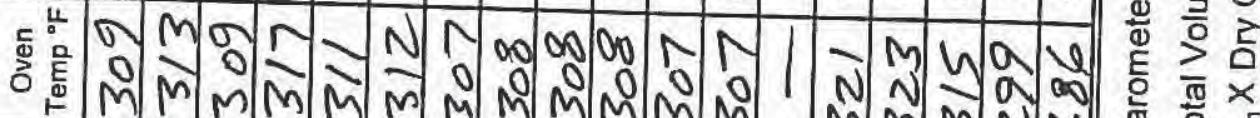

ㄴ.

V.

. $N$ N M M M N N N N O

oy

$\vdash$

5 के

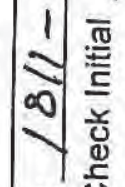

¿0

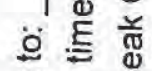

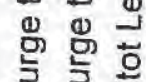

$\square^{E}$

है

Q

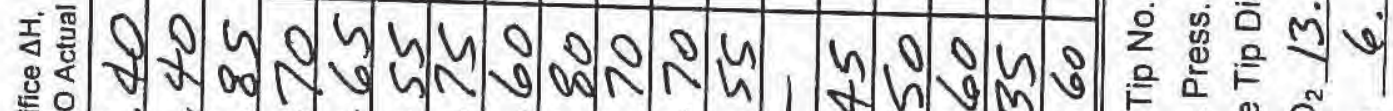

起到

$x^{\circ}$ : 0 (

든 은 0

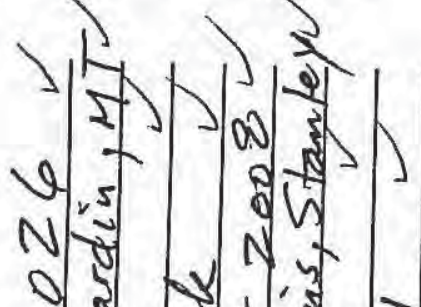

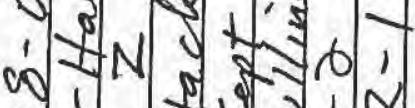

Qt 1

\& h

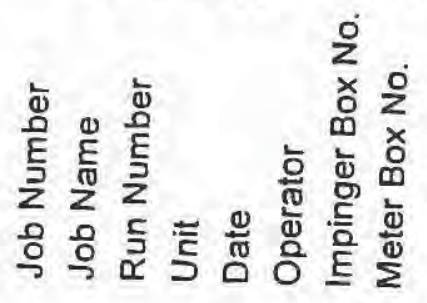

至

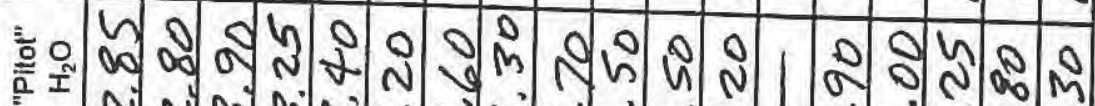

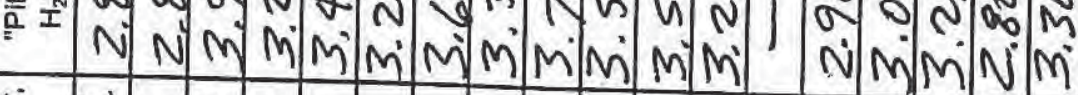

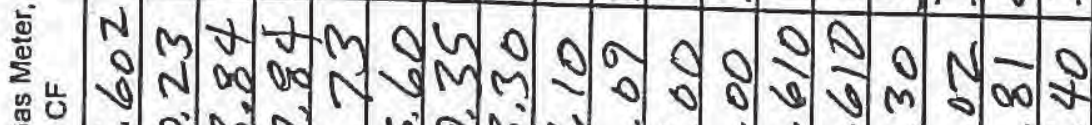

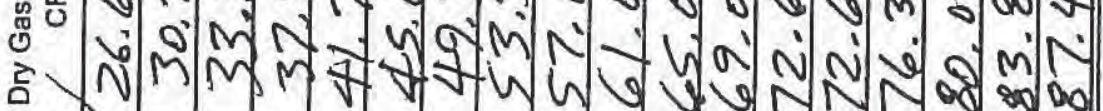

है जै के

है ज ज

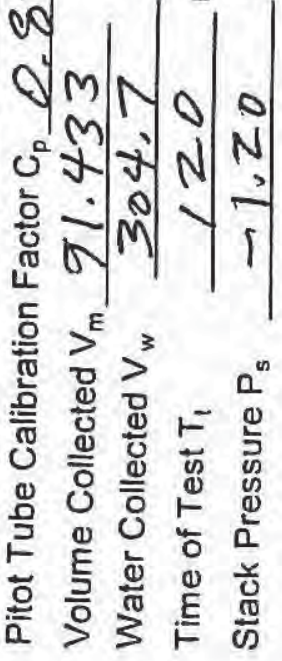




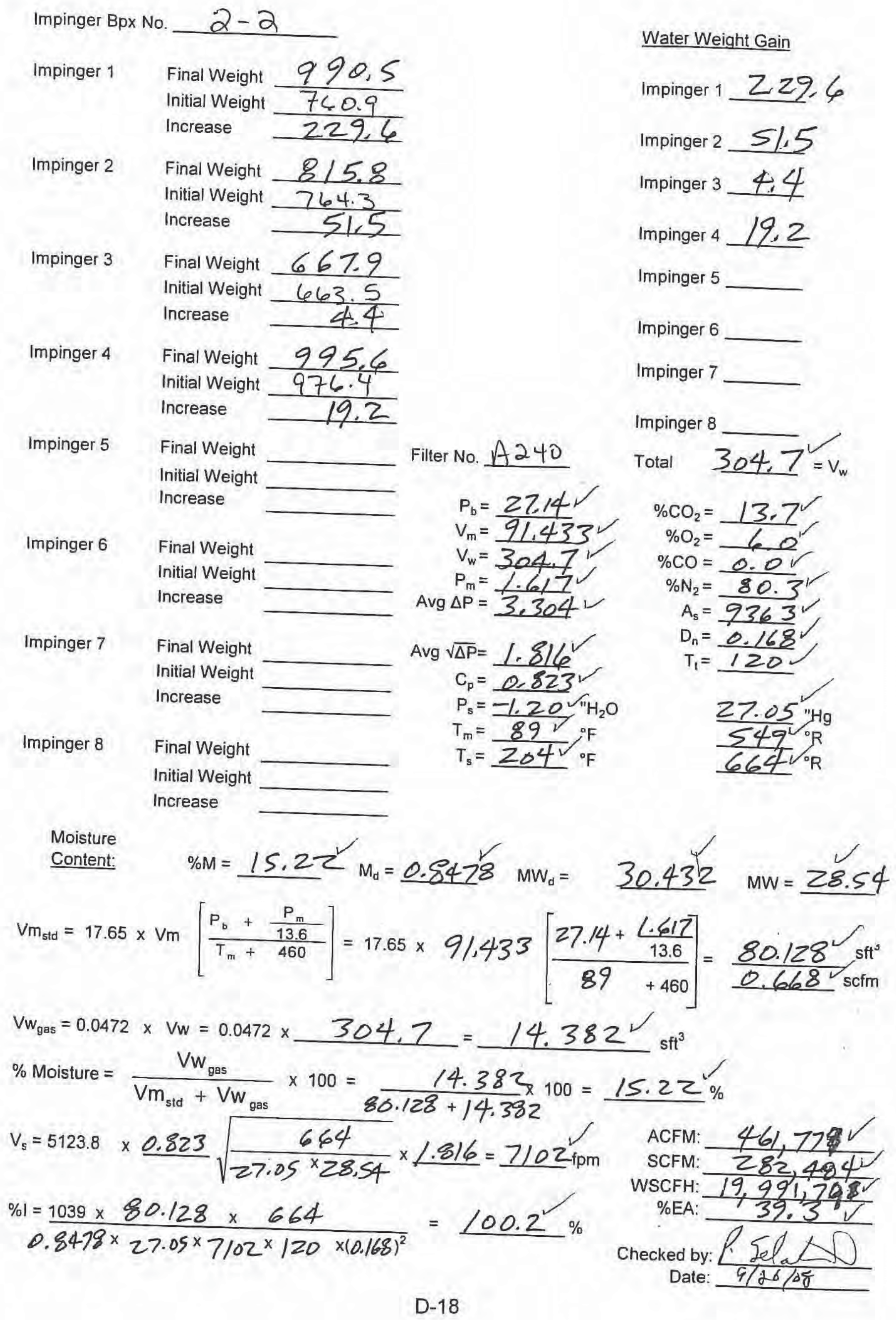




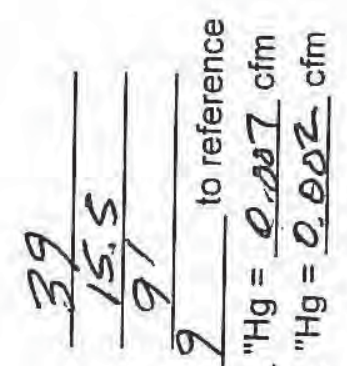

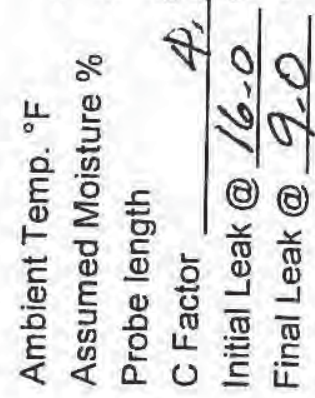

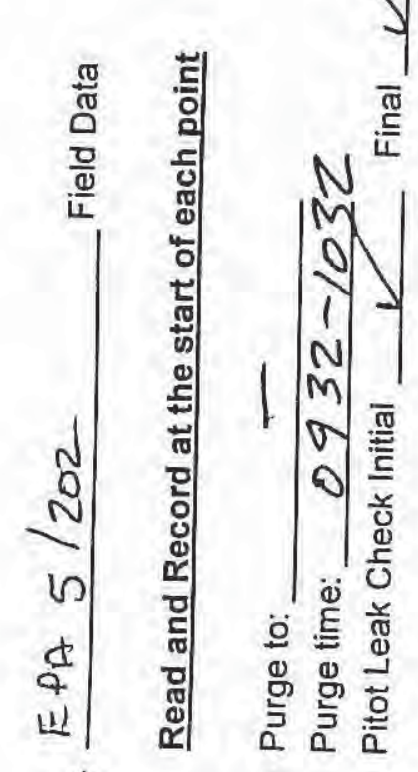

से। क्षे। है।

e. 3

$00 x+\frac{1}{4}$

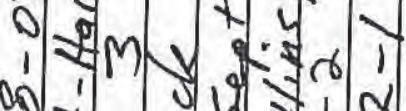

a) $*$ (1)

Q)

म $h$ और

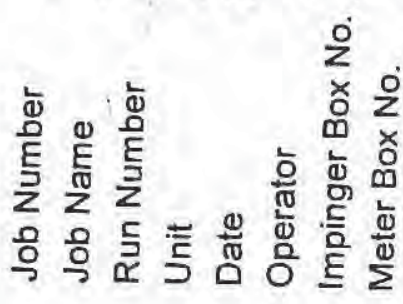

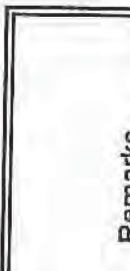

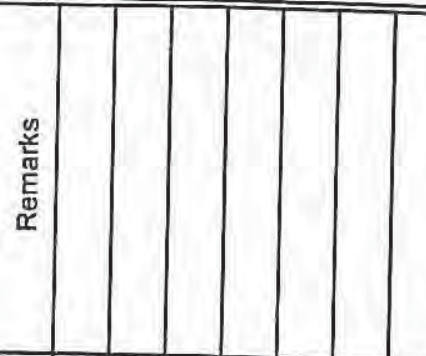

肴亭

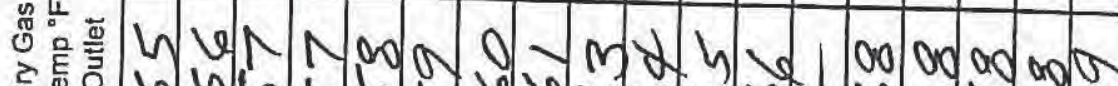

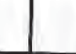

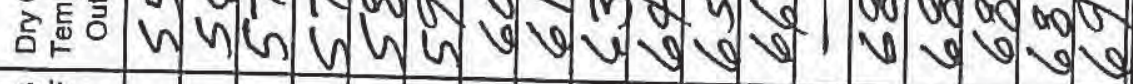

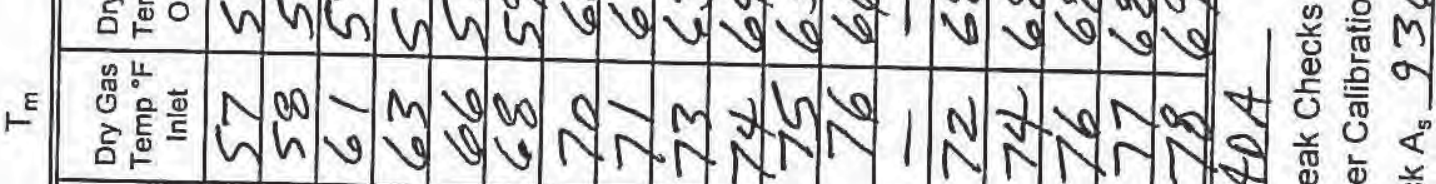

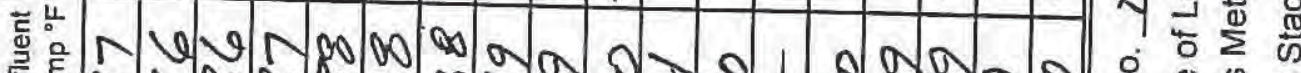

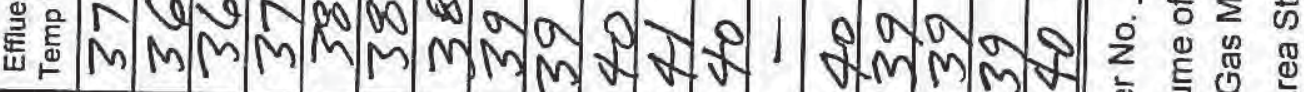

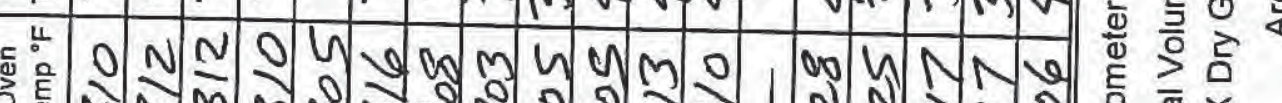

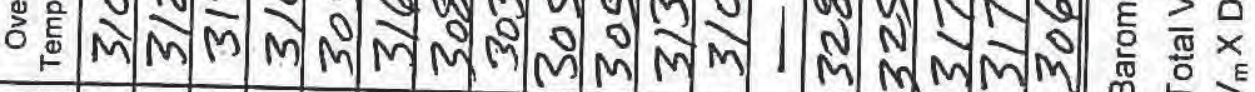

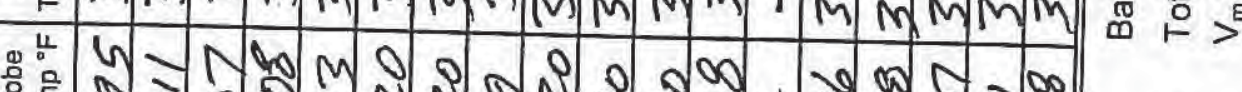

N M M M M M M M N NM 1 M

$\vdash$ क

का

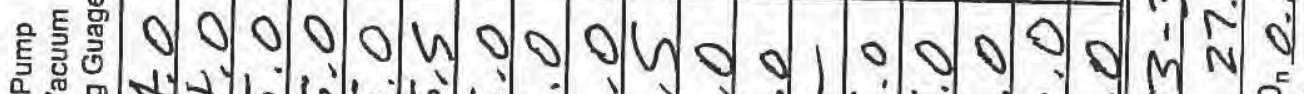

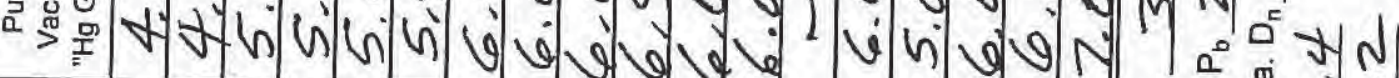

r

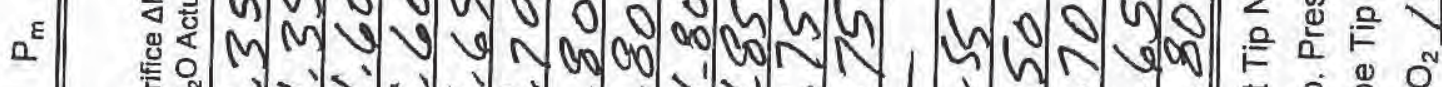

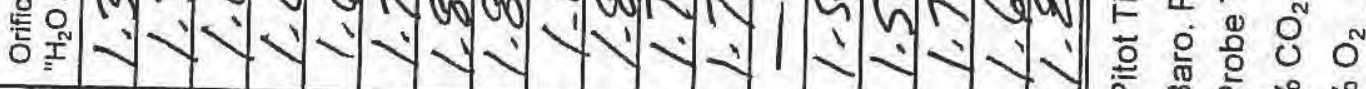

I

S.

造

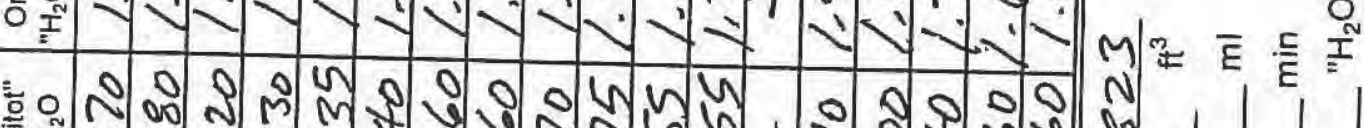

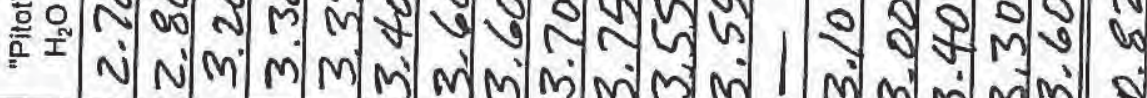

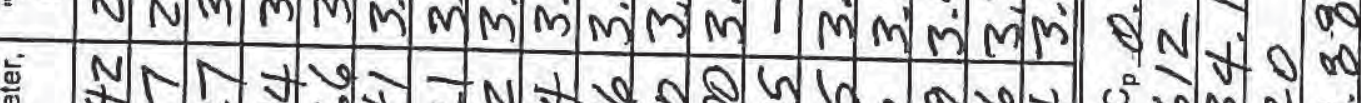

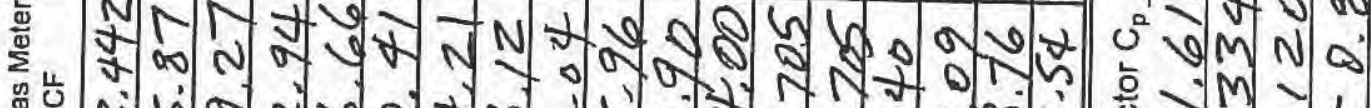

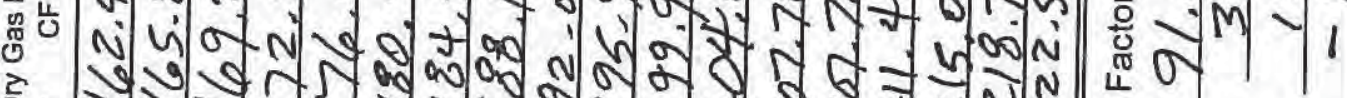

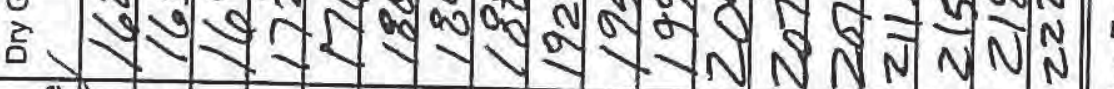

है

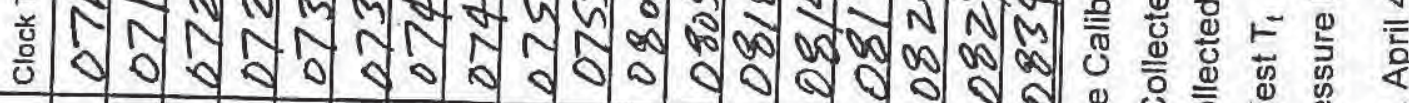

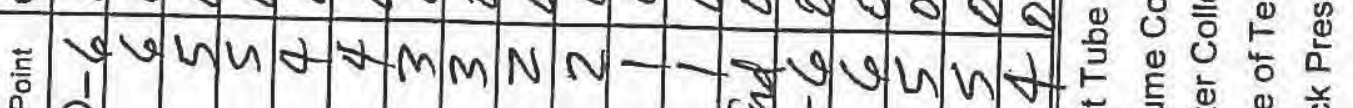

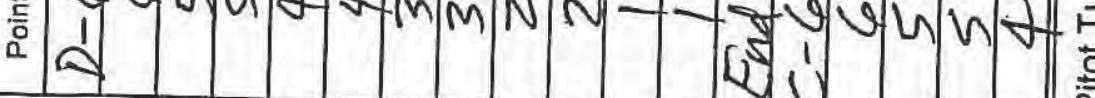

总 
Impinger Bpx No. $2-2$

$\begin{array}{ll}\text { Impinger } 1 & \text { Final Weight } \frac{972,1}{753.7} \\ & \begin{array}{l}\text { Initial Weight } \\ \text { Increase }\end{array}\end{array}$

Impinger 2 Final Weight 842.9

Initial Weight 759.9

Increase 83,0

Impinger 3 Final Weight 669.6

Initial Weight 463.8

Increase 5.8

Impinger $4 \quad \begin{aligned} & \text { Final Weight } \\ & \text { Initial Weight }\end{aligned} \frac{966,3}{944.4}$

Increase $\frac{.91 .9}{2}$

Impinger 5 Final Weight

Initial Weight

Increase

Impinger $6 \quad$ Final Weight

Initial Weight

Increase

lmpinger 7 Final Weight

Initial Weight

Increase

Final Weight

Initial Weight

Increase
Filter No. $A_{2}+1$

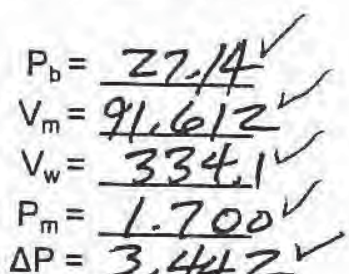

Avg $\Delta P=3.442$

Avg $\sqrt{\Delta \mathrm{P}}=1.853$

$c_{p}=0.823$

$P_{s}=0.88 \mathrm{~V} " \mathrm{H}_{2} \mathrm{O}$

$T_{m}=69 \mathrm{~V}{ }^{\circ} \mathrm{F}$

$T_{s}=210{ }^{\circ} \mathrm{F}$
Water Weight Gain

Impinger 1223.4

Impinger $2,83,0$

Impinger $3 \quad 5,8$

Impinger $4 \geq 1.9$

Impinger 5

Impinger 6

Impinger 7

Impinger 8

Total $\quad 334.1^{L}=v_{w}$

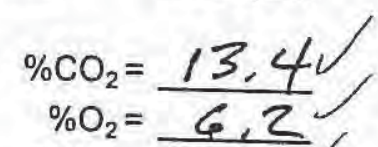

$\% \mathrm{CO}=0.0$

$\% \mathrm{~N}_{2}=80.4$

$A_{s}=9363$

$D_{n}=0.168$

$T_{1}=\angle 20$

$27.08^{2} \mathrm{Hg}$

$529^{\circ} \mathrm{R}$

$670 \mathrm{~N}$

Moisture

Content:

$$
\% \mathrm{M}=15.91^{2} \mathrm{~m}_{\mathrm{d}}=\underline{0.840^{2}} \mathrm{Mw}_{\mathrm{d}}=\underline{30.392} \quad \mathrm{MW}=\underline{28.42}
$$

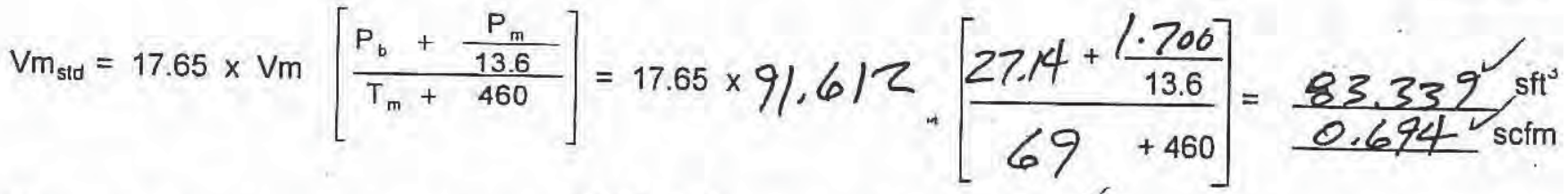

$V_{w_{\text {gas }}}=0.0472 \times V_{w}=0.0472 \times 334.1=15.770^{\circ} \mathrm{st}^{3}$

$\%$ Moisture $=\frac{V w_{\text {gas }}}{V m_{\text {std }}+V w_{\text {gas }}} \times 100=\frac{15,770}{83.339+15,770} \times 100=\left.15.9\right|^{/} \%$

$v_{s}=5123.8 \times 0.823 \sqrt{\frac{670}{27.08 \times 28.42}} \times 1.853=7291 \mathrm{fpm}$

$\% 1=1039 \times 83.339 \times 670 \quad=103.2^{\nu}$

$0.8409 \times 27.08 \times 7291 \times 120 \times(0.168)^{2}$

ACFM: 474,045

SCFM: 285381

WSCFH: $20,362,548$

$\% \mathrm{EA}$ :

Checked by: Selut

Date: $9 / 26 / \Delta 8$ 


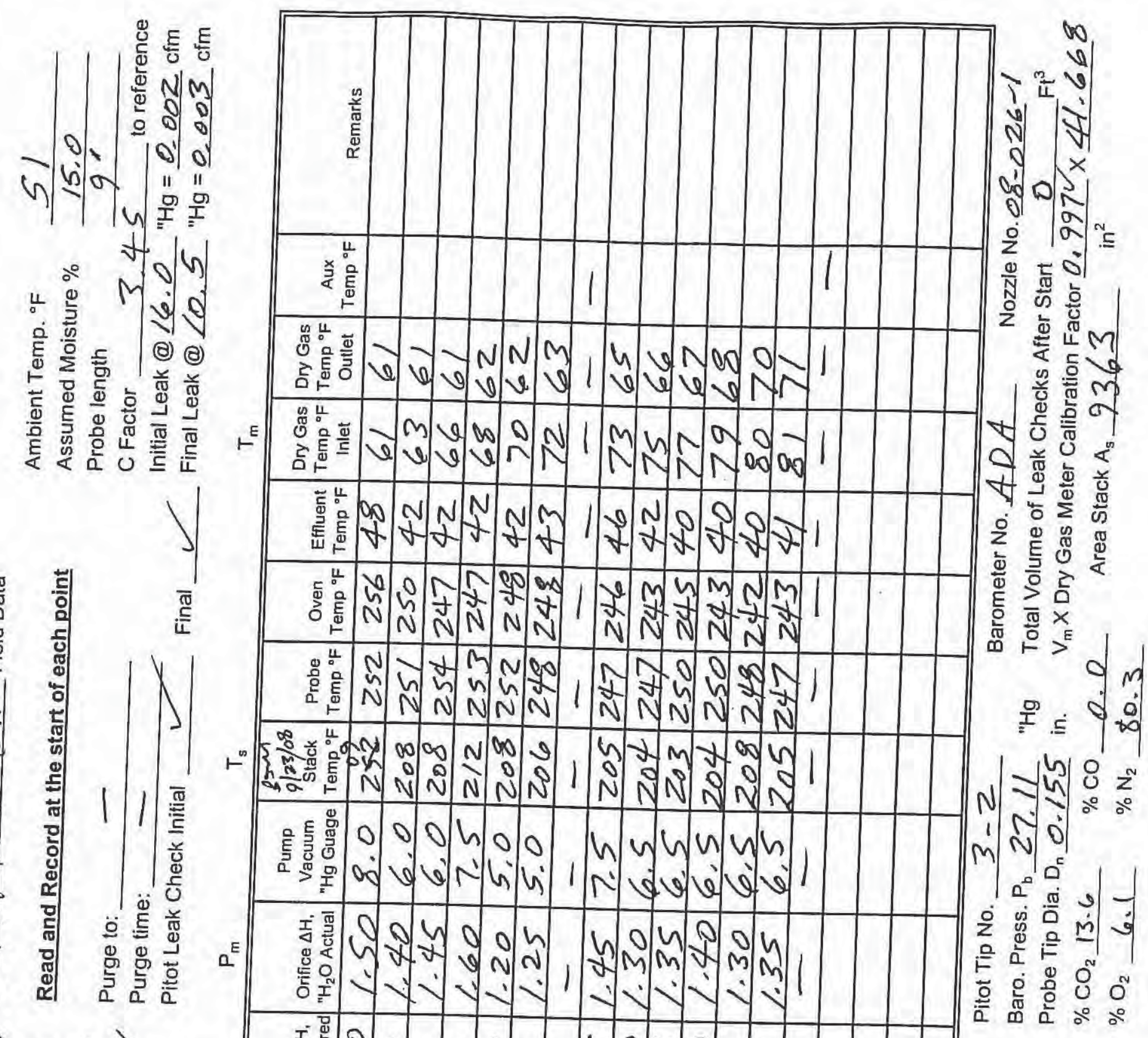

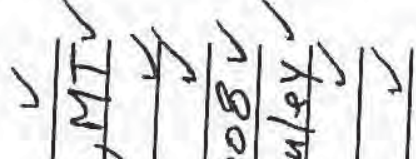

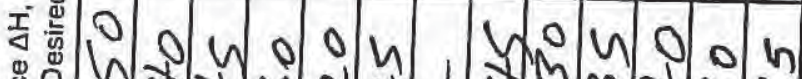

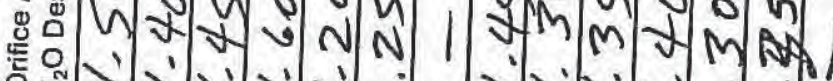

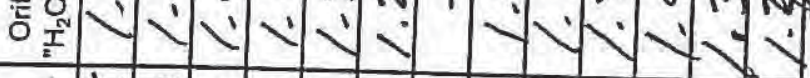

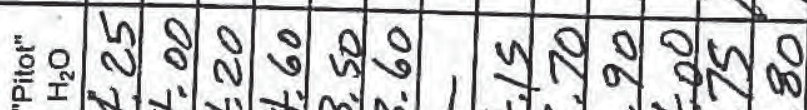

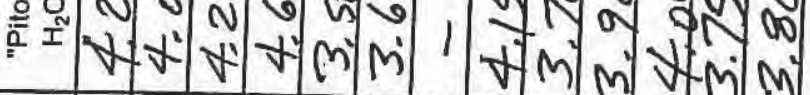

ปัँ

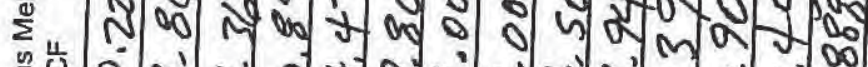

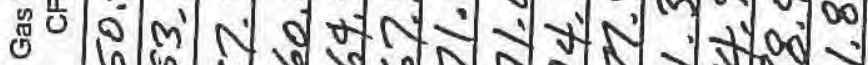

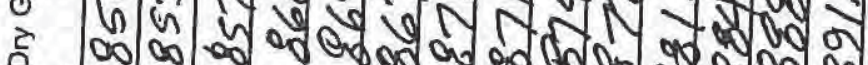

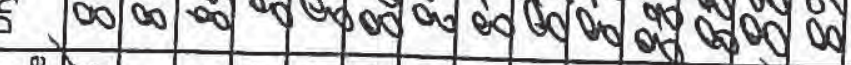

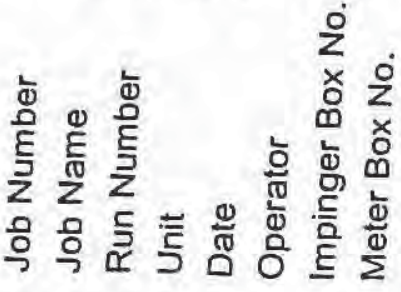

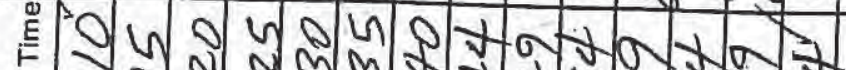

० न N $N$ N

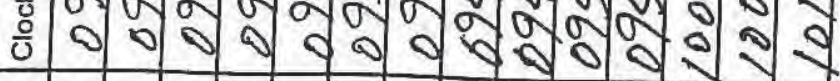

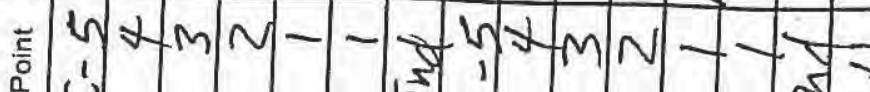

M르 E $\frac{\text { E }}{I^{N}}$

No

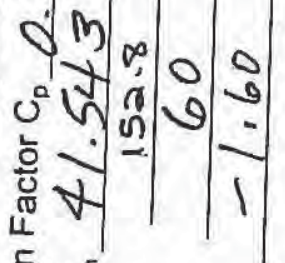

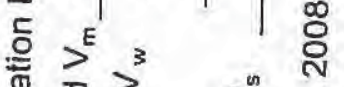

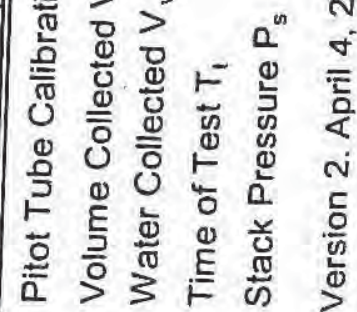


Impinger Bpx No.

2-

Impinger $1 \quad$ Final Weight Initial Weight Increase

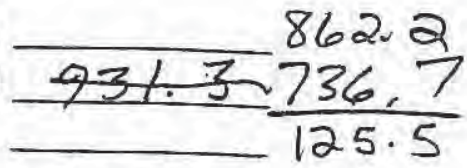

Impinger 2 Final Weight 769.5 Initial Weight $\frac{753.4}{16.1}$

Impinger 3

Final Weight Initial Weight Increase

$$
\frac{731.1}{730.4}
$$

Impinger 4

Final Weight Initial Weight Increase

Impinger 5

Final Weigh Initial Weight Increase

$$
\frac{\frac{740.9}{740.0}}{0.9}
$$

Impinger 6

Impinger 8

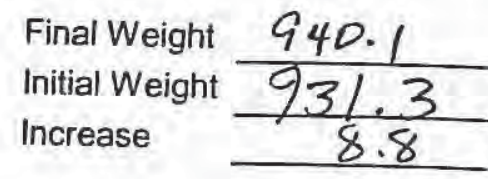

Final Weight

Initial Weight

Increase

Final Weight

Initial Weight Increase
Filter No. A-242

$P_{b}=27.11 \mathrm{~V}$

$v_{m}=41.543$

$v_{w}=152.8 \mathrm{~V}$

$P_{m}=1.379$ Avg $\Delta P=3.954 \mathrm{~V}$

Avg $v \overline{\Delta \mathrm{P}}=1.987$

$c_{p}=0.823 \mathrm{~V}$

$P_{\mathrm{s}}=-1.60 \mathrm{H}_{2} \mathrm{O}$

$T_{m}=6 \%{ }^{\circ} \mathrm{F}$

$T_{s}=207{ }^{\circ} \mathrm{F}$
Water Weight Gain

Impinger 1125.5

Impinger $2+16.1$

Impinger $3 \quad 0.7$

Impinger $4 \quad 0.9$

Impinger $5 \quad 0.8$

Impinger $6 \quad 8.8$

Impinger 7

Impinger 8

Total

$152.8^{\prime}=v_{w}$

$\% \mathrm{CO}_{2}=13.6^{\prime}$

$\% \mathrm{O}_{2}=6 \cdot 1 \mathrm{r}$

$\% \mathrm{CO}=0.0$

$\% \mathrm{~N}_{2}=80.3 \mathrm{v}$

$A_{s}=9363 \mathrm{~V}$

$D_{n}=0.155 \mathrm{~V}$

$T_{1}=60$

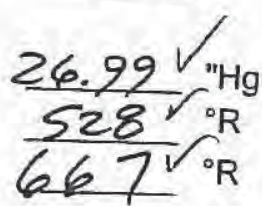

Moisture

Content:

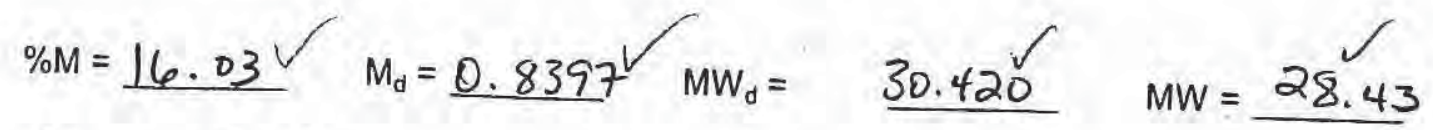

$\mathrm{Vm}_{\mathrm{std}}=17.65 \times \mathrm{Vm}\left[\frac{\mathrm{P}_{\mathrm{b}}+\frac{\mathrm{P}_{\mathrm{m}}}{13.6}}{\mathrm{~T}_{\mathrm{m}}+460}\right]=17.65 \times 41.543\left[\frac{27.11+\frac{T, 379}{13.6}}{68+460}\right]=\frac{37.788^{2}}{0.630^{\mathrm{scfm}}}$

$V w_{\text {gas }}=0.0472 \times V w=0.0472 \times 152.8=7.212 / \mathrm{sft}^{3}$

$\%$ Moisture $=\frac{V w_{\text {gas }}}{V m_{\text {std }}+V w_{\text {gas }}} \times 100=\frac{7.212}{7.212} \times 100=16.03^{1} \%$

$v_{5}=5123.8 \times 0.823 \sqrt{\frac{667}{26.99 \times 28.43} \times 1.987}=7812 \mathrm{fpm}$

$\% 1=\frac{1039 \times 37.788 \times 667}{0.8397 \times 26.99 \times 7812 \times 60 \times(0.155)^{2}}=102.6 \%$

ACFM: $\quad 507944$

SCFM: $305692 \mathrm{~V}$

WSCFH: 21842944

$\%$ EA:

Date: $5 / 26 / 08$ 


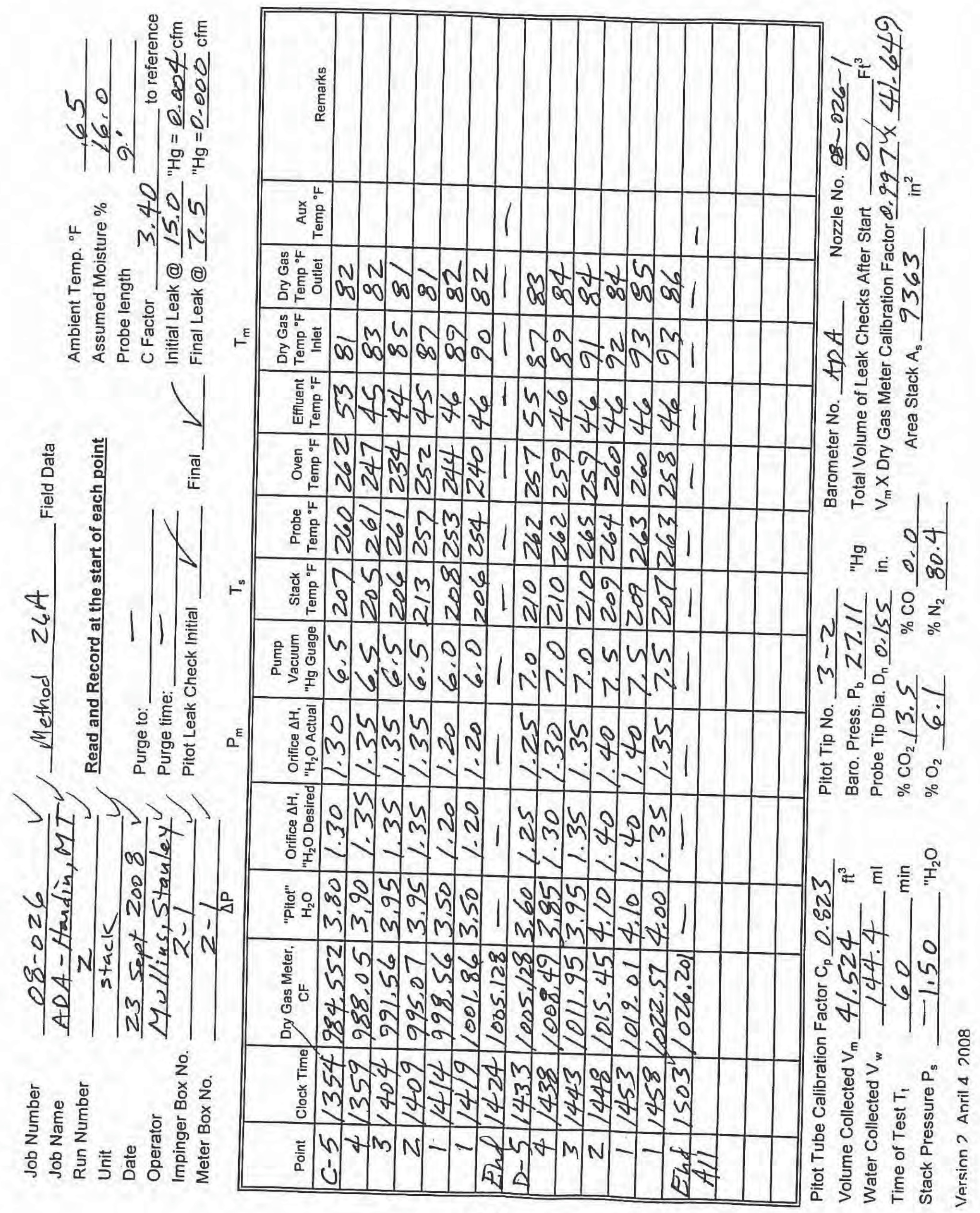


Impinger Bpx No.

$2-1$

Water Weight Gain

$\begin{array}{ll}\text { Impinger 1 } & \begin{array}{l}\text { Final Weight } \\ \text { Initial Weight } \frac{873.3}{751.5} \\ \text { Increase }\end{array}\end{array}$

Impinger 2

Final Weight $\frac{777.0}{765.2}$
Initial Weight
Increase

Impinger 3

Final Weight Initial Weight

Increase

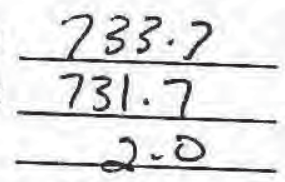

Impinger 4

Final Weight 748.8

Initial Weight $\rightarrow 47.9$

Increase $\frac{0.9}{0.9}$

Impinger 5

Final Weight

$\frac{\frac{644.9}{644.3}}{0.6}$

Filter No.

Initial Weig

Increase

0.6

Impinger 6

Final Weight

Initial Weight

Increase

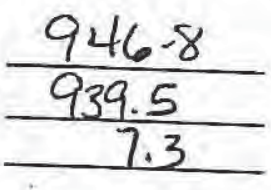

Impinger 7

Final Weight

Initial Weight

Increase

$$
\begin{aligned}
& P_{b}=27.11 \\
& V_{m}=\frac{41.524}{1.44 .4} \\
& V_{w}=\frac{1.317}{P_{m}}=1
\end{aligned}
$$

Avg $\triangle \mathrm{P}=2.3 .850^{\circ}$

Impinger $1 \underline{121.8}$

Impinger $3-2.0$

Impinger 4

Impinger $5 \quad 0.6$

Impinger 6

Impinger 7

Impinger 8

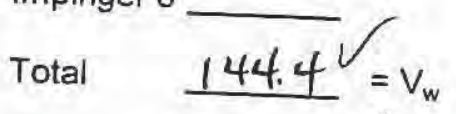

$\% \mathrm{CO}_{2}=13,5^{2}$

$\% \mathrm{O}_{2}=$

$\% \mathrm{CO}=0.0^{2}$

$\% \mathrm{~N}_{2}=80.4 \mathrm{~V}$

$A_{5}=9363$

$D_{n}=0.1552$

$T_{t}=$

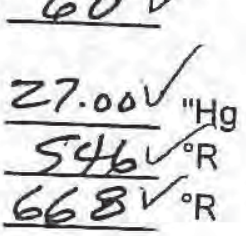

Impinger $8 \quad$ Final Weight

Initial Weight

Avg $\sqrt{\Delta \mathrm{P}}=1,96 \mathrm{~V}$

$\mathrm{C}_{\mathrm{p}}=0.823$

$P_{s}=-1.50^{2}=\mathrm{H}_{2} \mathrm{O}$

$T_{m}=\frac{86^{V}}{2} \mathrm{~F}$

$T_{s}=208^{\circ} \mathrm{F}$

Increase

Moisture

Content:

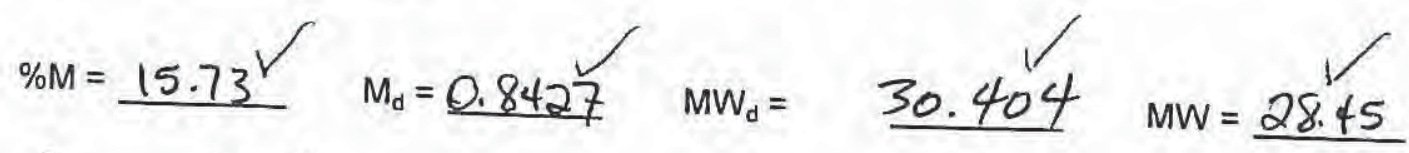

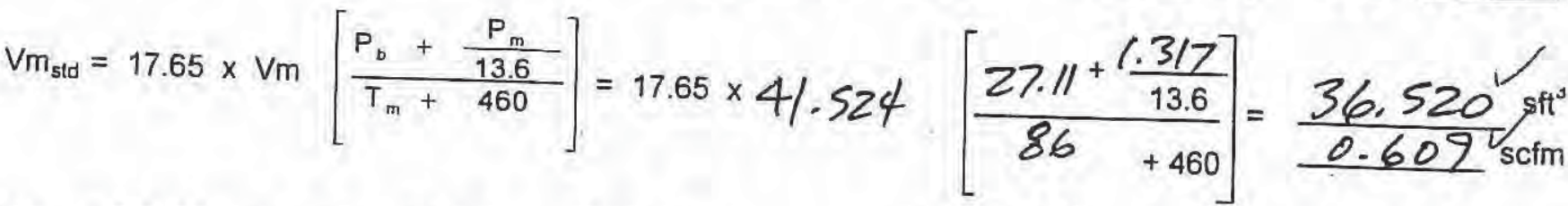

$V_{w_{\text {gas }}}=0.0472 \times V_{w}=0.0472 \times 144.4 \quad=6.816^{\nu} \mathrm{sft}^{3}$ $\%$ Moisture $=\frac{V w_{\text {gas }}}{V m_{\text {std }}+V w_{\text {gas }}} \times 100=\frac{6.816}{36,520+6.816} \times 100=15.73^{V} \%$

$v_{s}=5123.8 \times 0.823 \sqrt{\frac{668}{27.00 \times 28.45}} \times 1.961=7711 \mathrm{Lm}$

$\% 1=\frac{1039 \times 36,520 \times 668}{0.8427 \times 27.00 \times 7711 \times 60 \times(0.155)^{2}}=100.2 \mathrm{~V} \%$

ACFM:

SCFM: 302,476

WSCFH: $\quad 21.536,205$

$\% \mathrm{EA}$ :

40.12

Checked by: 150

Date: $9 / 26 / 08$ 


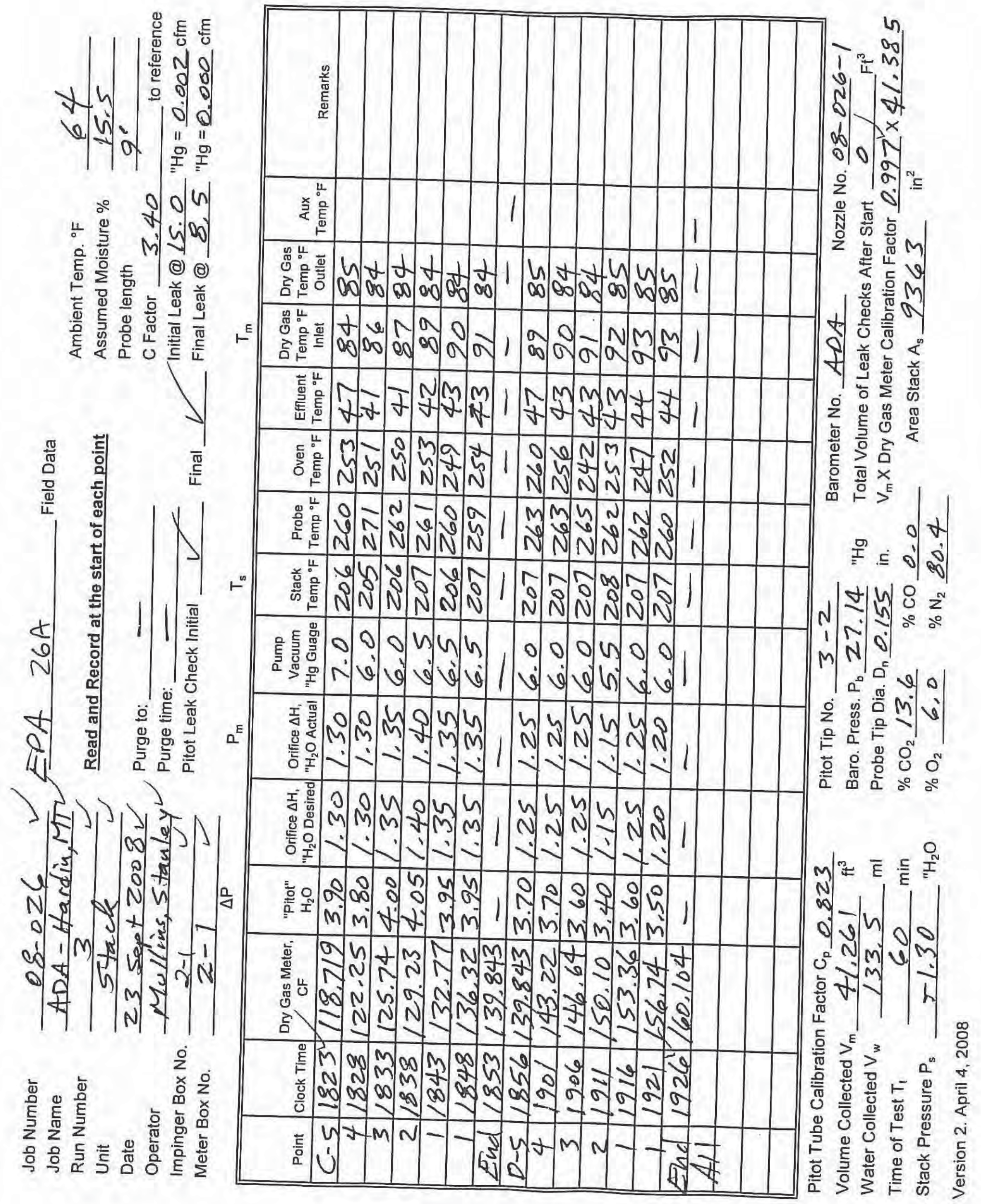




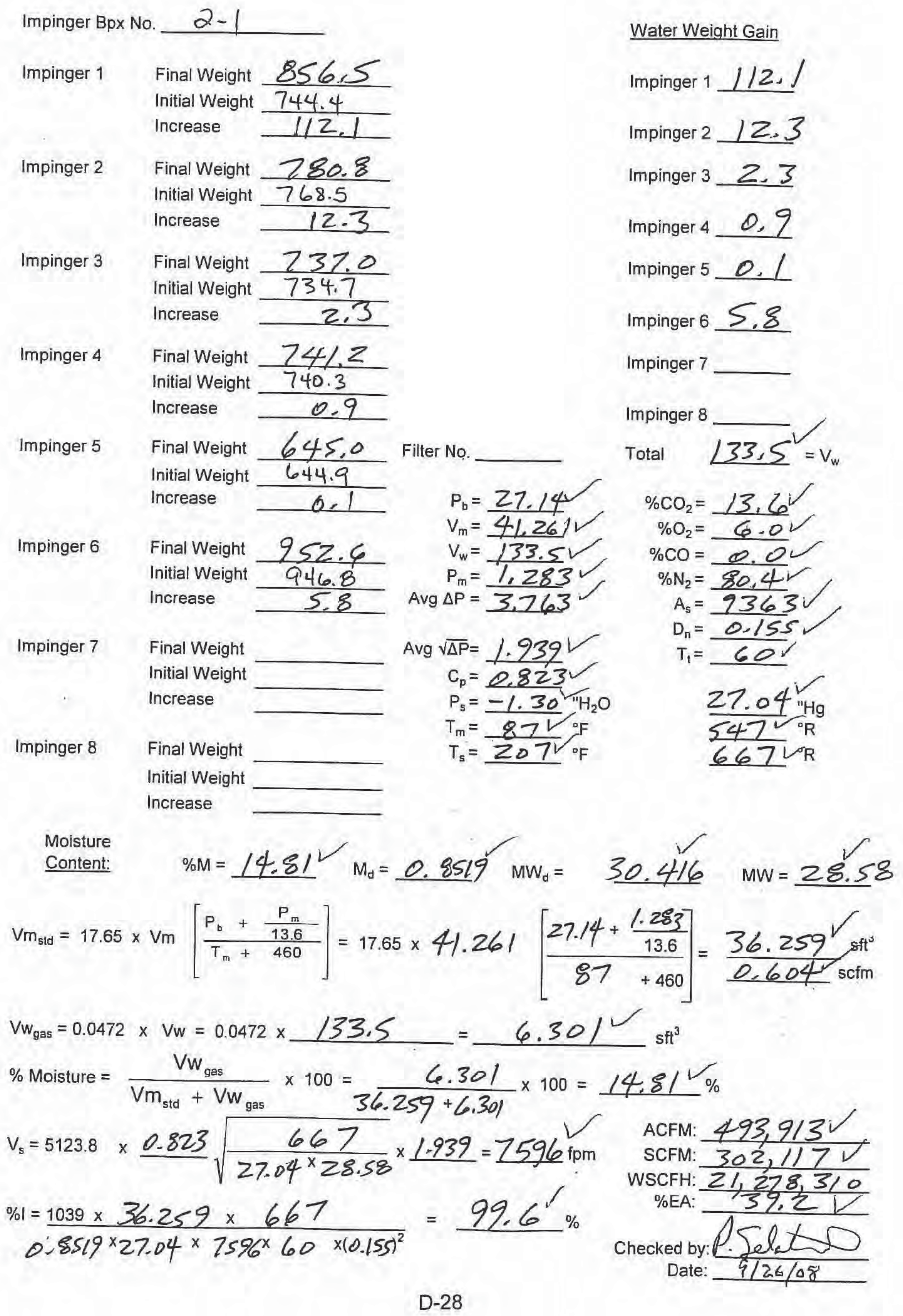


Impinger Bpx No. $3-1 \quad\left(C \subset T_{-1}\right)$

\begin{tabular}{|c|c|c|}
\hline \multirow[t]{3}{*}{ Impinger 1} & Final Weight & 790.9 \\
\hline & Initial Weight & 724.7 \\
\hline & Increase & 66.2 \\
\hline \multirow[t]{3}{*}{ Impinger 2} & Final Weight & .7 \\
\hline & Initial Weight & 739.4 \\
\hline & Increase & 1.5 \\
\hline \multirow[t]{3}{*}{ Impinger 3} & Final Weight & 628.2 \\
\hline & Initial Weight & 628.1 \\
\hline & Increase & 0.1 \\
\hline \multirow[t]{3}{*}{ Impinger 4} & Final Weight & 955.6 \\
\hline & Initial Weight & 952.0 \\
\hline & Increase & 3.6 \\
\hline
\end{tabular}

Impinger 5 Final Weight Initial Weight Increase

Impinger $6 \quad$ Final Weight

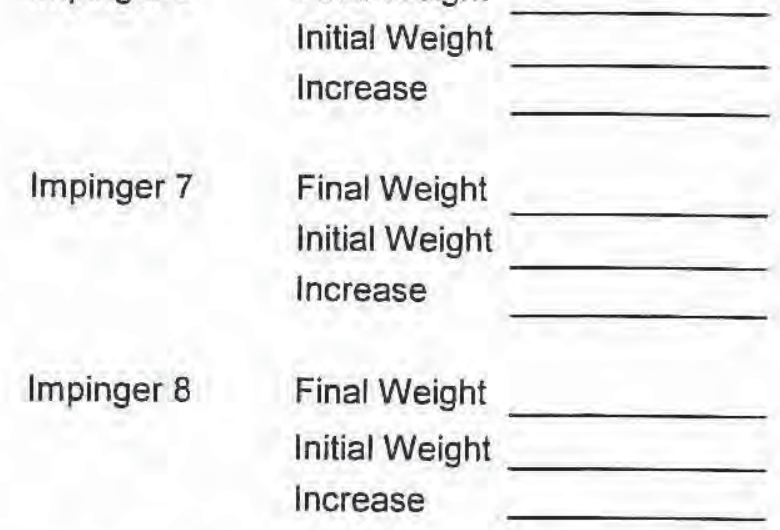

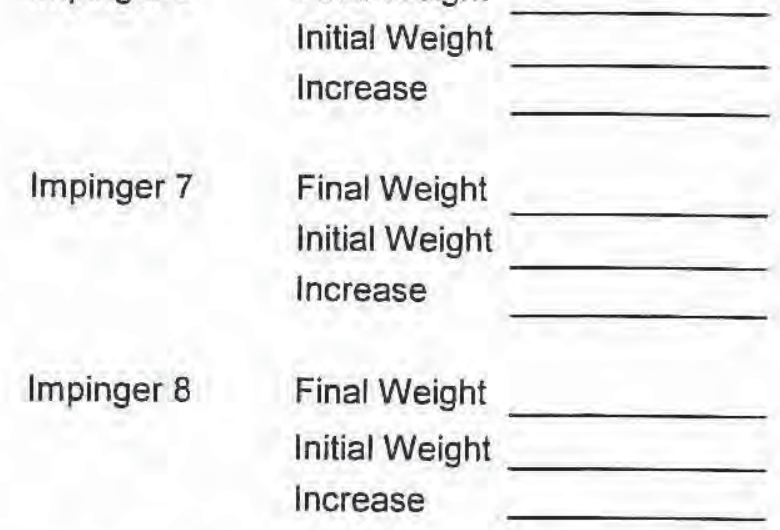

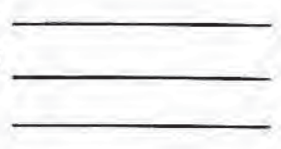

Filter No.

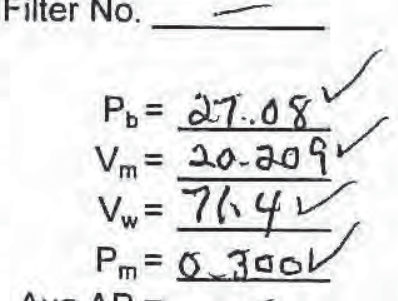

Avg $\triangle \mathrm{P}=$

$$
\begin{aligned}
& \text { Avg } \sqrt{\Delta \mathrm{P}}= \\
& \mathrm{C}_{\mathrm{p}}= \\
& P_{s}=-1 \mathrm{H}_{2} \mathrm{O} \\
& \mathrm{T}_{\mathrm{m}}=65 \mathrm{~V} \\
& T_{s}=\square{ }^{\circ} \mathrm{F}
\end{aligned}
$$

Water Weight Gain

Impinger 166,2

Impinger 21.5

Impinger $30-1$

Impinger $4,3,6$

Impinger 5

Impinger 6

Impinger 7

Impinger 8

Total $\underline{71.4}=v_{w}$

$\begin{aligned} \% \mathrm{CO}_{2} & =- \\ \% \mathrm{O}_{2} & =- \\ \% \mathrm{CO} & =- \\ \% \mathrm{~N}_{2} & =-\end{aligned}$

$A_{5}=4363$

$\begin{aligned} D_{n} & =\overline{60 \gamma} \\ T_{t} & =\overline{60 \gamma}\end{aligned}$

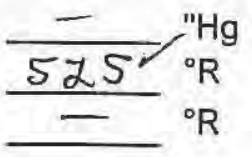

Moisture

Content:

$$
\% M=15.47^{2} M_{d}=0.8453^{2} \quad M W_{d}=
$$

$\mathrm{MW}=$

$V m_{\text {std }}=17.65 \times V m\left[\frac{P_{t}+\frac{P_{m}}{13.6}}{T_{m}+460}\right]=17.65 \times 20.209$

$\left[\frac{22.08+\frac{0.300}{13.6}}{65+460}\right]=\frac{18.413^{0.307} \mathrm{scfm}^{\circ}}{\mathrm{sft}^{\circ}}$

$V w_{\text {gas }}=0.0472 \times V w=0.0472 \times$ $=3.370$ $\mathrm{sft}^{3}$ $\%$ Moisture $=\frac{V w_{\text {gas }}}{V m_{\text {std }}+V w_{\text {gas }}} \times 100=\frac{3.370}{18.413+3.770} \times 100=1547^{2} \%$<smiles>[X][X]C([Y])(CC)C[R15]#[Y5]</smiles>

$\% 1=\underline{1039 x}$

$$
\mathrm{x}
$$
$x$

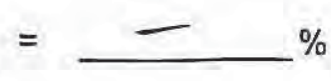
$\%$

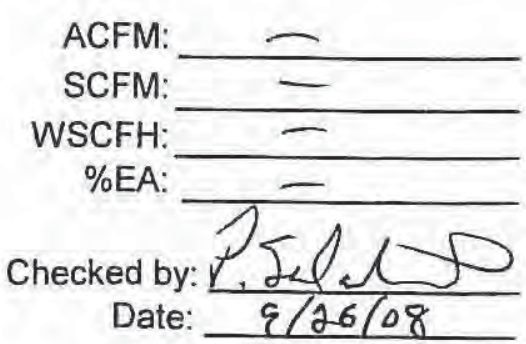




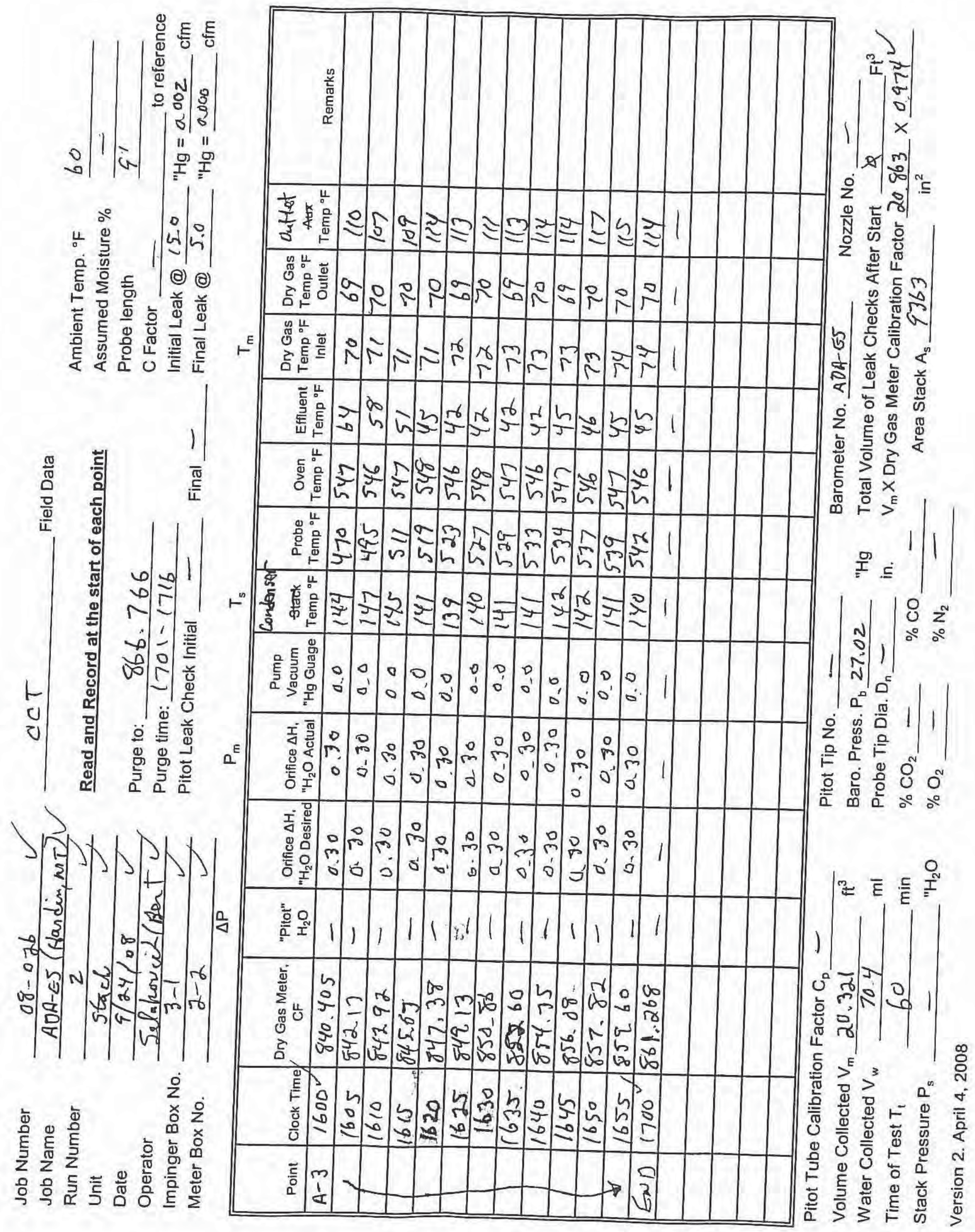


Impinger Bpx No.

$3-1$

Impinger 1 Final Weight 788.4 Initial Weight $\frac{723.5}{64.9}$
Increase

Impinger 2 Final Weight 7422 $\begin{array}{ll}\text { Initial Weight } & \frac{24(.9}{0.8} \\ \text { Increase } & \end{array}$

Impinger 3 Final Weight 628.5 Initial Weight $\frac{628.2}{0.3}$

Impinger 4 Final Weight 928.7 Initial Weight $\frac{95.16924 .3}{4.4}$

Impinger $5 \quad$ Final Weight Initial Weight Increase

Impinger $6 \quad$ Final Weight Initial Weight Increase

Impinger 7

Final Weight Initial Weight Increase

Impinger $8 \quad$ Final Weight Initial Weight Increase
Filter No.

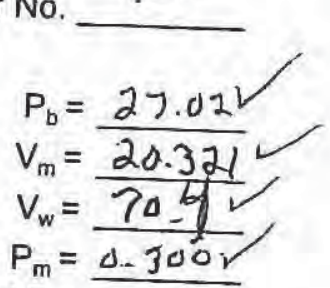

Avg $\triangle \mathrm{P}=$

Avg $v \overline{\Delta P}$

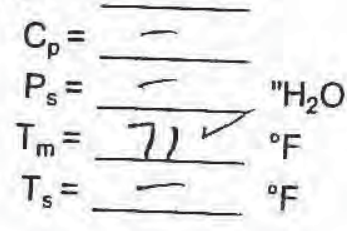

\section{Water Weight Gain}

Impinger 164.4

Impinger $2,4,8$

Impinger 3

Impinger $4 \quad 4.4$

Impinger 5

Impinger 6

Impinger 7

Impinger 8

Total $70.4^{-}=v_{w}$

$\begin{aligned} \% \mathrm{CO}_{2} & =- \\ \% \mathrm{O}_{2} & =- \\ \% \mathrm{CO} & =- \\ \% \mathrm{~N}_{2} & =-\end{aligned}$

$A_{5}=9363$

$D_{\mathrm{n}}=$

$T_{1}=60 \mathrm{~V}$

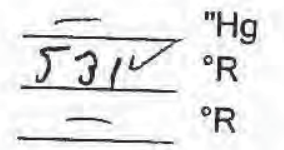

Moisture

Content:

$$
\% \mathrm{M}=15.39
$$$$
M_{d}=0.8461^{\prime} \quad \mathrm{MW}_{\mathrm{d}}=
$$

$\mathrm{MW}=$

$V m_{s t d}=17.65 \times V m\left[\frac{P_{b}+\frac{P_{m}}{13.6}}{T_{m}+460}\right]=17.65 \times 24.32$

$\left[\frac{27.02+\frac{\Delta .300}{13.6}}{71+460}\right]=\frac{18.266}{\Delta-304 / 5 \mathrm{sfm}}$

$V_{w_{\text {gas }}}=0.0472 \times V_{w}=0.0472 \times$

$=3.323^{2}$

$70-4$ $\mathrm{sft}^{3}$

$\%$ Moisture $=\frac{V w_{\text {gas }}}{V m_{\text {std }}+V w_{\text {gas }}} \times 100=\frac{7.373}{18.266+7.723} \times 100=15.39 \%$

$V_{s}=5123.8 \quad x$
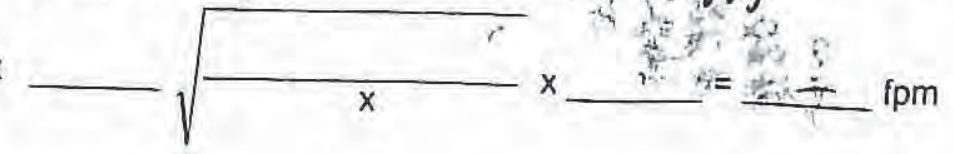

$\% 1=1039 x$

$$
\mathrm{x}
$$
$\mathrm{x}$

$$
=
$$

$\%$

Checked by:

ACFM:

SCFM:

WSCFH:

$\%$ EA:

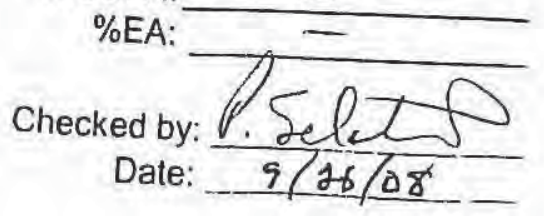




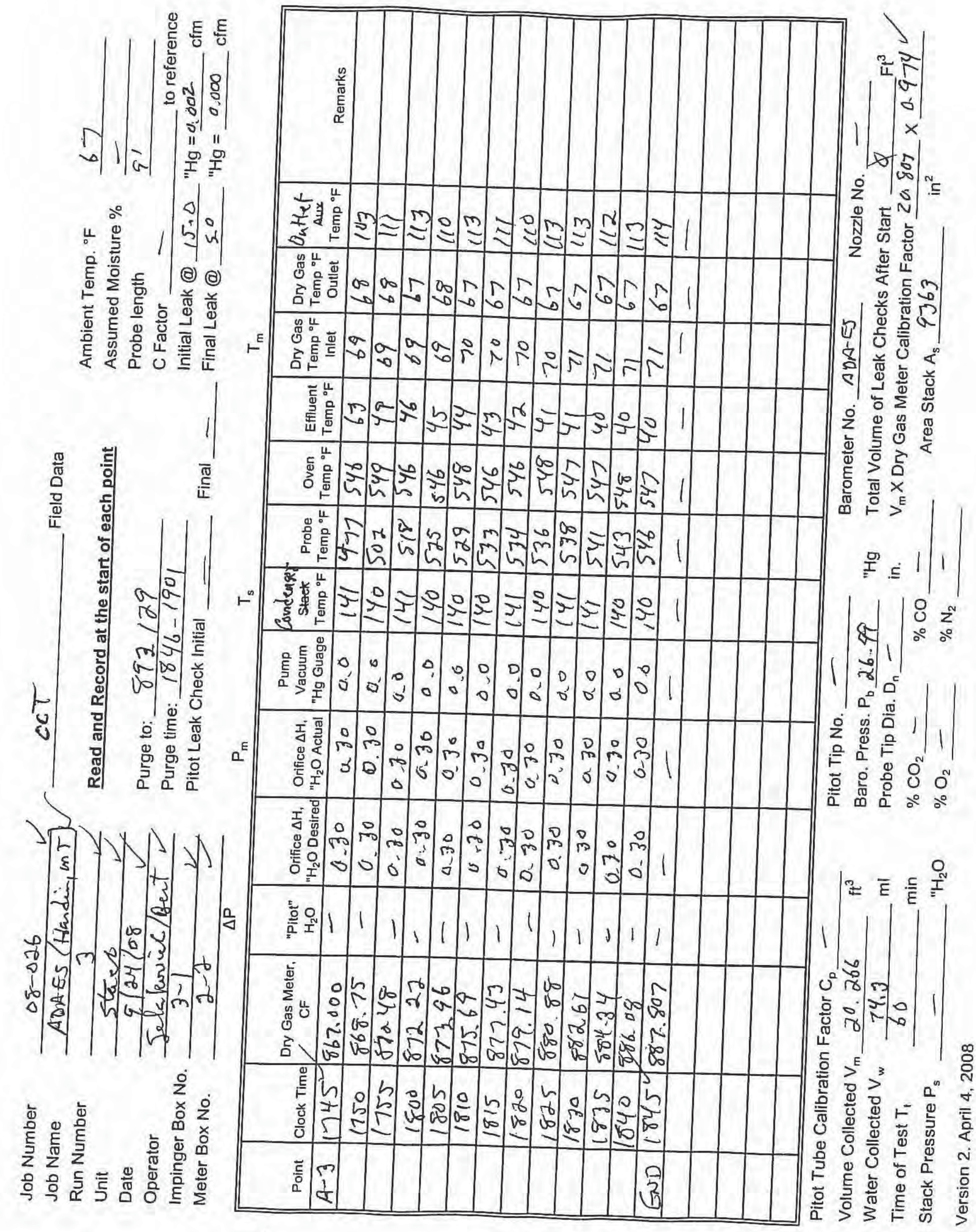


Impinger Bpx No. $3-1$

Water Weight Gain

$\begin{array}{ll}\text { Impinger } 1 & \begin{array}{l}\text { Final Weight } \\ \text { Initial Weight }\end{array} \\ \begin{array}{l}\text { Increase } \\ \text { Int.7 }\end{array} & \frac{78.7}{6.0}\end{array}$

Impinger 2 Final Weight 7449 Initial Weight 240,5 Increase

1.4

Impinger 3 Final Weight 628.8 Initial Weight 628.5 Increase 0.3

Impinger $1 \not 8.0$

Impinger $2 \ell y$

Impinger 30.3

Impinger 4 4.6

Impinger 5

Impinger 6

Impinger 4 Final Weight 933.3 Initial Weight $\frac{928.7}{4.6}$
Increase

Impinger 7

Impinger 8

Impinger $5 \quad$ Final Weight

Initial Weight

Filter No.

Increase

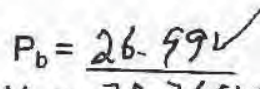

$V_{m}=20.266 \mathrm{~V}$

Impinger $6 \quad$ Final Weight

Initial Weight

$v_{w}=24.3 v$

$P_{m}=0.300 \mathrm{~L}$

Increase

Avg $\triangle \mathrm{P}=$

-

Impinger $7 \quad$ Final Weight

Initial Weight

Increase

Impinger $8 \quad$ Final Weight

Initial Weight

Increase

Avg $\vee \overline{\Delta \mathrm{P}}=$

$$
\begin{aligned}
& \mathrm{C}_{\mathrm{p}}=- \\
& \mathrm{P}_{\mathrm{s}}=-\frac{-}{69} \mathrm{H}_{2} \mathrm{O} \\
& T_{m}=69 \vee{ }^{\circ} \mathrm{F} \\
& T_{5}=\ldots{ }^{\circ} \mathrm{F}
\end{aligned}
$$$$
\text { Total } \underline{24.3 \mathrm{~V}}=\mathrm{v}_{\mathrm{w}}
$$$$
\begin{aligned}
\% \mathrm{CO}_{2} & =- \\
\% \mathrm{O}_{2} & =-
\end{aligned}
$$$$
\% \mathrm{CO}=-
$$$$
\% \mathrm{~N}_{2}=
$$$$
A_{s}=9363
$$$$
D_{n}=
$$$$
T_{1}=600
$$

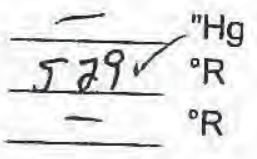

Moisture

Content:

$$
\% \mathrm{M}=16.11 \quad \mathrm{M}_{\mathrm{d}}=0.8389 \quad \mathrm{MW}_{\mathrm{d}}=
$$

$\mathrm{MW}=$

$V m_{s t d}=17.65 \times V m\left[\frac{P_{b}+\frac{P_{m}}{13.6}}{T_{m}+460}\right]=17.65 \times 20.266\left[\frac{2699+\frac{0.300}{13.6}}{69+460}\right]=\frac{18.265}{0.3045 s^{5} s^{5}}$

$V w_{\text {gas }}=0.0472 \times V w=0.0472 \times 7 / 4.3$

$=3.50 \mathrm{~J}$ $\mathrm{sft}^{3}$

$\%$ Moisture $=\frac{V w_{\text {gas }}}{V m_{\text {std }}+V w_{\text {gas }}} \times 100=\frac{3-507}{\mid 8.265+3.5 \sigma} \times 100=16.1,\left.\right|^{2} \%$

$V_{s}=5123.8 \times \sqrt{x} \times-\ldots i p m$

$\% 1=\underline{1039 x}$

$$
x
$$

$x$

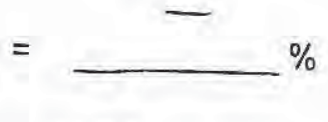

D-34
ACFM:

SCFM:

WSCFH:

$\%$ EA:

Checked by:

Date:

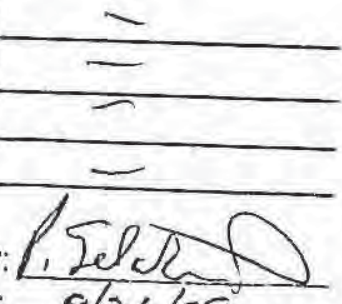

$9 / 26 / 08$ 


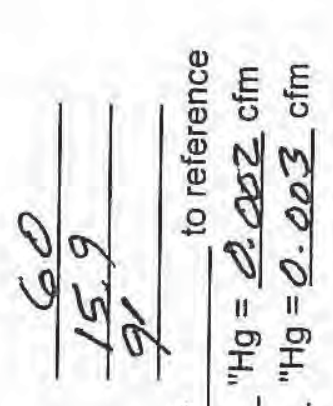

․․․

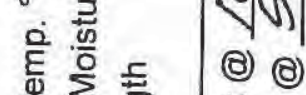

㲅

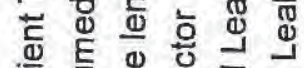

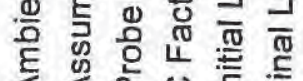

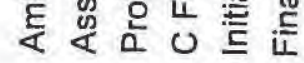

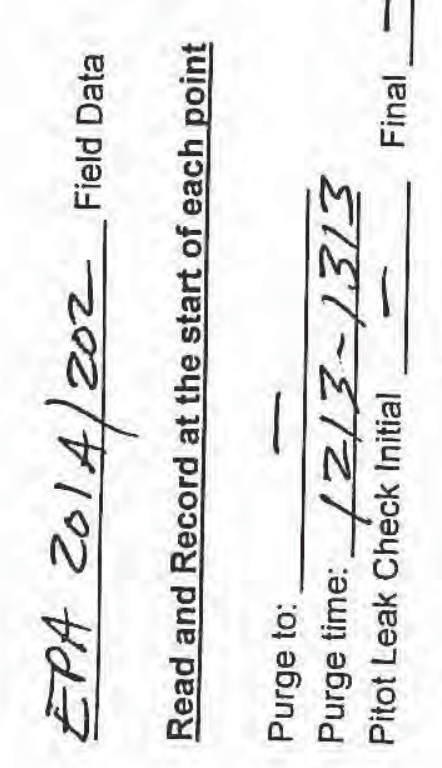

ㄴ. $\mid \log \frac{0}{8}$

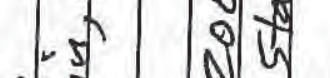

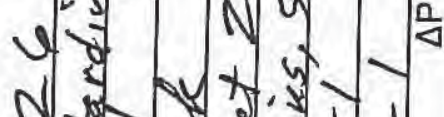

N (ún

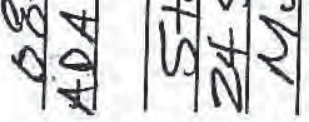

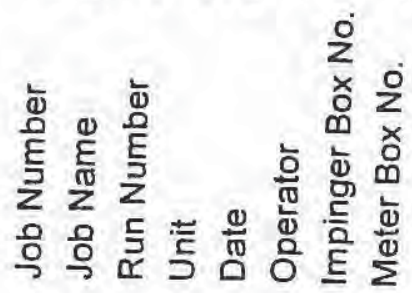

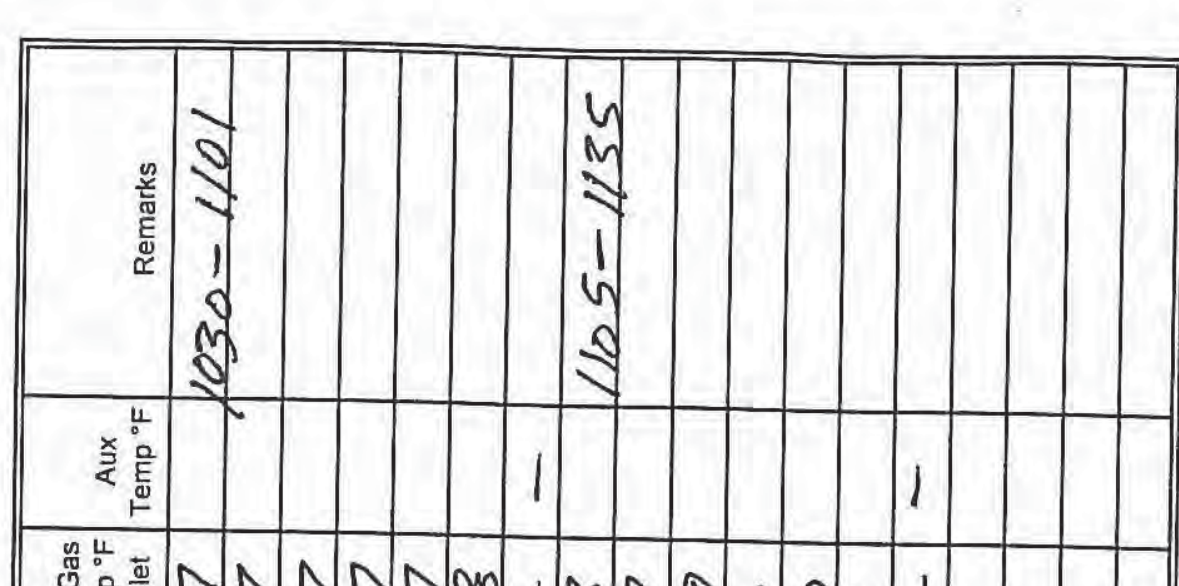

$N$

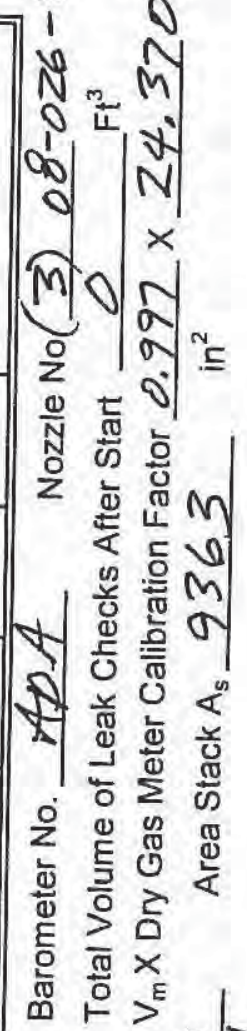

E

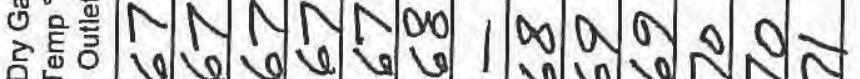

v v MN NNNNN

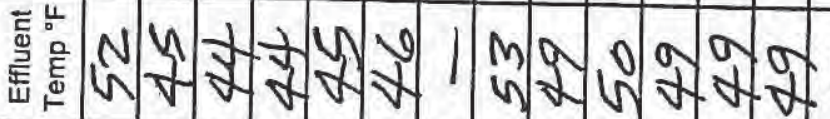

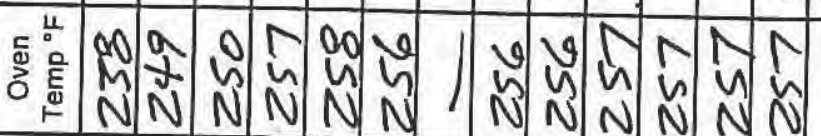

:

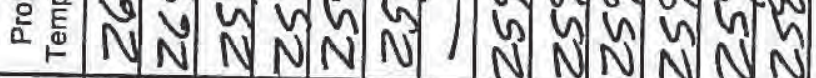

1 क

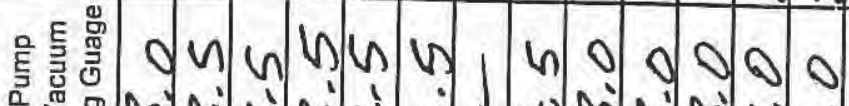

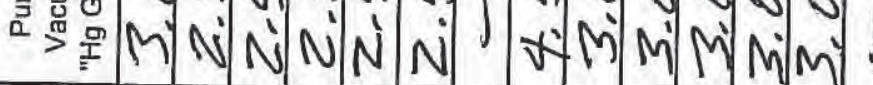

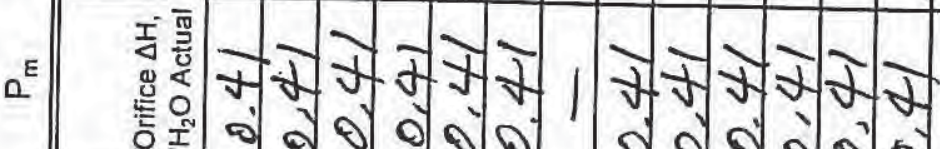

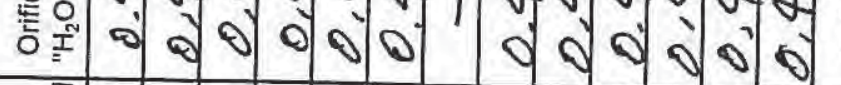

它总

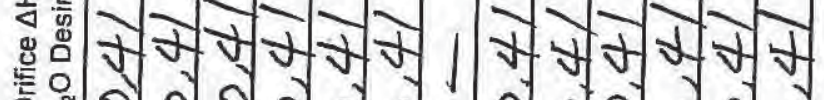

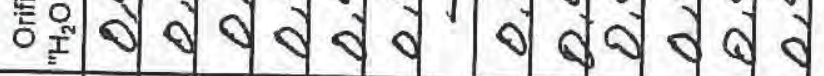

\%०

M M M M M M 1 N $M$ M $M$

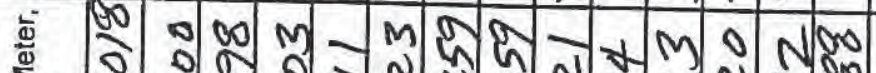

¿ 00 O

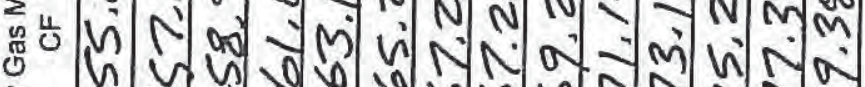

¿NNN 0 Vै

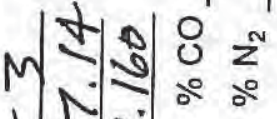

$$
\text { 몯ำ }
$$

1 Noj 0

m $\mathrm{N}$

1 造

之ं क्ष

은 은

\%

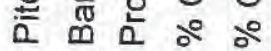


Impinger Bpx No. $\geq-1$

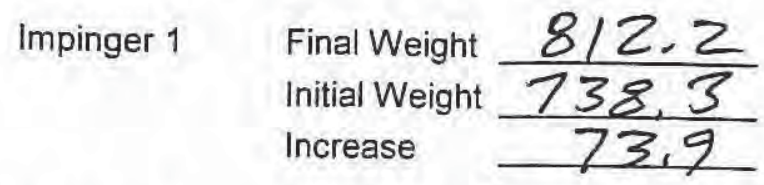

Impinger 2 Final Weight 764,0 Initial Weight 759,6
Increase

Impinger $3 \quad$ Final Weight $\quad 633.7$ Initial Weight 634.1 Increase $-0.4$

Impinger 4

Final Weight 957.1 Initial Weight 945.4 Increase

Impinger 5

Final Weight Initial Weight Increase

Impinger 6

Impinger 7

Impinger 8 Content:
Final Weight Initial Weight Increase

Final Weight Initial Weight Increase

Final Weight Initial Weight Increase
Water Weight Gain

Impinger $1,7,9$

Impinger 24,4

Impinger $3=5,4$

Impinger 411.7

Impinger 5

Impinger 6

Impinger 7

Impinger 8

Total $\quad 89,6^{2}=v_{w}$

Filter No. , Z/

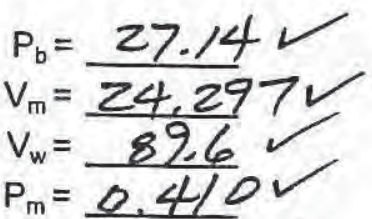

Avg $\Delta \mathrm{P}=3.3 .442$

Sind 4 कs

$A v g \sqrt{\Delta \mathrm{P}}=$

1.853

$c_{p}=0.8 z 3$

$P_{s}=-1.20{ }^{11} \mathrm{H}_{2} \mathrm{O}$

$\mathrm{T}_{\mathrm{m}}=70 \mathrm{~V}{ }^{\circ} \mathrm{F}$

$T_{\mathrm{s}}=210 / \mathrm{F}$
$\% \mathrm{CO}_{2}=13,4$

$\% \mathrm{O}_{2}=6,2$

$\% \mathrm{CO}=0.0$

$\% \mathrm{~N}_{2}=80.4$

$A_{5}=9363$

$D_{n}=0.160$

$T_{1}=61,2$

$27.05^{1 \mathrm{Hg}}$

$5300^{\circ}$

$670 \% \mathrm{R}$

$\mathrm{Vm}_{\mathrm{std}}=17.65 \times \mathrm{Vm}\left[\frac{\mathrm{P}_{\mathrm{b}}+\frac{\mathrm{P}_{\mathrm{m}}}{13.6}}{\mathrm{~T}_{\mathrm{m}}+460}\right]=17.65 \times 24.297\left[\frac{27.14+\frac{0.410}{13.6}}{70+460}\right]=\frac{21.984^{2}}{0.35 \mathrm{stm}^{\mathrm{s}}}$

$V_{w_{\text {gas }}}=0.0472 \times v_{w}=0.0472 \times 89.6=4.229 \vee \mathrm{stt}^{3}$

$\%$ Moisture $=\frac{V w_{\text {gas }}}{V m_{\text {std }}+V w_{\text {gas }}} \times 100=\frac{4.229}{21.984+4,229} \times 100=16,13 \%$

$v_{s}=5123.8 \times 0.823 \sqrt{\frac{670}{27.05 \times 28.39}} \times 1.853=7299 \mathrm{fpm}$

$\% 1=\frac{1039 \times 21.984 \times 670}{0.8387 \times 22.05 \times 7299 \times 61.2 \times(0.160)^{2}}=59.0 \%$

ACFM: 474560

SCFM: 284.6312

WSCFH: $20,362,315299$

$\% \mathrm{EA}$ :

Checked by:

Date:
$4 / .0$ 7.D 0 


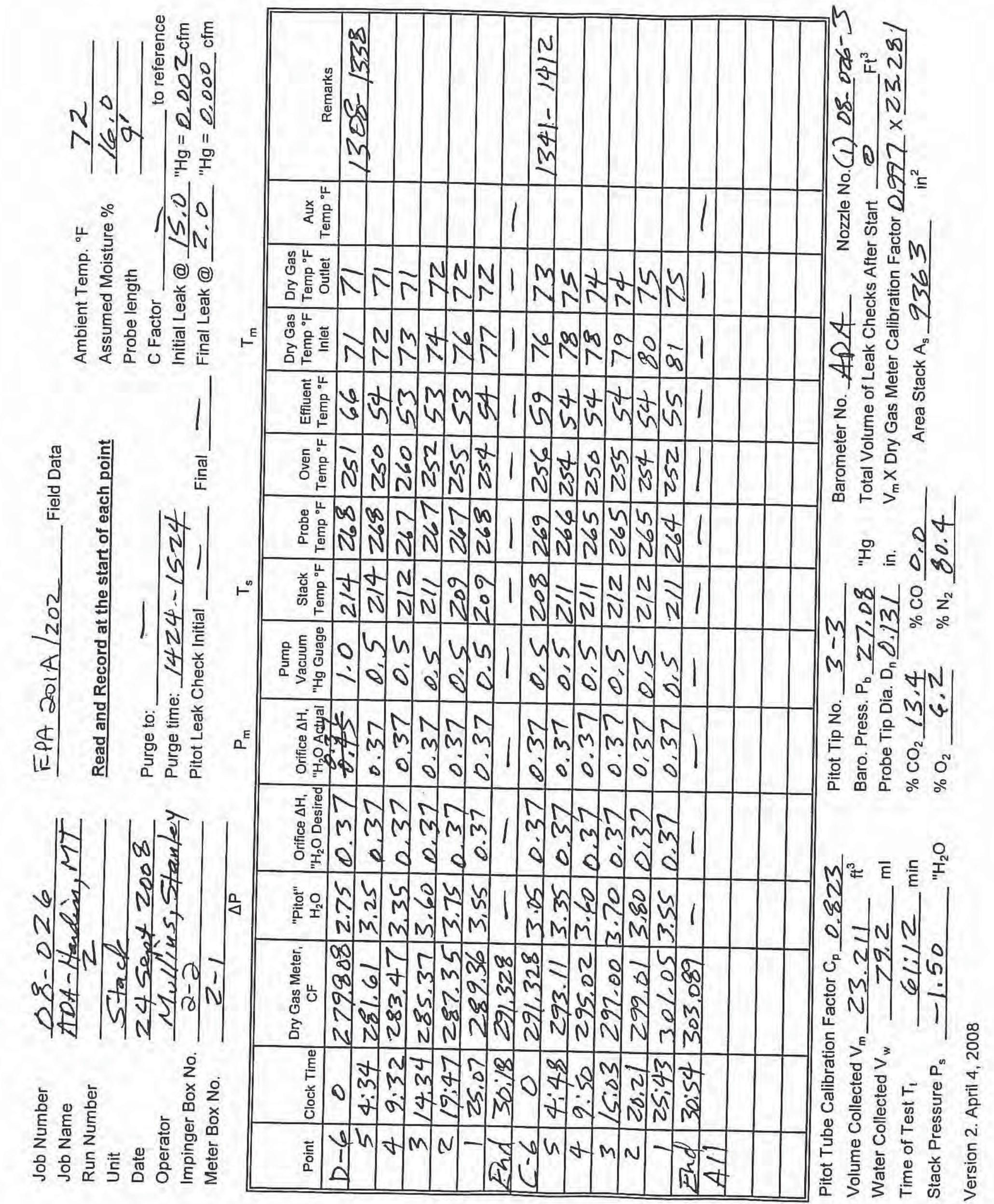


Impinger Bpx No. $2-2$

\begin{tabular}{|c|c|c|}
\hline \multirow[t]{2}{*}{ Impinger 1} & Final Weight & 822.0 \\
\hline & $\begin{array}{l}\text { Initial Weight } \\
\text { Increase }\end{array}$ & $\frac{753.8}{68.2}$ \\
\hline \multirow[t]{2}{*}{ Impinger 2} & Final Weight & 760.3 \\
\hline & $\begin{array}{l}\text { Initial Weight } \\
\text { Increase }\end{array}$ & $\begin{array}{r}755.4 \\
4.9\end{array}$ \\
\hline \multirow[t]{3}{*}{ Impinger 3} & Final Weight & 665.2 \\
\hline & Initial Weight & 664.8 \\
\hline & Increase & 0.4 \\
\hline \multirow[t]{3}{*}{ Impinger 4} & Final Weight & 971.5 \\
\hline & Initial Weight & 965.8 \\
\hline & Increase & \\
\hline
\end{tabular}

Impinger 5 Final Weight

Initial Weight Increase

Impinger $6 \quad$ Final Weight Initial Weight Increase

Impinger 7

Final Weight Initial Weight Increase

Impinger $8 \quad$ Final Weight Initial Weight Increase
Filter No. 22

$P_{b}=27.08$

$V_{m}=23.211$

$v_{w}=79,2$

$P_{m}=0.370$ Avg $\Delta \mathrm{P}=3.442 \mathrm{~V}$ Avg $\sqrt{\Delta \mathrm{P}}=1.853$

$C_{p}=0.823$

$\mathrm{P}_{\mathrm{s}}=-1.50$ " $\mathrm{H}_{2} \mathrm{O}$

$T_{m}=75{ }^{\circ} \mathrm{F}$

$T_{s}=211 / \mathrm{F}$
Water Weight Gain

Impinger $1,68,2$

Impinger 24,9

Impinger $3 \quad 0,4$

Impinger $4 \quad 5,7$

Impinger 5

Impinger 6

Impinger 7

Impinger 8

Total $\quad 79.2^{V}=v_{w}$

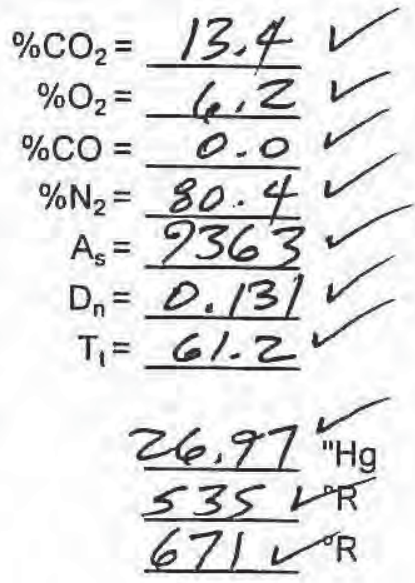

Moisture

Content:

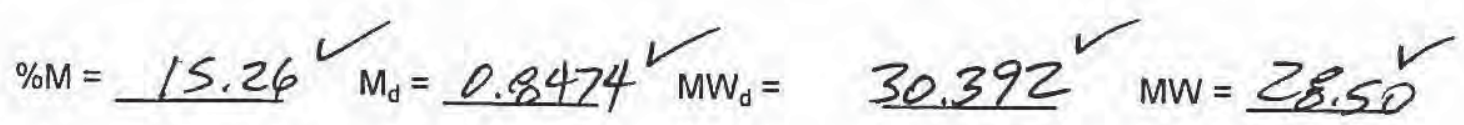

$\mathrm{Vm}_{\text {std }}=17.65 \times \mathrm{Vm}\left[\frac{\mathrm{P}_{\mathrm{b}}+\frac{\mathrm{P}_{\mathrm{m}}}{13.6}}{\mathrm{~T}_{\mathrm{m}}+460}\right]=17.65 \times 23.211\left[\frac{27.08+\frac{0.370}{13.6}}{75+460}\right]=\frac{20.757^{\mathrm{stt}}}{3.39 \mathrm{scfm}^{\mathrm{s}}}$

$V_{w_{\text {gas }}}=0.0472 \times V_{w}=0.0472 \times 79.2=3.738 \vee \mathrm{sft}^{3}$

$\%$ Moisture $=\frac{V w_{\text {gas }}}{V m_{\text {std }}+V w_{\text {gas }}} \times 100=\frac{3.738}{20.757+3.738} \times 100=15.26 \%$

$v_{s}=5123.8 \times 0.823 \sqrt{\frac{671}{26.97^{\times} 28.50}} \times 1.853=7301^{1} \mathrm{fm}$

$\% 1=\frac{1039 \times 20.757 \times 671}{82.6 \%}=8$

ACFM:

74,699

$\% E A$ :

Checked by: BSM

Date: $\frac{11 / 09 / 08}{1 / 08}$ 

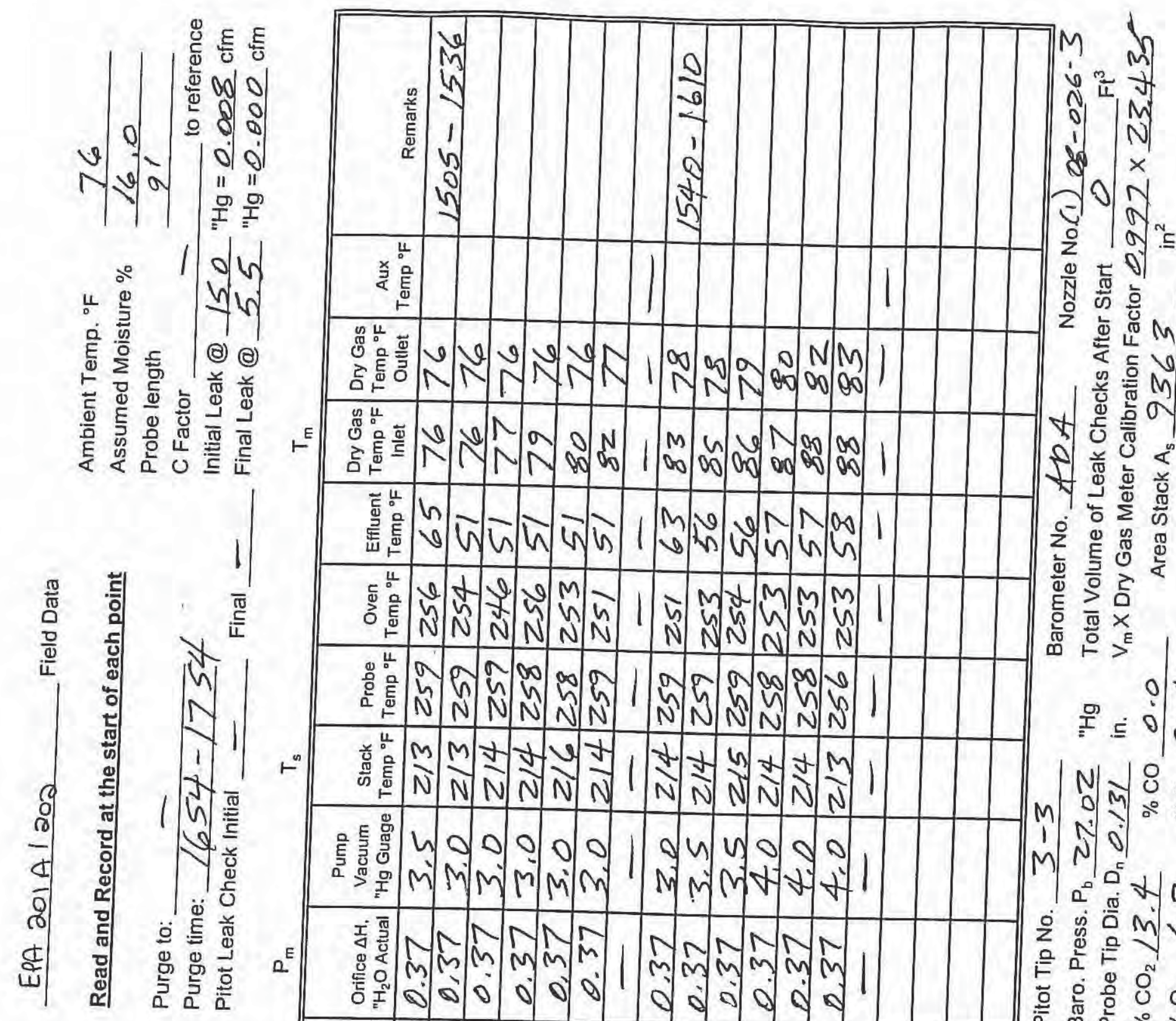

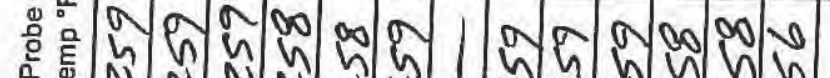

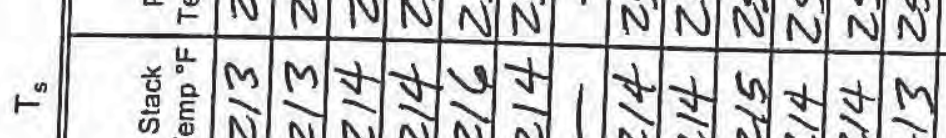

हE

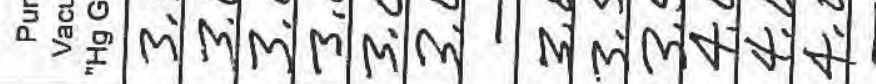

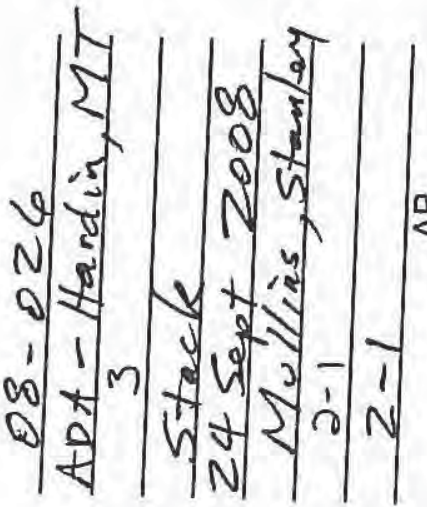

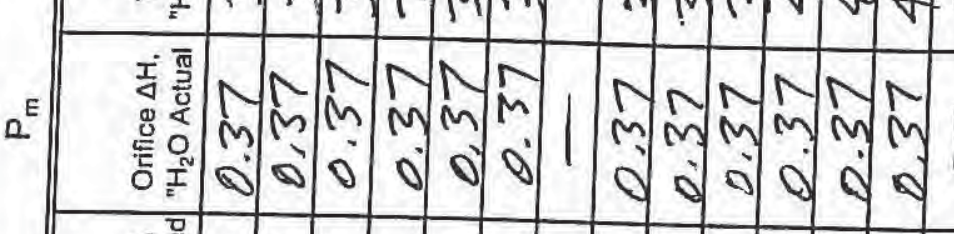

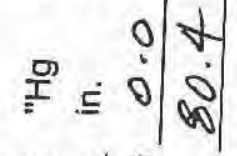
$M \mid$\begin{tabular}{c|c|cc}
$N$ & 0 & 0 & 0 \\
0 & $M$ & $z^{2}$ \\
0 & 0 & 0
\end{tabular}

$M N O$

SMNMN N N N N N N N

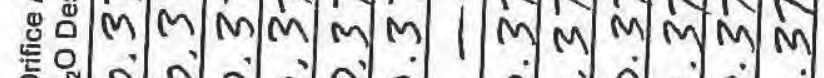

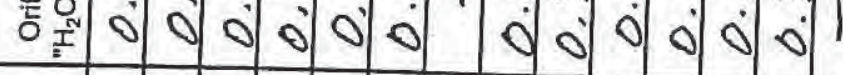

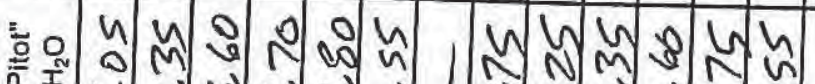

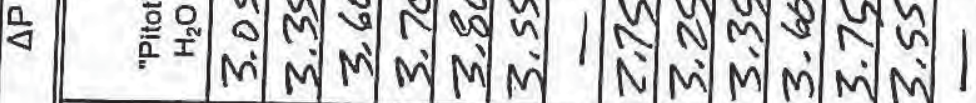

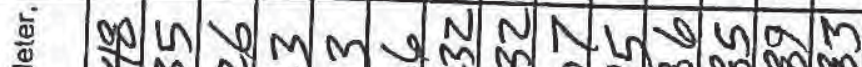

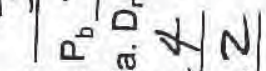

之

$\cong$ 는

은 000

范 范 。

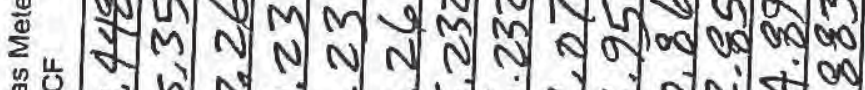

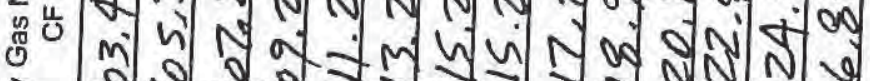

D M MN

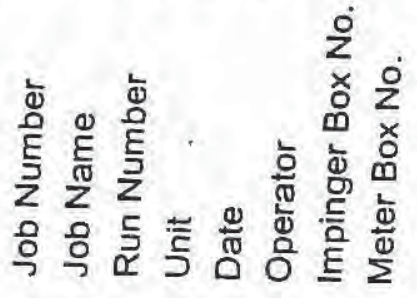

है $00 \mathrm{~m}$ मे

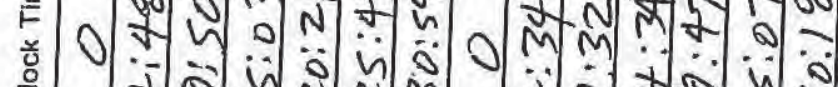

० में

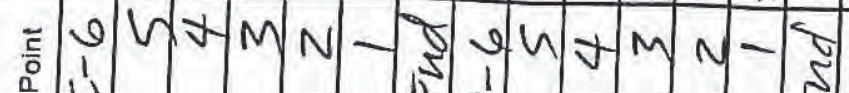

$M / \stackrel{\bar{E}}{\mathrm{~N}} \stackrel{\mathrm{O}}{\underline{\mathrm{N}}}$

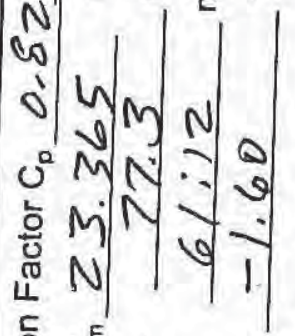

ᄃ ${ }^{\circ} 3$

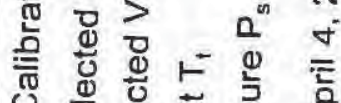

0 耐

¿ 0 广

: 1101010

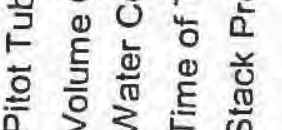


Imipinger Bpx No. 2-1

\begin{tabular}{|c|c|c|}
\hline \multirow[t]{3}{*}{ Impinger 1} & \multirow{3}{*}{$\begin{array}{l}\text { Final Weight } \\
\text { Initial Weight } \\
\text { Increase }\end{array}$} & 813.8 \\
\hline & & 746.1 \\
\hline & & 627 \\
\hline \multirow[t]{3}{*}{ Impinger 2} & \multirow{3}{*}{$\begin{array}{l}\text { Final Weight } \\
\text { Initial Weight } \\
\text { Increase }\end{array}$} & 760,0 \\
\hline & & 756.2 \\
\hline & & 38 \\
\hline \multirow[t]{3}{*}{ Impinger 3} & \multirow{3}{*}{$\begin{array}{l}\text { Final Weight } \\
\text { Initial Weight } \\
\text { Increase }\end{array}$} & 633.6 \\
\hline & & 632.9 \\
\hline & & 0.7 \\
\hline \multirow[t]{3}{*}{ Impinger 4} & Final Weight & 961.9 \\
\hline & \multirow{2}{*}{$\begin{array}{l}\text { Initial Weight } \\
\text { Increase }\end{array}$} & 956.8 \\
\hline & & \\
\hline
\end{tabular}

Impinger 5 Final Weight Initial Weight Increase

Impinger 6

Final Weight Initial Weight Increase

Impinger 7

Final Weight Initial Weight Increase
Final Weight Initial Weight Increase

Impinger 8

Initial Weight
Increase

\section{Filter No. 23 \\ Impinger 8 \\ Total $27,3^{\prime}=v_{w}$}

$P_{b}=27.02$

$v_{m}=23.365$

$v_{w}=72.3$

$P_{m}=0.370$

Avg $\triangle P=3.442$

$$
\text { Avg } \begin{aligned}
v \overline{\Delta \mathrm{P}} & =1.853 \mathrm{~V} \\
\mathrm{C}_{\mathrm{p}} & =0.823 \\
\mathrm{P}_{\mathrm{s}} & =1.60 \\
\mathrm{~T}_{\mathrm{m}} & =80 \mathrm{H}_{2} \mathrm{O} \\
\mathrm{T}_{\mathrm{s}} & =214 \mathrm{~V} \mathrm{~F}
\end{aligned}
$$

Water Weight Gain

Impinger 167.7

Impinger 23.8

Impinger $3 \quad 0,7$

Impinger 45.1

Impinger 5

Impinger 6

Impinger 7

$$
\begin{aligned}
\% \mathrm{CO}_{2} & =\frac{13.4}{6.2} \\
\% \mathrm{O}_{2} & =\frac{0.0}{\% \mathrm{CO}}=\frac{0.4}{8363} \\
\% \mathrm{~N}_{2} & =80.131 \\
\mathrm{~A}_{5} & =93 \\
\mathrm{D}_{\mathrm{n}} & =0.13 \\
\mathrm{~T}_{1} & =61.2
\end{aligned}
$$

Moisture

Content:

$$
\% M=15.01^{V} M_{d}=0.8499^{\circ} M W_{d}=
$$
$30.392^{\circ} \quad M w=28.53^{\circ}$

$V m_{\text {sld }}=17.65 \times V m\left[\frac{P_{b}+\frac{P_{m}}{13.6}}{T_{m}+460}\right]=17.65 \times$ $23.365\left[\frac{27.02^{+} \frac{0.370}{13.6}}{80+460}\right]=\frac{20.656}{0.338 \text { scfm }}$

$\mathrm{Vw}_{\mathrm{gas}}=0.0472 \times \mathrm{Vw}=0.0472 \times 77.3=3.649 \vee \mathrm{stt}^{3}$ $\%$ Moisture $=\frac{V w_{\text {gas }}}{V m_{\text {sid }}+V w_{\text {gas }}} \times 100=\frac{3.649}{20.656+3.649} \times 100=15.01 \%$

$v_{s}=5123.8 \times 0.823 \sqrt{\frac{674}{26.90 \times 28.53}} \times 1.853=2323 \mathrm{fpm}$ $\% 1=\frac{1039 \times 20.656 \times 674}{0.8499 \times 26.90 \times 7323 \times 6 / .2 \times(0.131)^{2}}=82.3^{\circ} \%$
ACFM:

SCFM: WSCFH:

\%EA:

Checked by: BTM

Date: $11 / 09 / 08$ 
Impinger Bpx No. $2-2$

$\begin{array}{ll}\text { Impinger 1 } & \text { Final Weight } \frac{828,6}{258,4} \\ & \begin{array}{l}\text { Initial Weight } \\ \text { Increase }\end{array}\end{array}$

Impinger 2 Final Weight $\frac{766,5}{763.5}$

Initial Weight 763.5

Increase

3. 0

Impinger 3 Final Weight -665.2

Initial Weight $\frac{664.5}{0.7}$
Increase

Impinger $4 \quad$ Final Weight $\frac{976,5}{971.4}$

Increase $\frac{9.1}{5.1}$

Impinger $5 \quad$ Final Weight

Initial Weight

Increase

Impinger $6 \quad$ Final Weight

Initial Weight

Increase

Impinger $7 \quad$ Final Weight

Initial Weight

Increase

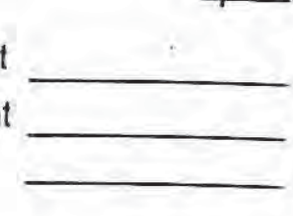

Filter No. 24

$P_{b}=26.99$

$v_{m}=23.0040$

$v_{w}=79,0$

$P_{m}=0.370 \mathrm{~V}$

Avg $\triangle \mathrm{P}=3.442$

Avg $v \overline{\Delta \mathrm{P}}=1.853$

$C_{p}=0.823$

$\mathrm{P}_{\mathrm{s}}=-1.10 \mathrm{H}_{2} \mathrm{O}$

$T_{m}=78{ }^{\circ} \mathrm{F}$

$T_{s}=2 / 1 /{ }^{\circ} \mathrm{F}$
Water Weight Gain

Impinger 1 70. 2

Impinger $2,3,0$

Impinger $3 \ldots 0.7$

Impinger 451

Impinger 5

Impinger 6

Impinger 7

Impinger 8

Total $29.0=v_{w}$

$\% \mathrm{CO}_{2}=13,4$

$\% \mathrm{O}_{2}=\frac{6,2}{6,2}$

$\% \mathrm{CO}=0,0$

$\% \mathrm{~N}_{2}=80,4$

$A_{s}=9363$

$D_{\mathrm{n}}=0.131$

$T_{1}=6 / .2$

$$
\frac{26.91 \mathrm{Hg}}{538}
$$

Initial Weight

Increase

Moisture

Content:

$$
\% M=15.46^{\prime} \quad M_{d}=0.84544^{\prime} \mathrm{MW}_{d}=
$$

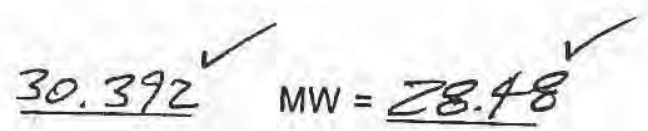

$V m_{s t d}=17.65 \times V m\left[\frac{P_{b}+\frac{P_{m}}{13.6}}{T_{m}+460}\right]=17.65 \times 23.004$

$$
\left[\frac{26.99+\frac{0.370}{13.6}}{78+460}\right]=\frac{20.389^{2} \mathrm{stt}^{\circ}}{0.333^{2}} \mathrm{scfm}
$$

$V w_{\text {gas }}=0.0472 \times V w=0.0472 \times$

79.0 $=3.729 \curvearrowleft \mathrm{sfi}^{3}$

$\%$ Moisture $=\frac{V W_{\text {gas }}}{V m_{\text {sid }}+V_{W_{\text {gas }}}} \times 100=\frac{3.729}{20.389+3.729} \times 100=15.46 \%$

$v_{s}=5123.8 \times 0.823 \sqrt{\frac{671}{26.91 \times 28.48}} \times 1.853=7311 \mathrm{fpm}$

$\% 1=1039 \times 20.38 \% \times 671$

$0.8454 \times 26.91 \times 7311 \times 61.2 \times(0.131)^{2}$
$=81.4 \%$

D-42
ACFM: $\quad 475,395$

SCFM:

285463

$\%$ EA: $20,259,99+172$ 41.0 ,

Checked by: Korel

Date: $11 / 09 / 08$ 


\section{Appendix E:}

EPA Test Methods 5B and 202 (Particulate Matter) Analytical Data Stack 
AIR

SAMPLING

ASSOCIATES, INC.

Air Sampling Associates, Inc.

Particulate Analysis Summary EPA Method $5 / 202$

Project Number: $08-026$

Project Name: ADA -ES
Date Analysis Completed: $10-13-08$

Unit Tested: Stack

Project Location: Hardin, MT

Run Number:

Particulate Matter on Filter (mg):

Particulate Matter in Front Wash (mg):

Total Particulate Matter in "Front-Half" - MF (mg):

Particulate Matter in “Back-Half” (mg):

Total Particulate Matter in Sample - MT (mg):

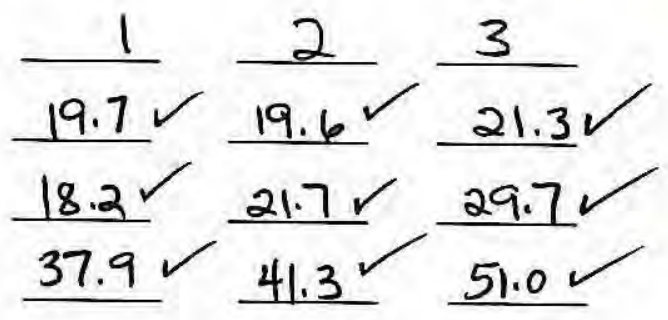

$365.7 \checkmark 373.8 \checkmark 3525$

$403.6^{\checkmark} 415.1^{\checkmark} 403.5^{\prime}$
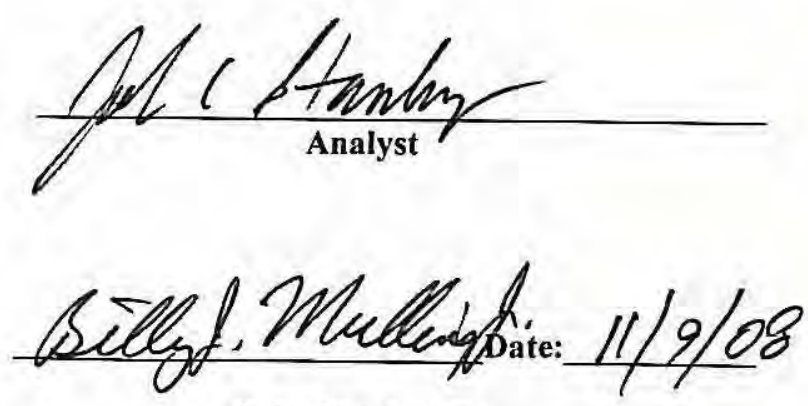

Data Checked By

Version No. 1 5/30/06

E-2 
Particulate Analysis EPA Method $5 / 202$

Stack Filters

Project No. $08-026$

Project Name ADA-ES
Location Handin, $m \pi$ Unit Tested

stack

\begin{tabular}{|c|cc|cc|c|c|c|}
\hline Desiccator Time In & $10-1$ & 0900 & 10.3 & 0908 & & & \\
\hline Desiccator Time Out & $10-3$ & 0900 & $10-6$ & 0900 & & & \\
\hline
\end{tabular}

\begin{tabular}{|c|c|c|c|c|}
\hline \multicolumn{2}{|c|}{ Run No. Iilter No. A239 } & \multicolumn{3}{c|}{ Sample I.D. } \\
\hline Filter \& Particulate (g) & 0.3955 & 0.3954 X & & \\
\hline
\end{tabular}

Filter \& Particulate Average (g) 0.3954 Initial Filter Weight $(\mathrm{g}) \quad 0.3757$. Total Particulate (mg) 19.7

\begin{tabular}{|c|c|c|c|}
\hline Run No. 2 & & Filter No. A 240 & Sample I.D. \\
\hline Filter \& Particulate $(\mathrm{g})$ & 0.3956 & 0.3956 & \\
\hline
\end{tabular}

Filter \& Particulate Average $(\mathrm{g}) \quad 0.3956$ Initial Filter Weight $(\mathrm{g})$

Total Particulate $(\mathrm{mg})$

0.3760 19.6

\begin{tabular}{|c|c|c|c|c|}
\hline \multicolumn{2}{|c|}{ Fun No. 3 No. A 24/ } & \multicolumn{1}{c|}{ Sample I.D. } \\
\hline Filter \& Particulate (g) & 0.3946 & 0.39514 & & \\
\hline
\end{tabular}

Filter \& Particulate Average (g) 0.3951 Initial Filter Weight $(\mathrm{g}) \quad 0.3738$ Total Particulate $(\mathrm{mg}) \quad 21.3$

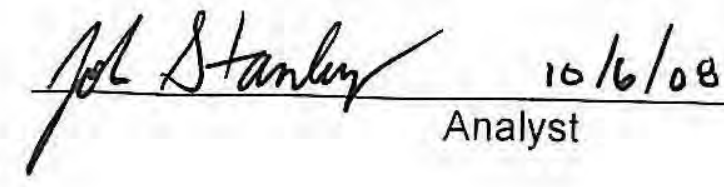


ASSOCIATES, INC.

Thimble Tare Weight Log

EPA Method 5

\begin{tabular}{|c|c|c|c|c|c|c|c|}
\hline \multirow{2}{*}{ Filter No. } & Into Desicator & Weight & Weight & Weight & Weight & Weight & \multirow{2}{*}{$\begin{array}{c}\text { Weight } \\
\text { Used }\end{array}$} \\
\hline & $\begin{array}{l}\text { Date } \\
\text { Time }\end{array}$ & \begin{tabular}{l|l} 
Date \\
Tin
\end{tabular} & \begin{tabular}{|l} 
Date \\
\end{tabular} & Date & Date & Date & \\
\hline$A$ & Time & $\frac{\text { Time }}{0.3433}$ & $\frac{\text { Time }}{10.3422}$ & Time & Time & Time & $\frac{\text { Used }}{0.3436}$ \\
\hline 226 & $10 / 1107$ & $12 / 7 / 34$ & $12 / 7 / 07$ & & & & \\
\hline 226 & 0800 & 1030 & 1645 & & & & \\
\hline$A$ & & 0,3489 & 0.3490 & $\swarrow$ & & & 0.3490 \\
\hline 227 & $\begin{array}{l}10 / 1107 \\
0800\end{array}$ & $\frac{12 / 7 / 07}{1041}$ & $\frac{12 / 7 / 07}{1645}$ & & & & \\
\hline$A$ & & 0.3461 & 0.3462 & 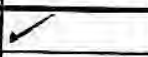 & & & 0.3462 \\
\hline 228 & $\frac{10 / 1 / 07}{0860}$ & $\begin{array}{l}12 / 7 / 67 \\
1040\end{array}$ & $12 / 7 / 07$ & & & & \\
\hline$A$ & & 0.3630 & $\frac{646}{0.3630}$ & $\checkmark$ & & & $0363 n$ \\
\hline & $10 / 1107$ & $12 / 7 / 07$ & $1 2 \longdiv { 7 1 0 7 }$ & & & & \\
\hline 227 & 6800 & 1040 & 1647 & & & & \\
\hline$A$ & & 0.3711 & 0.3710 & 7 & & & 0.3710 \\
\hline 230 & $\frac{10 / 1 / 07}{0800}$ & $12 / 7 / 071$ & $\frac{12,2107}{1645}$ & & & & \\
\hline$A$ & & 0.3701 & 0.3700 & $V$ & & & 0.3700 \\
\hline 231 & $\frac{10 / 1107}{6800}$ & $|2 / 7 / 07|$ & $\frac{12 / 7 / 07}{1648}$ & & & & \\
\hline$A$ & & 0.3706 & 0.3106 & $r$ & & & 0.3706 \\
\hline 232 & $10 / 1 / 01$ & $12 / 7 / 07$ & $12 / 7 / 07$ & & & & \\
\hline$A$ & 0800 & $\frac{1638}{0.3631}$ & $\frac{1645}{0.3629}$ & & & & $107 / 20$ \\
\hline 233 & $10 / 1107$ & 227107 & $12 \sqrt{2107}$ & & & & \\
\hline$A$ & 6800 & 1635 & 1648 & & & & \\
\hline 234 & 101107 & 0.3774 & 0.3773 & $レ$ & & & 0.3773 \\
\hline 234 & 0800 & 1635 & $2 / 7709$ & & & & \\
\hline 235 & & $0,3800 \mathrm{~K}$ & 0.3800 & $\checkmark$ & & & 0.3800 \\
\hline 235 & $10 / 1 / 07$ & $12 / 2) 07$ & $12 / 7 / 07$ & & & & \\
\hline$A$ & & 0.374 & $\frac{1650}{0.3798}$ & $\checkmark$ & & & $0 \pi 796$ \\
\hline 236 & $10 / 1107$ & $1213 / 07$ & $12 / 2107$ & & & & \\
\hline$\frac{\operatorname{cs} 6}{A}$ & 0800 & lo3n & $165 \mid$ & & & & \\
\hline 237 & 10110 os 7 & $\frac{0.3790}{213 \ln 7}$ & 0.3793 & $\checkmark$ & & & 0.3793 \\
\hline$\frac{231}{A}$ & 0800 & 41030 & $\frac{14101}{1652}$ & & & & \\
\hline $\begin{array}{c}A \\
238\end{array}$ & & 0.3739 & 0.3739 & & & & 0,3739 \\
\hline 238 & 0220108 & $02 / \lambda_{1} / p^{9}$ & $02 / 2160$ & & & & \\
\hline$A$ & 8900 & $\frac{0950}{0.3757}$ & $\frac{14.50}{0.3757}$ & & & & $5>2,7$ \\
\hline 239 & $02 / 20 / 09$ & 1212103 & 021.21168 & & & & 23157 \\
\hline & 6960 & 0950 & 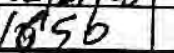 & & & & \\
\hline A & कhal & 0.3708 & 0.37064 & 0.3760 & 3260 & $Z$ & 33706 \\
\hline 240 & $\begin{array}{c}02 / 20100 \\
1900\end{array}$ & $\frac{22 / 216810}{0950}$ & $\frac{02 / 210002}{1 / 651}$ & $2 / 21 / 08$ & $\frac{2121108}{658}$ & & \\
\hline
\end{tabular}




\section{AIR \\ SAMIPLING}

ASSOCIATES, INC.

Thimble Tare Weight Log

EPA Method 5

\begin{tabular}{|c|c|c|c|c|c|c|c|}
\hline \multirow[b]{3}{*}{ Filter No. } & Into Desicator & Weight & Weight & Weight & Weight & Weight & \multirow{3}{*}{$\begin{array}{l}\text { Weight } \\
\text { Used }\end{array}$} \\
\hline & Date & Date & Date & Date & Date & Date & \\
\hline & Time & Time & Time & Time & Time & Time & \\
\hline$A$ & & 0.3739 & 0.3738 & 7 & & & 0.3738 \\
\hline 241 & $32 / 20 / 08$ & $02 / 21108$ & $02 / 21 / 0^{8}$ & & & & \\
\hline$A$ & $0 / 00$ & 0.3707 & 0.37064 & 7 & & & 0.3706 \\
\hline 242 & $02 / 20 / 08$ & $02 / 21 / 0$ & be/21/06 & & & & \\
\hline$A$ & 0900 & $\frac{0953}{6.3737}$ & $\frac{1655}{0.3739}$ & & & & 03729 \\
\hline il? & $02 / 20 / 08$ & $2 2 \longdiv { 2 1 1 0 8 }$ & $02 / 2170$ & & & & \\
\hline 245 & 0900 & 0954 & $1655^{t}$ & & & & \\
\hline$A$ & $02 / 20108$ & 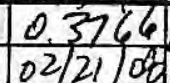 & $\frac{03765}{102721 / 8}$ & 7 & & & 0.3765 \\
\hline 244 & 0900 & 0955 & $\begin{array}{l}102 / 21 / 08 \\
1655\end{array}$ & $\zeta$ & & & \\
\hline$A$ & (5) & $0.37 x_{44}$ & 0.3792 & & & & \\
\hline 45 & $082 / 267.8$ & $5 / 29608$ & $5(3668)$ & & & & \\
\hline 243 & 12500900 & 1300 & 1059 & & & & \\
\hline D & & $0.37 / 4$ & 0.37134 & & & & \\
\hline & 02120108 & $5 / 29108$ & $6 / 30 / 08$ & & & & \\
\hline 246 & 0900 & 1300 & 1059 & & & & \\
\hline$A$ & & 0.3758 & 0.37564 & & & & \\
\hline 247 & $02 / 20108$ & $5 / 29108$ & $5 / 30 / 08$ & & & & \\
\hline & 0900 & 1301 & 1058 & & & & \\
\hline A & & 0.3780 & 0.37784 & & & & \\
\hline 248 & $02 / 20108$ & $s(29 / 08)$ & $530 / 08$ & & & & \\
\hline 248 & 0900 & 1302 & 1057 & & & & \\
\hline & $02(20 / 68$ & $\frac{0.377}{5 \% 2908}$ & $\frac{0.3775^{5}}{5 / 30 / 08}$ & & & & \\
\hline 249 & $0.90 \Delta$ & 1303 & 1056 & & & & \\
\hline$A$ & & 0.3763 & 0.37604 & & & & \\
\hline 150 & $02 / 20 / 08$ & $5 / 29108$ & $5730 \% 8$ & & & & \\
\hline 250 & 0900 & 1305 & 1055 & & & & \\
\hline$A$ & & 0.3736 & 0.37327 & & & & \\
\hline 251 & $02 / 20 / 08$ & $6 / 29108$ & $5 / 30 / 08$ & & & & \\
\hline a) & 0900 & 1306 & 1104 & & & & \\
\hline$H$ & $02 / 20 / 08$ & $\begin{array}{l}0,375) \\
5 / 2968\end{array}$ & $\begin{array}{l}0.3749^{5} \\
5130108\end{array}$ & & & & \\
\hline 252 & 0900 & 1307 & 1,03 & & & & \\
\hline & & 0.3784 & 0,37800 & & & & \\
\hline & $02 / 2008$ & 5729108 & $5 \sqrt{30} 0 \mathrm{~kg}$ & & & & \\
\hline & 0900 & 1308 & 1102 & & & & \\
\hline$A$ & & 0,3758 & $13755^{\circ}$ & & & & \\
\hline 254 & $02 / 20 / 08$ & $5 / 25108$ & $5 / 30 / 08$ & & & & \\
\hline & $0\{\infty$ & 1310 & 1101 & $\angle$ & & & \\
\hline A & & 0.3806 & 0,38034 & & & & \\
\hline & $\frac{02 / 20 / 08}{0900}$ & $\frac{5(29 / 08}{13 \sqrt{3}}$ & $\frac{5 / 30 / 68}{1100}$ & & & & \\
\hline
\end{tabular}




\section{Im

Particulate Analysis EPA Method $5 / 202$

Front Wash

Project No. $08-026$

Project Name ADA-ES

Location Hardin, MT
Unit Tested Stack

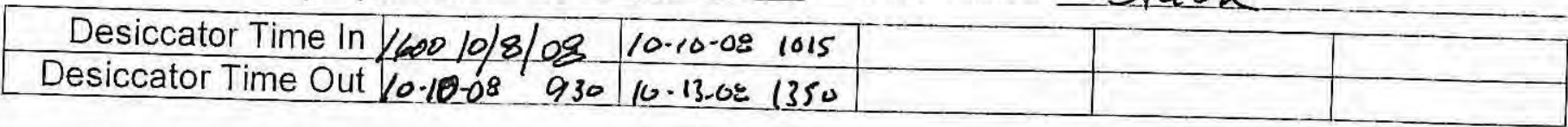

\begin{tabular}{|c|c|c|c|c|}
\hline Run No. I & \multicolumn{3}{|c|}{ Volume (ml) 83} & \\
\hline Final Weight $(\mathrm{g})$ & 103.4265 & 103.4267 & & \\
\hline Initial Weight $(\mathrm{g})$ & & 103.4082 & & \\
\hline Particulate Weight $(\mathrm{g})$ & & 0.0185 & & \\
\hline
\end{tabular}

Particulate Average (mg)

Less Acetone Blank (mg)

Total Particulate $(\mathrm{mg})$

18.5

Q.3

\begin{tabular}{|c|c|c|c|}
\hline Run No. 2 & & Volume $(\mathrm{ml}) \quad 108$ & Sample ID \\
\hline Final Weight (g) & 113.7187 & 113.7189 & \\
\hline Initial Weight (g) & & $113.6967 \%$ & \\
\hline Particulate Weight (q) & & 0.0222 & \\
\hline
\end{tabular}

Particulate Average (mg)

Less Acetone Blank (mg)

22.2

Total Particulate (mg)

0.5

21.7

\begin{tabular}{|c|c|c|c|c|}
\hline \multicolumn{3}{|c|}{ Run No. 3 } & \multicolumn{3}{|c|}{ Solume $(\mathrm{ml})$} & 86 & \\
\hline Final Weight $(\mathrm{g})$ & 103.6238 & 103.6238 & & \\
\hline Initial Weight $(\mathrm{g})$ & & 103.5937 & & \\
\hline Particulate Weight $(\mathrm{g})$ & & 0.0301 & & \\
\hline
\end{tabular}

Particulate Average (mg) Less Acetone Blank (mg)

Total Particulate $(\mathrm{mg})$

30.1

0.4

29.7

\begin{tabular}{|c|c|c|c|c|}
\hline \multicolumn{2}{|l|}{ Acetone Blank } & Volume $(\mathrm{ml}) \quad 190$ & \multicolumn{2}{|l|}{ Sample I.D. } \\
\hline Final Weight $(\mathrm{g})$ & 113.7708 & $113.7706 \mathrm{~J}$ & & \\
\hline Initial Weight (g) & & $113.7698 \mathrm{~W}$ & & \\
\hline Particulate Weight (g) & & 0.00084 & & \\
\hline te: if greater than 7.9 & & & $\begin{array}{r}\text { Average (mg) } \\
{ }^{*} \mathrm{mg} / \mathrm{l}\end{array}$ & $\begin{array}{l}0.8 \\
4.2 \quad\end{array}$ \\
\hline
\end{tabular}




\section{II}

Particulate Analysis EPA Method $5 / 202$

Front Half Tare Weights

Project No. $08-026$

Project Name ADA-ES

Location Handin, MT Unit Tested Stack

\begin{tabular}{|c|cc|cc|cc|cc|c|}
\hline Desiccator Time In & $10-1$ & 0845 & $10-2$ & 1043 & $10-3$ & 840 & $10-6$ & 0915 & \\
\hline Desiccator Time Out & $10-2$ & 1039 & $10-3$ & 835 & $10-6$ & 0900 & $10-7$ & 0830 & \\
\hline
\end{tabular}

\begin{tabular}{|l|}
\hline Run No. 1 \\
\hline Weigh (g)
\end{tabular} Weight (g)

$103.4079103 .40824 \quad$ Sample I.D.

Run No. 2 Weight (g)

\begin{tabular}{c|c} 
Sample I.D. \\
6962 & $113.6967 \mathrm{~V}$
\end{tabular}

\begin{tabular}{l|l|l|l} 
Run No.3 & Sample I.D. \\
Weight (g) & 103.5934 & $103.5937 \mathrm{X}$ &
\end{tabular}

Acetone Blank Weight (g)

$113.6958 \mid 113.6975$
$103.5934 \mid 103.5937$

\begin{tabular}{|c|}
\hline Run No.3 \\
\hline Weight $(\mathrm{g})$ \\
\hline
\end{tabular} $k$ $1 / 13.7694 \mid 1 / 3.76$ Sample I.D.

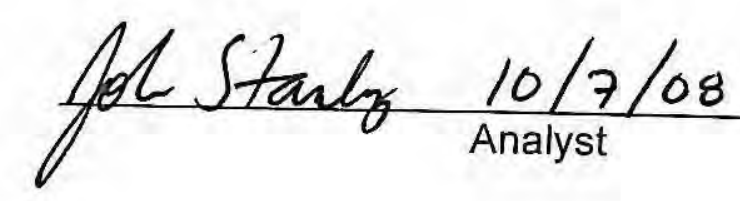




\section{Methylene Chloride Rinse}

\begin{abstract}
Job Number
Client Name

Unit Name
\end{abstract}

08-4117

Air Sampling Associates- Hardin MT

Stack-Impingers 1 \& 2

$\begin{array}{rr}\text { BEAKER } & \text { A } 2372 \\ & 109.3843 \\ 109.3776 \\ 0.0067\end{array}$

Run Date $9 / 23-24 / 08$

Method: 202
BEAKER

RUN 2

9/23/2008

Beaker + Particulate (g)

Beaker Tare (g)

Particulate Weight (g)

\section{A 2374 \\ 96.5967 \\ 96.5957 \\ 0.0010}

\begin{tabular}{|c|c|c|}
\hline $\begin{array}{l}\text { RUN 3 } \\
\text { 9/24/2008 }\end{array}$ & BEAKER & A 2376 \\
\hline Beaker + Particulate $(g$ & & 104.2502 \\
\hline Beaker Tare (g) & & 104.2492 \\
\hline Particulate Weight (g) & & 0.0010 \\
\hline
\end{tabular}

0.0010
RUN 1
9/23/2008
Beaker + Particulate $(g)$
Beaker Tare $(g)$
Particulate Weight $(g)$

VOLUME
109.3845
109.3779
0.0066

VOLUME

96.5966

96.5957

0.0009

VOLUME

104.2503

104.2492

0.0011
AVERAGE

109.3844

109.3778

0.0066

\section{AVERAGE}

96.5967

96.5957

0.0010

AVERAGE

104.2503

104.2492

0.0011

$9 / 25 / 2008$

$10 / 12 / 2008$ 


\section{Methylene Chloride Rinse}

\begin{abstract}
Job Number
Client Name

Unit Name
\end{abstract}

08-4117

Air Sampling Associates- Hardin, MT Stack-Reagent Blank

Method: 202

Run Date $9 / 24 / 2008$

BLANK

BEA

BEAKER

A 2386

112.7456

Beaker + Particu
Beaker Tare (g)

Particulate Weight (g)
112.7449

0.0007

VOLUME
112.7457
112.7448
0.0009

$\mathrm{ml} \quad 230$

AVERAGE

112.7457

112.7449

0.0008

Analyst

KP
Started

Completed
$9 / 25 / 2008$

$10 / 12 / 2008$ 


\section{Water Analysis (Back)}

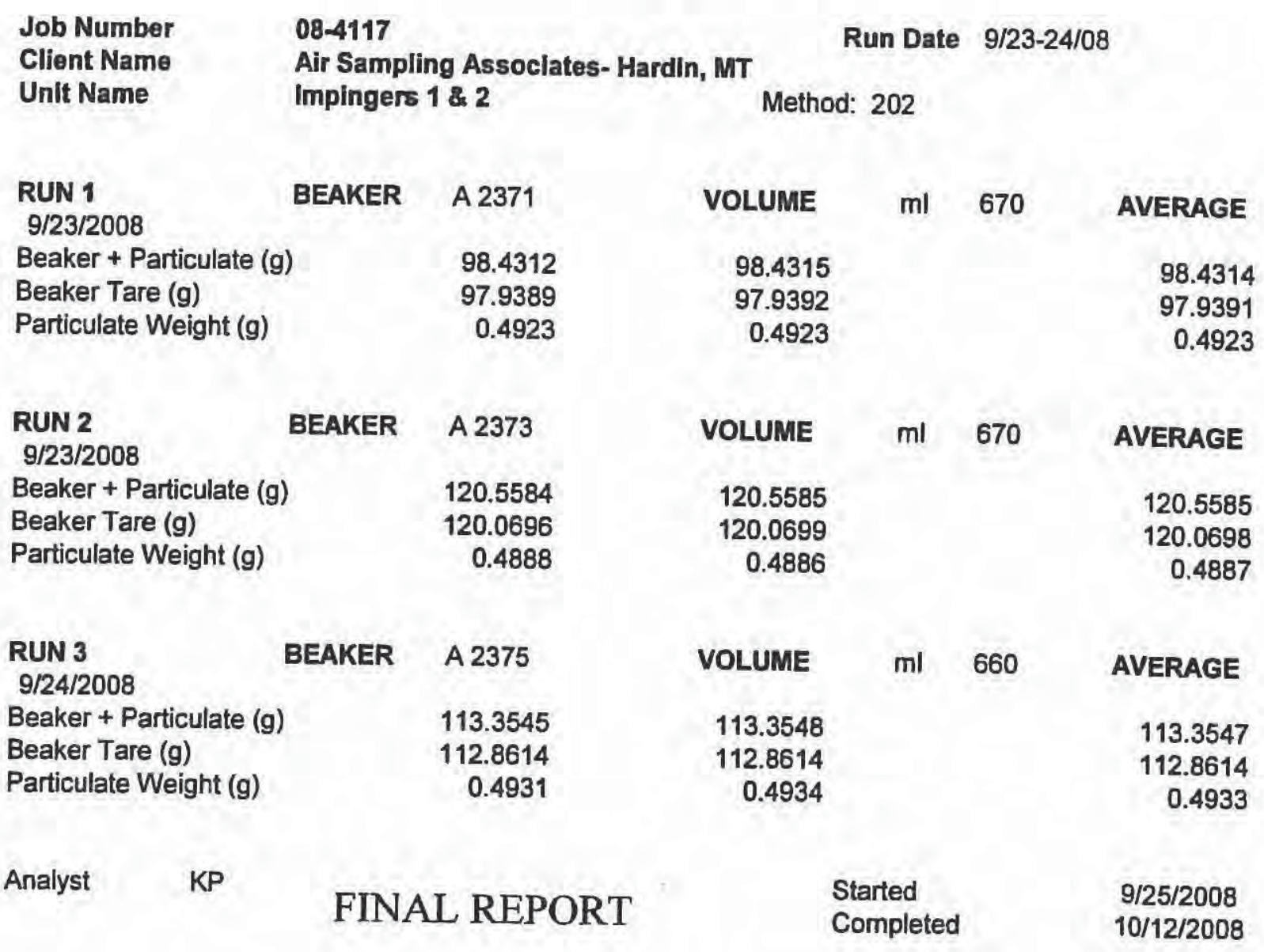


DI Water Analysis (Back)

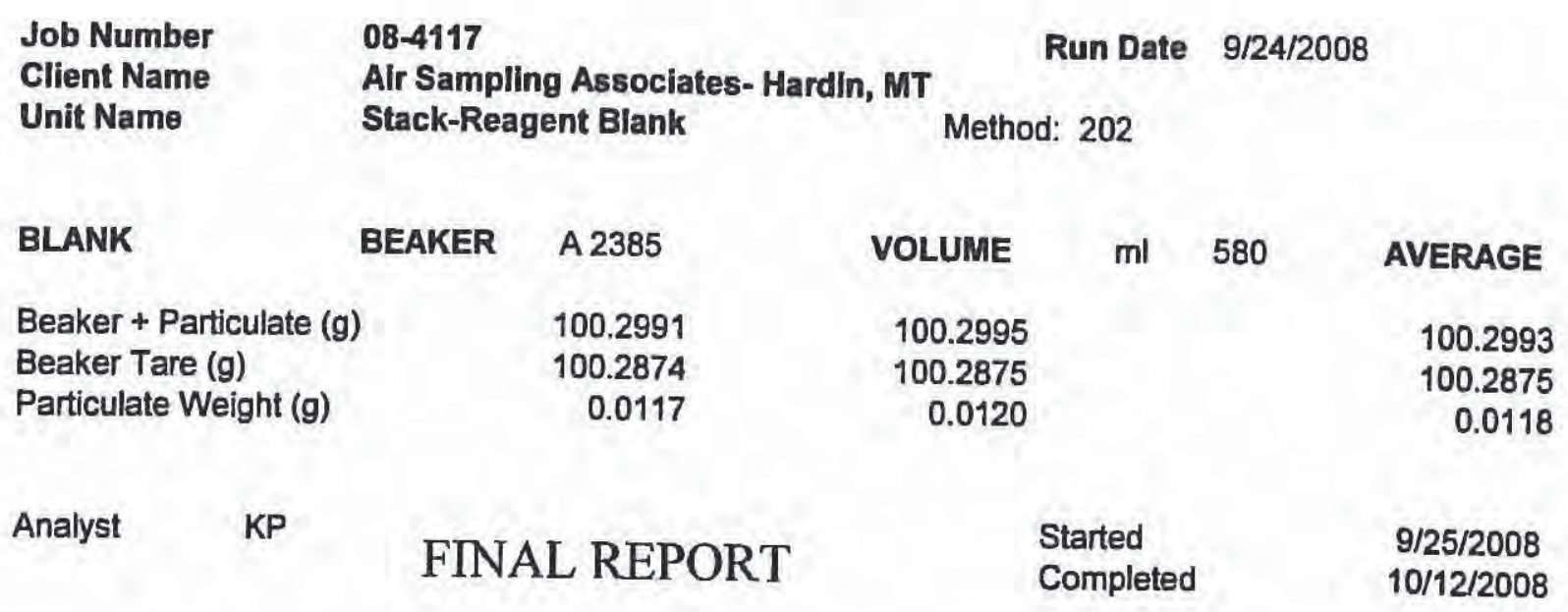


Sulfate

ADA-ES Run $1_{\text {Stack }}^{9 / 23-24 / 2008} 5 B / 202$

Run 2 Stack

Run 3 Stack

ADA-ES $\operatorname{Run}^{9 / 24 / 2008} \underset{\text { Stack }}{\mathrm{Z}} \mathrm{Ol} A / 202$

Run 2

Run 3

Run 4
Stack

Stack

Stack

$08-4117$

$\begin{array}{cccc}\text { Sample } & \text { Lab } & \text { Lab } & \text { Final } \\ \text { Volume, } & \text { Volume, Results, Results, }\end{array}$

Sample No. 4117-1

4117-2

$4117-3$ $\mathrm{ml}$

590

590

580

320

320

4117-5

$4117-6$

4117-7

$4117-4$

320

320

320 $\mathrm{ml}$

20

20

20

Sample No.
20

20

20

20 $\mathrm{mg} / \mathrm{l}$

569

118.841

$486 \quad 101.506$

$622 \quad 127.709$

243

252

307

204

23.109 
Chloride

$08-4117$

Chloride

08-4117

ADA-ES

$\begin{array}{ll}\text { Run } 1 & \text { Stack } \\ \text { Run } 2 & \text { Stack } \\ \text { Run } 3 & \text { Stack }\end{array}$

A2371 0.58

A2473 ND

A2375 ND

Final

Results,

Lab

Results,

$\mathrm{mg}$

0.58

0.001

ND

ND

\begin{tabular}{|c|c|c|c|c|c|}
\hline \multirow[t]{2}{*}{ ADA-ES } & \multicolumn{5}{|c|}{$201 \mathrm{~A} / 202$} \\
\hline & Run 1 & Stack & A2377 & ND & ND \\
\hline & Run 2 & Stack & A2379 & ND & ND \\
\hline & Run 3 & Stack & A2381 & ND & ND \\
\hline & Run 4 & Stack & A2383 & ND & ND \\
\hline
\end{tabular}




\section{ANALYTICAL SUMMARY REPORT}

October 20, 2008

Westem Environmental

913 Foster Rd

Casper, WY 82601

Workorder No.: $\mathrm{C} 08100503$

Project Name: $\quad 08-4117$

Energy Laboratories, Inc. received the following 8 samples from Westem Environmental on 10/10/2008 for analysis.

\begin{tabular}{|c|c|c|c|c|c|}
\hline Sample ID & Cllent Sample ID & Collect Date & Recelve Date & Matrix & Test \\
\hline C08100503-001 & $\begin{array}{l}\text { 08-4117-1 Stack } \\
\text { Impringers } 182\end{array}$ & \multicolumn{2}{|c|}{$09 / 23 / 0800: 0010 / 10 / 08$} & Aqueous & E300.0 Anions \\
\hline C08100503-002 & $\begin{array}{l}\text { 08-4117-2 Stack- } \\
\text { Impringers } 182\end{array}$ & \multicolumn{2}{|c|}{$09 / 23 / 0800: 0010 / 10 / 08$} & Aqueous & Same As Above \\
\hline C08100503-003 & $\begin{array}{l}\text { 08-4117-3 Stack } \\
\text { Impringers } 1 \& 2\end{array}$ & \multicolumn{2}{|c|}{ 09/24/08 00:00 10/10/08 } & Aqueous & Same As Above \\
\hline C08100503-004 & $\begin{array}{l}08-4117-4 \text { Stack } \\
\text { Impringers } 182 \text { \&BH }\end{array}$ & \multicolumn{2}{|c|}{ 09/24/08 00:00 10/10/08 } & Aqueous & Same As Above \\
\hline C08100503-005 & $\begin{array}{l}\text { 08-4117-5 Stack } \\
\text { Impringers } 18.2 \text { \&BH }\end{array}$ & \multicolumn{2}{|c|}{$09 / 24 / 0800: 0010 / 10 / 08$} & Aqueous & Same As Above \\
\hline C08100503-006 & $\begin{array}{l}\text { 08-4117-6 Stack } \\
\text { Impringers } 182 \text { \&BH }\end{array}$ & \multicolumn{2}{|c|}{$09 / 24 / 0800: 0010 / 10 / 08$} & Aqueous & Same As Above \\
\hline C08100503-007 & $\begin{array}{l}\text { 08-4117-7 Stack } \\
\text { Impringers } 182 \text { \&BH }\end{array}$ & \multicolumn{2}{|c|}{$09 / 24 / 0800: 0010 / 10 / 08$} & Aqueous & Same As Above \\
\hline C08100503-008 & 08-4117-8 Blank & \multicolumn{2}{|c|}{$09 / 24 / 0800: 0010 / 10 / 08$} & Aqueous & Same As Above \\
\hline
\end{tabular}

As appropriate, any exceptions or problems with the analyses are noted in the Laboratory Analytical Report, the QAVC Summary Report, or the Case Narrative.

If you have any questions regarding these tests results, please call.

Report Approved By:

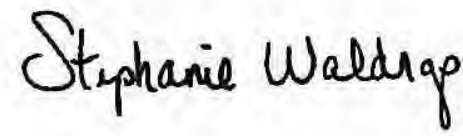




\section{LABORATORY ANALYTICAL REPORT}

Client: Westem Environmental

Site Name: $\quad$ 08-4117

Report Date: 10/20/08

Lab ID:

C08100503-001

Cllent Sample ID: 08-4117-1 Stack Impringers 182

Collection Date: 09/23/08

Matrix:

Aqueous

DateRecelved: $10 / 10 / 08$

\begin{tabular}{lcccccc}
\hline Analyses & Result Units & Qualifiors & RL & $\begin{array}{c}\text { MCL } \\
\text { QCL }\end{array}$ Mothod Analysis Date/By \\
\hline $\begin{array}{l}\text { MAJOR IONS } \\
\text { Sulfate }\end{array}$ & $569 \quad \mathrm{mg} / \mathrm{L}$ & 1 & E300.0 & $10 / 14 / 08$ 17:28/ ji
\end{tabular}

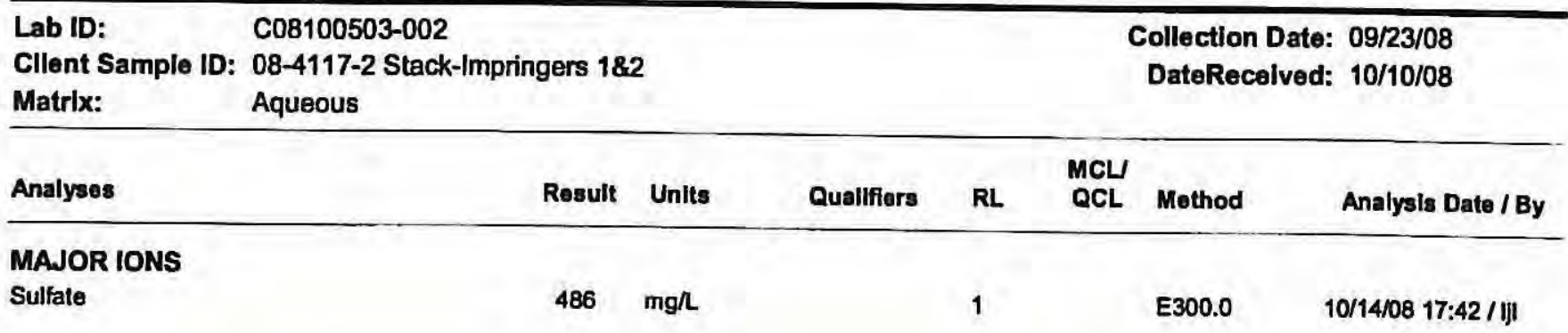

Lab ID: $\quad$ C08100503-003

Cllent Sample ID: 08-4117-3 Stack Impringers 182

Matrix:

Aqueous
Collectlon Date: 09/24/08

DateRecelved: $10 / 10 / 08$

\begin{tabular}{lccccc}
\hline $\begin{array}{l}\text { Analyses } \\
\text { MAJOR IONS }\end{array}$ & Result & Units & Quallfiers & RL & $\begin{array}{c}\text { MCL } \\
\text { QCL }\end{array}$ Mothod Analysis Date/By \\
$\begin{array}{l}\text { Sulfate } \\
\text { M }\end{array}$ & 622 & $\mathrm{mg} / \mathrm{L}$ & 1 & E300.0 & $10 / 14 / 08$ 17:56/lji
\end{tabular}

Lab ID:

C08100503-004

Client Sample ID: 08-4117-4 Stack Impringers $1 \& 2$ \&BH

Collection Date: 09/24/08

Matrix:

Aqueous

DateRecelved: 10/10/08

\begin{tabular}{|c|c|c|c|c|c|c|c|}
\hline Analyses & Result & Units & Quallfiers & $\mathbf{R L}$ & $\begin{array}{l}\text { MCL } \\
\text { QCL }\end{array}$ & Mothod & Analysis Date / By \\
\hline \multicolumn{8}{|c|}{ MAJOR IONS } \\
\hline Sulfate & 243 & $\mathrm{mg} / \mathrm{L}$ & & 1 & & $E 300.0$ & 10/14/08 18:11/Ji \\
\hline
\end{tabular}

Report RL - Analyte reporting limit.

Definitions: QCL - Quality control IImit.
MCL - Maximum contaminant level.

ND - Not detected at the reporting limit. 


\section{LABORATORY ANALYTICAL REPORT}

Client: Westem Environmental

Site Name: 08-4117

Report Date: 10/20/08

Lab ID: C08100503-005

Client Sample ID: 08-4117-5 Stack Impringers 1\&2 \&BH

Matrix: Aqueous

\begin{tabular}{|c|c|c|c|c|c|c|c|}
\hline Analyses & Rosult & Units & Quallfiers & $\mathbf{R L}$ & $\begin{array}{l}\text { MCLU } \\
\text { QCL }\end{array}$ & Method & Analysis Dato / By \\
\hline \multicolumn{8}{|c|}{ MAWOR IONS } \\
\hline Sulfate & 252 & $\mathrm{mg} / \mathrm{L}$ & & 1 & & E300.0 & 10/14/08 18:25/五 \\
\hline
\end{tabular}

\section{Lab ID: $\quad$ C08100503-006}

Cllent Sample ID: 08-4117-6 Stack impringers $1 \& 2$ \&BH

Matrix:

Aqueous
Collection Date: 09/24/08

DateReceived: $10 / 10 / 08$

\begin{tabular}{lccccc}
\hline $\begin{array}{l}\text { Analyses } \\
\text { MAJOR IONS }\end{array}$ & Rosult Units & Qualifiers & RL & $\begin{array}{c}\text { MCL } \\
\text { QCL }\end{array}$ & Method Analyzis Date/By \\
$\begin{array}{l}\text { Sulfate } \\
\text { M }\end{array}$ & 307 & $\mathrm{mg} / \mathrm{L}$ & 1 & E300.0 & $10 / 14 / 0818: 40 / \mathrm{jl}$
\end{tabular}

Lab ID: $\quad$ C08100503-007

Cllent Sample ID: 08-4117-7 Stack Impringers 182 \&BH

Matrix: Aqueous
Collection Date: 09/24/08

DateRecelved: $10 / 10 / 08$

\begin{tabular}{|c|c|c|c|c|c|c|c|}
\hline Analyses & Result & Unkts & Qualifiers & $\mathbf{R L}$ & $\begin{array}{l}\text { MCL } \\
\text { QCL }\end{array}$ & Mothod & Analysis Date / By \\
\hline \multicolumn{8}{|c|}{ MAJOR IONS } \\
\hline Sulfate & 204 & $\mathrm{mg} / \mathrm{L}$ & & 1 & & $E 300.0$ & 10/14/08 18:54/ 1 1 \\
\hline
\end{tabular}

Lab ID: $\quad$ C08100503-008

Client Sample ID: 08-4117-8 Blank

Matrix:

Aqueous
Collectlon Date: 09/24/08

DateRecelved: $10 / 10 / 08$

\begin{tabular}{|c|c|c|c|c|c|c|c|}
\hline Analyses & Result & Units & Quallfiors & $\mathbf{R L}$ & $\begin{array}{l}\text { MCL } \\
\text { QCL }\end{array}$ & Method & Analysis Date / By \\
\hline \multicolumn{8}{|c|}{ MAJOR IONS } \\
\hline Sulfate & ND & mgll & & 1 & & E300.0 & |j/ 10/14/08 19:08 \\
\hline
\end{tabular}

MCL - Maximum contaminant level. ND - Nol detected at the reporting limit. 


\section{QAVC Summary Report}

Client: Westem Environmental

Report Date: 10/20/08

Project: 08-4117

Work Order: C08100503

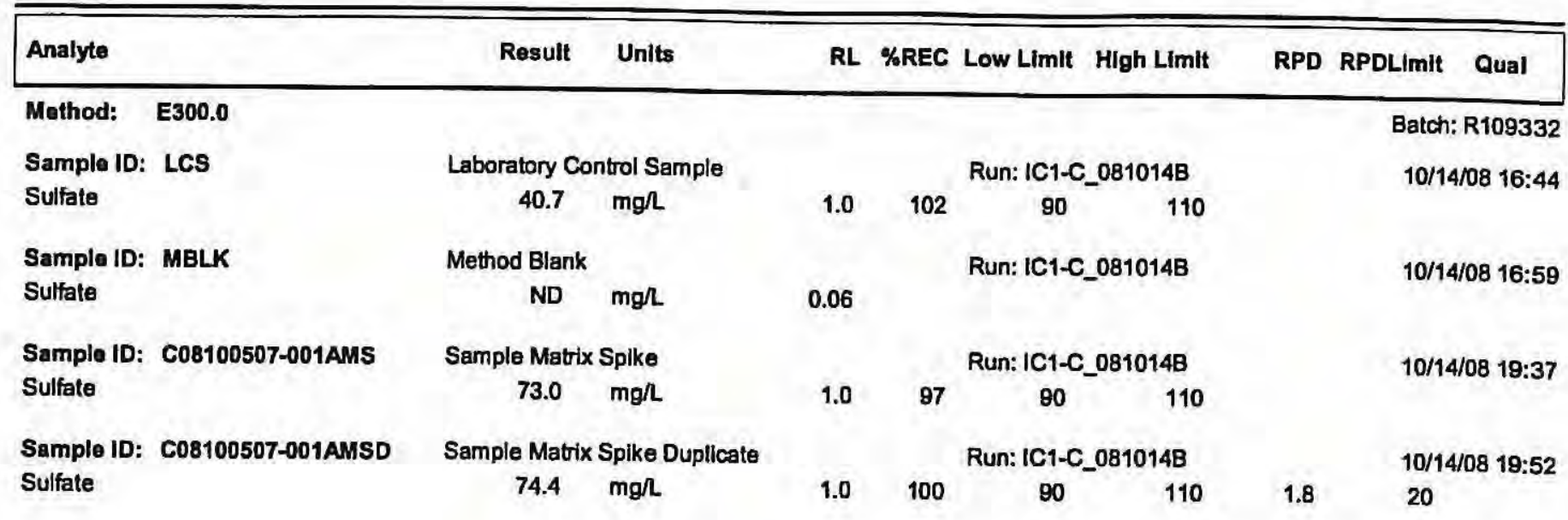

\section{Quallfiers:}

RL - Analyte reporting limit.

ND - Not detected at the reporting limit. 

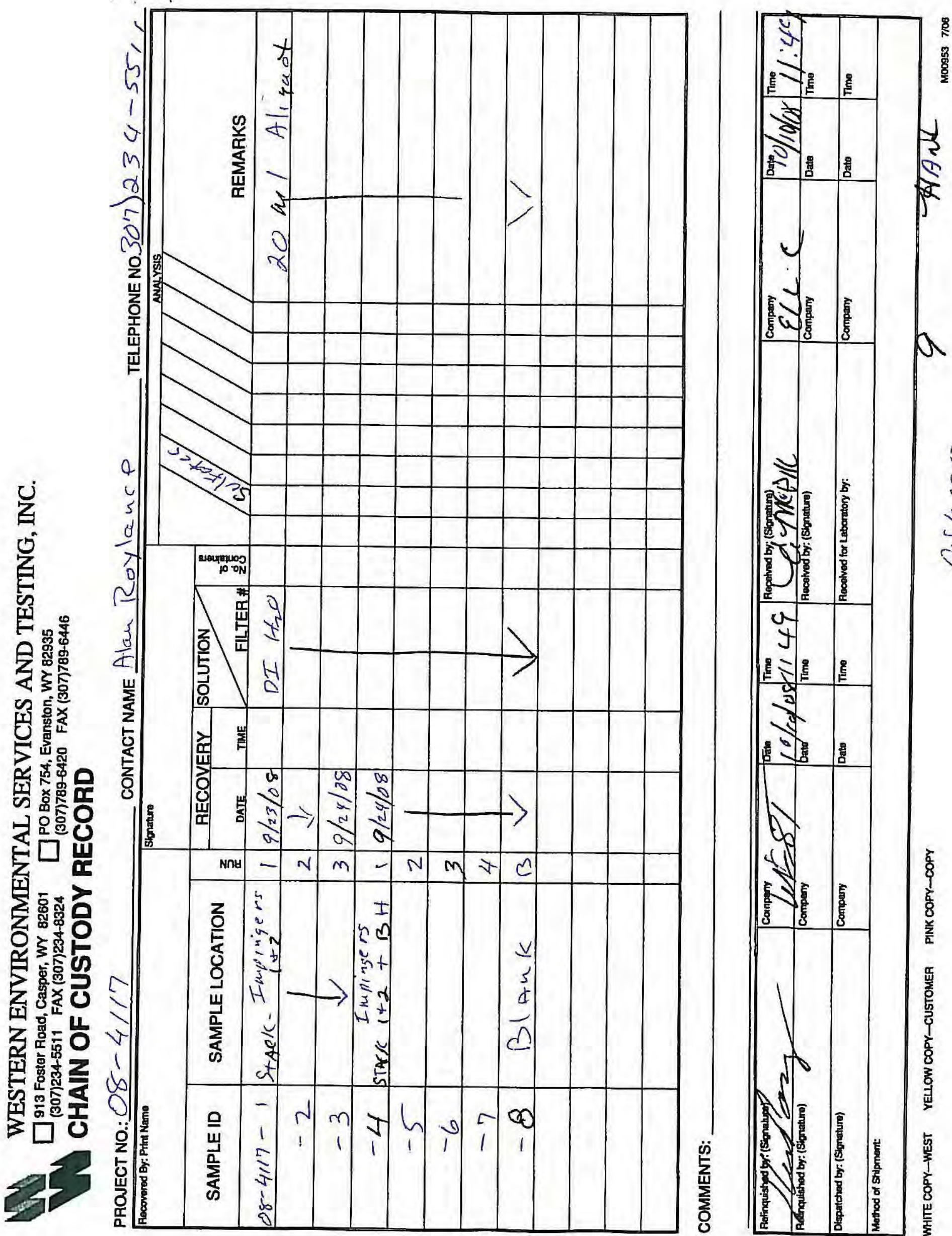

$\frac{8}{2}$
8
8
8
$\frac{8}{2}$

3

$\frac{\pi}{2}$

$N$

$\sum_{\substack{\infty \\ \infty}}^{n}$

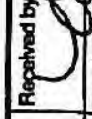

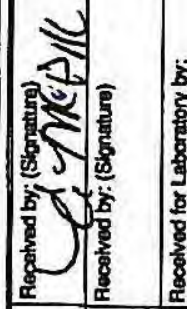

$y$

है출

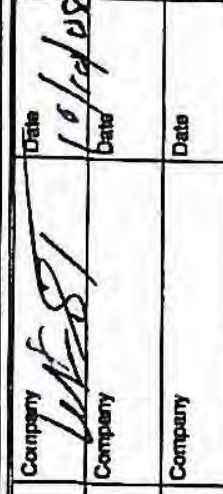

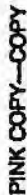

|

总 


\section{Energy Laboratories Inc Workorder Receipt Checklist}

\section{Western Environmental}

Login completed by: Edith McPike

Reviewed by:

Reviewed Date:

Shipping container/cooler in good condition?
Custody seals intact on shipping container/cooler?
Custody seals intact on sample bottles?
Chain of custody present?
Chain of custody slgned when relinquished and received?
Chain of custody agrees with sample labels?
Samples in proper container/bottle?
Sample containers intact?
Sufficient sample volume for indicated test?
All samples received within holding time?
Container/Temp Blank temperature;
Water - VOA vials have zero headspace?
Water - pH acceptable upon recaipt?

$\begin{array}{lll}\text { Yes } \square & \text { No } \square & \text { Not Present } \square \\ \text { Yes } \square & \text { No } \square & \text { Not Present } \square \\ \text { Yes } \square & \text { No } \square & \text { Not Present } \square \\ \text { Yes } \square & \text { No } \square & \\ \text { Yes } \square & \text { No } \square \\ \text { Yes } \square & \text { No } \square \\ \text { Yes } \square & \text { No } \square \\ \text { Yes } \square & \text { No } \square \\ \text { Yes } \square & \text { No } \square & \\ \text { Yes } \square & \text { No } \square & \\ \text { goc } & & \\ \text { Yes } \square & \text { No } \square & \text { No VoA vials submitted } \\ \text { Yes } \square & \text { No } \square & \text { Not Applicable } \square\end{array}$

Yes $\square$

Date and Time Received: 10/10/2008 11:49 AM Received by: em

Carrier name: Hand Del

Contact and Corrective Action Comments:

Headspace in vials; but not pertinant for this analysis 
CLIENT: Westem Environmental

Project: $\quad 08-4117$

Sample Delivery Group: C08100503
Date: 20-0ct-08

\section{CASE NARRATIVE}

The following Case Narrative contains exceptions or comments pertaining to the analysis of samples submitted by
Western Environmental on 10/10/2008 11:49:00. These samples were assigned C08100503.

ORIGINAL SAMPLE SUBMITTAL(S)

All original sample submittals have been retumed with the data package.

SAMPLE TEMPERATURE COMPLIANCE: $4^{\circ} \mathrm{C}\left( \pm 2^{\circ} \mathrm{C}\right)$

Temperature of samples received may not be considered properly preserved by accepted standards. Samples that are hand delivered immediately after collection shall be considered acceptable if there is evidence that the chilling process has begun.

GROSS ALPHA ANALYSIS

Method 900.0 for gross alpha and gross beta is intended as a drinking water method for low TDS waters. Data provided by this method for non potable waters should be viewed as inconsistent.

RADON IN AIR ANALYSIS

The desired exposure time is 48 hours (2 days). The time delay in retuming the canister to the laboratory for processing should be as short as possible to avoid excessive decay. Maximum recommended delay between end of exposure to beginning of counting should not exceed 8 days.

\section{SOILISOLID SAMPLES}

All samples reported on an as recelved basis unless otherwise indicated.

ATRAZINE, SIMAZINE AND PCB ANALYSIS USING EPA 505

Data for Atrazine and SImazine are reported from EPA 525.2, not from EPA 505. Data reported by ELI using EPA method 505 reflects the results for seven individual Aroclors. When the results for all seven are ND (not detected), the sample meets EPA compliance criteria for PCB monitoring.

\section{SUBCONTRACTING ANALYSIS}

Subcontracting of sample analyses to an outside laboratory may be required. If so, ENERGY LABORATORIES will utilize its branch laboratories or qualified contract laboratories for this service. Any such laboratories will be indicated within the Laboratory Analytical Report.

\section{BRANCH LABORATORY LOCATIONS}

eli-b - Energy Laboratories, Inc. - Billings, MT

eli-g - Energy Laboratories, Inc. - Gillette, WY

eli-h - Energy Laboratories, Inc. - Helena, MT

eli-r - Energy Laboratories, Inc. - Rapid City, SD

eli-t - Energy Laboratories, Inc. - College Station, TX

\section{CERTFICATIONS:}

USEPA: WY00002; FL-DOH NELAC: E87641; Califomia: 02118CA

Oregon: WY200001; Utah: 3072350515; Virginia: 00057 ; Washington: $\mathrm{C} 1903$

\section{ISO 17025 DISCLAIMER:}

The results of this Analytical Report relate only to the items submitted for analysis.

ENERGY LABORATORIES, INC. - CASPER,WY certifies that certain method selections contained in this report meet requirements as set forth by the above accrediting authorities. Some results requested by the cllent may not be covered under these certifications. All analysis data to be submitted for regulatory enforcement should be certified in the sample state of origin. Please verify ELl's certification coverage by visiting www.energylab.com

ELI appreciates the opportunity to provide you with this analytical service. For additional information and services visit our web page www.energylab.com.

THIS IS THE FINAL PAGE OF THE LABORATORY ANALYTICAL REPORT 


\section{ANALYTICAL SUMMARY REPORT}

October 24, 2008

Western Environmental

913 Foster Rd

Casper, WY 82601

Workorder No.: C08100558

Quote ID: C2258 - Chlorides

Project Name: Not Indicated

Energy Laboratories, Inc. received the following 8 samples from Western Environmental on 10/13/2008 for analysis.

\begin{tabular}{lllll}
\hline Sample ID & \multicolumn{1}{c}{ Client Sample ID } & Collect Date Receive Date & Matrix & Test \\
\hline \hline C08100558-001 08-4117-A2371 & 09/23/08 00:00 10/13/08 & Solid & $\begin{array}{l}\text { E300.0 Anions } \\
\text { DI Water Soil Extract }\end{array}$ \\
\hline C08100558-002 08-4117-A2373 & $09 / 23 / 08$ 00:00 10/13/08 & Solid & Same As Above \\
\hline C08100558-003 08-4117-A2375 & $09 / 24 / 0800: 0010 / 13 / 08$ & Solid & Same As Above \\
\hline C08100558-004 08-4117-A2377 & $09 / 24 / 0800: 0010 / 13 / 08$ & Solid & Same As Above \\
\hline C08100558-005 08-4117-A2379 & $09 / 24 / 0800: 0010 / 13 / 08$ & Solid & Same As Above \\
\hline C08100558-006 08-4117-A2381 & $09 / 24 / 0800: 0010 / 13 / 08$ & Solid & Same As Above \\
\hline C08100558-007 08-4117-A2383 & $09 / 24 / 0800: 00 ~ 10 / 13 / 08$ & Solid & Same As Above \\
\hline C08100558-008 08-4117-A2385 & $09 / 24 / 0800: 0010 / 13 / 08$ & Solid & Same As Above \\
\hline
\end{tabular}

As appropriate, any exceptions or problems with the analyses are noted in the Laboratory Analytical Report, the QAVC Summary Report, or the Case Narrative.

If you have any questions regarding these lests results, please call.

Report Approved By:

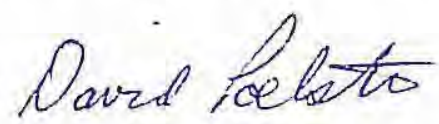




\section{LABORATORY ANALYTICAL REPORT}

Client: Western Environmental

$\begin{array}{lll}\text { Site Name: } & \text { Not Indicated } & \text { Report Date: 10/24/08 }\end{array}$

\begin{tabular}{|c|c|c|c|c|c|c|c|c|}
\hline $\begin{array}{l}\text { Lab ID: } \\
\text { Client Sample ID: } \\
\text { Matrix: }\end{array}$ & $\begin{array}{l}\text { C08100558-001 } \\
08-4117-A 2371 \\
\text { Solid }\end{array}$ & & & & & \multicolumn{3}{|c|}{$\begin{array}{r}\text { Collection Date: } 09 / 23 / 08 \\
\text { DateReceived: } 10 / 13 / 08\end{array}$} \\
\hline Analyses & & Result & Units & Qualifiers & $\mathbf{R L}$ & $\begin{array}{l}\text { MCL } \\
\text { QCL }\end{array}$ & Method & Analysis Date / By \\
\hline \multicolumn{9}{|l|}{ MAJOR IONS } \\
\hline Chloride & & 0.58 & mg/beaker & & 0.05 & & E300.0 & 10/15/08 01:52/ 1 \\
\hline
\end{tabular}

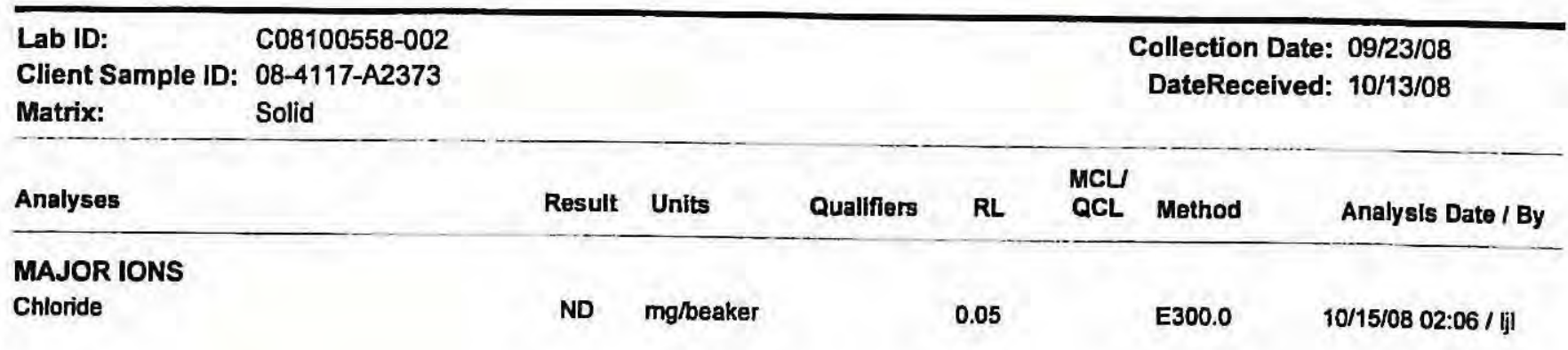

\begin{tabular}{|c|c|c|c|c|c|c|c|c|}
\hline $\begin{array}{l}\text { Lab ID: } \\
\text { Client Sample ID: } \\
\text { Matrix: }\end{array}$ & $\begin{array}{l}\text { C08100558-003 } \\
08-4117-A 2375 \\
\text { Solid }\end{array}$ & & & & & \multicolumn{3}{|c|}{$\begin{array}{r}\text { Collection Date: } 09 / 24 / 08 \\
\text { DateReceived: } 10 / 13 / 08\end{array}$} \\
\hline Analyses & & Result & Units & Quallflers & $\mathbf{R L}$ & $\begin{array}{l}\text { MCL } \\
\text { QCL }\end{array}$ & Method & Analysis Date / By \\
\hline $\begin{array}{l}\text { MAJOR IONS } \\
\text { Chloride }\end{array}$ & & ND & mg/beaker & & & & Eann & \\
\hline
\end{tabular}

\begin{tabular}{|c|c|c|c|c|c|c|c|c|}
\hline $\begin{array}{l}\text { Lab ID: } \\
\text { Client Sample ID: } \\
\text { Matrix: }\end{array}$ & $\begin{array}{l}\text { C08100558-003 } \\
08-4117-A 2375 \\
\text { Solid }\end{array}$ & & & & & \multicolumn{3}{|c|}{$\begin{array}{r}\text { Collection Date: } 09 / 24 / 08 \\
\text { DateReceived: } 10 / 13 / 08\end{array}$} \\
\hline Analyses & & Result & Units & Quallflers & $\mathbf{R L}$ & $\begin{array}{l}\text { MCL } \\
\text { QCL }\end{array}$ & Method & Analysis Date / By \\
\hline \multicolumn{9}{|l|}{ MAJOR IONS } \\
\hline Chloride & & ND & mg/beaker & & 0.05 & & $E 300.0$ & 10/15/08 02:21/ jil \\
\hline
\end{tabular}

\begin{tabular}{l} 
Client: \\
Site Name: \\
\hline Lab ID: \\
Client Sample \\
Matrix: \\
Analyses \\
MAJOR IONS \\
Chloride
\end{tabular}

(Dample $08-4117-A 2$

Matrix:

Solid
Collection Date: 09/23/08

DateReceived: $10 / 13 / 08$
Lab ID: $\quad$ C08100558-004

Client Sample ID: 08-4117-A2377

Matrix: Solid
Collection Date: 09/24/08

DateReceived: $10 / 13 / 08$

\begin{tabular}{|c|c|c|c|c|c|c|c|}
\hline Analyses & Result & Units & Qualifiers & $\mathbf{R L}$ & $\begin{array}{l}\text { MCL } \\
\text { QCL }\end{array}$ & Method & Analysis Date / By \\
\hline \multicolumn{8}{|c|}{ MAJOR IONS } \\
\hline Chloride & ND & mg/beaker & & 0.05 & & E300.0 & 10/15/08 02:35/ jl \\
\hline
\end{tabular}

Report RL - Analyte reporting limit.

Definitions: QCL - Quality control limit.
$\mathrm{MCL}$ - Maximum contaminant level.

ND - Not detected at the reporting limit. 


\section{LABORATORY ANALYTICAL REPORT}

Client: Western Environmental

Site Name: $\quad$ Not Indicated

Report Date: 10/24/08

\begin{tabular}{llr}
\hline Lab ID: & C08100558-005 & Collection Date: 09/24/08 \\
Client Sample ID: & $08-4117-A 2379$ & DateReceived: $10 / 13 / 08$ \\
Matrix: & Solid &
\end{tabular}

\begin{tabular}{|c|c|c|c|c|c|c|c|}
\hline Analyses & Result & Units & Quallfiers & RL & $\begin{array}{l}\text { MCU } \\
\text { QCL }\end{array}$ & Method & Analysis Date / By \\
\hline \multicolumn{8}{|c|}{ MAJOR IONS } \\
\hline Chloride & ND & mg/beaker & & 0.05 & & $E 300.0$ & 10/15/08 02:50/ \\
\hline
\end{tabular}

\begin{tabular}{llr}
\hline Lab ID: & C08100558-006 & Collection Date: 09/24/08 \\
Client Sample ID: & 08-4117-A2381 & DateReceived: $10 / 13 / 08$ \\
Matrix: & Solid &
\end{tabular}

Matrix:

Analyses
MAJOR IONS
Chloride

Chloride
DateReceived: $10 / 13 / 08$

\begin{tabular}{|c|c|c|c|c|c|c|}
\hline Result & Units & Qualifiers & RL & $\begin{array}{l}\text { MCLI } \\
\text { QCL }\end{array}$ & Method & Analysis Date / By \\
\hline ND & mg/beaker & & 0.05 & & E300.0 & 10/15/08 03:33 / lj \\
\hline
\end{tabular}

\begin{tabular}{llr}
\hline Lab ID: & C08100558-007 & Collection Date: 09/24/08 \\
Client Sample ID: $08-4117-A 2383$ & DateReceived: $10 / 13 / 08$ \\
Matrix: & Solid &
\end{tabular}

\begin{tabular}{|c|c|c|c|c|c|c|c|}
\hline Analyses & Result & Units & Quallfiers & RL & $\begin{array}{l}\text { MCL } \\
\text { QCL }\end{array}$ & Mothod & Analysis Date / By \\
\hline \multicolumn{8}{|c|}{ MAJOR IONS } \\
\hline Chloride & ND & mg/beaker & & 0.05 & & $E 300.0$ & 10/15/08 03:47 / ljl \\
\hline
\end{tabular}

\begin{tabular}{ll}
\hline Lab ID: & C08100558-008 \\
Client Sample ID: & 08-4117-A2385 \\
Matrix: & Solid
\end{tabular}

Collection Date: 09/24/08

DateReceived: 10/13/08

\begin{tabular}{lllllll}
\hline Analyses & Result & Units & Qualifiers & RL & $\begin{array}{c}\text { MCLI QCL Method } \\
\text { Analysis Date/By }\end{array}$ \\
$\begin{array}{l}\text { MAJOR IONS } \\
\text { Chloride }\end{array}$ & 0.16 & $\mathrm{mg} /$ beaker & 0.05 & E300.0 & $10 / 15 / 0804: 16 / \mathrm{mI}$
\end{tabular}

$\begin{array}{ll}\text { Report } & R L \text { - Analyte reporting limit. } \\ \text { Definitions: } & \text { QCL - Quality control limit. }\end{array}$
MCL - Maximum contaminant level.

ND - Not delected at the reporting limit. 


\section{QA/QC Summary Report}

Client: Westem Environmental

Report Date: 10/24/08

Project: Not Indicated

Work Order: C08100558

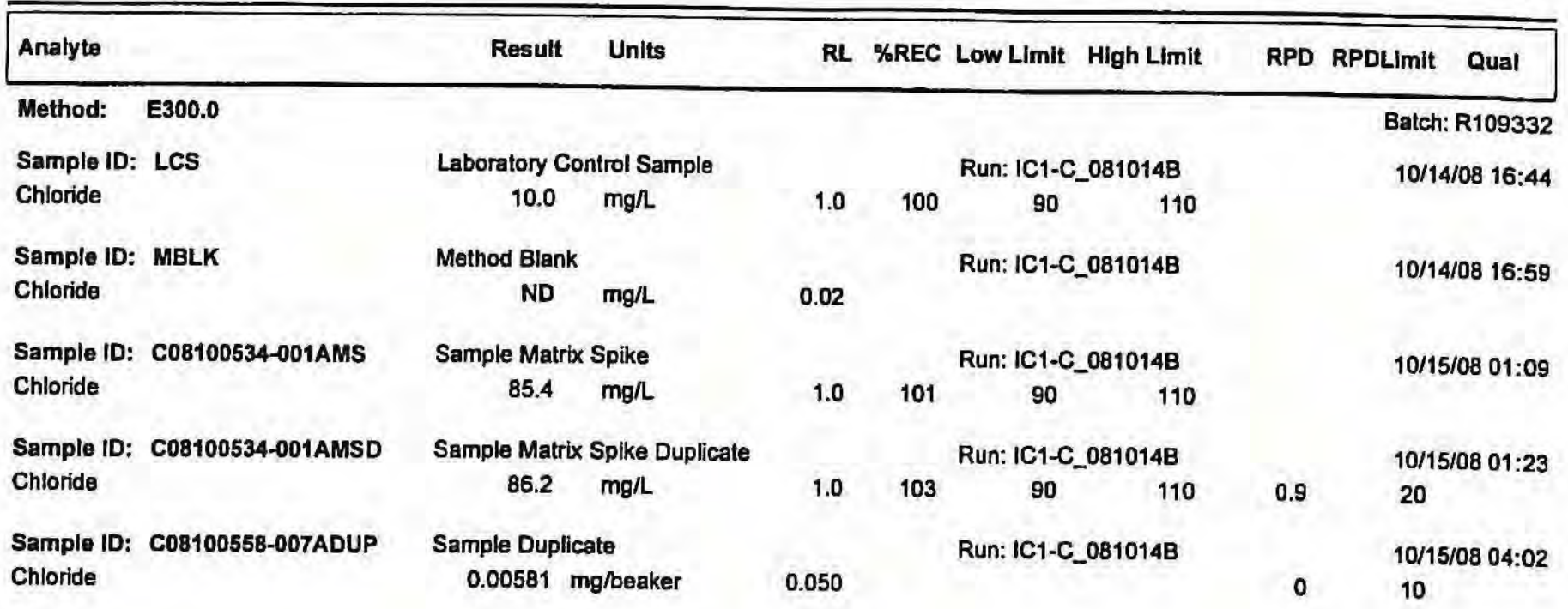

Quallfiers:

$\mathrm{RL}$ - Analyte reporting limit.

ND - Not detected at the reporting limit. 


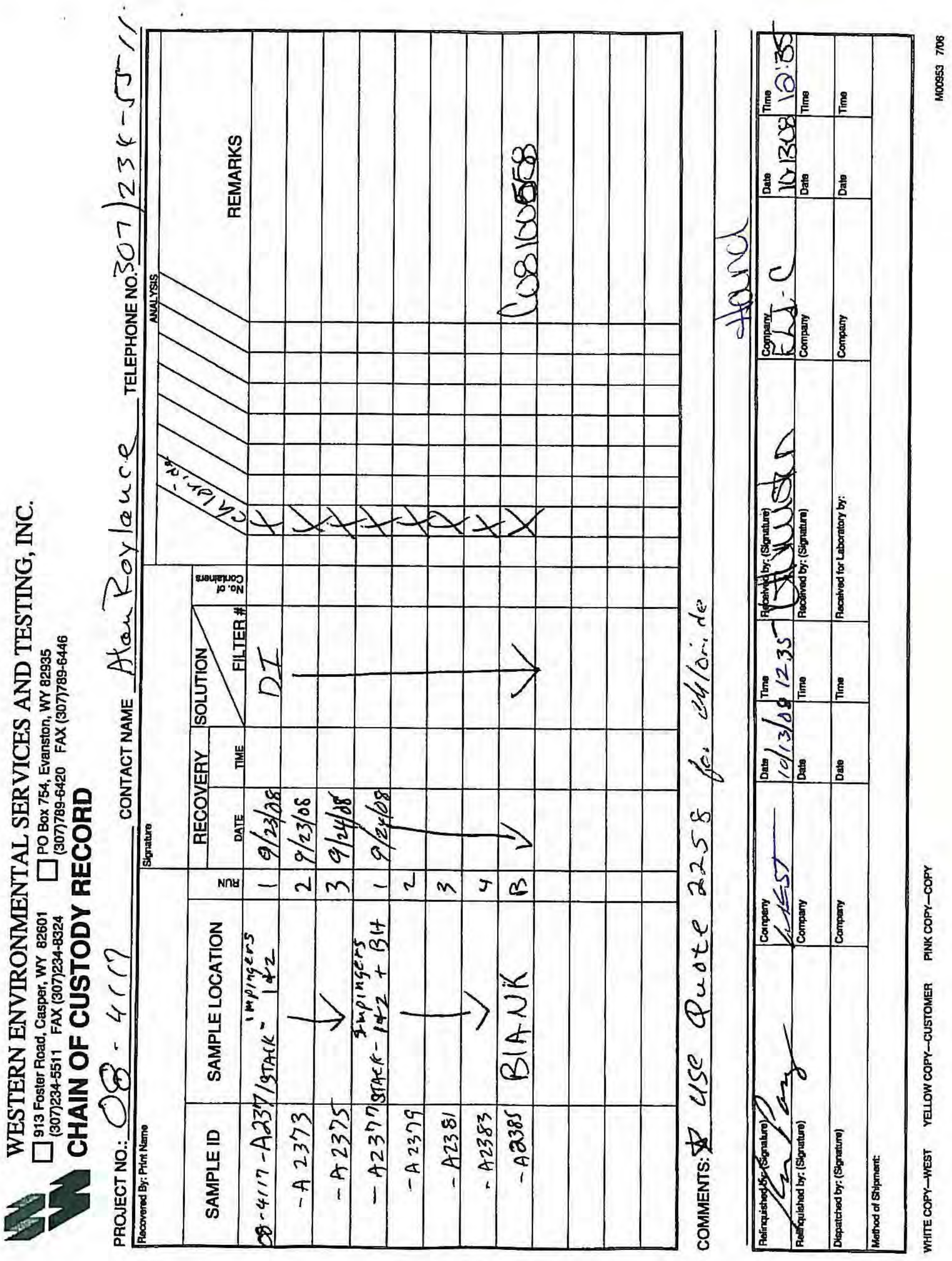




\section{Energy Laboratories Inc Workorder Receipt Checklist}

\section{Western Environmental}

Login completed by: Kimberly Humiston

Reviewed by:

Reviewed Date:

Shipping container/cooler in good condition?
Custody seals intact on shipping container/cooler?
Custody seals intact on sample bottles?
Chain of custody present?
Chain of custody signed when relinquished and received?
Chain of custody agrees with sample labels?
Samples in proper container/botlle?
Sample containers intact?
Sufficient sample volume for indicated test?
All samples received within holding time?
Container/Temp Blank temperature:
Water - VOA vials have zero headspace?
Water - pH acceptable upon receipt?

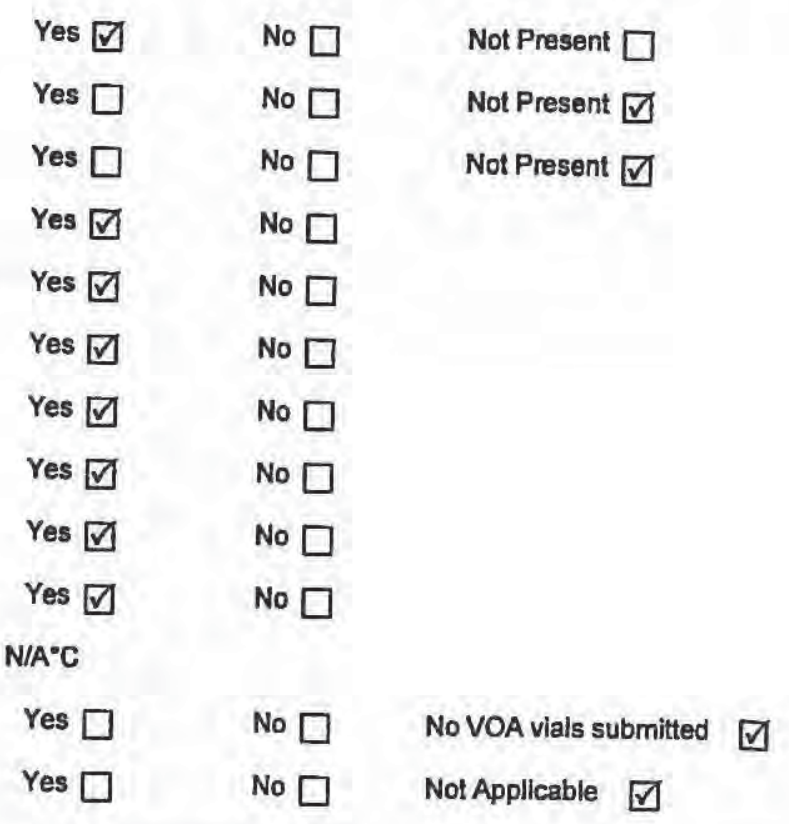

Yes $\square$

Date and Time Received: 10/13/2008 12:35 PM Received by: kh

Carrier name: Hand Del 
Project: Not Indicated

Sample Delivery Group: C08100558

The following Case Narrative contains exceptions or comments pertaining to the analysis of samples submitted by Westem Environmental on 10/13/2008 12:35:00. These samples were assigned ELI Workorder Number C08100558.

ORIGINAL SAMPLE SUBMITTAL(S)

All original sample submittals have been returned with the data package.

SAMPLE TEMPERATURE COMPLIANCE: $4^{\circ} \mathrm{C}\left( \pm 2^{\circ} \mathrm{C}\right)$

Temperature of samples received may not be considered properly preserved by accepted standards. Samples that are hand delivered immediately after collection shall be considered acceptable if there is evidence that the chilling process has begun.

GROSS ALPHA ANALYSIS

Method 900.0 for gross alpha and gross beta is intended as a drinking water method for low TDS waters. Data provided by this method for non potable waters should be viewed as inconsistent.

\section{RADON IN AIR ANALYSIS}

The desired exposure time is 48 hours ( 2 days). The time delay in returning the canister to the laboratory for processing should be as short as possible to avoid excessive decay. Maximum recommended delay between end of exposure to beginning of counting should not exceed 8 days.

\section{SOILSOLID SAMPLES}

All samples reported on an as received basis unless otherwise indicated.

ATRAZINE, SIMAZINE AND PCB ANALYSIS USING EPA 505

Data for Atrazine and Simazine are reported from EPA 525.2, not from EPA 505. Data reported by ELI using EPA method 505 reflects the results for seven individual Aroclors. When the results for all seven are ND (not detected), the sample meets EPA compliance criteria for PCB monitoring.

\section{SUBCONTRACTING ANALYSIS}

Subcontracting of sample analyses to an outside laboratory may be required. If so, ENERGY LABORATORIES will utilize its branch laboratories or qualified contract laboratories for this service. Any such laboratories will be indicated within the Laboratory Analytical Report.

\section{BRANCH LABORATORY LOCATIONS}

eli-b - Energy Laboratories, Inc. - Billings, MT

eli-g - Energy Laboratories, Inc. - Gillette, WY

eli-h - Energy Laboratories, Inc. - Helena, MT

eli-r - Energy Laboratories, Inc. - Rapid City, SD

eli-t - Energy Laboratories, Inc. - College Station, TX

CERTFICATIONS:

USEPA: WY00002; FL-DOH NELAC: EB7641; California: 02118CA

Oregon: WY200001; Utah: 3072350515; Virginia: 00057; Washington: C1903

ISO 17025 DISCLAIMER:

The results of this Analytical Report relate only to the items submitted for analysis.

ENERGY LABORATORIES, INC. - CASPER,WY certifies that certain method selections contained in this report meet requirements as set forth by the above accrediting authorities. Some results requested by the client may not be covered under these certifications. All analysis data to be submitted for regulatory enforcement should be certified in the sample state of origin. Please verify ELl's certification coverage by visiting www.energylab.com

ELI appreciates the opportunity to provide you with this analytical service. For additional information and services visit our web page www.energylab.com. 
Appendix F:

EPA Test Methods 8A, 26A, and CTM027 (Sulfur Trioxide, Hydrogen Halides \& Halogens, and Ammonia) Analytical Data - SDA Inlet Duct and Stack 


\title{
Air Sampling Associates 407-2B Holford's Prairie Rd Lewisville, TX 75056-9582
}

\author{
ADA - ES
}

Client \# 08-026

\author{
Analytical Report \\ (1008-22)
}

\section{EPA CTM-027 \\ Ammonia}

\section{EPA Method 26A}

Hydrogen bromide, Hydrogen chloride, Hydrogen fluoride Bromide, Chloride

\section{NCASI Method 8A-Type (HIPLC)}

Sulfate

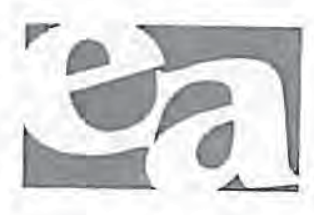

\section{Enthalpy Analytical, Inc.}

Phone: (919) 850 - 4392 / Fax: (919) 850 - 9012 / www.enthalpy.com 2202 Ellis Road Durham, NC $27703-5518$ 
I certify that to the best of my knowledge all analytical data presented in this report:

- Have been checked for completeness

- Are accurate, error-free, and legible

- Have been conducted in accordance with approved protocol, and that all deviations and analytical problems are summarized in the appropriate narrative(s)

- This analytical report was prepared in Portable Document Format (.PDF) and contains 267 pages.
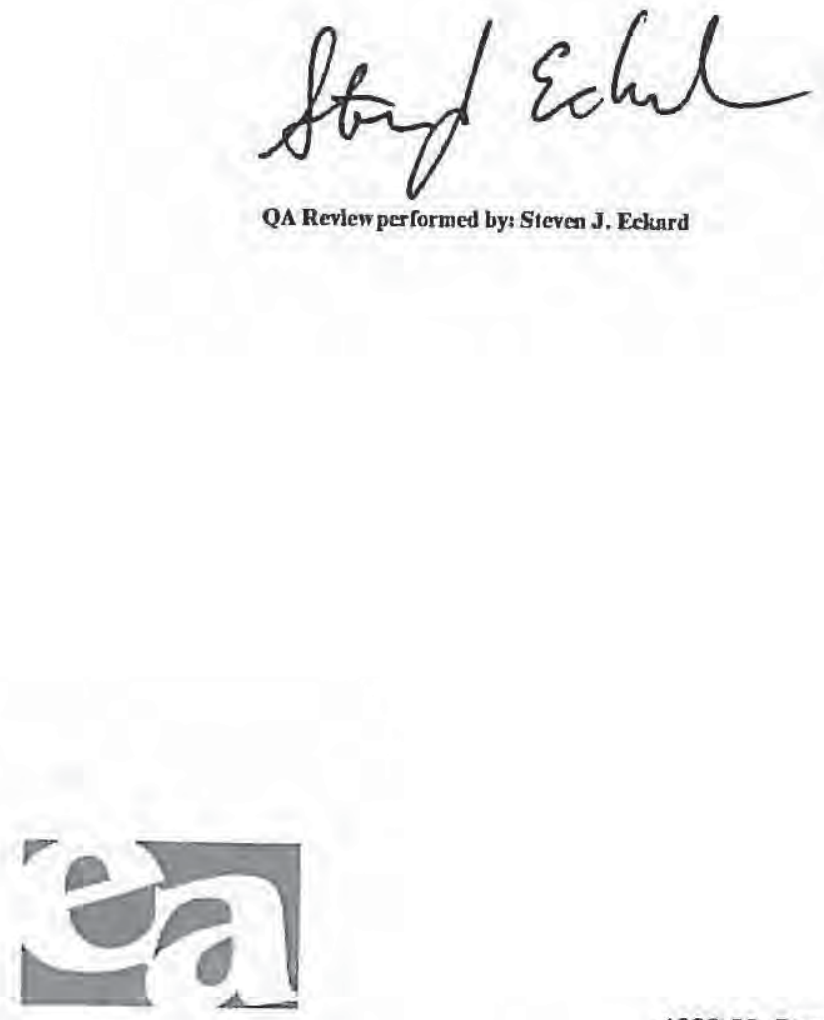


\section{Summary of Results}

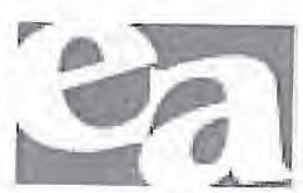




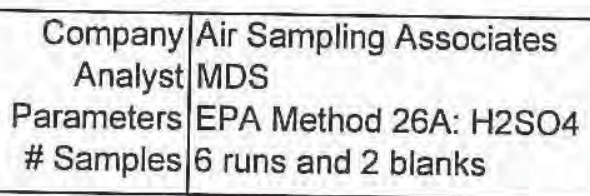

\begin{tabular}{|lccc|}
\hline Compound & \multicolumn{3}{c|}{ Sample ID / Catch Weight (ug) } \\
\hline & \multicolumn{4}{c}{ SDA Inlet Duct } \\
Hydrogen Fluoride & In R1 C3 & In R2 C3 & In R3 C3 \\
Hydrogen Chloride & $12.3 \mathrm{ND}$ & $13.4 \mathrm{ND}$ & $55.2 \mathrm{~J}$ \\
Hydrogen Bromide & 1118 & 317 & 592 \\
& $22.4 \mathrm{ND}$ & $24.4 \mathrm{ND}$ & $26.7 \mathrm{ND}$ \\
& & & \\
& & Stack & \\
Hydrogen Fluoride & Stk R1 C3 & Stk R2 C3 & Stk R3 C3 \\
Hydrogen Chloride & $16.6 \mathrm{ND}$ & $18.1 \mathrm{ND}$ & $17.3 \mathrm{ND}$ \\
Hydrogen Bromide & 761 & $116 \mathrm{~J}$ & $31.5 \mathrm{ND}$ \\
& $30.3 \mathrm{ND}$ & $33.1 \mathrm{ND}$ & $31.5 \mathrm{ND}$ \\
& & & \\
& & & \\
& & Blanks & \\
Hydrogen Fluoride & In C6 & $20.8 \mathrm{ND}$ & \\
Hydrogen Chloride & $25.3 \mathrm{ND}$ & $37.9 \mathrm{ND}$ & \\
Hydrogen Bromide & $25.3 \mathrm{ND}$ & $37.9 \mathrm{ND}$ & \\
& & & \\
& & &
\end{tabular}




\begin{tabular}{r|l|} 
Company & Air Sampling Associates \\
Analyst & MDS \\
Parameters & EPA Method $26 \mathrm{~A}: \mathrm{NaOH}$ \\
\# Samples & 6 runs and 2 blanks \\
\hline
\end{tabular}

\begin{tabular}{|r|l|}
\hline Client \# & $08-026$ \\
Job \# & $1008-22$ \\
PO \# & Verbal \\
Report Date & $10 / 18 / 2008$ \\
\hline
\end{tabular}

\section{Compound Sample ID / Catch Weight (ug)}

\begin{tabular}{lccc} 
& \multicolumn{3}{c}{ SDA Duct Inlet } \\
Chloride & In R1 C4 & In R2 C4 & In R3 C4 \\
Bromide & 790 & $126 \mathrm{~J}$ & 918 \\
& $76.8 \mathrm{ND}$ & $80.7 \mathrm{ND}$ & $80.7 \mathrm{ND}$ \\
& & & \\
& & & \\
Chloride & Stk R1 C4 & Stack 2 C4 & Stack R3 C4 \\
Bromide & $514 \mathrm{~J}$ & $507 \mathrm{~J}$ & $464 \mathrm{~J}$ \\
& $87.0 \mathrm{ND}$ & $84.2 \mathrm{ND}$ & $75.8 \mathrm{ND}$
\end{tabular}

Chloride

Bromide

In C7
$209 \mathrm{~J}$
$82.4 \mathrm{ND}$

Blanks

Stk C7

$80.0 \mathrm{~J}$

$76.9 \mathrm{ND}$ 


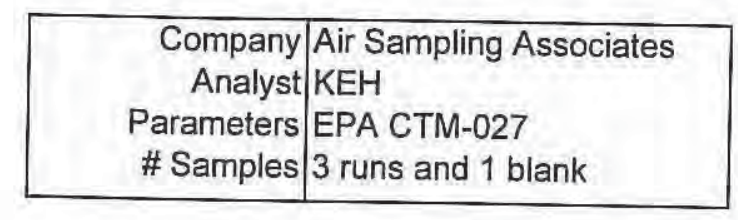

\begin{tabular}{|r|l|}
\hline Client \# & $08-026$ \\
Job \# & $1008-22$ \\
PO \# & Verbal \\
Report Date & $10 / 18 / 2008$ \\
\hline
\end{tabular}

\begin{tabular}{|c|c|c|c|}
\hline Compound & Sam & ple ID / Catch Weight (ug) & \\
\hline Ammonia & $\begin{array}{c}\text { Run } 1 \\
52,431 \\
\text { H2SO4 Blank } \\
7.99 \text { ND }\end{array}$ & $\begin{array}{c}\text { SDA Inlet Duct Container } 3 \\
\text { Run } 2 \\
23,750\end{array}$ & $\begin{array}{c}\text { Run } 3 \\
13,939\end{array}$ \\
\hline
\end{tabular}




\section{Company Air Sampling Associates Analyst MDS \\ Parameters NCASI Method 8A-Type \\ \# Samples 6 runs and 2 blanks}

\begin{tabular}{|c|c|c|c|}
\hline Compound & Sar & I Catch Weight (us & \\
\hline Sulfate & $\begin{array}{l}\text { In R1 C2 } \\
5,606\end{array}$ & $\begin{array}{c}\text { SDA Inlet Duct } \\
\quad \text { In R2 C2 } \\
22,952\end{array}$ & $\begin{array}{l}\text { In } R 3 \text { C2 } \\
2,606\end{array}$ \\
\hline Sulfate & $\begin{array}{c}\text { Stk R1 C2 } \\
2,233\end{array}$ & $\begin{array}{c}\text { Stack } \\
\text { Stk R2 C2 } \\
1,490\end{array}$ & $\begin{array}{c}\text { Stk R3 C2 } \\
2,561\end{array}$ \\
\hline Sulfate & $\begin{array}{l}\text { In } C 4 \\
13.7 \mathrm{ND}\end{array}$ & $\begin{array}{l}\text { Blanks } \\
\text { Stk C4 } \\
13.7 \mathrm{ND}\end{array}$ & \\
\hline
\end{tabular}




\section{Results}

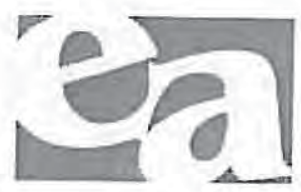




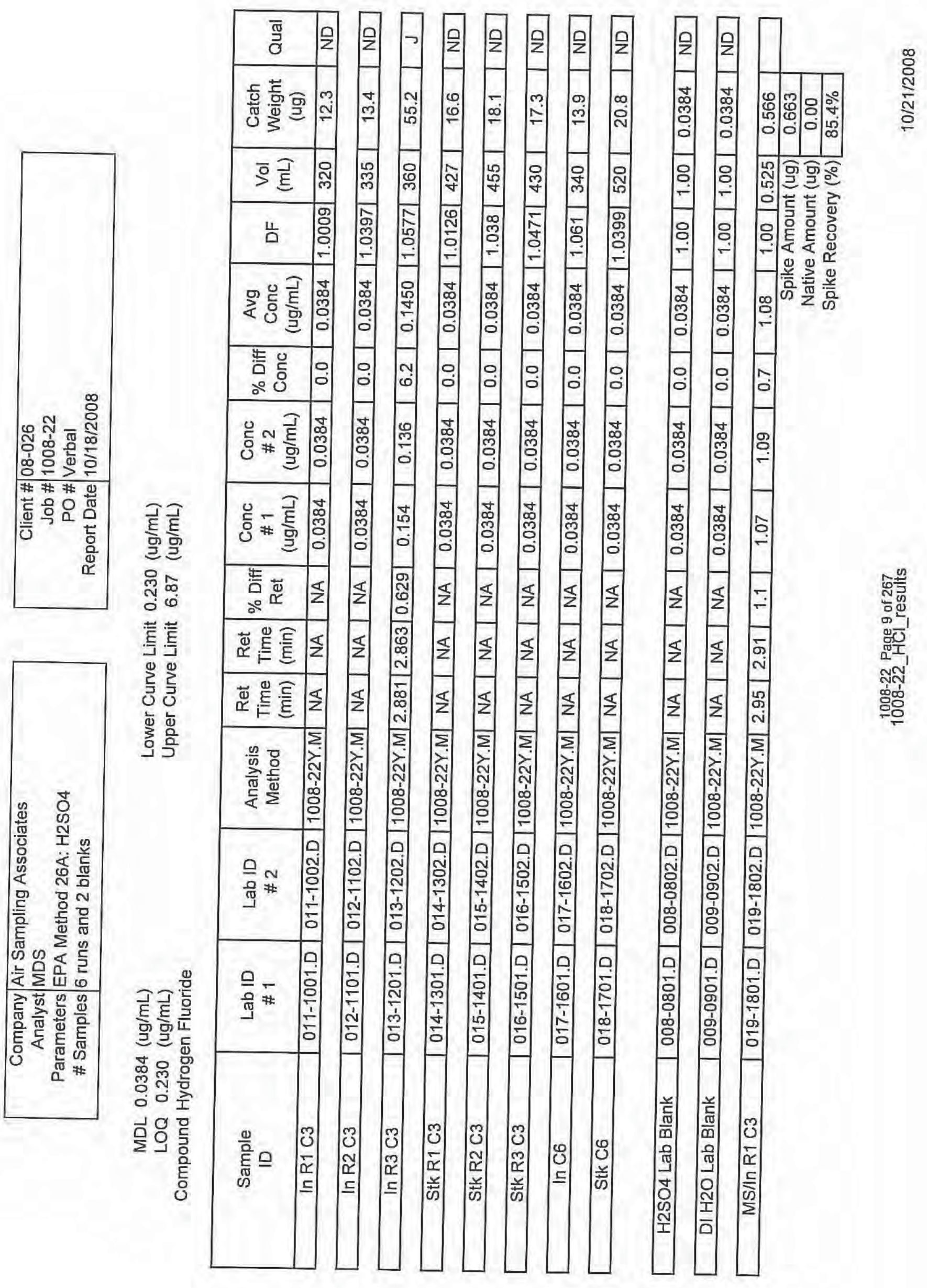




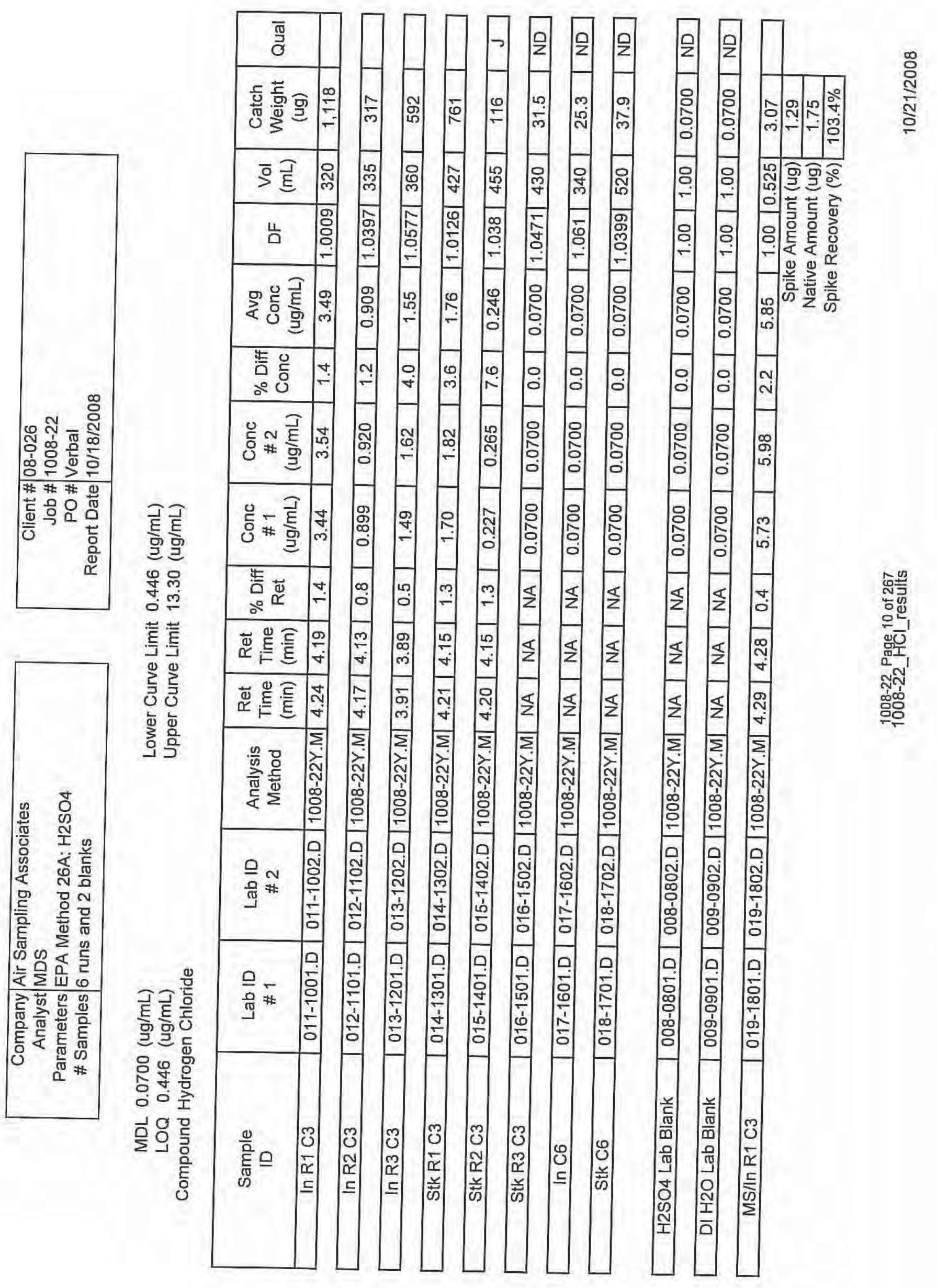




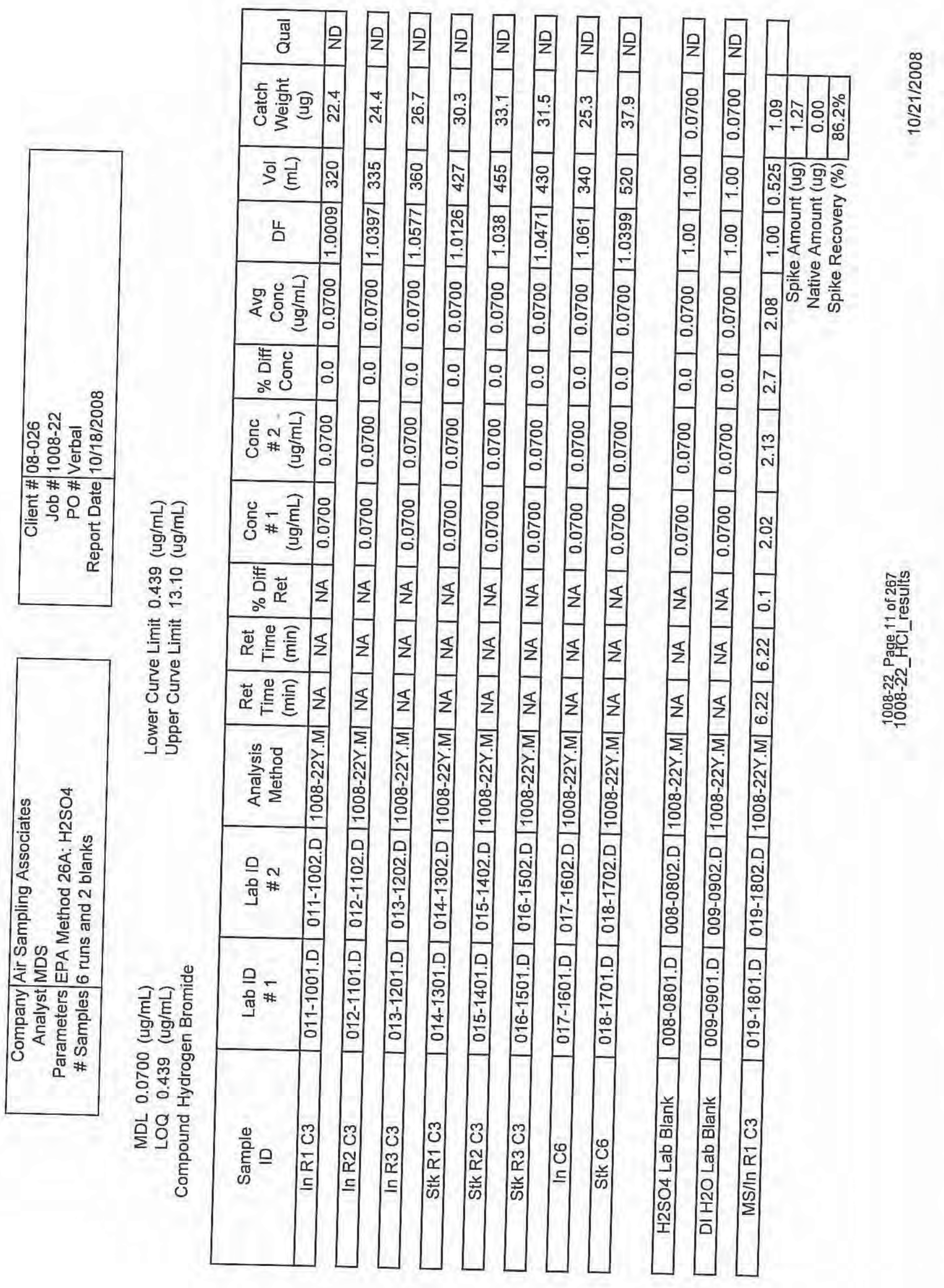




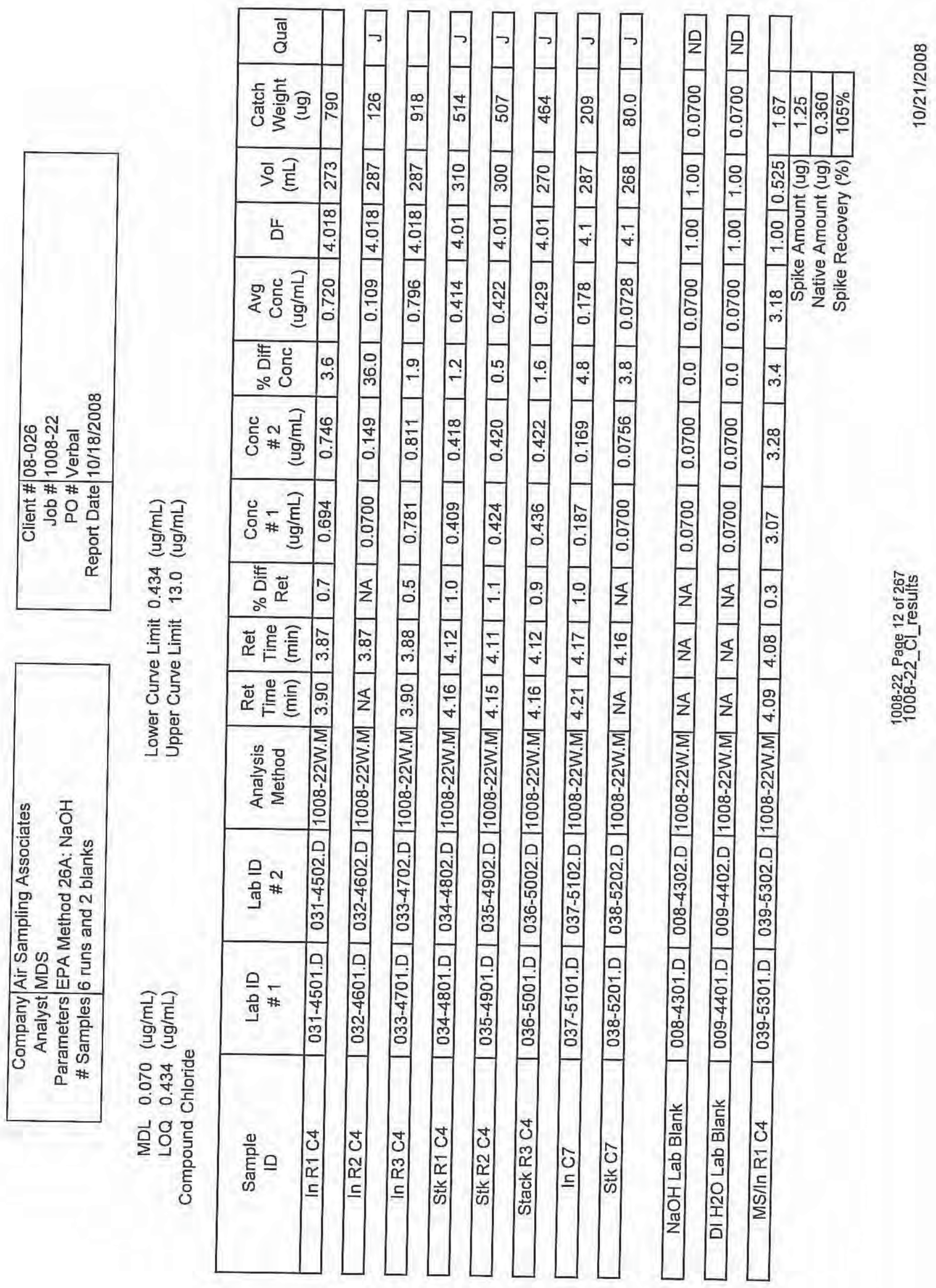




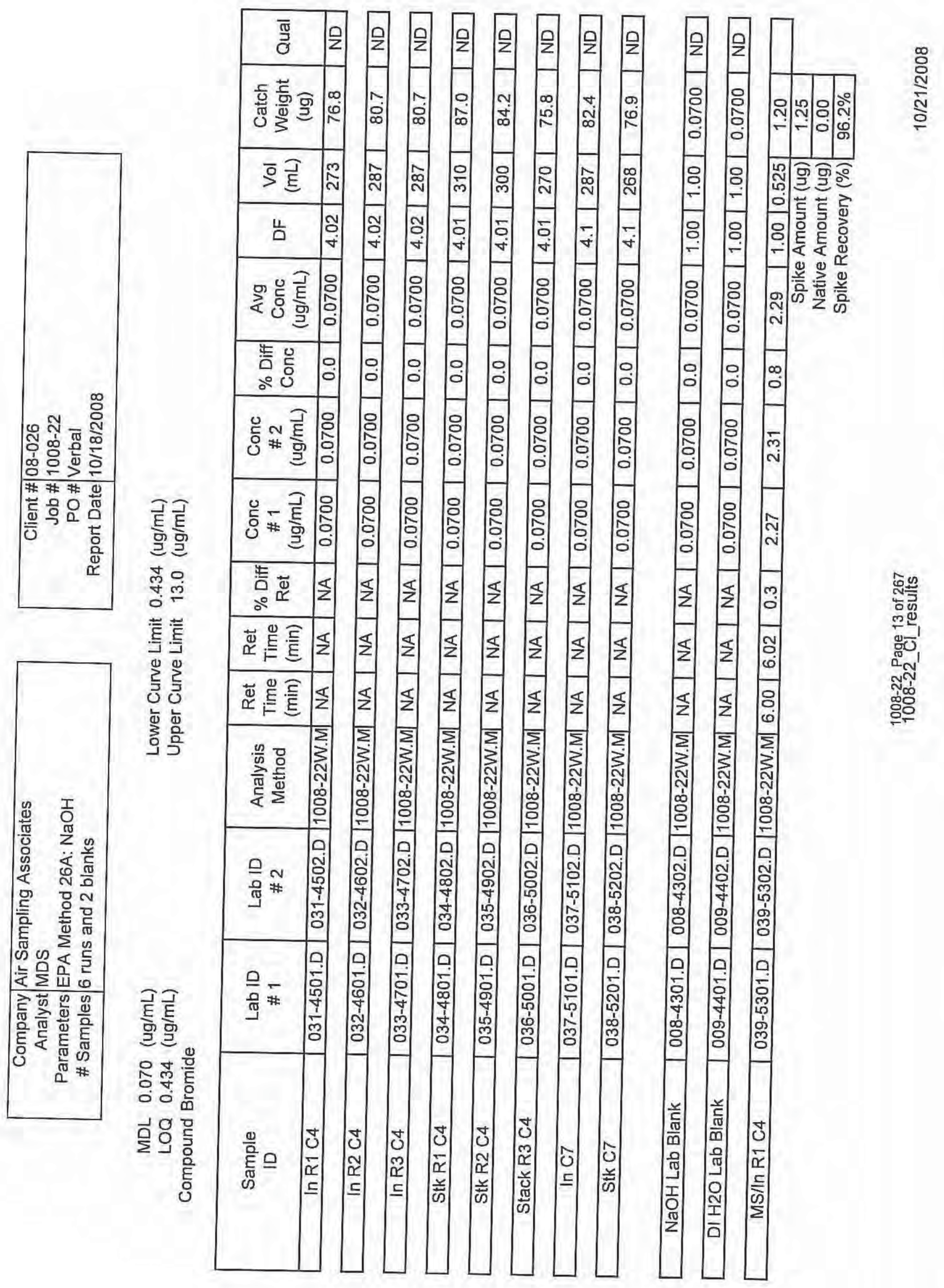




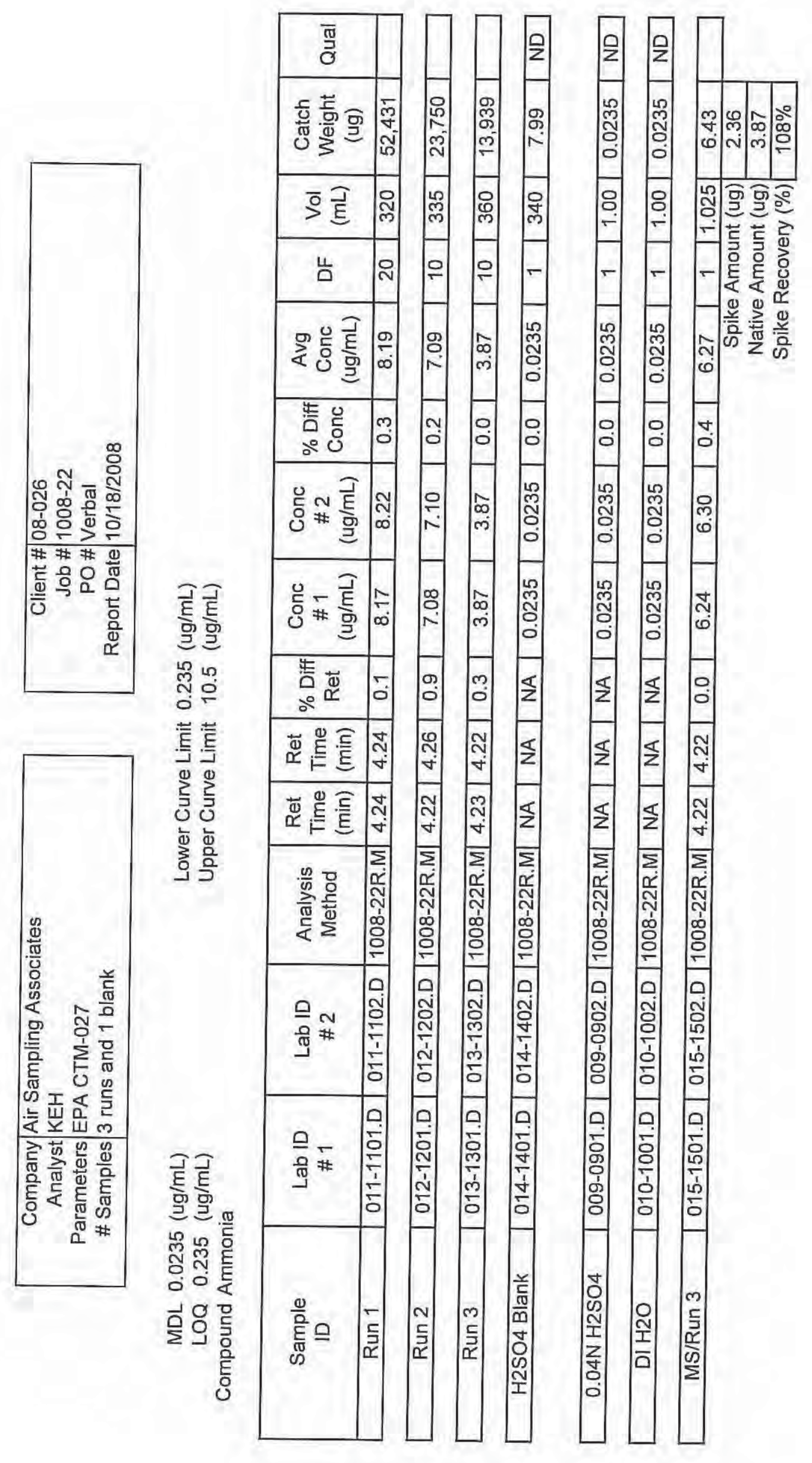

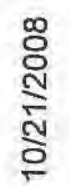

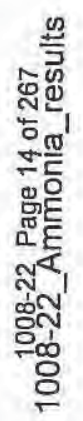




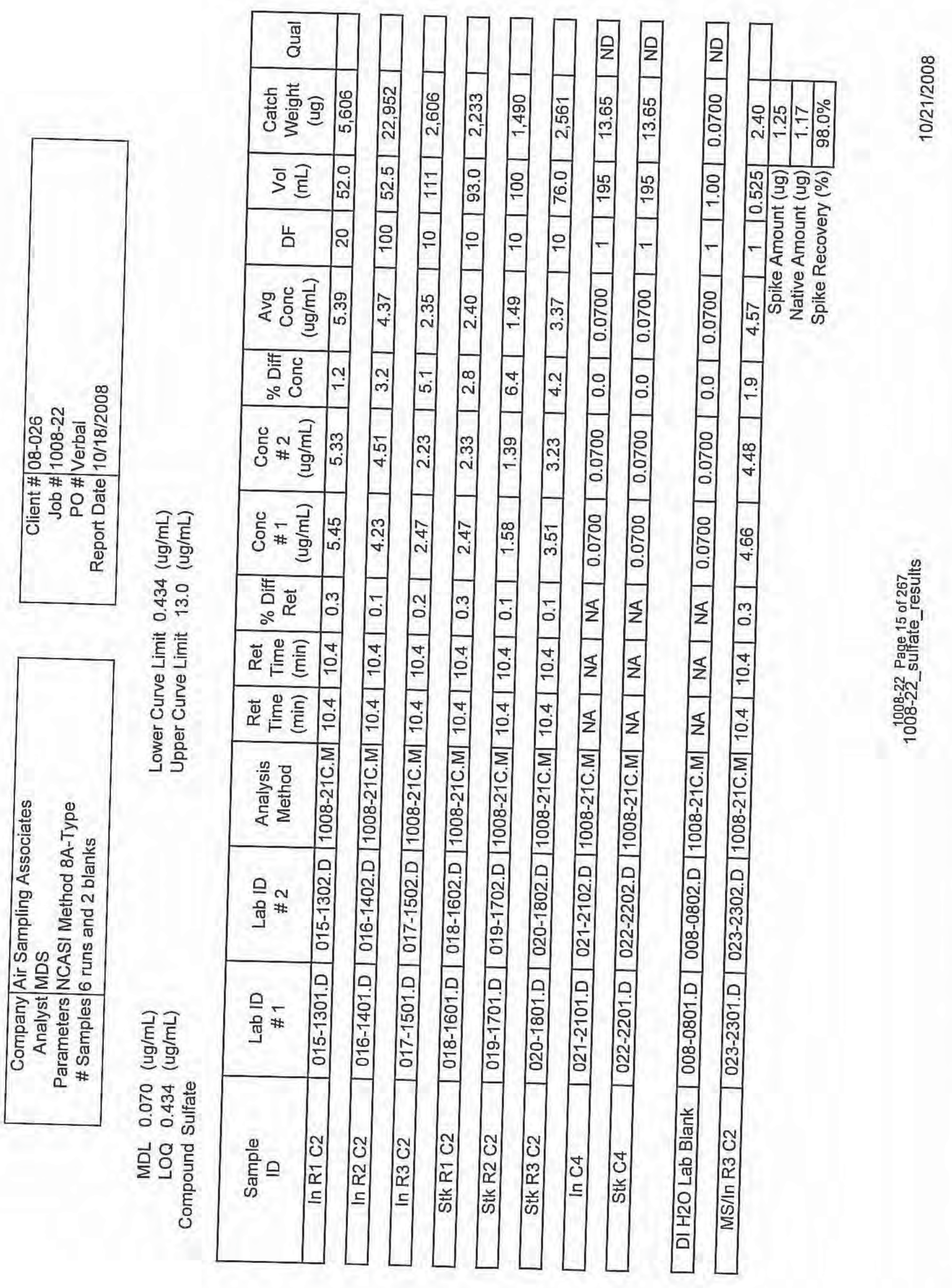




\section{Narrative Summary}

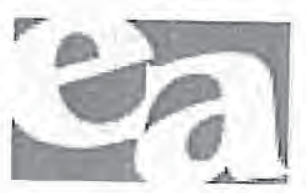




\section{Enthalpy Analytical Narrative Summary}

\begin{tabular}{|r|l|}
\hline Company & Air Sampling Associates \\
\hline Analyst & MDS \\
\hline Parameters & EPA Method 26A: H2SO4 \\
\hline \# Samples & 6 runs and 2 blanks \\
\hline
\end{tabular}

\begin{tabular}{|r|l|}
\hline Client \# & $08-026$ \\
\hline Job \# & $1008-22$ \\
\hline PO\# & Verbal \\
\hline Report Date & October 18, 2008 \\
\hline
\end{tabular}

Custody Tara Grayson received the samples on 10/2/08 after being relinquished by Air Sampling Associates. The samples were received at $19.6^{\circ} \mathrm{C}$ and were in good condition. Prior to and during analysis, the samples were kept under lock with access only to authorized personnel by Enthalpy Analytical, Inc.

Analysis The samples were analyzed for Hydrogen fluoride, Hydrogen chloride and Hydrogen bromide using the analytical procedures in EPA Method 26A, Determination of Hydrogen Halide and Halogen Emissions from Stationary Sources Isokinetic Method (40 CFR Part 60, Appendix A).

The samples were analyzed following the procedures in Section 11.0, Analytical Procedures. All samples and standards are prepared, stored, and analyzed using high-density polyethylene containers.

The Agilent Model 1100, High Performance Liquid Chromatograph ("Gonzo") was equipped as follows:

Front: Dionex CD20 Conductivity Detector.

Front: Dionex Ion Pac AS14A, 4 x 250 mm (S/N006255)

Rear: Dionex CD20 Conductivity Detector

Rear: Dionex Ion Pac AS14A, 4 x 250 mm (S/N006256)

Calibration The calibration curves are located in the back of this report and referenced in the Analysis Method column on the Detailed Results page.

For each calibration curve used, the first page of the curve contains all method specific parameters (i.e., curve type, origin, weight, etc.) used to quantify the samples. The calibration curve section also includes a table with the Retention Time (RetTime), Level (Lvl), Amount (corresponding units), Area, Response Factor (Amt/Area) and the analyte Name. The calibration table is used to identify (by retention time) and quantify each target compound.

Chromatographic The acquisition method (COLSWTCH.M) is included in the Curve/QA Conditions

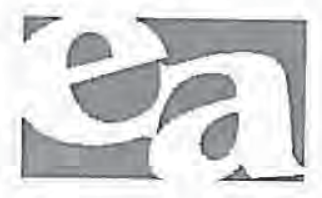
Chromatograms section of this report. 


\section{Enthalpy Narrative Summary (Cont.)}

QC Notes As required in Section 7.2.2, Absorbing Solution Blanks, clientprovided reagent blanks were analyzed. Additionally, a quality control check sample was analyzed at the same time as the blanks and samples. All method required acceptance criteria were met.

All sample preparation and analytical holding times specified in the method were met. Section, 13.2, Sample Stability, specifies an analytical holding time of four weeks.

Reporting Notes The $\mathrm{H}_{2} \mathrm{SO}_{4}$ matrix samples were analyzed for $\mathrm{Cl}^{-}$and $\mathrm{F}^{-}$but are reported as $\mathrm{HCl}$ and HF. The peaks listed in the "Sample Chromatograms" section are labeled hydrogen chloride and hydrogen fluoride. The additional molecular weight contribution from the $\mathrm{H}^{+}$is included in the results listed on each chromatogram and throughout the report.

Enthalpy Analytical, Inc. is accredited to perform this method for compliance purposes by the National Environmental Laboratory Accreditation Conference (NELAC) through the Louisiana Environmental Laboratory Accreditation Program (LELAP), certificate number 04010 .

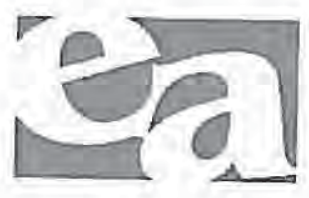




\section{Enthalpy Analytical Narrative Summary}

\begin{tabular}{|r|l|}
\hline Company & Air Sampling Associates \\
\hline Analyst & MDS \\
\hline Parameters & EPA Method $26 \mathrm{~A}: \mathrm{NaOH}$ \\
\hline \# Samples & 6 runs and 2 blanks \\
\hline
\end{tabular}

\begin{tabular}{|r|l|}
\hline Client \# & $08-026$ \\
\hline Job \# & $1008-22$ \\
\hline PO \# & Verbal \\
\hline Report Date & October 18, 2008 \\
\hline
\end{tabular}

Custody Tara Grayson received the samples on 10/2/08 after being relinquished by Air Sampling Associates. The samples were received at $19.6^{\circ} \mathrm{C}$ and were in good condition. Prior to and during analysis, the samples were kept under lock with access only to authorized personnel by Enthalpy Analytical, Inc.

Analysis The samples were analyzed for Bromide and Chloride using the analytical procedures in EPA Method 26A, Determination of Hydrogen Halide and Halogen Emissions from Stationary Sources Isokinetic Method (40 CFR Part 60, Appendix A).

The samples were analyzed following the procedures in Section 11.0, Analytical Procedures. All samples and standards are prepared, stored, and analyzed using high-density polyethylene containers.

The Agilent Model 1100, High Performance Liquid Chromatograph

("Gonzo") was equipped as follows:

Front: Dionex CD20 Conductivity Detector.

Front: Dionex Ion Pac AS14A, 4 x 250 mm (S/N006255)

Rear: Dionex CD20 Conductivity Detector

Rear: Dionex Ion Pac AS14A, 4 x 250 mm (S/N006256)

Calibration The calibration curves are located in the back of this report and referenced in the Analysis Method column on the Detailed Results page.

For each calibration curve used, the first page of the curve contains all method specific parameters (i.e., curve type, origin, weight, etc.) used to quantify the samples. The calibration curve section also includes a table with the Retention Time (RetTime), Level (Lvl), Amount (corresponding units), Area, Response Factor (Amt/Area) and the analyte Name. The calibration table is used to identify (by retention time) and quantify each target compound.

Chromatographic Conditions The acquisition method (CHLSWTCH.M) is included in the Curve/QA
Chromatograms section of this report.

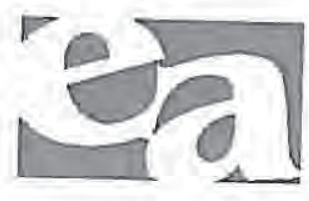




\section{Enthalpy Narrative Summary (Cont.)}

QC Notes As required in Section 7.2.2, Absorbing Solution Blanks, clientprovided reagent blanks were analyzed. Additionally, a quality control check sample was analyzed at the same time as the blanks and samples. All method required acceptance criteria were met.

All sample preparation and analytical holding times specified in the method were met. Section. 13.2, Sample Stability, specifies an analytical holding time of four weeks.

Reporting Notes Enthalpy Analytical, Inc. is accredited to perform this method for compliance purposes by the National Environmental Laboratory Accreditation Conference (NELAC) through the Louisiana Environmental Laboratory Accreditation Program (LELAP), certificate number 04010 . 


\section{Enthalpy Analytical Narrative Summary}

\begin{tabular}{|r|l|}
\hline Company & Air Sampling Associates \\
\hline Analyst & KEH \\
\hline Parameters & EPA CTM-027 \\
\hline \# Samples & 3 runs and 1 blank \\
\hline
\end{tabular}

\begin{tabular}{|r|l|}
\hline Client \# & $08-026$ \\
\hline Job \# & $1008-22$ \\
\hline PO \# & Verbal \\
\hline Report Date & October 18, 2008 \\
\hline
\end{tabular}

Custody Tara Grayson received the samples on 10/2/08 after being relinquished by Air Sampling Associates. The samples were received at $19.6^{\circ} \mathrm{C}$ and were in good condition. Prior to and during analysis, the samples were kept under lock with access only to authorized personnel by Enthalpy Analytical, Inc.

Analysis The samples were analyzed for Ammonia using the analytical procedures in EPA Conditional Test Method 027, Procedure for Collection and Analysis of Ammonia in Stationary Sources.

The DIONEX Model 500, High Performance Liquid Chromatograph ("Pugsly") was equipped with DIONEX ED40 Electrochemical Detector and a Dionex Ion Pac CS12, 4 x $250 \mathrm{~mm}$ column (S/N 008848).

Calibration The calibration curve is located in the back of this report and referenced in the Analysis Method column on the Detailed Results page.

For each calibration curve used, the first page of the curve contains all method specific parameters (i.e., curve type, origin, weight, etc.) used to quantify the samples. The calibration curve section also includes a table with the Retention Time (RetTime), Level (Lvl), Amount (corresponding units), Area, Response Factor (Amt/Area) and the analyte Name. The calibration table is used to identify (by retention time) and quantify each target compound.

Chromatographic The acquisition method (IC2.M) is included in the Curve/QA Conditions Chromatograms section of this report.

QC Notes As required in section 4.2.3, Quality Control and Quality Assurance Enthalpy periodically analyzes independently prepared standards and blank checks reagents.

All sample preparation and analytical holding times specified in the method were met. In Section. 4.1, Sample Preparation, the specified analytical holding time is two weeks from sampling date.

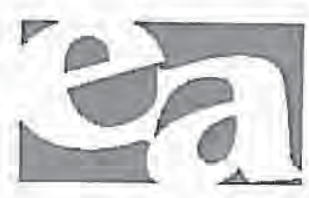




\section{Enthalpy Narrative Summary (Cont.)}

Reporting Notes Enthalpy Analytical, Inc. is accredited to perform this method for compliance purposes by the National Environmental Laboratory Accreditation Conference (NELAC) through the Louisiana Environmental Laboratory Accreditation Program (LELAP), certificate number 04010 .

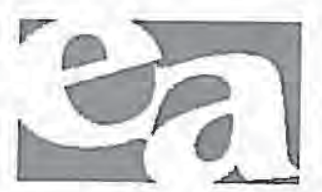




\section{Enthalpy Analytical Narrative Summary}

\begin{tabular}{|r|l|}
\hline Company & Air Sampling Associates \\
\hline Analyst & MDS \\
\hline Parameters & NCASI Method 8A-Type \\
\hline \# Samples & 6 runs and 2 blanks \\
\hline
\end{tabular}

\begin{tabular}{|r|l|}
\hline Client \# & $08-026$ \\
\hline Job \# & $1008-22$ \\
\hline PO \# & Verbal \\
\hline Report Date & October 18, 2008 \\
\hline
\end{tabular}

Custody Tara Grayson received the samples on 10/2/08 after being relinquished by Air Sampling Associates. The samples were received at $19.6^{\circ} \mathrm{C}$ and were in good condition. Prior to and during analysis, the samples were kept under lock with access only to authorized personnel by Enthalpy Analytical, Inc.

Analysis The samples were analyzed for Sulfate using the analytical procedures in Generic HPLC Sample analysis.

The Agilent Model 1100, High Performance Liquid Chromatograph ("Gonzo") was equipped as follows:

Front: Dionex CD20 Conductivity Detector.

Front: Dionex Ion Pac AS14A, 4 x 250 mm (S/N006255)

Rear: Dionex CD20 Conductivity Detector

Rear: Dionex Ion Pac AS14A, 4 x 250 mm (S/N006256)

Calibration The calibration curve is located in the back of this report and referenced in the Analysis Method column on the Detailed Results page.

For each calibration curve used, the first page of the curve contains all method specific parameters (i.e., curve type, origin, weight, etc.) used to quantify the samples. The calibration curve section also includes a table with the Retention Time (RetTime), Level (Lvl), Amount (corresponding units), Area, Response Factor (Amt/Area) and the analyte Name. The calibration table is used to identify (by retention time) and quantify each target compound.

Chromatographic

Conditions

QC Notes None

Reporting Notes

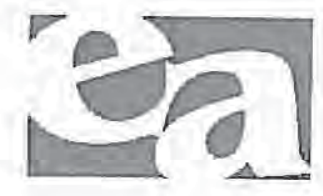

The acquisition method (COLSULF.M) is included in the Curve/QA Chromatograms section of this report.

Enthalpy Analytical, Inc. is accredited to perform this method for compliance purposes by the National Environmental Laboratory Accreditation Conference (NELAC) through the Louisiana Environmental Laboratory Accreditation Program (LELAP), certificate number 04010 . 


\section{General Reporting Notes}

The following are general reporting notes that are applicable to all Enthalpy Analytical, Inc. reports, unless specifically noted otherwise.

- The symbol $M D L$ represents the Minimum Detection Limit. Below this value the laboratory cannot confim the presence of the analyte of interest reliably.

- The symbol $L O Q$ represents the Limit of Quantification. Below this value the laboratory cannot quantitate the analyte of interest within the criteria of the method.

- The symbol ND following a value indicates a non-detect or analytical result below the MDL.

- The symbol $\boldsymbol{J}$ following a value indicates an analytical result between the MDL and the LOQ. A J flag indicates that the laboratory can positively identify the analyte of interest as present, but the value should be considered an estimate.

- The symbol $\boldsymbol{E}$ following a value indicates an analytical result exceeding $100 \%$ of the highest calibration point.

- The symbol $\boldsymbol{D F}$ represents a Dilution Factor. This number represents dilutions during the extraction and/or laboratory stages of sample treatment. The analytical result taken from a laboratory instrument is multiplied by the DF to get final results.

- The Sample ID $\boldsymbol{M S}$ represents a Matrix Spike. An aliquot of an actual sample is spiked with a known amount of analyte so that a percent recovery value can be determined. This shows what effect the sample matrix may have on the target analyte, i.e. whether or not anything in the sample matrix prohibits analysis for the analyte(s).

- The Sample ID MSD represents a Matrix Spike Duplicate. Prepared in the same manner as an MS, the use of duplicate matrix spikes allows further confirmation of laboratory quality by showing the consistency of results gained by performing the same steps multiple times. Most methods performed by Enthalpy do not require analysis of an MSD.

- The Sample ID BS represents a Blind Spike. A member of the Quality Assurance department has created BS samples for many of the analytes Enthalpy tests for, and only QA and the Enthalpy Analytical ownership have access to the actual values of these samples. The laboratory analyzes them without knowledge of the actual value, and the spreadsheets get completed for these samples solely by the QA group.

- The Sample ID $\boldsymbol{L C S}$ represents a Laboratory Control Sample. Whenever spikes are prepared for our clients more spikes are prepared than needed. The extras (randomly chosen) are kept in-house at the appropriate temperature conditions. When the spike samples come back from the client for analysis, the LCSs (usually two are saved) are analyzed to confirm that the analyte could be recovered from the media, separate from the spike samples which were used on the project and which may have had issues caused during collection and/or transport.

- Significant Figures: Where the reported value is much greater than unity (1.00) in the units expressed (specifically values of 1,000 or greater), the number is rounded to a whole number of units, rather than to 3 significant figures. For example, a value of $10,456.45$ ug catch is rounded to 10,456 ug. There are five significant digits reported, but no confidence should be placed on more than three significant digits.

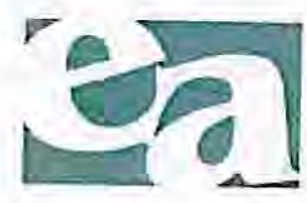




\section{Sample Custody}

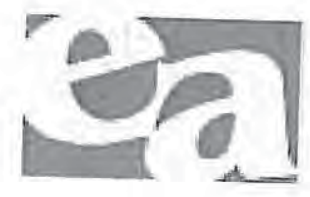




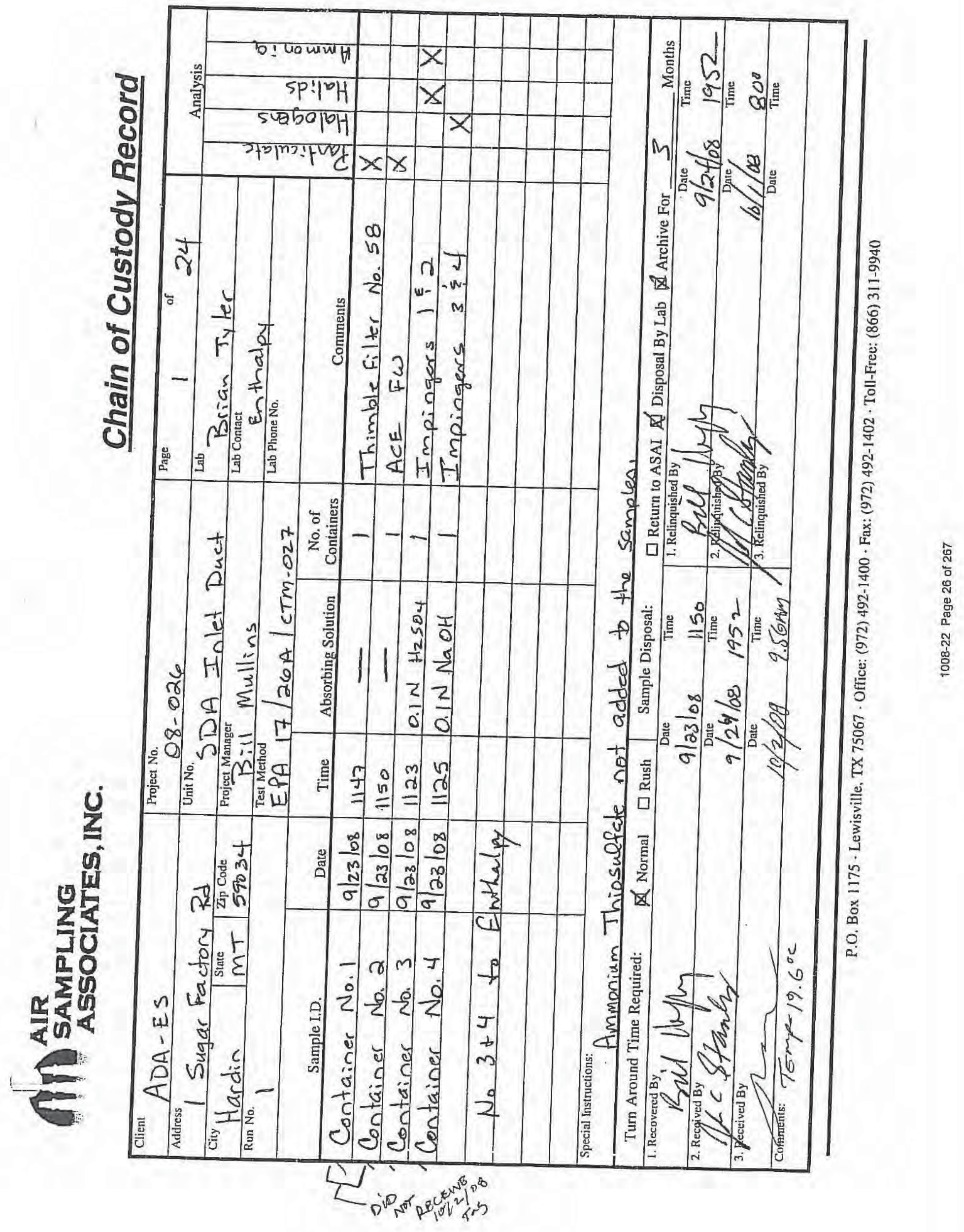




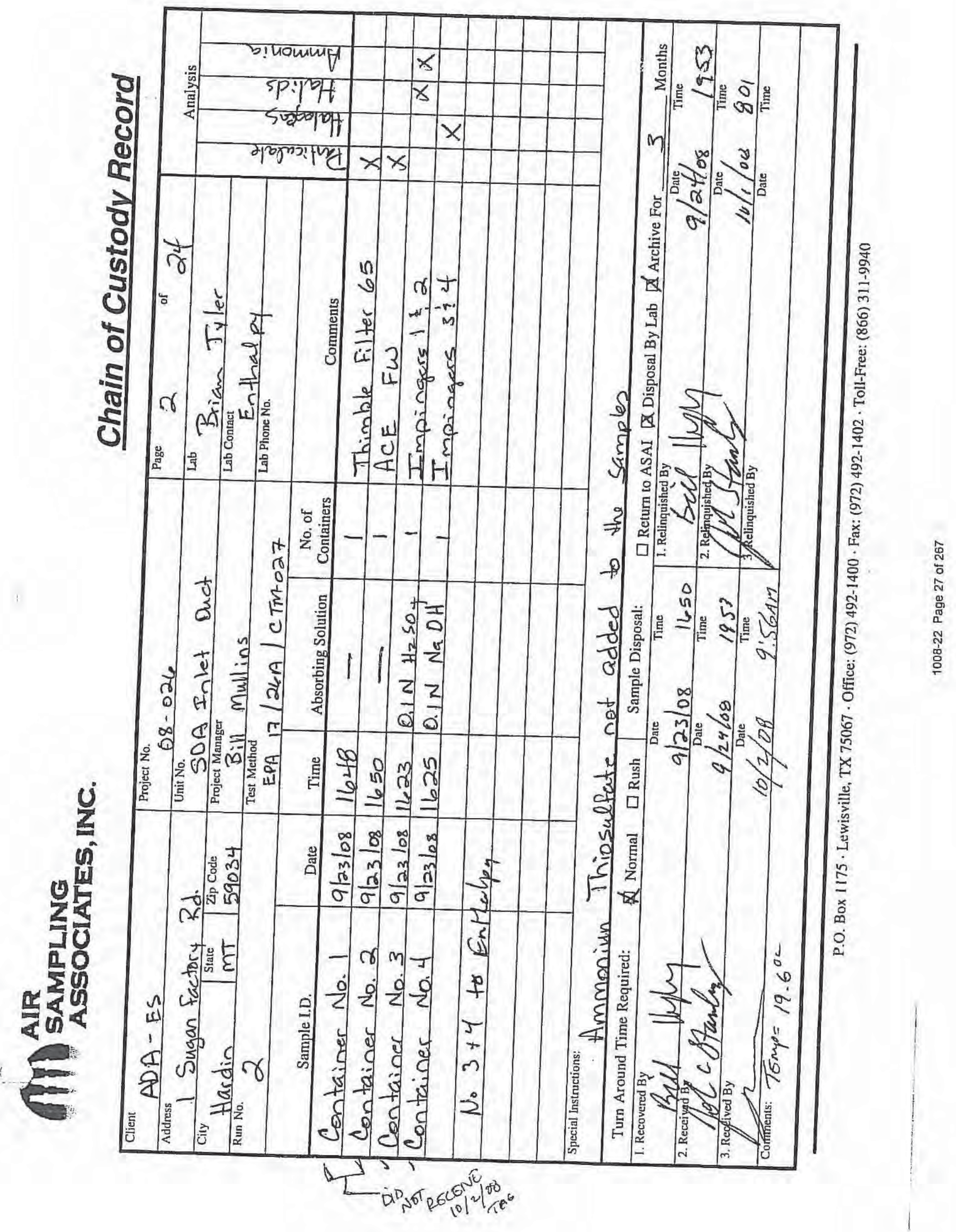




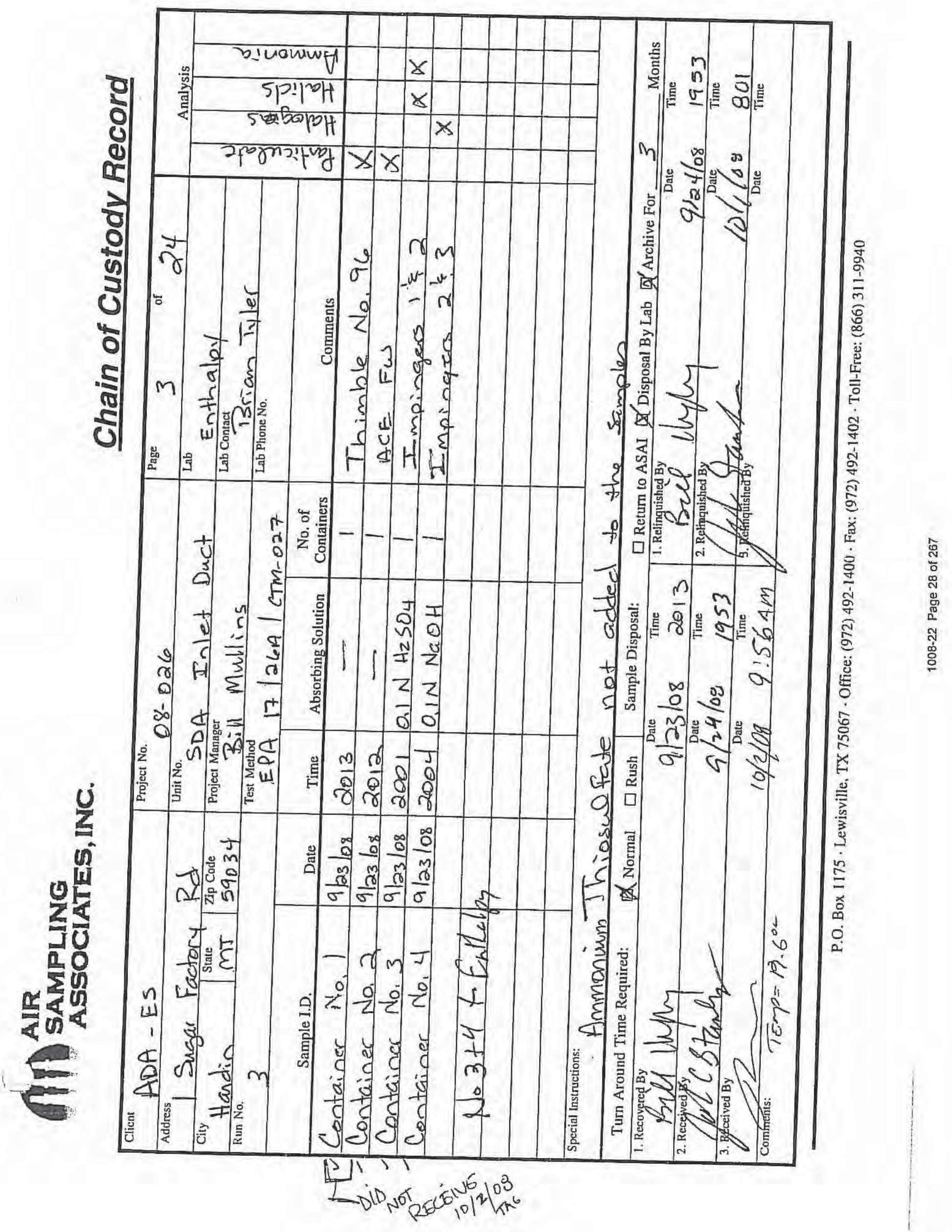




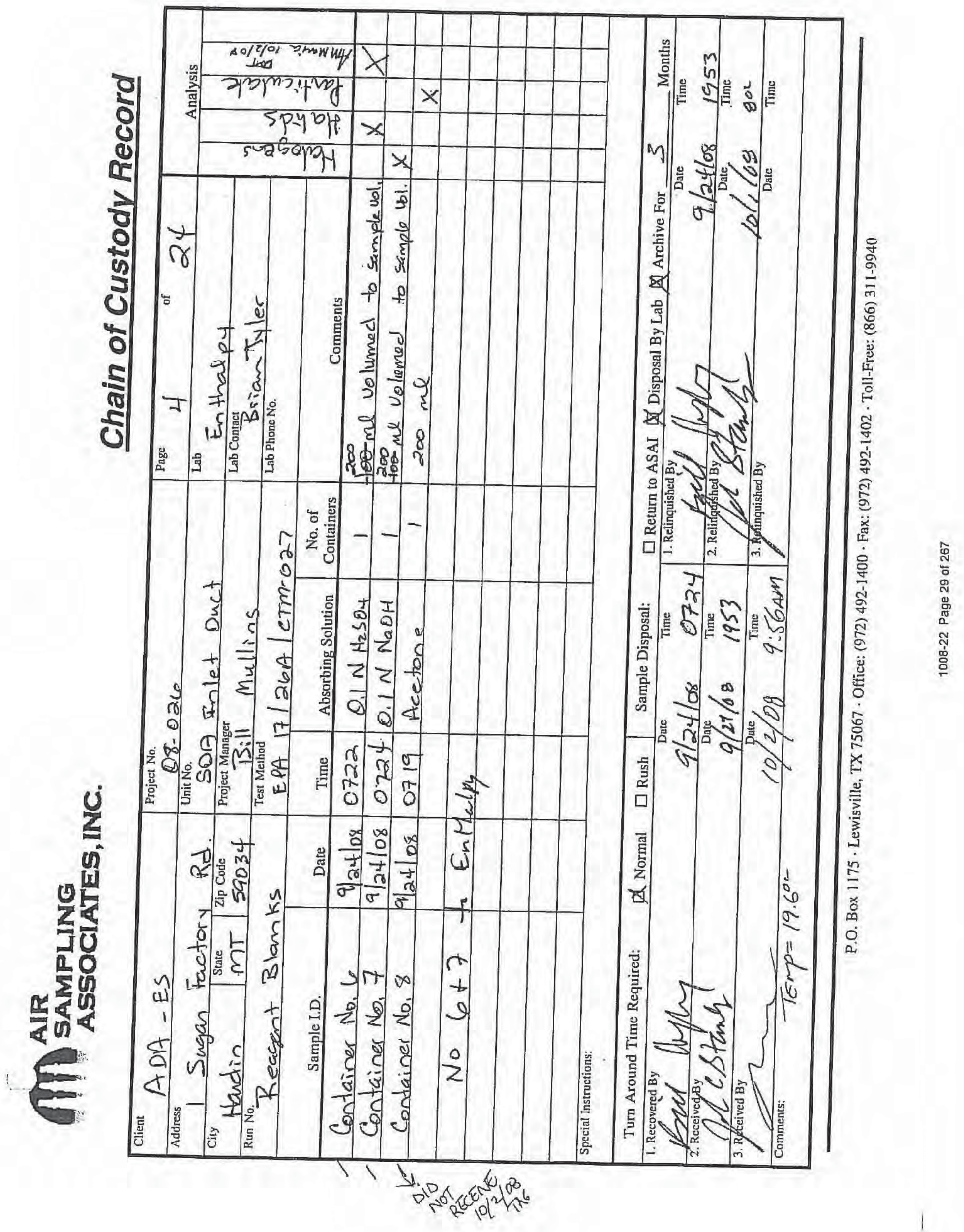




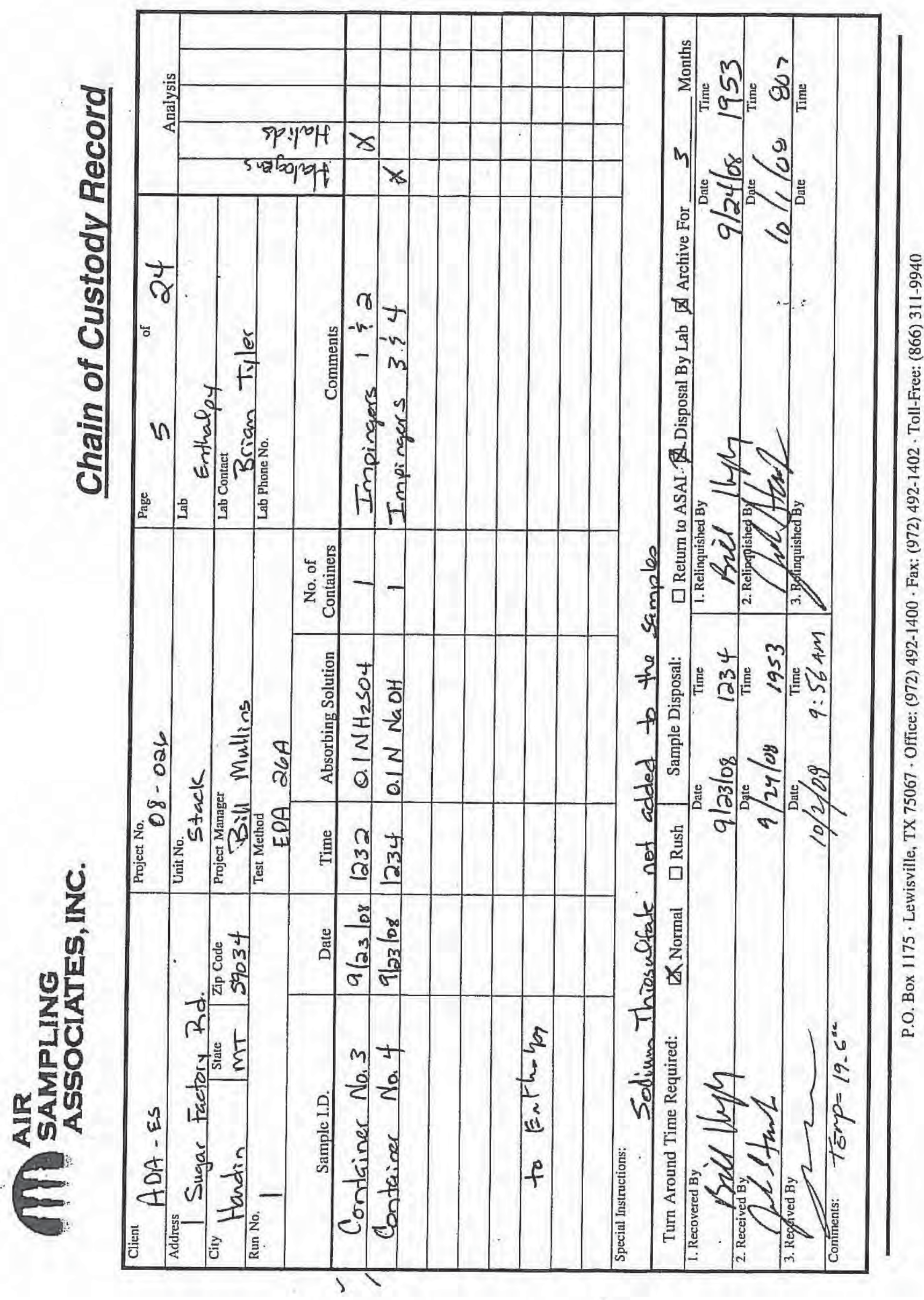

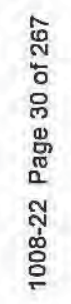




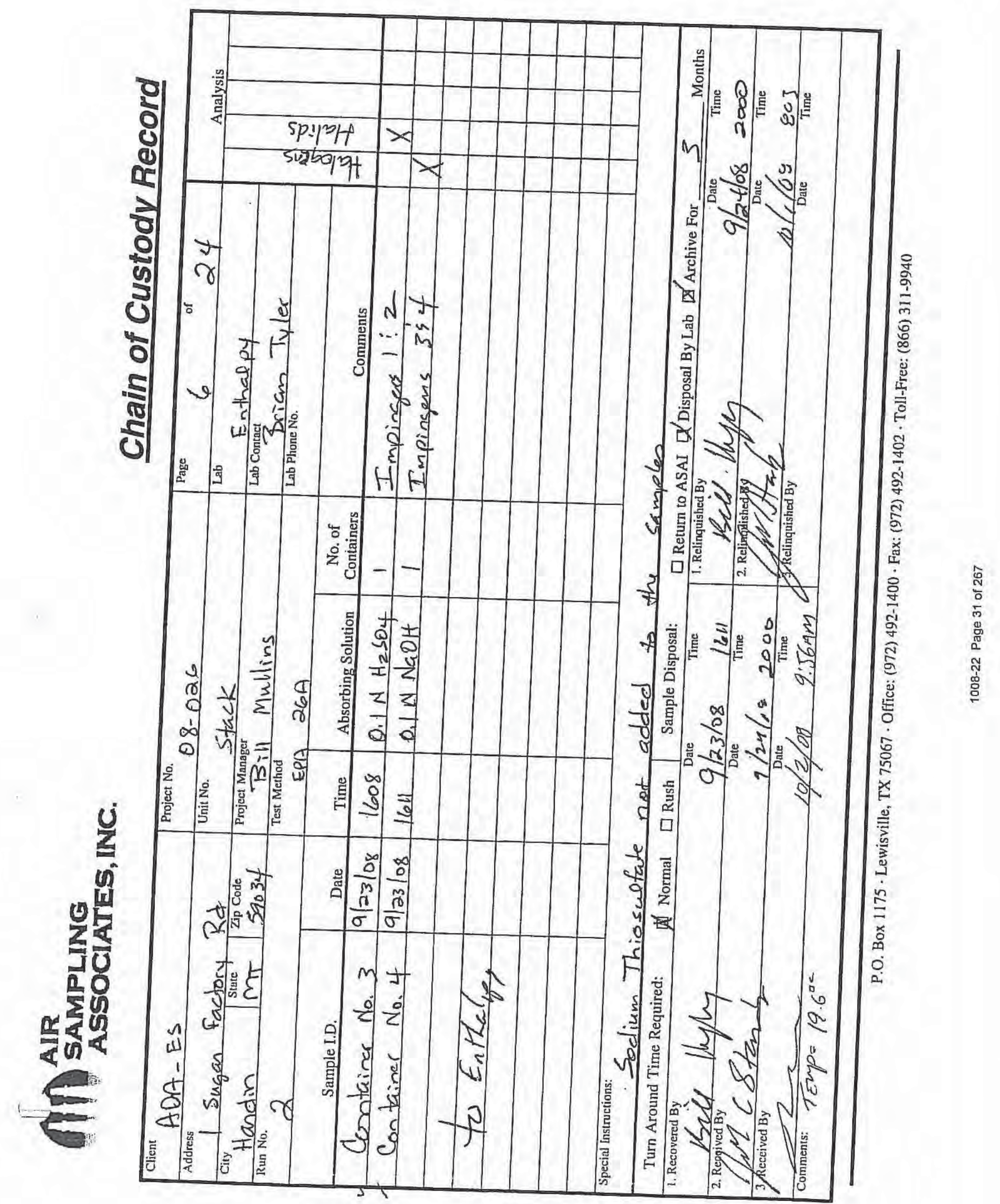




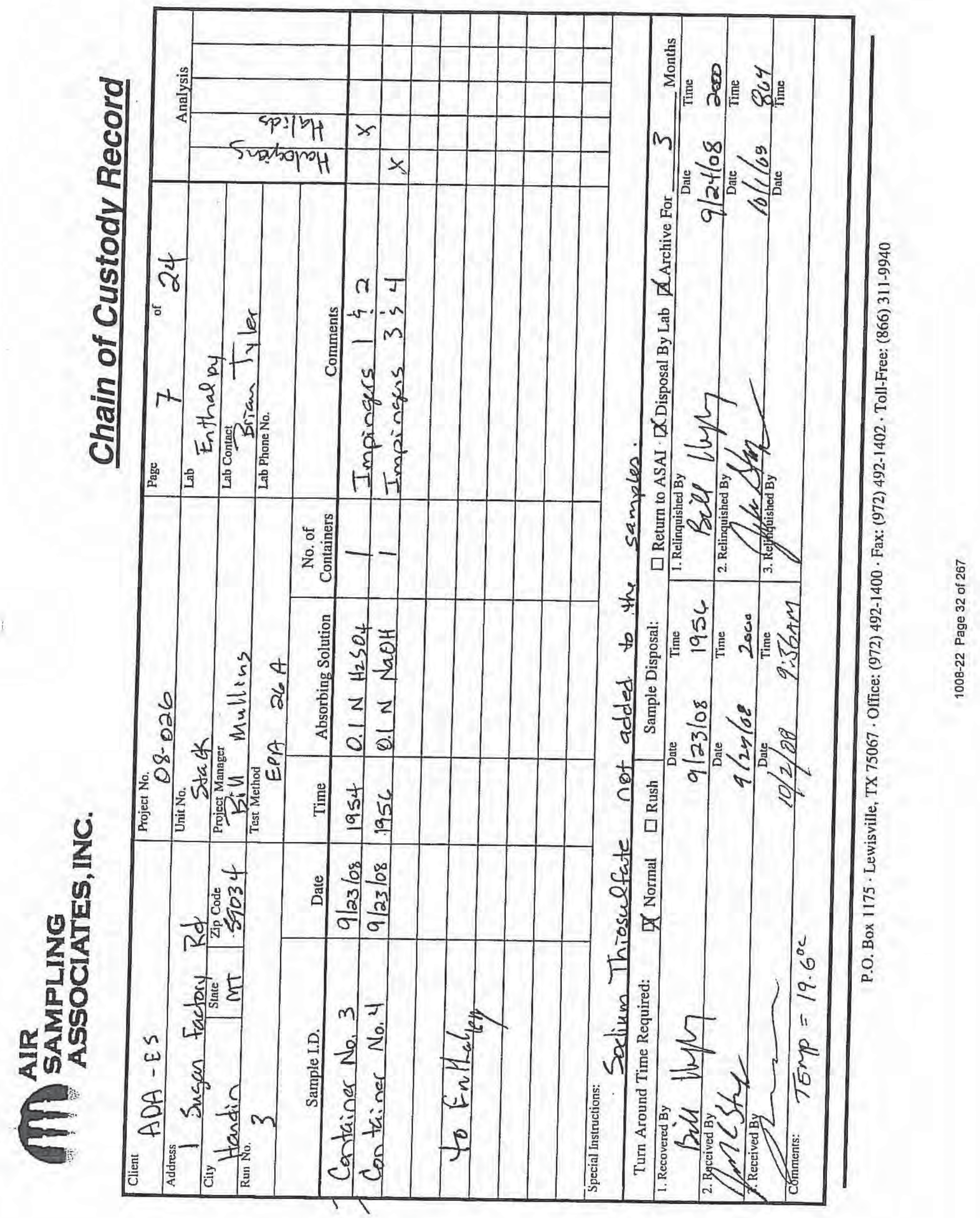




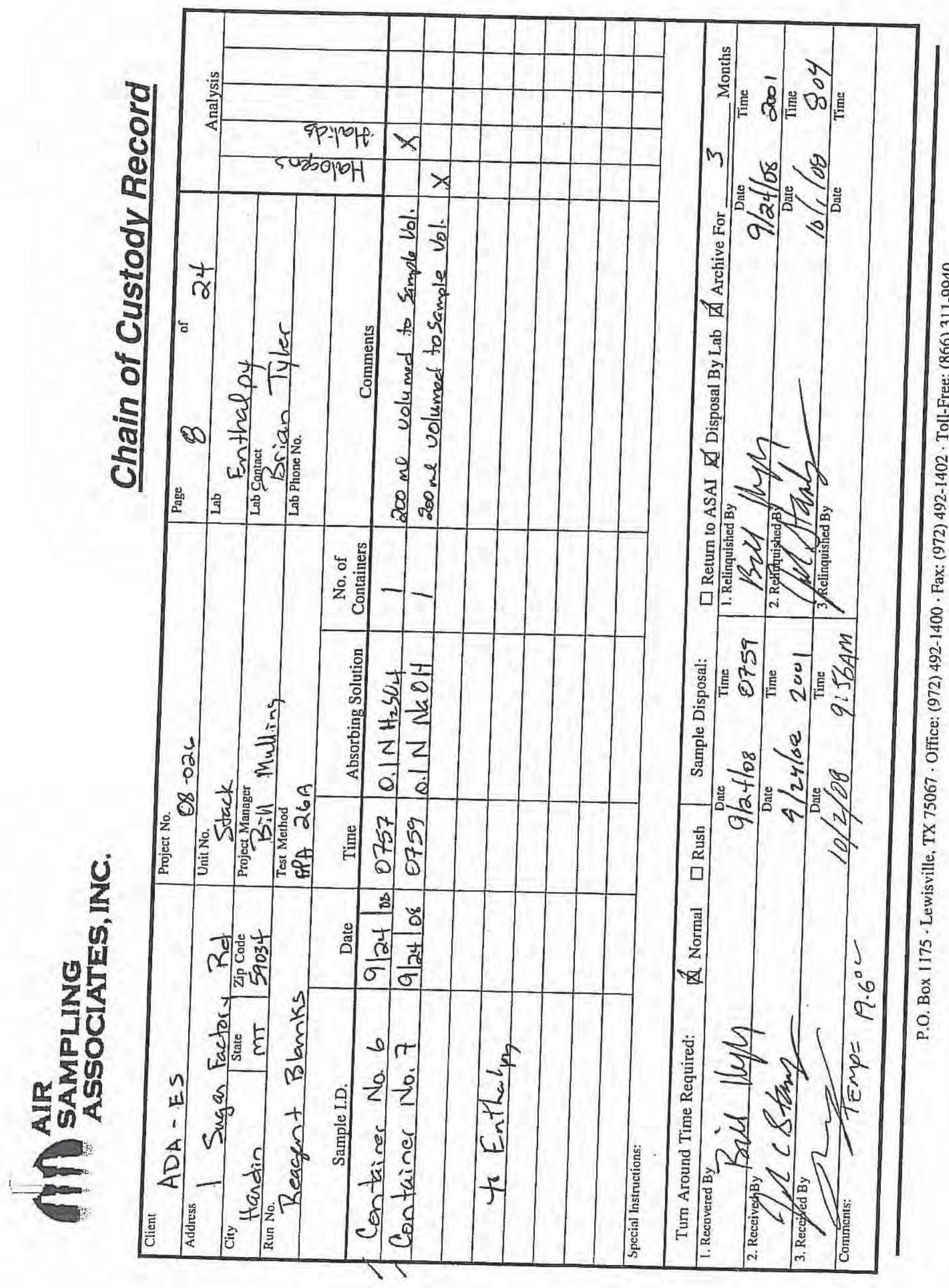




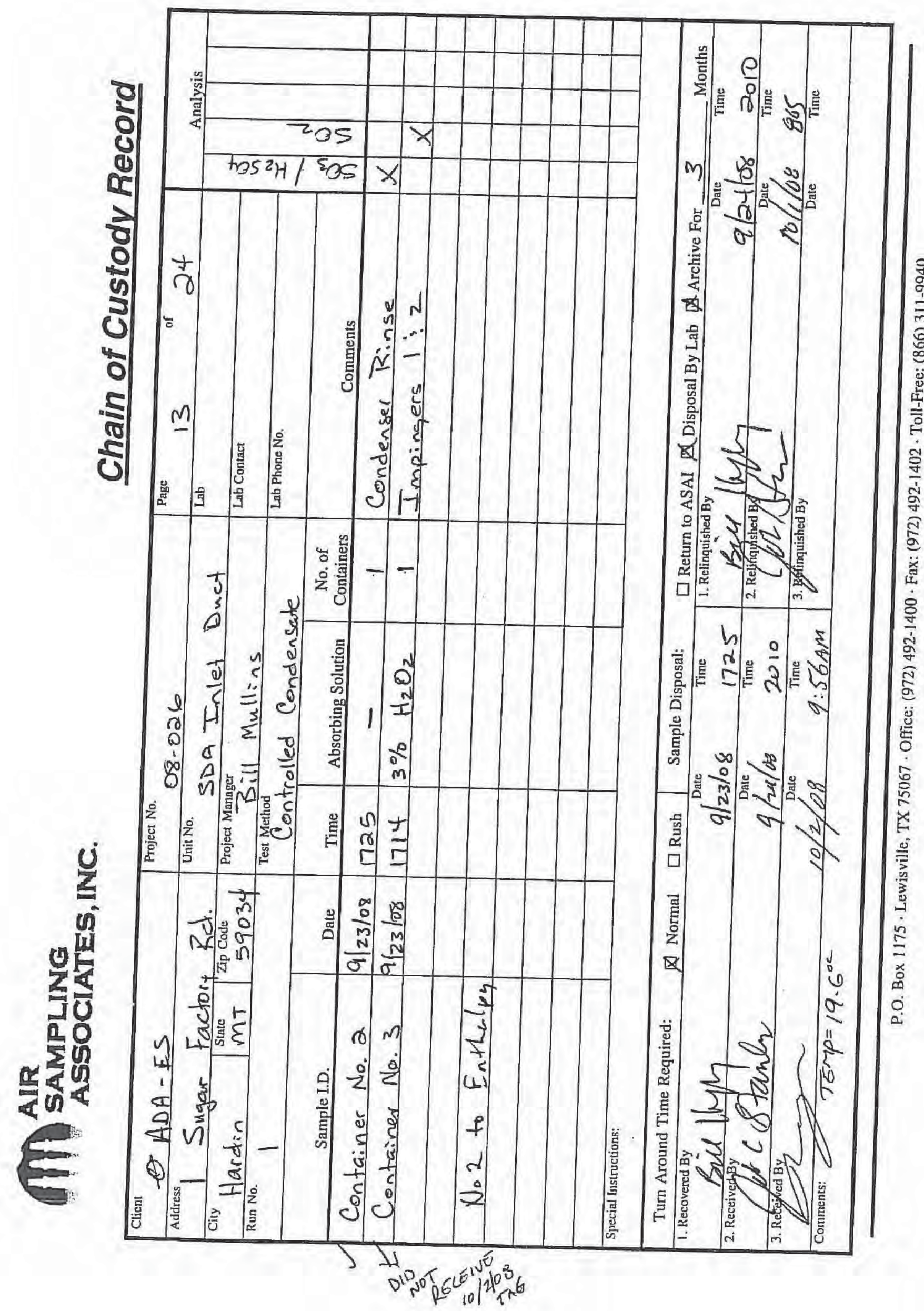




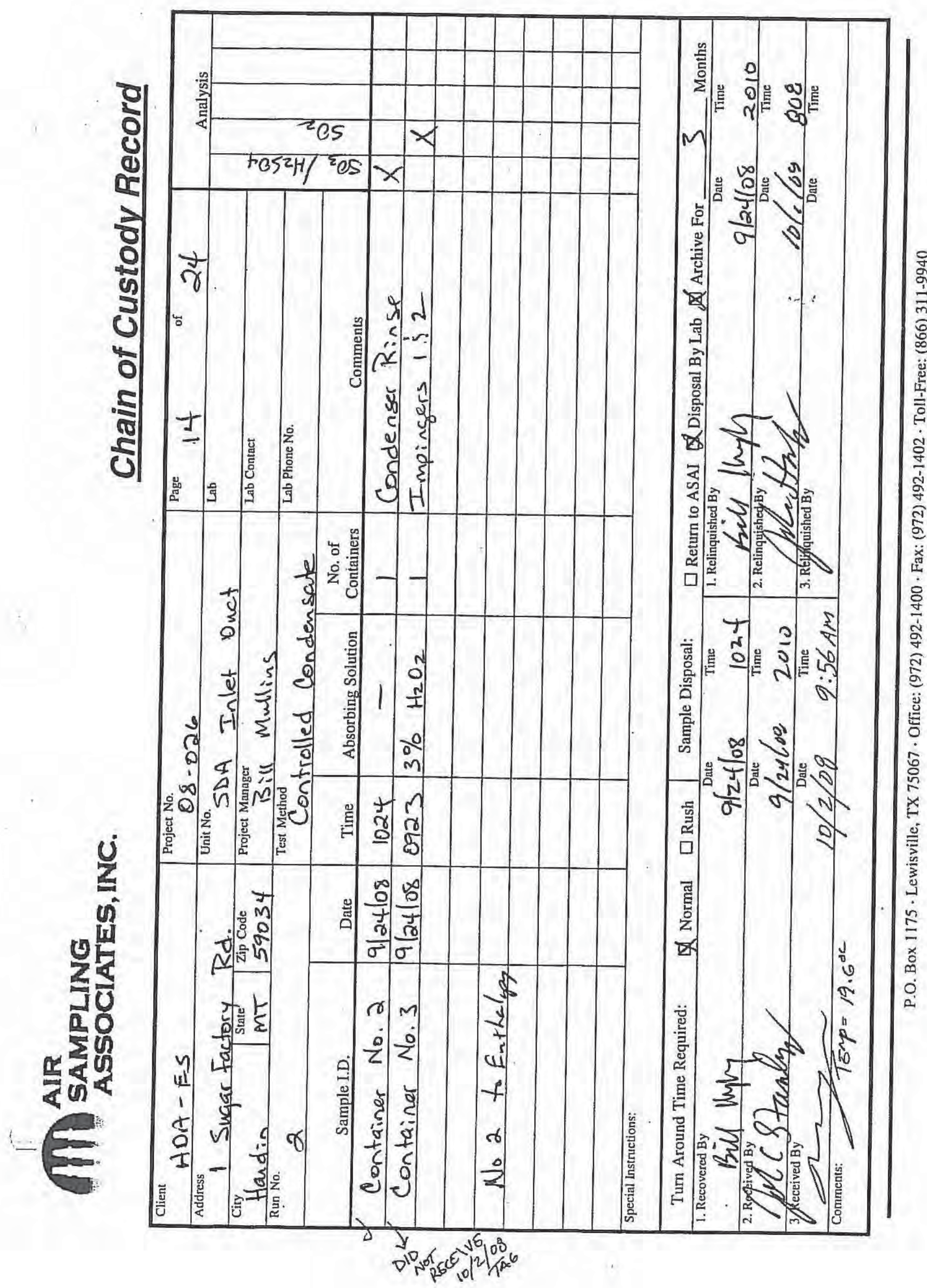




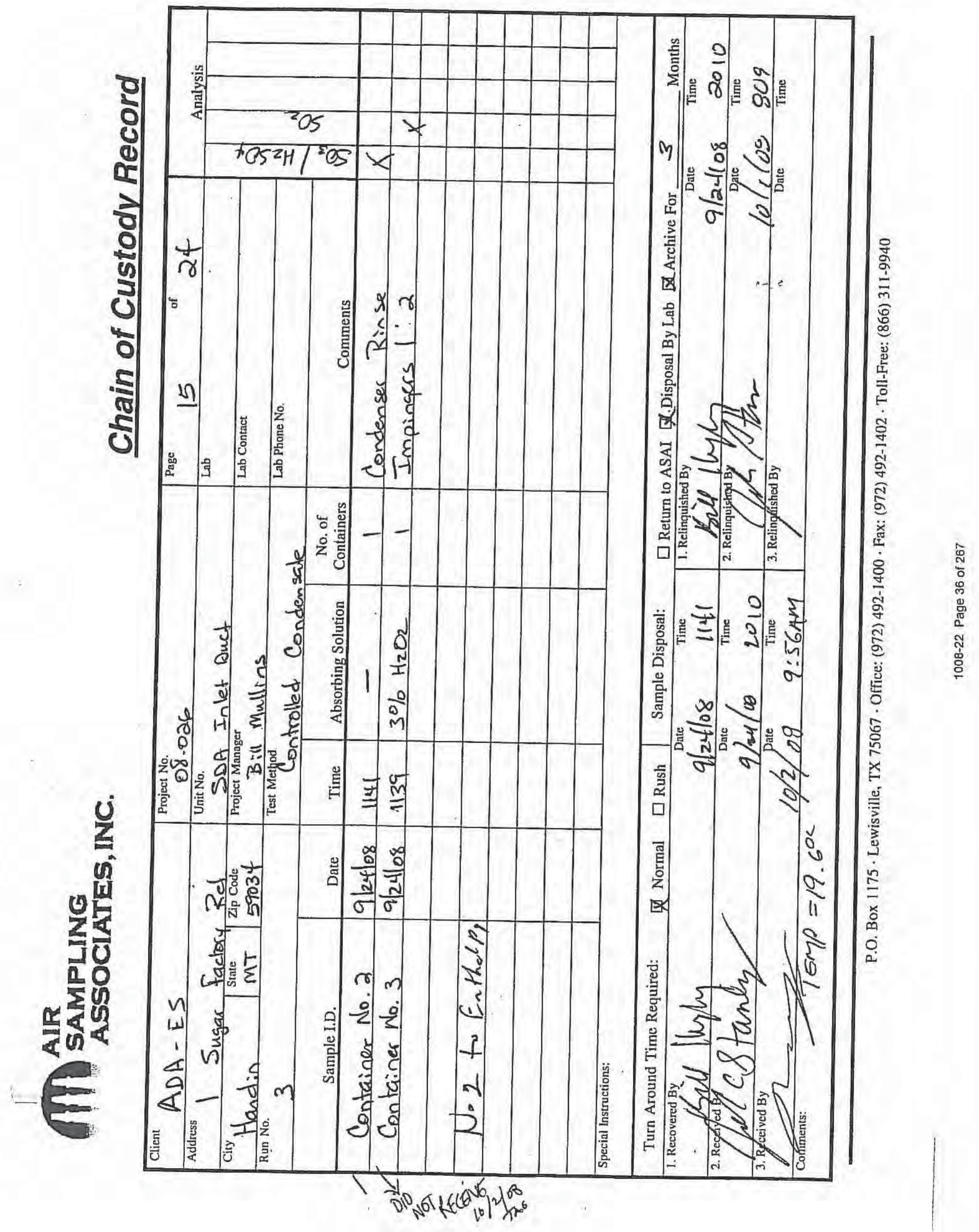




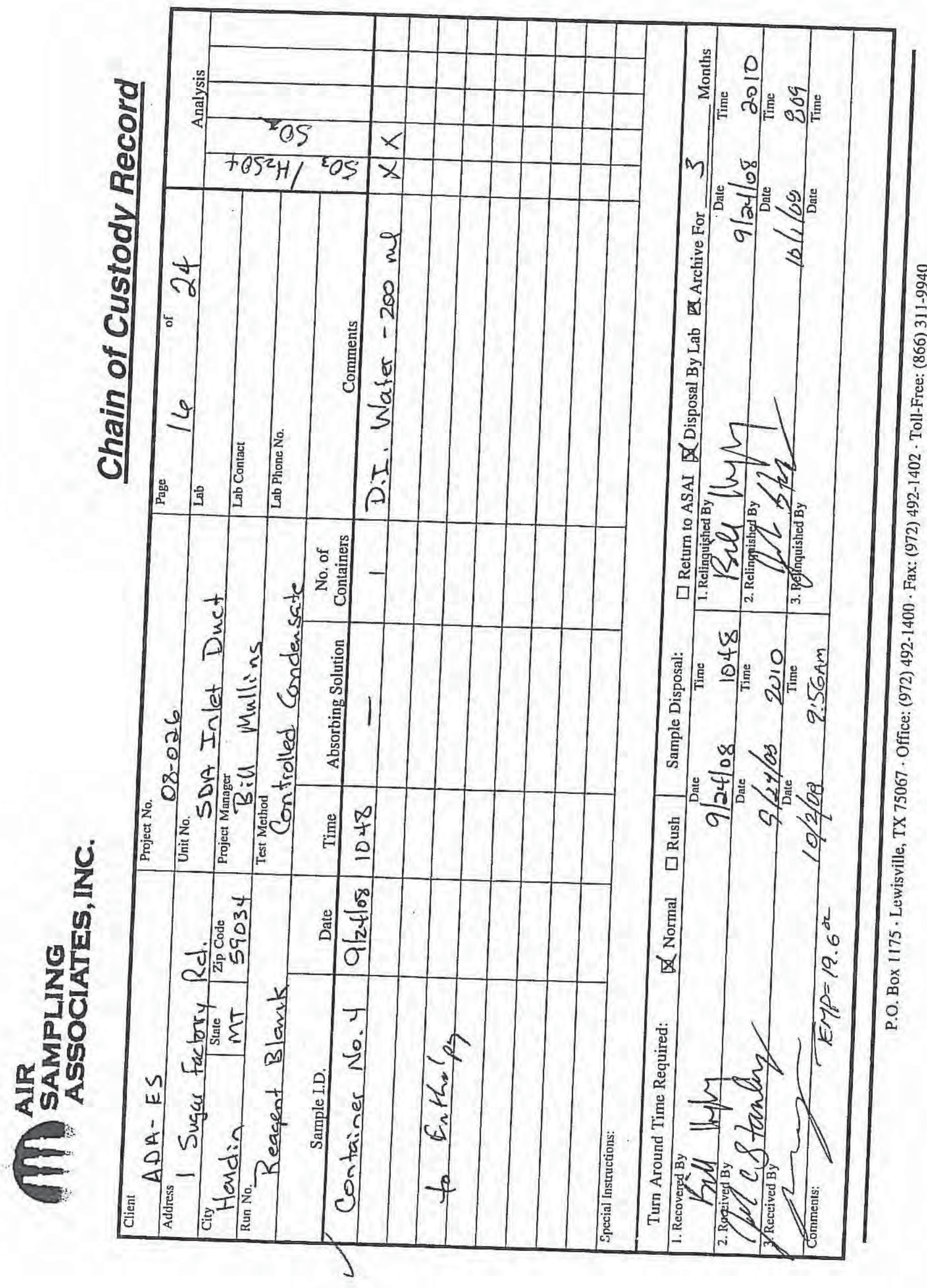

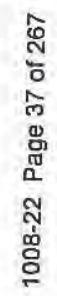




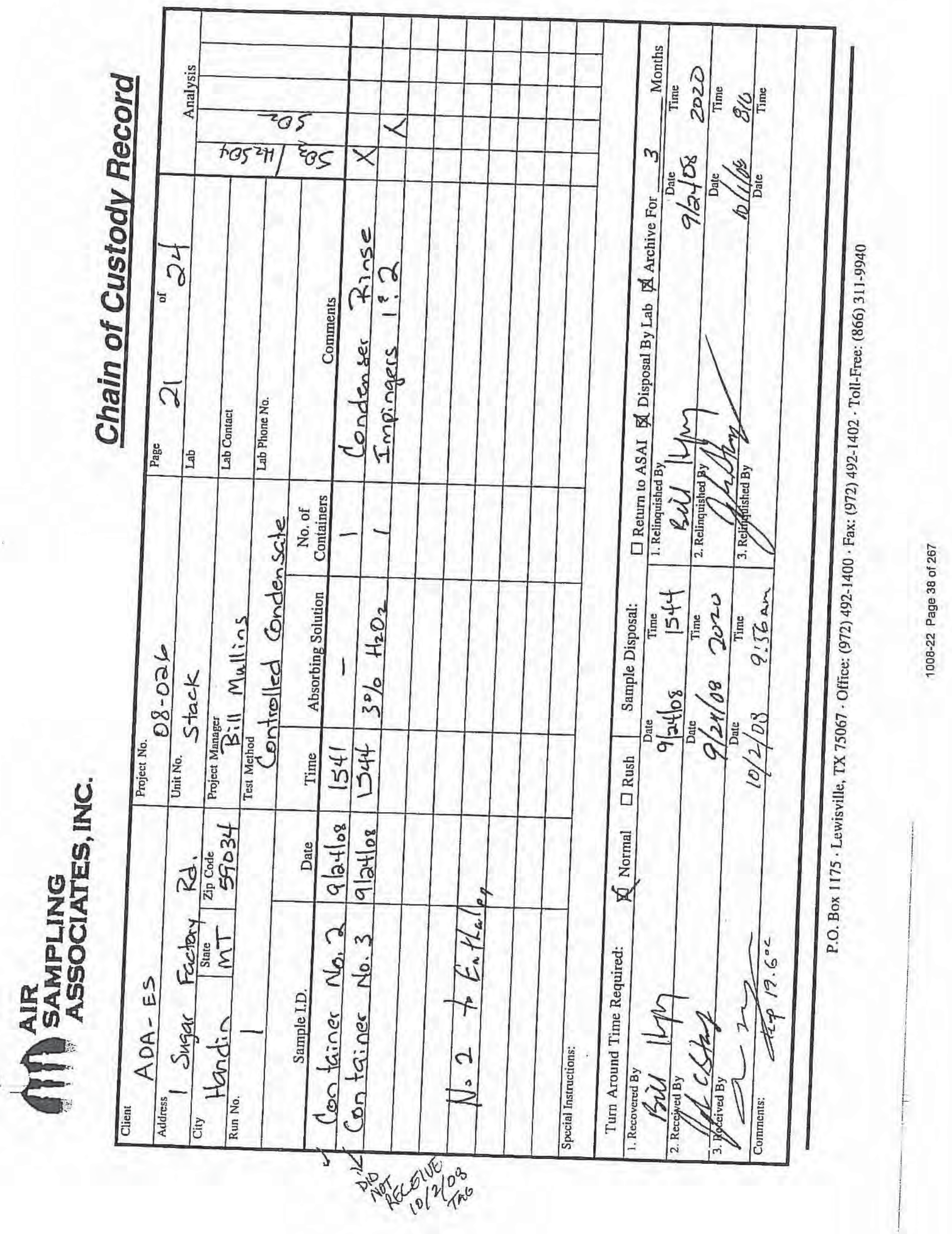




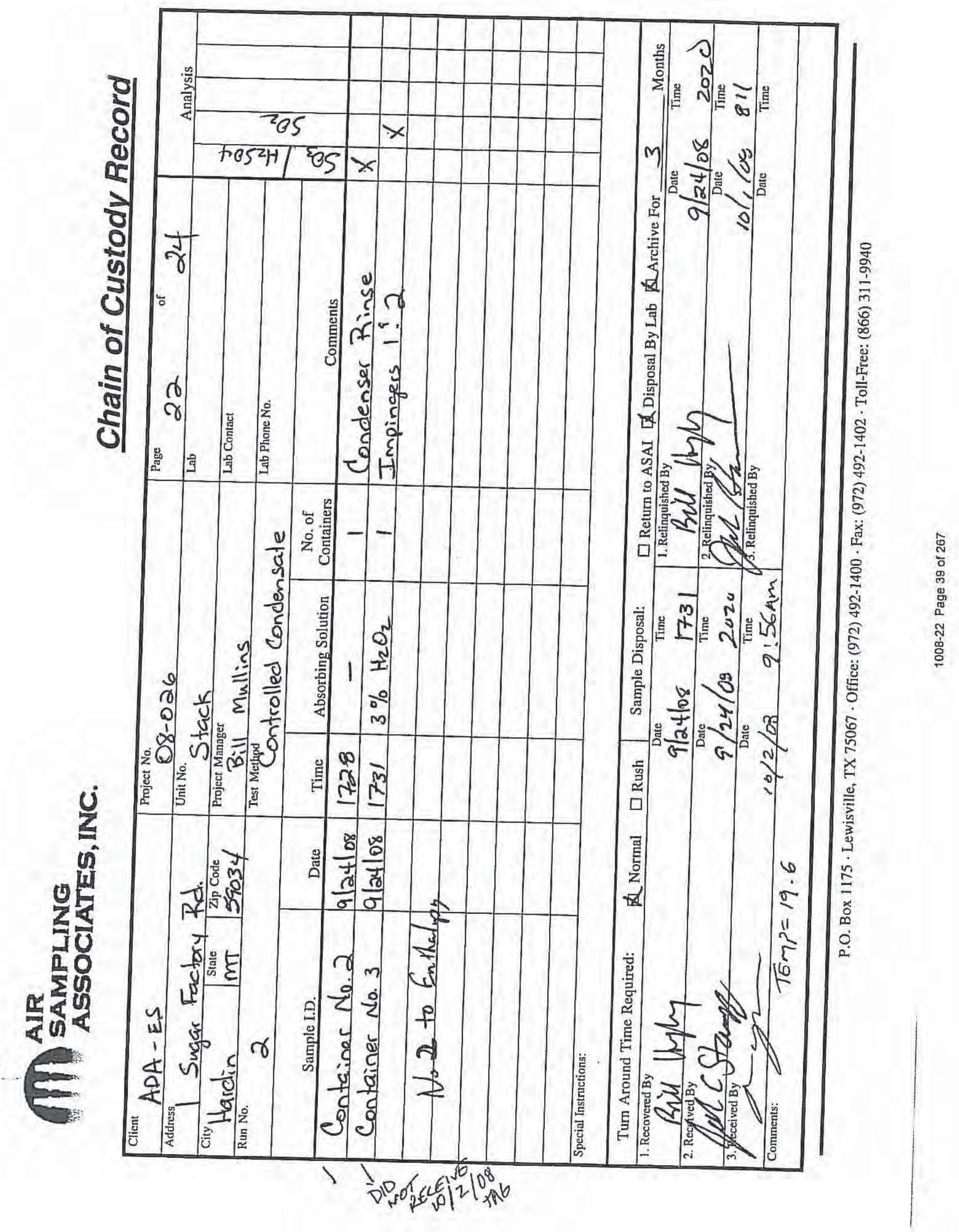




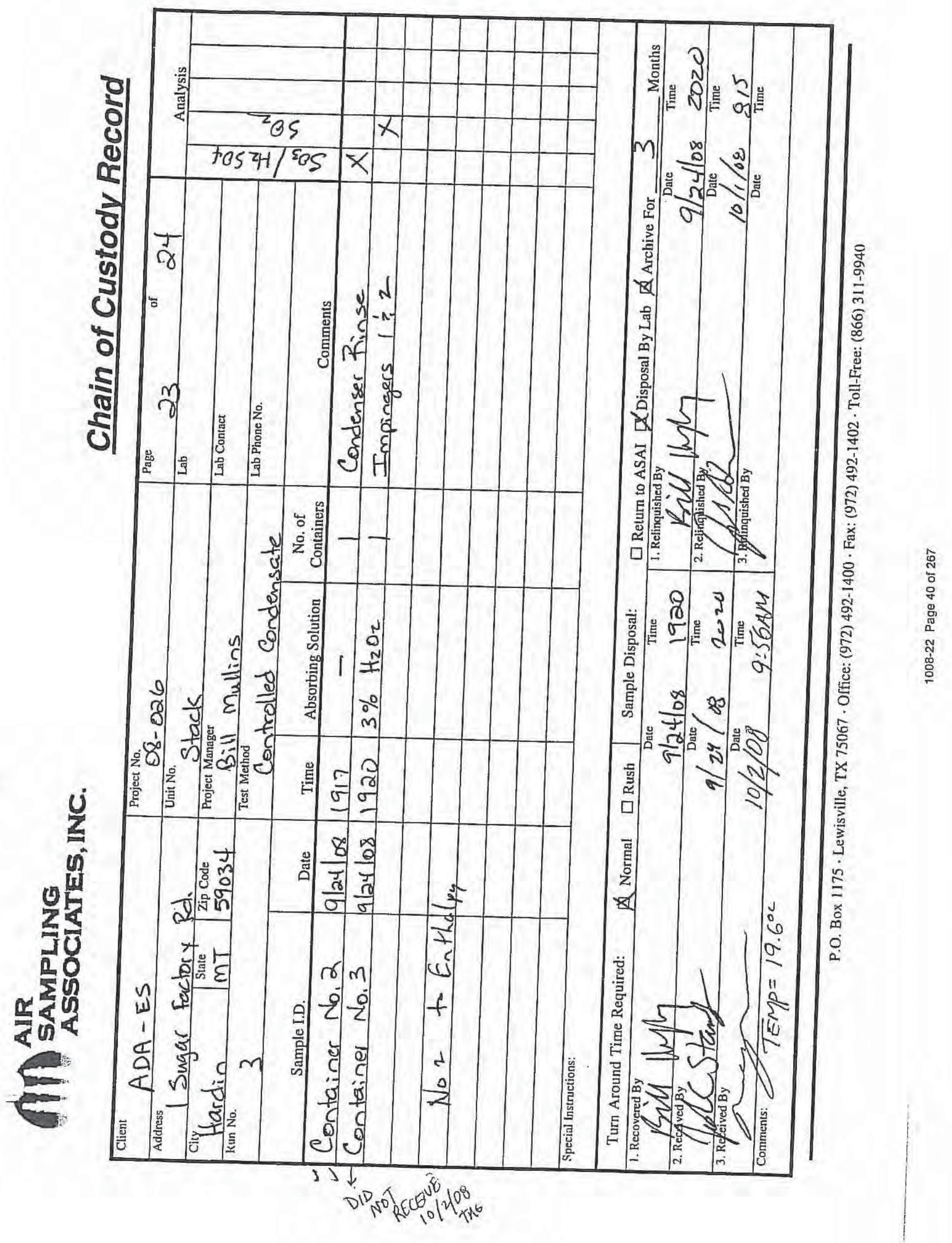




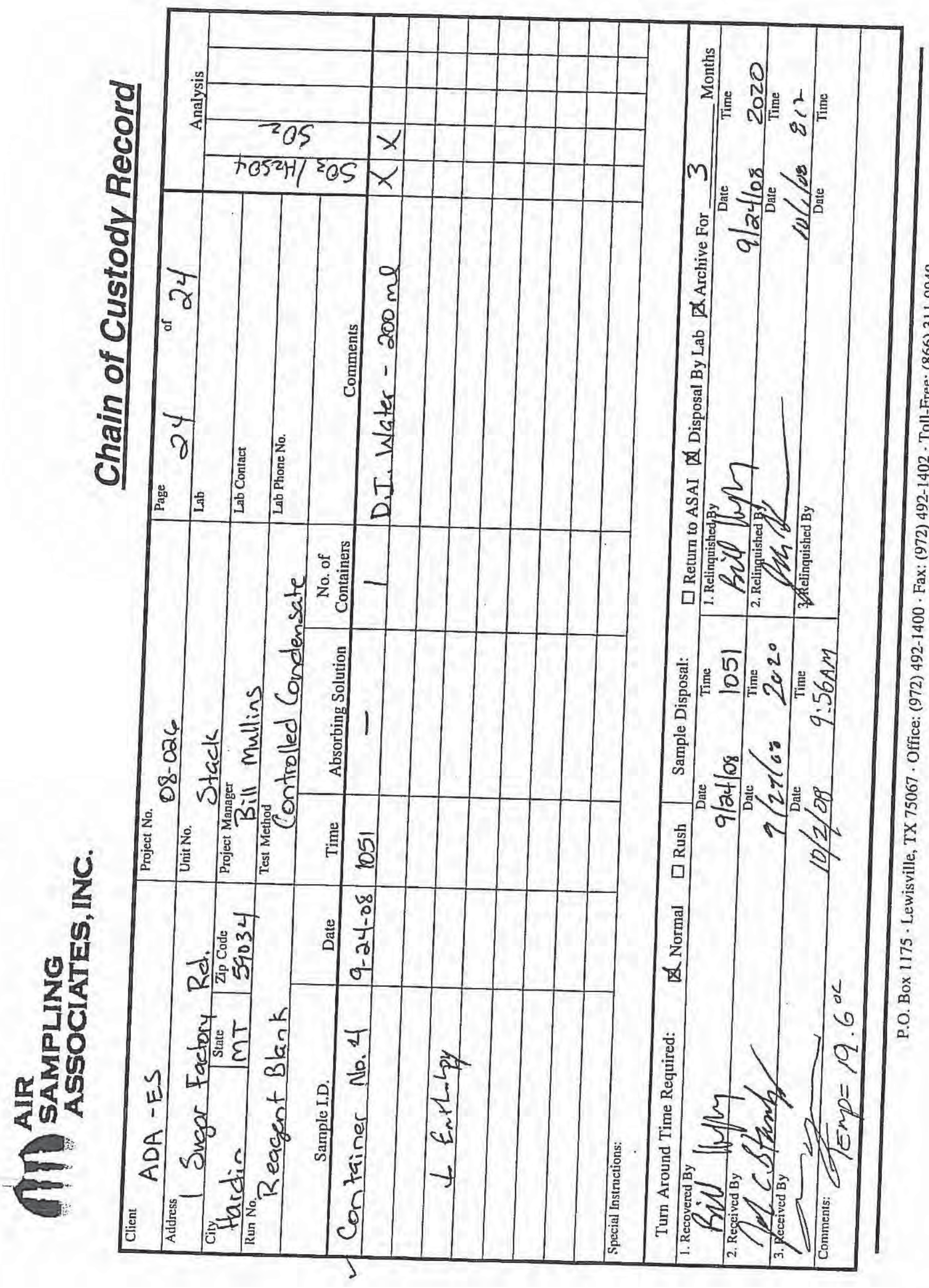

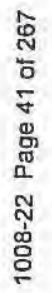




\section{Sample Chromatograms}

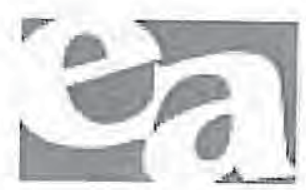



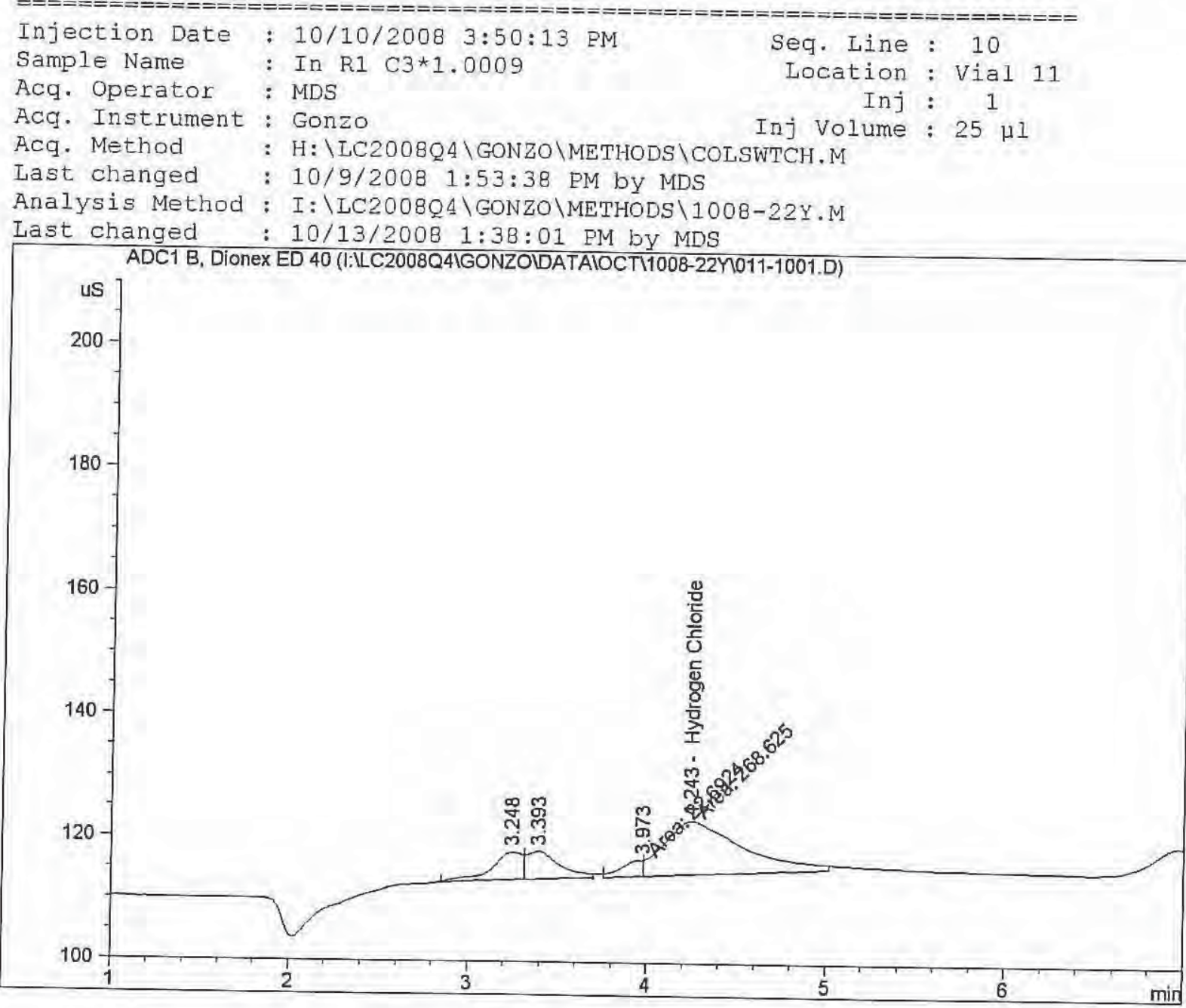

External Standard Report

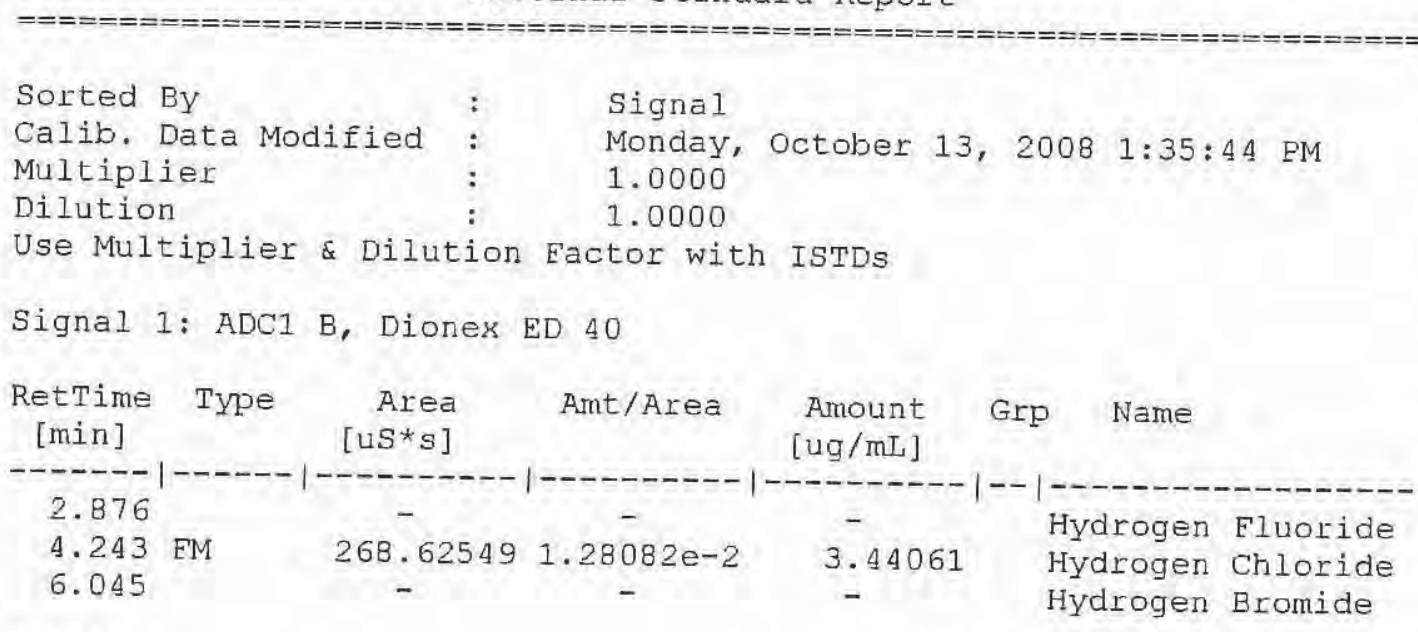

Totals :

3.44061

Results obtained with enhanced integrator! 1 Warnings or Errors :

Warning : Calibrated compound(s) not found 


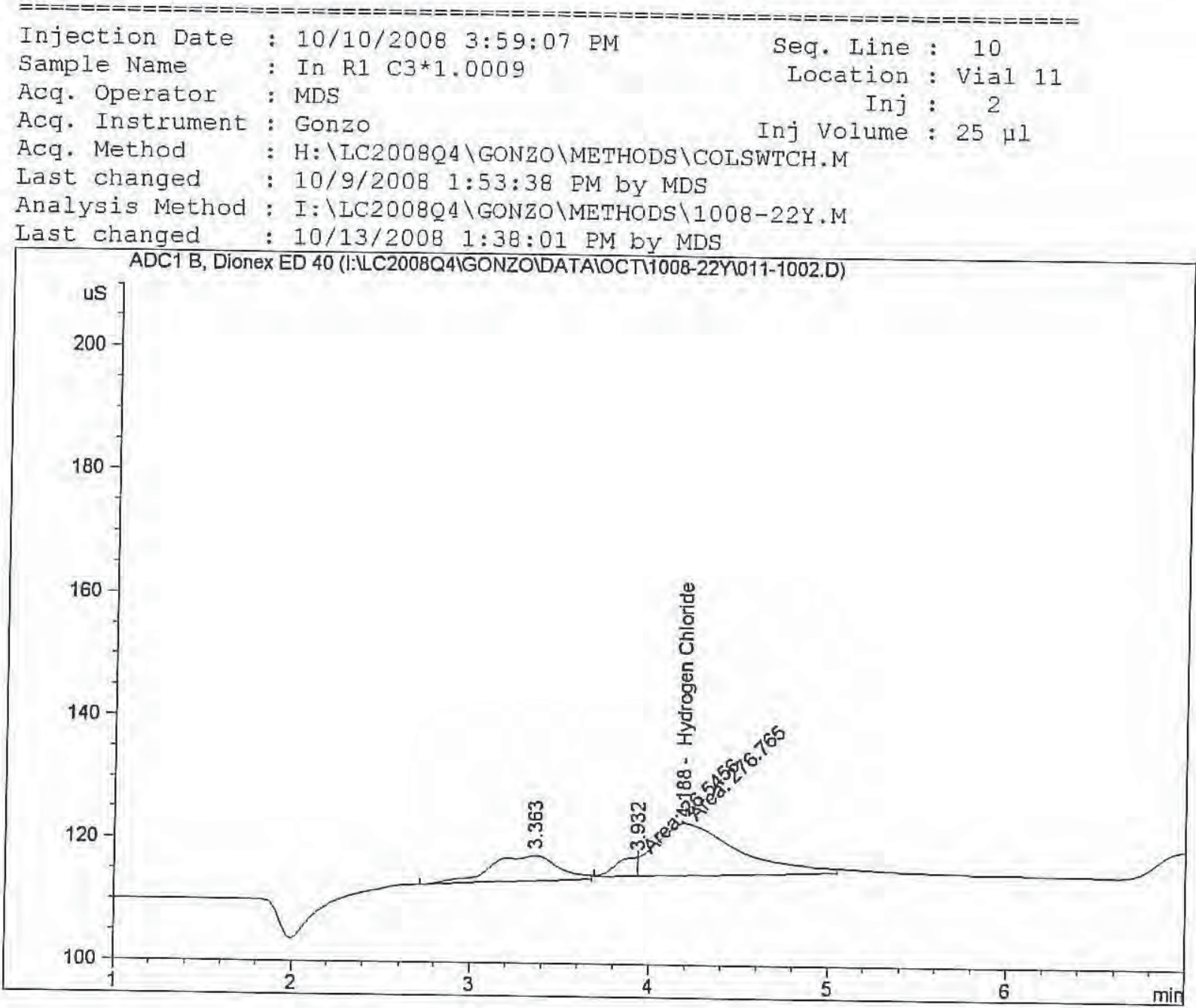

External Standard Report

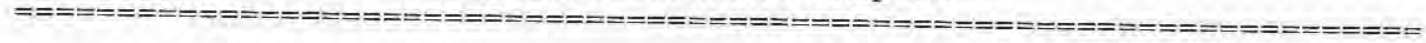

Sorted By

Calib. Data Modified

Multiplier : 1.0000

Dilution

October 13, 2008 1:35:44 PM

Use Multiplier

1.0000

Signal 1: ADC1 B, Diones ED 40

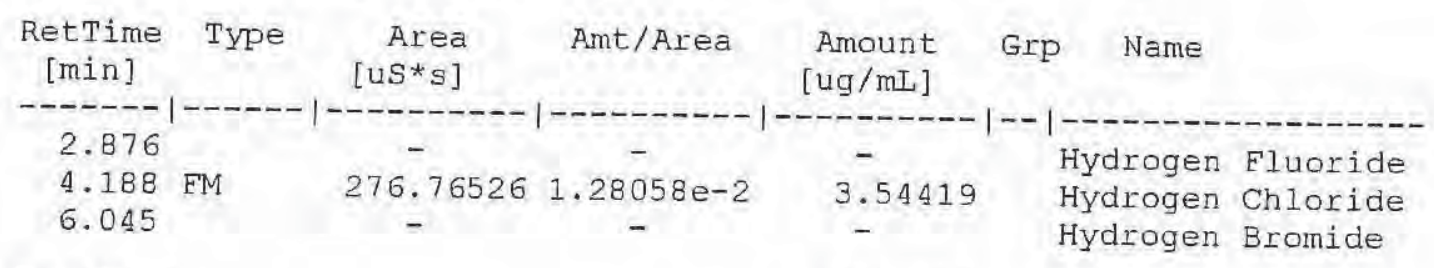

Totals :

3.54419

Results obtained with enhanced integrator!

1 warnings or Errors :

Warning : Calibrated compound(s) not found 
$1008-22$

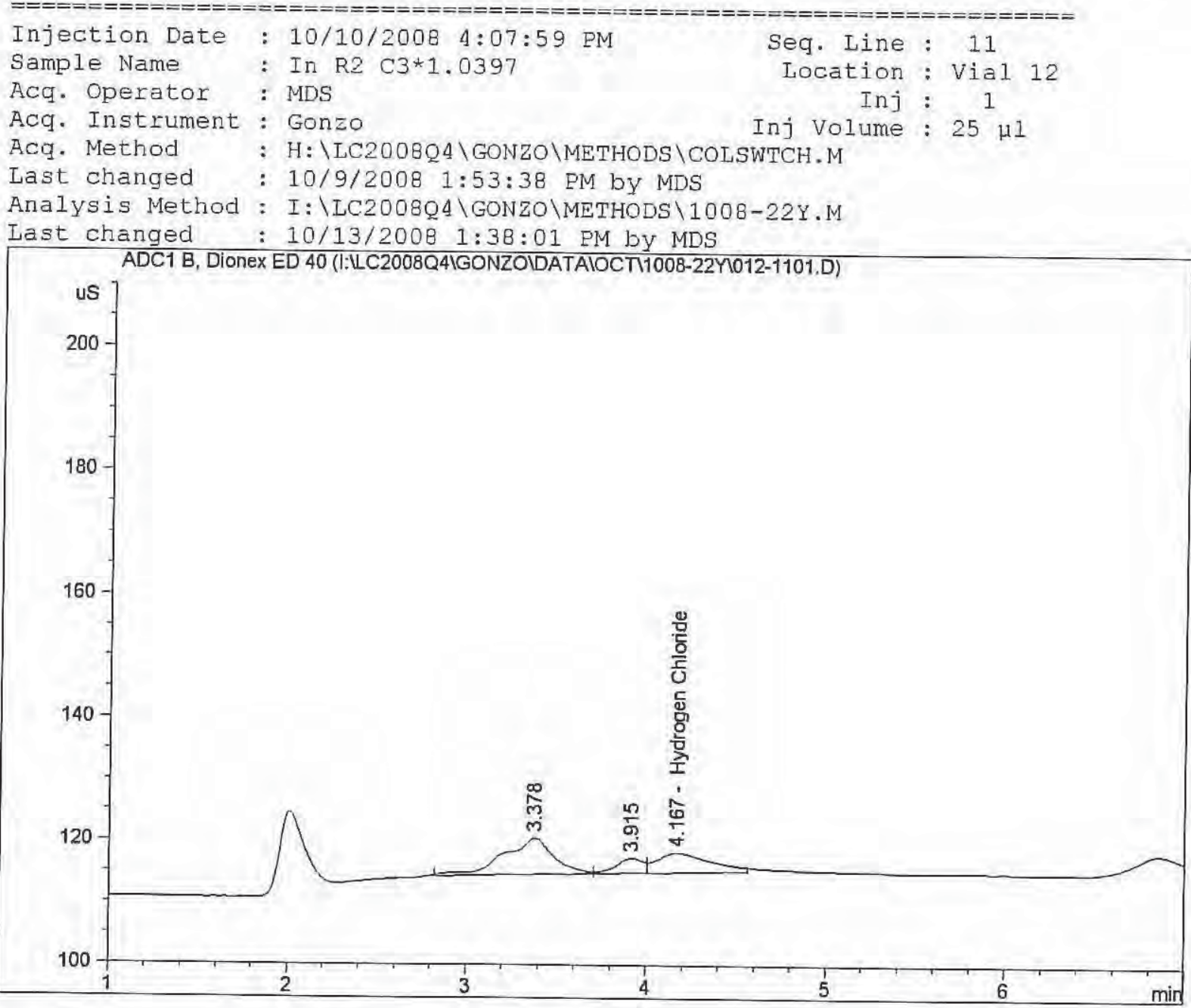

External Standard Report

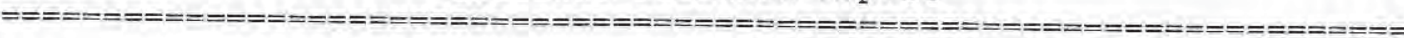

$\begin{array}{lll}\text { Sorted By } & : & \text { Signal } \\ \text { Calib. Data Modified : } & \text { Monday, October 13, } 2008 \text { 1:35:44 PM } \\ \text { Multiplier } & : & 1.0000 \\ \text { Dilution } & 1.0000 \\ \text { Use Multiplier \& Dilution Factor with }\end{array}$

Use Multiplier \& Dilution Eactor with ISTDS

Signal 1: ADC1 B, Diones ED 40

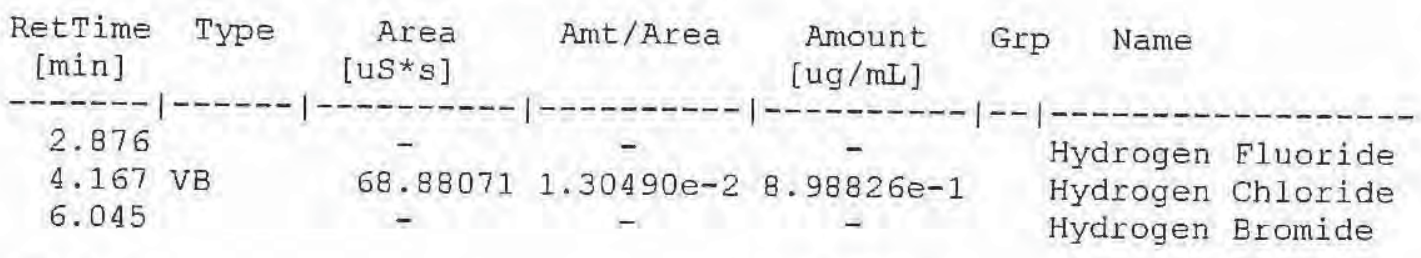

Totals :

$8.98826 e-1$

Results obtained with enhanced integrator!

1 Warnings or Errors :

Warning : Calibrated compound(s) not found 
$1008-22$

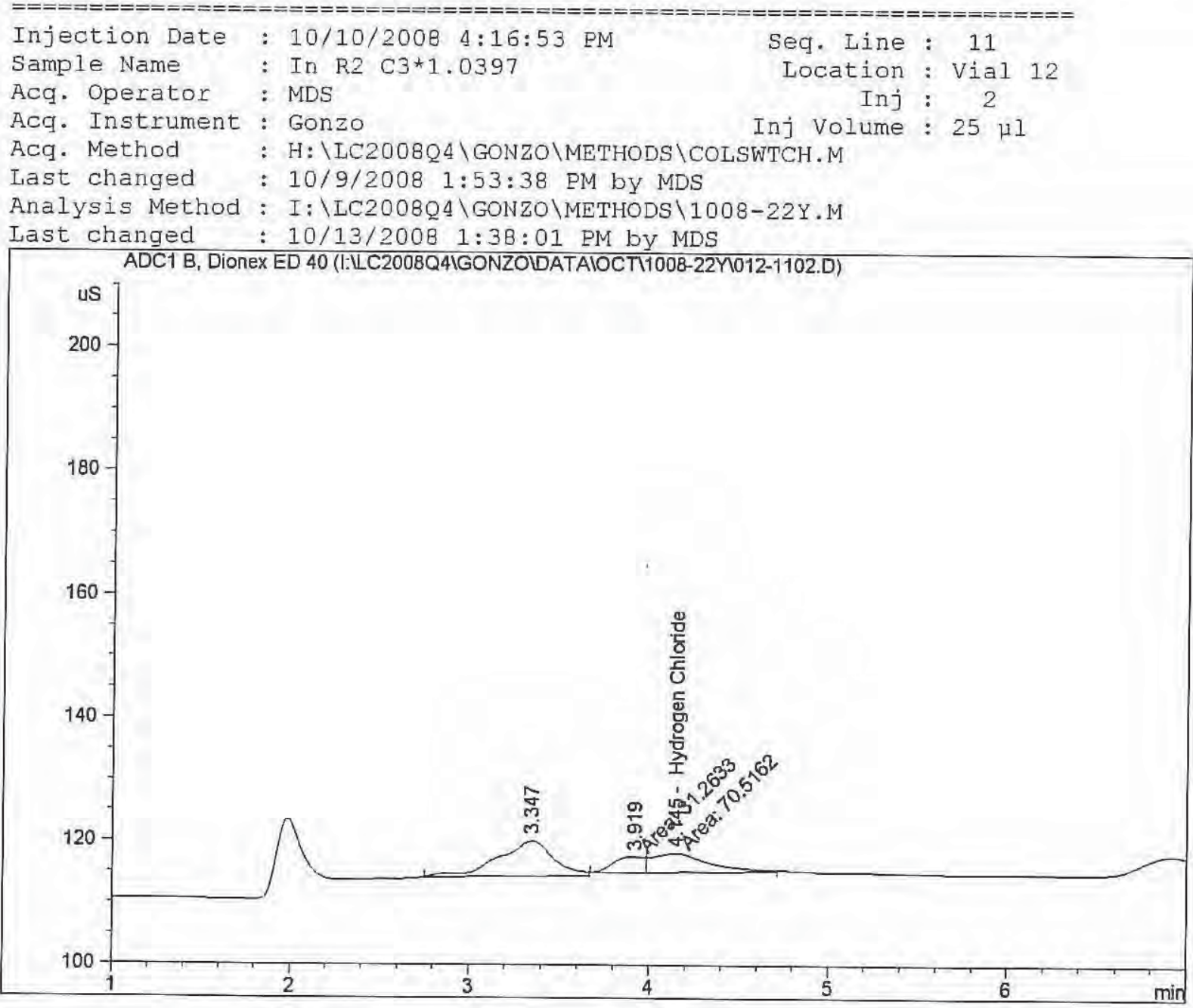

External Standard Report

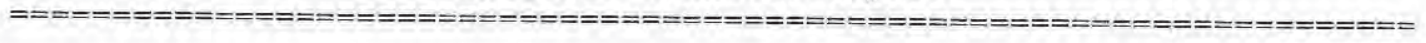

Sorted By

Signal

Calib. Data Modified : Monday, October 13, 2008 1:35:44 PM

Multiplier

Dilution

1.0000

Use Multiplier \& Dilution Eactor with ISTDs

Signal 1: ADC1 B, Diones ED 40

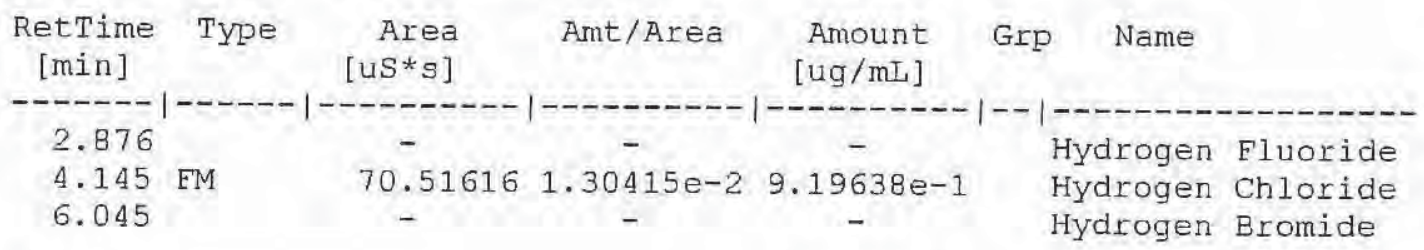

Totals :

\section{$9.19638 e-1$}

Results obtained with enhanced integrator!

1 Warnings or Errors :

Warning : Calibrated compound(s) not found 


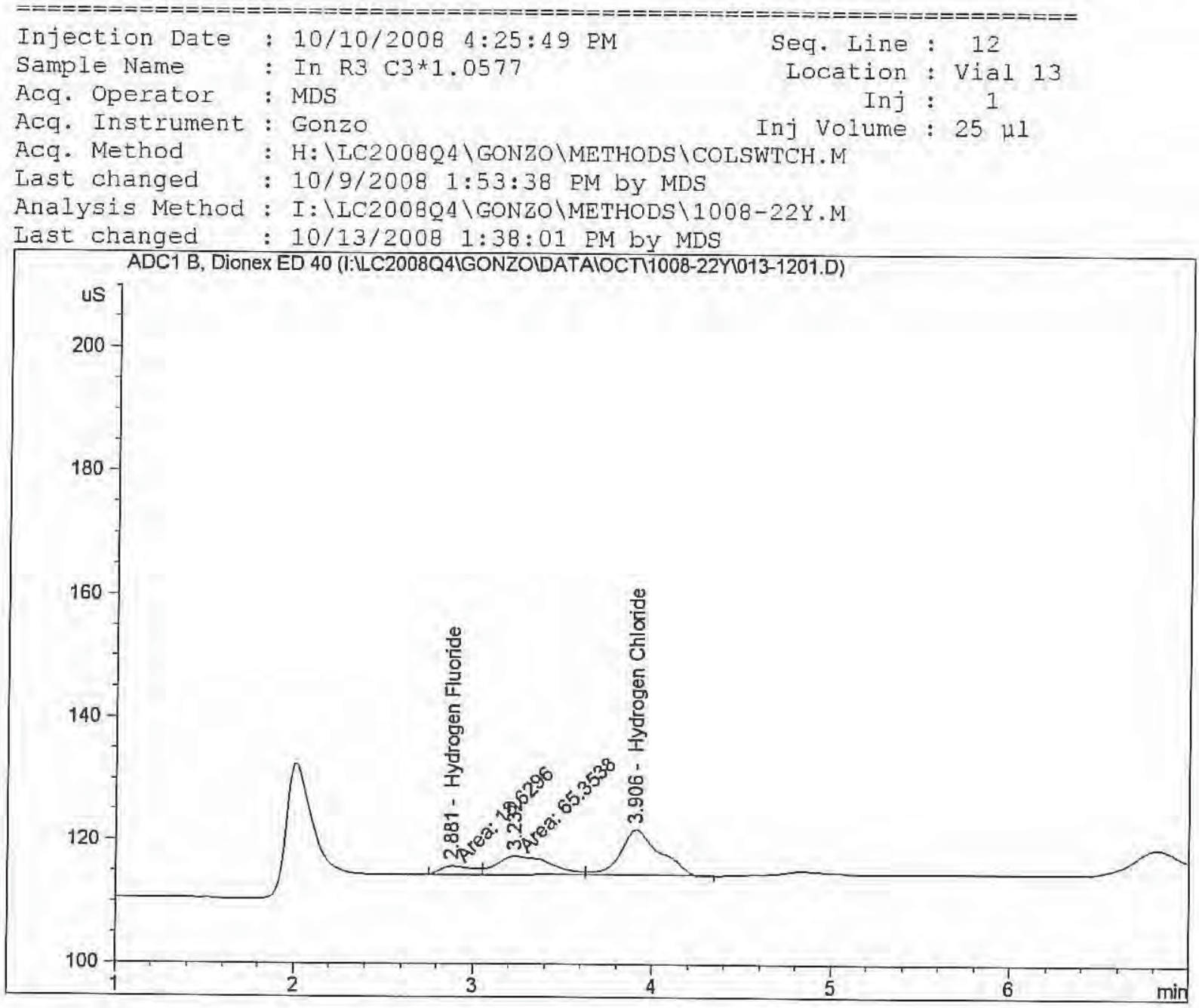

\section{External Standard Report}

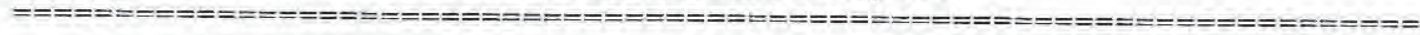

$\begin{array}{lcc}\text { Sorted By } & : & \text { Signal } \\ \text { Calib. Data Modified } & : & \text { Monday, October 13, 2008 1:35:44 PM } \\ \text { Multiplier } & \vdots & 1.0000 \\ \text { Dilution } & \vdots & 1.0000 \\ \text { Use Multiplier \& Dilution Eactor with ISTDS }\end{array}$

Signal 1: ADC1 B, Dionex ED 40

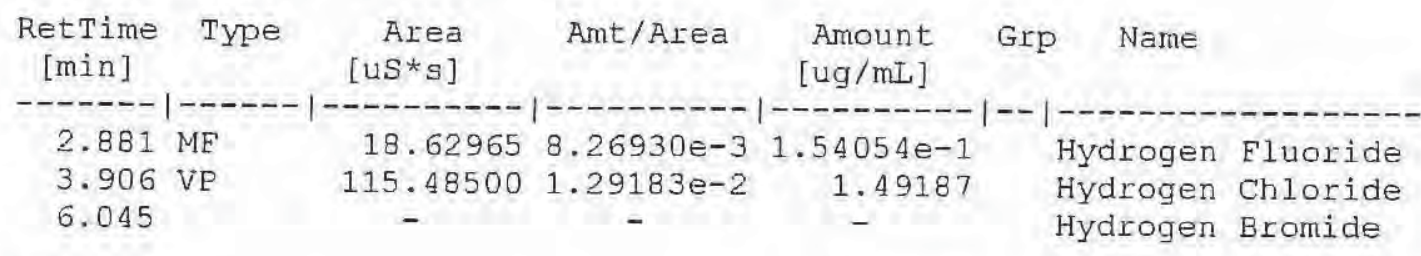

Totals :

1.64593

Results obtained with enhanced integrator!

1 Warnings or Errors :

Warning : Calibrated compound(s) not found 

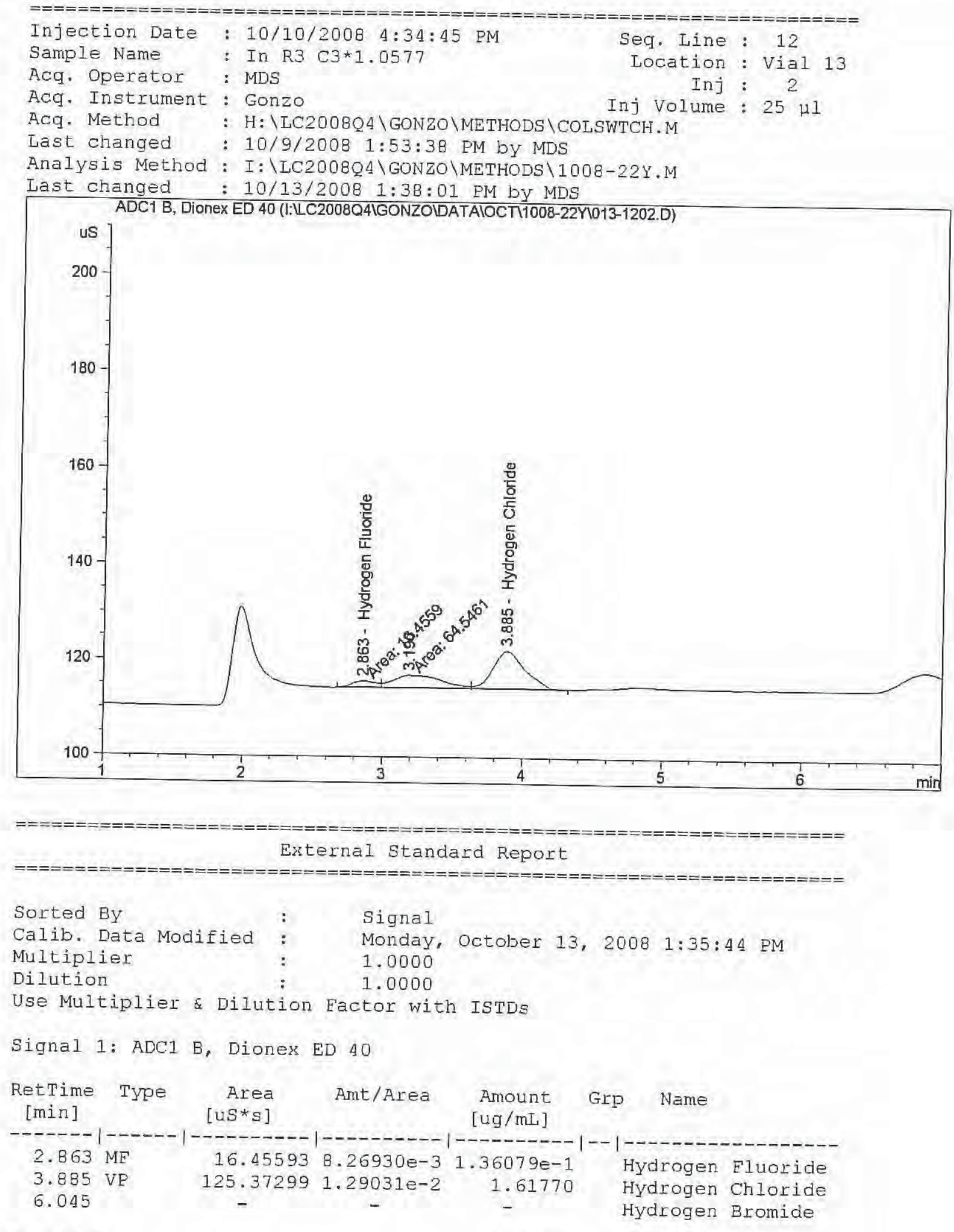

Results obtained with enhanced integrator! 1 Warnings or Errors :

Warning : Calibrated compound(s) not found 
$1008-22$

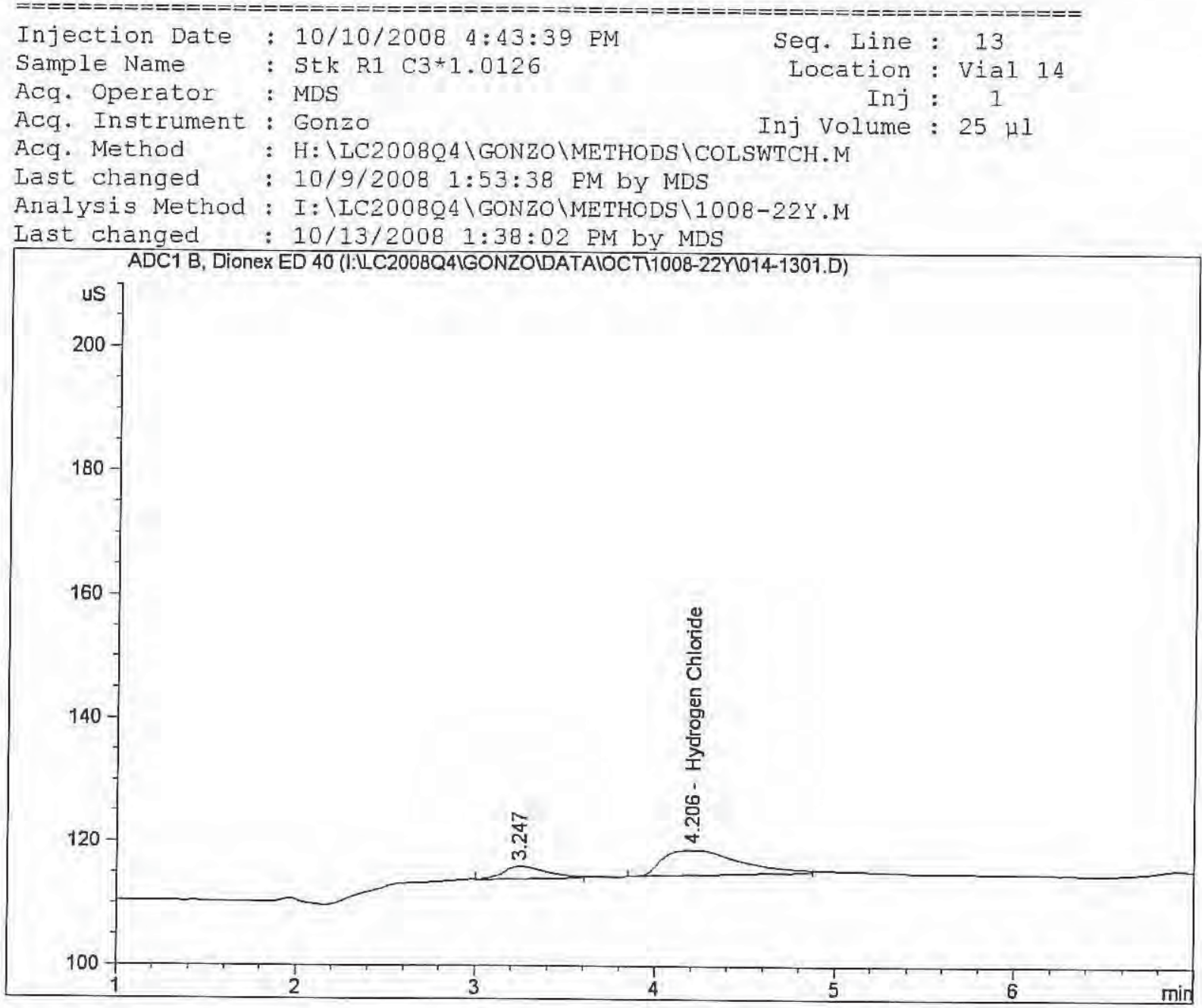

External standard Report

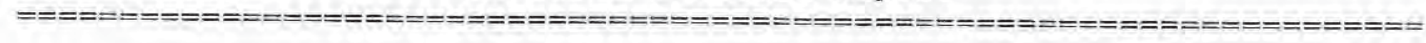

Sorted By

Signal

Calib. Data Modified

Multiplier

Dilution

Monday, October 13, 2008 1:35:44 PM

Use Multipl

1.0000

1.0000

Signal 1: ADC1 B, Dionex ED 40

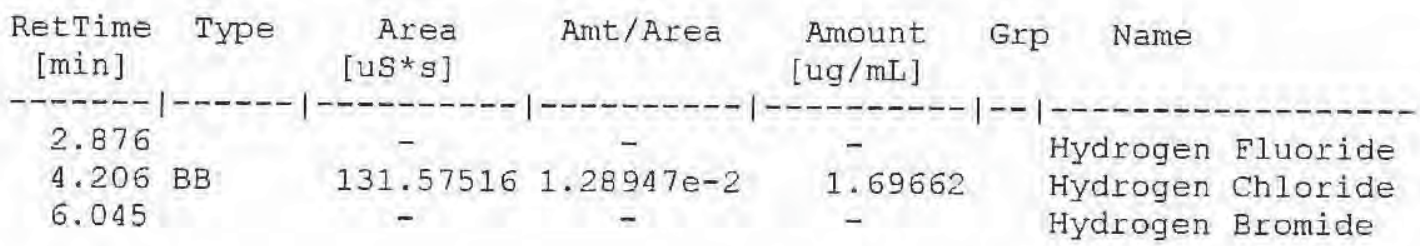

Totals :

1.69662

Results obtained with enhanced integrator!

1 Warnings or Errors :

Warning : Calibrated compound(s) not found 
$1008-22$
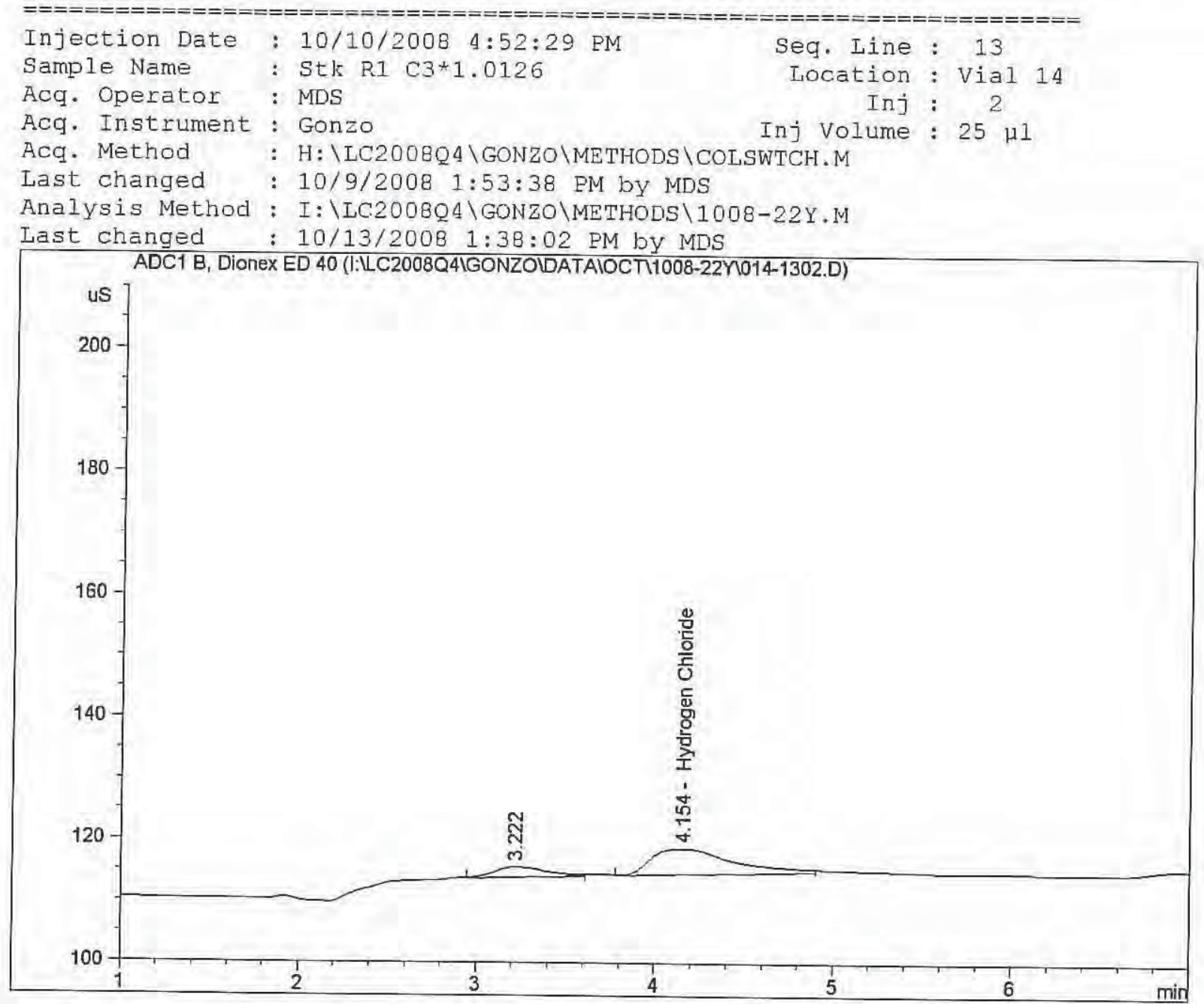

External Standard Report

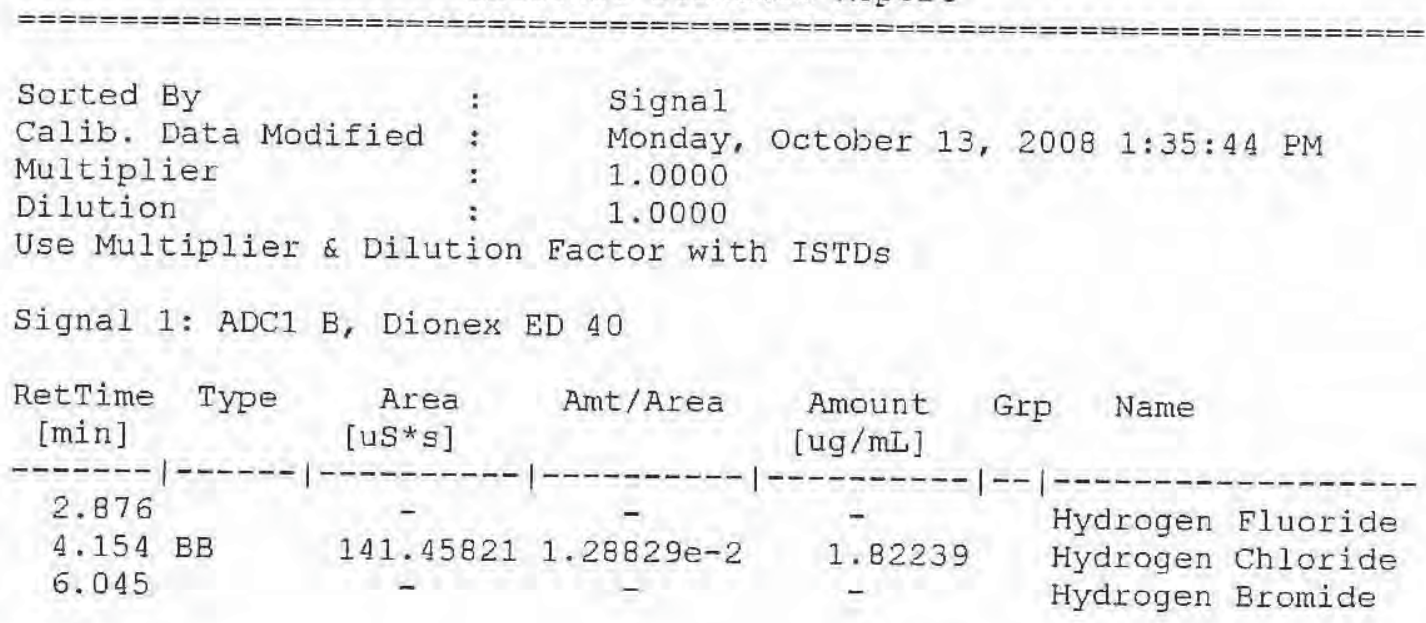

Totals :

1.82239

Results obtained with enhanced integrator!

1 Warnings or Errors :

Warning : Calibrated compound(s) not found 
$1008-22$

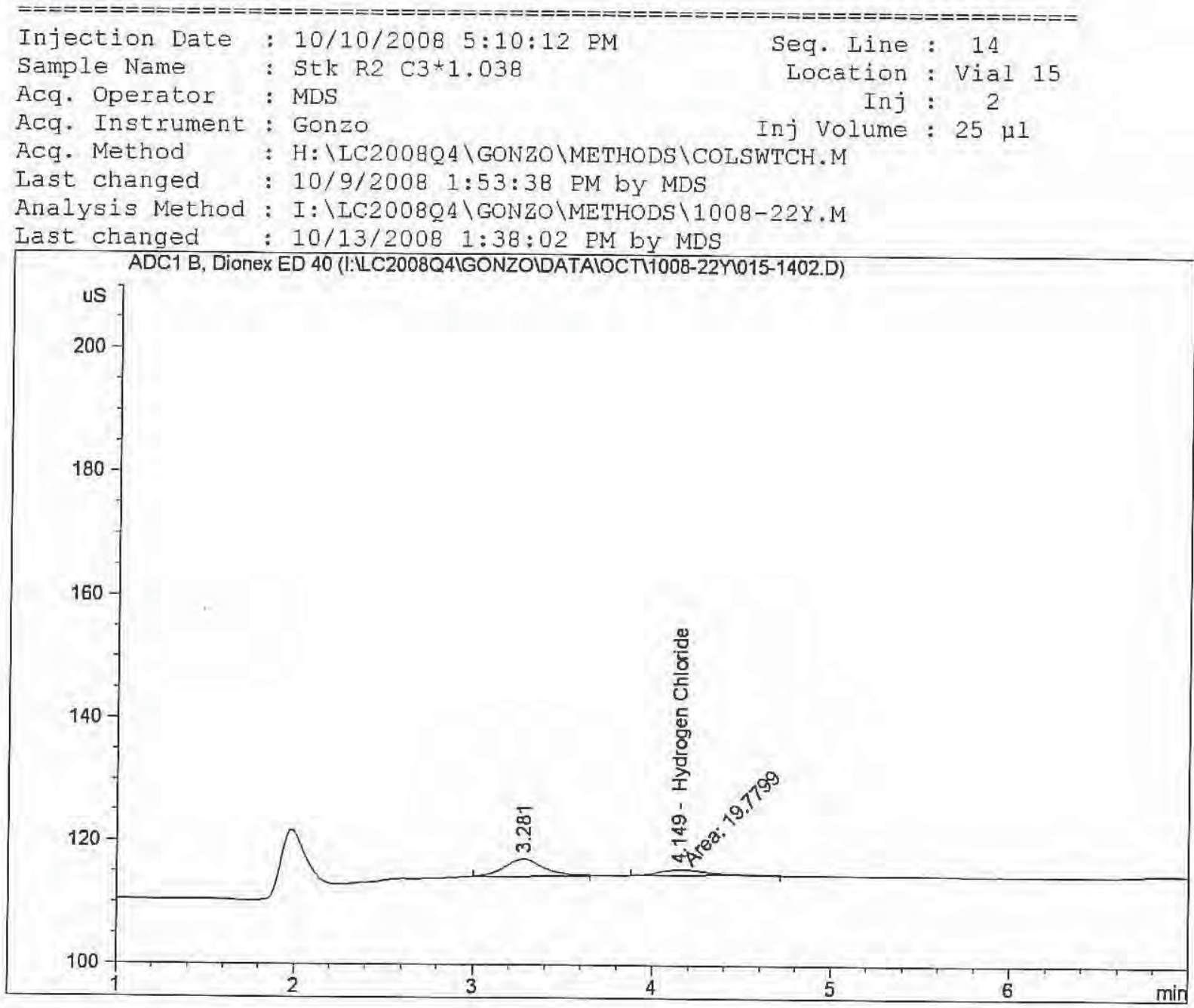

External Standard Report

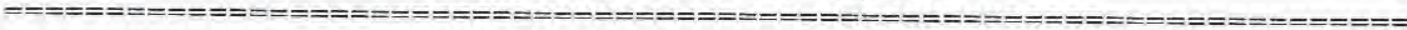

$\begin{array}{lll}\text { Sorted By } & : & \text { Signal } \\ \text { Calib. Data Modified } & \text { Monday, October 13, } 2008 \text { 1:35:44 PM } \\ \text { Multiplier } & : & 1.0000 \\ \text { Dilution } & \vdots & 1.0000 \\ \text { Use Multiplier \& Dilution Factor with ISTDs }\end{array}$

Signal 1: ADC1 B, Diones ED 40

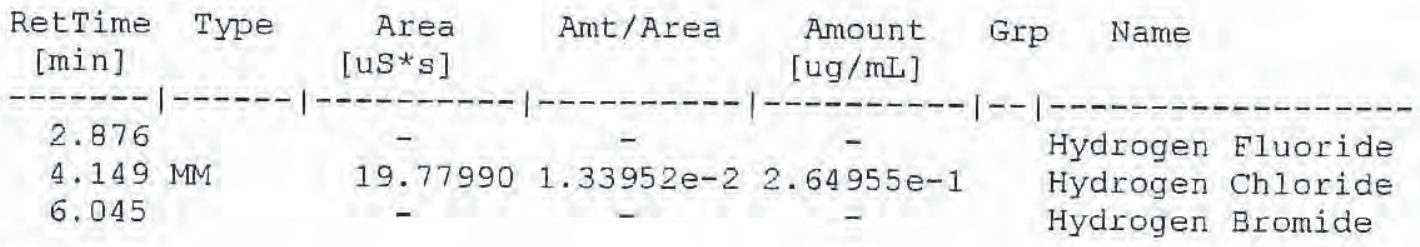

Totals: $\quad 2.64955 e-1$

Results obtained with enhanced integrator!

1 Warnings or Errors :

Warning : Calibrated compound(s) not found 
$1008-22$

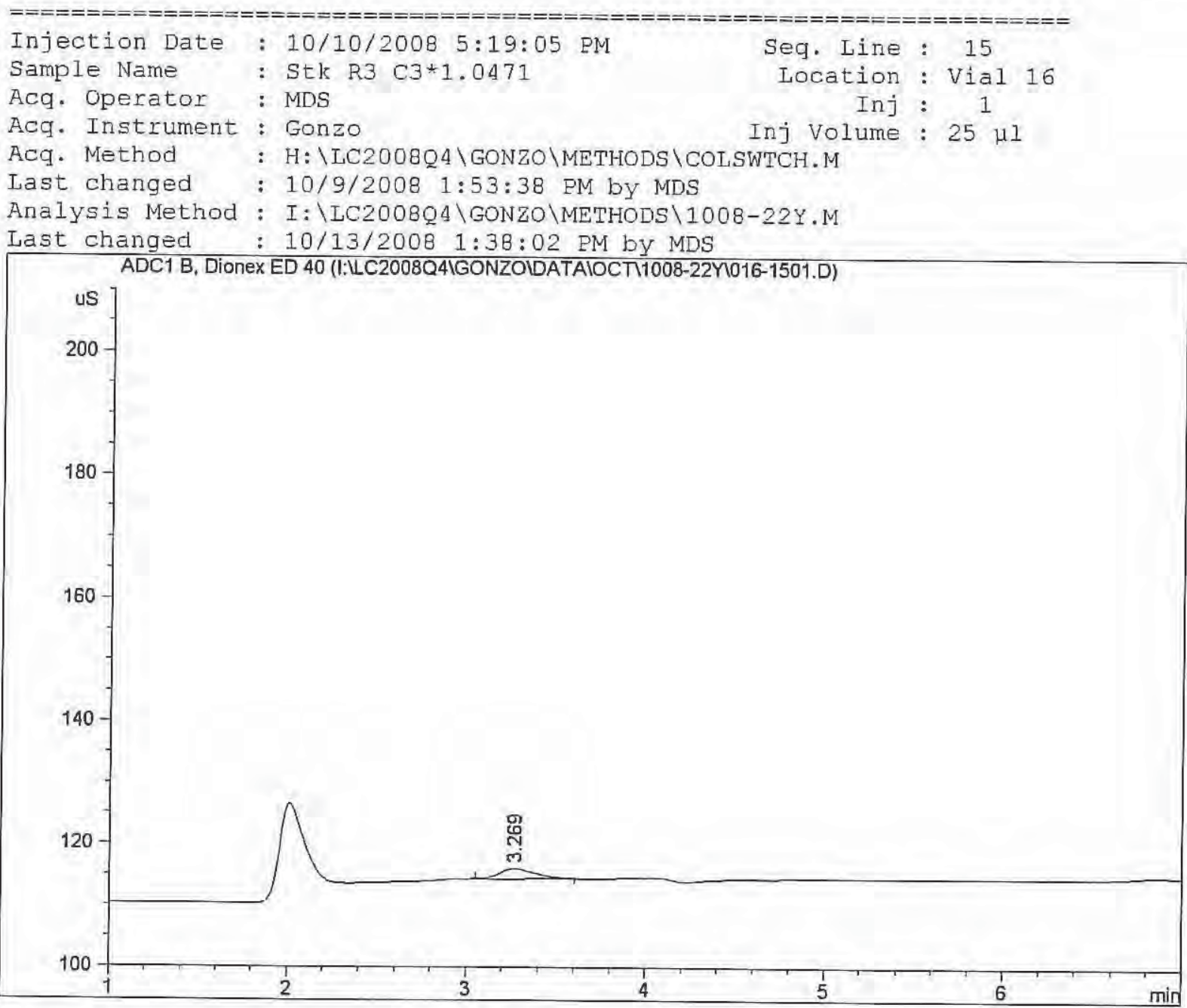

External Standard Report

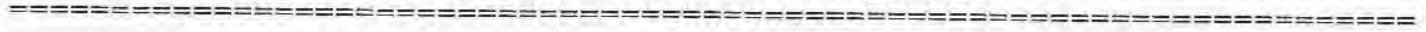

Sorted By

Calib. Data Modified

Multiplier

Dilution

Signal

Monday, October 13, 2008 1:35:44 PM

Use Multiplier \& Dilution Eactor with ISTDs

Signal 1: ADC1 B, Dionex ED 40

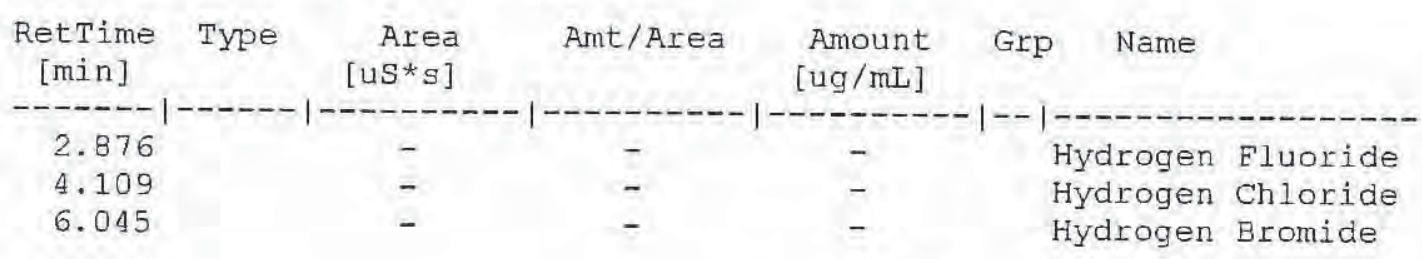

Totals :

0.00000

Results obtained with enhanced integrator!

1 Warnings or Errors :

Warning : Calibrated compound(s) not found 
$1008-22$

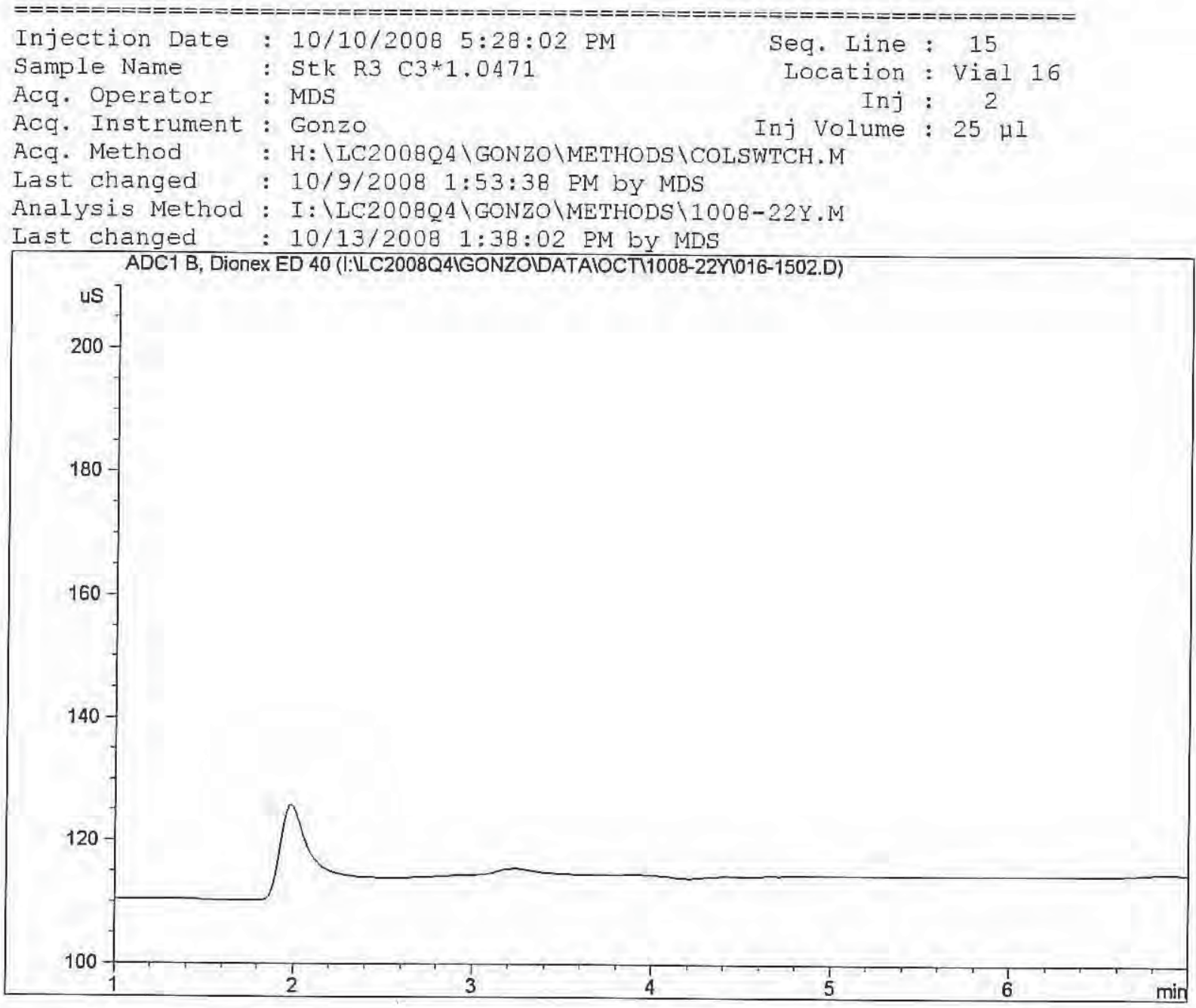

External Standard Report

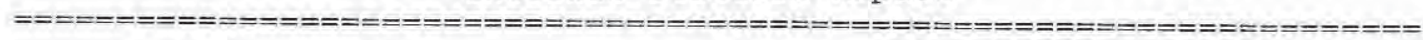

$\begin{array}{lll}\text { Sorted By } & : & \text { Signal } \\ \text { Calib. Data Modified } & : & \text { Monday, October 13, } 2008 \text { 1:35:44 PM } \\ \text { Multiplier } & : & 1.0000 \\ \text { Dilution } & : & 1.0000 \\ \text { Use Multiplier \& Dilution Eactor with ISTDs }\end{array}$

Signal 1: ADC1 B, Dionex ED 40

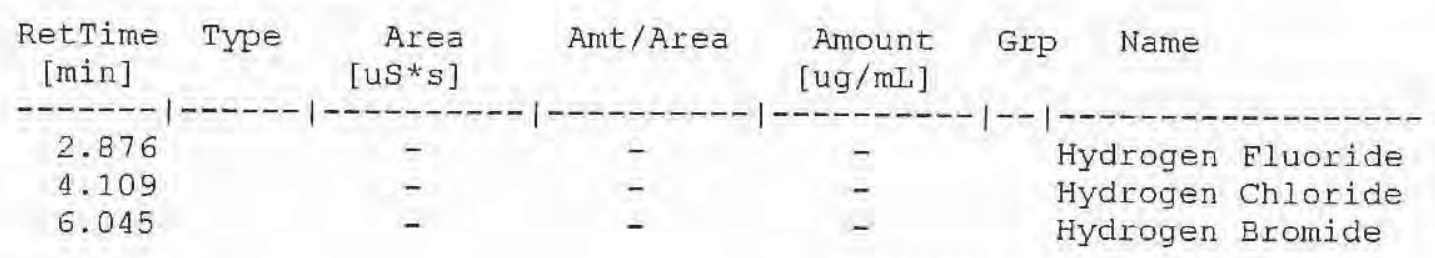

Totals : $\quad 0.00000$

Results obtained with enhanced integrator!

1 Warnings or Errors :

Warning : Calibrated compound(s) not found 
1008-22 Sample Blank

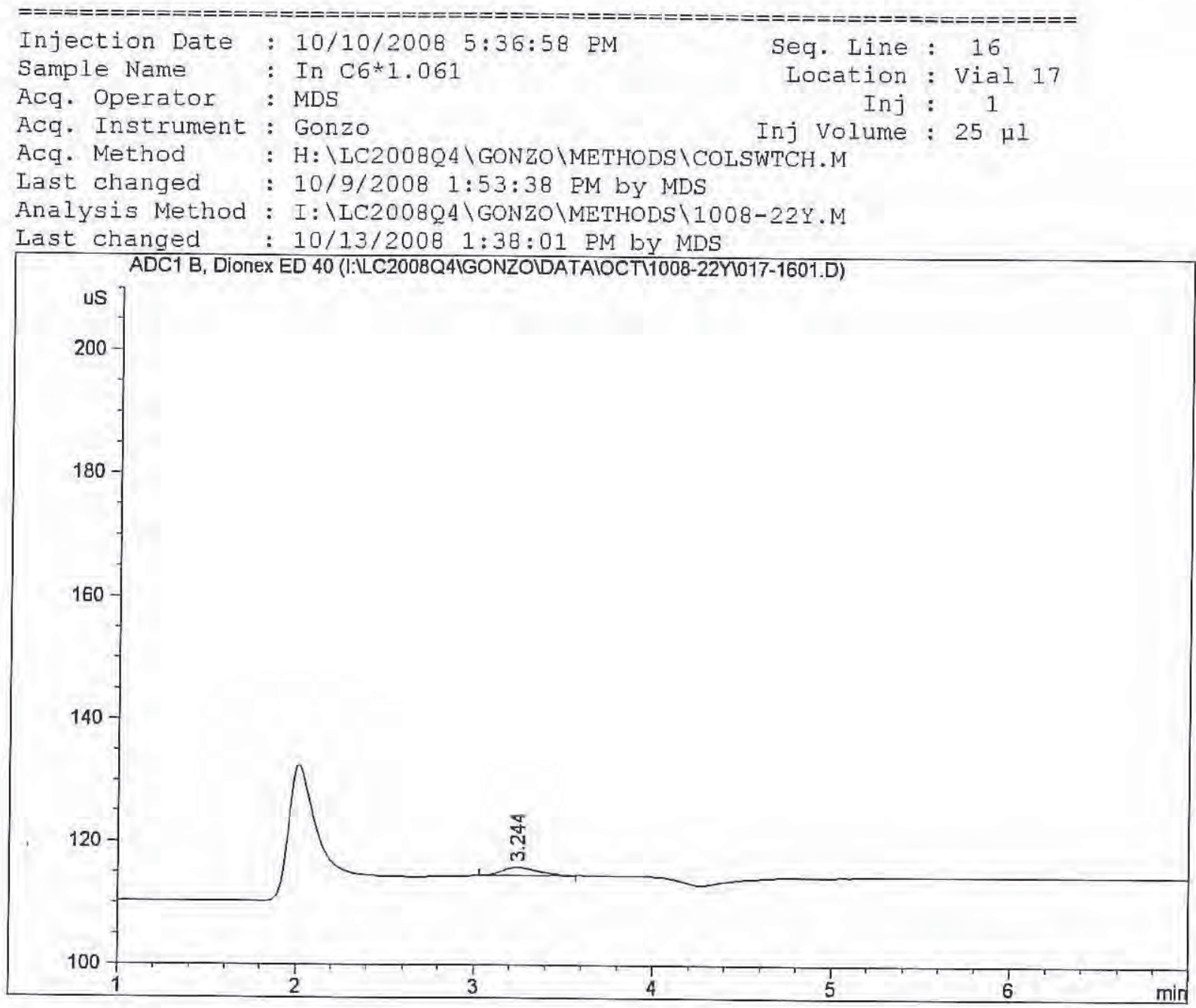

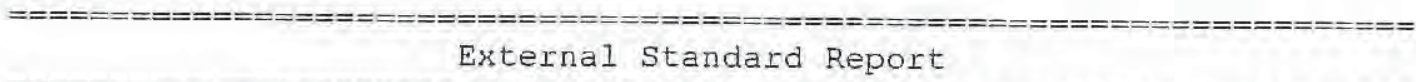

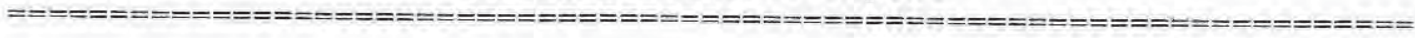

Sorted By : Signal

Calib. Data Modified : Monday, October 13, 2008 1:35:44 PM

Multiplier : 1.0000

Dilution : 1.0000

Use Multiplier \& Dilution Factor with ISTDs

Signal 1: ADC1 B, Dionex ED 40

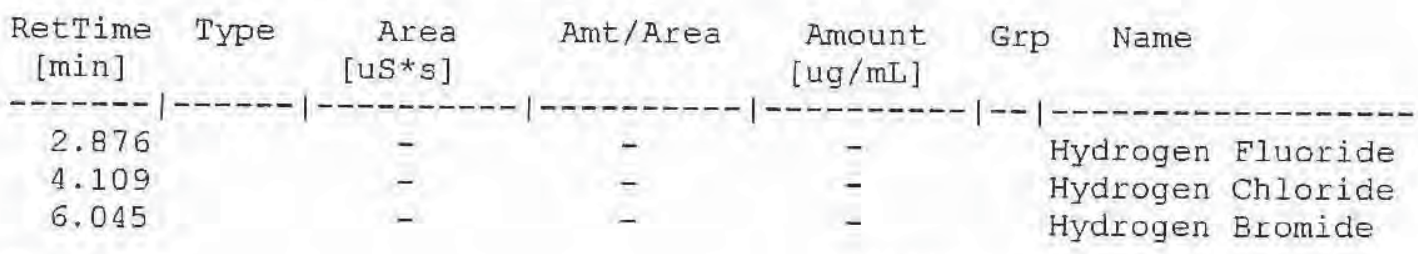

Totals :

0.00000

Results obtained with enhanced integrator!

1 Warnings or Errors :

Warning : Calibrated compound(s) not found 
1008-22 Sample Blank

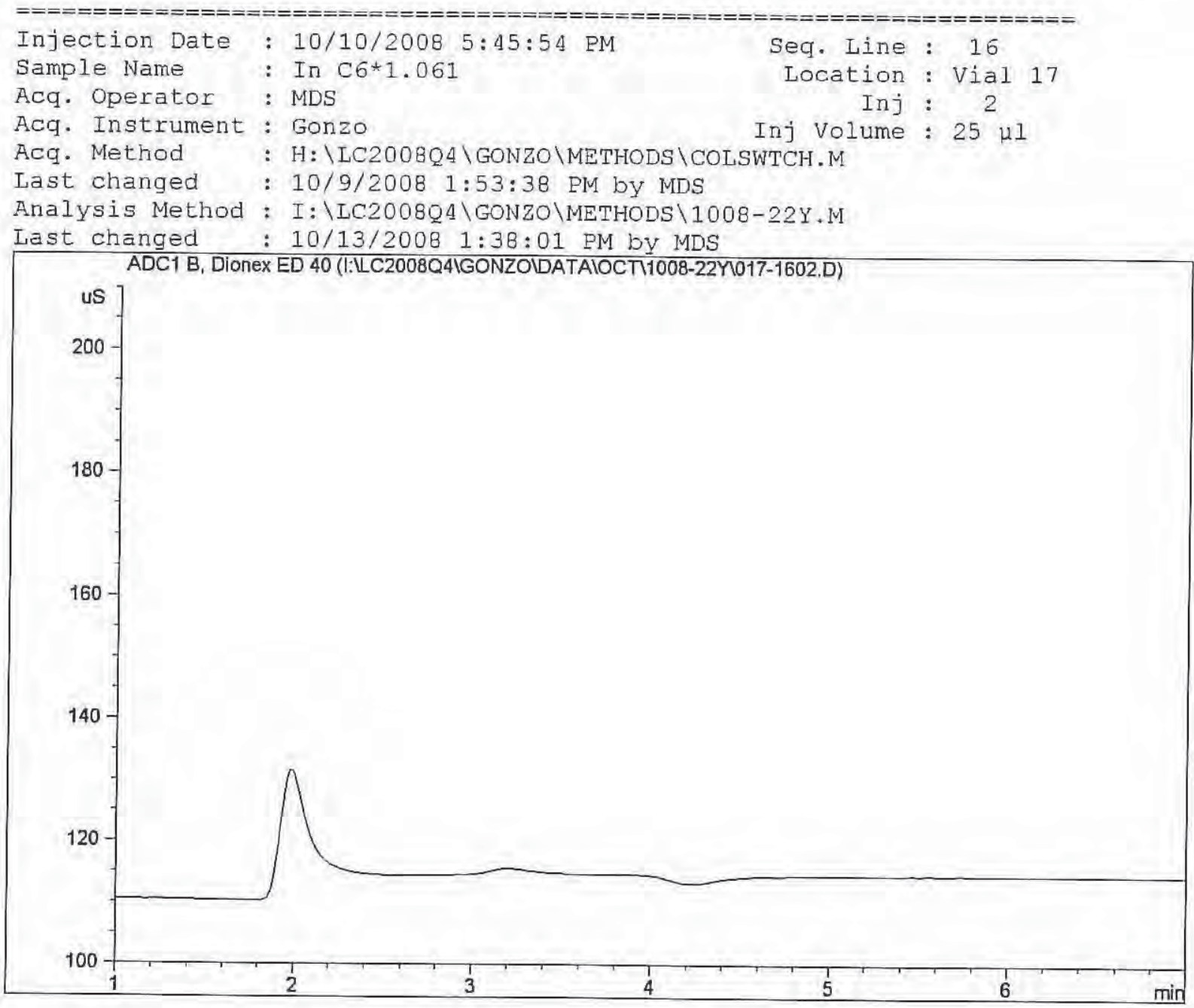

External Standard Report

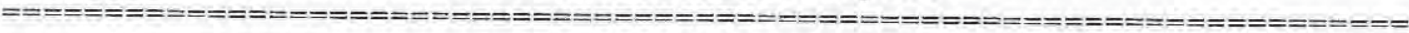

$\begin{array}{lll}\text { Sorted By } & : & \text { Signal } \\ \text { Calib. Data Modified } & : & \text { Monday, October 13, } 2008 \text { 1:35:44 PM } \\ \text { Multiplier } & : & 1.0000 \\ \text { Dilution } & : & 1.0000\end{array}$

Use Multiplier \& Dilution Factor with ISTDs

Signal 1: ADC1 B, Dionex ED 40

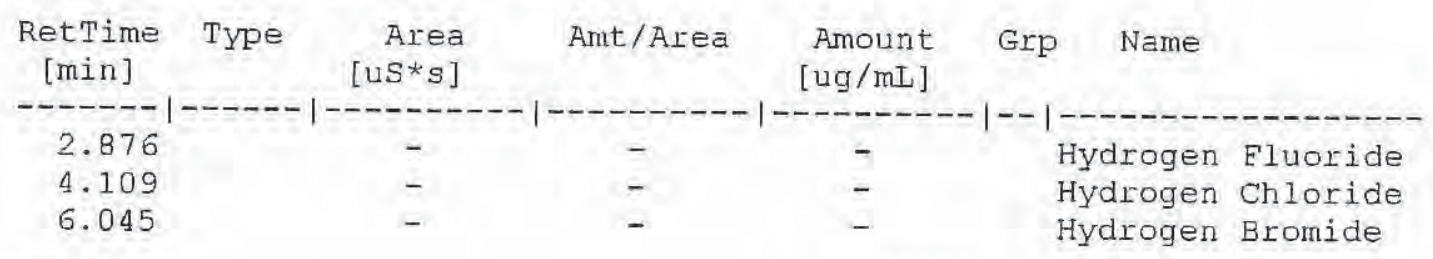

Totals: $\quad 0.00000$

Results obtained with enhanced integrator!

1 Warnings or Errors :

Warning : Calibrated compound(s) not found 


\section{8-22 Sample Blank}

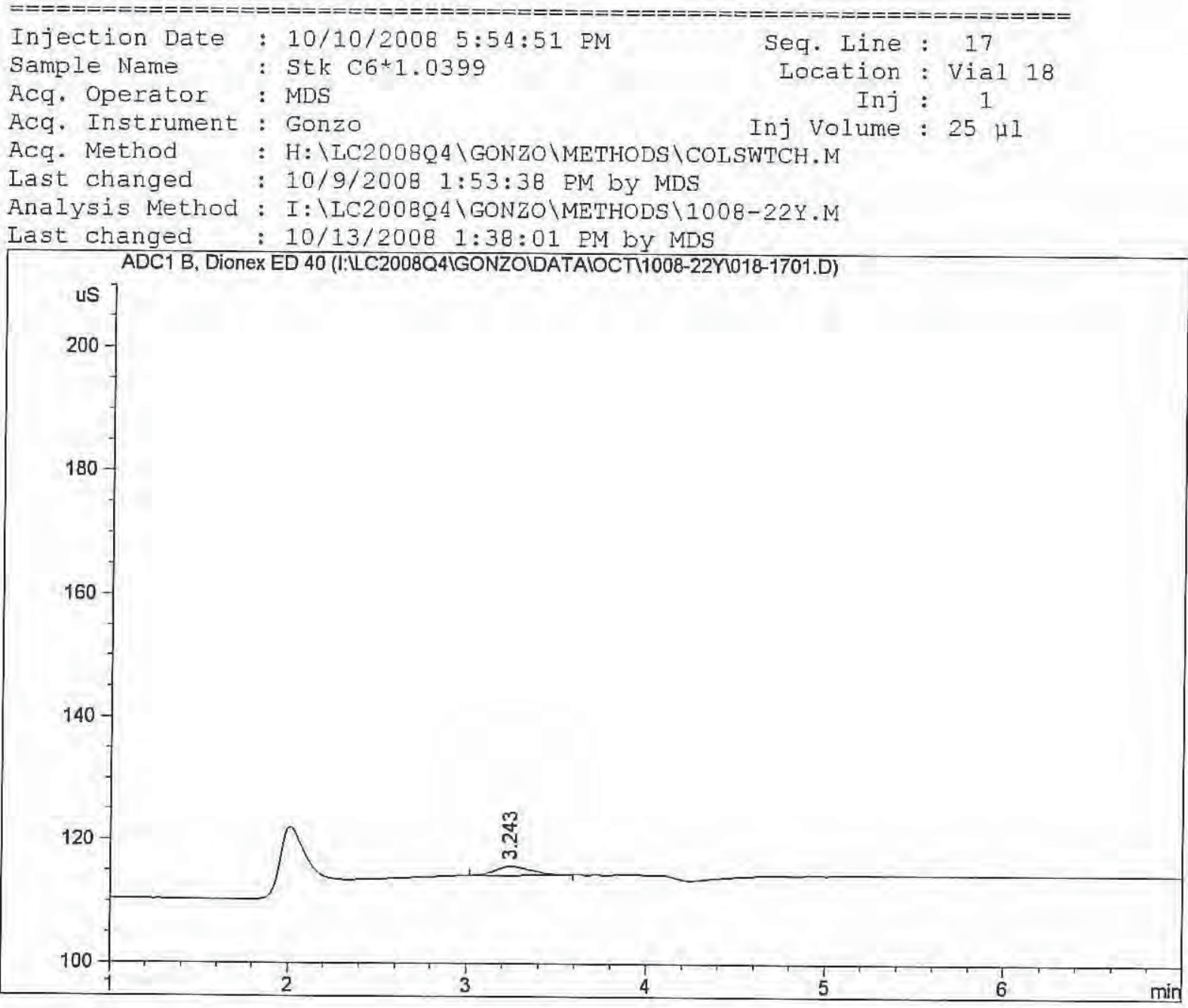

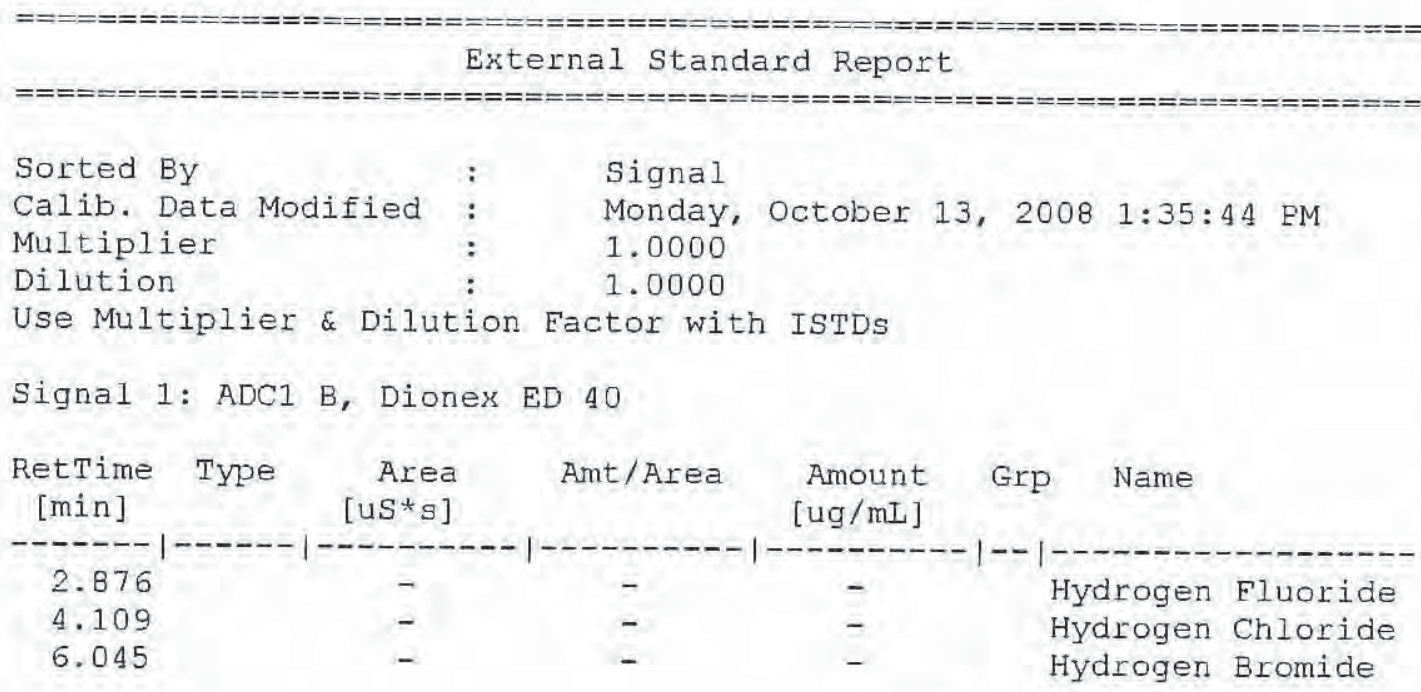

Totals :

0.00000

Results obtained with enhanced integrator!

1 Warnings or Errors :

Warning : Calibrated compound(s) not found 
1008-22 Sample Blank

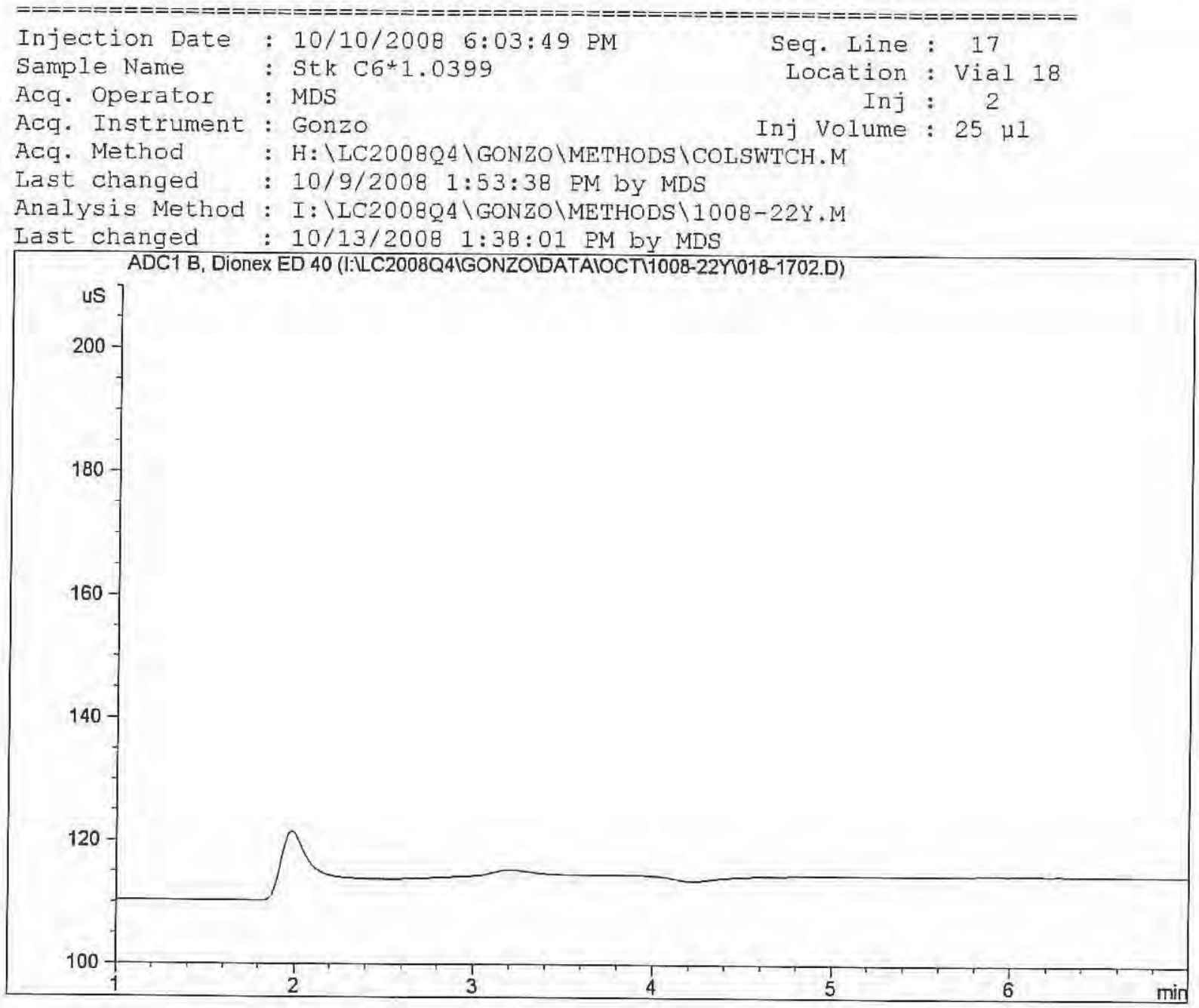

External Standard Report

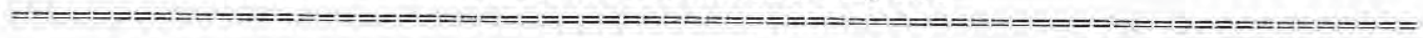

$\begin{array}{lcc}\text { Sorted By } & : & \text { Signal } \\ \text { Calib. Data Modified } & : & \text { Monday, October 13, } 2008 \text { 1:35:44 BM } \\ \text { Multiplier } & : & 1.0000 \\ \text { Dilution } & : & 1.0000 \\ \text { Use Multiplier \& Dilution Eactor with ISTDs }\end{array}$

Signal 1: ADC1 B, Dionex ED 40

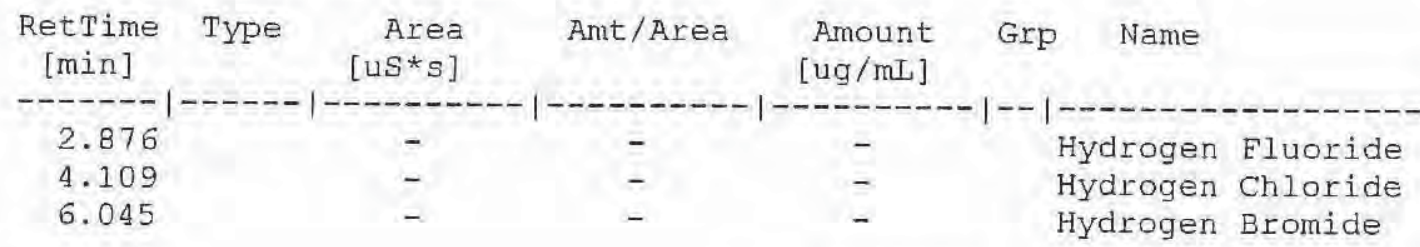

Totals :

0.00000

Results obtained with enhanced integrator!

1 Warnings or Errors :

Warning : Calibrated compound(s) not found 
$0.01 \mathrm{~N} \mathrm{H} 2 \mathrm{SO} 4$, used to prepare standards
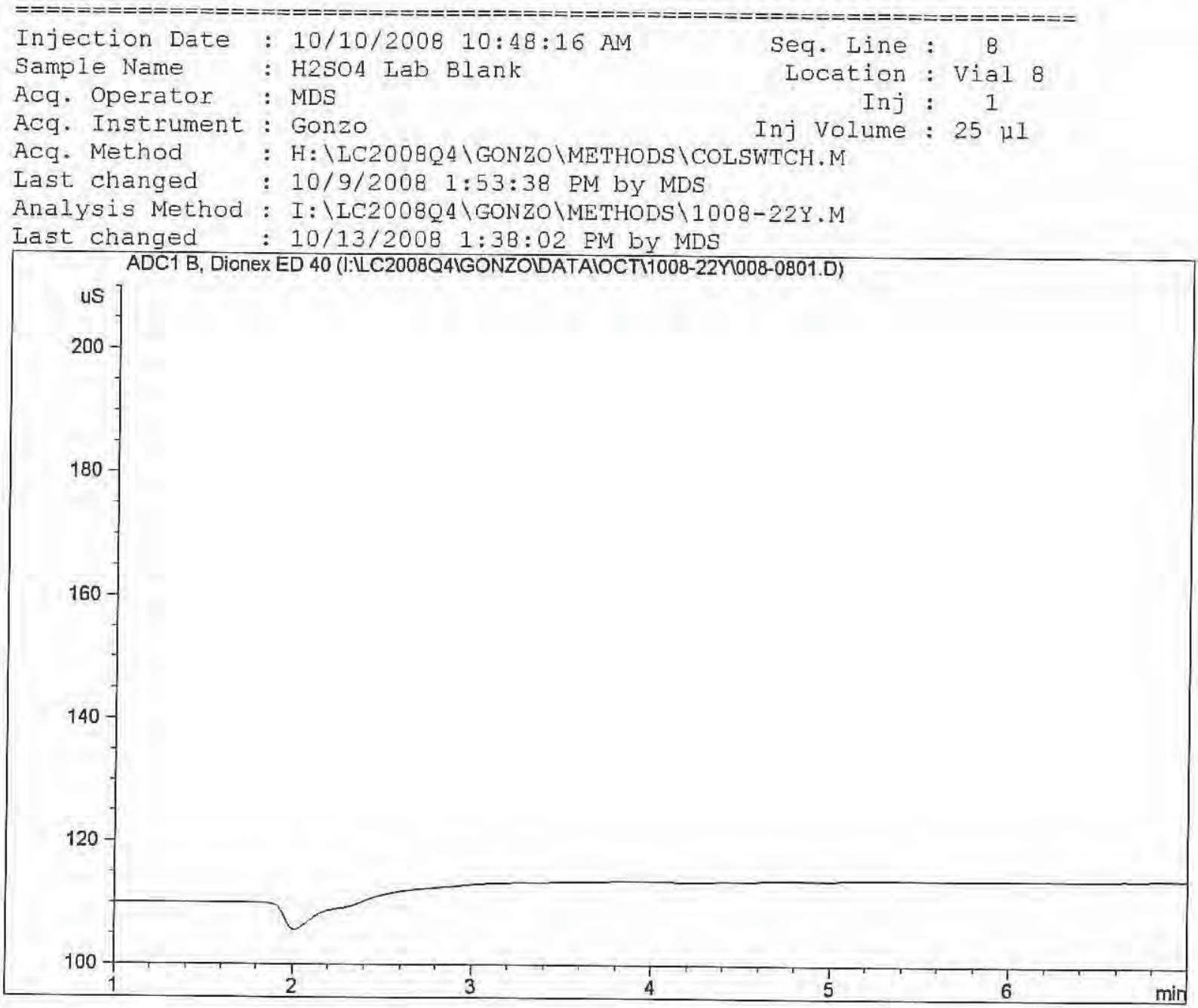

External Standard Report

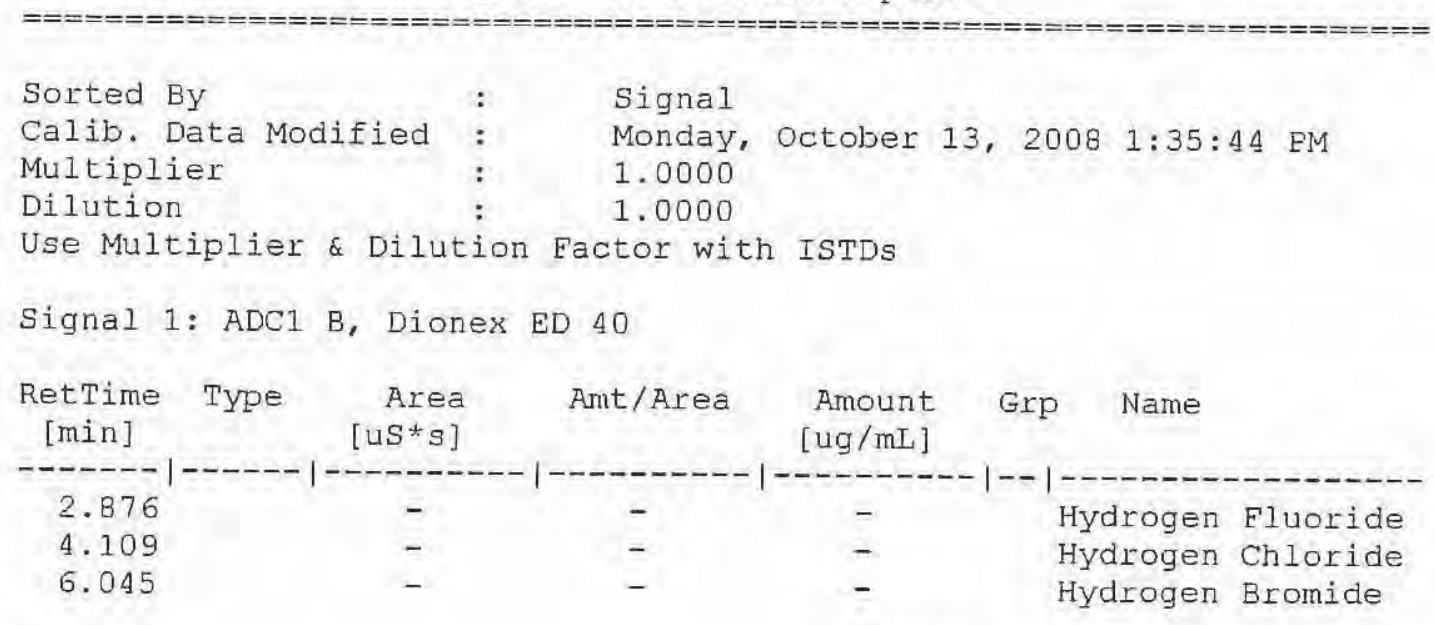


$0.01 \mathrm{~N} \mathrm{H} 2 \mathrm{SO}$, used to prepare standards

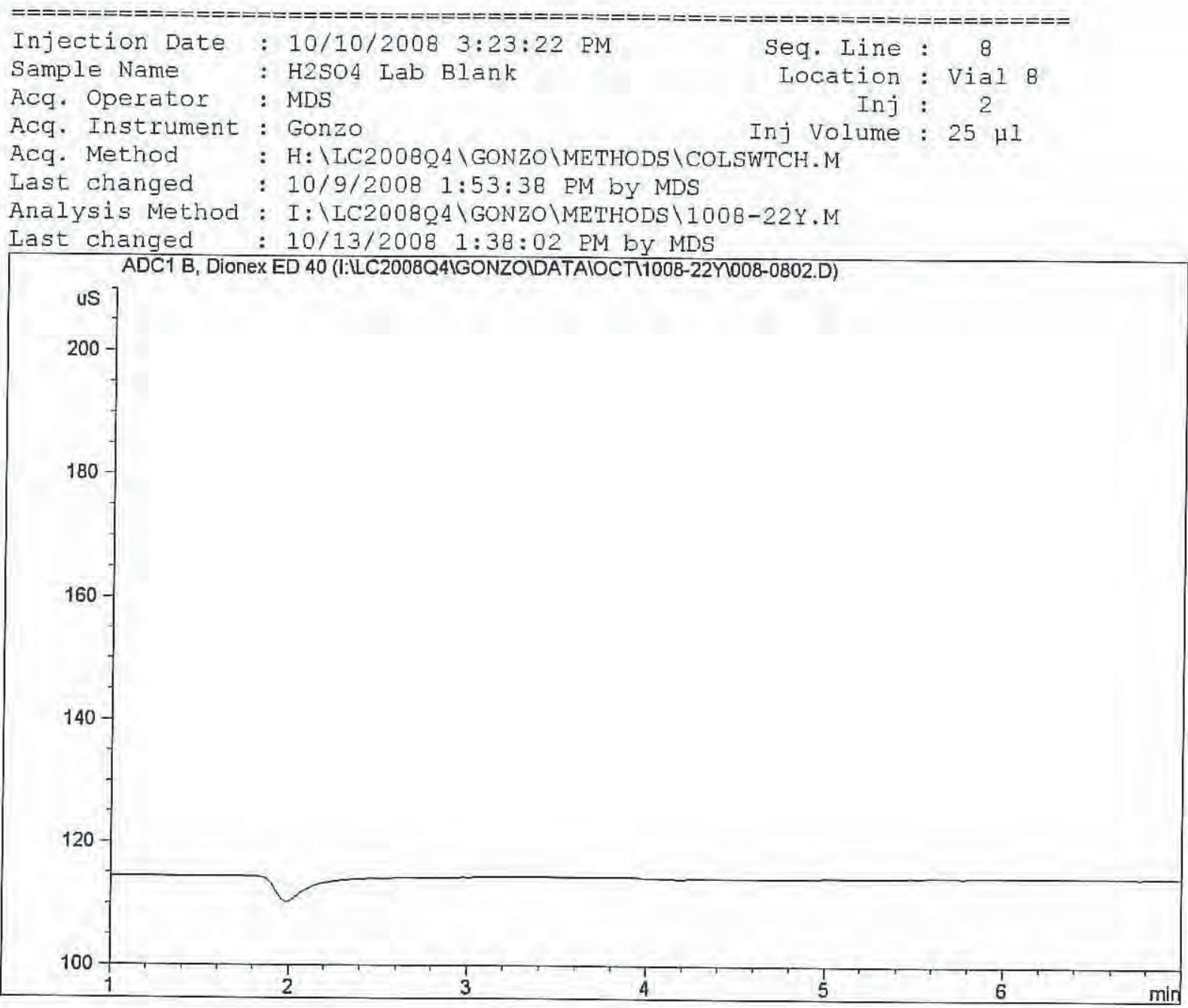

External Standard Report

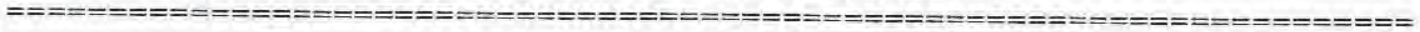

Sorted By

Calib. Data Modified

Multiplier

Dilution

Signal

Monday, October 13, 2008 1:35:44 PM

Use Multiplier \& Dilution Factor with ISTDS

Signal 1: ADC1 B, Dionex ED 40

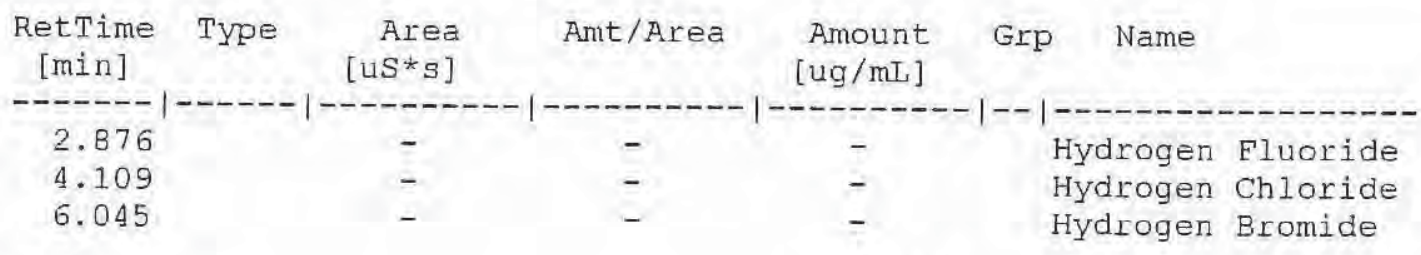

Totals :

0.00000

Results obtained with enhanced integrator!

1 Warnings or Errors :

Warning : Calibrated compound(s) not found 
used to dilute samples

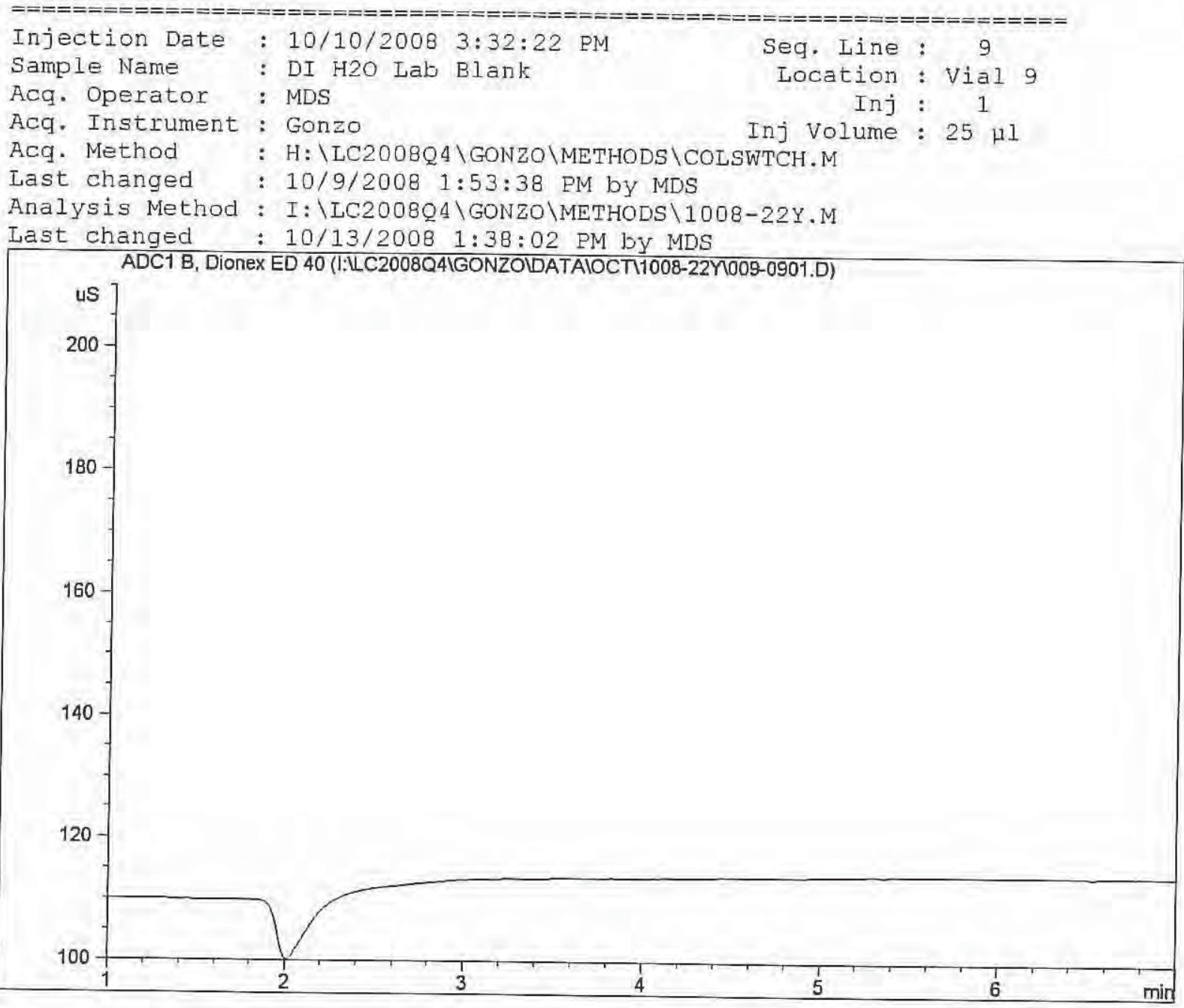

External standard Report

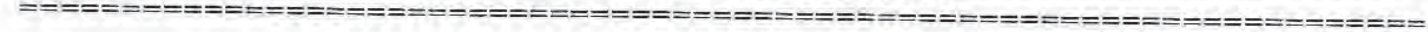

$\begin{array}{lll}\text { Sorted By } & \text { Signal } \\ \text { Calib. Data Modified } & : & \text { Monday, October 13, 2008 1:35:44 PM } \\ \text { Multiplier } & : & 1.0000 \\ \text { Dilution } & : & 1.0000\end{array}$

Use Multiplier \& Dilution Factor with ISTDS

Signal 1: ADC1 B, Dionex ED 40

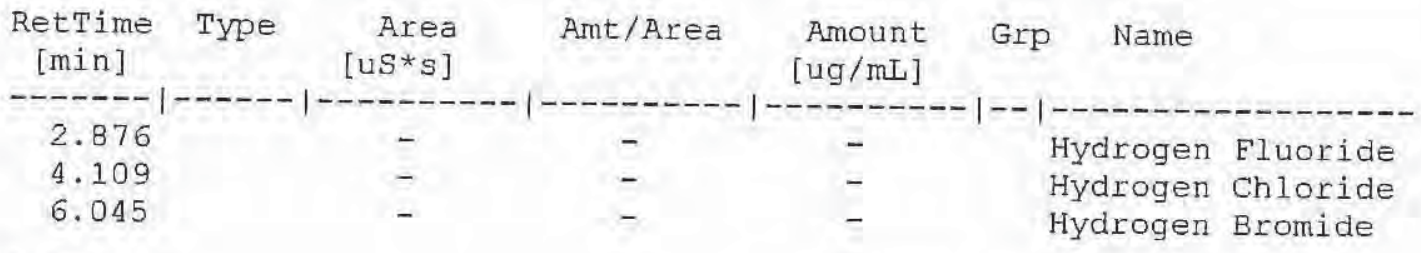

Totals :

0.00000

Results obtained with enhanced integrator!

1 Warnings or Errors :

Warning : Calibrated compound(s) not found 
used to dilute samples

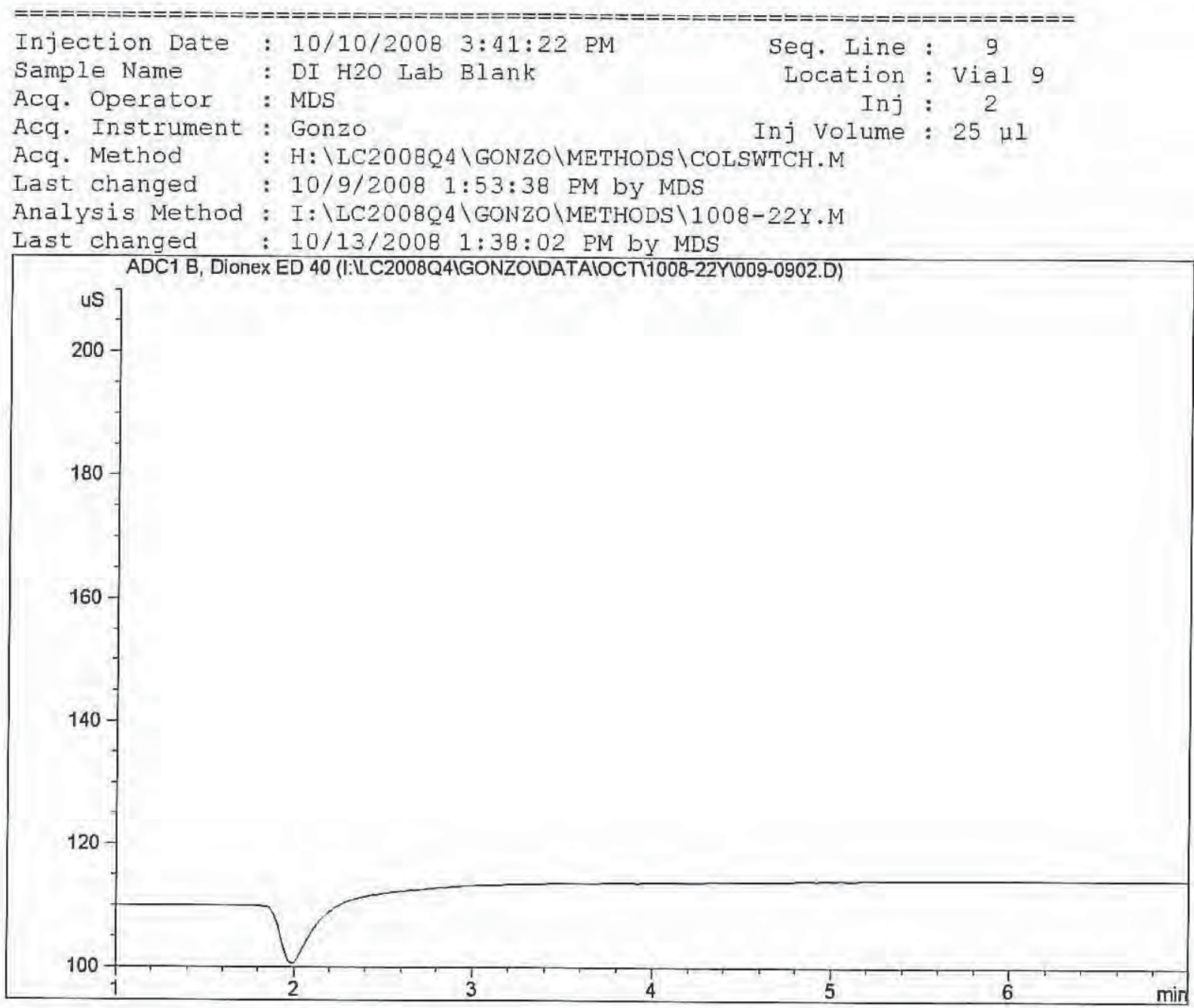

External Standard Report

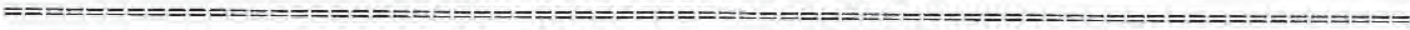

$\begin{array}{lll}\text { Sorted By } & : & \text { Signal } \\ \text { Calib. Data Modified } & : & \text { Monday, October 13, 2008 1:35:44 PM } \\ \text { Multiplier } & : & 1.0000 \\ \text { Dilution } & \vdots & 1.0000\end{array}$

Use Multiplier \& Dilution Factor with ISTDS

Signal 1: ADC1 B, Dionex ED 40

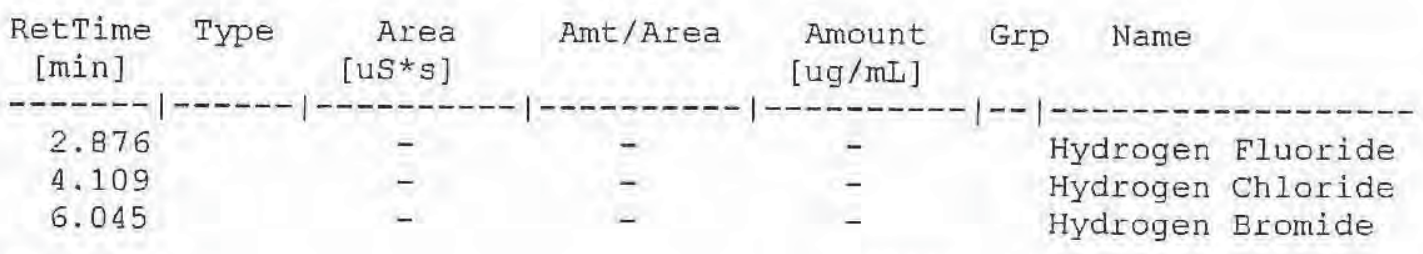

Totals :

0.00000

Results obtained with enhanced integrator!

1 Warnings or Errors :

Warning : Calibrated compound(s) not found 
1008-22 Matrix Spike

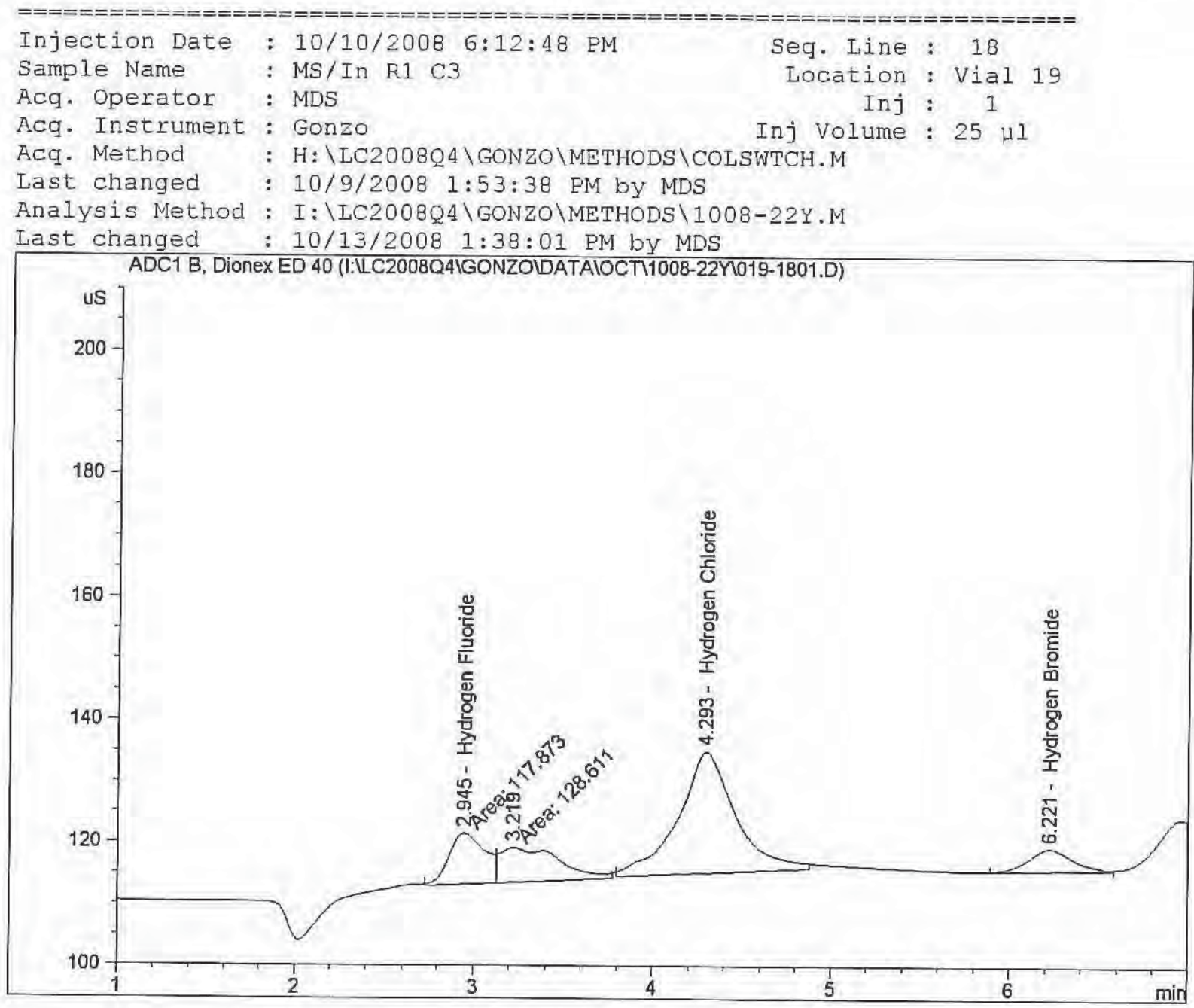

External Standard Report

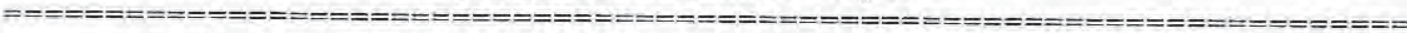

$\begin{array}{lll}\text { Sorted By } & : & \text { Signal } \\ \text { Calib. Data Modified } & : & \text { Monday, October 13, } 2008 \text { 1:35:44 BM } \\ \text { Multiplier } & : & 1.0000 \\ \text { Dilution } & \vdots & 1.0000 \\ \text { Use Multiplier \& Dilution Eactor with ISTDs }\end{array}$

Use Multiplier \& Dilution Eactor with ISTDs

Signal 1: ADC1 B, Diones ED 40

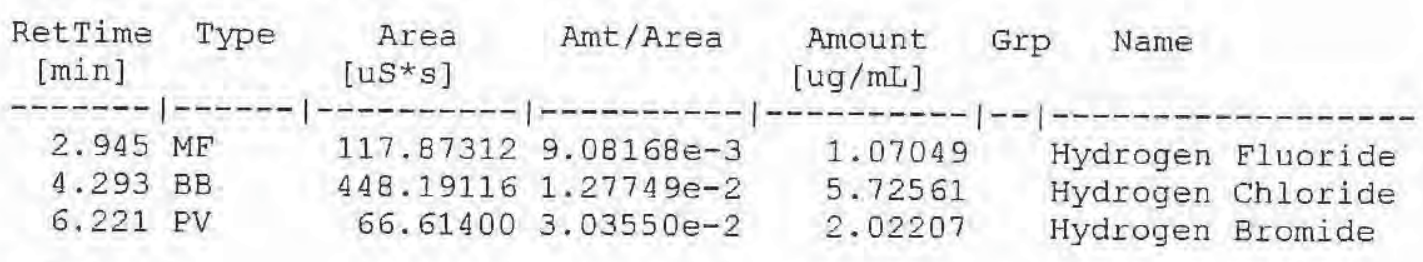

Totals :

8.81816

Results obtained with enhanced integrator!

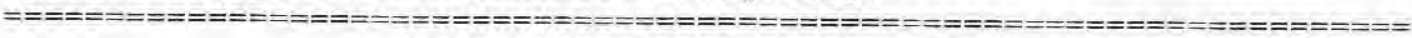

$* * *$ End of Report $* * *$ 
1008-22 Matrix Spike

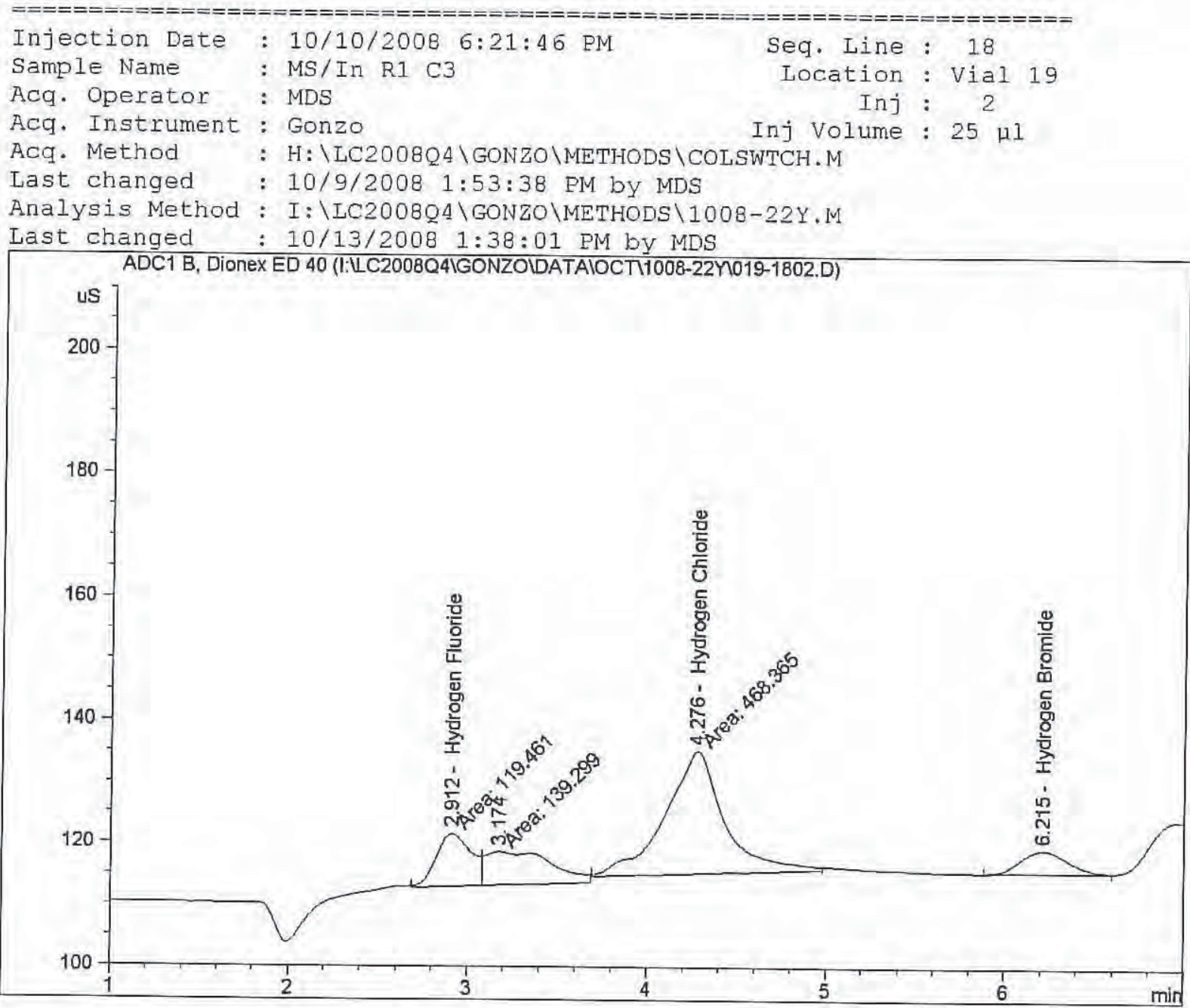

External Standard Report

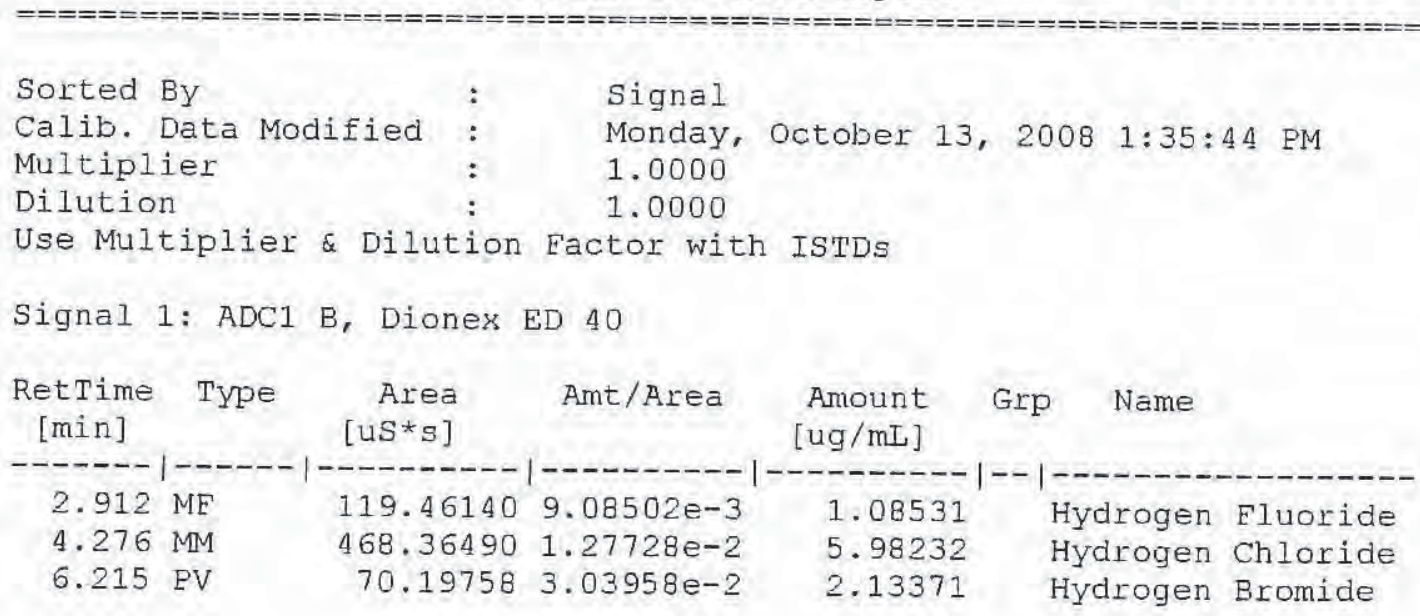

Totals :

9.20134

Results obtained with enhanced integrator! 


\section{Curve(s)/QA Point(s) Chromatograms}

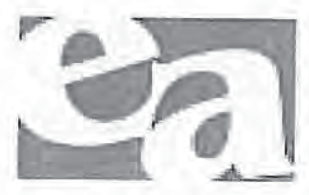




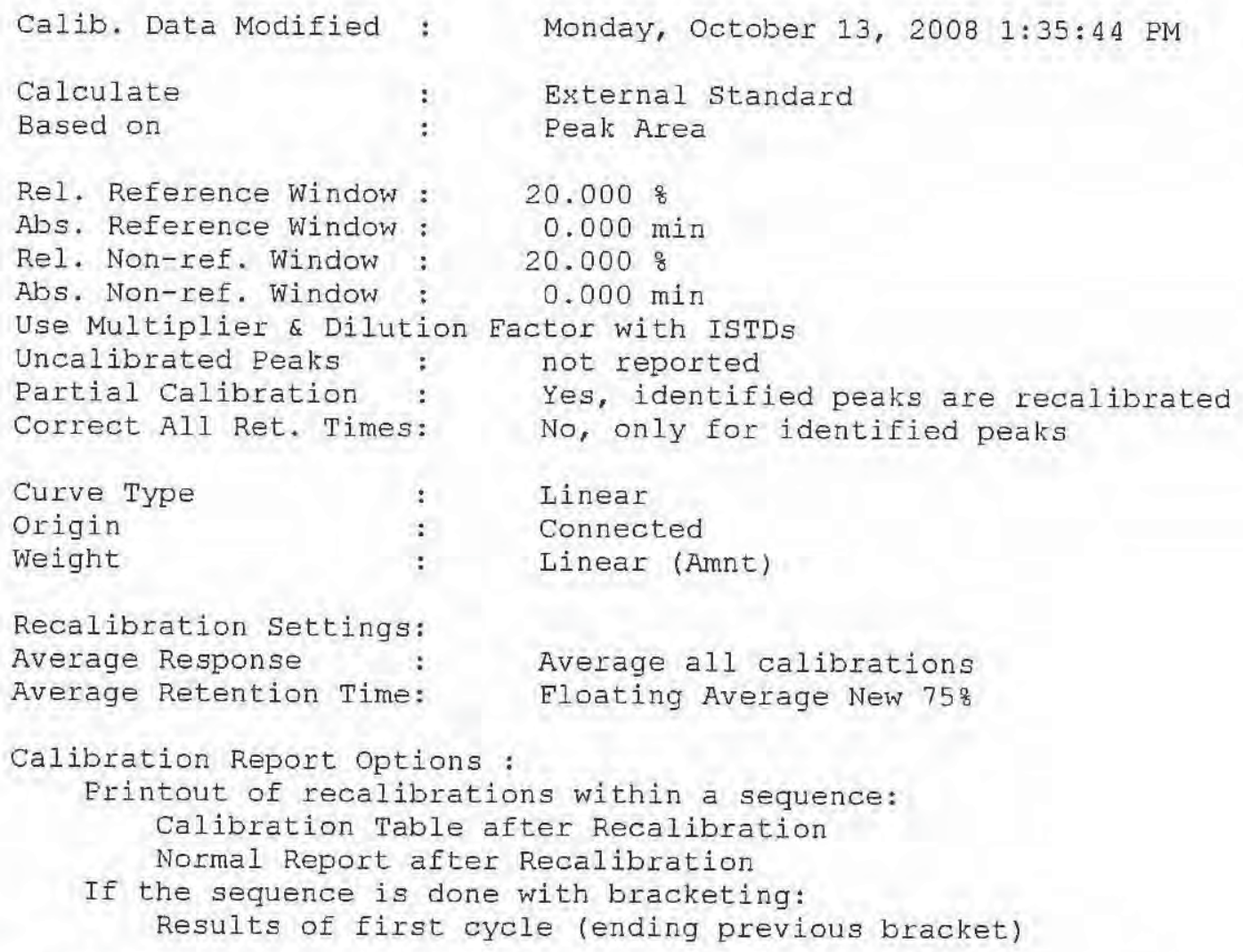

Recalibration Settings: Average Response : Average Retention Time:

Calibration Report Options :

Frintout of recalibrations within a sequence: Calibration Table after Recalibration Normal Report after Recalibration

If the sequence is done with bracketing: Results of first cycle (ending previous bracket)

Signal 1: ADC1 B, Dionex ED 40

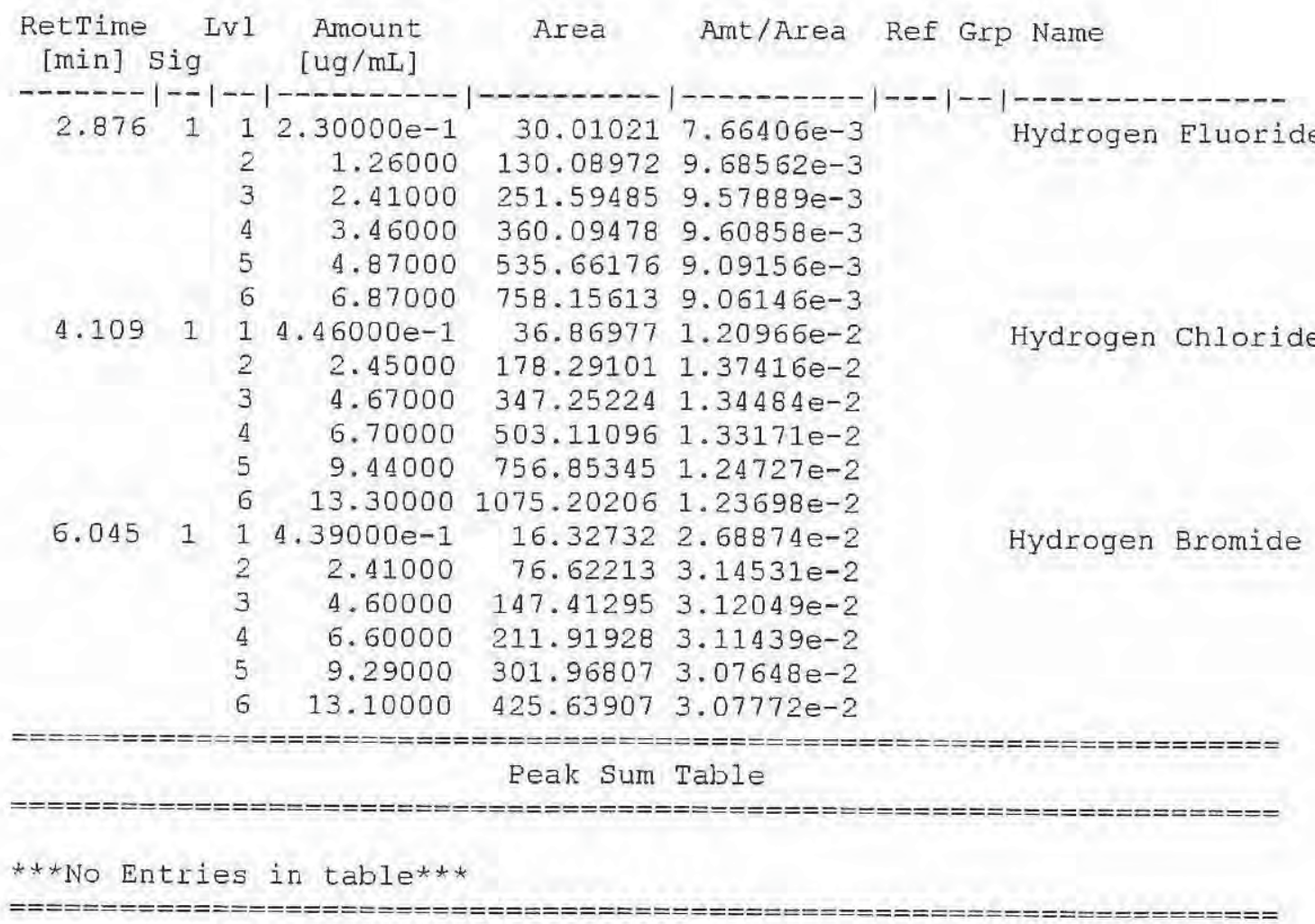



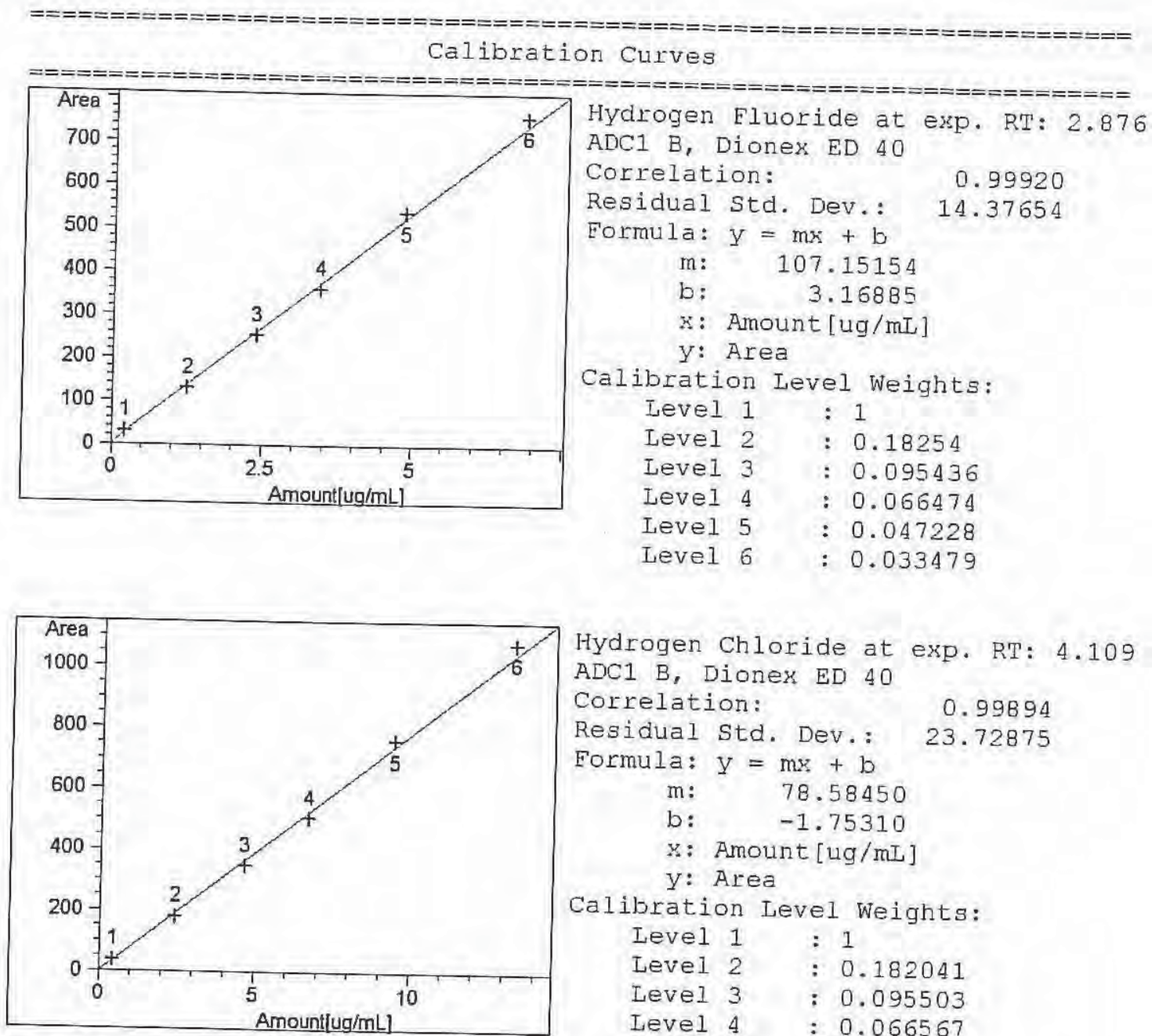

Hydrogen Chloride at exp. RT: 4.109 ADC1 B, Dionex ED 40 Correlation:

0.99894

Formula: $y=m x+b$ 23.72875

$\mathrm{m}: \quad 78.58450$

b: $\quad-1.75310$

$\mathrm{x}$ : Amount [ug/mL] y: Area

Calibration Level Weights:

Level $1: 1$

Level $2: 0.182041$

Level 3 : 0.095503

Level $4: 0.066567$

Level $5: 0.047246$

Level $6: 0.033534$

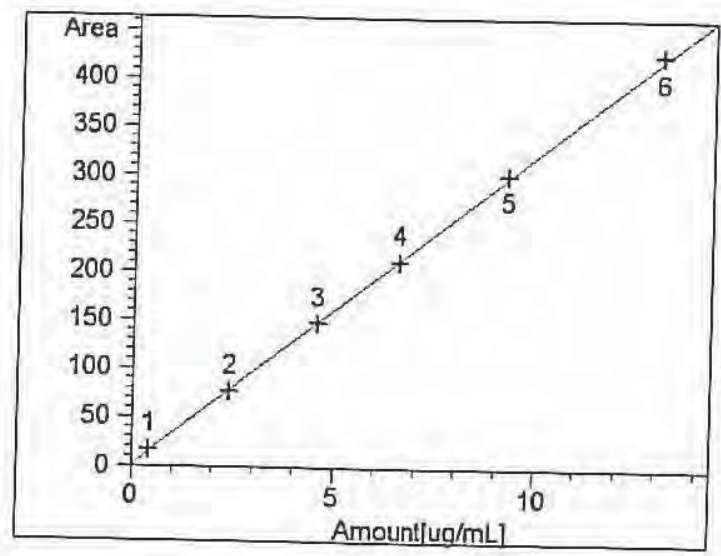

Hydrogen Bromide at exp. RT: 6.045 ADC1 B, Dionex ED 40

Correlation:

0.99989

Residual Std. Dev.: 2.68486

Formula: $y=m x+b$

m: $\quad 32.09848$

b: $\quad 1.70873$

x: Amount $[\mathrm{ug} / \mathrm{mL}]$

y: Area

Calibration Level Weights:

Leve1 $1: 1$

Level $2: 0.182158$

Level $3: 0,095435$

Level $4: 0.066515$

Level $5: 0.047255$

Level $6: 0.033511$ 

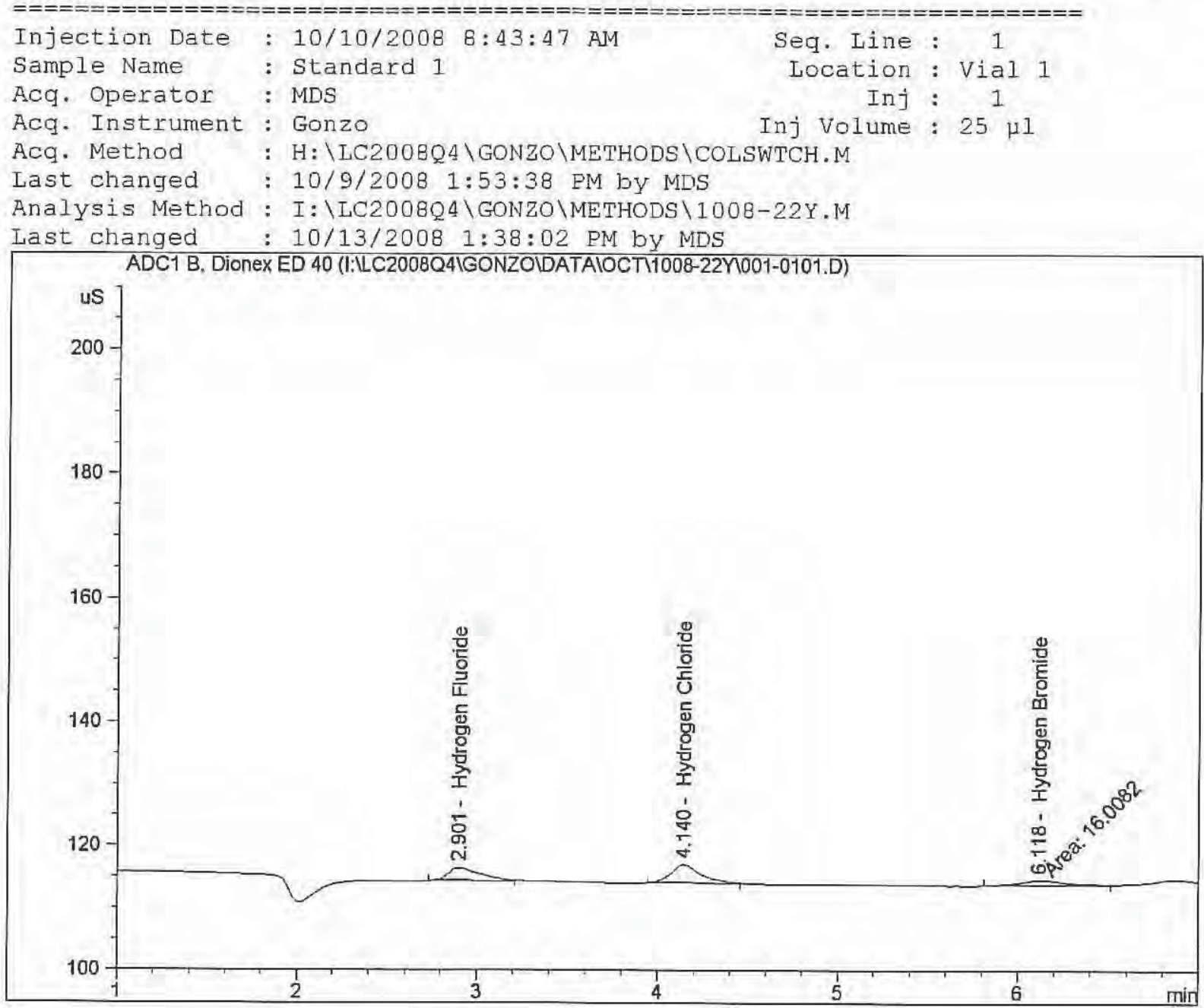

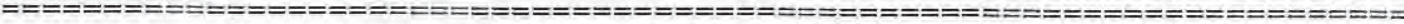

External Standard Report

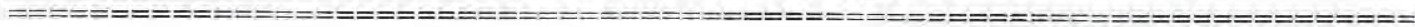

$\begin{array}{lcc}\text { Sorted By } & : & \text { Signal } \\ \text { Calib. Data Modified } & \text { Monday, October 13, } 2008 \text { 1:35:44 PM } \\ \text { Multiplier } & : & 1.0000 \\ \text { Dilution } & 1.0000 \\ \text { Use Multiplier \& Dilution Eactor with ISTDs }\end{array}$

Signal 1: ADC1 B, Dionex ED 40

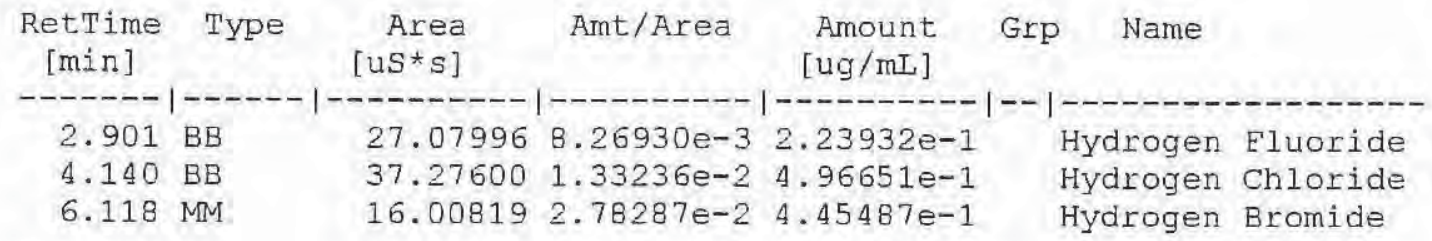

Totals :

1.16607

Results obtained with enhanced integrator!

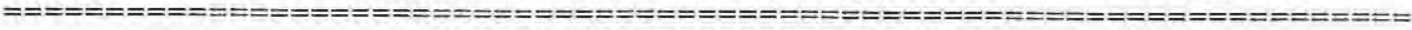

$\star \star *$ End of Report $* * *$ 


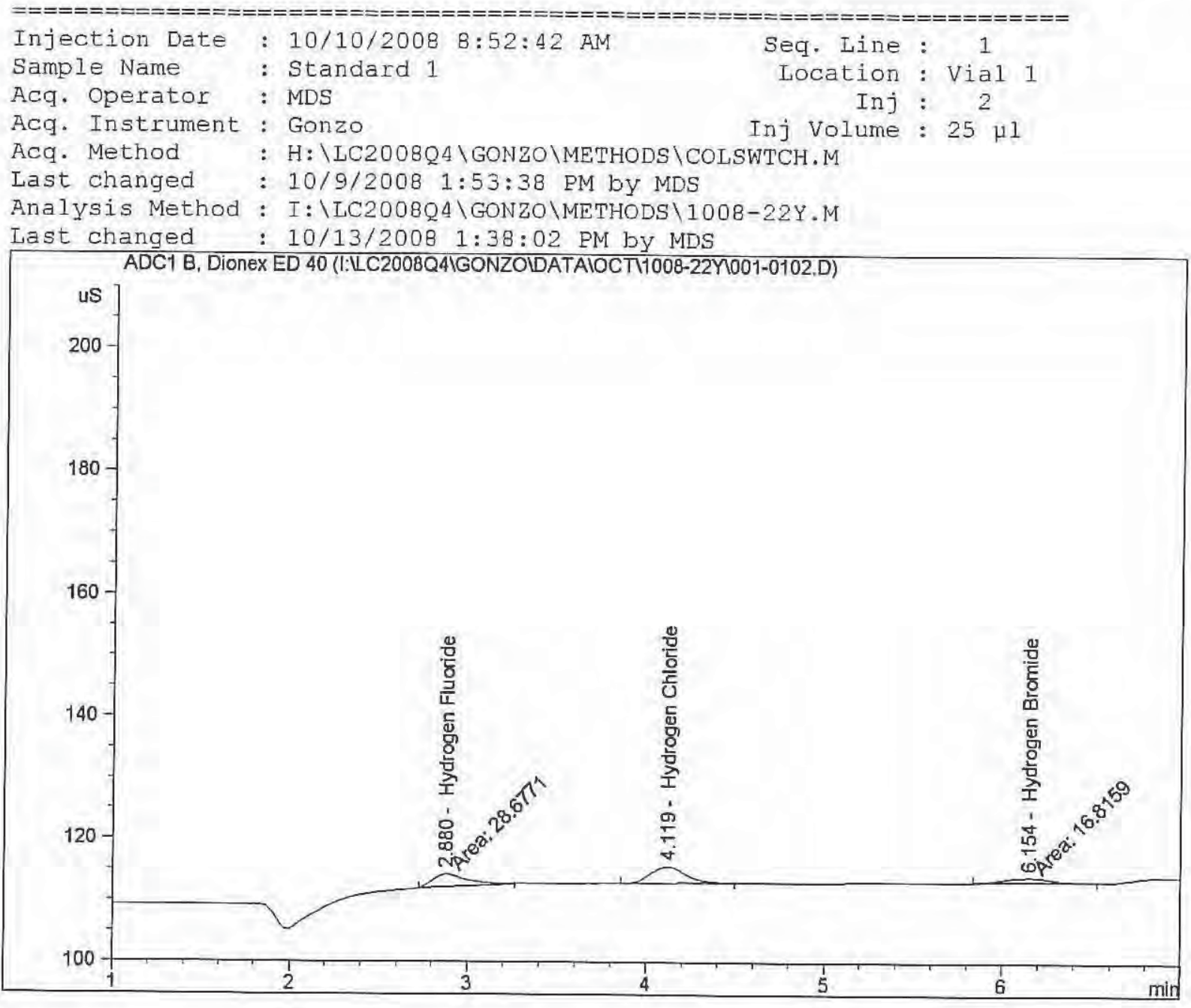

External standard Report

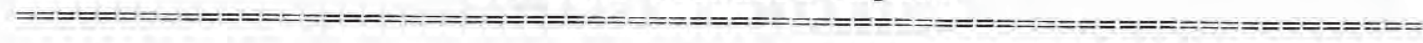

\begin{tabular}{|c|c|c|c|c|}
\hline Sorted By & Signal & & & \\
\hline Calib. Data Modified & Monday, & October 13, & 2008 & $1: 35: 44$ \\
\hline Multiplier & 1.0000 & & & \\
\hline Dilution & 1.0000 & & & \\
\hline
\end{tabular}

Signal 1: ADC1 B, Dionex ED 40

\begin{tabular}{|c|c|c|c|c|c|c|}
\hline $\begin{array}{l}\text { RetTime } \\
\text { [min] }\end{array}$ & Type & $\begin{array}{r}\text { Area } \\
\text { [us*s] }\end{array}$ & Amt/Area & $\begin{array}{l}\text { Amount } \\
\text { [ug/mL] }\end{array}$ & Name & \\
\hline & & & & ----1 & & \\
\hline 880 & MM & 28.67711 & 8. $30132 e-3$ & 2. $38058 e-1$ & Hydrogen & Eluoride \\
\hline 4.119 & $\mathrm{BB}$ & 37.22715 & 1. $33244 \mathrm{e}-2$ & 4. $96030 e-1$ & Hydrogen & Chloride \\
\hline 6.154 & MM & 16.81589 & $2.79884 e-2$ & $4.70650 \mathrm{e}-1$ & Hydrogen & Bromide \\
\hline
\end{tabular}

Totals :

1.20474

Results obtained with enhanced integrator!

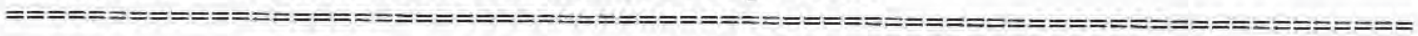

*** End of Report $\star * \star$ 

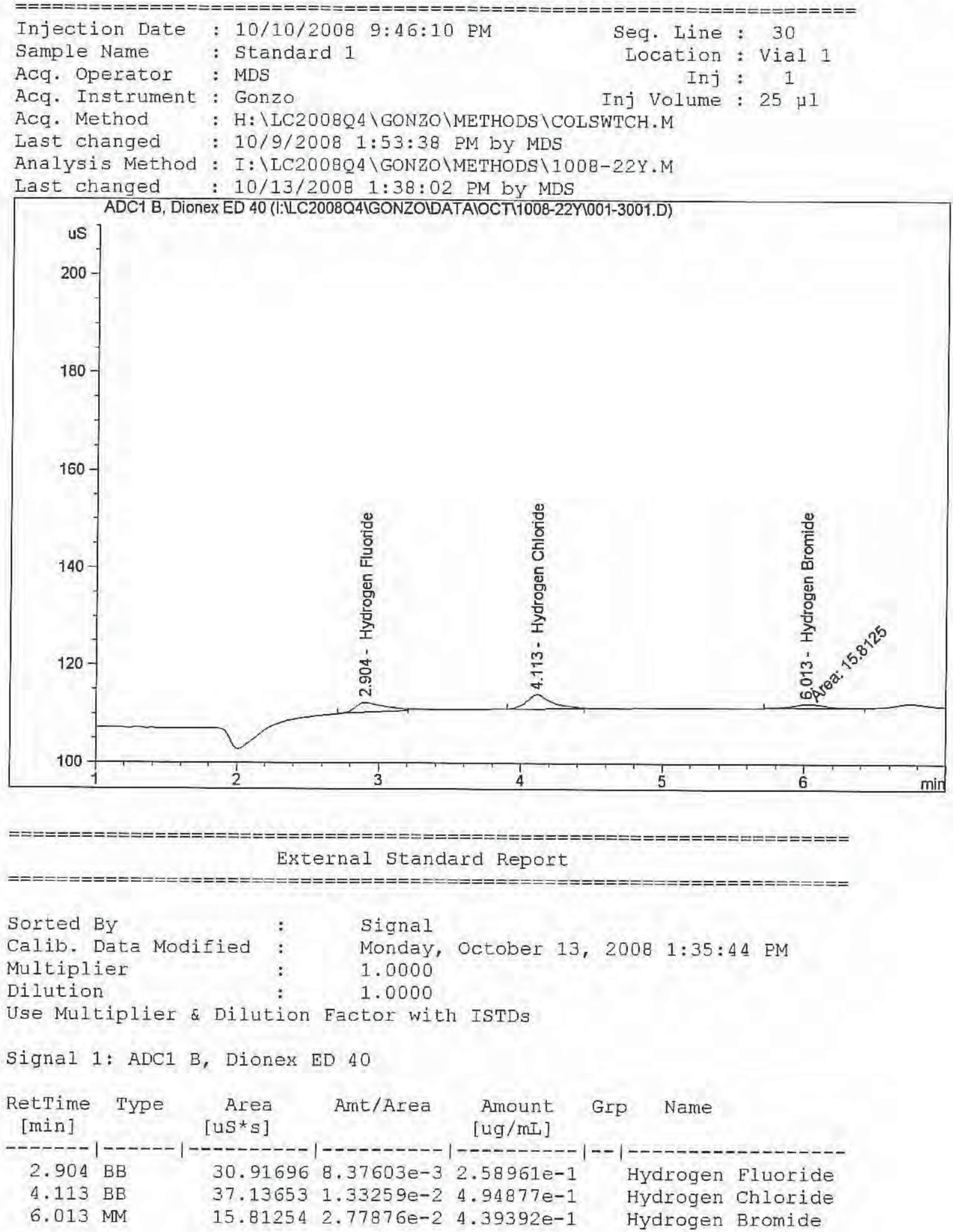

Totals :

1.19323

Results obtained with enhanced integrator! 


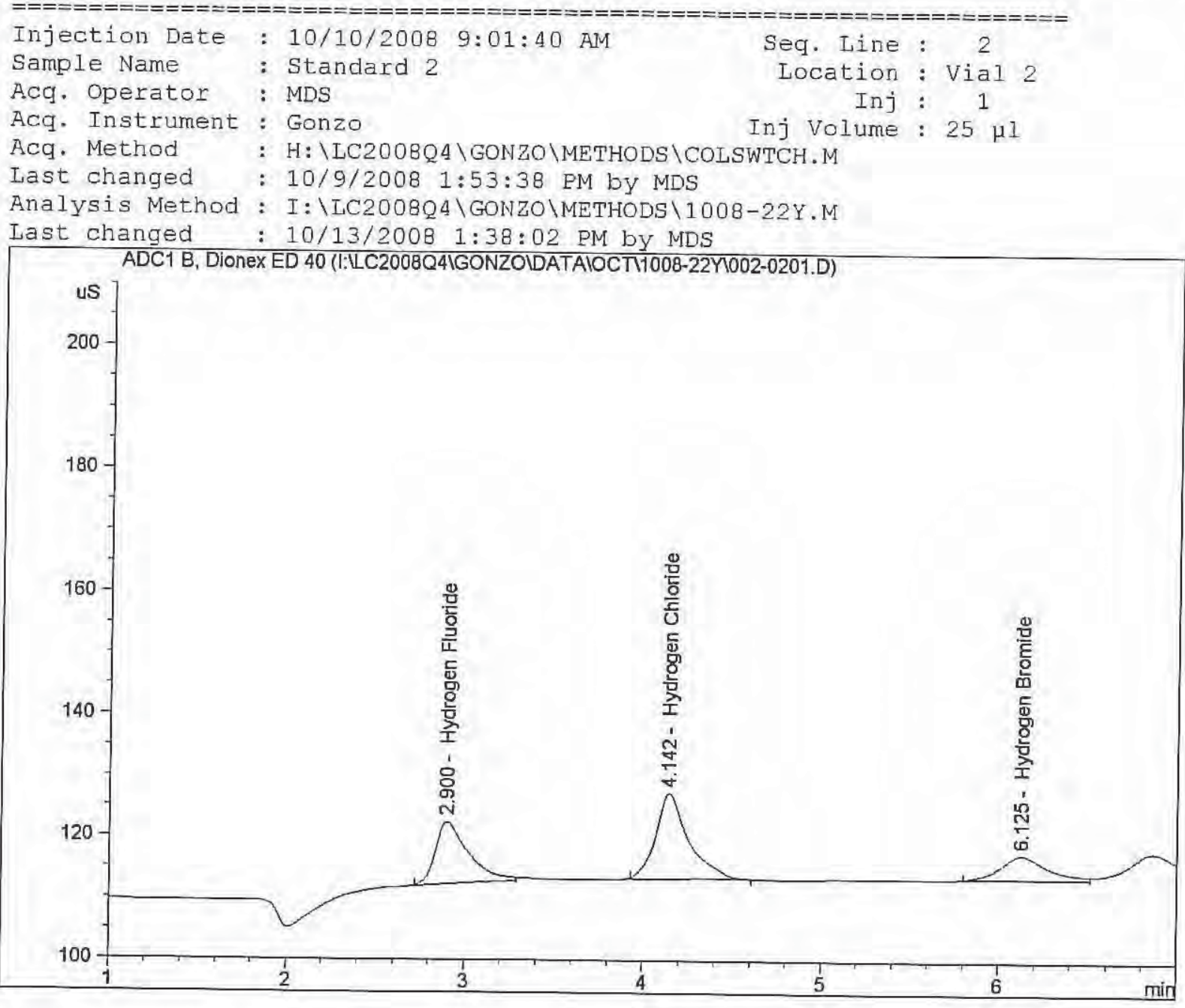

External Standard Report

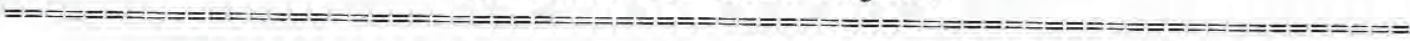

$\begin{array}{lll}\text { Sorted By } & : & \text { Signal } \\ \text { Calib. Data Modified : } & \text { Monday, October 13, } 2008 \text { 1:35:44 PM } \\ \text { Multiplier } & : & 1.0000 \\ \text { Dilution } & : & 1.0000\end{array}$

Use Multiplier \& Dilution Eactor with ISTDs

Signal 1: ADC1 B, Dionex ED 40

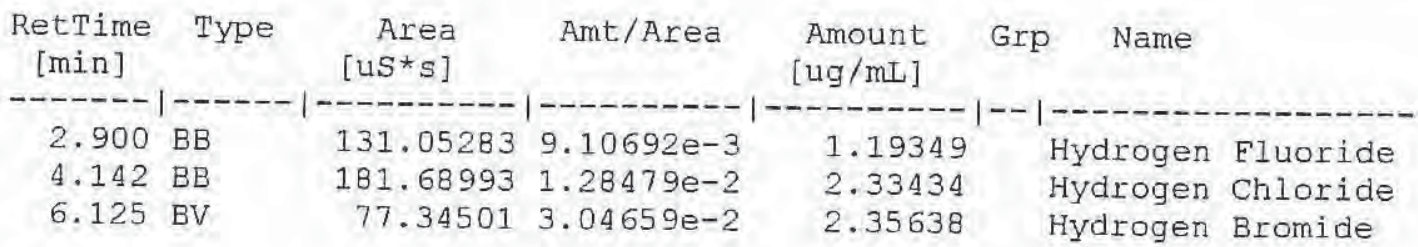

Totals :

5.88421

Results obtained with enhanced integrator! 

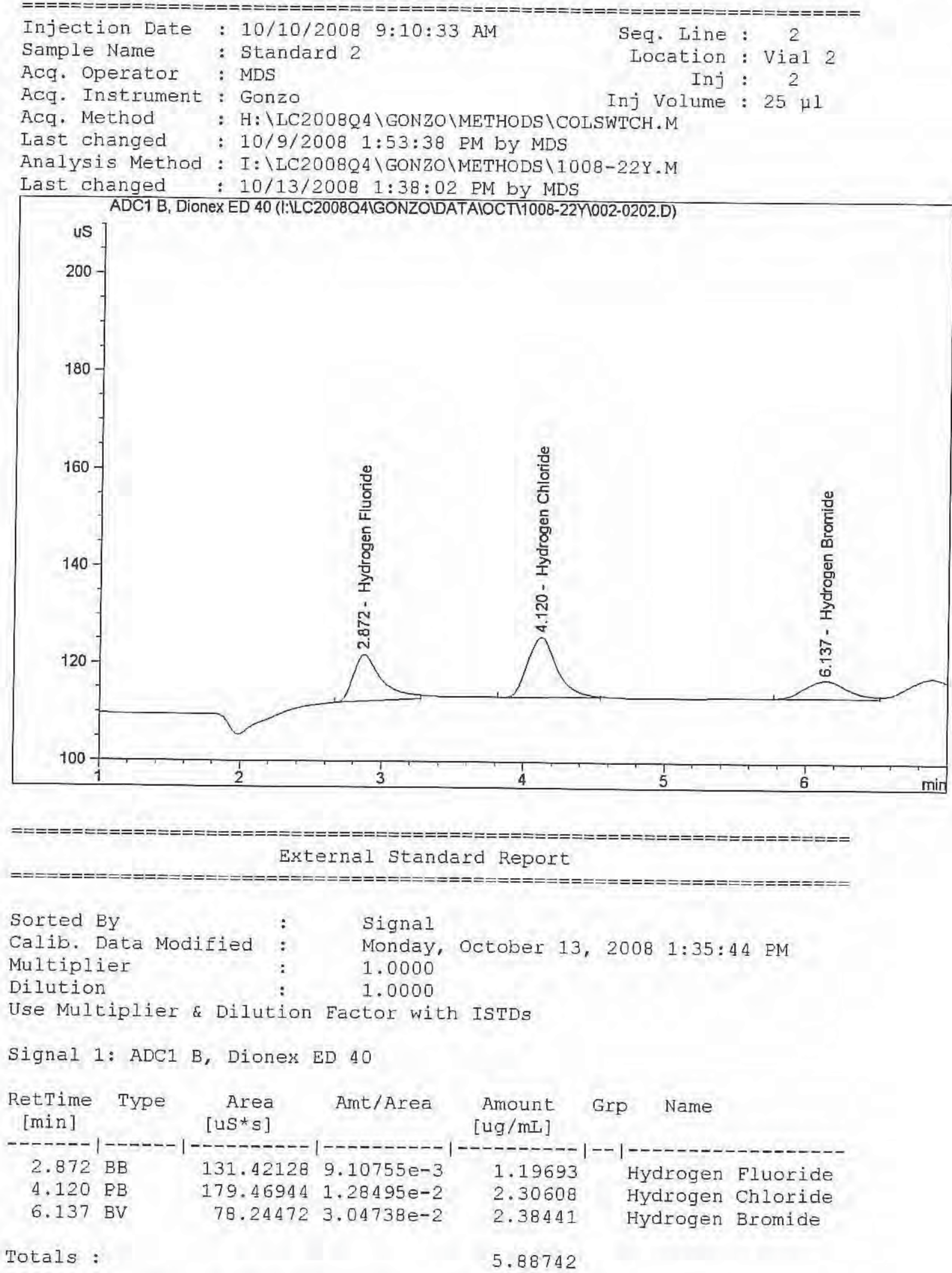

Results obtained with enhanced integrator! 


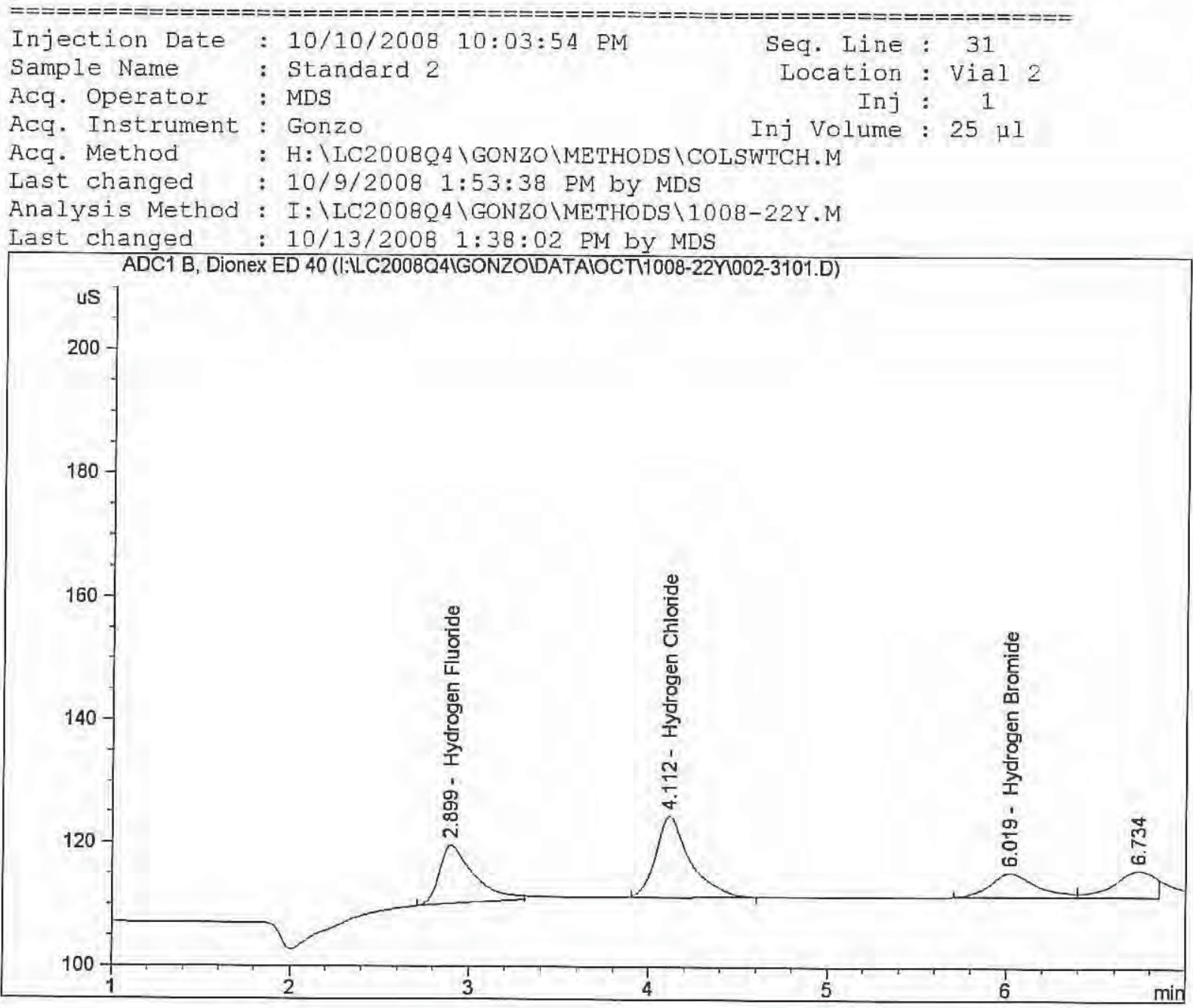

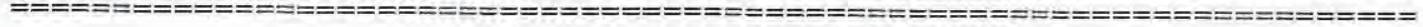

External Standard Report

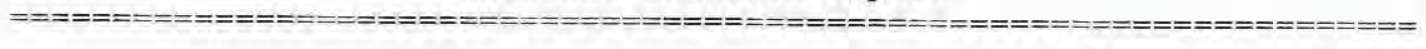

$\begin{array}{lll}\text { Sorted By } & \text { Signal } \\ \text { Calib. Data Modified } & : & \text { Monday, October 13, 2008 1:35:44 PM } \\ \text { Multiplier } & : & 1.0000 \\ \text { Dilution } & : & 1.0000\end{array}$

Use Multiplier \& Dilution Eactor with ISTDs

Signal 1: ADC1 B, Dionex ED 40

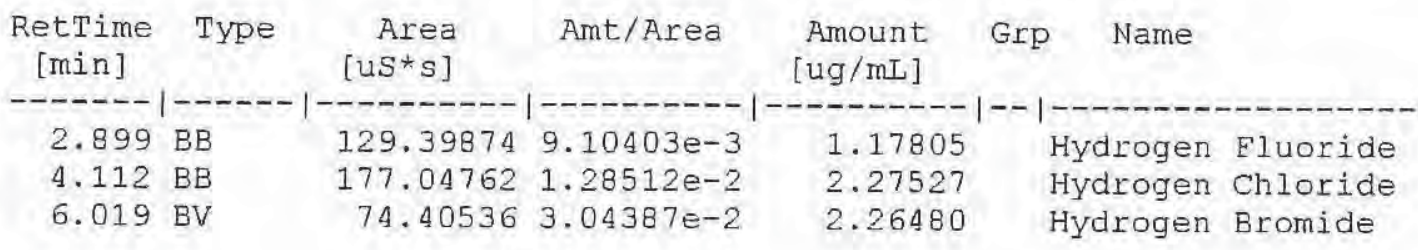

Totals :

5.71812

Results obtained with enhanced integrator!

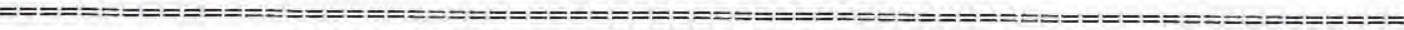
$\star \star \star$ End of Report ***
} 


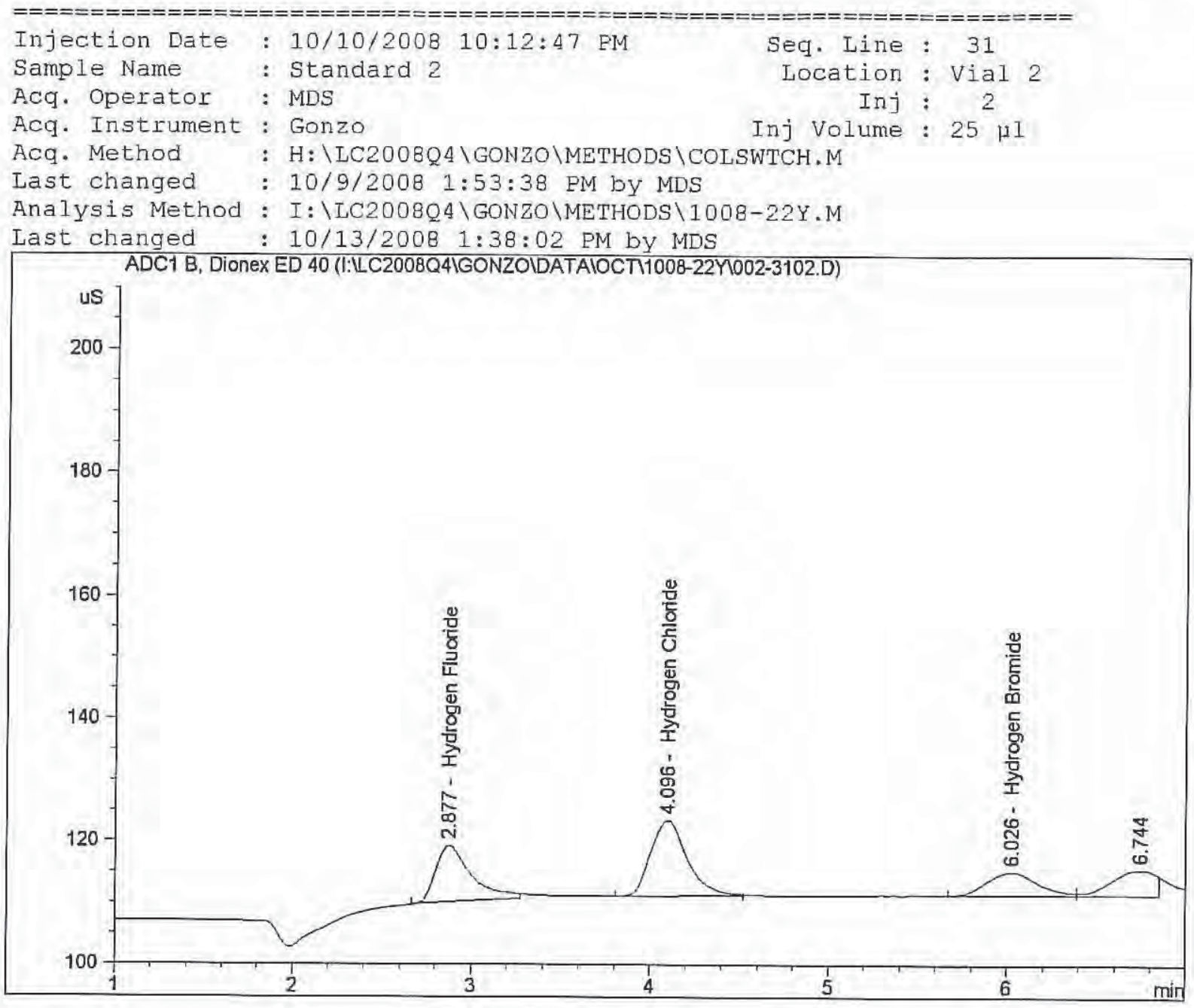

External standard Report

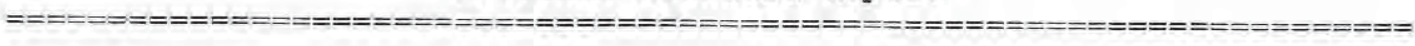

$\begin{array}{lll}\text { Sorted By } & : & \text { Signal } \\ \text { Calib. Data Modified } & \text { Monday, October 13, } 20081: 35: 44 \text { PM } \\ \text { Multiplier } & : & 1.0000 \\ \text { Dilution } & : & 1.0000\end{array}$

Use Multiplier \& Dilution Factor with ISTDS

Signal 1: ADC1 B, Dioner ED 40

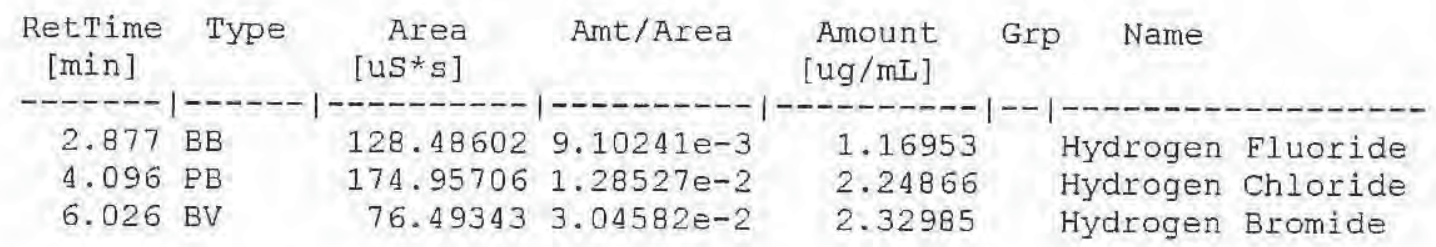

Totals :

5.74805

Results obtained with enhanced integrator!

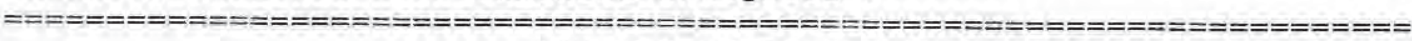
*** End of Report *** 


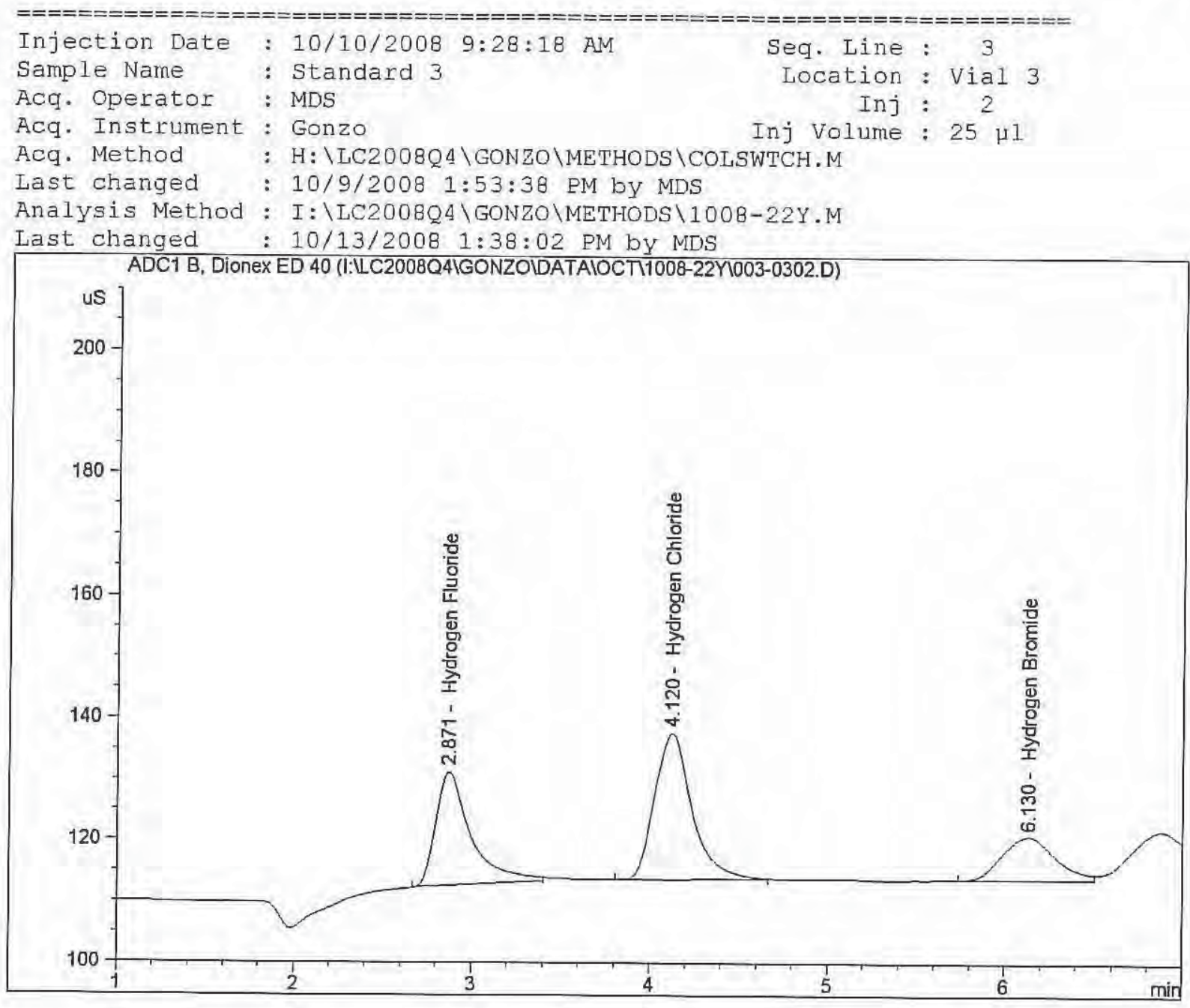

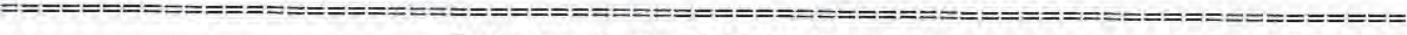
External standard Report

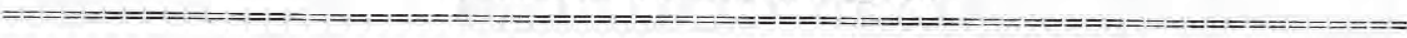

$\begin{array}{lcc}\text { Sorted By } & : & \text { Signal } \\ \text { Calib. Data Modified } & \text { Monday, october 13, } 2008 \text { 1:35:44 PM } \\ \text { Multiplier } & \vdots & 1.0000 \\ \text { Dilution } & 1.0000 \\ \text { Use Multiplier \& Dilution Eactor with ISTDs }\end{array}$

Use Multiplier \& Dilution Eactor with ISTDS

Signal 1: ADC1 B, Dionex ED 40

\begin{tabular}{|c|c|c|c|c|c|c|}
\hline $\begin{array}{c}\text { RetTime } \\
\text { [min] }\end{array}$ & Type & $\begin{array}{r}\text { Area } \\
{\left[\mathrm{uS}^{\star} \mathrm{s}^{-}\right]}\end{array}$ & Amt/Area & $\begin{array}{l}\text { Amount } \\
{[\mathrm{ug} / \mathrm{mL}]}\end{array}$ & Name & \\
\hline & & & & & & \\
\hline 2.871 & BB & 256.14951 & $9.21712 e-3$ & 2.36096 & Hydrogen & Elu \\
\hline 4. 12 & PB & 351.24615 & 1. $278 B 7 e-2$ & 4. 49197 & Hydrogen & Chloride \\
\hline 6.130 & BV & 150.02679 & $3.07993 e-2$ & 4. 62072 & Hydrogen & Bromide \\
\hline
\end{tabular}

Totals :

11. 47365

Results obtained with enhanced integrator!

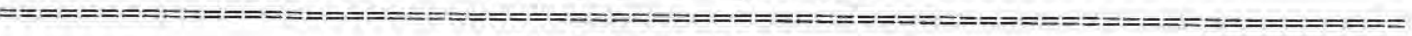

*** End of Report ***
} 


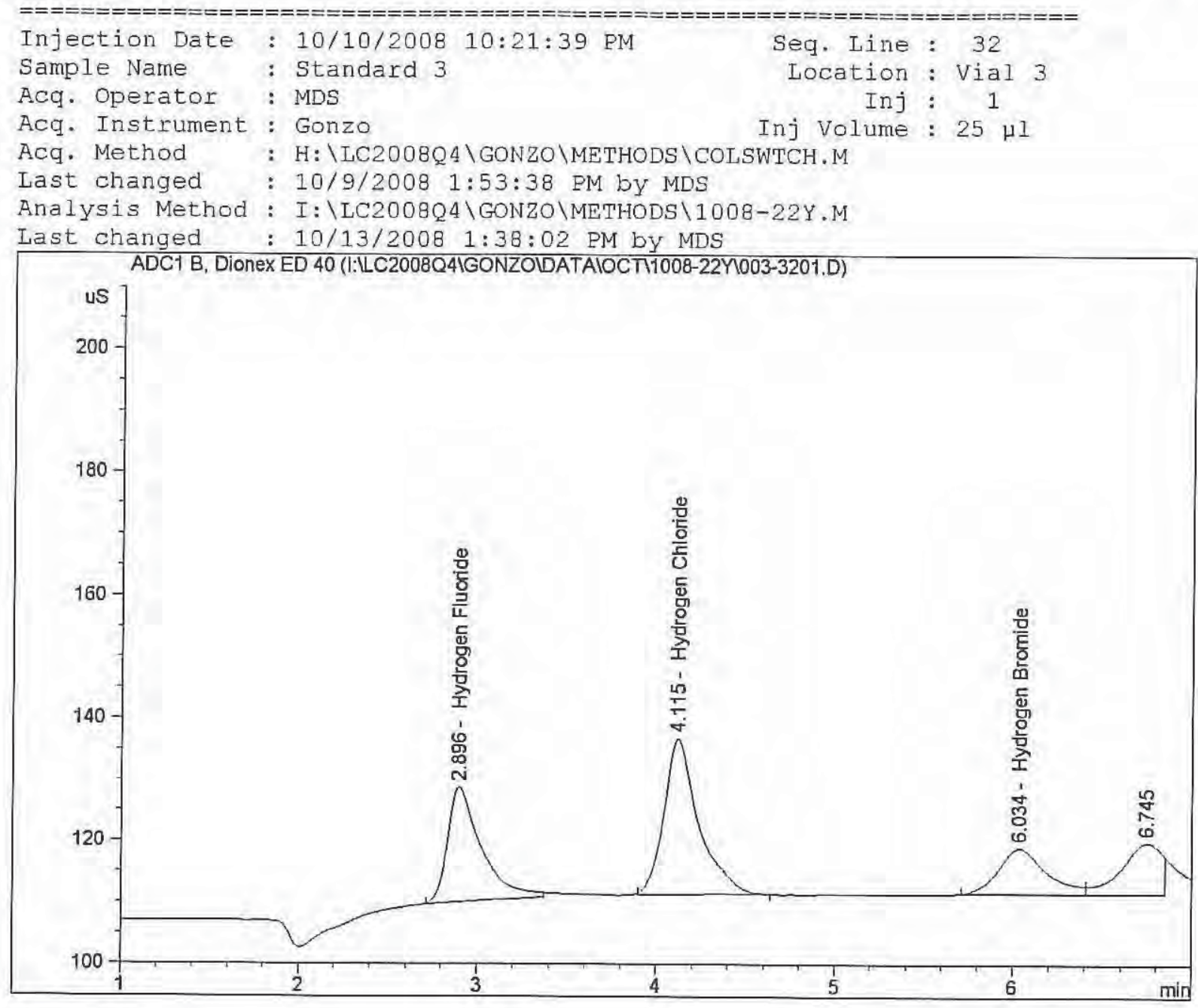

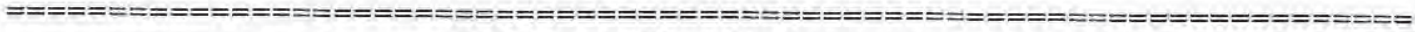
External standard Report

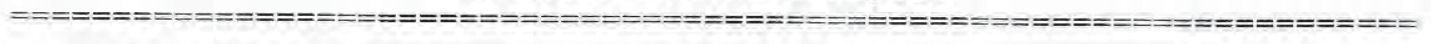

$\begin{array}{lcc}\text { Sorted By } & : & \text { Signal } \\ \text { Calib. Data Modified : } & \text { Monday, October 13, } 2008 \text { 1:35:44 PM } \\ \text { Multiplier } & : & 1.0000 \\ \text { Dilution } & \text { : } & 1.0000 \\ \text { Use Multiplier \& Dilution Factor with ISTDs }\end{array}$

Signal 1: ADC1 B, Dionex ED 40

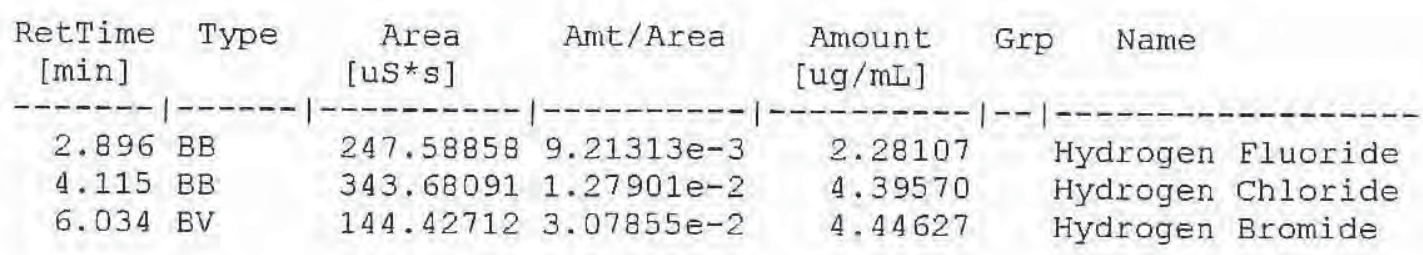

Totals:

11.12303

Results obtained with enhanced integrator!

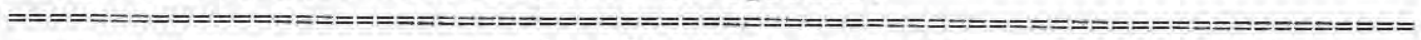
$* * *$ End of Report $* * *$
} 


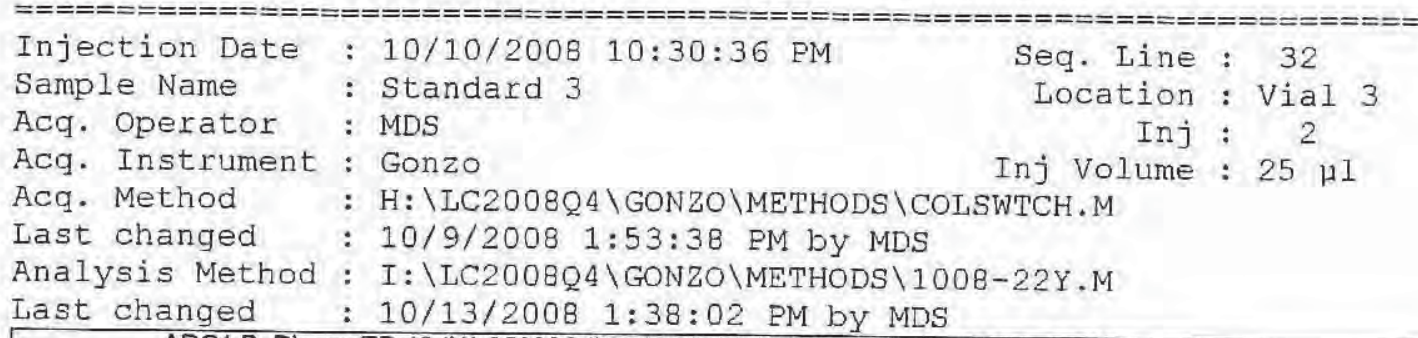

Results obtained with enhanced integrator! 


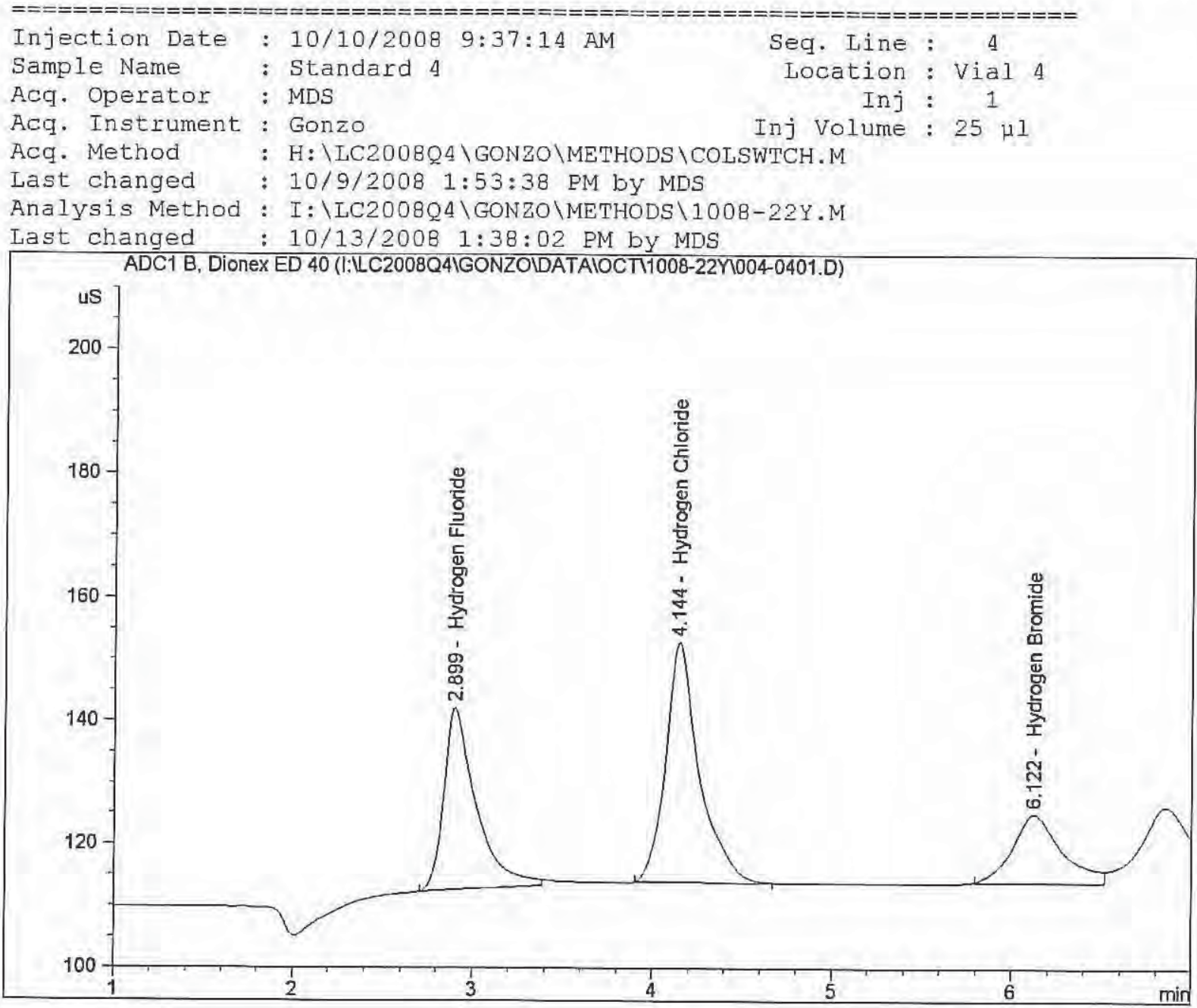

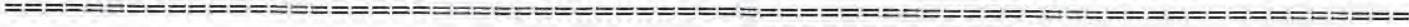

External Standard Report

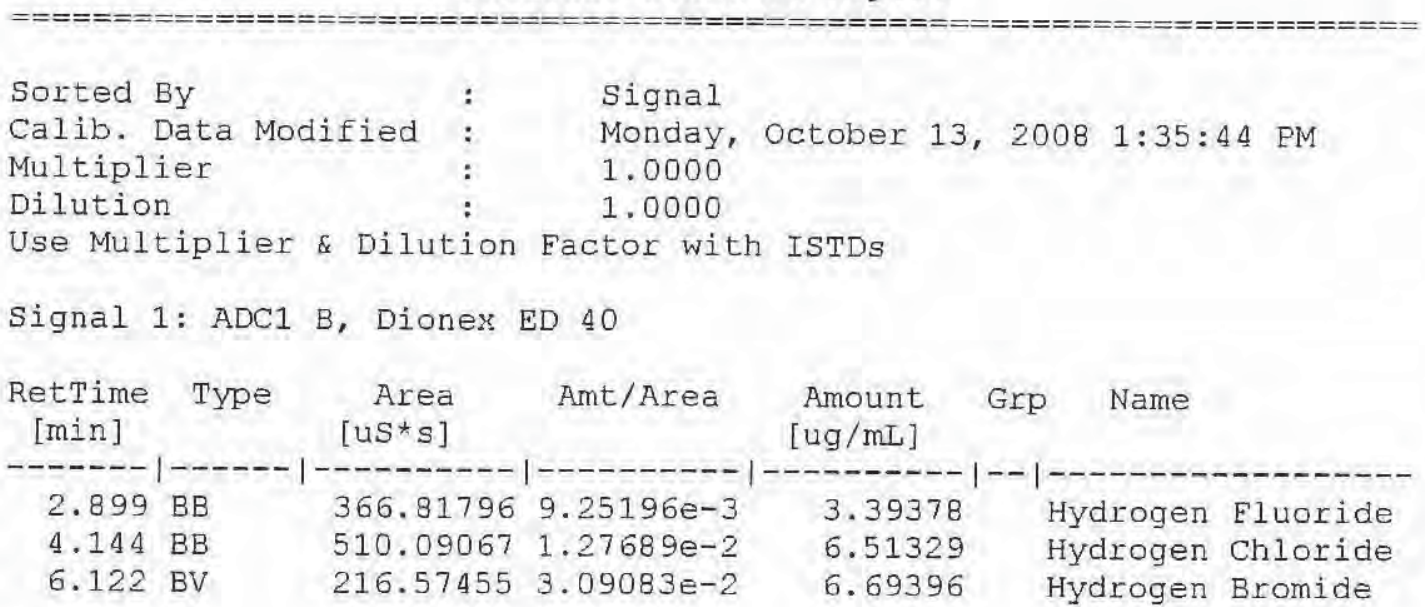

Totals :

16.60103

Results obtained with enhanced integrator!
} 


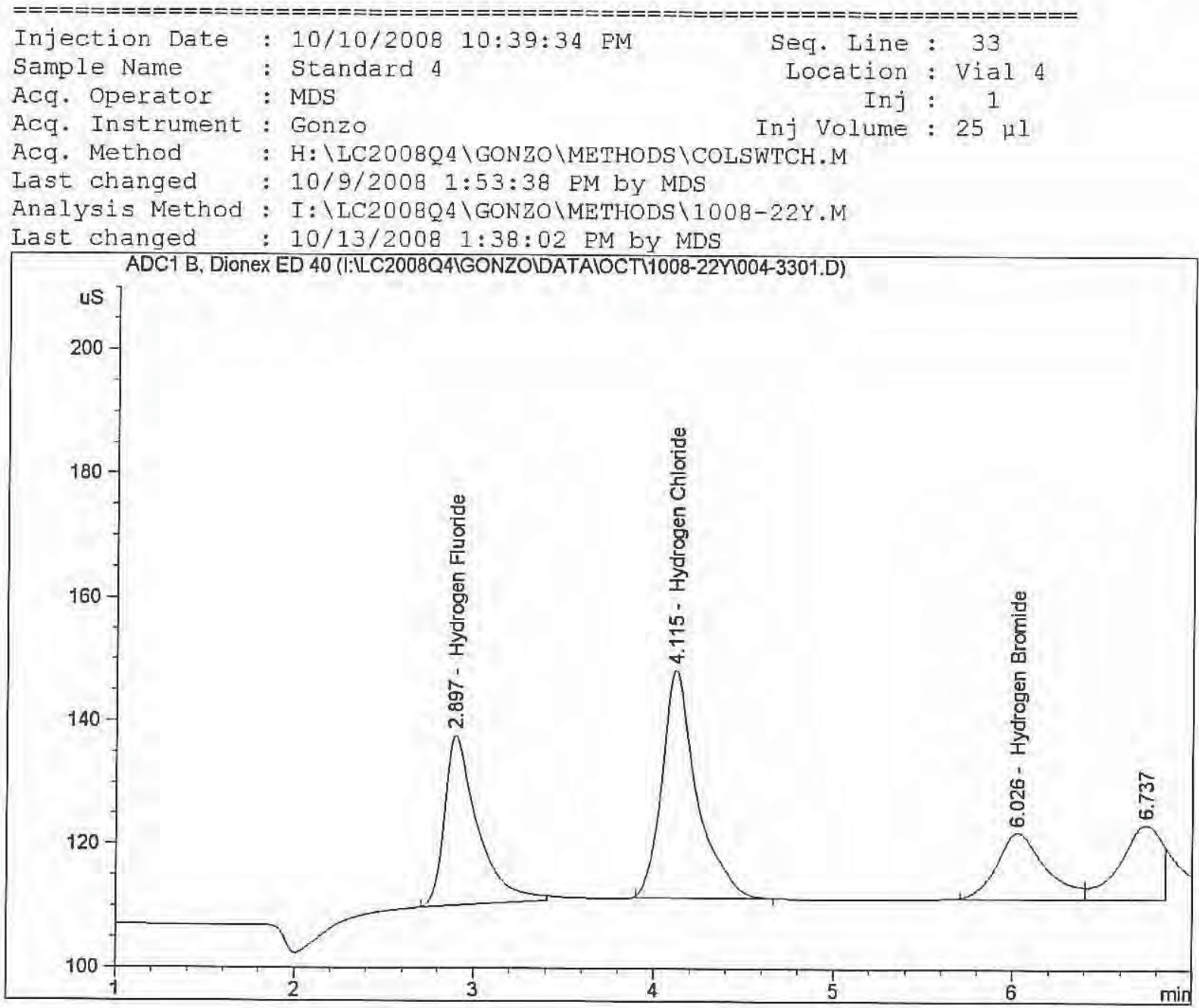

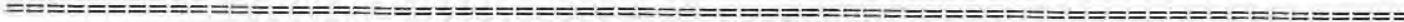

External standard Report

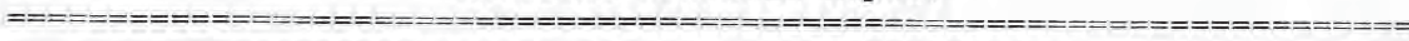

Sorted By : Signal

Calib. Data Modified : Monday, October 13, 2008 1:35:44 PM

Multiplier : 1.0000

Dilution : 1.0000

Use Multiplier \& Dilution Factor with ISTDS

Signal 1: ADC1 B, Dionex ED 40

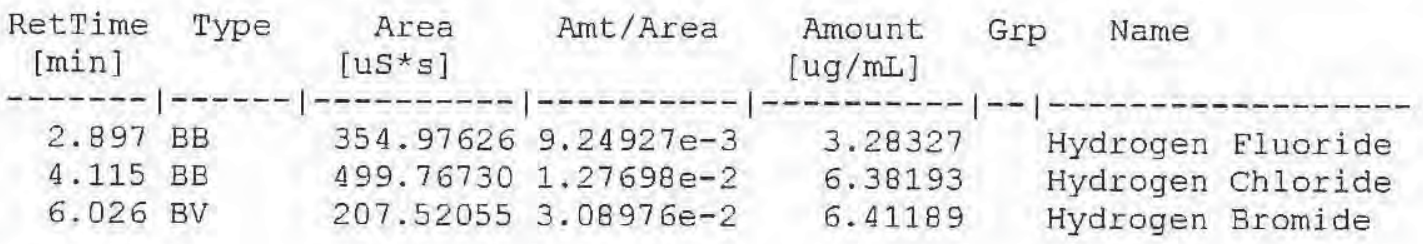

Totals :

16.07708

Results obtained with enhanced integrator!

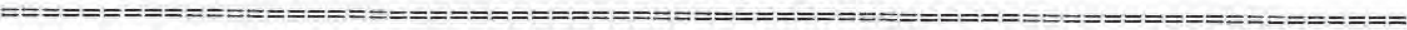
*** End of Report *** 


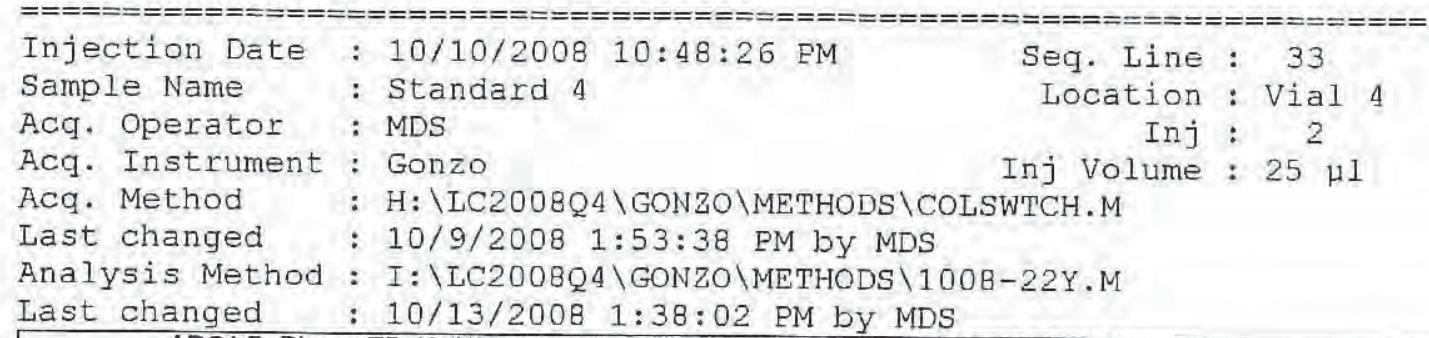

Results obtained with enhanced integrator! 


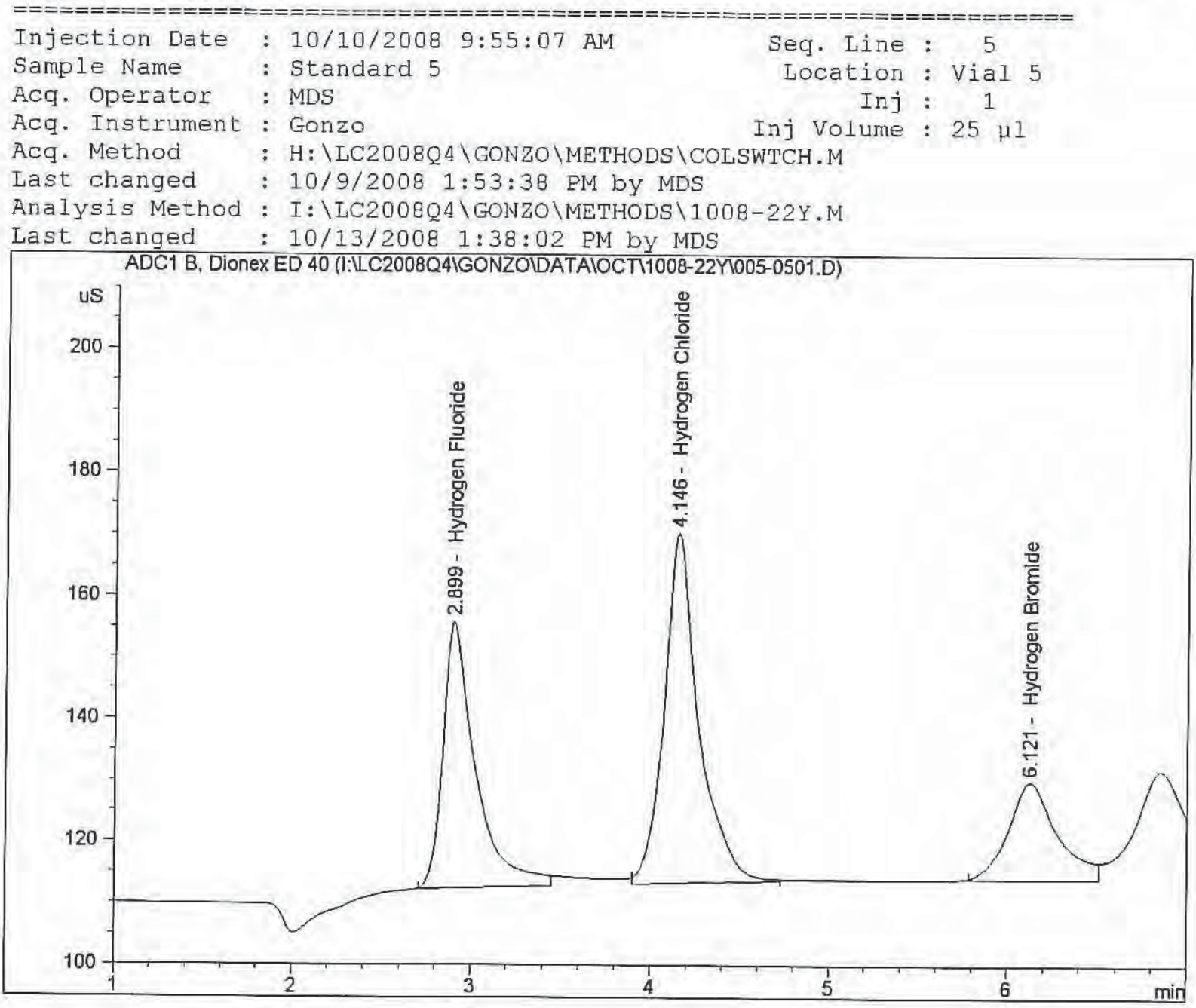

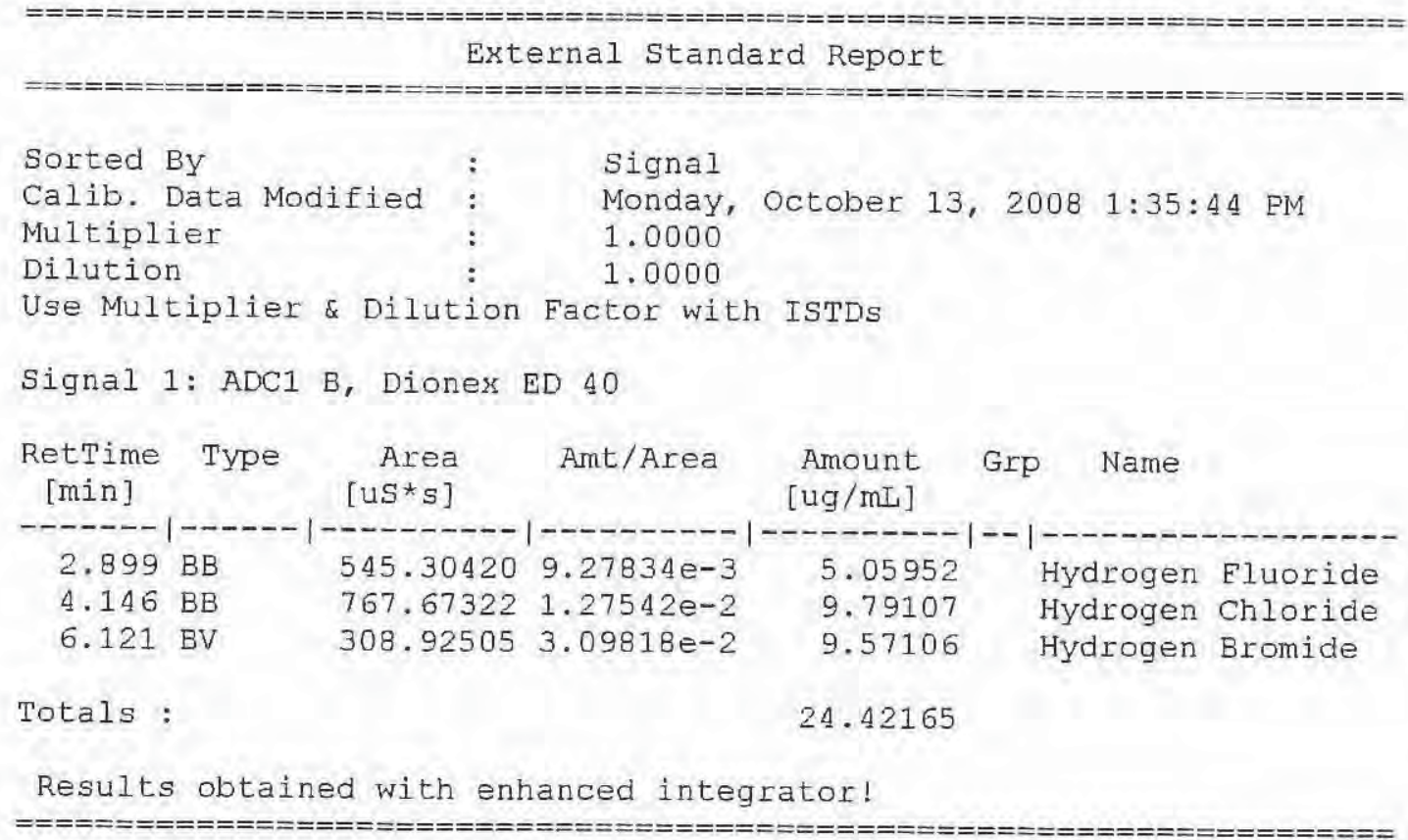

*** End of Report *** 


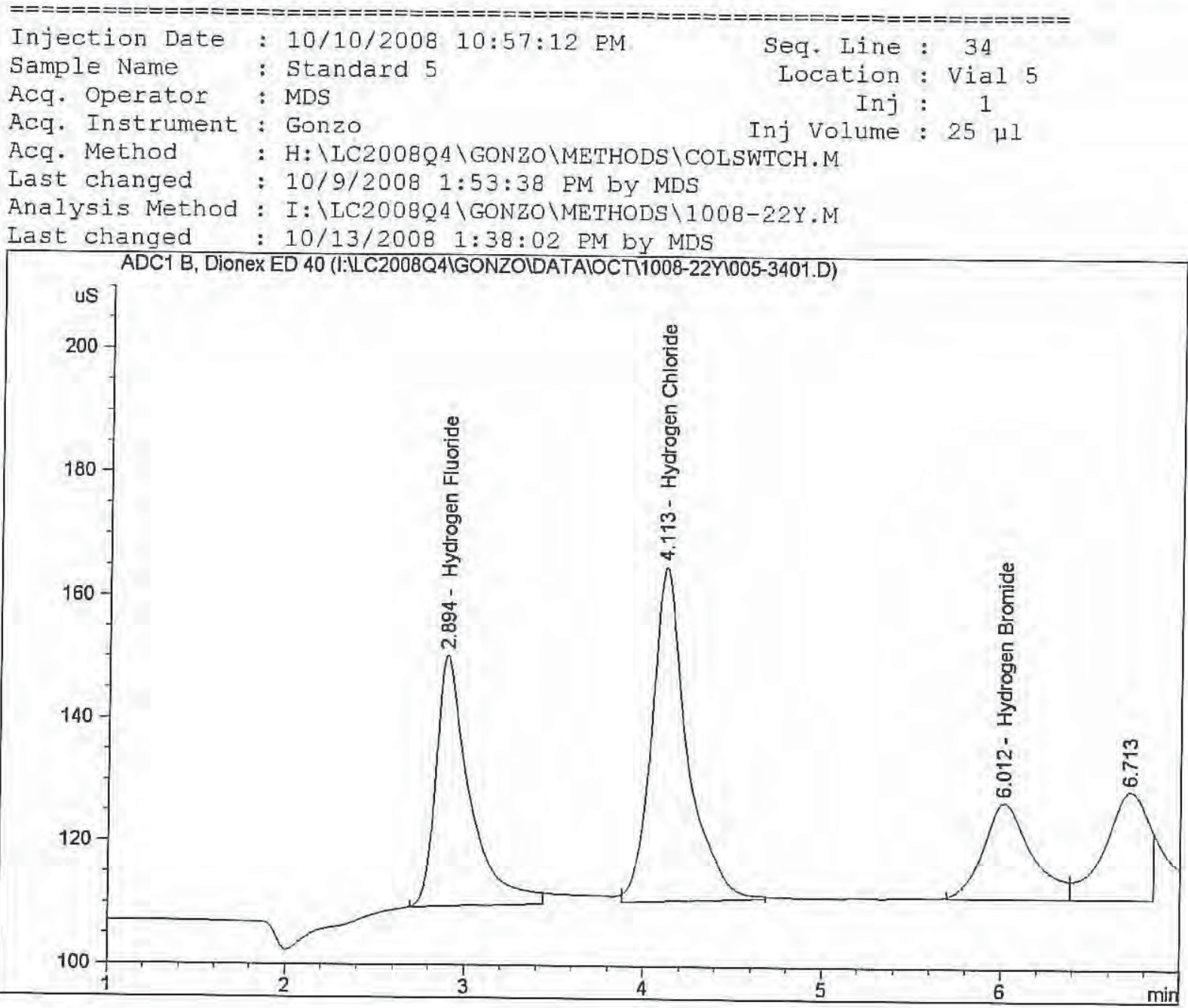

\footnotetext{
External standard Report

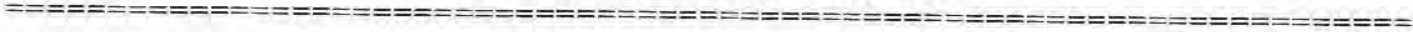

$\begin{array}{lll}\text { Sorted By } & \text { Signal } \\ \text { Calib. Data Modified : } & \text { Monday, October 13, 2008 1:35:44 PM } \\ \text { Multiplier } & : & 1.0000 \\ \text { Dilution } & 1.0000 \\ \text { Use Multiplier \& Dilution Factor with }\end{array}$

Use Multiplier \& Dilution Factor with ISTDs

Signal 1: ADC1 B, Dionex ED 40

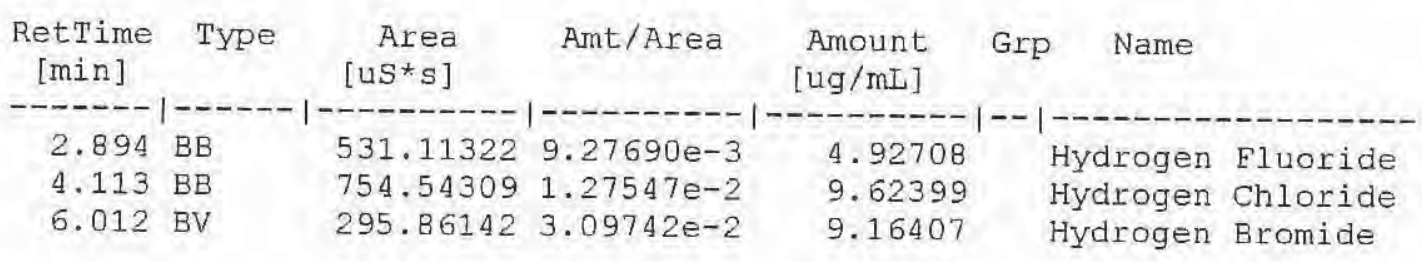

Totals :

$$
23.71514
$$

Results obtained with enhanced integrator! 


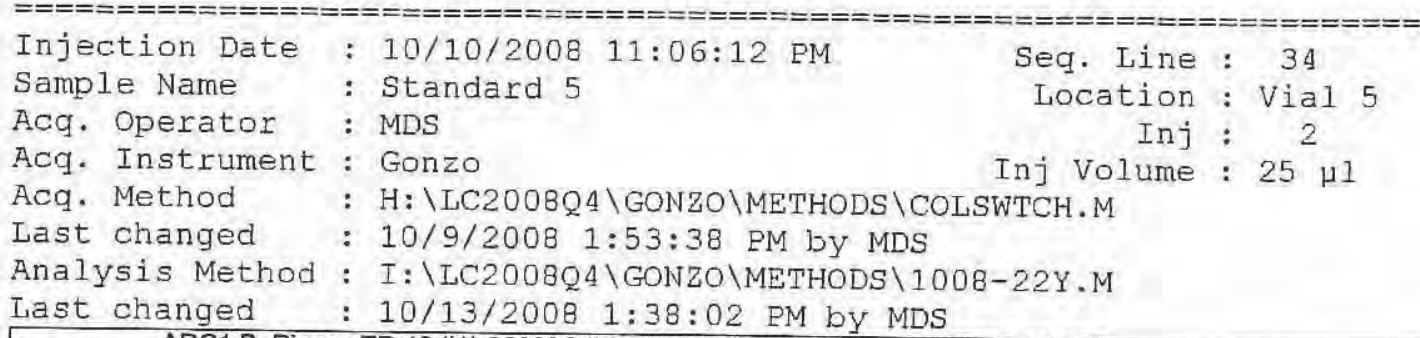

Results obtained with enhanced integrator! 


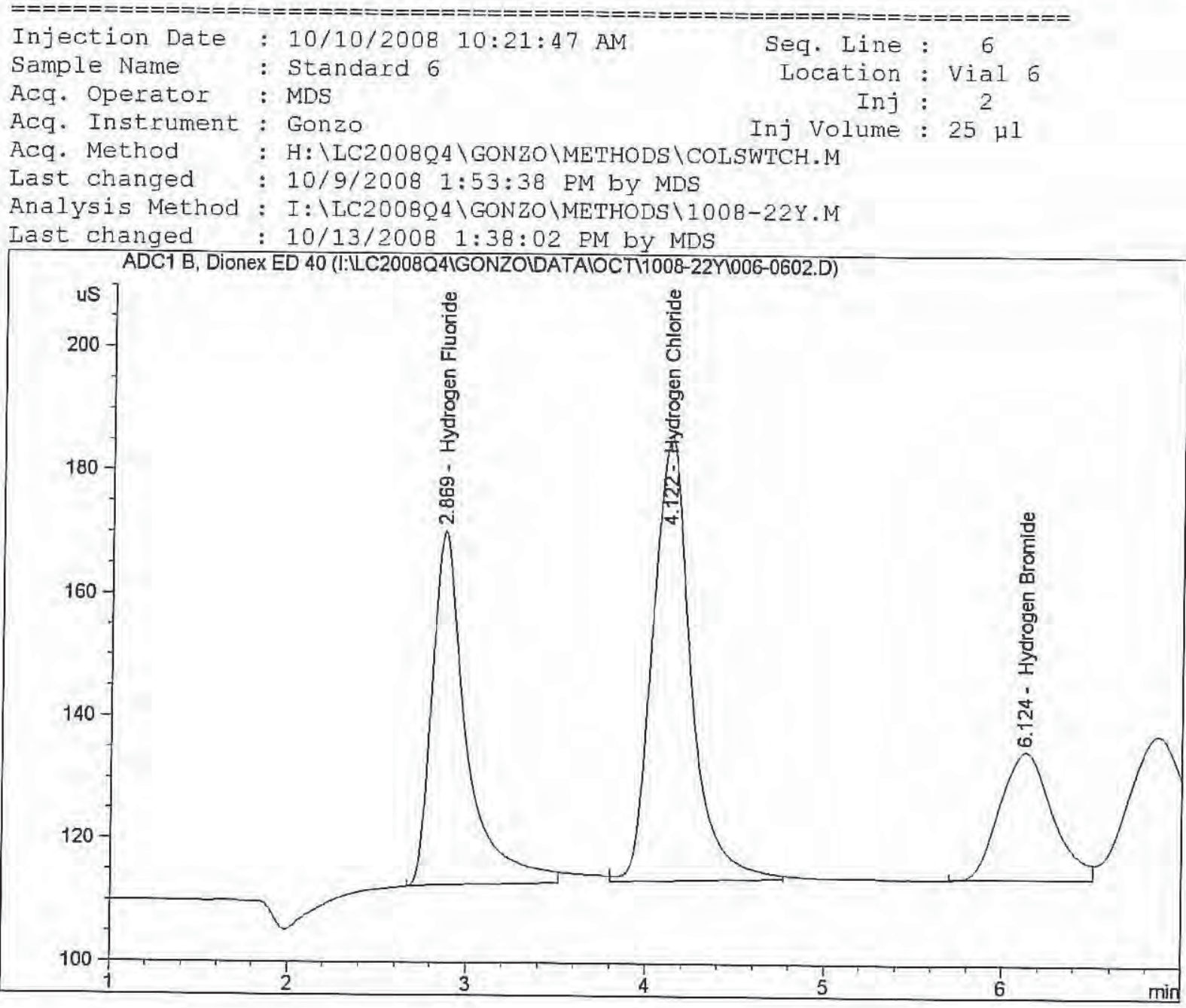

External Standard Report

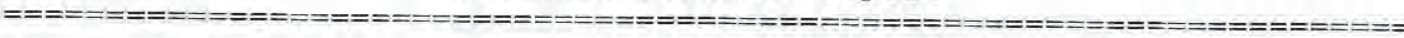

$\begin{array}{lll}\text { Sorted By } & : & \text { Signal } \\ \text { Calib. Data Modified } & : & \text { Monday, October 13, 2008 1:35:44 PM } \\ \text { Multiplier } & : & 1.0000 \\ \text { Dilution } & : & 1.0000\end{array}$

Use Multiplier \& Dilution Eactor with ISTDS

Signal 1: ADC1 B, Dioner ED 40

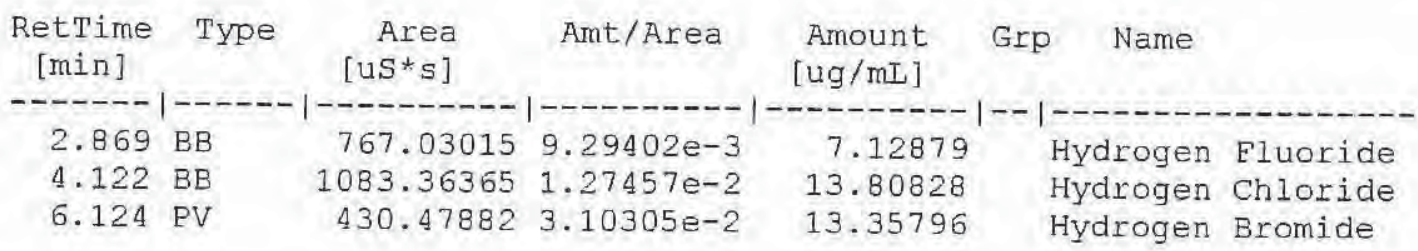

Totals :

34.29503

Results obtained with enhanced integrator!

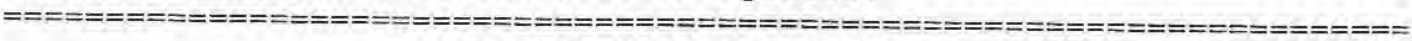
$\star * \star$ End of Report *** 


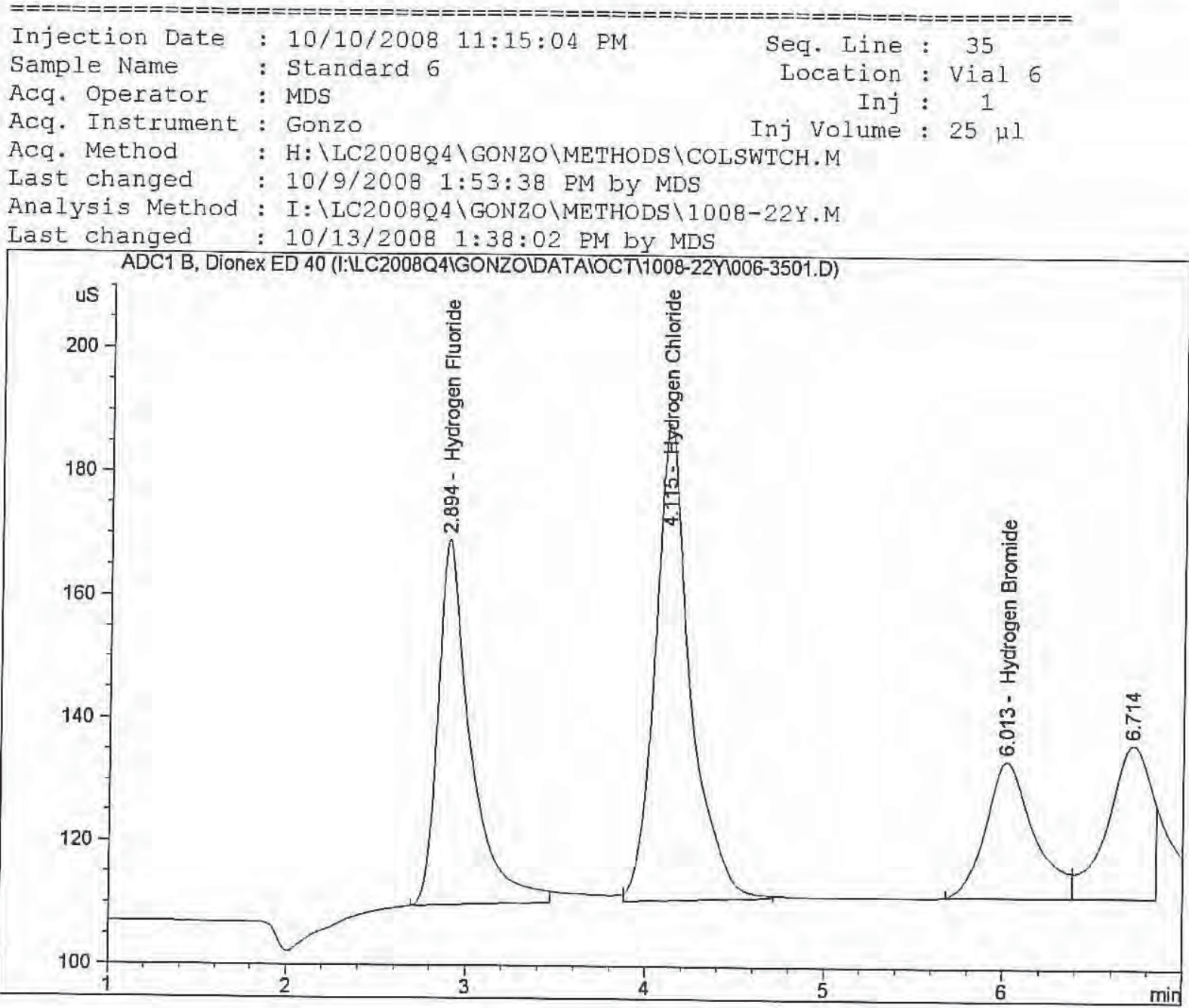

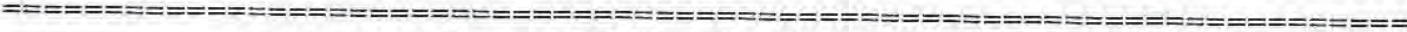
External Standard Report

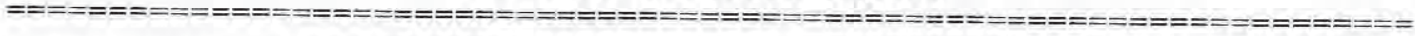

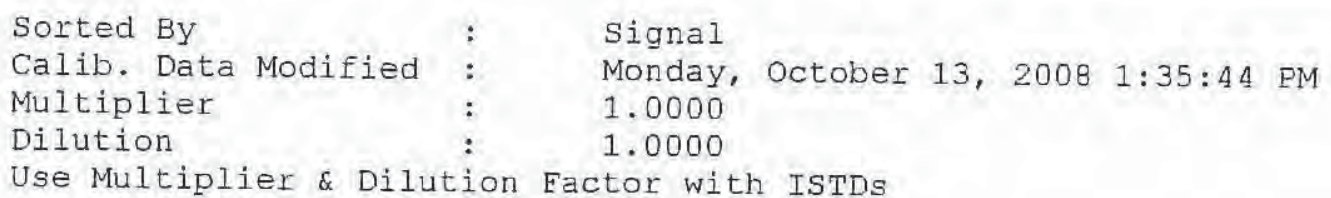

Use Multiplier \& Dilution Eactor with ISTDS

Signal 1: ADC1 B, Dionex ED 40

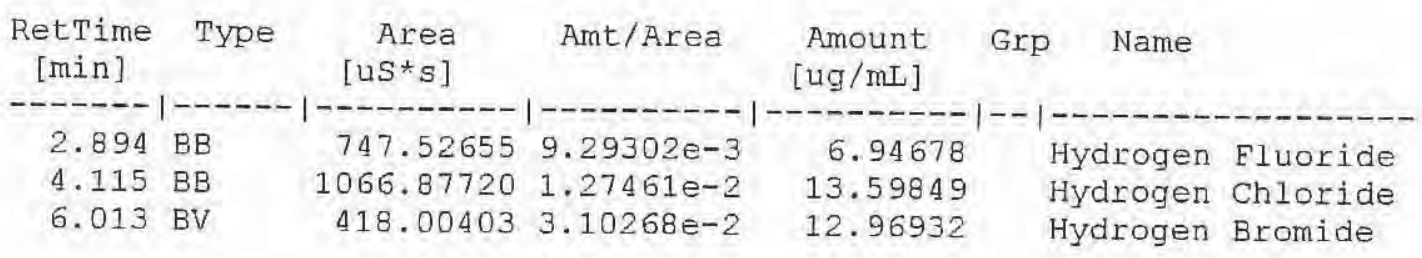

Totals :

33.51458

Results obtained with enhanced integrator!

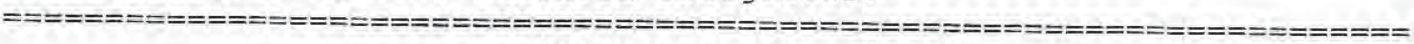

$\star * \star$ End of Report $* * *$
} 

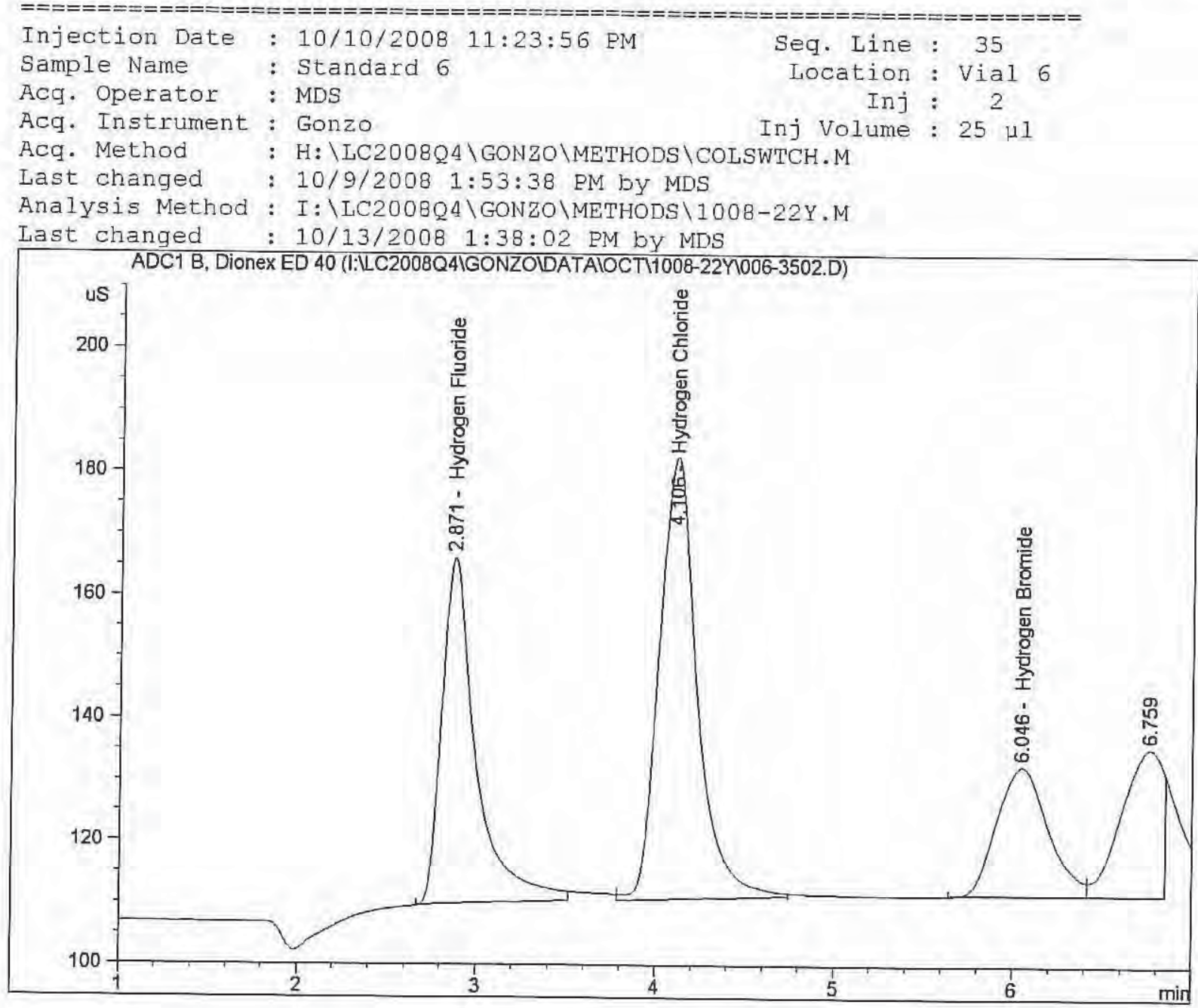

External Standard Report

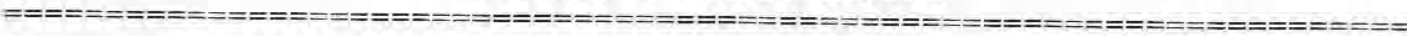

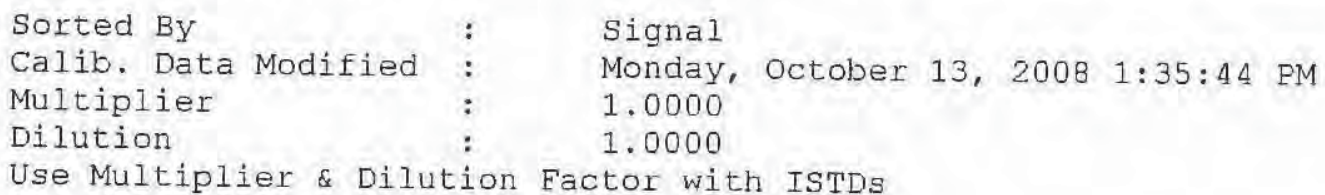

Use Multiplier \& Dilution Factor with ISTDs

Signal 1: ADC1 B, Dionex ED 40

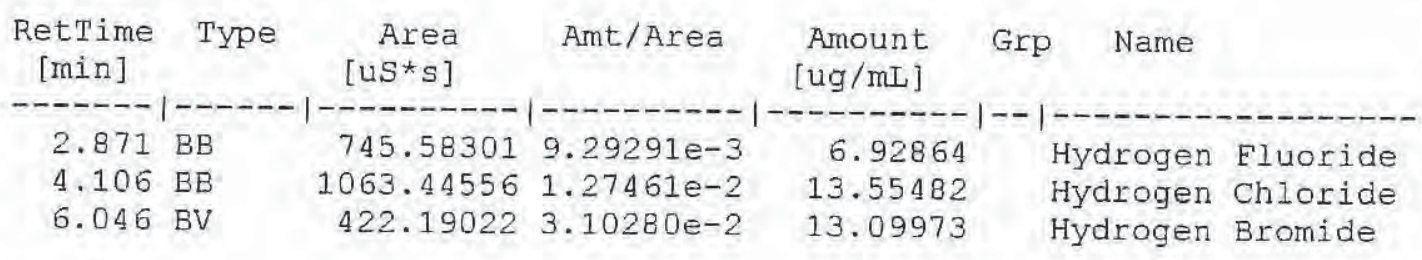

Totals :

33.58319

Results obtained with enhanced integrator! 


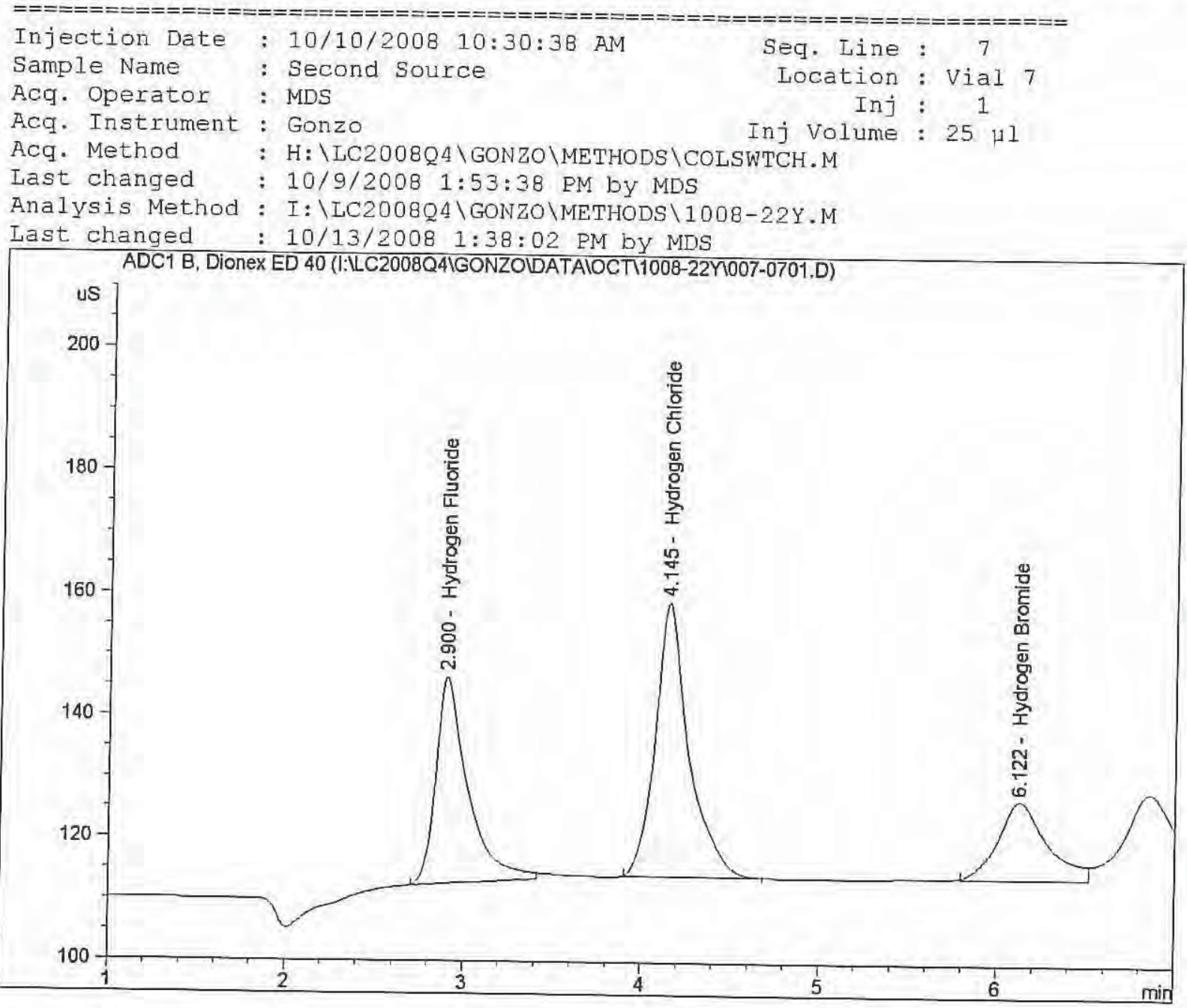

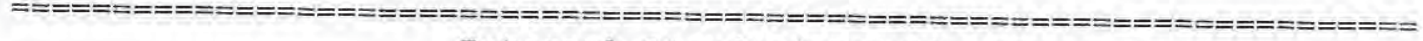
External Standard Report

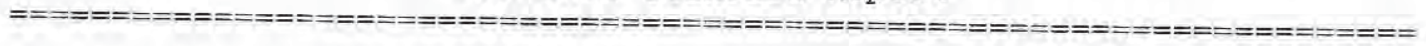

$\begin{array}{lcc}\text { Sorted By } & : & \text { Signal } \\ \text { Calib. Data Modified }: & \text { Monday, october 13, 2008 1:35:44 EM } \\ \text { Multiplier } & \vdots & 1.0000 \\ \text { Dilution } & 1.0000 \\ \text { Use Multiplier \& Dilution Factor with ISTDs }\end{array}$

Signal 1: ADC1 B, Dionex ED 40

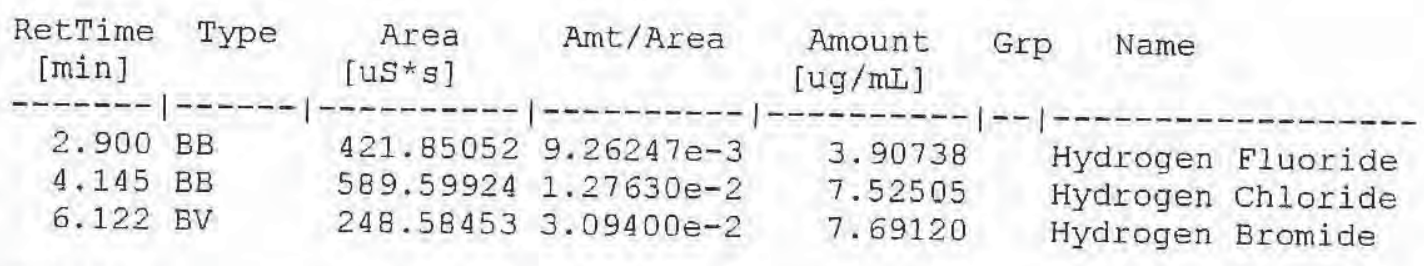

Totals :

19.12363

Results obtained with enhanced integrator!

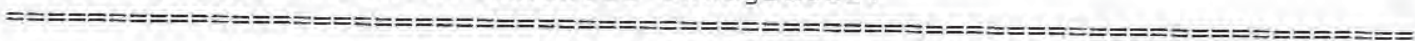

$* * t$ End of Report $* * *$
} 


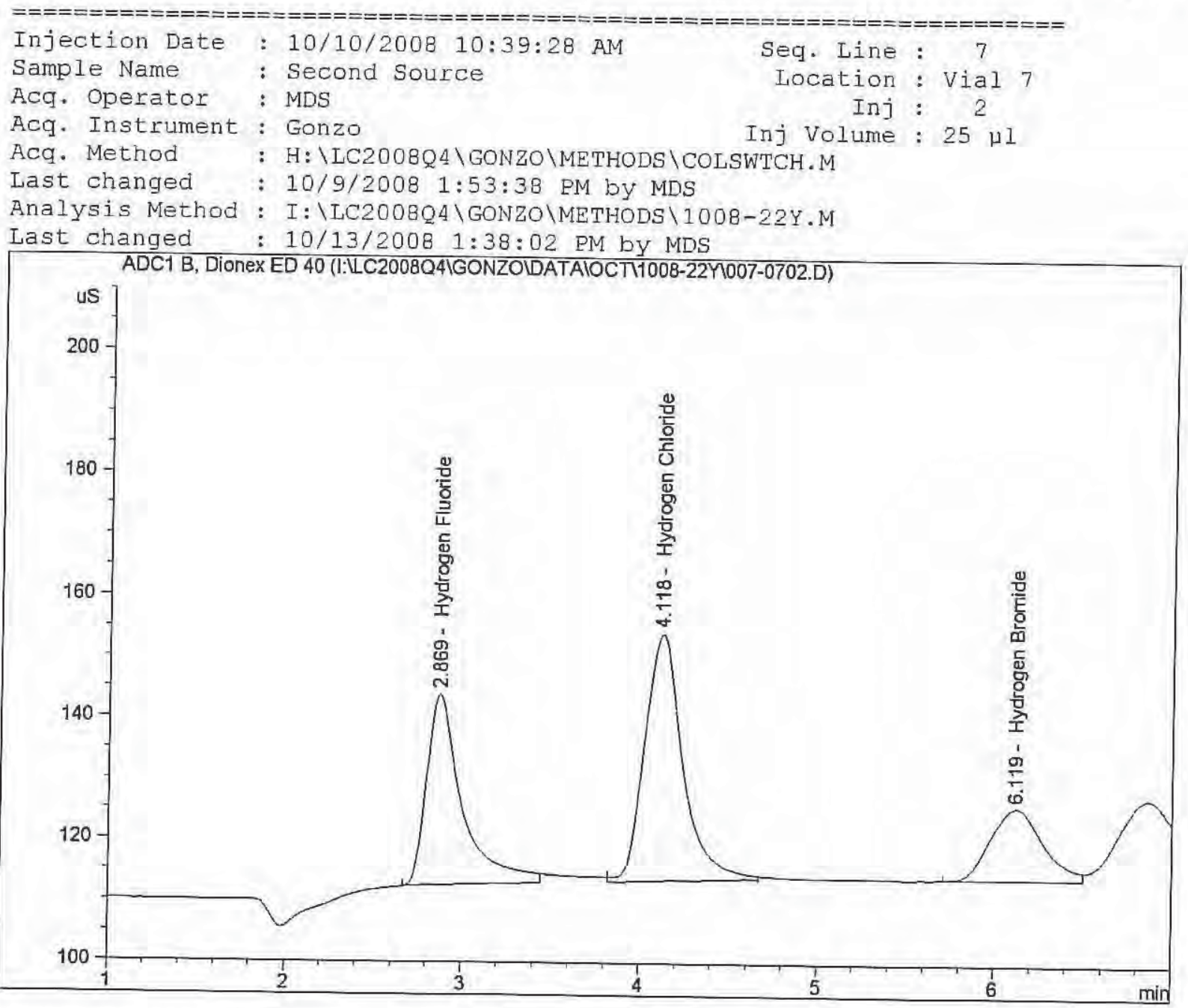

External standard Report

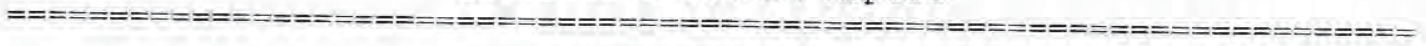

$\begin{array}{lcc}\text { Sorted By } & : & \text { Signal } \\ \text { Calib. Data Modified : } & \text { Monday, October 13, } 2008 \text { 1:35:44 PM } \\ \text { Multiplier } & : & 1.0000 \\ \text { Dilution } & \text { : } & 1.0000 \\ \text { Use Multiplier \& Dilution Factor with ISTDS }\end{array}$

Use Multiplier \& Dilution Factor with ISTDS

Signal 1: ADC1 B, Dionex ED 40

\begin{tabular}{ccccc}
$\begin{array}{c}\text { RetTime Type } \\
\text { [min] }\end{array}$ & $\begin{array}{c}\text { Area } \\
\text { [uS*S] }\end{array}$ & Amt/Area & $\begin{array}{c}\text { Amount } \\
\text { [ug/mL] }\end{array}$ & Grp Name \\
\hline $2.869 \mathrm{BB}$ & 436.20340 & $9.26478 \mathrm{e}-3$ & 4.04133 & Hydrogen Fluoride \\
$4.118 \mathrm{BB}$ & 610.33618 & $1.27617 \mathrm{e}-2$ & 7.78893 & Hydrogen Chloride \\
$6.119 \mathrm{BV}$ & 245.87801 & $3.09376 \mathrm{e}-2$ & 7.60688 & Hydrogen Bromide
\end{tabular}

Totals :

19.43714

Results obtained with enhanced integrator! 


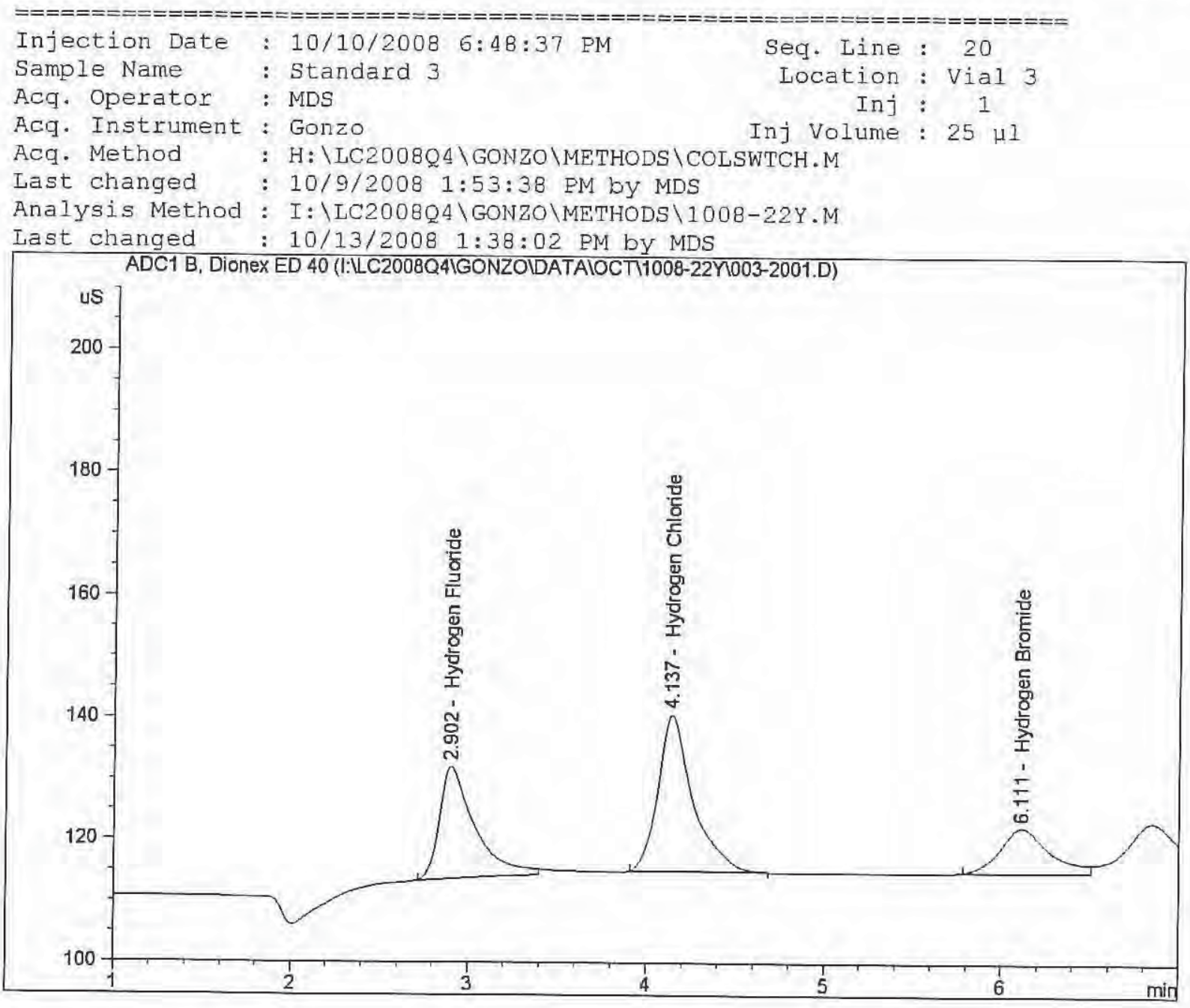

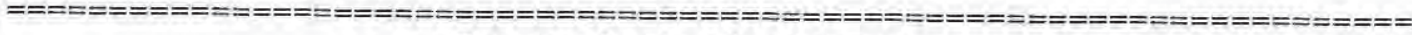
External standard Report

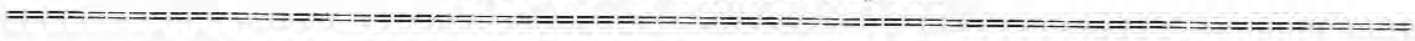

$\begin{array}{lcc}\text { Sorted By } & : & \text { Signal } \\ \text { Calib. Data Modified } & : & \text { Monday, October 13, } 2008 \text { 1:35:44 PM } \\ \text { Multiplier } & \vdots & 1.0000 \\ \text { Dilution } & \vdots & 1.0000 \\ \text { Use Multiplier \& Dilution Eactor with ISTDs }\end{array}$

Signal 1: ADC1 B, Dionex ED 40

\begin{tabular}{|c|c|c|c|c|c|}
\hline $\begin{array}{l}\text { RetTime } \\
\text { [min] }\end{array}$ & Type & $\begin{array}{r}\text { Area } \\
\text { [us* }{ }^{*} \text { ] }\end{array}$ & Ant/Area & $\begin{array}{l}\text { Amount } \\
{[\mathrm{ug} / \mathrm{mL}]}\end{array}$ & Name \\
\hline 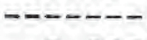 & & & & & \\
\hline 2.902 & $\mathrm{BB}$ & 251. & $9.21516 e-3$ & 2.32098 & gen Eluorid \\
\hline 4.13 & $\mathrm{BB}$ & 3641 & $1.27883 e-2$ & 4.51602 & Hydrogen Chloride \\
\hline 6.111 & BV & 146.92604 & $3.07918 e-2$ & 4.52412 & Hydrogen Bromide \\
\hline
\end{tabular}

Totals :

11.36112

Results obtained with enhanced integrator!

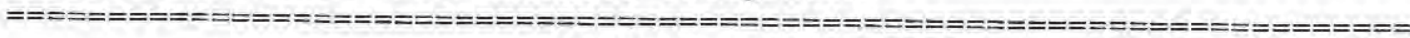
*** End of Report ***
} 

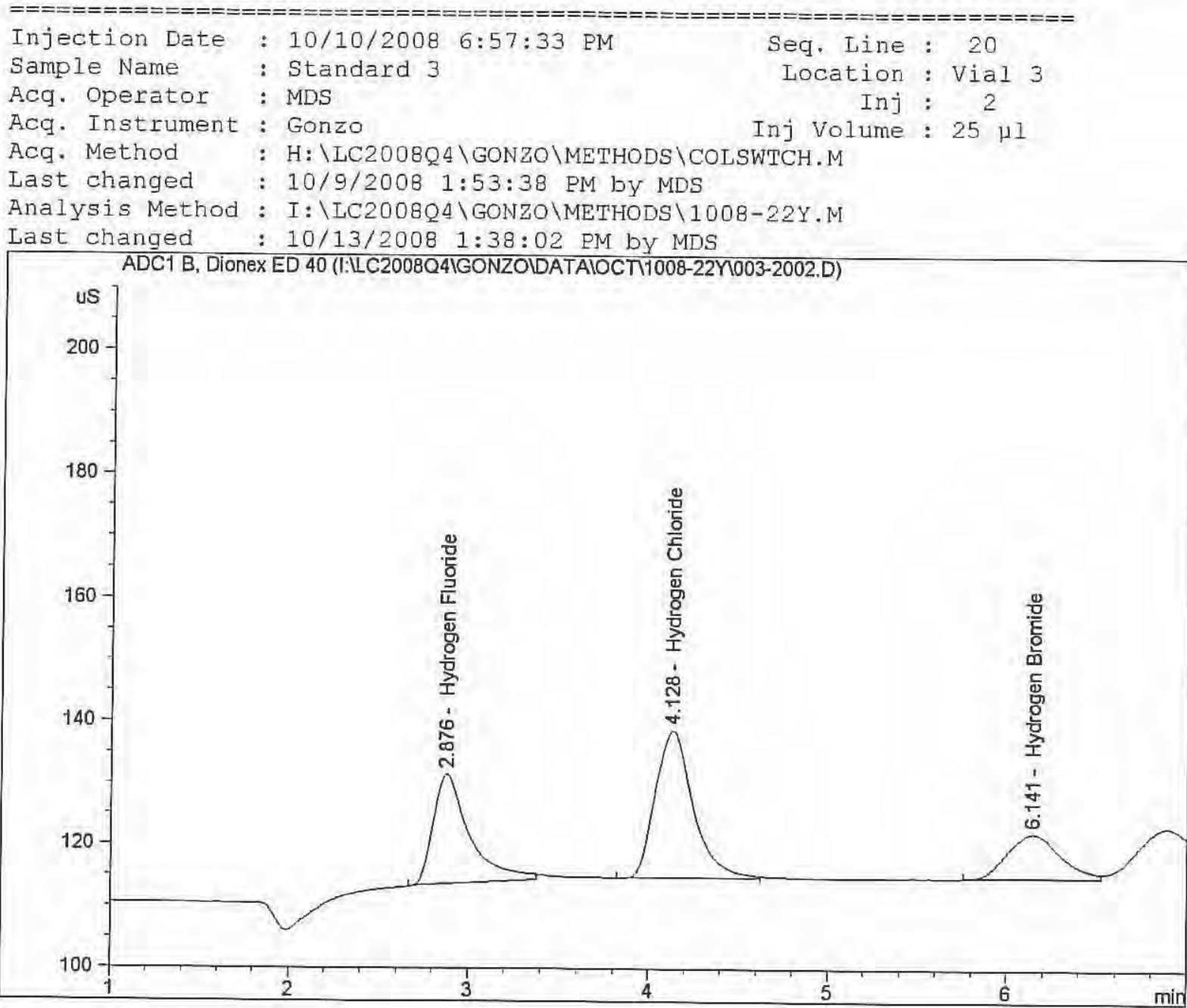

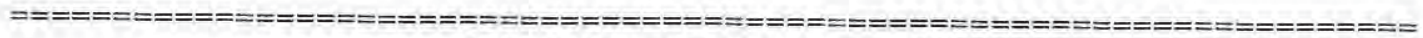

External Standard Report

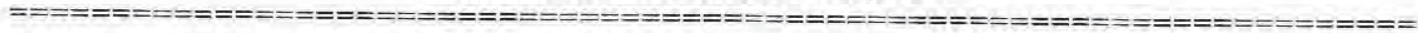

Sorted By

Signal

Calib. Data Modified : Monday, October 13, 2008 1:35:44 PM

Multiplier

Dilution

1.0000

1.0000

Use Multiplier \& Dilution Eactor with ISTDs

Signal 1: ADC1 B, Dionex ED 40

\begin{tabular}{lllll}
$\begin{array}{c}\text { RetTime Type } \\
\text { [min] }\end{array}$ & $\begin{array}{c}\text { Area } \\
{[\text { uS*s] }}\end{array}$ & Ant/Area & $\begin{array}{c}\text { Amount } \\
\text { [ug/mL] }\end{array}$ & Grp Name \\
\hline 2.876 BB & 249.48161 & $9.21404 \mathrm{e}-3$ & 2.29873 & Hydrogen Fluoride \\
$4.128 \mathrm{~PB}$ & 350.58881 & $1.27888 \mathrm{e}-2$ & 4.48361 & Hydrogen Chloride \\
$6.141 \mathrm{BV}$ & 149.40140 & $3.07978 \mathrm{e}-2$ & 4.60124 & Hydrogen Bromide
\end{tabular}

Totals :

11.38357

Results obtained with enhanced integrator!

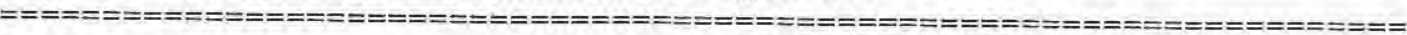

$\star * \star$ End of Report $* \star \star$ 


\section{Method Information}

Dionex IonPac AS-14A 4-mm analytical (4x250mm) column.

Flow rate $1.2 \mathrm{~mL} / \mathrm{min}$

$8 \mathrm{mM} \mathrm{Na} 2 \mathrm{CO} 3 / \mathrm{ImM} \mathrm{NaHCO} 3$ mobile phase

Suppressed anion conductivity detection

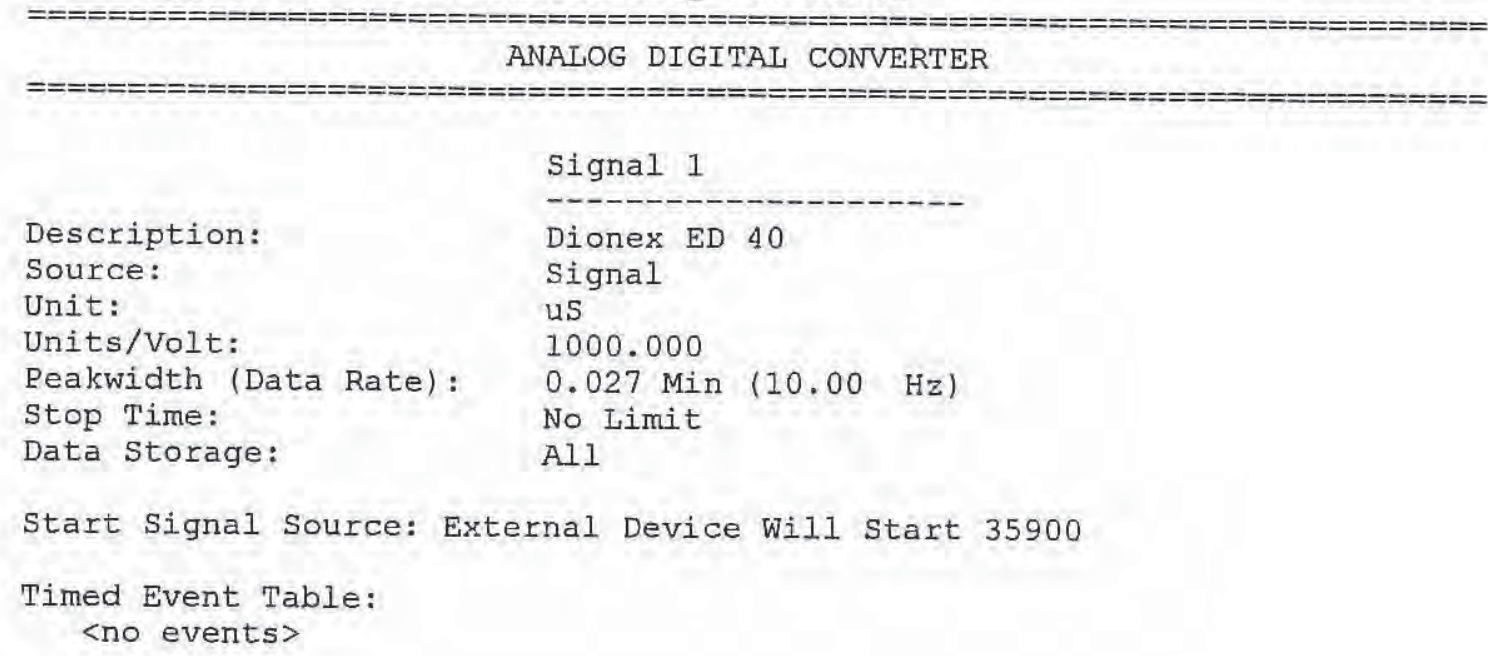




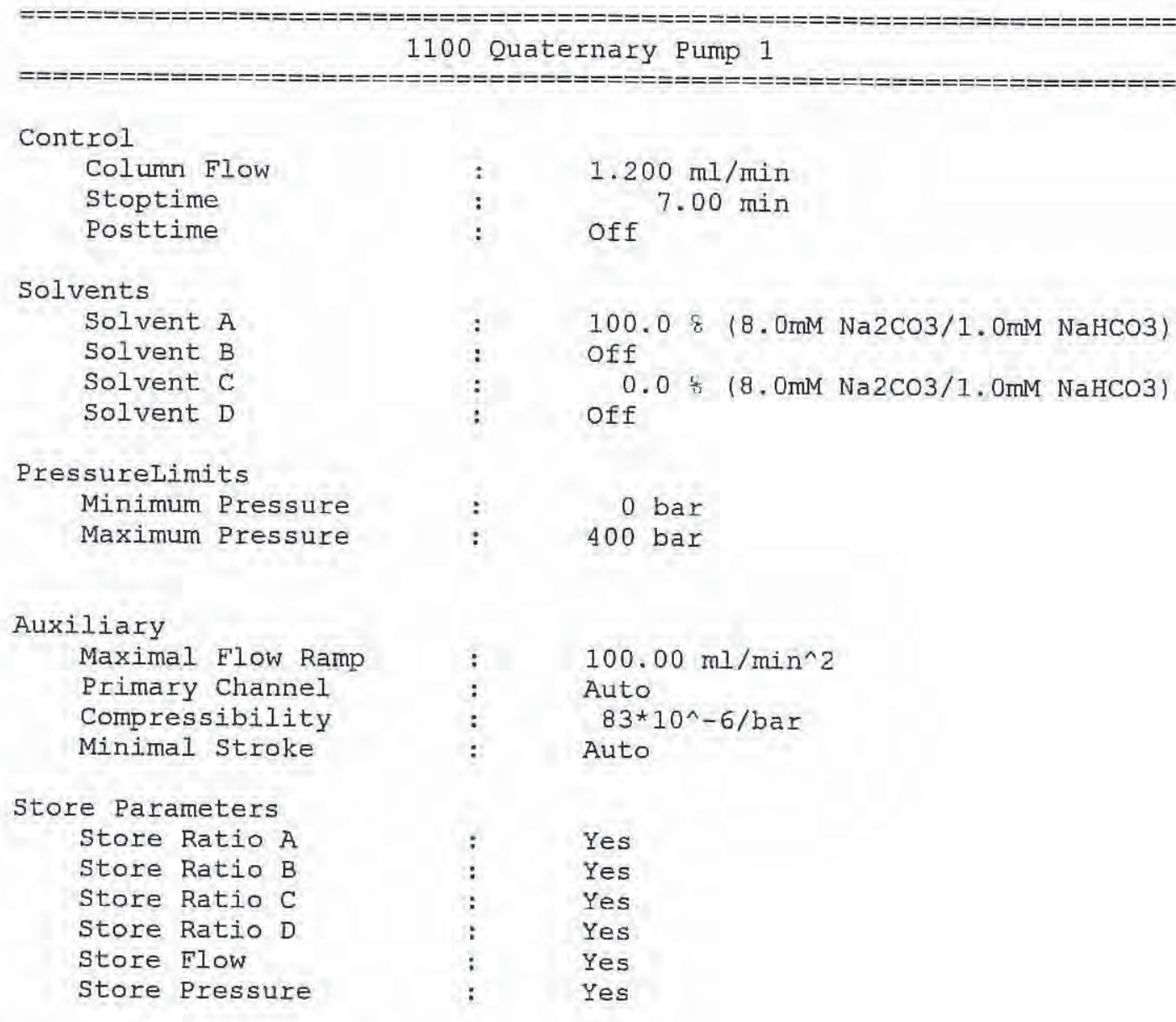

Agilent 1100 Contacts Option

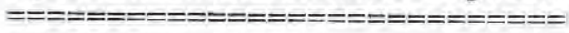

Contact 1

Contact 2

Contact 3

Contact 4

Open

Open

Open

Open

1100 Quaternary Pump 2

Control

Column Elow

Stoptime

Posttime

Solvents

Solvent $A$

Solvent $B$

Solvent $C$

Solvent $D$

Pressurelimits

Minimum Pressure

Maximum Pressure

Auxiliary

Maximal Flow Ramp

Primary Channel

$$
\begin{aligned}
& \begin{array}{l}
1.200 \mathrm{ml} / \mathrm{min} \\
7.00 \mathrm{~min}
\end{array} \\
& \text { off }
\end{aligned}
$$

: Off

$\begin{array}{ll}: & 0.0 \div(1) \\ : & \text { off } \\ : & \text { off } \\ & 100.0 \div \quad(8 \mathrm{mMNa} 2 \mathrm{CO} 3 \backslash 1 \mathrm{mMNaHCO})\end{array}$

: 0 bar

: 400 bar 
Method: H: \LC2008Q4\GONZO\METHODS\COLSWTCH.M of 10/9/2008 1:53:38 PM

Compressibility

Minimal Stroke

Store Parameters

Store Ratio A

Store Ratio B

Store Ratio C

Store Ratio D

Store Flow

Store Pressure
$100 * 10^{\wedge}-6 /$ bar

Auto

Yes

Yes

Yes

Yes

Yes

Yes

Agilent 1100 Contacts Option

$========================$

Contact 1

Contact 2

Contact 3

Contact 4

$\begin{array}{ll}: & \text { Open } \\ : & \text { Open } \\ : & \text { Open } \\ : & \text { Open }\end{array}$

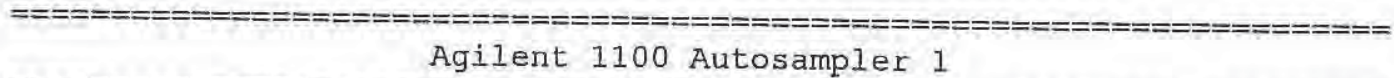

Injection

Injection Mode

Injector volume

Wash Vial

Optimization

Needle Wash

$25.00 \mu 1$

100

none

Auxiliary

Drawspeed

Ejectspeed

Draw position

$100 \mu 1 / \mathrm{min}$

$1000 \mathrm{\mu l} / \mathrm{min}$

$2.0 \mathrm{~mm}$

Time

Stoptime : : As Pump

Postime : Off

\section{Agilent 1100 Column Thermostat 1}

Temperature settings

Left temperature

Right temperature

Enable analysis

Store left temperature :

Store right temperature:

Time

Stoptime

Posttime

Column Switching Valve $30.0^{\circ} \mathrm{C}$

Same as left

When Temp. is within setpoint $+1-0.8^{\circ} \mathrm{C}$

No

No

As pump

Off

Use current 
Sequence Table:

Method and Injection Info Part:

\begin{tabular}{|c|c|c|c|c|c|c|c|c|}
\hline $\begin{array}{l}\text { Iine } \\
====\end{array}$ & $\begin{array}{l}\text { Locat } \\
=====\end{array}$ & $\begin{array}{l}\text { Eion } \\
====\end{array}$ & $\begin{array}{l}\text { SampleName } \\
\text { =========-===== }\end{array}$ & $\begin{array}{l}\text { Method } \\
=======\end{array}$ & $\begin{array}{l}\text { Inj } \\
===\end{array}$ & $\begin{array}{l}\text { SampleType I } \\
=========\end{array}$ & $\begin{array}{l}\text { InjVolume } \\
=======\end{array}$ & $\begin{array}{l}\text { DataFile } \\
=========\end{array}$ \\
\hline 1 & Vial & 1 & Standard 1 & COLSWTCH & 2 & Sample & & \\
\hline 2 & Vial & 2 & standard 2 & COLSWTCH & 2 & Sample & & \\
\hline 3 & Vial & 3 & Standard 3 & COLSWTCH & 2 & Sample & & \\
\hline 4 & Vial & 4 & Standard 4 & COLSWTCH & 2 & Sample & & \\
\hline 5 & Vial & 5 & Standard 5 & COLSWTCH & 2 & Sample & & \\
\hline 6 & Vial & 6 & Standard 6 & COLSWTCH & 2 & Sample & & \\
\hline 7 & Vial & 7 & Second Source & COLSWTCH & 2 & Sample & & \\
\hline 8 & Vial & 8 & H2SO4 Lab Blank & COLSWTCH & 2 & Sample & & \\
\hline 9 & Vial & 9 & DI H20 Lab Blank & COLSWTCH & 2 & Sample & & \\
\hline 10 & Vial & 11 & In $\mathrm{R} 1 \mathrm{C} 3 * 1.0009$ & COLSWTCH & 2 & Sample & & \\
\hline 11 & Vial & 12 & In $\mathrm{R} 2 \mathrm{C} 33^{*} 1.0397$ & COLSWTCH & 2 & Sample & & \\
\hline 12 & Vial & 13 & In $\mathrm{R} 3 \mathrm{C} 3 * 1.0577$ & COLSWTCH & 2 & Sample & & \\
\hline 13 & Vial & 14 & Stk R1 C $3 * 1.0126$ & COLSWTCH & 2 & Sample & & \\
\hline 14 & Vial & 15 & Stk R2 C $3 * 1.038$ & COLSWTCH & 2 & Sample & & \\
\hline 15 & Vial & 16 & stk R3 C3*1.0471 & COLSWTCH & 2 & Sample & & \\
\hline 16 & Vial. & 17 & In $\mathrm{C} 6 * 1.061$ & COLSWTCH & 2 & Sample & & \\
\hline 17 & Vial & 18 & Stk $C 6 * 1.0399$ & COLSWTCH & 2 & Sample & & \\
\hline 18 & Vial & 19 & $\mathrm{MS} / \mathrm{In} \mathrm{R} 1 \mathrm{C} 3$ & COLSWTCH & 2 & Sample & & \\
\hline 19 & Vial & 20 & $T-1 * 1.1245$ & COLSWTCH & 2 & Sample & & \\
\hline 20 & Vial & 3 & Standard 3 & COLSWTCH & 2 & Sample & & \\
\hline 21 & Vial & 4 & Standard $A$ & COLSWTCH & 2 & Sample & & \\
\hline 22 & Vial & 21 & $\mathrm{~T}-2 \star 1.1288$ & COLSWTCH & 2 & Sample & & \\
\hline 23 & Vial & 22 & $\mathrm{~T}-3 * 1.1384$ & COLSWTCH & 2 & Sample & & \\
\hline 24 & Vial & 23 & $T-4 * 1.1461$ & COLSWTCH & 2 & Sample & & \\
\hline 25 & Vial & 24 & $\mathrm{MS} / \mathrm{T}-1$ & COLSWTCH & 2 & Sample & & \\
\hline 26 & Vial & 25 & $\mathrm{Cl}-\mathrm{A} 25$ & COLSWTCH & 2 & Sample & & \\
\hline 27 & Vial & 26 & $\mathrm{Cl}-\mathrm{A} 26$ & COLSWTCH & 2 & Sample & & \\
\hline 28 & Vial & 27 & $\mathrm{Cl}-\mathrm{A}_{2} 2$ & COLSWTCH & 2 & Sample & & \\
\hline 29 & Vial & 28 & $\mathrm{Cl}-\mathrm{A} 28$ & COLSWTCH & 2 & Sample & & \\
\hline 30 & Vial & 1 & Standard 1 & COLSWTCH & 2 & Sample & & \\
\hline 31 & Vial & 2 & Standard 2 & COLSWTCH & 2 & Sample & & \\
\hline 32 & Vial & 3 & Standard 3 & COLSWTCH & 2 & Sample & & \\
\hline 33 & Vial & 4 & Standard 4 & COLSWTCH & 2 & Sample & & \\
\hline 34 & Vial & 5 & Standard 5 & COLSWTCH & 2 & Sample & & \\
\hline 35 & Vial & 6 & Standard 6 & COLSWTCH & 2 & Sample & & \\
\hline 36 & Vial & 1 & Standard 1 & COLSWTCH & 2 & Sample & & \\
\hline 37 & Vial & 2 & Standard 2 & COLSWTCH & 2 & Sample & & \\
\hline 38 & Vial & 3 & Standard 3 & COLSWTCH & 2 & Sample & & \\
\hline 39 & Vial & 4 & Standard 4 & COLSWTCH & 2 & Sample & & \\
\hline 40 & Vial & 5 & Standard 5 & COLSWTCH & 2 & Sample & & \\
\hline 41 & Vial & 6 & Standard 6 & COLSWTCH & 2 & Sample & & \\
\hline 42 & Vial & 7 & Second Source & COLSWTCH & 2 & Sample & & \\
\hline 43 & Vial & 8 & $\mathrm{NaOH}$ Lab Blank & COLSWTCH & 2 & Sample & & \\
\hline 44 & Vial & 9 & DI H2O Lab Blank & COLSWTCH & 2 & Sample & & \\
\hline 45 & Vial & 31 & In $\mathrm{R} 1 \mathrm{C} 4 * 4.018$ & CHLSWTCH & 2 & Sample & & \\
\hline 46 & Vial & 32 & In $\mathrm{R} 2 \mathrm{C} 4 * 4.018$ & CHLSWTCH & 2 & Sample & & \\
\hline 47 & Vial & 33 & In $\mathrm{R} 3 \mathrm{C} 4 * 4.018$ & CHLSWTCH & 2 & Sample & & \\
\hline 48 & Vial & 34 & Stk R1 $C 4 * 4.01$ & CHLSWTCH & 2 & Sample & & \\
\hline 49 & Vial & 35 & Stk R2 $C 4 * 4.01$ & CHLSWTCH & 2 & Sample & & \\
\hline 50 & Vial & 36 & Stack R3 C4*4.01 & CHLSWTCH & 2 & Sample & & \\
\hline 51 & Vial & 37 & In $C 7 \star 4.1$ & CHLSWTCH & 2 & Sample & & \\
\hline 52 & Vial & 38 & Stk $C 7 * 4.1$ & CHLSWTCH & 2 & Sample & & \\
\hline 53 & Vial & 39 & $\mathrm{MS} / \mathrm{In} \mathrm{R} 1 \mathrm{C} 4$ & CHLSWTCH & 2 & Sample & & \\
\hline 54 & Vial & 1 & Standard 1 & COLSWTCH & 2 & Sample & & \\
\hline 55 & Vial & 2 & Standard 2 & COLSWTCH & 2 & Sample & & \\
\hline 56 & Vial & 3 & Standard 3 & COLSWTCH & 2 & Sample & & \\
\hline 57 & Vial & 4 & Standard 4 & COLSWTCH & 2 & Sample & & \\
\hline 58 & Vial & 5 & Standard 5 & COLSWTCH & 2 & Sample & & \\
\hline 59 & Vial & 6 & Standard 6 & COLSWTCH & 2 & Sample & & \\
\hline 60 & Vial & 9 & DI H2O Lab Blank & SHUTDOW1PO & 22 & Psgentiole 267 & & \\
\hline
\end{tabular}




\section{Sample Chromatograms}

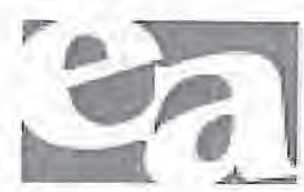




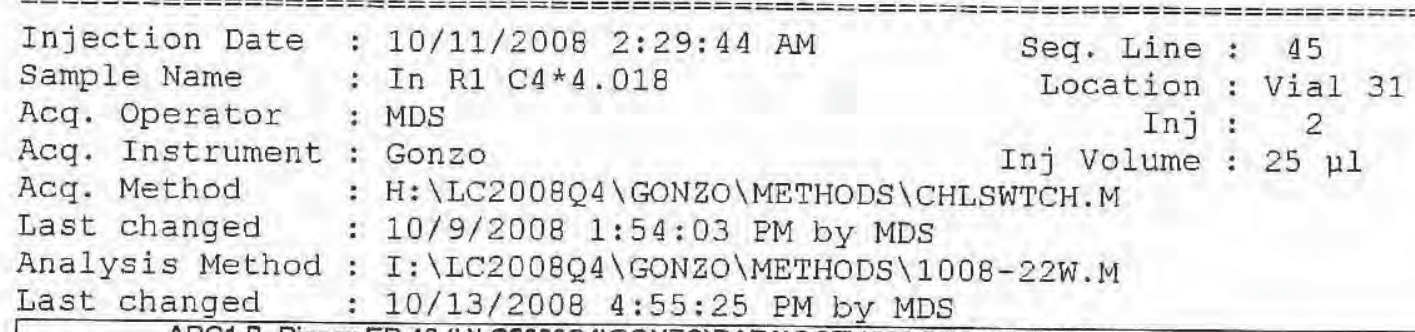

: Gonzo Inj Volume : $25 \mu l$

: H: \LC2008Q4 \GONZO\METHODS \CHLSWTCH.M

Analysis Method : I: \LC2008Q4 \GONZO\METHODS $\backslash 1008-22$ W.M

\section{Seq. Line : 45}

Location : Vial 31

Inj : 2

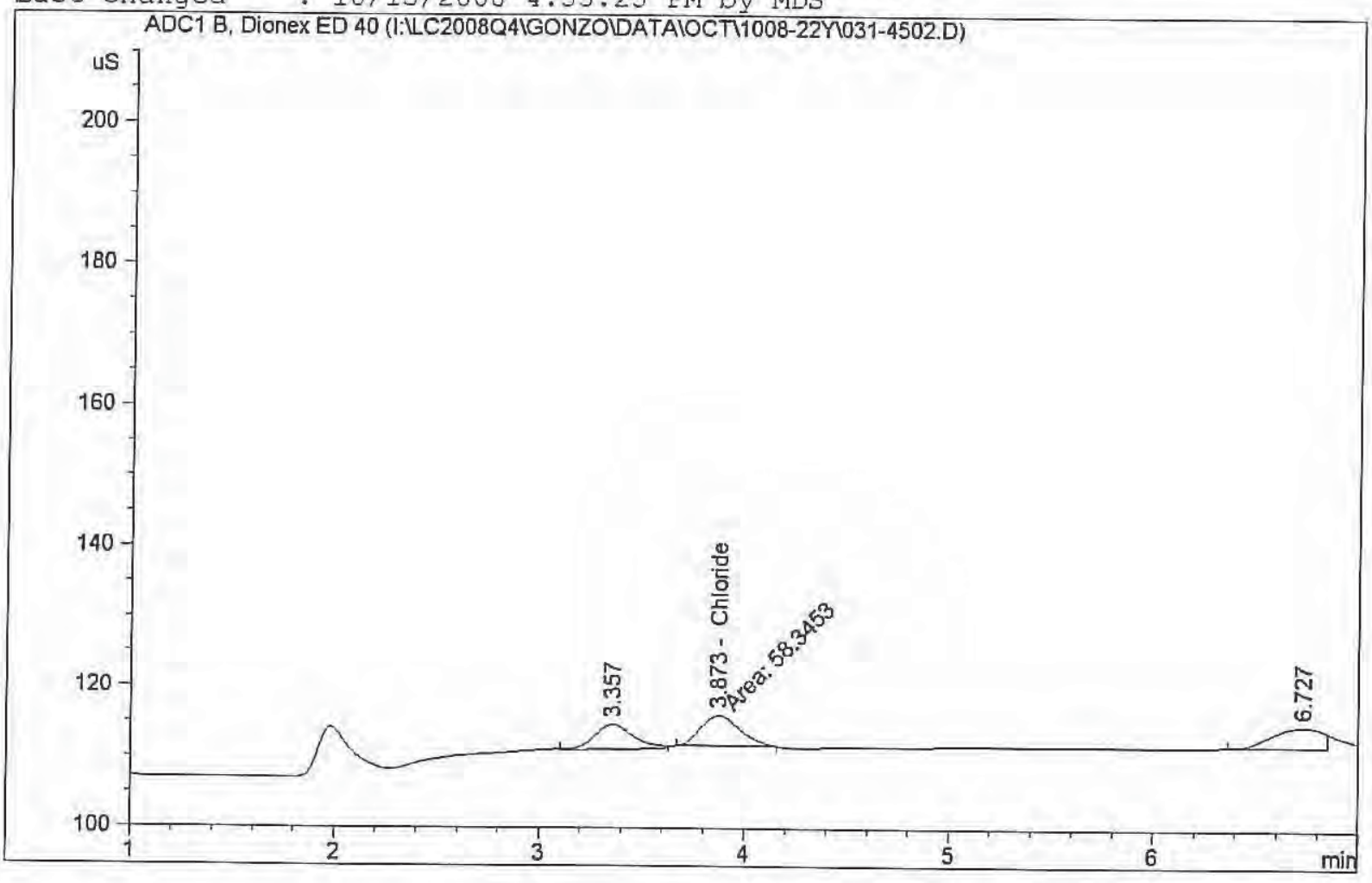

External standard Report

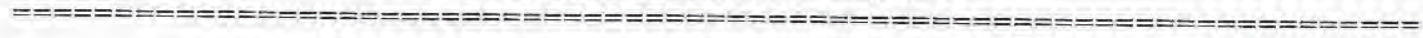

Sorted By

Calib. Data Modified :

Multiplier

Dilution

Use Multiplier \& Dilution Eactor with ISTDs

Signal 1: ADC1 B, Dionex ED 40 
$1008-22$

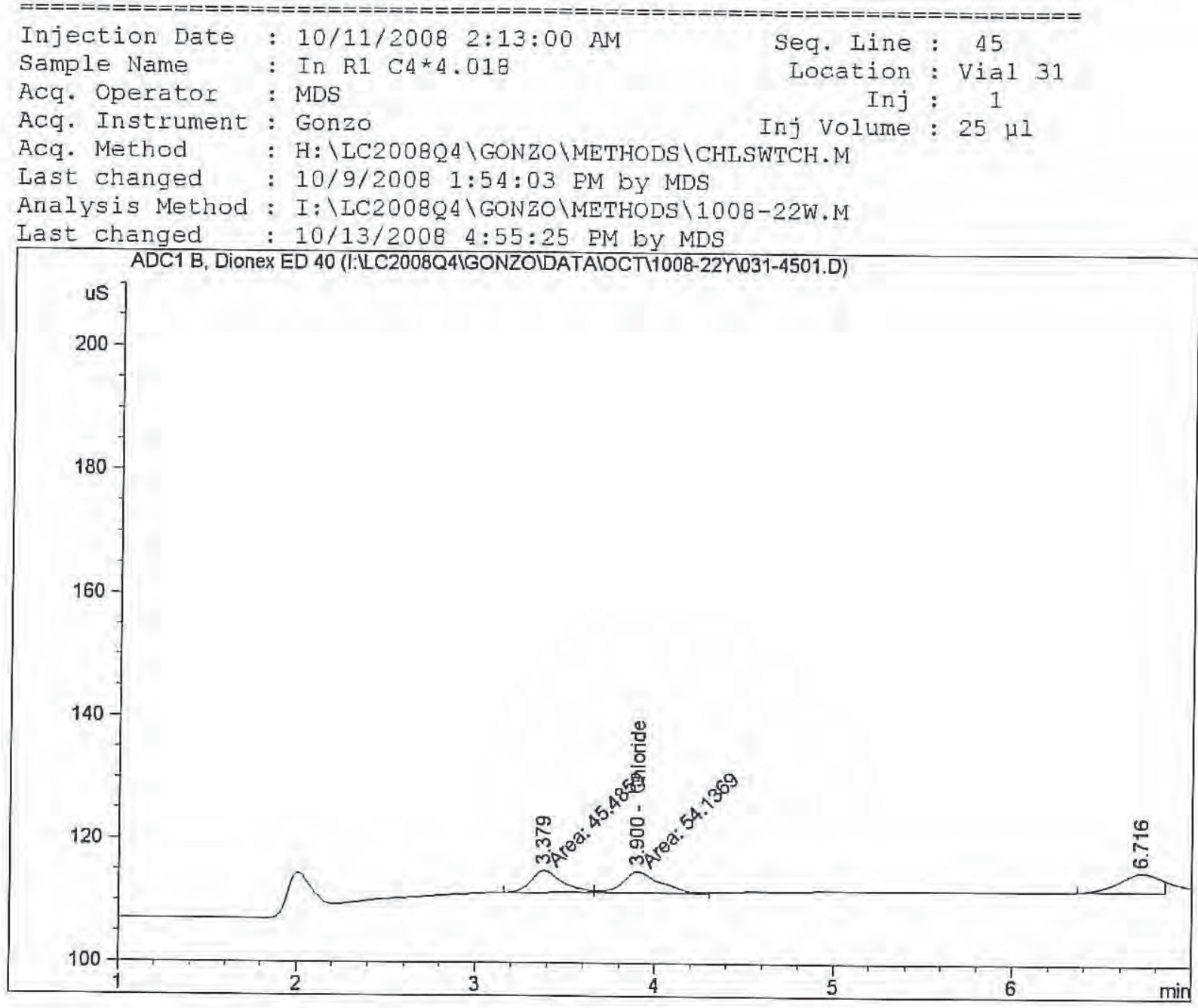

External Standard Report

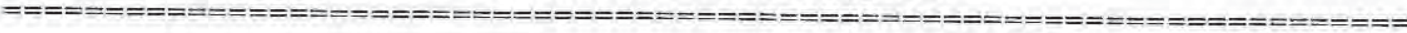

Sorted By

Multiplier : $\quad 1.0000$

Dilution : 1.0000

Use Multiplier \& Dilution Factor with ISTDS

Signal 1: ADC1 B, Dionex ED 40

\begin{tabular}{|c|c|c|c|c|c|}
\hline $\begin{array}{c}\text { RetTime } \\
\text { [min] }\end{array}$ & Type & $\begin{array}{c}\text { Area } \\
\text { [uS*s] }\end{array}$ & Amt/Area & $\begin{array}{c}\text { Amount } \\
{[\mathrm{ug} / \mathrm{mL}]}\end{array}$ & Name \\
\hline $\begin{array}{l}3.900 \\
6.083\end{array}$ & EM & $\begin{array}{c}54.13694 \\
-\end{array}$ & $\begin{array}{c}1.28247 e-2 \\
-\end{array}$ & $6.94290 e-1$ & $\begin{array}{l}\text { Chloride } \\
\text { Bromide }\end{array}$ \\
\hline
\end{tabular}
Totals :
$6.94290 e-1$

Results obtained with enhanced integrator!

1 Warnings or Errors :

Warning : Calibrated compound(s) not found

\section{*** End of Repoort 22 Page 102 of 267}




\section{$1008-22$}

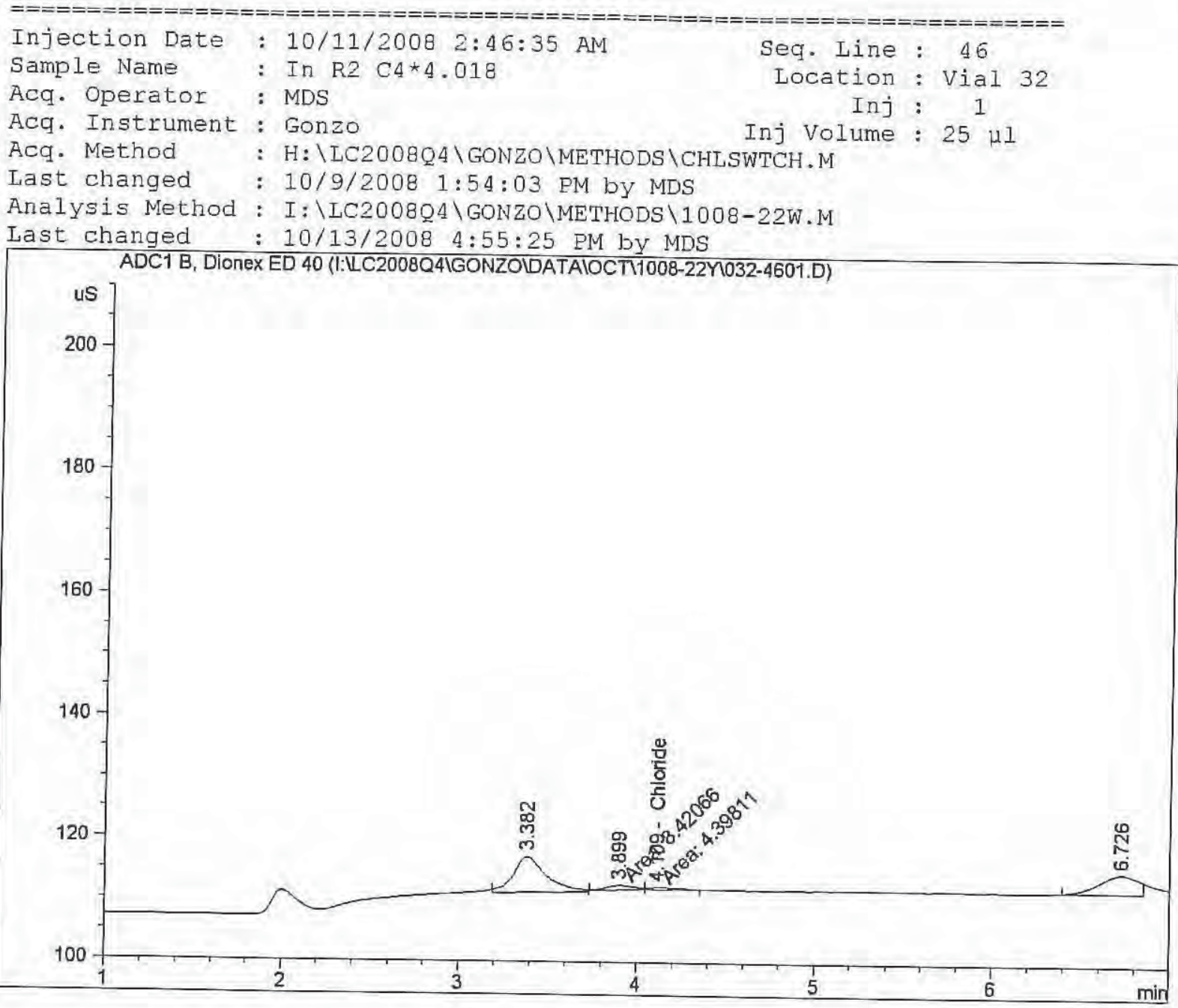

\section{External Standard Report}

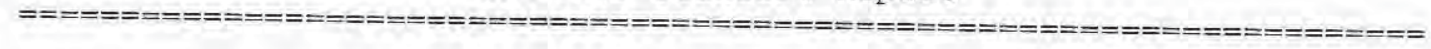

$\begin{array}{lll}\text { Sorted By } & : & \text { Signal } \\ \text { Calib. Data Modified } & : & \text { Monday, October 13, } 2008 \text { 4:55:23 PM } \\ \text { Multiplier } & : & 1.0000 \\ \text { Dilution } & : & 1.0000\end{array}$

Use Multiplier \& Dilution Eactor with ISTDs

Signal 1: ADC1 B, Dionex ED 40

\begin{tabular}{|c|c|c|c|c|c|}
\hline $\begin{array}{l}\text { RetTime } \\
\text { [min] }\end{array}$ & Type & $\begin{array}{r}\text { Area } \\
\text { [uS*s] }\end{array}$ & Amt/Area & $\begin{array}{r}\text { Amount } \\
\text { [ug/mL] }\end{array}$ & Grp Name \\
\hline $\begin{array}{l}4.109 \\
6.083\end{array}$ & FM & $\begin{array}{l}4.39811 \\
-\end{array}$ & $\begin{array}{c}1.31240 e-2 \\
-\end{array}$ & $\begin{array}{c}5.77206 e-2 \\
-\end{array}$ & $\begin{array}{l}\text { Chloride } \\
\text { Bromide }\end{array}$ \\
\hline
\end{tabular}

Totals : $\quad 5.77206 \mathrm{e}-2$

Results obtained with enhanced integrator!

1 Warnings or Errors :

Warning : Calibrated compound(s) not found 


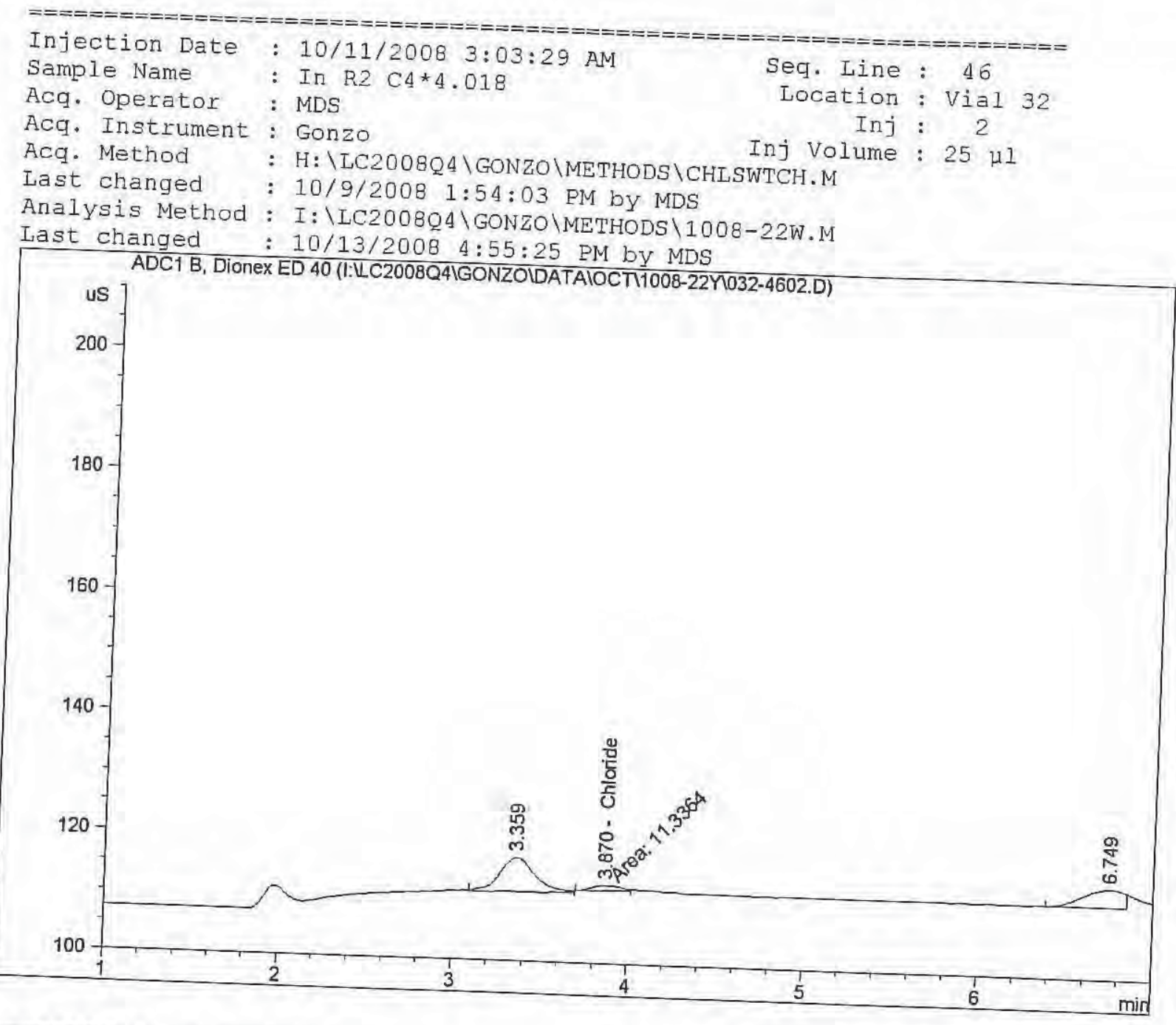

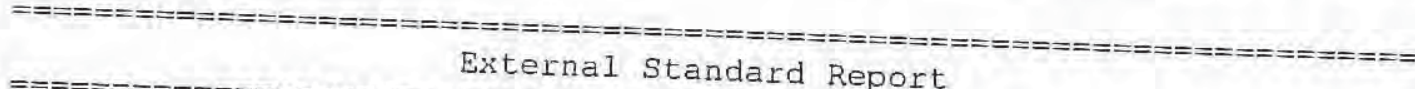

Results obtained with enhanced integrator!

I Warnings or Errors :

Warning : Calibrated compound(s) not found 


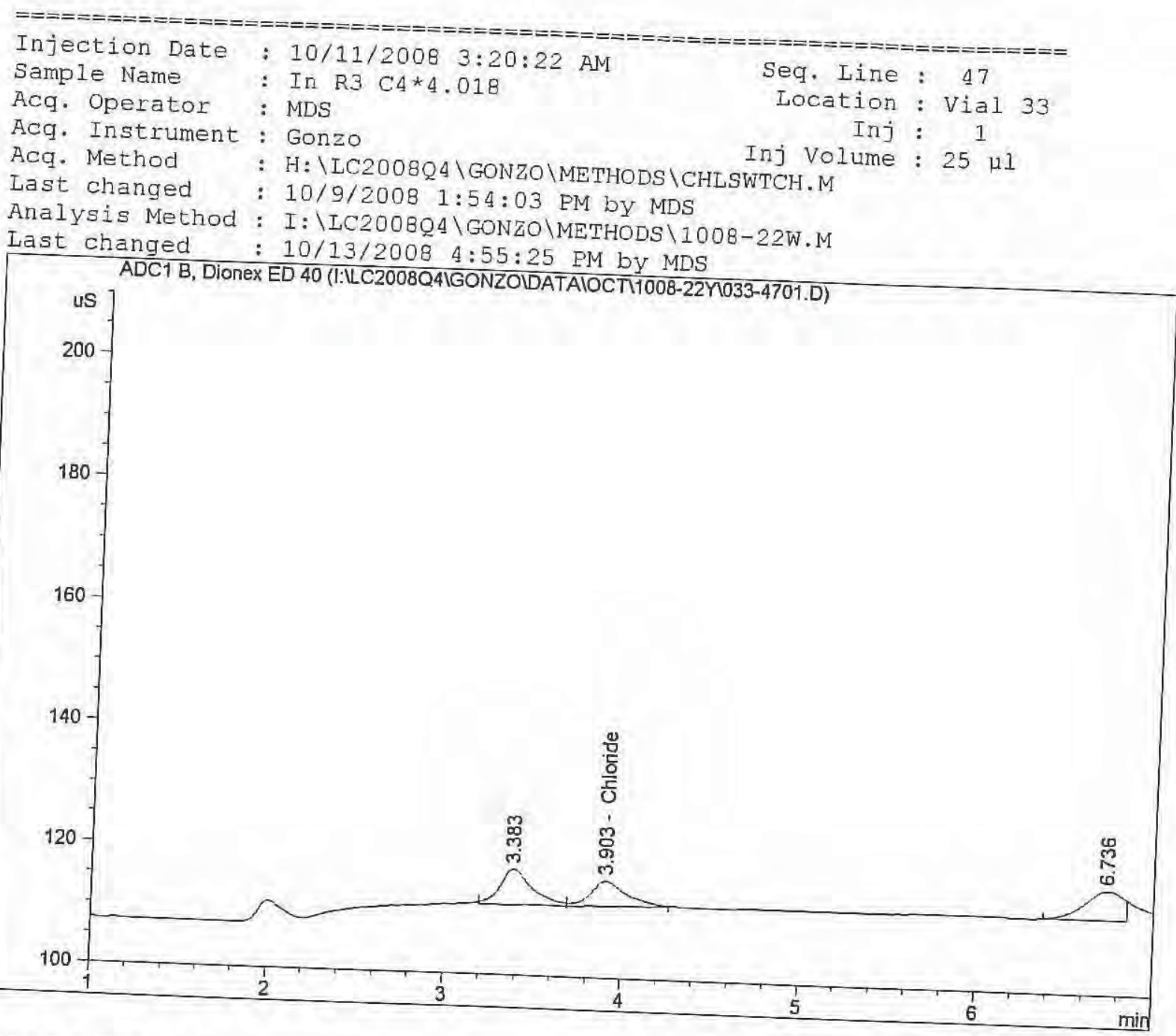

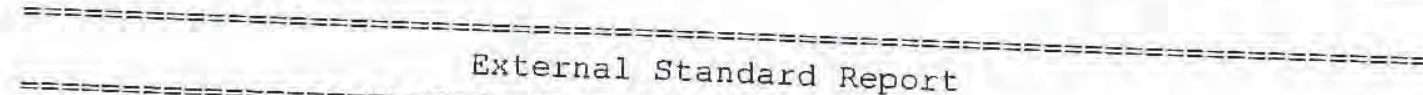
Sorted By
Calib. Data Modified : Signal
Multiplier
Dilution
Monday, October 13, 2008 4:55:23 PM
$\begin{array}{lll}\text { Dilution } & : & 1.0000 \\ \text { Use Multiplier \& Dilution } & 1.0000\end{array}$


Signal 1: ADC1 B, Dionex ED 40

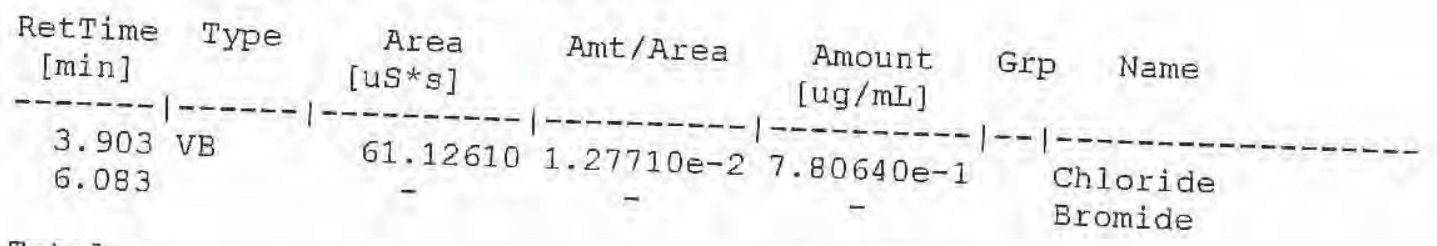

Totals :

$$
7.80640 \mathrm{e}-1
$$

Results obtained with enhanced integrator!

1 Warnings or Errors :

Warning : Calibrated compound(s) not found 


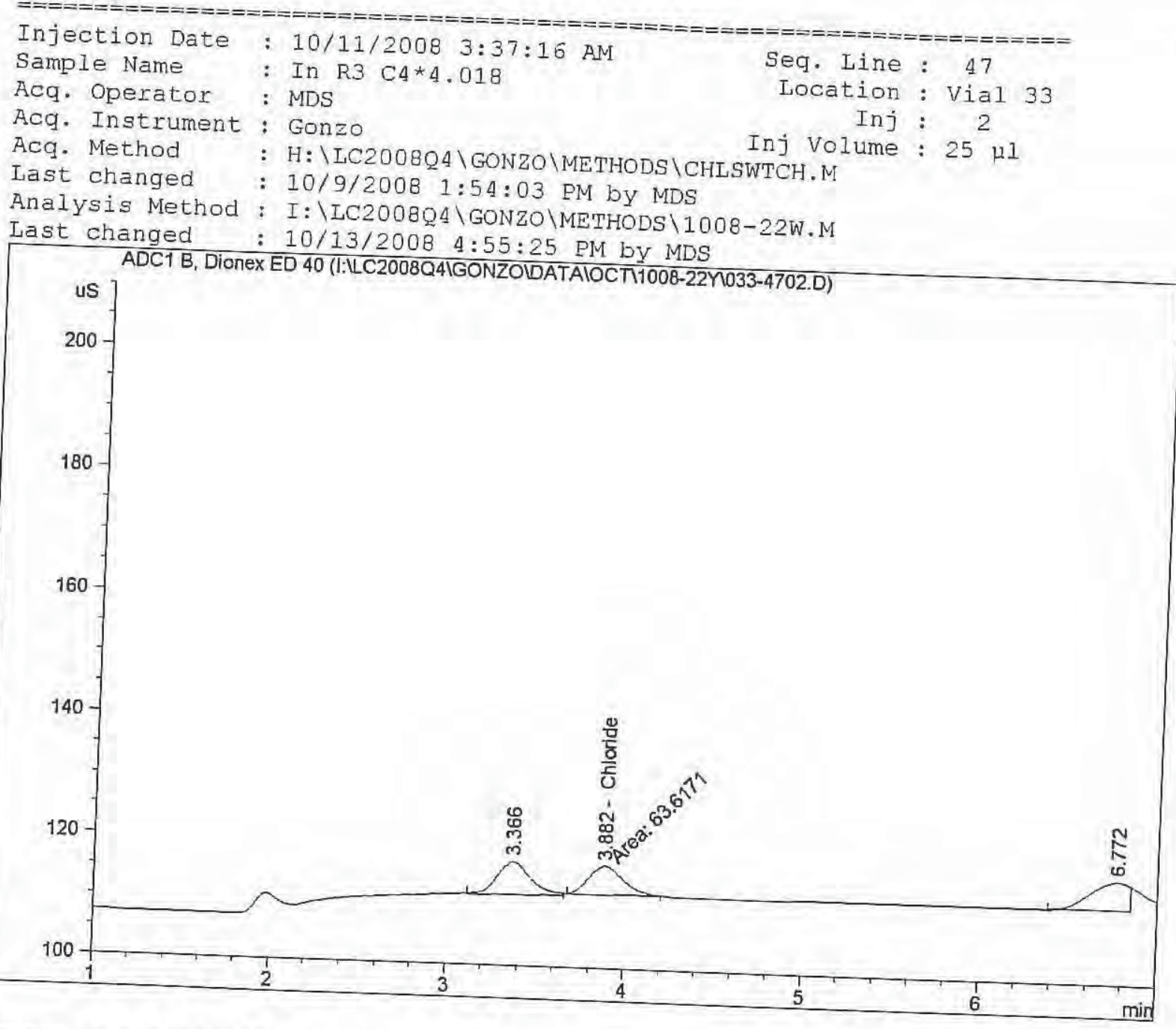

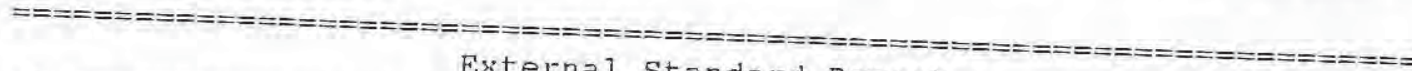
External standard Report

Sorted By

Calib. Data Modified : $\quad$ Signal

Multiplier : $\quad 1$ Monday, October 13, 2008 4:55:23 PM

Dilution : $\quad 1.0000$

Use Multiplier \& Dilution Eactor with ISTDs

Signal 1: ADC1 B, Diones ED 40

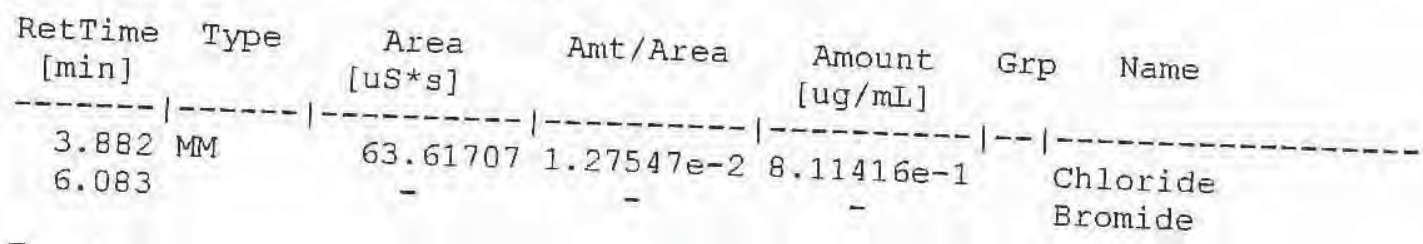

Totals :

$$
\text { 8. } 11416 \mathrm{e}-1
$$

Results obtained with enhanced integrator!

1 Warnings or Errors :

Warning : Calibrated compound(s) not found 


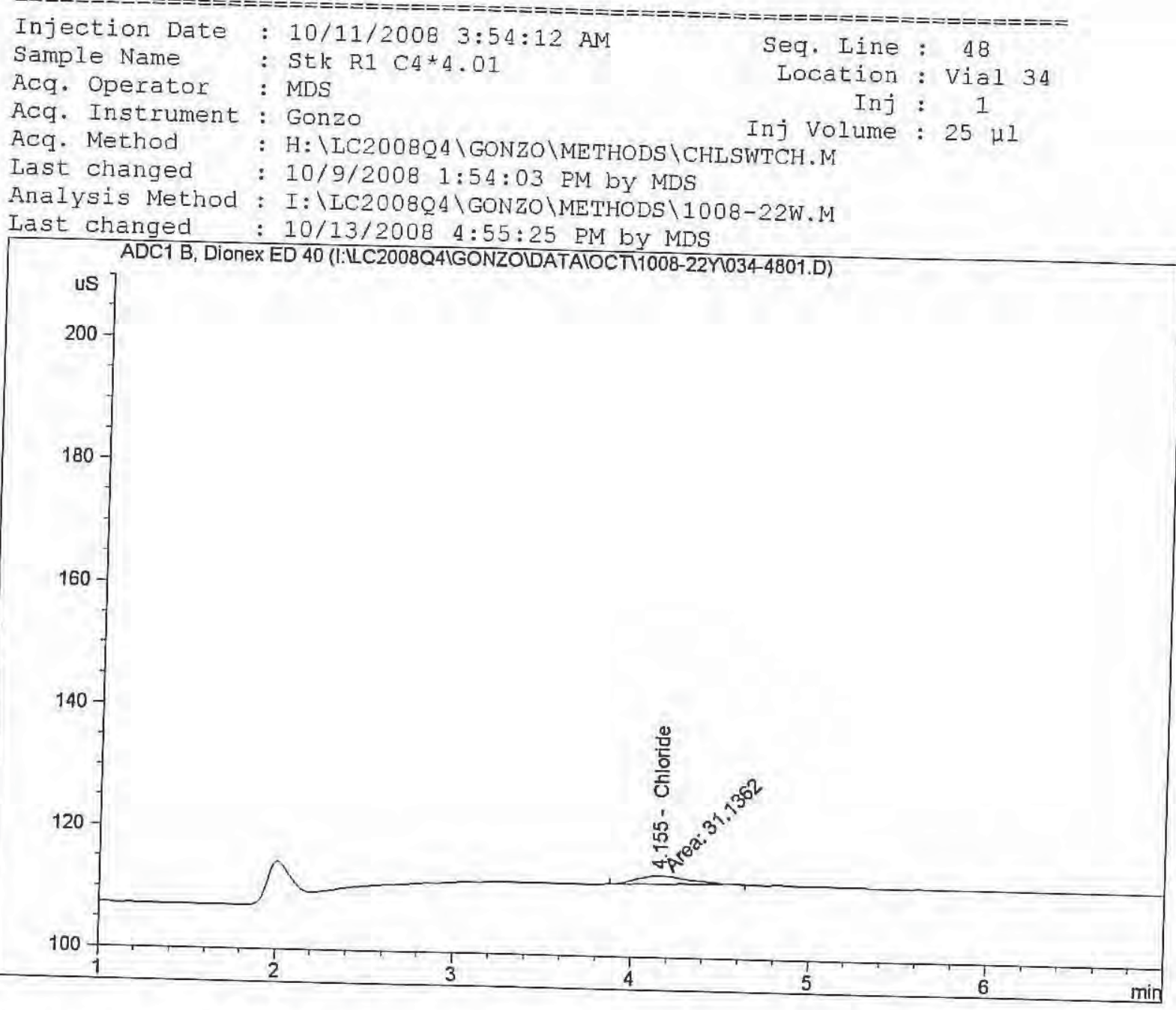

External Standard Report

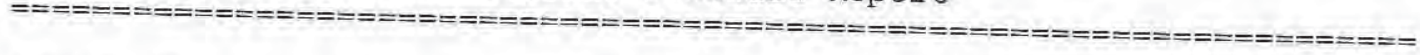

$\begin{array}{lcl}\text { Sorted By } & : & \text { Signal } \\ \text { Calib. Data Modified } & \vdots & \text { Monday, October 13, 2008 4:55:23 PM } \\ \text { Multiplier } & \vdots & 1.0000 \\ \text { Dilution } & \vdots & 1.0000 \\ \text { Use Multiplier \& Dilution Eactor with ISTDS }\end{array}$

Signal 1: ADC1 B, Dionex ED 40

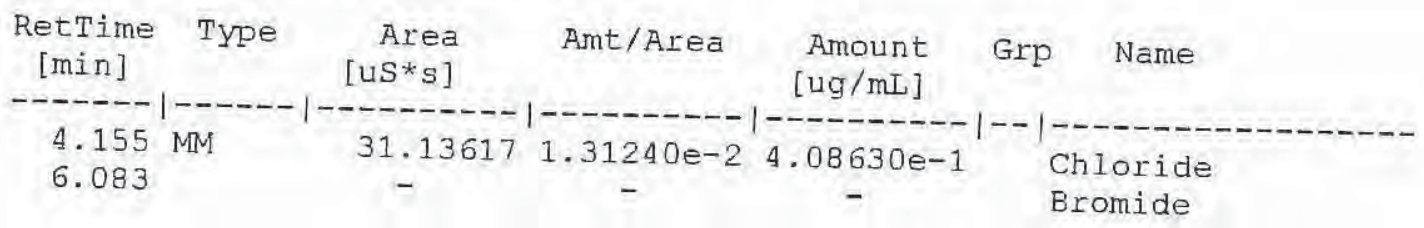

Totals : $4.08630 \mathrm{e}-1$

Results obtained with enhanced integrator!

1 Warnings or Errors :

Warning : Calibrated compound(s) not found 


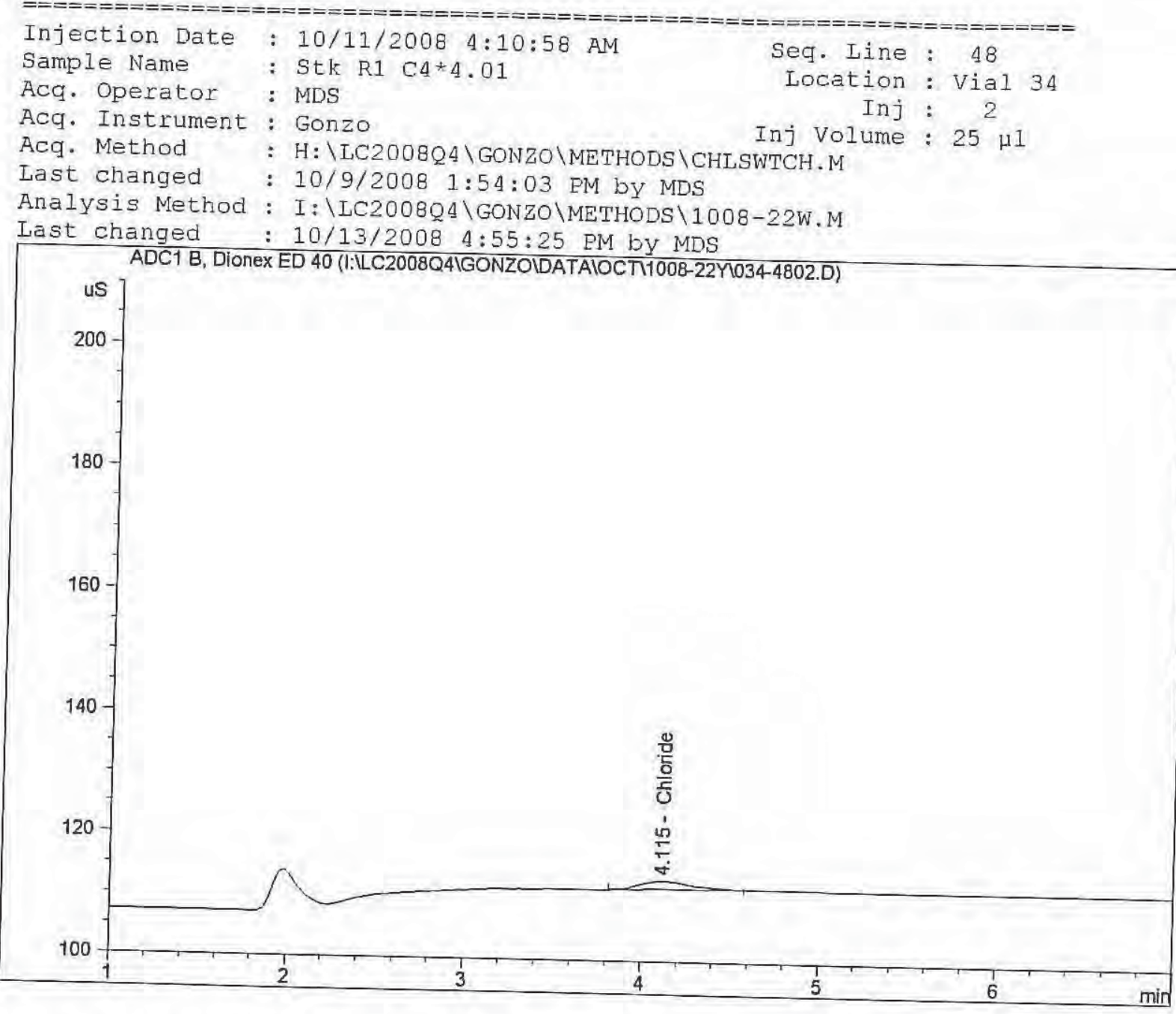

External Standard Report

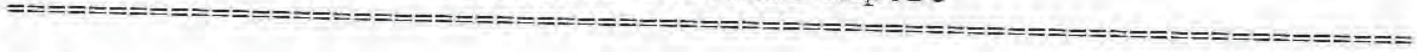

Sorted BY

Calib. Data Modified : Signal

Multiplier : $\quad 1.0000$

Dilution : 1.0000

Use Multiplier \& Dilution Factor with ISTDs

Signal 1: ADC1 B, Diones ED 40

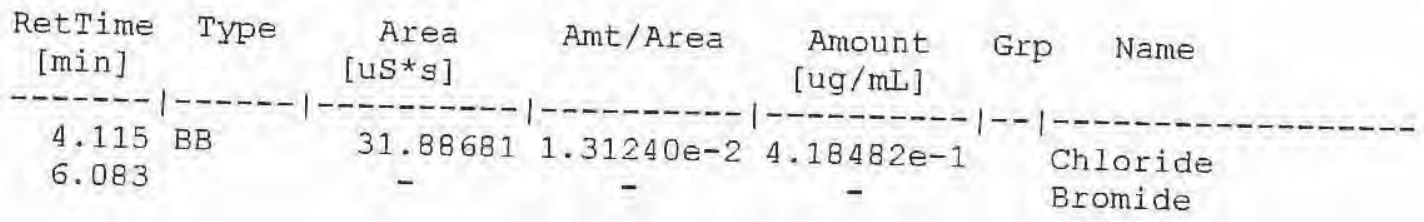

Totals :

4. $18482 e-1$

Results obtained with enhanced integrator!

1 Warnings or Errors :

Warning : Calibrated compound(s) not found 


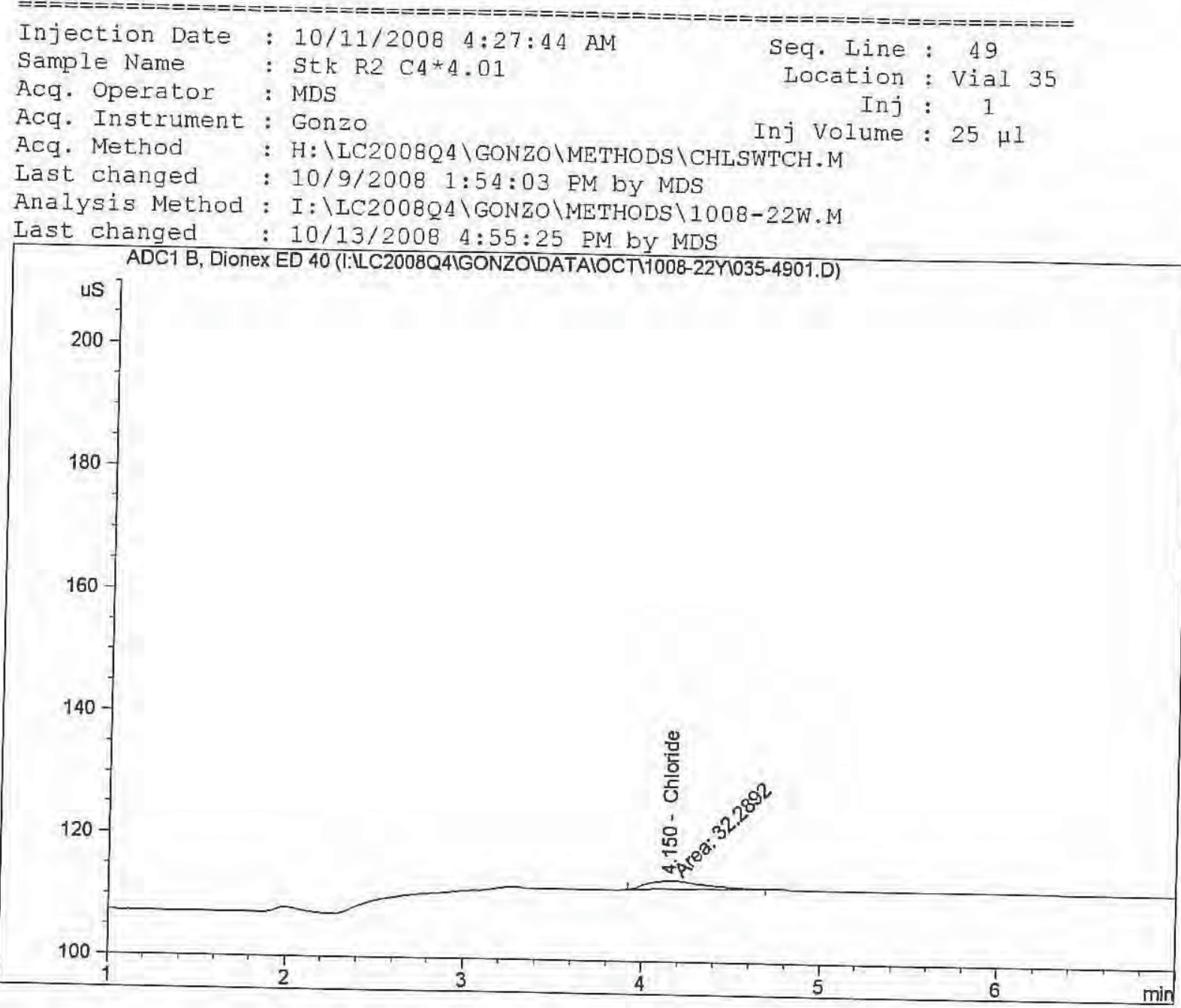

External Standard Report

Sorted By

Calib. Data Modified : Signal

Multiplier : 1.0000

Dilution : 1.0000

Use Multiplier \& Dilution Eactor with ISTDs

Signal 1: ADC1 B, Dionex ED 40

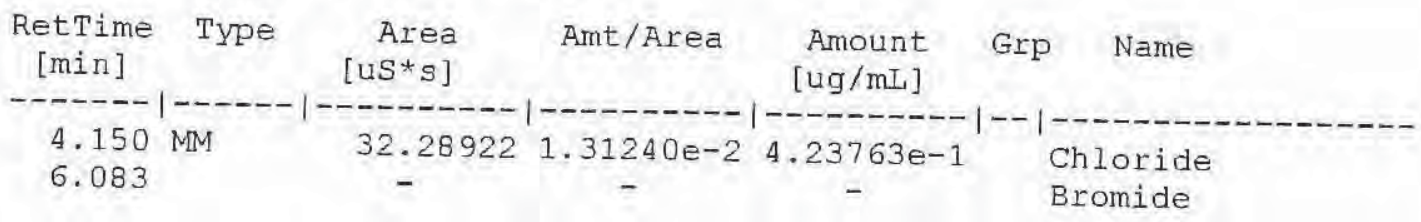

Totals : $\quad 4.23763 e-1$

Results obtained with enhanced integrator!

1 Warnings or Errors :

Warning : Calibrated compound(s) not found 

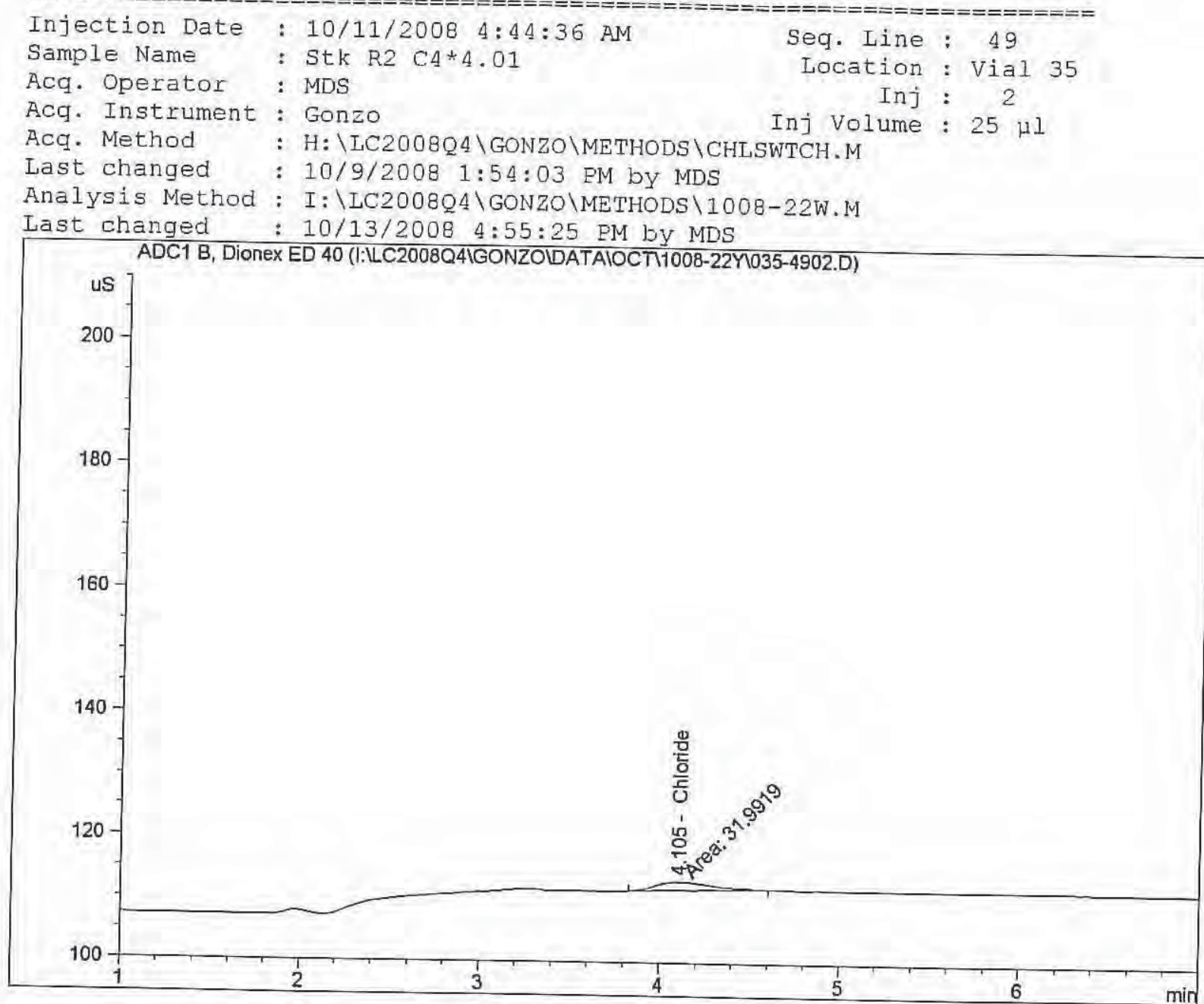

Sorted By Calib. Data Modified Multiplier

Dilution

: Signal

: 1.0000

, October 13, 2008 4:55:23 PM

Use Multiplier

Signal 1: ADC1 B, Diones ED 40
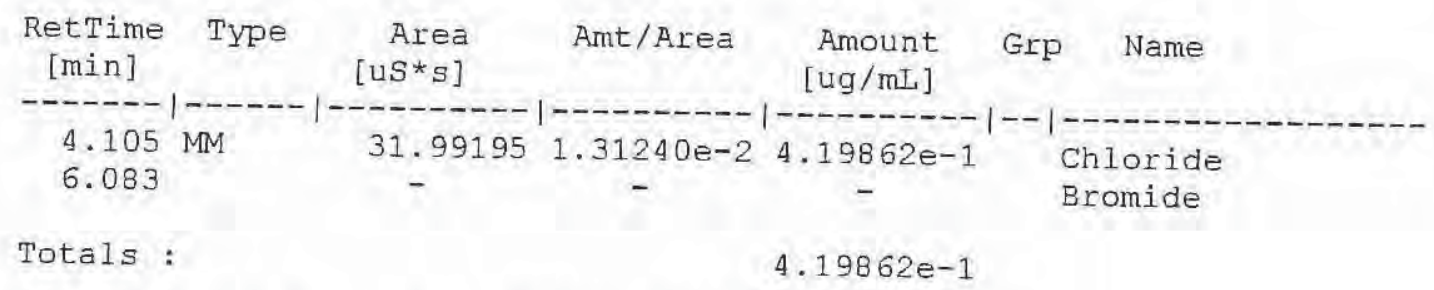

Totals: 4. $19862 \mathrm{e}-1$

Results obtained with enhanced integrator!

1 Warnings or Errors :

Warning : Calibrated compound(s) not found 


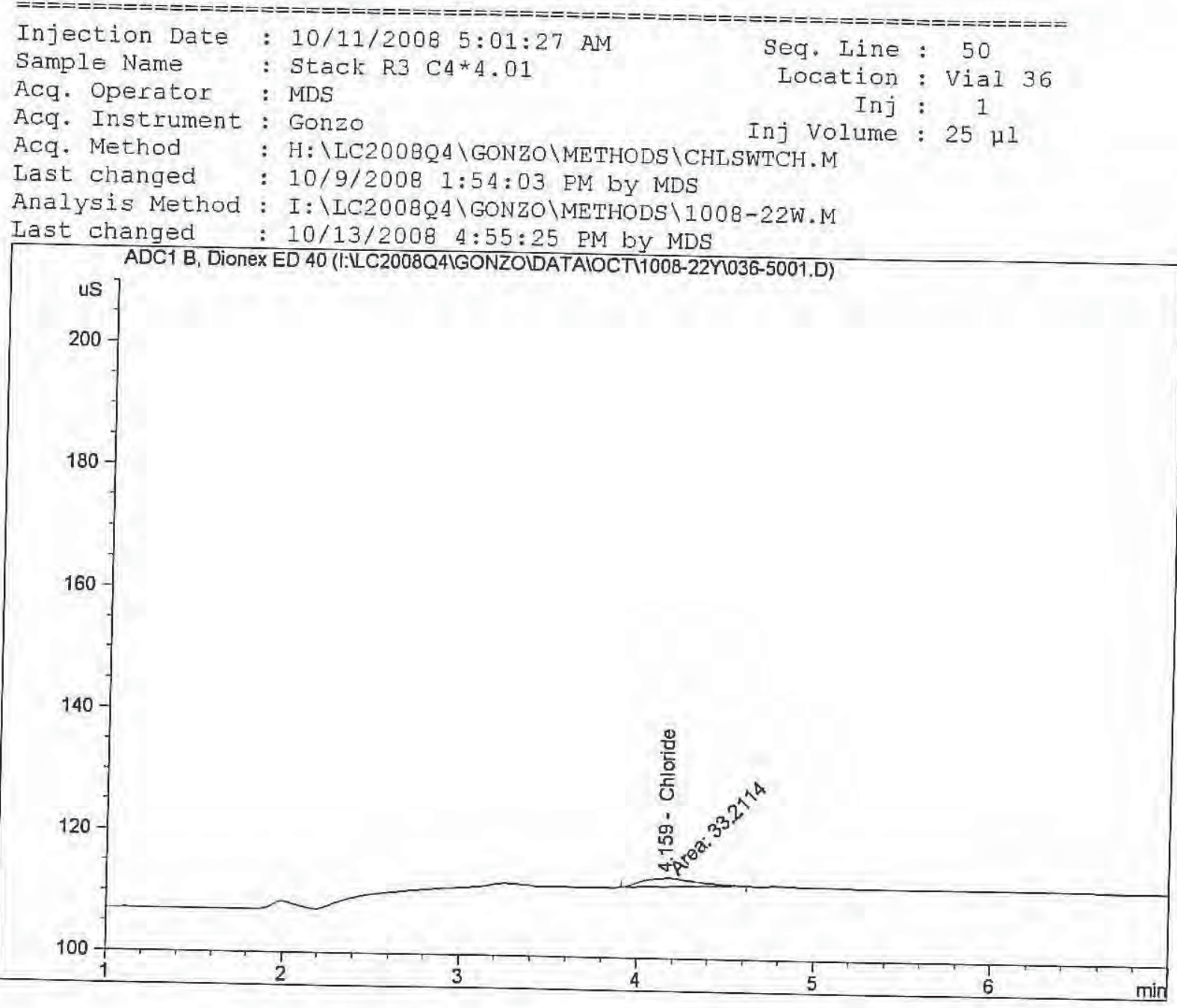

\section{External Standard Report}

Sorted By

Calib. Data Modified : Signal

Multiplier

Dilution

Monday, October 13, 2008 4:55:23 PM

1. 0000

Use Multiplier \& Dilution Factor with ISTDS

Signal 1: ADC1 B, Dionex ED 40

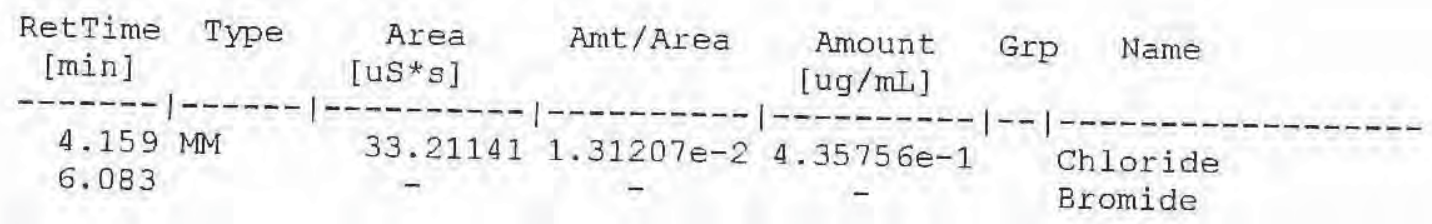

Totals :

$$
\text { 4. } 35756 e-1
$$

Results obtained with enhanced integrator!

1 Warnings or Errors :

Warning : Calibrated compound(s) not found 
1008-22 Sample Blank

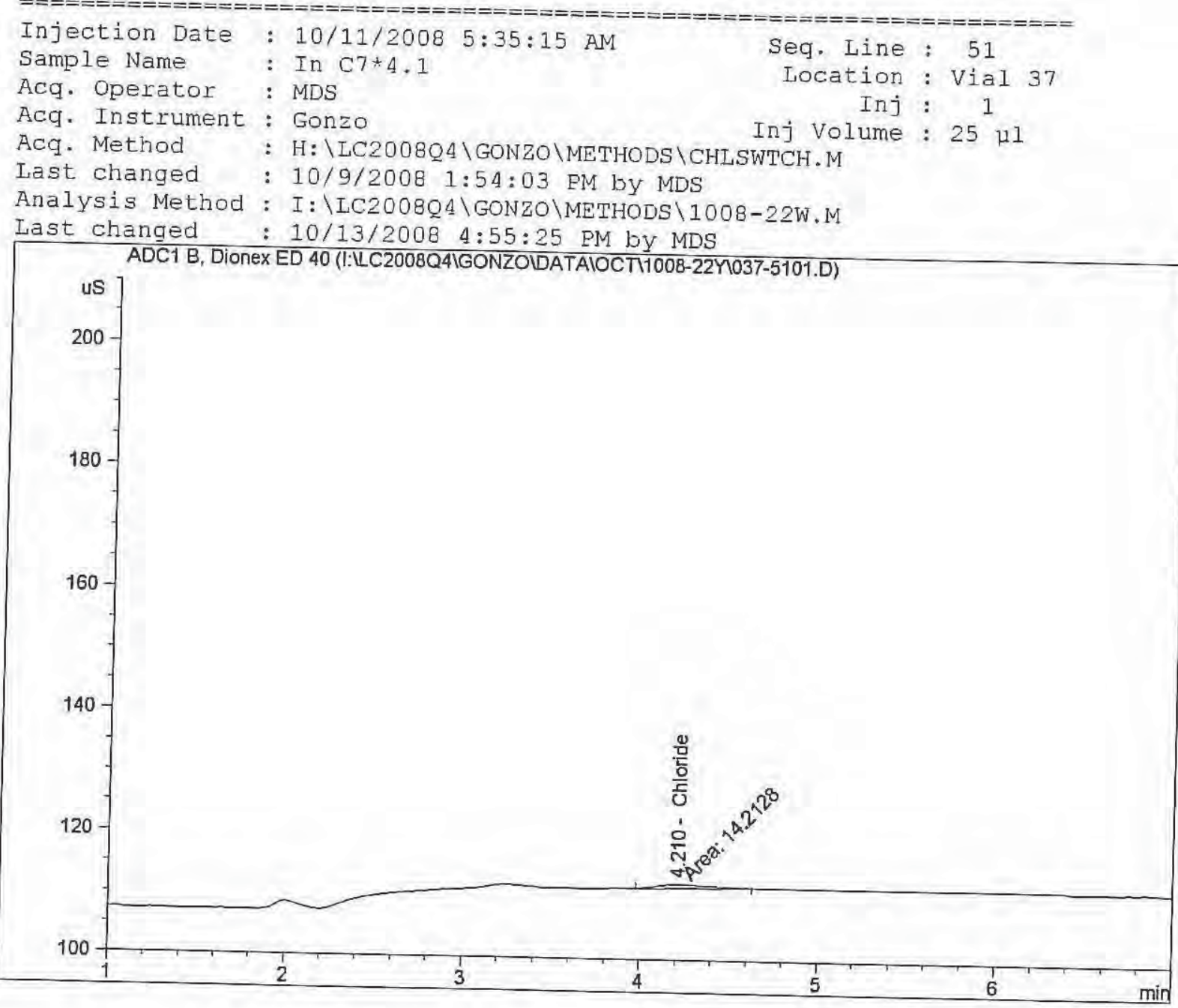

External Standard Report

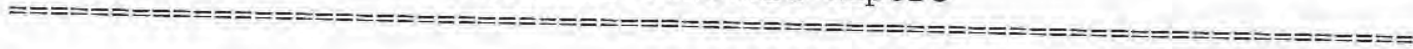

Sorted By

Calib. Data Modified : Signal

Multiplier : $\quad 1.0000$

Dilution : 1.0000

Use Multiplier \& Dilution Factor with ISTDs

Signal 1: ADC1 B, Dionex ED 40

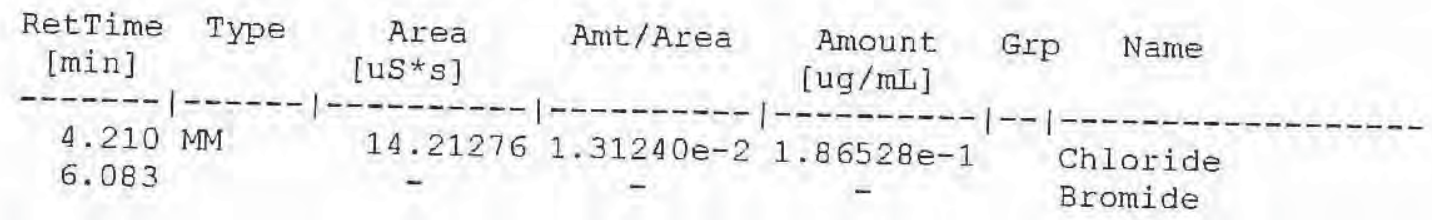

Totals:

1. $86528 e-1$

Results obtained with enhanced integrator!

1 Warnings or Errors :

Warning : Calibrated compound(s) not found 


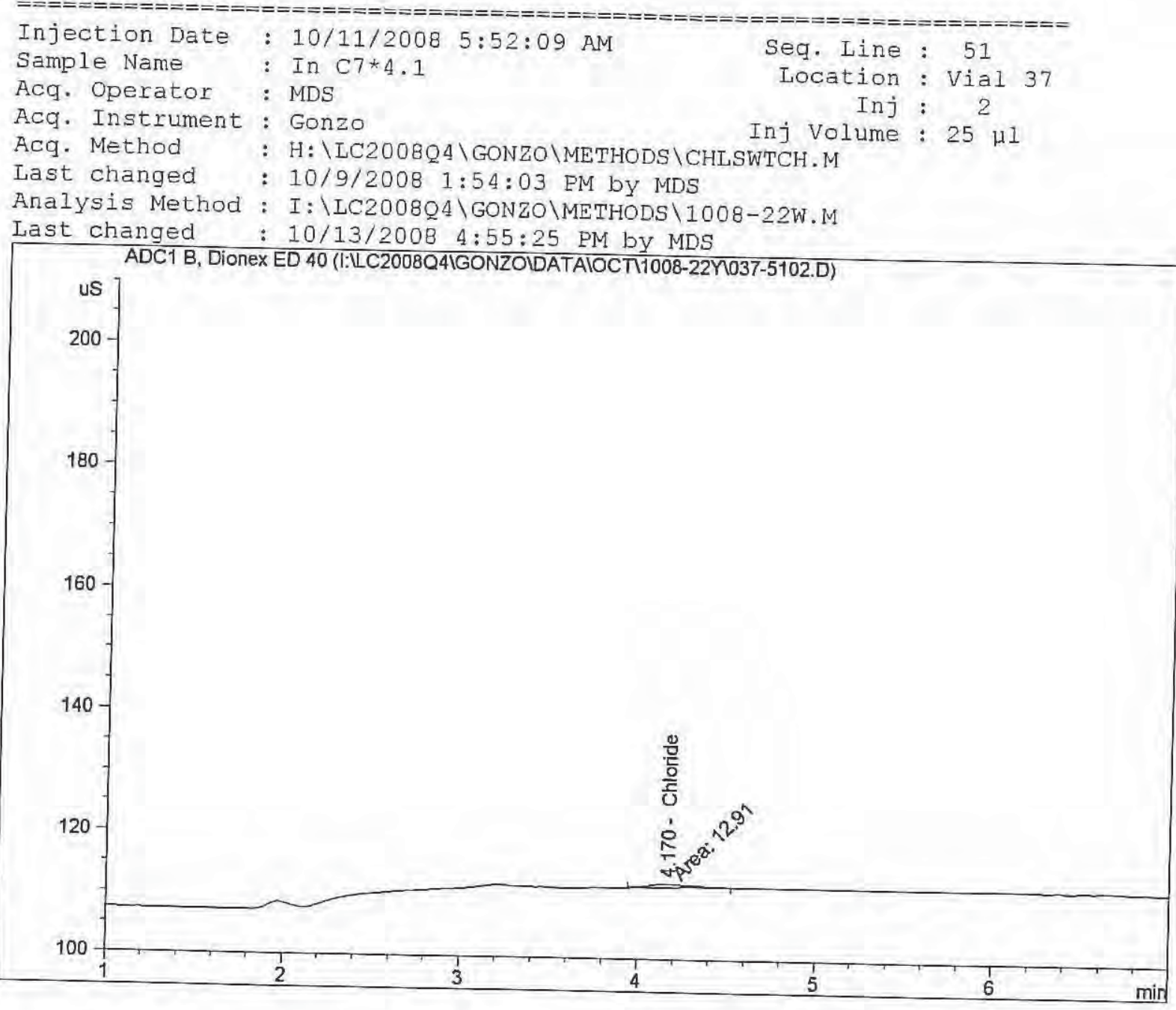

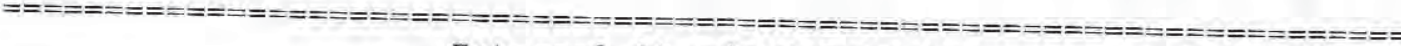

External Standard Report

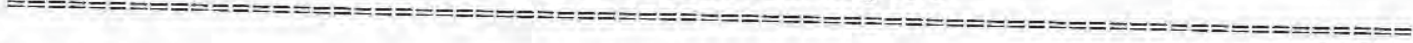

Sorted By

Calib. Data Modified Signal

Multiplier : : 1.0000

Dilution : 1.0000

Use Multiplier \& Dilution Factor with ISTDS

Signal 1: ADC1 B, Diones ED 40

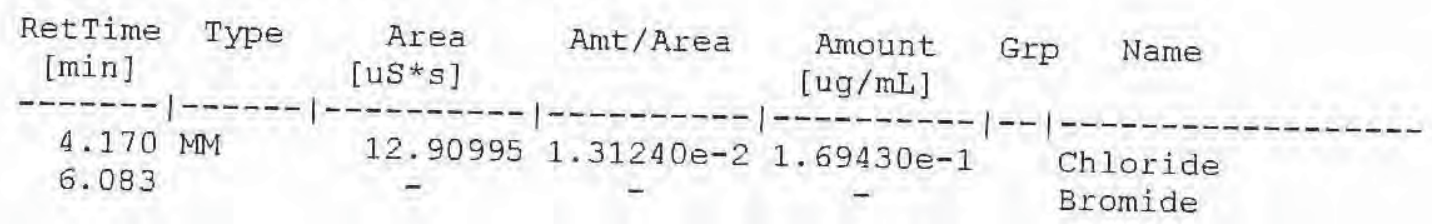

Totals :

1. $69430 \mathrm{e}-1$

Results obtained with enhanced integrator!

1 Warnings or Errors :

Warning : Calibrated compound(s) not found
} 
$1008-22$

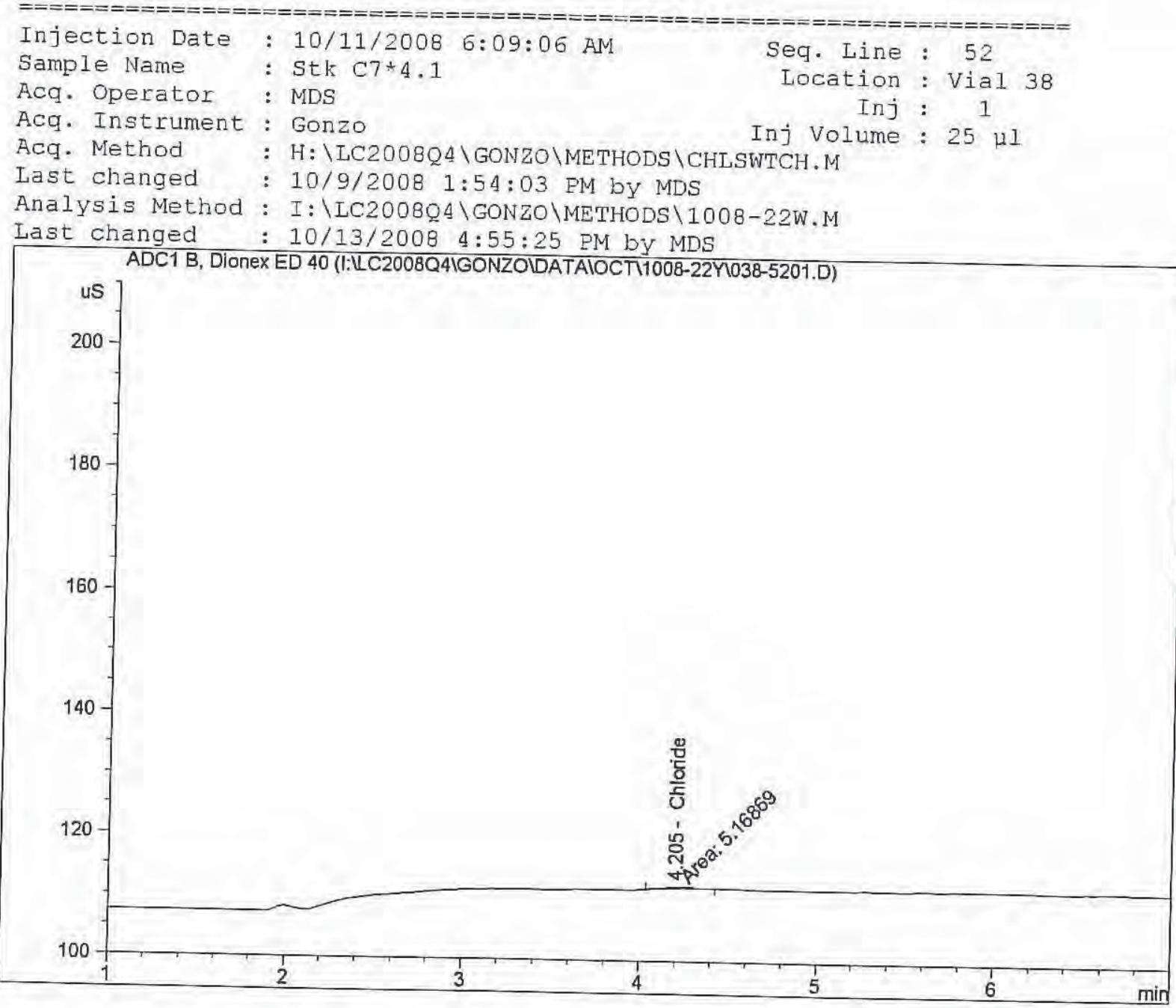

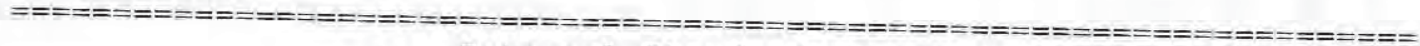

External Standard Report

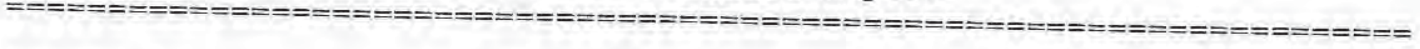

Sorted By

Calib. Data Modified: Signal

Multiplier : 1.0000

Dilution : 1.0000

Use Multiplier \& Dilution Eactor with ISTDS

Signal 1: ADC1 B, Dionex ED 40

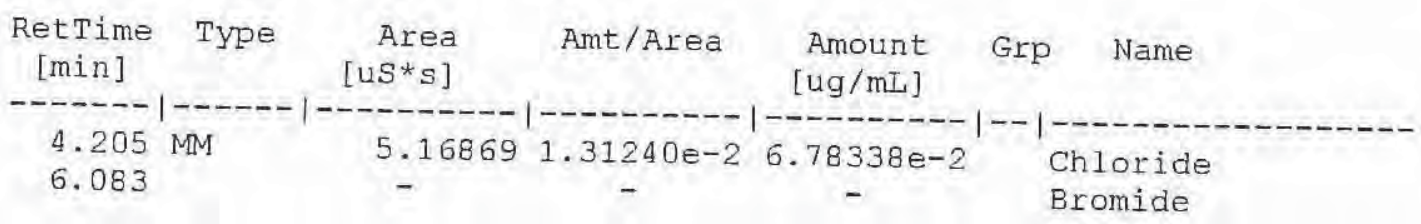

Totals :

$6.78338 \mathrm{e}-2$

Results obtained with enhanced integrator!

1 Warnings or Errors :

Warning : Calibrated compound(s) not found
} 

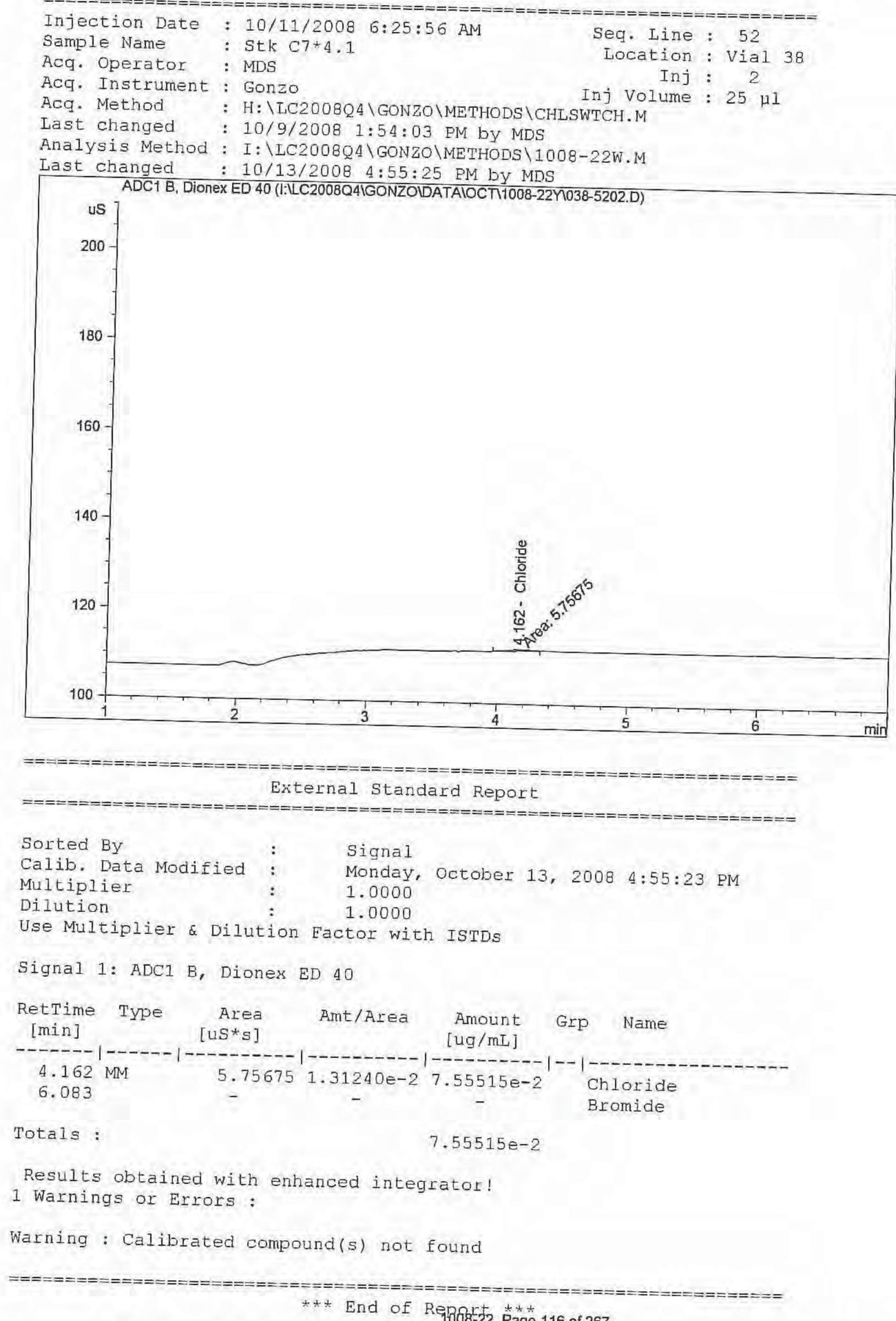
$0.01 \mathrm{~N} \mathrm{NaOH}$, used to prepare standards
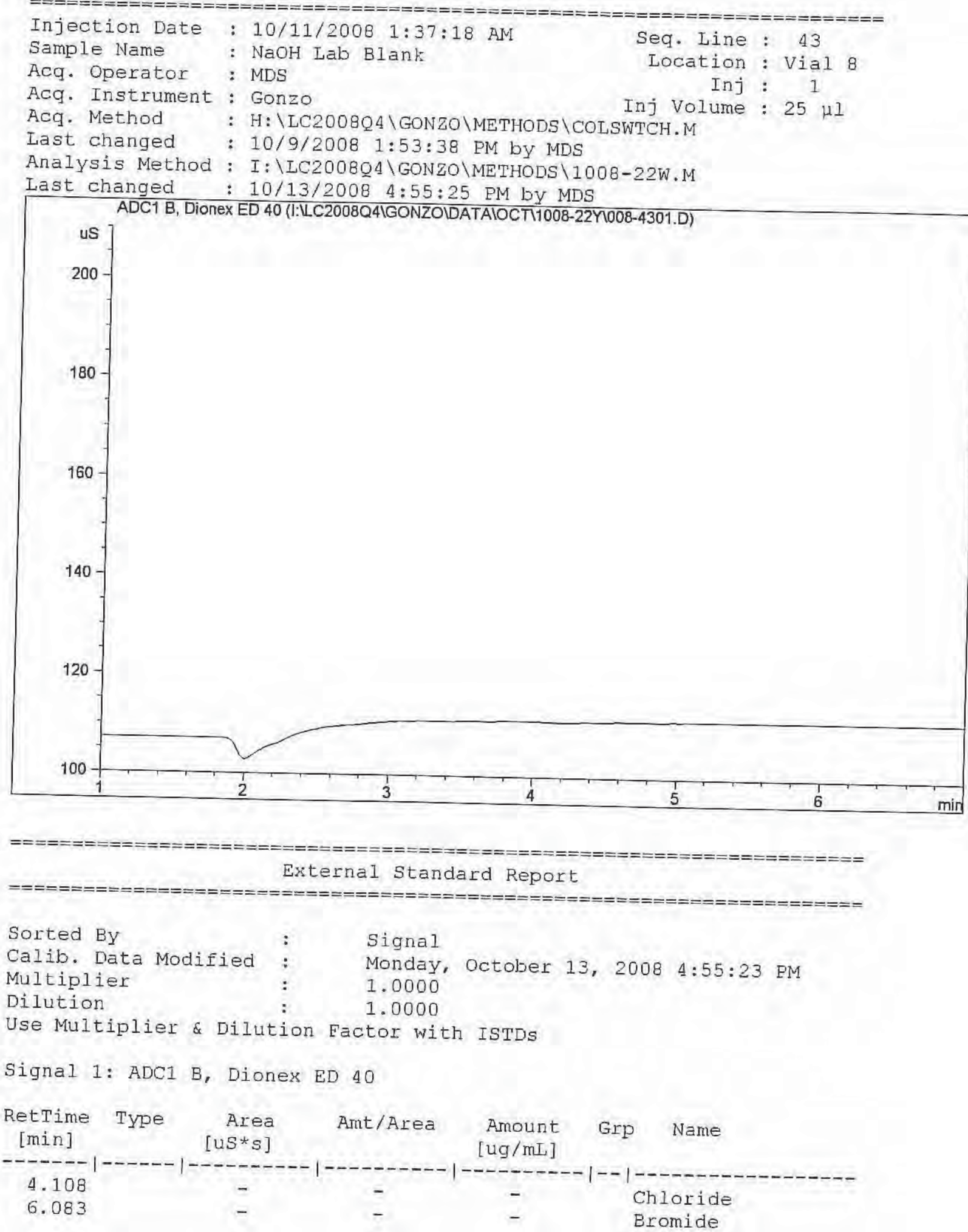

Totals :

0.00000

Results obtained with enhanced integrator!

1 Warnings or Errors :

Warning : Calibrated compound(s) not found 
$0.01 \mathrm{~N} \mathrm{NaOH}$, used to prepare standards

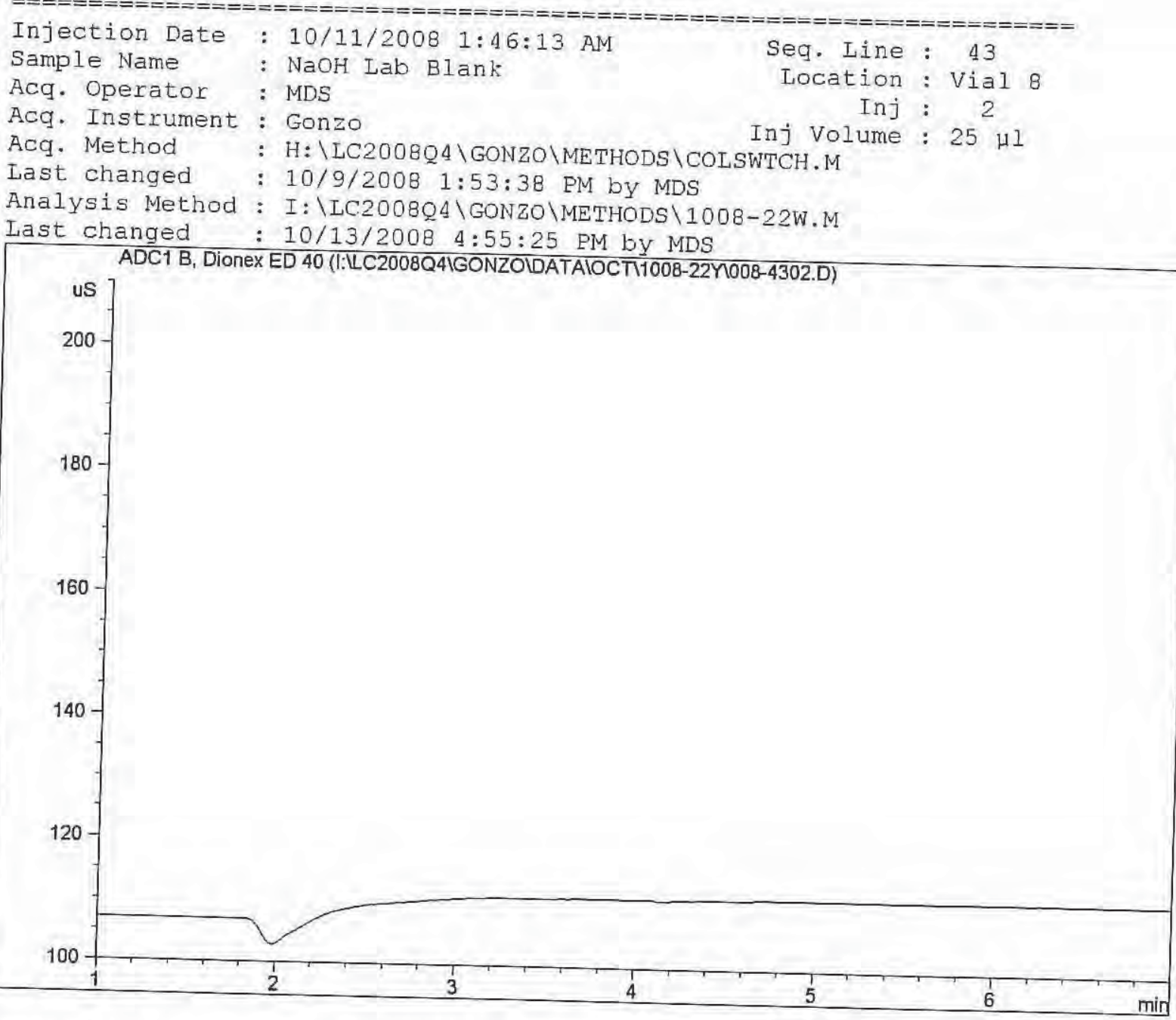

\section{External Standard Report}

Sorted By

Calib. Data Modified : Signal

Multiplier : $\quad 1.0000$

Dilution : 1.0000

Use Multiplier \& Dilution Factor with ISTDs

Signal 1: ADC1 B, Diones ED 40

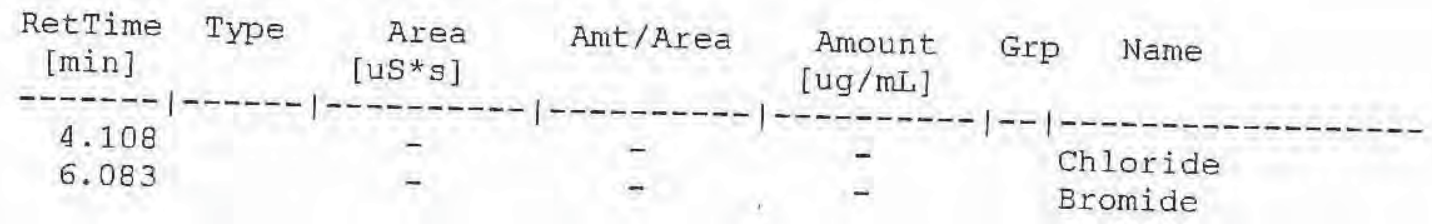

Totals:

0.00000

Results obtained with enhanced integrator!

1 Warnings or Errors :

Warning : Calibrated compound(s) not found 
used to dilute samples

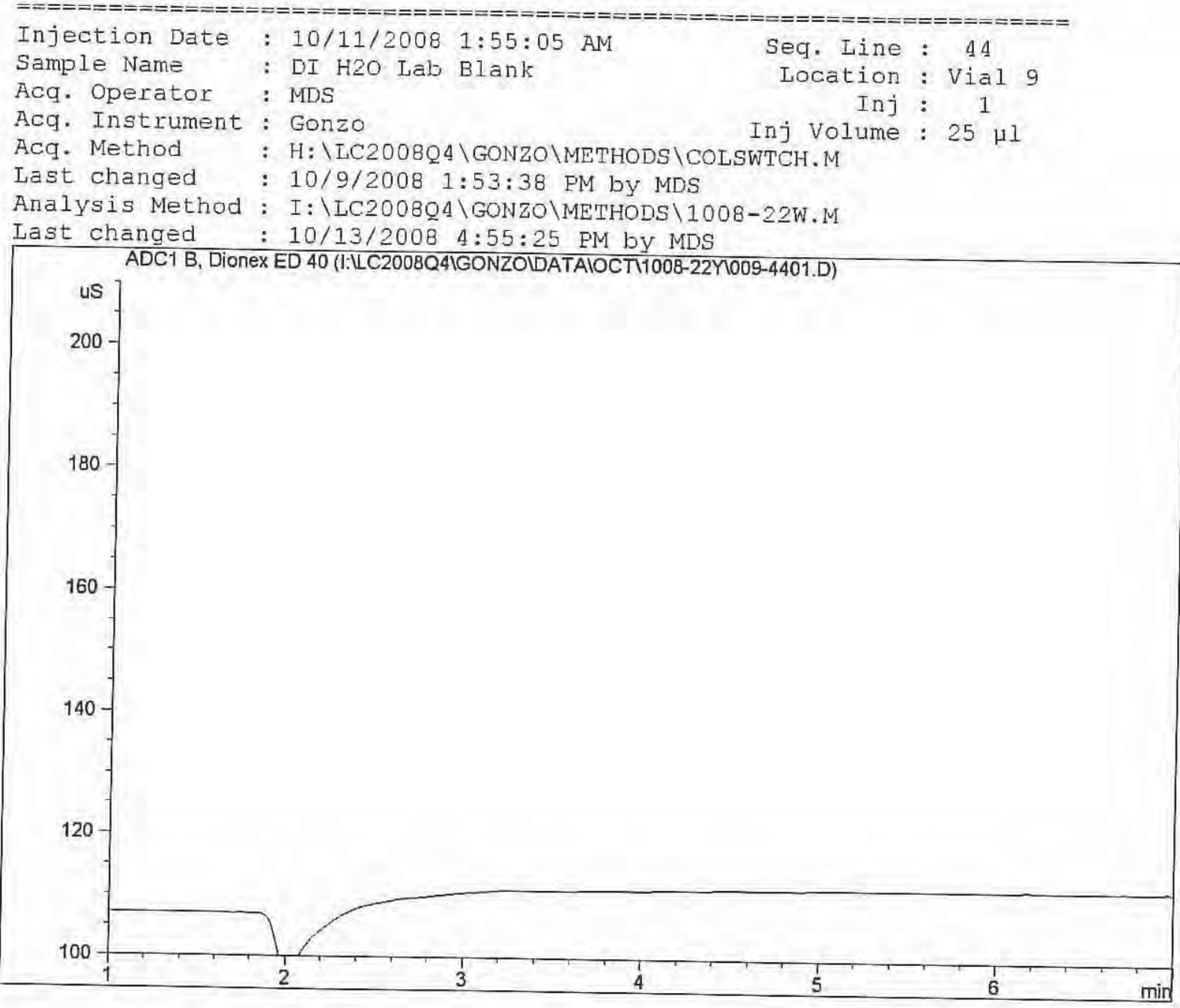

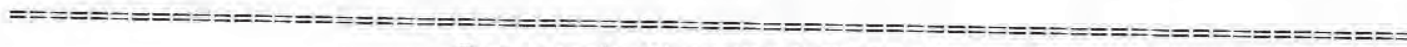

External Standard Report

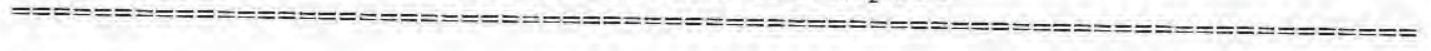

Sorted BY

Signal

Calib. Data Modified : Monday, October 13, 2008 4:55:23 PM

Multiplier : 1.0000

Dilution : 1.0000

Use Multiplier \& Dilution Factor with ISTDS

Signal 1: ADC1 B, Dionex ED 40

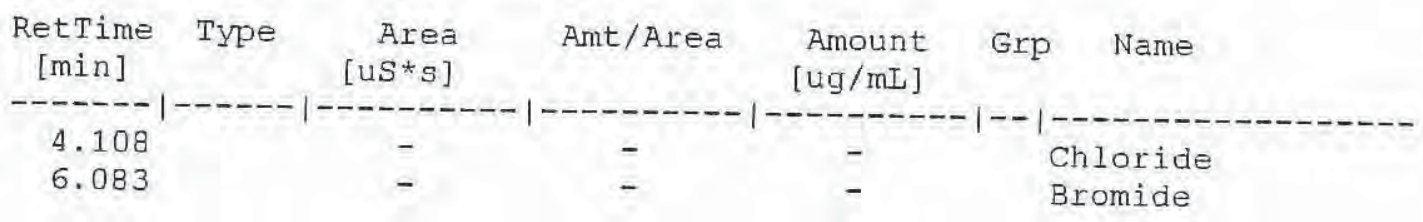

Totals :

0.00000

Results obtained with enhanced integrator!

1 Warnings or Errors :

Warning : Calibrated compound(s) not found
} 
used to dilute samples

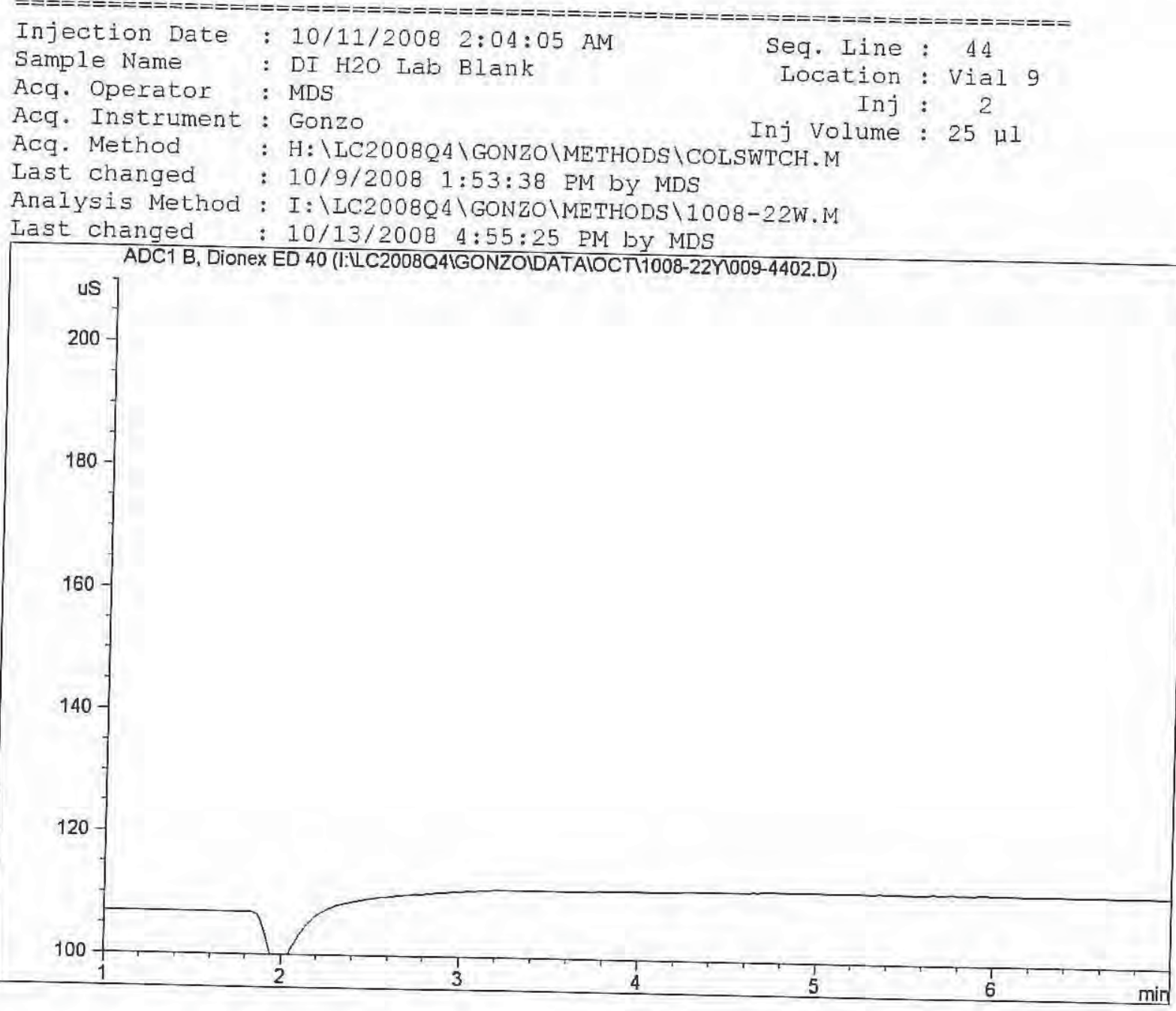

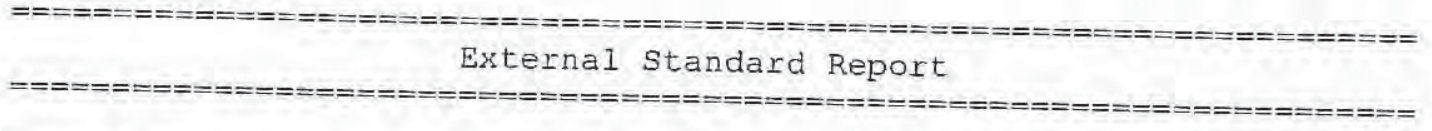

Sorted By

Calib. Data Modified Signal

Multiplier : $\quad 1.0000$

Dilution : 1.0000

Use Multiplier \& Dilution Factor with ISTDS

Signal 1: ADC1 B, Dionex ED 40

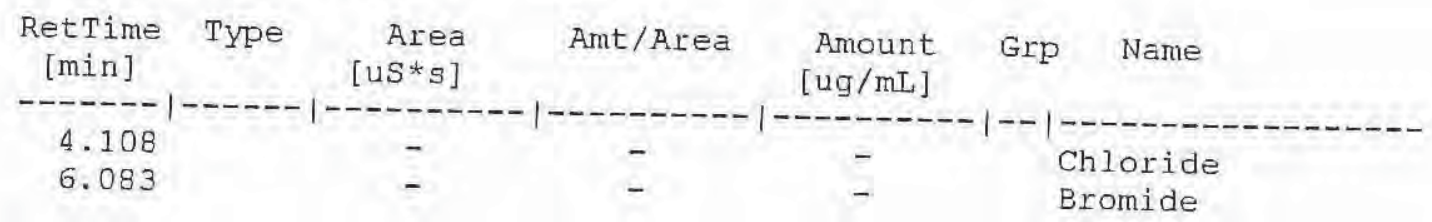

Totals:

0.00000

Results obtained with enhanced integrator!

1 Warnings or Errors :

Warning : Calibrated compound(s) not found 
1008-22 Matrix Spike

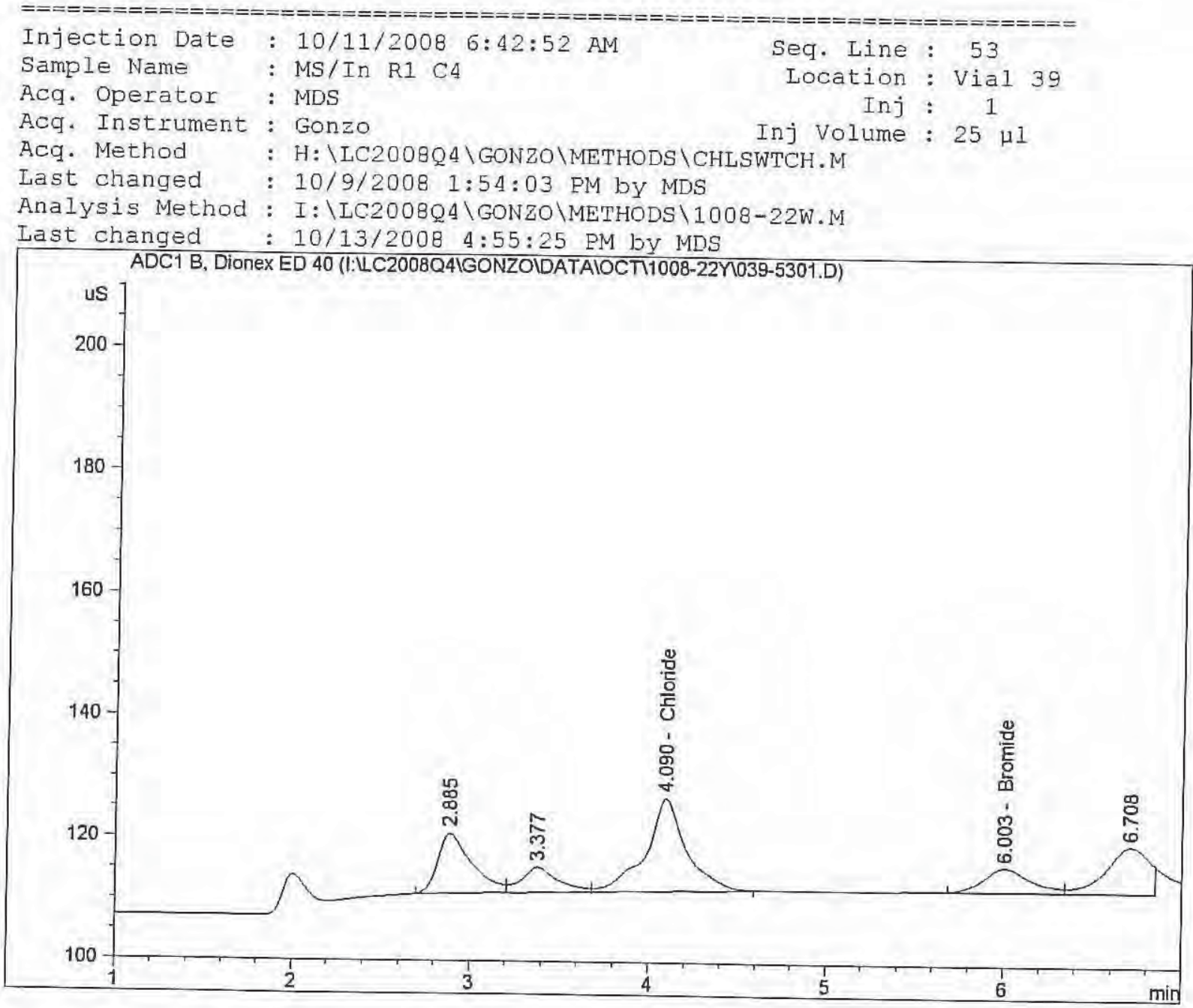

External Standard Report

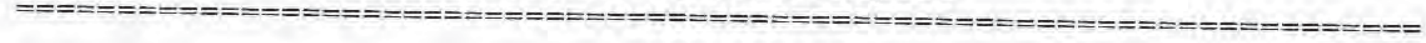

$\begin{array}{lll}\text { Sorted By } & : & \text { Signal } \\ \text { Calib. Data Modified } & : & \text { Monday, October 13, } 2008 \text { 4:55:23 PM } \\ \text { Multiplier } & : & 1.0000 \\ \text { Dilution } & : & 1.0000\end{array}$

Use Multiplier \& Dilution Factor with ISTDs

Signal 1: ADC1 B, Diones ED 40

\begin{tabular}{ccccc}
$\begin{array}{c}\text { RetTime Type } \\
\text { [min] }\end{array}$ & $\begin{array}{c}\text { Area } \\
\text { [uS*s] }\end{array}$ & Ant/Area & $\begin{array}{c}\text { Amount Grp Name } \\
{[\text { Ug/mL] }}\end{array}$ & \\
\hdashline .090 VB & 246.30531 & $1.24582 e-2$ & 3.06851 & Chloride \\
6.003 BV & 75.76545 & $2.99869 e-2$ & 2.27197 & Bromide
\end{tabular}

Totals :

5.34049

Results obtained with enhanced integrator!

*** End of Report *** 
1008-22 Matrix Spike

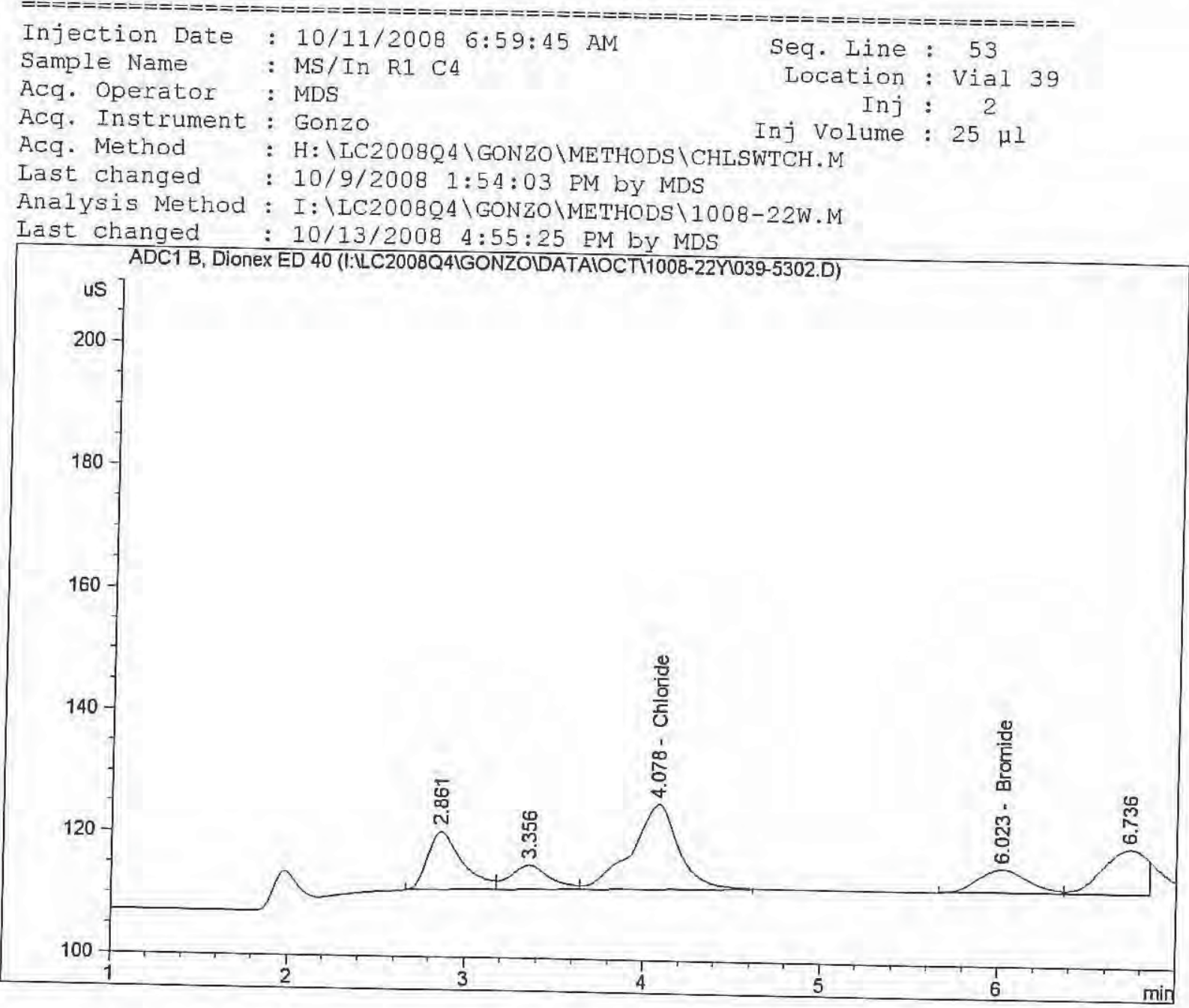

External Standard Report

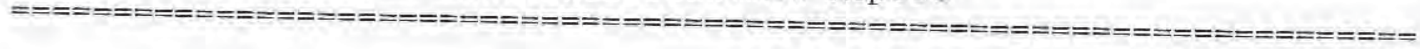

Sorted By
Calib. Data Modified :
Multiplier
Dilution

Signal 1: ADC1 B, Dionex ED 40

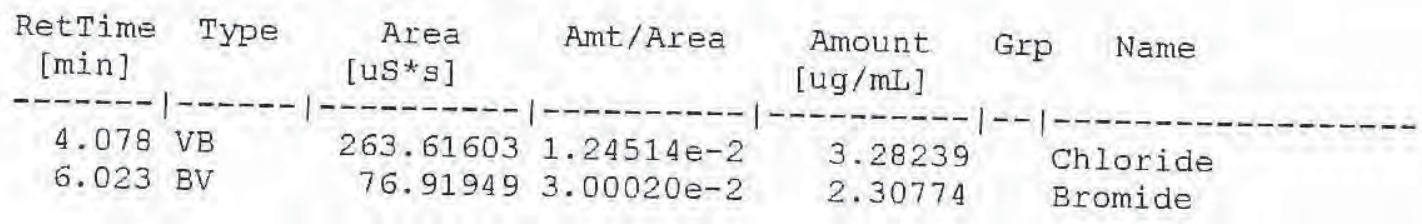

Totals :

5.59012

Results obtained with enhanced integrator!

$$
* * * \text { End of Report *** }
$$




\section{Curve(s)/QA Point(s) Chromatograms}

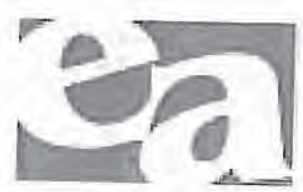




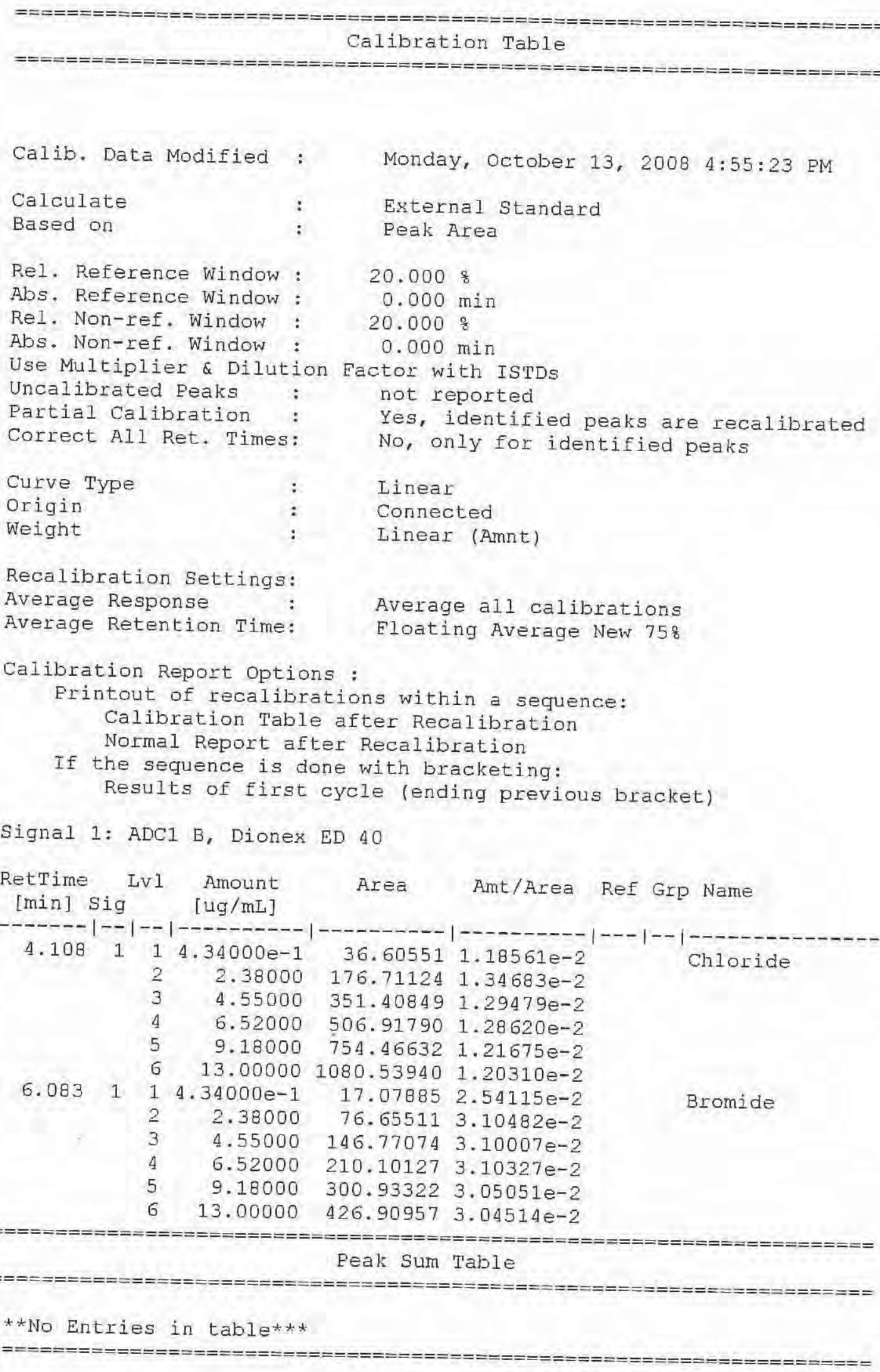




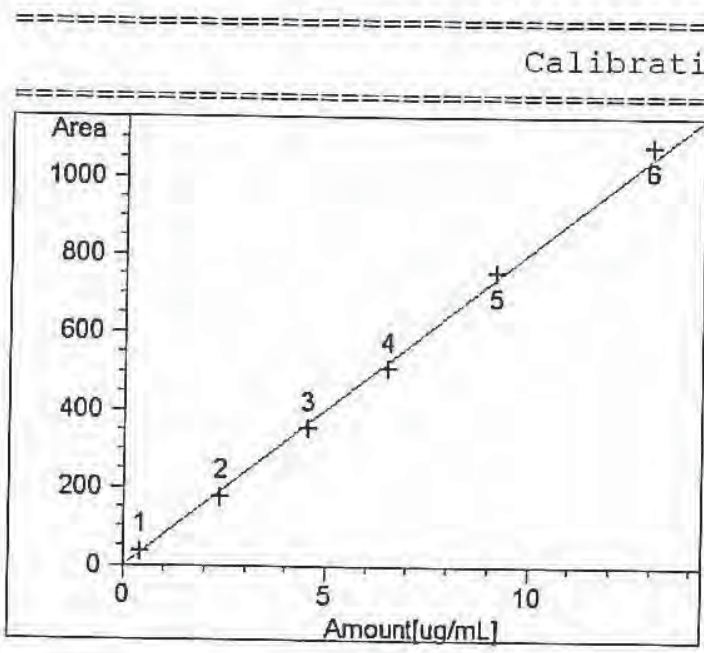

Chloride at exp. RT: 4.108

ADC1 B, Dionex ED 40

Correlation:

0.99908

Residual Std. Dev.: 21.68574

Formula: $\mathrm{y}=\mathrm{m} x+\mathrm{b}$

$$
\begin{array}{ll}
\mathrm{m}: & 80.93943 \\
\mathrm{~b}: & -2.05847 \\
\mathrm{x}: & \text { Amount }[\mathrm{ug} / \mathrm{mL}] \\
\mathrm{y}: & \text { Area }
\end{array}
$$

Calibration Level Weights:

Level $1:$ : 1

Level $2: 0.182353$

Level $3: 0.095385$

Level $4: 0.066564$

Level $5: 0.047277$

Level $6: 0.033385$

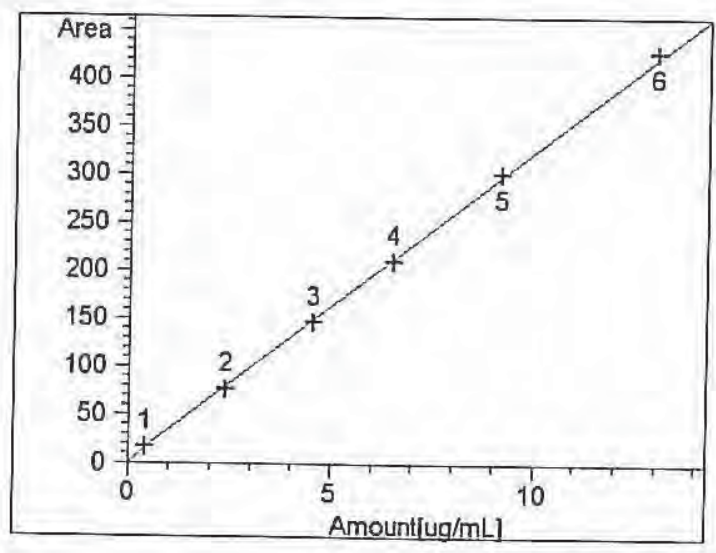

Bromide at exp. RT: 6.083

ADC1 B, Diones ED 40

Correlation:

0.99983

Residual Std. Dev.:

3.55845

Eormula: $y=m x+b$

$$
\mathrm{m}: \quad 32.26868
$$

b: $\quad 2.45182$

$\mathrm{x}$ : Amount [ug/mL]

$y$ : Area

Calibration Level Weights:

Level 1 : 1

Level $2: 0.182353$

Leve1 $3: 0.095385$

Level 4:0.066564

Level $5: 0.047277$

Level $6: 0.033385$ 


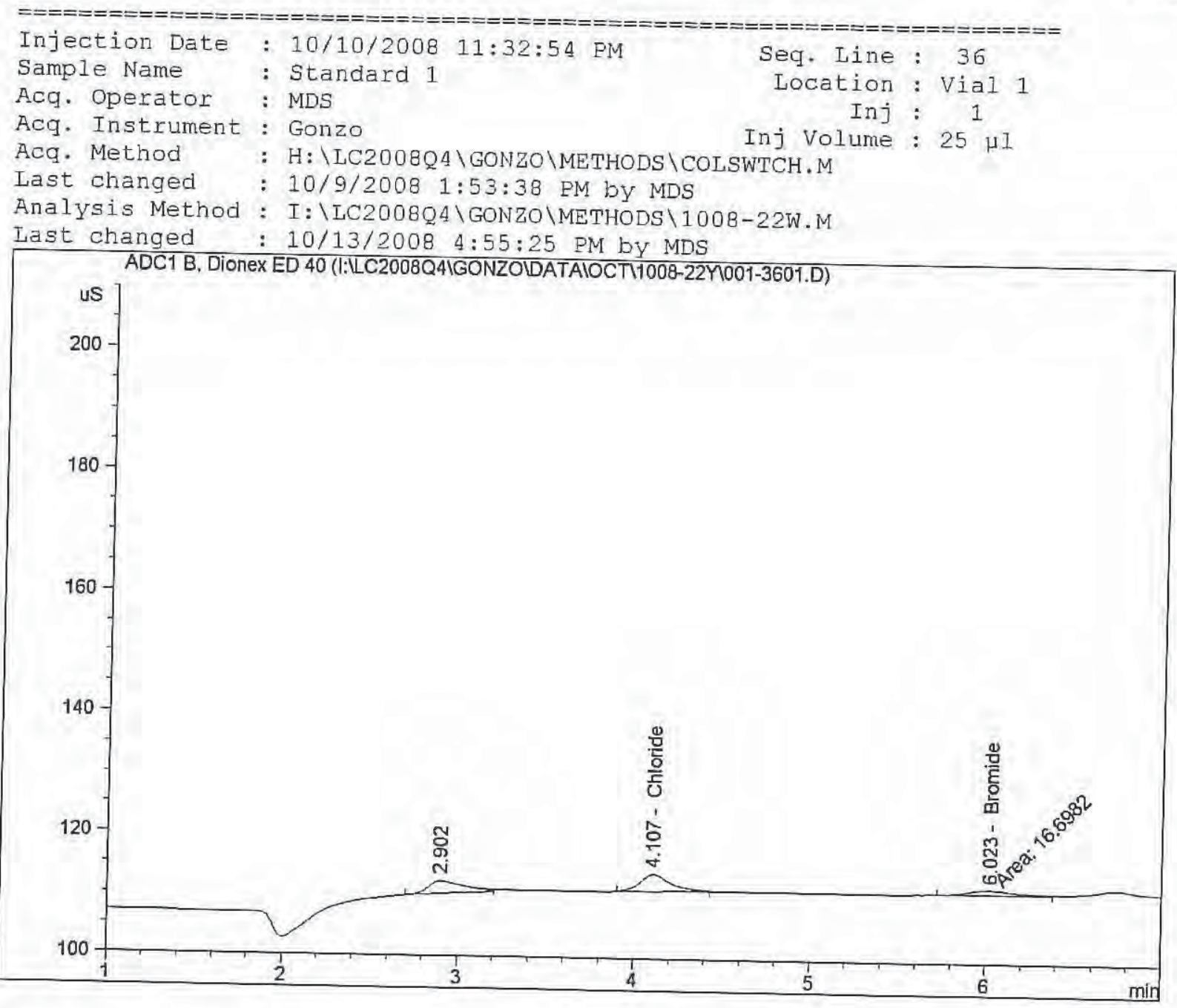

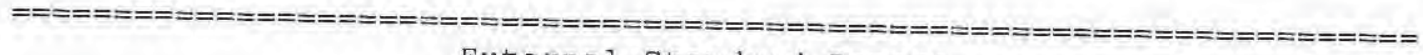

External Standard Report

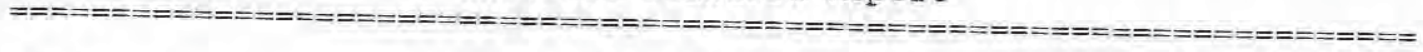

$\begin{array}{lll}\text { Sorted By } & : & \text { Signal } \\ \text { Calib. Data Modified } & : & \text { Monday, October 13, } 2008 \text { 4:55:23 PM } \\ \text { Multiplier } & : & 1.0000 \\ \text { Dilution } & : & 1.0000\end{array}$

Use Multiplier \& Dilution Factor with ISTDS

Signal 1: ADC1 B, Dionex ED 40

\begin{tabular}{|c|c|c|c|c|c|c|}
\hline $\begin{array}{l}\text { RetTime } \\
\text { [min] }\end{array}$ & Type & $\begin{array}{r}\text { Area } \\
{\left[u{ }^{*} s\right]}\end{array}$ & Ant/Area & $\begin{array}{l}\text { Amount } \\
{[\mathrm{ug} / \mathrm{mL}]}\end{array}$ & Grp & Name \\
\hline $\begin{array}{l}4.107 \\
6.023\end{array}$ & $\begin{array}{l}\mathrm{BB} \\
\mathrm{MM}\end{array}$ & $\begin{array}{l}36.69376 \\
16.69819\end{array}$ & $\begin{array}{l}\text { 1. } 30480 e-2 \\
\text { 2. } 64395 e-2\end{array}$ & $\begin{array}{l}4.78781 \mathrm{e}-1 \\
4.41492 \mathrm{e}-1\end{array}$ & & de \\
\hline
\end{tabular}

Totals :

$9.20273 e-1$

Results obtained with enhanced integrator!

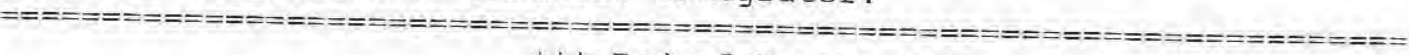

$\star * *$ End of Report $* * \star$
} 


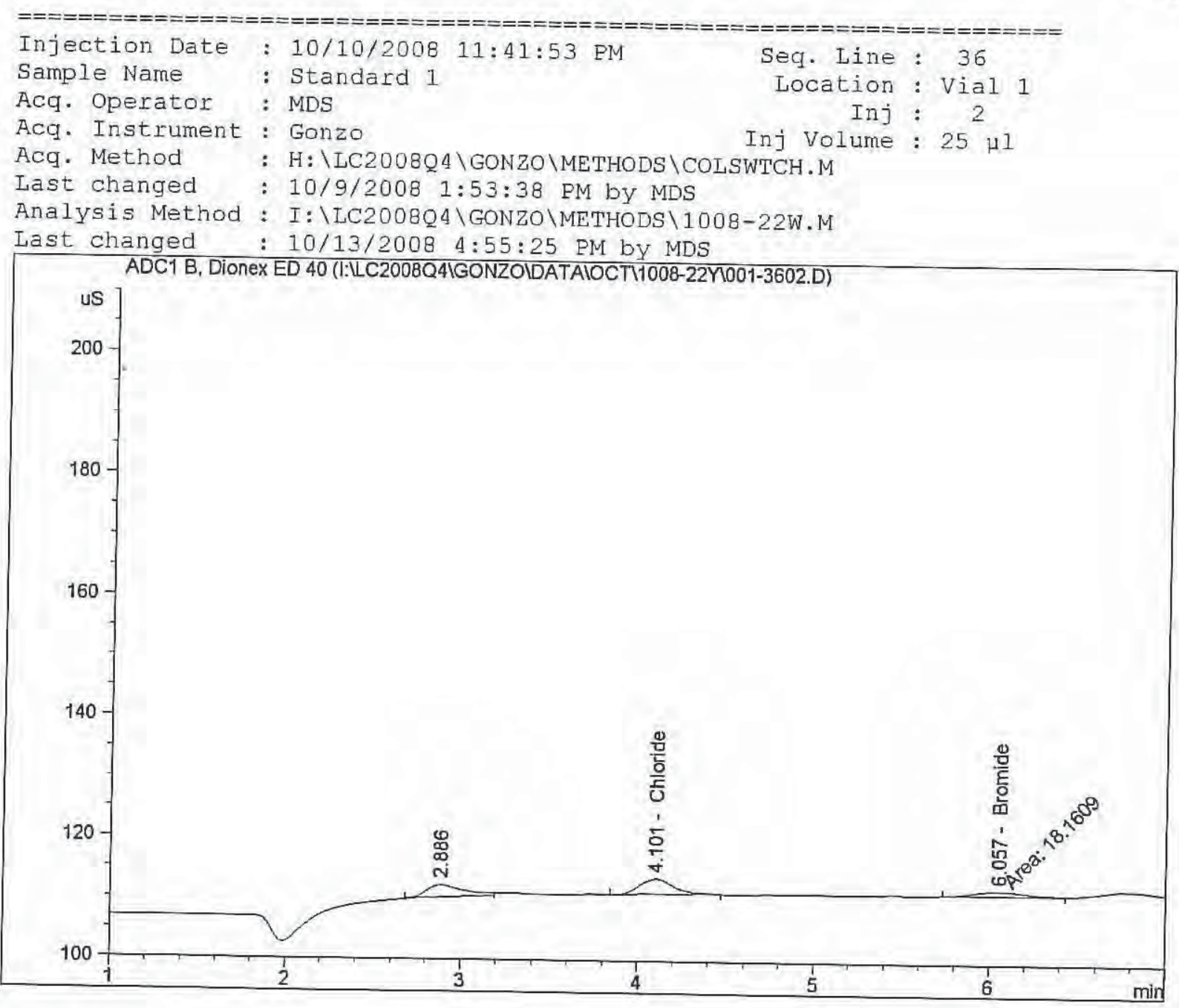

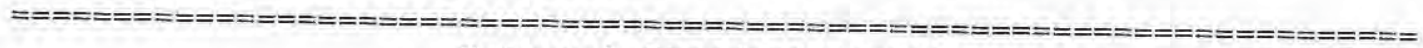

External standard Report

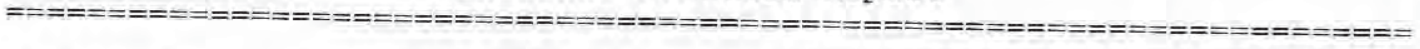

$\begin{array}{lll}\text { Sorted By } & : & \text { Signal } \\ \text { Calib. Data Modified } & : & \text { Monday, October 13, 2008 4:55:23 PM } \\ \text { Multiplier } & : & 1.0000 \\ \text { Dilution } & : & 1.0000\end{array}$

Use Multiplier \& Dilution Factor with ISTDS

Signal 1: ADCl B, Dionex ED 40

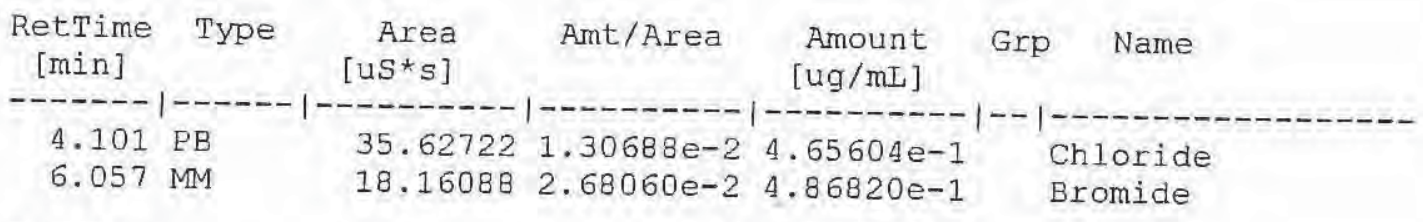

Totals : $\quad 9.52424 \mathrm{e}-1$

Results obtained with enhanced integrator!

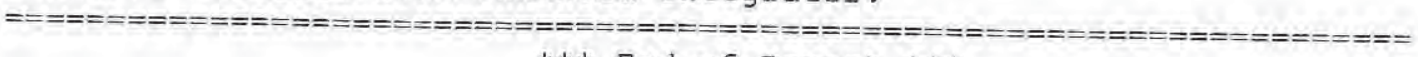

$* \star *$ End of Report ***
} 


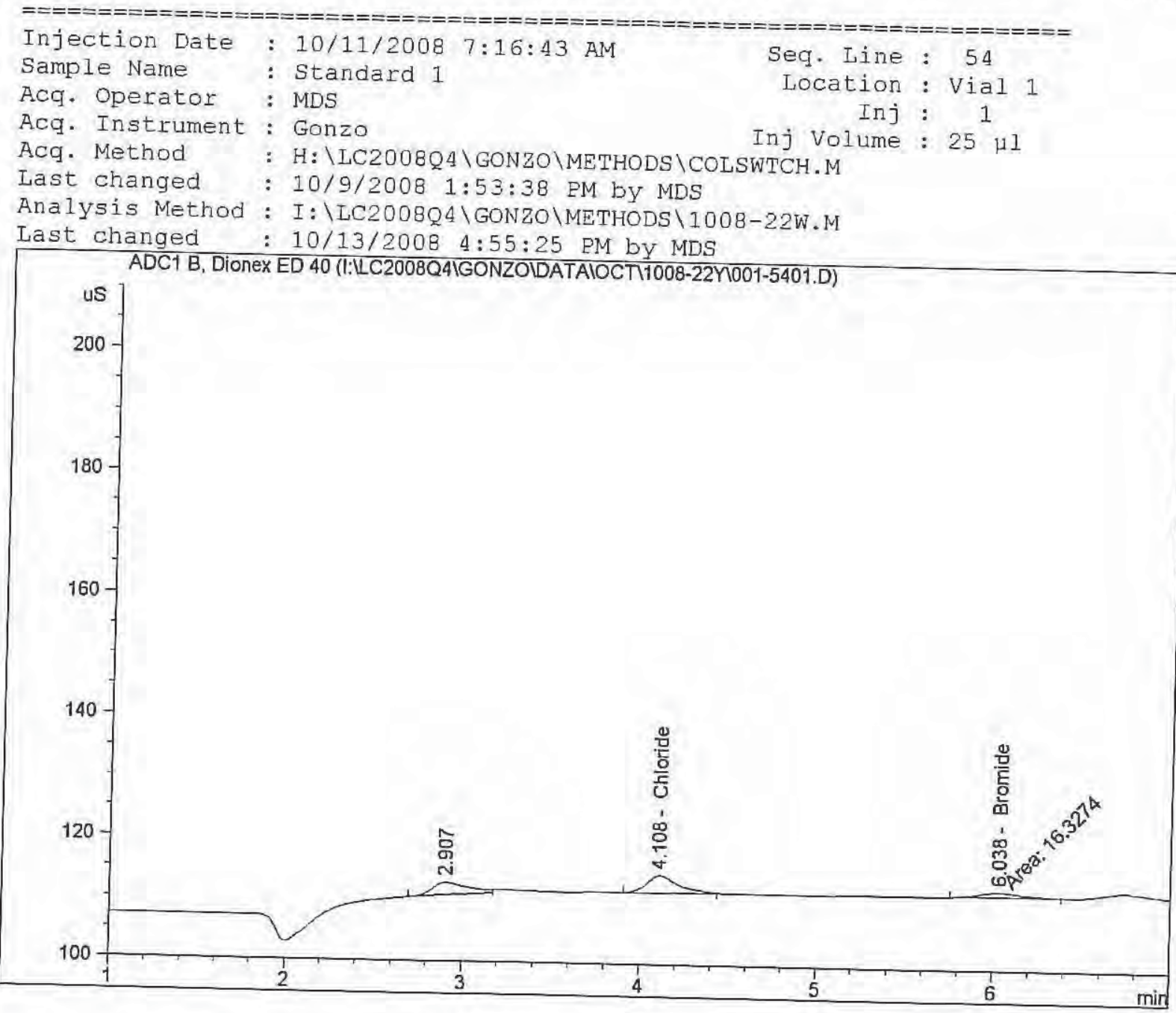

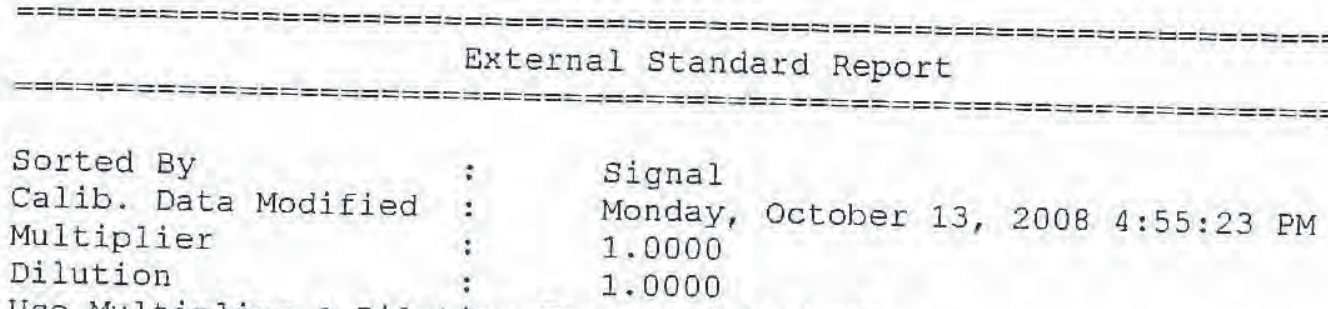

Use Multiplier \& Dilution Eactor with ISTDs

Signal 1: ADC1 B, Dionex ED 40

\begin{tabular}{|c|c|c|c|c|c|}
\hline $\begin{array}{l}\text { RetTime } \\
\text { [min] }\end{array}$ & Type & $\begin{array}{r}\text { Area } \\
{\left[u S^{\star} s\right]}\end{array}$ & Ant/Area & $\begin{array}{l}\text { Amount } \\
{[\mathrm{ug} / \mathrm{mL}]}\end{array}$ & Name \\
\hline$-2---20$ & $1--$ & $-------\infty$ & 1 - - - - - - - & $1--\cdots-\cdots$ & \\
\hline $\begin{array}{l}4.108 \\
6.038\end{array}$ & $\begin{array}{l}\mathrm{BB} \\
\mathrm{MM}\end{array}$ & $\begin{array}{l}36.85690 \\
16.32742\end{array}$ & $\begin{array}{l}1.30449 e-2 \\
2.63727 e-2\end{array}$ & $\begin{array}{l}\text { 4. } 80796 e-1 \\
\text { 4. } 30598 \mathrm{e}-1\end{array}$ & $\begin{array}{l}\text { Chloride } \\
\text { Bromide }\end{array}$ \\
\hline Totals : & & & & $9.11394 \mathrm{e}-1$ & \\
\hline
\end{tabular}

Results obtained with enhanced integrator! 


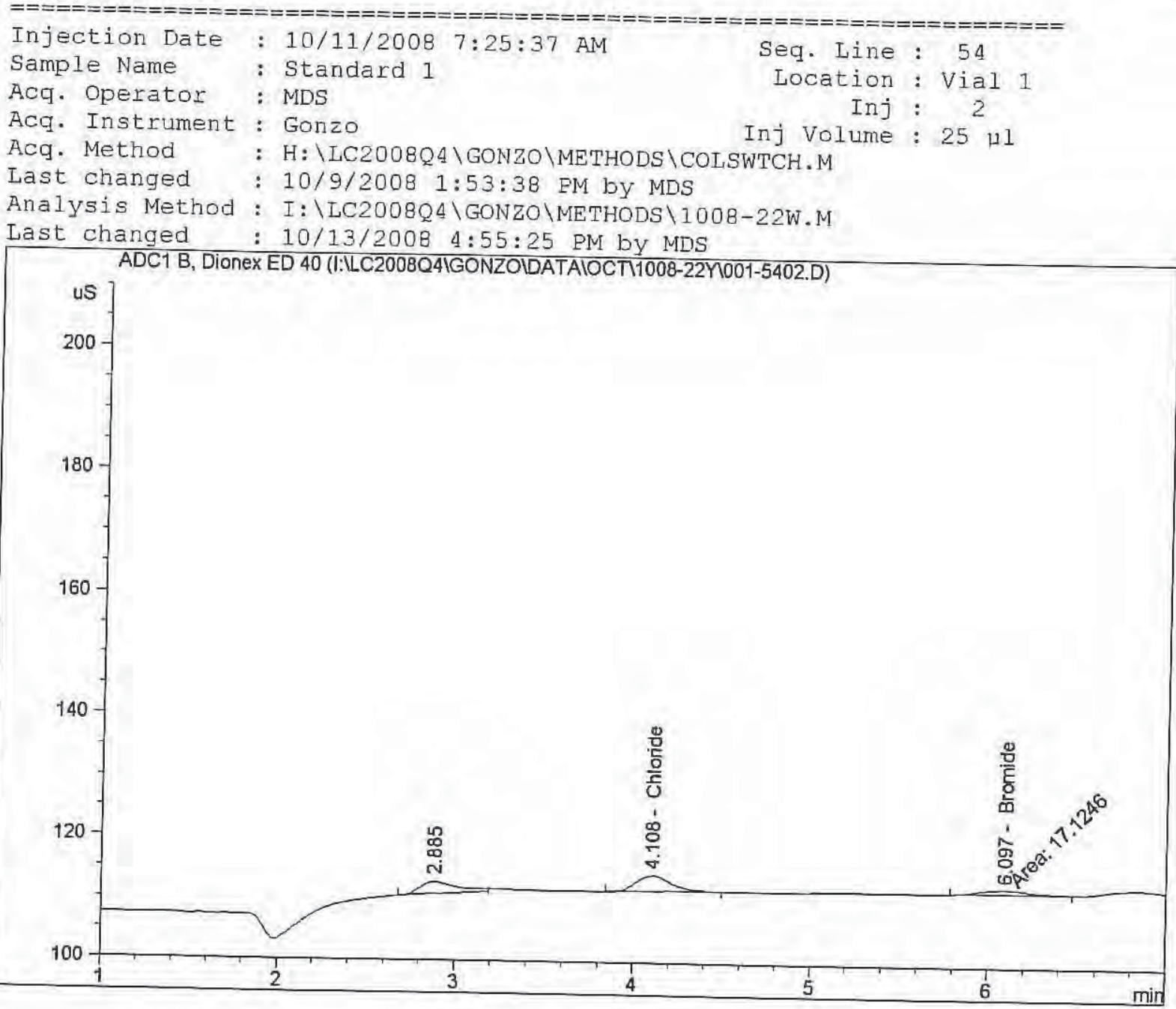

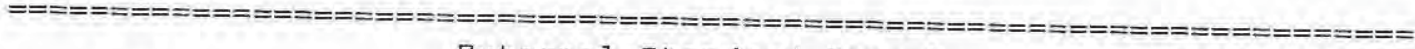

External Standard Report

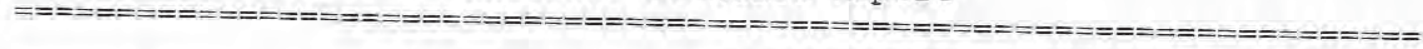

$\begin{array}{lll}\text { Sorted By } & : & \text { Signal } \\ \text { Calib. Data Modified } & : & \text { Monday, October 13, 2008 4:55:23 PM } \\ \text { Multiplier } & : & 1.0000 \\ \text { Dilution } & : & 1.0000\end{array}$

Use Multiplier \& Dilution Factor with ISTDs

Signal 1: ADC1 B, Dionex ED 40

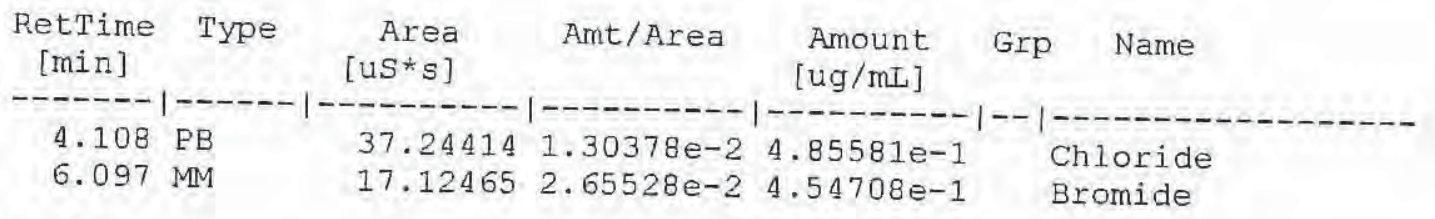

Totals : $\quad 9.40288 \mathrm{e}-1$

Results obtained with enhanced integrator!

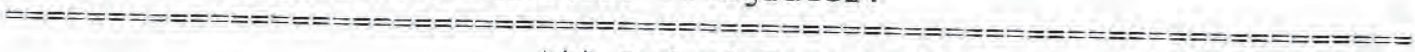

$* * *$ End of Report ***
} 


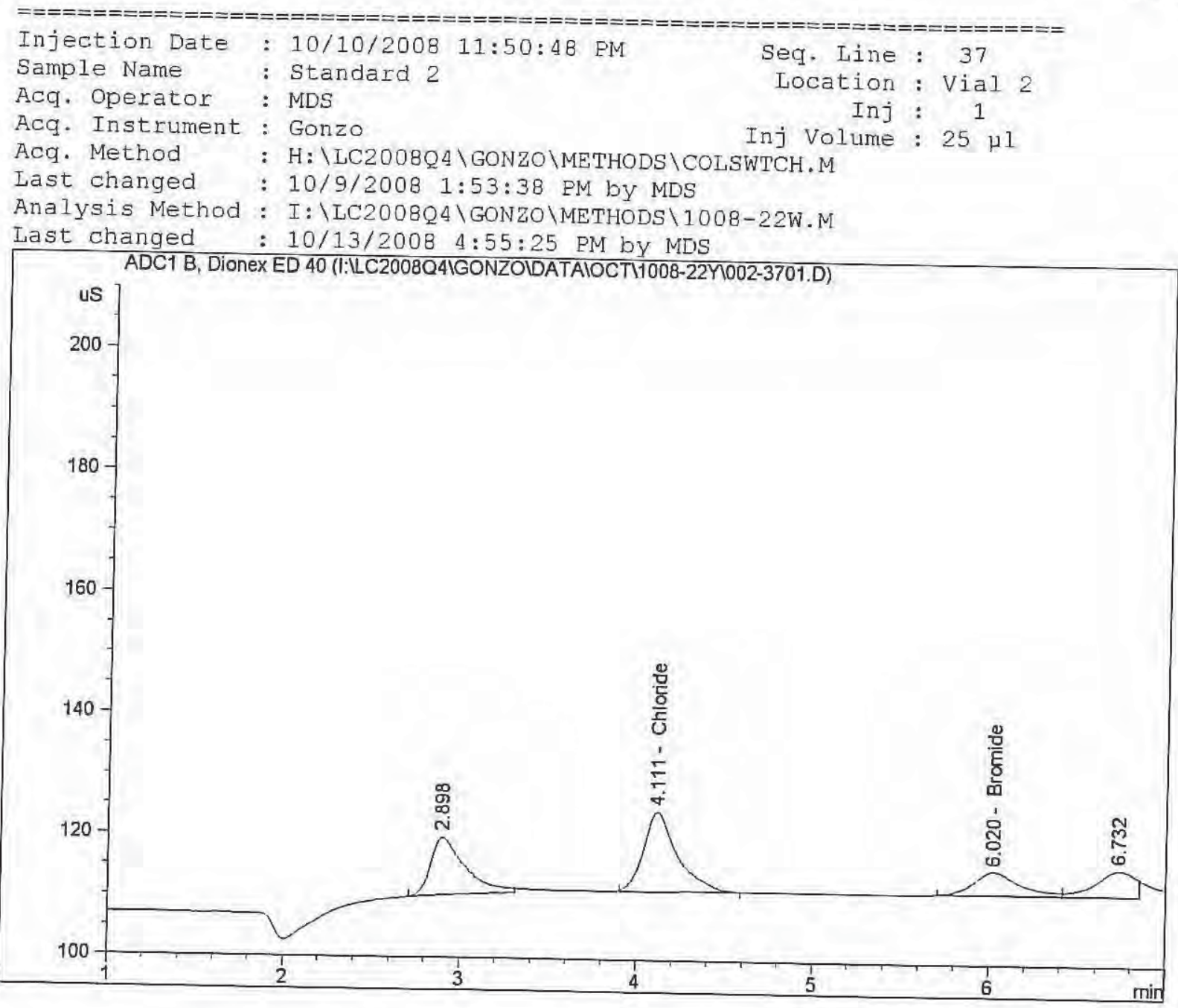

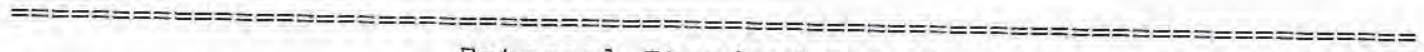
External Standard Report

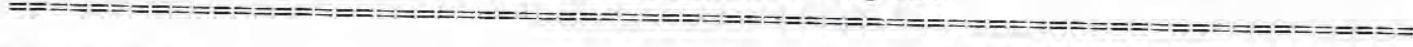

Sorted By : Signal

Calib. Data Modified : Monday, October 13, 2008 4:55:23 PM

Multiplier : 1.0000

Dilution : 1.0000

Use Multiplier \& Dilution Factor with ISTDs

Signal 1: ADC1 B, Dionex ED 40

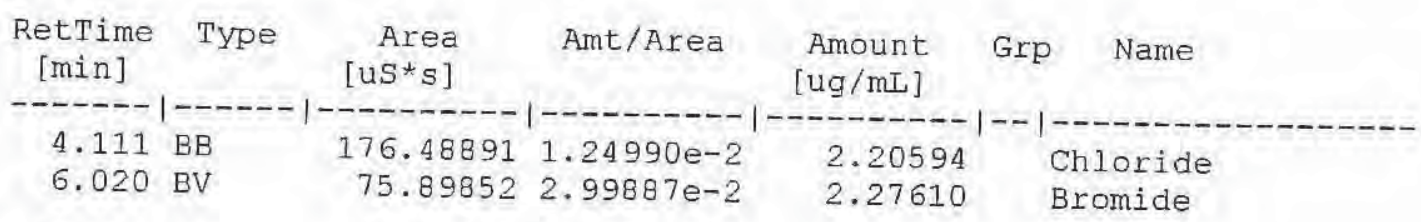

Totals :

$$
4.48204
$$

Results obtained with enhanced integrator!

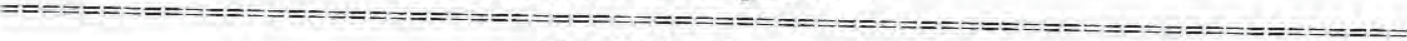

$$
\text { *** End of Report *** }
$$
}




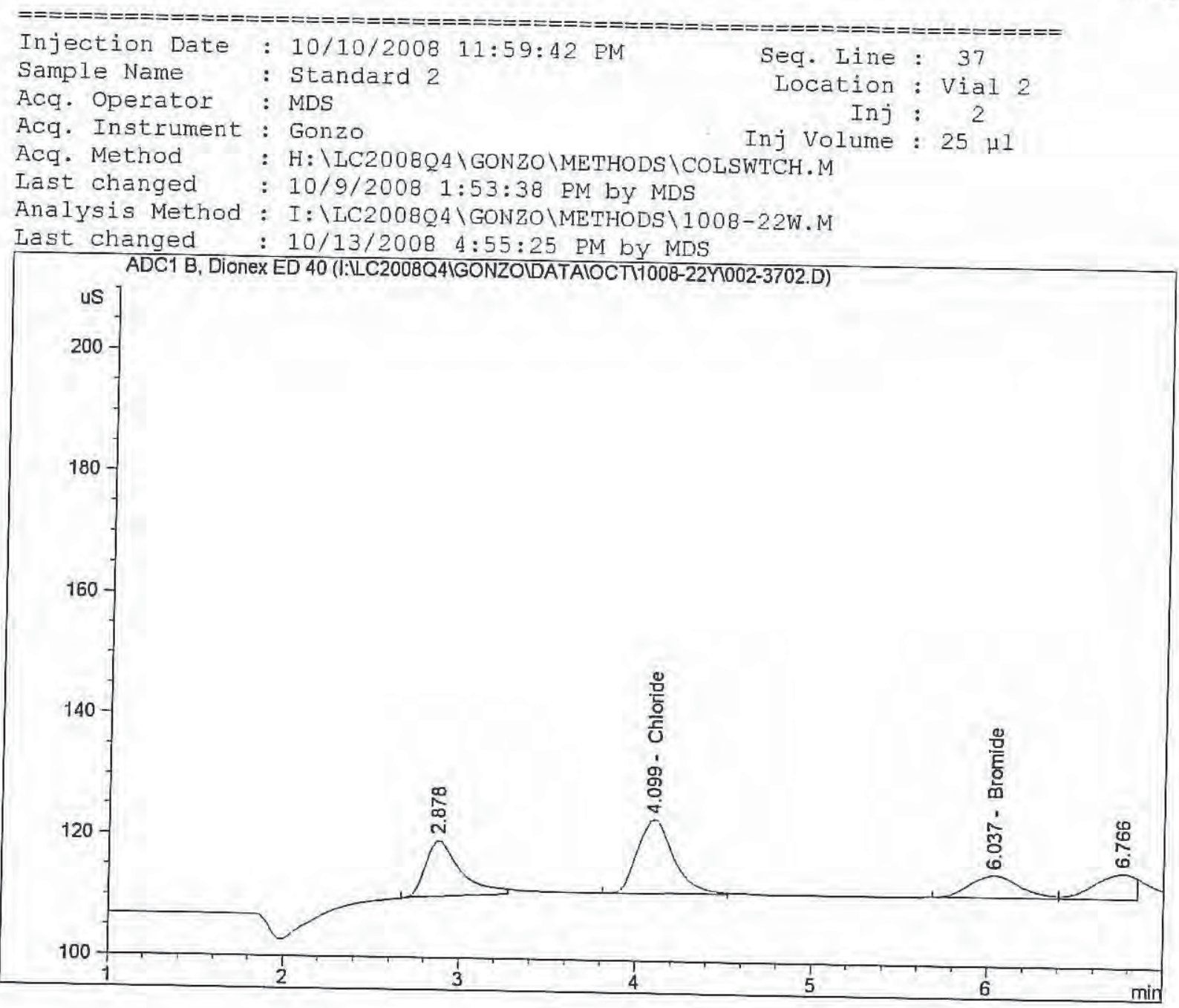

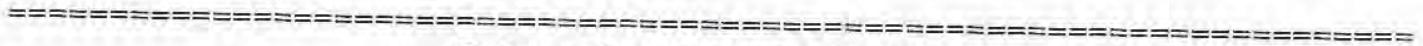

External Standard Report

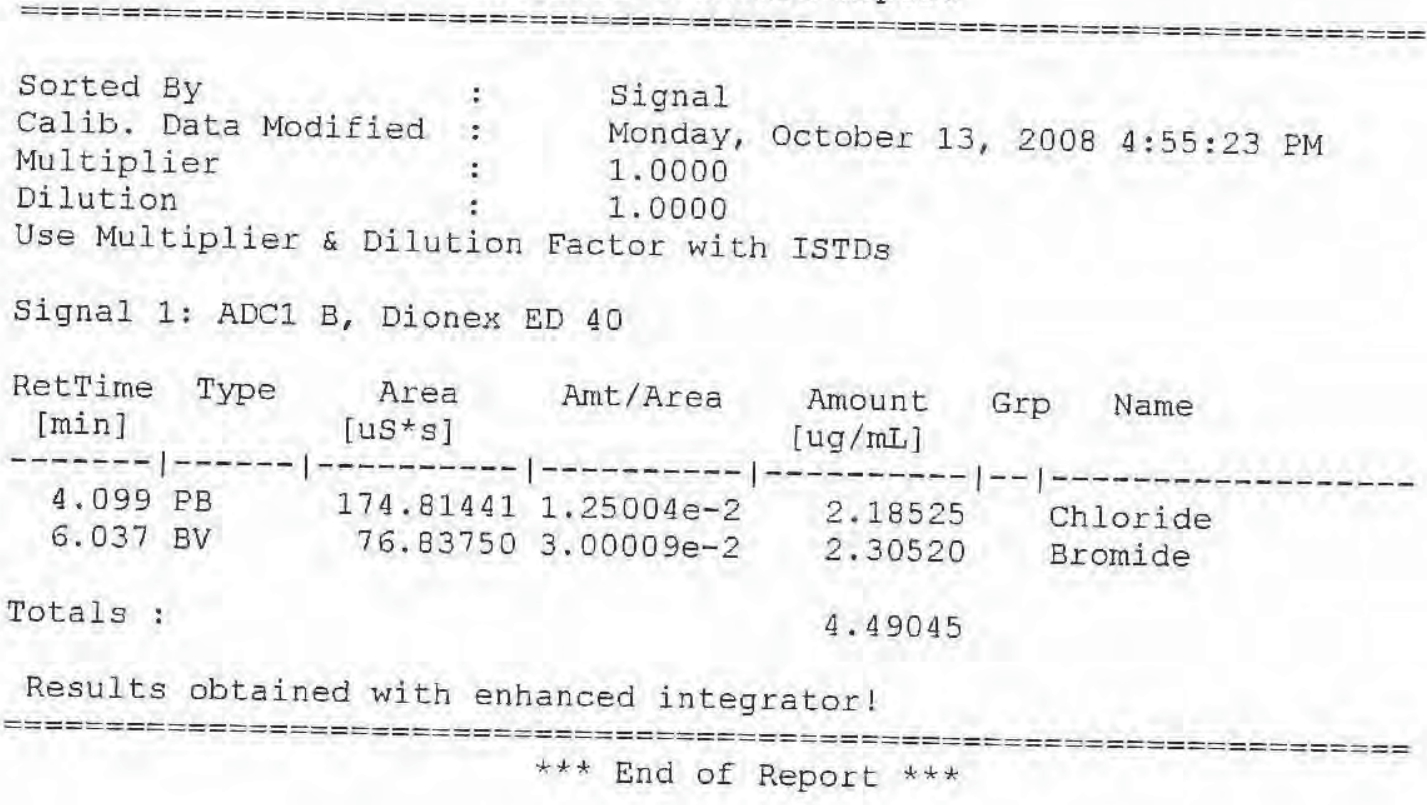




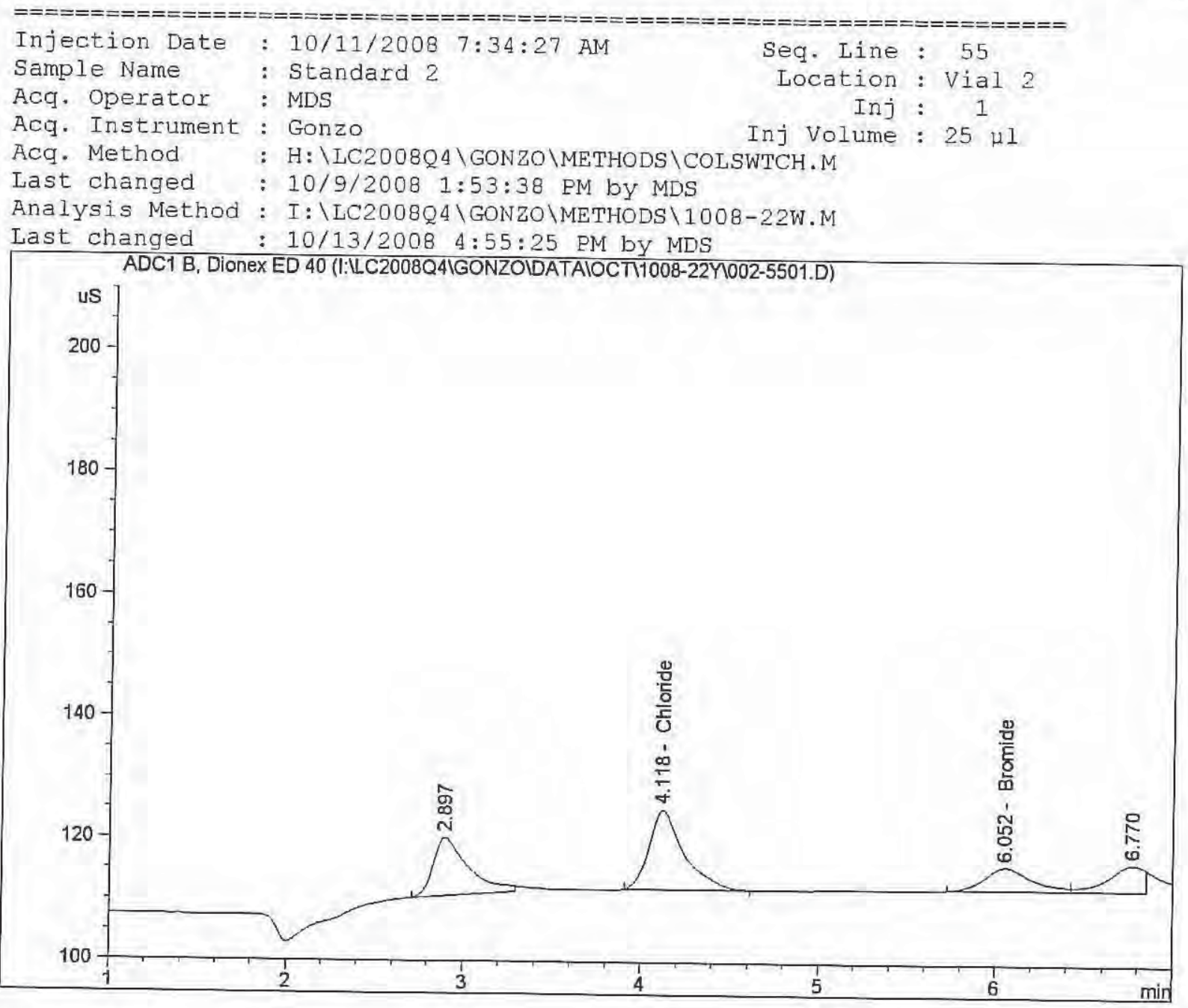

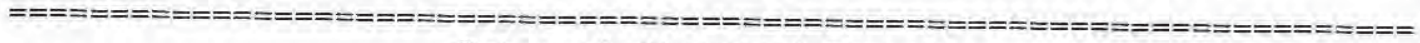
External Standard Report

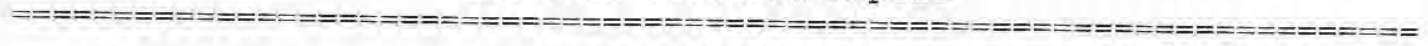

$\begin{array}{lll}\text { Sorted By } & : & \text { Signal } \\ \text { Calib. Data Modified } & : & \text { Monday, October 13, } 2008 \text { 4:55:23 PM } \\ \text { Multiplier } & : & 1.0000 \\ \text { Dilution } & : & 1.0000\end{array}$

Use Multiplier \& Dilution Eactor with ISTDs

Signal 1: ADC1 B, Dionex ED 40

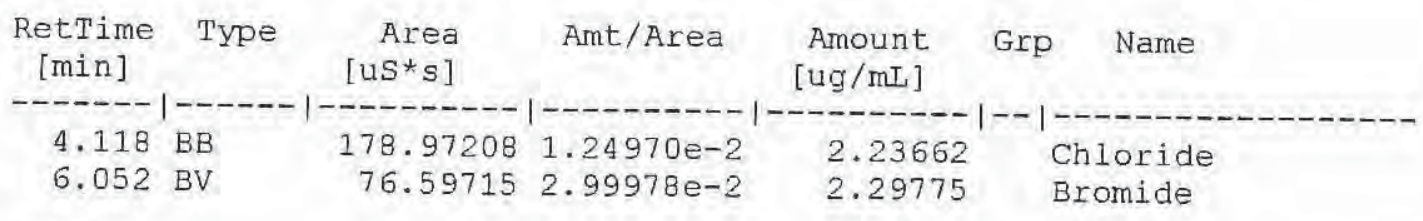

Totals:

4.53437

Results obtained with enhanced integrator!

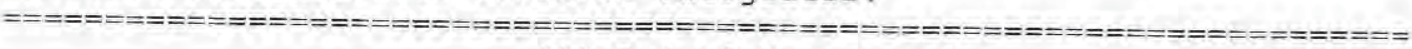
*** End of Report ***
} 


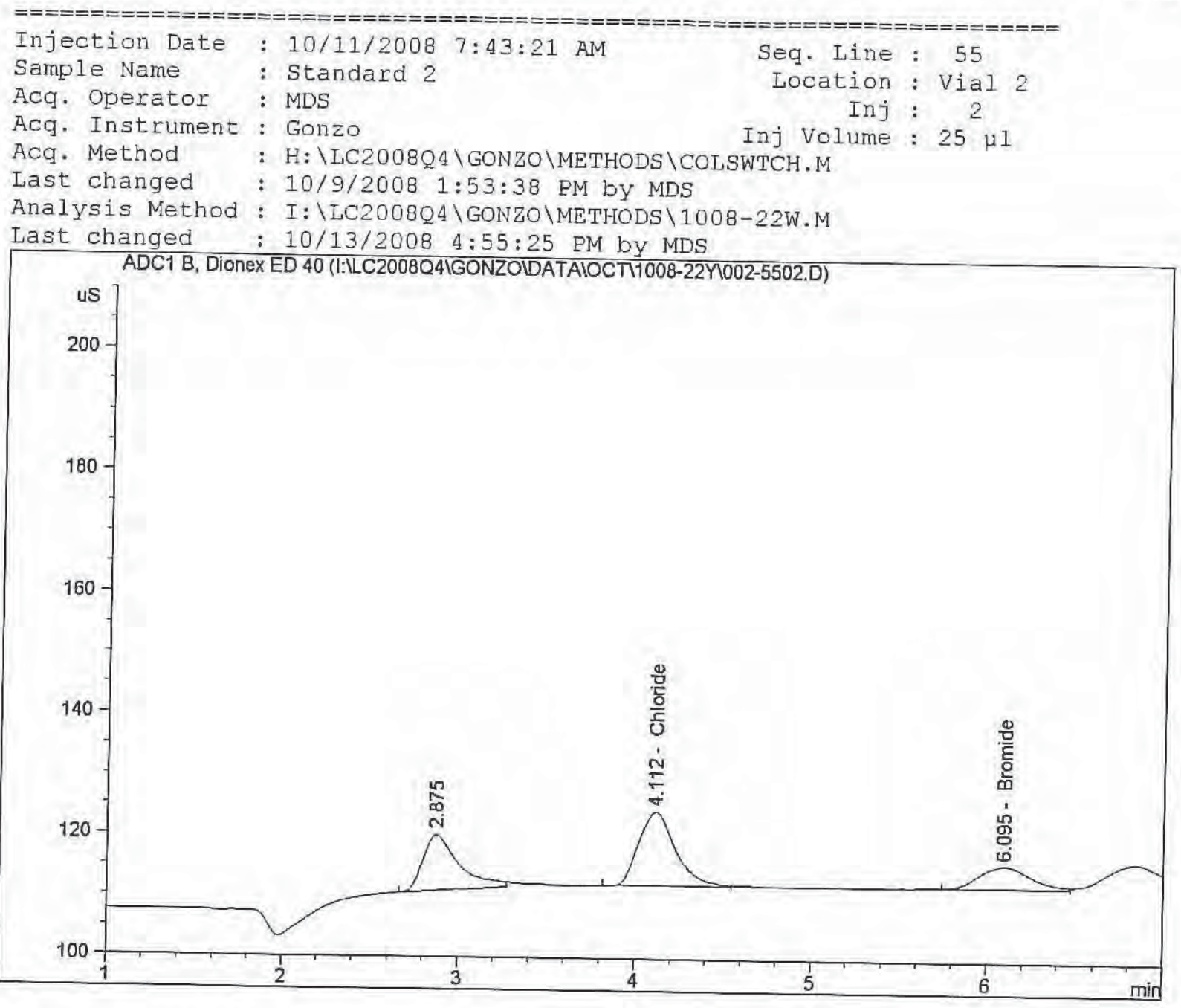

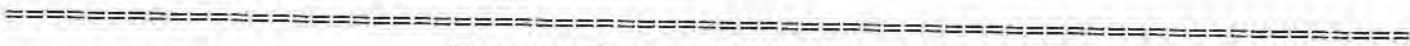

External Standard Report

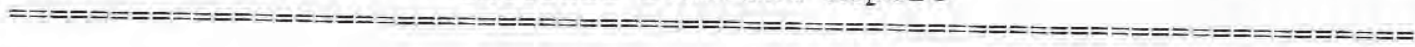

$\begin{array}{lll}\text { Sorted By } & : & \text { Signal } \\ \text { Calib. Data Modified } & : & \text { Monday, October 13, 2008 4:55:23 PM } \\ \text { Multiplier } & : & 1.0000 \\ \text { Dilution } & : & 1.0000\end{array}$

Use Multiplier \& Dilution Eactor with ISTDS

Signal 1: ADC1 B, Dionex ED 40

\begin{tabular}{|c|c|c|c|c|c|c|}
\hline $\begin{array}{c}\text { RetTime } \\
\text { [min] }\end{array}$ & Type & $\begin{array}{r}\text { Area } \\
\text { [us*s] }\end{array}$ & Ant/Area & $\begin{array}{l}\text { Amount } \\
{[\mathrm{ug} / \mathrm{mL}]}\end{array}$ & Grp & Name \\
\hline 4. 112 & PB & 176.56955 & $1.24990 e-2$ & 2.20693 & & \\
\hline 6.095 & $B V$ & 77.28726 & $3.00067 e-2$ & 2.31914 & & omide \\
\hline
\end{tabular}

Totals :

$$
4.52607
$$

Results obtained with enhanced integrator! 


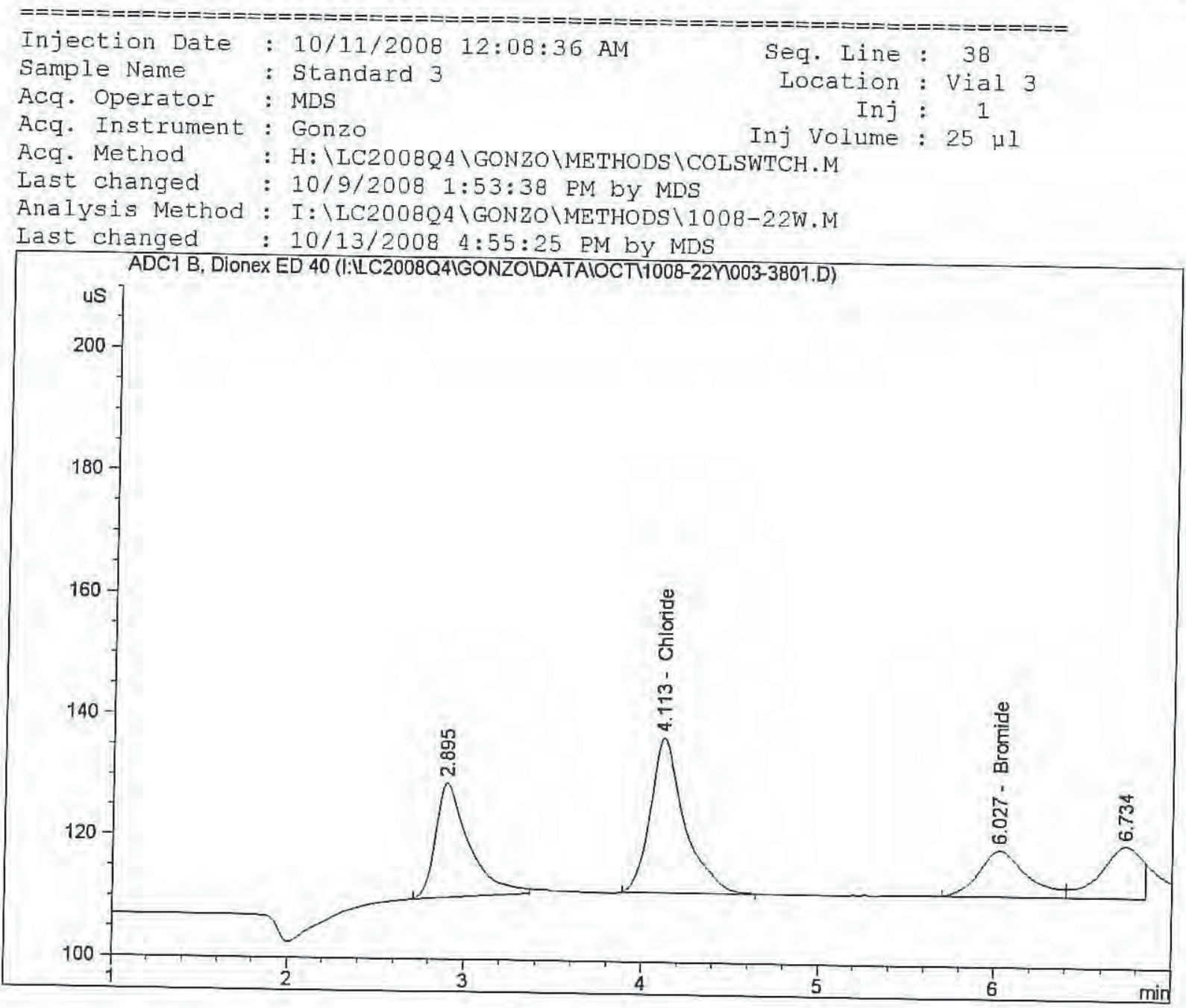

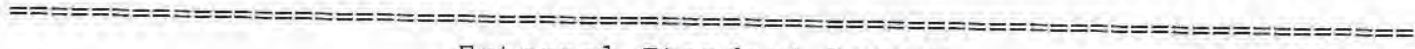

External Standard Report

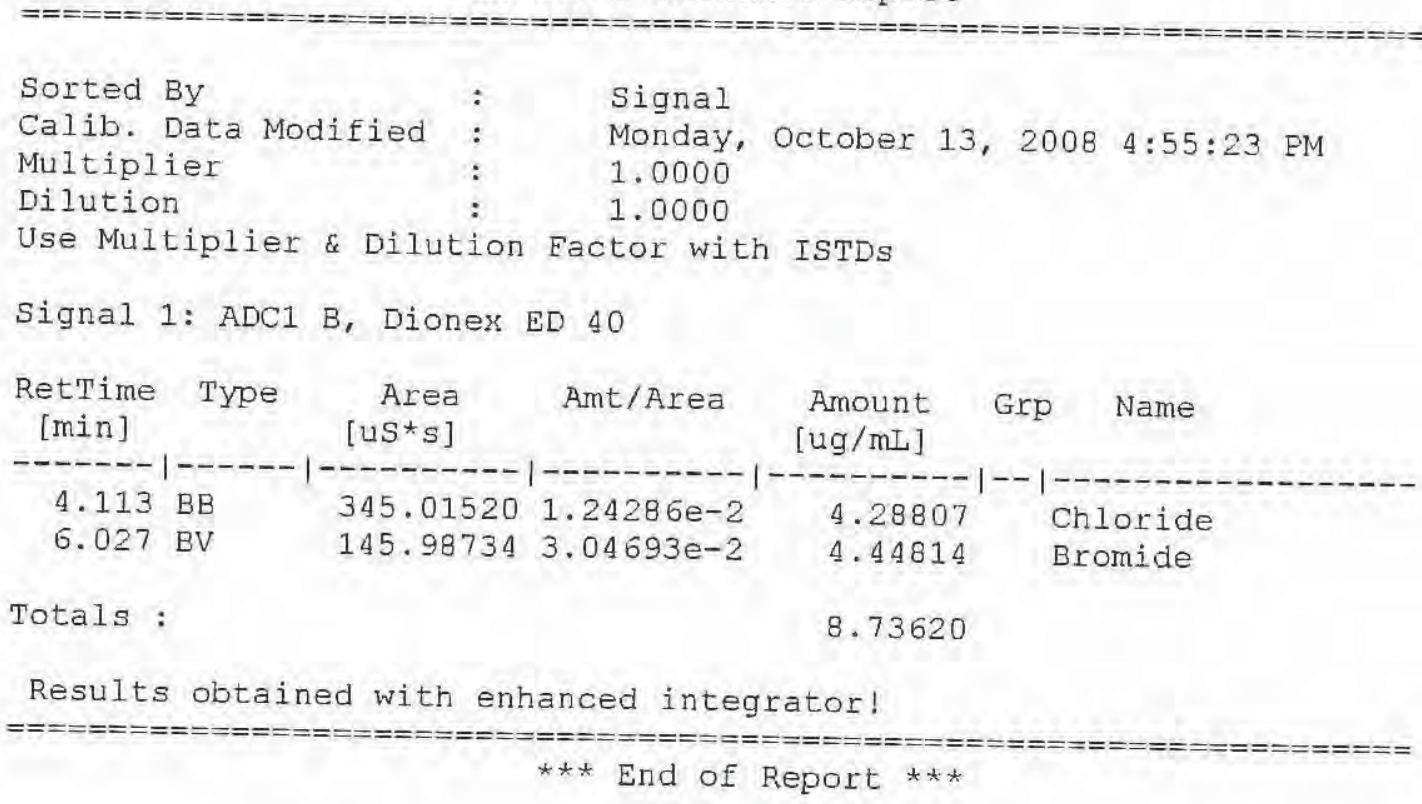




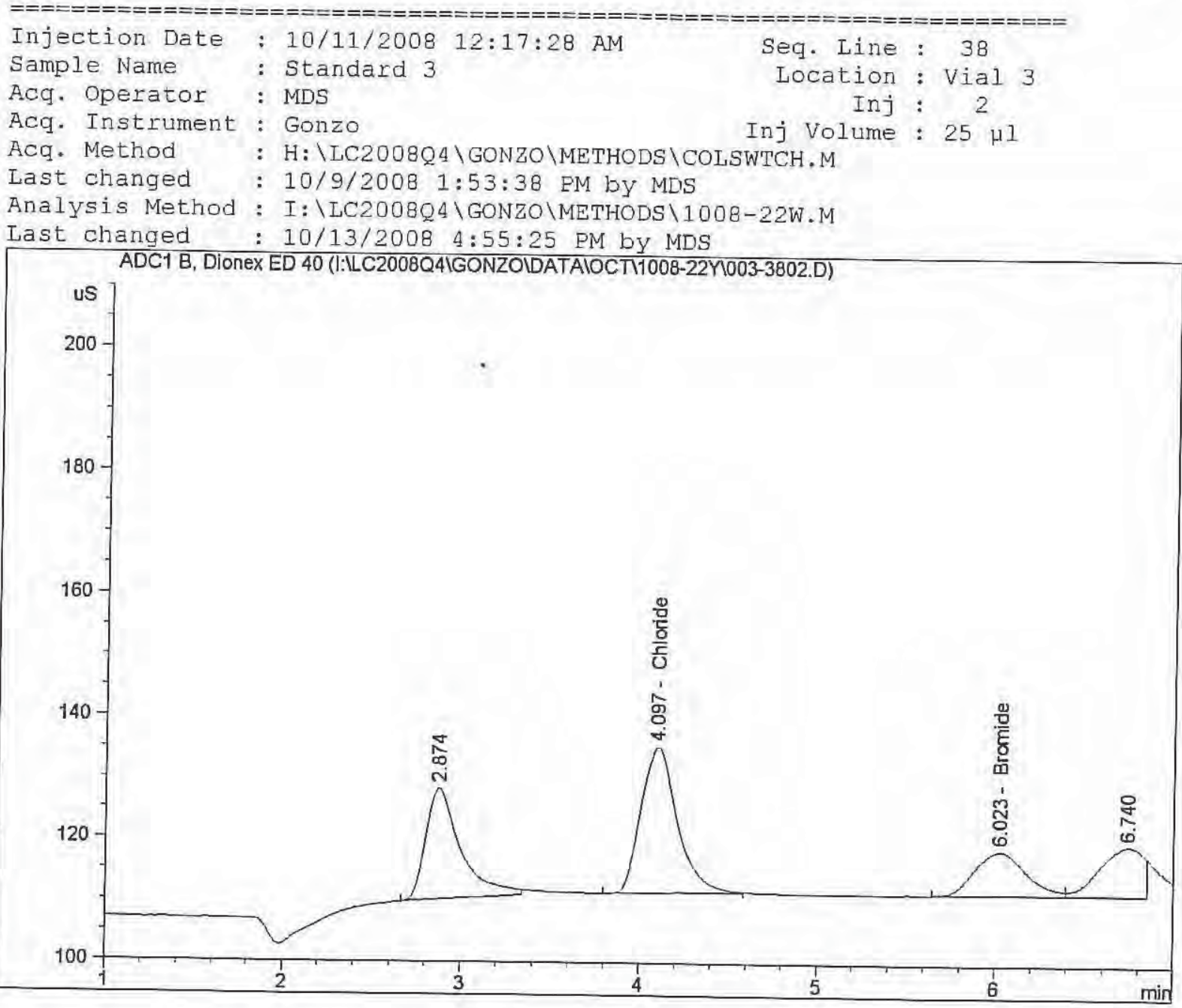

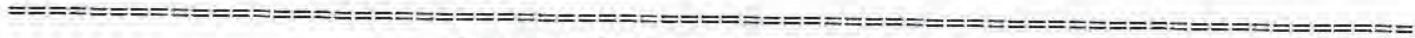
External Standard Report

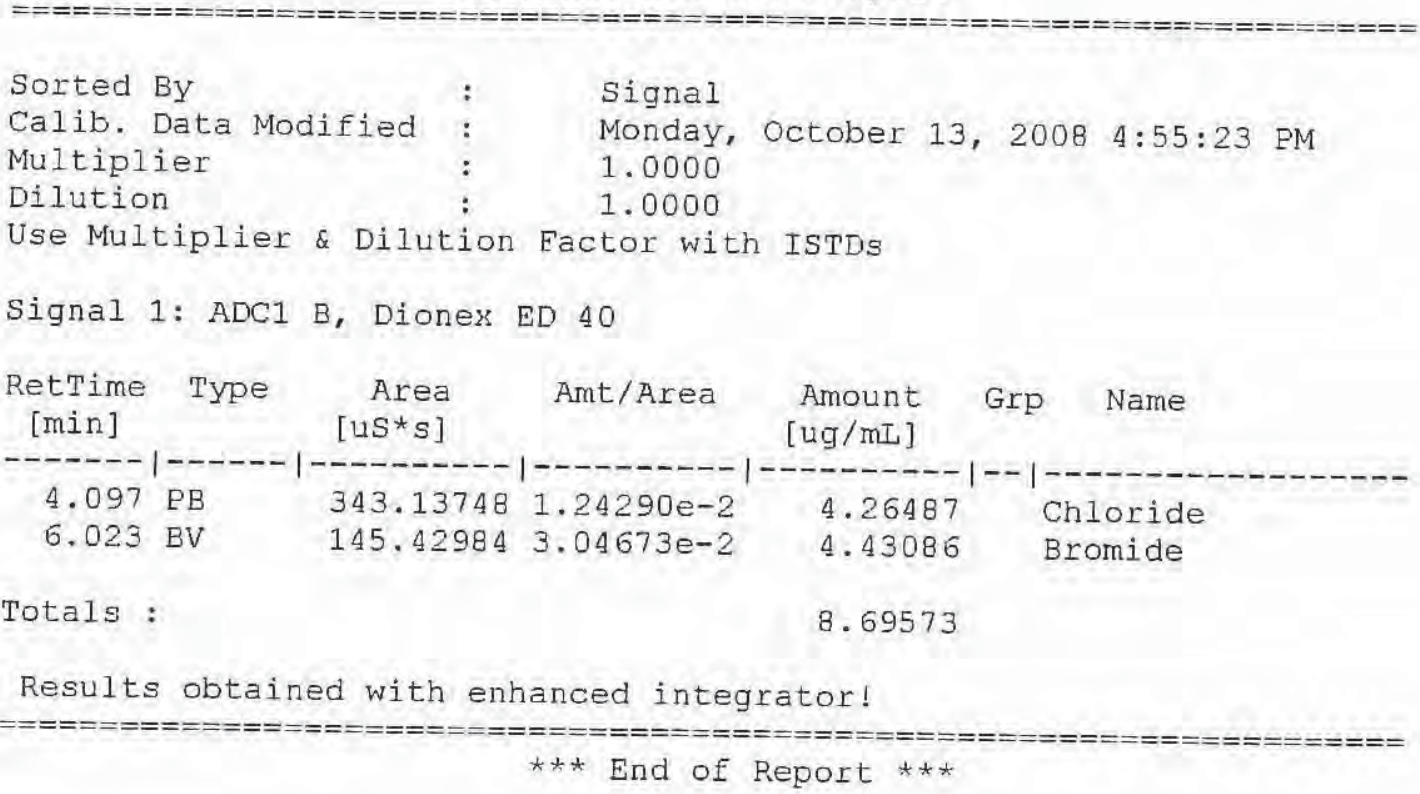




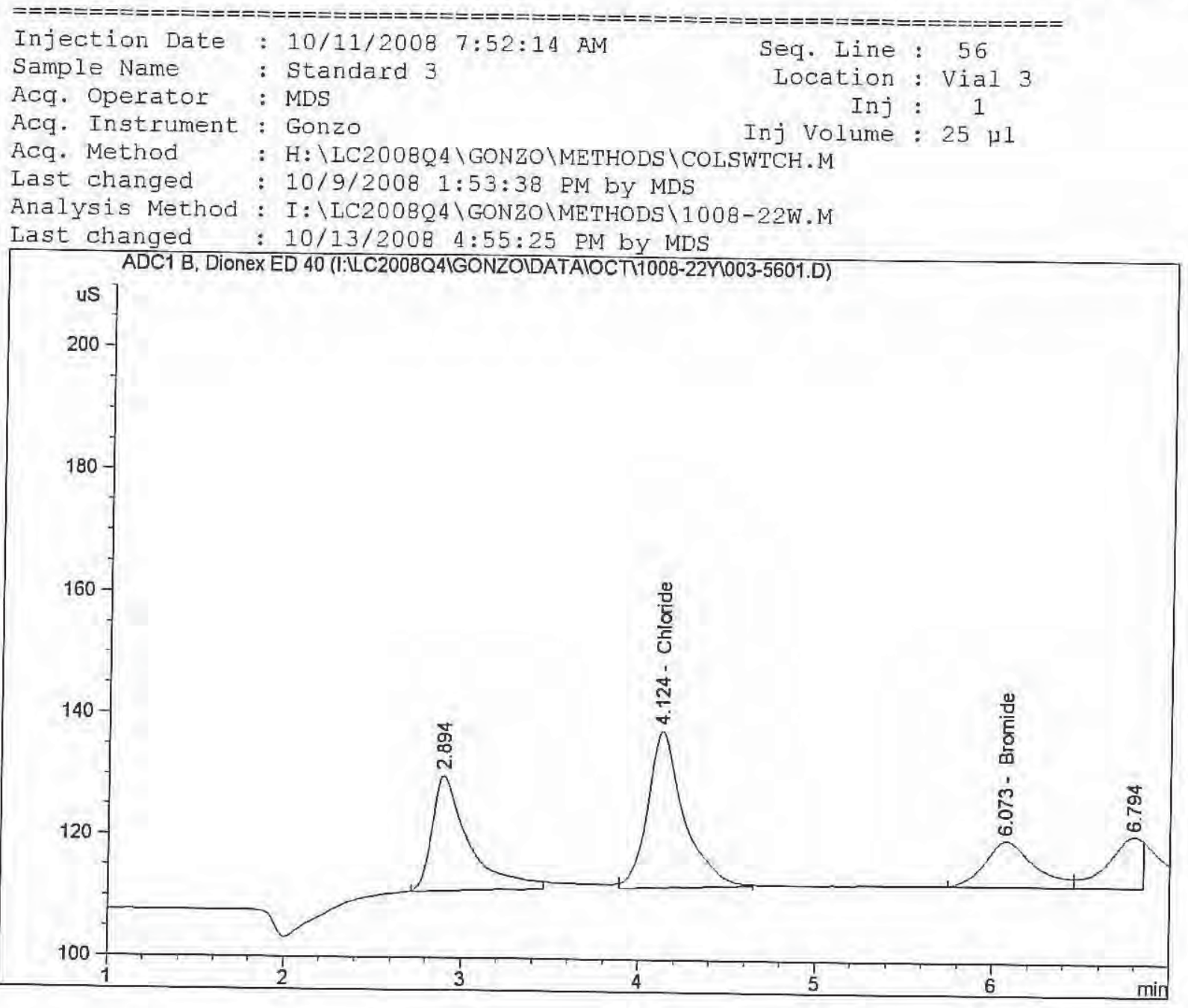

\section{External Standard Report}

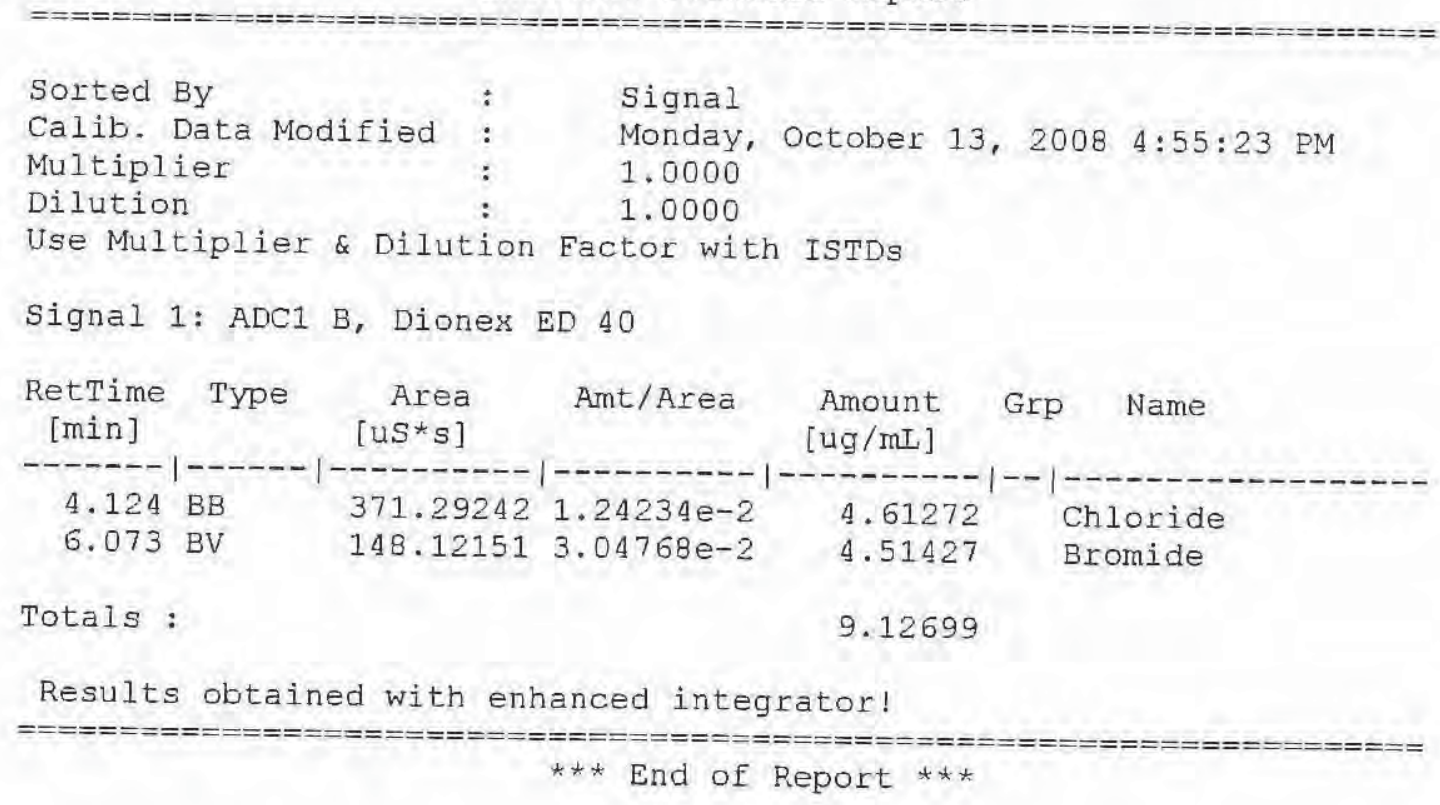




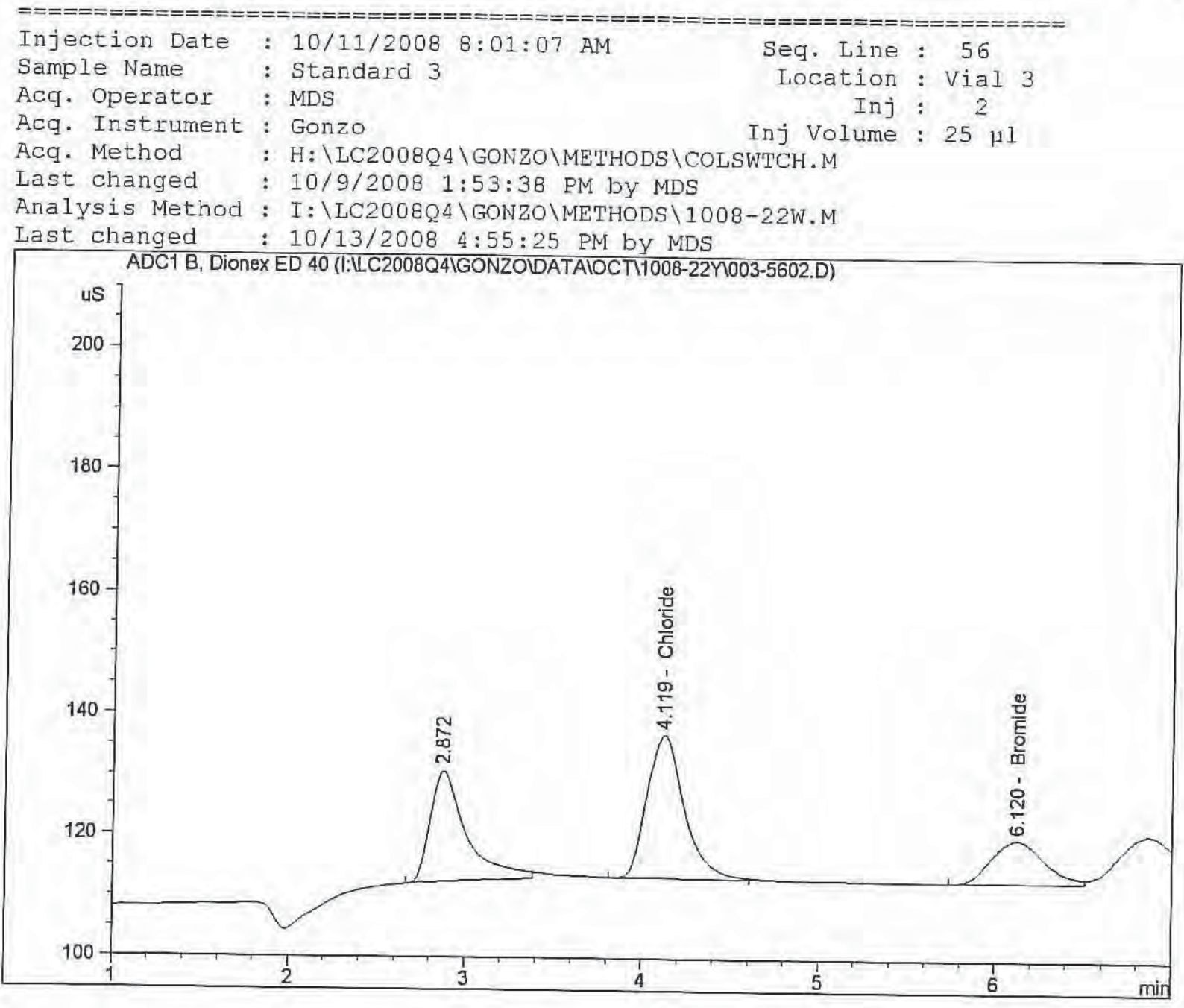

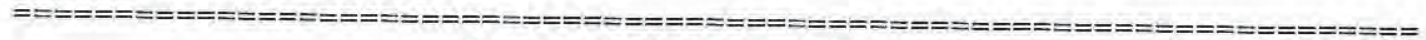
External Standard Report

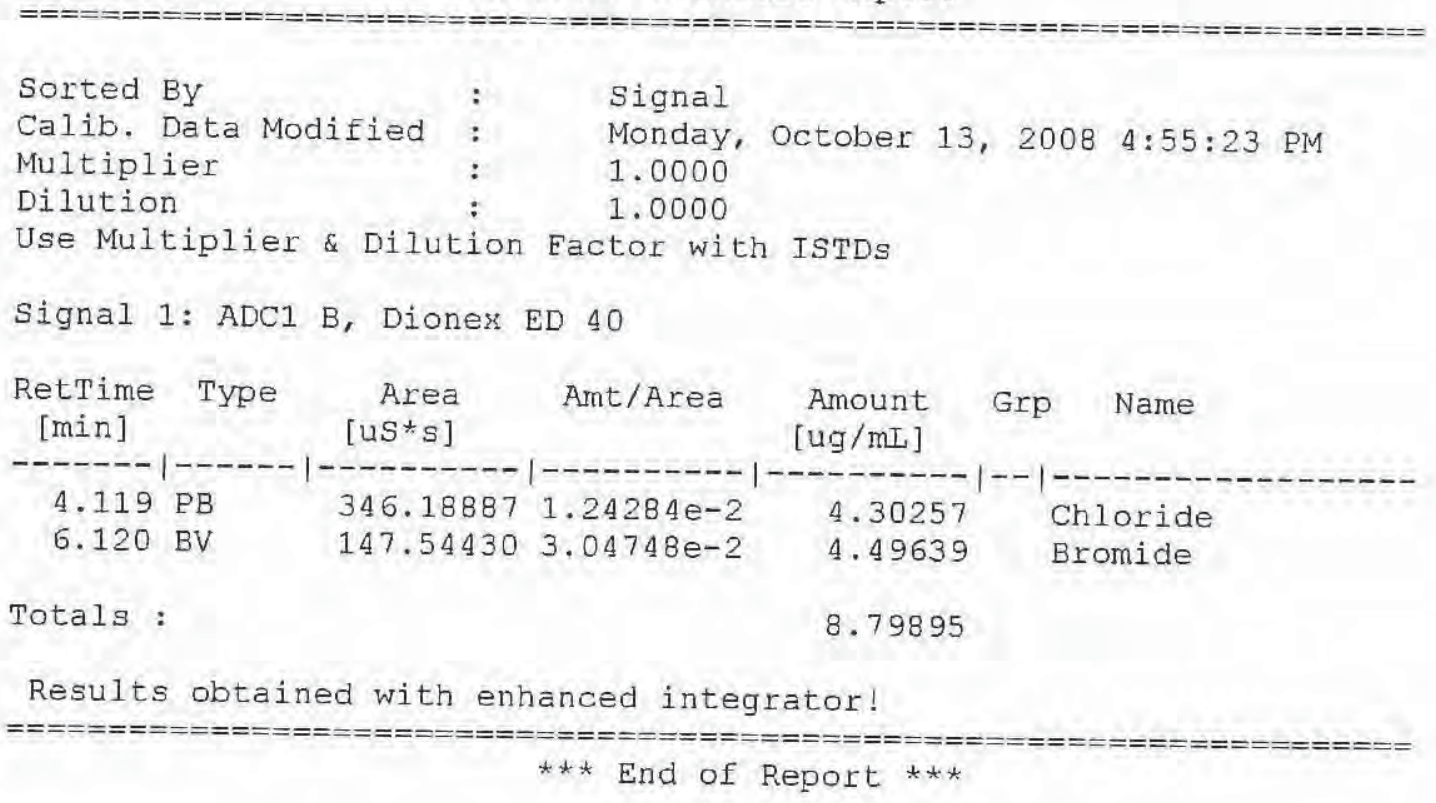




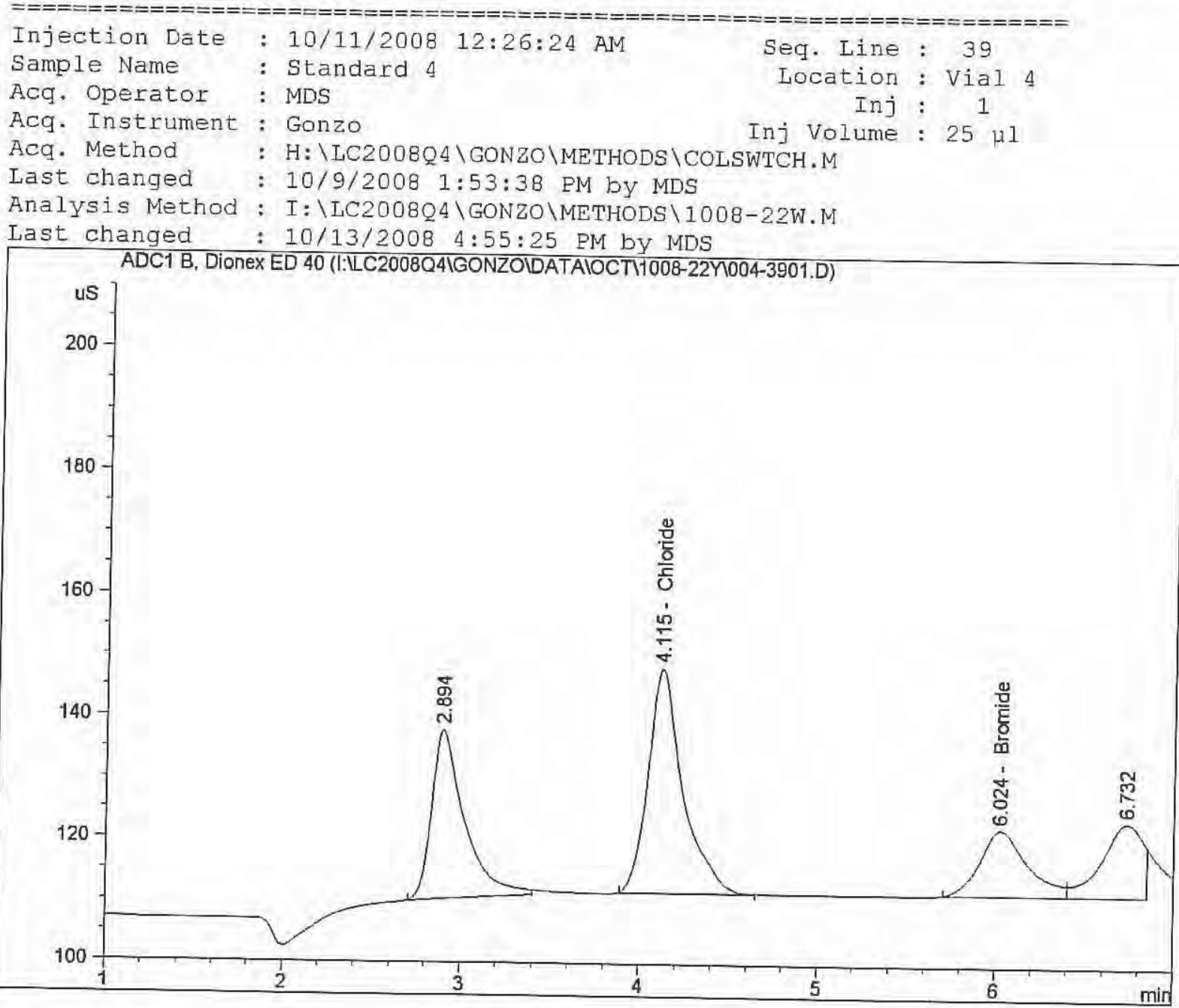

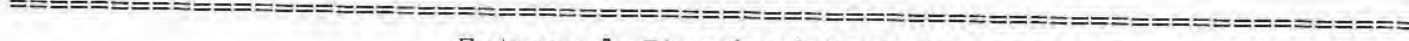
External Standard Report

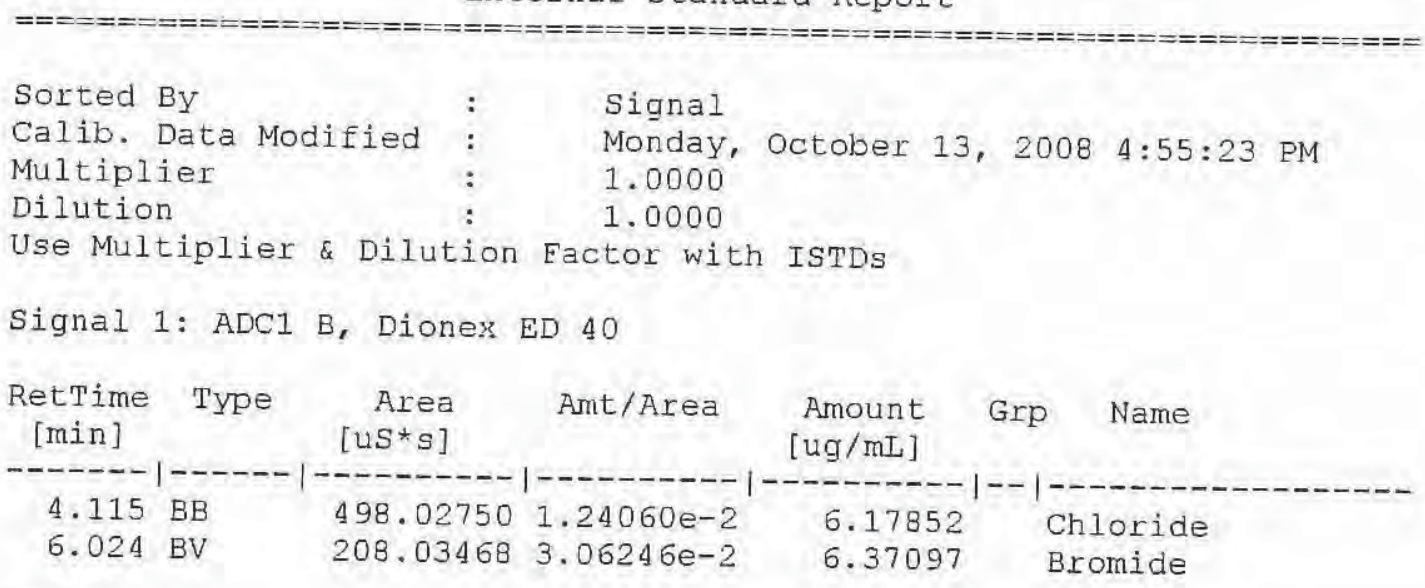

Totals :

12.54949

Results obtained with enhanced integrator!

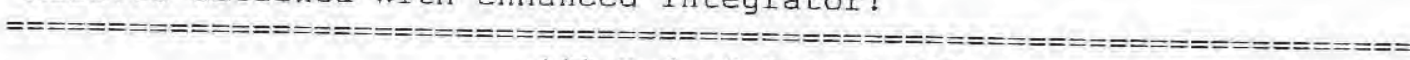

$\star \star *$ End of Report $* * *$
} 


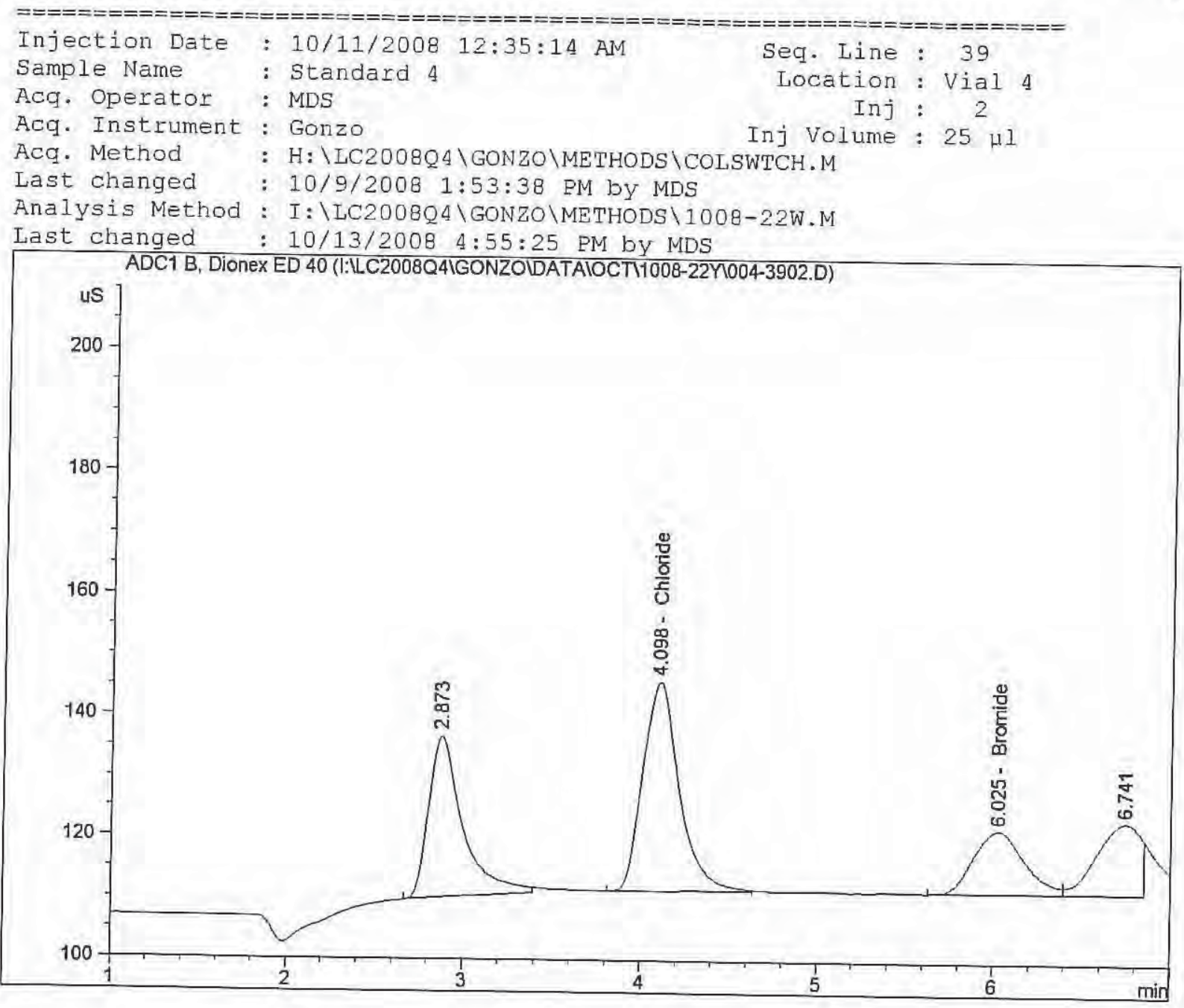

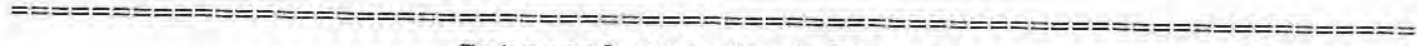
External standard Report

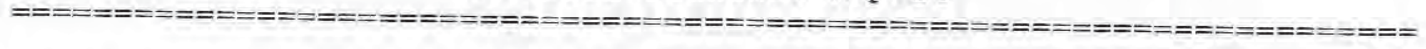

Sorted By
Calib. Data Modified $:$ Signal
Multiplier
Dilution
Use Multiplier \& Dilution Eactor with ISTDs

Signal 1: ADC1 B, Dionex ED 40

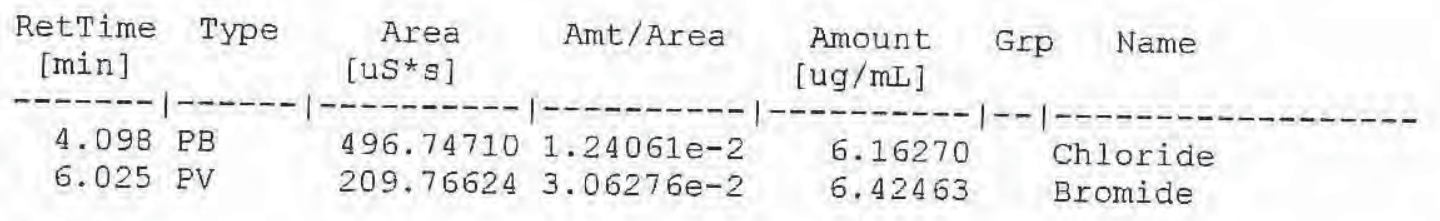

Totals : 12.58733

Results obtained with enhanced integrator!

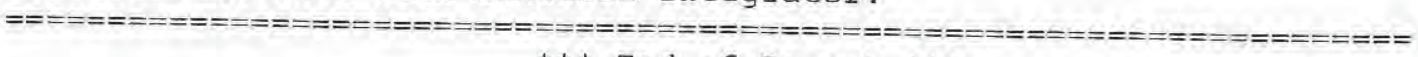
$* * *$ End of Report $* * *$
} 


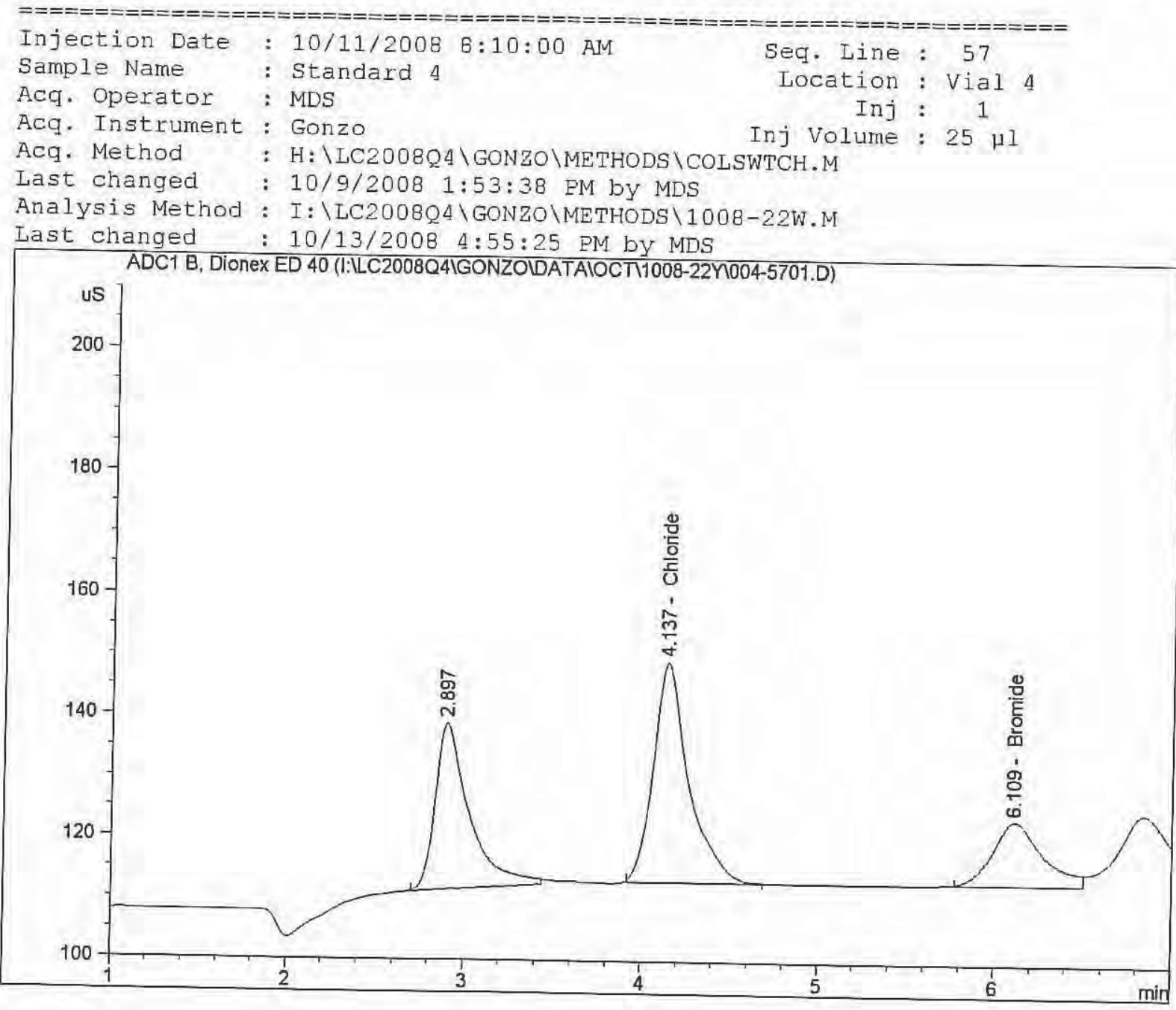

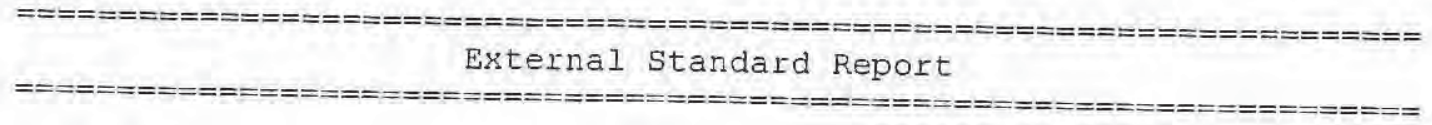

Sorted By

Calib. Data Modified : Signal

Multiplier

Dilution

Monday, October 13, 2008 4:55:23 PM

Use Multiplier \& Dilution Eactor with ISTDS

Signal 1: ADC1 B, Diones ED 40

\begin{tabular}{|c|c|c|c|c|c|c|}
\hline $\begin{array}{l}\text { RetTime } \\
\text { [min] }\end{array}$ & Type & $\begin{array}{r}\text { Area } \\
\text { [us*s] }\end{array}$ & Amt/Area & $\begin{array}{l}\text { Amount } \\
{[\mathrm{ug} / \mathrm{mL}]}\end{array}$ & Grp & Name \\
\hline $\begin{array}{l}4.137 \\
6.109\end{array}$ & $\begin{array}{l}B B \\
B V\end{array}$ & $\begin{array}{l}505.14505 \\
210.29846\end{array}$ & $\begin{array}{l}1.24053 \mathrm{e}-2 \\
3.06285 \mathrm{e}-2\end{array}$ & $\begin{array}{l}6.26646 \\
6.44113\end{array}$ & & $\begin{array}{l}\text { Ioride } \\
\text { comide }\end{array}$ \\
\hline
\end{tabular}

Totals :

12.70758

Results obtained with enhanced integrator!

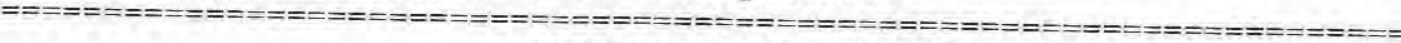

$\star * *$ End of Report $* * *$ 


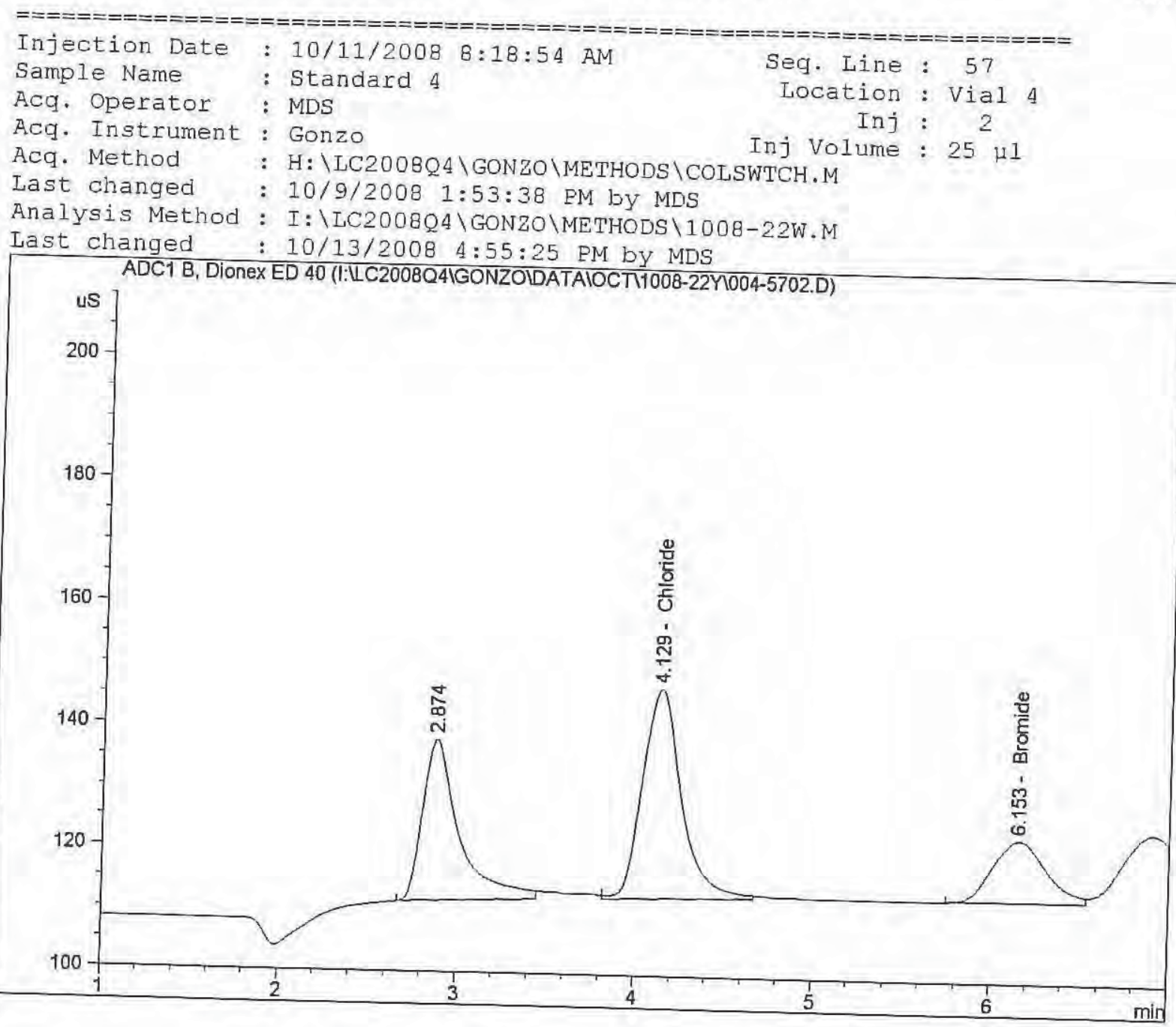

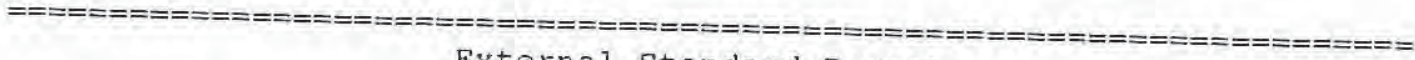

External standard Report

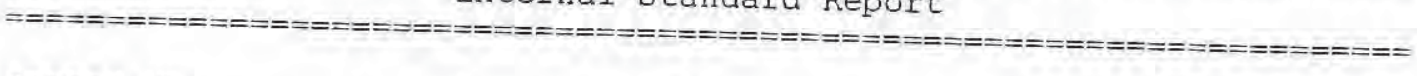

$\begin{array}{lll}\text { Sorted By } & : & \text { Signal } \\ \text { Calib. Data Modified } & : & \text { Monday, October 13, } 2008 \text { 4:55:23 PM } \\ \text { Multiplier } & : & 1.0000 \\ \text { Dilution } & \vdots & 1.0000\end{array}$

Use Multiplier \& Dilution Factor with ISTDS

Signal 1: ADC1 B, Dionex ED 40

\begin{tabular}{ccccc}
$\begin{array}{c}\text { RetTime Type } \\
\text { [min] }\end{array}$ & $\begin{array}{c}\text { Area } \\
{\left[\mathrm{uS}^{*} \mathrm{~s}\right]}\end{array}$ & Amt/Area & $\begin{array}{c}\text { Amount } \\
\text { [ug/mL] }\end{array}$ & Grp Name \\
\hline $4.129 \mathrm{BB}$ & 527.75195 & $1.24031 \mathrm{e}-2$ & 6.54576 & Chloride \\
$6.153 \mathrm{PV}$ & 212.30571 & $3.06319 \mathrm{e}-2$ & 6.50333 & Bromide
\end{tabular}

Totals :

$$
13.04909
$$

Results obtained with enhanced integrator!

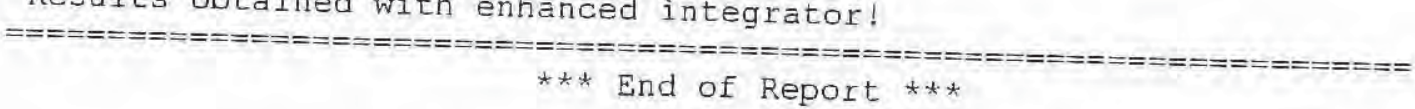




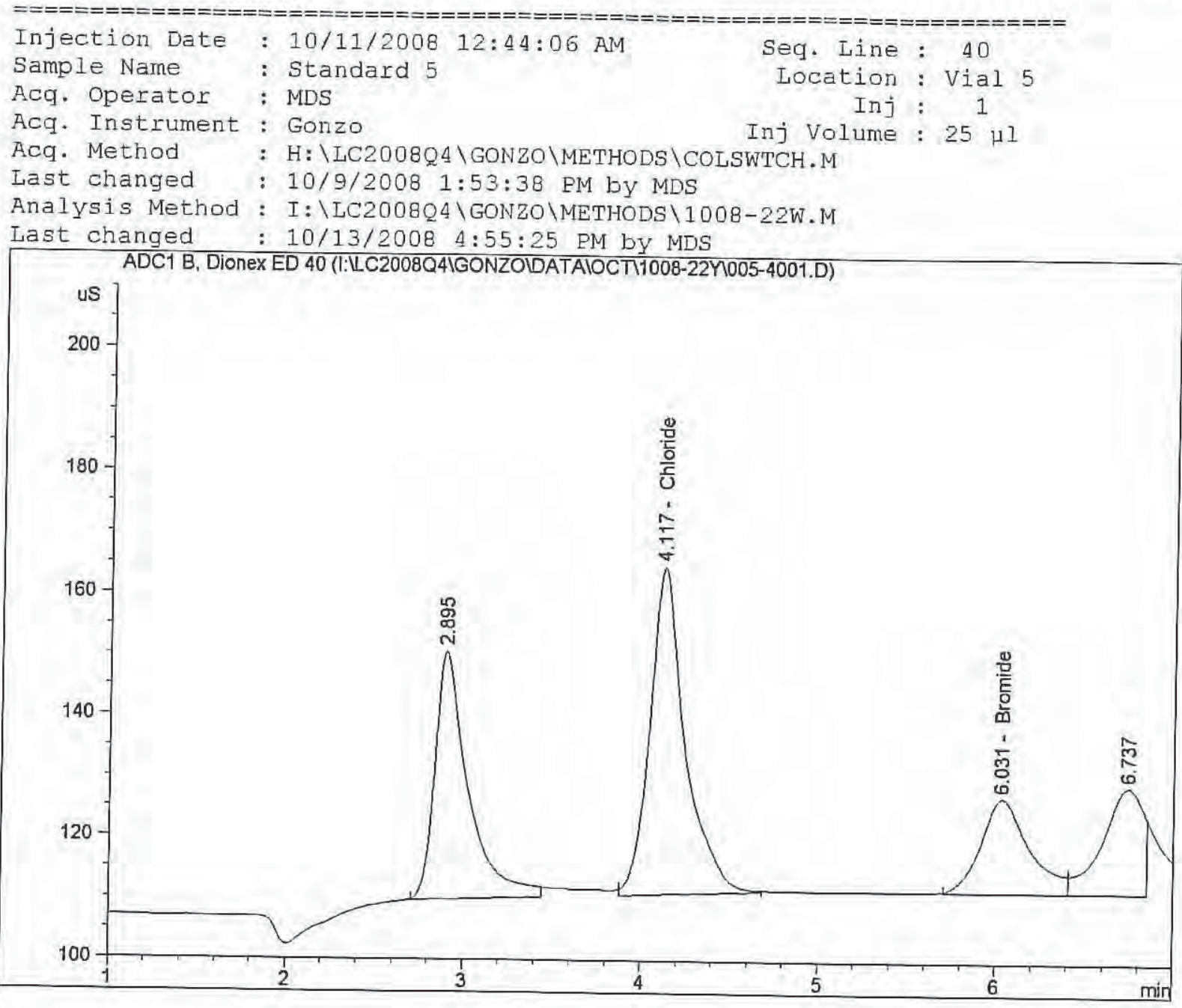

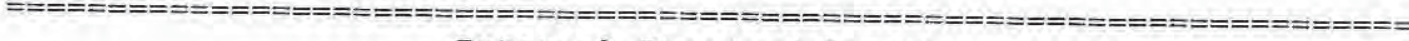
External standard Report

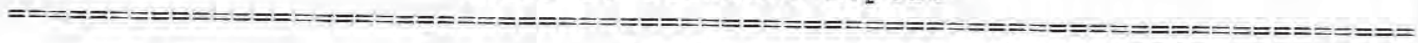

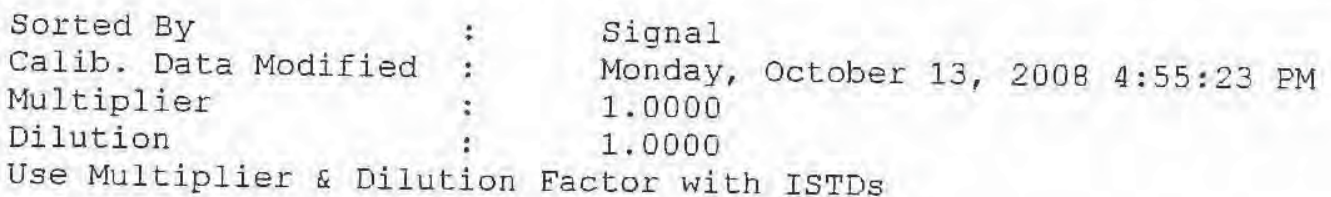

Use Multiplier \& Dilution Eactor with ISTDs

Signal 1: ADC1 B, Dionex ED 40

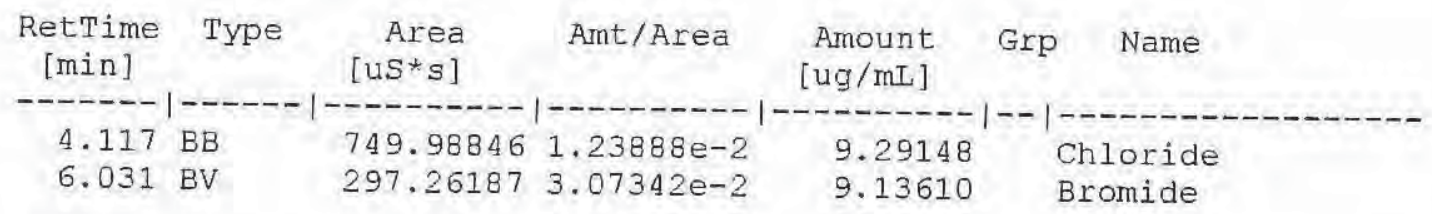

Totals :

18. 42758

Results obtained with enhanced integrator!

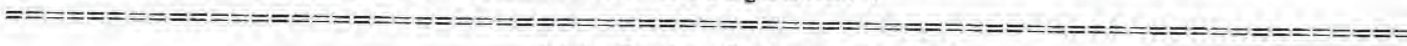

$* * *$ End of Report $* * *$
} 

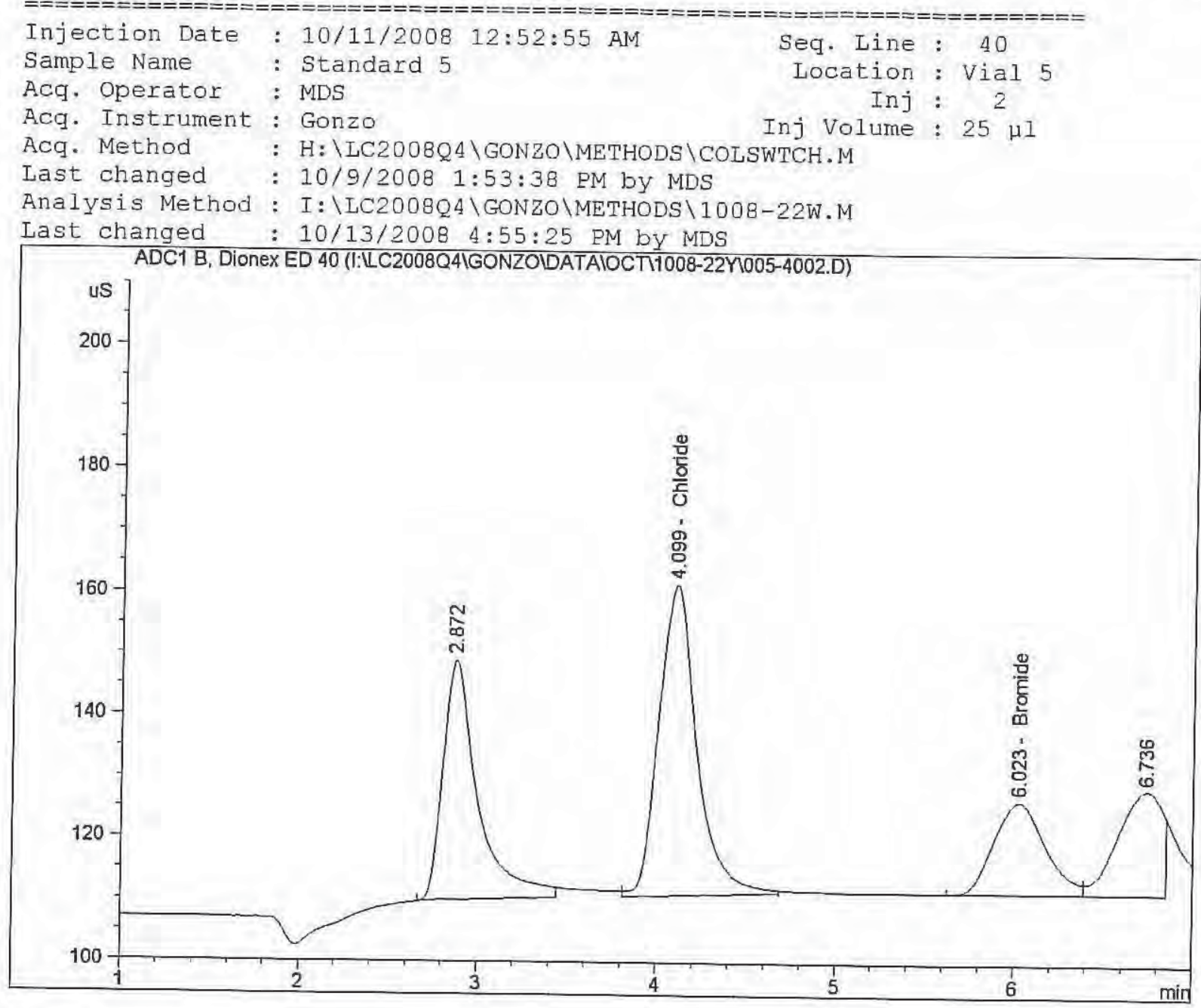

External Standard Report

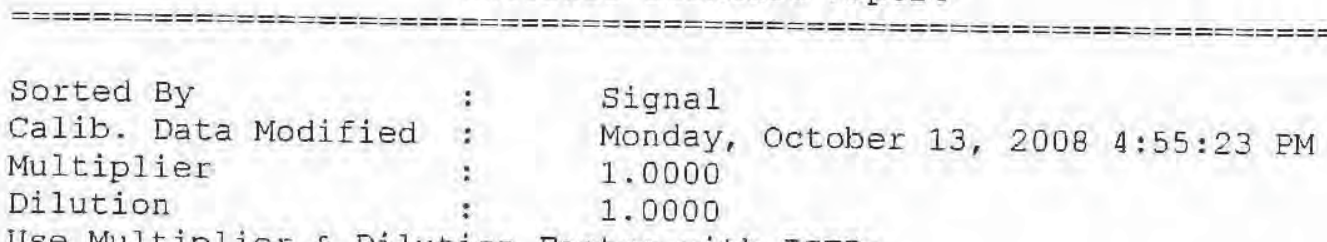

Use Multiplier \& Dilution Factor with IsTDs

Signal 1: ADC1 B, Dionex ED 40

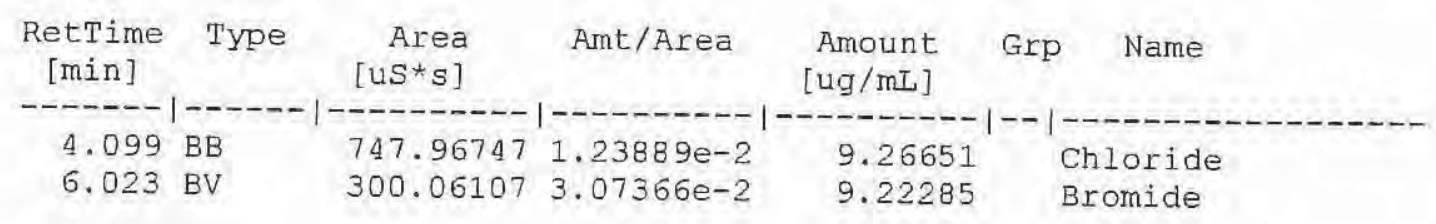

Totals : 18.48936

Results obtained with enhanced integrator! 


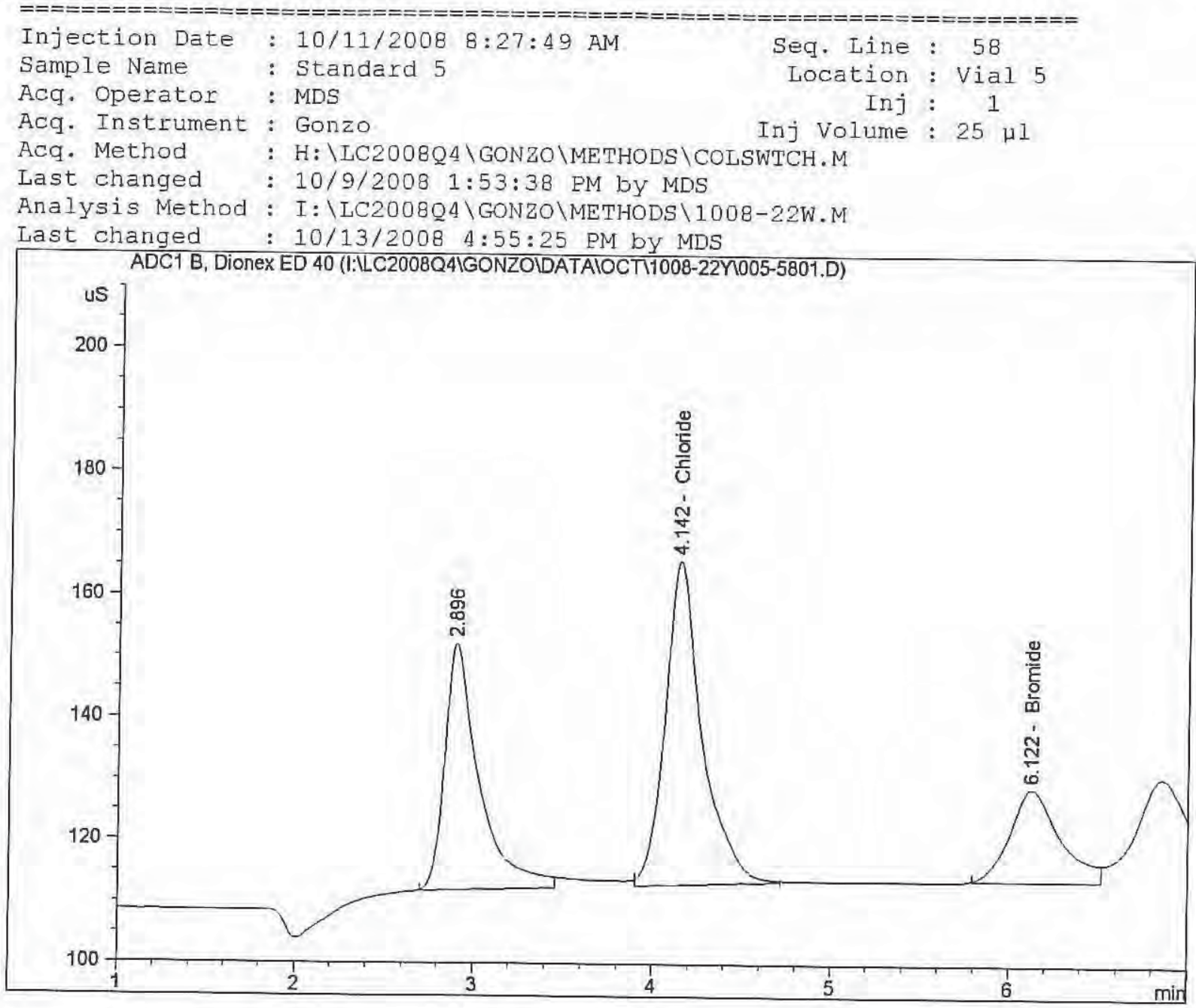

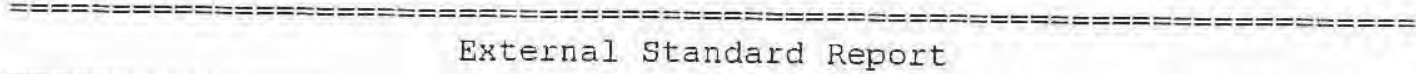

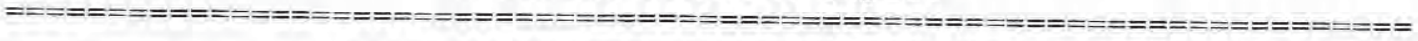

$\begin{array}{lll}\text { Sorted By } & : & \text { Signal } \\ \text { Calib. Data Modified } & : & \text { Monday, October 13, } 2008 \text { 4:55:23 PM } \\ \text { Multiplier } & : & 1.0000 \\ \text { Dilution } & \text { : } & 1.0000 \\ \text { Use Multiplier \& Dilution Eactor with ISTDs }\end{array}$

Use Multiplier \& Dilution Factor with ISTDS

Signal 1: ADC1 B, Dionex ED 40

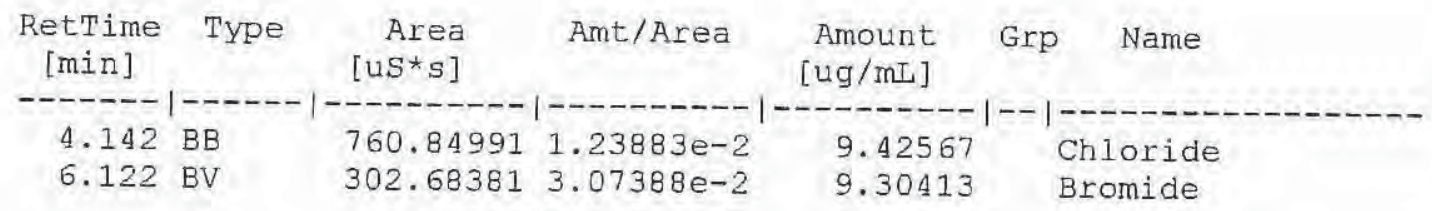

Totals :

18.72980

Results obtained with enhanced integrator!

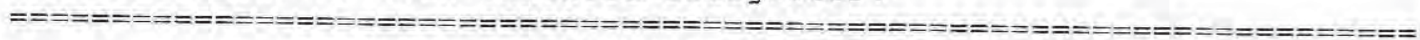

$\star * \star$ End of Report $* * *$
} 

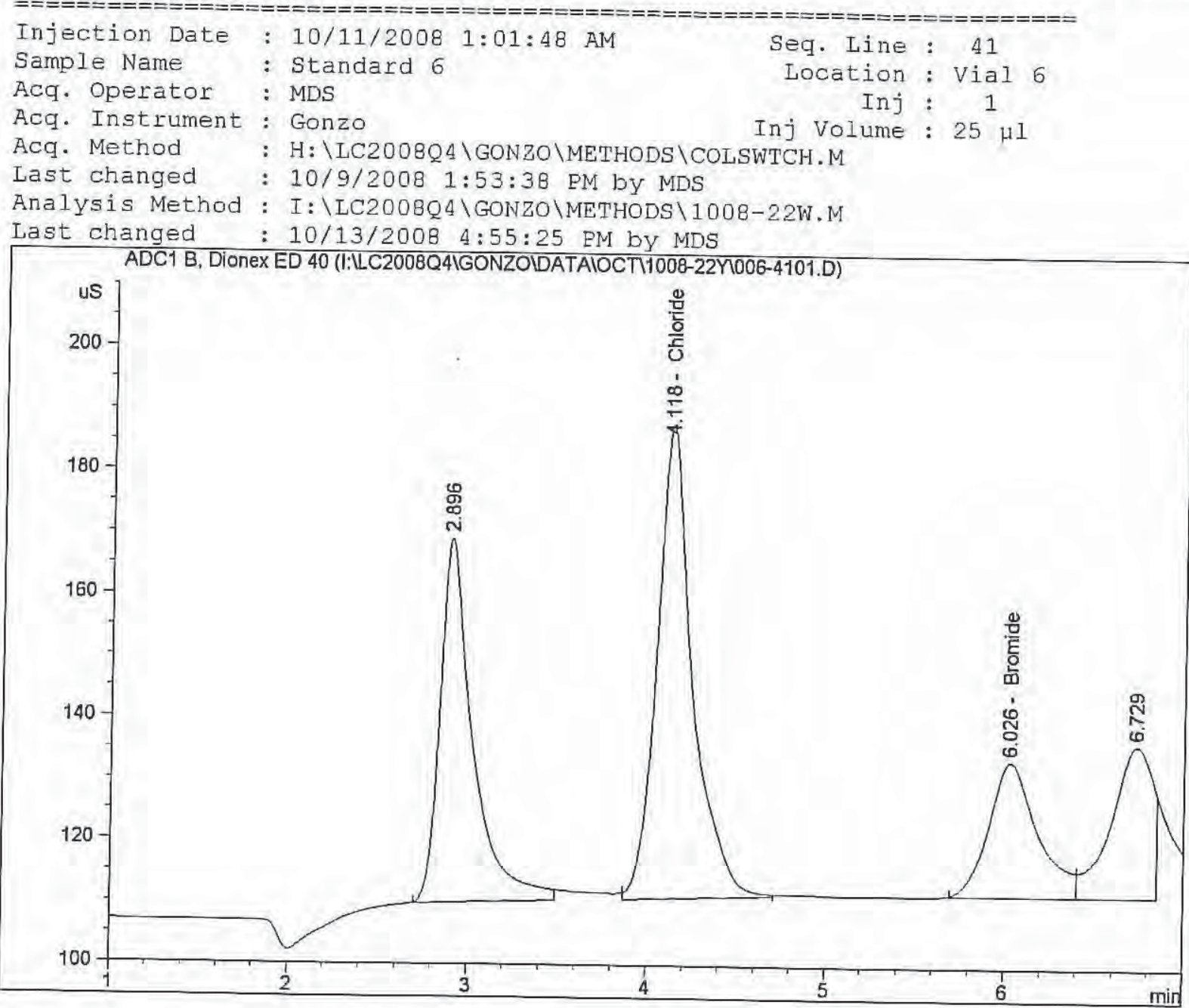

External Standard Report

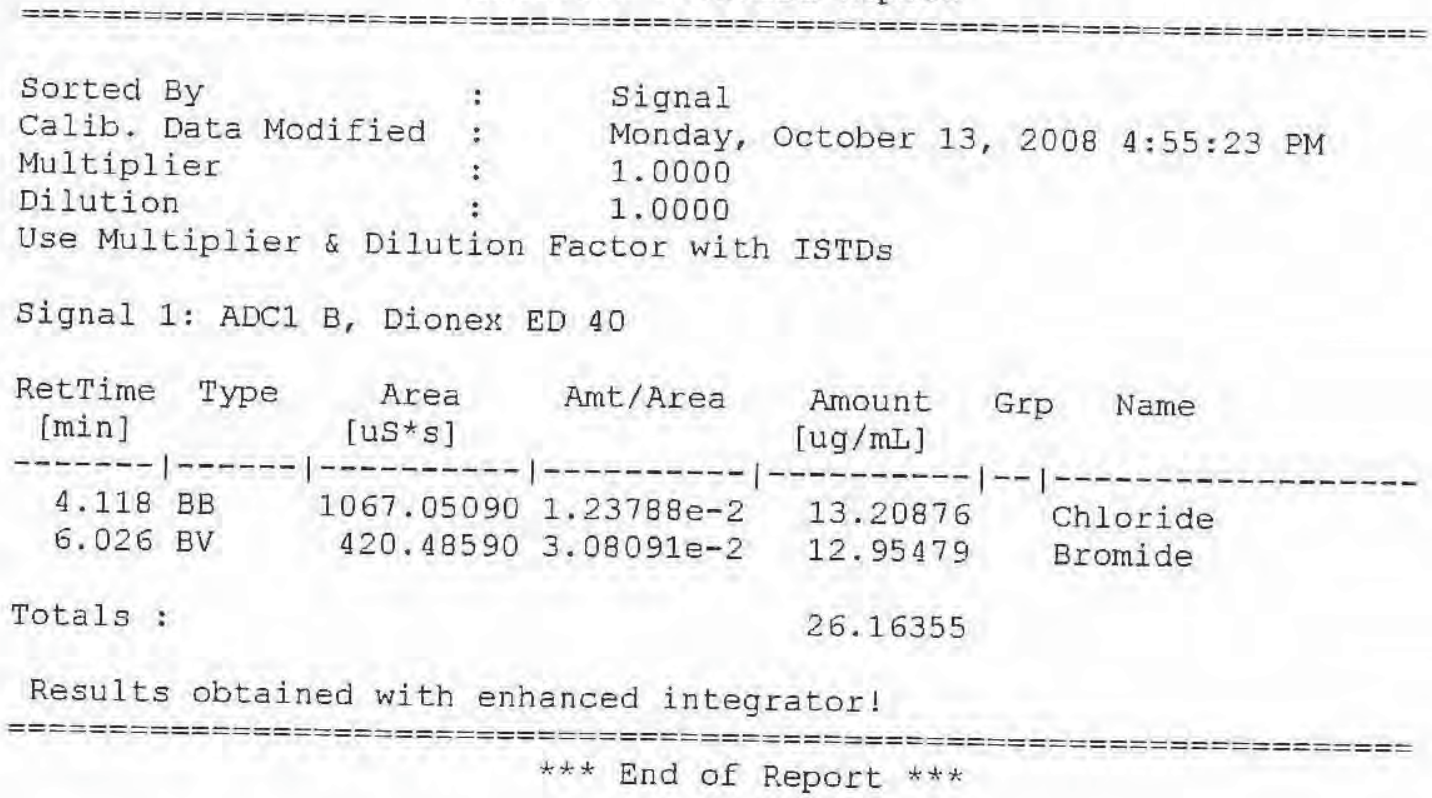

Results obtained with enhanced integrator! 


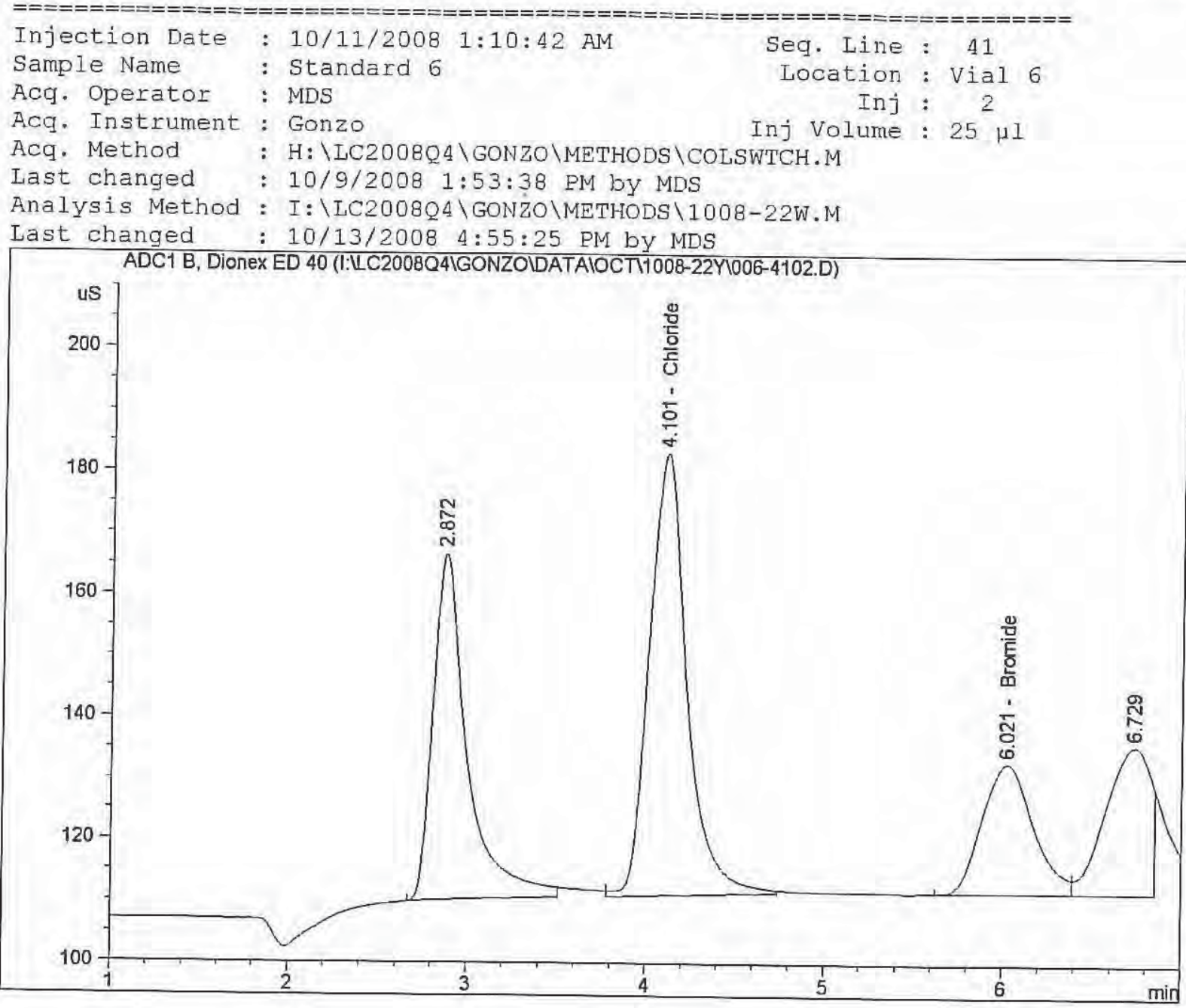

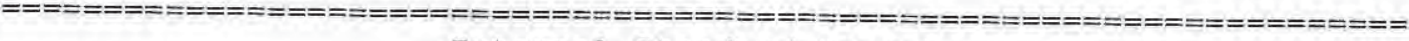
External Standard Report

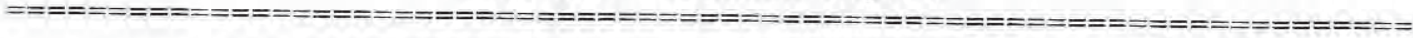

$\begin{array}{lll}\text { Sorted By } & : & \text { Signal } \\ \text { Calib. Data Modified } & : & \text { Monday, October 13, } 2008 \text { 4:55:23 PM } \\ \text { Multiplier } & : & 1.0000 \\ \text { Dilution } & \vdots & 1.0000\end{array}$

Use Multiplier \& Dilution Eactor with ISTDs

Signal 1: ADC1 B, Dionex ED 40

\begin{tabular}{|c|c|c|c|c|c|}
\hline $\begin{array}{c}\text { RetTime } \\
\text { [min] }\end{array}$ & Type & $\begin{array}{r}\text { Area } \\
\text { [uS*s] }\end{array}$ & Ant/Area & $\begin{array}{l}\text { Amount } \\
{[\mathrm{ug} / \mathrm{mL}]}\end{array}$ & Name \\
\hline 4.10 & $B R$ & ---0 & & 216 & $1-1$ \\
\hline 6.021 & BV & 424.88870 & 3.08110e-2 & $\begin{array}{l}13.20216 \\
13.09123\end{array}$ & $\begin{array}{l}\text { Chloride } \\
\text { Bromide }\end{array}$ \\
\hline
\end{tabular}

Totals :

26.29340

Results obtained with enhanced integrator! 


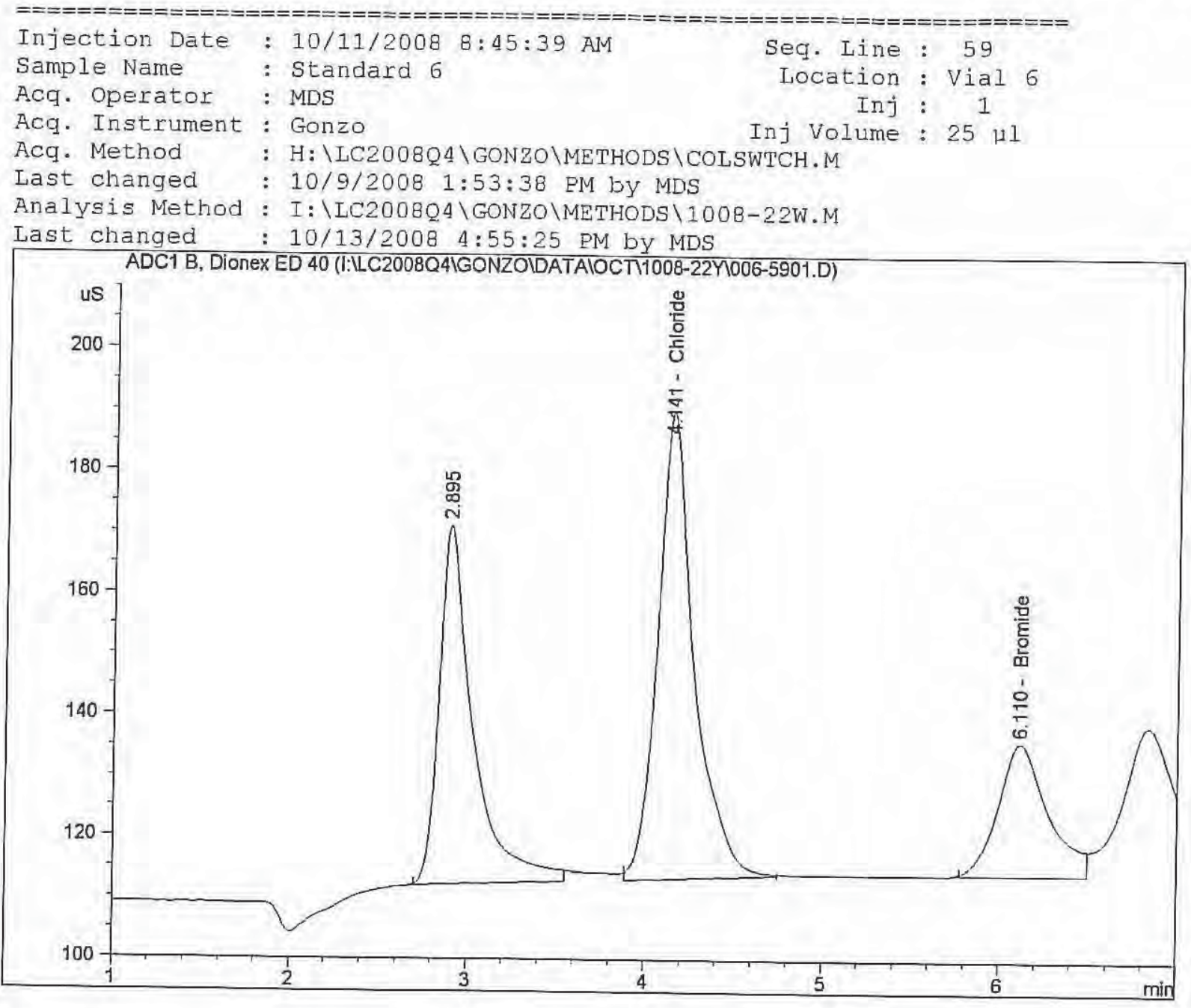

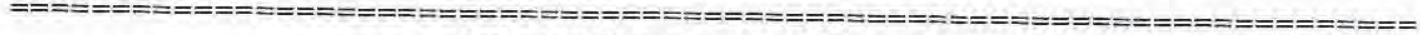
External standard Report

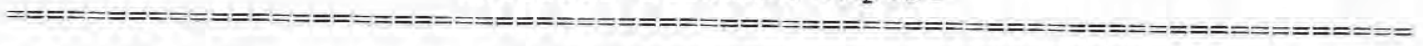

Sorted By
Calib. Data Modified :
Multiplier
Dilution

Signal 1: ADC1 B, Dionex ED 40

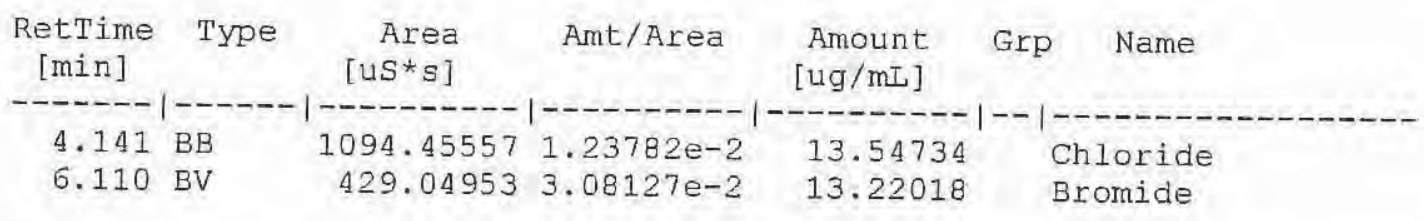

Totals : 26.76752

Results obtained with enhanced integrator!

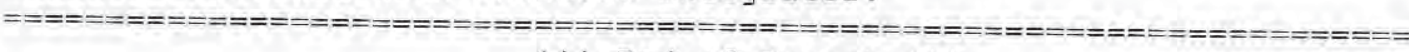
$* \star *$ End of Report ***
} 


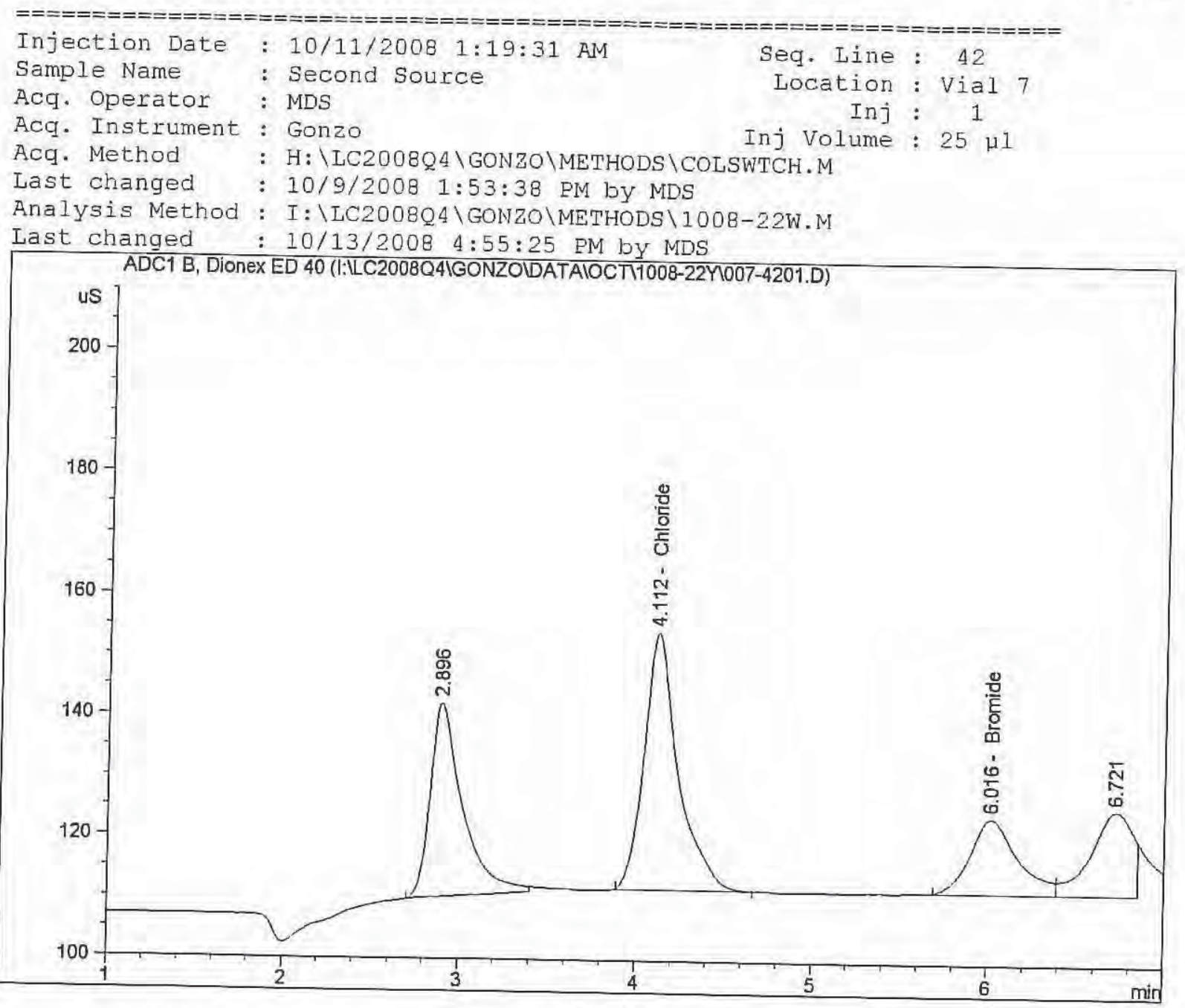

\section{External standard Report}

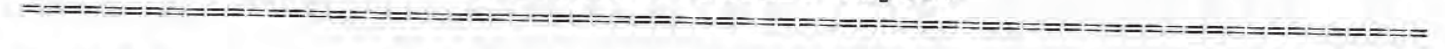

$\begin{array}{lll}\text { Sorted By } & \text { Signal } \\ \text { Calib. Data Modified } & \text { Monday, October 13, } 2008 \text { 4:55:23 PM } \\ \text { Multiplier } & : & 1.0000 \\ \text { Dilution } & 1.0000\end{array}$

Use Multiplier \& Dilution Factor with ISTDS

Signal 1: ADCI B, Diones ED 40

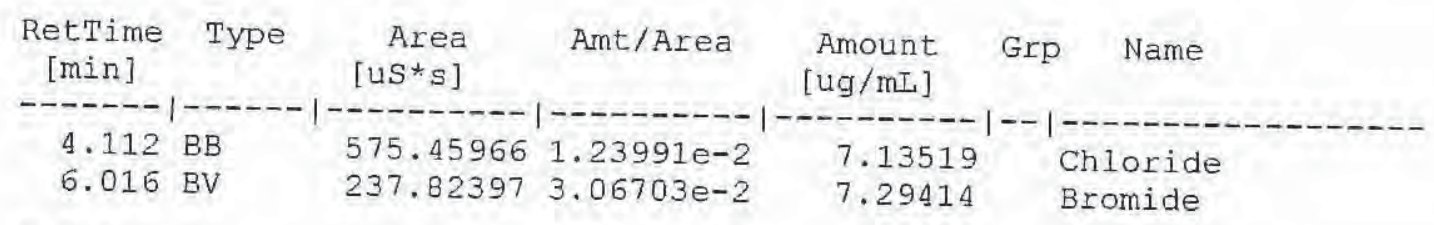

Totals :

14.42932

Results obtained with enhanced integrator!

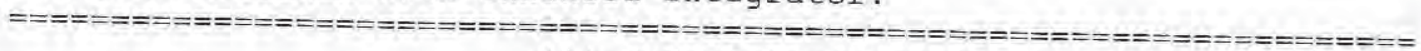

$* * *$ End of Report *** 


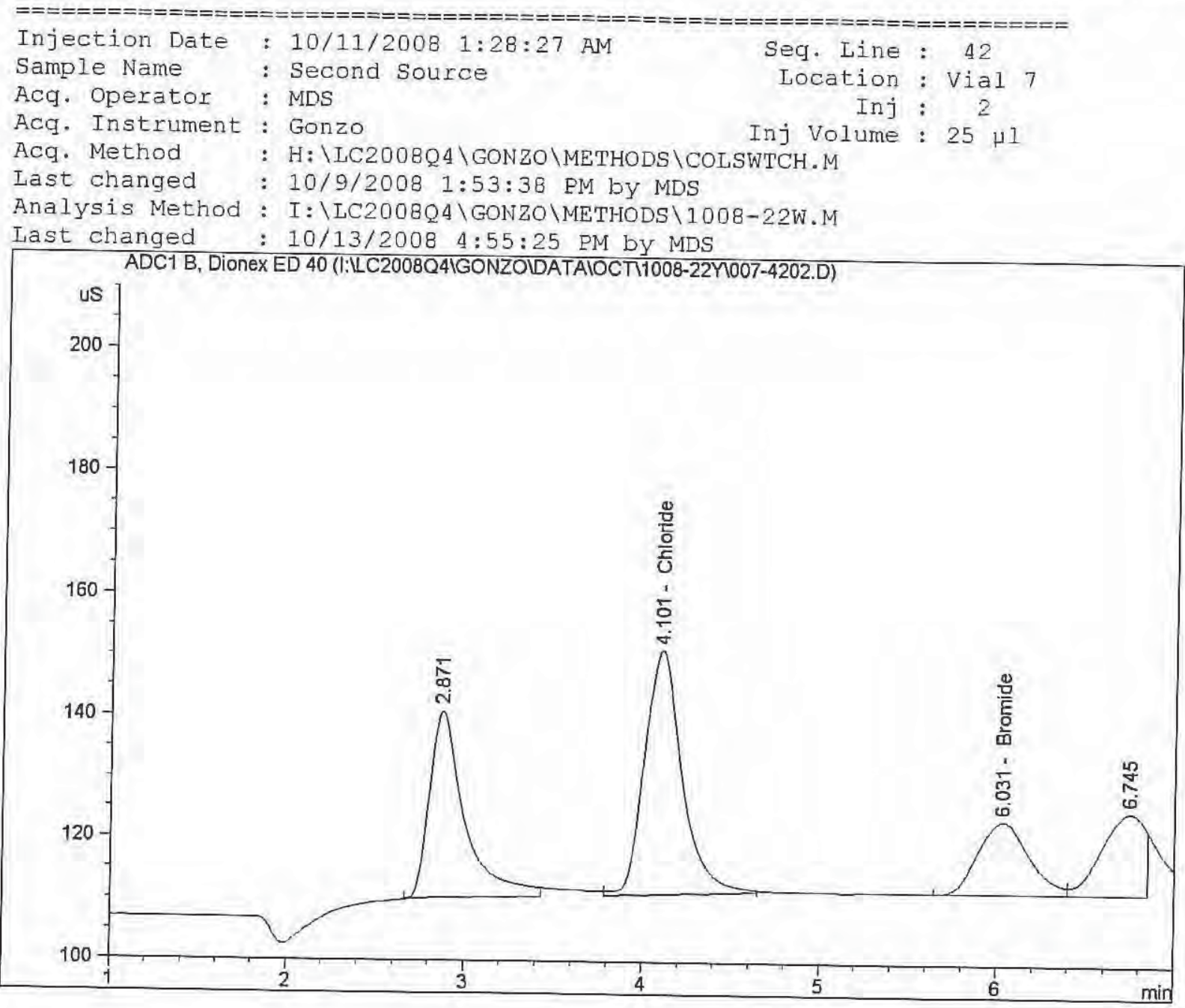

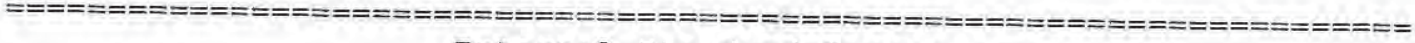
External Standard Report

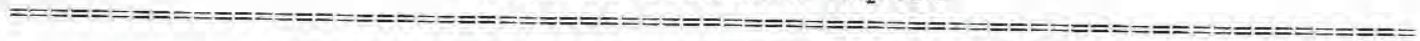

$\begin{array}{lcc}\text { Sorted By } & : & \text { Signal } \\ \text { Calib. Data Modified } & : & \text { Monday, October 13, 2008 4:55:23 PM } \\ \text { Multiplier } & : & 1.0000 \\ \text { Dilution } & \text { : } & 1.0000 \\ \text { Use Multiplier \& Dilution Eactor with ISTDs }\end{array}$

Signal 1: ADC1 B, Diones ED 40

\begin{tabular}{|c|c|c|c|c|c|c|}
\hline $\begin{array}{c}\text { RetTime } \\
\text { [min] }\end{array}$ & Type & $\begin{array}{r}\text { Area } \\
\text { [us*s] }\end{array}$ & Amt/Area & $\begin{array}{l}\text { Amount } \\
{[u g / m L]}\end{array}$ & Grp & Name \\
\hline 4.101 & $\mathrm{BB}$ & 596.61646 & & 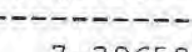 & & \\
\hline 6.031 & BV & 240.11308 & 3. $06734 \mathrm{e}-2$ & $\begin{array}{l}7.39658 \\
7.36507\end{array}$ & & omide \\
\hline
\end{tabular}

Totals :

$$
14.76165
$$

Results obtained with enhanced integrator!

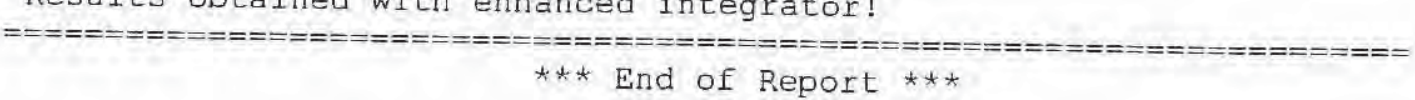

$$
\star \star * \text { End of Report } \star * \star
$$
}


Method Information

Dionex IonPac AS-14A 4-mm analytical (4x250mm) column.

Flow rate $1.2 \mathrm{~mL} / \mathrm{min}$

$8 \mathrm{mM} \mathrm{Na} 2 \mathrm{CO} 3 / 1 \mathrm{mM} \mathrm{NaHCO} 3$ mobile phase

Suppressed anion conductivity detection

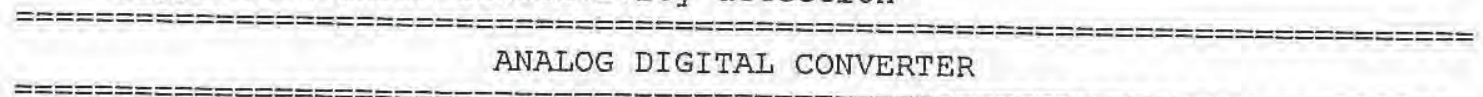

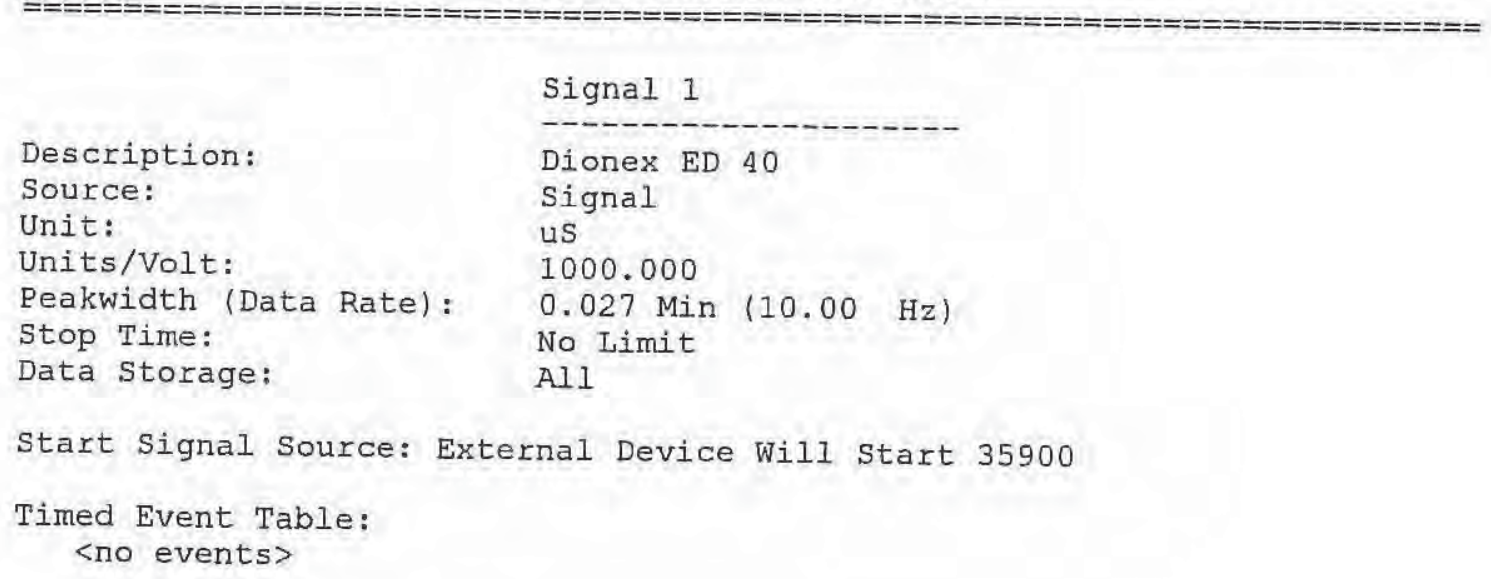




\begin{tabular}{|c|c|}
\hline \multicolumn{2}{|l|}{ Control } \\
\hline Column Flow & $1.200 \mathrm{ml} / \mathrm{min}$ \\
\hline Stoptime & $7.00 \mathrm{~min}$ \\
\hline Posttime & Off \\
\hline \multicolumn{2}{|l|}{ Solvents } \\
\hline Solvent $A$ & 100.0 \& (8.0mM $\left.\mathrm{Na} 2 \mathrm{CO} 3 / 1.0 \mathrm{mM} \mathrm{NaHCO}_{3}\right)$ \\
\hline Solvent $B$ & Off \\
\hline Solvent $\mathrm{C}$ & 0.0 \& (8.0mM Na2CO3/1.0mM NaHCO3) \\
\hline Solvent $D$ & Off \\
\hline \multicolumn{2}{|l|}{ Pressurelimits } \\
\hline Minimum Pressure & 0 bar \\
\hline Maximum Pressure & 400 bar \\
\hline \multicolumn{2}{|l|}{ Auxiliary } \\
\hline Maximal Flow Ramp & $100.00 \mathrm{ml} / \mathrm{min}^{\wedge} 2$ \\
\hline Primary Channel & Auto \\
\hline Compressibility & $83 * 10^{\wedge}-6 /$ bar \\
\hline Minimal Stroke & Auto \\
\hline \multicolumn{2}{|l|}{ Store Parameters } \\
\hline Store Ratio A & Yes \\
\hline Store Ratio B & Yes \\
\hline Store Ratio C & Yes \\
\hline Store Ratio D & Yes \\
\hline Store Flow & Yes \\
\hline Store Pressure & Yes \\
\hline
\end{tabular}

Agilent 1100 Contacts Option $========================0$

Contact 1

Contact 2

Contact 3

Contact 4

Open

Open

Open

Open

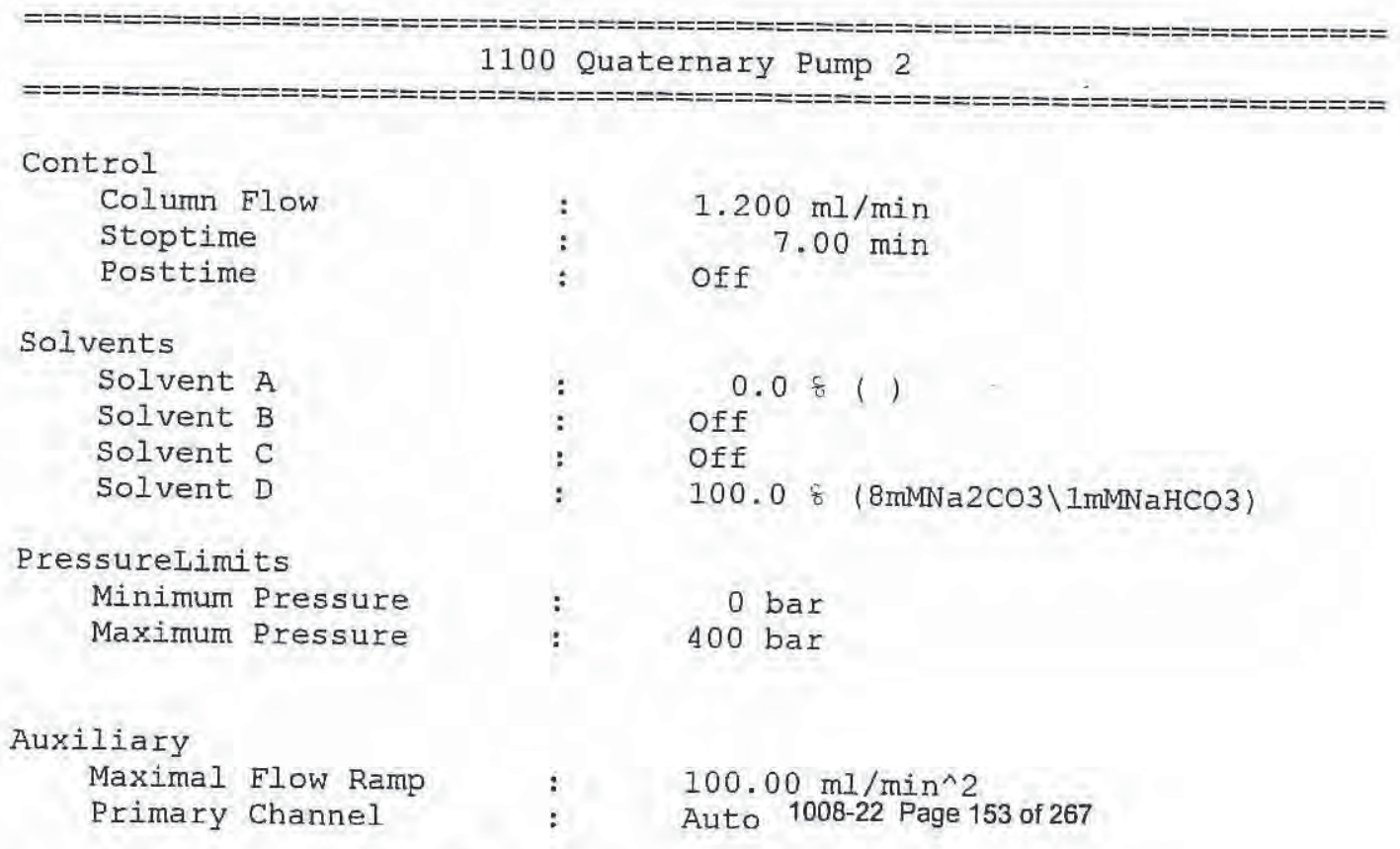


Method: H: \LC2008Q4\GONZO\METHODS\COLSWTCH.M of 10/9/2008 1:53:38 PM

$\begin{array}{ccc}\text { Compressibility } & : & 10{ }^{\star} \\ \text { Minimal Stroke } & : & \text { Auto } \\ \text { Store Parameters } & : & \text { Yes } \\ \text { Store Ratio A } & : & \text { Yes } \\ \text { Store Ratio B } & : & \text { Yes } \\ \text { Store Ratio C } & : & \text { Yes } \\ \text { Store Ratio D } & : & \text { Yes } \\ \text { Store Flow } & : & \text { Yes } \\ \text { Store Pressure } & & \\ & & \\ \text { Agilent } 1100 \text { Contacts Option } & \\ ====================== & \\ \text { Contact } 1 & : & \text { Open } \\ \text { Contact } 2 & : & \text { Open } \\ \text { Contact } 3 & : & \text { Open } \\ \text { Contact } 4 & \end{array}$

Agilent 1100 Autosampler 1

\begin{tabular}{|c|c|c|}
\hline \multicolumn{3}{|l|}{ Injection } \\
\hline Injection Mode & : & Needle Wash \\
\hline Injector volume & : & $25.00 \mu 1$ \\
\hline Wash Vial & : & 100 \\
\hline Optimization & : & none \\
\hline \multicolumn{3}{|l|}{ Auxiliary } \\
\hline Drawspeed & : & $100 \mu 1 / \mathrm{min}$ \\
\hline Ejectspeed & : & $1000 \mu \mathrm{l} / \mathrm{min}$ \\
\hline Draw position & : & $2.0 \mathrm{~mm}$ \\
\hline \multicolumn{3}{|l|}{ Time } \\
\hline Stoptime & : & As Pump \\
\hline Posttime & : & Off \\
\hline \\
\hline 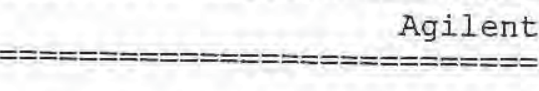 & $\begin{array}{l}t 1100 \\
======\end{array}$ & 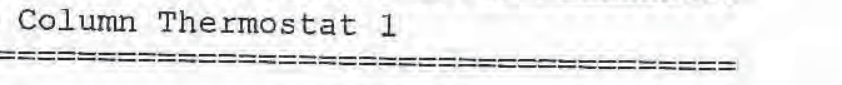 \\
\hline \multicolumn{3}{|l|}{ Temperature settings } \\
\hline Left temperature & : & $30.0^{\circ} \mathrm{C}$ \\
\hline Right temperature & : & Same as left \\
\hline Enable analysis & : & When Temp. is within setpoint $+1-$ \\
\hline Store left temperature & : & No \\
\hline Store right temperature & e: & No \\
\hline \multicolumn{3}{|l|}{ ime } \\
\hline Stoptime & : & As pump \\
\hline Posttime & : & off \\
\hline olumn Switching Valve & : & Use current \\
\hline
\end{tabular}


Method Information

Dionex IonPac AS-14A 4-mm analytical (4x250mm) column.

Flow rate $1.2 \mathrm{~mL} / \mathrm{min}$

$8 \mathrm{mM} \mathrm{Na} 2 \mathrm{CO} 3 / 1 \mathrm{mM} \mathrm{NaHCO} 3$ mobile phase

Suppressed anion conductivity detection

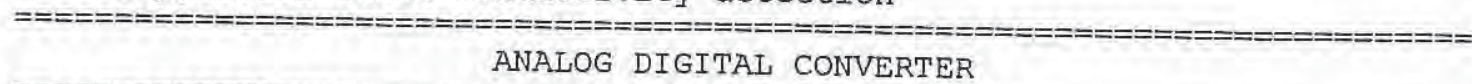

ANALOG DIGITAL CONVERTER

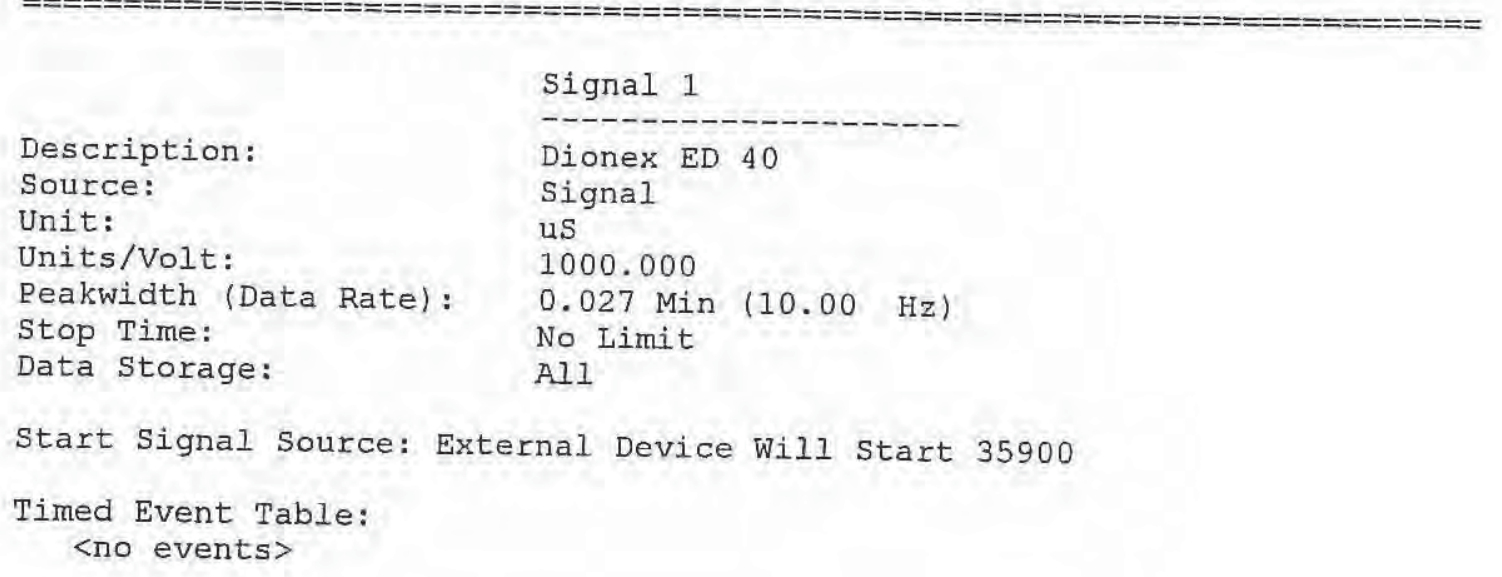




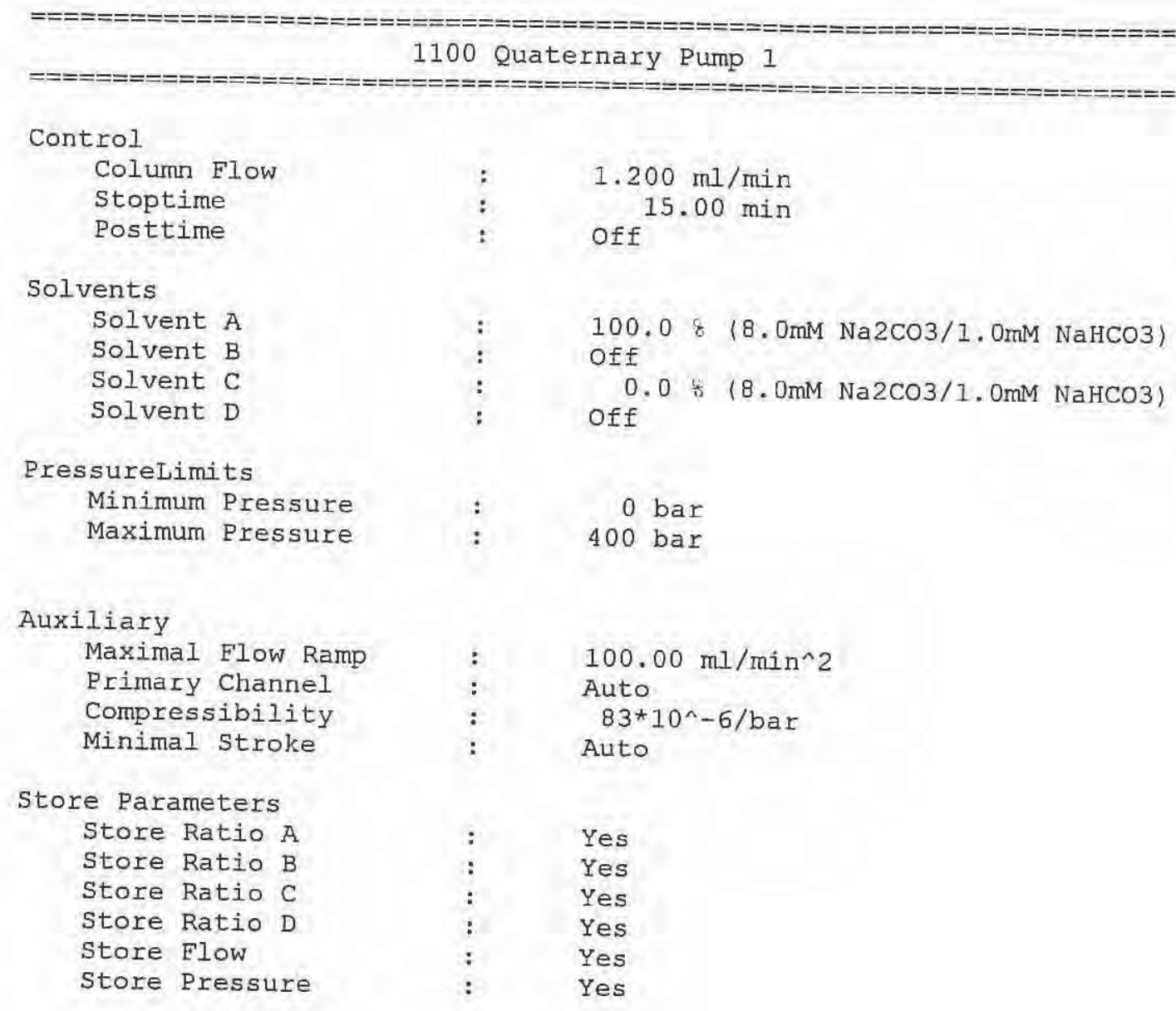

Agilent 1100 Contacts Option $======================$

Contact 1

Contact 2

Contact 3

Contact 4

Open

Open

Open

Open

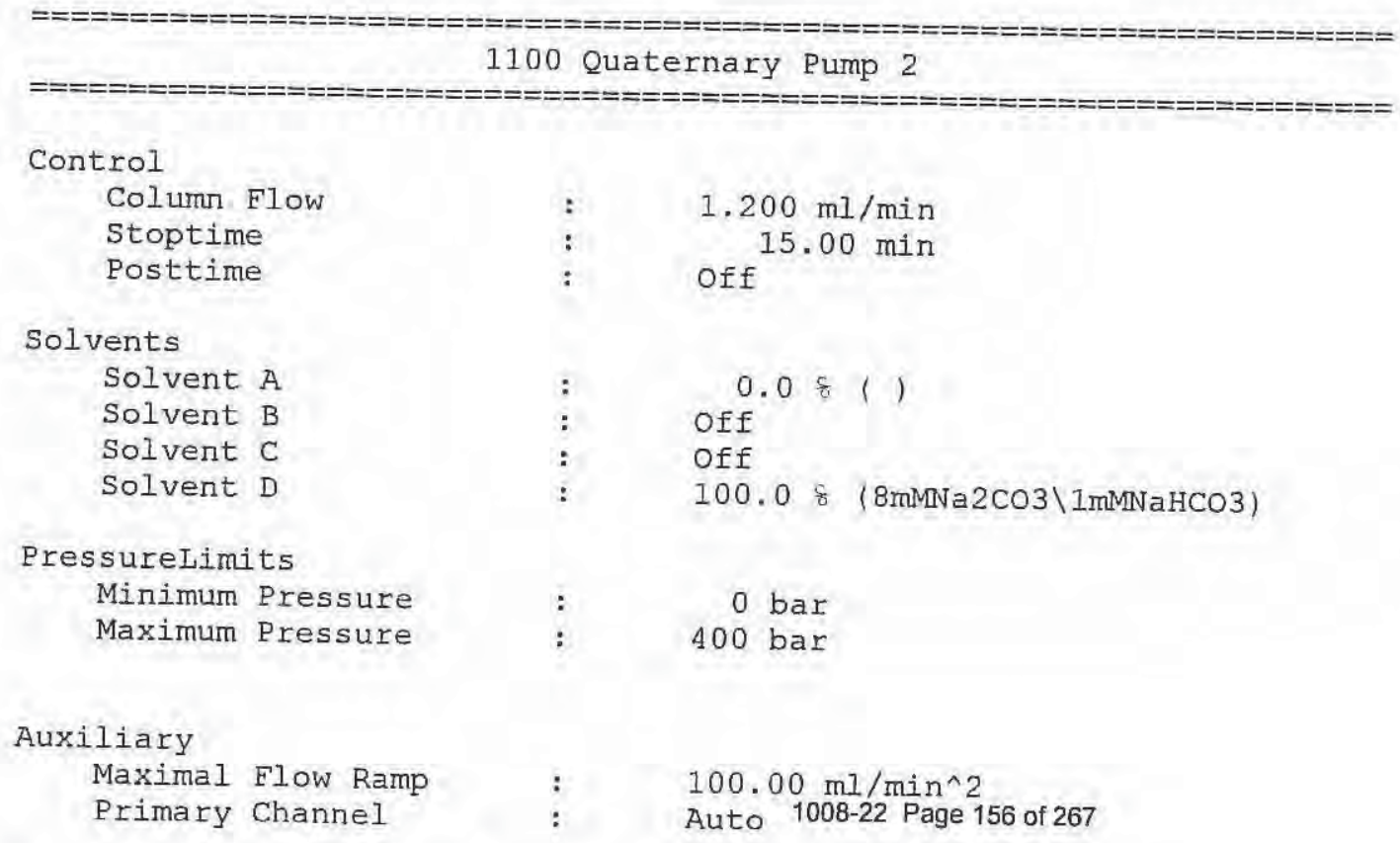


Method: H: \LC2008Q4 \GONZO\METHODS\CHLSWTCH.M of 10/9/2008 1:54:03 PM

$\begin{array}{lll}\text { Compressibility } & : & 100 * 10^{\wedge}-6 / \text { bar } \\ \text { Minimal Stroke } & : & \text { Auto }\end{array}$

$\begin{array}{lll}\text { Store Parameters } & & \\ \text { Store Ratio A } & : & \text { Yes } \\ \text { Store Ratio B } & : & \text { Yes } \\ \text { Store Ratio C } & : & \text { Yes } \\ \text { Store Ratio D } & : & \text { Yes } \\ \text { Store Flow } & : & \text { Yes } \\ \text { Store Pressure } & \text { Y } & \text { Yes }\end{array}$

Agilent 1100 Contacts Option

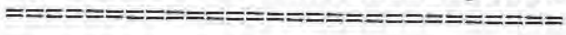

Contact 1

Contact 2

Contact 3

Contact 4

Open

Open

Open

Open

\section{Agilent 1100 Autosampler 1}

Injection

Injection Mode

Injector volume

Wash Vial

Optimization

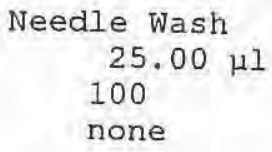

none

Auxiliary

Drawspeed

Ejectspeed

Draw position

$100 \mu \mathrm{I} / \mathrm{min}$

$1000 \mu \mathrm{l} / \mathrm{min}$

$2.0 \mathrm{~mm}$

Time

Stoptime : As Pump

Postime : : off

\section{Agilent 1100 Column Thermostat 1}

Temperature settings

Left temperature

Right temperature

Enable analysis

$30.0^{\circ} \mathrm{C}$

Same as left

When Temp. is within setpoint $+1-0.8^{\circ} \mathrm{C}$

Store left temperature : No

Store right temperature: No

Time

Stoptime

Posttime

Column Switching Valve : Use current 
Sequence Table:

Method and Injection Info Part:

\begin{tabular}{|c|c|c|c|c|c|c|c|c|}
\hline $\begin{array}{l}\text { Line } \\
====\end{array}$ & $\begin{array}{l}\text { Locat } \\
=====\end{array}$ & $\begin{array}{l}\text { tion } \\
====\end{array}$ & $\begin{array}{l}\text { SampleName } \\
==========-=====\end{array}$ & $\begin{array}{l}\text { Method } \\
=======\end{array}$ & $\begin{array}{l}\text { Inj } \\
===\end{array}$ & $\begin{array}{l}\text { SampleType I. } \\
========\end{array}$ & $\begin{array}{l}\text { InjVolume } \\
=======\end{array}$ & $\begin{array}{l}\text { DataFile } \\
=========\end{array}$ \\
\hline 1 & Vial & 1 & Standard 1 & COLSWTCH & 2 & Sample & & \\
\hline 2 & Vial & 2 & Standard 2 & COLSWTCH & 2 & Sample & & \\
\hline 3 & Vial & 3 & Standard 3 & COLSWTCH & 2 & Sample & & \\
\hline 4 & Vial & 4 & Standard 4 & COLSWTCH & 2 & Sample & & \\
\hline 5 & Vial & 5 & Standard 5 & COLSWTCH & 2 & Sample & & \\
\hline 6 & Vial & 6 & Standard 6 & COLSWTCH & 2 & Sample & & \\
\hline 7 & Vial & 7 & Second Source & COLSWTCH & 2 & Sample & & \\
\hline 8 & Vial & 8 & H2SO4 Iab Blank & COLSWTCH & 2 & Sample & & \\
\hline 9 & Vial & 9 & DI H2O Lab Blank & COLSWTCH & 2 & Sample & & \\
\hline 10 & Vial & 11 & In R1 $\mathrm{C} 3 * 1.0009$ & COLSWTCH & 2 & Sample & & \\
\hline 11 & Vial & 12 & In $\mathrm{R} 2 \mathrm{C}^{*}{ }^{*} 1.0397$ & COLSWTCH & 2 & Sample & & \\
\hline 12 & Vial & 13 & In $\mathrm{R} 3 \mathrm{C} 3 * 1.0577$ & COLSWTCH & 2 & Sample & & \\
\hline 13 & Vial & 14 & Stk R1 C3*1.0126 & COLSWTCH & 2 & Sample & & \\
\hline 14 & Vial & 15 & Stk R2 $\mathrm{C} 3 * 1.038$ & COLSWTCH & 2 & Sample & & \\
\hline 15 & Vial & 16 & Stk R3 $\mathrm{C}^{*} 1.0471$ & COLSWTCH & 2 & Sample & & \\
\hline 16 & Vial & 17 & In $C 6 * 1.061$ & COLSWTCH & 2 & Sample & & \\
\hline 17 & Vial & 18 & Stk C6*1.0399 & COLSWTCH & 2 & Sample & & \\
\hline 18 & Vial & 19 & $\mathrm{MS} / \mathrm{In} \mathrm{R} 1 \mathrm{C} 3$ & COLSWTCH & 2 & Sample & & \\
\hline 19 & Vial & 20 & $\mathrm{~T}-1 \star 1.1245$ & COLSWTCH & 2 & Sample & & \\
\hline 20 & Vial & 3 & Standard 3 & COLSWTCH & 2 & Sample & & \\
\hline 21 & Vial & 4 & Standard 4 & COLSWTCH & 2 & Sample & & \\
\hline 22 & Vial & 21 & $T-2 * 1.1288$ & COLSWTCH & 2 & Sample & & \\
\hline 23 & Vial & 22 & $T-3 * 1.1384$ & COLSWTCH & 2 & Sample & & \\
\hline 24 & Vial & 23 & $\mathrm{~T}-4 * 1.1461$ & COLSWTCH & 2 & Sample & & \\
\hline 25 & Vial & 24 & $\mathrm{MS} / \mathrm{T}-1$ & COISWTCH & 2 & Sample & & \\
\hline 26 & Vial & 25 & $\mathrm{Cl}-\mathrm{A} 25$ & COLSWTCH & 2 & Sample & & \\
\hline 27 & Vial & 26 & $\mathrm{Cl}-\mathrm{A} 26$ & COLSWTCH & 2 & Sample & & \\
\hline 28 & Vial & 27 & $\mathrm{Cl}-\mathrm{A} 27$ & COLSWTCH & 2 & Sample & & \\
\hline 29 & Vial & 28 & $\mathrm{Cl}-\mathrm{A} 28$ & COLSWTCH & 2 & Sample & & \\
\hline 30 & Vial & 1 & Standard 1 & COLSWTCH & 2 & Sample & & \\
\hline 31 & Vial & 2 & Standard 2 & COLSWTCH & 2 & Sample & & \\
\hline 32 & Vial & 3 & Standard 3 & COLSWTCH & 2 & Sample & & \\
\hline 33 & Vial & 4 & Standard 4 & COLSWTCH & 2 & Sample & & \\
\hline 34 & Vial & 5 & Standard 5 & COLSWTCH & 2 & Sample & & \\
\hline 35 & Vial & 6 & Standard 6 & COLSWTCH & 2 & Sample & & \\
\hline 36 & Vial & 1 & Standard 1 & COLSWTCH & 2 & Sample & & \\
\hline 37 & Vial & 2 & Standard 2 & COLSWTCH & 2 & Sample & & \\
\hline 38 & Vial & 3 & Standard 3 & COLSWTCH & 2 & Sample & & \\
\hline 39 & Vial & 4 & Standard 4 & COLSWTCH & 2 & Sample & & \\
\hline 40 & Vial & 5 & Standard 5 & COLSWTCH & 2 & Sample & & \\
\hline 41 & Vial & 6 & Standard 6 & COLSWTCH & 2 & Sample & & \\
\hline 42 & Vial & 7 & Second Source & COLSWTCH & 2 & Sample & & \\
\hline 43 & Vial & 8 & $\mathrm{NaOH}$ Lab Blank & COLSWTCH & 2 & Sample & & \\
\hline 44 & Vial & 9 & DI H2O Lab Blank & COLSWTCH & 2 & Sample & & \\
\hline 45 & Vial & 31 & In R1 $\mathrm{C} 44^{*} 4.018$ & CHLSWTCH & 2 & Sample & & \\
\hline 46 & Vial & 32 & In $\mathrm{R} 2 \mathrm{C} 4 * 4.018$ & CHLSWTCH & 2 & Sample & & \\
\hline 47 & Vial & 33 & In $\mathrm{R} 3 \mathrm{C} 4 * 4.018$ & CHLSWTCH & 2 & Sample & & \\
\hline 48 & Vial & 34 & Stk RI C $4 * 4.01$ & CHLSWTCH & 2 & Sample & & \\
\hline 49 & Vial & 35 & Stk R2 C4*4.01 & CHLSWTCH & 2 & Sample & & \\
\hline 50 & Vial & 36 & Stack R3 $\mathrm{C} 4 * 4.01$ & CHLSWTCH & 2 & Sample & & \\
\hline 51 & Vial & 37 & In $\mathrm{C} 7 * 4.1$ & CHLSWTCH & 2 & Sample & & \\
\hline 52 & Vial & 38 & Stk $C 7 * 4.1$ & CHLSWTCH & 2 & Sample & & \\
\hline 53 & Vial & 39 & $\mathrm{MS} /$ In R1 C4 & CHLSWTCH & 2 & Sample & & \\
\hline 54 & Vial & 1 & Standard 1 & COLSWTCH & 2 & Sample & & \\
\hline 55 & Vial & 2 & Standard 2 & COLSWTCH & 2 & Sample & & \\
\hline 56 & Vial & 3 & Standard 3 & COLSWTCH & 2 & Sample & & \\
\hline 57 & Vial & 4 & Standard 4 & COLSWTCH & 2 & Sample & & \\
\hline 58 & Vial & 5 & Standard 5 & COLSWTCH & 2 & Sample & & \\
\hline 59 & Vial & 6 & Standard 6 & COLSWTCH & 2 & Sample & & \\
\hline 60 & Vial & 9 & DI H2O Lab Blank & SHUTDOWึPO & $822 P$ & Pagentsi of 267 & & \\
\hline
\end{tabular}




\section{Sample Chromatograms}

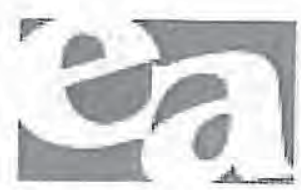



$1008-22$
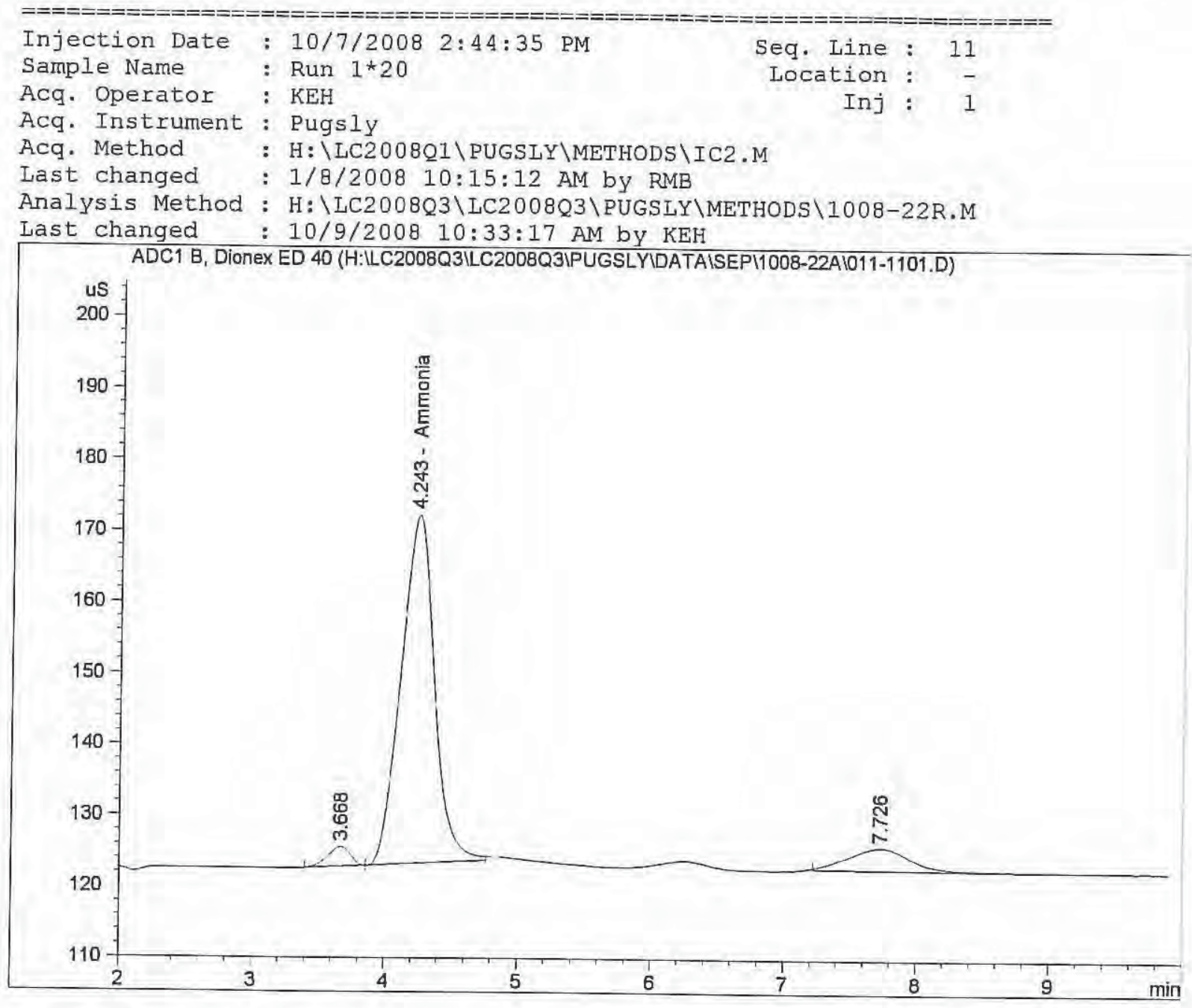

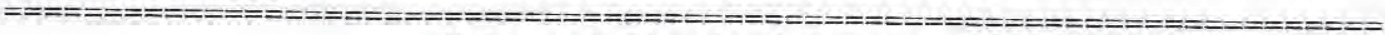

External standard Report

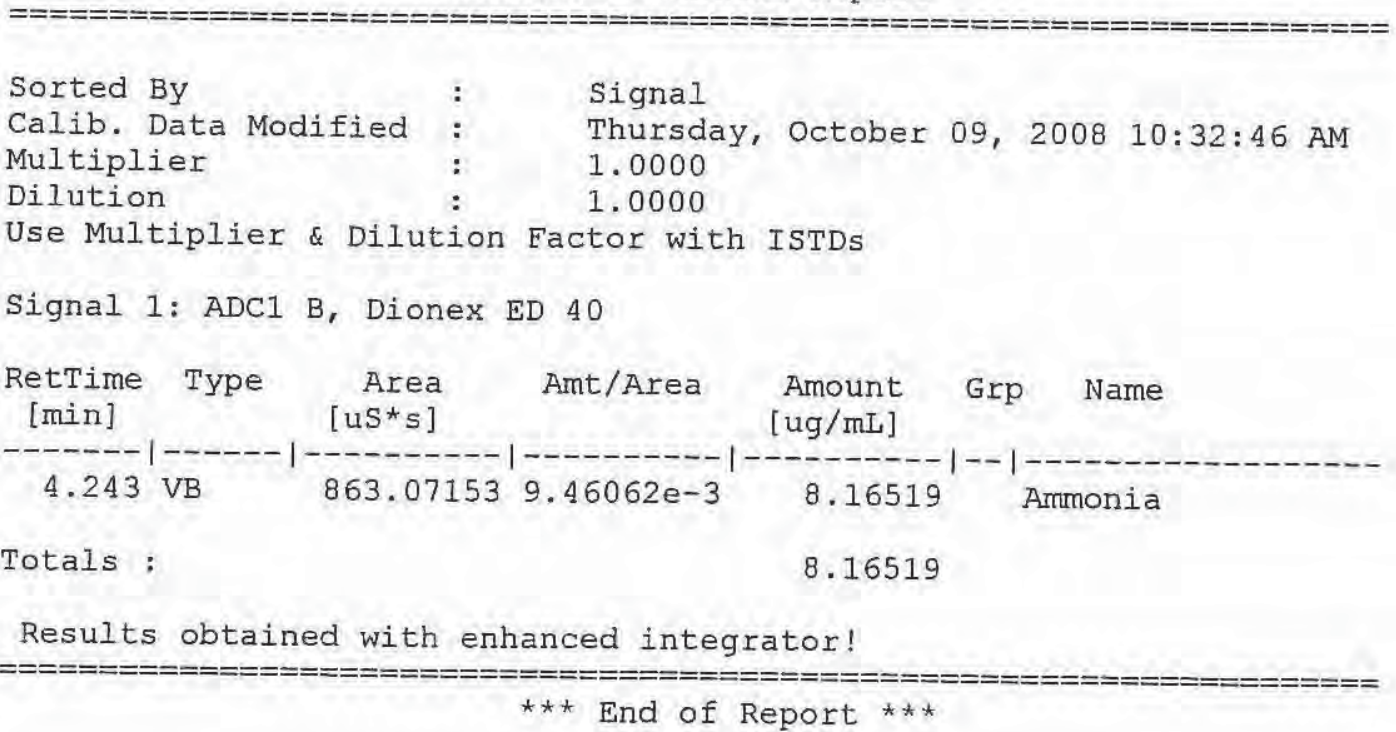




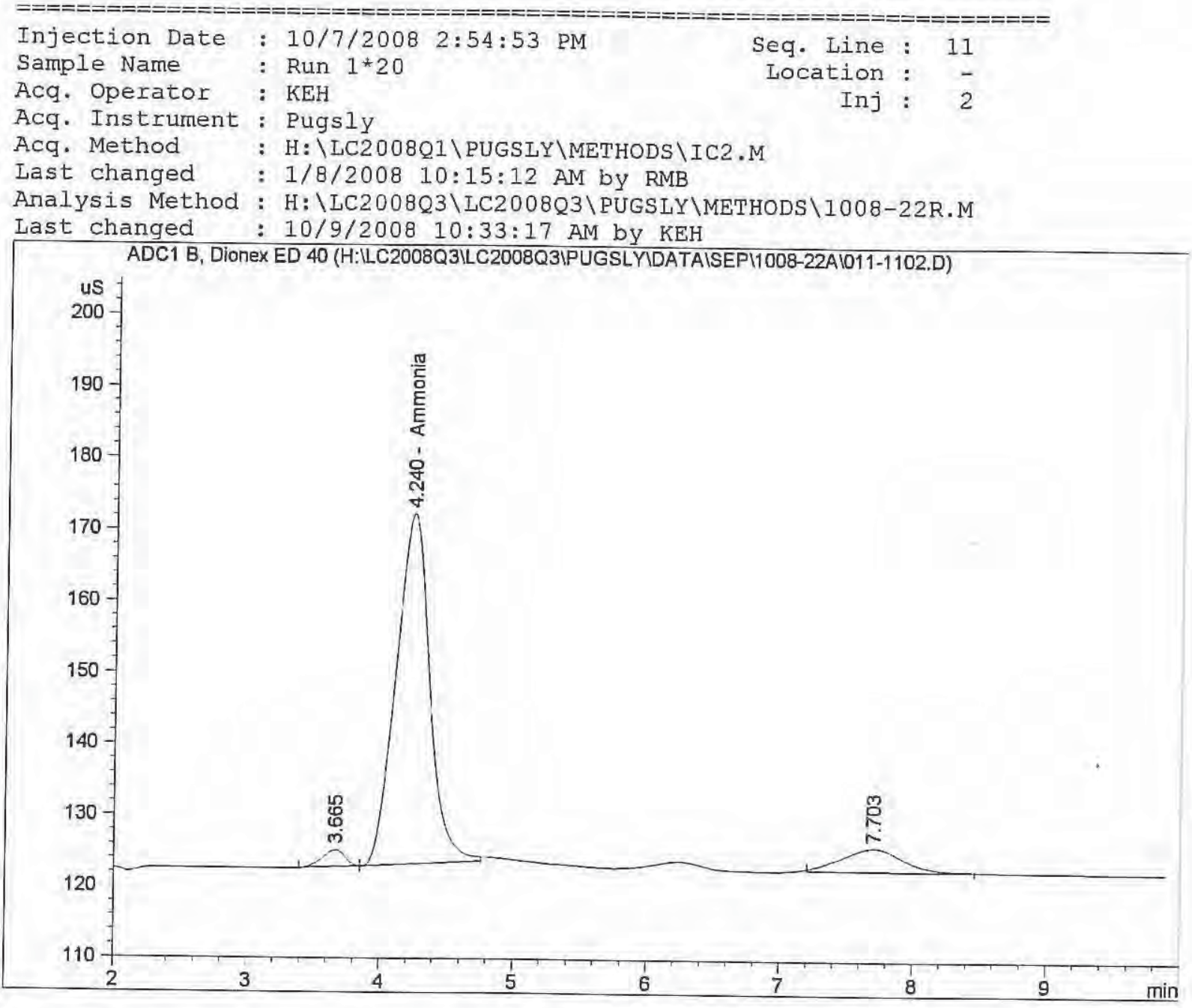

External standard Report

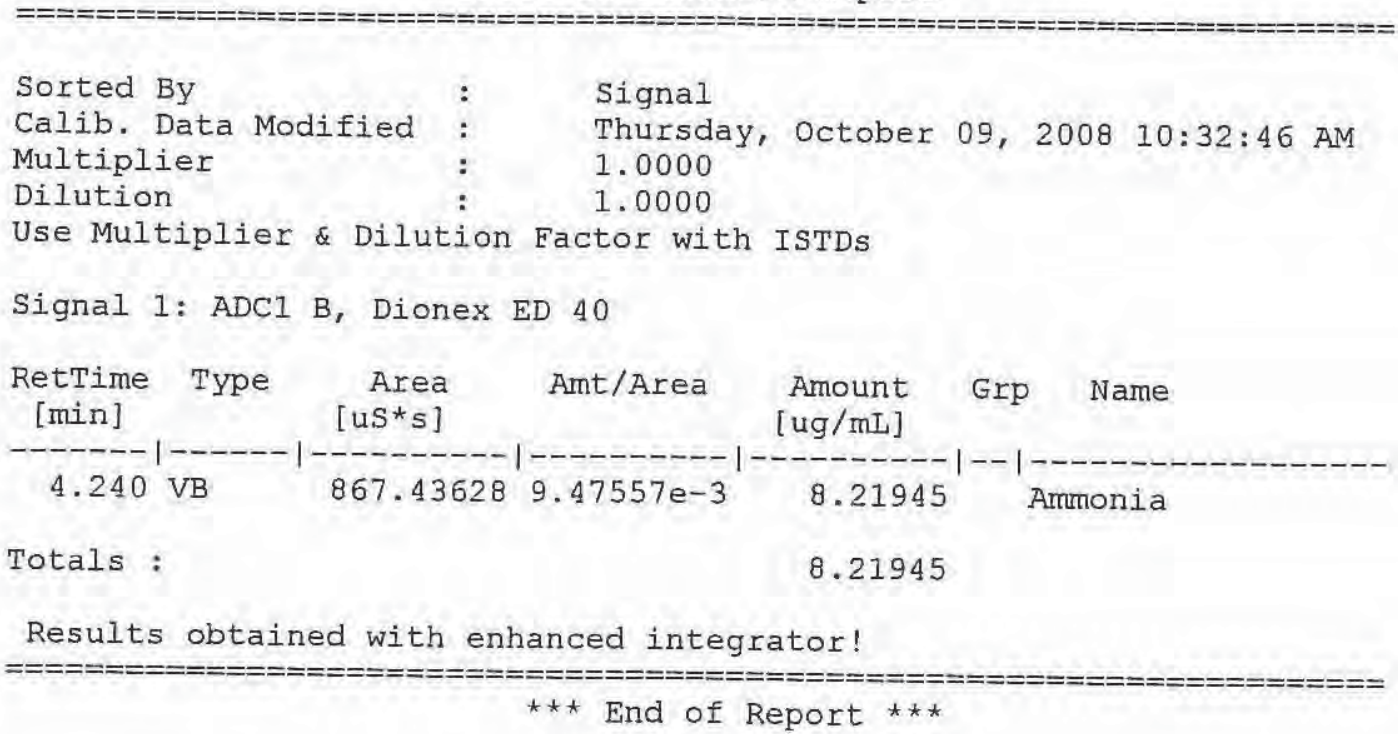


$1008-22$

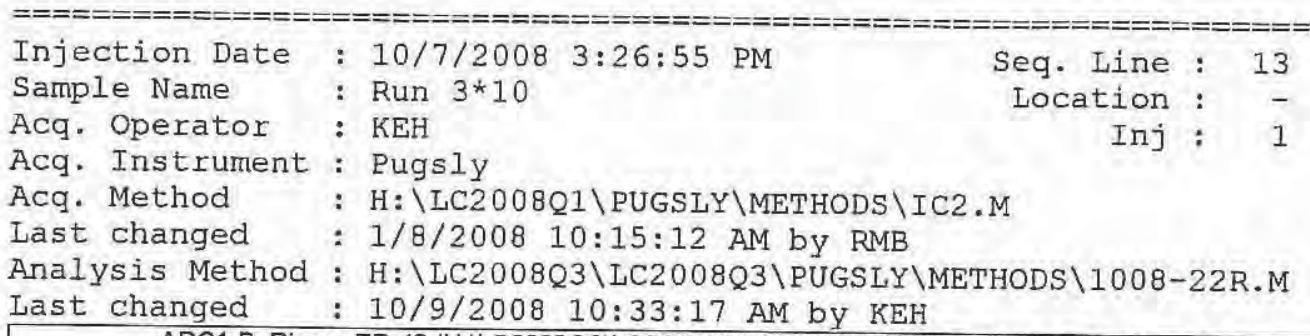

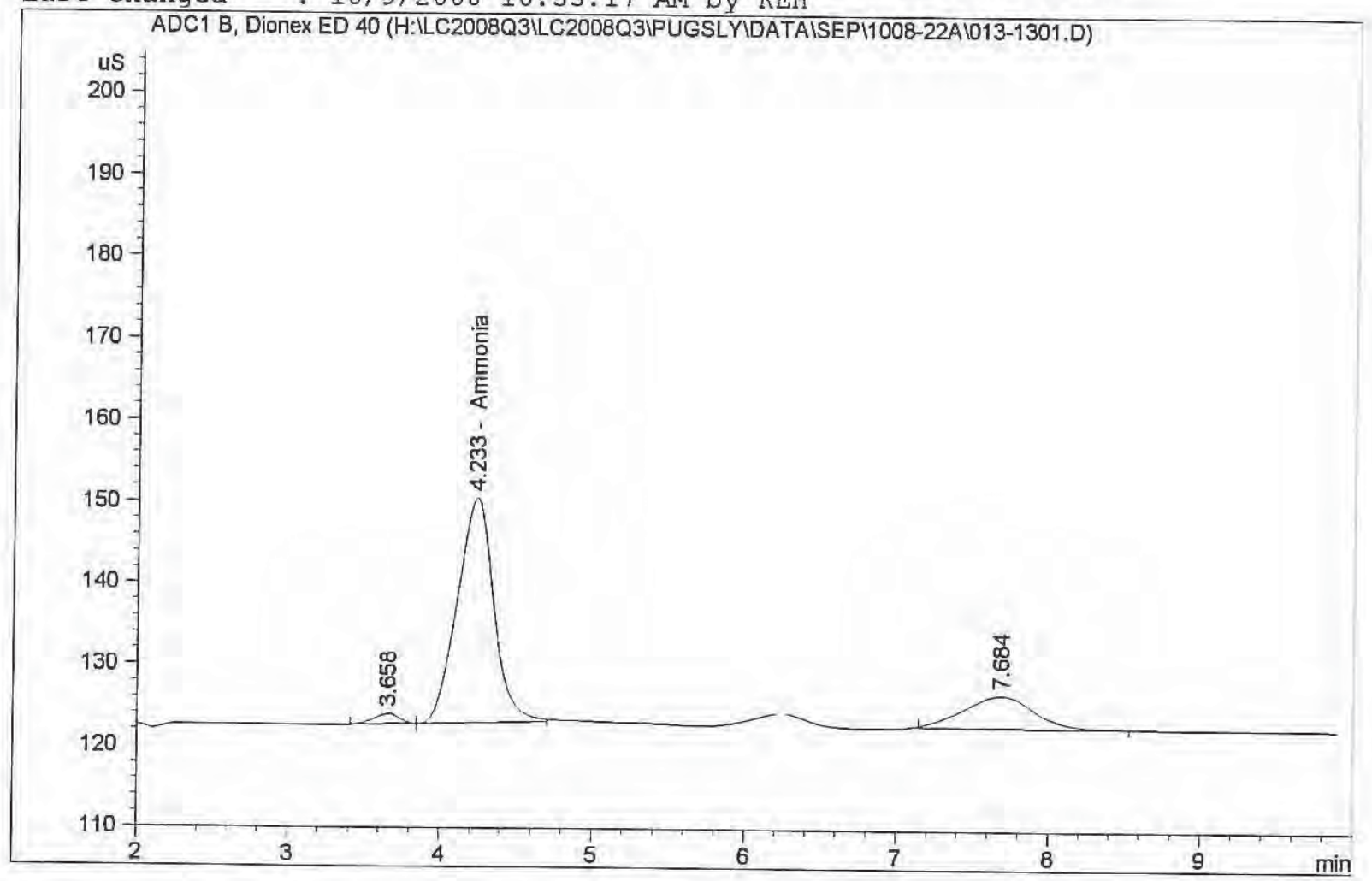

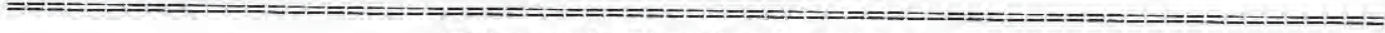

External Standard Report

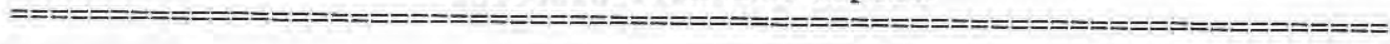

$\begin{array}{lll}\text { Sorted By } & : & \text { Signal } \\ \text { Calib. Data Modified } & : & \text { Thursday, October 09, 2008 10:32:46 AM } \\ \text { Multiplier } & : & 1.0000 \\ \text { Dilution } & : & 1.0000\end{array}$

Use Multiplier \& Dilution Factor with ISTDs

Signal 1: ADC1 B, Dionex ED 40

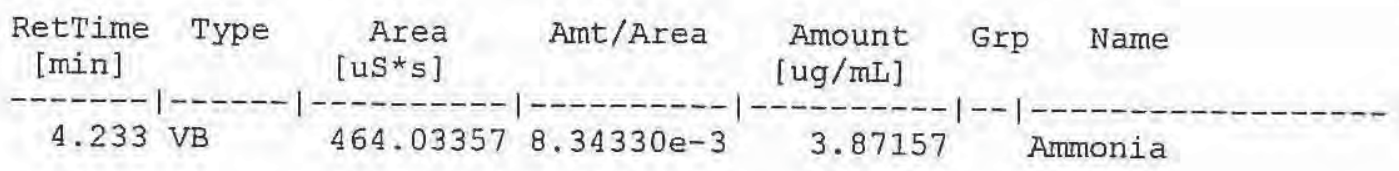

Totals :

3.87157

Results obtained with enhanced integrator!

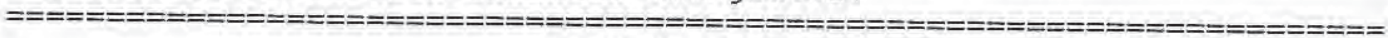
*** End of Report ***
} 


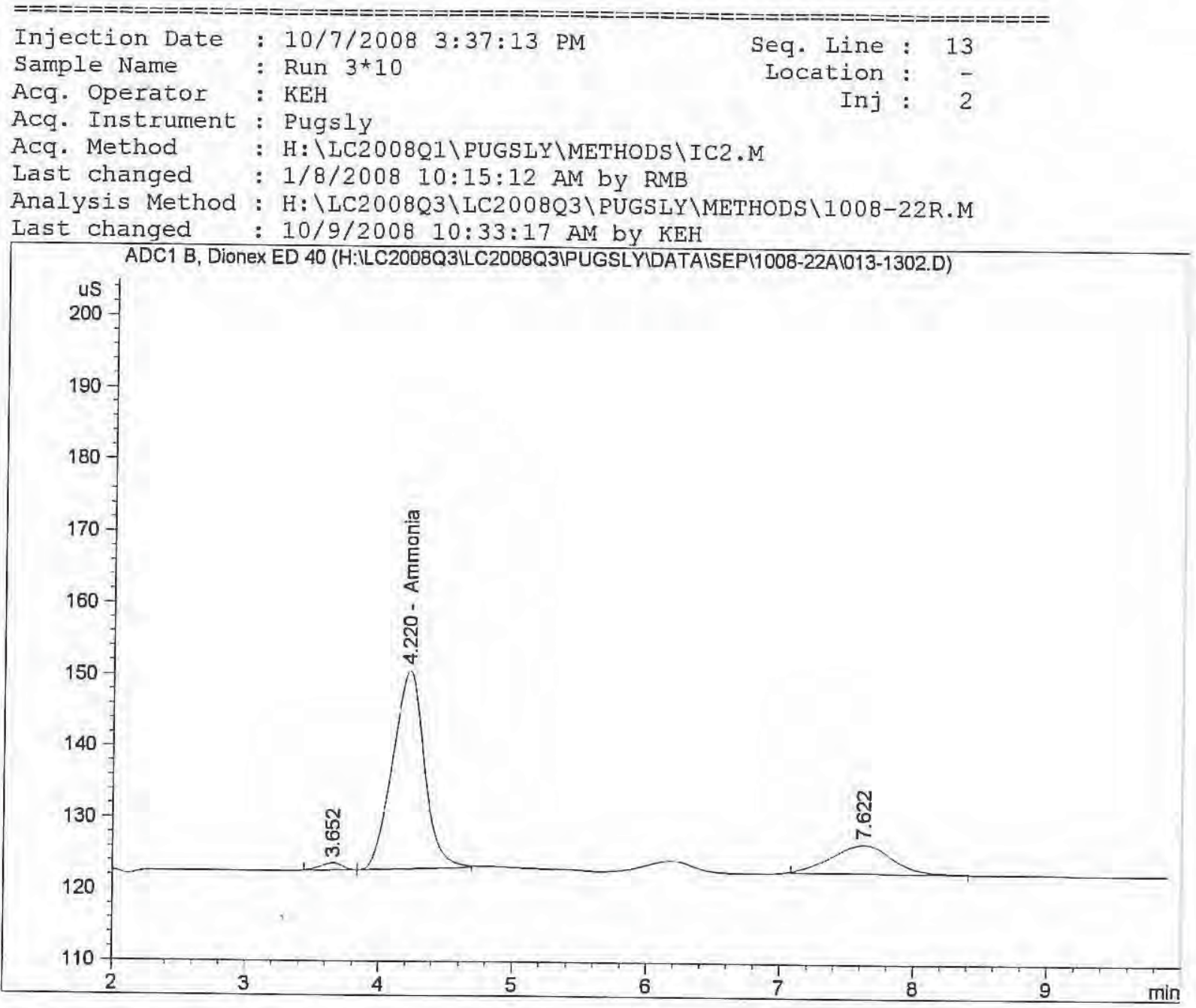

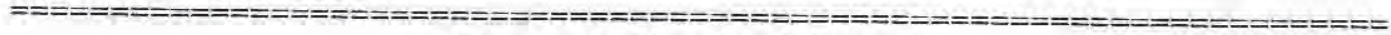

External Standard Report

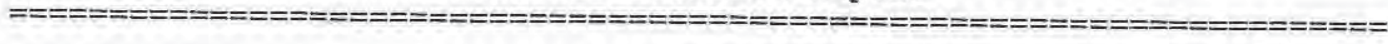

Sorted By : Signal

Calib. Data Modified : Thursday, October 09, 2008 10:32:46 AM

Multiplier : 1.0000

Dilution : 1.0000

Use Multiplier \& Dilution Factor with ISTDs

Signal 1: ADC1 B, Dionex ED 40

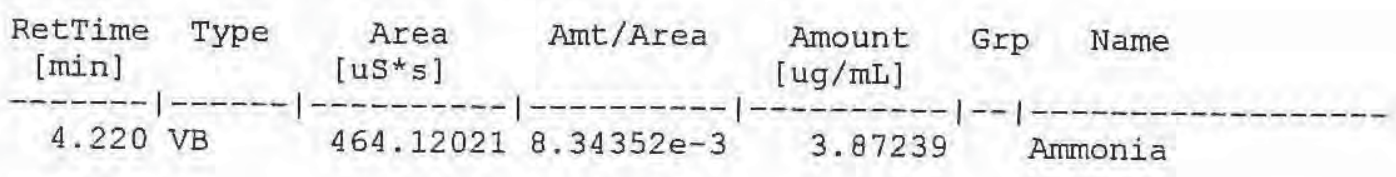

Totals :

3.87239

Results obtained with enhanced integrator!

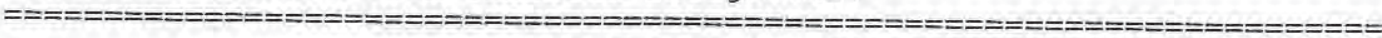
$\star \star *$ End of Report *** 
1008-22 Sample Blank

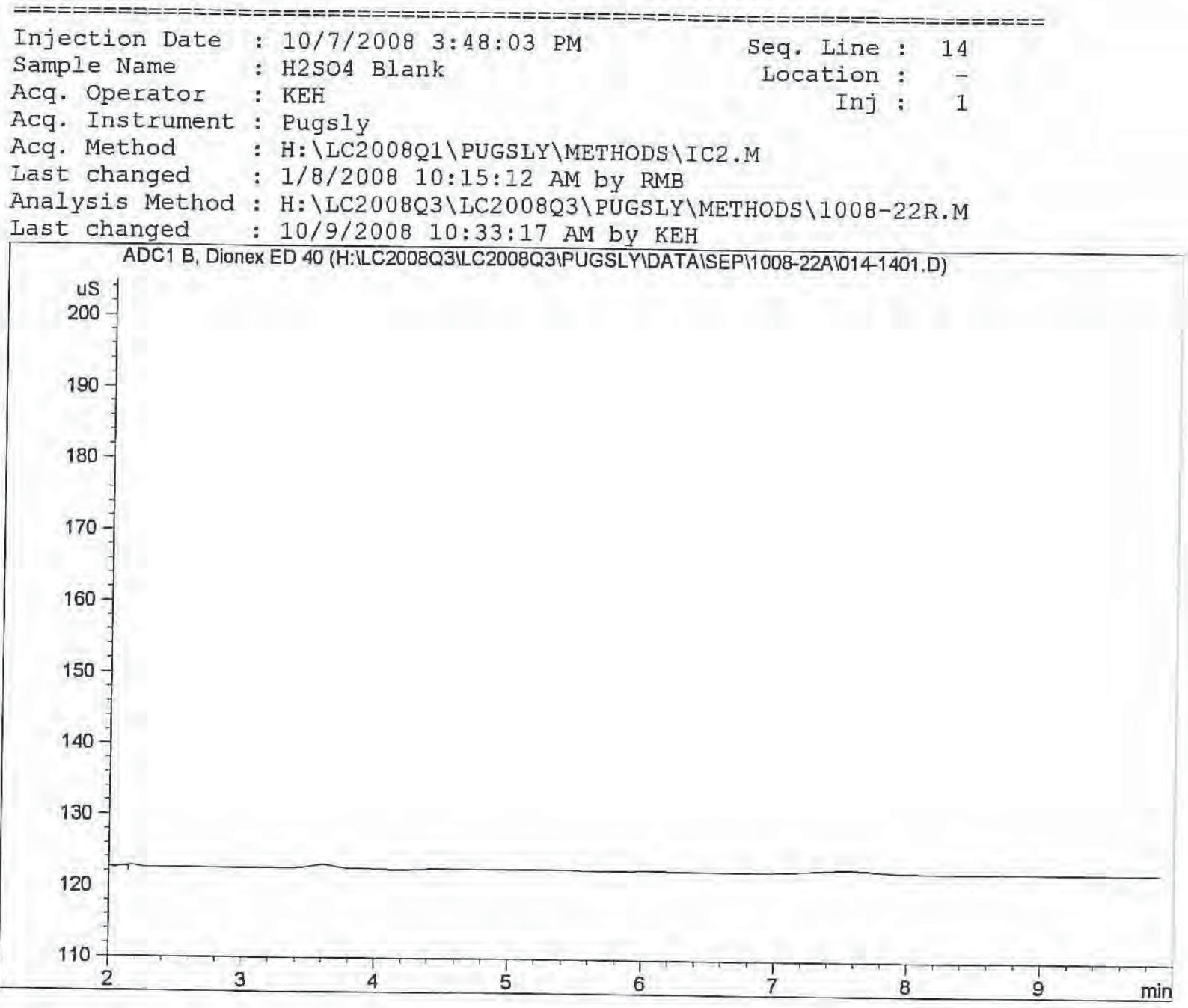

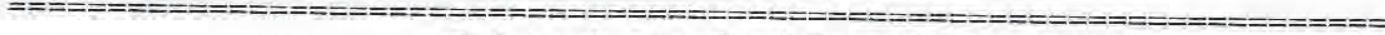

External standard Report

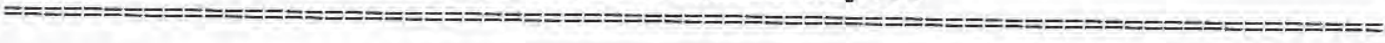

$\begin{array}{lll}\text { Sorted By } & : & \text { Signal } \\ \text { Calib. Data Modified } & : & \text { Thursday, October 09, 2008 10:32:46 AM } \\ \text { Multiplier } & : & 1.0000 \\ \text { Dilution } & : & 1.0000\end{array}$

Use Multiplier \& Dilution Factor with ISTDs

Signal 1: ADC1 B, Dionex ED 40

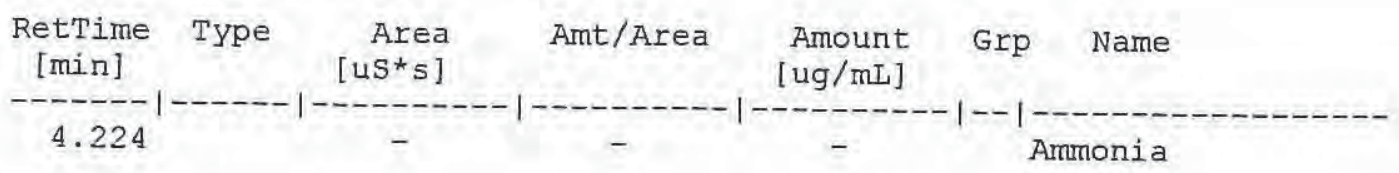

Totals : $\quad 0.00000$

Results obtained with enhanced integrator!

I Warnings or Errors :

Warning : Calibrated compound(s) not found
} 
Lab Blank $0.04 \mathrm{~N}$ H2SO4

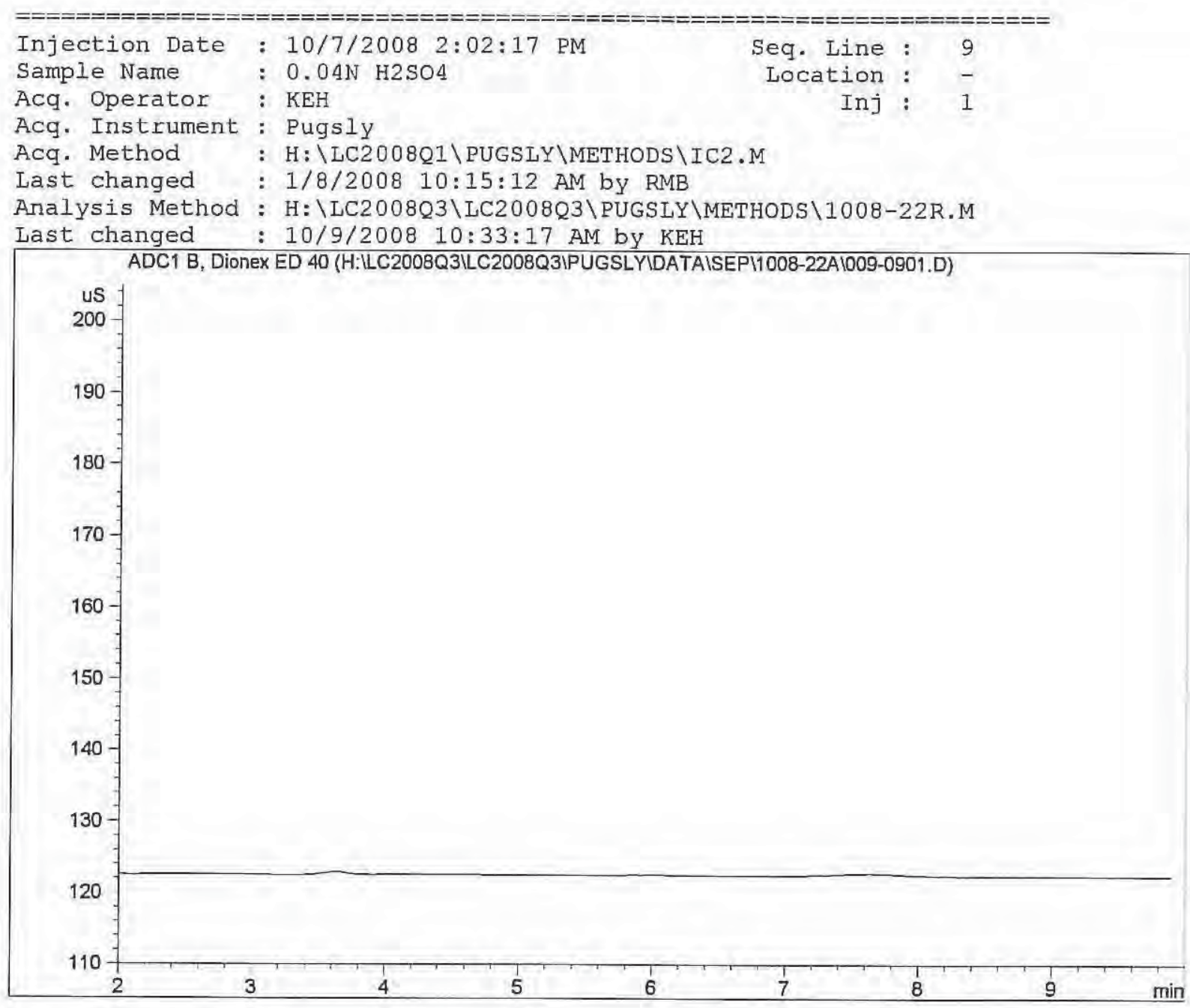

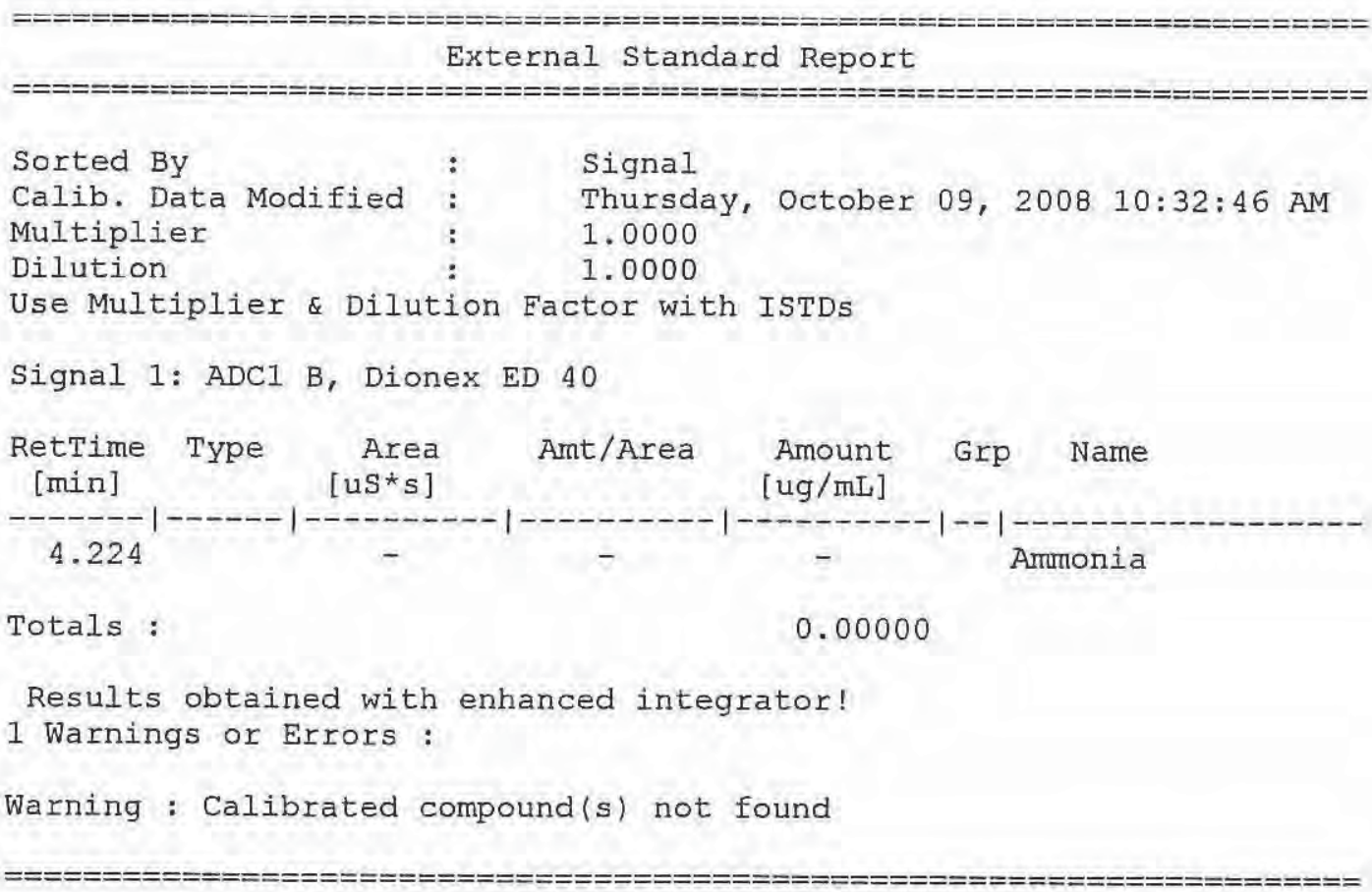


Lab Blank $0.04 \mathrm{~N} \mathrm{H2SO4}$

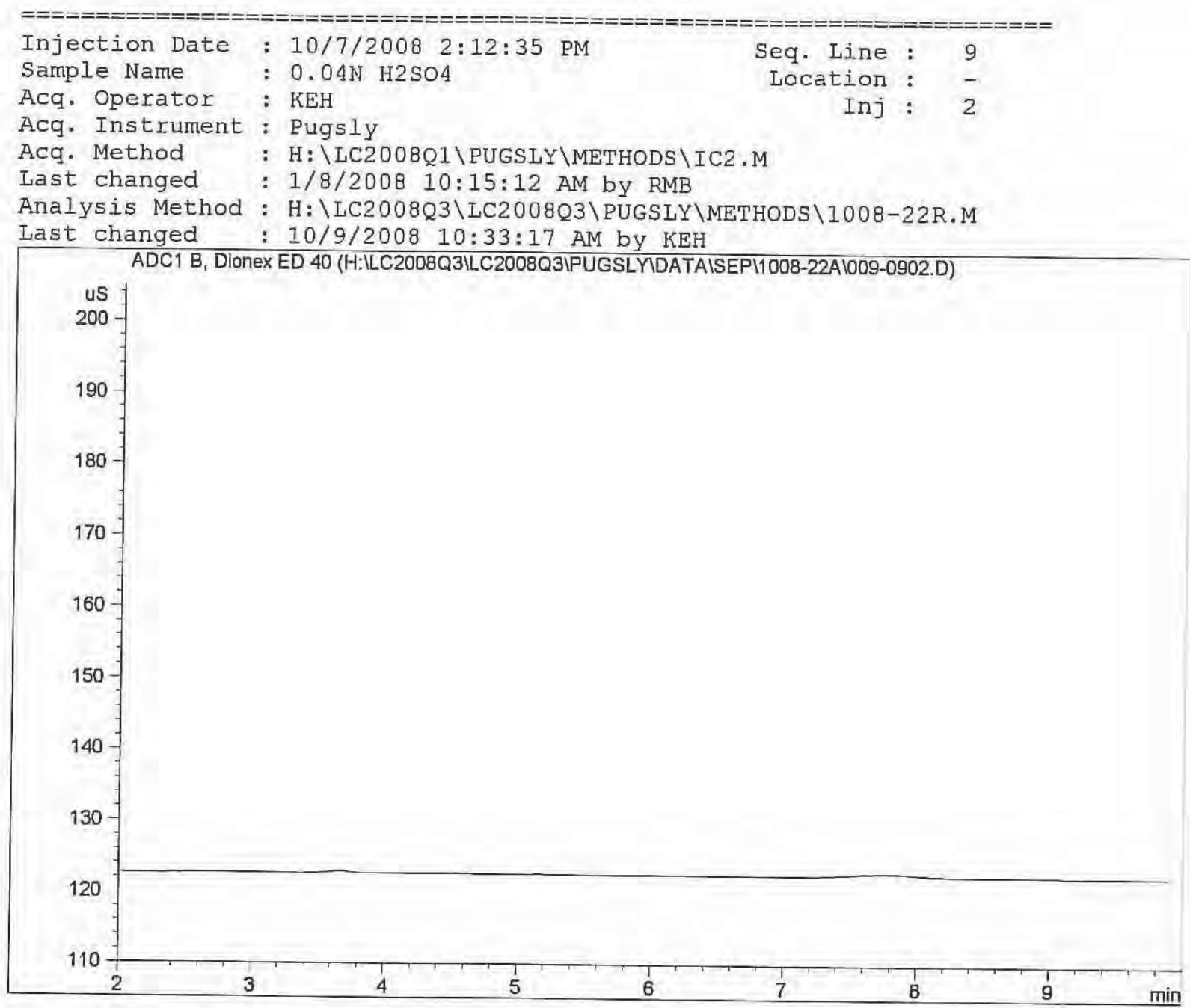

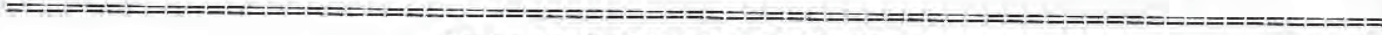

External Standard Report

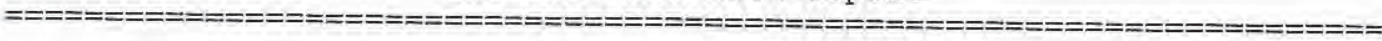

$\begin{array}{lll}\text { Sorted By } & : & \text { Signal } \\ \text { Calib. Data Modified } & : & \text { Thursday, October 09, } 200810: 32: 46 \text { AM } \\ \text { Multiplier } & : & 1.0000 \\ \text { Dilution } & : & 1.0000\end{array}$

Use Multiplier \& Dilution Factor with ISTDS

Signal 1: ADC1 B, Dionex ED 40

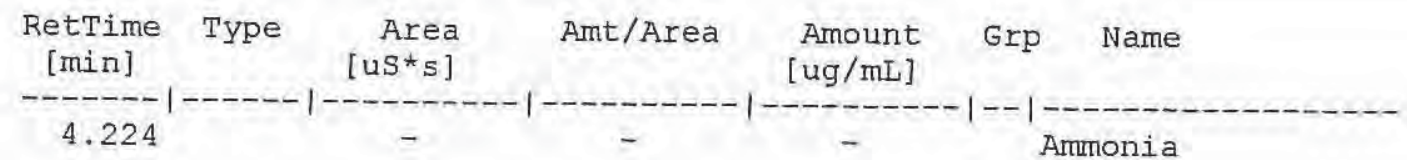

Totals :

0.00000

Results obtained with enhanced integrator!

1 Warnings or Errors :

Warning : Calibrated compound(s) not found
} 
Lab Blank DI H2O
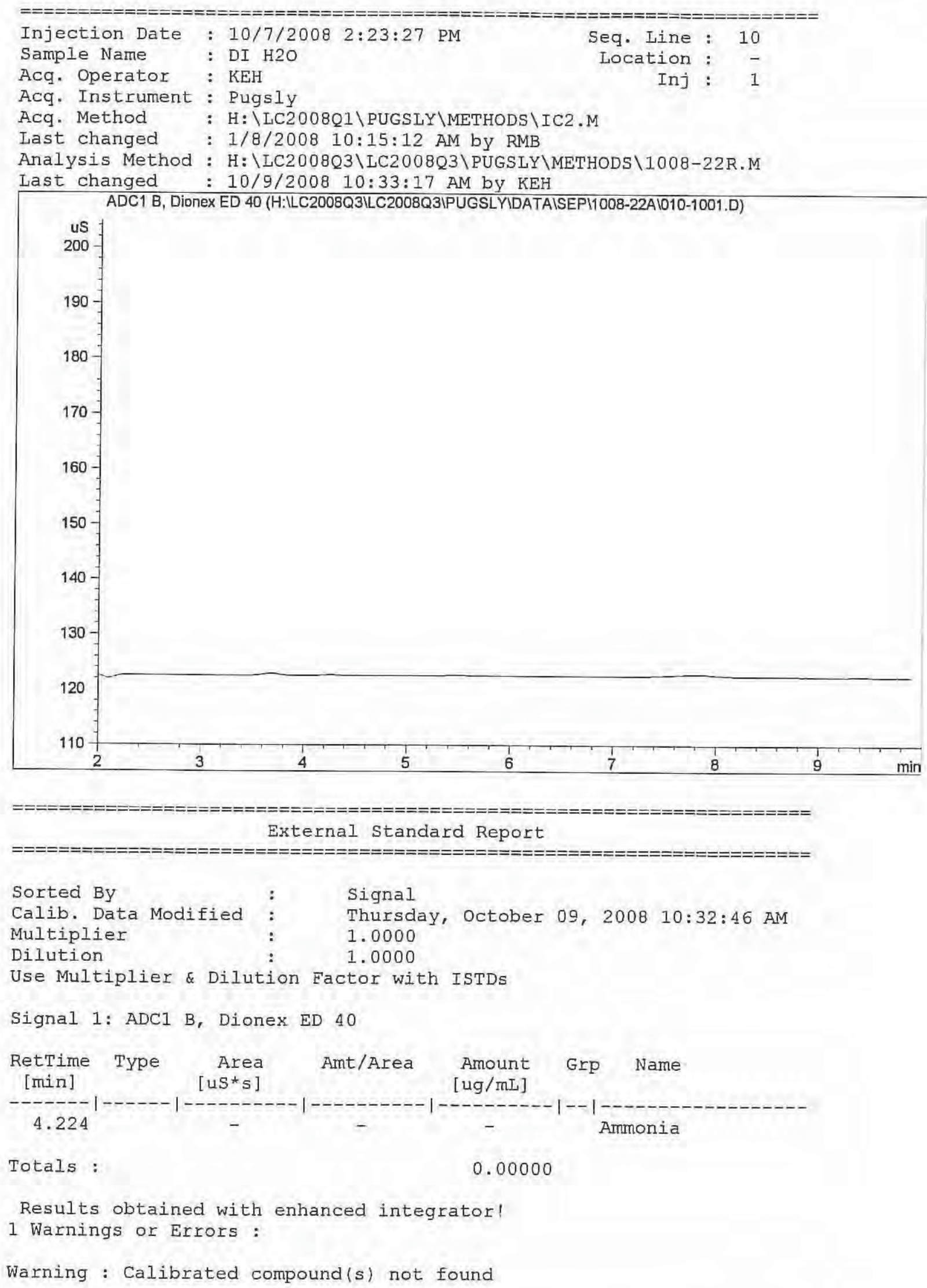

Results obtained with enhanced integrator!

1 Warnings or Errors :

Warning : Calibrated compound(s) not found 
Lab Blank DI H2O
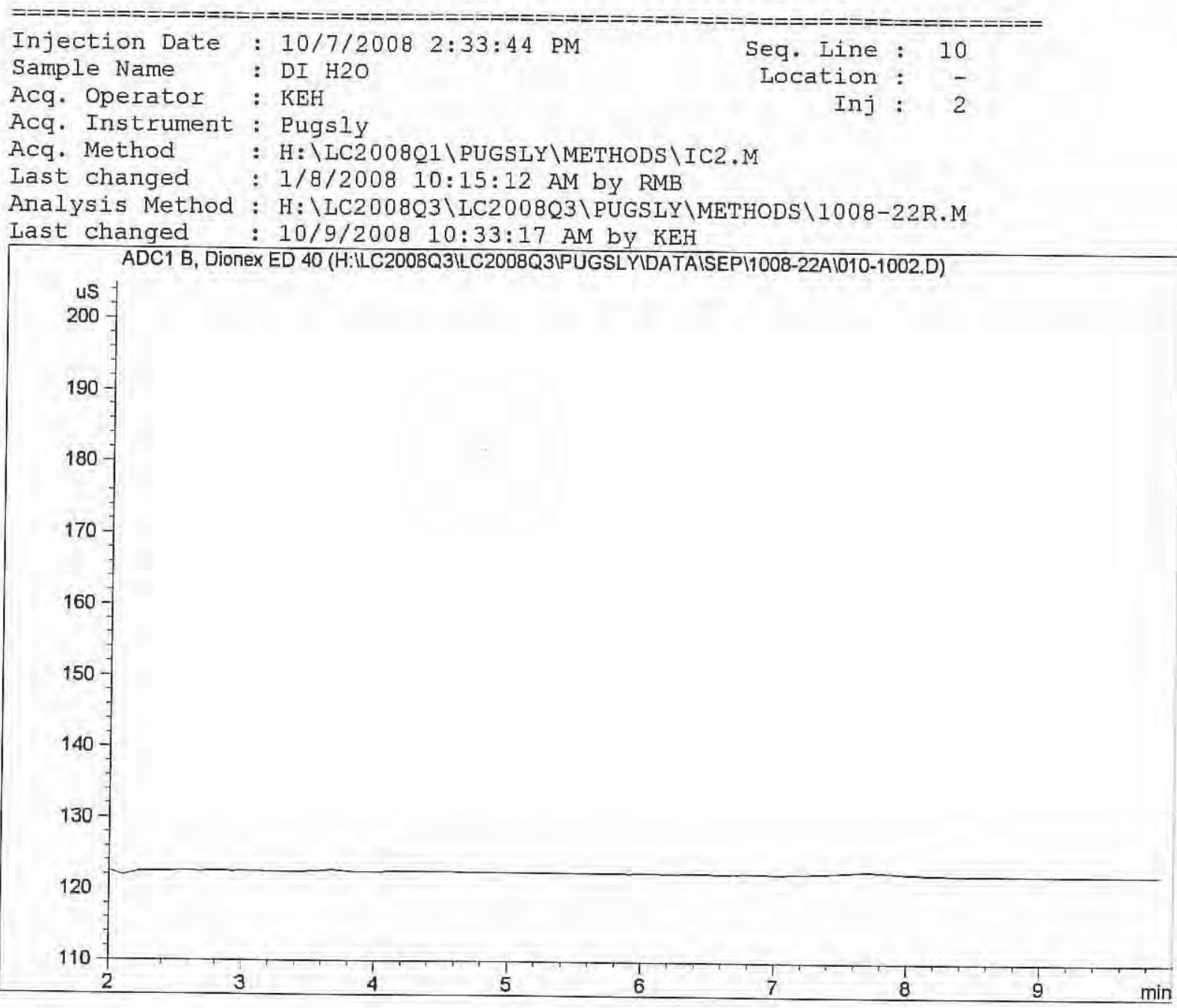

External Standard Report

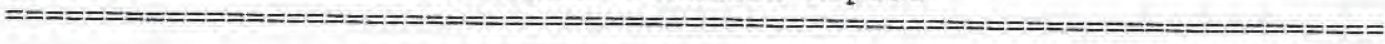

$\begin{array}{lll}\text { Sorted By } & : & \text { Signal } \\ \text { Calib. Data Modified } & \vdots & \text { Thursday, October 09, 2008 10:32:46 AM } \\ \text { Multiplier } & : & 1.0000 \\ \text { Dilution } & : & 1.0000\end{array}$

Use Multiplier \& Dilution Factor with ISTDs

Signal 1: ADCl B, Dionex ED 40

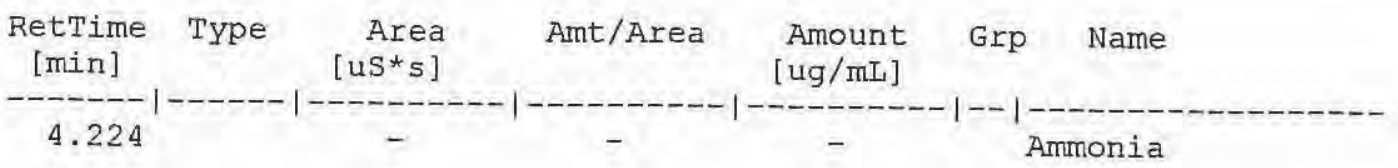

Totals :

0.00000

Results obtained with enhanced integrator!

I Warnings or Errors :

Warning : Calibrated compound(s) not found 

1008-22 Matrix spike
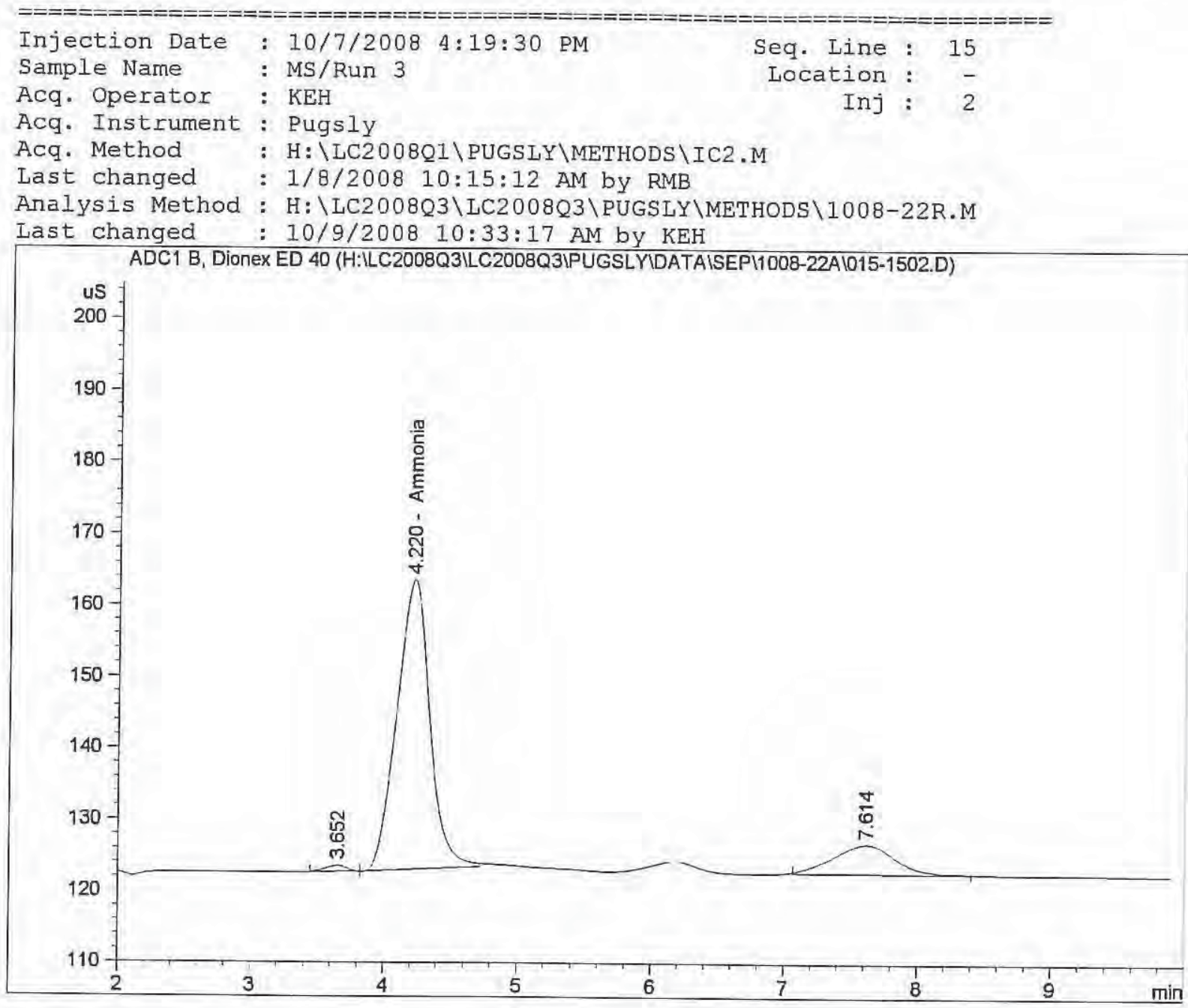

External Standard Report

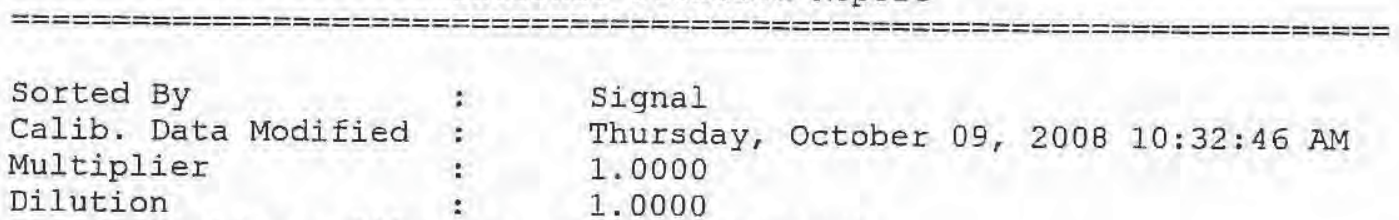

Use Multiplier \& Dilution Eactor with ISTDS

Signal 1: ADC1 B, Dionex ED 40

\begin{tabular}{|c|c|c|c|c|c|c|}
\hline $\begin{array}{c}\text { RetTime } \\
\text { [min] }\end{array}$ & Type & $\begin{array}{r}\text { Area } \\
\text { [us*s] }\end{array}$ & Amt/Area & $\begin{array}{l}\text { Amount } \\
\text { [ug/mL] }\end{array}$ & Grp & Name \\
\hline 4.220 & VB & 702.77374 & $8.96487 e-3$ & 6.30028 & & monia \\
\hline
\end{tabular}

Totals :

6.30028

Results obtained with enhanced integrator! 


\section{Curve(s)/QA Point(s) Chromatograms}

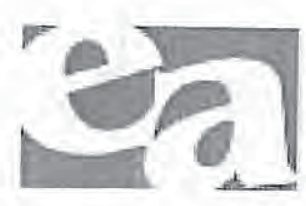




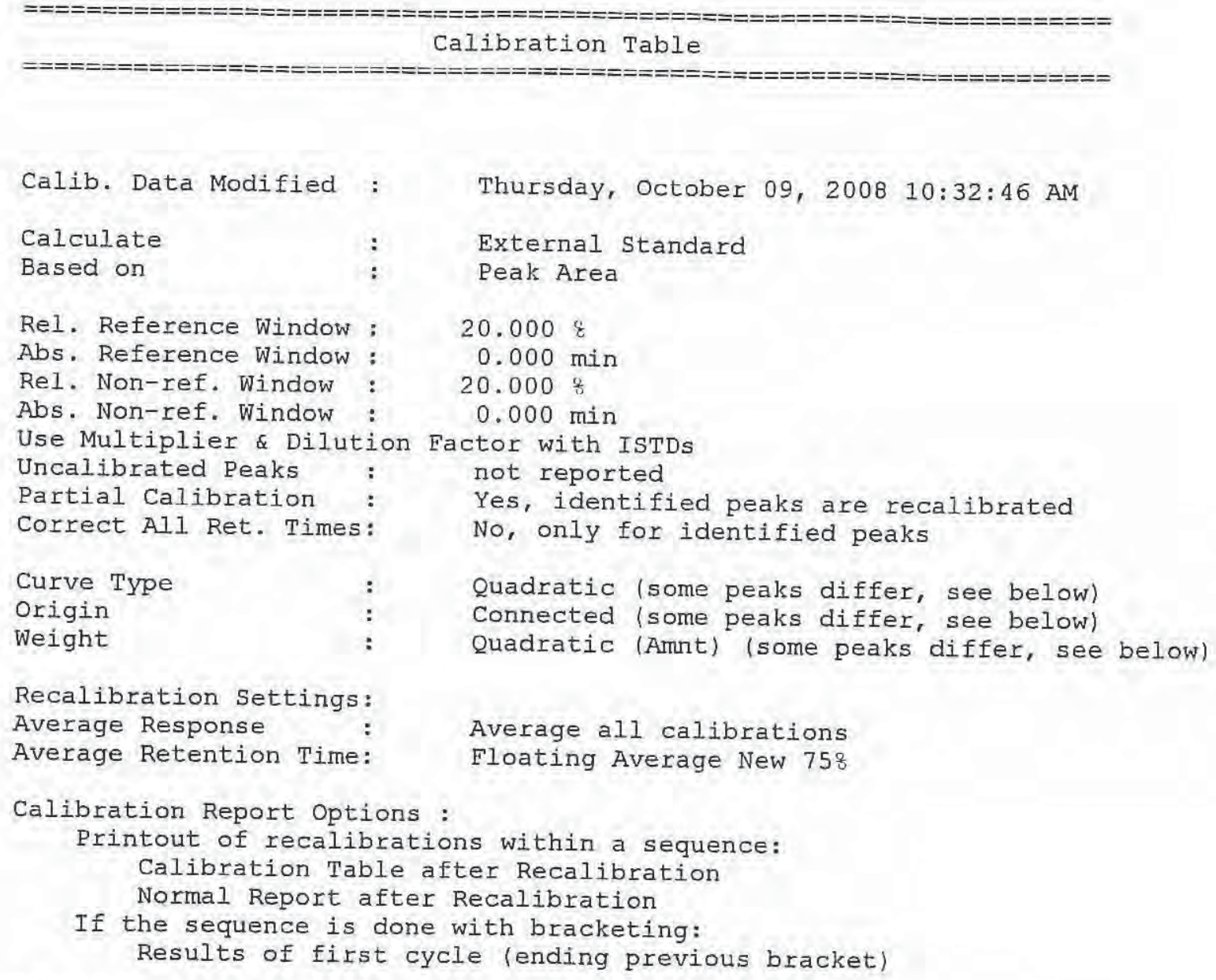

Recalibration Settings:

Average Response : Average all calibrations

Average Retention Time: Floating Average New 75 ?

Calibration Report Options :

Printout of recalibrations within a sequence:

Calibration Table after Recalibration

Normal Report after Recalibration

If the sequence is done with bracketing:

Results of first cycle (ending previous bracket)

Signal 1: ADC1 B, Dionex ED 40

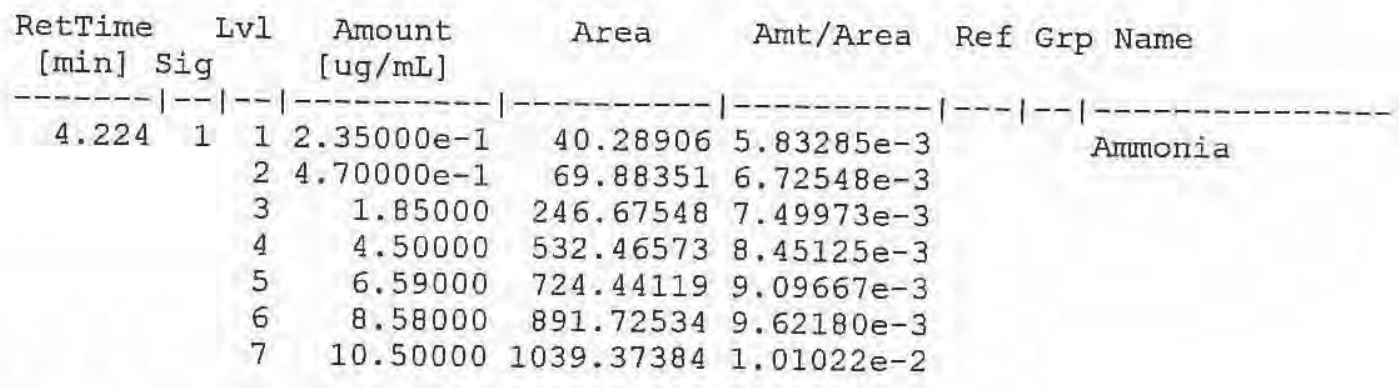

More compound-specific settings:

Compound: Ammonia

$\begin{array}{ll}\text { Curve Type } & \text { : Quadratic } \\ \text { Origin } & \text { : Connected }\end{array}$

Calibration Level Weights:/

Level 1 : 1

Level 2 : 1

Level $3 \quad: 1$

Level $4: 1$

Level $5: 1$

Level $6 \quad: 1$

Level $7: 1$ 
Method H: \IC2008Q3\LC2008Q3\PUGSIY\METHODS \1008-22R.M

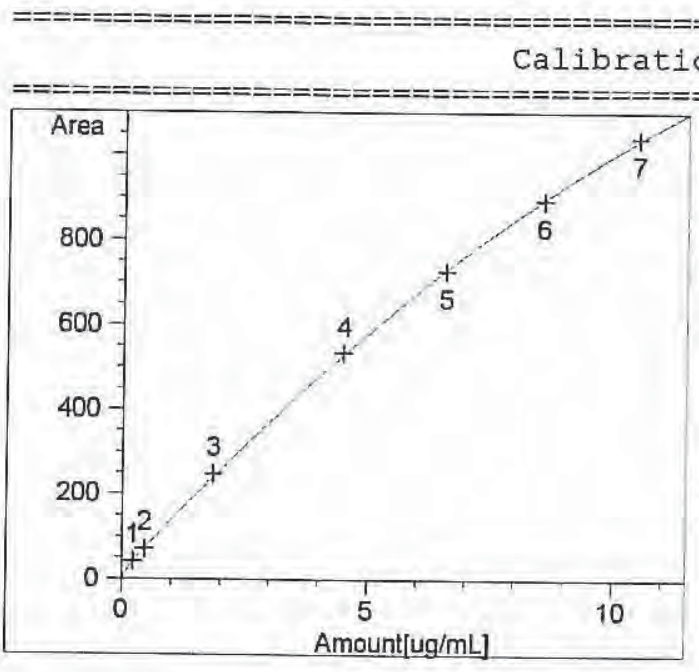

Ammonia at exp. RT: 4.224

ADC1 B, Dionex ED 40

Correlation:

0.99993

Residual Std. Dev.: 5.15949

Formula: $y=a x^{2}+b x+c$

$$
\begin{array}{lr}
\text { a: } & -2.87518 \\
\text { b: } & 127.54534 \\
\text { c: } & 13.32901
\end{array}
$$

$x$ : Amount $[\mathrm{ug} / \mathrm{mL}]$

$y$ : Area

Calibration Level Weights:

Level $1: 1$

Level $2: 1$

Level $3: 1$

Level $4: 1$

Level $5: 1$

Level $6: 1$

Level $7: 1$ 


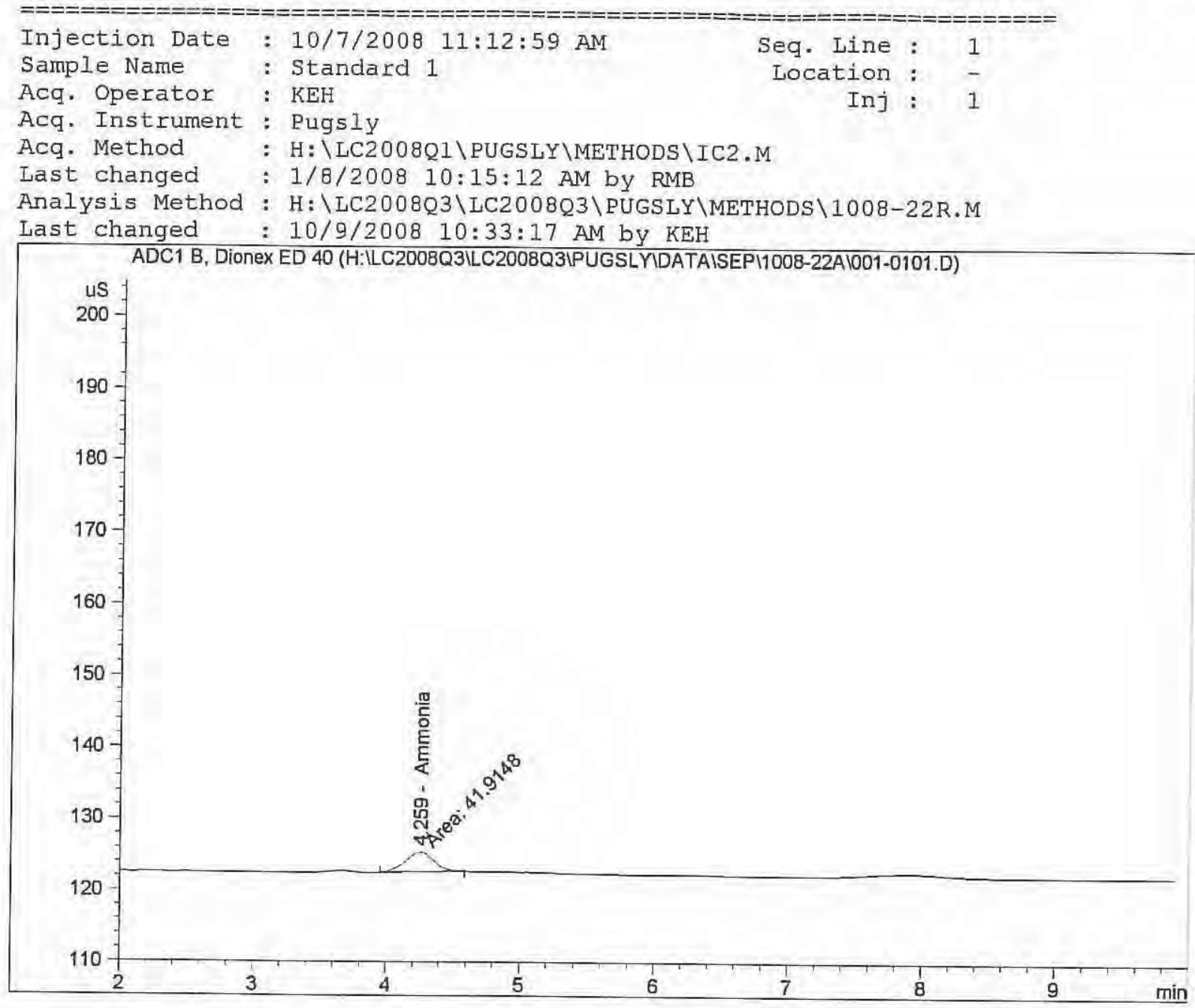

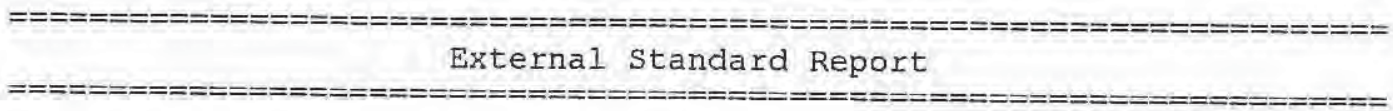

$\begin{array}{lll}\text { Sorted By } & : & \text { Signal } \\ \text { Calib. Data Modified } & : & \text { Thursday, October 09, 2008 10:32:46 AM } \\ \text { Multiplier } & : & 1.0000 \\ \text { Dilution } & : & 1.0000\end{array}$

Use Multiplier \& Dilution Factor with ISTDS

Signal 1: ADC1 B, Dionex ED 40

\begin{tabular}{|c|c|c|c|c|c|c|}
\hline $\begin{array}{c}\text { RetTime } \\
\text { [min] }\end{array}$ & Type & $\begin{array}{r}\text { Area } \\
{\left[u S^{*} s\right]}\end{array}$ & Amt/Area & $\begin{array}{l}\text { Amount } \\
{[\mathrm{ug} / \mathrm{mL}]}\end{array}$ & Grp & Name \\
\hline 4.259 & & 41.916 & $5.44695 e^{-3}$ & $2.28308 \mathrm{e}-$ & & monia \\
\hline
\end{tabular}

Totals : $\quad 2.28308 \mathrm{e}-1$

Results obtained with enhanced integrator!

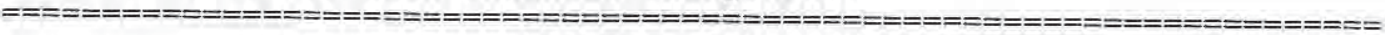

*** End of Report *** 


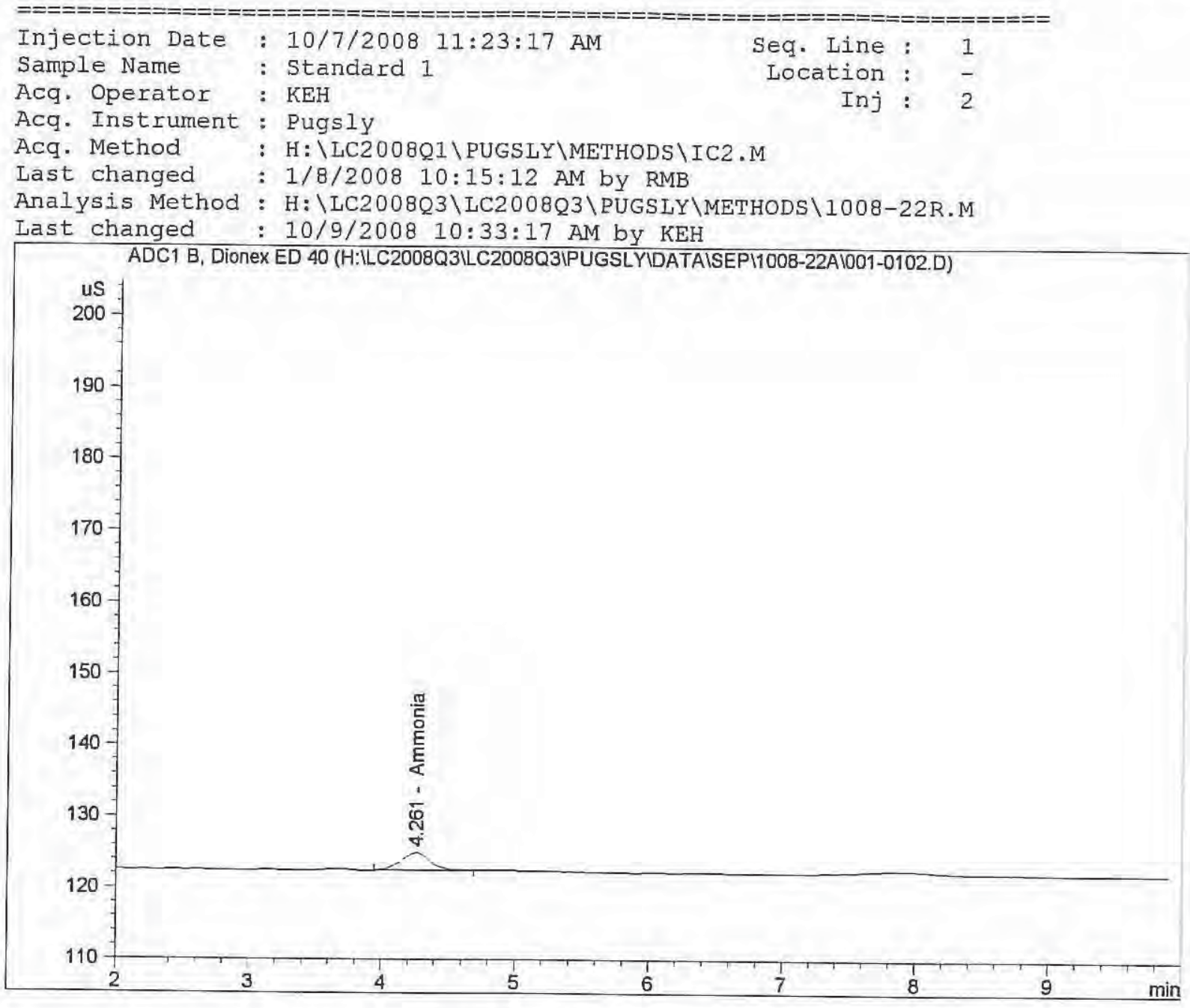

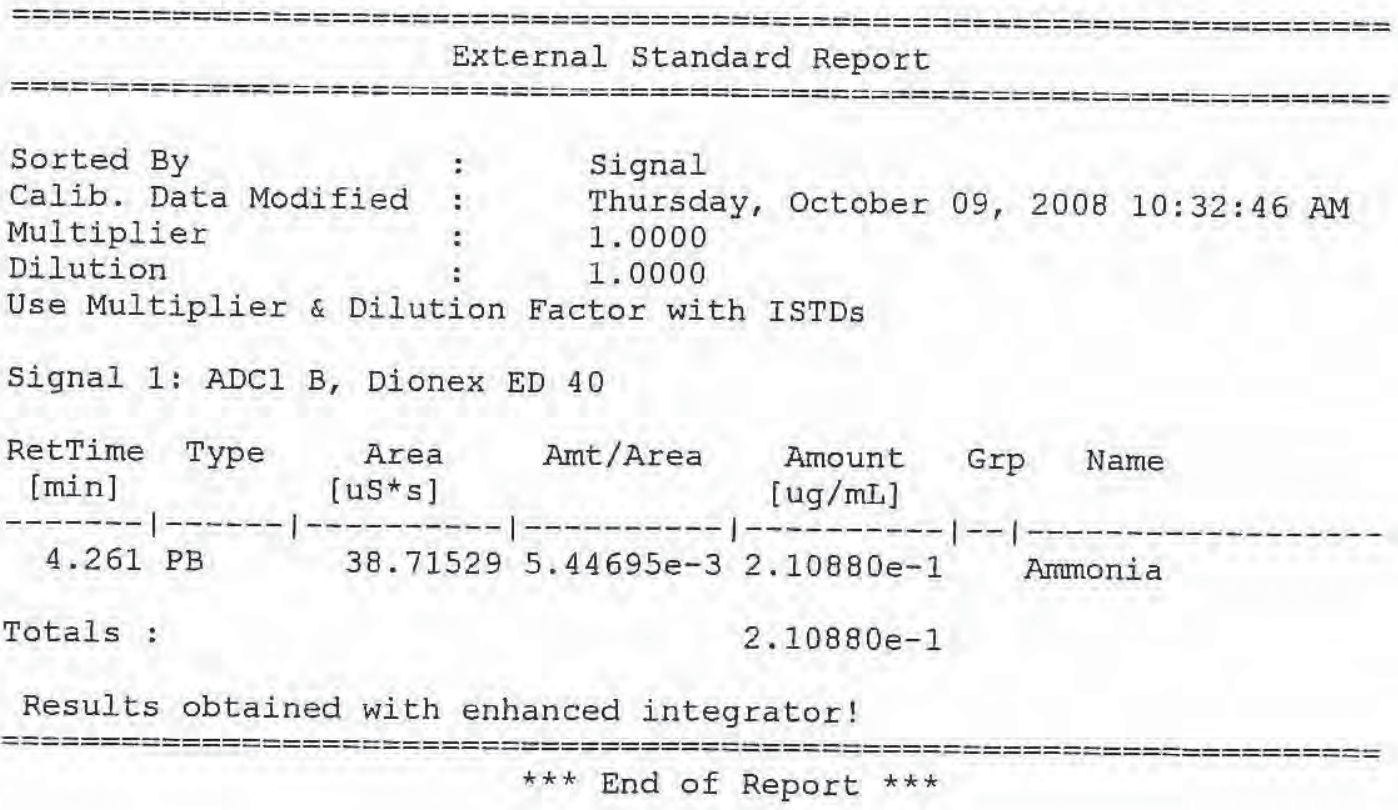




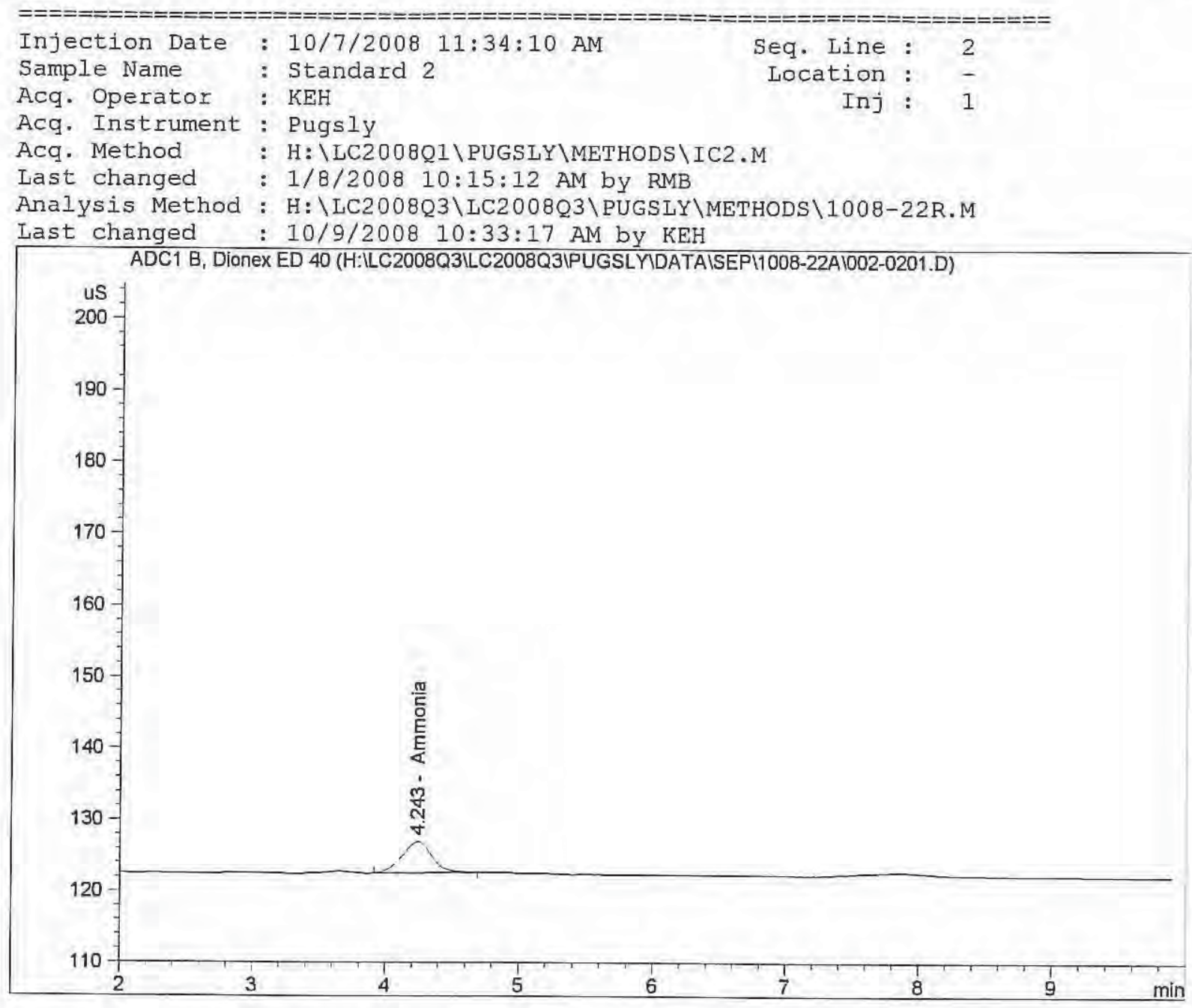

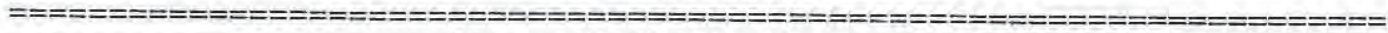

External Standard Report

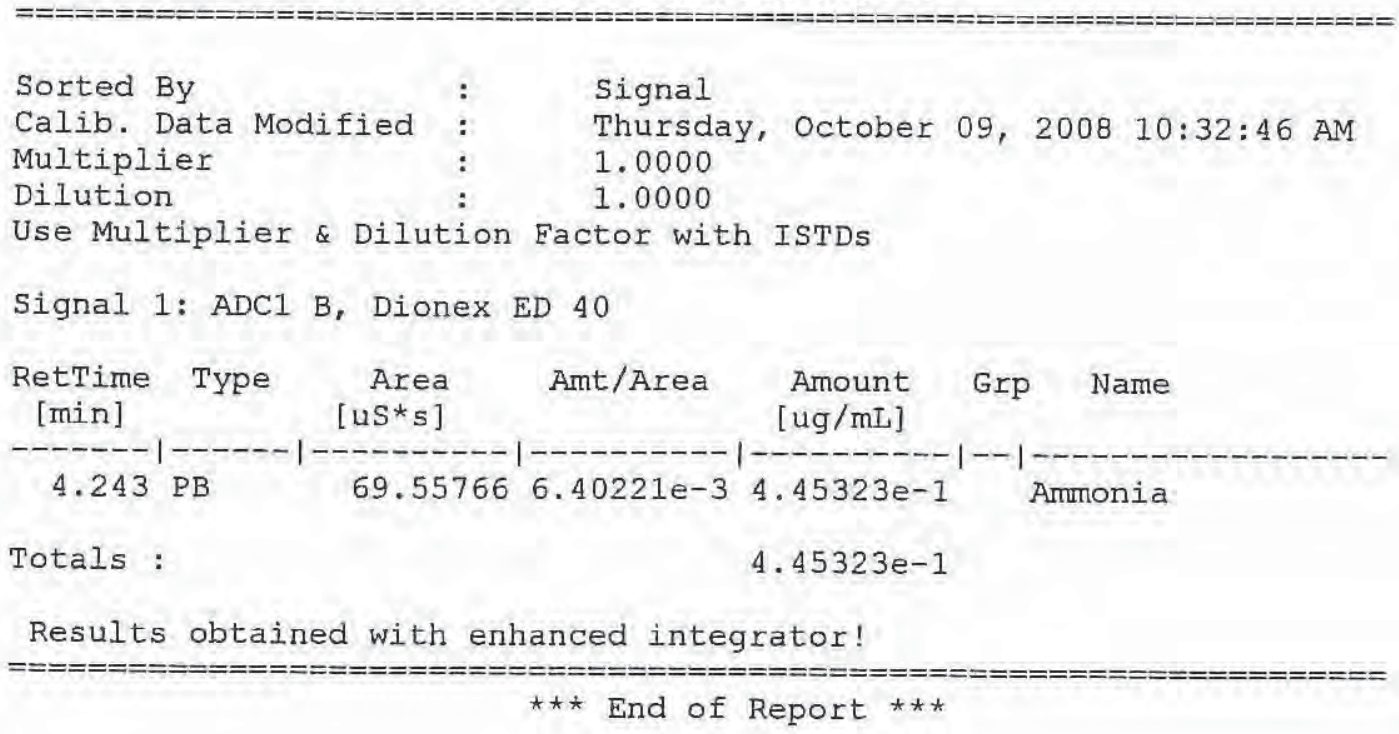




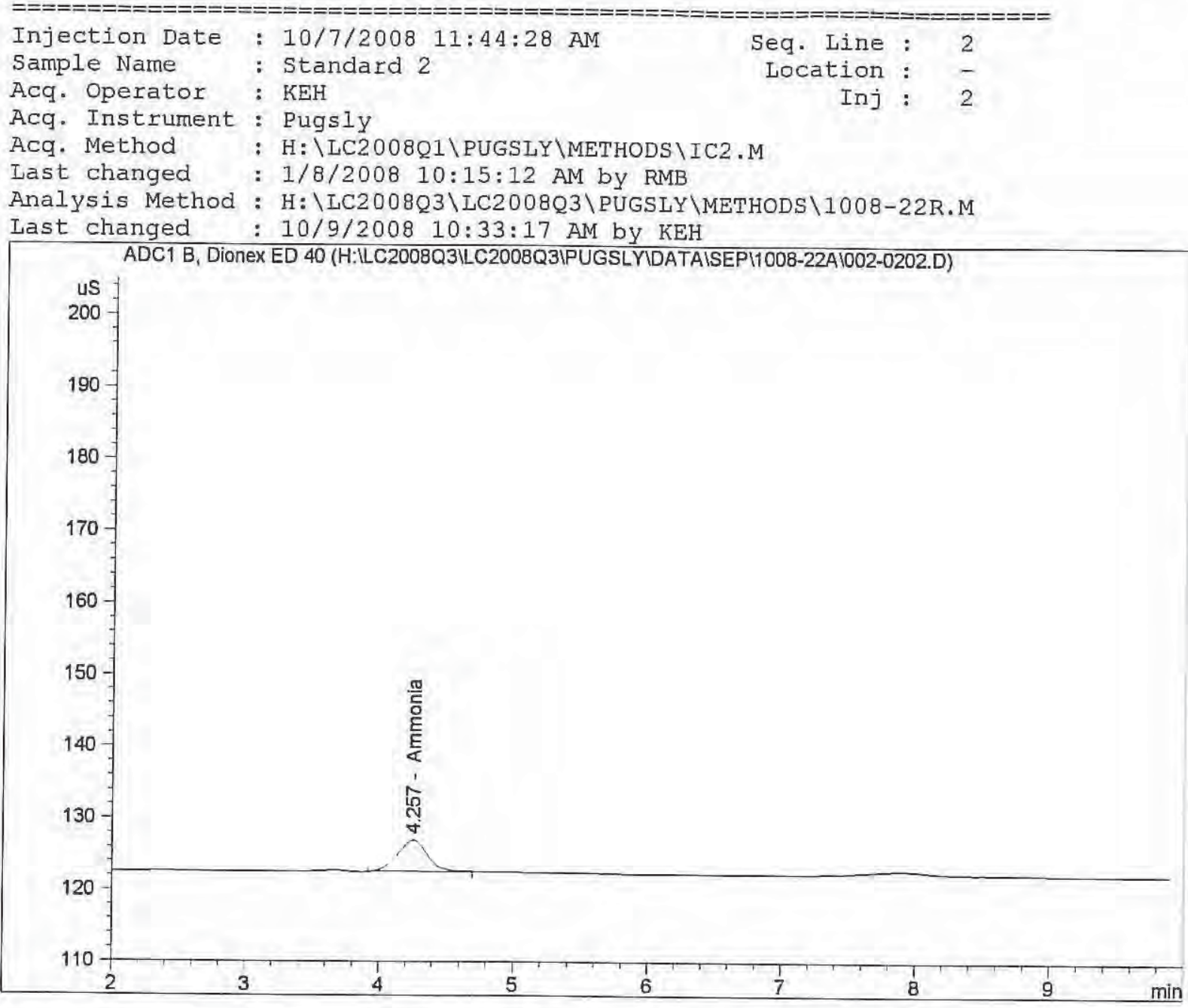

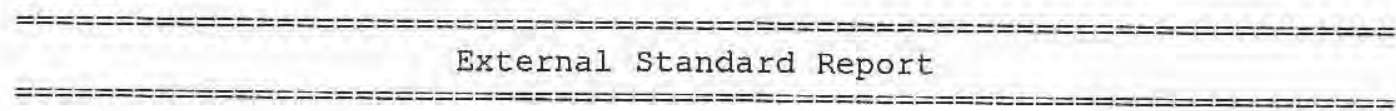

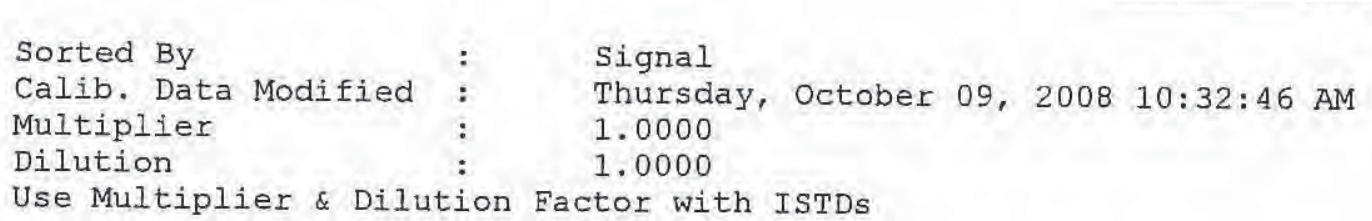

Use Multiplier \& Dilution Eactor with ISTDs

Signal 1: ADCl B, Dionex ED 40

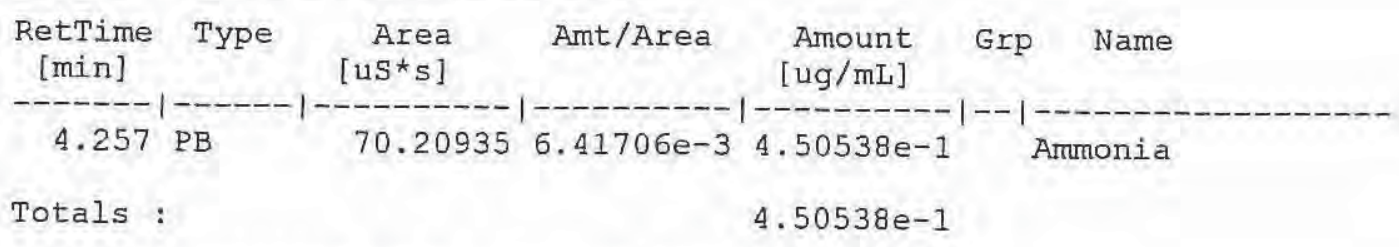

Results obtained with enhanced integrator!

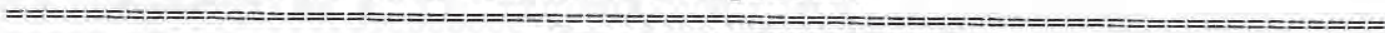
$\star \star \star$ End of Report $* * *$ 


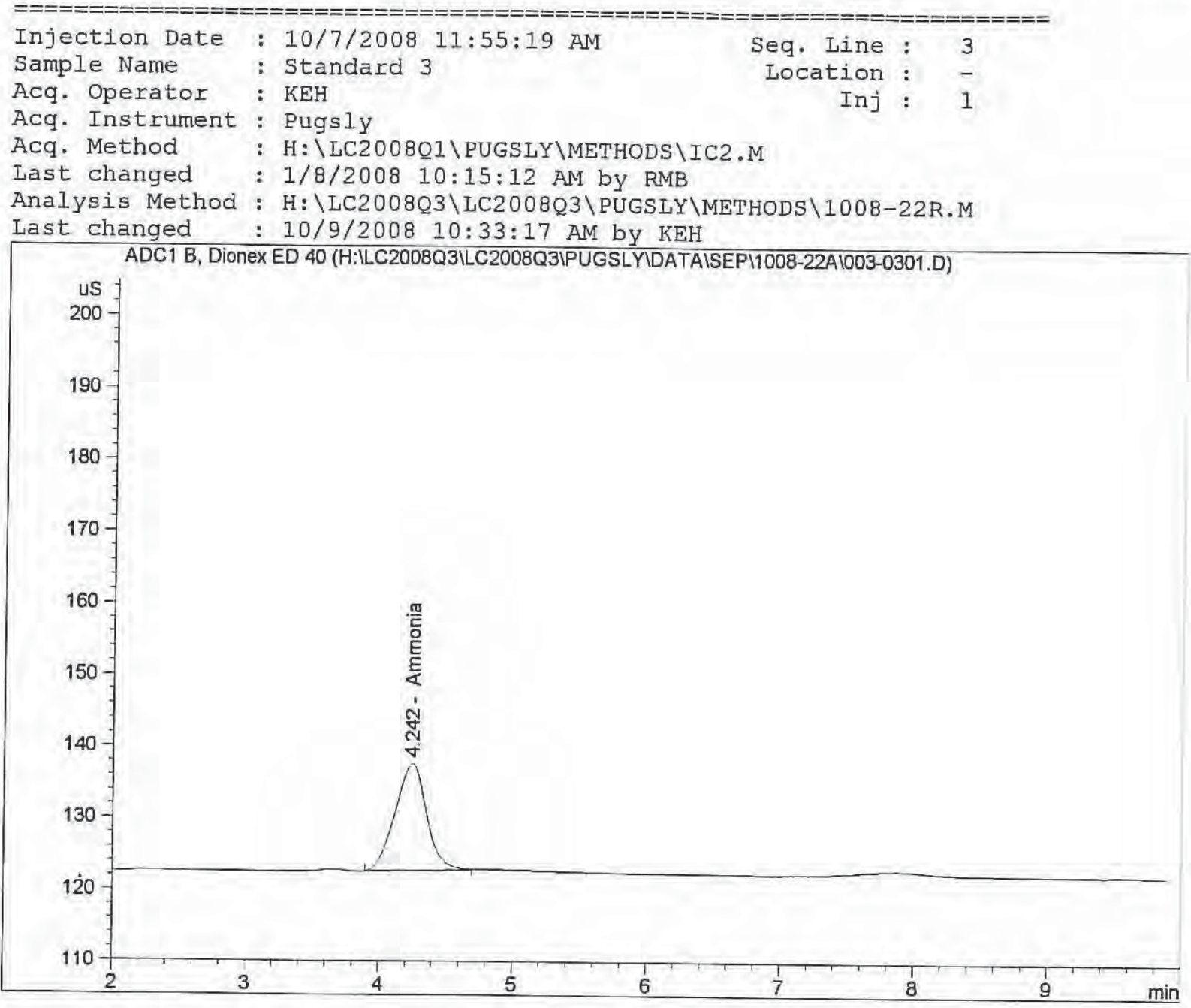

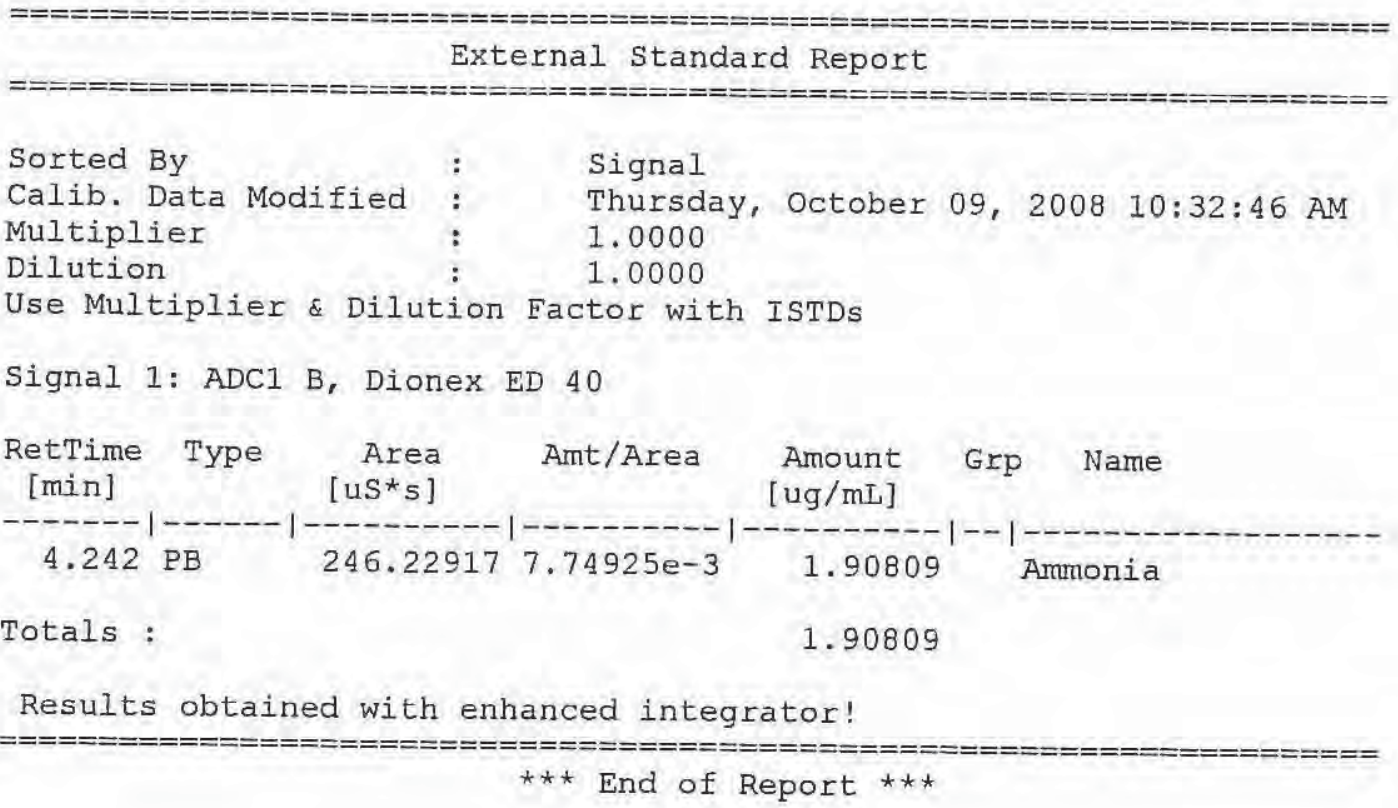




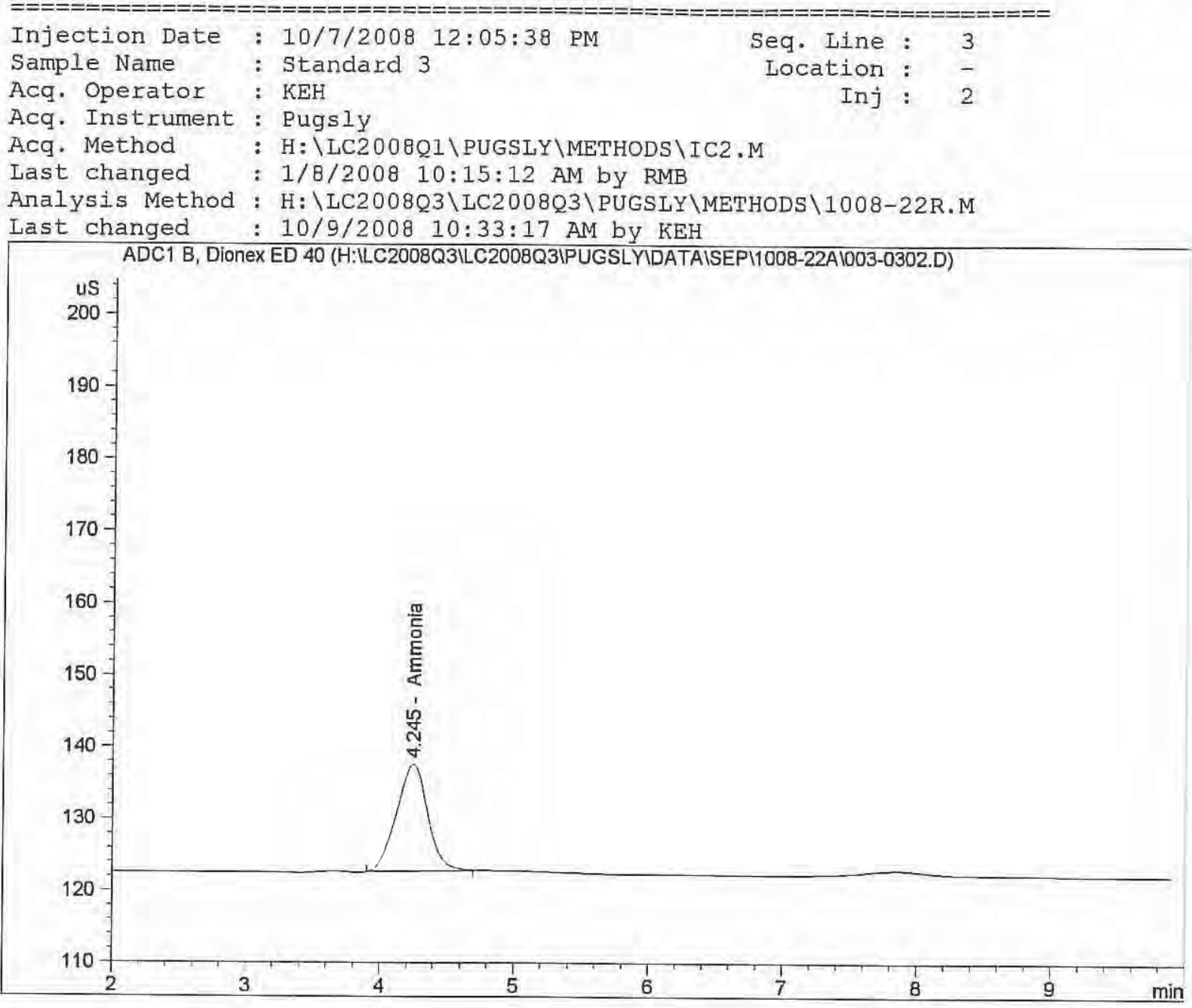

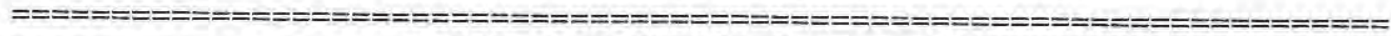

External Standard Report

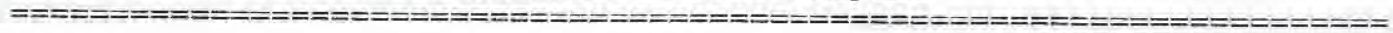

$\begin{array}{lll}\text { Sorted By } & : & \text { Signal } \\ \text { Calib. Data Modified } & : & \text { Thursday, October 09, } 2008 \text { 10:32:46 AM } \\ \text { Multiplier } & : & 1.0000 \\ \text { Dilution } & : & 1.0000\end{array}$

Use Multiplier \& Dilution Factor with ISTDs

Signal 1: ADC1 B, Dionex ED 40

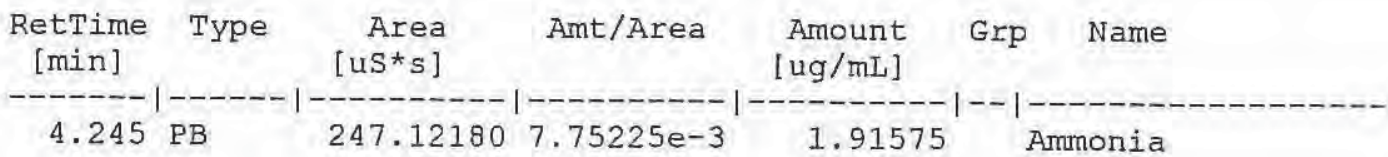

Totals : $\quad 1.91575$

Results obtained with enhanced integrator!

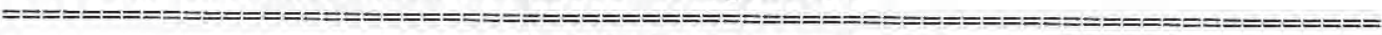

*** End of Report $* \star *$ 


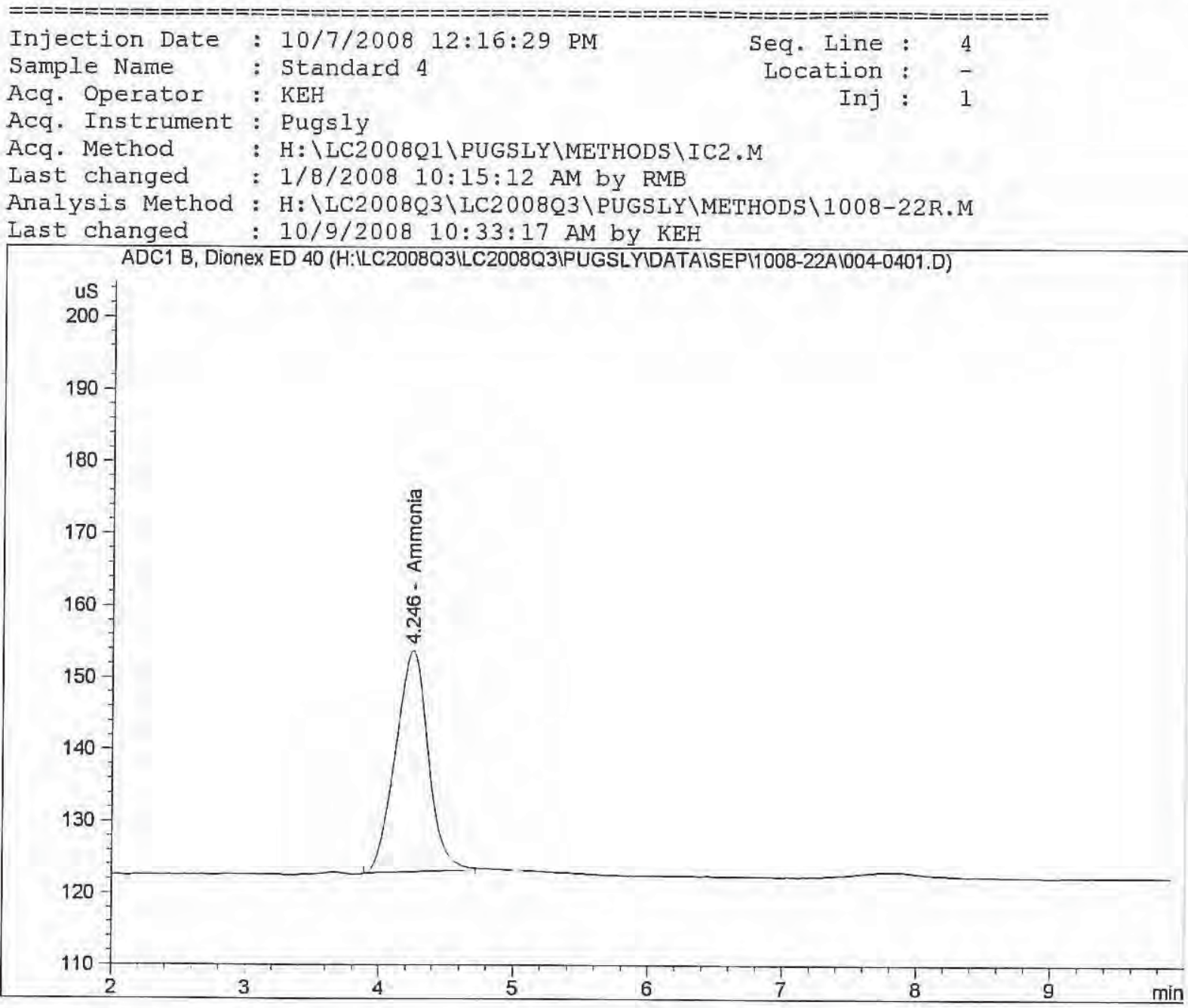

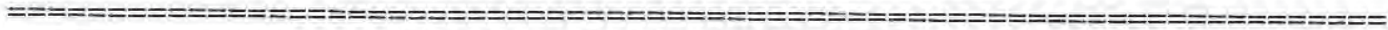

External Standard Report

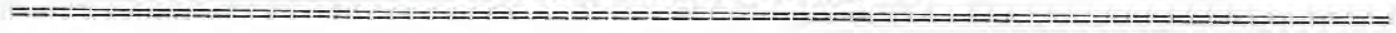

Sorted By : Signal

Calib. Data Modified : $\quad$ Thursday, October 09, 2008 10:32:46 AM

Multiplier : 1.0000

Dilution : 1.0000

Use Multiplier \& Dilution Factor with ISTDs

Signal 1: ADC1 B, Dionex ED 40

\begin{tabular}{|c|c|c|c|c|c|c|}
\hline $\begin{array}{l}\text { RetTime } \\
\text { [min] }\end{array}$ & Type & $\begin{array}{r}\text { Area } \\
\text { [uS*s] }\end{array}$ & Amt/Area & $\begin{array}{r}\text { Amount } \\
{[\mathrm{ug} / \mathrm{mL}]}\end{array}$ & Grp & Name \\
\hline 4.246 & $P B$ & 531.84253 & $8.51264 e-3$ & 4.52738 & & monia \\
\hline
\end{tabular}

Totals :

4.52738

Results obtained with enhanced integrator!

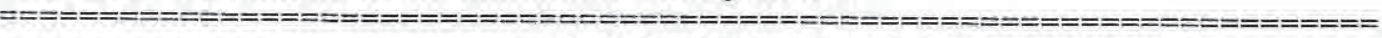

$\star \star \star$ End of Report *** 


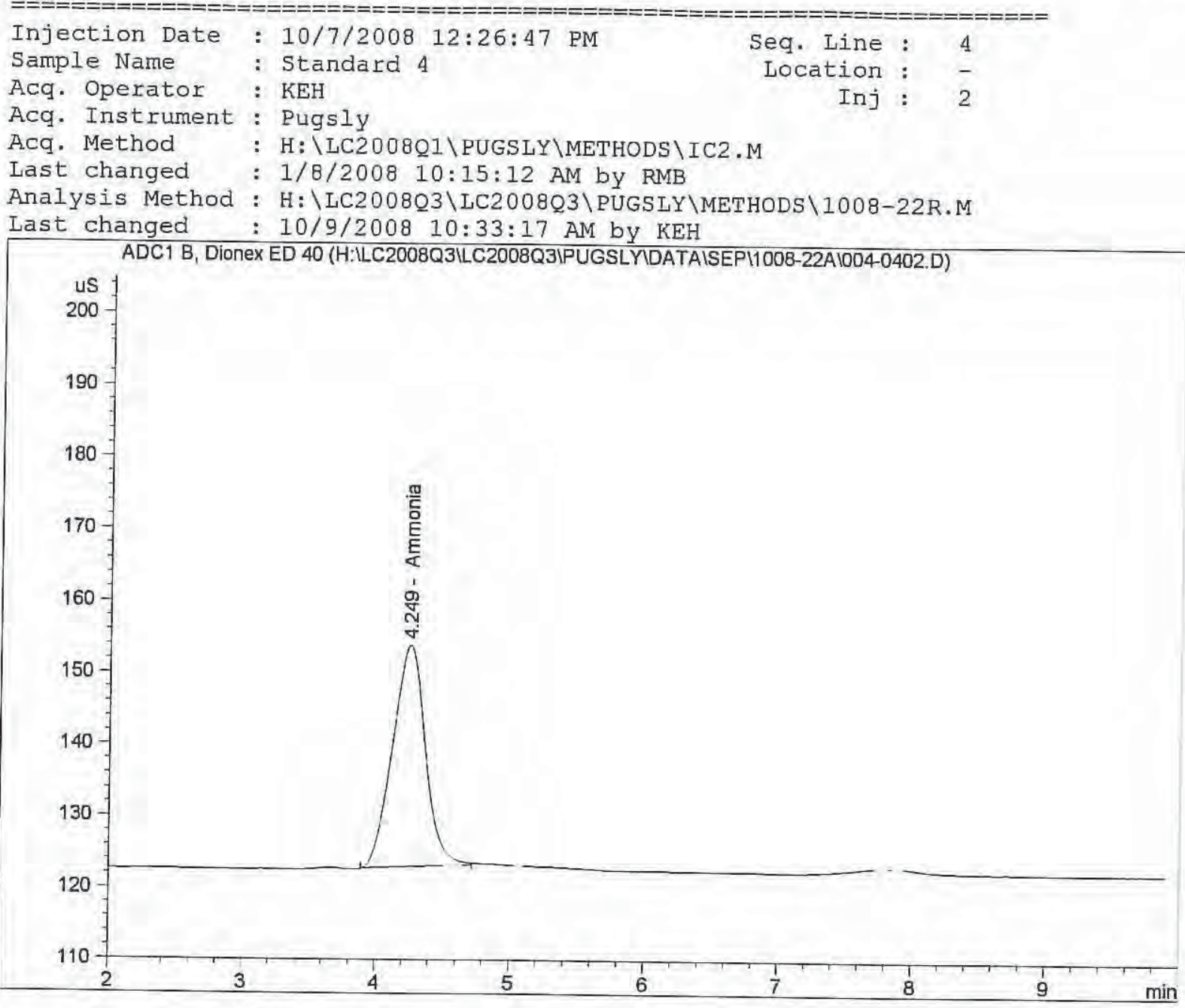

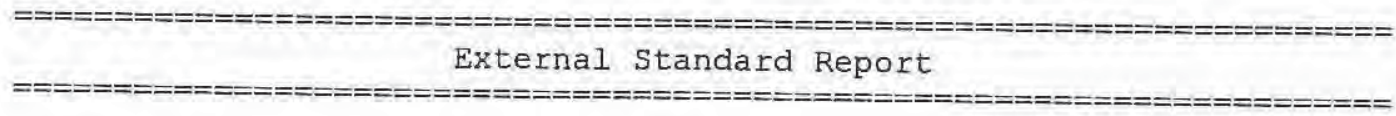

Sorted By : : Signal

Calib. Data Modified : Thursday, October 09, 2008 10:32:46 AM

Multiplier

Dilution

1.0000

Use Multiplier \& Dilution Eactor with ISTDs

Signal 1: ADCl B, Dionex ED 40

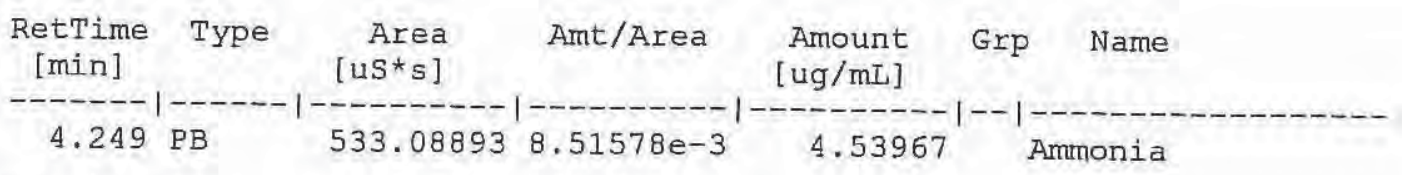

Totals :

4.53967

Results obtained with enhanced integrator!

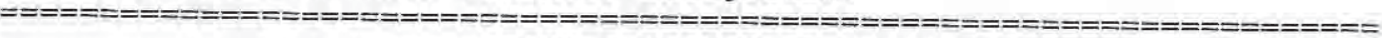

*** End of Report *** 


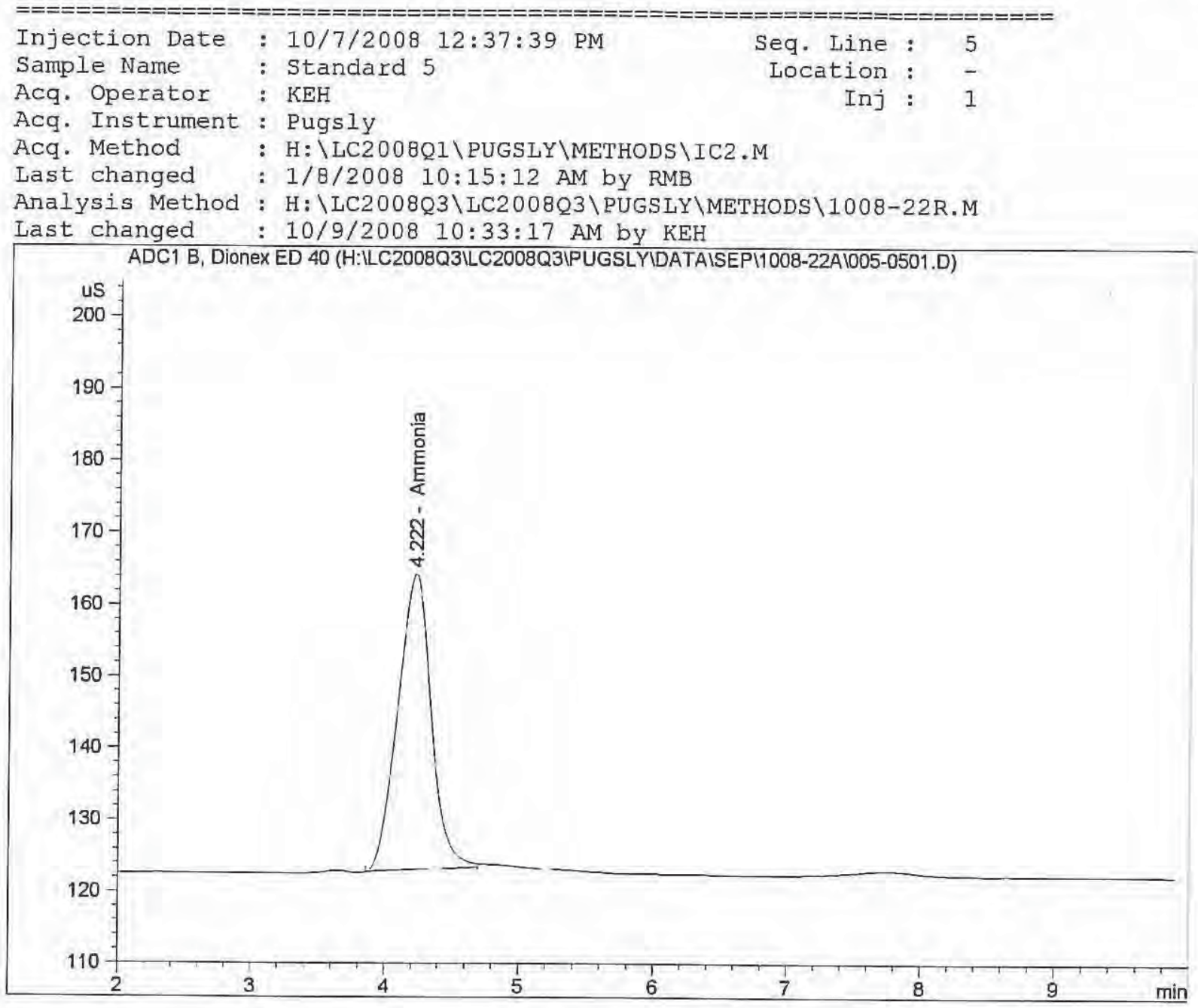

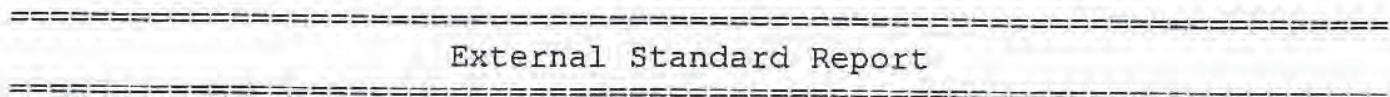

Sorted By

Calib. Signal

Multiplier : $\quad 1.0000$

Dilution : 1.0000

Use Multiplier \& Dilution Factor with ISTDS

Signal 1: ADC1 B, Dionex ED 40

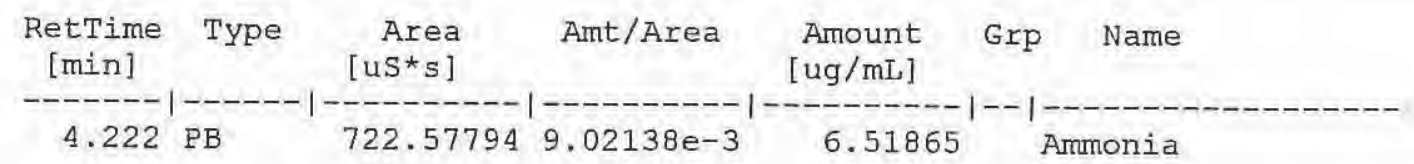

Totals : $\quad 6.51865$

Results obtained with enhanced integrator!

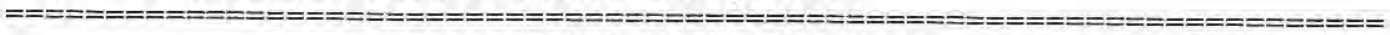

*** End of Report $* * *$ 

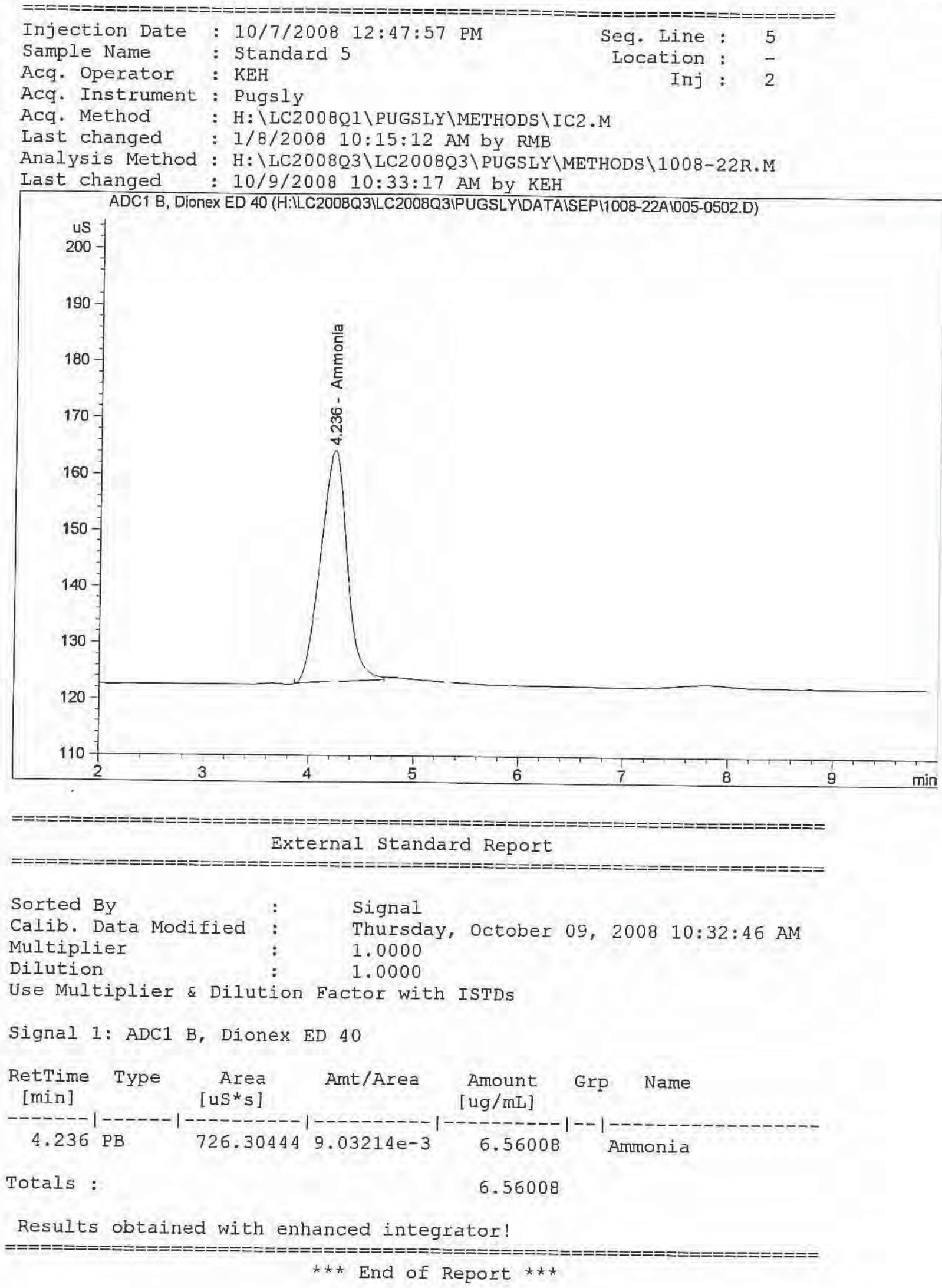


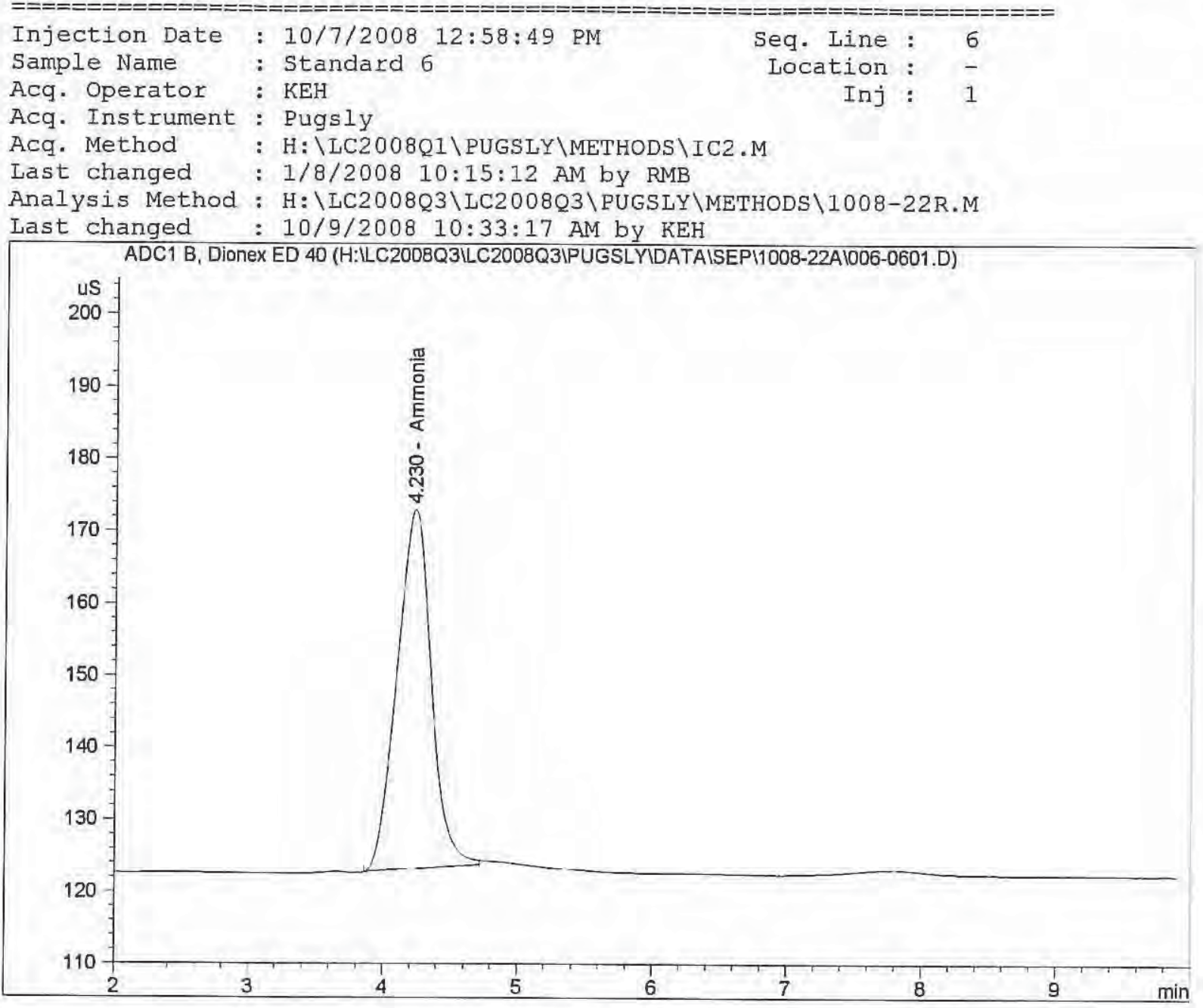

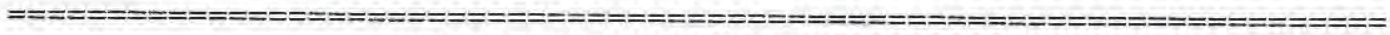

External Standard Report

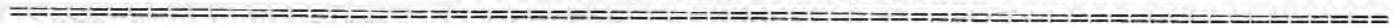

$\begin{array}{lll}\text { Sorted By } & : & \text { Signal } \\ \text { Calib. Data Modified } & : & \text { Thursday, October 09, 2008 10:32:46 AM } \\ \text { Multiplier } & : & 1.0000 \\ \text { Dilution } & : & 1.0000\end{array}$

Use Multiplier \& Dilution Factor with ISTDs

Signal 1: ADC1 B, Dionex ED 40

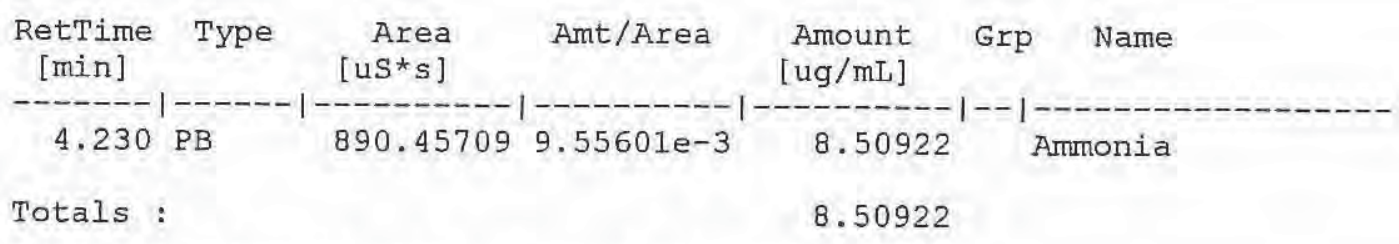

Results obtained with enhanced integrator!

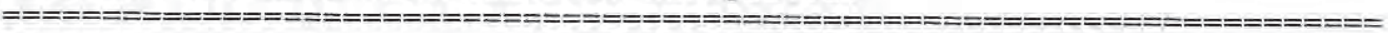
$\star \star \star$ End of Report $* \star \star$
} 

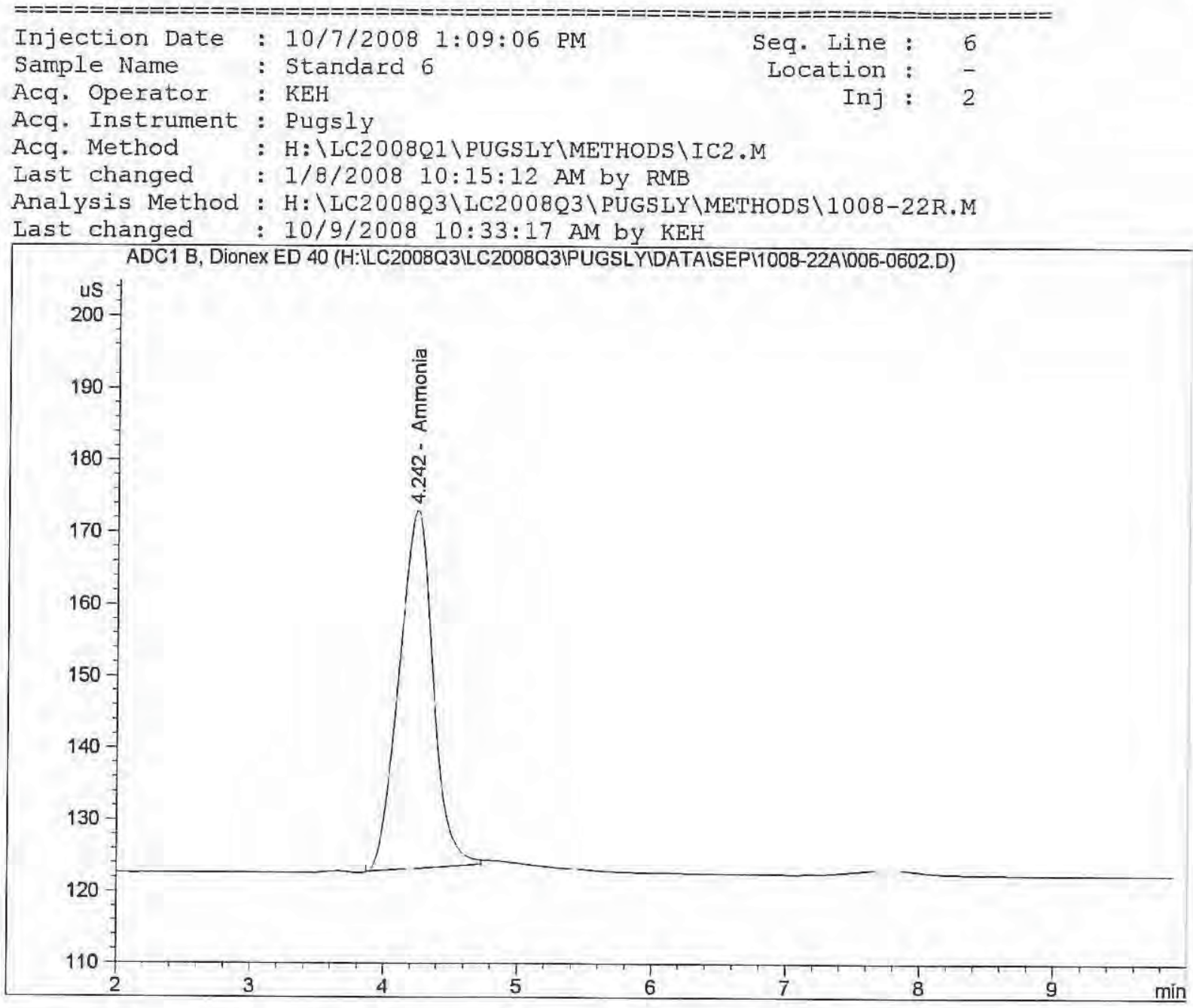

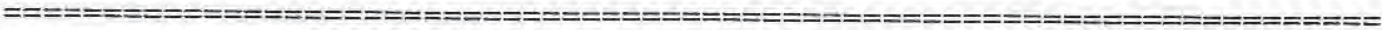

External Standard Report

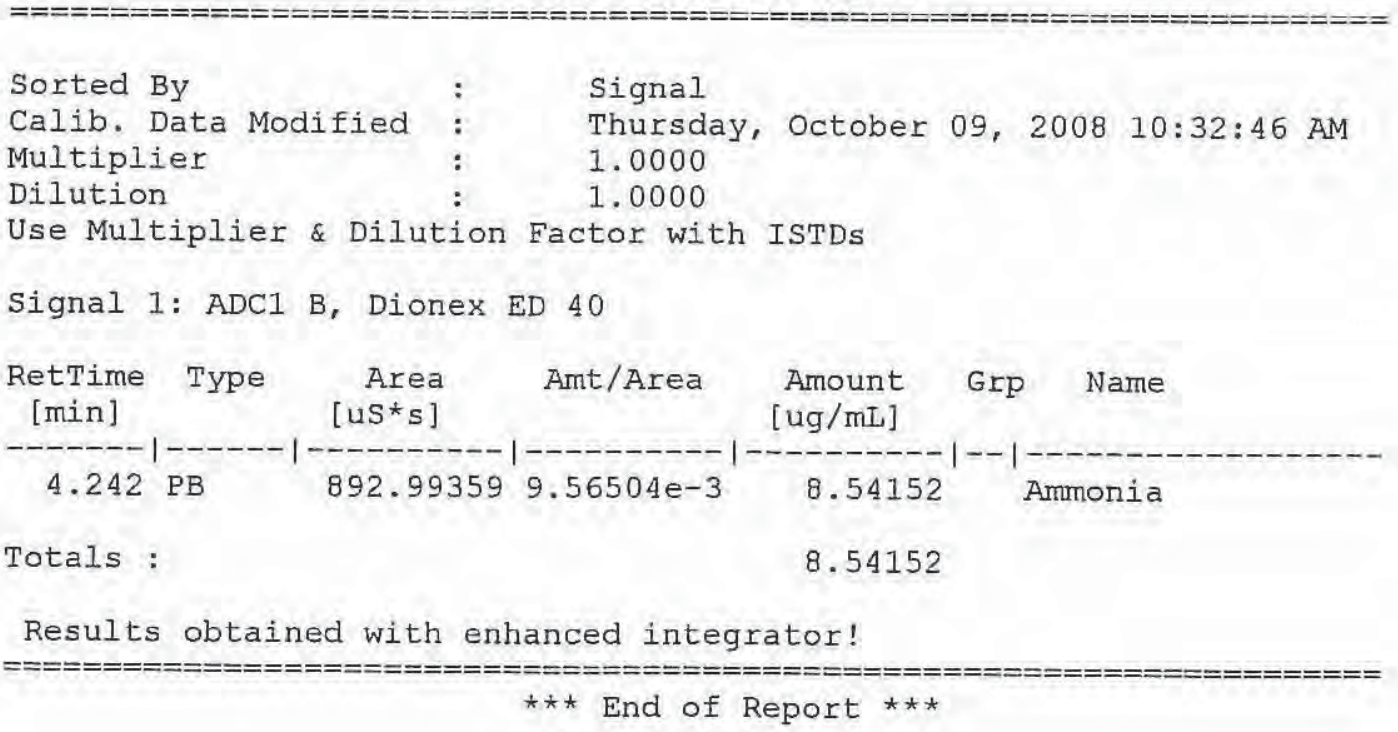



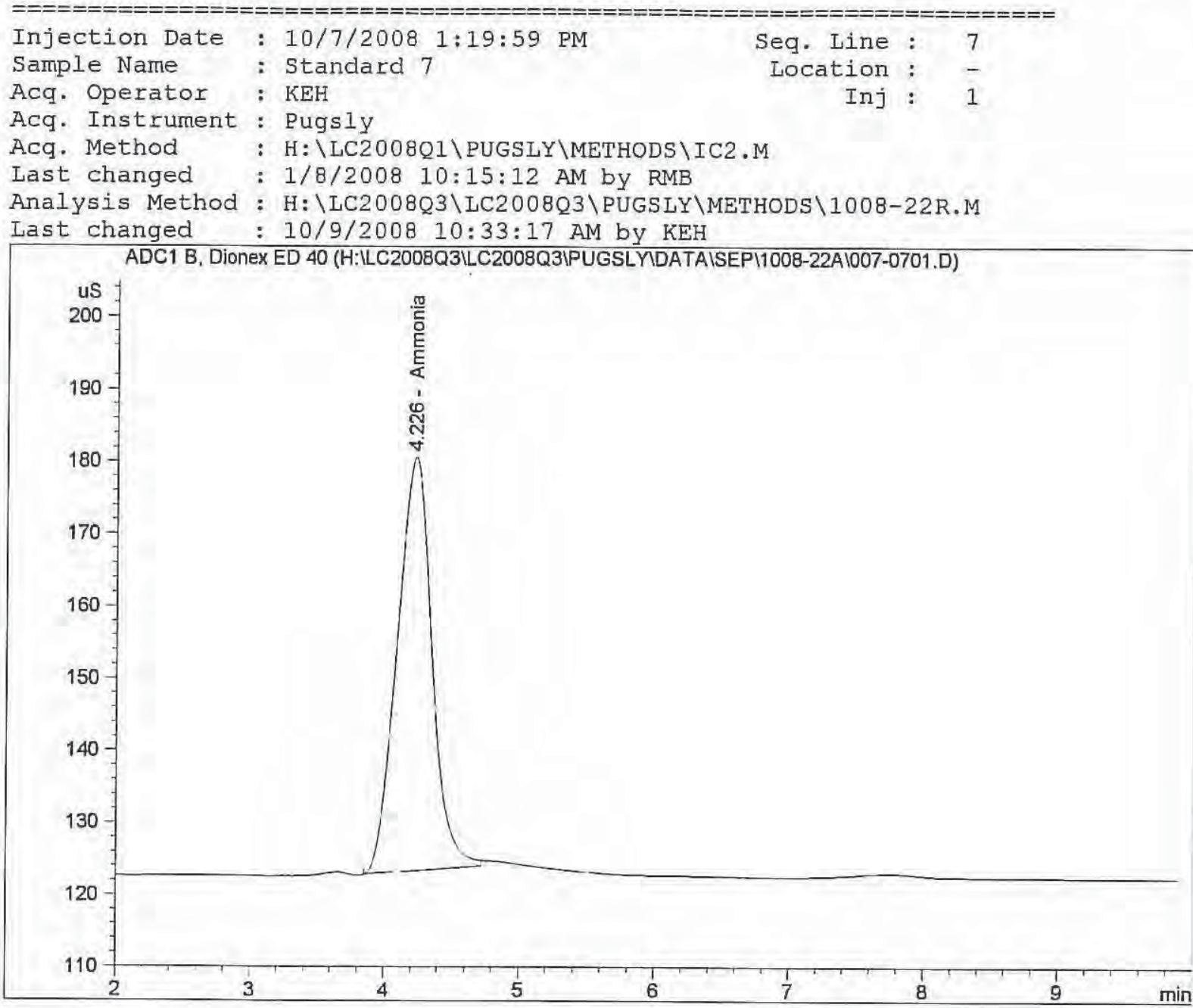

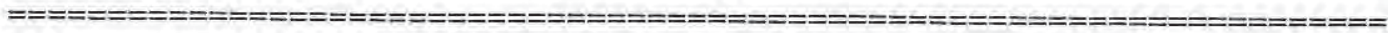

\section{External Standard Report}

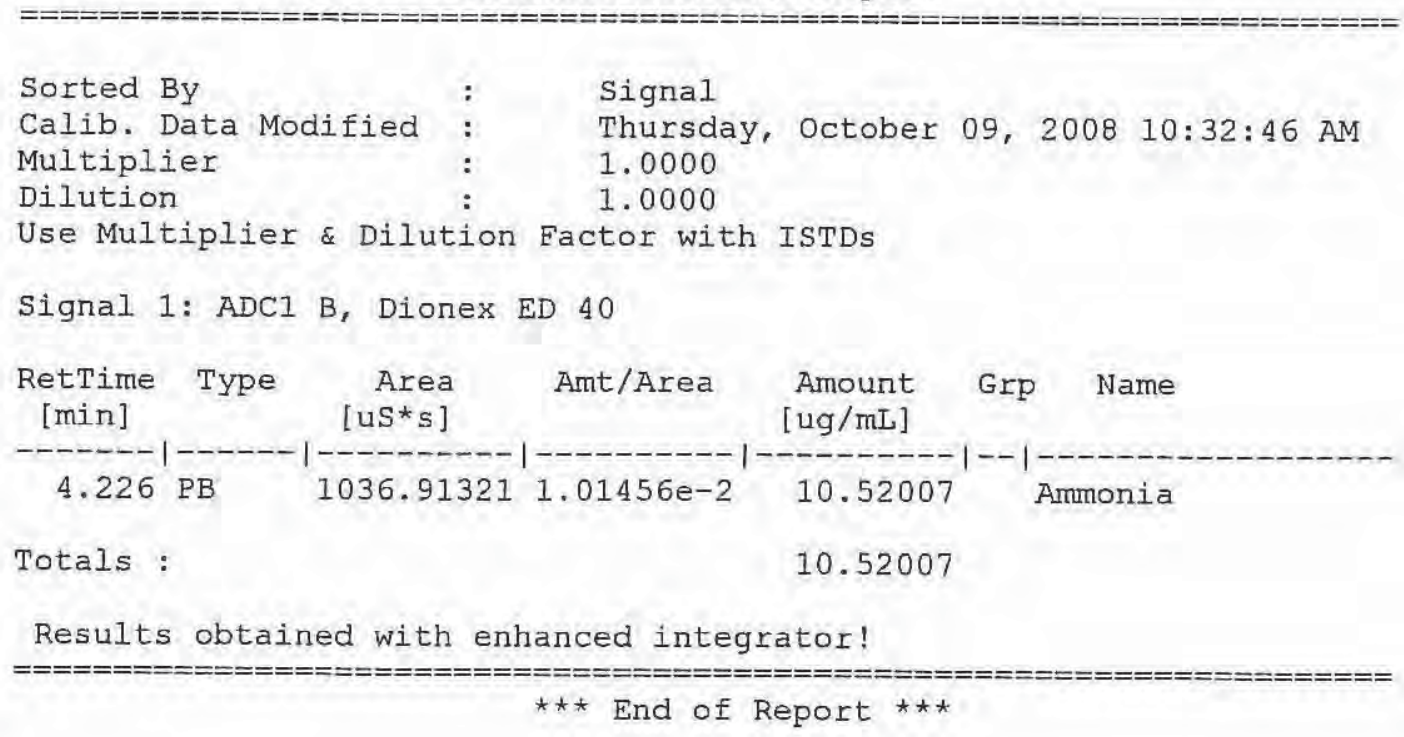




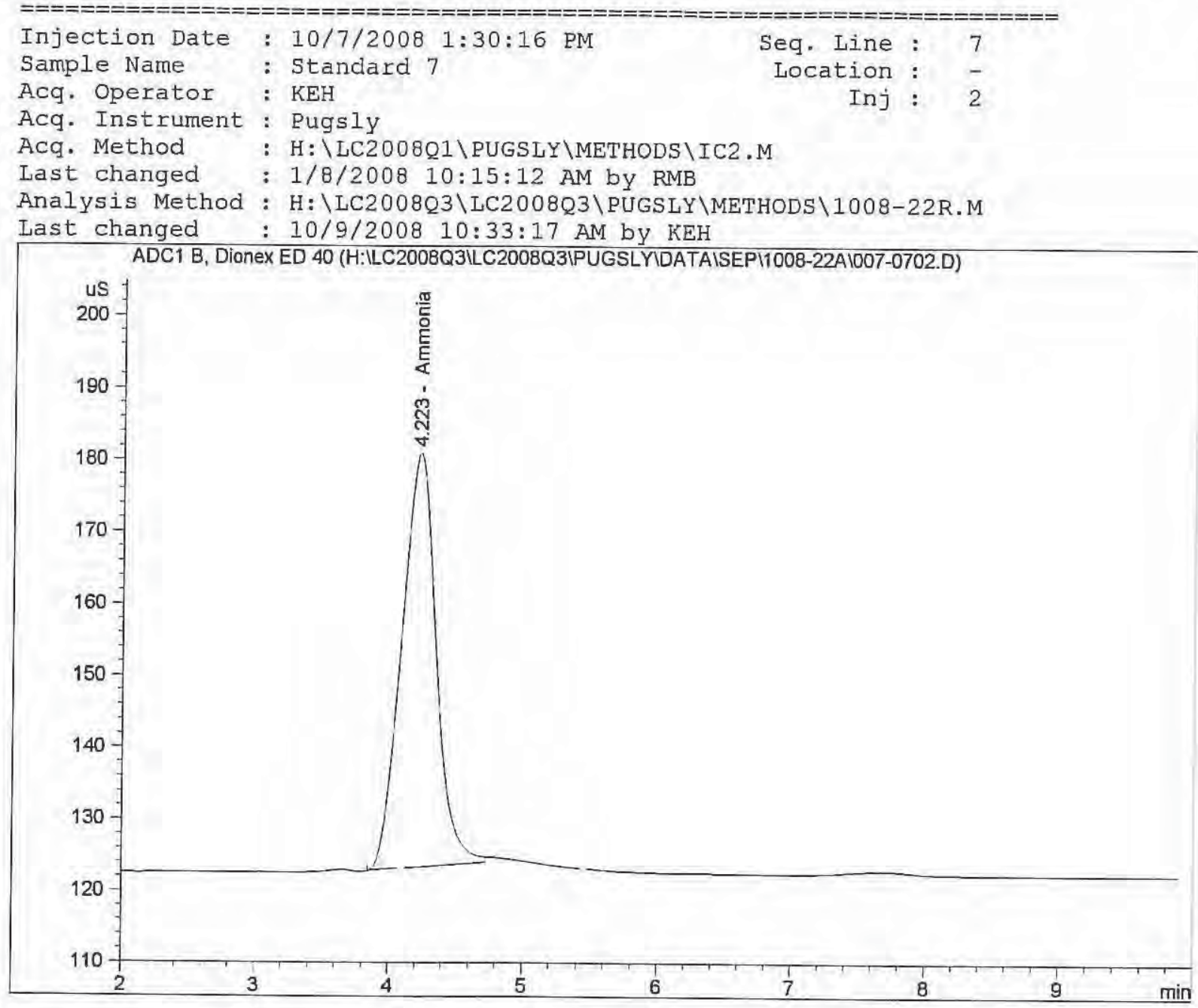

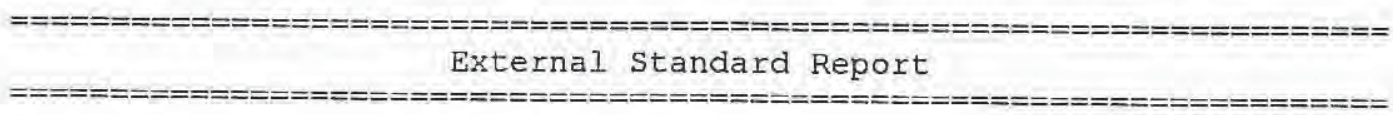

$\begin{array}{lll}\text { Sorted By } & : & \text { Signal } \\ \text { Calib. Data Modified } & : & \text { Thursday, October 09, 2008 10:32:46 AM } \\ \text { Multiplier } & : 1.0000 \\ \text { Dilution } & \text { 1.0000 } \\ \text { Use Multiplier \& Dilution Factor with ISTDs }\end{array}$

Signal 1: ADC1 B, Dionex ED 40

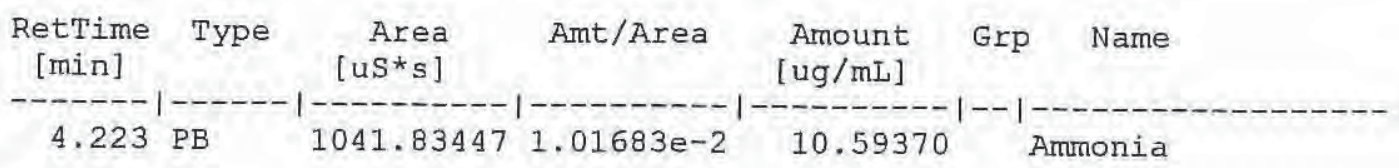

Totals : $\quad 10.59370$

Results obtained with enhanced integrator!

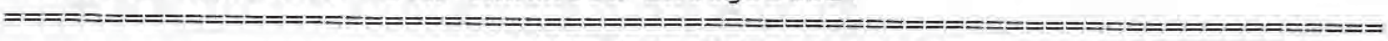

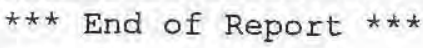




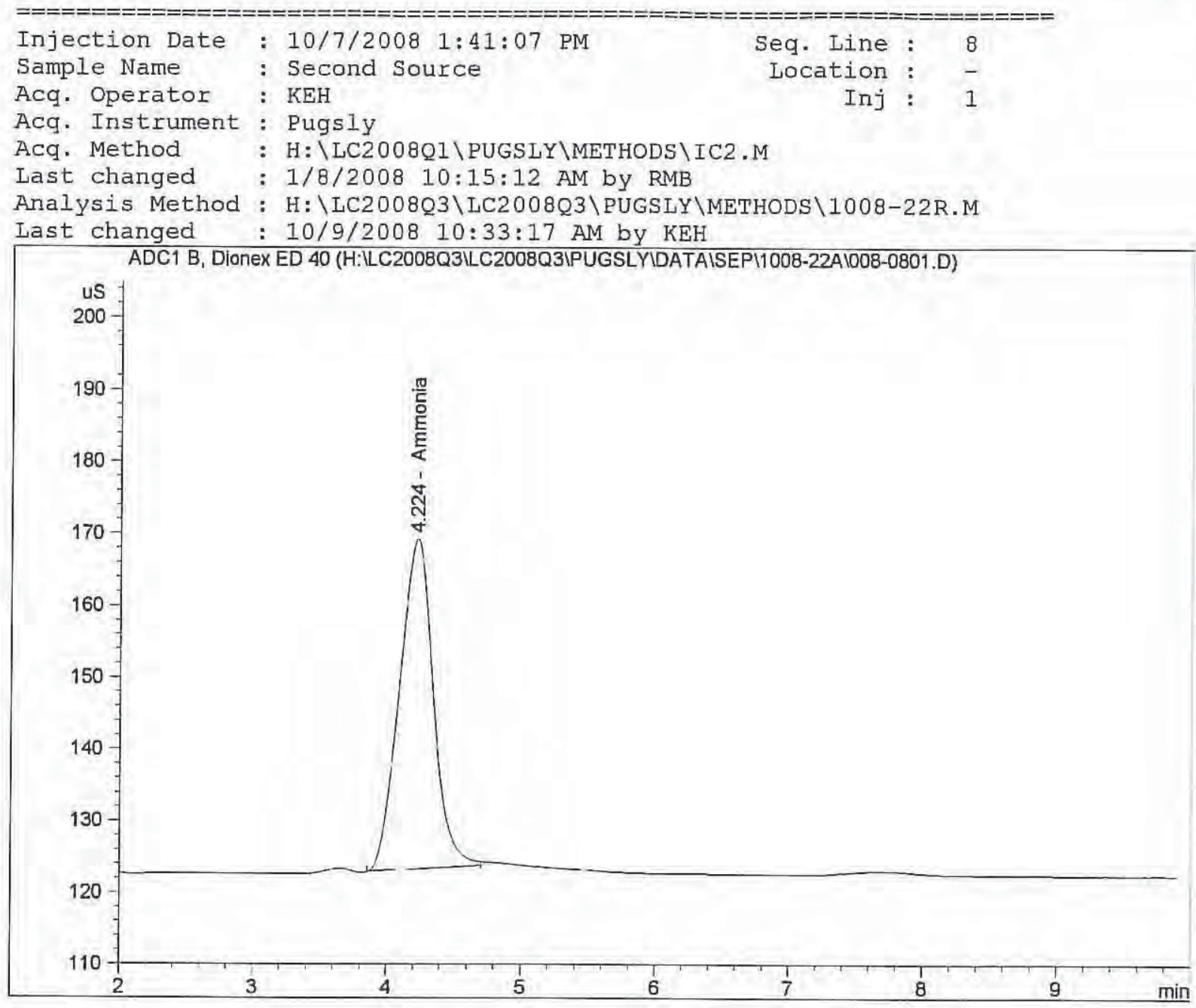

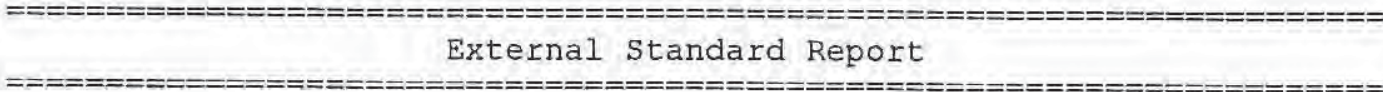

$\begin{array}{lcc}\text { Sorted By } & : & \text { Signal } \\ \text { Calib. Data Modified } & : & \text { Thursday, October 09, 2008 10:32:46 AM } \\ \text { Multiplier } & \vdots & 1.0000 \\ \text { Dilution } & \vdots & 1.0000 \\ \text { Use Multiplier \& Dilution Factor with ISTDs }\end{array}$

Signal 1: ADC1 B, Dionex ED 40

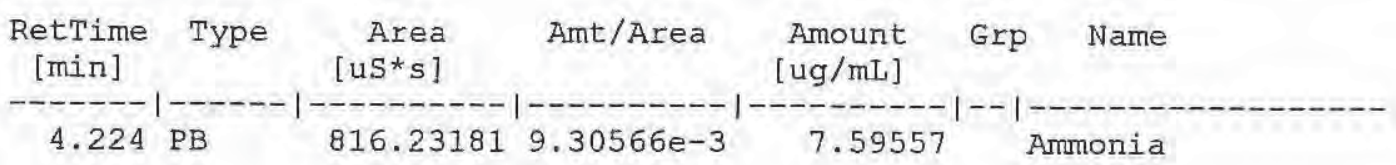

Totals :

7.59557

Results obtained with enhanced integrator!

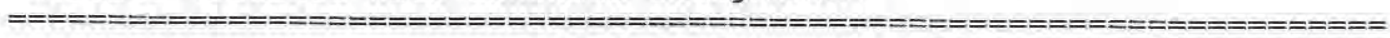

$\star \star \star$ End of Report $* \star \star$ 


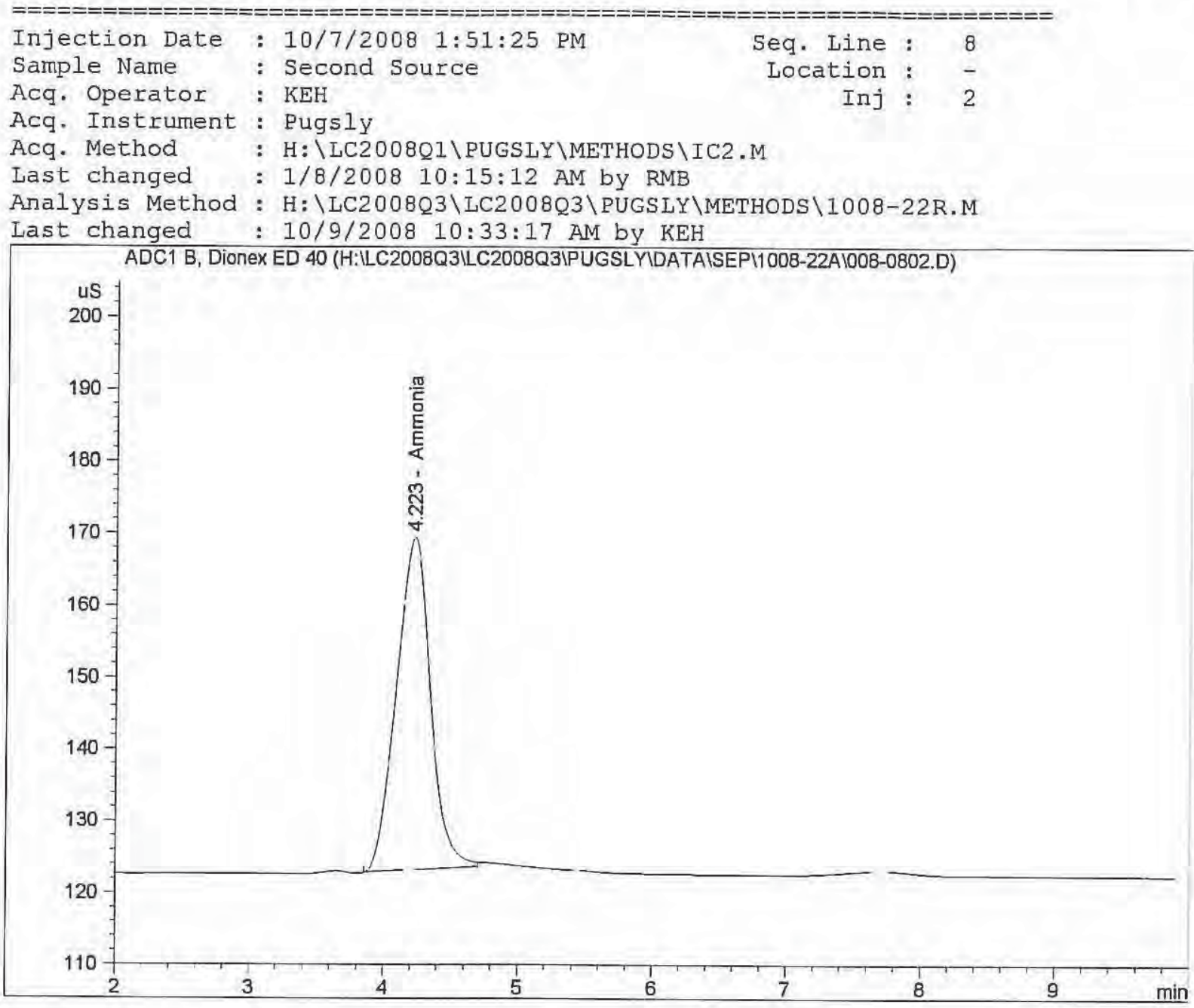

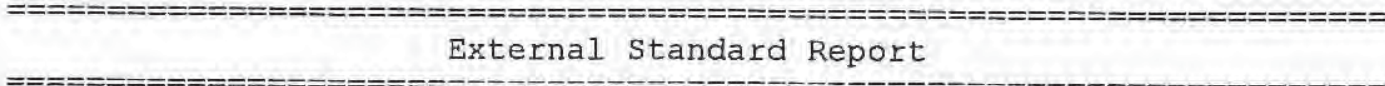

Sorted By

Signal

Calib. Data Modified : Thursday, October 09, 2008 10:32:46 AM

Multiplier

1.0000

Dilution

1.0000

Use Multiplier \& Dilution Factor with ISTDS

Signal 1: ADC1 B, Dionex ED 40

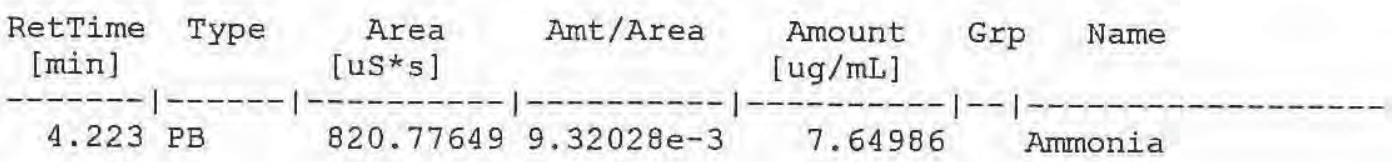

$\begin{array}{ll}\text { Totals : } & 7.64986\end{array}$

Results obtained with enhanced integrator!

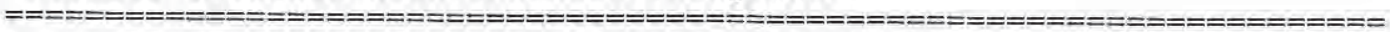

*** End of Report *** 


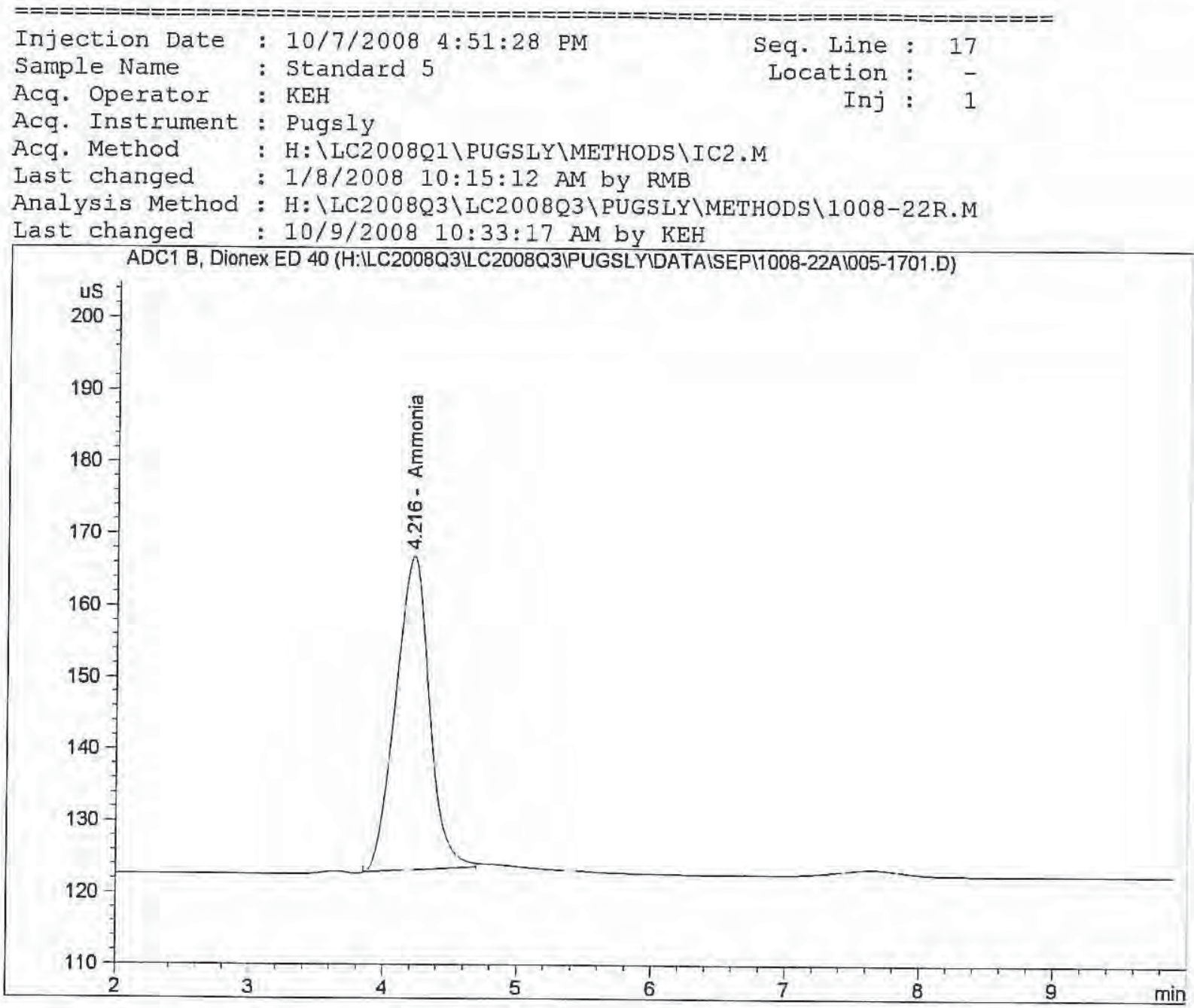

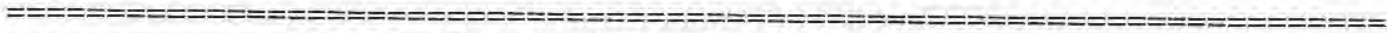

External Standard Report

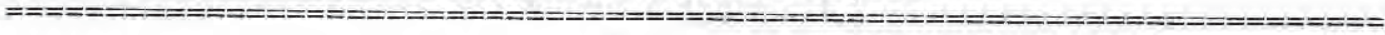

$\begin{array}{lll}\text { Sorted By } & : & \text { Signal } \\ \text { Calib. Data Modified } & : & \text { Thursday, October 09, } 2008 \text { 10:32:46 AM } \\ \text { Multiplier } & \vdots & 1.0000 \\ \text { Dilution } & \text { : } & 1.0000 \\ \text { Use Multiplier \& Dilution Factor with ISTDs }\end{array}$

Use Multiplier \& Dilution Factor with ISTDs

Signal 1: ADC1 B, Dionex ED 40

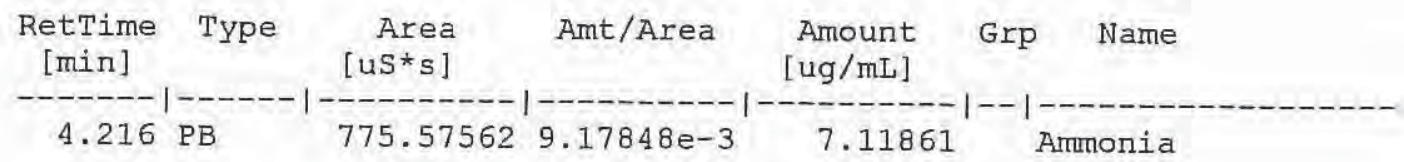

Totals : $\quad 7.11861$

Results obtained with enhanced integrator!

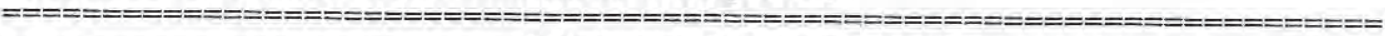

$* \star *$ End of Report $\star \star *$ 


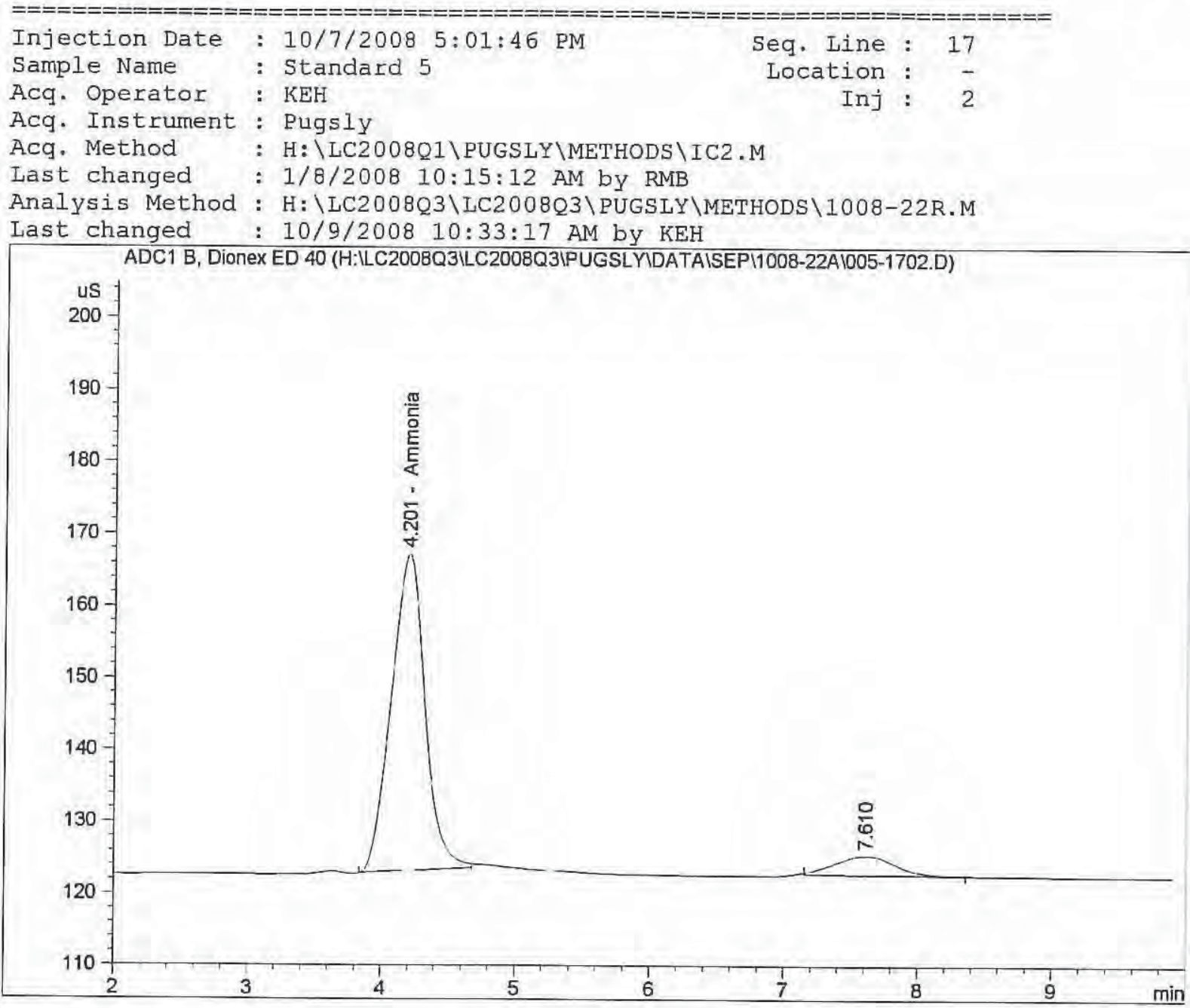

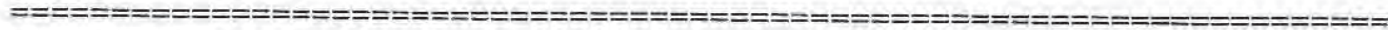

External Standard Report

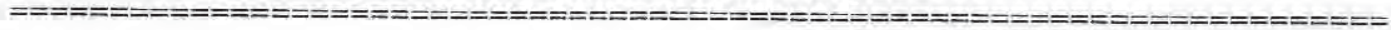

$\begin{array}{lll}\text { Sorted By } & : & \text { Signal } \\ \text { Calib. Data Modified } & : & \text { Thursday, October 09, } 2008 \text { 10:32:46 AM } \\ \text { Multiplier } & : & 1.0000 \\ \text { Dilution } & : & 1.0000\end{array}$

Use Multiplier \& Dilution Factor with ISTDs

Signal 1: ADC1 B, Dionex ED 40

\begin{tabular}{ccccc}
$\begin{array}{c}\text { RetTime Type } \\
\text { [min] }\end{array}$ & $\begin{array}{c}\text { Area } \\
{\left[\mathrm{uS}^{*} \mathrm{~s}\right]}\end{array}$ & Amt/Area & $\begin{array}{c}\text { Amount } \\
{[\mathrm{ug} / \mathrm{mL}]}\end{array}$ & Grp Name \\
\hline 4.201 PB & 776.92407 & $9.18260 \mathrm{e}-3$ & 7.13419 & Ammonia
\end{tabular}

Totals :

7.13419

Results obtained with enhanced integrator!

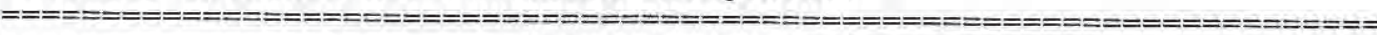

$\star \star *$ End of Report *** 


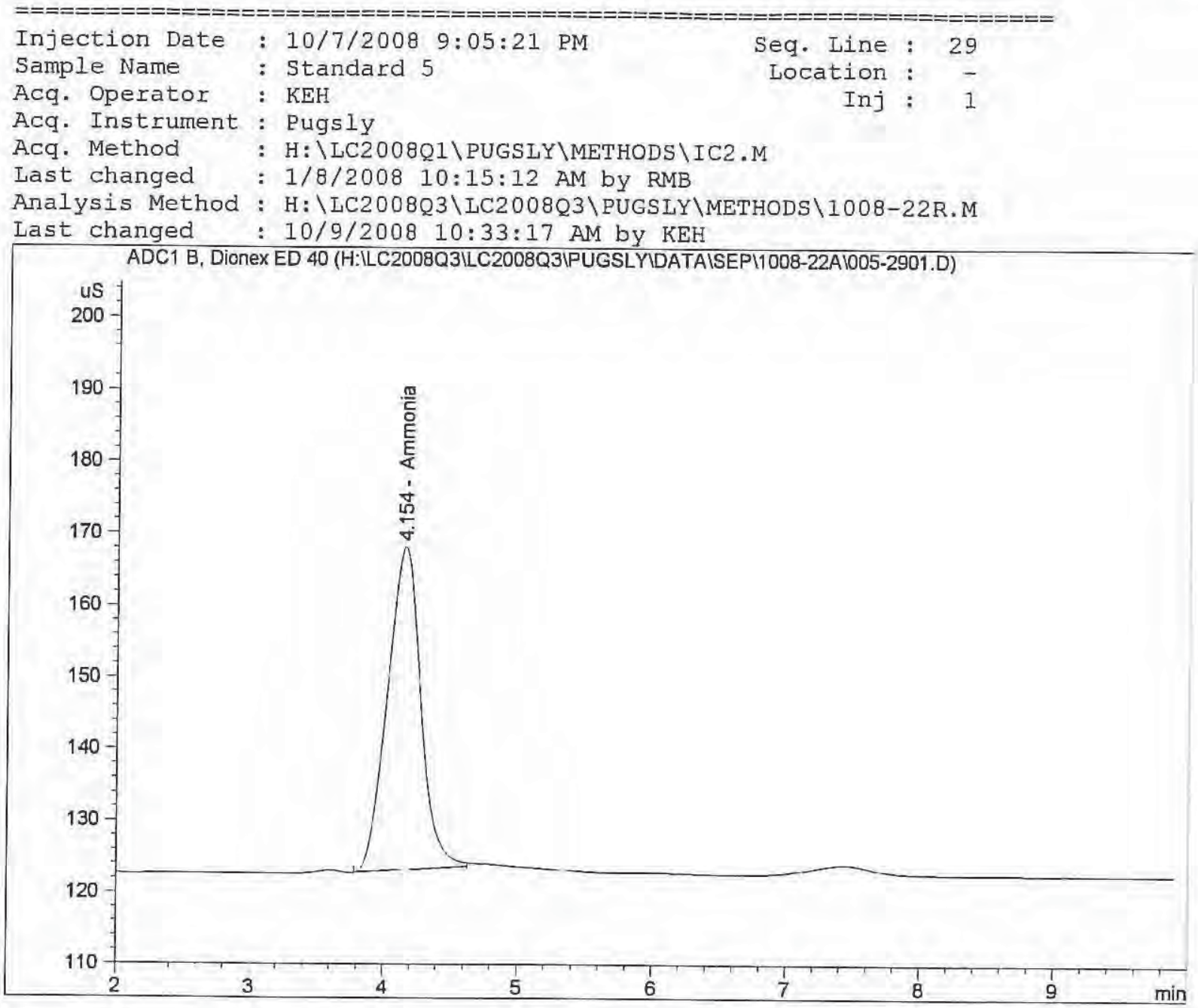

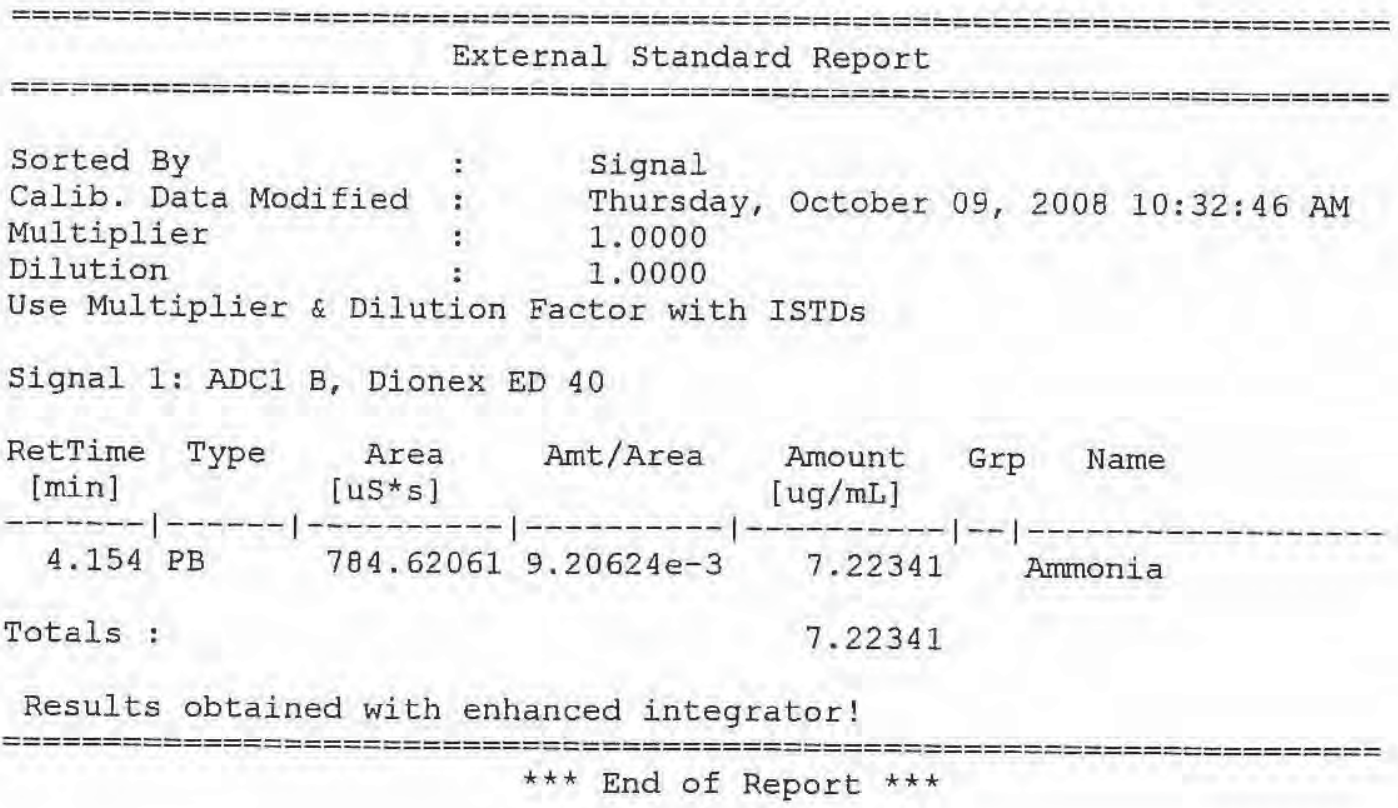



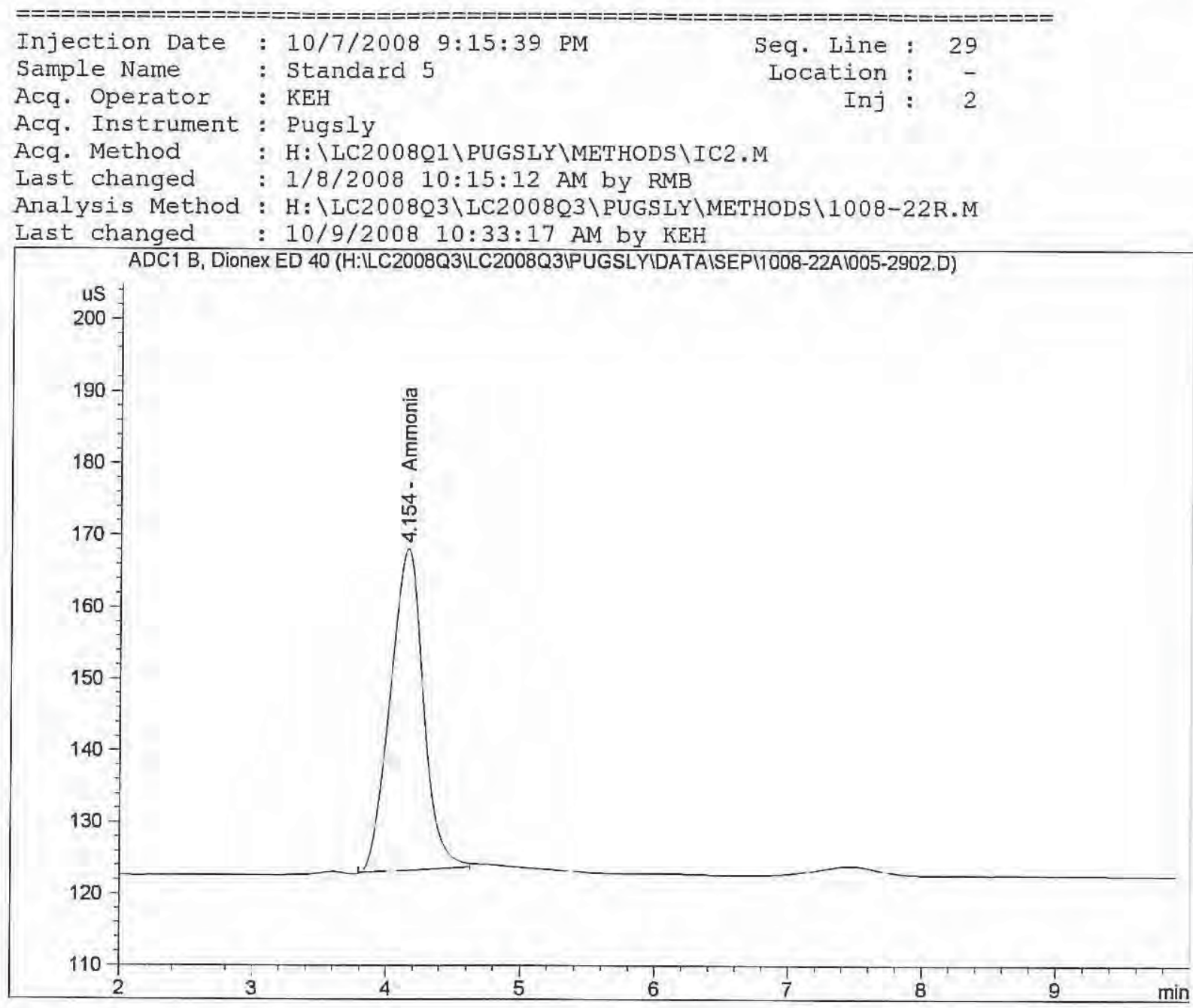

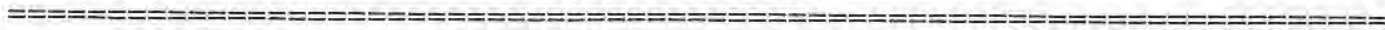

External Standard Report

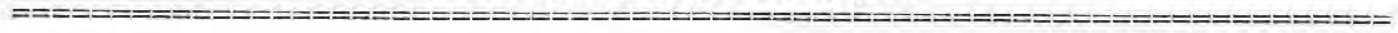

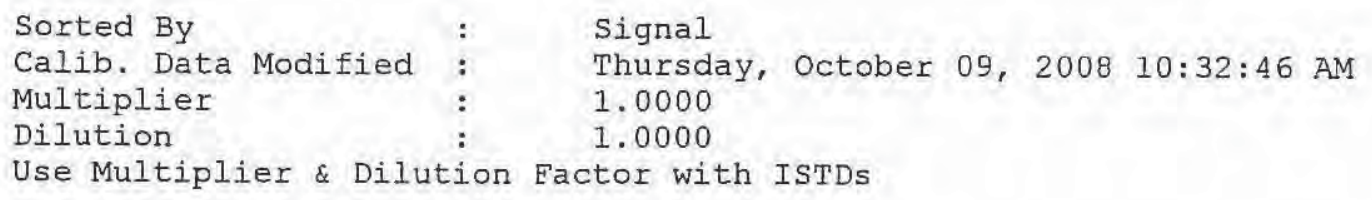

Signal 1: ADC1 B, Dionex ED 40

\begin{tabular}{|c|c|c|c|c|c|c|}
\hline $\begin{array}{l}\text { RetTime } \\
\text { [min] }\end{array}$ & Type & $\begin{array}{r}\text { Area } \\
{\left[\mathrm{uS}^{*} \mathrm{~s}\right]}\end{array}$ & Amt/Area & $\begin{array}{l}\text { Amount } \\
{[\mathrm{ug} / \mathrm{mL}]}\end{array}$ & Grp & Name \\
\hline 4. 154 & PB & 781.69250 & $9.19722 e-3$ & 7.18940 & & monia \\
\hline
\end{tabular}

Totals :

7.18940

Results obtained with enhanced integrator!

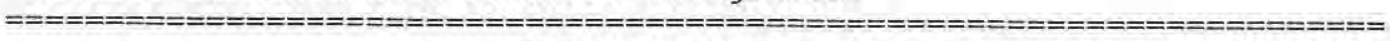

*** End of Report *** 


\begin{tabular}{|c|c|c|c|c|c|c|}
\hline Metho & od and In & jection Info Part: & & & & \\
\hline $\begin{array}{l}\text { Iine } \\
====\end{array}$ & $\begin{array}{l}\text { Location } \\
======\end{array}$ & $\begin{array}{l}\text { SampleName } \\
===============\end{array}$ & $\begin{array}{l}\text { Method } \\
=======\end{array}$ & $\begin{array}{l}\text { Inj } \\
===\end{array}$ & $\begin{array}{l}\text { SampleType } \\
========\end{array}$ & $\begin{array}{l}\text { InjVolume DataFile } \\
=============\end{array}$ \\
\hline 1 & Vial 1 & Standard 1 & IC2 & 2 & Sample & \\
\hline 2 & Vial 2 & standard 2 & IC2 & 2 & Sample & \\
\hline 3 & Vial 3 & Standard 3 & IC2 & 2 & Sample & \\
\hline 4 & Vial 4 & Standard 4 & IC2 & 2 & Sample & \\
\hline 5 & Vial 5 & Standard 5 & IC2 & 2 & Sample & \\
\hline 6 & Vial 6 & Standard 6 & IC2 & 2 & Sample & \\
\hline 7 & Vial 7 & Standard 7 & IC2 & 2 & Sample & \\
\hline 8 & Vial 8 & Second Source & IC2 & 2 & Sample & \\
\hline 9 & Vial 9 & $0.04 \mathrm{~N}$ H2SO4 & IC2 & 2 & Sample & \\
\hline 10 & Vial 10 & DI $\mathrm{H} 2 \mathrm{O}$ & IC2 & 2 & Sample & \\
\hline 11 & Vial 11 & Run $1 * 20$ & IC2 & 2 & Sample & \\
\hline 12 & Vial 12 & Run $2 * 10$ & IC2 & 2 & Sample & \\
\hline 13 & Vial 13 & Run $3 * 10$ & IC2 & 2 & Sample & \\
\hline 14 & Vial 14 & H2SO4 Blank & IC2 & 2 & Sample & \\
\hline 15 & Vial 15 & MS/Run 3 & IC2 & 2 & Sample & \\
\hline 16 & Vial 4 & Standard 4 & IC2 & 2 & Sample & \\
\hline 17 & Vial 5 & Standard 5 & IC2 & 2 & Sample & \\
\hline 18 & Vial 16 & Run 1 & IC2 & 2 & Sample & \\
\hline 19 & Vial 17 & Run 2 & IC2 & 2 & Sample & \\
\hline 20 & Vial 18 & Run 3 & IC2 & 2 & Sample & \\
\hline 21 & Vial 19 & Run 4 & IC2 & 2 & Sample & \\
\hline 22 & Vial 20 & Run 5 & IC2 & 2 & Sample & \\
\hline 23 & Vial 21 & Run 6 & IC2 & 2 & Sample & \\
\hline 24 & Vial 22 & Run 7 & IC2 & 2 & Sample & \\
\hline 25 & Vial 23 & Run 8 & IC2 & 2 & Sample & \\
\hline 26 & Vial 24 & Run 9 & IC2 & 2 & Sample & \\
\hline 27 & Vial 25 & Blank & IC2 & 2 & Sample & \\
\hline 28 & Vial 4 & Standard 4 & IC2 & 2 & Sample & \\
\hline 29 & Vial 5 & Standard 5 & IC2 & 2 & Sample & \\
\hline
\end{tabular}


Sample Chromatograms

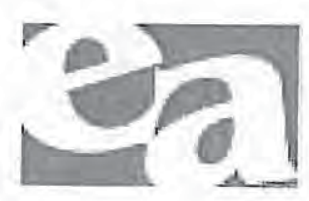


$1008-22$

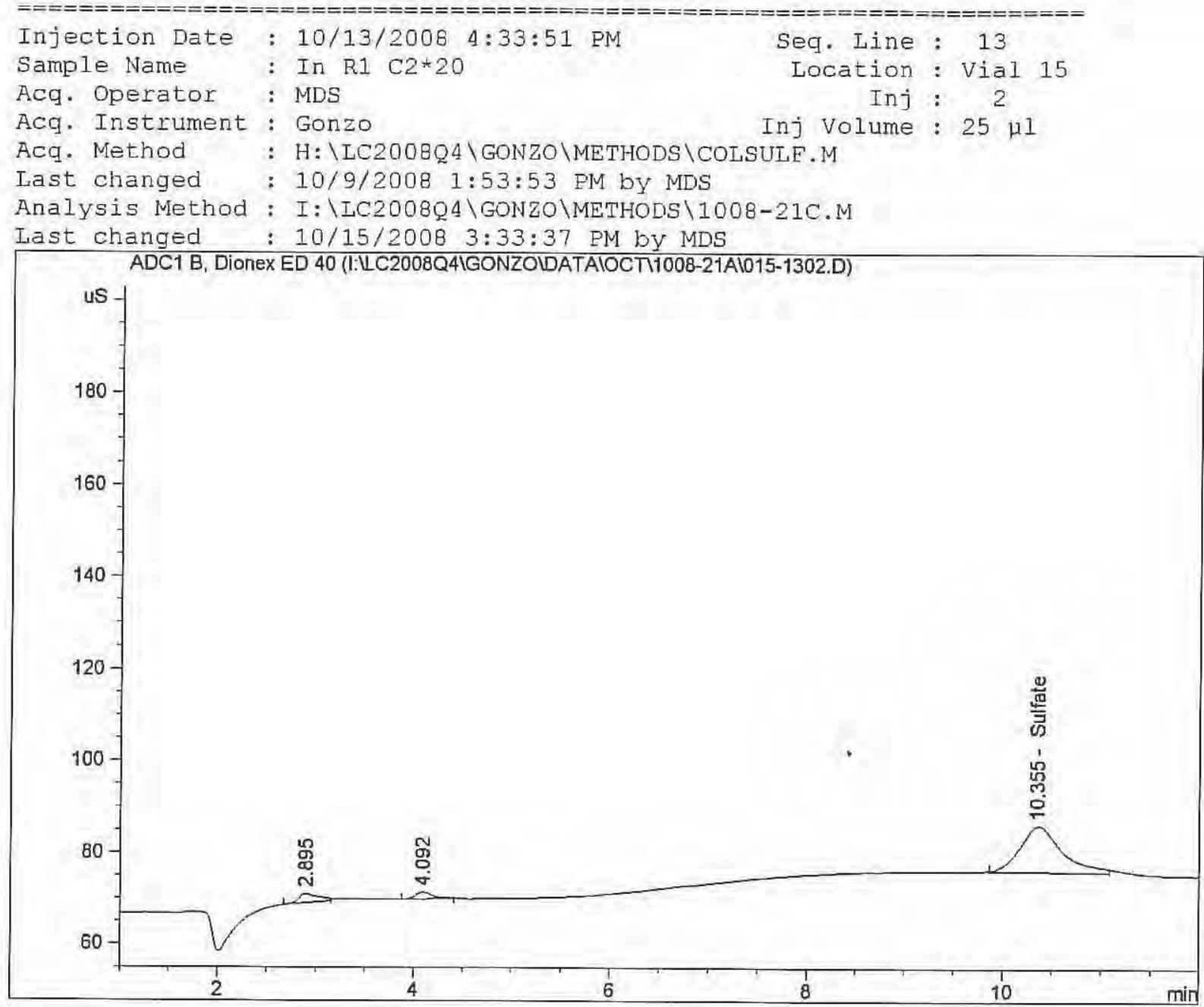

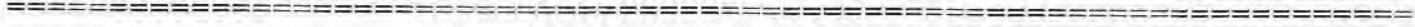

External Standard Report

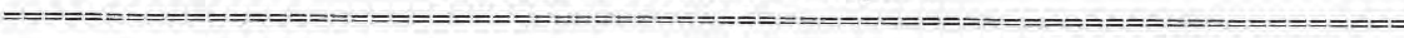

Sorted BY

Signal

Calib. Data Modified : $10 / 15 / 2008$ 3:33:29 PM

Multiplier : $\quad 1.0000$

Dilution : 1.0000

Use Multiplier \& Dilution Eactor with ISTDS

Signal 1: ADCl B, Dionex ED 40

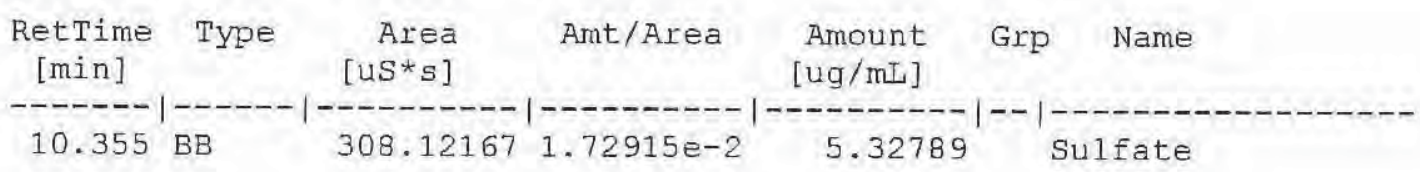

Totals :

5.32789

Results obtained with enhanced integrator!

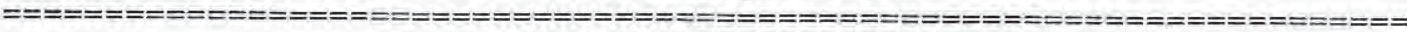

$\star * \star$ End of Report $* * *$
} 
$1008-22$

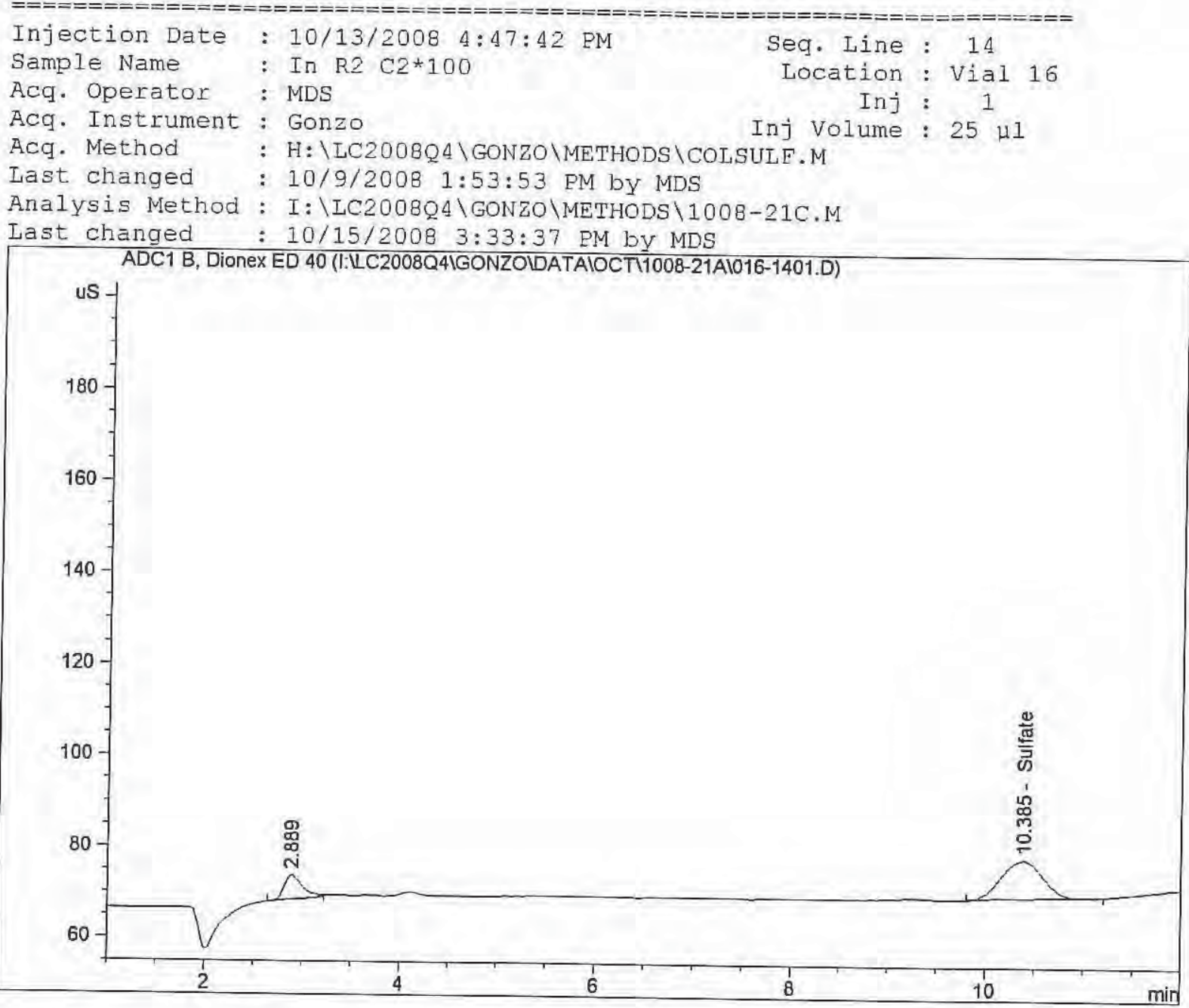

External Standard Report

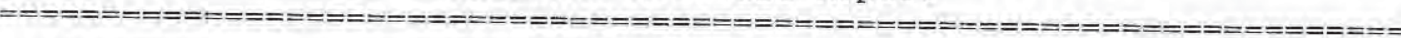

Sorted By

Multiplier : 1.0000

Dilution : 1.0000

Use Multiplier \& Dilution Factor with ISTDs

Signal 1: ADC1 B, Dioner ED 40

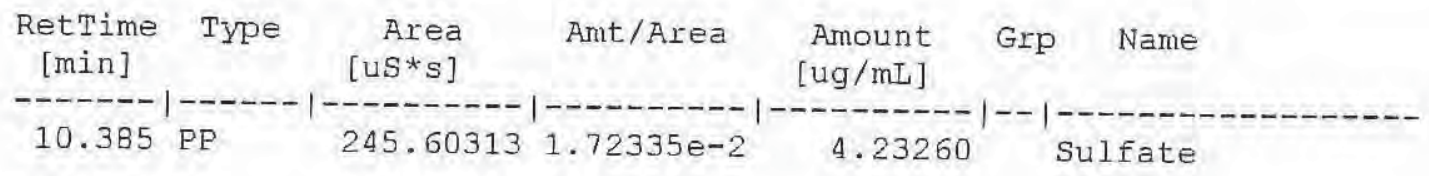

Totals :

4.23260

Results obtained with enhanced integrator!

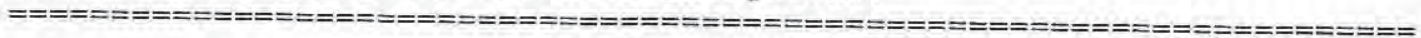

$\star \star \star$ End of Report $* * *$ 


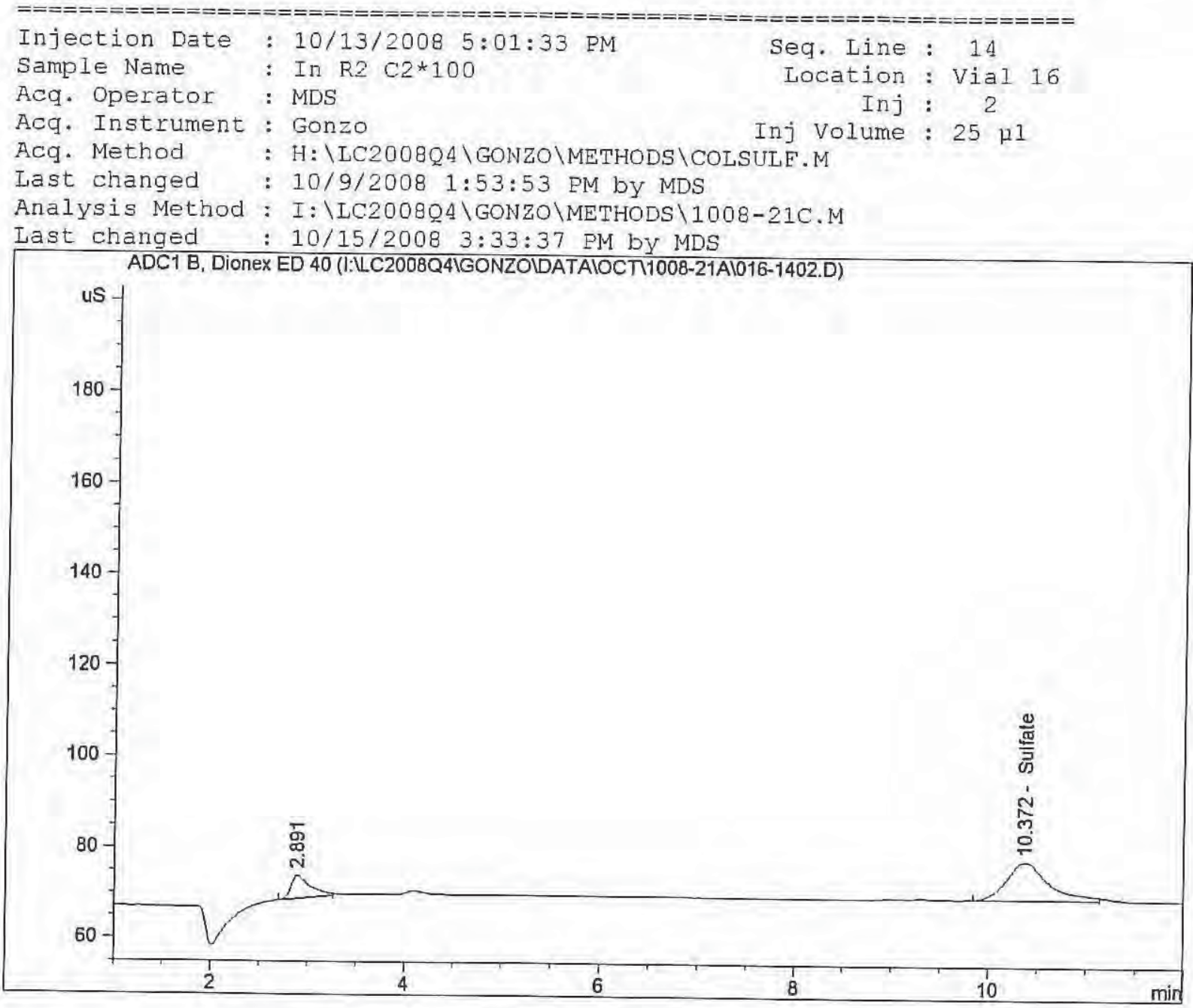

External Standard Report

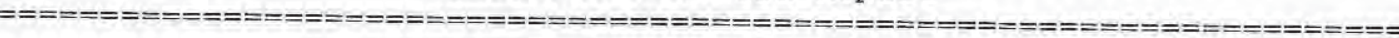

$\begin{array}{lcc}\text { Sorted By } & : & \text { Signal } \\ \text { Calib. Data Modified } & : & 10 / 15 / 2008 \text { 3:33:29 PM } \\ \text { Multiplier } & : & 1.0000 \\ \text { Dilution } & \text { 1.0000 } \\ \text { Use Multiplier \& Dilution Eactor with ISTDs }\end{array}$

Signal 1: ADC1 B, Dionex ED 40

\begin{tabular}{|c|c|c|c|c|c|c|}
\hline $\begin{array}{l}\text { RetTime } \\
\text { [min] }\end{array}$ & Type & $\begin{array}{r}\text { Area } \\
\text { [us*s] }\end{array}$ & Amt/Area & $\begin{array}{l}\text { Amount } \\
\text { [ug/mL] }\end{array}$ & Grp & Name \\
\hline 10.372 & & 261.490 & $1.72509 \mathrm{e}-$ & 4.510 & & \\
\hline
\end{tabular}

Totals :

4.51093

Results obtained with enhanced integrator!

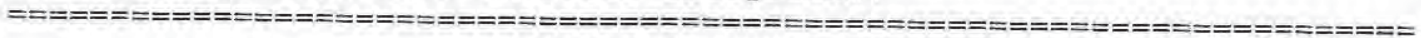
$\star \star *$ End of Report $* * *$ 
$1008-22$

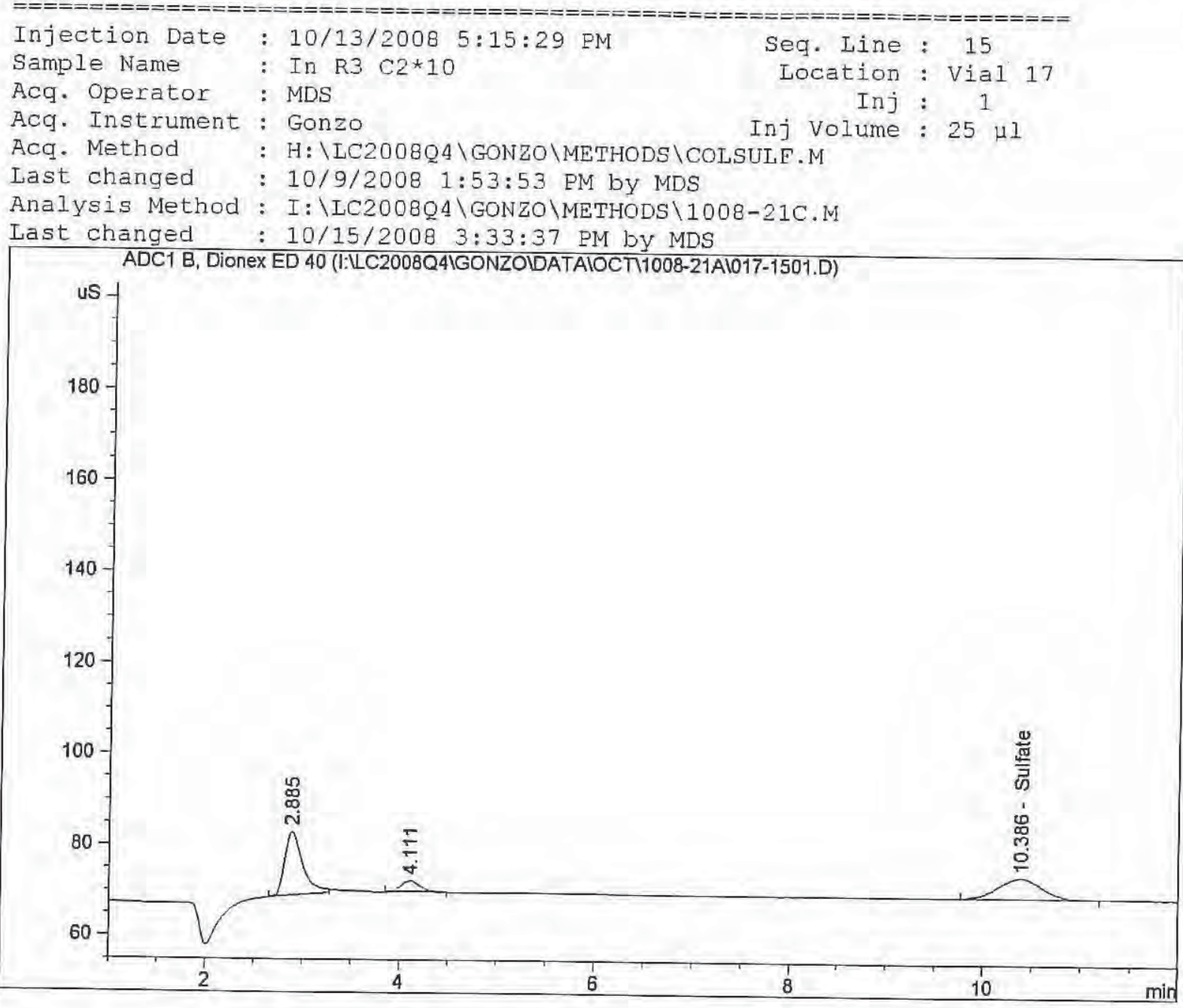

ExternaI Standard Report

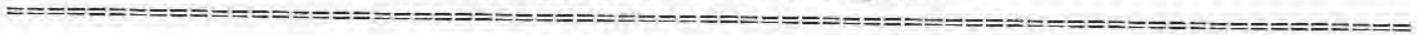

$\begin{array}{lcc}\text { Sorted By } & : & \text { Signal } \\ \text { Calib. Data Modified } & : & 10 / 15 / 2008 \quad 3: 33: 29 \text { PM } \\ \text { Multiplier } & : & 1.0000 \\ \text { Dilution } & : & 1.0000 \\ \text { Use Multiplier \& Dilution Factor with ISTDs }\end{array}$

Signal 1: ADC1 B, Diones ED 40

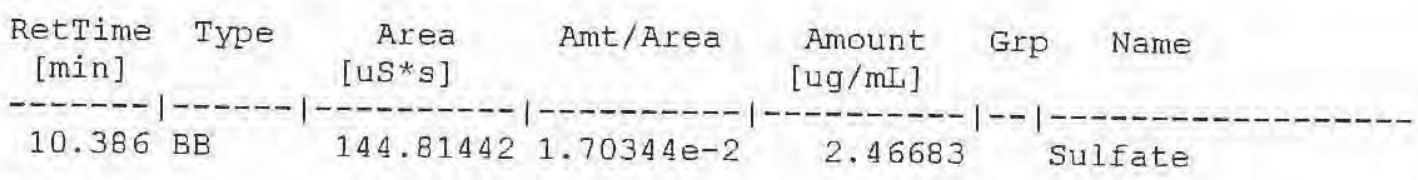

Totals : $\quad 2.46683$

Results obtained with enhanced integrator!

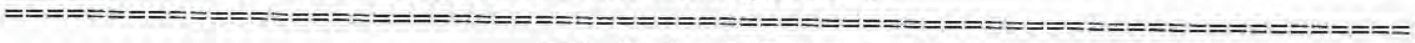
$\star \star \star$ End of Report $* * \star$ 
$1008-22$

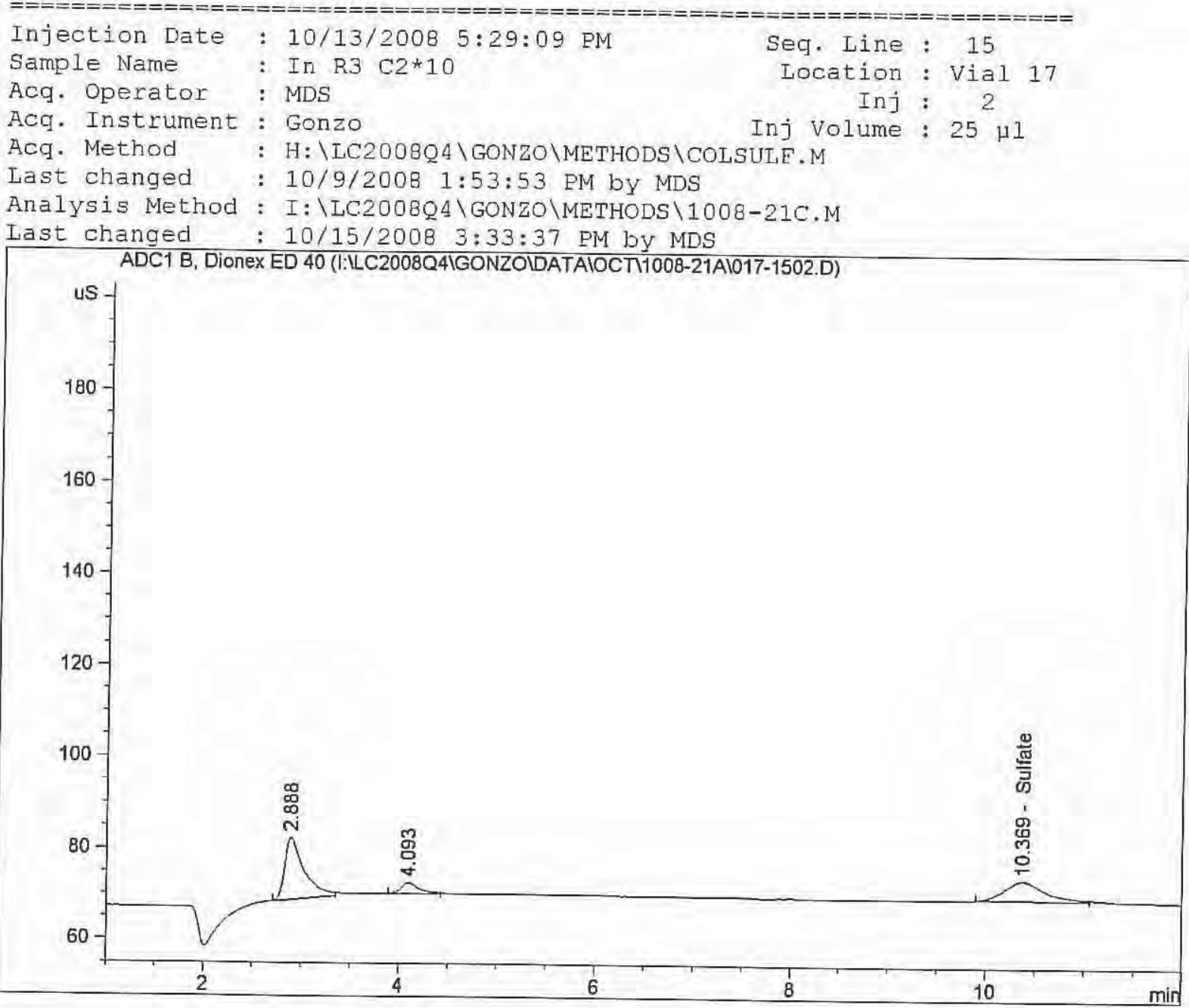

External Standard Report

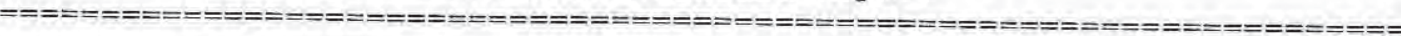

$\begin{array}{lcc}\text { Sorted By } & : & \text { Signal } \\ \text { Calib. Data Modified } & : & 10 / 15 / 2008 \text { 3:33:29 PM } \\ \text { Multiplier } & : & 1.0000 \\ \text { Dilution } & : & 1.0000 \\ \text { Use Multiplier \& Dilution Eactor with ISTDs }\end{array}$

Signal 1: ADC1 B, Diones ED 40

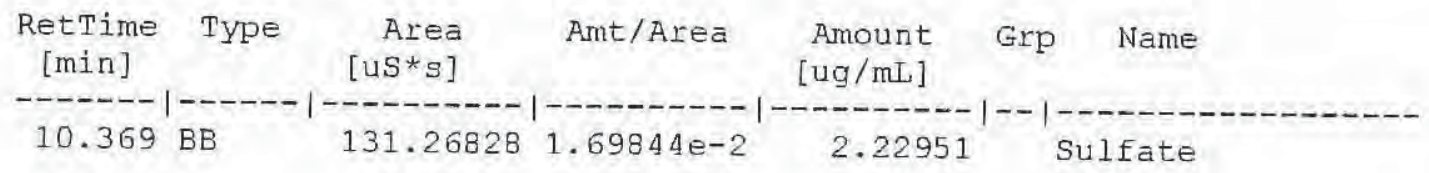

Totals :

2.22951

Results obtained with enhanced integrator!

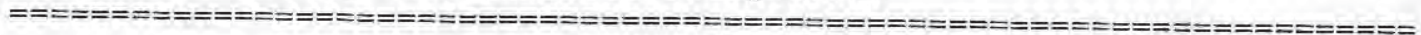

$\star \star \star$ End of Report $* \star *$ 
$1008-22$

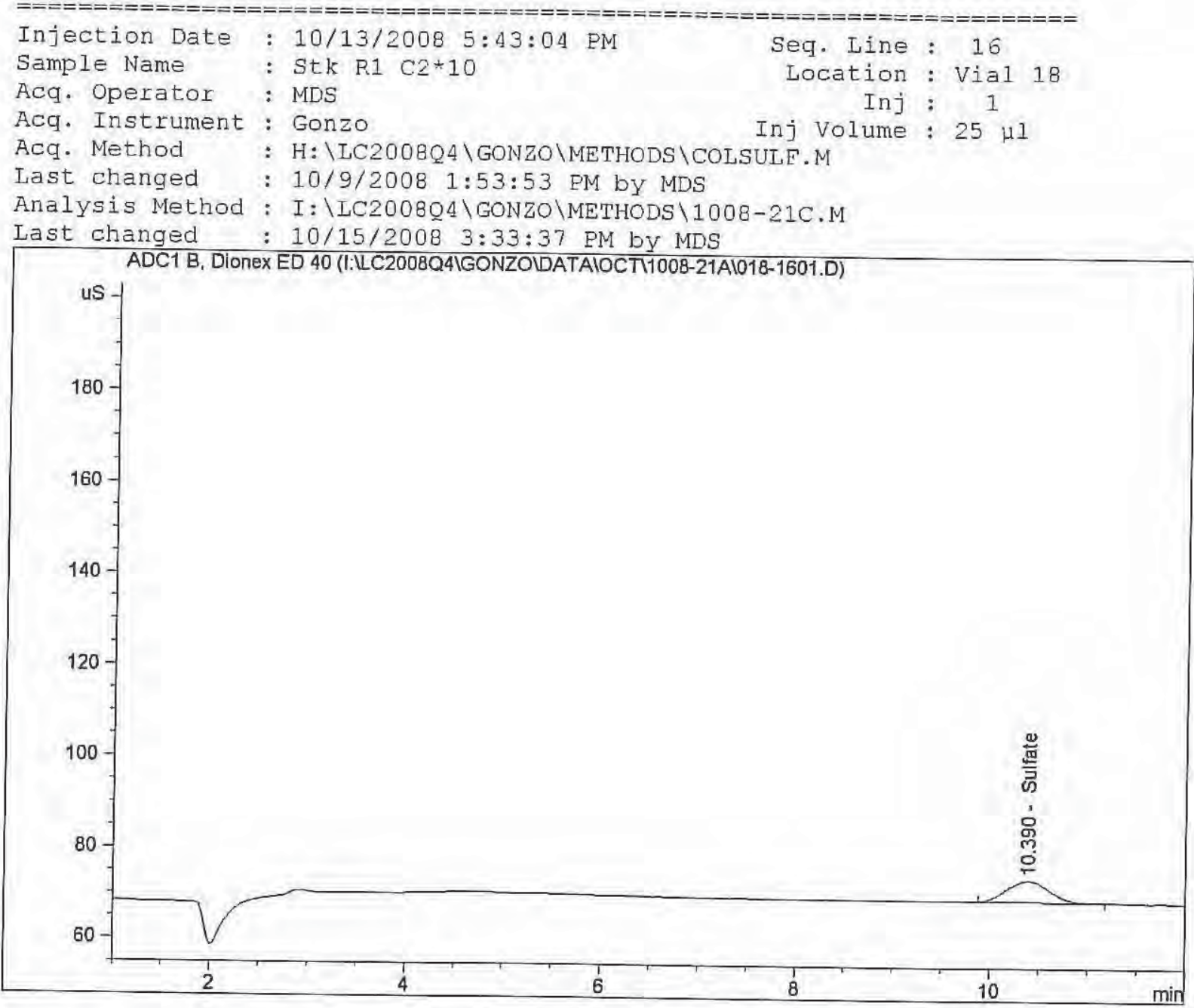

External standard Report

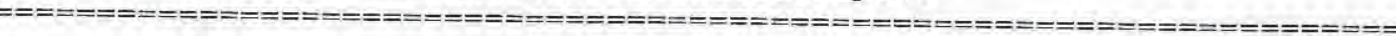

$\begin{array}{lcc}\text { Sorted By } & : & \text { Signal } \\ \text { Calib. Data Modified } & : & 10 / 15 / 2008 \quad 3: 33: 29 \text { PM } \\ \text { Multiplier } & : & 1.0000 \\ \text { Dilution } & \vdots & 1.0000 \\ \text { Use Multiplier \& Dilution Eactor with ISTDs }\end{array}$

Multiplier \& Dilution Eactor with ISTDs

Signal 1: ADC1 B, Diones ED 40

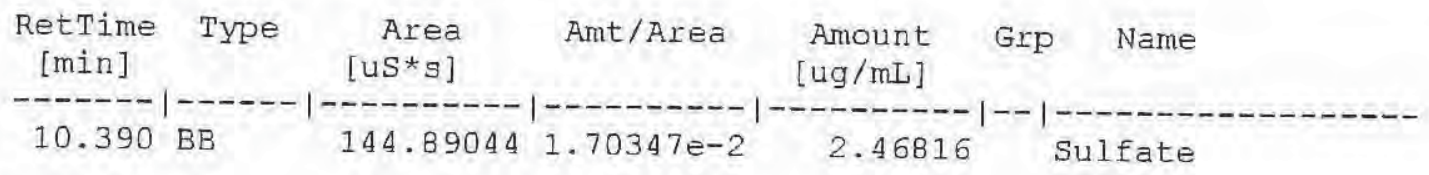

Totals:

2.46816

Results obtained with enhanced integrator!

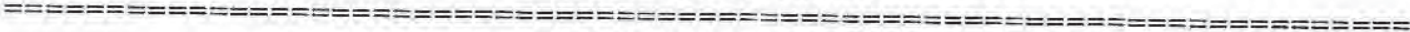

$$
\star * \star \text { End of Report } * \star \star
$$


$1008-22$
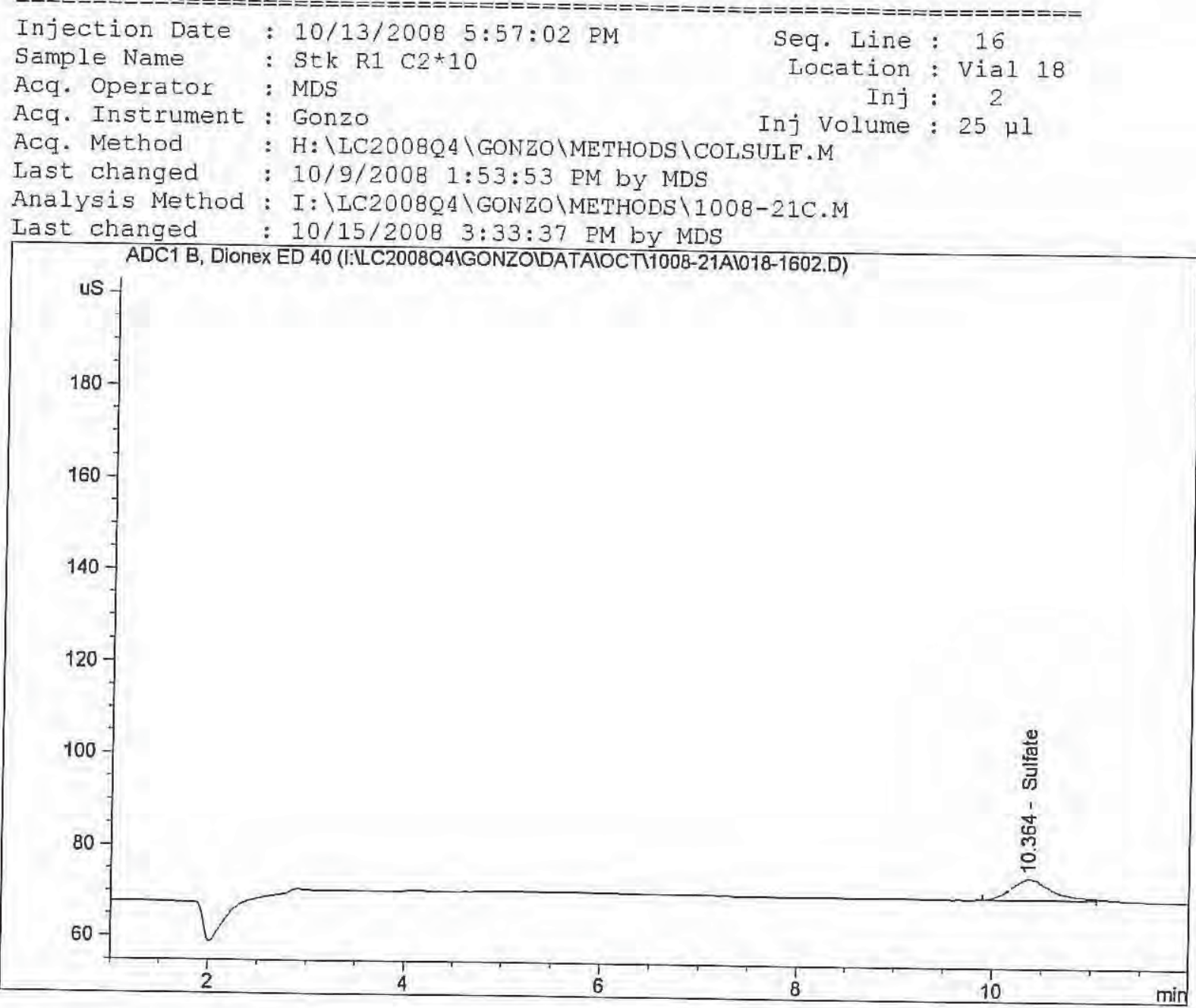

External standard Report

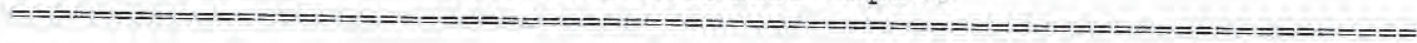

Sorted By

Calib. Data Modified

Multiplier

Dilution

Signal

10/15/2008 $3: 33: 29$ PM

Use Mult ipl

1.0000

1.0000

Signal 1: ADC1 B, Dionex ED 40

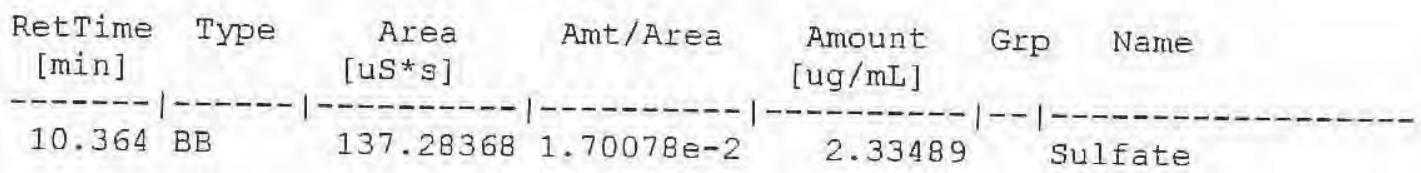

Totals :

2.33489

Results obtained with enhanced integrator!

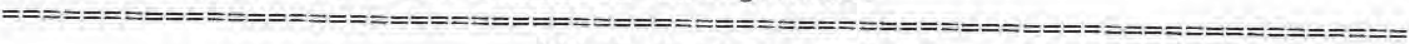

$$
* * * \text { End of Report *** }
$$




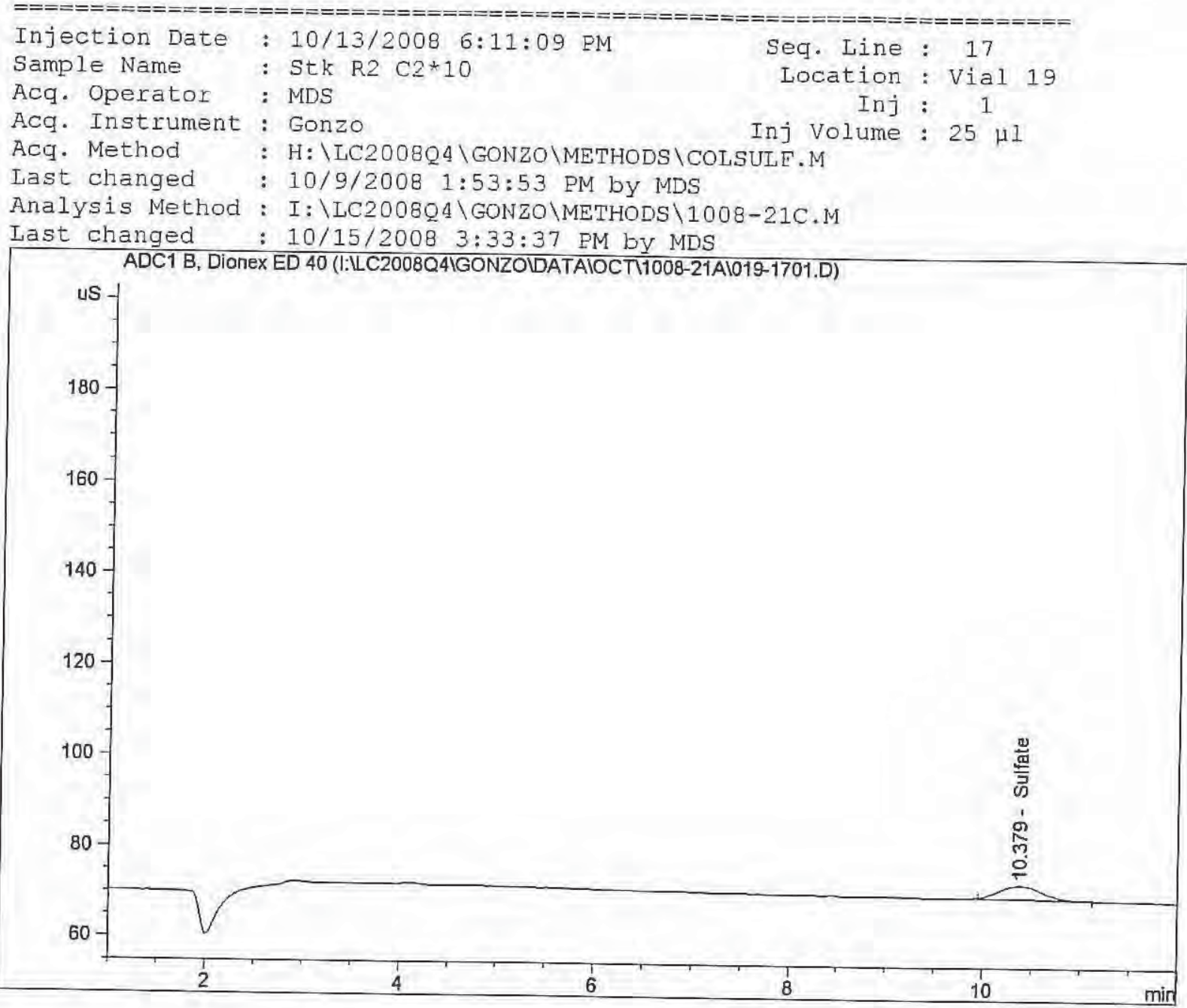

External Standard Report

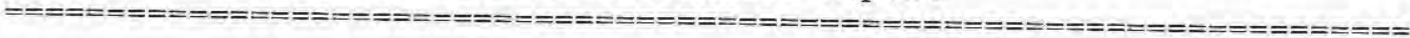

Sorted By

Calib. Data Modified :

Multiplier

Dilution

Signal

10/15/2008 $3: 33: 29$ PM

Use Multiplier \& Dilution Factor with ISTDS

Signal 1: ADC1 B, Dionex ED 40

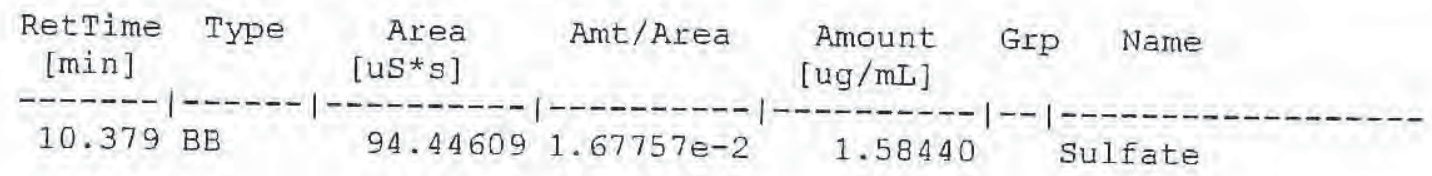

Totals :

1.58440

Results obtained with enhanced integrator!

$\star * *$ End of Report *** 


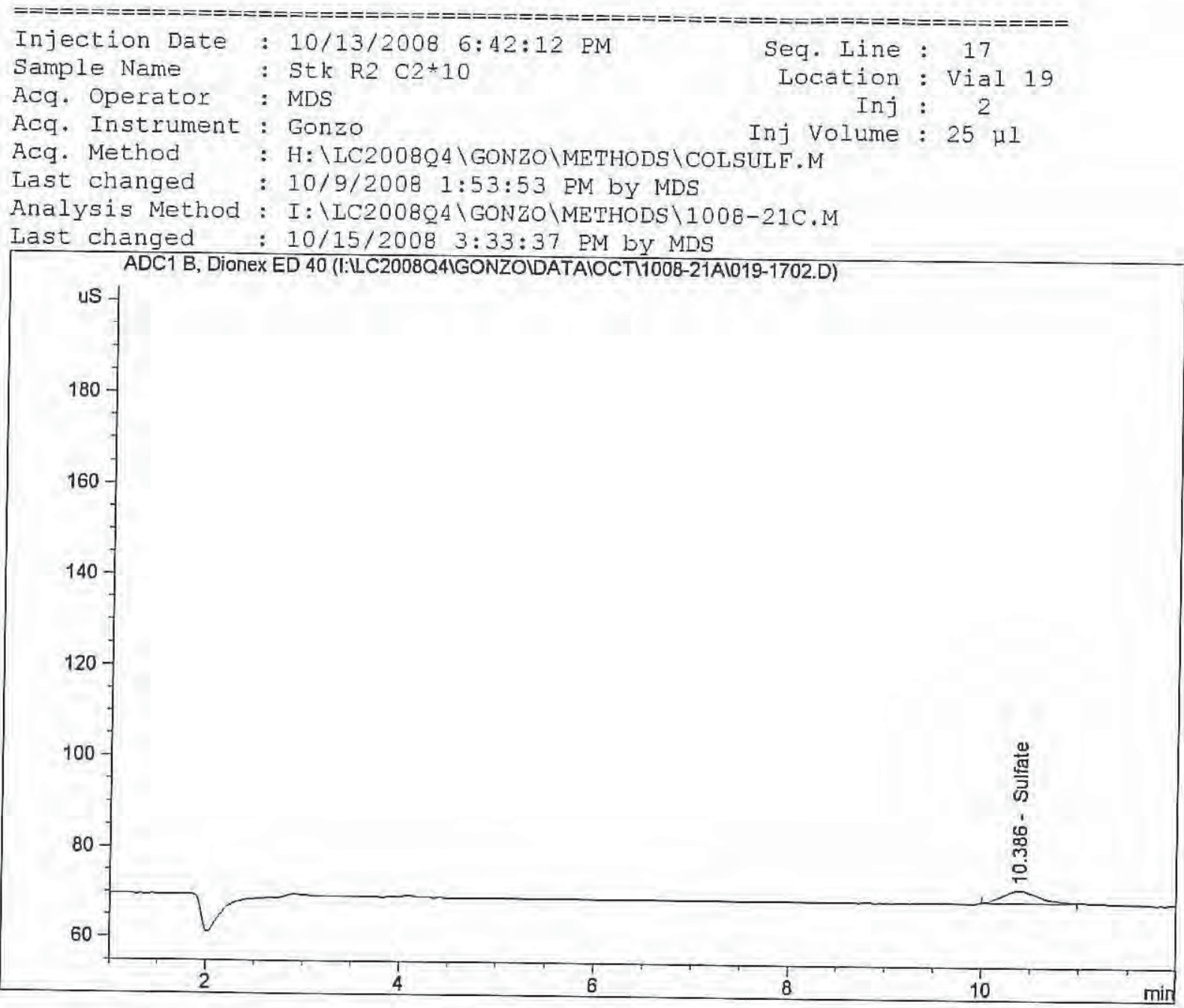

External Standard Report

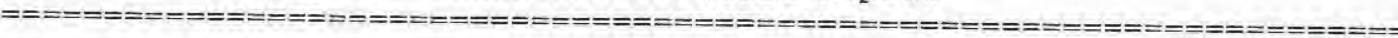

Sorted By

Multiplier : 1.0000

Dilution : 1.0000

Use Multiplier \& Dilution Eactor with ISTDs

Signal 1: ADCI B, Diones ED 40

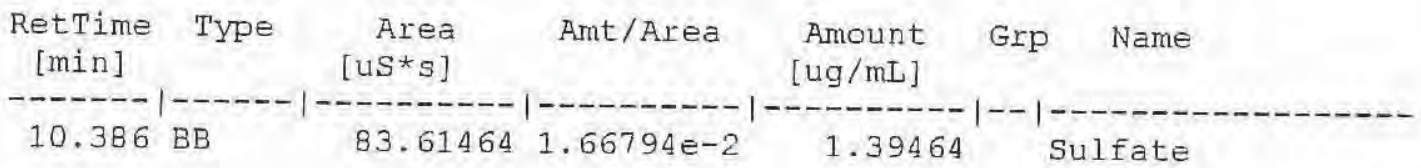

Totals :

1.39464

Results obtained with enhanced integrator!

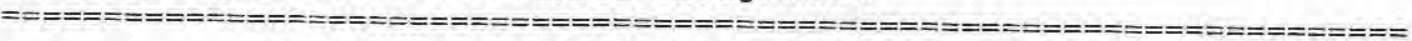
$\star \star *$ End of Report $* \star \star$ 
$1008-22$

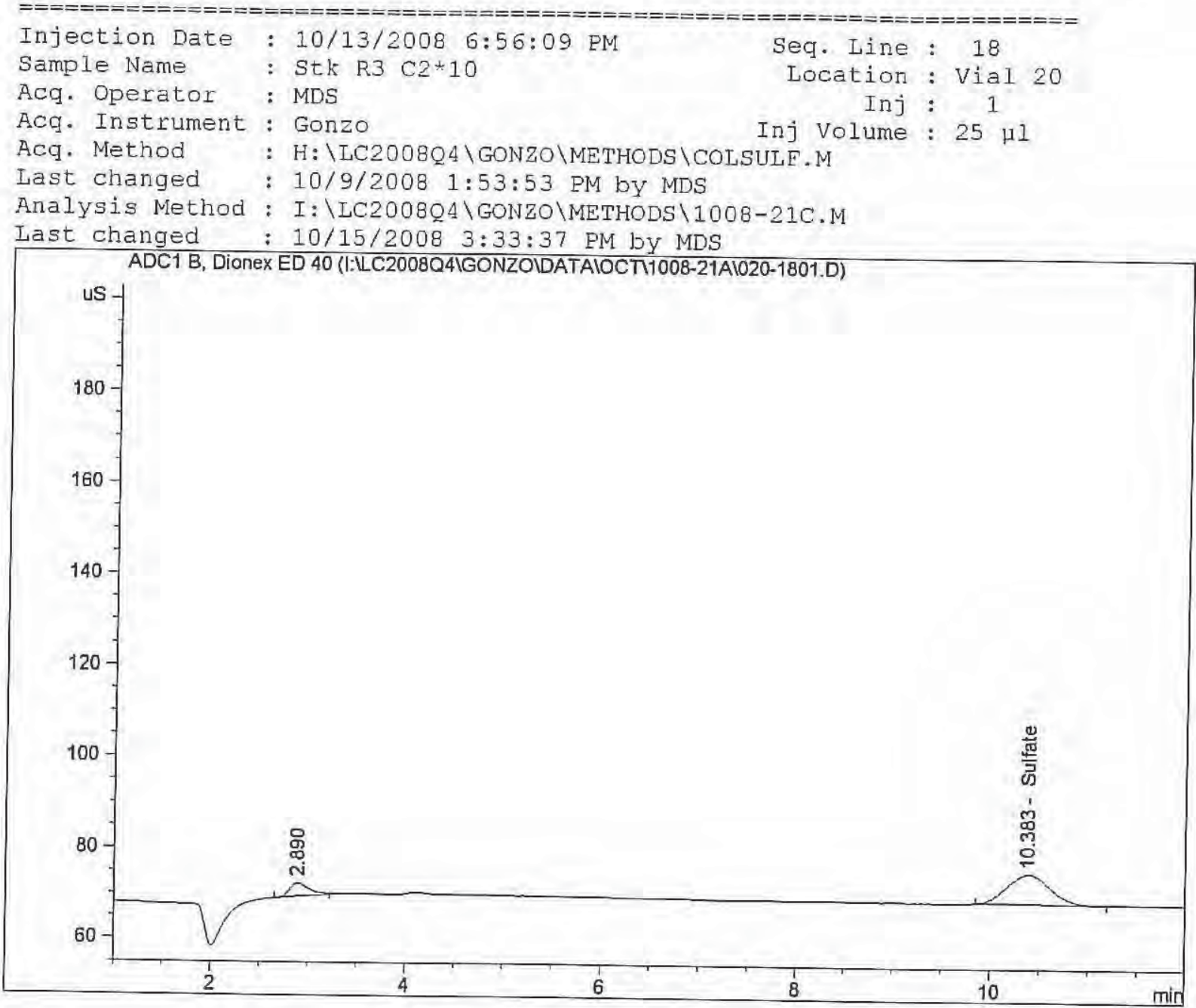

External Standard Report

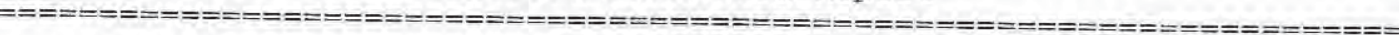

Sorted By

Signal

Calib. Data Modified : $10 / 15 / 2008$ 3:33:29 PM

Multiplier : 1.0000

Dilution : 1.0000

Use Multiplier \& Dilution Factor with ISTDs

Signal 1: ADC1 B, Diones ED 40

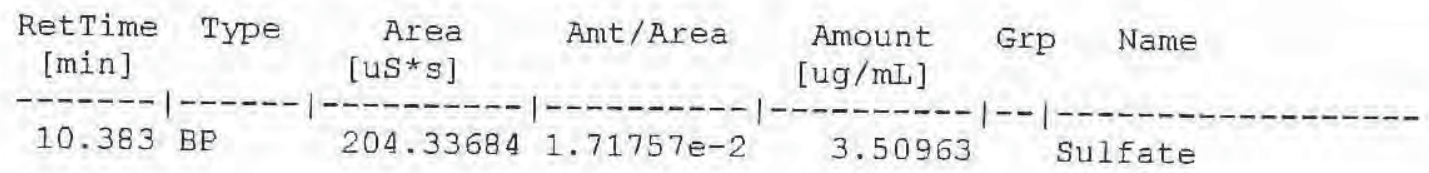

Totals :

3.50963

Results obtained with enhanced integrator!

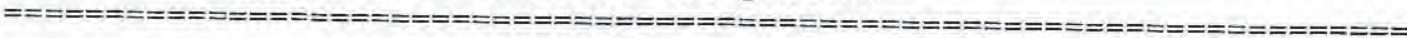
$\star * \star$ End of Report $* \star \star$ 
$1008-22$

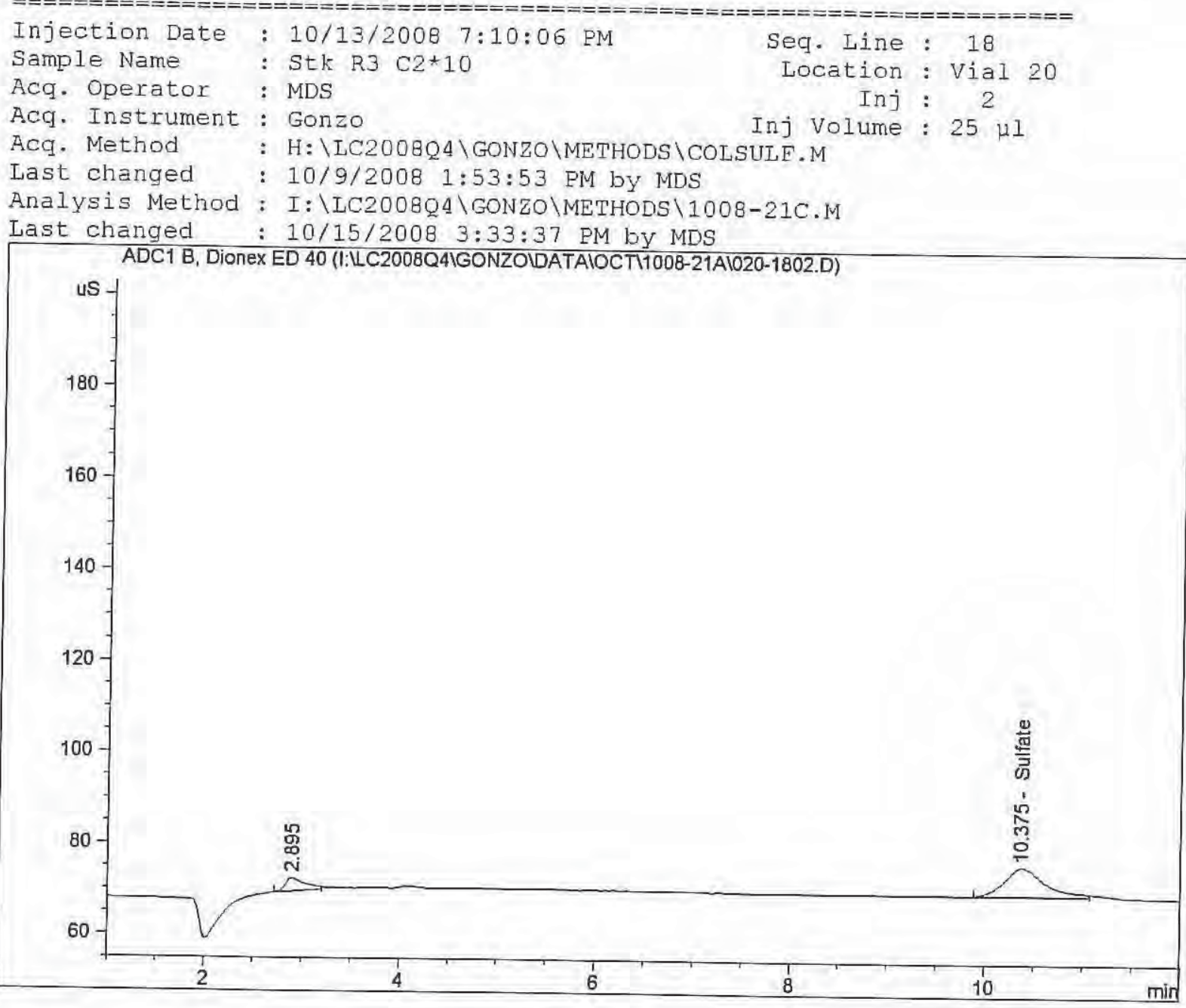

External Standard Report

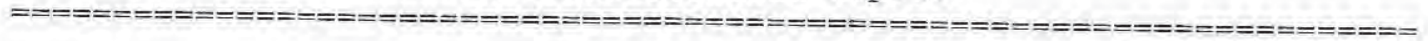

Sorted By

Calib. Data Modified : Signal

Multiplier : 1.0000

Dilution : 1.0000

Use Multiplier \& Dilution Factor with ISTDS

Signal 1: ADC1 B, Dionex ED 40

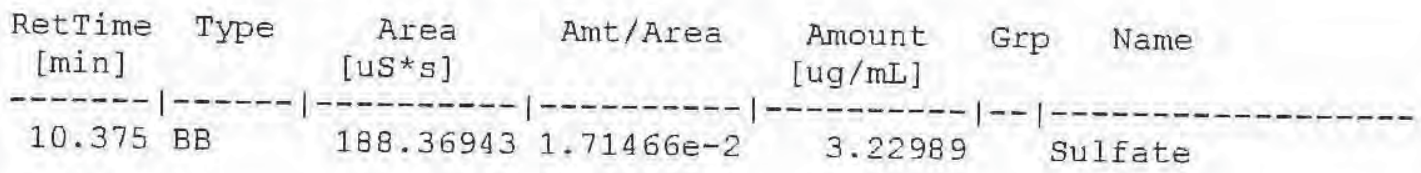

Totals :

3. 22989

Results obtained with enhanced integrator!

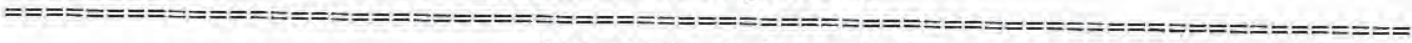

$\star \star \star$ End of Report *** 
1008-22 Sample Blank

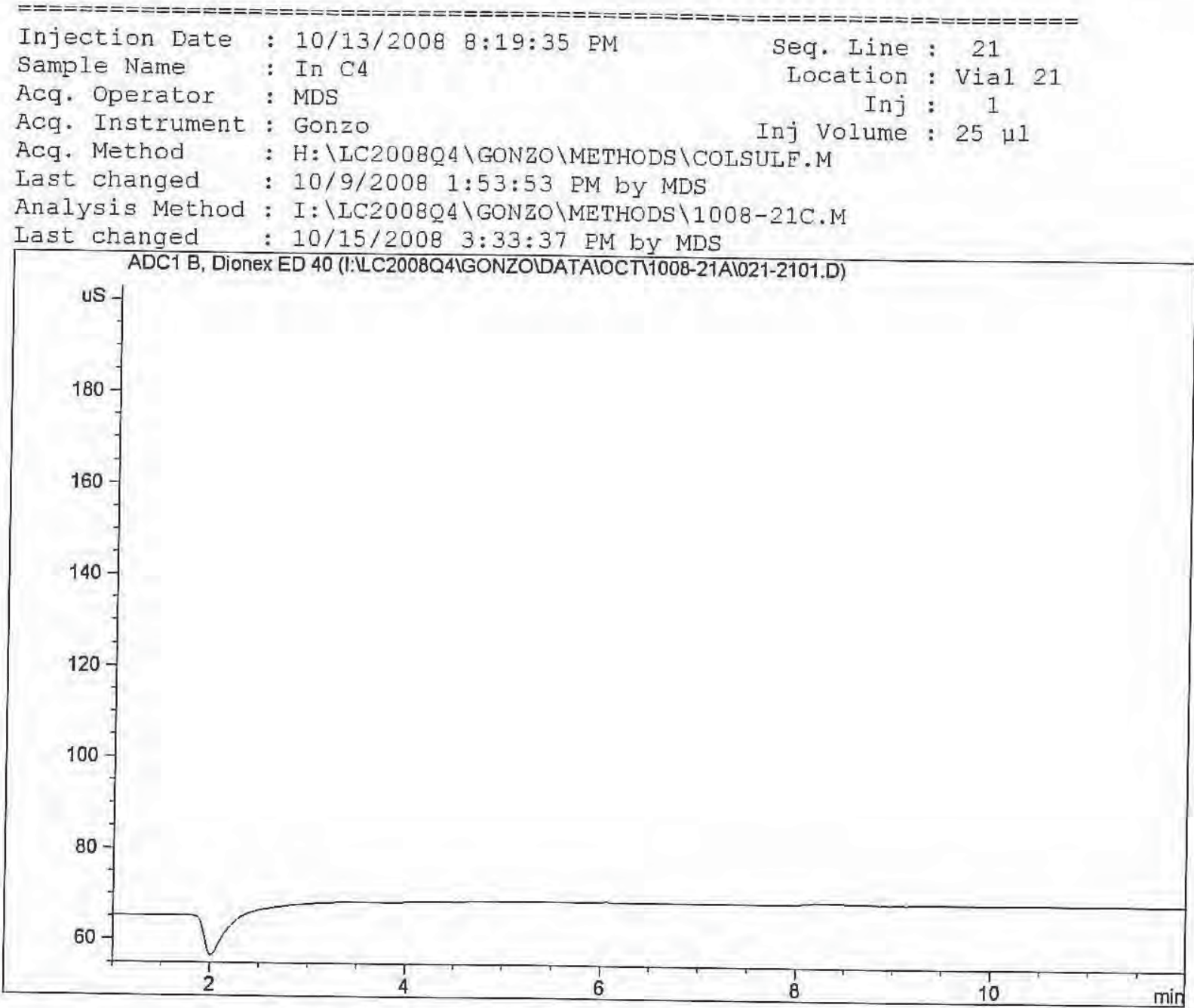

External Standard Report

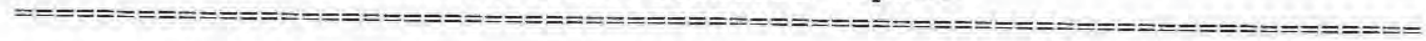

Sorted BY

Multiplier : 1.0000

Dilution : 1.0000

Use Multiplier \& Dilution Factor with ISTDS

Signal 1: ADC1 B, Diones ED 40

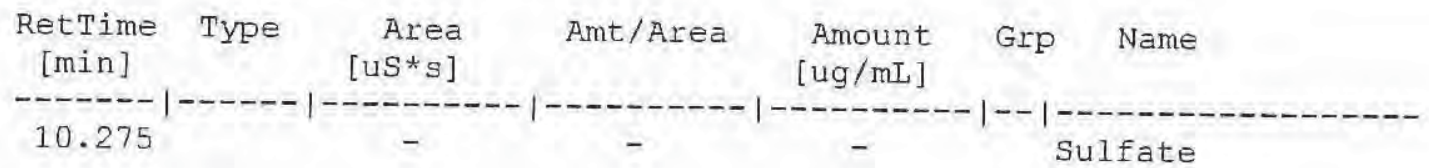

10.275

Totals :

0.00000

Results obtained with enhanced integrator!

1 Warnings or Errors :

Warning : Calibrated compound(s) not found 
1008-22 Sample Blank

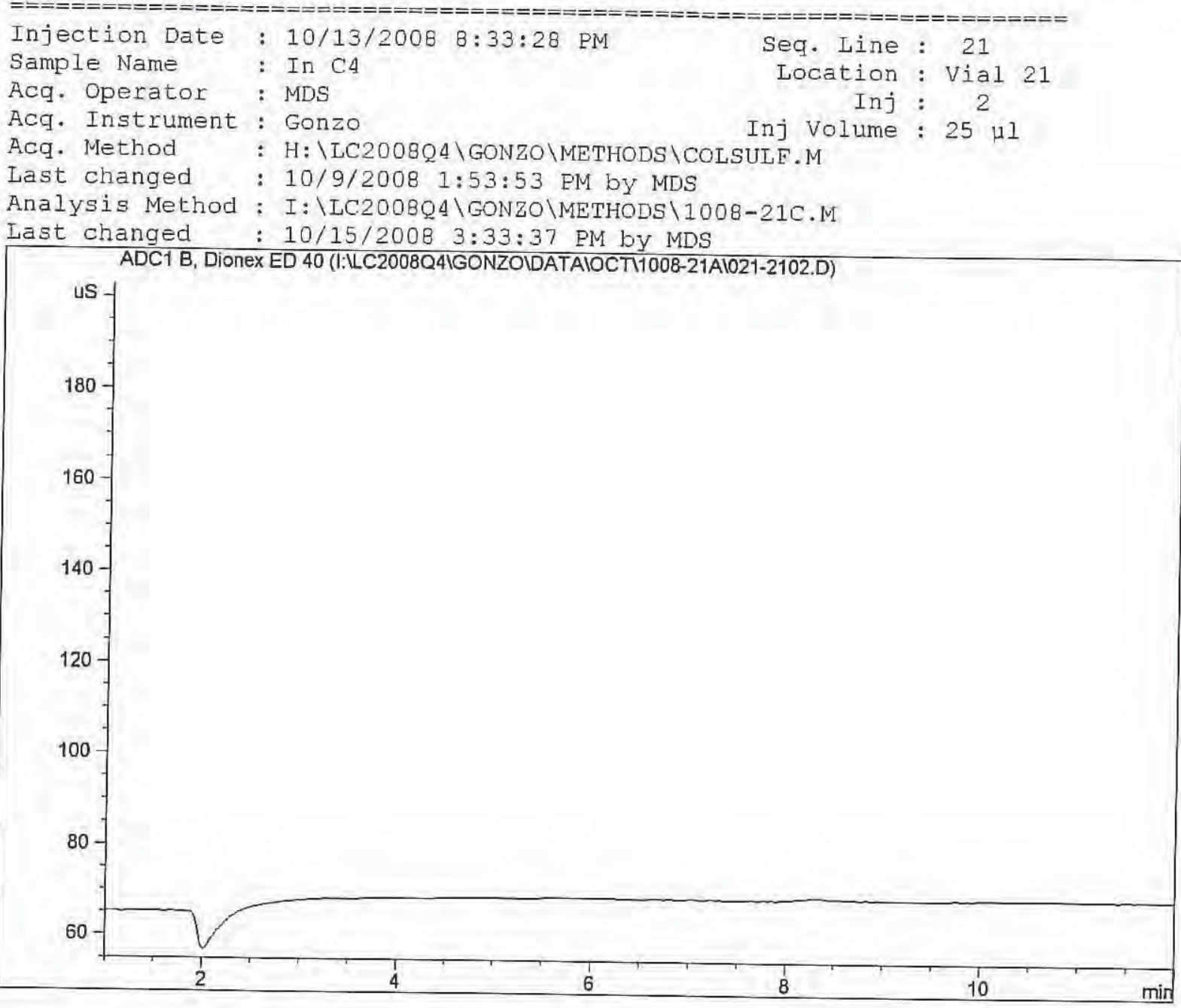

External Standard Report

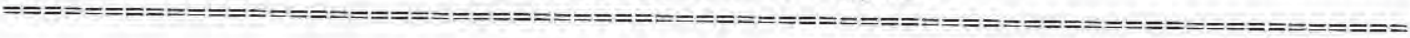

$\begin{array}{lll}\text { Sorted By } & : & \text { Signal } \\ \text { Calib. Data Modified } & : & 10 / 15 / 2008 \quad 3: 33: 29 \text { PM } \\ \text { Multiplier } & : & 1.0000 \\ \text { Dilution } & : & 1.0000\end{array}$

Use Multiplier \& Dilution Factor with ISTDS

Signal 1: ADC1 B, Dionex ED 40

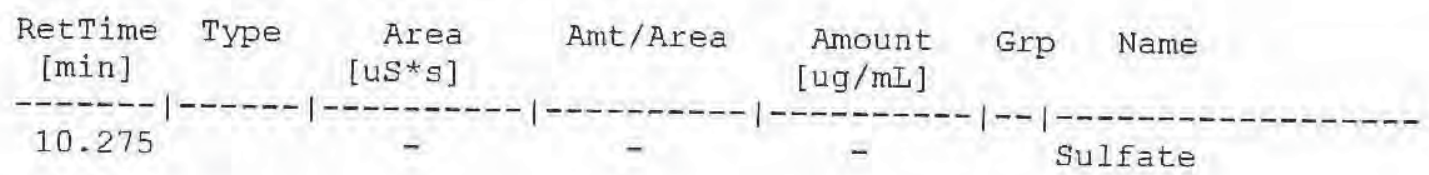

Totals : $\quad 0.00000$

Results obtained with enhanced integrator!

1 Warnings or Errors :

Warning : Calibrated compound(s) not found 
1008-22 Sample Blank
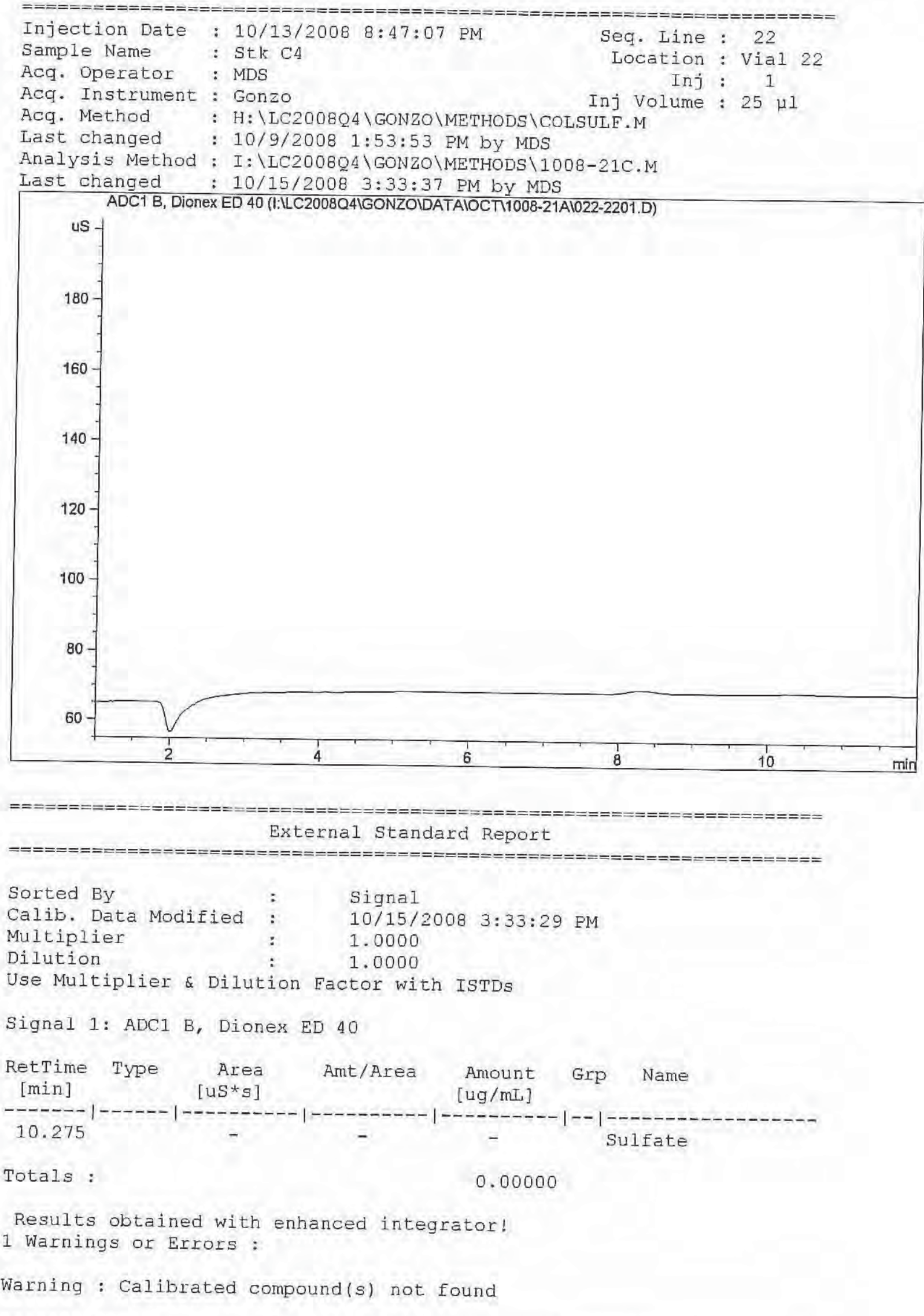
1008-22 Sample Blank

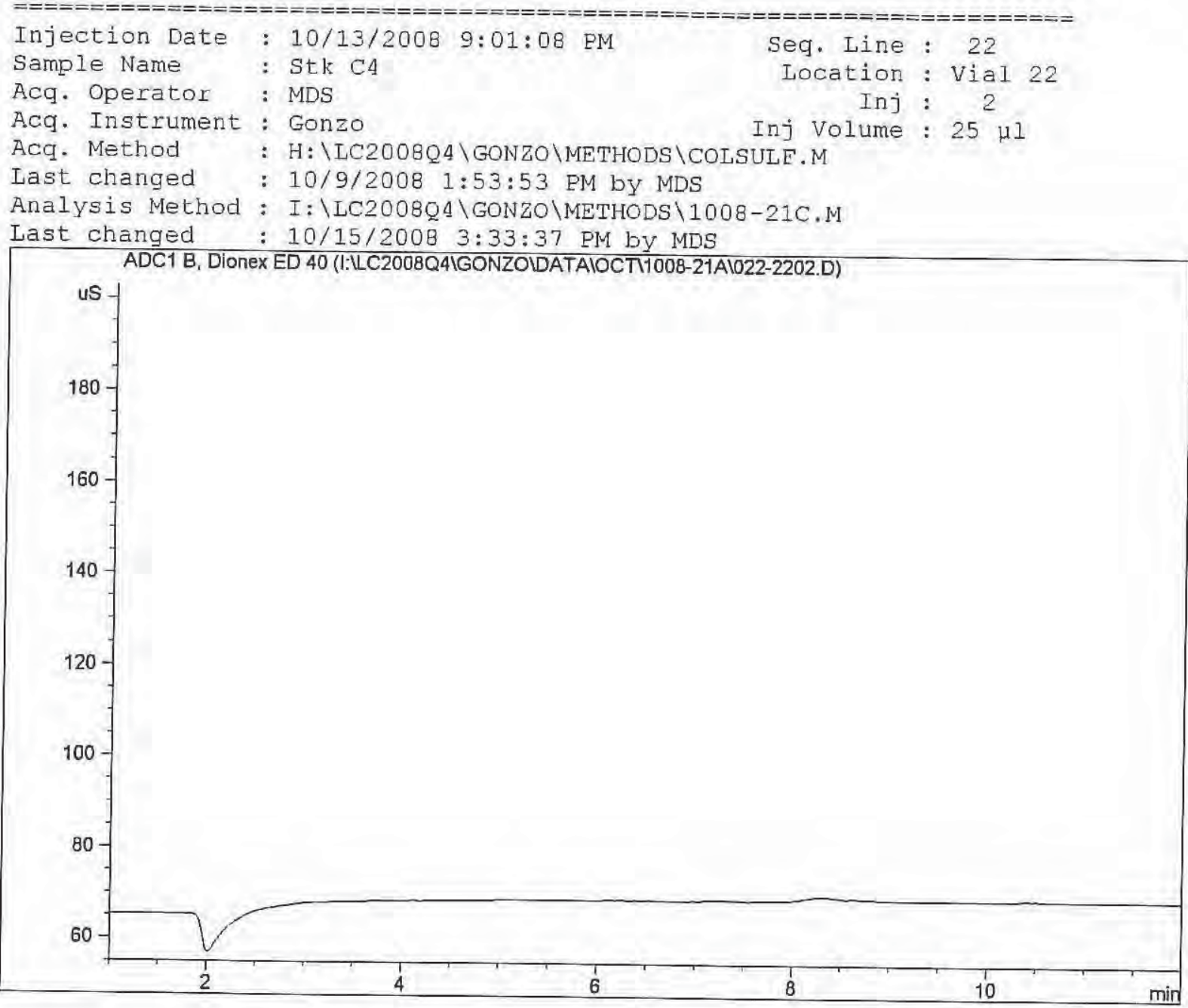

External standard Report

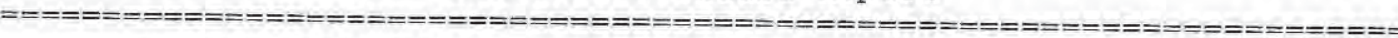

Sorted By

Calib. Data Modified

Multiplier

Dilution

Signal

10/15/2008 3:33:29 PM

Dilution

1.0000

1.0000

Use Multiplier \& Dilution Eactor with ISTDs

Signal 1: ADC1 B, Diones ED 40

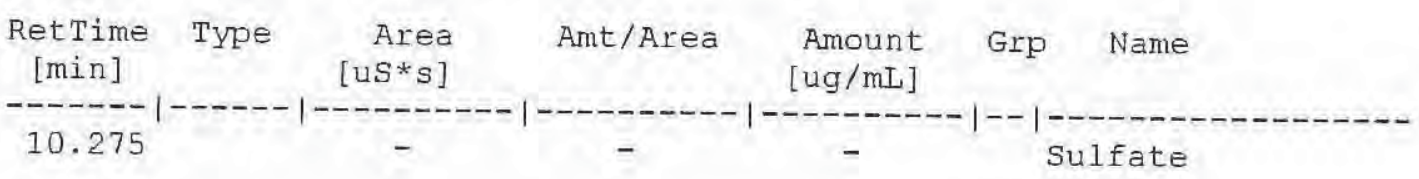

Totals :

0.00000

Results obtained with enhanced integrator!

1 Warnings or Errors :

Warning : Calibrated compound(s) not found 
used to prepare standards and dilute samples

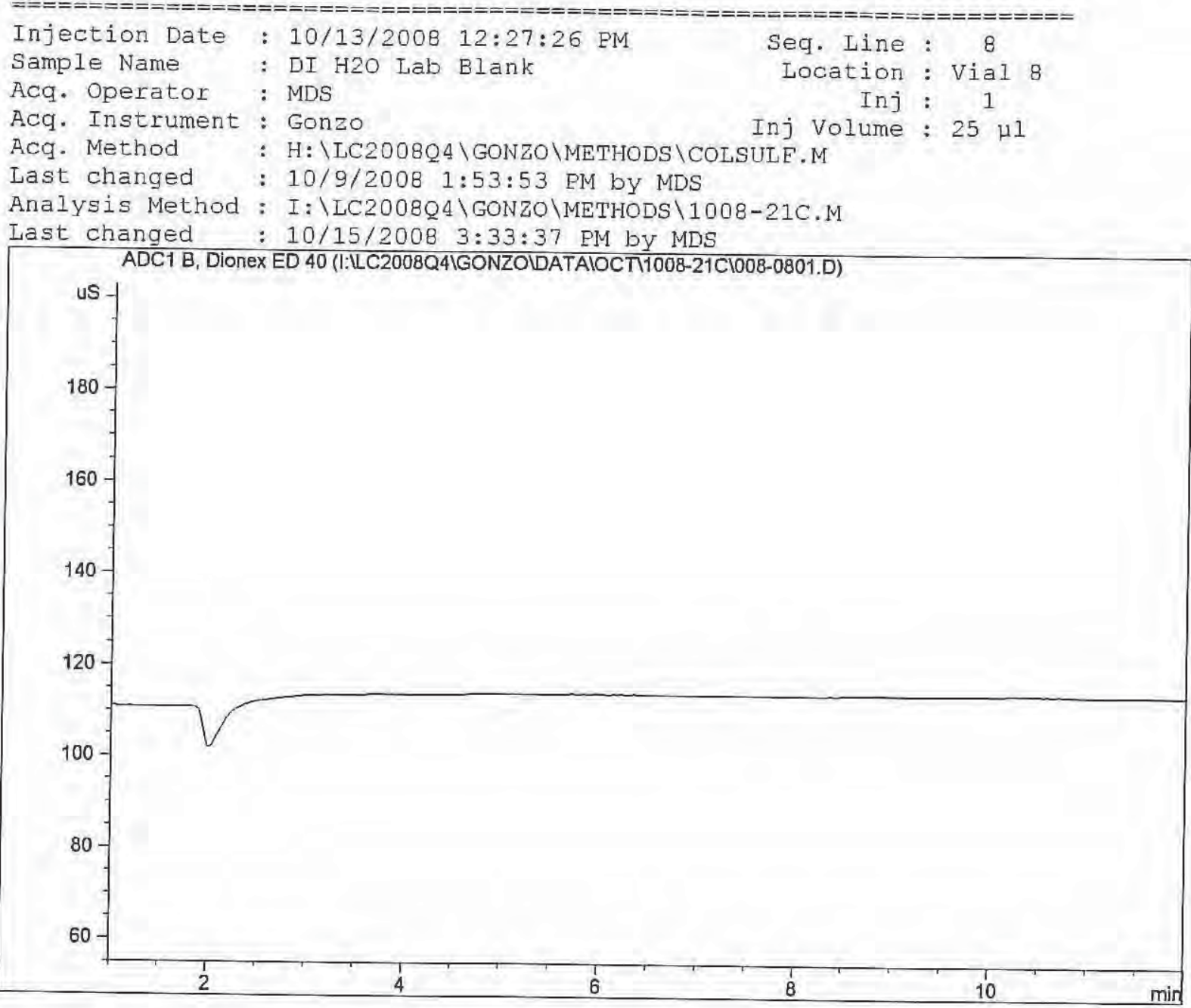

External Standard Report

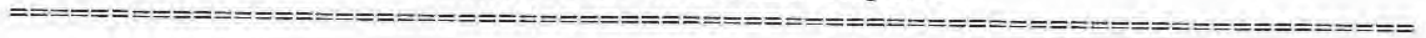

Sorted BY

Signal

Calib. Data Modified : $10 / 15 / 2008$ 3:33:29 PM

Multiplier : 1.0000

Dilution : 1.0000

Use Multiplier \& Dilution Factor with ISTDs

Signal 1: ADC1 B, Dionex ED 40

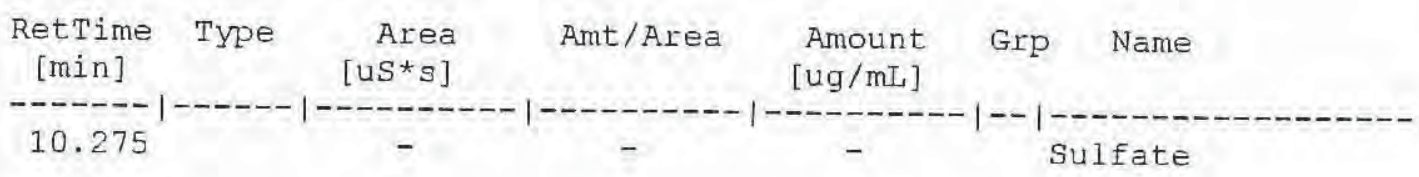

Totals :

0.00000

Results obtained with enhanced integrator!

1 Warnings or Errors :

Warning : Calibrated compound(s) not found 
used to prepare standards and dilute samples
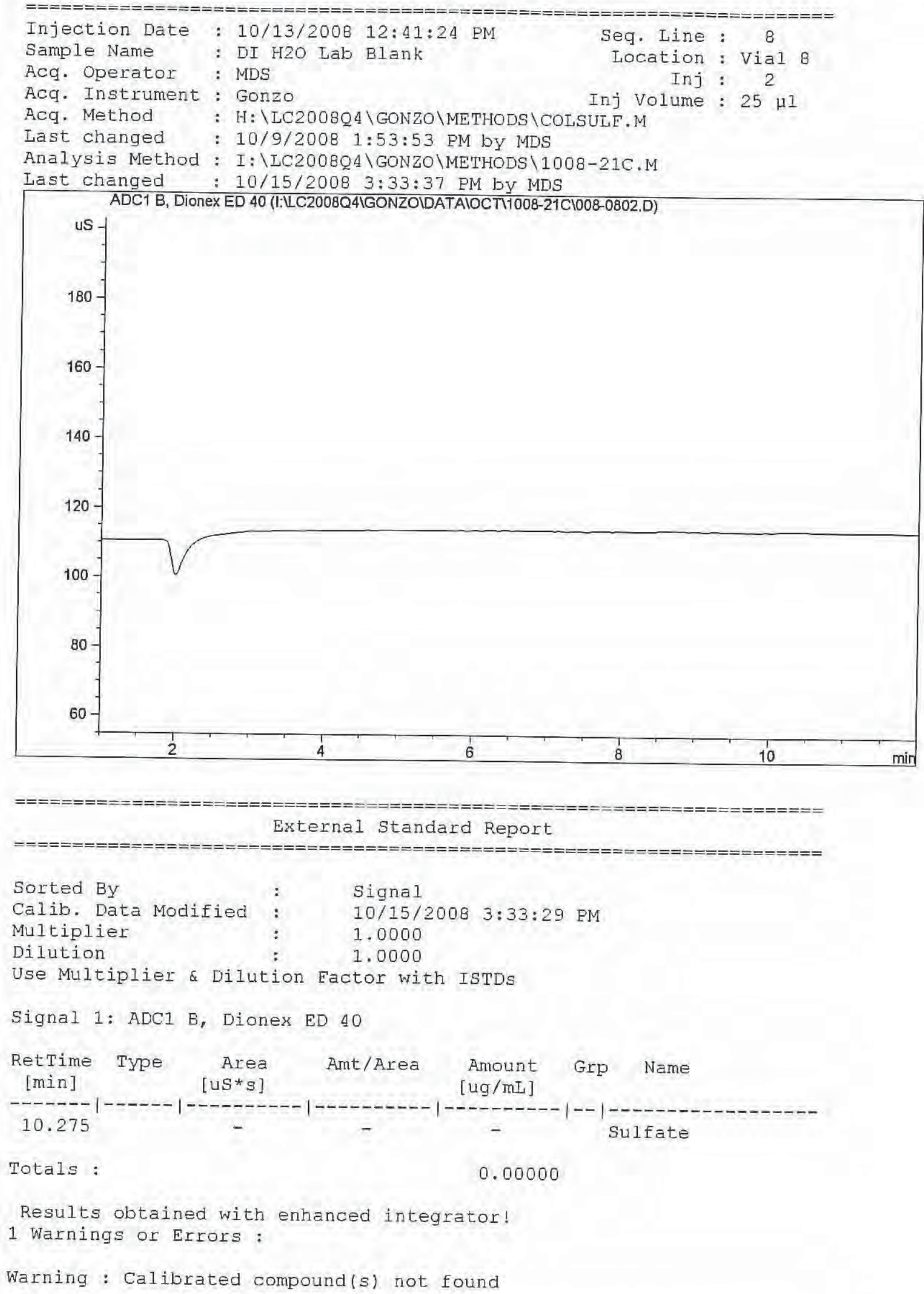
1008-22 Matrix Spike

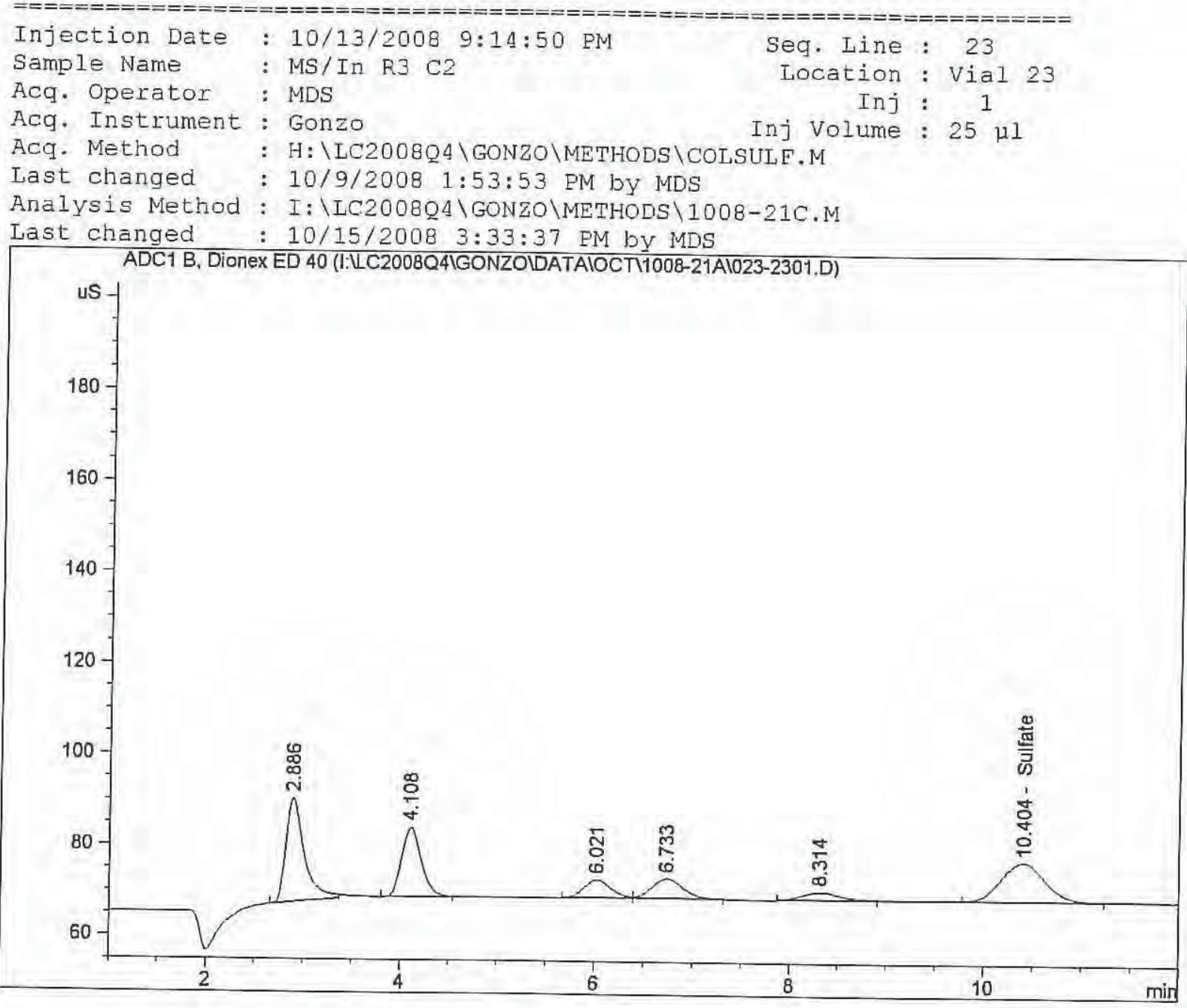

External Standard Report

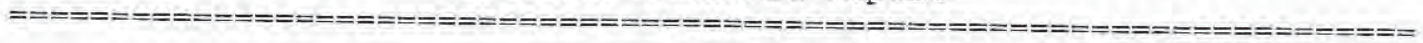

Sorted By

Signal

Calib. Data Modified : $10 / 15 / 2008$ 3:33:29 PM

Multiplier

Dilution

1.0000

Use Multiplier \& Dilution Eactor with ISTDS

Signal 1: ADC1 B, Dionex ED 40

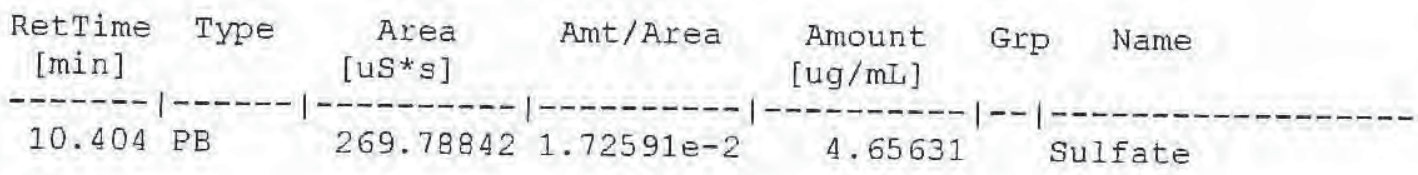

Totals :

4.65631

Results obtained with enhanced integrator!

$\star * \star$ End of Report $* * *$ 
1008-22 Matrix spike

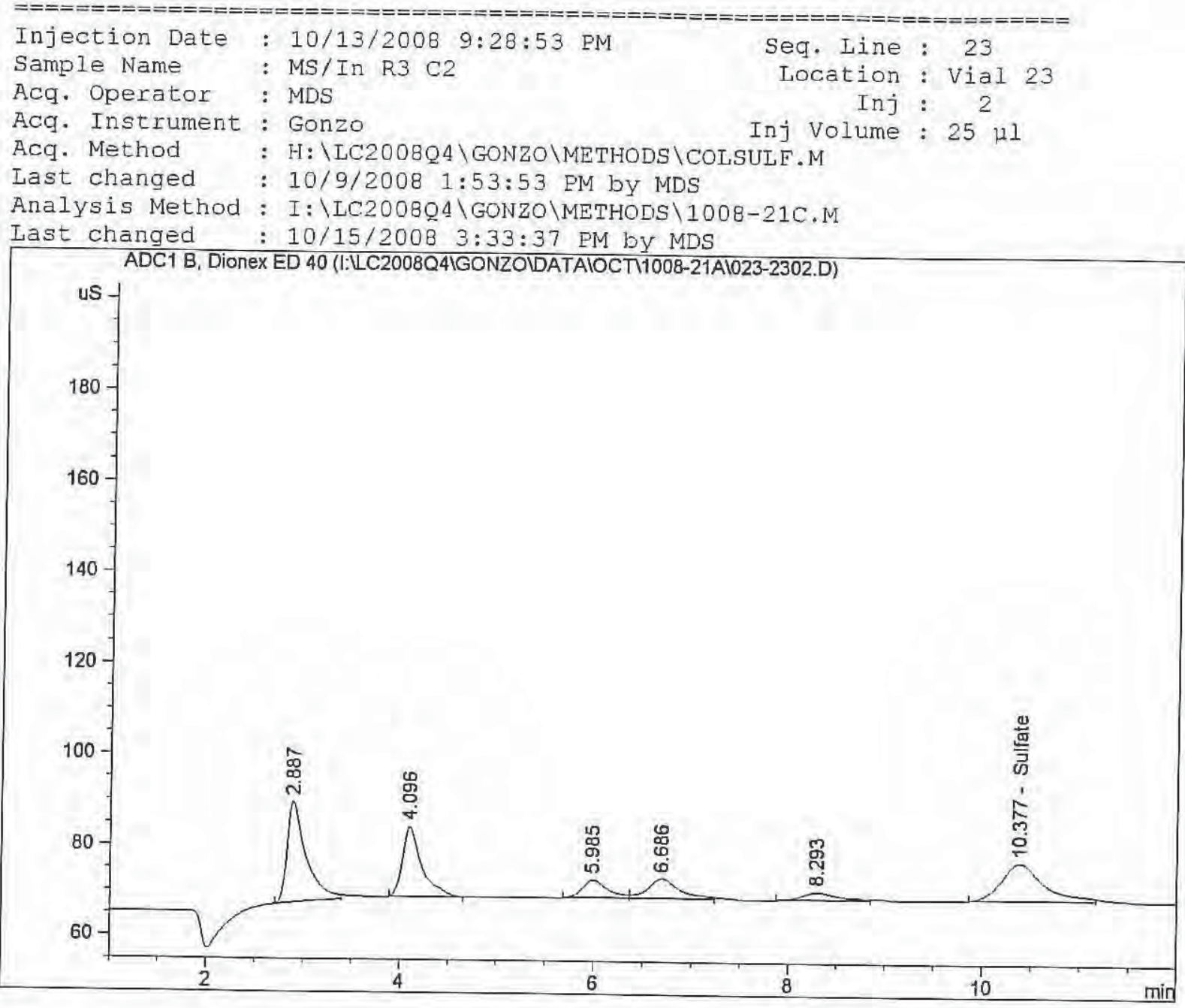

External Standard Report

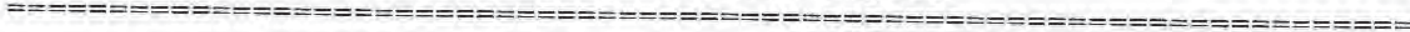

$\begin{array}{lll}\text { Sorted By } & : & \text { Signal } \\ \text { Calib. Data Modified : } & 10 / 15 / 2008 \quad 3: 33: 29 \text { PM } \\ \text { Multiplier } & : & 1.0000 \\ \text { Dilution } & 1.0000 \\ \text { Use Multiplier \& Dilution Factor with ISTDs }\end{array}$

Use Multiplier \& Dilution Eactor with ISTDs

Signal 1: ADC1 B, Dionex ED 40

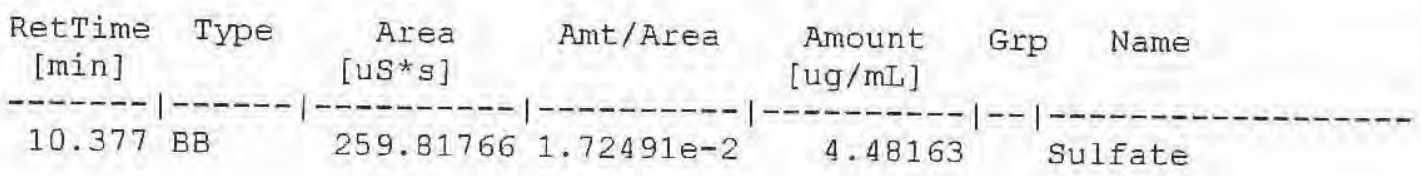

Totals :

4.48163

Results obtained with enhanced integrator!

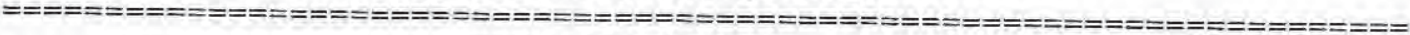

$\star \star \star$ End of Report $* * *$ 


\section{Curve(s)/QA Point(s) Chromatograms}

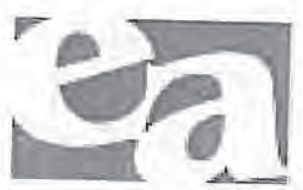




\section{Calibration Table}

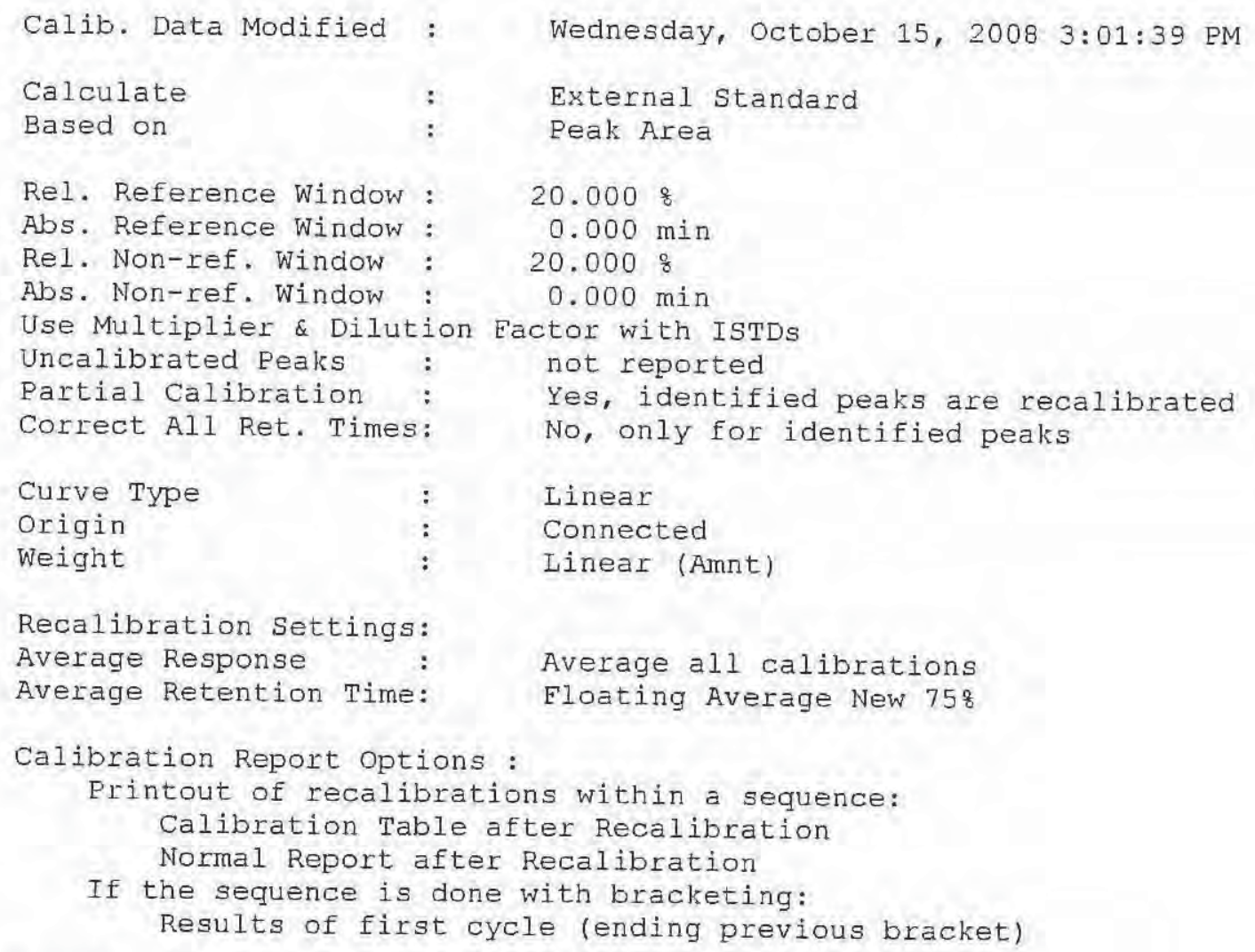




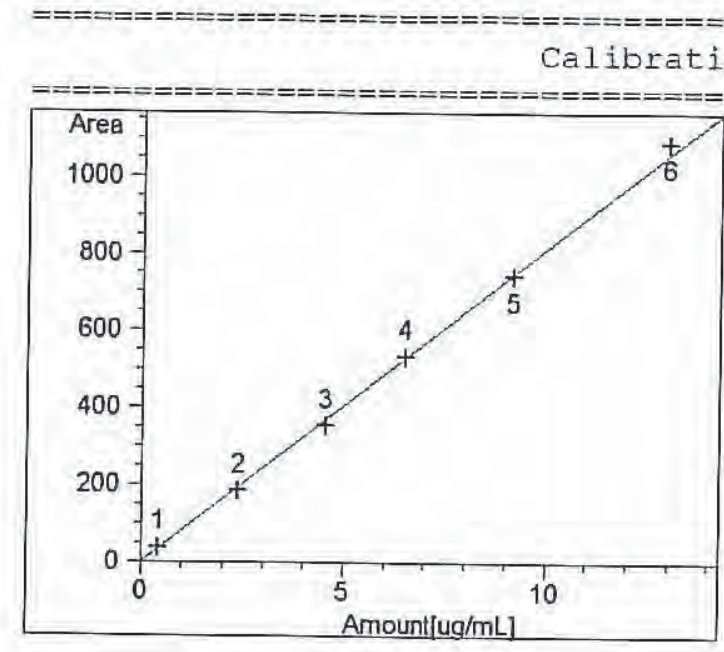

Calibration Curves

Chloride at exp. RT: 4.077

ADC1 B, Dionex ED 40

Correlation:

0.99952

Residual Std. Dev.: 16.54705

Formula: $y=m x+b$

$$
\begin{aligned}
& \mathrm{m}: \quad 81.86062 \\
& \mathrm{~b}: \quad-3.25525 \\
& \mathrm{x}: \text { Amount }[\mathrm{ug} / \mathrm{mL}] \\
& \mathrm{y} \text { : Area }
\end{aligned}
$$

Calibration Level Weights:

Level $1: 1$

Level $2: 0.182353$

Level $3: 0.095385$

Level $4: 0.066564$

Level $5: 0.047277$

Level $6: 0.033385$

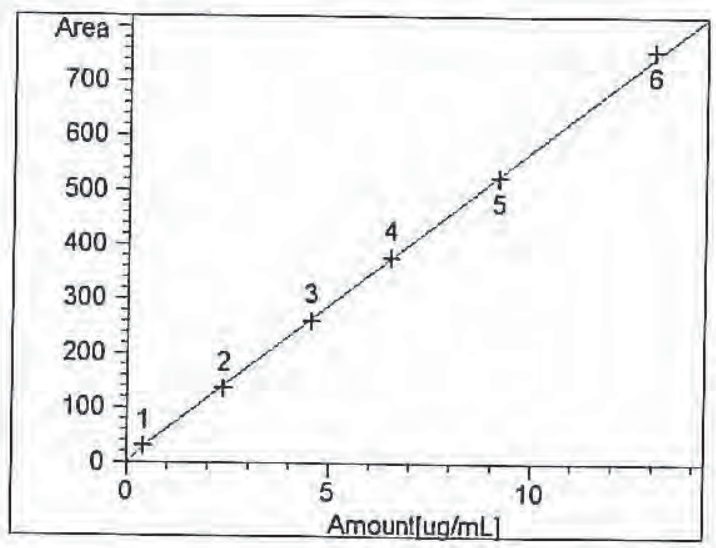

Sulfate at exp. RT: 10.275

$A D C 1$ B, Dionex ED 40

Correlation:

0.99987

Residual Std. Dev.:

6.05844

Formula: $y=m s+b$

$$
\begin{array}{lr}
\text { m: } & 57.07929 \\
\mathrm{~b}: & 4.00958 \\
\mathrm{x}: & \text { Amount }[\mathrm{ug} / \mathrm{mL}] \\
\mathrm{y}: & \text { Area }
\end{array}
$$

Calibration Level Weights:

Level $1: 1$

Ievel $2: 0.182353$

Level $3: 0.095385$

Level $4: 0.066564$

Level $5: 0.047277$

Level $6: 0.033385$ 


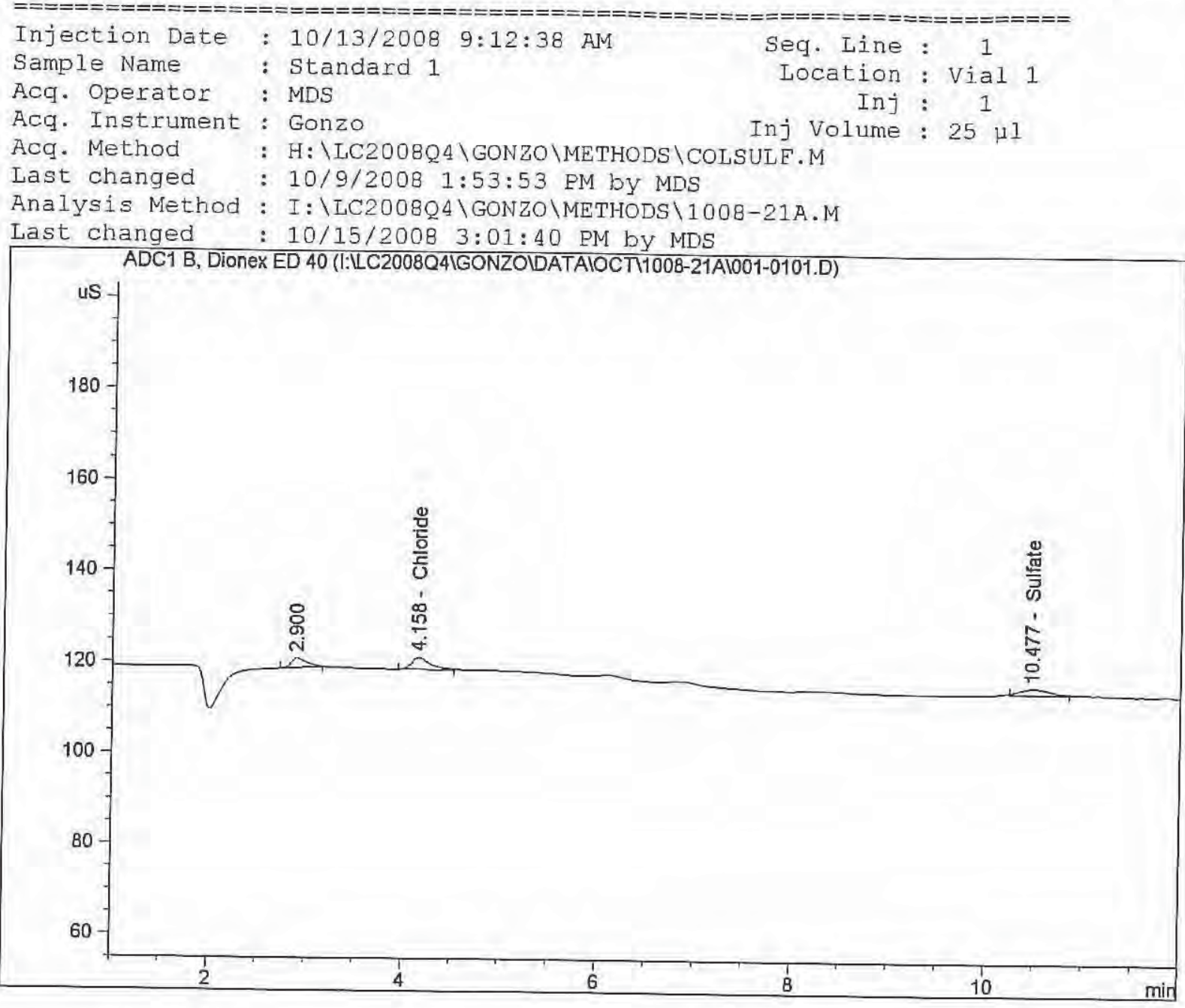

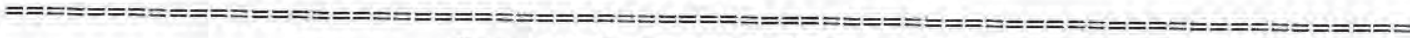
External Standard Report

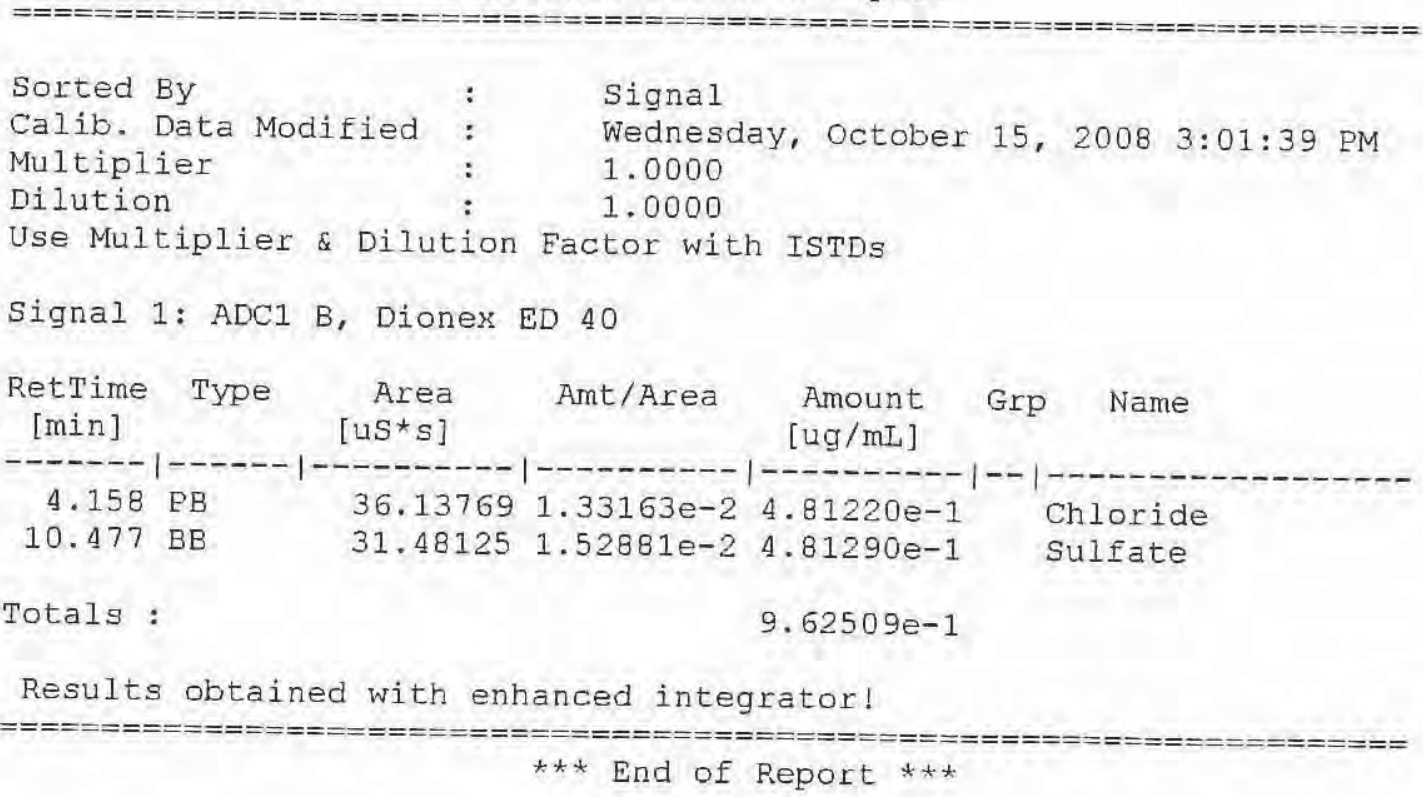




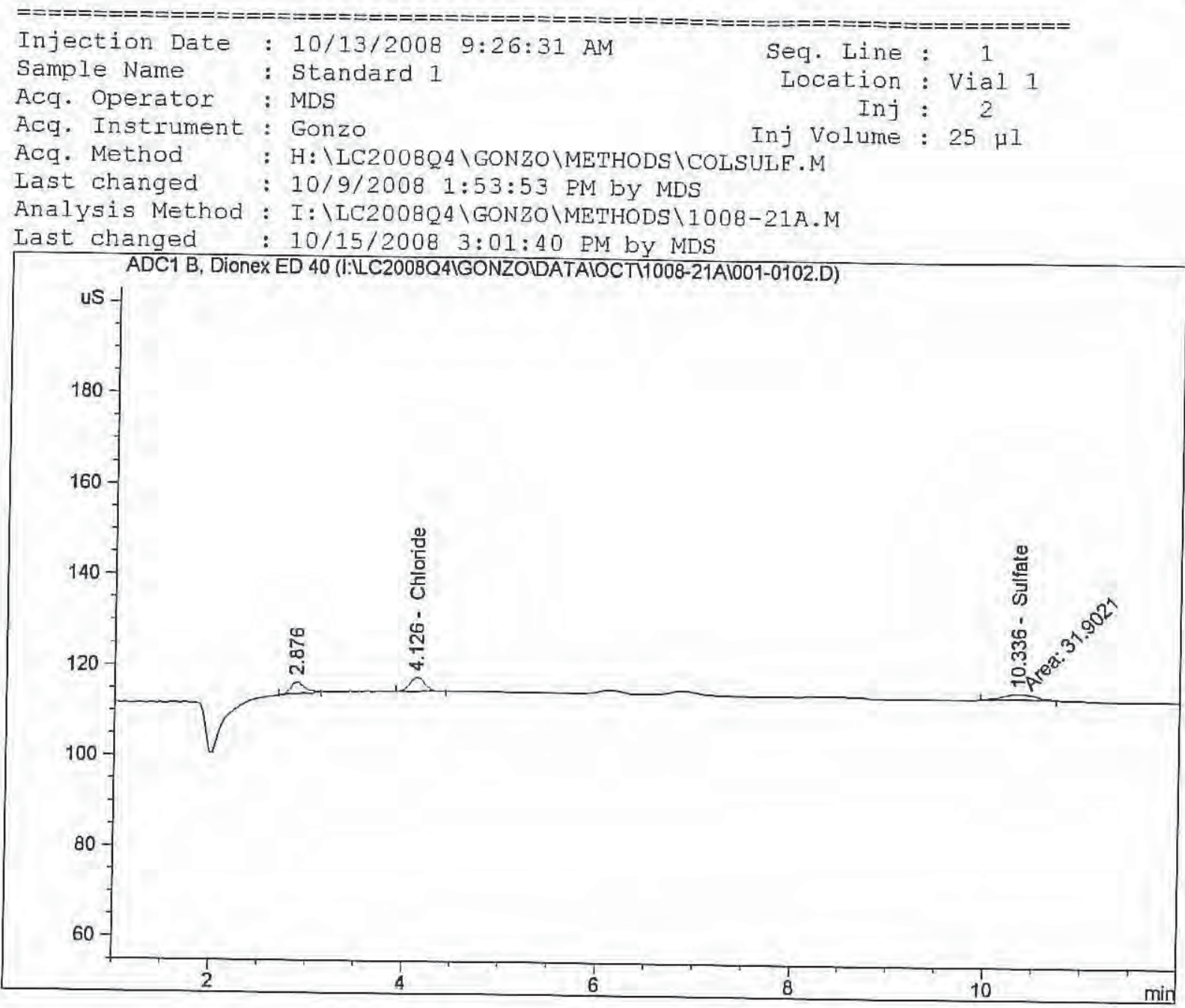

\section{External standard Report}

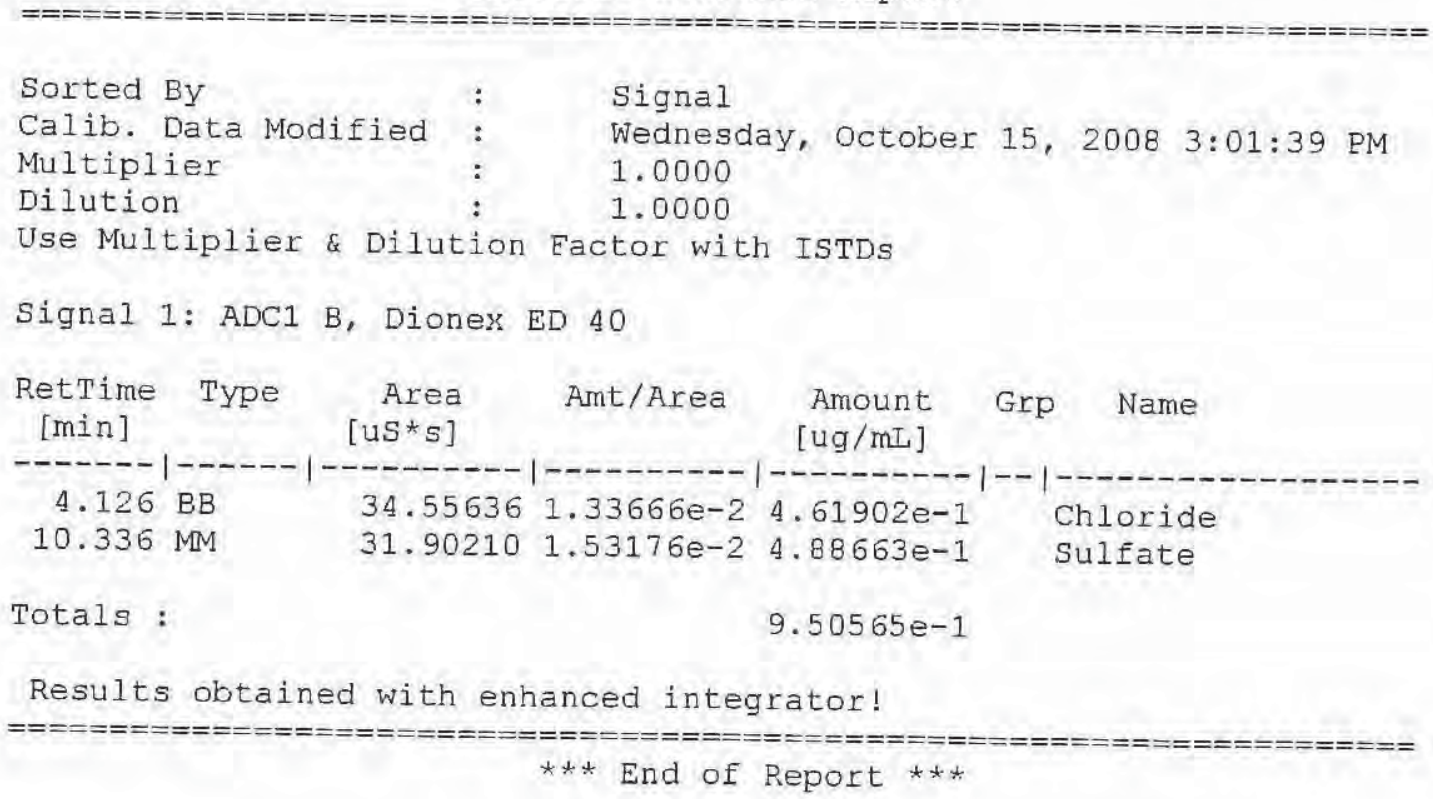




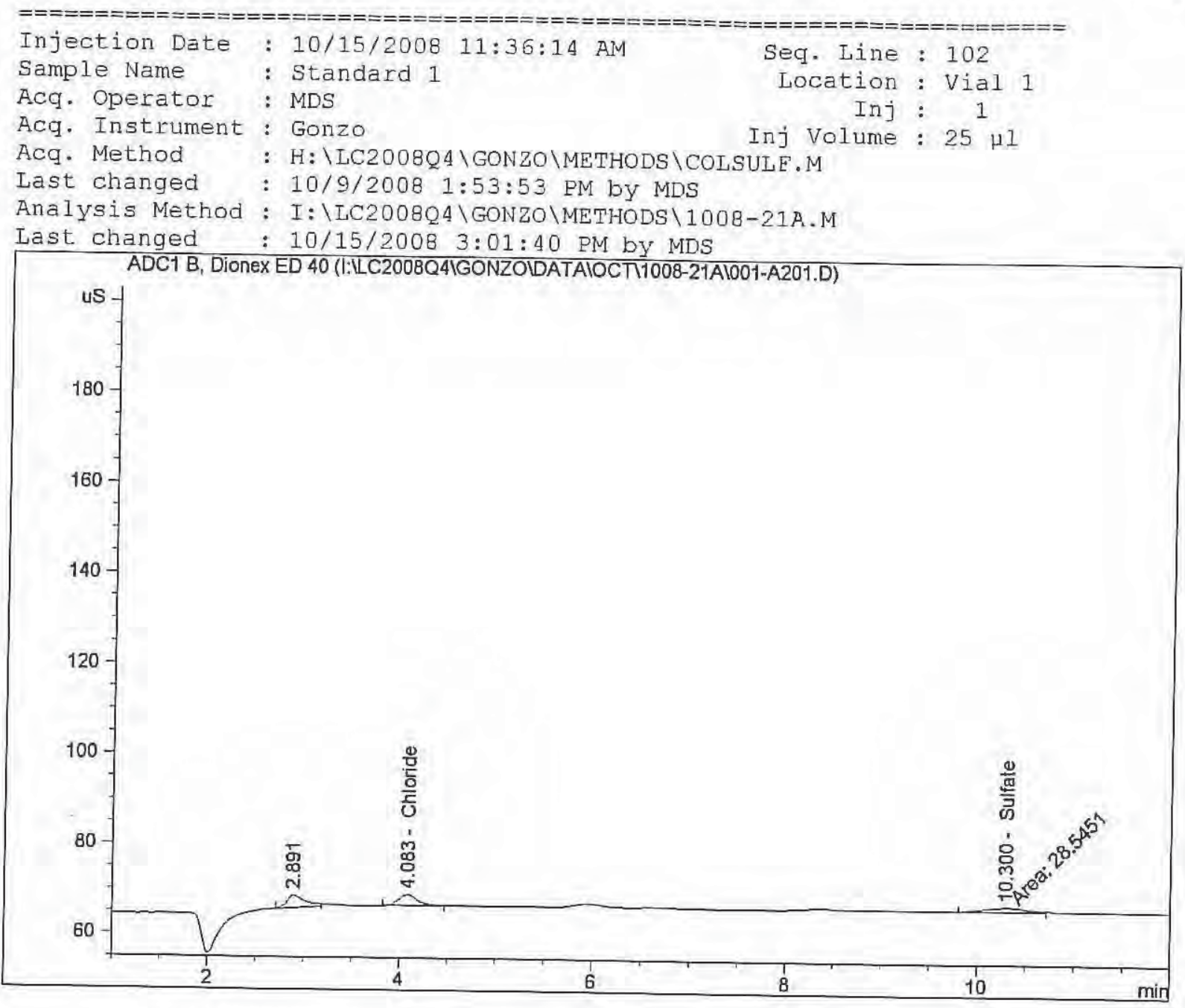

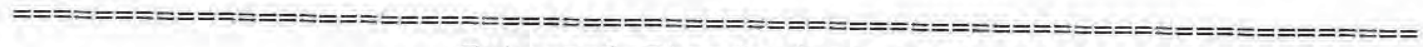

External standard Report

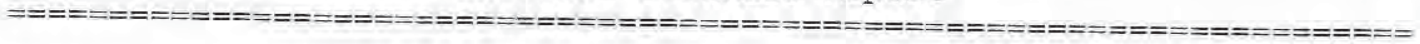

$\begin{array}{lll}\text { Sorted By } & : & \text { Signal } \\ \text { Calib. Data Modified } & : & \text { Wednesday, October 15, 2008 3:01:39 PM } \\ \text { Multiplier } & : & 1.0000 \\ \text { Dilution } & : & 1.0000\end{array}$

Use Multiplier \& Dilution Factor with ISTDS

Signal 1: ADC1 B, Dionex ED 40

\begin{tabular}{|c|c|c|c|c|c|c|}
\hline $\begin{array}{c}\text { RetTime } \\
\text { [min] }\end{array}$ & Type & $\begin{array}{r}\text { Area } \\
{\left[u S^{\star} s\right]}\end{array}$ & Ant/Area & $\begin{array}{l}\text { Amount } \\
{[\mathrm{ug} / \mathrm{mL}]}\end{array}$ & Grp & Name \\
\hline & & 33.96087 & & & & \\
\hline 10.300 & $\begin{array}{l}B B \\
M M\end{array}$ & $\begin{array}{l}33.96087 \\
28.54506\end{array}$ & $\begin{array}{l}1.33868 \mathrm{e}-2 \\
1.50789 \mathrm{e}-2\end{array}$ & $\begin{array}{l}4.54628 e-1 \\
4.30427 e-1\end{array}$ & & $\begin{array}{l}\text { loride } \\
\text { lfate }\end{array}$ \\
\hline
\end{tabular}

Totals : $\quad 8.85055 \mathrm{e}-1$

Results obtained with enhanced integrator!

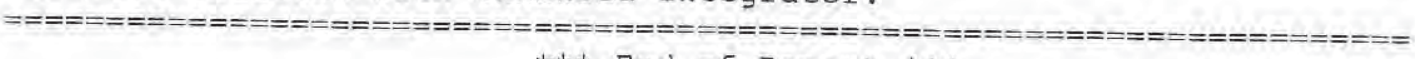

*** End of Report ***
} 

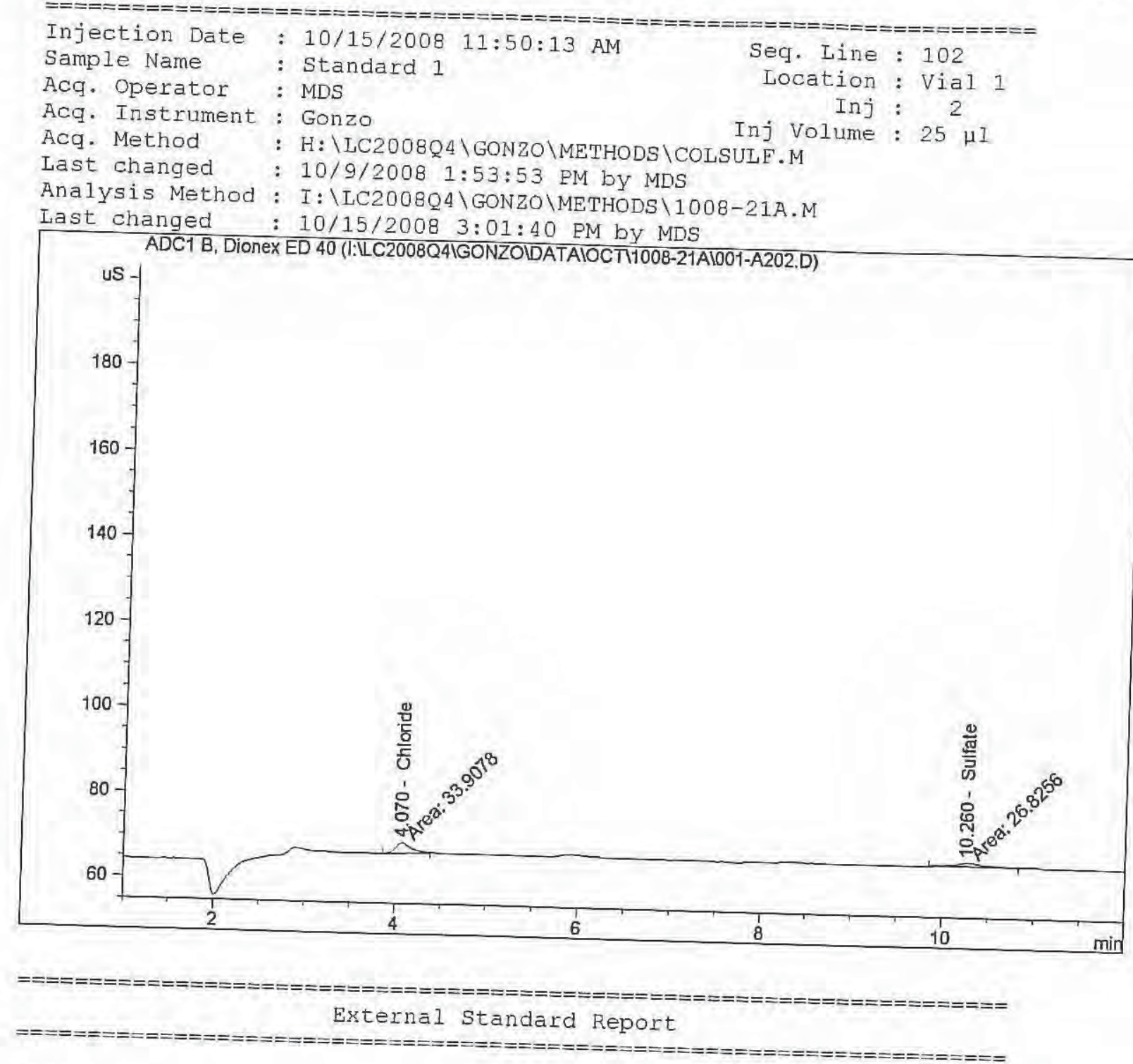

\section{Sorted By}

Modified:

Dilution

Signal

Wednesday, October 15, 2008 3:01:39 PM

Signal 1: ADC1 B, Dionex ED 40

\begin{tabular}{|c|c|c|c|c|c|}
\hline $\begin{array}{l}\text { RetTime } \\
\text { [min] }\end{array}$ & Type & $\begin{array}{r}\text { Area } \\
{[\mathrm{u} S * \mathrm{~s}]}\end{array}$ & Amt/Area & $\begin{array}{l}\text { Amount } \\
{[\mathrm{ug} / \mathrm{mL}]}\end{array}$ & Name \\
\hline $\begin{array}{r}4.070 \\
10.260\end{array}$ & $\begin{array}{l}\text { MM } \\
\text { MM }\end{array}$ & $\begin{array}{l}33.90780 \\
26.82557\end{array}$ & $\begin{array}{l}1.33886 \mathrm{e}-2 \\
1.50789 \mathrm{e}-2\end{array}$ & $\begin{array}{l}4.53980 e-1 \\
4.04499 e-1\end{array}$ & $\begin{array}{l}\text { Chloride } \\
\text { Sulfate }\end{array}$ \\
\hline
\end{tabular}

Totals :

$$
8.58479 e-1
$$

Results obtained with enhanced integrator!

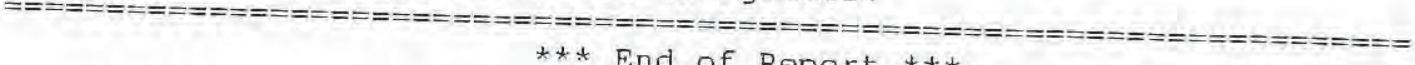

$* * *$ End of Report *** 

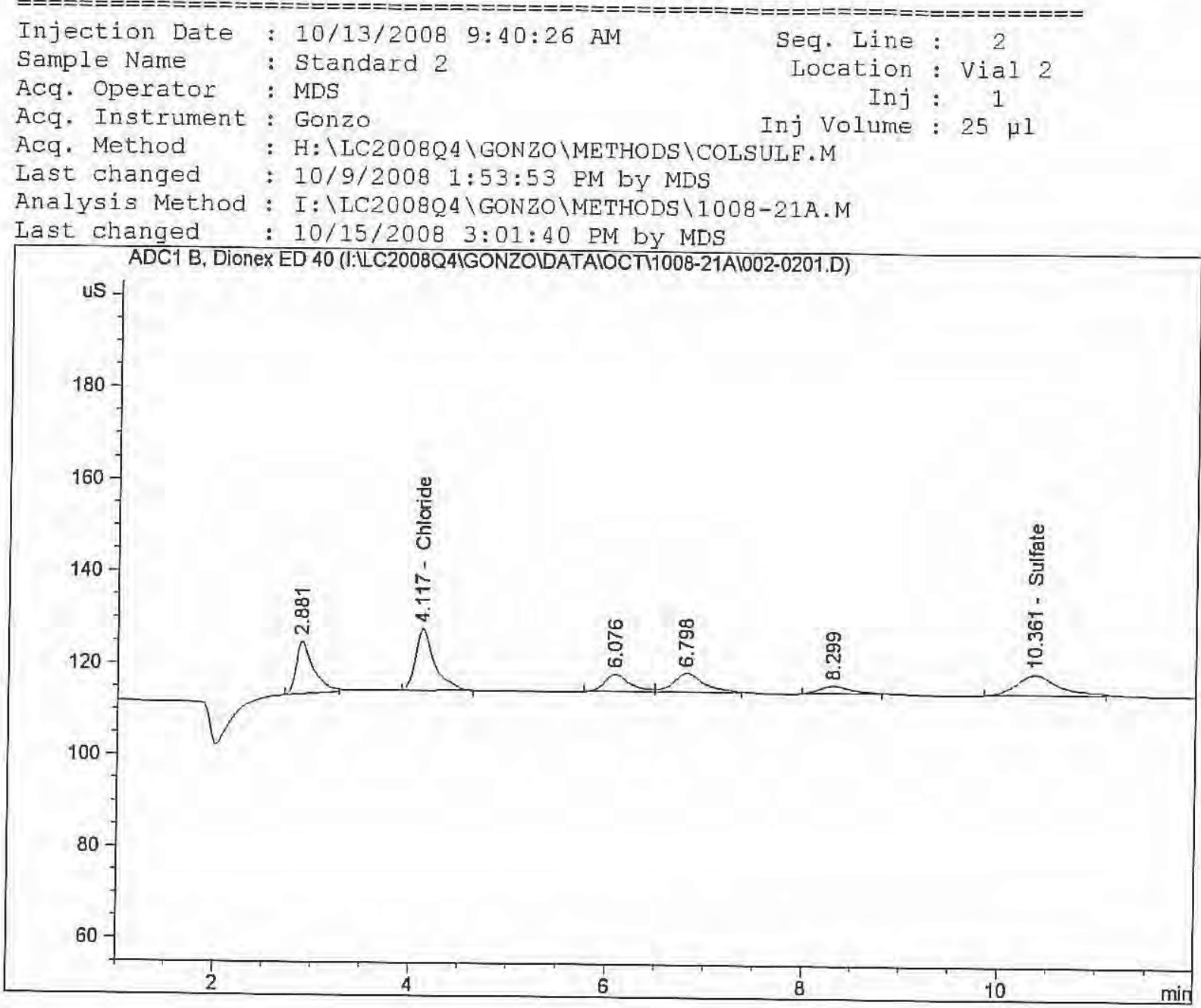

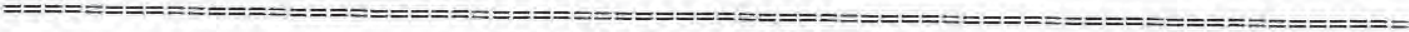

External Standard Report

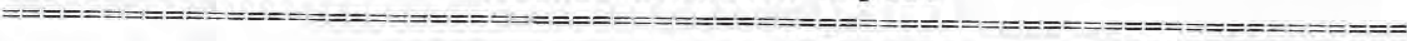

Sorted By : Signal

Calib. Data Modified : Wednesday, October 15, 2008 3:01:39 PM

Multiplier : 1.0000

Dilution : 1.0000

Use Multiplier \& Dilution Factor with ISTDs

Signal 1: ADC1 B, Dionex ED 40

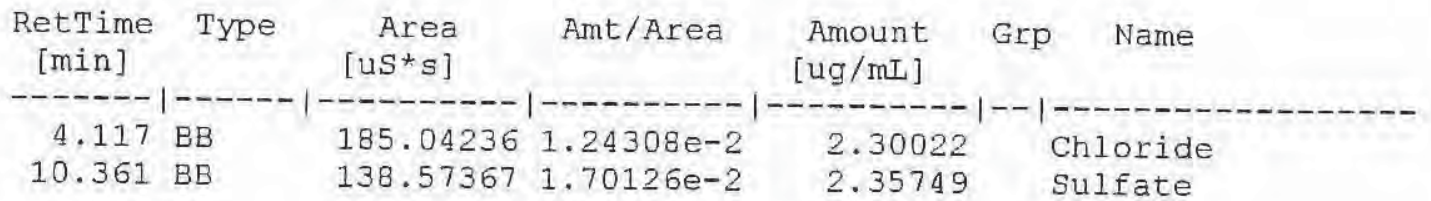

Totals :

4.65772

Results obtained with enhanced integrator!

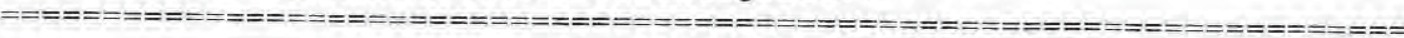
$\star \star \star$ End of Report $* * \star$ 


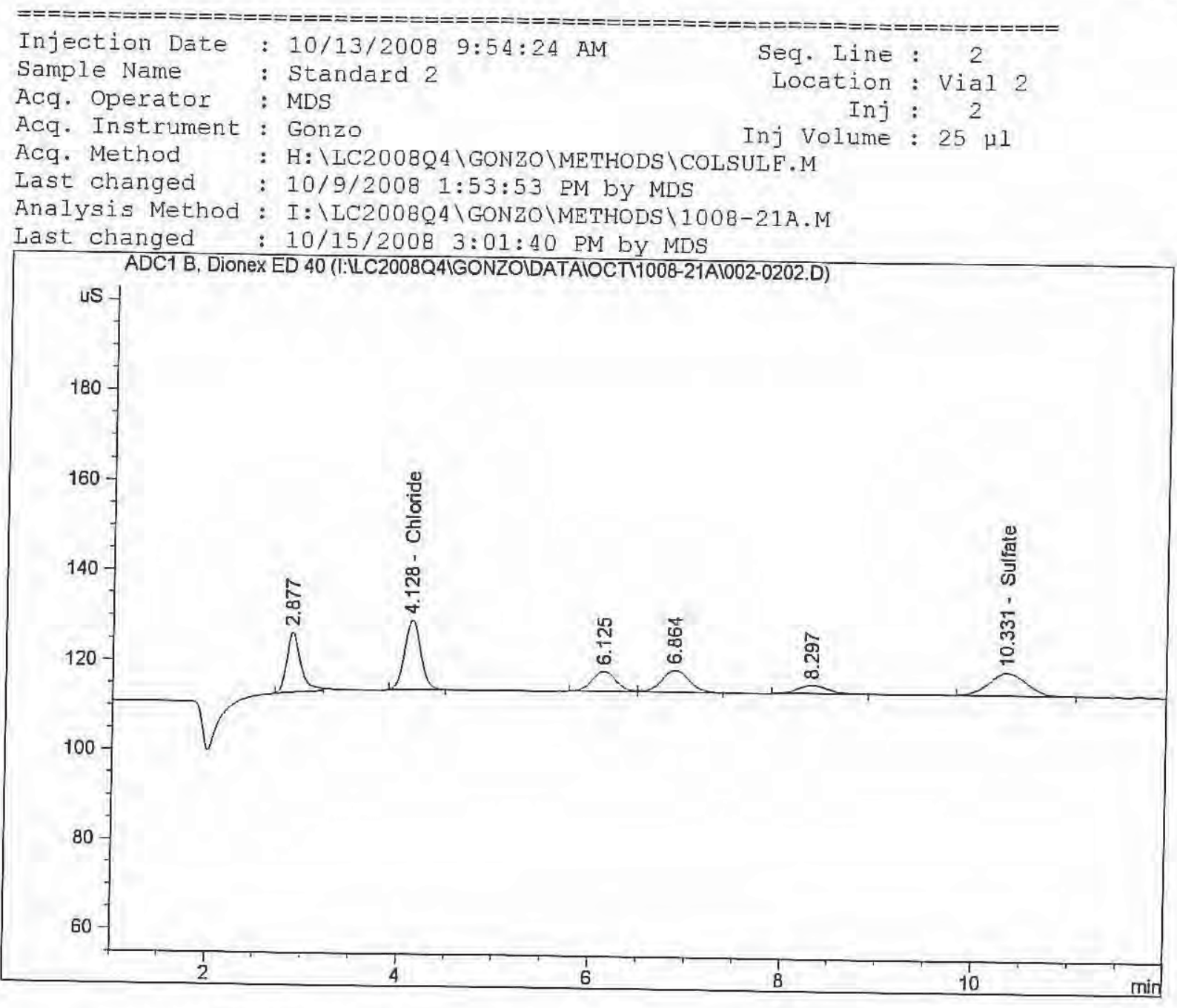

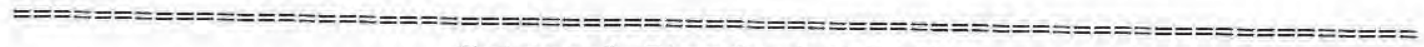

External standard Report

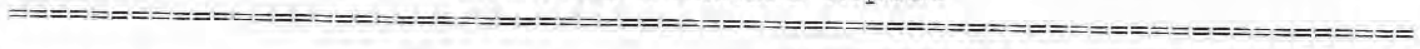

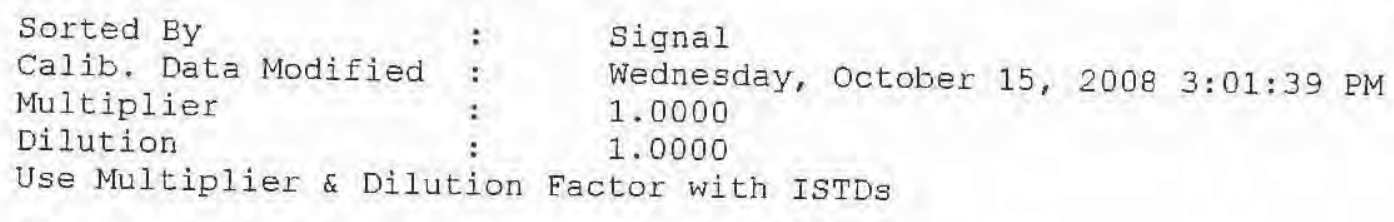

Signal 1: ADC1 B, Dionex ED 40

\begin{tabular}{ccccc}
$\begin{array}{c}\text { RetTime Type } \\
\text { [min] }\end{array}$ & $\begin{array}{c}\text { Area } \\
\text { [uS*s] }\end{array}$ & Amt/Area & $\begin{array}{c}\text { Amount Grp Name } \\
{[\mathrm{ug} / \mathrm{mL}]}\end{array}$ \\
\hdashline $.128 \mathrm{BB}$ & 184.75447 & $1.24311 \mathrm{e}-2$ & 2.29671 & Chloride \\
$10.331 \mathrm{BB}$ & 142.20760 & $1.70255 \mathrm{e}-2$ & 2.42116 & Sulfate
\end{tabular}

Totals :

4.71786

Results obtained with enhanced integrator!

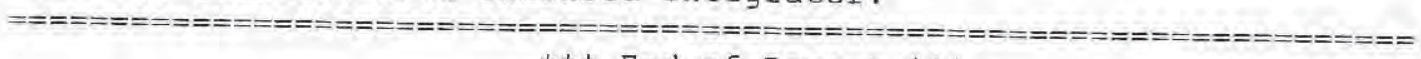

$* * \star$ End of Report $* * *$
} 


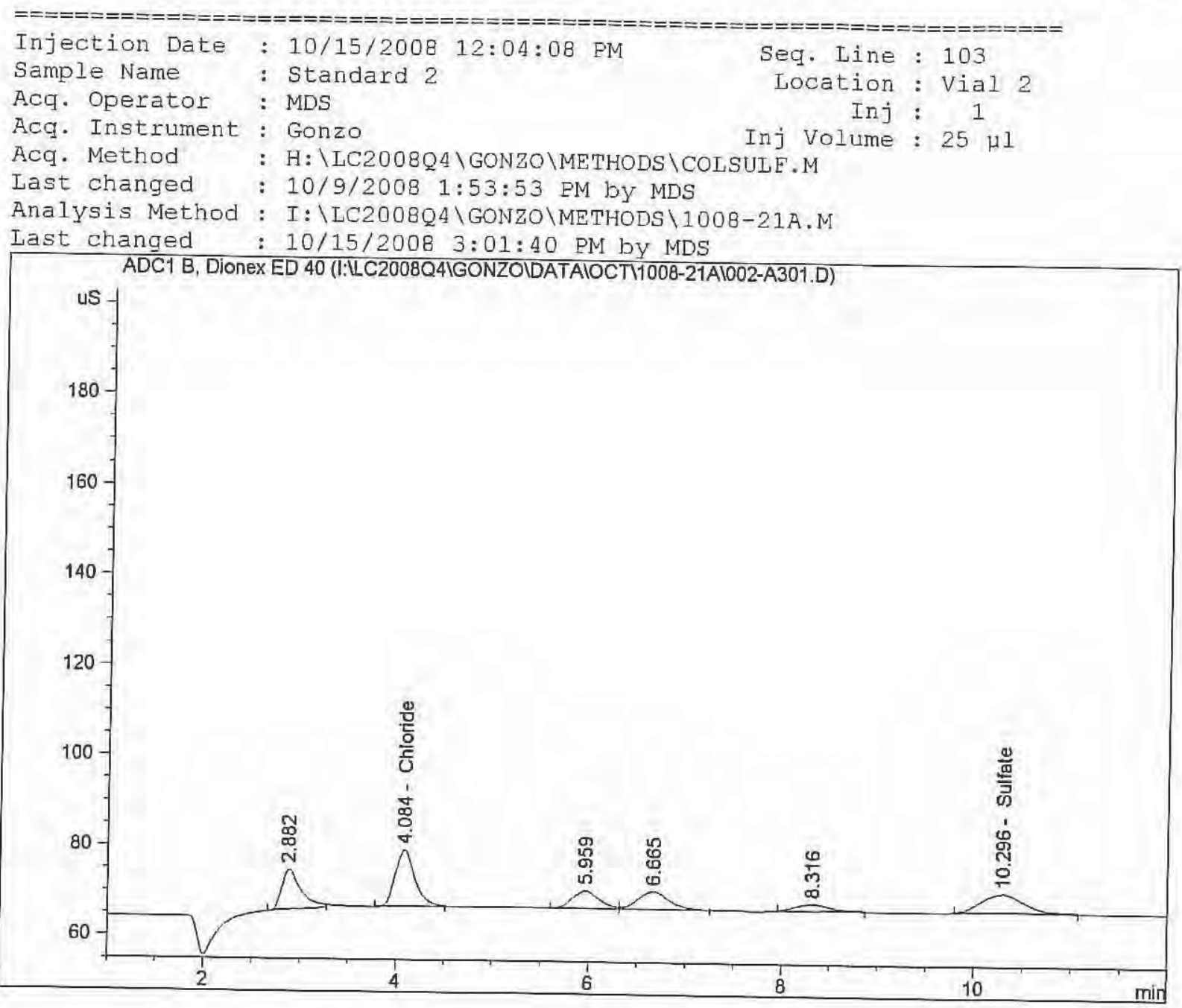

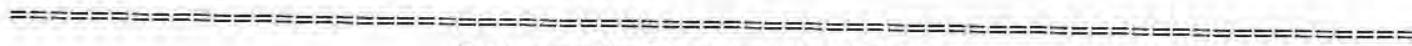

External standard Report

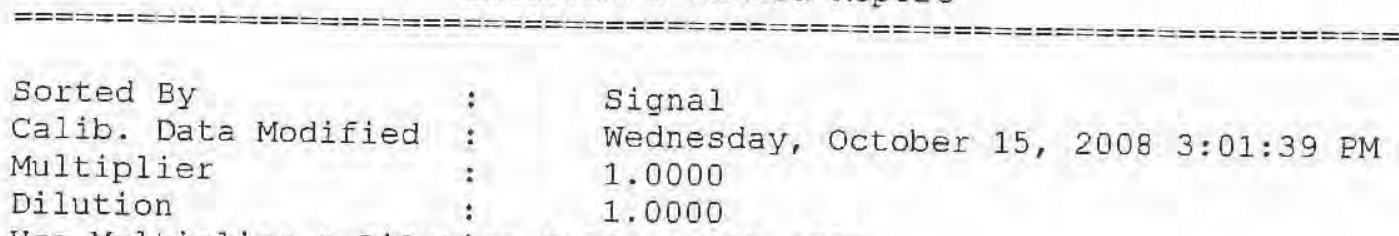

Use Multiplier \& Dilution Factor with ISTDs

Signal 1: ADC1 B, Dionex ED 40

\begin{tabular}{|c|c|c|c|c|c|c|}
\hline $\begin{array}{l}\text { RetTime } \\
\text { [min] }\end{array}$ & Type & $\begin{array}{r}\text { Area } \\
\text { [us*s] }\end{array}$ & Ant/Area & $\begin{array}{l}\text { Amount } \\
\text { [ug/mL] }\end{array}$ & Grp & Name \\
\hline 4.084 & $\mathrm{~PB}$ & 181.06718 & 1. $24355 e-2$ & 2.25166 & & hlo \\
\hline 10.296 & BB & 133.96823 & 1. $69951 \mathrm{e}-2$ & 2.27681 & & llfate \\
\hline
\end{tabular}

Totals :$$
\text { 4. } 52847
$$

Results obtained with enhanced integrator! 


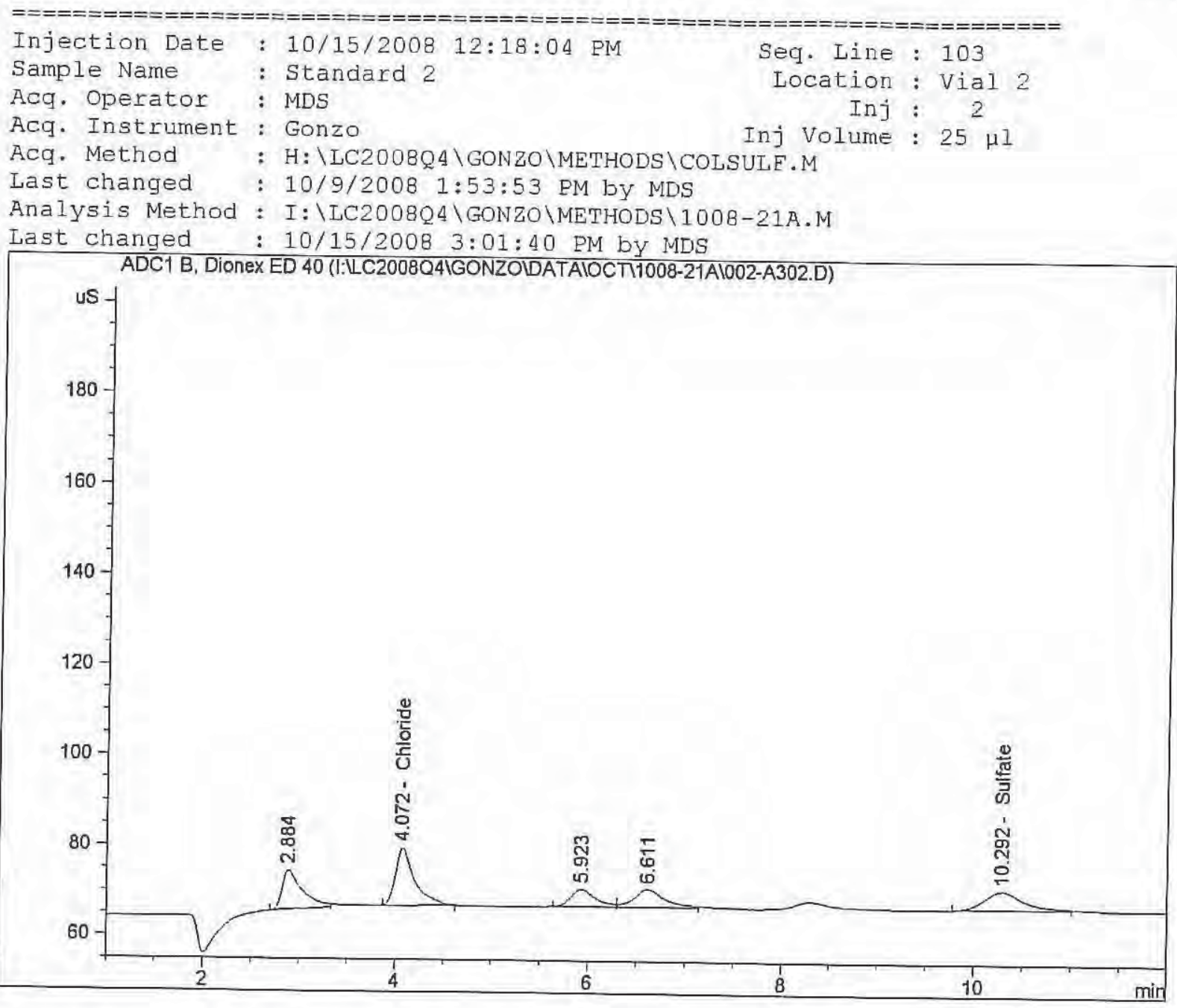

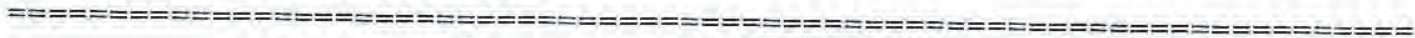

External standard Report

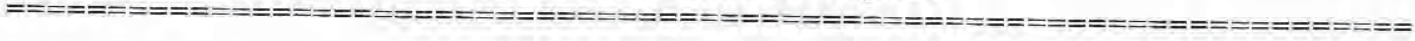

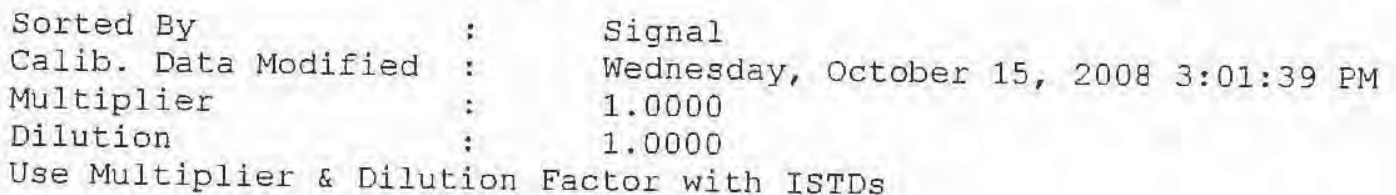

Signal 1: ADC1 B, Dionex ED 40

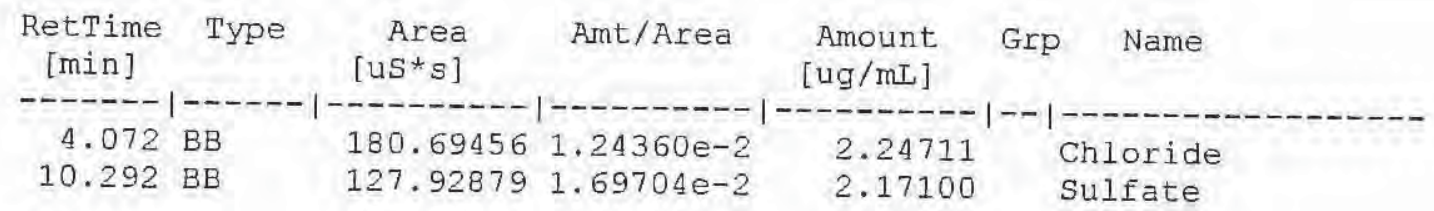

Totals:

4. 41811

Results obtained with enhanced integrator!

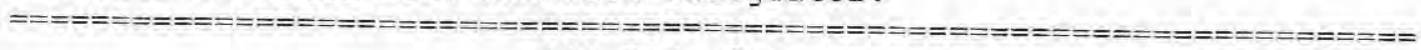

$\star \star *$ End of Report $\star * \star$ 


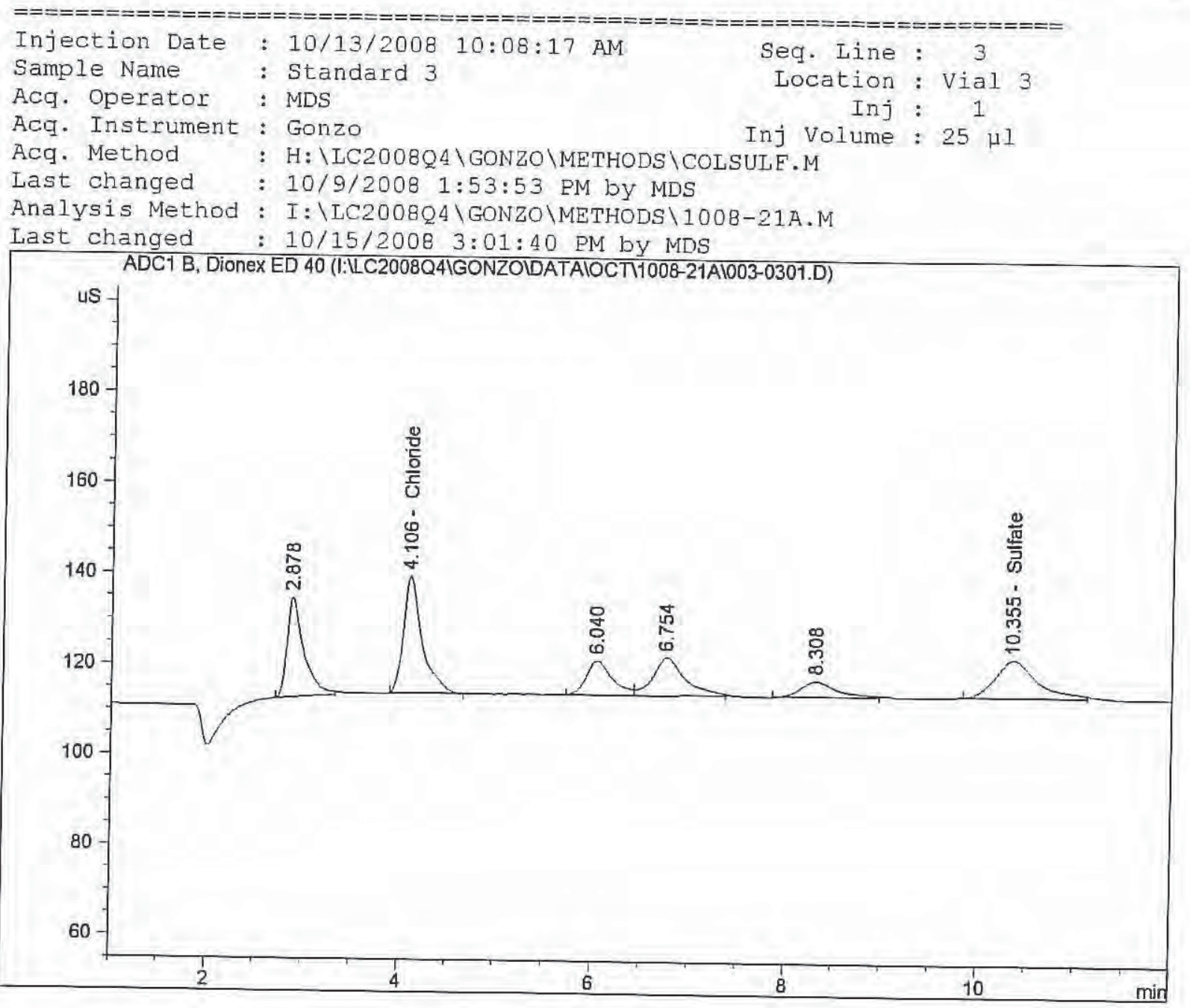

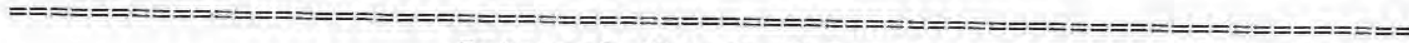
External Standard Report

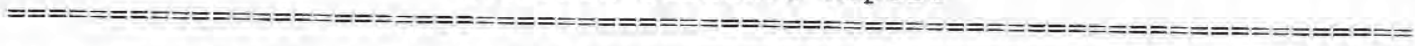

$\begin{array}{lll}\text { Sorted By } & : & \text { Signal } \\ \text { Calib. Data Modified } & : & \text { Wednesday, October 15, 2008 3:01:39 PM } \\ \text { Multiplier } & : & 1.0000 \\ \text { Dilution } & \vdots & 1.0000 \\ \text { Use Multiplier \& Dilution Factor with ISTDs }\end{array}$

Use Multiplier \& Dilution Factor with ISTDS

Signal 1: ADC1 B, Dionex ED 40

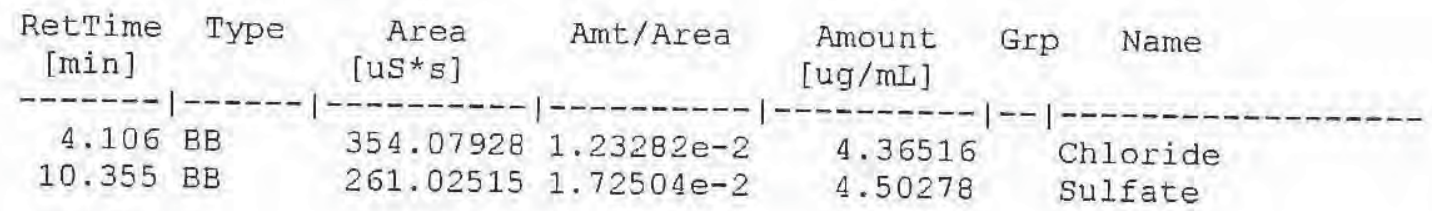

Totals :

8.86794

Results obtained with enhanced integrator!

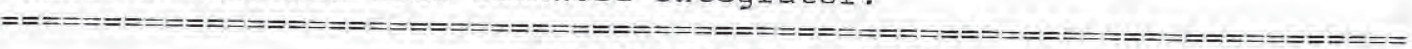

$\star \star \star$ End of Report $\star \star \star *$
} 


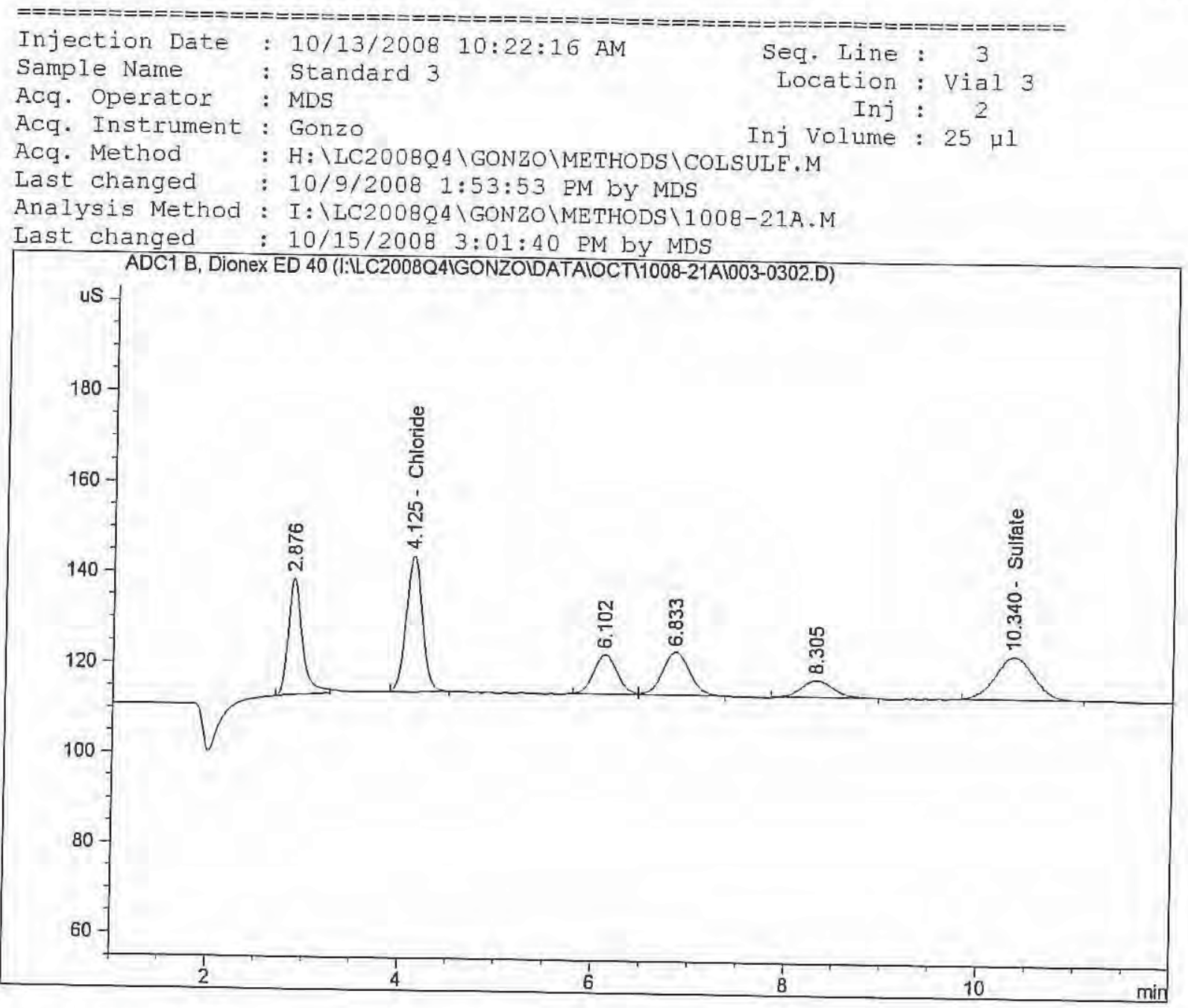

External Standard Report

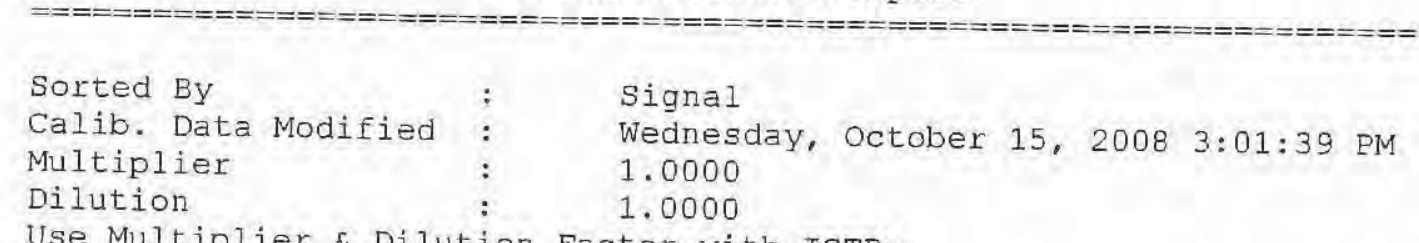

Use Multiplier \& Dilution Factor with ISTDs

Signal 1: ADC1 B, Dionex ED 40

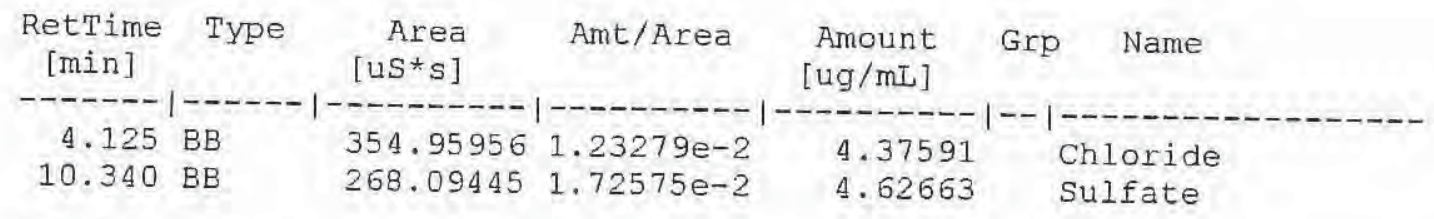
Totals :
9.00254

Results obtained with enhanced integrator!

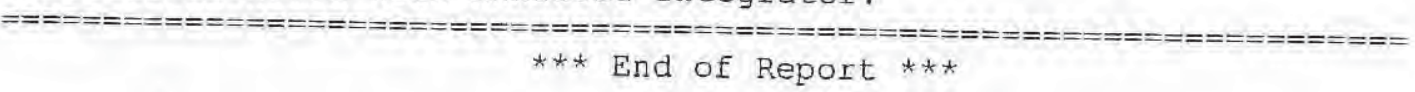




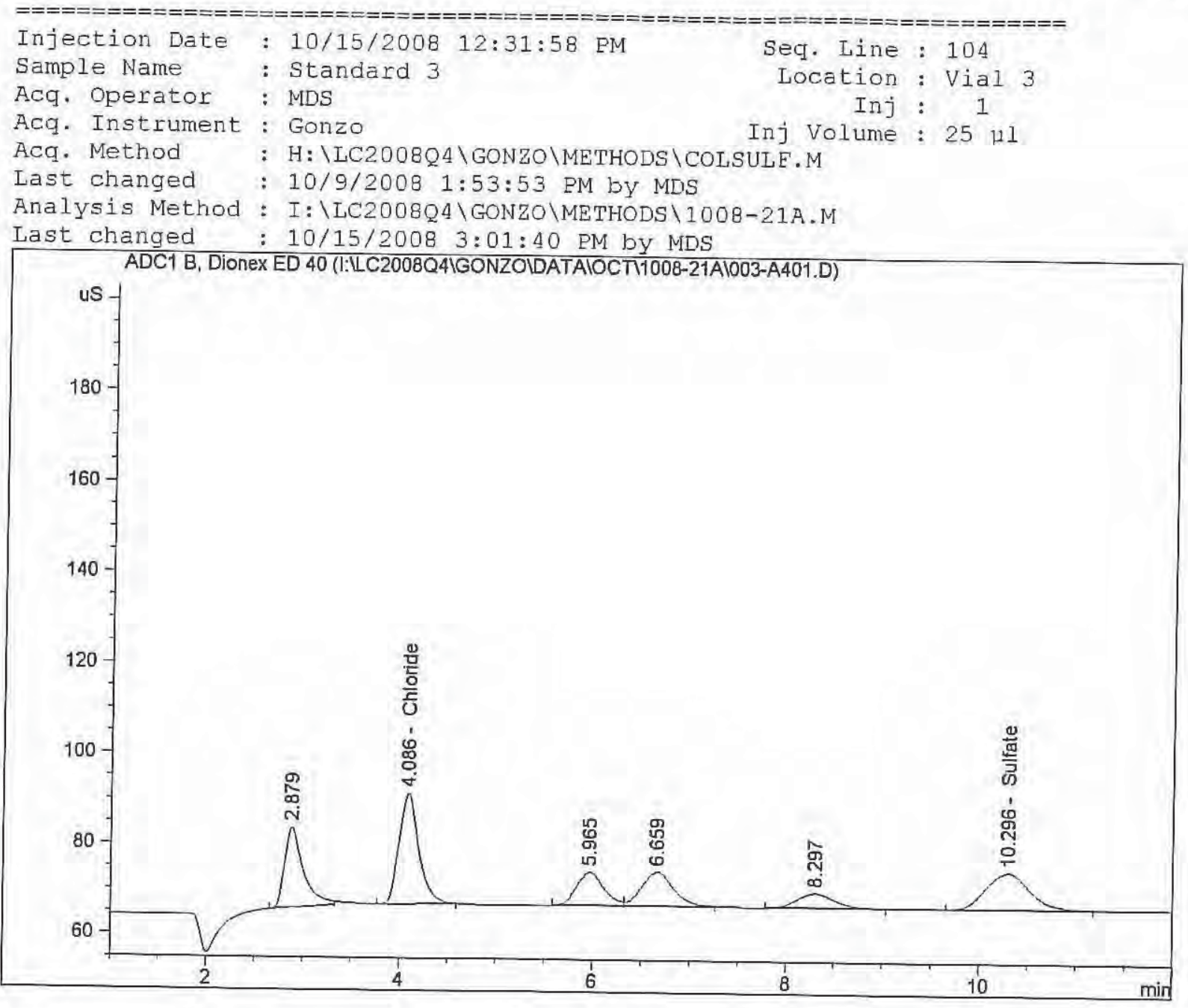

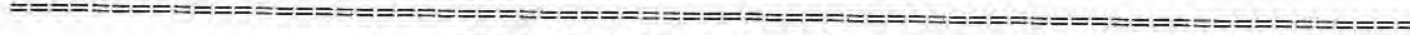
External Standard Report

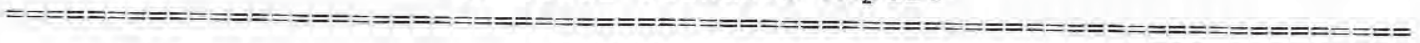

$\begin{array}{lll}\text { Sorted By } & : & \text { Signal } \\ \text { Calib. Data Modified : } & \text { Wednesday, October 15, 2008 3:01:39 PM } \\ \text { Multiplier } & : & 1.0000 \\ \text { Dilution } & \text { Use Multiplier \& Dilution Eactor with ISTDs }\end{array}$

Use Multiplier \& Dilution Eactor with ISTDS

Signal 1: ADC1 B, Dionex ED 40

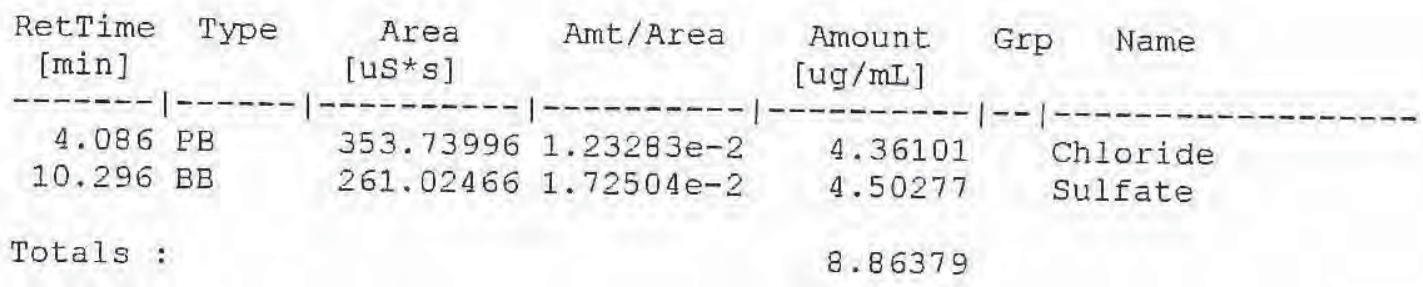

Results obtained with enhanced integrator!

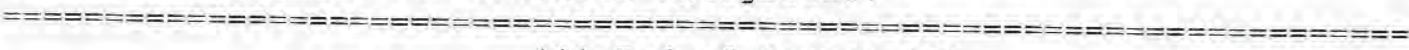

*** End of Report $* * *$
} 


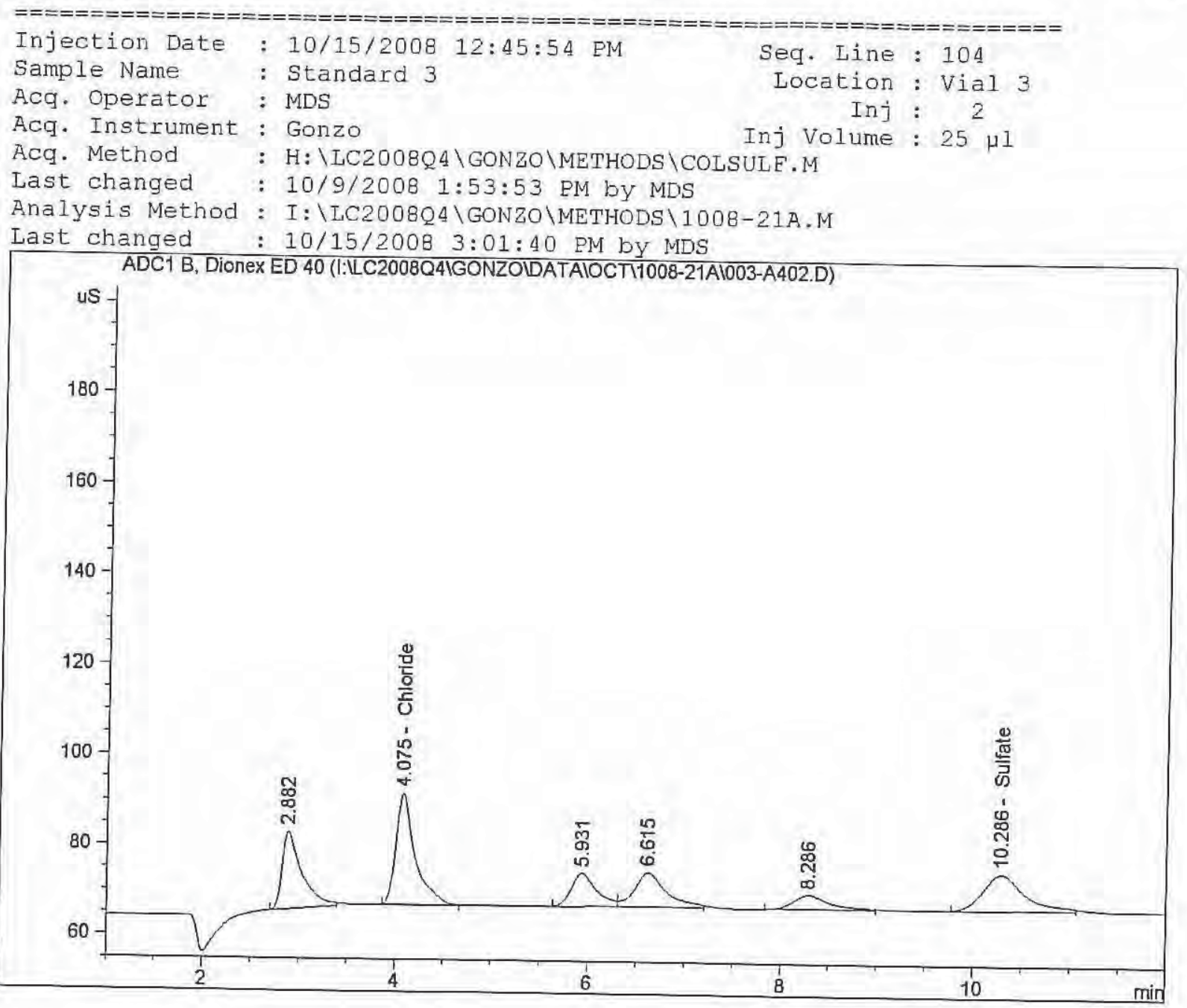

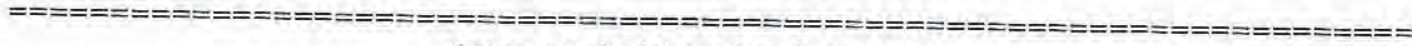
External Standard Report

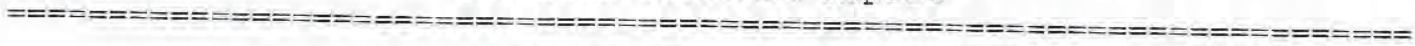

Sorted BY

Calib. Data Modified

Multiplier

Dilution

Signal

Wednesday, October 15, 2008 3:01:39 PM

1.0000

1.0000

Use Multiplier \& Dilution Factor with ISTDS

Signal 1: ADC1 B, Dionex ED 40

\begin{tabular}{|c|c|c|c|c|c|c|}
\hline $\begin{array}{l}\text { RetTime } \\
\text { [min] }\end{array}$ & Type & $\begin{array}{r}\text { Area } \\
{\left[u S^{*} s\right]}\end{array}$ & Amt/Area & $\begin{array}{l}\text { Amount } \\
\text { [ug/mU] }\end{array}$ & Grp & Name \\
\hline 4.075 & $B B$ & 350.23236 & 1. $23294 \mathrm{e}-2$ & 4.31816 & & \\
\hline 10.286 & $\mathrm{BB}$ & 249.71764 & 1. $723 \mathrm{~B} 2 \mathrm{e}-2$ & 4.30468 & & Ifate \\
\hline
\end{tabular}

Totals :

8.62284

Results obtained with enhanced integrator!

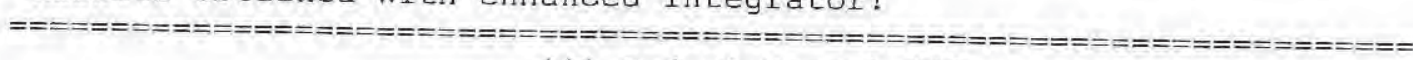

*** End of Report $* * *$
} 

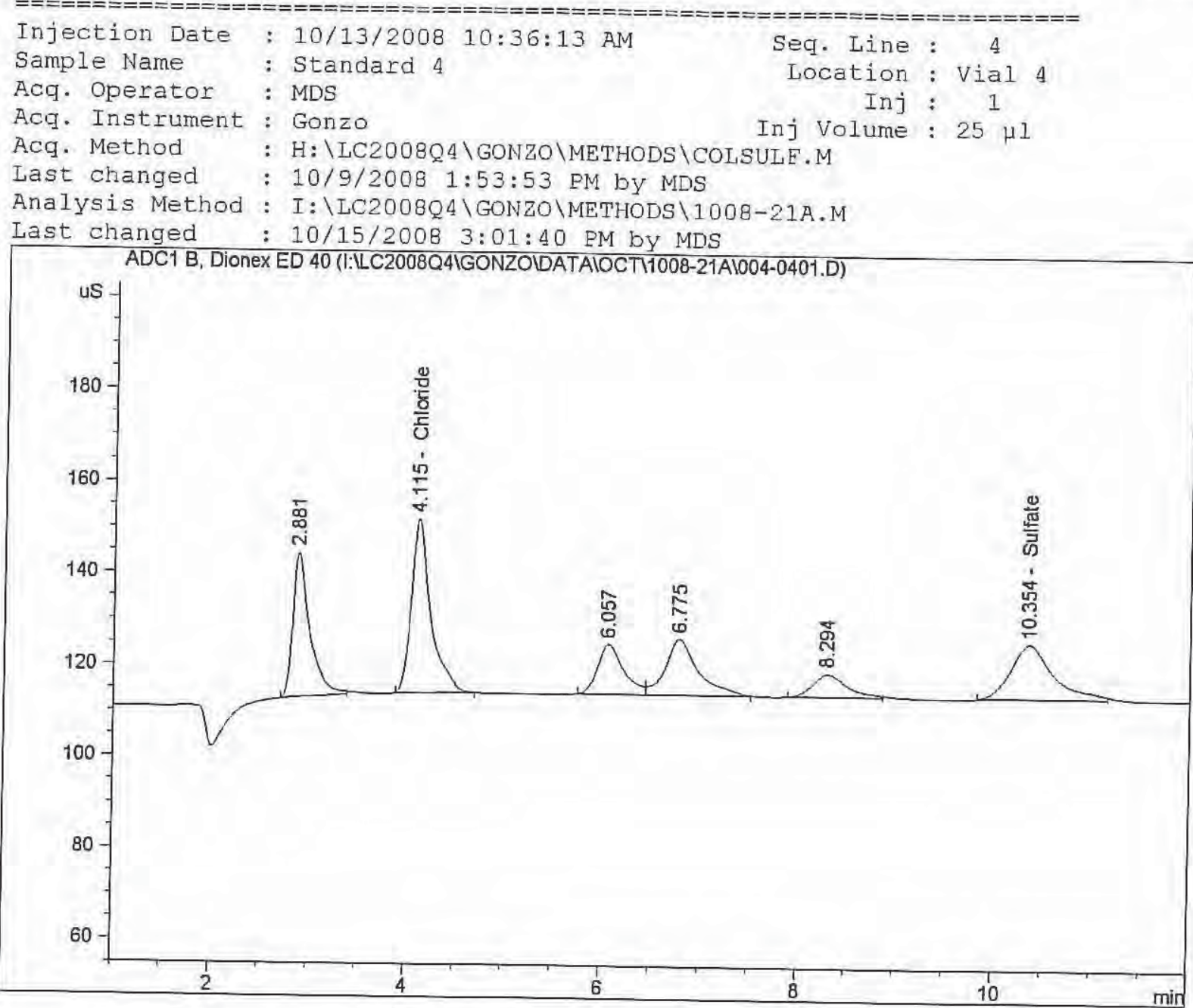

External Standard Report

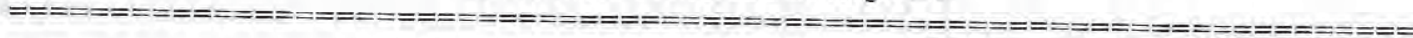

Sorted By

Signal

Calib. Data Modified : Wednesday, October 15, 2008 3:01:39 PM

Multiplier : 1.0000

Dilution : 1.0000

Use Multiplier \& Dilution Eactor with ISTDS

Signal 1: ADC1 B, Dionex ED 40

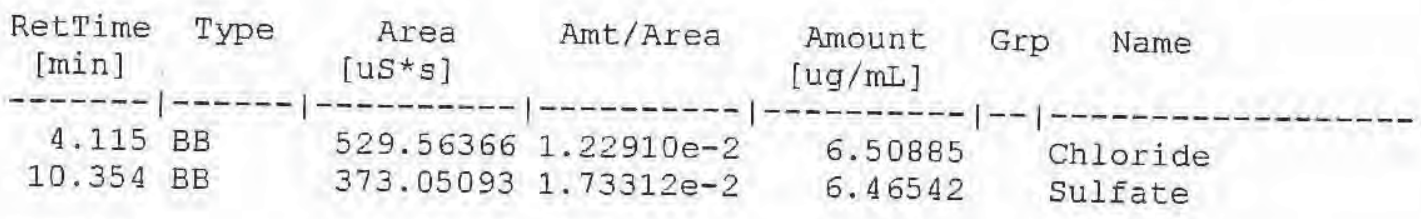

Totals :

12. 97427

Results obtained with enhanced integrator!

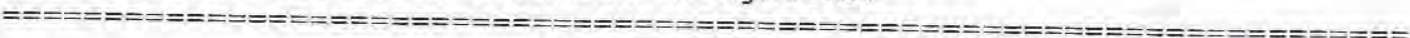

$* \star *$ End of Report *** 


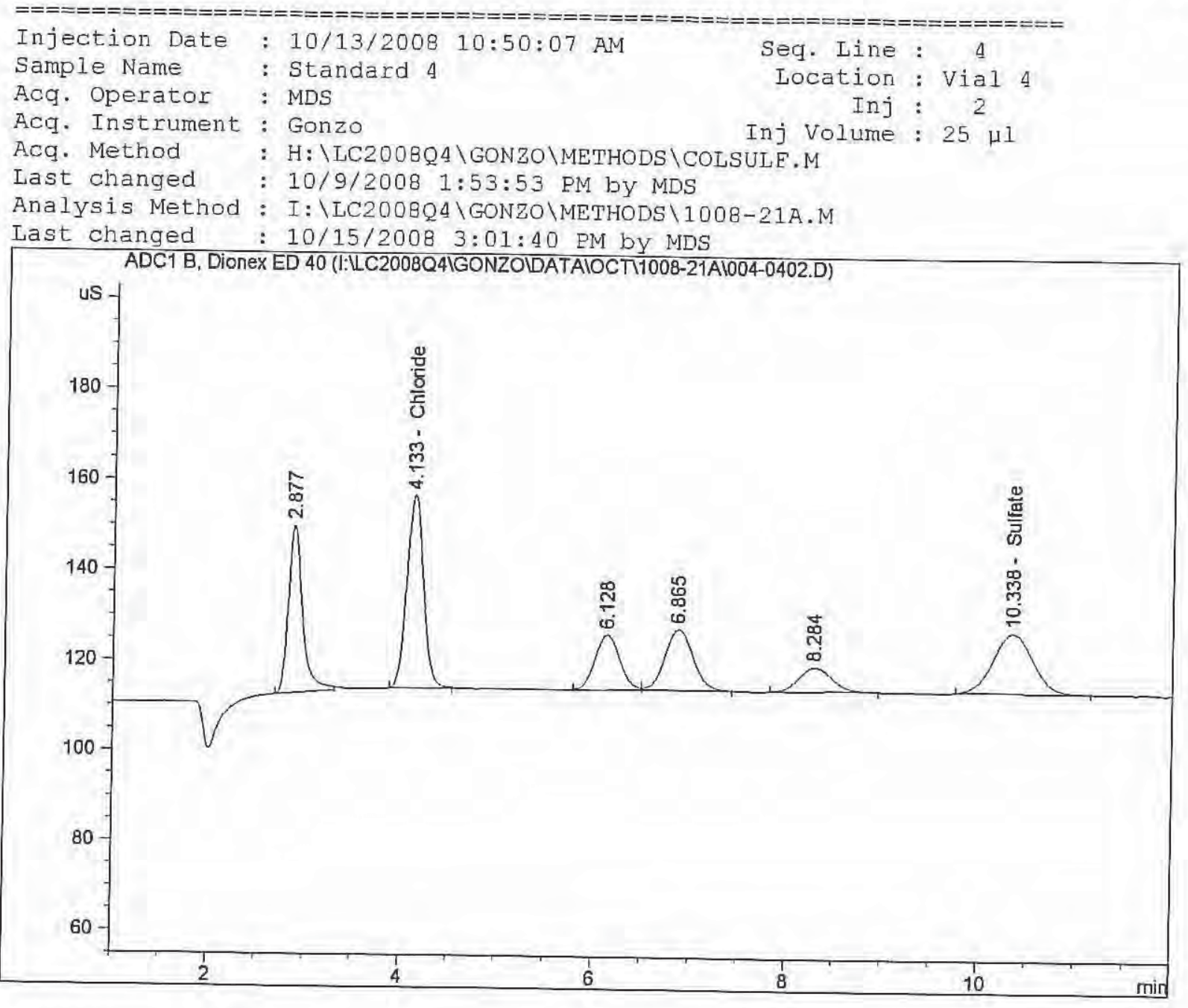

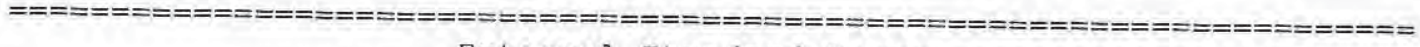

External Standard Report

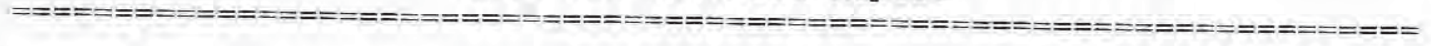

$\begin{array}{lcl}\text { Sorted By } & : & \text { Signal } \\ \text { Calib. Data Modified : } & \text { Wednesday, October 15, 2008 3:01:39 PM } \\ \text { Multiplier } & : & 1.0000 \\ \text { Dilution } & \text { : } & 1.0000 \\ \text { Use Multiplier \& Dilution Factor with ISTDs }\end{array}$

Use Multiplier \& Dilution Factor with ISTDS

Signal 1: ADC1 B, Dionex ED 40

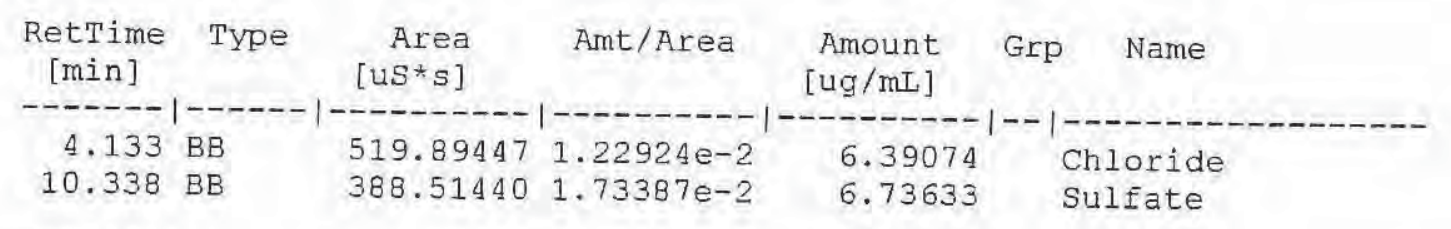

Totals : $\quad 13.12707$

Results obtained with enhanced integrator!

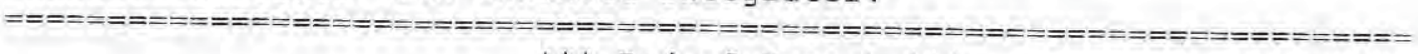

$\star * *$ End of Report $* * *$
} 


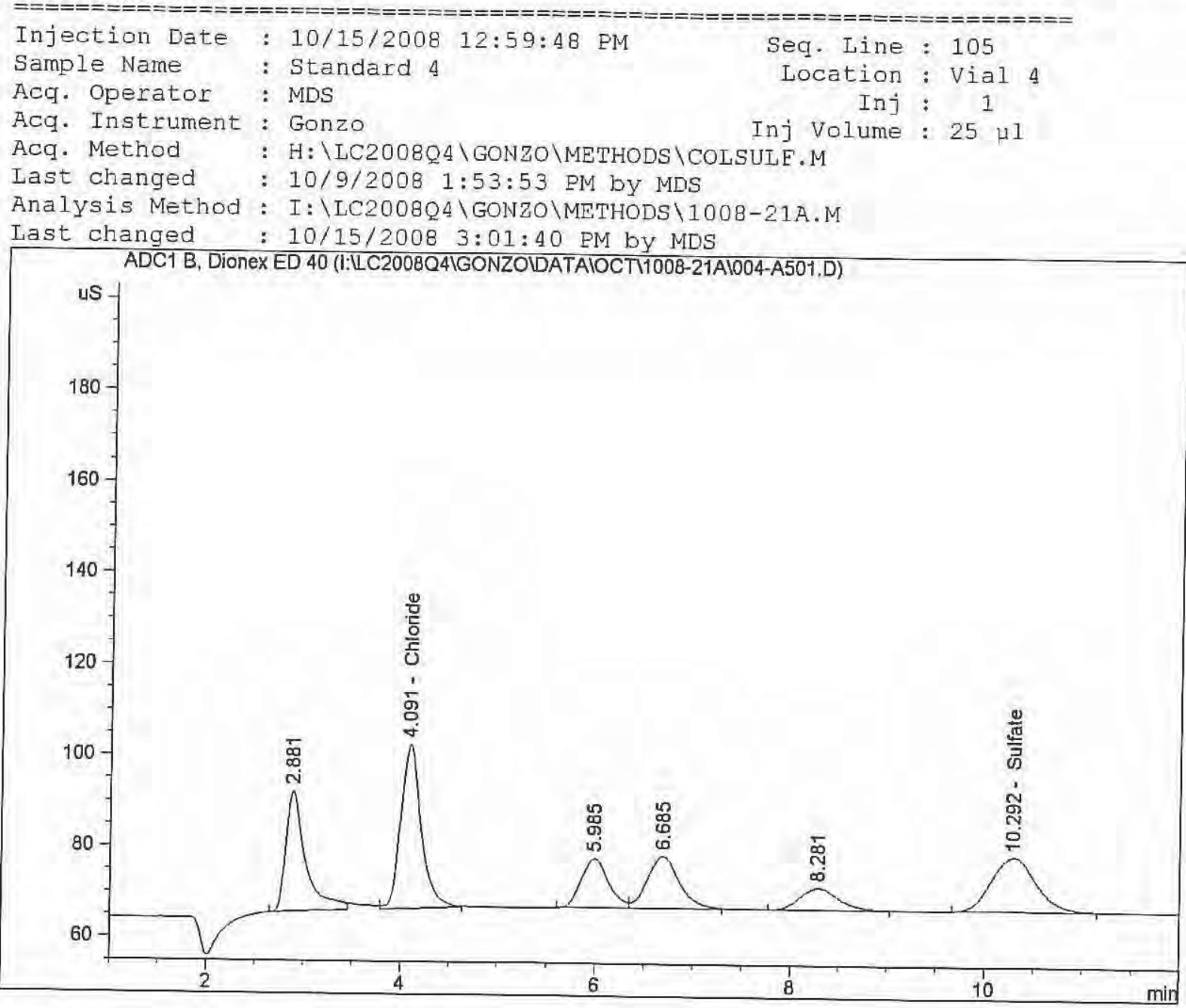

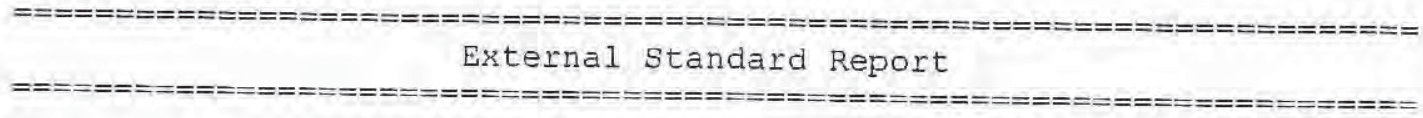

Sorted By

Multiplier : $\quad 1.0000$

Dilution : 1.0000

Use Multiplier \& Dilution Eactor with ISTDS

Signal 1: ADC1 B, Dionex ED 40

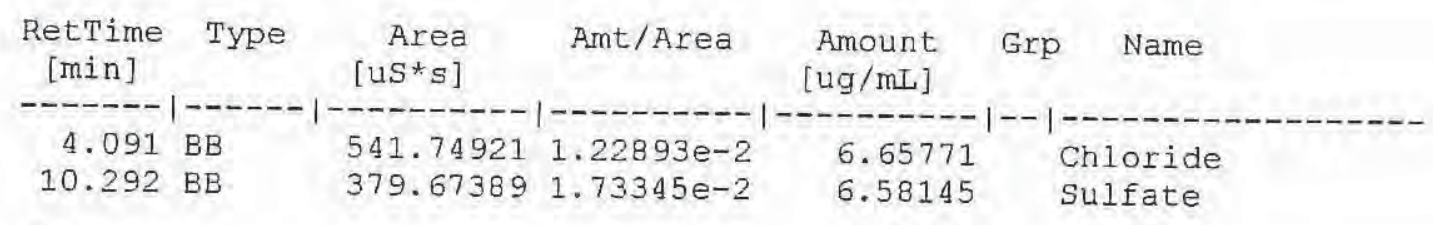

Totals :

13.23916

Results obtained with enhanced integrator! 


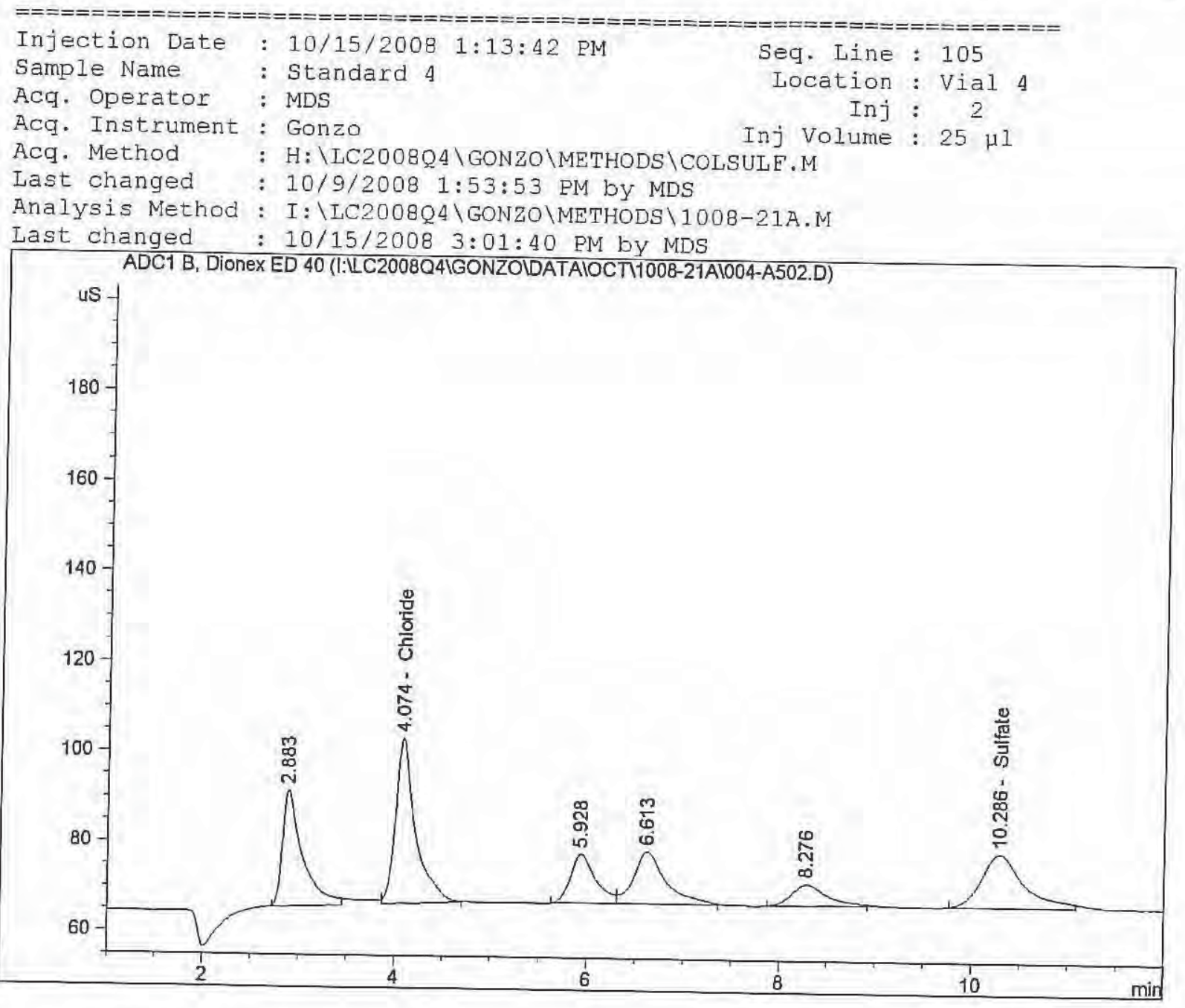

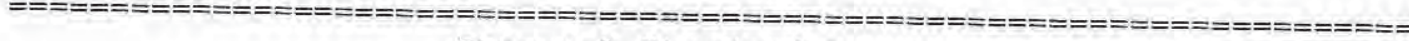
External standard Report

Sorted By Calib. Data Modified Multiplier

Dilution

SignaI

Wednesday, October 15, 2008 3:01:39 PM

1.0000

1.0000

Use Multiplier \& Dilution Eactor with ISTDS

Signal 1: ADC1 B, Dionex ED 40

\begin{tabular}{|c|c|c|c|c|c|}
\hline $\begin{array}{c}\text { RetTime } \\
\text { [min] }\end{array}$ & Type & $\begin{array}{r}\text { Area } \\
\text { [us*s] }\end{array}$ & Amt/Area & $\begin{array}{l}\text { Amount } \\
{[\mathrm{ug} / \mathrm{mL}]}\end{array}$ & Name \\
\hline 4.074 & $B B$ & 541.60791 & $1.228930-2$ & & 1 \\
\hline 10.286 & BB & 365.29208 & $\begin{array}{l}1.22893 \mathrm{e}-2 \\
1.73272 \mathrm{e}-2\end{array}$ & $\begin{array}{l}6.65599 \\
6.32949\end{array}$ & $\begin{array}{l}\text { Chloride } \\
\text { Sulfate }\end{array}$ \\
\hline
\end{tabular}

Totals :

12.98547

Results obtained with enhanced integrator!

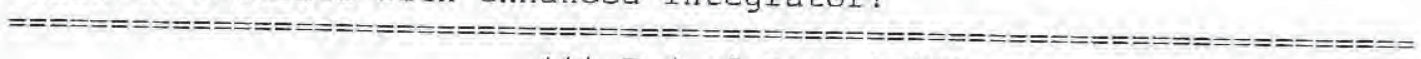

$* * *$ End of Report ***
} 


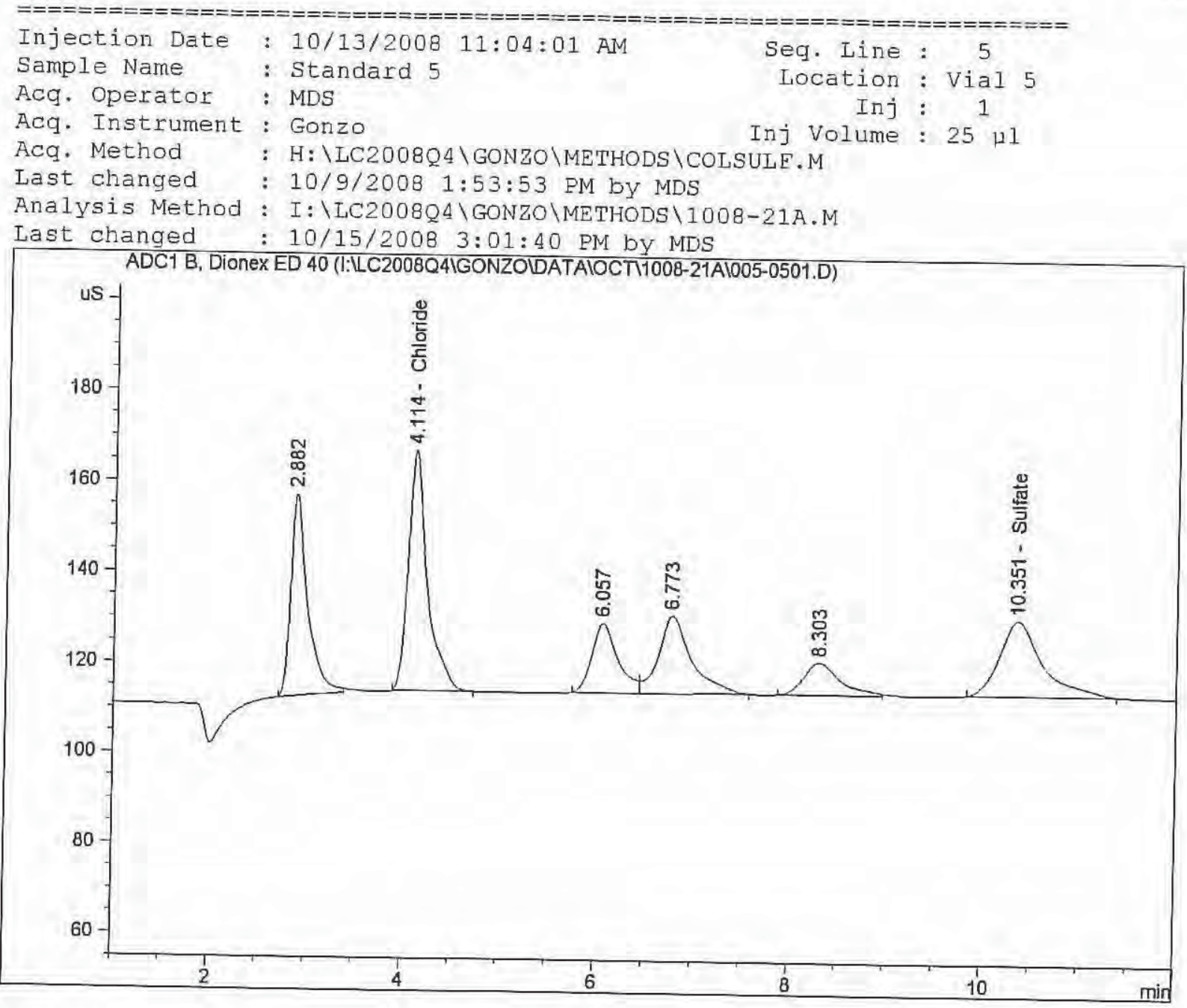

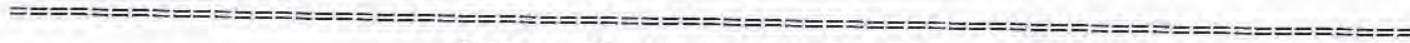

External Standard Report

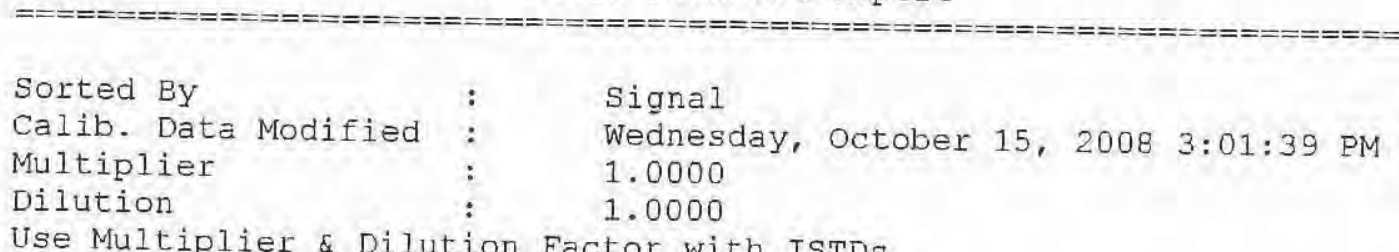

Use Multiplier \& Dilution Factor with ISTDS

Signal 1: ADC1 B, Dionex ED 40

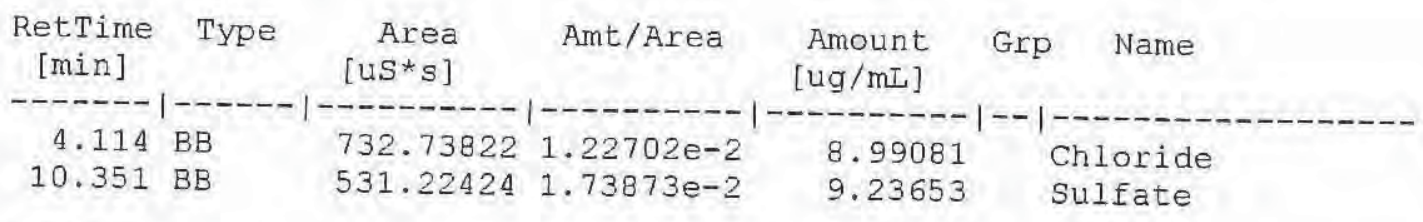

Totals :

18.22734

Results obtained with enhanced integrator!

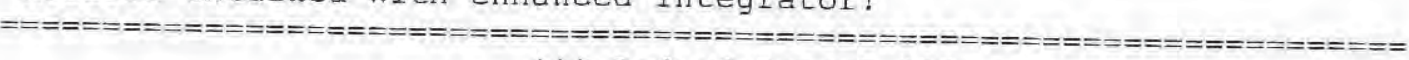
$* * *$ End of Report ***
} 


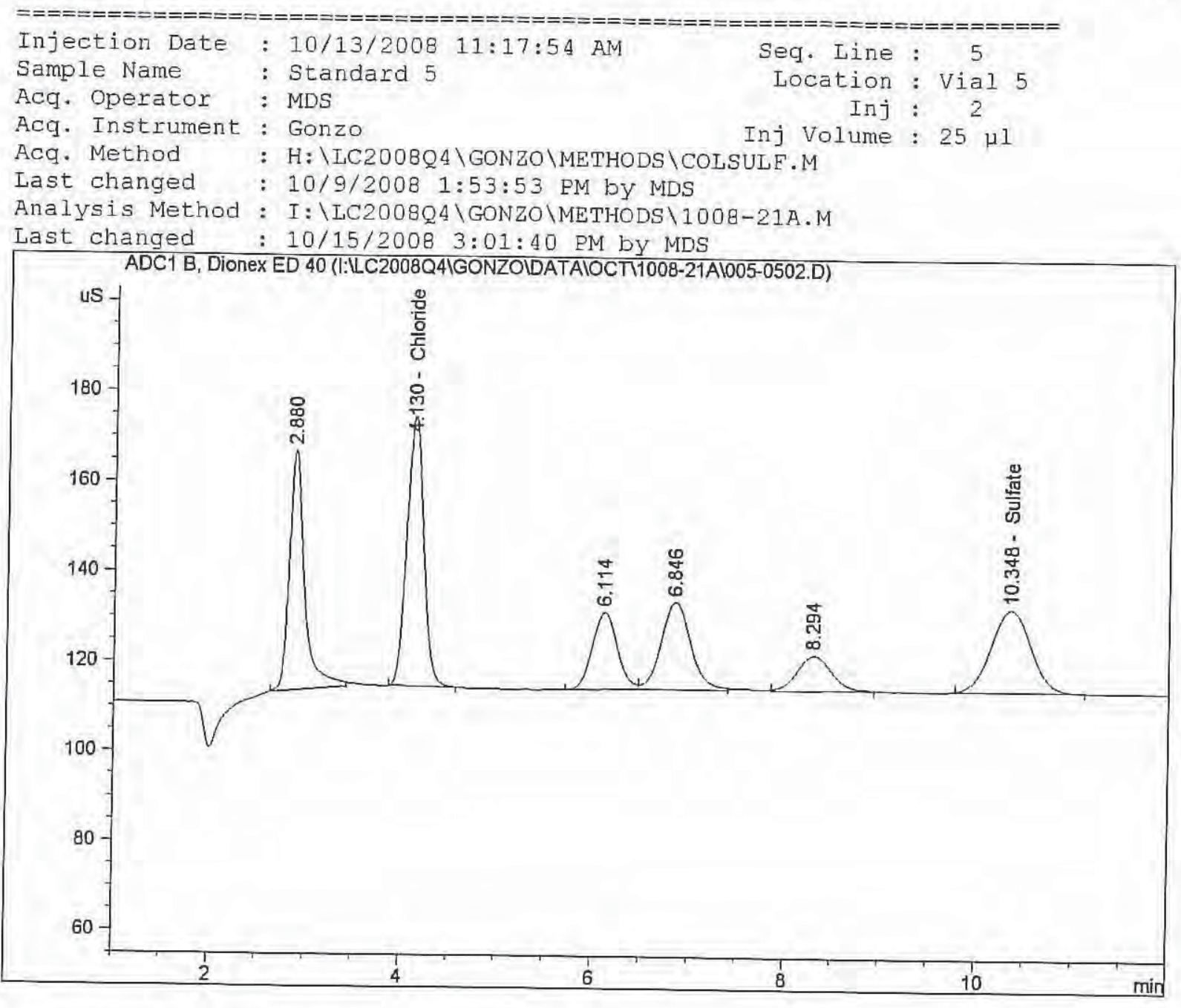

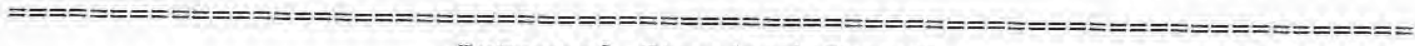
External Standard Report

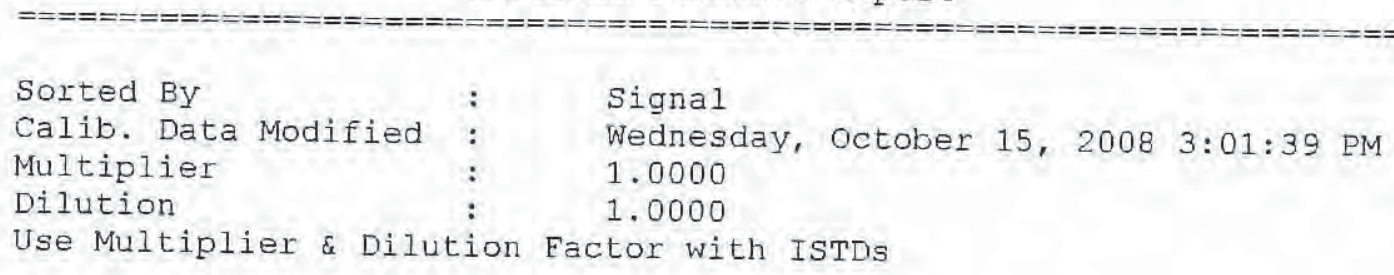

Signal 1: ADC1 B, Dionex ED 40

\begin{tabular}{|c|c|c|c|c|c|}
\hline $\begin{array}{l}\text { RetTime } \\
\text { [min] }\end{array}$ & Type & $\begin{array}{c}\text { Area } \\
{\left[u S^{*} s\right]}\end{array}$ & Amt/Area & $\begin{array}{l}\text { Amount } \\
{[\mathrm{ug} / \mathrm{mL}]}\end{array}$ & Grp Name \\
\hline $\begin{array}{r}4.130 \\
10.348\end{array}$ & $\begin{array}{l}\mathrm{PB} \\
\mathrm{BB}\end{array}$ & $\begin{array}{l}734.13550 \\
533.77448\end{array}$ & $\begin{array}{l}1.22701 e-2 \\
1.73879 e-2\end{array}$ & $\begin{array}{l}9.00788 \\
9.28121\end{array}$ & $\begin{array}{l}\text { Chloride } \\
\text { Sulfate }\end{array}$ \\
\hline
\end{tabular}

Totals :

$$
18.28909
$$

Results obtained with enhanced integrator!

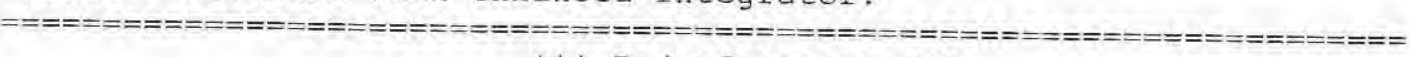

$* * *$ End of Report ***
} 


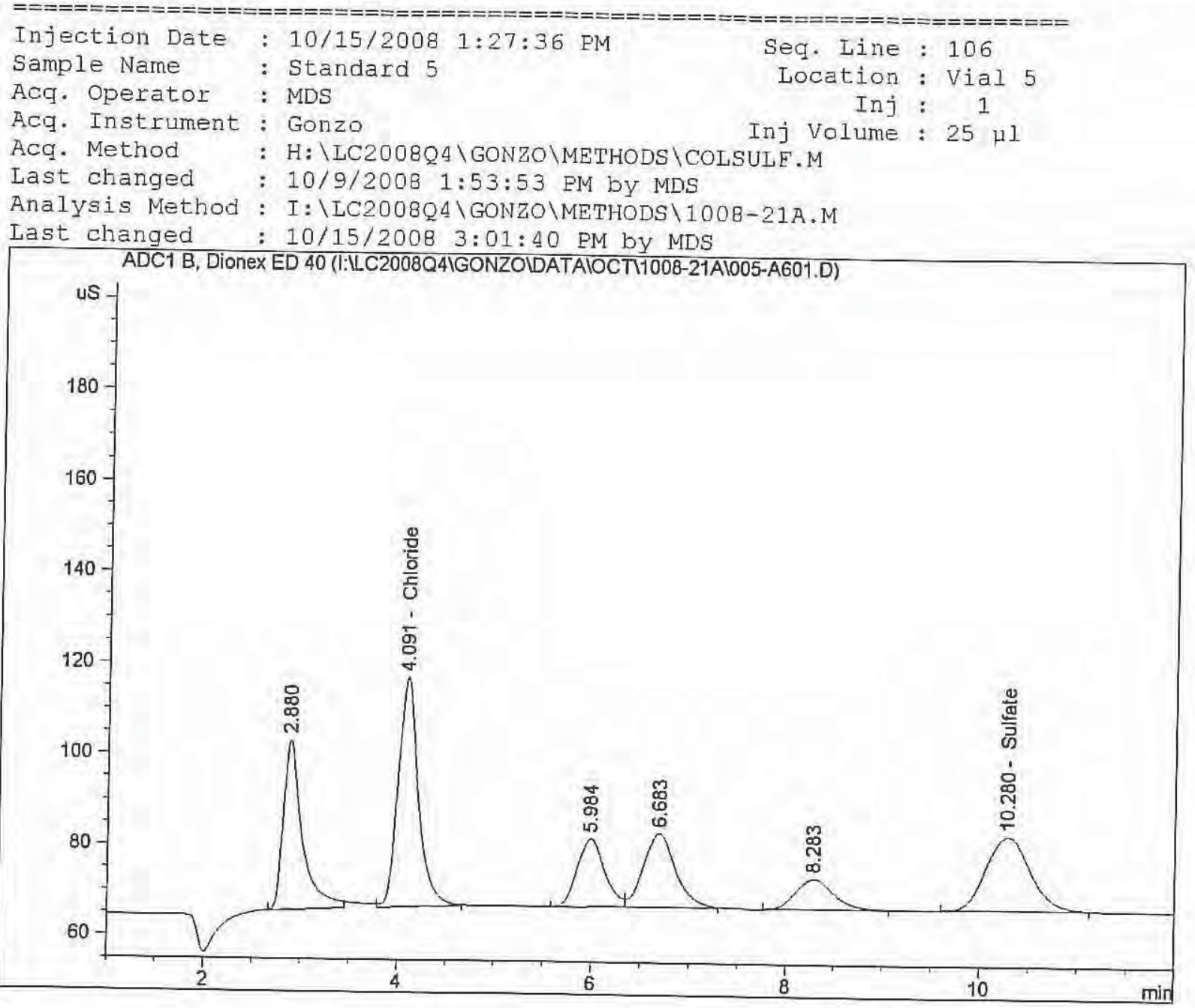

External Standard Report

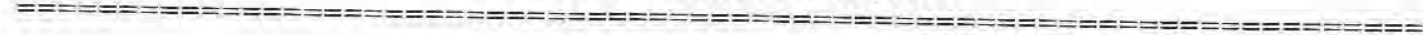

$\begin{array}{lll}\text { Sorted By } & : & \text { Signal } \\ \text { Calib. Data Modified : } & \text { Wednesday, October 15, 2008 3:01:39 PM } \\ \text { Multiplier } & \vdots & 1.0000 \\ \text { Dilution } & 1.0000 \\ \text { Use Multiplier } & \text { Dilution Factor with }\end{array}$

Use Multiplier \& Dilution Eactor with ISTDs

Signal 1: ADC1 B, Dionex ED 40

\begin{tabular}{|c|c|c|c|c|c|}
\hline $\begin{array}{l}\text { RetTime } \\
\text { [min] }\end{array}$ & Type & $\begin{array}{r}\text { Area } \\
{\left[\mathrm{us}^{*} \mathrm{~s}\right]}\end{array}$ & Amt/Area & $\begin{array}{l}\text { Amount } \\
{[\mathrm{ug} / \mathrm{mL}]}\end{array}$ & Name \\
\hline 4.091 & $\mathrm{BB}$ & 749.82336 & 1. $22689 \mathrm{e}-2$ & 9.19052 & Chloricio \\
\hline 10.280 & $\mathrm{~PB}$ & 521.70685 & 1. $73848 e-2$ & 9.06979 & $\begin{array}{l}\text { Chloride } \\
\text { Sulfate }\end{array}$ \\
\hline
\end{tabular}

Totals :

18.26931

Results obtained with enhanced integrator!

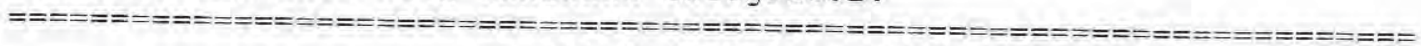

$* * *$ End of Report *** 


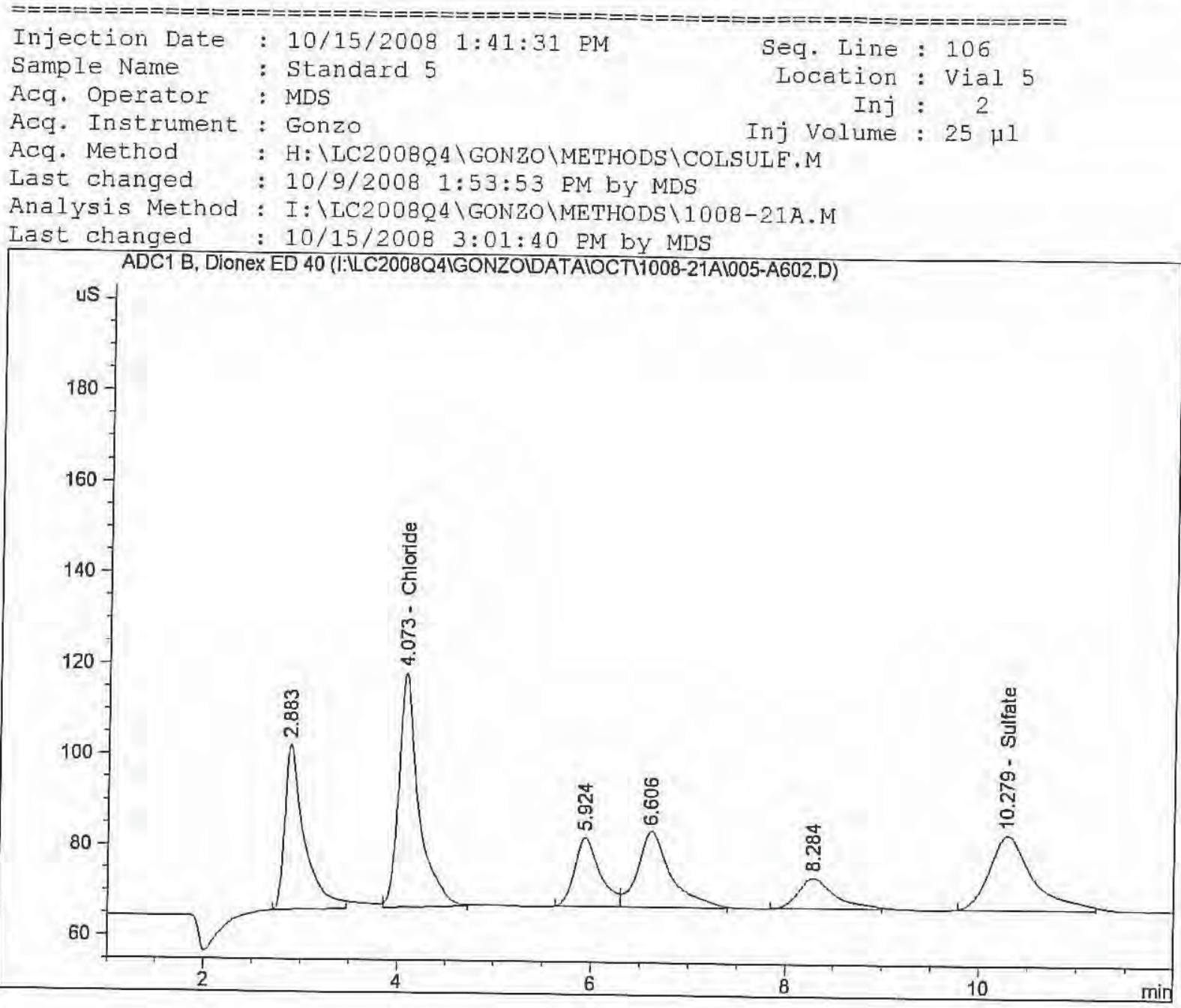

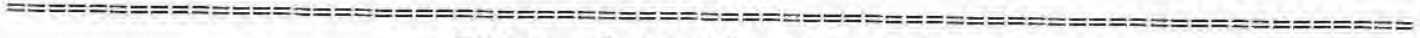
External standard Report

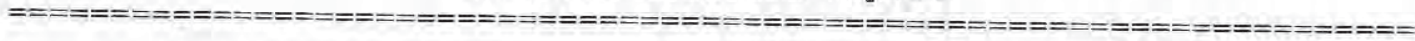

Sorted By : Signal

Calib. Data Modified : Wednesday, October 15, 2008 3:01:39 PM

Multiplier : 1.0000

Dilution : 1.0000

Use Multiplier \& Dilution Eactor with ISTDs

Signal 1: ADC1 B, Dionex ED 40

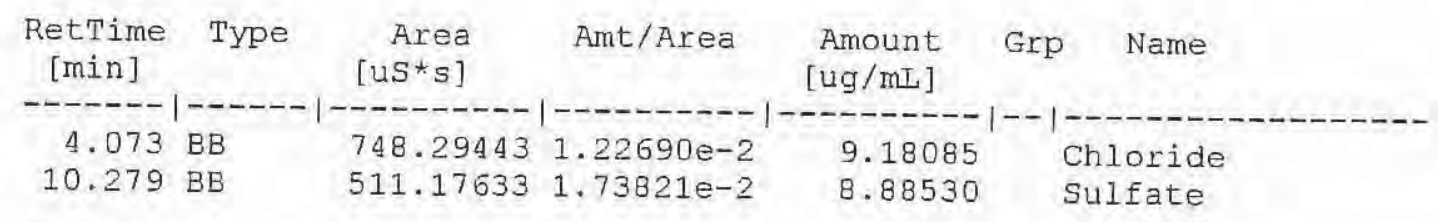

Totals :

18.06615

Results obtained with enhanced integrator!

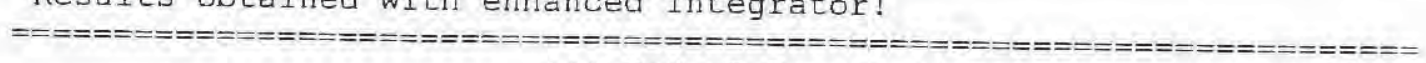

$$
\star * * \text { End of Report } * * *
$$
}




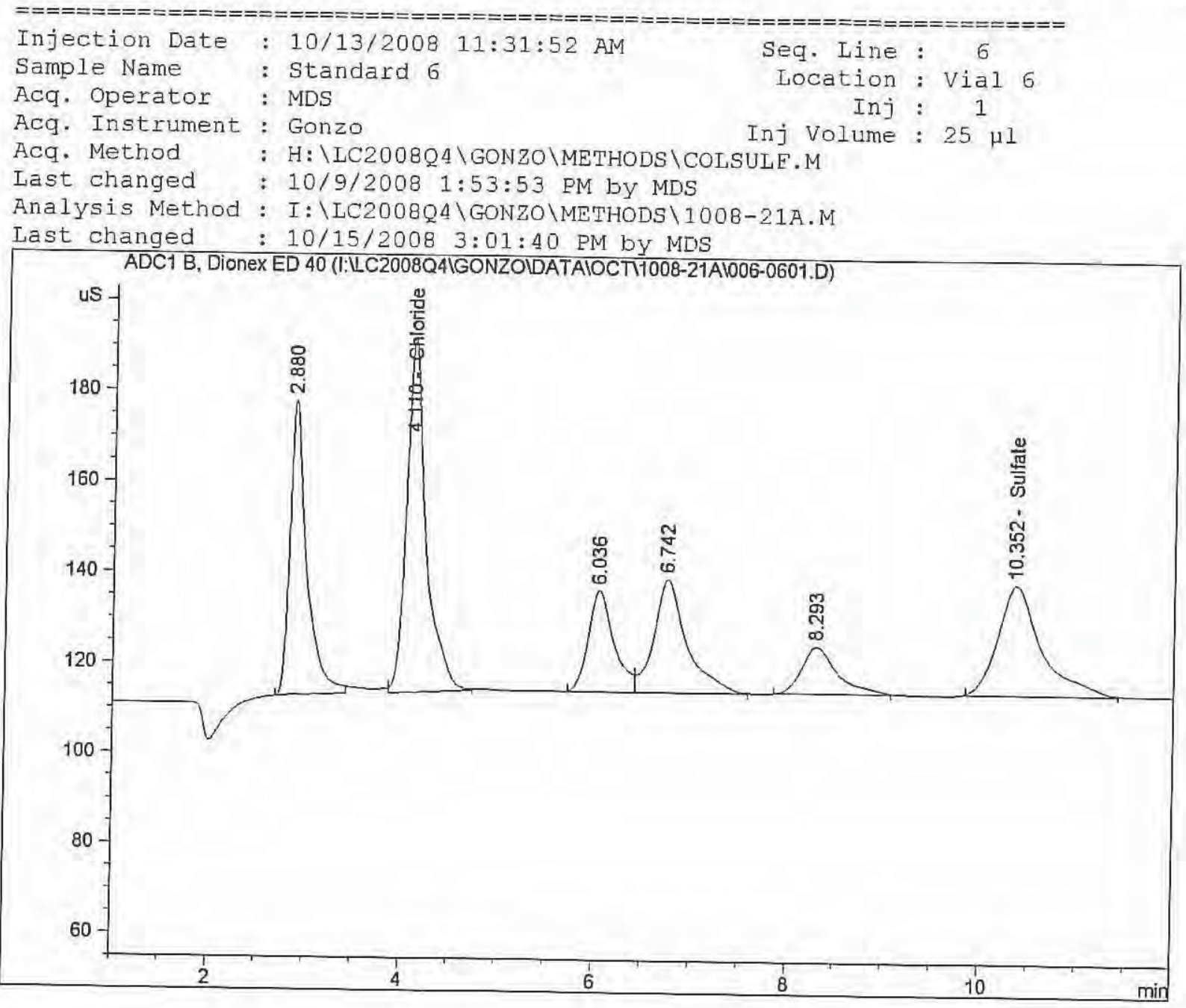

\section{External Standard Report}

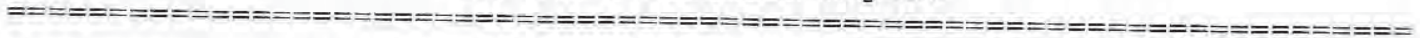

$\begin{array}{lll}\text { Sorted By } & : & \text { Signal } \\ \text { Calib. Data Modified } & : & \text { Wednesday, October 15, } 2008 \text { 3:01:39 PM } \\ \text { Multiplier } & : & 1.0000 \\ \text { Dilution } & \vdots & 1.0000 \\ \text { Use Multiplier \& Dilution Factor with ISTDs }\end{array}$

Signal 1: ADC1 B, Diones ED 40

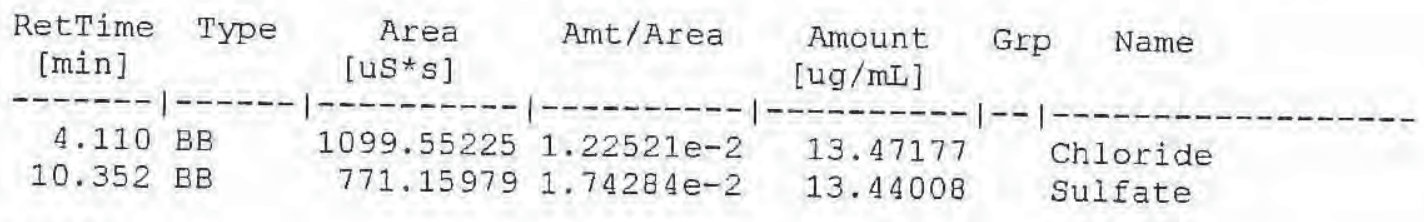

Totals : $\quad 26.91185$

Results obtained with enhanced integrator!

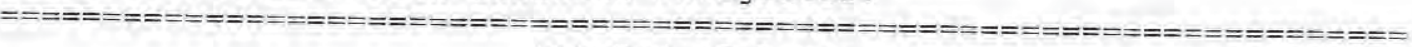
$* * *$ End of Report *** 


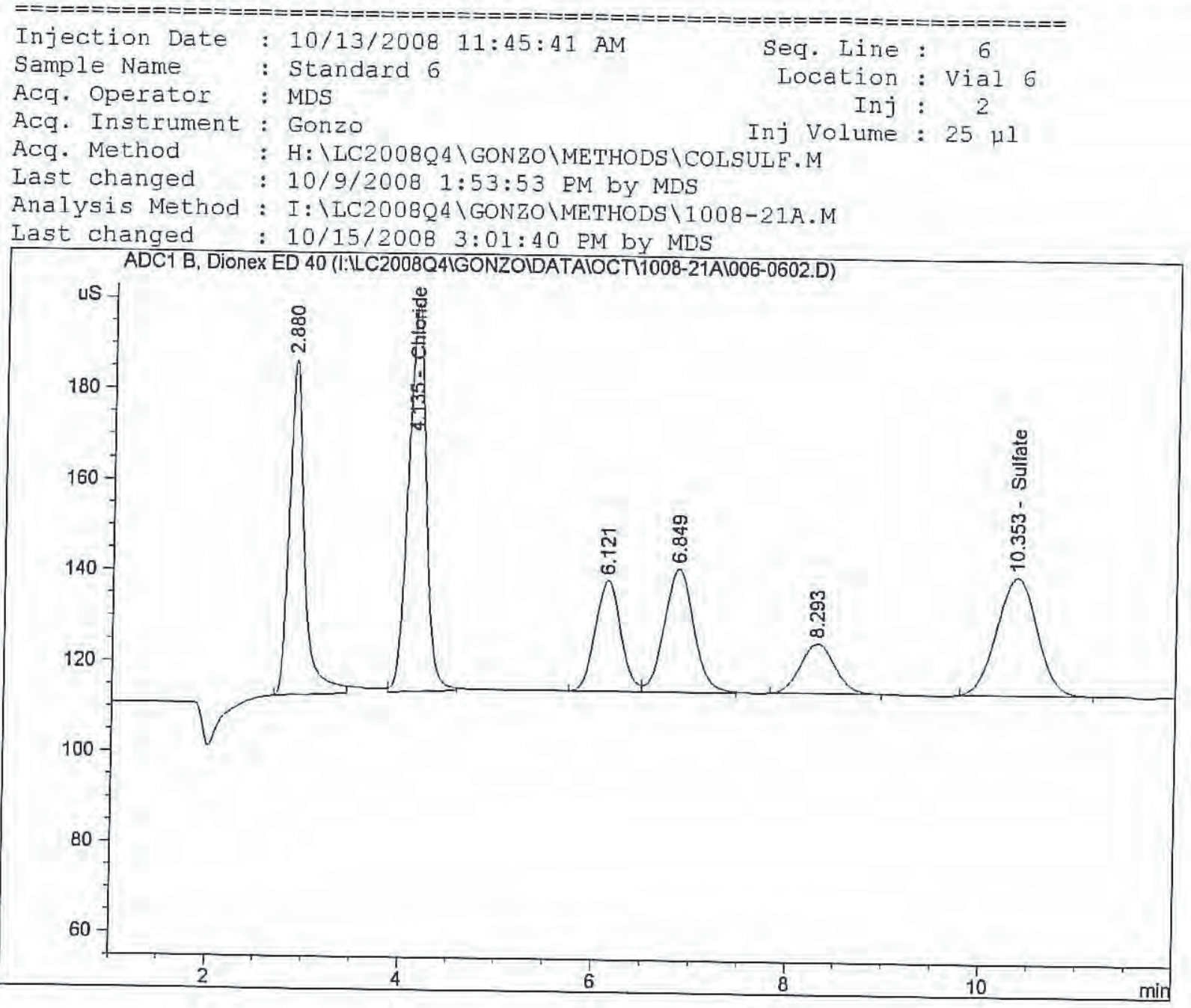

External Standard Report

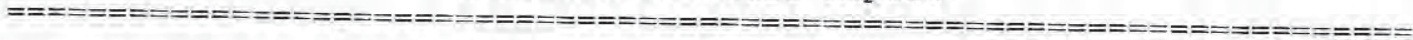

Sorted By

Calib. Data Modified

Multiplier : $\quad 1.0000$

Dilution : 1.0000

Use Multiplier \& Dilution Eactor with ISTDS

Signal 1: ADC1 B, Dionex ED 40

\begin{tabular}{|c|c|c|c|c|c|c|}
\hline $\begin{array}{l}\text { RetTime } \\
\text { [min] }\end{array}$ & Type & $\begin{array}{r}\text { Area } \\
\text { [us*s] }\end{array}$ & Amt/Area & $\begin{array}{l}\text { Amount } \\
\text { [ug/mL] }\end{array}$ & Grp & Name \\
\hline 4. 135 & $B B$ & 1095,18262 & & 17 1 & & \\
\hline 10.353 & $\begin{array}{l}B \mathrm{~B} \\
\mathrm{BB}\end{array}$ & $\begin{array}{r}1095.18262 \\
769.98358\end{array}$ & $\begin{array}{l}\text { 1. } 22522 e-2 \\
1.74283 e-2\end{array}$ & $\begin{array}{l}13.41839 \\
13.41947\end{array}$ & & $\begin{array}{l}\text { loride } \\
\text { lifate }\end{array}$ \\
\hline
\end{tabular}

Totals :

26.83787

Results obtained with enhanced integrator!

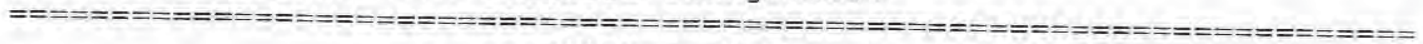

$\star \star \star$ End of Report $* * *$ 


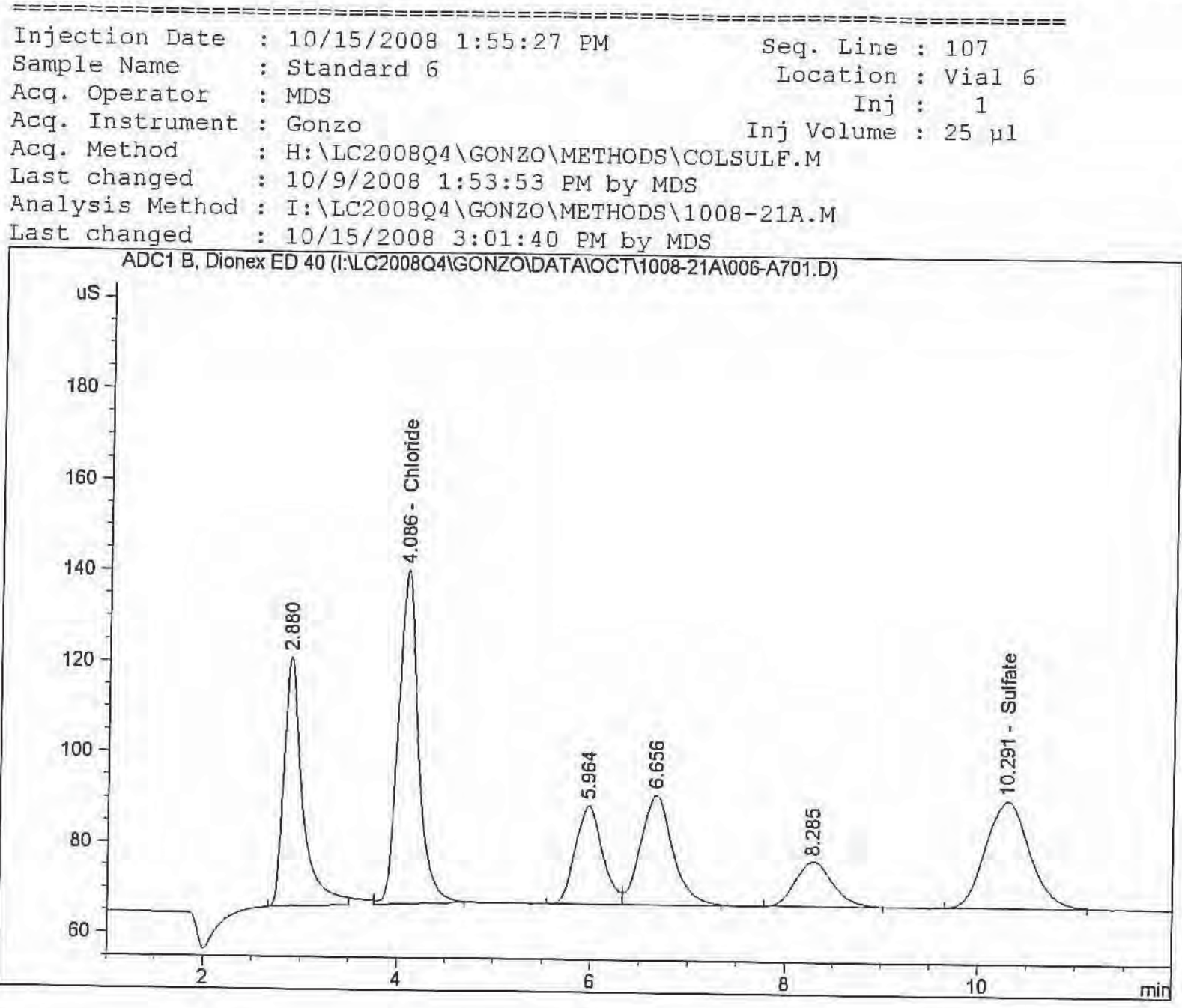

External standard Report

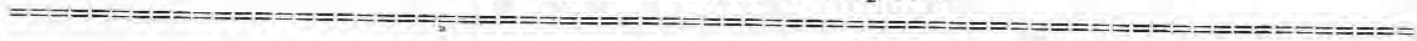

Sorted By

Signal

Calib. Data Modified : Wednesday, october 15, 2008 3:01:39 PM

Multiplier : 1.0000

Dilution : 1.0000

Use Multiplier \& Dilution Eactor with ISTDS

Signal 1: ADC1 B, Dionex ED 40

\begin{tabular}{|c|c|c|c|c|c|c|}
\hline $\begin{array}{l}\text { RetTime } \\
\text { [min] }\end{array}$ & Type & $\begin{array}{r}\text { Area } \\
\text { [uS*s] }\end{array}$ & Amt/Area & $\begin{array}{l}\text { Amount } \\
{[\mathrm{ug} / \mathrm{mL}]}\end{array}$ & Grp & Name \\
\hline 4006 & & | & & $-2---2-2$ & & \\
\hline $\begin{array}{r}4.086 \\
10.291\end{array}$ & $\begin{array}{l}B B \\
B B\end{array}$ & $\begin{array}{r}1081.24670 \\
747.60205\end{array}$ & $\begin{array}{l}1.22527 e-2 \\
1.74255 e-2\end{array}$ & $\begin{array}{l}13.24815 \\
13.02736\end{array}$ & & Ifate \\
\hline
\end{tabular}

Totals :

26.27551

Results obtained with enhanced integrator!

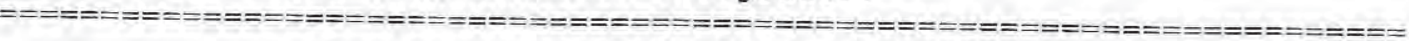

$$
\star \star * \text { End of Report } * * *
$$




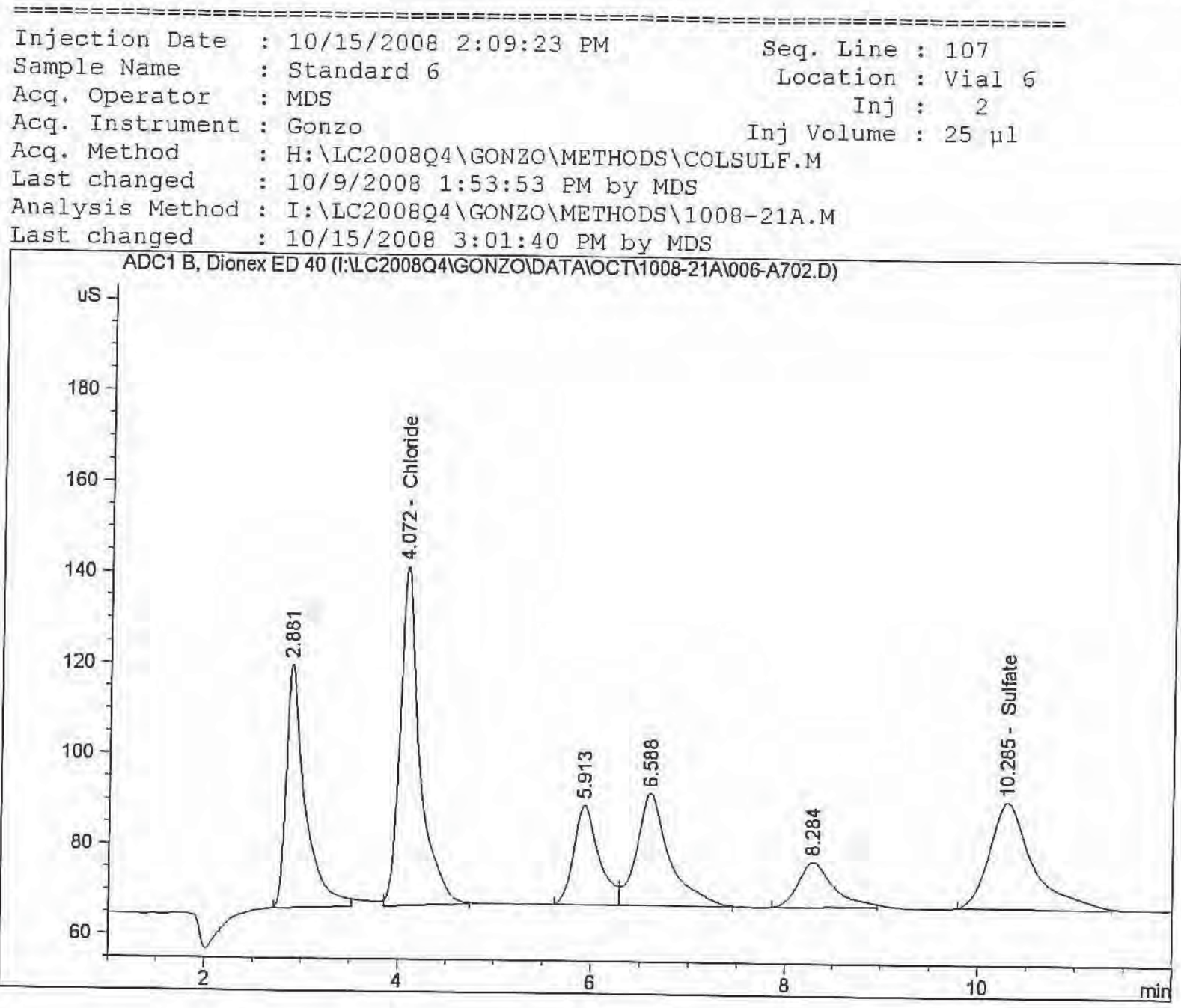

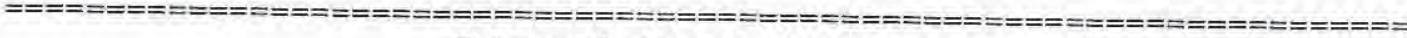
External Standard Report

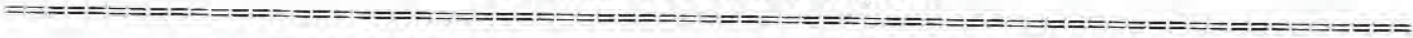

Sorted By

Multiplier : 1.0000

Dilution : 1.0000

Use Multiplier \& Dilution Eactor with ISTDS

Signal 1: ADC1 B, Dionex ED 40

\begin{tabular}{|c|c|c|c|c|c|}
\hline $\begin{array}{c}\text { RetTime } \\
\text { [min] }\end{array}$ & Type & $\begin{array}{r}\text { Area } \\
{[u S * s]}\end{array}$ & Amt/Area & $\begin{array}{l}\text { Amount } \\
\text { [ug/mL] }\end{array}$ & Name \\
\hline -ー・ー-ー- & & $-1-0-1--1$ & & & \\
\hline $\begin{array}{r}4.072 \\
10.285\end{array}$ & $\begin{array}{l}B B \\
B B\end{array}$ & $\begin{array}{r}1073.83899 \\
735.74823\end{array}$ & $\begin{array}{l}1.22529 \mathrm{e}-2 \\
1.74240 \mathrm{e}-2\end{array}$ & $\begin{array}{l}13.15766 \\
12.81969\end{array}$ & $\begin{array}{l}\text { Chloride } \\
\text { Sulfate }\end{array}$ \\
\hline
\end{tabular}

Totals :

25.97735

Results obtained with enhanced integrator!

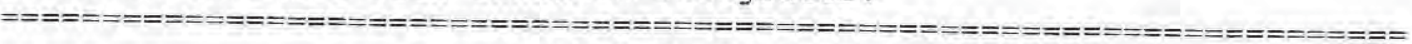

$$
\star * \star \text { End of Report *** }
$$
}




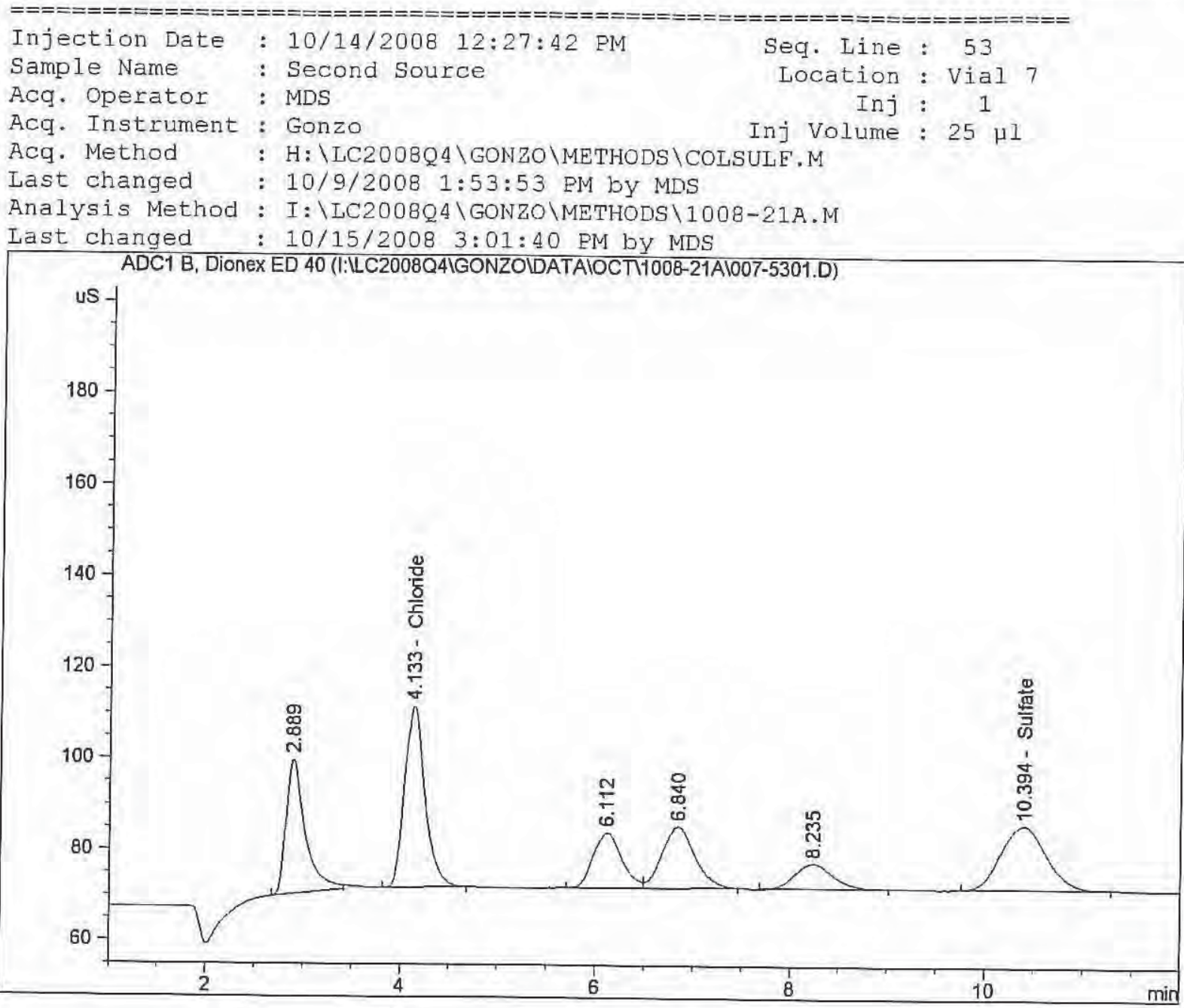

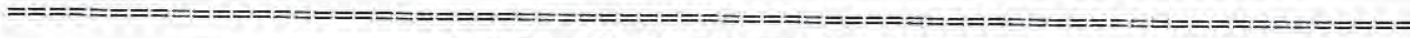
External Standard Report

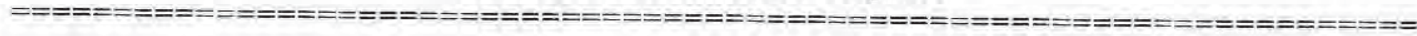

$\begin{array}{lll}\text { Sorted By } & : & \text { Signal } \\ \text { Calib. Data Modified } & : & \text { Wednesday, October 15, 2008 3:01:39 PM } \\ \text { Multiplier } & : & 1.0000 \\ \text { Dilution } & : & 1.0000\end{array}$

Use Multiplier \& Dilution Factor with ISTDs

Signal 1: ADC1 B, Dionex ED 40

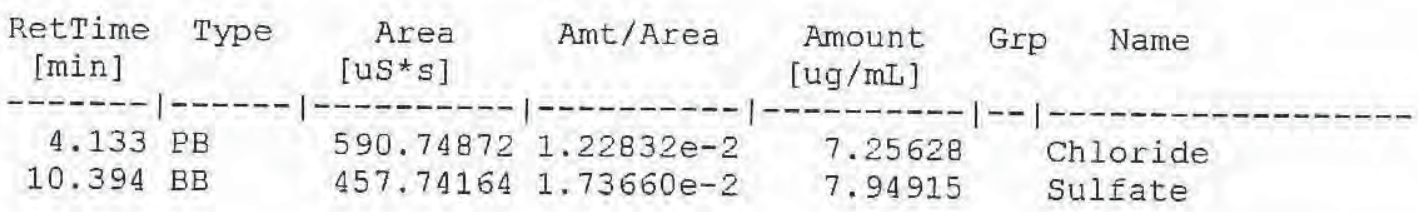

Totals :

15.20544

Results obtained with enhanced integrator!

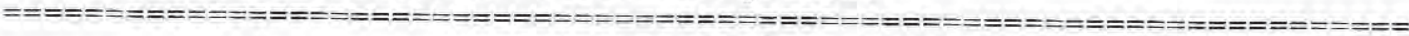

$* * *$ End of Report $* * *$ 


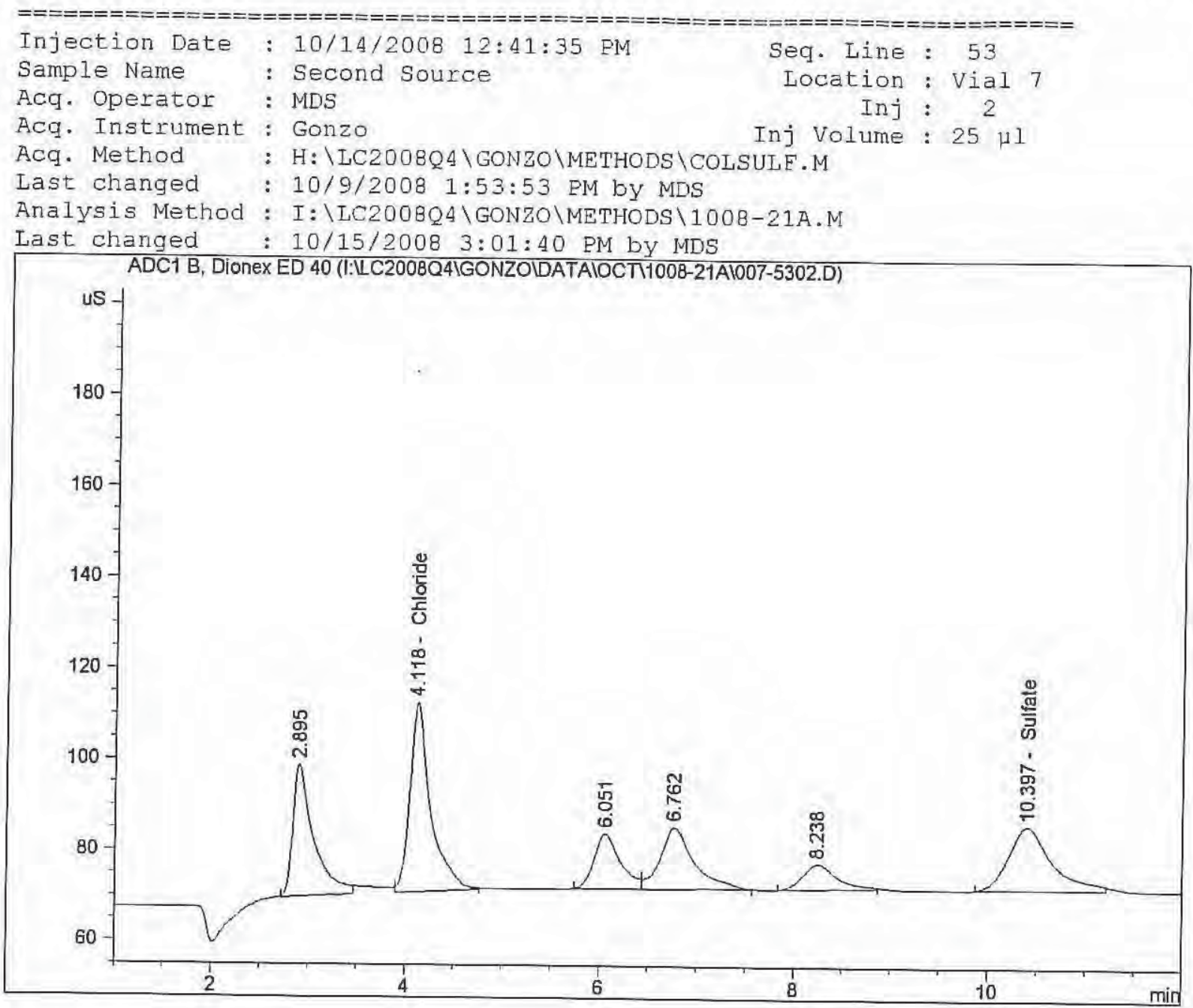

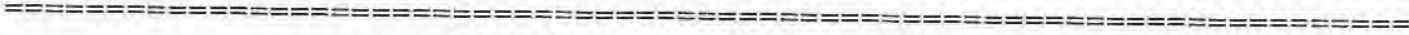

External Standard Report

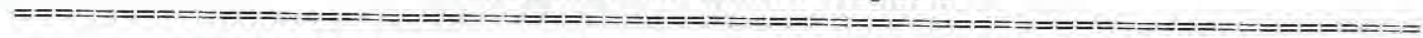

$\begin{array}{lll}\text { Sorted By } & : & \text { Signal } \\ \text { Calib. Data Modified } & : & \text { Wednesday, October 15, 2008 3:01:39 PM } \\ \text { Multiplier } & : & 1.0000 \\ \text { Dilution } & : & 1.0000\end{array}$

Use Multiplier \& Dilution Eactor with ISTDS

Signal 1: ADC1 B, Dionex ED 40

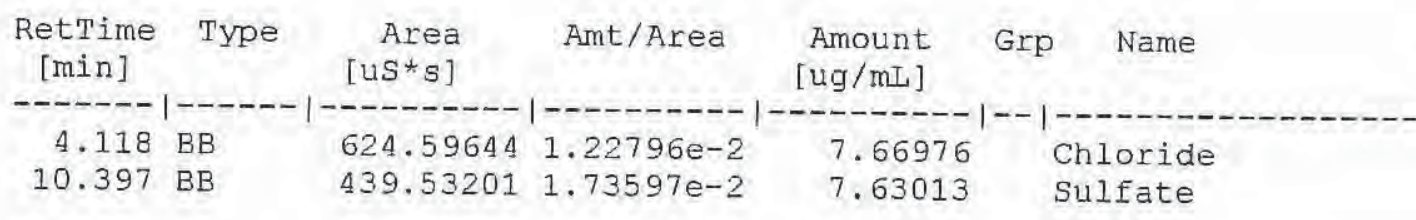

Totals :

15.29990

Results obtained with enhanced integrator!

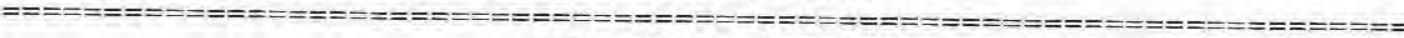
$* \star \star$ End of Report $* * *$
} 


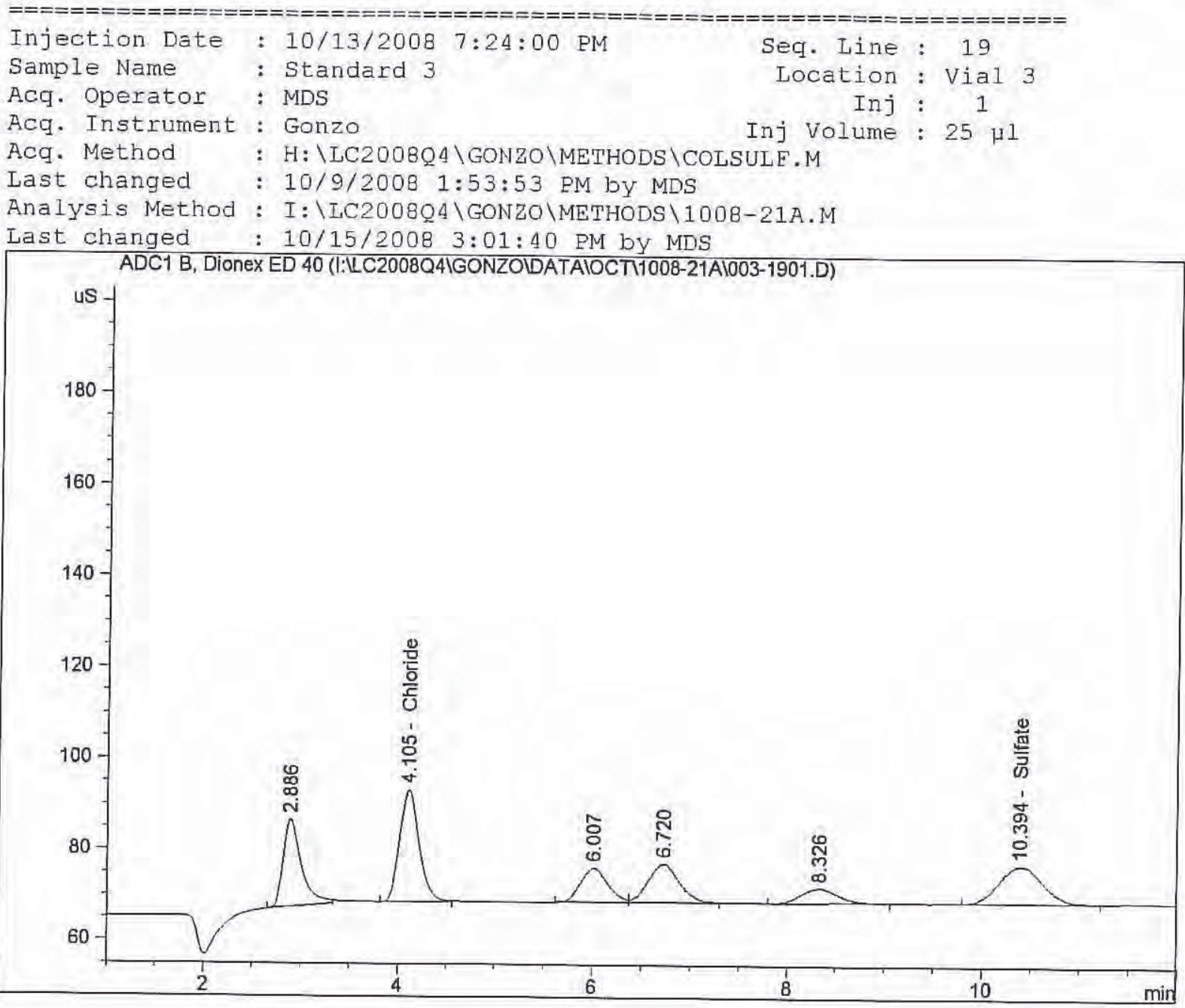

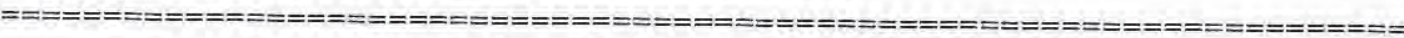

External Standard Report

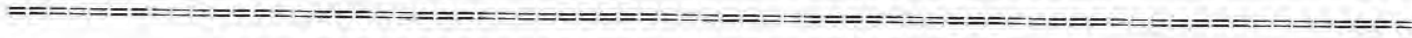

$\begin{array}{lll}\text { Sorted By } & : & \text { Signal } \\ \text { Calib. Data Modified } & : & \text { Wednesday, October 15, 2008 3:01:39 PM } \\ \text { Multiplier } & : & 1.0000 \\ \text { Dilution } & : & 1.0000\end{array}$

Use Multiplier \& Dilution Factor with ISTDs

Signal 1: ADC1 B, Dionex ED 40

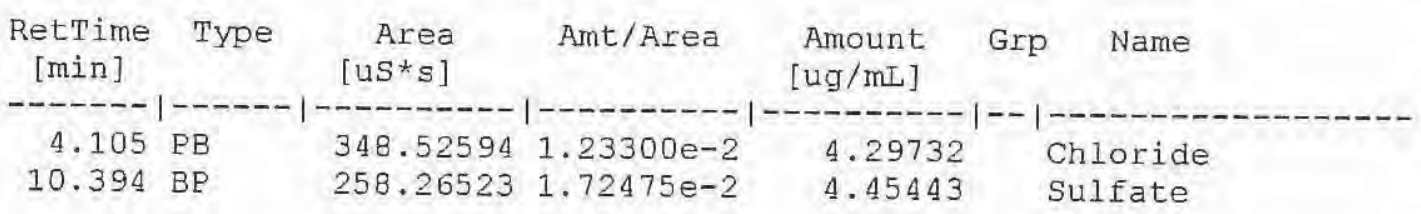

Totals :

8.75175

Results obtained with enhanced integrator! 

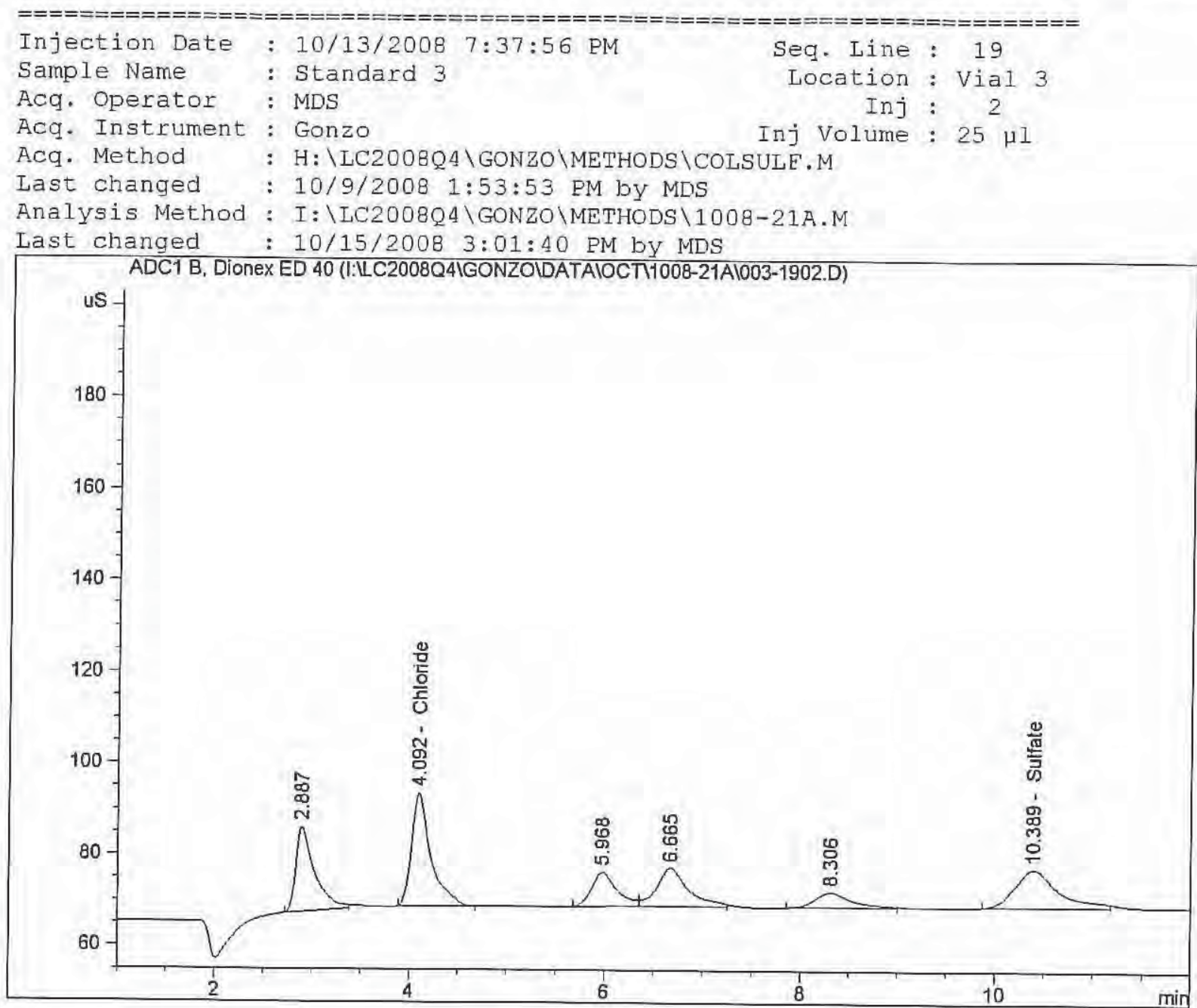

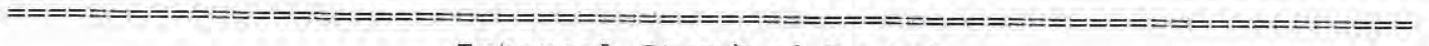
External Standard Report

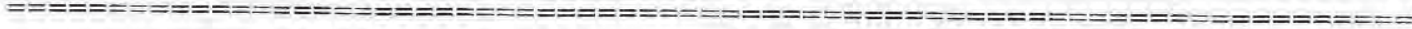

$\begin{array}{lcc}\text { Sorted By } & : & \text { Signal } \\ \text { Calib. Data Modified : } & \text { Wednesday, October 15, 2008 3:01:39 PM } \\ \text { Multiplier } & : & 1.0000 \\ \text { Dilution } & \text { : } & 1.0000 \\ \text { Use Multiplier \& Dilution Factor with ISTDs }\end{array}$

Use Multiplier \& Dilution Factor with ISTDS

Signal 1: ADC1 B, Dionex ED 40

\begin{tabular}{|c|c|c|c|c|c|}
\hline $\begin{array}{c}\text { RetTime } \\
\text { [min] }\end{array}$ & Type & $\begin{array}{r}\text { Area } \\
\left.\text { [us* } S^{*}\right]\end{array}$ & Amt/Area & $\begin{array}{l}\text { Amount } \\
{[\mathrm{ug} / \mathrm{mL}]}\end{array}$ & Name \\
\hline------ & ----1 & --------- & -------- & - - & $--1---\cdots-\cdots$ \\
\hline 4.092 & BB & 346.03046 & 1. $23308 e-2$ & 4.26683 & Chloride \\
\hline 10.389 & $\mathrm{BB}$ & 259.69534 & $1.72490 \mathrm{e}-2$ & 4.47948 & Sulfate \\
\hline Totals : & & & & 8.74632 & \\
\hline
\end{tabular}

Results obtained with enhanced integrator! 


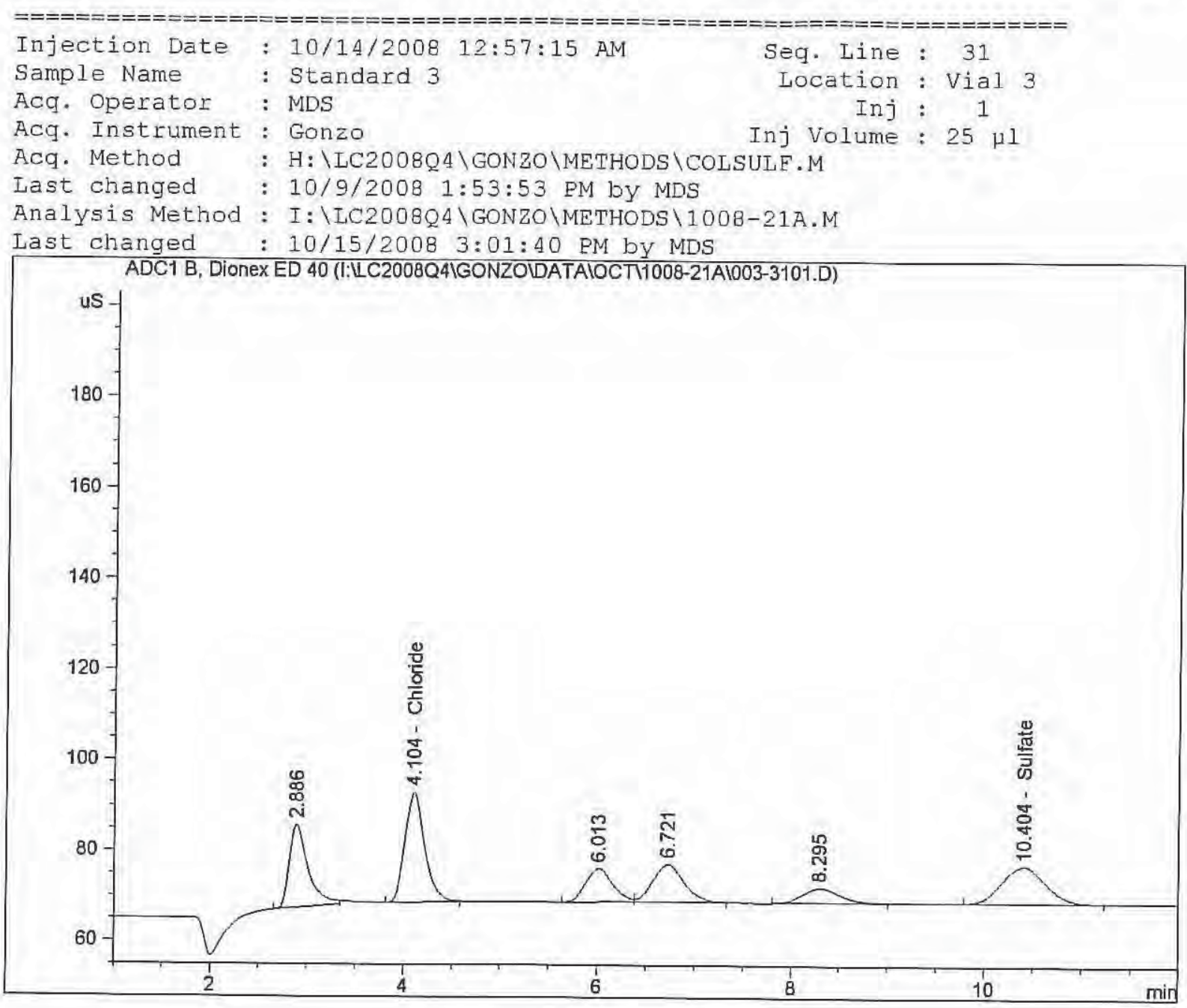

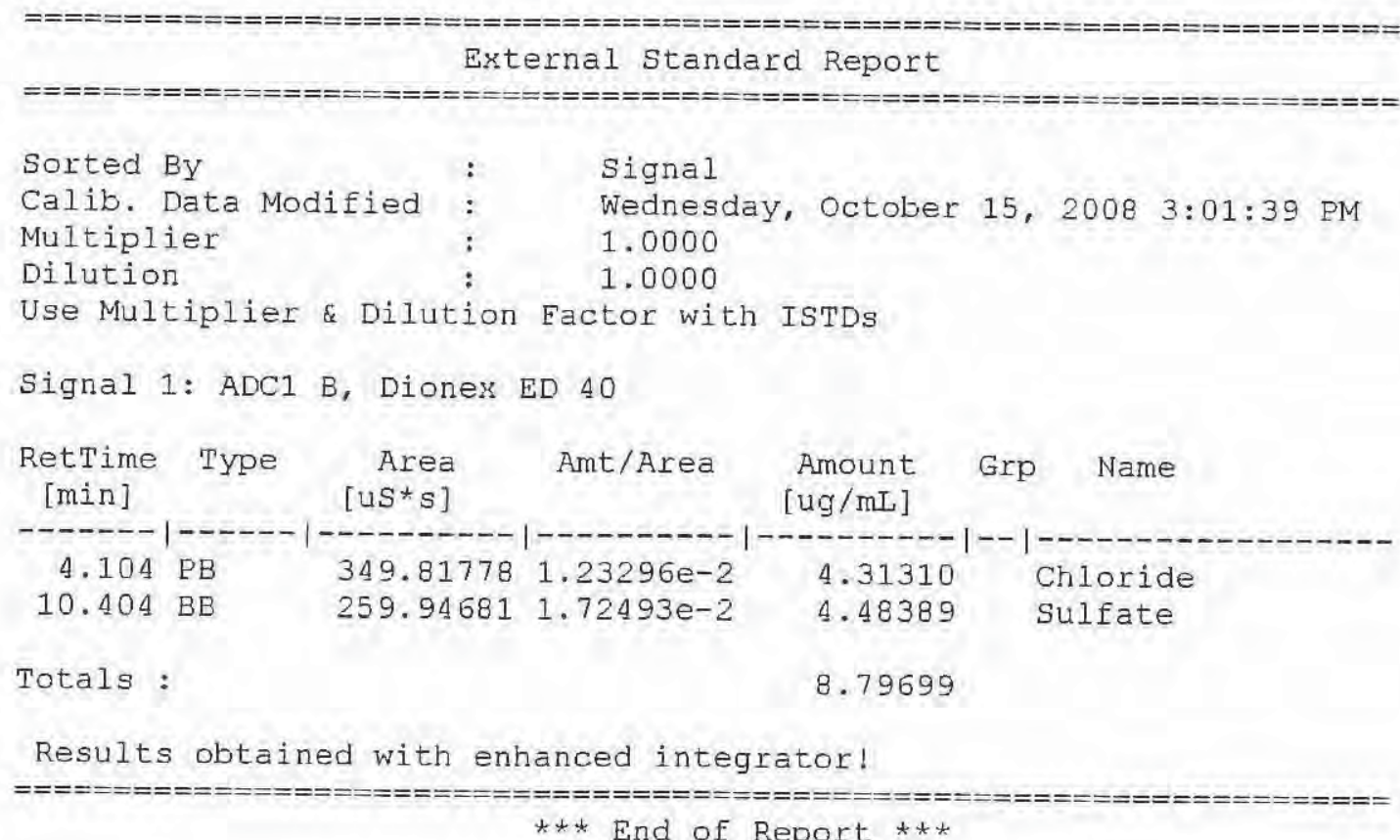



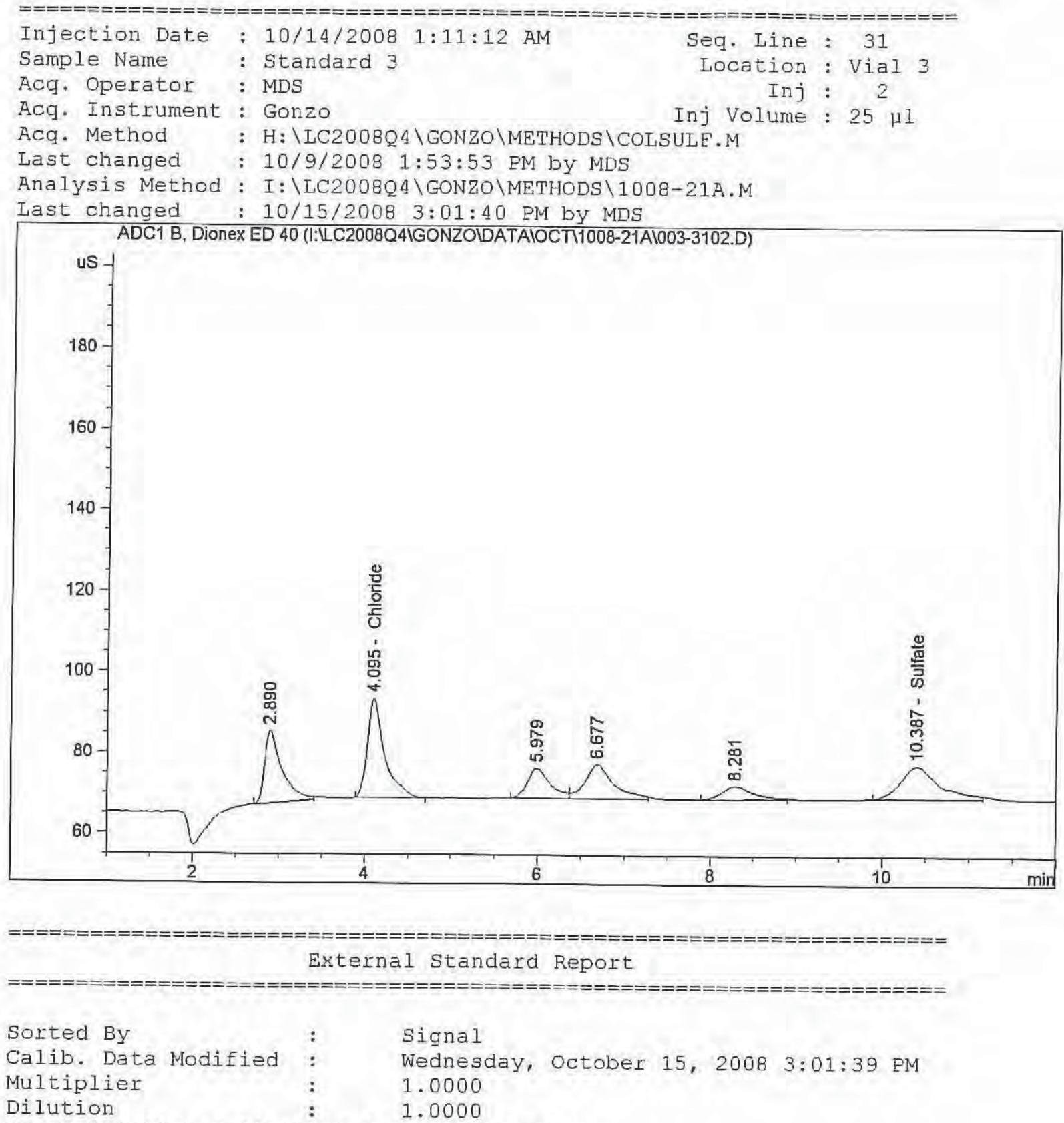

Use Multiplier \& Dilution Factor with ISTDS

Signal 1: ADC1 B, Dionex ED 40

\begin{tabular}{|c|c|c|c|c|c|}
\hline $\begin{array}{l}\text { RetTime } \\
\text { [min] }\end{array}$ & Type & $\begin{array}{r}\text { Area } \\
\text { [uS*s] }\end{array}$ & Amt/Area & $\begin{array}{l}\text { Amount } \\
{[\mathrm{ug} / \mathrm{mL}]}\end{array}$ & Name \\
\hline $\begin{array}{r}4.095 \\
10.387\end{array}$ & $\begin{array}{l}\mathrm{BB} \\
\mathrm{BB}\end{array}$ & $\begin{array}{l}346.47870 \\
257.92206\end{array}$ & $\begin{array}{l}1.23307 \mathrm{e}-2 \\
1.72471 \mathrm{e}-2\end{array}$ & $\begin{array}{l}4.27231 \\
4.44842\end{array}$ & $\begin{array}{l}\text { Chloride } \\
\text { Sulfate }\end{array}$ \\
\hline Totals & & & & 8.72073 & \\
\hline
\end{tabular}

Results obtained with enhanced integrator! 

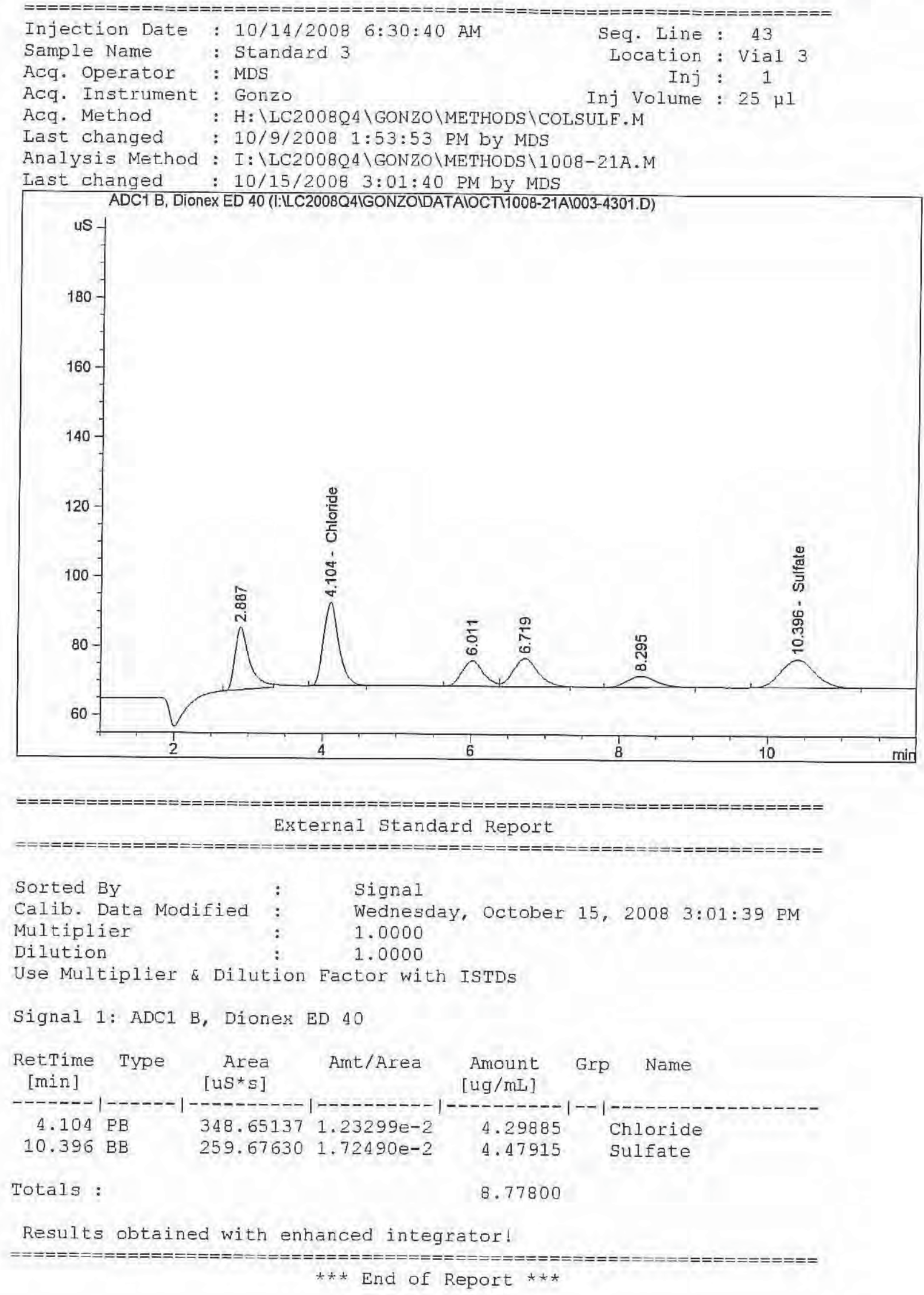

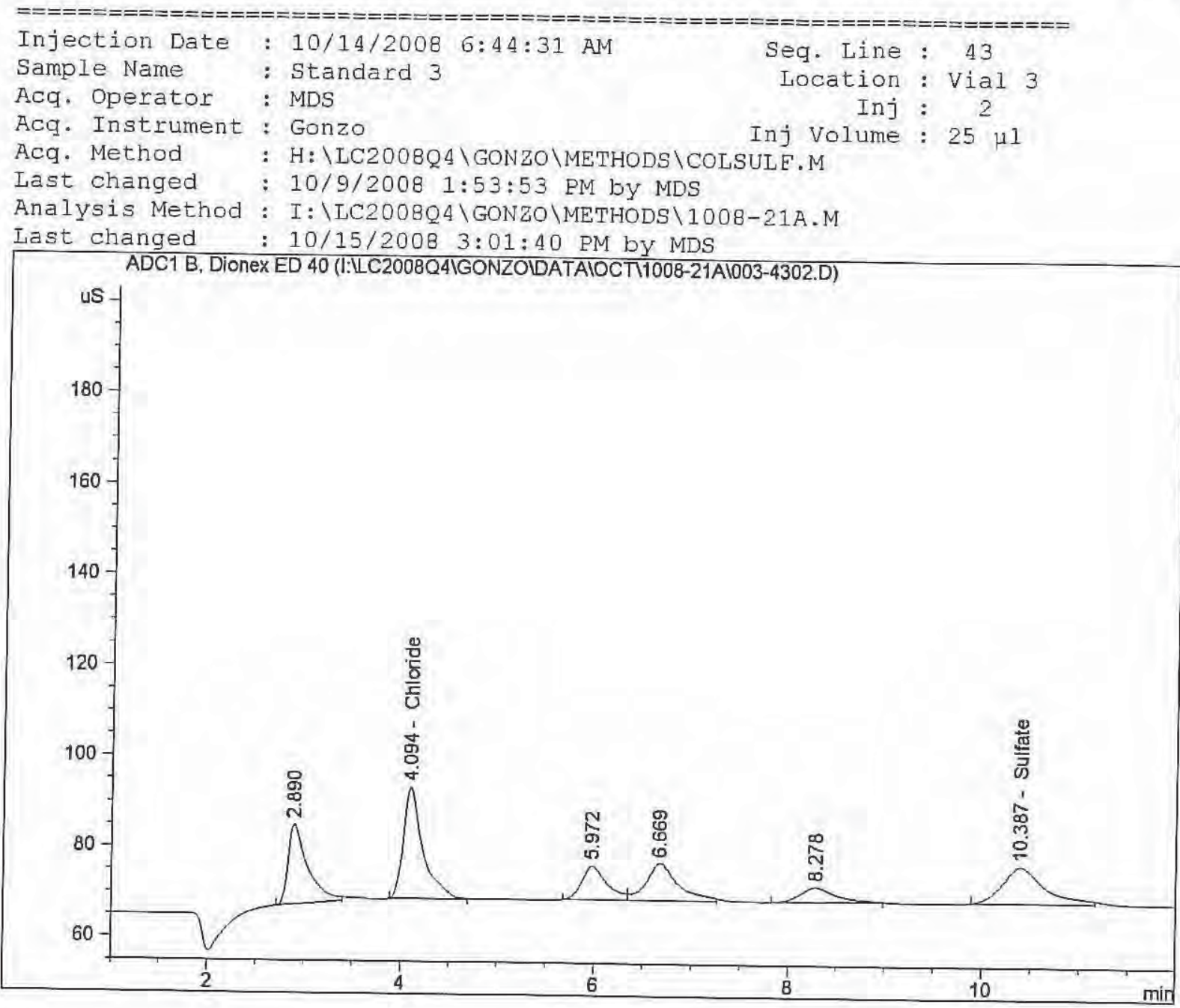

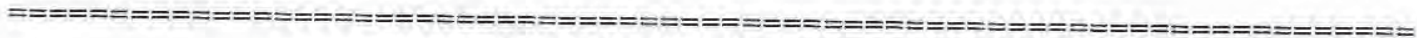

External standard Report

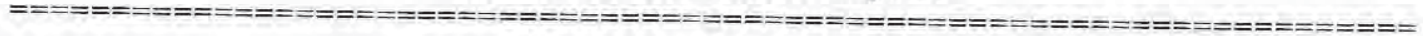

Sorted By

Signal

$\begin{array}{lll}\text { Calib. Data Modified : } & \text { Wednesday, October 15, 2008 3:01:39 PM } \\ \text { Multiplier } & : & 1.0000\end{array}$

Dilution

1.0000

Use Multiplier \& Dilution Factor with ISTDS

Signal 1: ADC1 B, Dioner ED 40

\begin{tabular}{|c|c|c|c|c|c|}
\hline $\begin{array}{l}\text { RetTime } \\
\text { [min] }\end{array}$ & Type & $\begin{array}{r}\text { Area } \\
\text { [us*s] }\end{array}$ & Amt/Area & $\begin{array}{l}\text { Amount } \\
\text { [ug/mL] }\end{array}$ & Name \\
\hline $\begin{array}{r}4.094 \\
10.387\end{array}$ & $\begin{array}{l}\mathrm{BB} \\
\mathrm{BB}\end{array}$ & $\begin{array}{l}346.92133 \\
252.15431\end{array}$ & 1. $23305 e-2$ & 4. 27772 & Chloride \\
\hline
\end{tabular}

Totals:

8.62509

Results obtained with enhanced integrator!

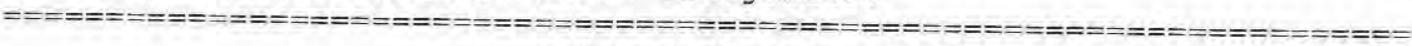

*** End of Report *** 

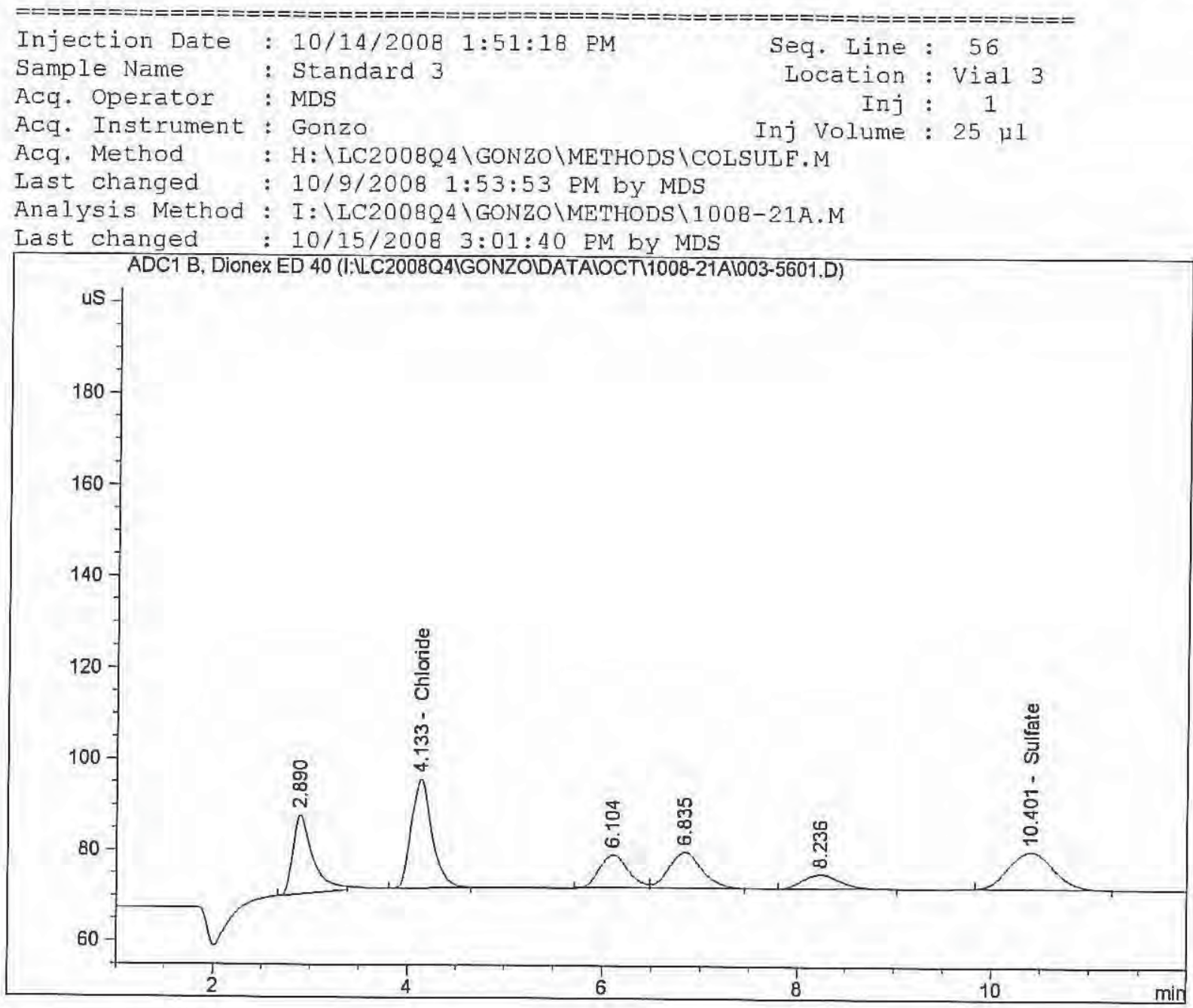

External Standard Report

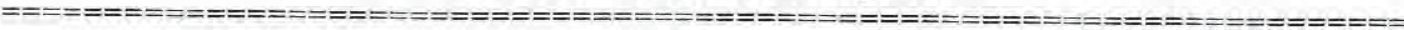

$\begin{array}{lcl}\text { Sorted By } & : & \text { Signal } \\ \text { Calib. Data Modified } & : & \text { Wednesday, October 15, 2008 3:01:39 PM } \\ \text { Multiplier } & : & 1.0000 \\ \text { Dilution } & \vdots & 1.0000 \\ \text { Use Multiplier \& Dilution Factor with ISTDs }\end{array}$

Use Multiplier \& Dilution Eactor with ISTDs

Signal 1: ADC1 B, Dionex ED 40

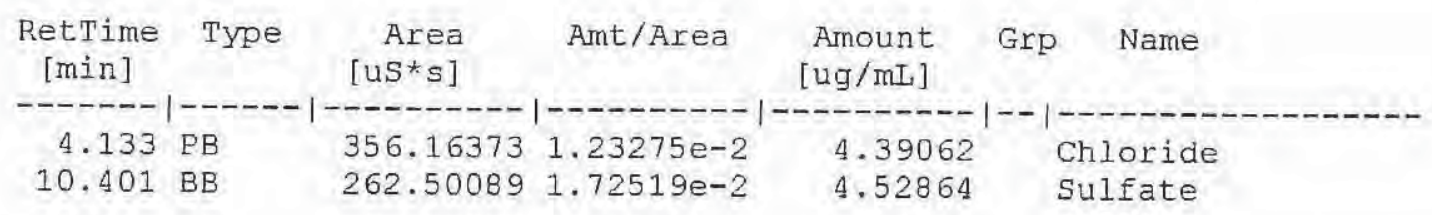

Totals:

8. 91926

Results obtained with enhanced integrator! 


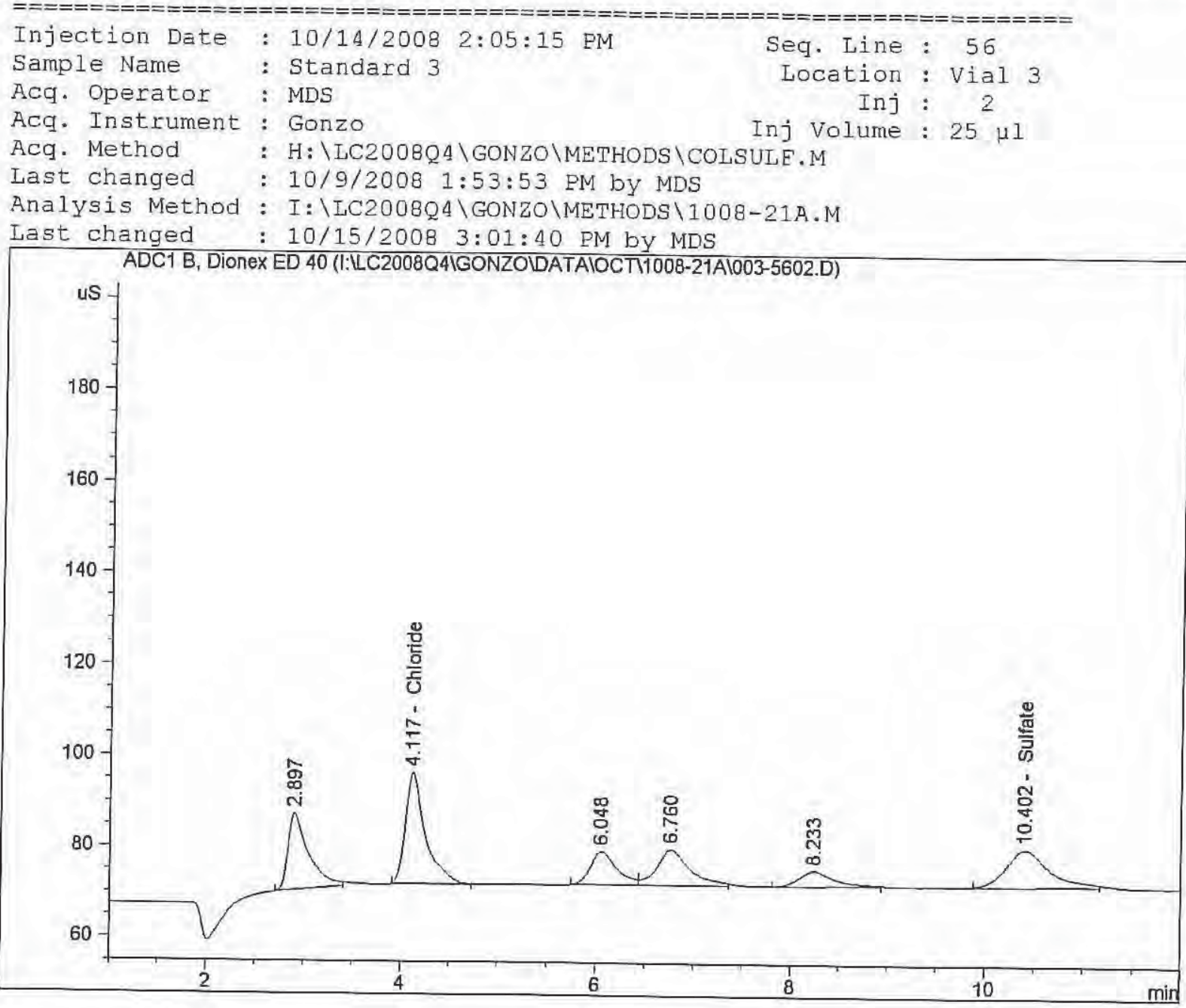

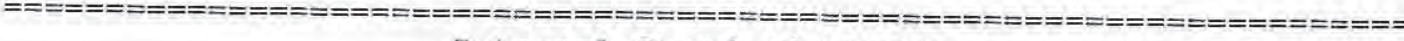
External Standard Report

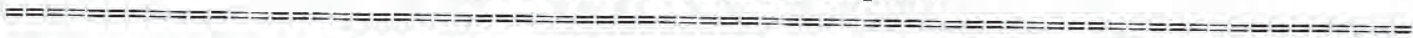

Sorted By

Signal

$\begin{array}{lll}\text { Calib. Data Modified : } & \text { Wednesday, October 15, 2008 3:01:39 PM } \\ \text { Multiplier } & 1.0000\end{array}$

Dilution : 1.0000

Use Multiplier \& Dilution Factor with ISTDS

Signal 1: ADC1 B, Dionex ED 40

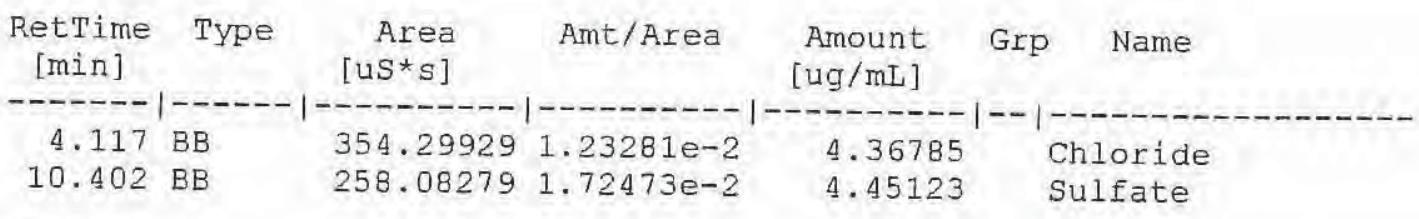

Totals :

8.81908

Results obtained with enhanced integrator!

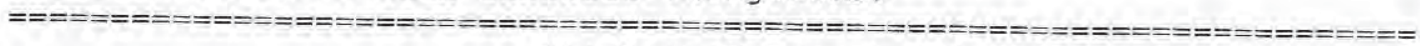

$\star \star \star$ End of Report $\star \star \star ~$
} 

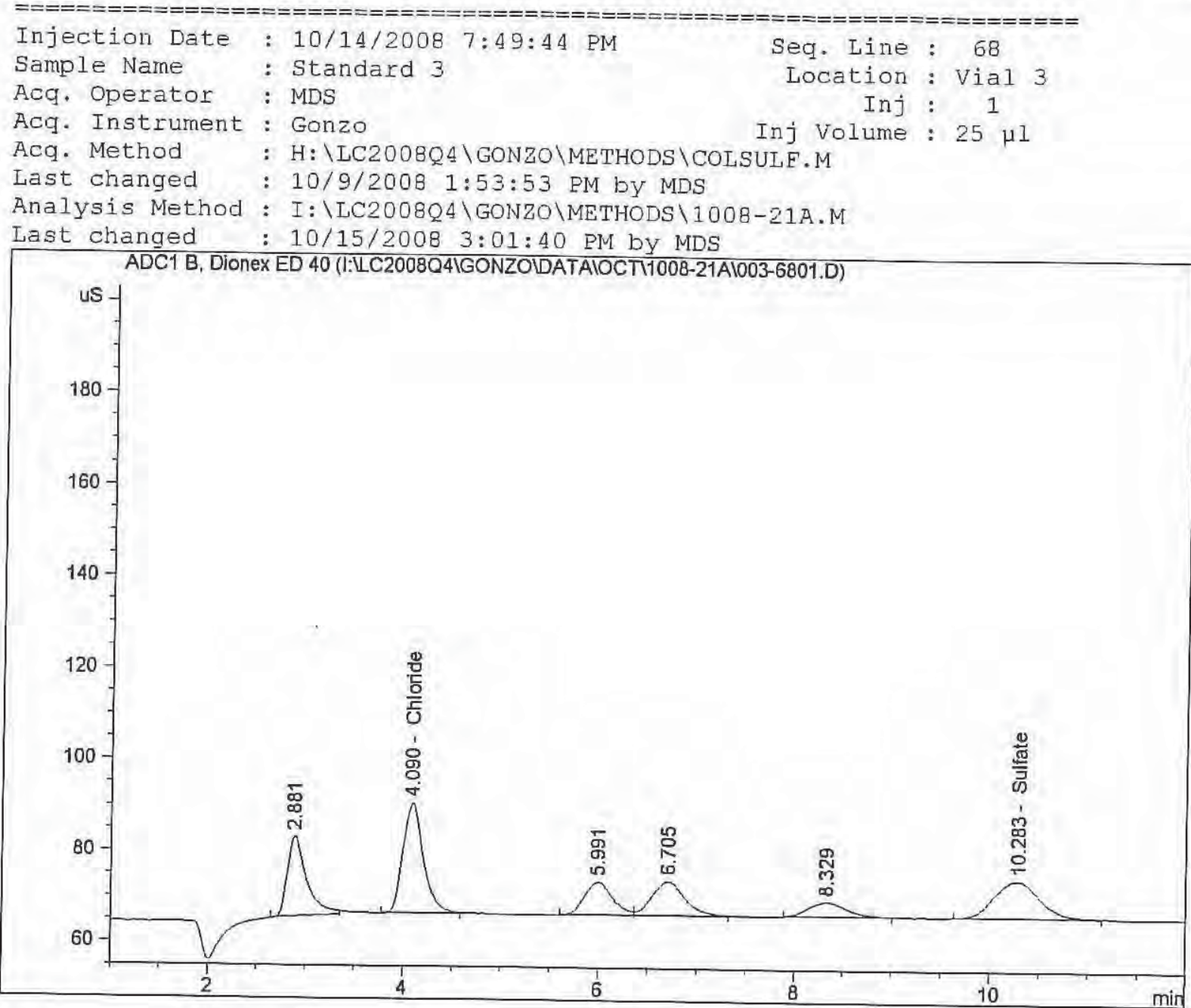

External Standard Report

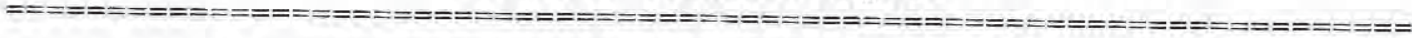

Sorted By

Signal

Calib. Data Modified : Wednesday, october 15, 2008 3:01:39 PM

Multiplier : 1.0000

Dilution : 1.0000

Use Multiplier \& Dilution Factor with ISTDs

Signal 1: ADC1 B, Dionex ED 40

\begin{tabular}{|c|c|c|c|c|c|c|}
\hline $\begin{array}{l}\text { RetTime } \\
\text { [min] }\end{array}$ & Type & $\begin{array}{r}\text { Area } \\
\text { [us*s] }\end{array}$ & Amt/Area & $\begin{array}{l}\text { Amount } \\
\text { [ug/mI] }\end{array}$ & Grp & Name \\
\hline 4.090 & $\mathrm{~PB}$ & 353.14325 & $1.23285 e-2$ & 4.35372 & & \\
\hline 10.283 & PB & 264.67578 & $1.72541 e-2$ & 4.56674 & & lifate \\
\hline
\end{tabular}

Totals:

8.92046

Results obtained with enhanced integrator!

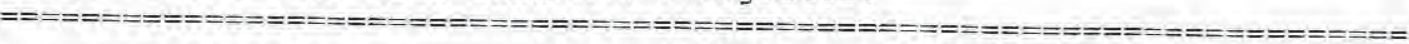
$* \star *$ End of Report $* * *$ 


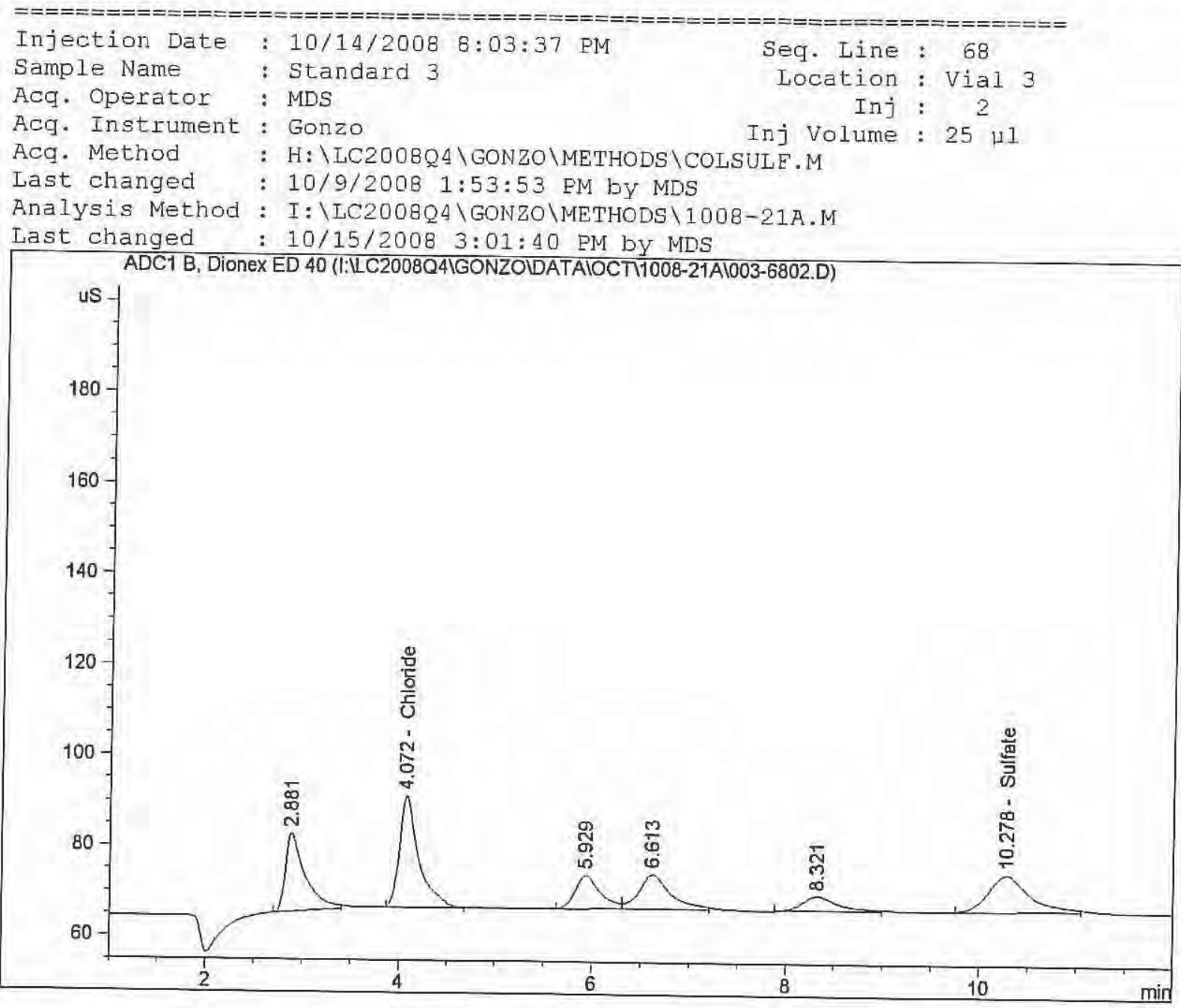

External Standard Report

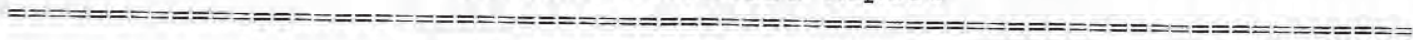

Sorted By

Signal

Calib. Data Modified : Wednesday, October 15, 2008 3:01:39 PM

Multiplier

Dilution

1.0000

Use Multiplier \& Dilution Eactor with ISTDs

Signal 1: ADC1 B, Dionex ED 40

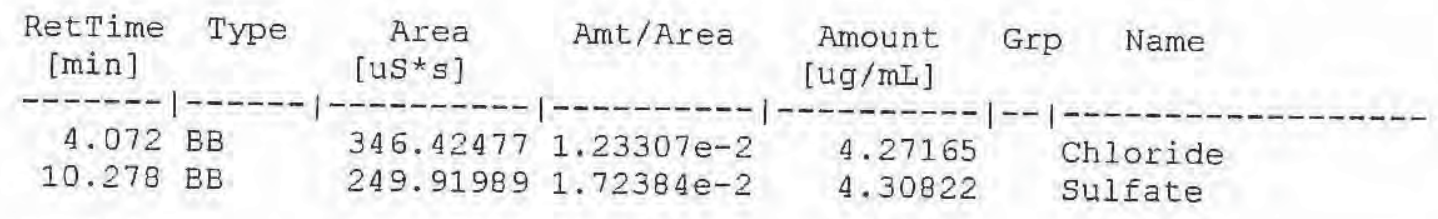

Totals :

8.57987

Results obtained with enhanced integrator!

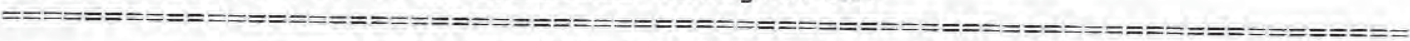

*** End of Report *** 


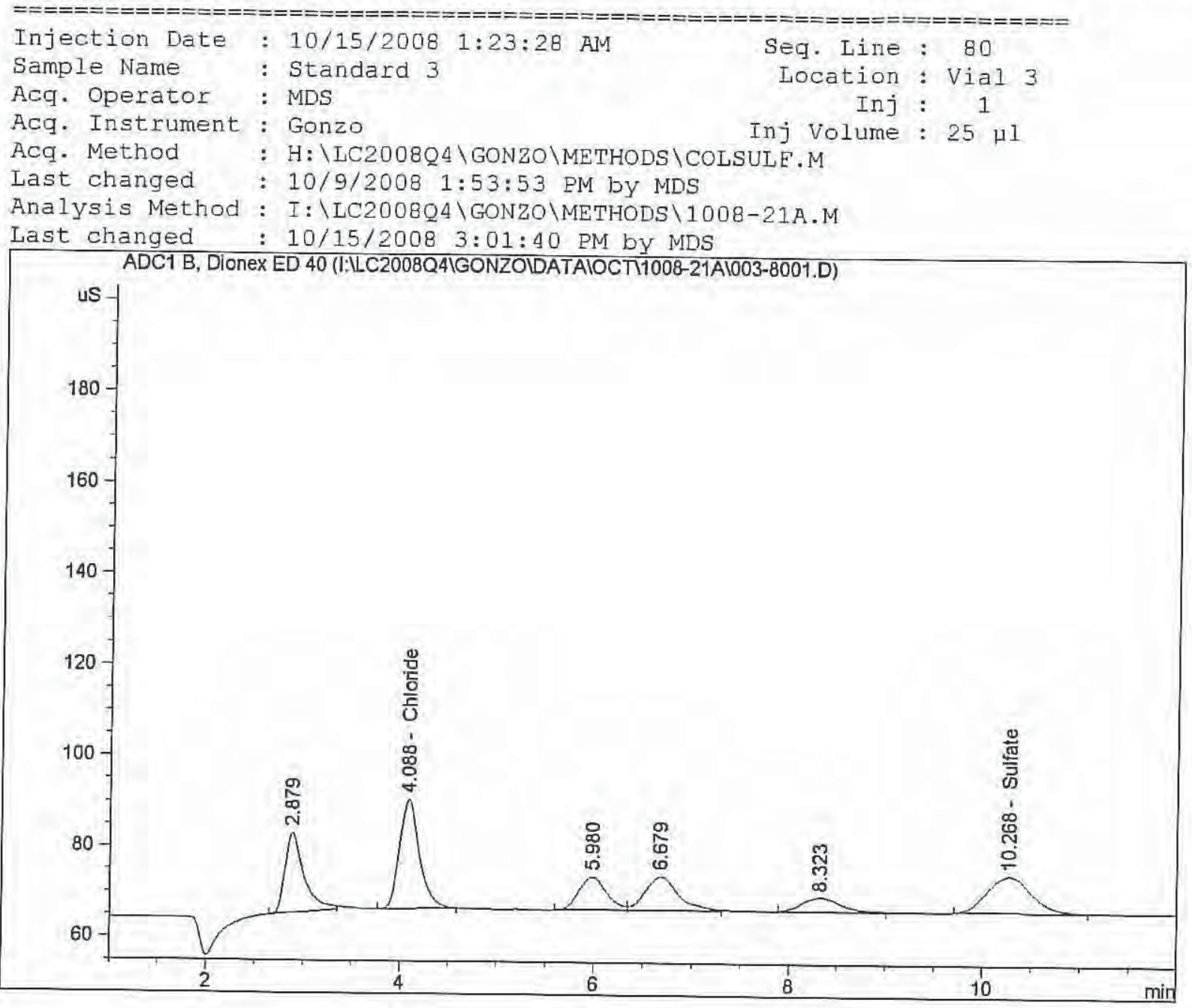

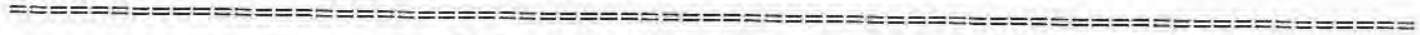
External Standard Report

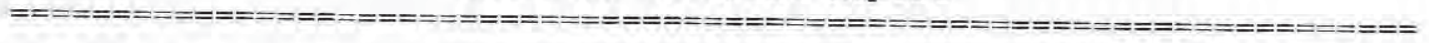

Sorted By

Signal

Calib. Data Modified : Wednesday, October 15, 2008 3:01:39 PM

Multiplier : 1.0000

Dilution : $\quad 1,0000$

Use Multiplier \& Dilution Factor with ISTDS

Signal 1: ADC1 B, Dionex ED 40

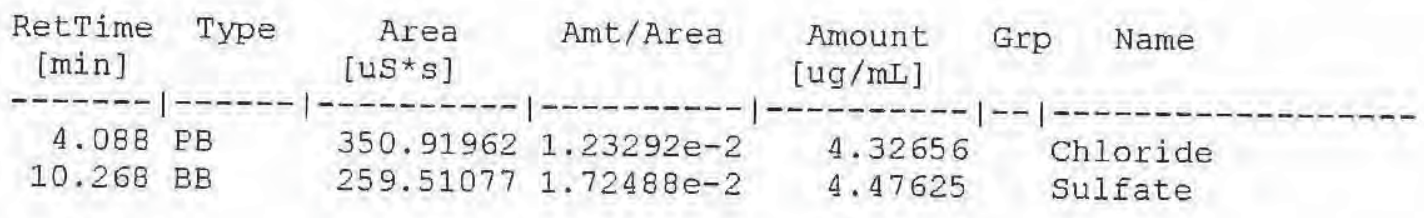

Totals :

8.80281

Results obtained with enhanced integrator!

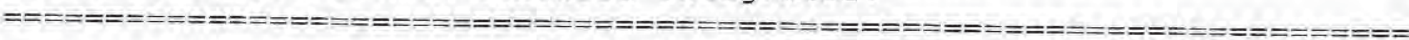
$\star * *$ End of Report $* * *$
} 

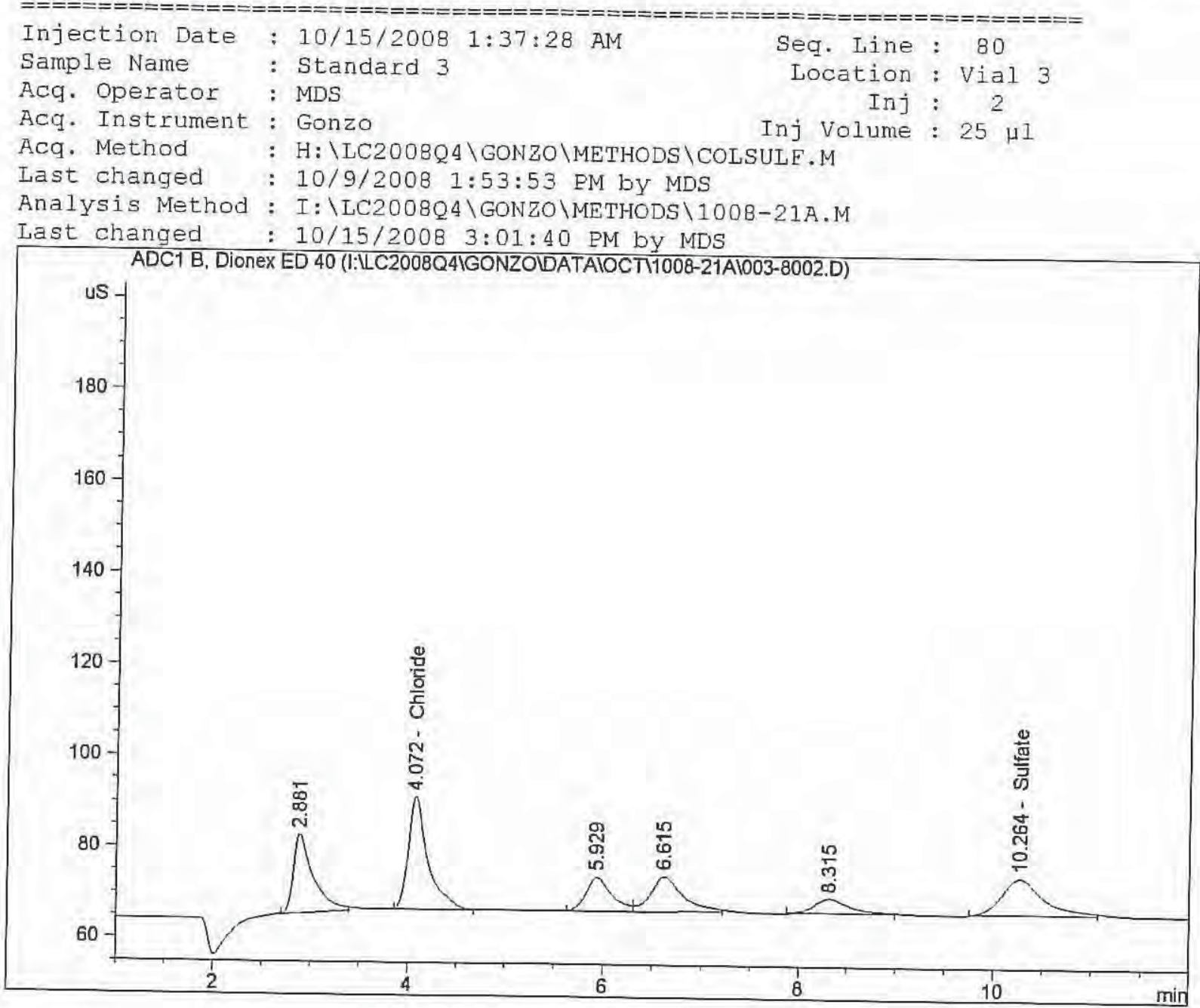

External Standard Report

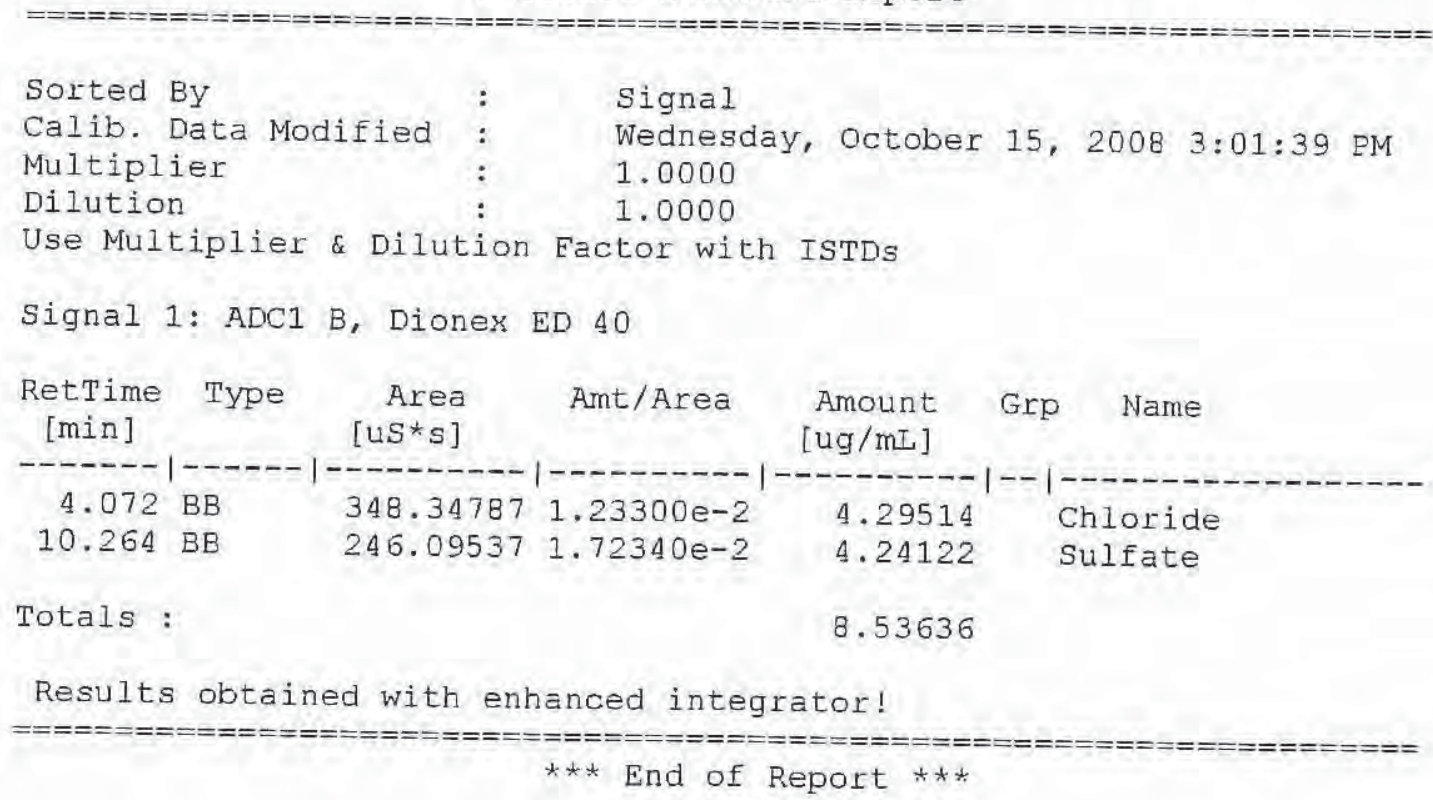

Results obtained with enhanced integrator! 


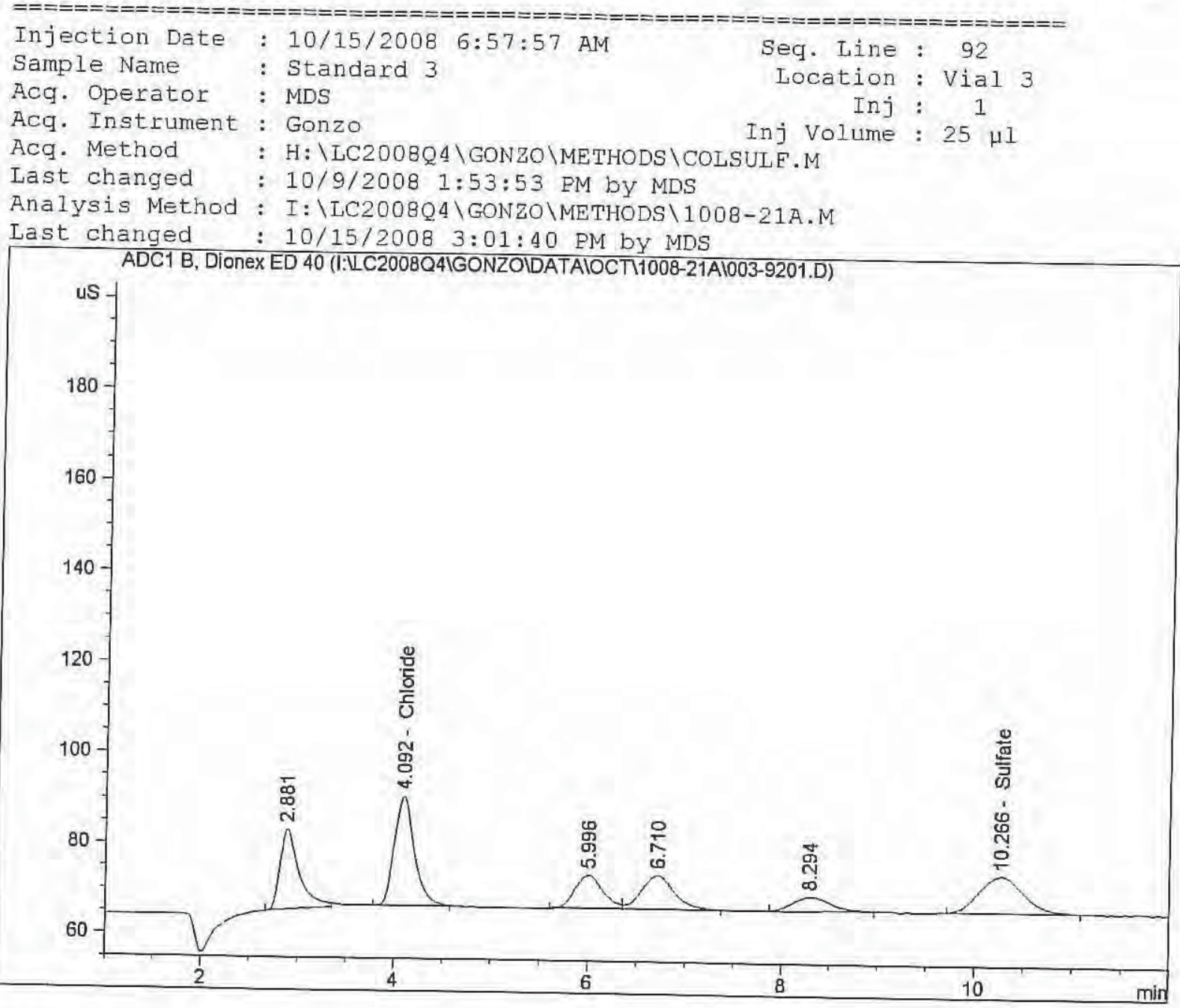

External Standard Report

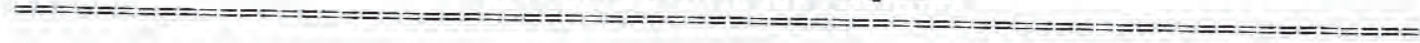

Sorted By

Calib. Data Modified: Signal

Multiplier : : 1.0000

Dilution : 1.0000

Use Multiplier \& Dilution Factor with ISTDS

Signal 1: ADC1 B, Dionex ED 40

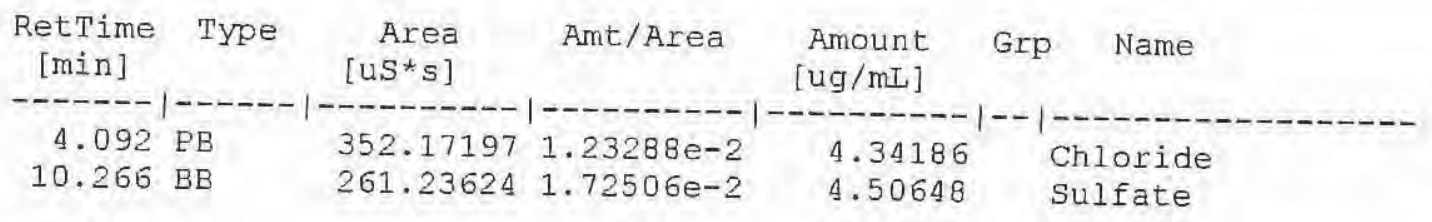

Totals :

8.84834

Results obtained with enhanced integrator!

$$
\star * * \text { End of Report *** }
$$



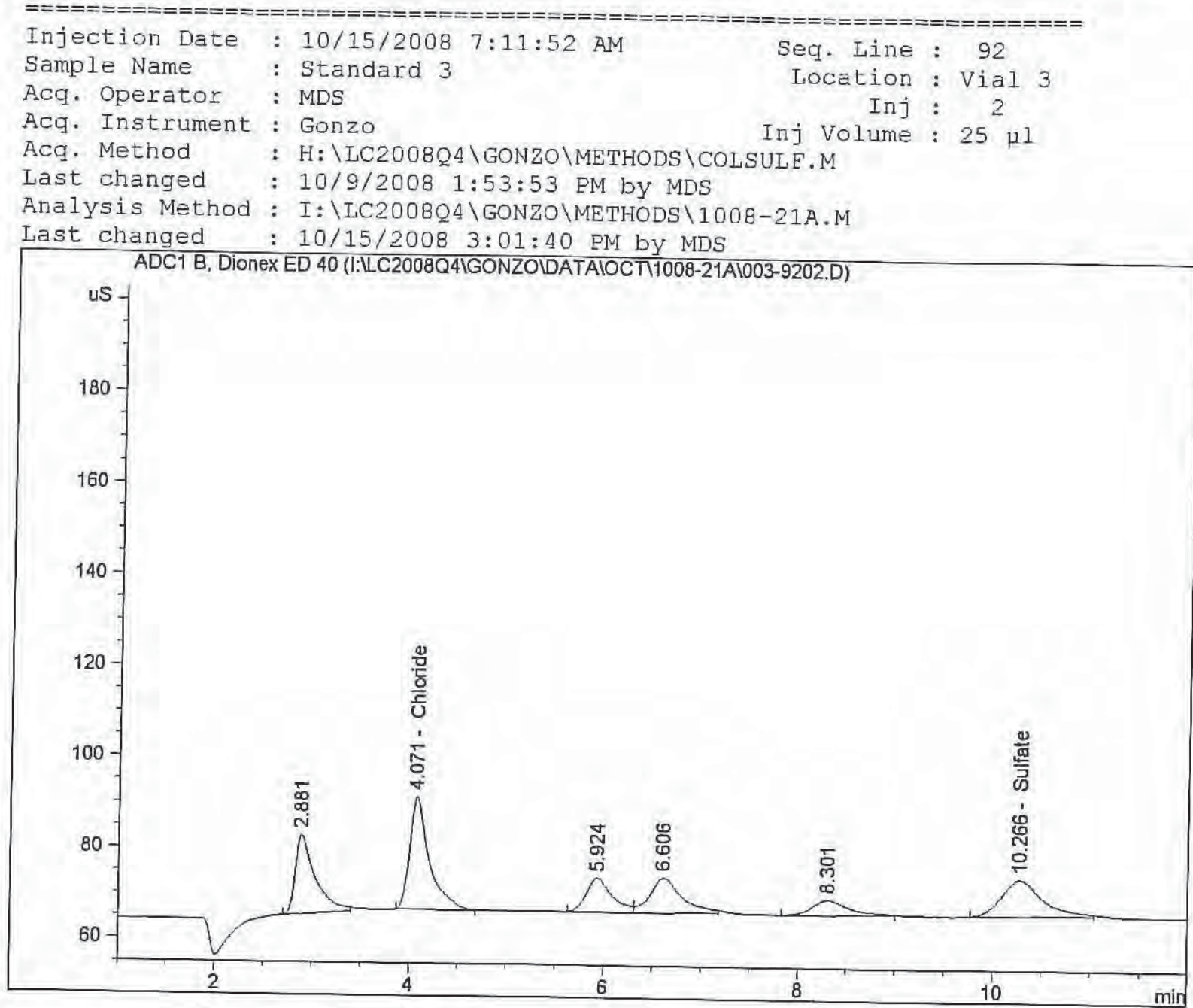

External Standard Report

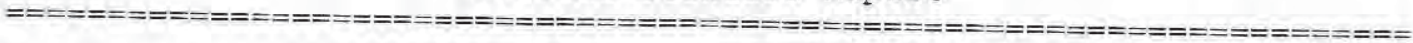

$\begin{array}{lll}\text { Sorted By } & : & \text { Signal } \\ \text { Calib. Data Modified } & : & \text { Wednesday, October 15, 2008 3:01:39 PM } \\ \text { Multiplier } & \vdots & 1.0000 \\ \text { Dilution } & \vdots & 1.0000\end{array}$

Use Multiplier \& Dilution Factor with ISTDS

Signal 1: ADC1 B, Dionex ED 40

\begin{tabular}{|c|c|c|c|c|c|c|}
\hline $\begin{array}{l}\text { RetTime } \\
\text { [min] }\end{array}$ & Type & $\begin{array}{r}\text { Area } \\
{\left[\mathrm{uS}^{*} \mathrm{~S}\right]}\end{array}$ & Amt/Area & $\begin{array}{l}\text { Amount } \\
{[\mathrm{ug} / \mathrm{mL}]}\end{array}$ & Grp & Name \\
\hline $\begin{array}{r}4.071 \\
10.266\end{array}$ & $\begin{array}{l}\mathrm{BB} \\
\mathrm{BB}\end{array}$ & $\begin{array}{l}351.14844 \\
247.36397\end{array}$ & $\begin{array}{l}1.23291 \mathrm{e}-2 \\
1.72355 \mathrm{e}-2\end{array}$ & $\begin{array}{l}4.32935 \\
4.26344\end{array}$ & & $\begin{array}{l}\text { Ioride } \\
\text { iffate }\end{array}$ \\
\hline
\end{tabular}

Totals:

8.59280

Results obtained with enhanced integrator!

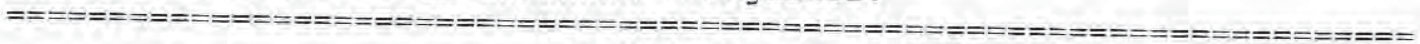
$* * *$ End of Report *** 
Method Information

Dionex IonPac AS-14A 4-mm analytical ( $4 \times 250 \mathrm{~mm})$ column.

Flow rate $1.2 \mathrm{~mL} / \mathrm{min}$

$8 \mathrm{mM}$ Na2 $\mathrm{CO} 3 / 1 \mathrm{mM}$ NaHCO3 mobile phase

Suppressed anion conductivity detection

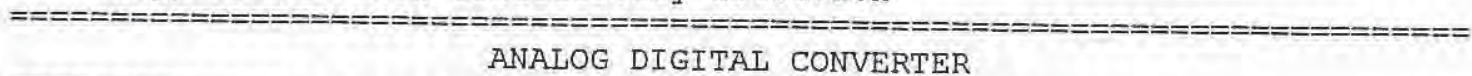

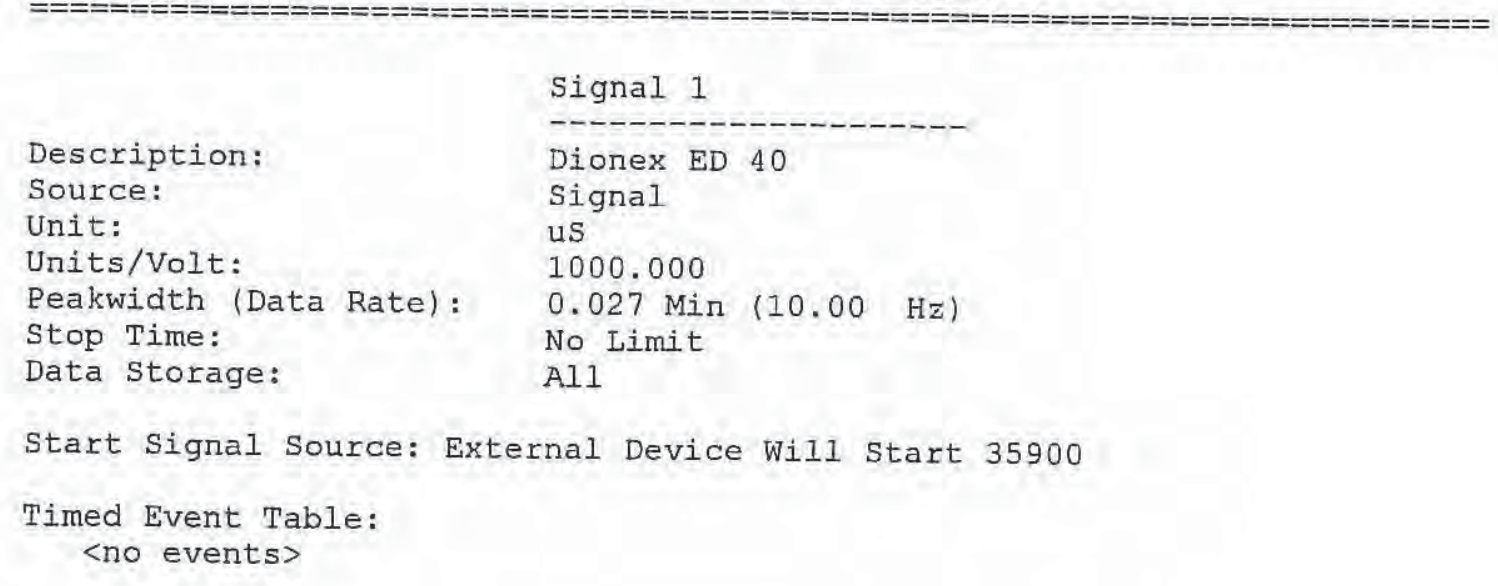




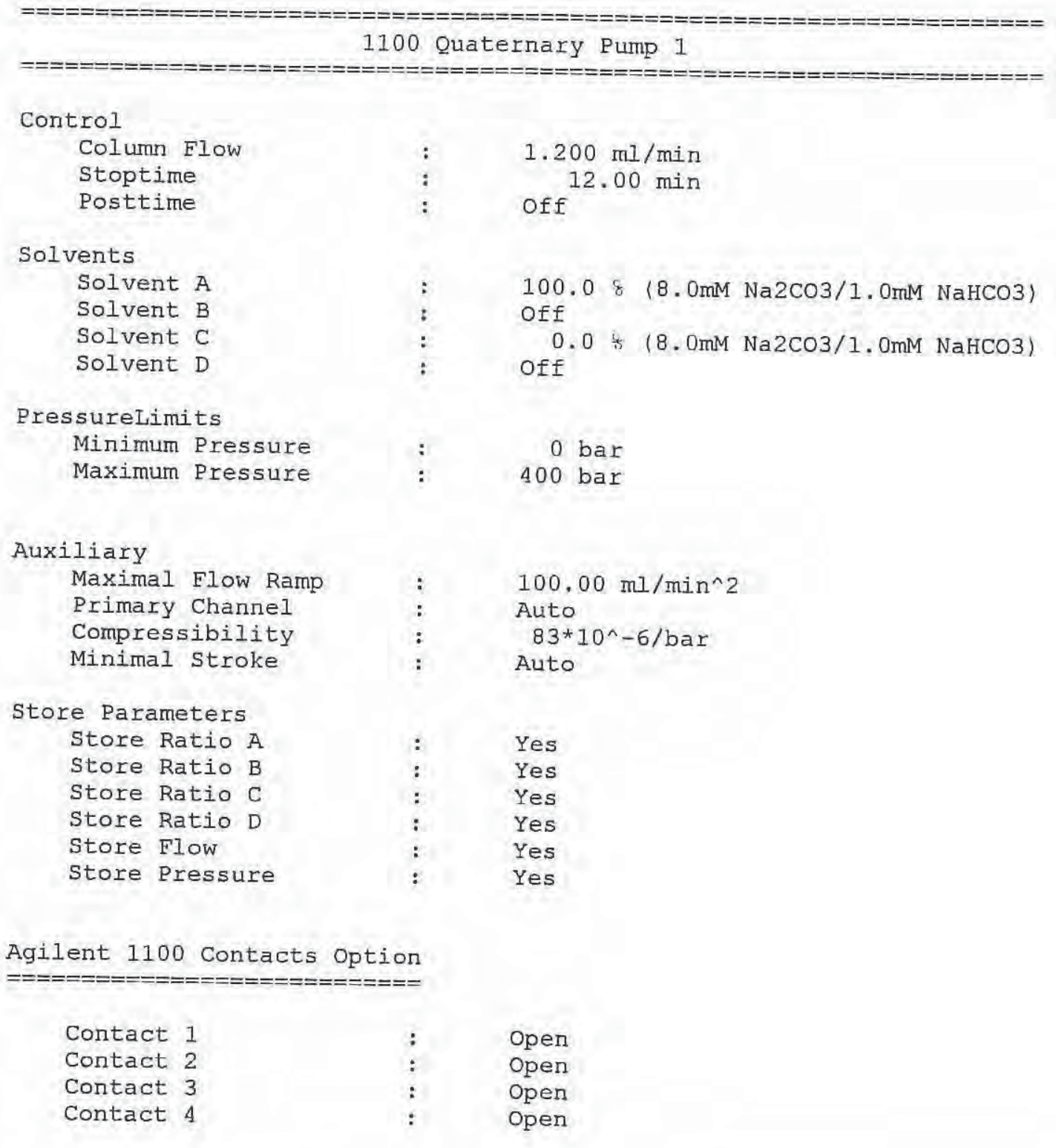

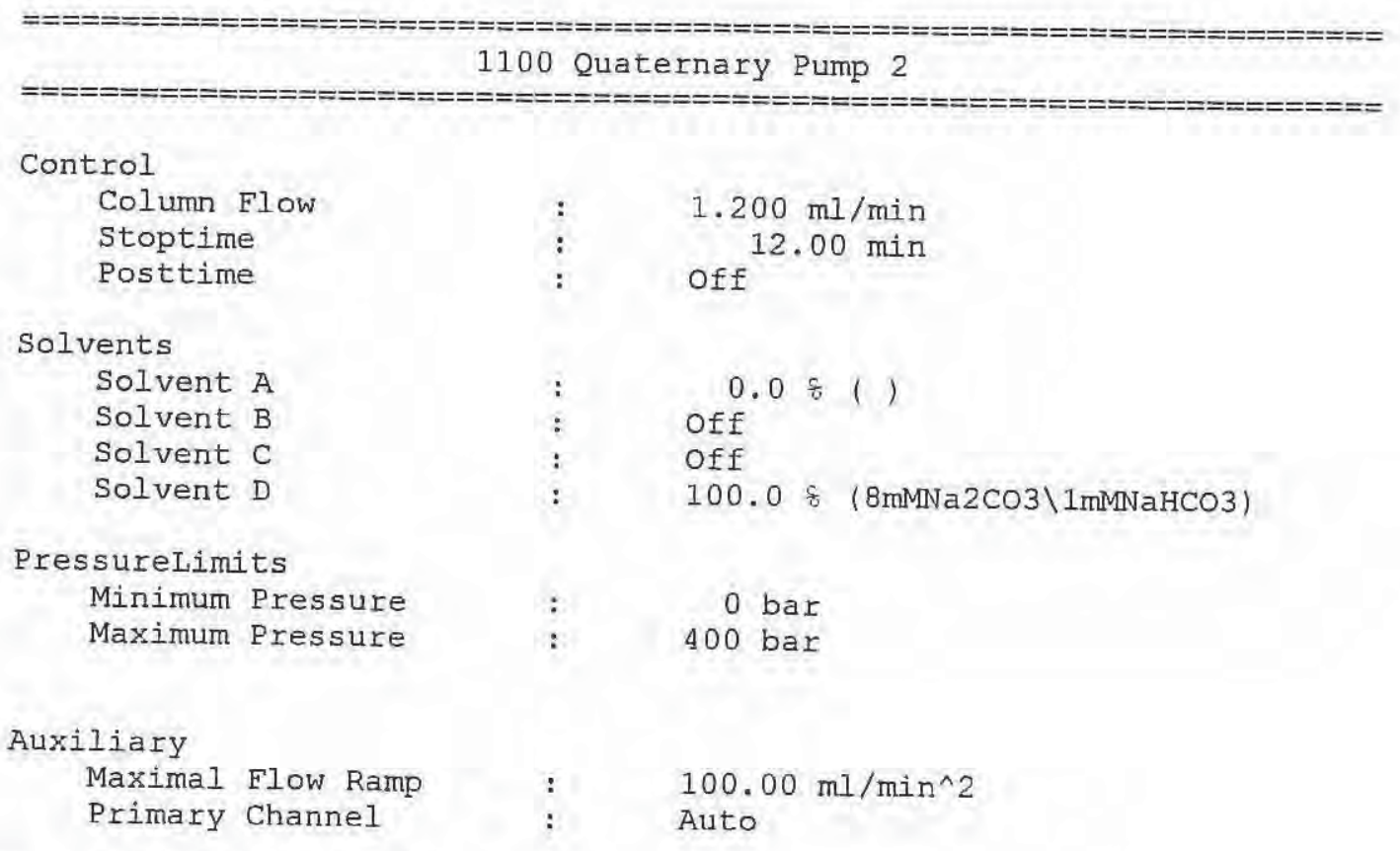


Method: H: \LC200BQ4\GONZO\METHODS\COLSULF.M of 10/9/2008 1:53:53 PM

$\begin{array}{lll}\text { Compressibility } & : & 100 * 10^{\wedge}-6 / \text { bar } \\ \text { Minimal Stroke } & \text { Auto }\end{array}$

Store Parameters

Store Ratio A : : Yes

Store Ratio B : : Yes

Store Ratio C : Yes

Store Ratio D : Yes

Store Flow : Yes

Store Pressure : Yes

Agilent 1100 Contacts Option

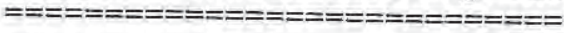

Contact 1

Contact 2

Contact 3

Contact 4

Open

Open

Open

Open

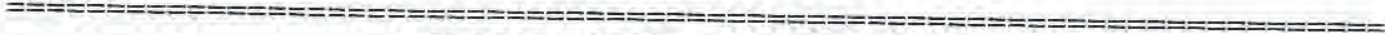

Agilent 1100 Autosampler 1
\end{abstract}

\begin{tabular}{|c|c|c|}
\hline \multicolumn{3}{|l|}{ Injection } \\
\hline Injection Mode & : & Needle Wash \\
\hline Injector volume & : & $25.00 \mu 1$ \\
\hline Wash Vial & : & 100 \\
\hline Optimization & : & none \\
\hline
\end{tabular}

Auxiliary

Drawspeed

Ejectspeed

Draw position

$100 \mu 1 / \mathrm{min}$
$1000 \mu \mathrm{m} / \mathrm{min}$
$2.0 \mathrm{~m}$

Time

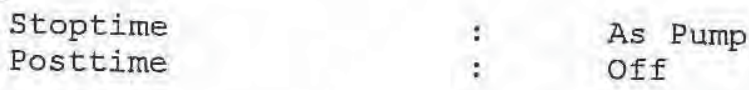

Agilent 1100 Column Thermostat 1

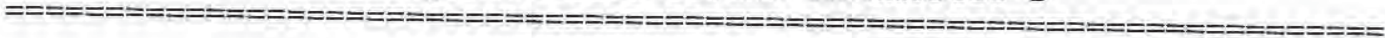

Temperature settings

Left temperature

Right temperature

Enable analysis

Store left temperature:

Store right temperature:

$30.0^{\circ} \mathrm{C}$

Same as left

When Temp. is within setpoint $+/-0.8^{\circ} \mathrm{C}$

No

No

Time

Stoptime

Posttime

As pump

Column Switching Valve

Off

Use current 
Sequence Table:

Method and Injection Info Part:

Iine Location SampleName

$== \pm======$
Method Inj SampleType InjVolume DataFile

1 Vial 1

Standard 2

Standard 3

Standard 4

Standard 5

Standard 6

Second Source

DI H2O Lab Blank

KR6521-1

KR6521-2

KR6521-3

MS/KR6521-1

In RI $\mathrm{C} 2 * 20$

In $\mathrm{R} 2 \quad \mathrm{C} 2 * 100$

In R3 $\mathrm{C} 2 * 10$

Stk R1 C2*10

Stk R2 C $2 * 10$

Stk R3 C2* 10

standard 3

Standard 4

In $\mathrm{C} 4$

Vial 4

Vial 21

Vial 22

Vial 23

Vial 24

Vial 25

Stk C4

$\mathrm{MS} / \mathrm{In} \mathrm{R} 3 \mathrm{C} 2$

$\mathrm{U} 4-\mathrm{E} 1-1 * 10$

U $4-E 1-1 * 10$

Vial 26 U4-E $1-2 * 100$

Vial 27

$\mathrm{U} 4-\mathrm{EI}-2 * 100$

Vial 28

$\mathrm{U} 4-\mathrm{E} 1-3 * 100$

$\mathrm{U} 4-\mathrm{E} 1-4 * 100$

$\mathrm{U} 4-\mathrm{E} 1-8 * 100$

Vial 30

Vial 3

Standard 3

Standard 4

Vial 4

$\mathrm{U} 4-\mathrm{E} 1-16 * 100$

$U 4-E 2-2 * 100$

$\mathrm{U} 4-\mathrm{E} 2-3 * 20$

$\mathrm{U} 4-\mathrm{E} 2-4$ * 100

$\mathrm{U} 4-\mathrm{E} 2-4 * 100$

$\mathrm{U} 4-\mathrm{E} 2-8 * 20$

$\mathrm{U} 4-\mathrm{E} 2-15 * 100$

$\mathrm{U} 4-\mathrm{E} 3-2 * 10$

$\mathrm{U} 4-\mathrm{E} 3-3 * 10$

U $4-E 4-1 * 10$

Standard 3

Standard 4

U $4-W 3-3 * 100$

U4 $-W 4-1100$

U $4-W 4-2 * 100$

MSI/U 4-E1-3

U 4-EI-1-4* 100

U $4-E 1-1-4 * 100$

U $4-E 1-1-4 * 100$

$\mathrm{U} 4-\mathrm{E} 1-1-4 * 100$

Second source

$\mathrm{U} 4-\mathrm{E} 1-1-6 * 100$

$\mathrm{U} 4-\mathrm{E} 1-5-6 * 100$

Standard 3

Standard 4

$\mathrm{U} 4-\mathrm{E} 1-7-9 * 100$

$\mathrm{U} 4-\mathrm{E} 1-7-12 * 100$

$\mathrm{U} 4-\mathrm{E} 1-13-15 * 100$
$======$

COLSULF 2 sample

COLSULF 2 Sample

COLSULF 2 Sample

COLSULF 2 Sample

COLSULE 2 Sample

COLSUlF 2 sample

COLSUlF 2 Sample

Sample

Sample

Sample

Sample

Sample

Sample

Sample

Sample

Sample

Sample

Sample

Sample

Sample

Sample

Sample

Sample

Sample

Sample

Sample

Sample

Sample

Sample

Sample

Sample

Sample

Sample

Sample

Sample

Sample

Sample

Sample

Sample

Sample

Sample

Sample

Sample

Sample

Sample

Sample

Sample

Sample

Sample

Sample

Sample

Sample

Sample

Sample

Sample

Sample

Sample

Sample

Sample

Sample 
Sequence: H: \LC2008Q4 \GONZO\SEQUENCE \1008-21A.S

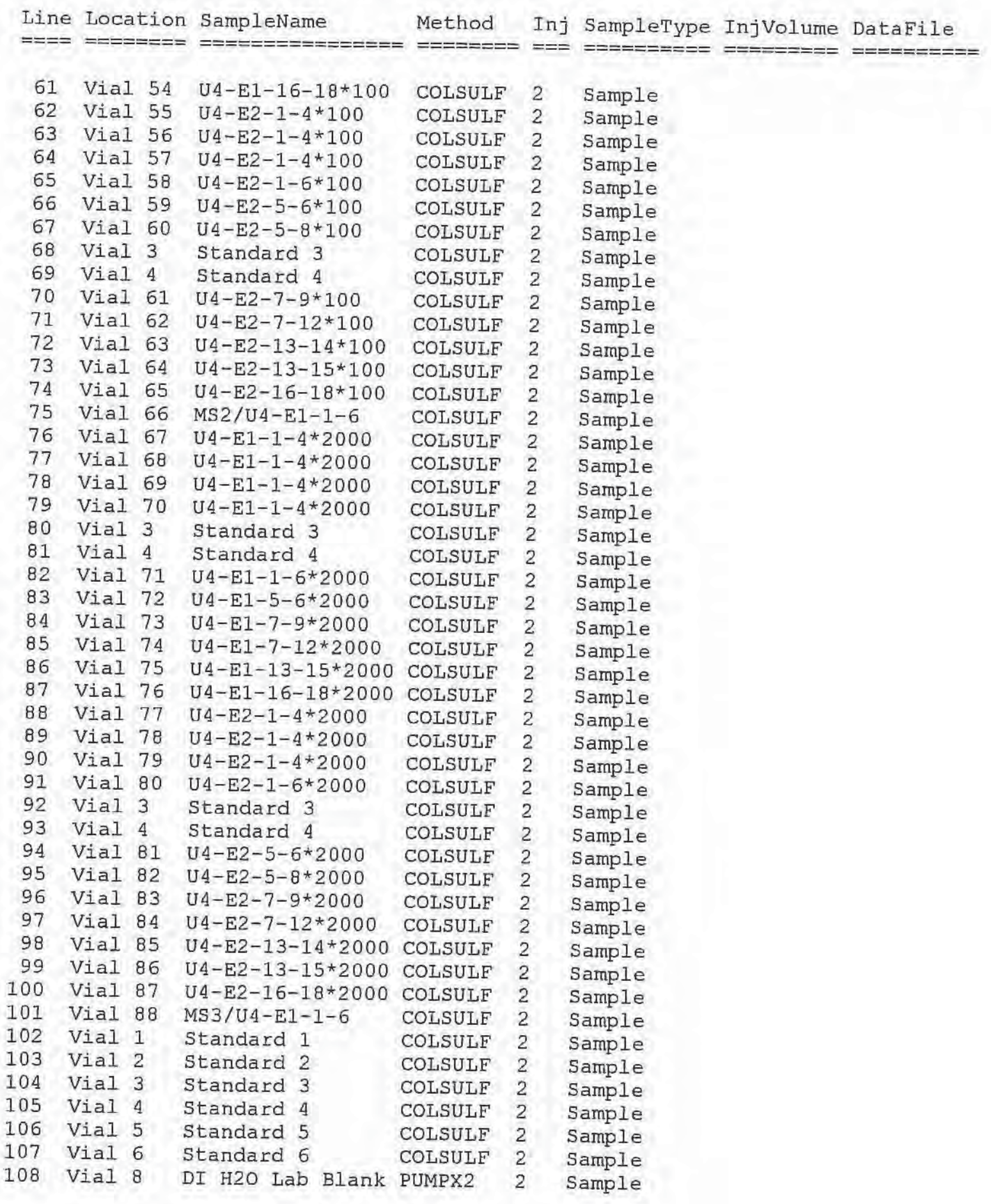




\section{This Is The Last Page Of This Report.}

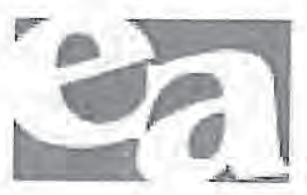




\section{Appendix G:}

\section{EPA Test Method 17 (Particulate Matter) Analytical Data - SDA Inlet} Duct 
Air Sampling Associates, Inc.

\section{Particulate Analysis Summary \\ EPA Method 17}

Project Number: $08-026$

Date Analysis Completed: $10 / / 4 / 08$

Project Name: ADA-ES

Unit Tested: SDA Inlet Duct

Project Location: Hardin, MT

Run Number:

Particulate Matter on Filter (mg):

Particulate Matter in Front Wash (mg):

Total Particulate Matter in "Front-Half" - MF (mg):

Particulate Matter in "Back-Half" (mg):

Total Particulate Matter in Sample - MT (mg):
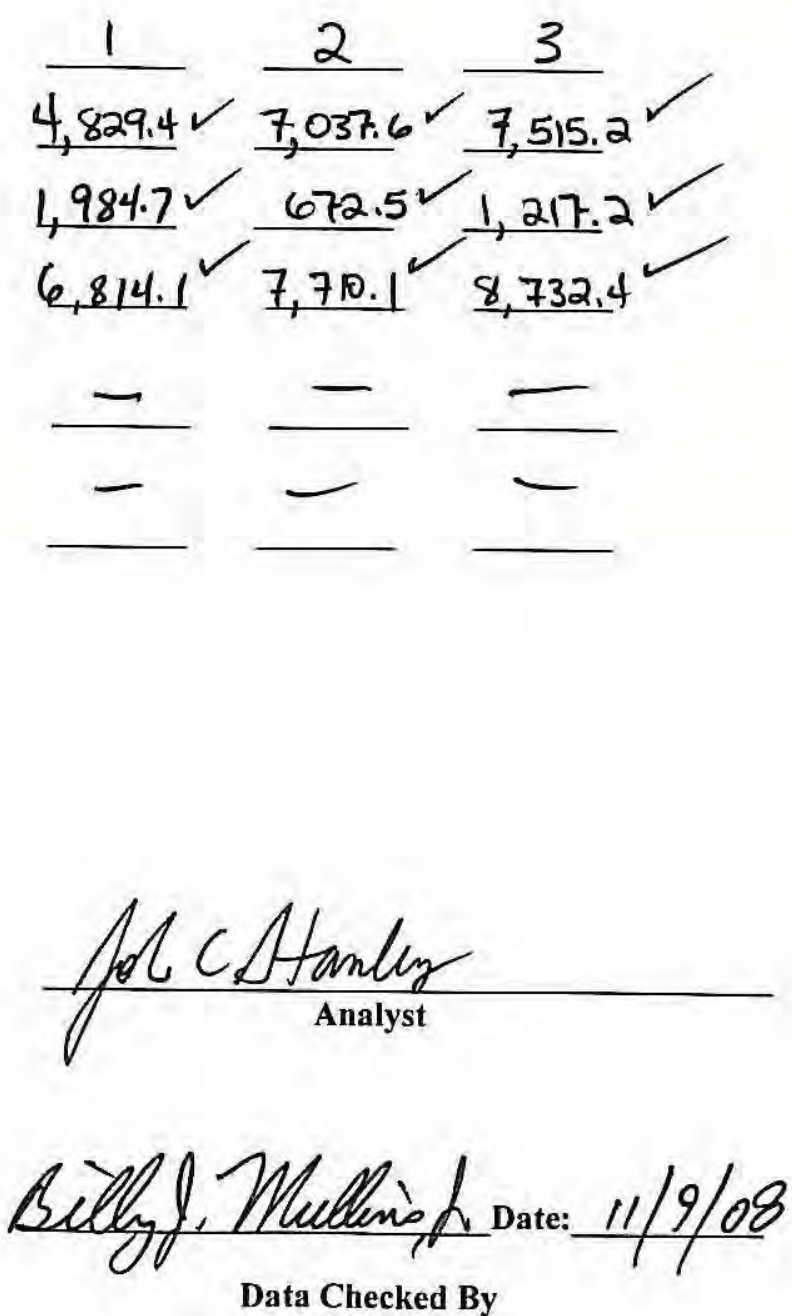

Data Checked By

Version No. 1 5/30/06 


\section{Particulate Analysis EPA Method 17}

\section{Stack Filters}

Project No. $08-026$

Project Name ADA.ES
Location Unit Tested

$\frac{\text { Hardin, } M T}{\text { SDA Inlet Duct }}$

\begin{tabular}{|c|cc|cc|cc|cc|c|}
\hline Desiccator Time In & 10.1 .08 & 0900 & 10.3 .08 & 0911 & $10-6.08$ & 0930 & $10 \cdot 10.08$ & 1020 & \\
\hline Desiccator Time Out & 10.3 .08 & 0907 & 10.6 .08 & 0900 & 10.10 .08 & 0900 & 10.13 .08 & 1350 & \\
\hline
\end{tabular}

\begin{tabular}{|l|l|l|l|l|l|}
\hline \multicolumn{2}{|c|}{ Run No. I I I ter No. Thimble 58 Sample I.D. } \\
\hline Filter \& Particulate (g) & 8.2994 & 8.2955 & 8.2950 & \\
\hline
\end{tabular}

Filter \& Particulate Average (g) 8.2950 Initial Filter Weight $(\mathrm{g}) 3.4656$ Total Particulate $(\mathrm{mg}) \quad 4,829.4$

\begin{tabular}{|c|c|c|c|c|}
\hline \multicolumn{2}{|c|}{ Run No. 2} & Filter No. Thim 66e 65 Sample I.D. \\
\hline Filter \& Particulate (g) & 9.6750 & 9.6759 & 9.6757 & \\
\hline
\end{tabular}

Filter \& Particulate Average (g) 9.6757 Initial Filter Weight (g) Total Particulate $(\mathrm{mg}) \quad 7,037.6$ $2.6381 V$

Run No. 3 Filter \& Particulate (g)
9.9758
Filter No. Thimble 9.9693

9.966

Sample I.D.

9.9670

Filter \& Particulate Average $(\mathrm{g})$ $\frac{9.9670 V}{\frac{2.4518}{7,515.2 \%}}$ Initial Filter Weight $(\mathrm{g}) \quad 2.4518$ Total Particulate $(\mathrm{mg})$

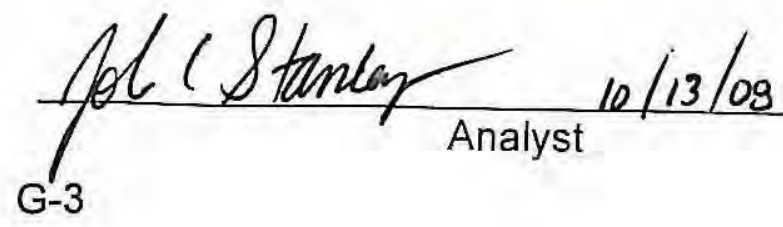




\section{III \\ SAMPLING \\ ASSOCIATES, INC.}

Thimble Tare Weight Log

EPA Method 17

\begin{tabular}{|c|c|c|c|c|c|c|c|}
\hline \multirow[b]{3}{*}{ Filter No. } & Into Desicator & Weight & Weight & Weight & Weight & Weight & \multirow{3}{*}{$\begin{array}{l}\text { Weight } \\
\text { Used }\end{array}$} \\
\hline & Date & Date & Date & Date & Date & Date & \\
\hline & Time & Time & \begin{tabular}{|l|} 
Time \\
\end{tabular} & Time & Time & Time & \\
\hline \multirow{3}{*}{45} & & 2.7 .941 & 2.7944 & & & & $2.79+4$ \\
\hline & 9129100 & $1012 / 0$ & $10 / 3104$ & & & & \\
\hline & 1200 & 1635 & 1100 & & & & \\
\hline \multirow{3}{*}{46} & 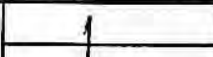 & 2.7987 & 2.7990 & & & & 2.7990 \\
\hline & & $10 / 2 / 06$ & $10 / 3 / 04$ & & & & \\
\hline & & 1636 & 1101 & & & & \\
\hline \multirow{3}{*}{47} & & 2,5514 & 2.55147 & & & & 2.5514 \\
\hline & & $10 / 2 / 06$ & 1013106 & & & & \\
\hline & & 1637 & 1102 & & & & \\
\hline \multirow{3}{*}{48} & & 2.7781 & 2.77857 & & & & 2.7785 \\
\hline & & $10 / 2 / 06$ & $10 / 3106$ & & & & \\
\hline & & 1638 & 1103 & & & & \\
\hline \multirow{3}{*}{49} & & 2.7795 & 2,7799 & & & & 2.7799 \\
\hline & & $10 / 2 / 06$ & $10 / 3106$ & & & & \\
\hline & & 1639 & \begin{tabular}{|l|l|}
1104 \\
\end{tabular} & & & & \\
\hline \multirow{3}{*}{50} & & 2.6485 & 2.6487 & & & & 2.6487 \\
\hline & & $10 / 2 / 06$ & 1013106 & & & & \\
\hline & & 1640 & 1105 & & & & \\
\hline \multirow{3}{*}{51} & & 2.8600 & 2.8605 & & & & 2.8605 \\
\hline & & $10 / 2106$ & 1013106 & & & & \\
\hline & & 1641 & 1106 & & & & \\
\hline \multirow{3}{*}{52} & & 2.8470 & 2.8474 & & & & 2.8474 \\
\hline & & $10 / 2 / 06$ & $10 / 3 / 04$ & & & & \\
\hline & & 1642 & 1107 & & & & \\
\hline \multirow{3}{*}{53} & & 2,7349 & 2.7352 & E & & & 2.7352 \\
\hline & t & $10 / 2 / 06$ & 1013106 & & & & \\
\hline & & 1643 & 408 & & & & \\
\hline \multirow{3}{*}{54} & & 3.5794 & 3,5795 & $\angle$ & & & 3,5795 \\
\hline & 11) 20106 & $11 / 21 / \alpha_{6}$ & 11/22/06 & & & & \\
\hline & 1360 & 1350 & 6745 & & & & \\
\hline \multirow{3}{*}{55} & & 2.8785 & 2,8788 & $\angle$ & & & 2.8788 \\
\hline & & $11 / 21 / 06$ & $14 / 22 / 06$ & & & & \\
\hline & & $|35|$ & $07+6$ & & & & \\
\hline \multirow{3}{*}{56} & & 2.4725 & 2.9724 & E & & & 2.4724 \\
\hline & & $11 / 21 / 06$ & $11 / 22 / 06$ & & & & \\
\hline & & 1352 & 6747 & & & & \\
\hline \multirow{2}{*}{57} & & $2.99 ; 6$ & 2.99114 & E & & & 2.9911 \\
\hline & & $\frac{11 / 21 / 06}{1253}$ & $11 / 22 / 06$ & & & & \\
\hline \multirow{3}{*}{58} & & 3.4660 & $\frac{0746}{3.46567}$ & & & & 3,4656 \\
\hline & & $11 / 21 / 06$ & $11 / 22 / 06$ & & & & \\
\hline & & 1354 & 0749 & & & & \\
\hline \multirow{3}{*}{59} & & 3,1165 & 3.1160 & E & & & 3.1160 \\
\hline & & $11 / 21 / 06$ & $11 / 22 / 106$ & & & & \\
\hline & $\underline{4}$ & 1355 & 16750 & & & & \\
\hline
\end{tabular}




\section{AIR}

SAMPLING

ASSOCIATES, INC.

Thimble Tare Weight Log

EPA Method 17

\begin{tabular}{|c|c|c|c|c|c|c|c|}
\hline \multirow[b]{3}{*}{ Filter No. } & Into Desicator & Weight & Weight & Weight & Weight & Weight & \multirow{3}{*}{$\begin{array}{l}\text { Weight } \\
\text { Used }\end{array}$} \\
\hline & Date & Date & Date & Date & Date & Date & \\
\hline & Time & Time & Time & Time & Time & Time & \\
\hline \multirow{3}{*}{60} & & 2.7215 & 2,7210 & 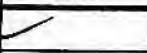 & & & 2.7210 \\
\hline & $11 / 20 / 06$ & $11 / 21 / 06$ & $1 / 122 / 86$ & & & & \\
\hline & $1 / 1300$ & 1358 & 0752 & & & & \\
\hline \multirow{3}{*}{61} & & 2.7531 & 2,75304 & $F$ & & & 2.7530 \\
\hline & $11 / 20 / 06$ & $11 / 21 / 06$ & $11 / 22 / 06$ & & & & \\
\hline & 1300 & 1359 & 02531 & & & & \\
\hline \multirow{3}{*}{62} & & 2,7516 & 2.7514 & $=$ & & & 2.7514 \\
\hline & $11 / 20 / 06$ & $11 / 21 / 06$ & $(1 / 22 / 06$ & & & & \\
\hline & $1 / 360$ & 1400 & 0754 & & & & \\
\hline \multirow{3}{*}{63} & & 2.7876 & 2,78484 & 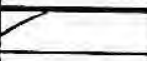 & & & 2.7848 \\
\hline & $11 / 20 / 06$ & $11 / 21 / 06$ & $11 / 22 / 06$ & & & & \\
\hline & $\angle 300$ & 1401 & 0755 & & & & \\
\hline \multirow{3}{*}{64} & & 2.9993 & 2.9992 & $\approx$ & & & 2.9992 \\
\hline & $11 / 20 / 06$ & $11 / 21 / 06$ & $11 / 22 / 06$ & & & & \\
\hline & 1360 & 1402 & 0756 & & & & \\
\hline \multirow{3}{*}{65} & & 2.6385 & 2.63814 & 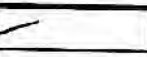 & & & 2.6381 \\
\hline & $11 / 20 / 06$ & $\mid 1 / 21 / 06$ & $11 / 2 z 106$ & & & & \\
\hline & 1360 & 1403 & 6757 & & & & \\
\hline \multirow{3}{*}{66} & & 2.8663 & 2,86604 & $=$ & & & 2.8660 \\
\hline & $11 / 20 / 06$ & $11 / 21 / 06$ & $11 / 22 / \alpha$ & & & & \\
\hline & 1300 & 1404 & 6860 & & & & \\
\hline \multirow{3}{*}{67} & & 2.9442 & 2.94394 & 7 & & & 2,9439 \\
\hline & $11 / 20 / 06$ & $11 / 21 / 06$ & $11 / 22 / 06$ & & & & \\
\hline & 1300 & 1404 & 0801 & & & & \\
\hline \multirow{3}{*}{68} & & 2.5183 & 2.5182 & $\angle$ & & & 2.5182 \\
\hline & $11 / 20 / 06$ & $11 / 2106$ & $11 / 22 / 06$ & & & & \\
\hline & 1300 & 1405 & 0802 & & & & \\
\hline \multirow{3}{*}{69} & & 2,7293 & 2.72914 & 7 & & & 2,729 \\
\hline & $11 / 20 / 06$ & $11 / 21 / 06$ & $11 / 22 / 06$ & & & & \\
\hline & 1300 & 1406 & 6803 & & & & \\
\hline \multirow{3}{*}{70} & & 2,5250 & 2.52484 & 5 & & & 2,5248 \\
\hline & $11 / 20 / 06$ & $11 / 2106$ & $11 / 22 / 06$ & & & & \\
\hline & $1 / 360$ & 1407 & 0804 & & & & \\
\hline \multirow{3}{*}{71} & & 2.4725 & 2.47214 & & & & 2.4721 \\
\hline & $1 / / 20 / 06$ & $1 /\left.2\right|_{\infty}$ & $11 / 22 / 06$ & & & & \\
\hline & 1300 & 1408 & 2805 & & & & \\
\hline \multirow{3}{*}{72} & & 2.7991 & 2,79884 & ᄃ & & & 2.7988 \\
\hline & $11 / 20 / 06$ & $11 / 21 / 06$ & $11 / 22 / 06$ & & & & \\
\hline & $1 / 300$ & 1409 & 0806 & & & & \\
\hline \multirow{3}{*}{73} & & 3.1339 & 3,13364 & 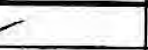 & & & 3.1336 \\
\hline & $11 / 20 / 06$ & $11 / 21 / 6$ & $11 / 22 / 06$ & & & & \\
\hline & 1300 & 1410 & 0807 & & & $=$ & \\
\hline \multirow{3}{*}{74} & & 2.8562 & 2.85614 & 7 & & & 2.8561 \\
\hline & $11 / 20 / 06$ & $11 / 21 / 06$ & $11 / 22 / 06$ & & & & \\
\hline & & $2 / 410$ & 0808 & & & & \\
\hline
\end{tabular}


ASSOCIATES, INC.

Thimble Tare Weight Log

EPA Method 17

\begin{tabular}{|c|c|c|c|c|c|c|c|}
\hline \multirow[b]{3}{*}{ Filter No. } & Into Desicator & Weight & Weight & Weight & Weight & Weight & \multirow{3}{*}{$\begin{array}{l}\text { Weight } \\
\text { Used }\end{array}$} \\
\hline & Date & Date & Date & Date & Date & Date & \\
\hline & Time & Time & Time & Time & Time & Time & \\
\hline \multirow{3}{*}{90} & & 2.2367 & 2.2364 & $\checkmark$ & & & 2.2364 \\
\hline & $8 / 10 / 07$ & $8 / 4 / 0)$ & $8 \longdiv { 1 3 \longdiv { 0 7 } }$ & & & & \\
\hline & 0700 & 0737 & 0805 & & & & \\
\hline \multirow{3}{*}{91} & & 2.8053 & 2.8050 & $\checkmark$ & & & 2.8050 \\
\hline & $18160(0)$ & $8 / 17 / 57$ & $8 / 13 / 07$ & & & & \\
\hline & 5700 & 0738 & 0807 & & & & \\
\hline \multirow{3}{*}{92} & & 2.3373 & 2.3369 & $<$ & & & 2.3369 \\
\hline & $8 / 10 / 57$ & $8 / 4 / \mathrm{m}$ & $8 / 13 / 07$ & & & & \\
\hline & 0700 & $074_{0}$ & 0810 & & & & \\
\hline \multirow{3}{*}{93} & & 2.2907 & 2.2904 & $\angle$ & & & 2.2904 \\
\hline & $8 / 10 / 27$ & $8 / 4 / 07$ & $8 / 13 / 07$ & & & & \\
\hline & 0700 & $0 J 41$ & 0812 & & & & \\
\hline \multirow{3}{*}{94} & 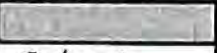 & 2.3437 & 2.3436 & $F$ & & & 2.3436 \\
\hline & 0600 & $8 / 13107$ & $8 / 13 / 08$ & & & & ${ }^{2}$ \\
\hline & $8 / 12107$ & 0800 & 1430 & & & & ( \\
\hline \multirow{3}{*}{95} & $x_{-1}$ & 2.4924 & 2.4926 & $F$ & & & 2.4724 \\
\hline & 0600 & 81 is lot & 8113107 & & & & \begin{tabular}{|l|l|}
$\pi$ \\
\end{tabular} \\
\hline & $8 / 12107$ & 0900 & 143,0 & & & & $\square$ \\
\hline \multirow{3}{*}{96} & & 2,4515 & 2.4518 & 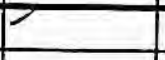 & & & 2.4518 \\
\hline & 0600 & 8113107 & $8 / 13107$ & & & & \\
\hline & 8112107 & 0800 & 1430 & & & & \\
\hline \multirow{3}{*}{97} & प1 & 2.4844 & 2.4846 & $E$ & & & 2.4846 \\
\hline & 0600 & 8113107 & $8 / .3 / 6^{2}$ & & & & \\
\hline & 8112107 & 0800 & 1430 & & $\zeta$ & & 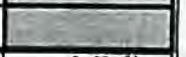 \\
\hline \multirow{3}{*}{98} & & $2.68+8$ & 2.6580 & 2.65814 & & & 2.6581 \\
\hline & 1155 & $6 / 5 / 08$ & & $6 / 6 / \Delta 8$ & & & \\
\hline & $5 / 580 / 08$ & +24906 & 80 & $124^{\circ}$ & & & \\
\hline \multirow{3}{*}{99} & & 2.3832 & 2.38354 & & & & 2.3835 \\
\hline & $5130 / 08$ & $6 / 6 / 08$ & $6 / 6 / 08$ & & & & \\
\hline & 1155 & 125306 & $\$ 0122$ & L & & & \\
\hline \multirow{3}{*}{100} & & 2.1985 & 2.19834 & & & & 2.1983 \\
\hline & $5 / 30 / 08$ & $6 / 6 / 08$ & $6 / 6 / 08$ & & & & \\
\hline & 1155 & 0633 & 1224 & & & & \\
\hline \multirow{3}{*}{101} & & 2.4363 & 2.4364 & & & & 2.4364 \\
\hline & $5 / 30 / 08$ & $6 / 6 / 08$ & $6 / 6 / 08$ & & & & \\
\hline & 1155 & 0635 & 1246 & & & & \\
\hline \multirow{3}{*}{102} & & 2.1281 & 2.12854 & & & & 2.1285 \\
\hline & 5130108 & $6 / 6 / 08$ & $6 / 6 / 08$ & & & & \\
\hline & 1155 & 0638 & 1248 & & & & \\
\hline \multirow{3}{*}{103} & & 2.1338 & $2.1340^{2}$ & & & & 2.1340 \\
\hline & 5130108 & $6 / 6 / 08$ & $6 / 608$ & & & & \\
\hline & 1155 & 8640 & 1250 & & & & \\
\hline \multirow{3}{*}{104} & & 2.5574 & 2.55734 & & & & 2.5573 \\
\hline & 5130108 & $6 / 6 / 08$ & $6 / 6 / 08$ & & & & \\
\hline & 1155 & 0644 & 1251 & & & & \\
\hline
\end{tabular}


Particulate Analysis EPA Method 17

Front Wash

Project No. $08 \cdot 026$

Project Name ADA-ES

Location

Hardin, $m T$

Unit Tested SOA Inlef Duct

\begin{tabular}{l|ll|ll|lll} 
Desiccator Time In & 9.7 .08 & 0900 & 10.10 .08 & 1000 & 10.13 .08 & 1400
\end{tabular}

\begin{tabular}{l|lllllll} 
Desiccator Time Out & $10 \cdot 10.08$ & 0930 & $10 \cdot 13.08$ & 1350 & $10 \cdot 14.08$ & 1434
\end{tabular}

\begin{tabular}{|c|c|c|c|c|}
\hline Run No. 1 & & Volume (ml) & 230 & Sample I.D. \\
\hline Final Weight (g) & 107.4009 & 107.4031 & $107.4035 \mathrm{~V}$ & \\
\hline Initial Weight (g) & & tos. 418 , & 105.418, & \\
\hline Particulate Weight (g) & & +9850 & 1.9854 & \\
\hline
\end{tabular}

Particulate Average $(\mathrm{mg})$

Less Acetone Blank (mg)

Total Particulate $(\mathrm{mg})$

$1,985.4$

0.7

\begin{tabular}{|c|l|l|l|l|}
\hline \multicolumn{2}{|c|}{ Run No. 2 } & \multicolumn{1}{|c|}{ Sample I.D. } \\
\hline Final Weight (g) & $1 / 3.90 / 5$ & $1 / 3.90 / 7$ & & \\
\hline Initial Weight (g) & & $1 / 3.2288$ & & \\
\hline Particulate Weight (g) & & 0.6729 & & \\
\hline
\end{tabular}

Particulate Average (mg)

Less Acetone Blank (mg)

Total Particulate $(\mathrm{mg})$

672.9

$\frac{0.4}{672.5}$

\begin{tabular}{|c|c|c|c|}
\hline Run No. 3 & & Volume $(\mathrm{ml}) \quad 104$ & Sample I.D. \\
\hline Final Weight $(\mathrm{g})$ & 113.7768 & $113.7768 \psi$ & \\
\hline Initial Weight (g) & & 112.5589 & \\
\hline Particulate Weight $(\mathrm{g})$ & & 1.2190 & \\
\hline
\end{tabular}

Particulate Average (mg)

Less Acetone Blank (mg)

Total Particulate $(\mathrm{mg})$

0.3

1217.7

\begin{tabular}{|c|c|c|c|c|}
\hline Acetone Blank & & Volume (ml) 206 & Sample I.D. & \\
\hline Final Weight $(\mathrm{g})$ & 113.9258 & $113.9254 \measuredangle$ & & \\
\hline Initial Weight $(\mathrm{g})$ & & $113.9248 \mathrm{~V}$ & & \\
\hline Particulate Weight (g) & & 0.0006 & & \\
\hline te. if creat & & & $\begin{array}{r}\text { Average (mg) } \\
* \mathrm{mg} / \mathrm{l}\end{array}$ & $\frac{0.6 V}{2.9 V}$ \\
\hline
\end{tabular}

* Note: if greater than $7.9 \mathrm{mg} / \mathrm{l}$, use $7.9 \mathrm{mg} / \mathrm{l}$ 


\section{II}

Particulate Analysis EPA Method 17

Front Half Tare Weights

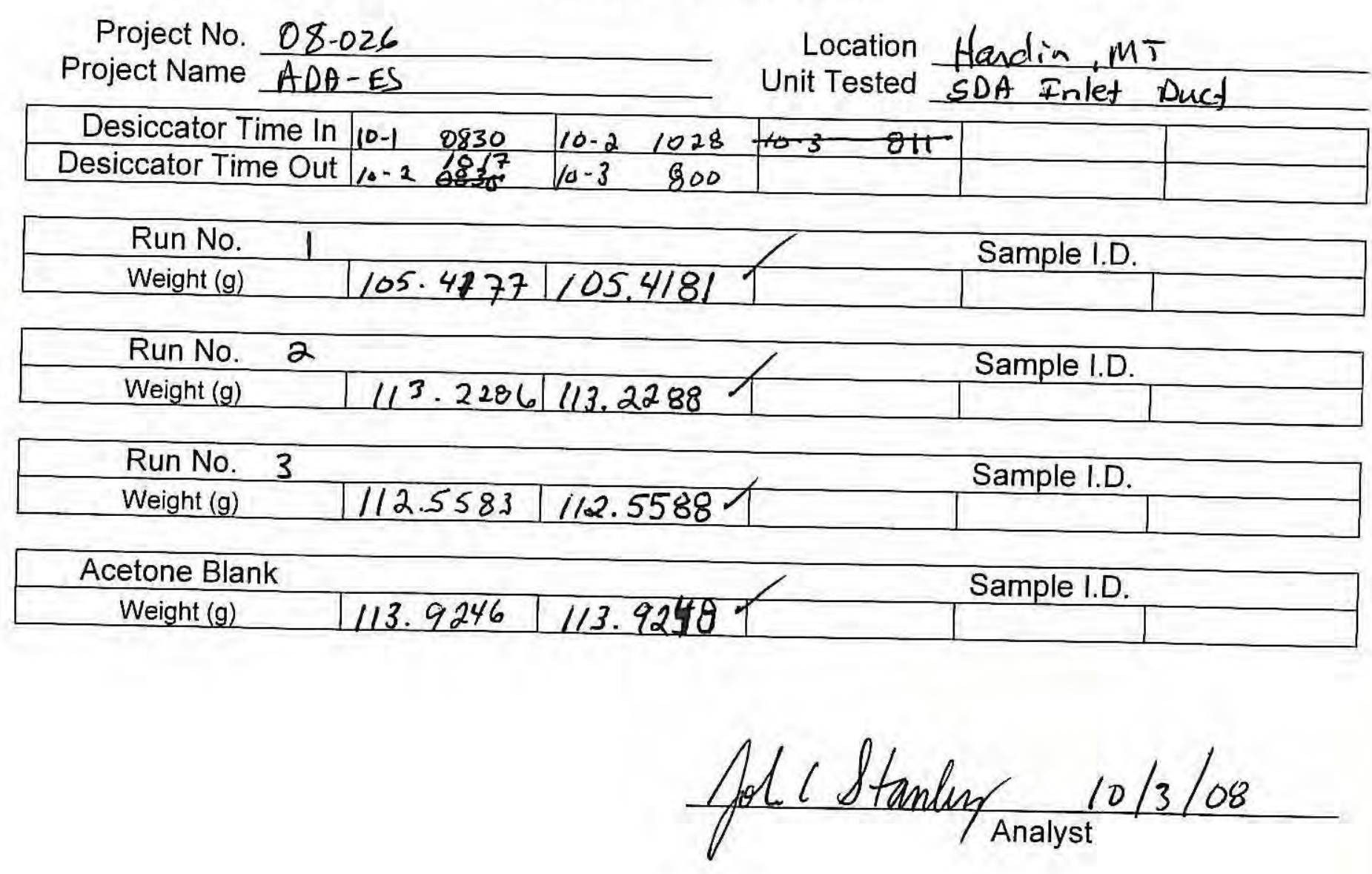




\section{Appendix H:}

EPA Test Methods 201A and 202 (PM10/2.5) Analytical Data- Stack 
AIR

SAMPLING

ASSOCIATES, INC.

Air Sampling Associates, Inc.

Particulate Analysis Summary

Project Number: $08-026$

Project Name: ADA -ES

Project Location: Hardin, MT
Date Analysis Completed: $10 / 30 / 08$

Unit Tested: Stack
Run Number:

Particulate Matter >PM10 (mas):

Particulate Matter < PM10 >PM2.5 (mss):

Particulate Matter PM2.5 Rinse (mss):

Particulate Matter PM2.5 Filter (mss):

Total Particulate Matter as PM2.5 (mss):

Particulate Matter in "Back-Half" (mos):

Total Particulate Matter in Sample - MT (mg):

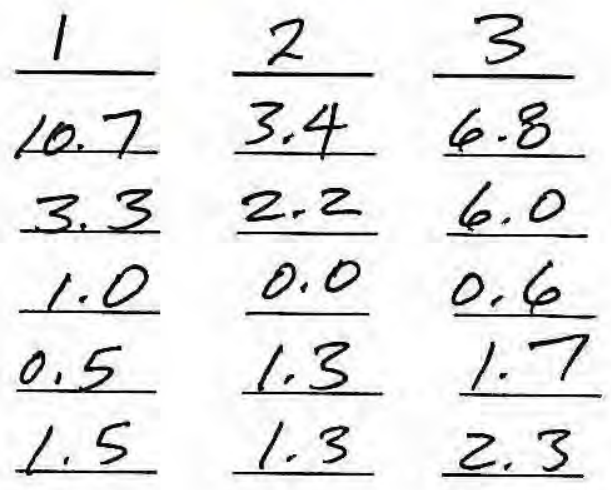

$\begin{array}{llll}79.2 & 74.9 & 90.3\end{array}$

$\begin{array}{lll}24.7 & 81.8 & 105.4\end{array}$

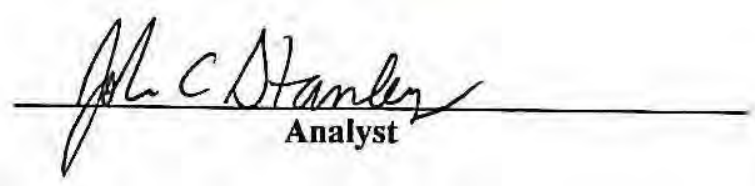

Billy \&. Mullingfpate:11/09/88

Data Checked By

$\mathrm{H}-2$ 


\section{Air Sampling Associates, Inc.}

\section{Particulate Analysis Summary}

Project Number: $08-026$

Date Analysis Completed:

Project Name: $A D A-E S$

Project Location: Hardin, MT

Unit Tested:

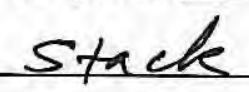

Run Number:

Particulate Matter >PM10 (mgs):

Particulate Matter < PM10 > PM2.5 (mgs):

Particulate Matter PM2.5 Rinse (mgs):

Particulate Matter PM2.5 Filter (mgs):

Total Particulate Matter as PM2.5 (mgs):

Particulate Matter in "Back-Half" (mgs):

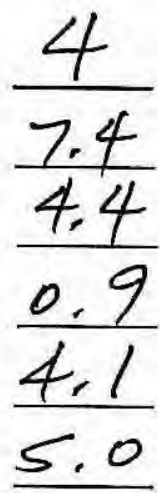

59,2

Total Particulate Matter in Sample-MT (mg):
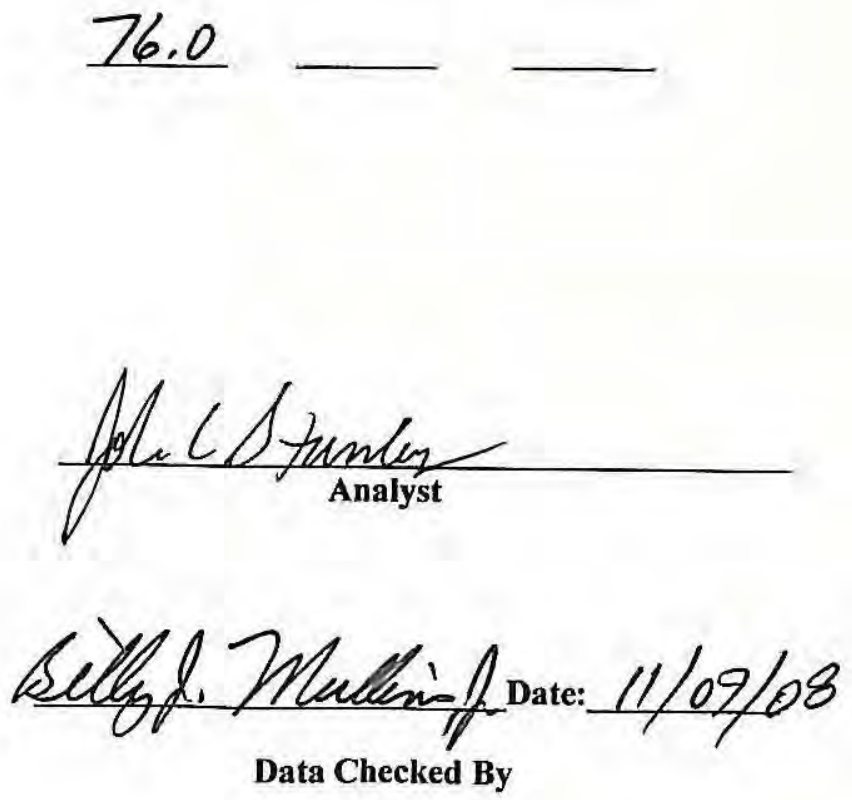


\section{Particulate Analysis EPA Method $201 \mathrm{~A} / 202$}

\section{Stack Filters}

Project No. $08-026$

Project Name ADA-ES
Location Handin, $M T$

Unit Tested Stack

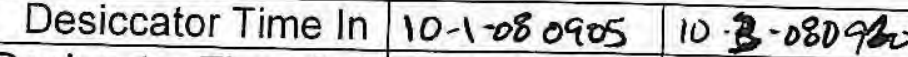

Desiccator Time Out $10 \cdot 3-08091010.6080900$

\begin{tabular}{|c|c|c|c|c|}
\hline \multicolumn{2}{|c|}{ Fun No. 1 No. 21 } & \multicolumn{1}{c|}{ Sample I.D. } \\
\hline Filter \& Particulate (g) & 0.1218 & 0.1219 of & & \\
\hline
\end{tabular}

Filter \& Particulate Average $(\mathrm{g})$ Initial Filter Weight $(\mathrm{g})$ Total Particulate $(\mathrm{mg})$

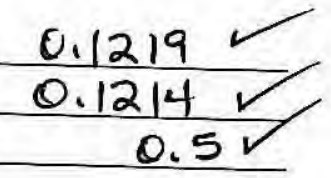

Run No.

Filter \& Particulate $(\mathrm{g})$

\section{Filter No. 22}

0.1105

Filter \& Particulate Average $(\mathrm{g})$ Initial Filter Weight (g)

Total Particulate (mg) Sample I.D.

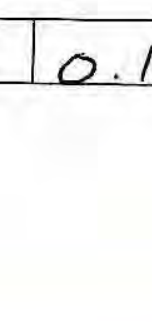

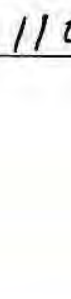

Run No. 3

Filter \& Particulate (g)

\section{Filter No. 23} Sample I.D.

Filter \& Particulate Average $(\mathrm{g}) \quad 0.1084$ Initial Filter Weight $(\mathrm{g}) \quad 0,1067$

Total Particulate $(\mathrm{mg})$

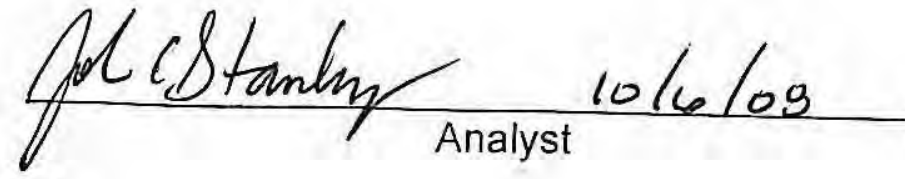




\section{Particulate Analysis EPA Method 201 A /202}

\section{Stack Filters}

Project No. O8-02C

Project Name ADA-ES
Location

Unit Tested
Handin, $M T$
Stack

\begin{tabular}{|r|r|r|r|r|r|}
\hline Desiccator Time In & $10-1.060905$ & $10.3-08$ 0920 & & & \\
\hline Desiccator Time Out & $10-3-080914$ & $10-6-08$ 0900 & & & \\
\hline
\end{tabular}

\begin{tabular}{|l|l|l|l|l|}
\hline \multicolumn{2}{|c|}{ Run No. 4} & Filter No. 24 & \multicolumn{2}{c|}{ Sample I.D. } \\
\hline Filter \& Particulate (g) & 0.1254 & 0.1253 丩 & & \\
\hline
\end{tabular}

Filter \& Particulate Average $(\mathrm{g}) \quad 0.1253$ Initial Filter Weight $(\mathrm{g})$ Total Particulate $(\mathrm{mg})$

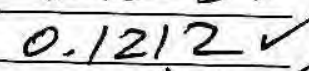

\section{Run No.}

Filter \& Particulate $(\mathrm{g})$

\section{Filter No.}

Sample I.D.

Filter \& Particulate Average (g) Initial Filter Weight $(\mathrm{g})$

Total Particulate $(\mathrm{mg})$

\section{Run No.}

Filter \& Particulate $(\mathrm{g})$
Filter No.

Sample I.D.

Filter \& Particulate Average (g) Initial Filter Weight $(\mathrm{g})$ Total Particulate $(\mathrm{mg})$

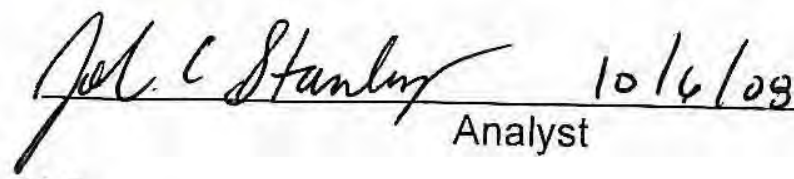




\section{AIR \\ SAMPLING \\ ASSOCIATES, INC.}

Filter Weight Log

\begin{tabular}{|c|c|c|c|c|c|c|c|}
\hline \multirow[b]{3}{*}{ Filter No. } & Into Desicator & Weinht & & & & & \\
\hline & Date & & Weight & Weight & Weight & Weight & \multirow{3}{*}{$\begin{array}{l}\text { Weight } \\
\text { Used }\end{array}$} \\
\hline & Time & $\begin{array}{l}\text { Date } \\
\text { Time }\end{array}$ & Date & Date & Date & Date & \\
\hline \multirow{3}{*}{16} & $8 / 1107$ & \begin{tabular}{c|} 
Time \\
0.12 .14
\end{tabular} & Time & \begin{tabular}{|l} 
Time \\
\end{tabular} & Time & Time & \\
\hline & 0800 & $\frac{0.1214}{8128107}$ & 0.1217 & $F$ & & & 0.1217 \\
\hline & & 0910 & $\frac{8 / 28 / 07}{1520}$ & & & & \\
\hline \multirow{3}{*}{17} & $8 / 1 / 07$ & 0.1084 & 0.1089 & $\checkmark$ & & & $b \ln 80$ \\
\hline & 0800 & $8 / 28 / 07$ & $8 / 28 / 01$ & & & & 2. 1088 \\
\hline & & 09,0 & 1520 & & & & \\
\hline \multirow{3}{*}{18} & 01107 & 0.1211 & 0.1213 & $\longleftarrow$ & & & 0.1213 \\
\hline & 0800 & $8 / 28 / 07$ & $8 / 28 / 07$ & & & & \\
\hline & & 0910 & 1520 & & & & \\
\hline \multirow{3}{*}{19} & $8 / 1107$ & 0.1075 & 0.1078 & 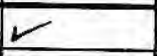 & & & D. 1079 \\
\hline & 6800 & $8 / 28 / 07$ & $8 / 28 / 07$ & Nat & 1 & & \\
\hline & & 0915 & 1525 & $\left\langle\omega^{\circ}\right| \sigma^{\infty}$ & & & \\
\hline \multirow{3}{*}{20} & $8 / 1 / 07$ & 0.1229 & otafor & $7<$ & & & 0.1232 \\
\hline & 0800 & $8 / 28 / 01$ & 0,1232 & & & & \\
\hline & & 6915 & 1525 & & & & \\
\hline \multirow{3}{*}{21} & $8 / 1107$ & $0.12 / 2$ & 0.1214 & $=$ & & & 0.1214 \\
\hline & 0800 & $8 / 2867$ & $8 / 28 / 47$ & & & & \\
\hline & & 0915 & 1525 & & & & \\
\hline \multirow{3}{*}{22} & $8 / 1 / 07$ & $0.10^{8} \pi$ & 0.1097 & $E$ & & & 0.1092 \\
\hline & 0800 & $8 / 28 / 07$ & $8 / 28107$ & & & & \\
\hline & & 0920 & 1530 & & & & \\
\hline \multirow{3}{*}{23} & $8 / 1107$ & 0.1067 & 0.1067 & $\checkmark$ & & & 0.1067 \\
\hline & 0800 & $3 / 28 / 07$ & $8 / 28 / 07$ & & & & \\
\hline & & 0220 & 1530 & & & & \\
\hline \multirow{3}{*}{24} & B/1/07 & $0.12 / 2$ & 0.1212 & 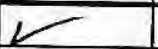 & & & b. 1212 \\
\hline & 0800 & $8 / 2817$ & $8 / 28 / 07$ & & & & \\
\hline & & 10220 & 1530 & & & & \\
\hline \multirow{3}{*}{25} & $8 / 1 / 07$ & 0.11881 & 0.11897 & & & & 2.1189 \\
\hline & 0.800 & $3 / 28 / 07$ & $8 / 28 / 07$ & & & & \\
\hline & & 0925 & 1535 & & & & \\
\hline \multirow{3}{*}{26} & $8 / 107$ & 0.1053 & 0.1053 & & & & 0.1053 \\
\hline & 6800 & $8 / 28 / 07$ & $8 / 28 / 27$ & & & & \\
\hline & & 0925 & 1535 & & & & \\
\hline \multirow{3}{*}{27} & 811107 & 0.1211 & $0.12 / 6$ & $r$ & & & 0.1216 \\
\hline & 0800 & $8 / 28 / 07$ & $8 / 28 / 27$ & & & & \\
\hline & & 0925 & 1535 & & & & \\
\hline \multirow{3}{*}{28} & & & & & & & \\
\hline & & & & & & & \\
\hline & & & & & & & \\
\hline \multirow{3}{*}{29} & & & & & & & \\
\hline & & & & & & & \\
\hline & & & & & & & \\
\hline \multirow{3}{*}{30} & & & & & & & \\
\hline & & & & & & & \\
\hline & & & & & & & \\
\hline
\end{tabular}


Front Wash

Project No. $08-026$ Project Name ADA = ES Location Hardia, MT M
Unit Tested Desiccator Time In 10. 13.08 1350 10.17.08 0840 Desiccator Time out 10.17 .08 0830/6.3.3.08 1360

\begin{tabular}{|c|c|c|}
\hline Run No. I & Volume (ml) \\
\hline Final Weight $(\mathrm{g})$ & 115.3016 & 115.3020 \\
\hline Initial Weight $(\mathrm{g})$ & & 115.2913 \\
\hline Particulate Weight $(\mathrm{g})$ & & 0.0107 \\
\hline
\end{tabular}

Particulate Average (mg) Less Acetone Blank (mg) Sample I.D. $>$ PM10 Total Particulate $(\mathrm{mg})$

\begin{tabular}{|c|l|l|l|l|}
\hline Run No. & \multicolumn{1}{|c|}{ Volume $(\mathrm{ml})$} & 19 & Sample I.D. <Pm $10>$ Pm.25 \\
\hline Final Weight $(\mathrm{g})$ & 104.2970 & 104.2973 & \\
\hline Initial Weight $(\mathrm{g})$ & & 104.2940 & \\
\hline Particulate Weight $(\mathrm{g})$ & & 0.0033 & & \\
\hline
\end{tabular}

Particulate Average (mg) Less Acetone Blank (mg) $3 \cdot 3$

Total Particulate (mg)

\begin{tabular}{|c|c|c|}
\hline Run No. I & \multicolumn{1}{|c|}{ Volume (ml) } \\
\hline Final Weight $(\mathrm{g})$ & 80.3041 & 80.3045 \\
\hline Initial Weight $(\mathrm{g})$ & & 80.3035 \\
\hline Particulate Weight $(\mathrm{g})$ & & 0.0010 \\
\hline
\end{tabular}

10.72

$9-$ Sample I.D. <Pm 2.5

\begin{tabular}{|c|}
\hline Acetone Blank \\
\hline Final Weight $(\mathrm{g})$ \\
\hline Initial Weight $(\mathrm{g})$ \\
\hline Particulate Weight $(\mathrm{g})$ \\
\hline
\end{tabular}

* Note: if greater than $7.9 \mathrm{mg} / \mathrm{l}$, use $7.9 \mathrm{mg} / \mathrm{l}$
Particulate Average (mg)

Total Particulate $(\mathrm{mg})$
Sample I.D. 
Particulate Analysis EPA Method 201A/202

Front Half Tare Weights

Project No.

Project Name

$08-026$

ADA-ES

Unit Tested Hardir, MT

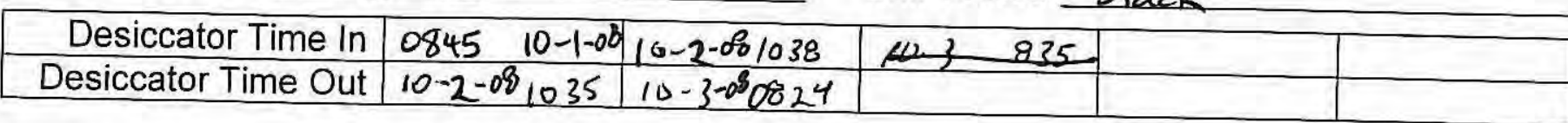

\begin{tabular}{|r}
\hline Run No. \\
\hline
\end{tabular} Weight (g) Sample I.D. $>P_{10} 10$

Run No. Weight (g) \begin{tabular}{|l|l|l|}
115.2910 & 115.2913 \\
\hline
\end{tabular}

ᄀ

Sample I.D. $<$ PM10 >PM 2.5

Run No. 1 Weight (g) $104.2940 \mid 104.2940$

Sample I.D. $<P M 2.5$

Acetone Blank

Weight (g) Sample I.D.

Aoh i Stanlin $10 / 3108$ 
Particulate Analysis EPA Method $201 \mathrm{~A} / 202$

Front Wash

Project No. $08-026$

Project Name ADA - ES

Location Hardia MT

Unit Tested .. Stack

Desiccator Time In $10 \cdot 13.08 \quad 135010.17 .08 \quad 0850$

Desiccator Time out 10.9 .09 0830 $10-36.68$ i300

Run No. 2

Final Weight (g)

Initial Weight $(g)$

Particulate Weight $(g)$

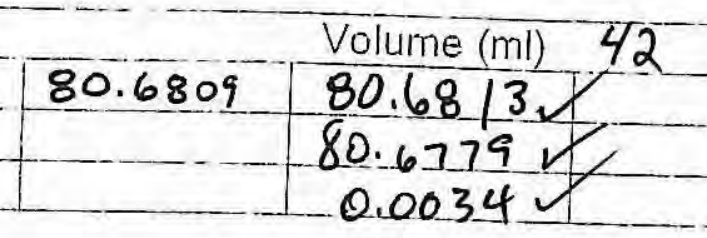

Sample I.D

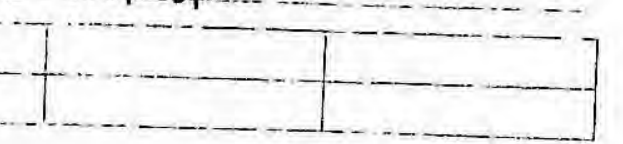

Particulate Average (mg)

Less Acetone Blank (mg)

Total Particulate $(\mathrm{mg})$

\begin{tabular}{|c|c|c|c|}
\hline Run No. 2 & & Volume $(\mathrm{ml}) 37$ & Sample 1.D. $\left\langle p_{m} 10>P_{m}\right.$ \\
\hline Final Weight $(\mathrm{g})$ & $106.1 / 68$ & $106.1172 \sqrt{ }$ & \\
\hline Initial Weight (g) & & $106.1150 \mathrm{~V}$ & \\
\hline Particulate Weight $(\mathrm{g})$ & & 0.00224 & \\
\hline
\end{tabular}

Particulate Average (ng)

Less Acetone Blank (mg)

2.2

Total Particulate ( $\mathrm{mg}$ )

\begin{tabular}{|c|c|c|c|}
\hline Run No. 2 & & Volume (ml) 21 & Sample 1.D. <Pm 2.5 \\
\hline Final Weight (g) & 111.9531 & $111.8533 \square$ & \\
\hline Initial Weight (g) & & 111.8537 & \\
\hline Particulate Weight (g) & & -0.0004 & \\
\hline
\end{tabular}

Particulate Average (mg)

Less Acetone Blank (mg)

0.0

Total Particulate (mg)

\begin{tabular}{|c|c|c|}
\hline Acetone Blank & Volume (ml) & Sample I.D. \\
\hline Final Weight $(g)$ & & \\
\hline Initial Weight $(g)$ & & \\
\hline Particulate Weight (g) & & \\
\hline
\end{tabular}

Average (mg)

* Note: if greater than $7.9 \mathrm{mg} / 1$ use $7.9 \mathrm{mg} / \mathrm{l}$

* $\mathrm{mg} / \mathrm{l}$ 


\section{Particulate Analysis EPA Method 201 A/202}

\section{Front Half Tare Weights}

Project No.

Project Name

$$
\frac{O 8-026}{\text { ADA-ES }} \begin{array}{r}
\text { Location Handin, MT } \\
\text { Unit Tested Stact }
\end{array}
$$

Desiccator Time In $10-1-080845$

Desiccator Time Out $10-2-681029$

$10-2-08 / 034$

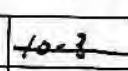

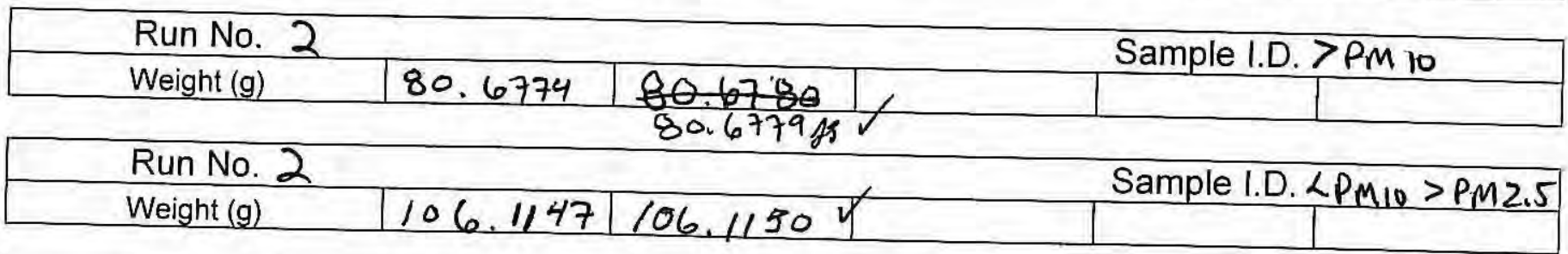

\begin{tabular}{|l|l|l|l|l|}
\hline \multicolumn{2}{|c|}{ Run No. 2 } & \multicolumn{2}{c|}{ Sample I.D. < PM 2.5 } \\
\hline Weight (g) & 111.8536 & $/ 1 / .8537 \leadsto$ & & \\
\hline
\end{tabular}

Acetone Blank

Weight (g)

\section{Sample I.D.}

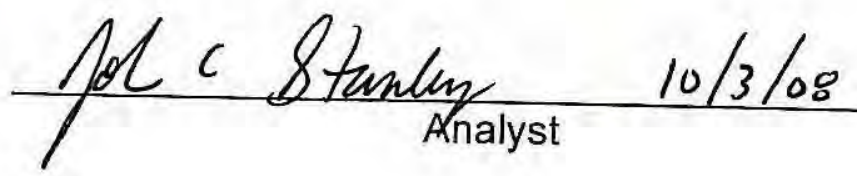




\section{IIIIIR

Particulate Analysis EPA Method $201 \mathrm{~A} / 202$

\section{Front Wash}

Project No. $08-026$

Project Name AOA-ES

Location Hardin, $m t$ Unit Tested - Stack

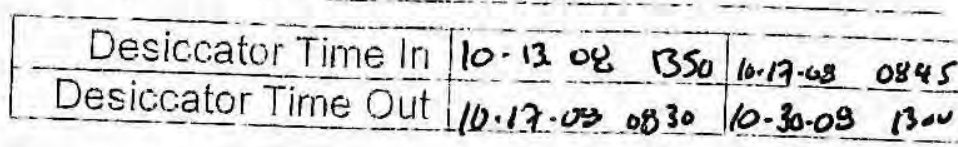

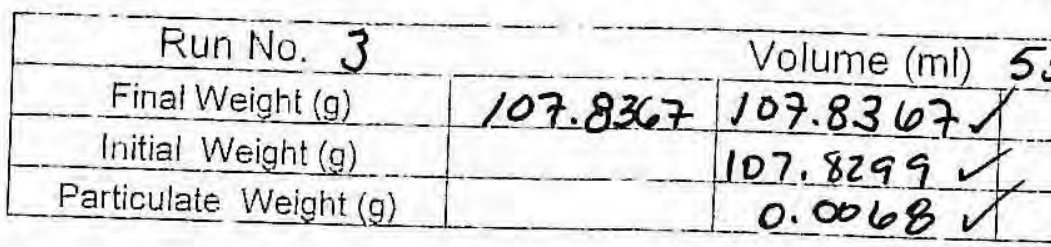

Particulate Average (mg)

Less Acetone Blank ( $\mathrm{mg}$ )

Sample 1.D. $>$ PM 10

Total Particulate $(\mathrm{mg})$

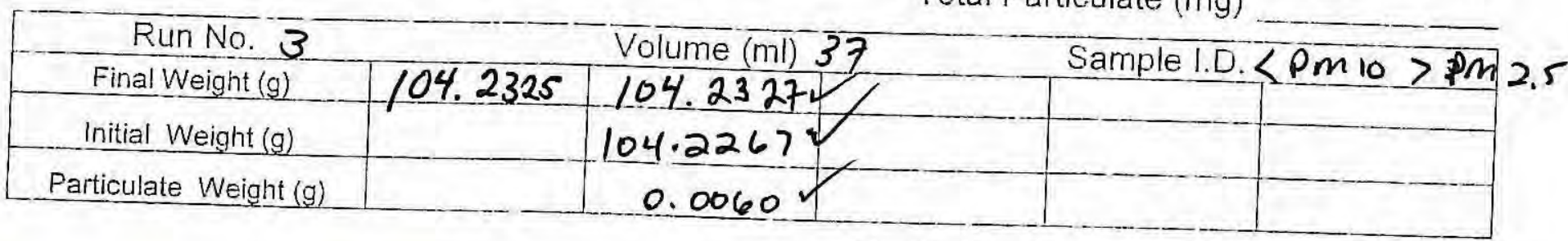

Particulate Average ( $\mathrm{mg}$ )

Less Acetone Blank (mg)

$6.0 \_$

Total Particulate $(\mathrm{mg})$

\begin{tabular}{|c|c|c|}
\hline Run No. 3 & \multicolumn{1}{|c|}{ Volume (ml) 32} \\
\hline Final Weight $(\mathrm{g})$ & 103.9187 & 103.9191 \\
\hline Initial Weight $(\mathrm{g})$ & & 103.9185 \\
\hline Particulate Weight $(\mathrm{g})$ & & 0.0006 \\
\hline
\end{tabular}

2 Sample I.D. $<$ Pm 2.5

\begin{tabular}{|c|}
\hline Acetone Blank \\
\hline Final Weight $(\mathrm{g})$ \\
\hline Initial Weight $(\mathrm{g})$ \\
\hline Particulate Weight $(\mathrm{g})$ \\
\hline
\end{tabular}
* Note: if greater than $7.9 \mathrm{mg} / \mathrm{l}$, use $7.9 \mathrm{mg} / \mathrm{l}$

Particulate Average (mg) Less Acetone Blank (mg)

Total Particulate (mg)

\begin{tabular}{|c|c|c|}
\hline Volume (ml) & Sample I.D. \\
\hline & & \\
\hline & Average $(\mathrm{mg})$ \\
& * $\mathrm{mg} / \mathrm{l}$
\end{tabular}

0.6 
Particulate Analysis EPA Method ZolA/202

Front Half Tare Weights

Project No. $08-026$

Project Name ADA-ES

Unit Tested Hardin, MT

\begin{tabular}{|r|r|r|}
\hline Desiccator Time In & $10-1.080850$ \\
\hline
\end{tabular}

Desiccator Time Out $10-2.081049$

\begin{tabular}{|l|l|}
$10-2781052$ & $10-3-080852$ \\
\hline 0
\end{tabular}

$10-3-08849 \quad 10-6-080900$

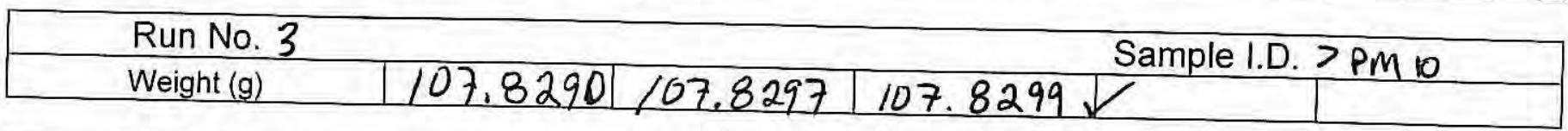

\begin{tabular}{|c|c|c|}
\hline Run No. 3 & & Sample I.D. $\angle P_{M_{10}}>P_{M} 2.5$ \\
\hline Weight $(g)$ & $104.2263 \quad 104.2267 \mathrm{~V}$ & \\
\hline
\end{tabular}

Run No. 3

Weight (g)

Sample I.D. $<$ Pm 2.5

Acetone Blank

Weight $(\mathrm{g})$

Sample I.D.

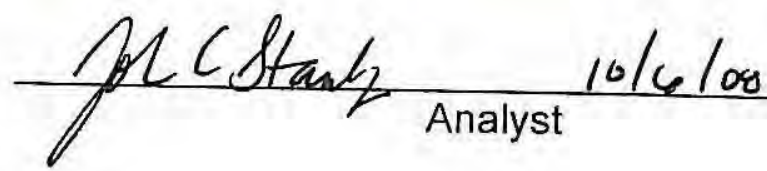


Particulate Analysis EPA Method 20/A/202

Front Wash

Project No. $08-026$

Project Name AOA-ES

Location Hardin, mT

Desiccator Time In $10.13-08 \quad 1350 \quad 10.73 .09$ cass

Desiccator Time Out 10.17 .00 o93 $10.30 .08 \quad 1300$

Unit Tested stack

\begin{tabular}{|c|c|c|}
\hline \multicolumn{2}{|c|}{ Run No. 4} & Volume $(\mathrm{ml})$ \\
\hline Final Weight $(\mathrm{g})$ & 106.6105 & 106.6109 \\
\hline Initial Weight $(\mathrm{g})$ & & 106.6035 \\
\hline Particulate Weight $(\mathrm{g})$ & & 0.0074 \\
\hline
\end{tabular}

Particulate Average (mg)

Less Acetone Blank (mg)

Total Particulate $(\mathrm{mg})$

\begin{tabular}{|c|c|c|c|c|}
\hline \multicolumn{2}{|c|}{ Run No. 4} & 81 me $(\mathrm{ml})$ & 28 & \\
\hline Final Weight $(\mathrm{g})$ & 81.1873 & 81.1878 & & \\
\hline Initial Weight $(\mathrm{g})$ & & 81.1834 & & \\
\hline Particulate Weight $(\mathrm{g})$ & & 0.0044 & & \\
\hline
\end{tabular}

Particulate Average ( $\mathrm{mg})$

Less Acetone Blank (mg)

4.4

Total Particulate $(\mathrm{mg})$

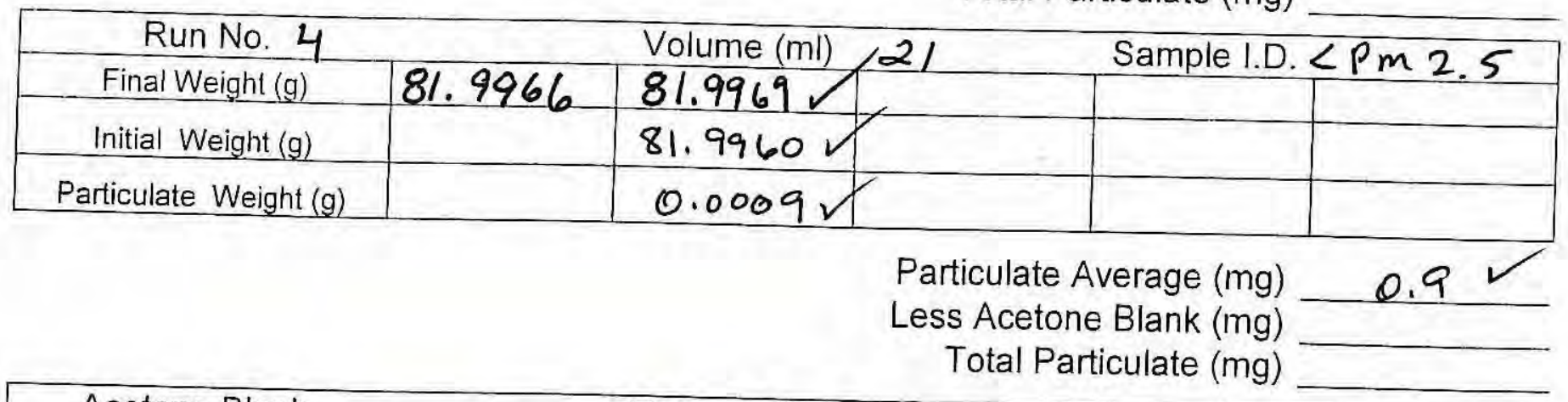

\begin{tabular}{|c|l|l|l|l|l|}
\hline Acetone Blank & \multicolumn{3}{|c|}{ Salume (ml) } & & \\
\hline Final Weight $(\mathrm{g})$ & & & & & \\
\hline Initial Weight $(\mathrm{g})$ & & & & & \\
\hline Particulate Weight $(\mathrm{g})$ & & & & \\
\hline
\end{tabular}

* Note: if greater than $7.9 \mathrm{mg} / \mathrm{l}$, use $7.9 \mathrm{mg} / \mathrm{l}$

Average (mg)

${ }^{*} \mathrm{mg} / \mathrm{l}$ 


\section{AlR

\section{Particulate Analysis EPA Method $201 \mathrm{~A} / 202$}

\section{Front Half Tare Weights}

Project No.

Project Name ADA-ES

O8-026
ADA-ES

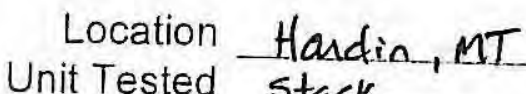

\begin{tabular}{|l|l|l|l|l|}
\hline Desiccator Time In & $10-1-080850$ & $10-2-08$ & 1057 & $10 \cdot 3-080858$
\end{tabular}

Desiccator Time Out $10-2-08 \quad 1053 \quad 10-3-08 \quad \& 52$

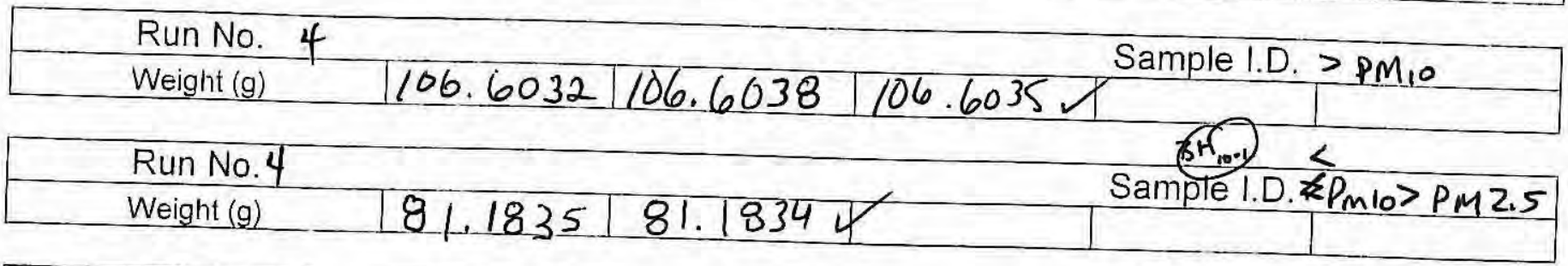

$\frac{\text { Run No.4 }}{\text { Weight (g) }}$

$81.9959 \mid 81.9960$ म

Sample I.D. $<$ P m 2.5

Acetone Blank Weight $(\mathrm{g})$

Sample I.D.

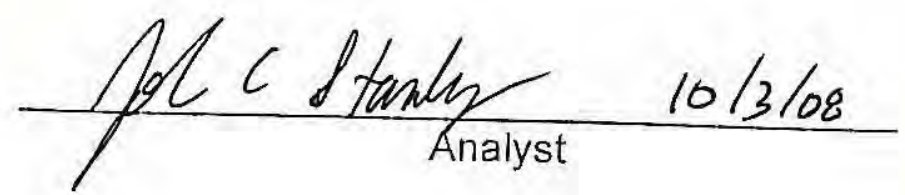


Methylene Chloride Rinse

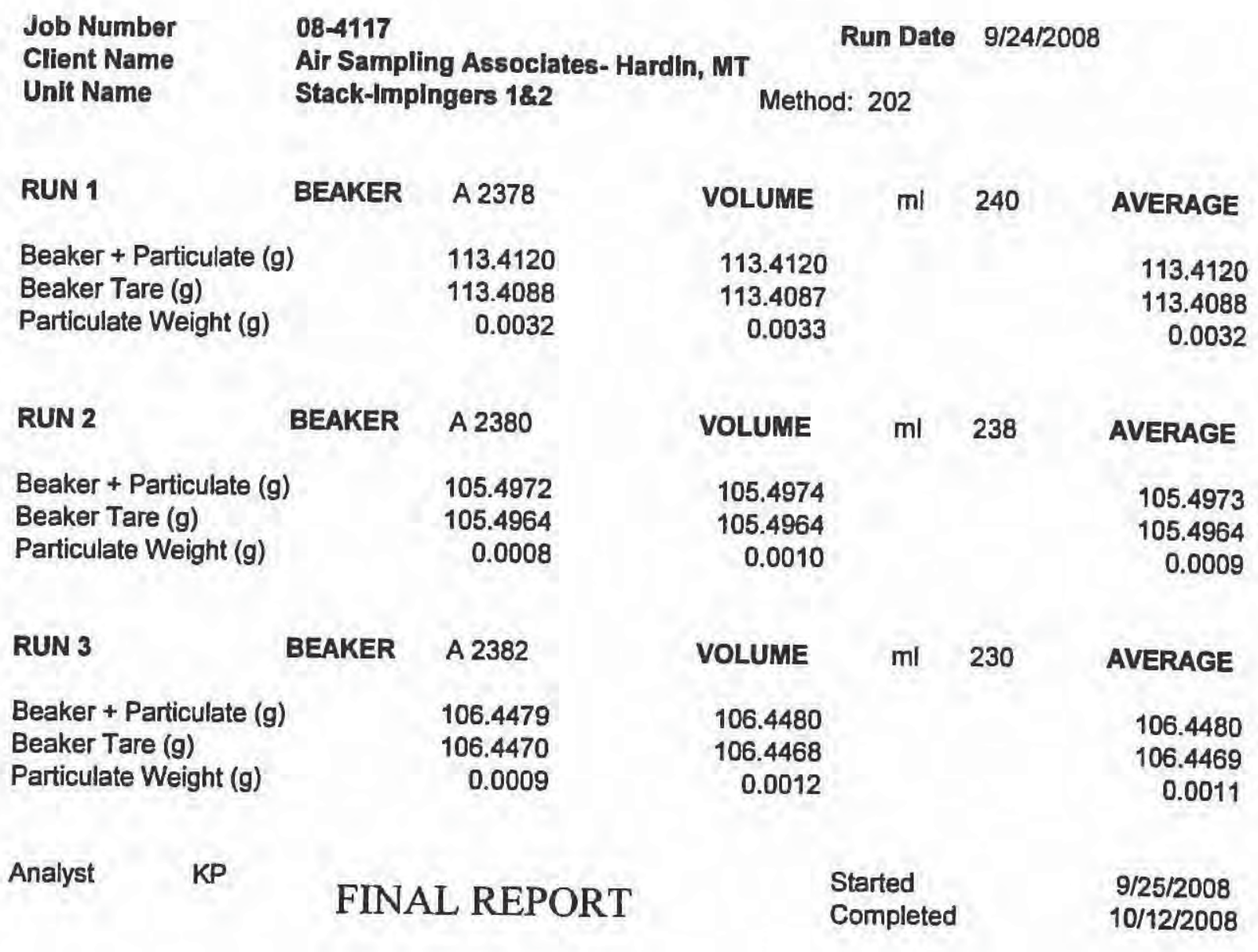




\section{Methylene Chloride Rinse}

\begin{abstract}
Job Number
Client Name

Unit Name
\end{abstract}

RUN 4

Beaker + Particulate (g)

Beaker Tare (g)

Particulate Weight (g)
$08-4117$

Air Sampling Assoclates- Hardin, MT

Stack-Impingers 1\& 2

Method: 202
Run Date $9 / 24 / 2008$

BEAKER A 2384

106.8155

106.8144

0.0011

\section{VOLUME \\ 106.8154 \\ 106.8145 \\ 0.0009}

$\mathrm{ml} 230$

815

106.8145

0.0010
FINAL REPORT
Started

Completed
$9 / 25 / 2008$

$10 / 12 / 2008$ 


\section{Methylene Chloride Rinse}

\begin{abstract}
Job Number Client Name Unit Name
\end{abstract}

BLANK

Beaker + Particulate $(g)$

Beaker Tare (g)

Particulate Weight (g)
08-4117

Air Sampling Associates- Hardin, MT Stack-Reagent Blank

BEAKER A 2386

112.7456

112.7449

0.0007

Run Date $9 / 24 / 2008$

Method: 202

$\begin{array}{rrrr}\text { VOLUME } & \mathrm{ml} & 230 & \text { AVERAGE } \\ & & & \\ 112.7457 & & & 112.7457 \\ 112.7448 & & & 112.7449 \\ 0.0009 & & & 0.0008\end{array}$

Analyst

KP

FINAL REPORT

Started

Completed

$9 / 25 / 2008$

$10 / 12 / 2008$ 


\section{Water Analysis (Back)}

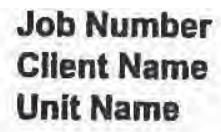

08-4117

Air Sampling Assoclates Impingers 1 \& 2 + BH

A 2377

119.0689

0.1125

Particulate Weight (g)

BEAKER A 2377

119.0689
118.9564
0.1125

118.9564

\section{BEAKER}

RUN 2

Beaker + Particulate $(\mathrm{g})$

Beaker Tare (g)

Particulate Weight (g)

A 2379
119.0768
118.9653
0.1115

A 2381

105.9272

105.7945

0.1327
Run Date 9/24/2008

Method: 202

$\begin{array}{rrrr}\text { VOLUME } & \mathrm{ml} & 400 & \text { AVERAGE } \\ & & & \\ 119.0688 & & & 119.0689 \\ 118.9565 & & & 118.9565 \\ 0.1123 & & & 0.1124\end{array}$

VOLUME $\mathrm{ml} \quad 400$

AVERAGE

119.0769

118.9657

0.1112

$\begin{array}{rrrr}\text { VOLUME } & \mathrm{ml} & 400 & \text { AVERAGE } \\ 105.9276 & & & 105.9274 \\ 105.7945 & & & 105.7945 \\ 0.1331 & & & 0.1329\end{array}$

Started

Completed
$9 / 25 / 2008$

119.0769

118.9655

0.1114

$10 / 12 / 2008$ 


\section{Water Analysis (Back)}

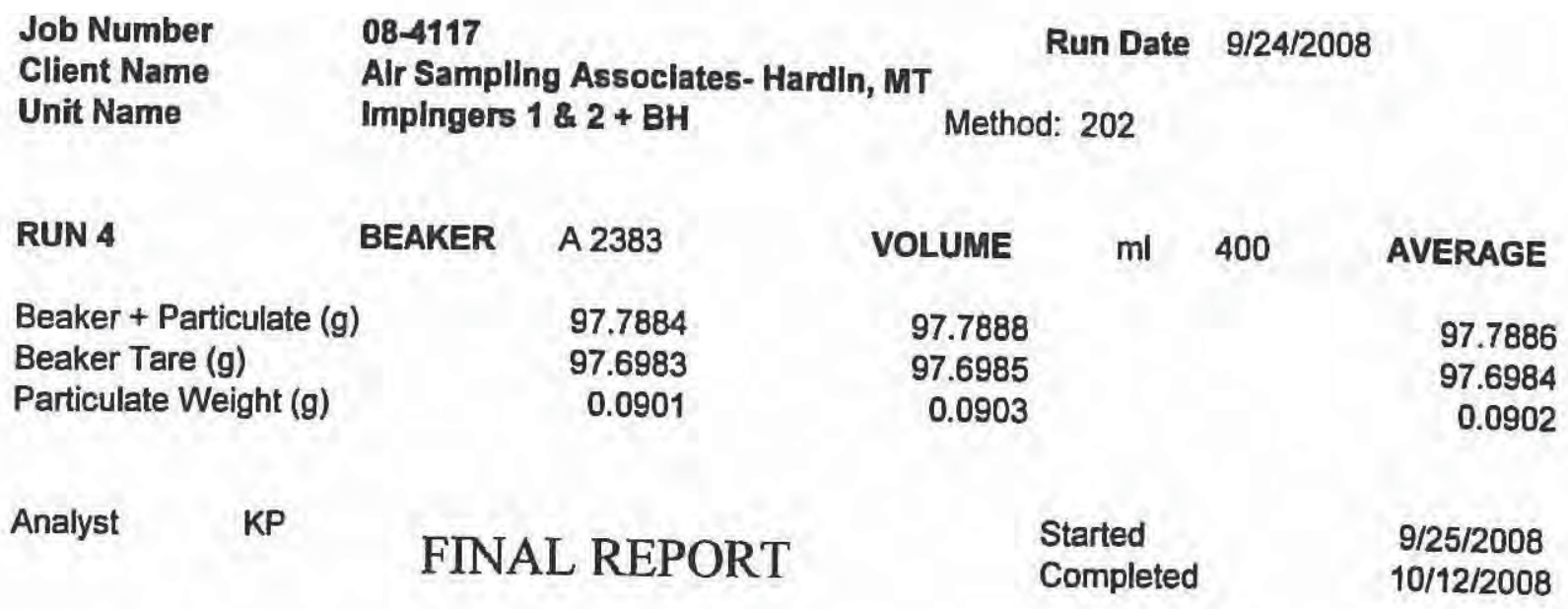




\section{Water Analysis (Back)}

Job Number
Client Name
Unit Name

BLANK

Beaker + Particulate $(g)$

Beaker Tare (g)

Particulate Weight (g)
08-4117

Air Sampling Associates- Hardin, MT

Stack-Reagent Blank

Method: 202

\section{Run Date $\quad 9 / 24 / 2008$}

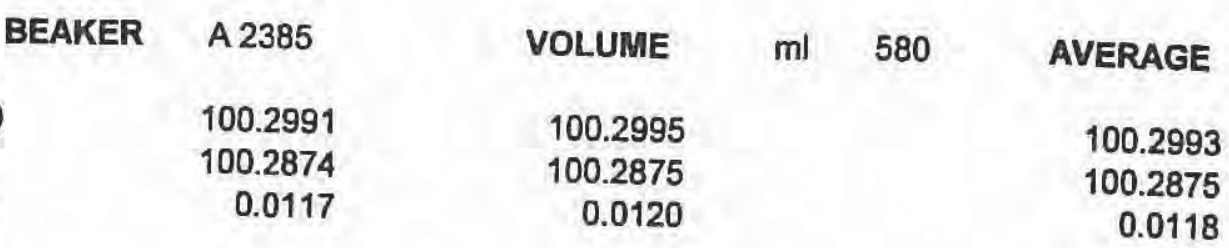

Started

$9 / 25 / 2008$

Completed

$10 / 12 / 2008$ 
Sulfate

ADA-ES Run $1^{9 / 23-24 / 2008} 5 B / 202$

Run 2 Stack

Run 3 Stack

ADA-ES $\operatorname{Run} 1^{9 / 24 / 2008} \underset{\text { stack }}{20 / A / 202}$

Run 2 Stack

Run 3 Stack

Run $4 \quad$ Stack
$08-4117$

Sample Lab Lab Final

Volume, Volume, Results, Results,

Sample No.

$\mathrm{ml} \quad \mathrm{ml} \mathrm{mg} / \mathrm{mg}$

$\begin{array}{lllll}4117-1 & 590 & 20 & 569 & 118.841\end{array}$

$\begin{array}{lllll}4117-2 & 590 & 20 & 486 & 101.506\end{array}$

4117-3

580

20

$622 \quad 127.709$

Sample No. $4117-4$

$\begin{array}{llll}320 & 20 & 243 & 27.527\end{array}$

4117-5

320

20

252

28.547

4117-6

320

20

307

34.777

4117-7

320

20

204

23.109 


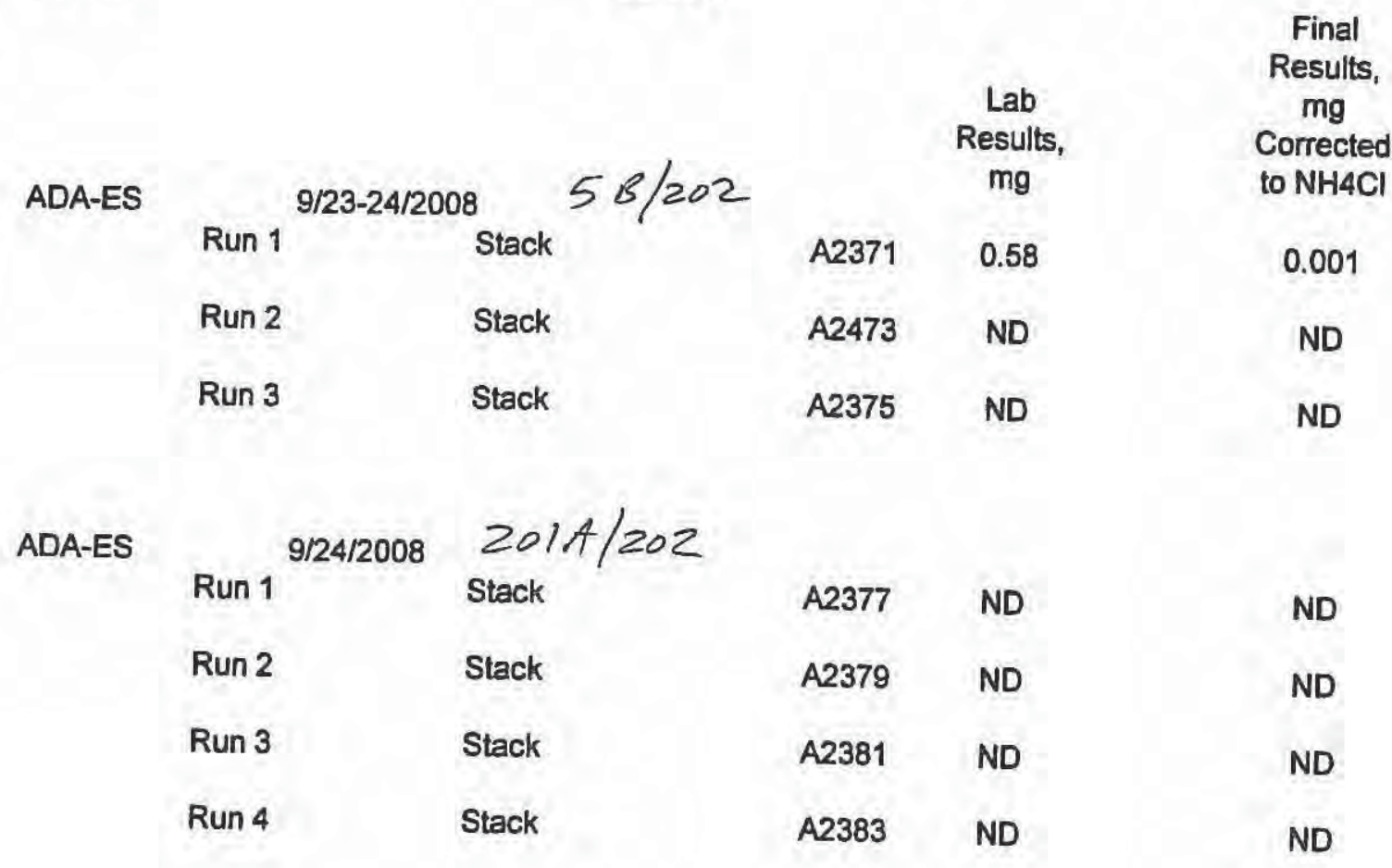




\section{ANALYTICAL SUMMARY REPORT}

October 20, 2008

Westem Environmental

913 Foster Rd

Casper, WY 82601

Workorder No.: $\mathrm{C} 08100503$

Project Name: $\quad 08-4117$

Energy Laboratories, Inc. received the following 8 samples from Westem Environmental on 10/10/2008 for analysis.

\begin{tabular}{|c|c|c|c|c|c|}
\hline Sample ID & Client Sample ID & Collect Date & Recelve Date & Matrix & Test \\
\hline C08100503-001 & $\begin{array}{l}\text { 08-4117-1 Stack } \\
\text { Impringers } 182\end{array}$ & 09/23/08 00:00 & $10 / 10 / 08$ & Aqueous & E300.0 Anions \\
\hline C08100503-002 & $\begin{array}{l}\text { 08-4117-2 Stack- } \\
\text { Impringers } 182\end{array}$ & $09 / 23 / 0800: 00$ & $10 / 10 / 08$ & Aqueous & Same As Above \\
\hline C08100503-003 & $\begin{array}{l}\text { 08-4117-3 Stack } \\
\text { Impringers 1\&2 }\end{array}$ & $09 / 24 / 0800: 00$ & $10 / 10 / 08$ & Aqueous & Same As Above \\
\hline C08100503-004 & $\begin{array}{l}\text { 08-4117-4 Stack } \\
\text { Impringers } 1 \& 2 \text { \&BH }\end{array}$ & $09 / 24 / 0800: 00$ & $10 / 10 / 08$ & Aqueous & Same As Above \\
\hline C08100503-005 & $\begin{array}{l}\text { 08-4117-5 Stack } \\
\text { Impringers } 1 \& 2 \text { \&BH }\end{array}$ & $09 / 24 / 0800: 00$ & $10 / 10 / 08$ & Aqueous & Same As Above \\
\hline C08100503-006 & $\begin{array}{l}\text { 08-4117-6 Stack } \\
\text { Impringers } 1 \& 2 \text { \&BH }\end{array}$ & $09 / 24 / 0800: 00$ & $10 / 10 / 08$ & Aqueous & Same As Above \\
\hline C08100503-007 & $\begin{array}{l}\text { 08-4117-7 Stack } \\
\text { Impringers } 1 \& 2 \mathrm{\& BH}\end{array}$ & $09 / 24 / 0800: 00$ & $10 / 10 / 08$ & Aqueous & Same As Above \\
\hline C08100503-008 & 08-4117-8 Blank & $09 / 24 / 0800: 00$ & $10 / 10 / 08$ & Aqueous & Same As Above \\
\hline
\end{tabular}

As appropriate, any exceptions or problems with the analyses are noted in the Laboratory Analytical Report, the QAVC Summary Report, or the Case Narrative.

If you have any questions regarding these tests results, please call.

Report Approved By: Styphanie Waldigp 


\section{LABORATORY ANALYTICAL REPORT}

Client: Westem Environmental

Slte Name: $\quad 08-4117$

Report Date: 10/20/08

Lab ID: $\quad$ C08100503-001

Client Sample ID: 08-4117-1 Stack Impringers 182

Matrix:

Aqueous

Collection Date: 09/23/08

DateRecelved: $10 / 10 / 08$

Analyses
$\begin{aligned} & \text { MAJOR IONS } \\ & \text { Sulfate }\end{aligned}$

Lab ID: $\quad$ C08100503-002

Cllent Sample ID: 08-4117-2 Stack-Impringers 182

Matrlx:

Aqueous

Collection Date: 09/23/08

DateReceived: $10 / 10 / 08$

\begin{tabular}{llllllll}
\hline $\begin{array}{l}\text { Analyses } \\
\text { MANOR IONS } \\
\text { Sulfate }\end{array}$ & Result Units & Qualfiers & RL & $\begin{array}{c}\text { MCLI } \\
\text { QCL Mothod }\end{array}$ & Analysia Date / By \\
\hline
\end{tabular}

Lab ID: $\quad$ C08100503-003

Client Sample ID: 08-4117-3 Stack Impringers 1 \&2

Matrix:

Aqueous
Collectlon Date: 09/24/08

DateRecelved: $10 / 10 / 08$

\begin{tabular}{|c|c|c|c|c|c|c|c|}
\hline Analyses & Result & Units & Quallfiors & $\mathbf{R L}$ & $\begin{array}{l}\text { MCL } \\
\text { QCL }\end{array}$ & Mothod & Analysis Date / By \\
\hline \multicolumn{8}{|c|}{$\begin{array}{l}\text { MAJOR IONS } \\
\text { Suffate }\end{array}$} \\
\hline Suffate & 622 & $\mathrm{mg} / \mathrm{L}$ & & 1 & & E300.0 & 10/14/08 17:56/jا \\
\hline
\end{tabular}

Lab ID:

C08100503-004

Client Sample ID: 08-4117-4 Stack Impringers 1\&.2 \&BH

Matrix:

Aqueous

Collection Date: 09/24/08

DateRecelved: $10 / 10 / 08$

\begin{tabular}{|c|c|c|c|c|c|c|c|}
\hline Analyses & Result & Units & Qualifiers & $\mathbf{R L}$ & $\begin{array}{c}\text { MCLI } \\
\text { QCL }\end{array}$ & Mothod & Analysis Date / By \\
\hline \multicolumn{8}{|c|}{$\begin{array}{l}\text { MAJOR IONS } \\
\text { Sulfate }\end{array}$} \\
\hline Sulfate & 243 & $\mathrm{mg} / \mathrm{L}$ & & 1 & & $E 300.0$ & 10/14/08 18:11/11 \\
\hline
\end{tabular}

\section{Report \\ Definitions: \\ $R \mathrm{~L}$ - Analyte reporting limit. \\ QCL - Quality control limit.}

MCL - Maximum contaminant level.

ND - Not detected at the reporting limit. 


\section{LABORATORY ANALYTICAL REPORT}

Client: Westem Environmental

Site Name: $\quad 08-4117$

Report Date: 10/20/08

Lab ID: $\quad$ C08100503-005

Client Sample ID: 08-4117-5 Stack Impringers 1\&2 \&BH

Matrix: Aqueous

\begin{tabular}{|c|c|c|c|c|c|c|c|}
\hline Analyses & Result & Units & Quallfiers & $\mathbf{R L}$ & $\begin{array}{l}\text { MCLI } \\
\text { QCL }\end{array}$ & Mothod & Analysis Date / By \\
\hline \multicolumn{8}{|c|}{ MAJOR IONS } \\
\hline Sulfale & 252 & $\mathrm{mgh}$ & & 1 & & $E 300.0$ & 10/14/08 18:25/ 1 \\
\hline
\end{tabular}

Lab ID: $\quad$ C08100503-006

Client Sample ID: 08-4117-6 Stack Impringers 1\&2 \&BH

Matrix:

Aqueous
Collection Date: 09/24/08

DateReceived: $10 / 10 / 08$

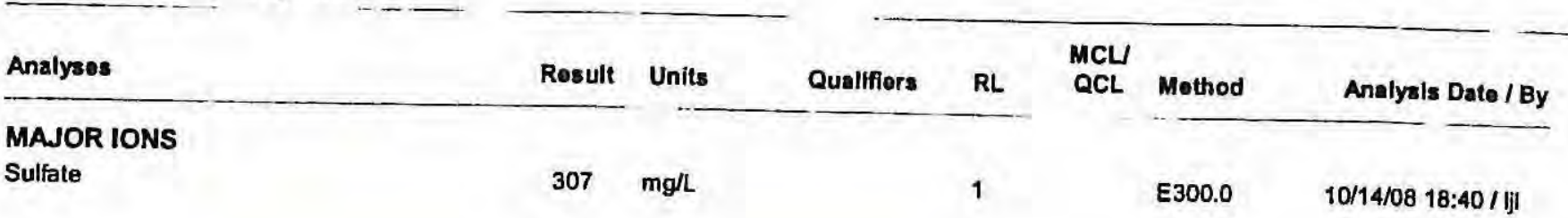

Lab ID: $\quad$ C08100503-007

Client Sample ID: 08-4117-7 Stack Impringers $182 \& \mathrm{BH}$

Matrix:

Aqueous
Collection Date: 09/24/08

DateReceived: $10 / 10 / 08$

\begin{tabular}{|c|c|c|c|c|c|c|c|}
\hline Analyeses & Result & Units & Quallfiers & $\mathbf{R L}$ & $\begin{array}{l}\text { MCL } \\
\text { QCL }\end{array}$ & Mothod & Aralysis Date / By \\
\hline \multicolumn{8}{|c|}{$\begin{array}{l}\text { MAJOR IONS } \\
\text { Sulfate }\end{array}$} \\
\hline Sulfate & 204 & $\mathrm{mg} h$ & & 1 & & $E 300.0$ & 10/14/08 18:54/ 1] \\
\hline
\end{tabular}

Lab ID:

C08100503-008

Client Sample ID: 08-4117-8 Blank

Collection Date: 09/24/08

Matrix:

Aqueous

DateRecelved: 10/10/08

\begin{tabular}{|c|c|c|c|c|c|c|c|}
\hline Analyses & Result & Units & Qualifiers & $\mathbf{R L}$ & $\begin{array}{l}\text { MCLI } \\
\text { QCL }\end{array}$ & Method & Analysis Date / By \\
\hline \multicolumn{8}{|c|}{$\begin{array}{l}\text { MAWOR IONS } \\
\text { Sulfate }\end{array}$} \\
\hline Sulfate & ND & $\mathrm{mg} / \mathrm{L}$ & & 1 & & $E 300.0$ & 10/14/08 19:08 / ا \\
\hline
\end{tabular}

\section{Roport}

$R L$ - Analyte reporting limit.

Dofinitions: $Q C L$ - Quality control limit.
$\mathrm{MCL}$ - Maximum contaminant level.

ND - Not detected at the reporting limit. 
QA/QC Summary Report

Cllent: Westem Environmental

Project: 08-4117

Report Date: 10/20/08

Work Order: C08100503

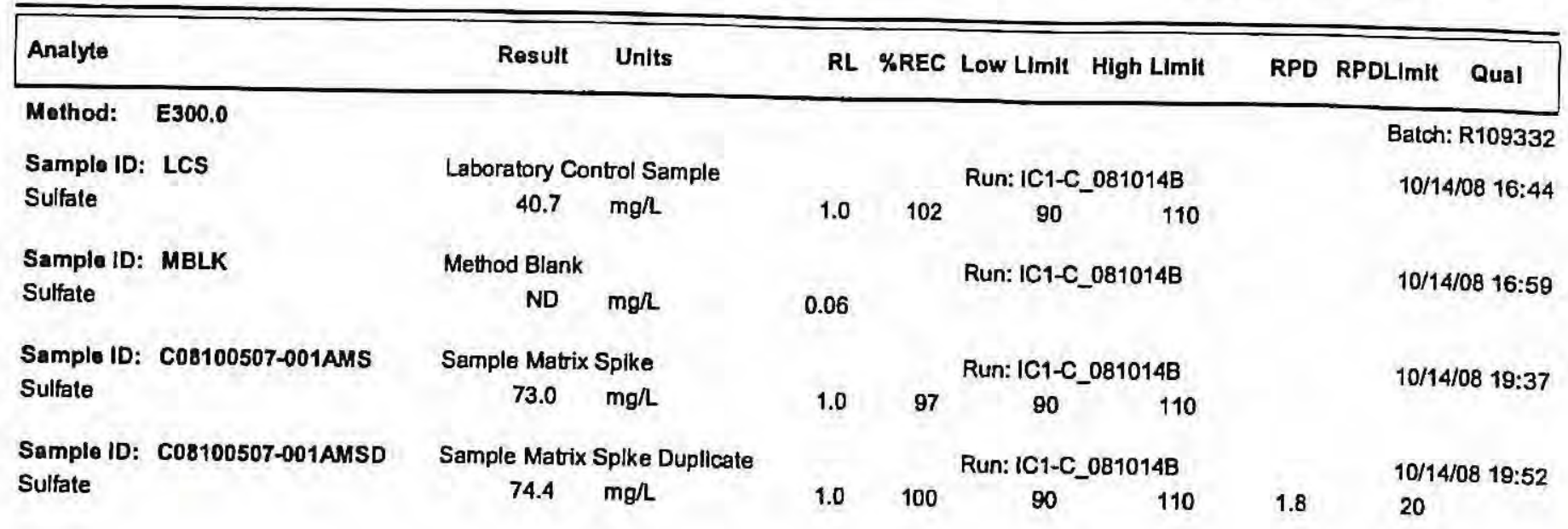

Qualifiers:

RL - Analyte reporting limit.
ND - Not detected at the reporting limit. 

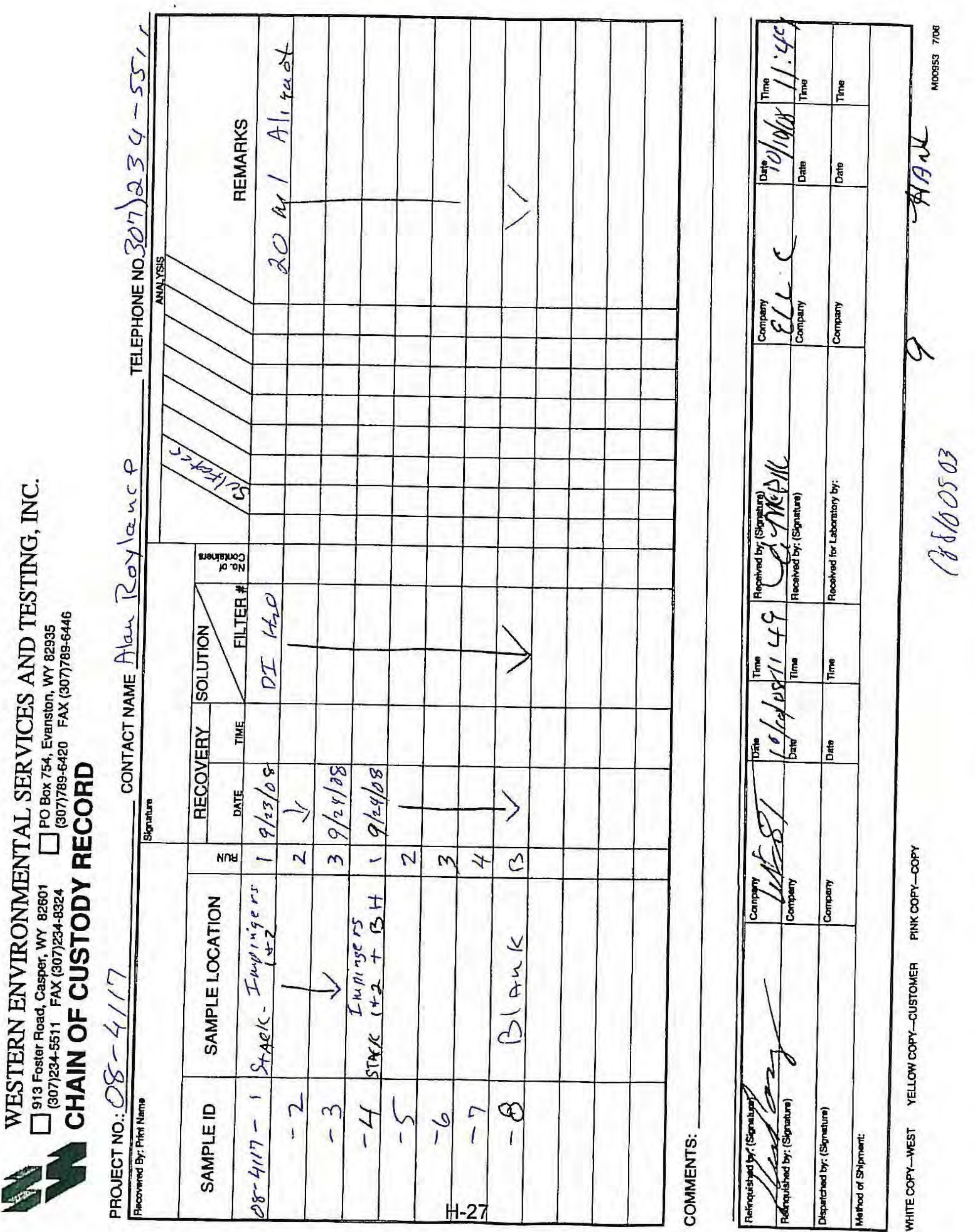


\section{Energy Laboratories Inc}

\section{Workorder Receipt Checklist}

\section{Western Environmental}

Login completed by: Edith McPike

Reviewed by:

Reviewed Date:

Shipping container/cooler in good condition?

Custody seals intact on shipping container/cooler?

Custody seals intact on sample bottles?

Chain of custody present?

Chain of custody signed when relinquished and received?

Chain of custody agrees with sample labels?

Samples in proper container/bottle?

Sample containers intact?

Sufficient sample volume for Indicated test?

All samples received within holding time?

Container/Temp Blank temperature:

Water - VOA vials have zero headspace?

Water - pH acceptable upon receipt?

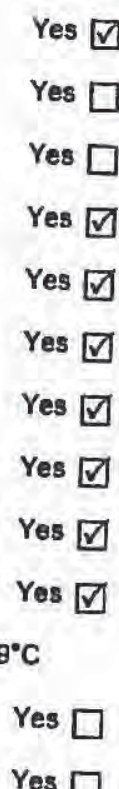

Yes

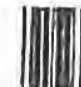

C08100503

Date and Time Received: 10/10/2008 11:49 AM

Received by: em

Carrier name: Hand Del

Contact and Corrective Action Comments:

Headspace in vials; but not pertinant for this analysis 


\section{CLIENT: Westem Environmental \\ Project: $\quad 08-4117$}

Sample Dellvery Group: C08100503
Date: $20-0 c 1-08$

\section{CASE NARRATIVE} The following Case Narrative contains exceptions or comments pertaining to the analysis of samples submitted by
Westem Environmental on 10/10/2008 11:49:00. These samples were assigned ELI Workorder Number
C08100503.

ORIGINAL SAMPLE SUBMITTAL(S)

All original sample submittals have been retumed with the data package.

SAMPLE TEMPERATURE COMPLIANCE: $4^{\circ} \mathrm{C}\left( \pm 2^{\circ} \mathrm{C}\right)$

Temperature of samples received may not be considered properly preserved by accepted standards. Samples that are hand delivered immediately after collection shall be considered acceptable if there is evidence that the chilling process has begun.

GROSS ALPHA ANALYSIS

Method 900.0 for gross alpha and gross beta is intended as a drinking water method for low TDS waters. Data provided by this method for non polable waters should be viewed as inconsistent.

RADON IN AIR ANALYSIS

The desired exposure time is 48 hours ( 2 days). The time delay in retuming the canister to the laboratory for processing beginning of counting should not exceed 8 days.

\section{SOILSSOLID SAMPLES}

All samples reported on an as recelved basis unless otherwise indicated.

\section{ATRAZINE, SIMAZINE AND PCB ANALYSIS USING EPA 505}

Data for Atrazine and Simazine are reported from EPA 525.2, not from EPA 505. Data reported by ELI using EPA method 505 refiects the results for seven individual Aroclors. When the results for all seven are ND (not detected), the sample

\section{SUBCONTRACTING ANALYSIS}

Subcontracting of sample analyses to an outside laboratory may be required. If so, ENERGY LABORATORIES will utilize its branch laboratories or qualified contract laboratories for this service. Any such laboratories will be indicated within the
Laboratory Analytical Report.

\section{BRANCH LABORATORY LOCATIONS}

eli-b - Energy Laboratories, Inc. - Billings, MT

eli-g - Energy Laboratories, Inc. - Gillette, WY

eli-h - Energy Laboratories, Inc. - Helena, MT

eli-r - Energy Laboratories, Inc. - Rapid City, SD

eli-t - Energy Laboratories, Inc. - College Station, TX

\section{CERTFICATIONS:}

USEPA: WY00002; FL-DOH NELAC: E87641; Califomia: 02118CA

Oregon: WY200001; Utah: 3072350515; Virginia: 00057; Washington: C1903

ISO 17025 DISCLAIMER:

The results of this Analytical Report relate only to the items submitted for analysis.

ENERGY LABORATORIES, INC. - CASPER,WY certifies that certain method selections contained in this report meet requirements as set forth by the above accrediting authorities. Some results requested by the client may not be covered under these certifications. All analysis data to be submitted for regulatory enforcement should be certified in the sample state of origin. Please verify ELI's certification coverage by visiting www.energylab.com ELI appreciates the opportunity to provide you with this anatytical service. For additional information and services visit our
web page www.energylab.com. 


\section{ANALYTICAL SUMMARY REPORT}

October 24,2008

Western Environmental

913 Foster Rd

Casper, WY 82601

Workorder No.: C08100558

Quote ID: C2258 - Chlorides

Project Name: Not Indicated

Energy Laboratories, Inc. received the following 8 samples from Westem Environmental on 10/13/2008 for analysis.

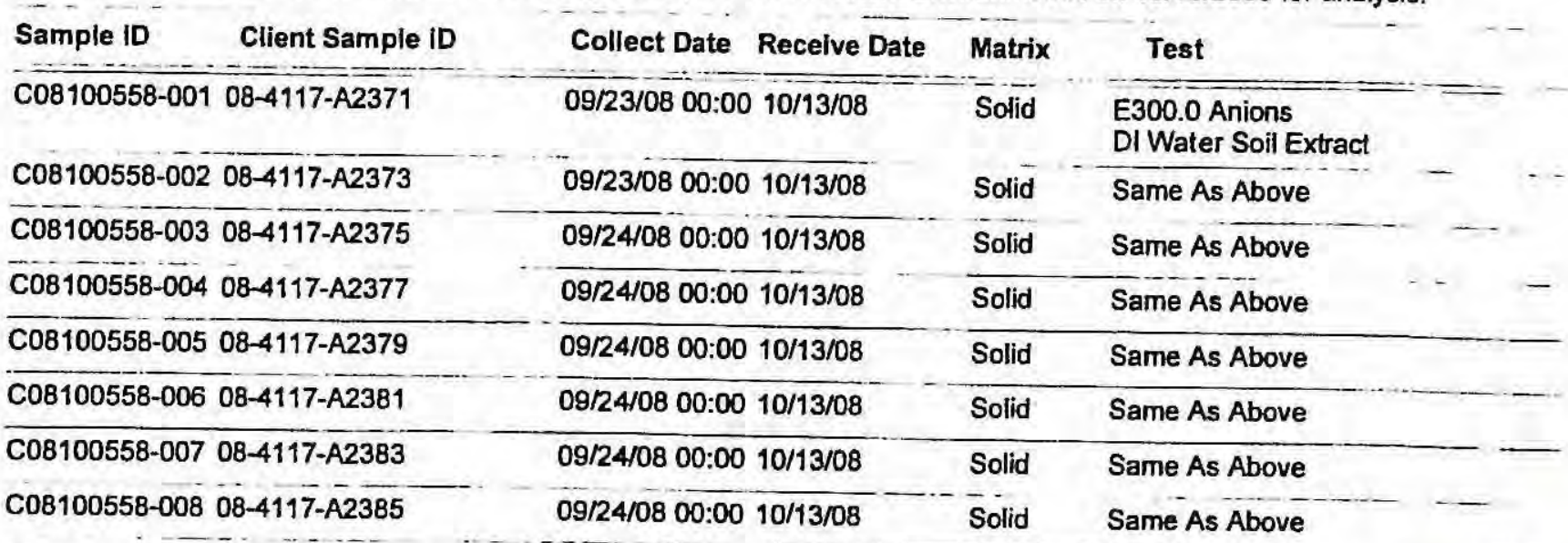

As appropriate, any exceptions or problems with the analyses are noted in the Laboratory Analytical Report, the QAVQC Summary Report, or the Case Narrative.

If you have any questions regarding these tests results, please call.

Report Approved By: 0 oved 100 la 


\section{LABORATORY ANALYTICAL REPORT}

Client: Western Environmental

Site Name: $\quad$ Not Indicated

Report Date: $10 / 24 / 08$

Lab ID: C08100558-001

Client Sample ID: 08-4117-A2371

Matrix:

Solid

Collection Date: 09/23/08

DateReceived: $10 / 13 / 08$

$\begin{array}{llllll}\text { Analyses } & \text { Result } & \text { Units } & \text { Qualifiers } & \text { RL } & \begin{array}{c}\text { MCL } \\ \text { QCL Method Analysis Date / By }\end{array} \\ \begin{array}{l}\text { MAJOR IONS } \\ \text { Chloride }\end{array} & & & & & \\ & 0.58 & \text { mg/beaker } & 0.05 & 10 / 15 / 0801: 52 / 51\end{array}$

Lab ID: C08100558-002

Client Sample ID: $08-4117-A 2373$

Matrix: Solid

Analyses

MAJOR IONS

Chloride

$$
\text { - }
$$

Collection Date: 09/23/08

DateReceived: $10 / 13 / 08$

ND mg/beaker Quallitiors

RL

MCL Analysis Date / By

QCL Method

0.05

E300.0

10/15/08 02:06 / ljil
Lab ID:
C08100558-003
Client Sample ID: 08-4117-A2375
Matrix:
Solid

\begin{tabular}{ll} 
Analyses \\
MAJOR IONS \\
Chloride \\
\\
\hline $\begin{array}{ll}\text { Lab ID: } & \text { C08100558-004 } \\
\text { Client Sample ID: } & 08-4117-A 2377 \\
\text { Matrix: } & \text { Solid }\end{array}$ \\
\hline
\end{tabular}

Collection Date: 09/24/08

DateReceived: $10 / 13 / 08$

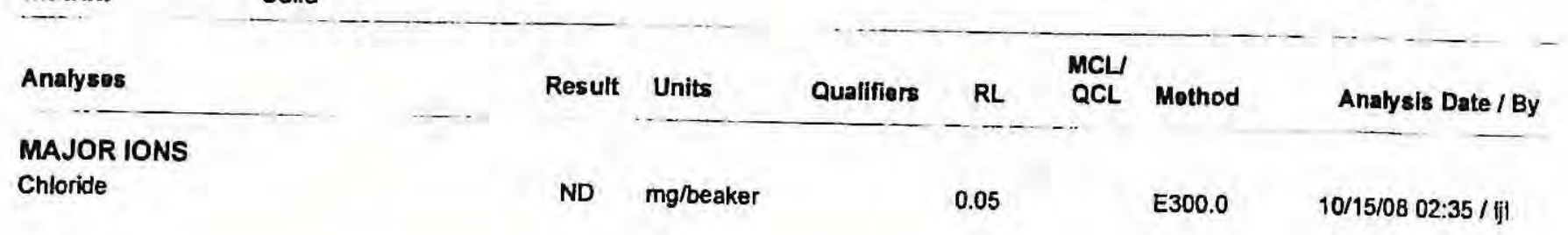

Report RL - Analyte reporting limit.

Definitions: $Q C L$ - Quality control limit.
ND mg/beaker
RL $\quad$ QCL

0.05

$E 300.0$

10/15/08 02:21/ II
Collection Date: 09/24/08

DateRecelved: $10 / 13 / 08$ 


\section{LABORATORY ANALYTICAL REPORT}

Client: Western Environmental

Site Name: $\quad$ Not Indicated

Report Date: 10/24/08

Lab ID: $\quad$ C08100558-005

Client Sample ID: 08-4117-A2379

Matrix:

Solid

Collection Date: 09/24/08

DateReceived: $10 / 13 / 08$

\begin{tabular}{|c|c|c|c|c|c|c|c|}
\hline Analyses & Result & Units & Quallfiers & RL & $\begin{array}{l}\text { MCL } \\
\text { QCL }\end{array}$ & Method & Analysis Date / By \\
\hline \multicolumn{8}{|l|}{$\begin{array}{l}\text { MAJOR IONS } \\
\text { Chloride }\end{array}$} \\
\hline Chloride & ND & mg/beaker & & 0.05 & & $E 300.0$ & 10/15/08 02:50/ \\
\hline
\end{tabular}

Lab ID: $\quad$ C08100558-006

Client Sample ID: 08-4117-A2381

Matrix: Solid

Analyses

\section{MAJOR IONS}

Chloride

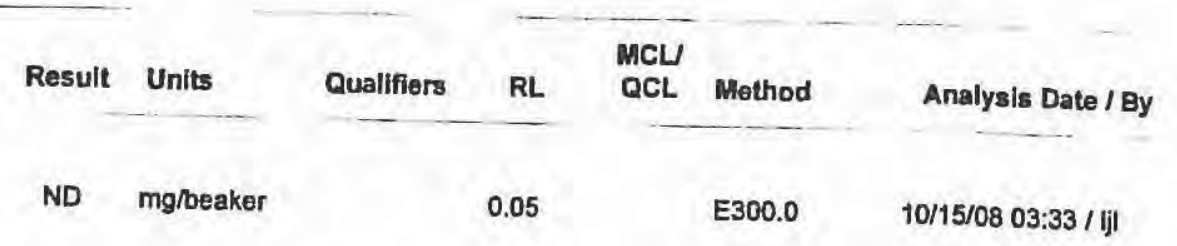

Collection Date: 09/24/08

DateReceived: $10 / 13 / 08$

Collection Date: 09/24/08

DateReceived: $10 / 13 / 08$

DateReceived. 10/13/08
Lab ID:
Client Sample ID: 08-4117-A2383
Matrix:
Solid

Analyses

MAJOR IONS

Chloride

$\begin{array}{lcccc}\text { Result Units } & \text { Qualifiers } & \text { RL } & \begin{array}{c}\text { MCL } \\ \text { QCL Method }\end{array} & \text { Analysis Date / By } \\ \text { ND mg/beaker } & 0.05 & \text { E300.0 } & 10 / 15 / 0803: 47 / \text { ji }\end{array}$

Lab ID:

C08100558-008

Client Sample ID: 08-4117-A2385

Matrix:

Solid

Collection Date: 09/24/08

DateReceived: 10/13/08

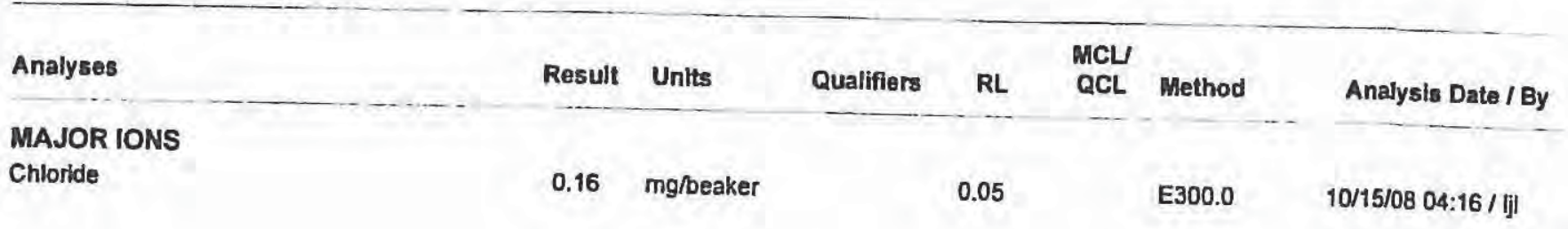

Report Definitions: RL - Analyte reporting limit. QCL - Quality control limit.
MCL - Maximum contaminant level.

ND - Not detected at the reporting limit. 


\section{QA/QC Summary Report}

Client: Westem Environmental

Project: Not Indicated

Report Date: 10/24/08

Work Order: C08100558

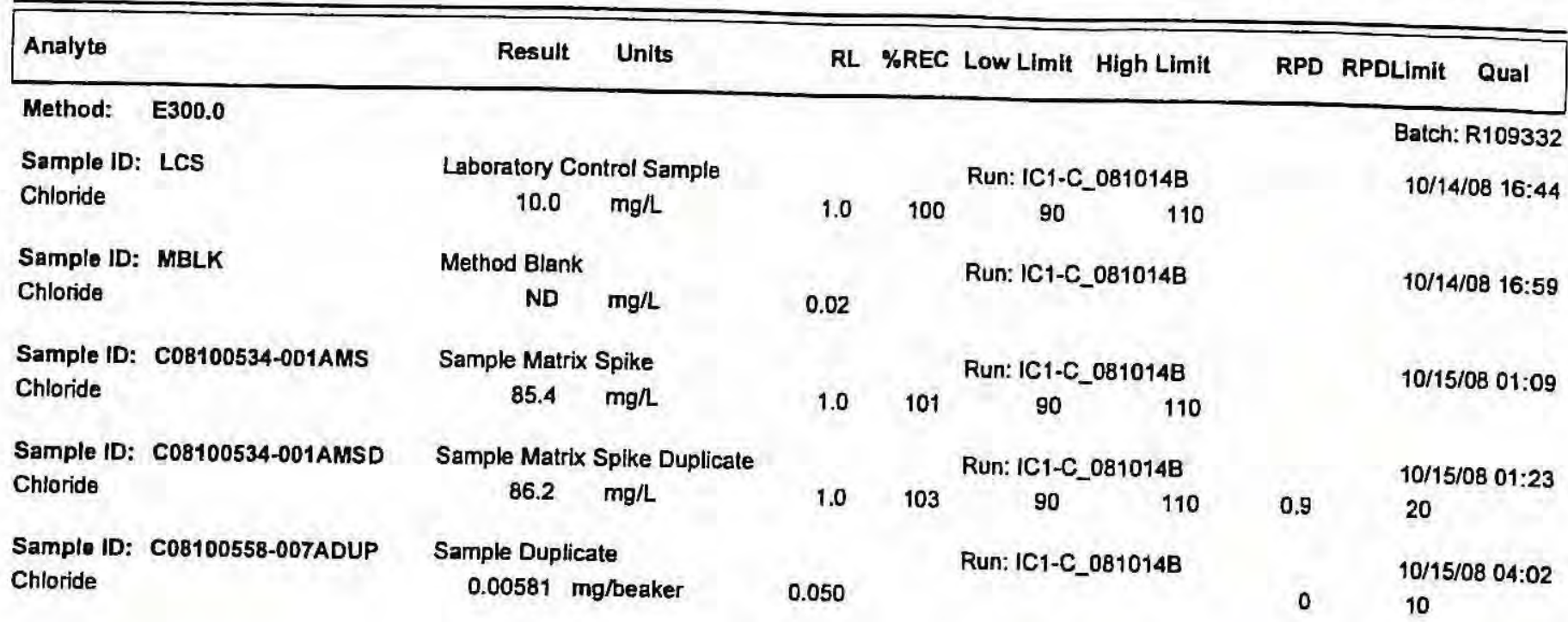




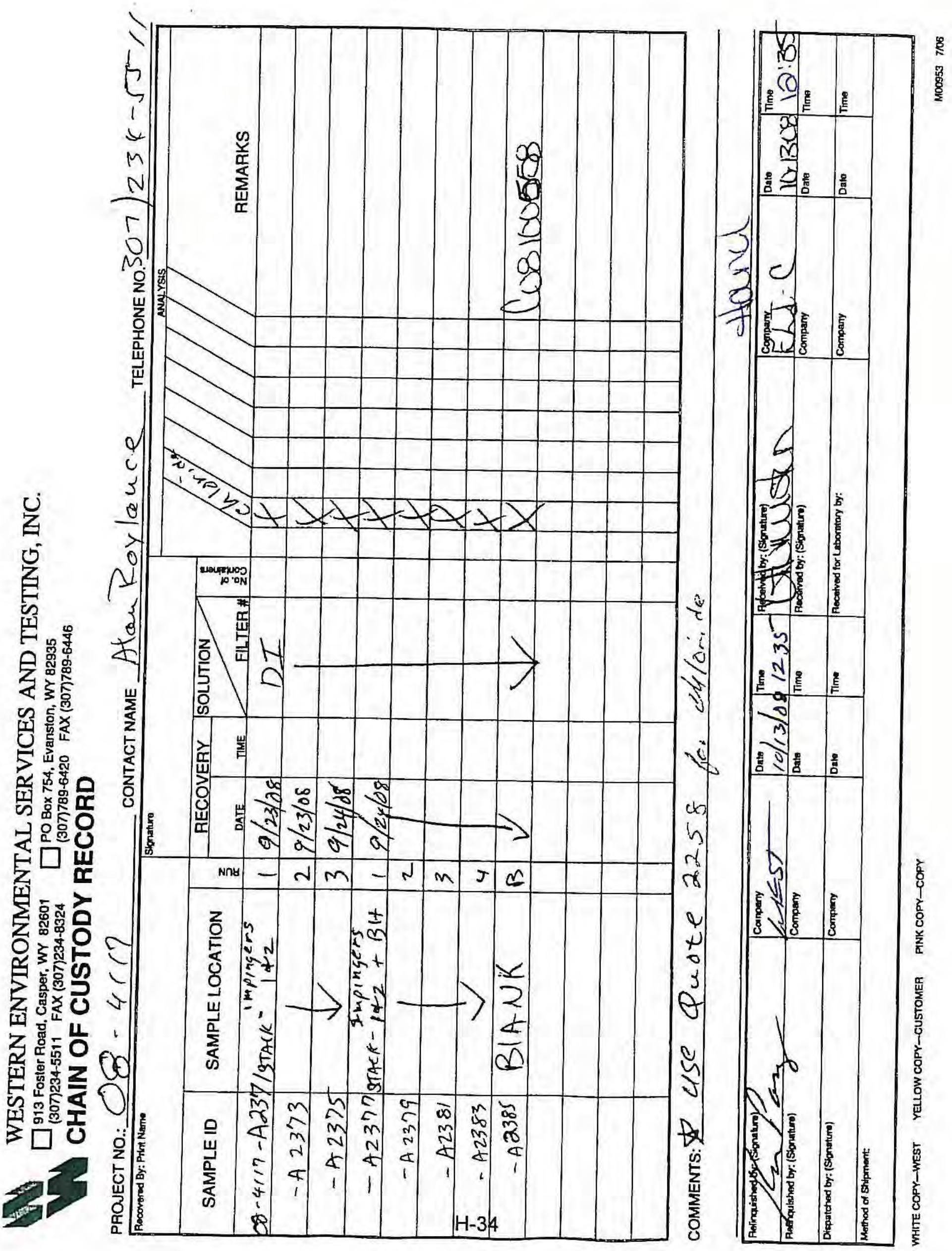




\section{Energy Laboratories Inc Workorder Receipt Checklist}

\section{Western Environmental}

Login completed by: Kimberly Humiston

Reviewed by:

Reviewed Date:

Shipping container/cooler in good condition?
Custody seals intact on shipping container/cooler?
Custody seals intact on sample bottles?
Chain of custody present?
Chain of custody signed when relinquished and received?
Chain of custody agrees with sample labels?
Samples in proper container/bottle?
Sample containers intact?
Sufficient sample volume for indicated test?
All samples recelved within holding time?
Container/Temp Blank temperature:
Water - VOA vials have zero headspace?
Water - pH acceptable upon receipt?

$\begin{array}{lll}\text { Yes } \square & \text { No } \square & \text { Not Present } \square \\ \text { Yes } \square & \text { No } \square & \text { Not Present } \square \\ \text { Yes } \square & \text { No } \square & \text { Not Present } \square \\ \text { Yes } \square & \text { No } \square \\ \text { Yes } \square & \text { No } \square \\ \text { Yes } \square & \text { No } \square \\ \text { Yes } \square & \text { No } \square \\ \text { Yes } \square & \text { No } \square \\ \text { Yes } \square & \text { No } \square \\ \text { Yes } \square & \text { No } \square & \\ \text { N/A C } & \\ \text { Yes } \square & \text { No } \square & \\ \text { Yes } \square & \text { No } \square & \text { Not VOA vials submitted } \square\end{array}$

Contact and Corrective Action Comments:

None

Date and Time Received: 10/13/2008 12:35 PM

Received by: kh

Carrier name: Hand Del

C08100558 
CLIENT: Westem Environmental

Project: Not Indicated

Sample Delivery Group: C08100558
Date: 240 ct-08

\section{CASE NARRATIVE} The following Case Narrative contains exceptions or comments pertaining to the analysis of samples submitted by
Western Environmental on 10/13/2008 12:35:00. These samples were assigned ELI Workorder Number
C08100558.

ORIGINAL SAMPLE SUBMITTAL(S)

All original sample submittals have been retumed with the data package.

SAMPLE TEMPERATURE COMPLIANCE: $4^{\circ} \mathrm{C}\left( \pm 2^{\circ} \mathrm{C}\right)$

Temperature of samples received may not be considered properly preserved by accepted standards. Samples that are hand delivered immediately after collection shall be considered acceptable if there is evidence that the chilling process has begun.

GROSS ALPHA ANALYSIS Method 900.0 for gross alpha and gross beta is intended as a drinking water method for low TDS waters. Data provided by
this method for non potable waters should be viewed as inconsistent.

\section{RADON IN AIR ANALYSIS}

The desired exposure time is 48 hours ( 2 days). The time delay in retuming the canister to the laboratory for processing beginning of counting should not exceed 8 days.

\section{SOILISOLID SAMPLES}

All samples reported on an as received basis unless otherwise indicated.

\section{ATRAZINE, SIMAZINE AND PCB ANALYSIS USING EPA 505}

Data for Atrazine and Simazine are reported from EPA 525.2, not from EPA 505. Data reported by ELI using EPA method 505 reflects the results for seven individual Aroclors. When the results for all seven are ND (not detected), the sample

SUBCONTRACTING ANALYSIS

Subcontracting of sample analyses to an outside laboratory may be required. If so, ENERGY LABORATORIES will utilize its branch laboratories or qualified contract laboratories for this service. Any such laboratories will be indicated within the
Laboratory Analytical Report.

BRANCH LABORATORY LOCATIONS

eli-b - Energy Laboratories, Inc. - Billings, MT

eli-g - Energy Laboratories, Inc. - Gillette, WY

eli-h - Energy Laboratories, Inc. - Helena, MT

eli-r - Energy Laboratories, Inc. - Rapid City, SD

eli-t - Energy Laboratories, Inc. - College Station, TX

\section{CERTFICATIONS:}

USEPA: WY00002; FL-DOH NELAC: E87641; California: 02118CA

Oregon: WY200001; Utah: 3072350515; Virginia: 00057; Washington: C1903

ISO 17025 DISCLAIMER:

The results of this Analytical Report relate only to the items submitted for analysis.

ENERGY LABORATORIES, INC. - CASPER,WY certifies that certain method selections contained in this report meet requirements as set forth by the above accrediting authorities. Some results requested by the client may not be covered under these certifications. All analysis data to be submitted for regulatory enforcement should be certified in the sample state of origin. Please verify ELl's certification coverage by visiting www.energylab.com ELI appreciates the opportunity to provide you with this analytical service. For additional information and services visit our
web page www.energylab.com. 
Appendix I:

Chain of Custodies 


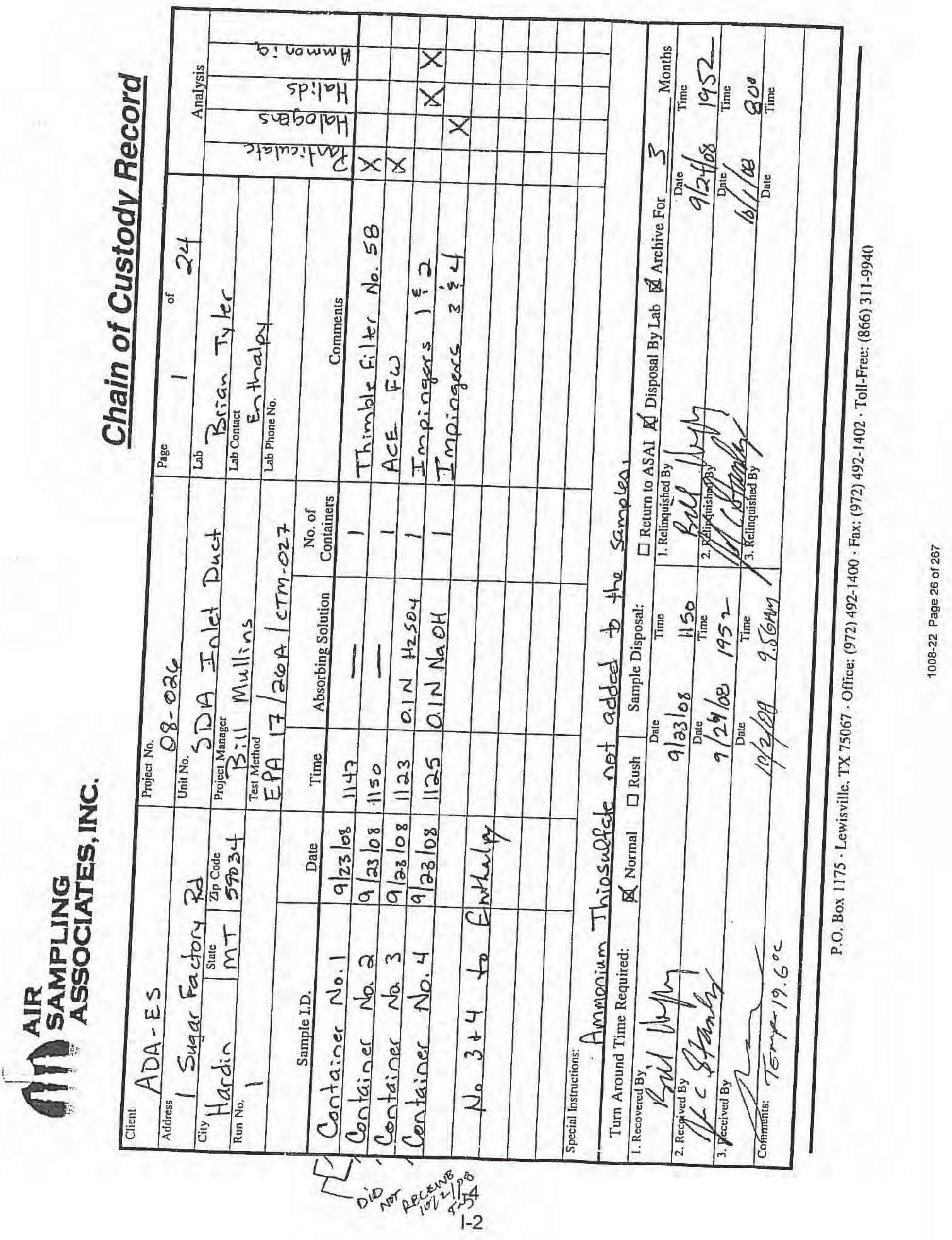




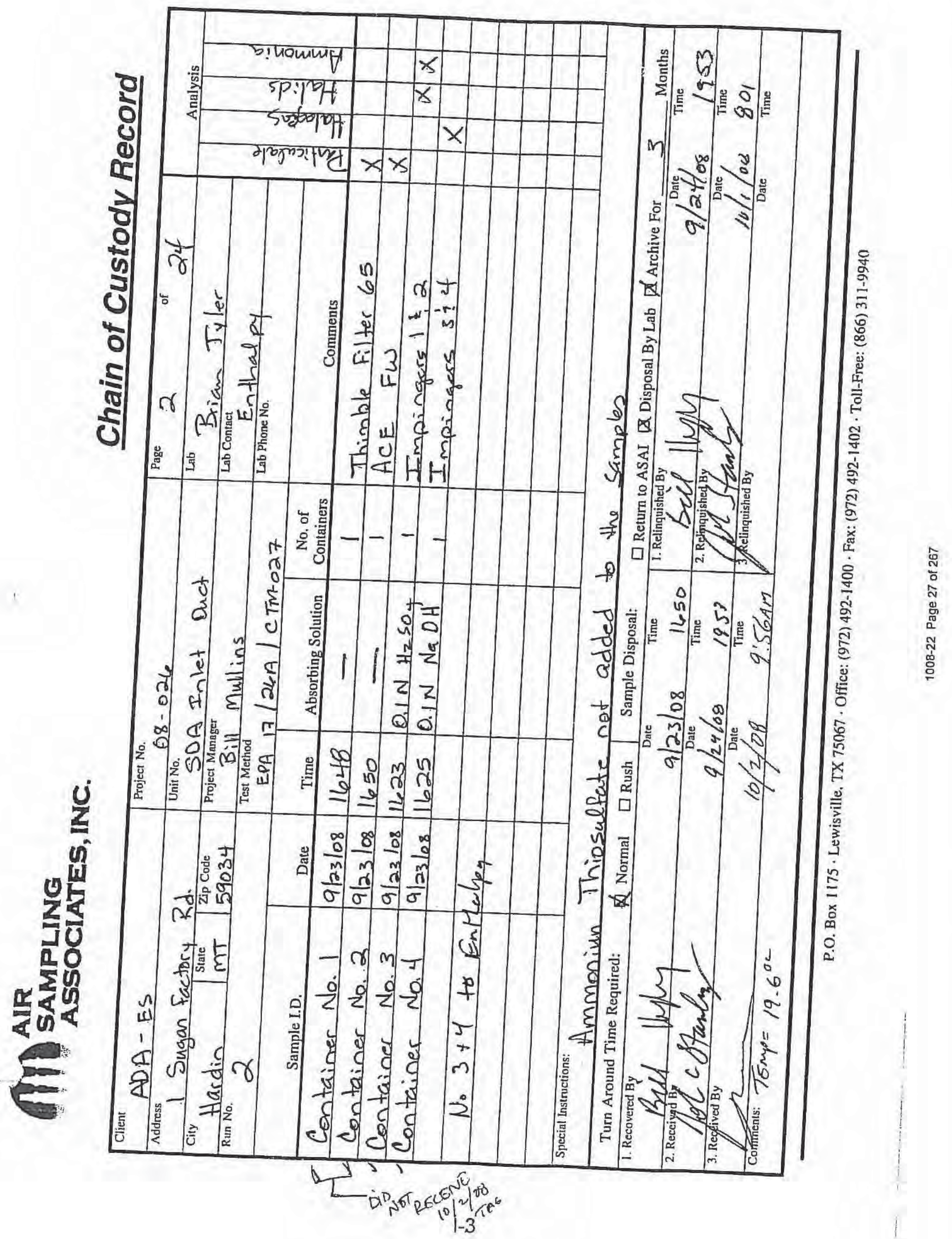




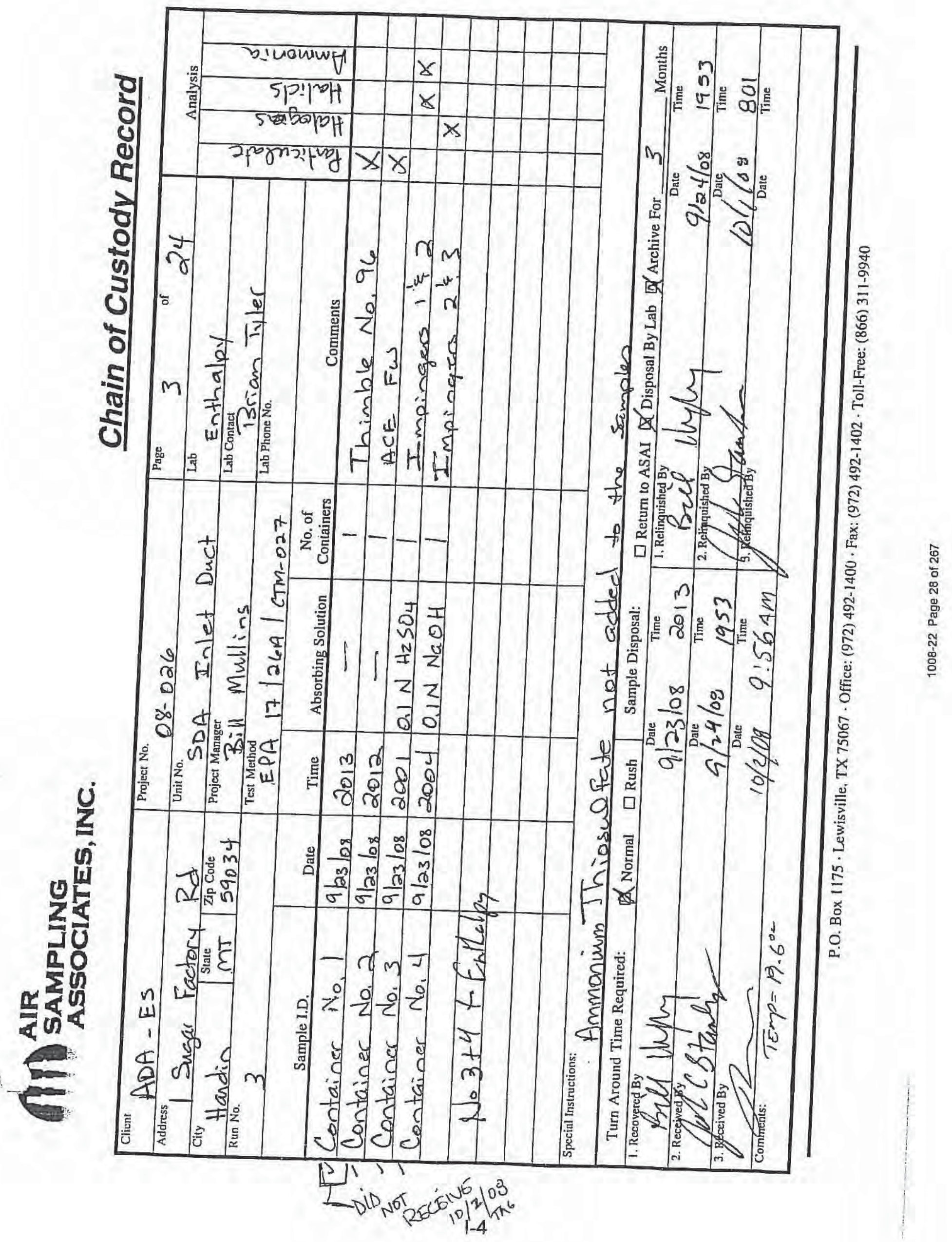




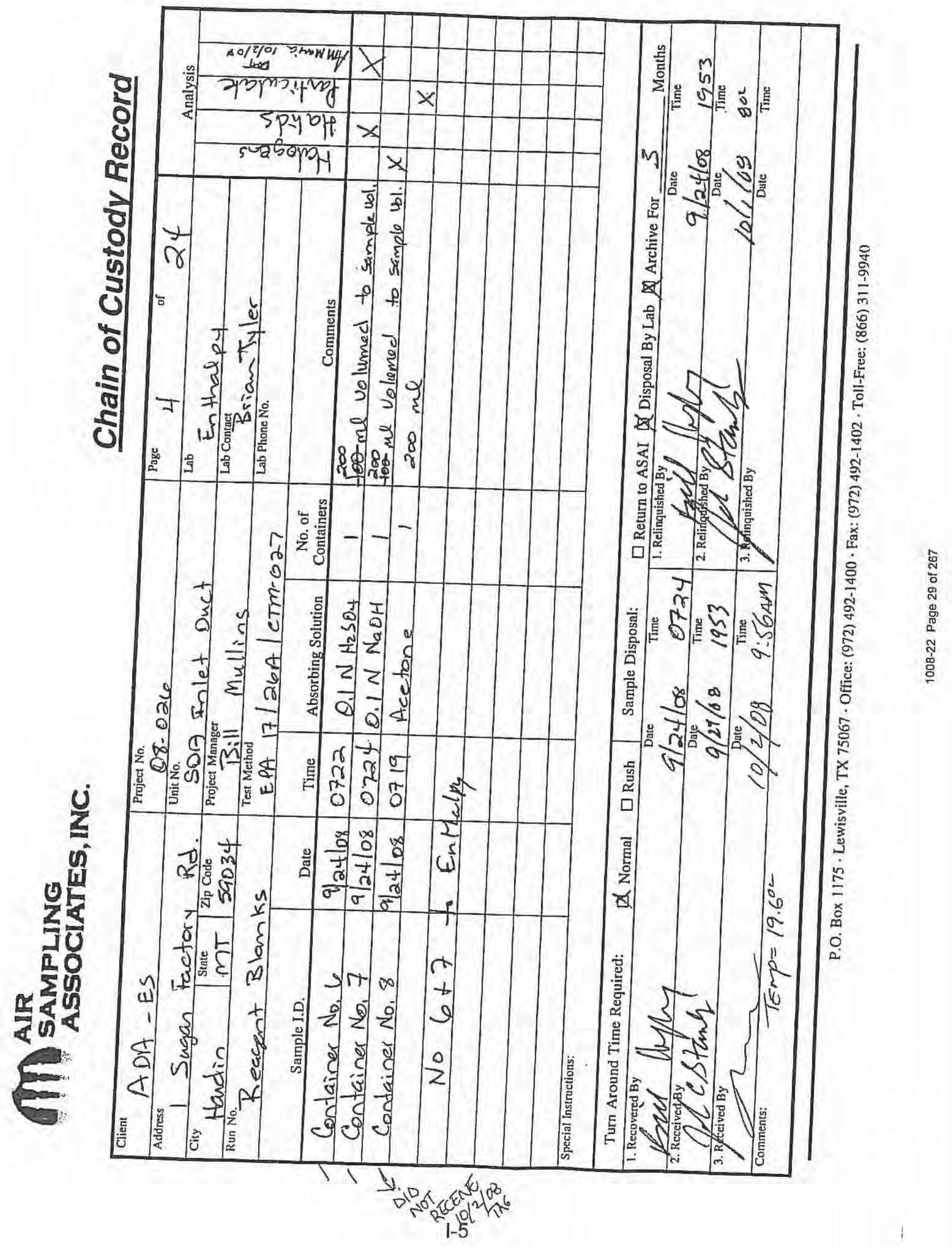




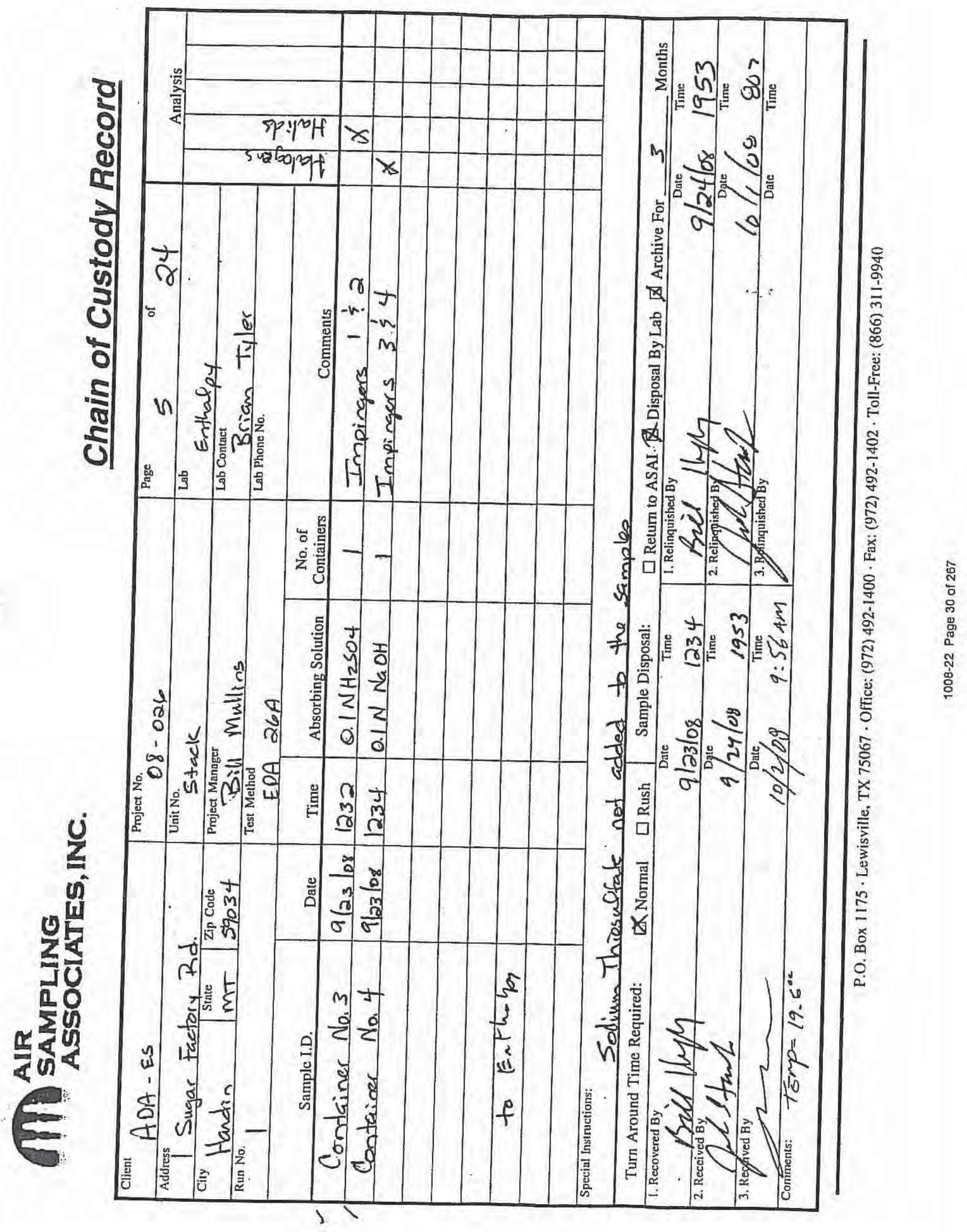




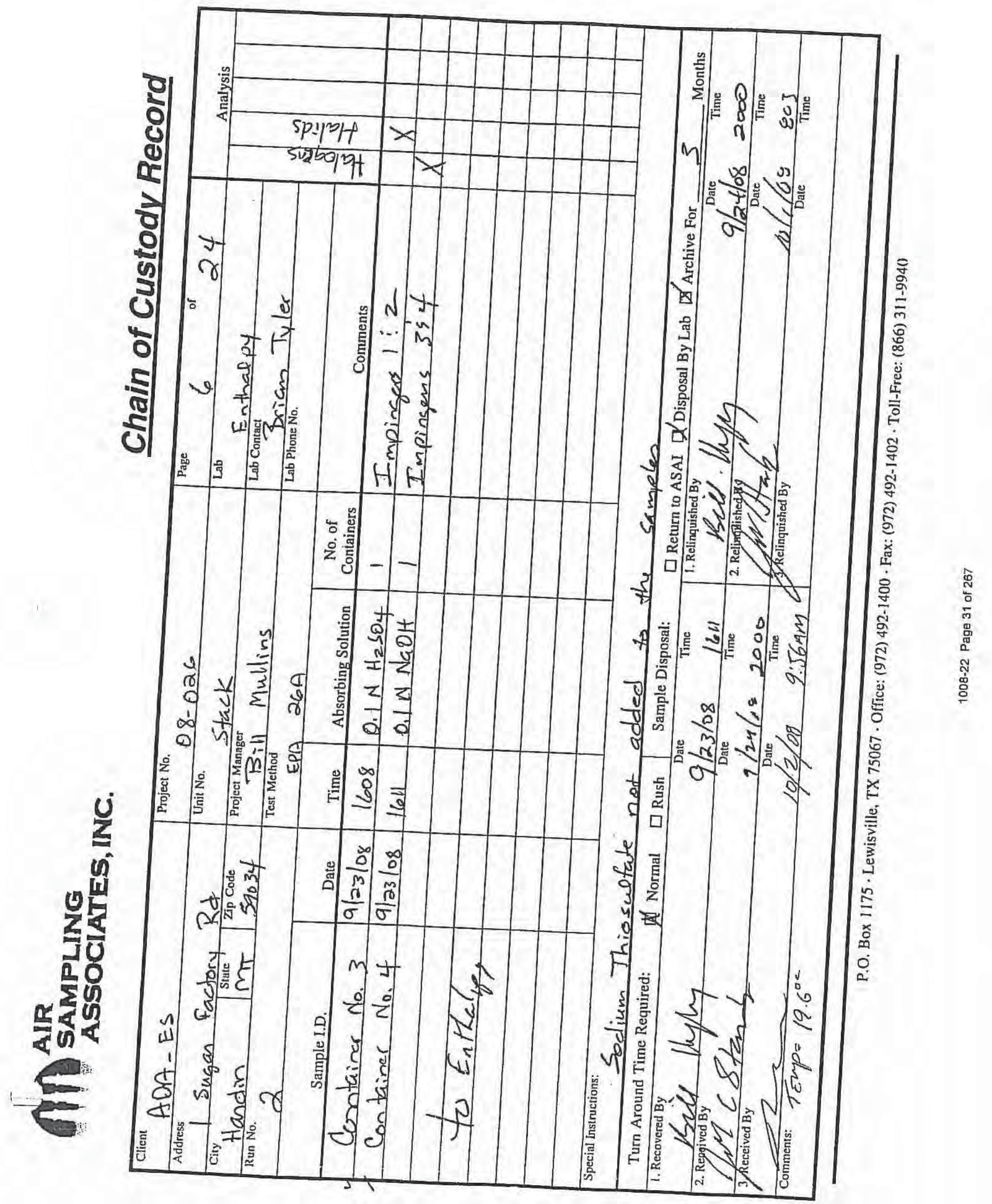




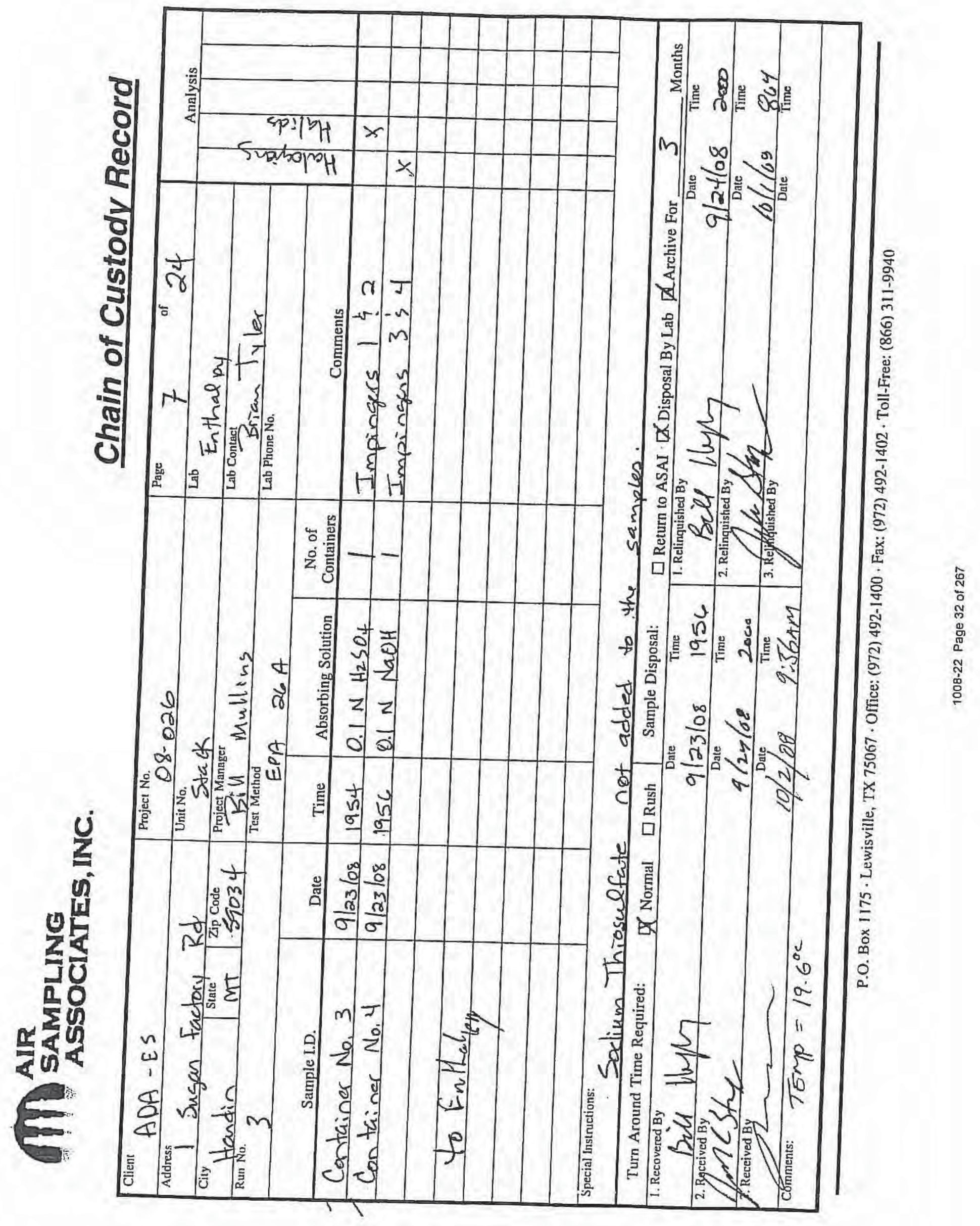




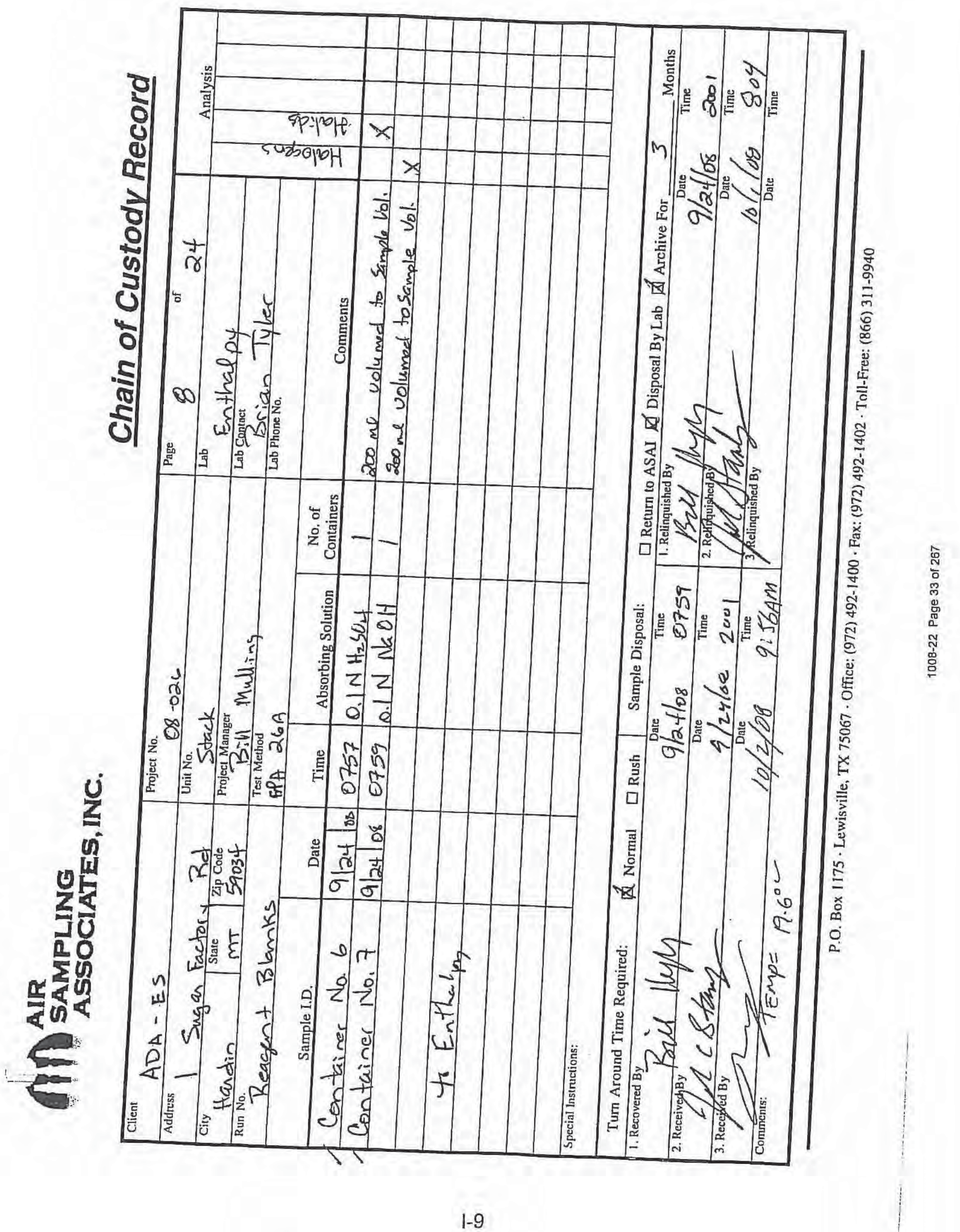




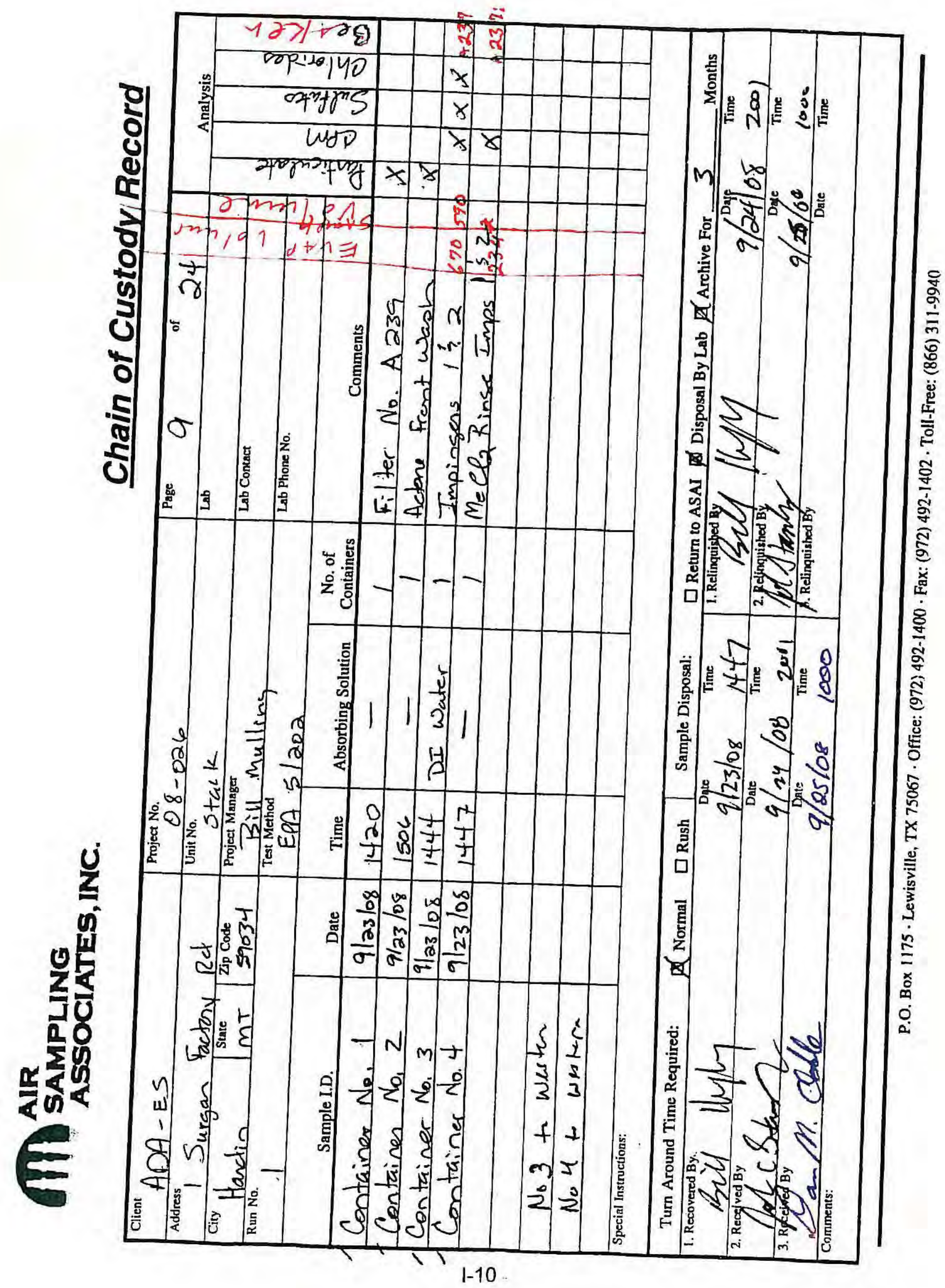




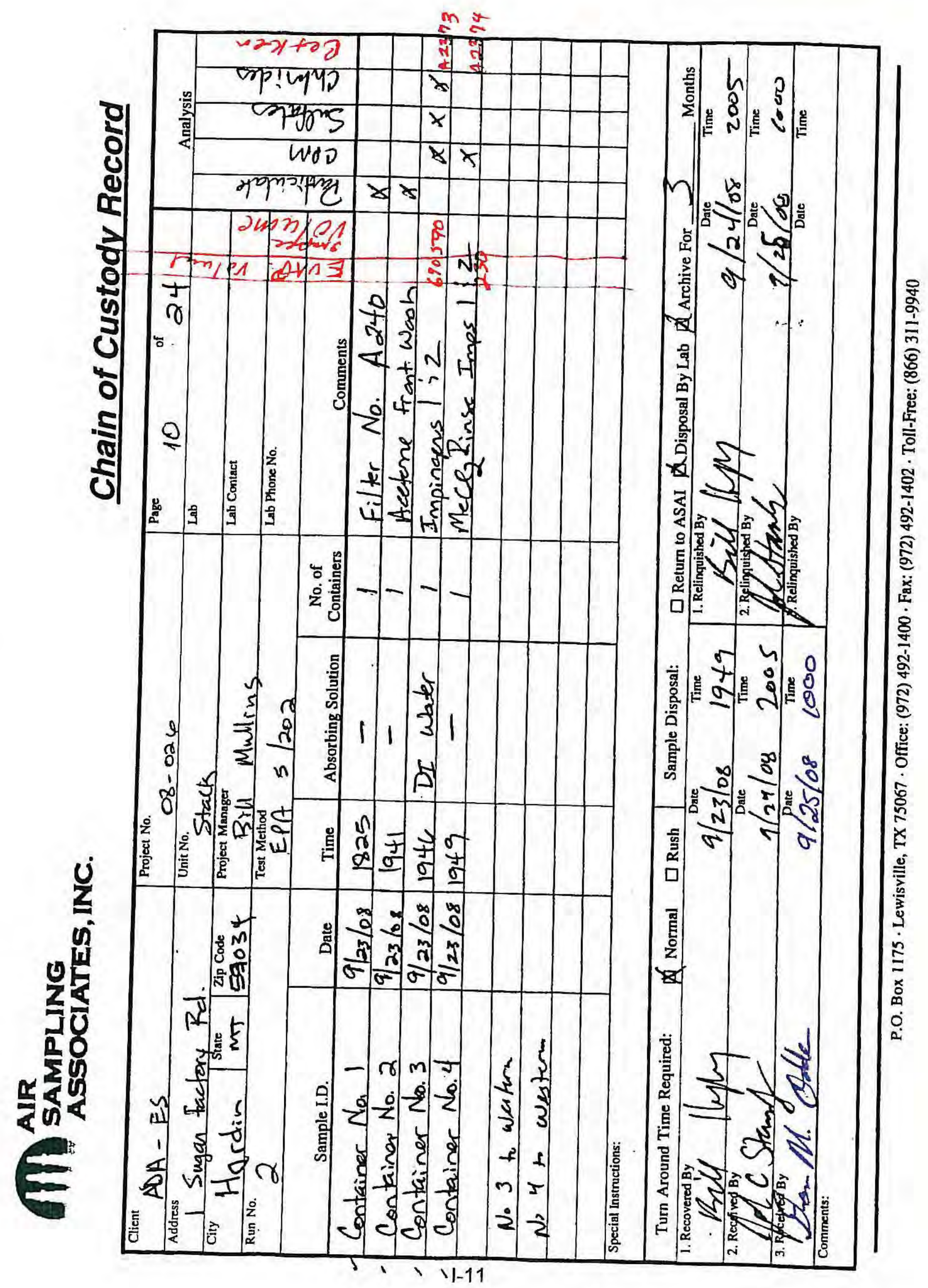




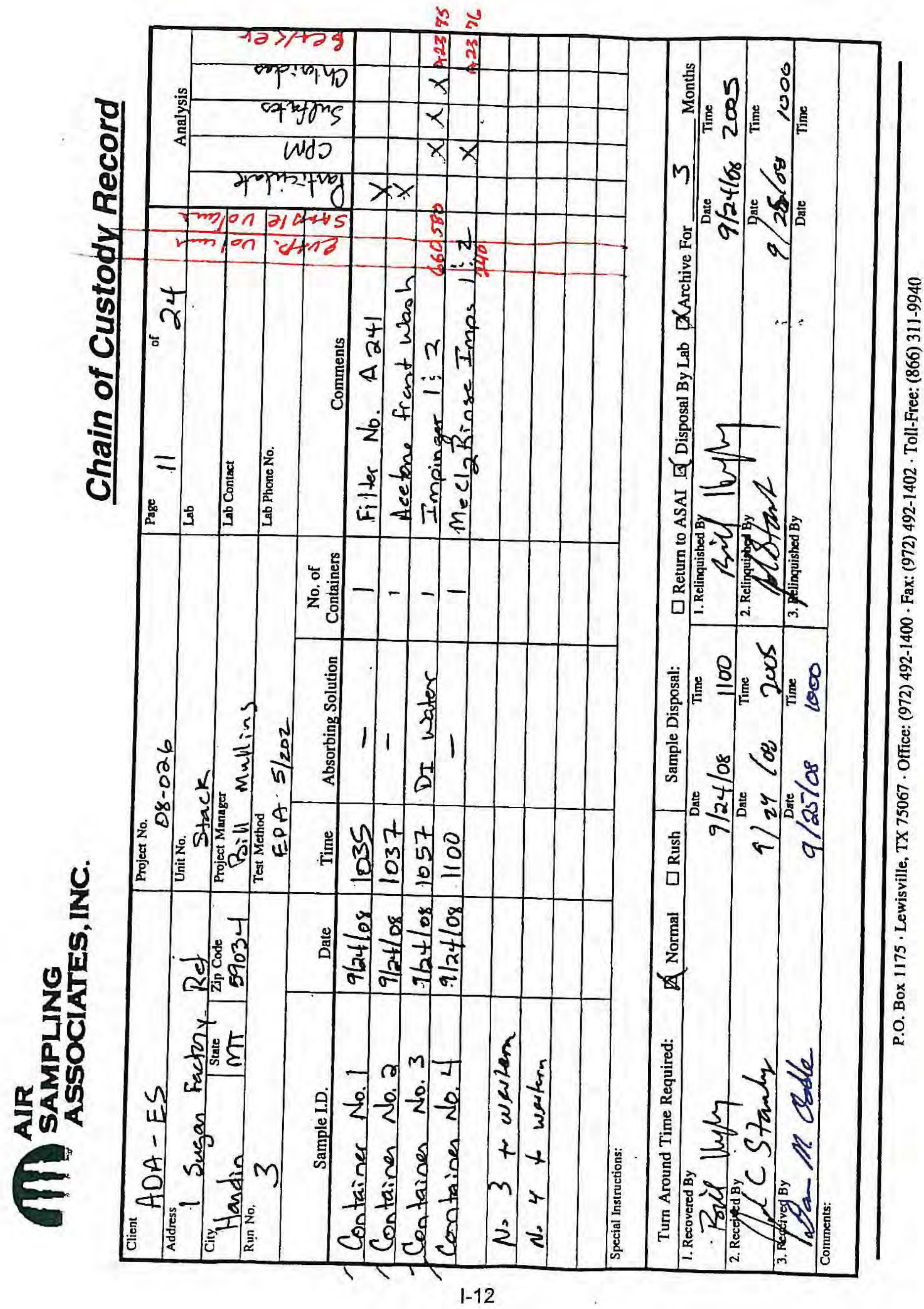




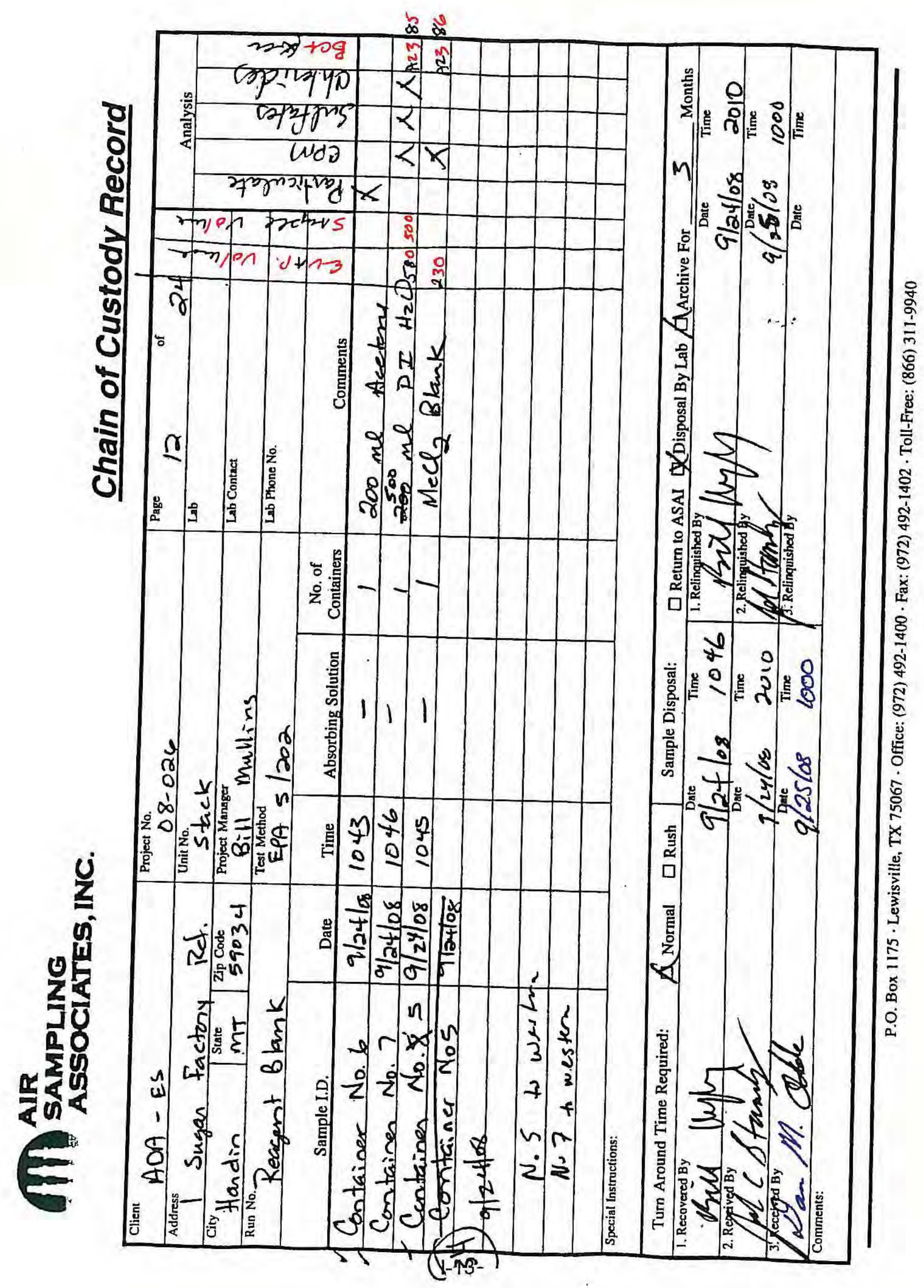




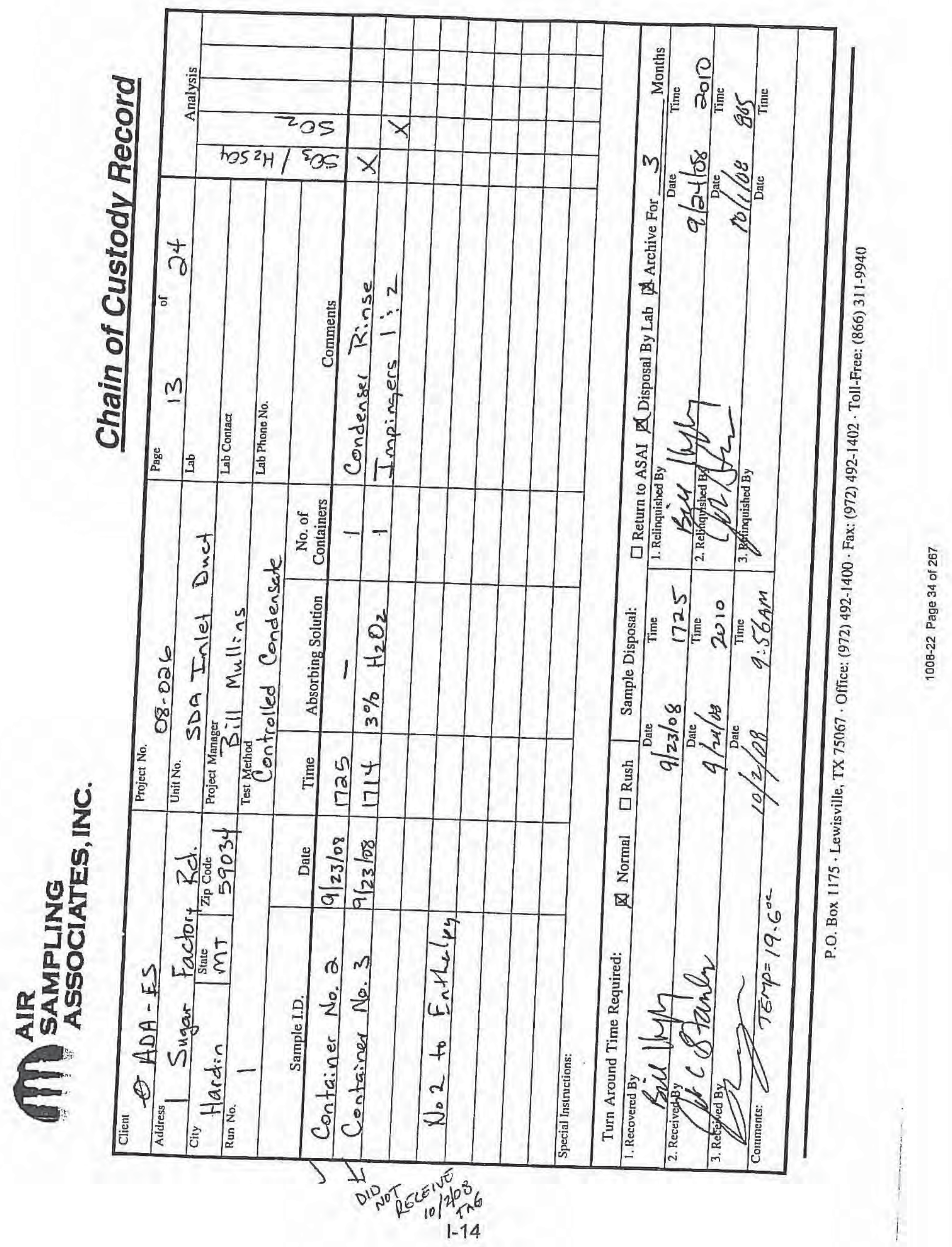




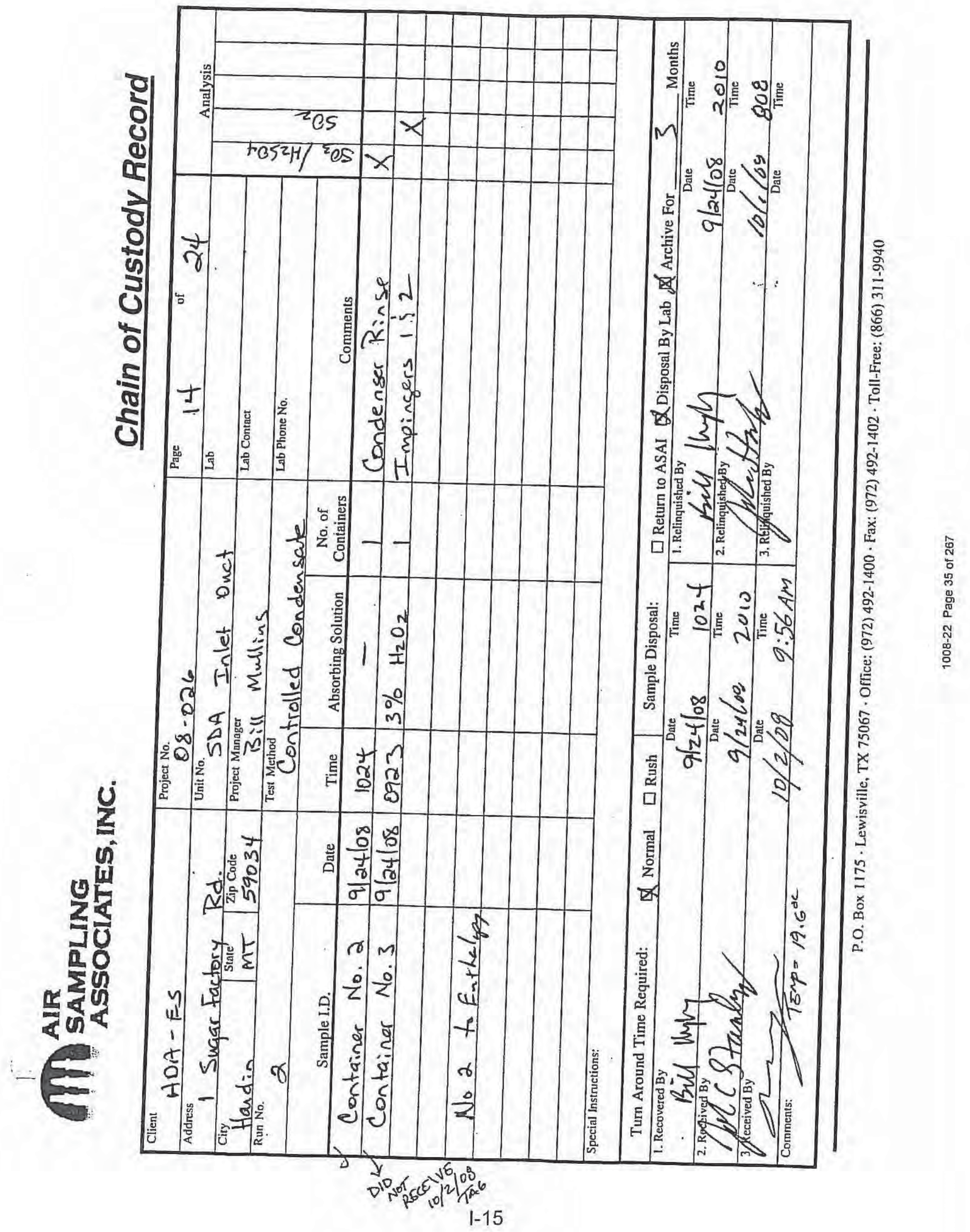




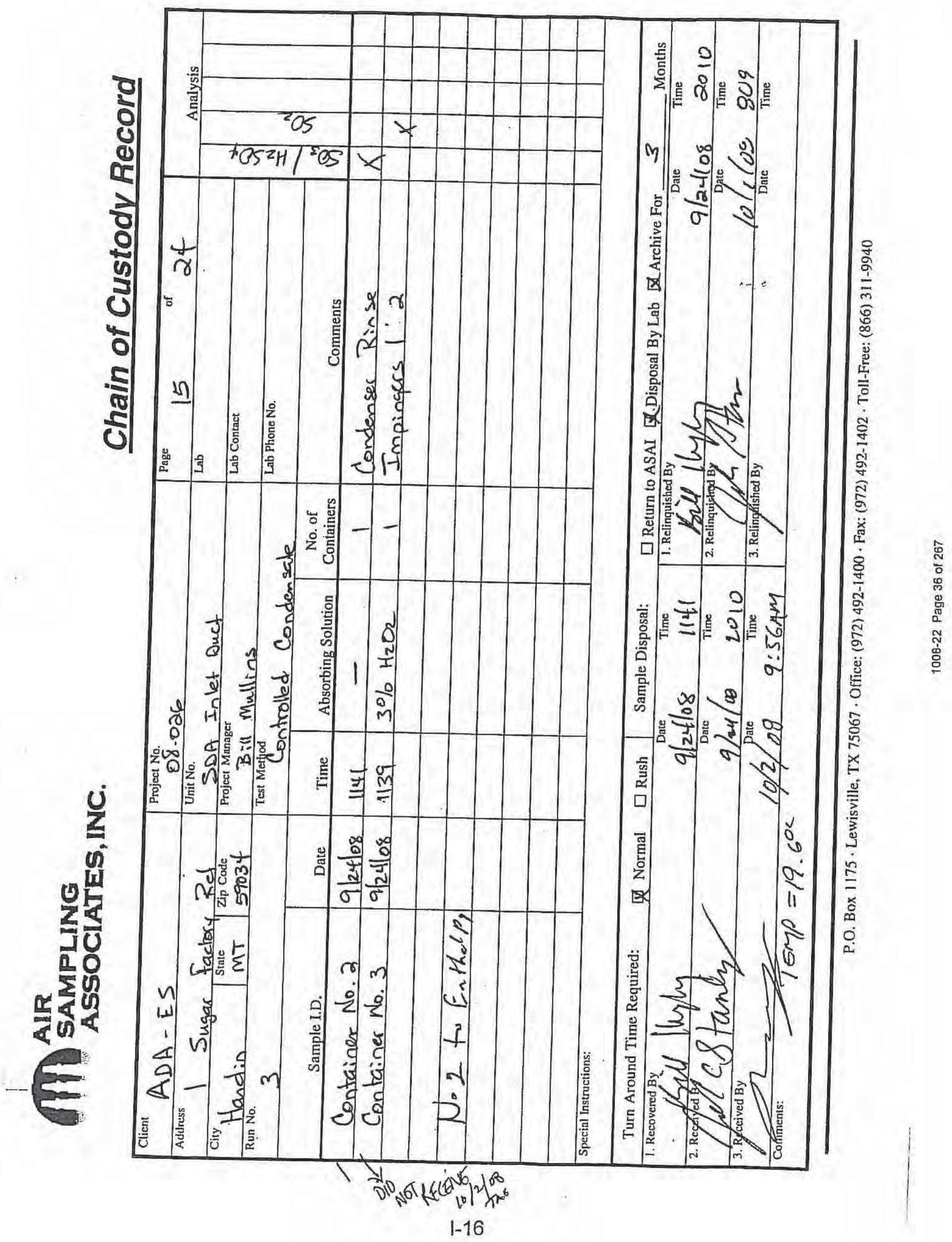




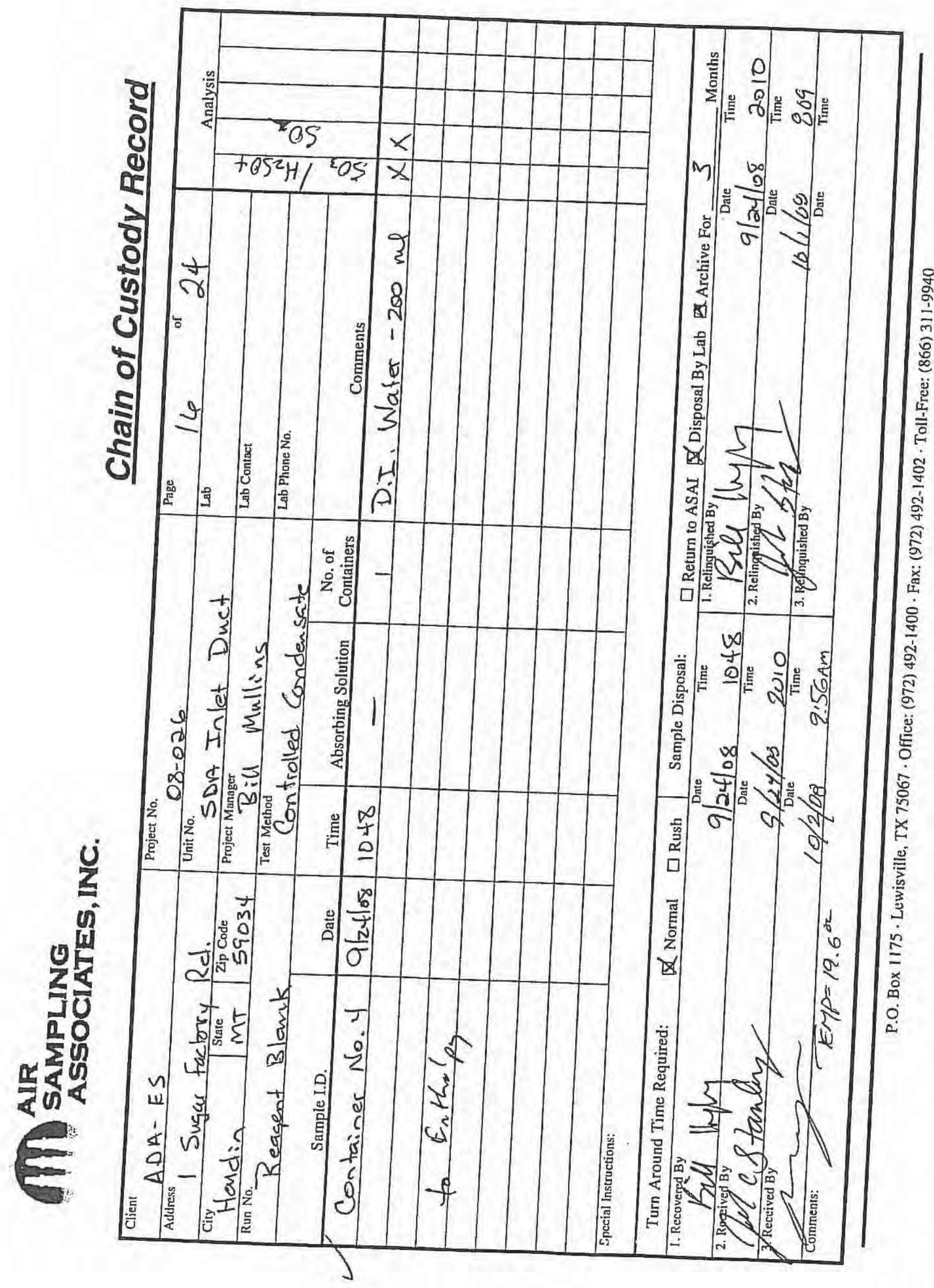

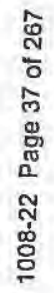




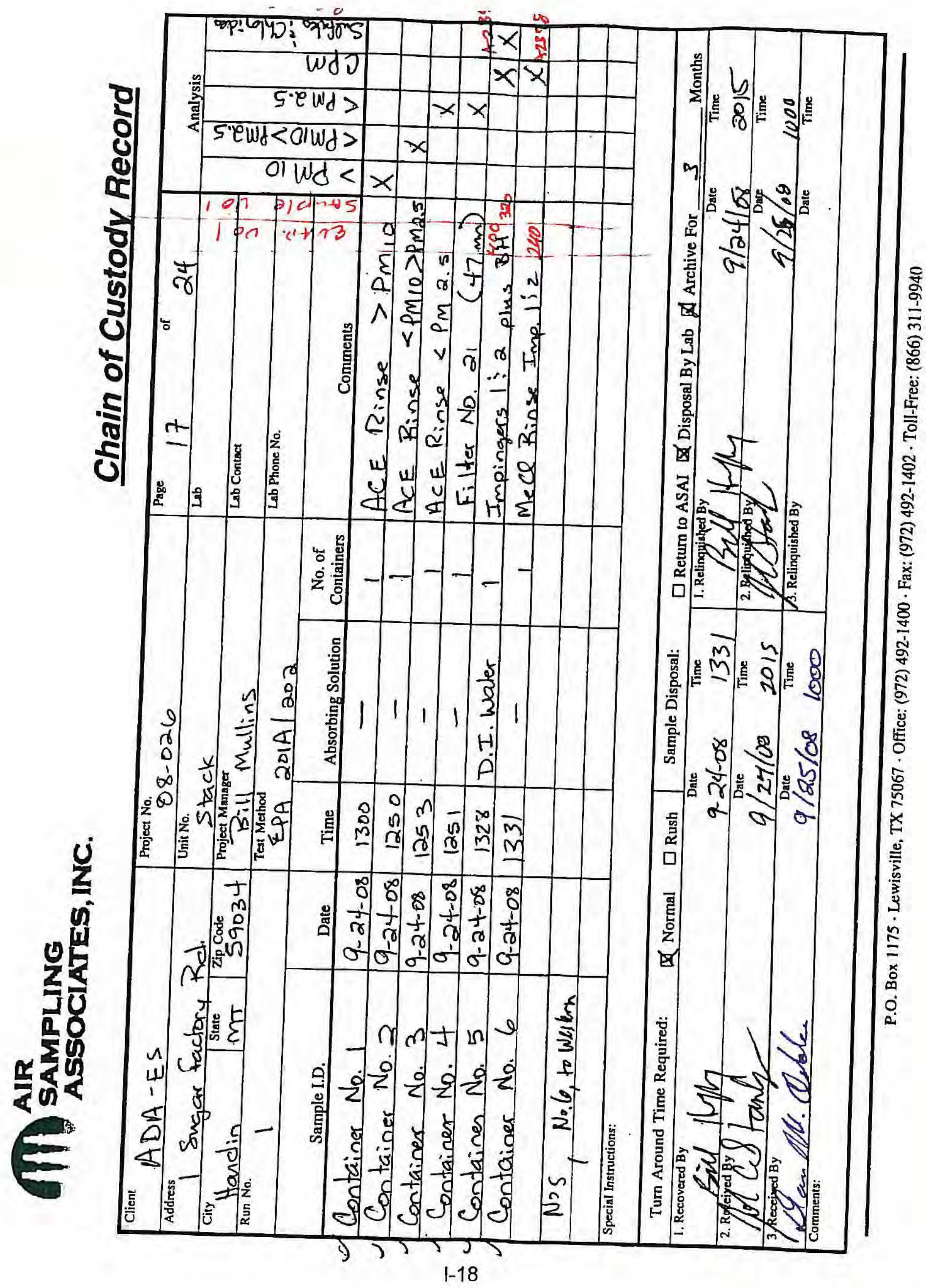




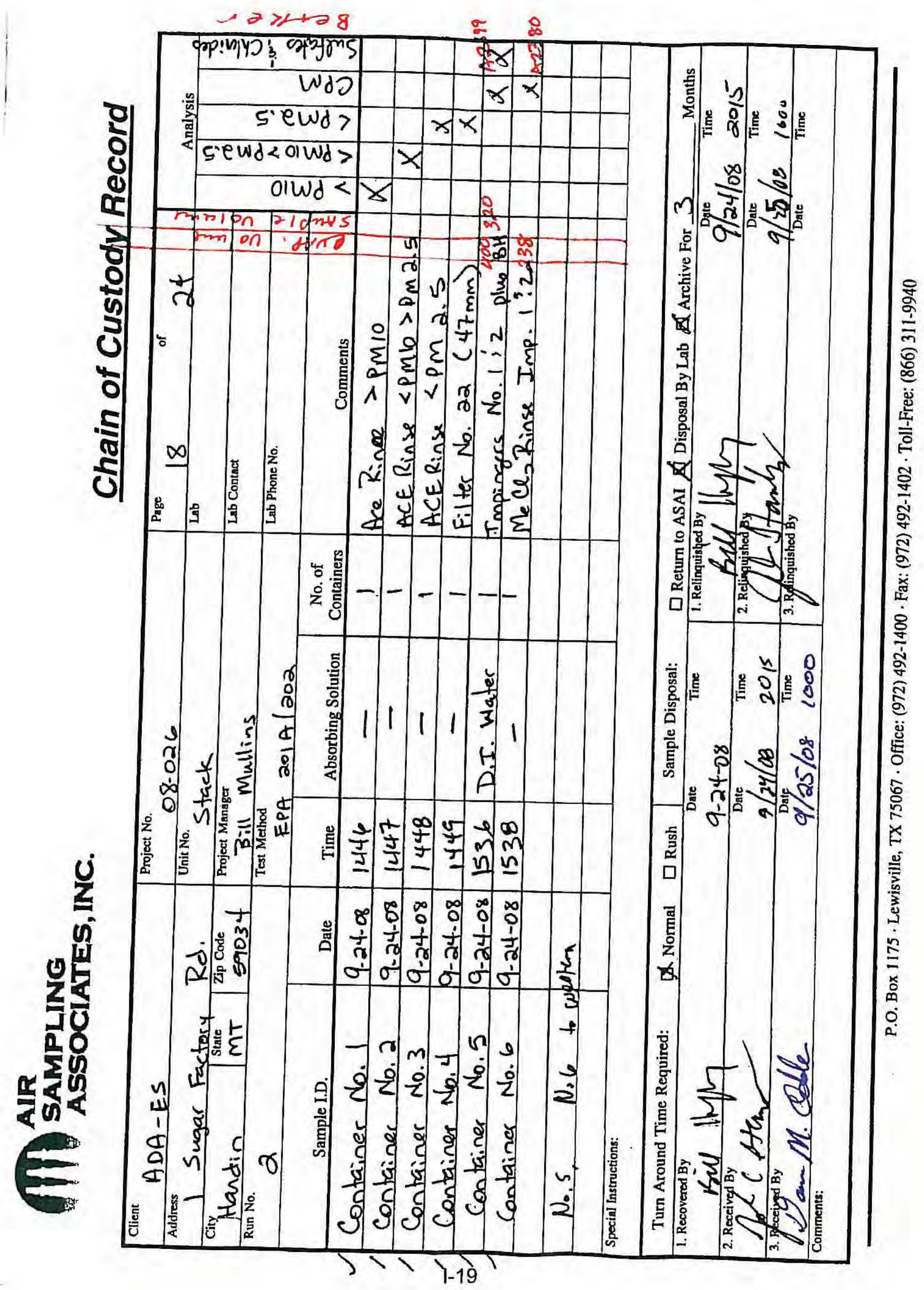




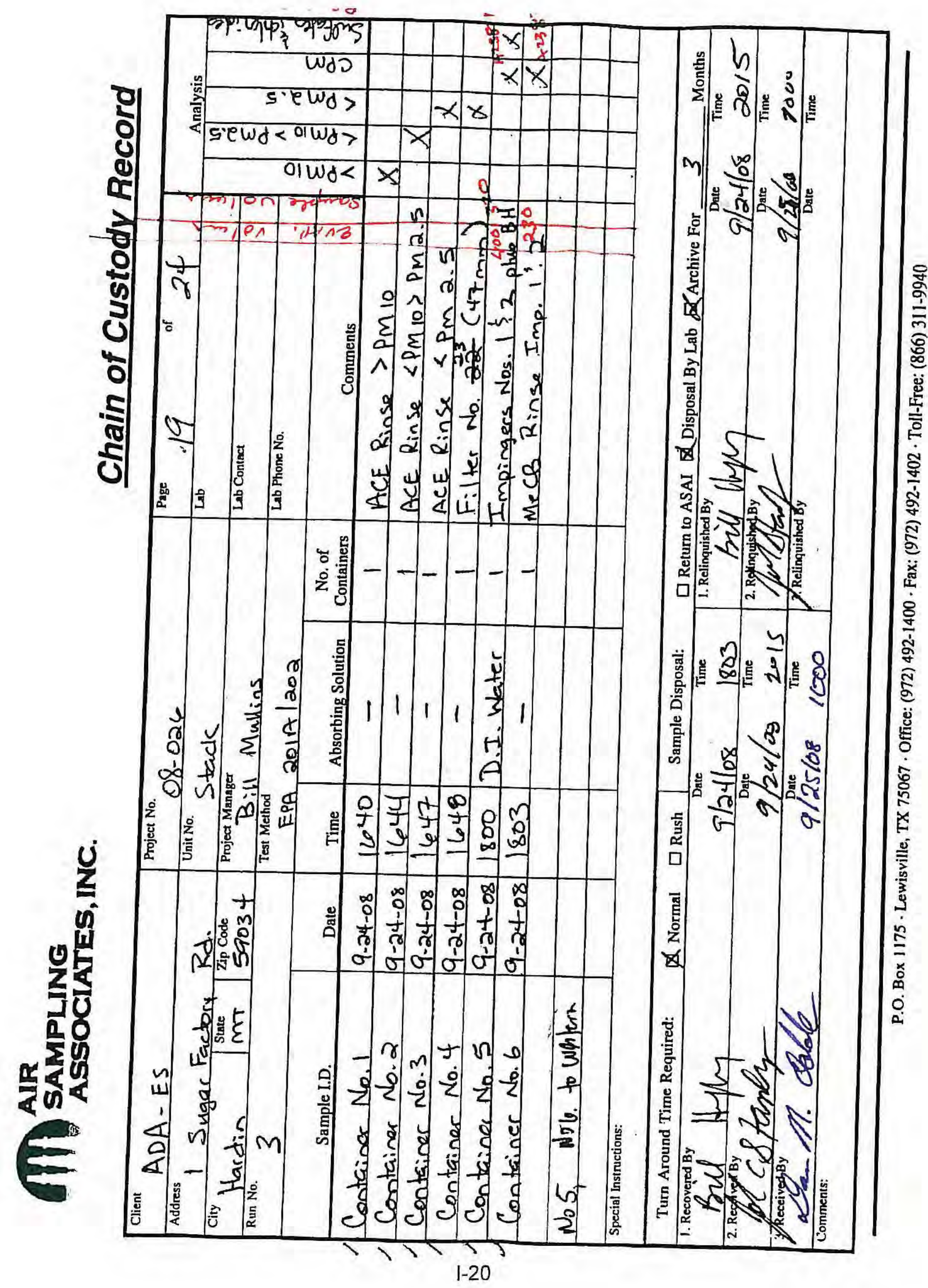




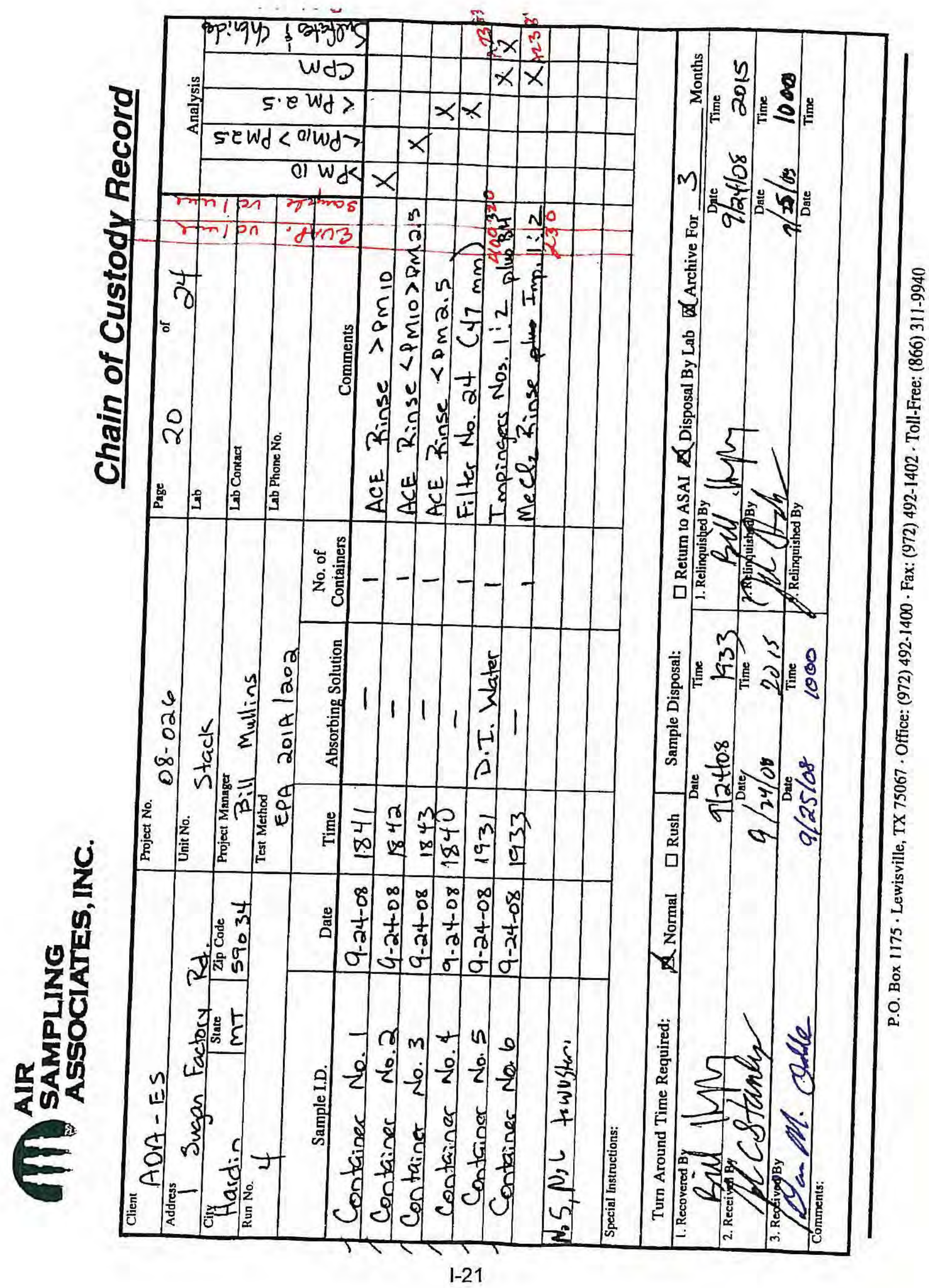




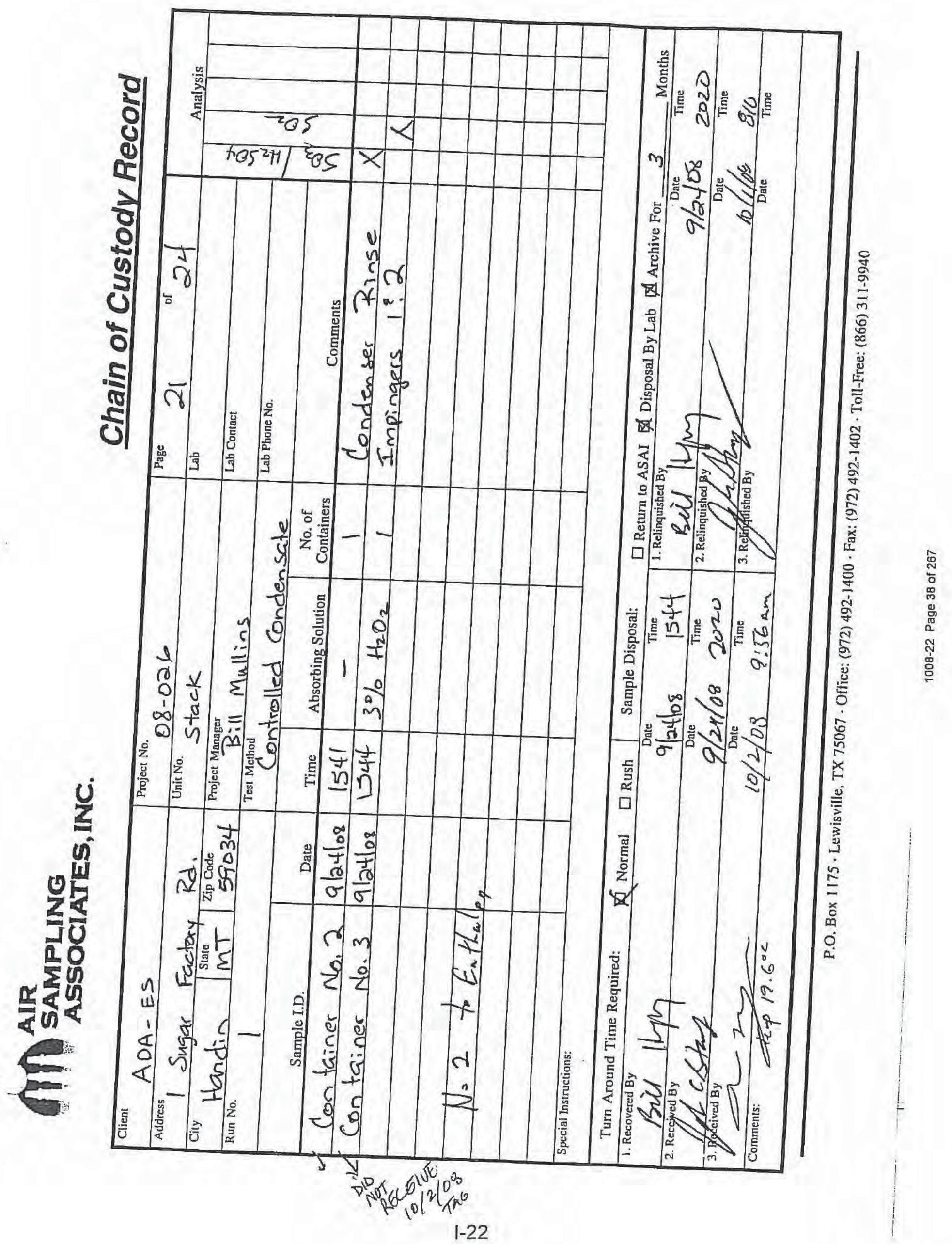




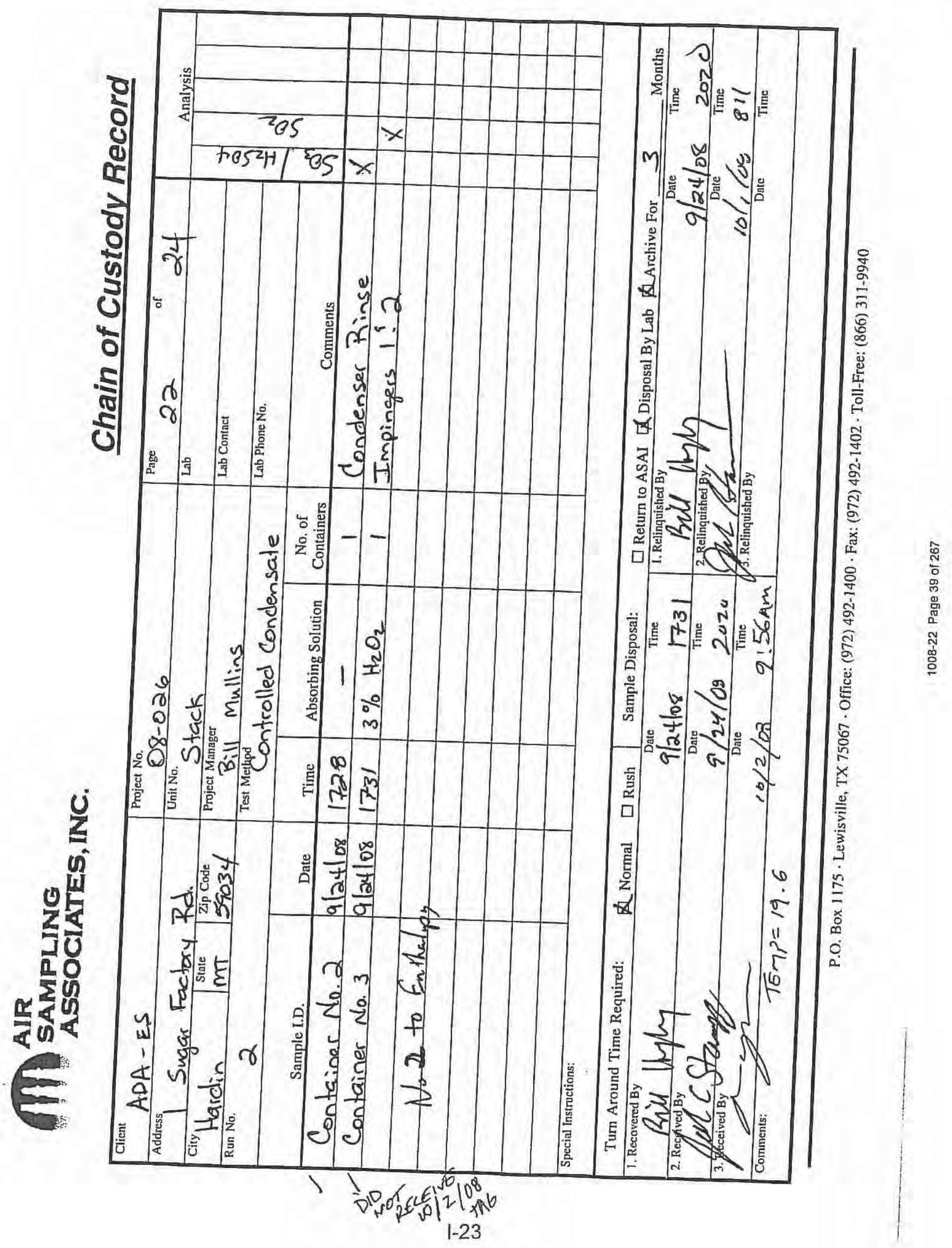




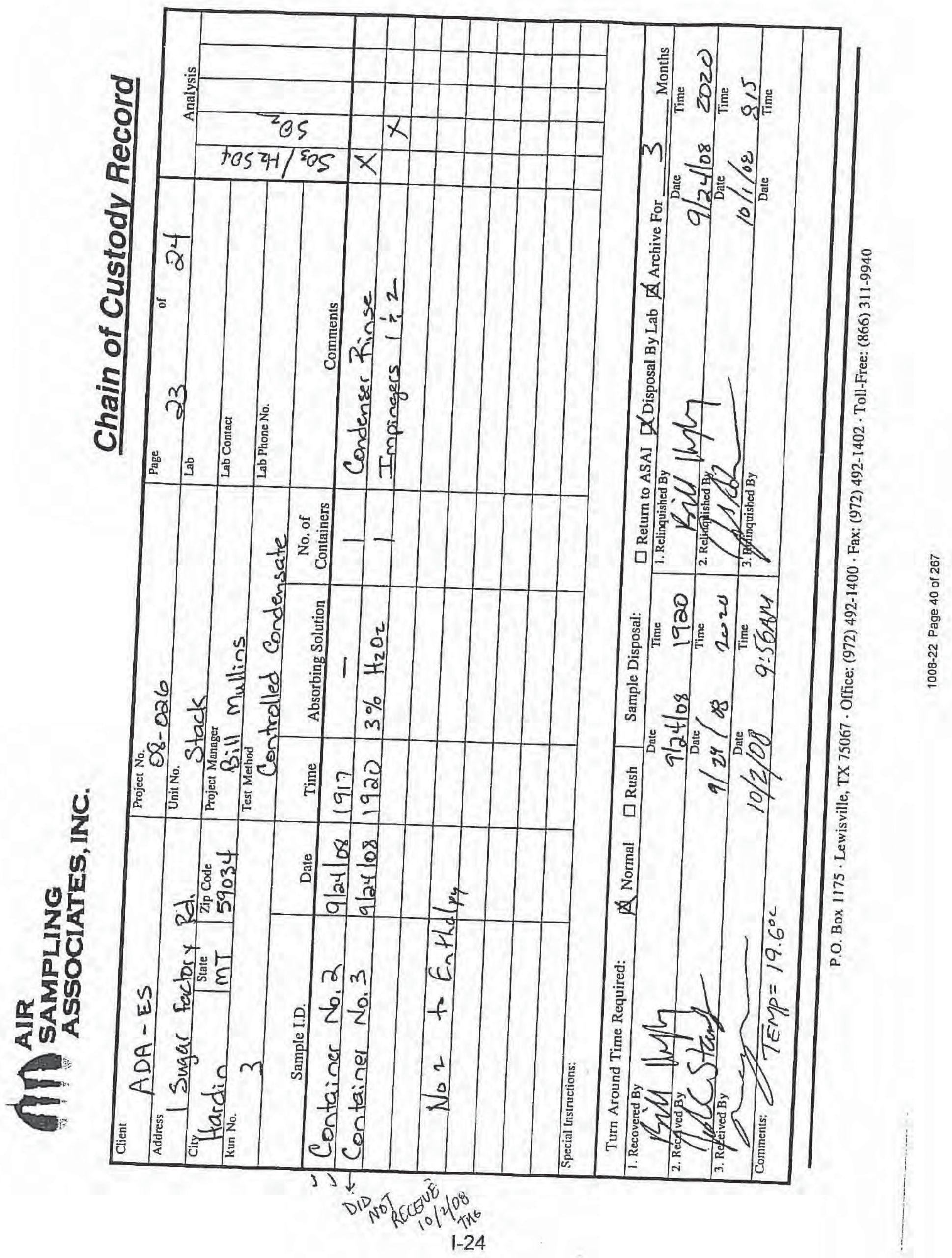




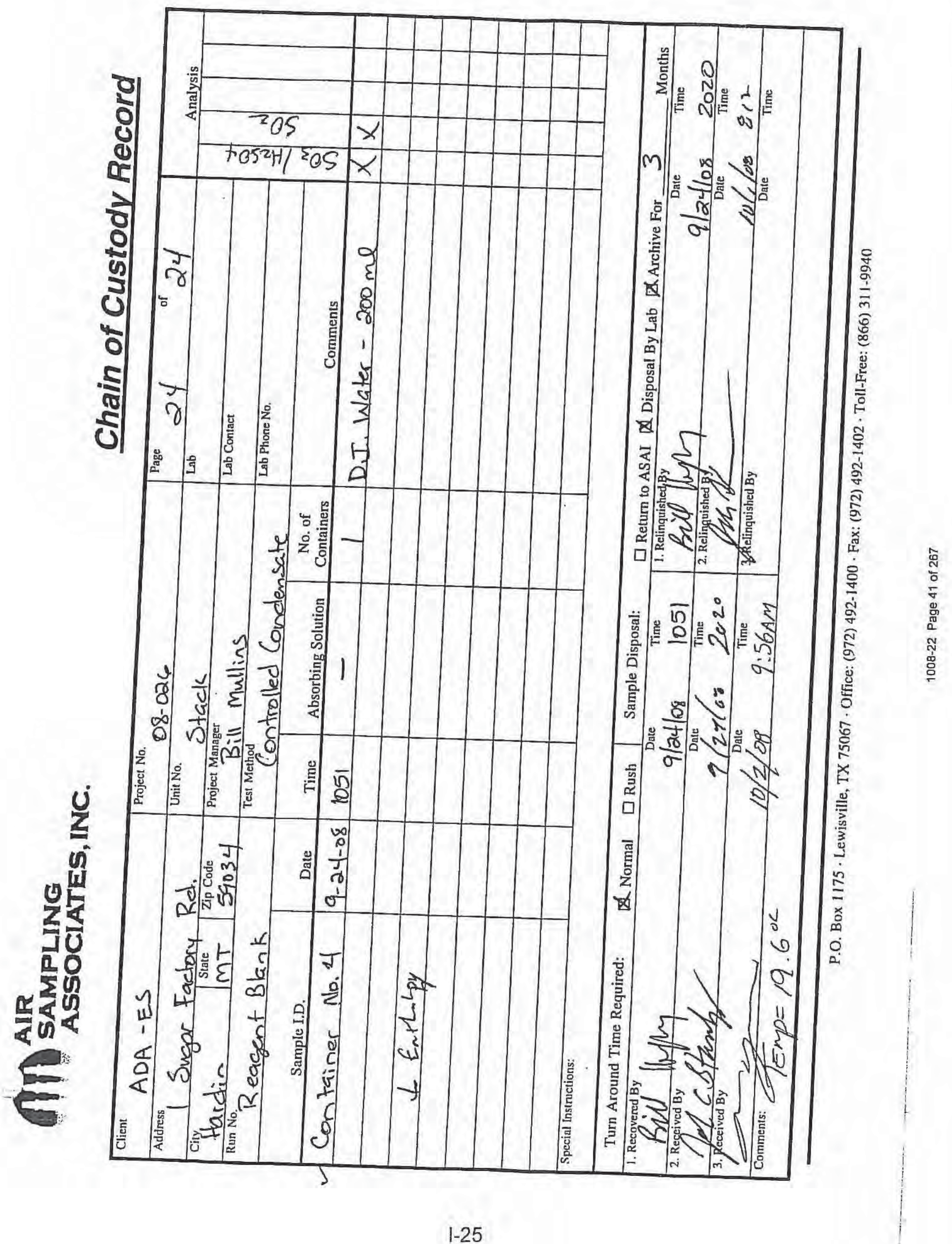


Appendix J:

Resumes of Test Personnel 
BILLY J. MULLINS, JR.; President

Education

Post Graduate Study Environmental Engineering at Southern Methodist University; Dallas, Texas 1970.

M.S. 1969, New York University; New York, New York, in Civil Engineering (Air Resources).

B.S. 1968, Texas Tech University; Lubbock, Texas, in Civil Engineering (Water Resources). Studies in Engineering at the U.S. Naval Academy; Annapolis, Maryland, 1963-1964

Professional Attended Short Course on Air Pollution Engineering at the University

Training of Texas at Austin, February 1970.

$\underline{\text { Courses }}$

Attended four-week management course presented by the American Management Association, 1976.

Certification Registered Professional Engineer

Certified Visible Emissions Evaluator

Licensed Private Pilot (Multi-Engine-Land, Instrument)

Diplomat in the American Academy of Environmental Engineers

Inductee into the Stack Sampling Hall of Fame

Certified as Qualified Environmental Professional (QEP)

Professional Air \& Waste Management Association - Past Chairman, Past Vice Memberships Chairman, and Past Board of Directors of North Texas Chapter and Southwest Section; Past Chairman, Consultants Committee; Past Chairman, Source Measurement Committee

Source Evaluations Society - Past President, Past Board of Directors

\section{American Management Association}


MULLINS (p. 2)

Publications Authored "Real World Experience with USEPA's New Sampling and Analytical Methods for Conducting Risk Burn," May 1998.

Co-authored "Sulfur Compound Emissions of the Petroleum Production Industry," December 1974.

Co-authored "Field Procedure for Stabilizing Hydrogen Sulfide Samples to be Analyzed Using Modified Methylene Blue Technique," presented at the Conference on Ambient Air Quality Measurements, Austin, Texas, March 1975.

Co-authored "Atmospheric Emissions Survey of the Sour Gas Industry," October 1975.

Co-authored "Technique for Insuring the Validity of Samples for High Concentrations of Sulfur Dioxide Using the EPA Method 5 Sampling Train," presented at the Third National Conference on Energy and the Environment, College Corner, Ohio, September 1975.

Teaching Experience
Conducted training seminars on sampling methods periodically since 1974 to present.

Conducted a one-day seminar on Part 75 Testing over ten times in 1993 and 1994.

Served as a lecturer in the Environmental Protection Agency's (EPA) training course number 450, "Source Sampling for Particulate Pollutants," for two years from January 1974 to October 1975 and March, 1992.

Conducted a two-day training course entitled "technical Assistance in Source Sampling" at lowa State University, Ames, lowa, for the Environmental Protection Agency (EPA), October 1974.

Conducted Environmental Protection Agency's (EPA) training course number 450, "Source Sampling for Particulate Pollutants," at Research Triangle Park, North Carolina, September 1975. 
MULLINS (p. 3)

Teaching

Experience

(Cont'd)
Technical

Experience
Conducted a two-day short course entitled "Performing and Observing Source Sampling," Dallas, Texas, July 1976, May 1977, October 1977, November 1987 and November 1988; Lake Charles, Louisiana, May 1977; Casper Wyoming, May 1977; Point Comfort, Texas, November 1992.

Served as a lecturer in the Environmental Protection Agency's twoday seminar entitled "Asphalt Industry Environmental Solutions," presented in Dallas, Texas, March 21-22, 1979.

Conducted a two-day short course entitled "Performing and Observing Source Sampling," Phoenix, Arizona, August, 1990, for the State of Arizona, Department of Environmental Quality; Lincoln, Nebraska, March 1980, for the State of Nebraska, Air Quality Control Division.

Directed and performed stack sampling on over 2000 sources of which over 500 were sampled simultaneously using more than one sampling train at several points in the flue gas stream; 1972-present.

Directed and performed over 200 short-term ambient air studies using mobile sampling vans and various ambient air sampling equipment; 1972-present.

Designed, directed and operated over 20 permanent ambient air networks of various size and duration for a variety of parameters; 1972-present.

Designed surface and underground drainage systems for residential subdivisions, public works projects, and shopping centers; 19691972.

Designed several residential subdivisions including lot layout, street design, drainage design, and utility design; 1969-1972. 
MULLINS (p. 4)

Research

Projects

Related

$\underline{\text { Projects }}$
Supervised and conducted a study made by the Hawaiian Sugar Planters' Association to characterize the emissions for several bagasse-fired boilers, April-May 1976.

Supervised and conducted a study made by the Rio Grande Valley Sugar Growers, Inc. to determine the area affected by the burning of sugarcane fields prior to harvesting, November 1974-April 1975.

Supervised and conducted a study by a lightweight aggregate manufacturer to develop a material balance around the process through sampling and analysis of several parameters, November 1973.

Conducted a study in New York City to attempt to develop a correlation in the ambient air between carbon dioxide and sulfur dioxide to provide a tool for predicting air pollution predicting air pollution episodes, January-May 1969.

Served as Chairman of the Engineering Foundation Conference on Stack Sampling and Source Evaluation in Destin, Florida, 2002, and Santa Barbara, California, 1985.

Served as Co-Chairman of the Engineering Foundation Conference on Stack Sampling and Source Evaluation in Destin, Florida, 2001.

Served as Session Chairman at the Engineering Foundation Conference on Stack Sampling and Source Evaluation in Hershey, Pennsylvania, 1984; San Diego, California, 1993; and in Palm Coast, Florida, 1994. 
BILLY L. HEFLEY; Associate

Education

Professional

Training

Certification

Professional

Memberships

Technical

Experience
B.S. 1992, East Central University; Ada, Oklahoma, in Environmental Science with a concentration in Environmental Management.

Attended 40-hour Occupational and Environmental Training Program on Hazardous Materials (CFR 1910.120) Dallas, Texas, April 1993.

Also attended an 8-hour refresher course January 1994, February 1995, January 1996, and May 2004.

Attended 8-hour Safe Hazardous Materials Transportation Training Program (HM-126F and HM-181) Dallas, Texas, October 1994.

Attended Bill Mullins' Performing and Observing Source Sampling Short Course; Dallas, Texas, January 1995.

Certified Visible Emissions Evaluator

\section{Air \& Waste Management Association Source Evaluations Society}

Participated in the sampling of over 750 sources, including several of which were sampled simultaneously using more than one sampling train. Thoroughly trained in all EPA testing procedures, 1992present.

Participated in an auditing program for a permanent eight-station sulfur dioxide ambient air network in East Texas, 1992-1993.

Participated in a semi-monthly ambient air monitoring survey for organic compounds at a petrochemical facility located in South Texas, 1992-1993.

Participated in EPA's Information Collection Request for Mercury conducting more than 46 simultaneous tests for Speciated Mercury using the "Standard Test Method for Elemental, Oxidized, ParticleBound, and Total Mercury in Flue Gas Generated from Coal-Fired Stationary Sources (Ontario Hydro Method)." September 1999-May 2000 . 
$\operatorname{HEFLEY~(p.~2)~}$

Experienced in the analysis of commercial calibration gas cylinders for $\mathrm{NO}_{x}, \mathrm{SO}_{2}, \mathrm{CO}_{2}$, and $\mathrm{O}_{2}$.

Experience with calibration techniques for all field testing equipment.

Thoroughly trained in the operation and routine maintenance of the following:

Anarad Model AR50-C Carbon Dioxide Analyzer

Anarad Model AR880 Oxides of Nitrogen Analyzer

Anarad Model AR23 Oxygen Analyzer

Anarad Model AR30C2 Sulfur Dioxide Analyzer

California Analytical Model 300-HFID Total Hydrocarbon Analyzer

Servomex Model 1440 Carbon Dioxide Analyzer

Servomex Model 1440 Oxygen Analyzer

Teledyne Model 326 Oxygen Analyzer

Thermo Environmental Model 10AR \& 10S Oxides of Nitrogen Analyzer

Thermo Electron Model 46C Oxides of Nitrogen Analyzer

Thermo Electron Model 48C Oxides of Nitrogen Analyzer

Western Research Model 721A Sulfur Dioxide Analyzer 
PATRICK SELAKOVICH; Associate

Education

Professional

Training

Professional Memberships

Technical Experience
B.S.B.A. 1992, University of Arkansas; Fayetteville, Arkansas, in General Business.

Attended 24-hour Occupational and Environmental Training Program on Hazardous Materials (CFR 1910.120) Dallas, Texas, April 1997.

Attended 40-hour Occupational and Environmental Training Program on Hazardous Materials (CFR 1910.120) \& 'Train the Trainer', Gettysburg, Pennsylvania, July 1998.

Also attended an 8-hour refresher course January 2000, January 2001, and May 2004.

Attended OSHA General Industry Safety and Health Training, May 1999.

\section{Society For Human Resource Management}

Participated in the sampling of over 150 sources, including several of which were sampled simultaneously using more than one sampling train. Thoroughly trained in all EPA testing procedures, 1996present.

Participated in EPA's 3-D probe study. May - August 1997.

Experience with calibration techniques for all field testing equipment.

Thoroughly trained in the operation and routine maintenance of the following:

California Analytical Model 300-HFID Total Hydrocarbon Analyzer Servomex Model 1440 Carbon Dioxide Analyzer

Servomex Model 1440 Oxygen Analyzer

Thermo Electron Model 46C Oxides of Nitrogen Analyzer Thermo Electron Model 48C Oxides of Nitrogen Analyzer

Western Research Model 721A Sulfur Dioxide Analyzer 
JOHN STANLEY; Associate

Education

B.A. June 1990, Hendrix College, in Business and Economics.

$\underline{\text { Certification }}$

Certified Visible Emissions Evaluator

Technical

Participated in the sampling of over 25 sources, including several of Experience which were sampled simultaneously using more than one sampling train.

Experience with calibration techniques for all field testing equipment.

Thoroughly trained in the operation and routine maintenance of the following:

California Analytical Model 300-HFID Total Hydrocarbon Analyzer Servomex Model 1440 Carbon Dioxide Analyzer

Servomex Model 1440 Oxygen Analyzer

Thermo Electron Model 42CHL Oxides of Nitrogen Analyzer

Thermo Electron Model 48C Carbon Monoxide Analyzer

Western Research Model 921A Sulfur Dioxide Analyzer 
DAN BERT; Associate

Education

Technical

Experience
Associates of Electronics Engineering Technology, National Education Center 1987.

Participated in the sampling of over 25 sources, including several of which were sampled simultaneously using more than one sampling train.

Experience with calibration techniques for all field testing equipment.

Thoroughly trained in the operation and routine maintenance of the following:

California Analytical Model 300-HFID Total Hydrocarbon Analyzer Servomex Model 1440 Carbon Dioxide Analyzer Servomex Model 1440 Oxygen Analyzer

Thermo Electron Model 42CHL Oxides of Nitrogen Analyzer Thermo Electron Model 48C Carbon Monoxide Analyzer Western Research Model 921A Sulfur Dioxide Analyzer 


\section{APPENDIX F: COAL AND ASH ANALYSES}




\section{Sample Analyses}

The tables below contain all results from the chemical analyses performed on the coal, ash and slurry samples.

Table 1 shows the results for some of the ash samples including Bottom Ash, taken from the drag change in the water seal below the boiler, Bottom+Econ Ash, taken from an ash pit that combines bottom ash and ash from the economizer hoppers, SDA Lime Slurry, taken from a sample port neat the rotary atomizer, and CEGRIT ash, which is ash taken in-flight at the SDA Inlet and after PAC injection by an in-situ sampler called a CEGRIT. Analyses include, moisture $(\% \mathrm{H} 2 \mathrm{O})$, mercury $(\mathrm{Hg})$, bromine $(\mathrm{Br})$, carbon by the loss-on-ignition $(\% \mathrm{LOI})$ test, total carbon $(\% \mathrm{C})$ and total organic carbon (TOC).

Table 2 contains the results for the same analyses as Table 1 but only for fly ash samples taken from the FF or from the rotary valve below the SDA hopper.

Table 3 contains coal analyses including halogens $(\mathrm{Br}, \mathrm{Cl}, \mathrm{F})$, moisture $(\% \mathrm{H} 2 \mathrm{O})$ and dry and as-received mercury $(\mathrm{Hg})$.

Table 4 contains the results of Proximate Analyses for coal.

Table 5 contains the results of Ultimate Analyses for coal.

Finally, Table 6 contains the results from analyses on PAC including moisture $(\% \mathrm{H} 2 \mathrm{O})$, leachable bromine ( $\mathrm{Br} \%$ ), grind size (PSS-325), Red Cap, Tap Density, and halogens (Br and I).

(ug/g)" 
Table 1. Analyses of Bottom Ash, SDA Lime Slurry and CEGRIT Ash.

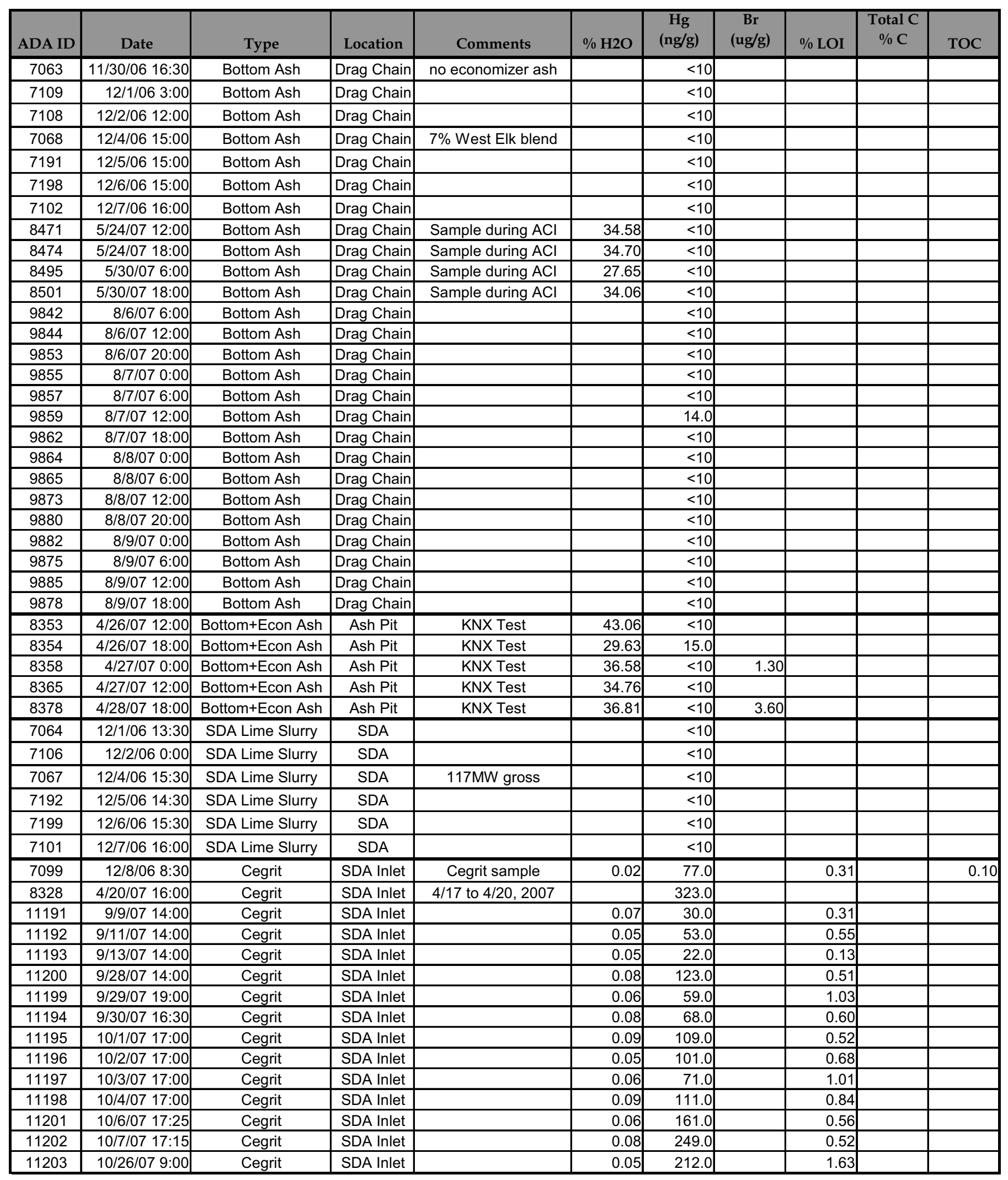


Table 2. Analyses of Fly Ash Samples.

\begin{tabular}{|c|c|c|c|c|c|c|c|c|c|c|}
\hline ADA ID & Date & Type & Location & Comments & $\% \mathrm{H} 2 \mathrm{O}$ & $\begin{array}{c}\mathrm{Hg} \\
(\mathrm{ng} / \mathrm{g})\end{array}$ & $\begin{array}{c}\mathrm{Br} \\
\text { (ug/g) }\end{array}$ & $\%$ LOI & $\begin{array}{c}\text { Total C } \\
\% \text { C }\end{array}$ & TOC \\
\hline 6836 & $11 / 3 / 06$ 0:00 & Fly Ash & FF - A & $117 \mathrm{MW}$ gross & 3.19 & 78.0 & & 8.46 & 1.25 & \\
\hline 6837 & 11/3/06 0:00 & Fly Ash & FF - B & $117 \mathrm{MW}$ gross & 3.58 & 73.0 & & 8.65 & 1.43 & \\
\hline 6838 & 11/3/06 0:00 & Fly Ash & FF - C & $117 \mathrm{MW}$ gross & 2.83 & 76.0 & & 8.40 & 1.13 & \\
\hline 6839 & 11/3/06 0:00 & Fly Ash & $F F-D$ & $117 \mathrm{MW}$ gross & 2.94 & 67.0 & & 8.63 & 1.56 & \\
\hline 6840 & $11 / 3 / 06$ 0:00 & Fly Ash & $F F-E$ & $117 \mathrm{MW}$ gross & 2.91 & 58.0 & & 9.52 & 1.76 & \\
\hline 6841 & 11/3/06 0:00 & Fly Ash & $F F-F$ & $117 \mathrm{MW}$ gross & 3.02 & 77.0 & & 8.66 & 1.47 & \\
\hline 7187 & 11/17/06 0:00 & Fly Ash & FF - B & $117 \mathrm{MW}$ gross & 6.06 & 66.0 & & 8.47 & & 0.68 \\
\hline 7055 & 11/30/06 15:30 & Fly Ash & FF - A & $117 \mathrm{MW}$ gross & 2.83 & 37.0 & & 7.25 & & 0.90 \\
\hline 7056 & 11/30/06 15:30 & Fly Ash & $F F-B$ & $117 \mathrm{MW}$ gross & 2.39 & 41.0 & & 7.21 & & 0.83 \\
\hline 7057 & $11 / 30 / 06$ 15:30 & Fly Ash & $F F-C$ & $117 \mathrm{MW}$ gross & 2.86 & 25.0 & & 7.68 & & 0.86 \\
\hline 7058 & $11 / 30 / 06$ 15:30 & Fly Ash & FF - D & $117 \mathrm{MW}$ gross & 4.77 & 57.0 & & 8.99 & & 0.97 \\
\hline 7059 & $11 / 30 / 06$ 15:30 & Fly Ash & $F F-E$ & $117 \mathrm{MW}$ gross & 2.61 & 36.0 & & 8.04 & & 0.91 \\
\hline 7060 & $11 / 30 / 06$ 15:30 & Fly Ash & $F F-F$ & $117 \mathrm{MW}$ gross & 8.73 & 42.0 & & 9.96 & & 0.59 \\
\hline 7061 & 12/1/06 11:30 & Fly Ash & $F F-B$ & $117 \mathrm{MW}$ gross & 3.53 & 36.0 & & 8.38 & & 0.90 \\
\hline 7107 & 12/2/06 12:00 & Fly Ash & $F F-B$ & & 2.18 & 67.0 & & 7.41 & & 0.39 \\
\hline 7190 & $12 / 5 / 06$ 15:00 & Fly Ash & FF - B & $117 \mathrm{MW}$ gross & 2.30 & 137.0 & & 8.84 & & 1.25 \\
\hline 7197 & 12/6/06 14:30 & Fly Ash & FF - B & & 2.40 & 46.0 & & 7.56 & & 0.73 \\
\hline 7105 & 12/7/06 16:00 & Fly Ash & FF - B & & 3.24 & 112.0 & & 9.06 & & 1.13 \\
\hline 8313 & $3 / 2 / 070: 00$ & Fly Ash & $F F-B$ & & & 47.0 & & & & \\
\hline 8314 & $3 / 13 / 07$ 0:00 & Fly Ash & FF - B & & & 111.0 & & & & \\
\hline 8352 & 4/26/07 12:00 & Fly Ash & $F F-B$ & KNX Test & & 49.0 & & & & 2.30 \\
\hline 8317 & 4/26/07 16:00 & Fly Ash & $F F-B$ & KNX Test & & 52.0 & 4.80 & & & 0.55 \\
\hline 8320 & 4/26/07 16:09 & Fly Ash & $F F-E$ & KNX Test & & 58.0 & 4.80 & & & 0.65 \\
\hline 8319 & 4/26/07 16:16 & Fly Ash & FF - D & KNX Test & & 52.0 & 2.60 & & & 0.57 \\
\hline 8316 & 4/26/07 16:25 & Fly Ash & FF - A & KNX Test & & 56.0 & 3.60 & & & 0.50 \\
\hline 8318 & 4/26/07 16:39 & Fly Ash & FF - C & KNX Test & & 50.0 & 4.80 & & & 0.56 \\
\hline 8321 & 4/26/07 16:43 & Fly Ash & $F F-F$ & KNX Test & & 49.0 & 4.50 & & & 0.69 \\
\hline 8356 & 4/26/07 18:00 & Fly Ash & $F F-B$ & KNX Test & & 18.0 & & & & 1.70 \\
\hline 8357 & 4/27/07 0:00 & Fly Ash & FF - B & KNX Test & & 31.0 & 2.80 & & & 1.80 \\
\hline 8364 & 4/27/07 12:00 & Fly Ash & FF - B & KNX Test & & 29.0 & & & & \\
\hline 8323 & 4/27/07 14:40 & Fly Ash & FF - B & KNX Test & & 140.0 & 190.00 & & & 0.96 \\
\hline 8326 & 4/27/07 14:46 & Fly Ash & $F F-E$ & KNX Test & & 157.0 & 173.10 & & & 1.50 \\
\hline 8327 & 4/27/07 14:49 & Fly Ash & $F F-F$ & KNX Test & & 151.0 & 156.10 & & & 1.50 \\
\hline 8324 & 4/27/07 14:52 & Fly Ash & FF - C & KNX Test & & 158.0 & 183.30 & & & 1.50 \\
\hline 8322 & 4/27/07 15:00 & Fly Ash & $F F-A$ & KNX Test & & 173.0 & 202.00 & & & 0.83 \\
\hline 8325 & 4/27/07 15:04 & Fly Ash & $F F-D$ & KNX Test & & 168.0 & 183.30 & & & 1.50 \\
\hline 8380 & 4/28/07 18:00 & Fly Ash & $F F-B$ & KNX Test & & 110.0 & 30.00 & & & 2.00 \\
\hline 8470 & 5/24/07 12:00 & Fly Ash & Detר & Sample during $\mathrm{ACl}$ & & 40.0 & 4.10 & & & 1.90 \\
\hline 8473 & 5/24/07 18:00 & Fly Ash & 年 & Sample during $\mathrm{ACl}$ & & 34.0 & 11.40 & & & 1.90 \\
\hline 8494 & 5/30/07 6:00 & Fly Ash & 2 & Sample during $\mathrm{ACl}$ & & 53.0 & 8.60 & & & 2.00 \\
\hline 8500 & 5/30/07 18:00 & Fly Ash & (2) & Sample during $\mathrm{ACl}$ & & 41.0 & 32.40 & & & 2.50 \\
\hline 9845 & $8 / 6 / 07$ 12:30 & Fly Ash & $F F-A$ & & & 71.0 & 4.60 & & & 0.47 \\
\hline 9846 & 8/6/07 12:30 & Fly Ash & $F F-B$ & Leach Test & & 74.0 & 4.30 & & & 0.90 \\
\hline 9847 & 8/6/07 12:30 & Fly Ash & FF - C & & & 68.0 & 3.80 & & & 0.61 \\
\hline 9848 & 8/6/07 12:30 & Fly Ash & $F F-D$ & & & 62.0 & 3.50 & & & 0.73 \\
\hline 9849 & $8 / 6 / 07$ 12:30 & Fly Ash & $F F-E$ & & & 66.0 & 4.50 & & & 0.09 \\
\hline 9850 & $8 / 6 / 07$ 12:30 & Fly Ash & $F F-F$ & & & 121.0 & 30.10 & & & 0.74 \\
\hline 9851 & 8/6/07 19:00 & Fly Ash & $\mathrm{FF}-\mathrm{B}$ & & & 208.0 & & & & 1.40 \\
\hline 9860 & 8/7/07 16:30 & Fly Ash & FF - B & & & 296.0 & & & & 0.78 \\
\hline 9866 & $8 / 8 / 07$ 10:30 & Fly Ash & $F F-A$ & & & 91.0 & 12.10 & & & 1.00 \\
\hline 9867 & $8 / 8 / 07$ 10:30 & Fly Ash & $F F-B$ & & & 90.0 & 13.20 & & & 0.84 \\
\hline 9868 & $8 / 8 / 07$ 10:30 & Fly Ash & FF - C & & & 94.0 & 12.10 & & & 1.00 \\
\hline 9869 & $8 / 8 / 07$ 10:30 & Fly Ash & $F F-D$ & & & 89.0 & 13.60 & & & 0.64 \\
\hline 9870 & $8 / 8 / 07$ 10:30 & Fly Ash & $F F-E$ & & & 90.0 & 12.70 & & & 1.30 \\
\hline 9871 & $8 / 8 / 07$ 10:30 & Fly Ash & $F F-F$ & & & 89.0 & 12.40 & & & 1.00 \\
\hline 9883 & $8 / 9 / 07$ 10:45 & Fly Ash & FF - B & & & 231.0 & & & & 0.70 \\
\hline 9876 & 8/9/07 17:00 & Fly Ash & $F F-B$ & & & 283.0 & & & & 0.91 \\
\hline 9886 & 8/10/07 8:00 & Fly Ash & $F F-B$ & & & 196.0 & 49.70 & & & 0.65 \\
\hline 9887 & 8/10/07 8:00 & Fly Ash & $\mathrm{FF}-\mathrm{C}$ & & & 142.0 & 30.00 & & & 0.57 \\
\hline 9888 & 8/10/07 8:00 & Fly Ash & $F F-F$ & & & 149.0 & 36.20 & & & 0.83 \\
\hline 11205 & $10 / 5 / 070: 00$ & Fly Ash & $F F-B$ & & & 208.0 & & & & \\
\hline 11204 & 10/26/07 9:00 & Fly Ash & FF - B & & & 241.0 & & & & \\
\hline
\end{tabular}


Table 3. Coal Mercury and Halogens.

\begin{tabular}{|c|c|c|c|c|c|c|c|c|c|c|}
\hline \multirow[b]{2}{*}{ ADA ID } & \multirow[b]{2}{*}{ Date } & \multirow[b]{2}{*}{ Type } & \multirow[b]{2}{*}{ Location } & \multirow[b]{2}{*}{ Comments } & \multicolumn{3}{|c|}{ Halogens } & \multicolumn{3}{|c|}{ Mercur Analysis } \\
\hline & & & & & $\begin{array}{c}\text { Br } \\
(\mathrm{ug} / \mathrm{g})\end{array}$ & $\begin{array}{c}\mathrm{Cl} \\
\text { (ug/g) }\end{array}$ & $\begin{array}{c}\text { F } \\
\text { (ug/g) }\end{array}$ & $\% \mathrm{H} 2 \mathrm{O}$ & $\begin{array}{c}\mathrm{Hg} \text { as rec } \\
\text { (ng/g) }\end{array}$ & $\begin{array}{c}\text { Hg dry } \\
\text { (ng/g) }\end{array}$ \\
\hline 6842 & $11 / 3 / 060: 00$ & Coal & Reclaim Coal Belt & Westmoreland sample 1102 & & 47 & & 12.3 & 53.9 & 61.5 \\
\hline 7188 & $11 / 17 / 060: 00$ & Coal & Reclaim Coal Belt & $117 \mathrm{~mW}$ gross & & 20 & & 11.5 & 48.8 & 55.1 \\
\hline 7062 & 11/30/06 16:00 & Coal & Reclaim Coal Belt & & 1.5 & 43 & 25 & 9.2 & 29.3 & 32.3 \\
\hline 7104 & 12/1/06 15:00 & Coal & Reclaim Coal Belt & absaloka & 1.9 & 21 & 60 & 11.3 & 61.6 & 69.5 \\
\hline 7110 & $12 / 2 / 0612: 00$ & Coal & Reclaim Coal Belt & Absaloka & & 19 & & 10.7 & 23.1 & 25.9 \\
\hline 7070 & $12 / 3 / 0621: 00$ & Coal & Blend conveyor & West Elk, $7 \%$ blend,from pile & & 96 & & 0 & 28.6 & 28.6 \\
\hline 7069 & 12/4/06 9:00 & Coal & Reclaim Coal Belt & Plant coal, $7 \%$ West Elk blend & & 17 & & 10.5 & 27.7 & 30.9 \\
\hline 7193 & $12 / 4 / 0614: 30$ & Coal & Reclaim Coal Belt & Absaloka-during 14\% WE & & 6 & & 11.5 & 12.0 & 13.6 \\
\hline 7194 & $12 / 4 / 0614: 30$ & Coal & Blend conveyor & West Elk 14\% Blend & & 86 & & 0.0 & 83.1 & 83.1 \\
\hline 7195 & $12 / 5 / 0617: 00$ & Coal & Blend conveyor & Bull Mountain $14 \%$ Blend & & 23 & & 7.2 & 42.4 & 45.7 \\
\hline 7196 & 12/5/06 17:00 & Coal & Reclaim Coal Belt & Absaloka-during 14\% BM & & $<5$ & & 11.1 & 54.1 & 60.9 \\
\hline 7100 & 12/6/06 16:00 & Coal & Reclaim Coal Belt & Absaloka - during 14\% BM & & 43 & & 10.9 & 57.4 & 64.4 \\
\hline 7103 & 12/6/06 16:00 & Coal & Blend conveyor & Bull Mtn, $7 \%$ Blend & & 30 & & 6.5 & 36.7 & 39.3 \\
\hline 8351 & $4 / 26 / 0712: 00$ & Coal & Reclaim Coal Belt & Sample during KNX testing & & $<10$ & & 15.7 & 41.2 & 48.9 \\
\hline 9472 & 6/11/07 9:00 & Coal & Reclaim Coal Belt & QC-8351 Dup-to FGS/HML & & $<10$ & & 14.5 & 36.9 & 43.2 \\
\hline 8355 & $4 / 26 / 0718: 00$ & Coal & Reclaim Coal Belt & Sample during KNX testing & & $<10$ & & 14.6 & 37.6 & 44 \\
\hline 8359 & 4/27/07 0:00 & Coal & Reclaim Coal Belt & Sample during KNX testing & 1.2 & $<10$ & & 15.8 & 43.0 & 51.1 \\
\hline 8379 & 4/28/07 18:00 & Coal & Reclaim Coal Belt & Sample during KNX testing & 2.8 & $<10$ & & 16.1 & 28.4 & 33.8 \\
\hline 8472 & $5 / 24 / 0712: 00$ & Coal & Reclaim Coal Belt & Sample during carbon Inj tests & & $<10$ & & 14.9 & 46.0 & 54.1 \\
\hline 8475 & $5 / 24 / 0718: 00$ & Coal & Reclaim Coal Belt & Sample during carbon Inj tests & & $<10$ & & 14.8 & 36.0 & 42.3 \\
\hline 8496 & $5 / 30 / 076: 00$ & Coal & Reclaim Coal Belt & Sample during carbon Inj tests & & $<10$ & & 13.5 & 14.5 & 16.8 \\
\hline 8502 & $5 / 30 / 07$ 18:00 & Coal & Reclaim Coal Belt & Sample during carbon Inj tests & & $<10$ & & 15.4 & 16.7 & 19.7 \\
\hline 9841 & 8/6/07 6:00 & Coal & Reclaim Coal Belt & & & & & 11.1 & 38.4 & 43.2 \\
\hline 9843 & $8 / 6 / 0712: 00$ & Coal & Reclaim Coal Belt & & & & & 10.4 & 48.3 & 53.9 \\
\hline 9852 & $8 / 6 / 0720: 00$ & Coal & Reclaim Coal Belt & & & & & 10.4 & 66.3 & 74 \\
\hline 9854 & $8 / 7 / 07$ 0:00 & Coal & Reclaim Coal Belt & & & & & 10.7 & 62.7 & 70.2 \\
\hline 9856 & $8 / 7 / 076: 00$ & Coal & Reclaim Coal Belt & & & & & 11.2 & 38.5 & 43.3 \\
\hline 9858 & 8/7/07 12:00 & Coal & Reclaim Coal Belt & & & & & 12.1 & 32.0 & 36.4 \\
\hline 9861 & $8 / 7 / 0718: 00$ & Coal & Reclaim Coal Belt & & & & & 11.1 & 60.4 & 67.9 \\
\hline 9863 & $8 / 8 / 070: 00$ & Coal & Reclaim Coal Belt & & & & & 10.6 & 25.8 & 28.9 \\
\hline 9872 & $8 / 8 / 0712: 00$ & Coal & Reclaim Coal Belt & & & & & 10.4 & 84.3 & 94.1 \\
\hline 9879 & 8/8/07 20:00 & Coal & Reclaim Coal Belt & & & & & 10.3 & 46.0 & 51.3 \\
\hline 9881 & 8/9/07 0:00 & Coal & Reclaim Coal Belt & & & & & 8.7 & 51.5 & 56.4 \\
\hline 9874 & 8/9/07 6:00 & Coal & Reclaim Coal Belt & & & & & 7.8 & 62.1 & 67.4 \\
\hline 9884 & 8/9/07 12:00 & Coal & Reclaim Coal Belt & & & & & 9.3 & 18.5 & 20.4 \\
\hline 9877 & 8/9/07 18:00 & Coal & Reclaim Coal Belt & & & & & 10.4 & 26.3 & 29.4 \\
\hline 12167 & $3 / 27 / 08$ 11:00 & Coal & Reclaim Coal Belt & & 1.2 & 77 & & 8.9 & 30.7 & 33.7 \\
\hline
\end{tabular}




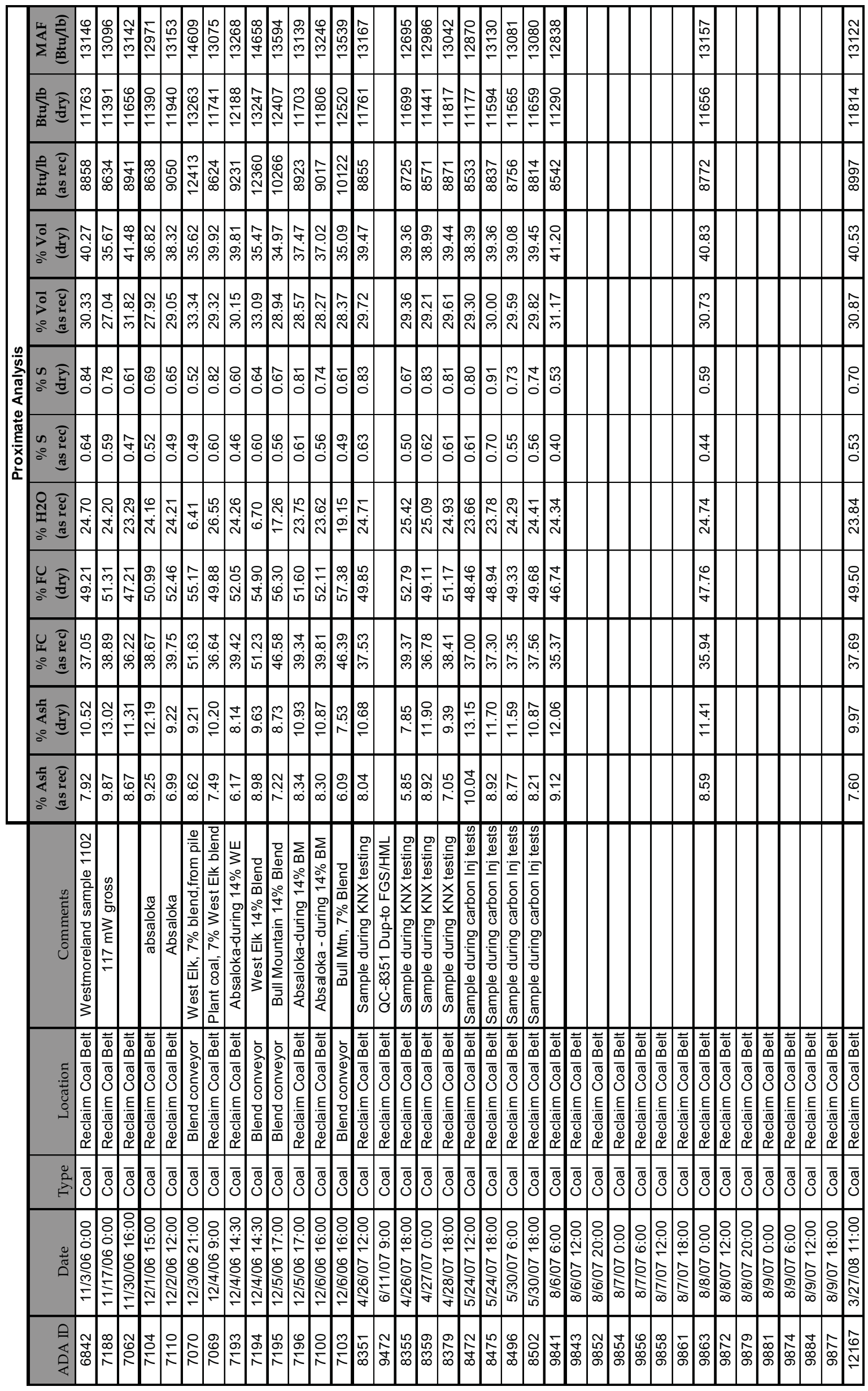




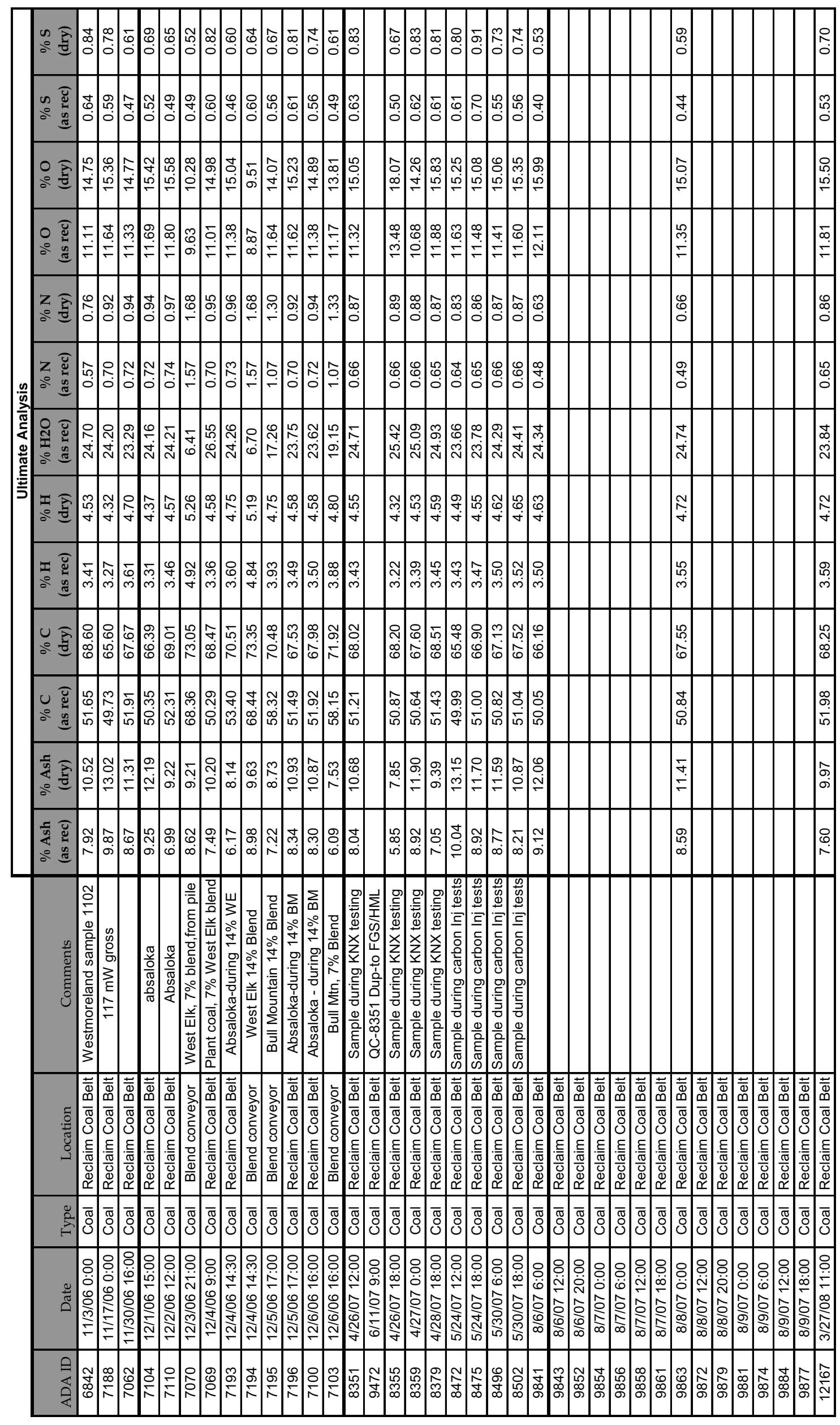


Table 6. PAC Analyses.

\begin{tabular}{|c|c|c|c|c|c|c|c|c|c|c|c|}
\hline ADA ID & Date & Type & Location & Comments & $\% \mathrm{H} 2 \mathrm{O}$ & $\begin{array}{c}\text { Leachable } \\
\text { Br }(\%)\end{array}$ & PSS -325 & Red Cap & $\begin{array}{c}\text { Tap } \\
\text { Density }\end{array}$ & $\begin{array}{c}\mathrm{Br} \\
\text { (ug/g) }\end{array}$ & $\begin{array}{c}\text { I } \\
\text { (ug/g) }\end{array}$ \\
\hline 12166 & $3 / 27 / 08$ 11:00 & $\mathrm{Hg}-\mathrm{LH}$ & PAC Silo & Questionable Batch & 4 & 2.6 & 96 & 12.5 & 0.57 & 30300 & 493 \\
\hline 12379 & $6 / 17 / 08$ 0:00 & $\mathrm{Hg}-\mathrm{LH}$ & PAC Silo & Questionable Batch \#2 & & & 97.6 & & 0.533 & 46200 & \\
\hline 12246 & 4/3/08 11:00 & $\mathrm{Hg}-\mathrm{LH}$ & PAC Silo & Darco Hg-LH & & & & & & 50000 & \\
\hline 9447 & $5 / 24 / 070: 00$ & $\mathrm{Hg}-\mathrm{LH}$ & PAC Silo & Darco Hg-LH & & & & & & 54000 & \\
\hline 9520 & 6/21/07 0:00 & $\begin{array}{l}\text { IVIC } \\
\mathrm{O} \\
\end{array}$ & PAC Silo & sample from liner mishap & & & & & & 42500 & \\
\hline 9519 & 6/20/07 0:00 & $\mathrm{PAC} \mathrm{Hg}$ & PAC Silo & sample from liner mishap & & & & & & 24100 & \\
\hline
\end{tabular}

\begin{tabular}{|c|c|c|c|c|c|c|c|c|c|c|c|}
\hline ADA ID & Date & Type & Location & Comments & $\begin{array}{l}\% \text { Ash } \\
\text { (as rec) }\end{array}$ & $\begin{array}{l}\% \mathrm{FC} \\
\text { (as rec) }\end{array}$ & $\begin{array}{l}\% \mathrm{H} 2 \mathrm{O} \\
\text { (as rec) }\end{array}$ & $\begin{array}{c}\% \mathrm{~S} \\
\text { (as rec) }\end{array}$ & $\begin{array}{l}\% \text { Vol } \\
\text { (as rec) }\end{array}$ & $\begin{array}{l}\text { BTU/lb } \\
\text { (as rec) }\end{array}$ & $\begin{array}{c}\text { MAF } \\
\text { (BTU/lb) }\end{array}$ \\
\hline 12379 & $6 / 17 / 080: 00$ & $\mathrm{Hg}-\mathrm{LH}$ & PAC Silo & Questionable Batch \#2 & 28.48 & 56.48 & 6.36 & 0.70 & 8.68 & 8539 & 13105 \\
\hline
\end{tabular}

